ORNL-4897

UC-34 - Physics

Contract No. W-7405-eng-26

SOLID STATE DIVISION

CROSS SECTIONS FOR ATOMIC DISPLACEMENTS

IN SOLIDS BY FAST ELECTRONS

0. S. Oen

\title{
NOTICE
}

This report was prepared as an account of work

sponsored by the United States Government. Neither

the United States nor the United States Atomic Energy

Commission, nor any of their employees, nor any of

their contractors, subcontractors, or their employees,

makes any warranty, express or implied, or assumes any

legal liability or responsibility for the accuracy, com-

pleteness or usefulness of any information, apparatus,

product or process disclosed, or represents that its use

would not infringe privately owned rights.

\section{AUGUST 1973}

OAK RIDGE NATIONAL LABORATORY

Oak Ridge, Tennessee 37830

operated by

UNION CARBIDE CORPORATION

for the

U.S. ATOMIC ENERGY COMMISSION 


\section{DISCLAIMER}

This report was prepared as an account of work sponsored by an agency of the United States Government. Neither the United States Government nor any agency Thereof, nor any of their employees, makes any warranty, express or implied, or assumes any legal liability or responsibility for the accuracy, completeness, or usefulness of any information, apparatus, product, or process disclosed, or represents that its use would not infringe privately owned rights. Reference herein to any specific commercial product, process, or service by trade name, trademark, manufacturer, or otherwise does not necessarily constitute or imply its endorsement, recommendation, or favoring by the United States Government or any agency thereof. The views and opinions of authors expressed herein do not necessarily state or reflect those of the United States Government or any agency thereof. 


\section{DISCLAIMER}

Portions of this document may be illegible in electronic image products. Images are produced from the best available original document. 
$\bullet$

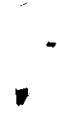

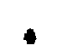

- 
ABSTRACT . . . . . . . . . . . . . . . . . . . . . 1

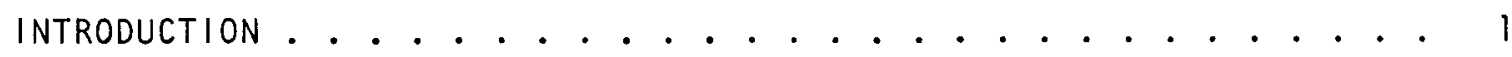

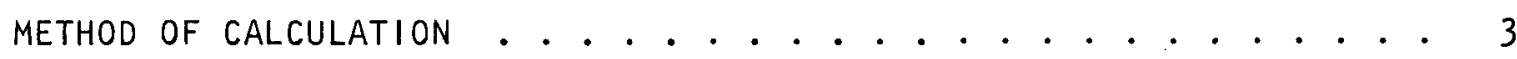

ADDITIONAL USE OF TABLES ......................... . . . 6

ACCURACY AND OTHER CALCULATIONS . . . . . . . . . . . . . . . . 6

DISCUSSION OF FIGURES AND TABLES ................... 7

FIGURES AND TABLES

Fig. 1. Comparison of the Displacement Cross Sections Computed from the Mott Series and the McKinley-Feshbach Formula for Gold vs Electron Energy . . . . . . . . . . . 10

Fig. 2. Ratio of the Primary Displacement Cross Section Computed from the Mott Series to that of the McKinley-Feshbach Formula for Uranium, Gold, Silver, and Copper vs Electron Energy

Fig. 3. The Ratio of Mott to Rutherford Scattering for Gold vs Electron Scattering Angle for Several Electron

Fig. 4. The Ratio of Mott to Rutherford Scattering vs Electron Scattering Angle for $Z=92,79,62,47$, and 29 for $2 \mathrm{MeV}$ Electrons

Fig. 5. The Ratio of Mott to Mckinley-Feshbach Scattering vs Electron Scattering Angle for Uranium, Gold, and

Fig. 6. The Maximum Energy Transferred to an Atomic Nucleus by an Electron in a Head-on Elastic Collision vs Electron Energy for Several Elements... . . . . . . . . . 15

Tables 1-37 Give Primary and Total Displacement Cross Sections Computed Using the Mott Series for Different Elements and Threshold Energies vs Electron Energy

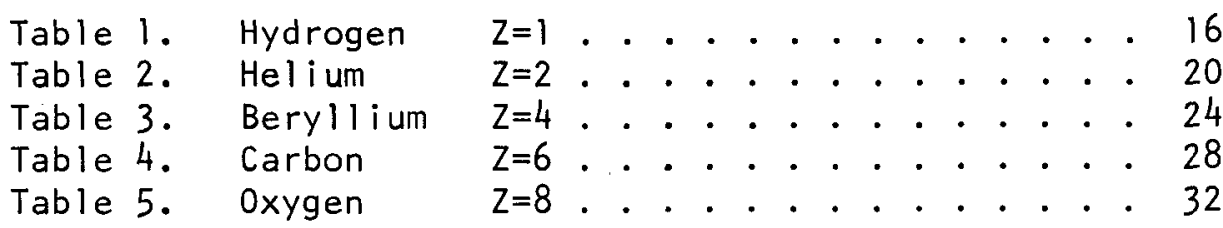


Page

Table 6. Magnesium $\mathrm{z}=12 \ldots \ldots$

Table 7. Aluminum $\mathrm{Z}=13$......... 40

Table 8. Silicon $z=14$............ 44

Table 9. Potassium $z=19$............ 48

Table 10. Titanium $\mathrm{Z}=22$.......... 52

Table 11. Vanadium $\mathrm{z}=23$............ 56

Table 12. Chromium $\mathrm{Z}=24$........... 60

Table 13. I ron $\mathrm{z}=26$.......... 64

Table 14. Nickel $\mathrm{Z}=28$.......... 68

Table 15. Copper $\mathrm{Z}=29$.......... . 72

Table 16. Zinc $\mathrm{Z}=30$.......... 76

Table 17. Germanium $Z=32$.......... 80

Table 18. Rubidium $\mathrm{Z}=37$.......... 84

Table 19. Zirconium $Z=40$.......... . 88

Table 20. Niobium $\mathrm{Z}=41$.......... 92

Table 21. Molybdenum $\mathrm{z}=42$........... 96

Table 22. Silver $\quad \mathrm{z}=47$........... 100

Table 23. Tin $\mathrm{Z}=50$.......... 104

Table 24. Cesium $\quad z=55$.......... 108

Table 25. Neodymium $\quad z=60$............ 112

Table 26. Terbium $\mathrm{z}=65$........... 116

Table 27. Ytterbium $Z=70$.......... 120

Table 28. Hafnium $\mathrm{Z}=72$......... 124

Table 29. Tantalum $\mathrm{z}=73$.......... 128

Table 30. Tungsten $\quad z=74$.......... 132

Table 31. Platinum $z=78$.......... 136

Table 32. Gold $\mathrm{Z}=79$.......... 140

Table 33. Lead $z=82$. . . . . . . . . 144

Table 34. Francium $\mathrm{z}=87$.......... 148

Table 35. Uranium $\mathrm{z}=92$.......... 152

Table 36. Plutonium $z=94$.......... 156

Table 37. Einsteinium $z=99$......... 160

Tables 38-55 Give the Ratio of Mott to Rutherford Scattering vs Electron Energies Ranging from 0.1 to $100 \mathrm{MeV}$ for the Following Elements:

Table 38. $z=1,2$............ 164

Table 39. $Z=4,6$.............. 165

Table 40. $z=8,12$............. 166

Table 41. $Z=13,14 . . . . . . . . . .167$

Table 42. $z=19,22$............. 168

Table 43. $Z=23,24 . . . . . . . . . . .169$

Table 44. $z=26,28$............. . . 170

Table 45. $z=29,30$.............. 171

Table 46. $z=32,37$............... 172

Table 47. $Z=40,41 . . . . . . . . . . .173$

Table 48. $z=42,47$.............. 174

Table 49. $z=50,55$................. 175 
Table 50. $\mathrm{Z}=60,65$............. 176

Table 51. $Z=70,72$.............. . 177

Table 52. $Z=73,74$. . . . . . . . . . 178

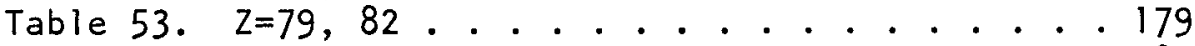

Table 54. $Z=87,92$............. 180

Table 55. $z=94,99 . . . . . . . . . . .181$

Tables 56-73 Give the Ratio of Mott to McKinley-Feshbach

Scattering vs Electron Scattering Angle for

Electron Energies from 0.1 to $100 \mathrm{MeV}$ for the

Following Elements:

Table 56. $z=1,2$.............. 182

Table 57. $\mathrm{Z}=4,6$............. . . 183

Table 58. $Z=8,12$............... 184

Table 59. $Z=13,14$.............. 185

Table 60. $z=19,22 . . . . . . . . . .186$

Table 61. $Z=23,24$............. 187

Table 62. $\mathrm{Z}=26,28$.............. 188

Table 63. $z=29,30 . . . . . . . . . .189$

Table 64. $Z=32,37$............. . 190

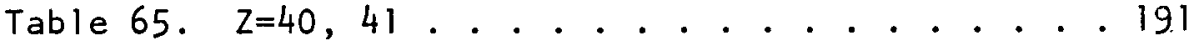

Table 66. $z=42,47 . . . . . . . . . .192$

Table 67. $Z=50,55 . . . . . . . . . . .193$

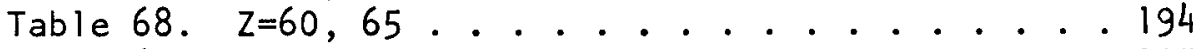

Table 69. $z=70,72 . . . . . . . . . .195$

Table 70. $Z=73,74$.............. 196

Table 71. $Z=79,82$.............. 197

Table 72. $Z=87,92$.............. 198

Table 73. $Z=94,99 . . . . . . . . . . . .199$

Table 74. The Differential Rutherford Scattering per Steradian with the $Z^{2}$ Factor Omitted vs Electron Scattering

Angle . . . . . . . . . . . . . . . 200 


\title{
CROSS SECTIONS FOR ATOMIC DISPLACEMENTS
}

IN SOLIDS BY FAST ELECTRONS

\author{
0. S. Oen
}

\begin{abstract}
The Mott series has been used to calculate the number of displacements produced in elastic collisions between fast electrons and atomic nuclei. Displacement cross sections of 37 different elements spanning the atomic table were calculated for electron energies ranging from threshold to about $150 \mathrm{MeV}$. The Kinchin and Pease model was used with atomic thresholds ranging from 4 to $96 \mathrm{eV}$ in steps of $4 \mathrm{eV}$. The results are compared with those using the less accurate Mckinley-Feshbach formula frequently used in radiation damage studies. In addition, tables giving the differential scattering cross sections in $5^{\circ}$ intervals for both the Mott series and the McKinley-Feshbach formula are included.
\end{abstract}

\section{INTRODUCTION}

The use of energetic electrons to produce radiation damage in solids is widespread. Their usefulness results from several factors: A monochromatic collimated beam can be readily obtained, they have a large penetrating ability, and it is believed that they produce a simpler type of damage than heavy particles such as neutrons or heavy ions. An earlier version of the present report has been widely used. 1 Because of a large number of requests it has seemed useful to produce an expanded version. This new version includes more values of the atomic displacement threshold, more electron energies, and a greater variety of elements. In particular a large number of threshold energies has been used since it has been

\footnotetext{
10. S. Oen, "Cross Sections for Atomic Displacements in Solids by Fast Electrons," ORNL-3813 (1965).
} 
recently shown ${ }^{2}$ that the displacement cross-section for a linear displacement probability can be obtained from the conventional sharp displacement cross-sections. The recent widespread use of the high energy electron microscope ${ }^{3}$ to introduce damage has prompted some aspects of the calculations. Some of the elements chosen in this work were selected because of their great potential in technological applications. The very high electron energy of the order $150 \mathrm{MeV}$ was selected since it represents the energy available in the Oak Ridge 1 inear electron accelerator.

In order to calculate the probability of displacing an atom in a solid from its normal lattice position by a fast electron it is necessary to know the scattering cross section between an electron and an atom. The scattering of a nonrelativistic electron by the Coulomb field of a point nucleus was first treated by Rutherford, and the relativistic extension was done by Mott ${ }^{4}$ using the Dirac theory of the electron. Since the electrons used in radiation damage are in the relativistic velocity range, it is necessary to use Mott's theory. Mott has expressed the scattering between a point nucleus and an electron as an infinite series of Legendre expansions. Since that series solution is cumbersome to evaluate, several authors have approximated it to get simpler, more tractable expressions. The so-called McKinley-Feshbach version of the Mott equation has been most frequently used to calculate atomic displacement cross sections for

20. S. Oen, in Radiation Effects in Semiconductors, edited by F. L. Vook (Plenum, New York, 1968), p. 264.

3.M. J. Makin, Phil. Mag. 18, 637 (1968).

${ }^{4}$ N. F. Mott, Proc. Roy. Soc. (London) A124, 426 (1929); A135, 429 (1932); N. F. Mott and H. S. W. Massey, The Theory of Atomic Colizisions (Oxford University Press, New York, 1965), 3rd ed., chap. IX. 
radiation damage research. Seitz and Koehler ${ }^{5}$ pointed out that the Mckinley-Feshbach formula should be sufficiently accurate for light elements $(z \leqslant 29)$, but that for heavy elements it becomes necessary to use the more accurate Mott series formulation.

\section{METHOD OF CALCULATION}

The method used to calculate the atomic displacement cross sections by fast electrons follows closely that of Seitz and Koehler. ${ }^{5}$ The calculational procedures are outlined in the paragraphs below.

The energy transferred to a heavy nucleus by an electron scattered through an angle $\theta$ is given by

$$
T=T_{m} \sin ^{2} \theta / 2
$$

where $T_{m}$, the maximum transferred energy which occurs for a head-on collision, is

$$
T_{m}=2 \frac{E}{M c^{2}}\left(E+2 m c^{2}\right)
$$

Here $E$ is the kinetic energy of the electron and $M$ is the mass of the target atom. The rest energy of the electron is $\mathrm{mc}^{2}=0.511 \mathrm{MeV}$.

The conventional sharp displacement threshold model has been used. It assumes that an atom can be displaced only if it receives an energy equal or greater than some threshold energy, $T_{d}$. Upon receiving such an

${ }^{5}$ F. Seitz and J. S. Koehler, P. 305 in Solid State Physics, vol. 2, ed. by F. Seitz and D. Turnbull (Academic Press, New York, 1956). See also J. W. Corbett, Electron Radiation Damage in Semiconductors and Metals (Academic Press, New York, 1966). 
energy, the probability that the atom is displaced is taken as unity. Furthermore, the primary displaced atom can, if sufficiently energetic, produce additional displaced atoms. The cascade model used is that of Kinchin and Pease ${ }^{6}$ which gives the average number of displacements, $v$, produced by a primary knock-on of energy $T$ as

$$
\begin{array}{rlrl}
\nu(T) & =1 & T_{d} \leq T \leq 2 T_{d} \\
& =T / 2 T_{d} & T>2 T_{d} .
\end{array}
$$

The total cross section (primary plus secondaries) for producing atomic displacements by an electron of energy $E$ can be written as

$$
\sigma_{\text {tot }}\left(E, T_{d}\right)=\int_{T_{d}}^{T_{m}} v(T) \frac{d \sigma}{d T} d T
$$

while the cross section for producing primary displacements only is

$$
\sigma_{p}\left(E, T_{d}\right)=\int_{T_{d}}^{T} \frac{d \sigma}{d T} d T
$$

In Eqs. (4) and (5) the quantity do/dT is the differential scattering cross section for transferring an energy $T$ to an atom by an electron of energy $E$. The above integrals can be done analytically for the Mckinley-Feshbach version of $\mathrm{d} \sigma / \mathrm{dT}$, but numerical methods become necessary when using the Mott series. Rewriting Eq. (4) more explicitly using the results of Eq. (3) gives

${ }^{6}$ G. H. Kinchin and R. S. Pease, Repts. Progr. Phys. 18, 1 (1955). 
$\begin{aligned} \sigma_{\text {tot }}\left(E, T_{d}\right) & =\frac{\pi Z^{2} e^{4}\left(1-\beta^{2}\right)}{m^{2} c^{4} \beta^{4}}\left\{\int_{T_{d} / T_{m}}^{l} \frac{d x}{x^{2}} M(x, E)\right\} T_{d} \leq T_{m} \leq 2 T_{d} \\ & =\frac{\pi Z^{2} e^{4}\left(1-\beta^{2}\right)}{m^{2} c^{4} \beta^{4}}\left\{\int_{T_{d} / T_{m}}^{2 T_{d} / T_{m}} \frac{M(x, E)}{x^{2}} d x+\int_{2 T_{d} / T_{m}}^{l} \frac{T_{m}}{2 T_{d}} \frac{M(x, E)}{x} d x\right\} T_{m}>2 T_{d}\end{aligned}$

where $\beta^{2}=E\left(E+2 m c^{2}\right) /\left(E+m c^{2}\right)^{2} . \quad Z$ is the atomic number of the target nucleus, $e$ is the electronic charge and $M(X, E)$ is the ratio of the Mott to the Rutherford cross section. The ratio $M(X, E)$ was calculated using the method of Doggett and Spencer ${ }^{7}$ and the integrals evaluated by 16 point Gauss-Legendre quadrature techniques. The integrand in the second integral of Eq. (4b) was transformed by the substitution $x=e^{-y}$ before evaluating the integral. The transformed integrand which varied less over the domain of integration proved easier to evaluate. The primary displacement cross section given by Eq. (5), when written out more explicitly, becomes identical to Eq. (4a) except that now there is no restriction on the magnitude of $T_{m}$ provided $i t$ is larger than $T_{d}$. The substitution, $x=1 / y$, facilitated the evaluation of the integral for the primary cross sections.

The differential Rutherford scattering cross section per steradian is

$$
\frac{d \sigma_{R}(\theta)}{d \Omega}=\frac{z^{2} e^{4}\left(1-\beta^{2}\right)}{m^{2} c^{4} \beta^{4} \sin ^{4} \frac{\theta}{2}} .
$$

The differential Mckinley-Feshbach scattering cross section per steradian is

7J. A. Doggett and L. V. Spencer, Phys. Rev. 103, 1597 (1956). 
$\frac{d \sigma_{M C K-F}(\theta)}{d \Omega}=\frac{d \sigma_{R}(\theta)}{d \Omega}\left[1-\beta^{2} \sin ^{2} \frac{\theta}{2}+\frac{\pi Z}{137} \beta \sin \frac{\theta}{2}\left\{1-\sin \frac{\theta}{2}\right\}\right]$.

\section{ADDITIONAL USE OF TABLES}

Tables of primary and total displacement cross sections constructed using Eqs. (4) and (5), respectively, are made more flexible by noting the following relationship. ${ }^{2}$ If $\sigma_{\text {tot }}\left(E, T_{d}\right)$ is the total displacement cross section, it is easy to show that the quantity

$$
c_{1}\left[\sigma_{\text {tot }}\left(E, \frac{T_{d}}{2}\right)-\sigma_{d}\left(E, \frac{T_{d}}{2}\right)\right]
$$

gives the displacement cross section for a linear displacement probability function of the form

$$
\begin{array}{rlrl}
P(T) & =0 & \text { for } & T \leq T_{d} \\
& =C_{1}\left(\frac{T-T_{d}}{T_{d}}\right) \text { for } T>T_{d}
\end{array}
$$

Here, $c_{1}$ is an adjustable slope parameter.

\section{ACCURACY AND OTHER CALCULATIONS}

All computations were performed on an IBM System/360 model 91 computer using long floating-point numbers (REAL $* 8$, about 16 decimal digits).

The Mott series was evaluated using the computer code of Doggett and spencer ${ }^{7}$ taking 36 terms in the Legendre sums. For those cases of overlap the present results, as expected, are in excellent agreement with theirs and therefore their comments on the accuracy of the method apply here also. They have made several comparisons with other calcula- 
calculations were made assuming a point nucleus. At ultra high electron energies and large scattering angles this assumption becomes less valid because of nuclear size effects. 4

For convenience and accuracy in reproducing, the tables have been printed as output by the computer and therefore some of the values contain more than the three or four figures which are significant.

All of the integrations were performed using 16 point Gauss-Legendre quadrature techniques. The integration routine was checked by numerically integrating the McKinley-Feshbach version of the Mott series and comparing that with the analytical result. Excellent agreement was found.

The calculated displacement cross sections for gold agree well with those of Khandelwal and Merzbacher. ${ }^{8}$ The results appear also to agree well with those of Burke, Grossbard and Lowe. 9 For the light elements they agree well with the McKinley-Feshbach formula. It is believed that the overall accuracy of the calculations should be good to within a few percent.

\section{DISCUSSION OF FIGURES AND TABLES}

A. Integral Displacement Cross Sections

a) Figure 1 shows a comparison of the Mott and McKinley-Feshbach displacement cross sections for gold. The primary displacement cross section using Mott and McKinley-Feshbach scattering is shown as a ratio in Fig. 2 .

${ }^{8}$ G. S. Khandelwal and E. Merzbacher, Phys. Rev. 130, 1822 (1963).

$9^{9}$. A. Burke, N. J. Grossbard, and L. F. Lowe, "Calculated Cross Section for Atomic Displacements Produced by Electrons in the 1.0-3.0 MeV Energy Range," Air Force Cambridge Research Laboratories, AFCRL-65-286 (1965). 
b) The first 37 tables give the primary and total displacement cross sections as a function of electron energy using several displacement threshold energies for 37 different elements spanning the atomic table. The Mott series is used for the electron-nuclear differential scattering and it was computed by the procedure al ready described.

\section{B. Differential Scattering Cross Sections}

For some purposes it is desirable to calculate displacement cross sections using a more refined, but specialized displacement model than that of Kinchin and Pease. This occurs, for instance, when one is studying atomic displacements as a function of crystal orientation. 10 For purposes such as these it would seem convenient to have available tables giving the Mott differential scattering.

a) Figures 3 and 4 give the ratio of Mott to Rutherford scattering for different elements.

b) Tables $38-55$ give the ratio of Mott to Rutherford scattering as a function of electron scattering angle.

c) Figure 5 gives the ratio of Mott to Mckinley-Feshbach scattering.

d) Tables 56-73 give the ratio of Mott to McKinley-Feshbach scattering as a function of electron scattering angle.

e) Table 74 gives the differential Rutherford scattering cross section in barns per steradian with the $z^{2}$ factor omitted.

${ }^{10}$. Jung and W. F. Schilling, Phys. Rev. B $\underline{5}, 2046$ (1972). 
9

C. Auxiliary Results

Figure 6 gives the maximum energy in electron volts transferred to an atom by an electron in a head-on collision (Eq. 2) as a function of electron energy for several different elements.

The author is indebted to J. J. Cone of the National Bureau of Standards for making available the computer code of J. A. Doggett and L. V. Spencer. He also wishes to thank J. H. Barrett, D. K. Holmes, M. S. Wechsler and especially M. T. Robinson for many stimulating discussions. 


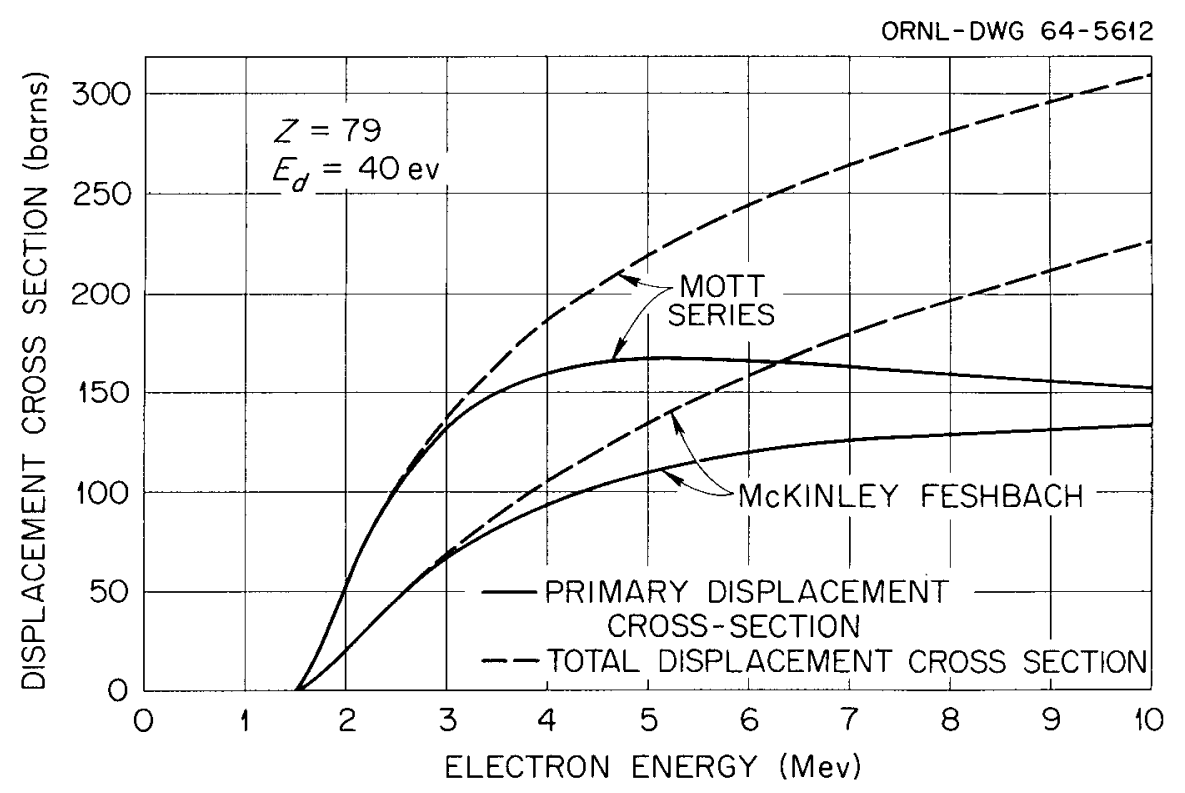

Fig. 1. Comparison of the Displacement Cross Sections Computer from the Mott Series and the McKinley-Feshbach Formula for Gold vs Electron Energy. 
11

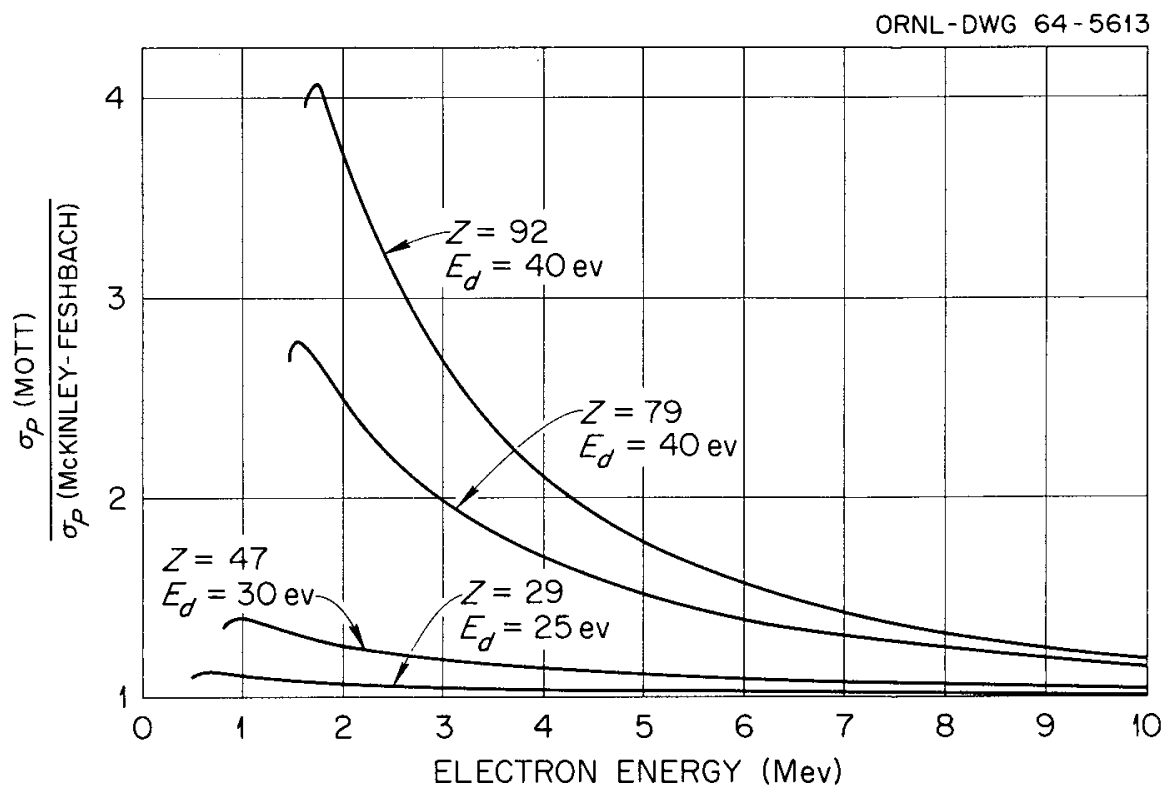

Fig. 2. Ratio of the Primary Displacement Cross Section Computed from the Mott Series to that of the McKinley-Feshbach Formula for Uranium, Gold, Silver, and Copper vs Electron Energy. 


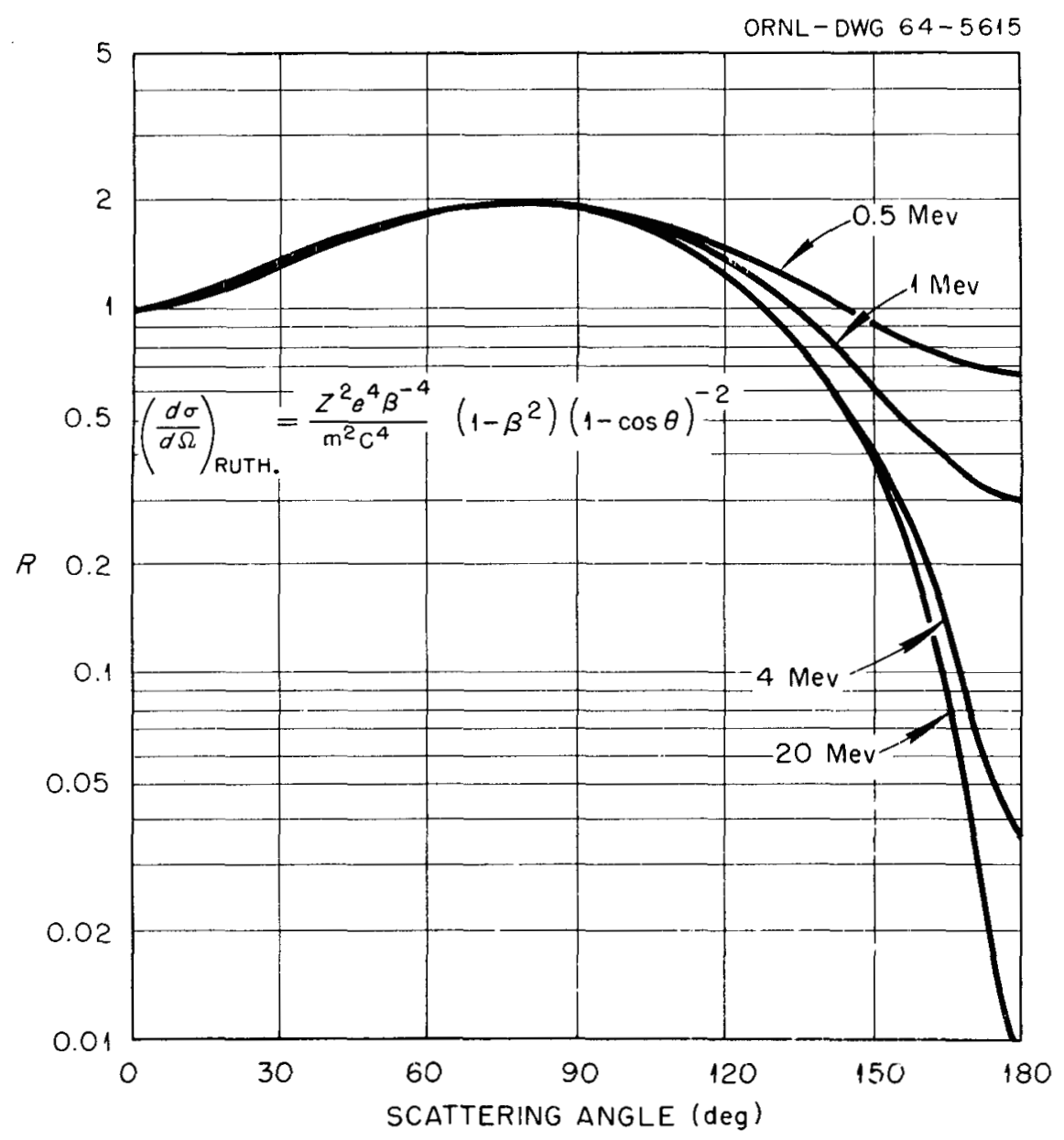

Fig. 3. The Ratio of Mott to Rutherford Scattering for Gold vs Electron Scattering Angle for Several Electron Energies. 


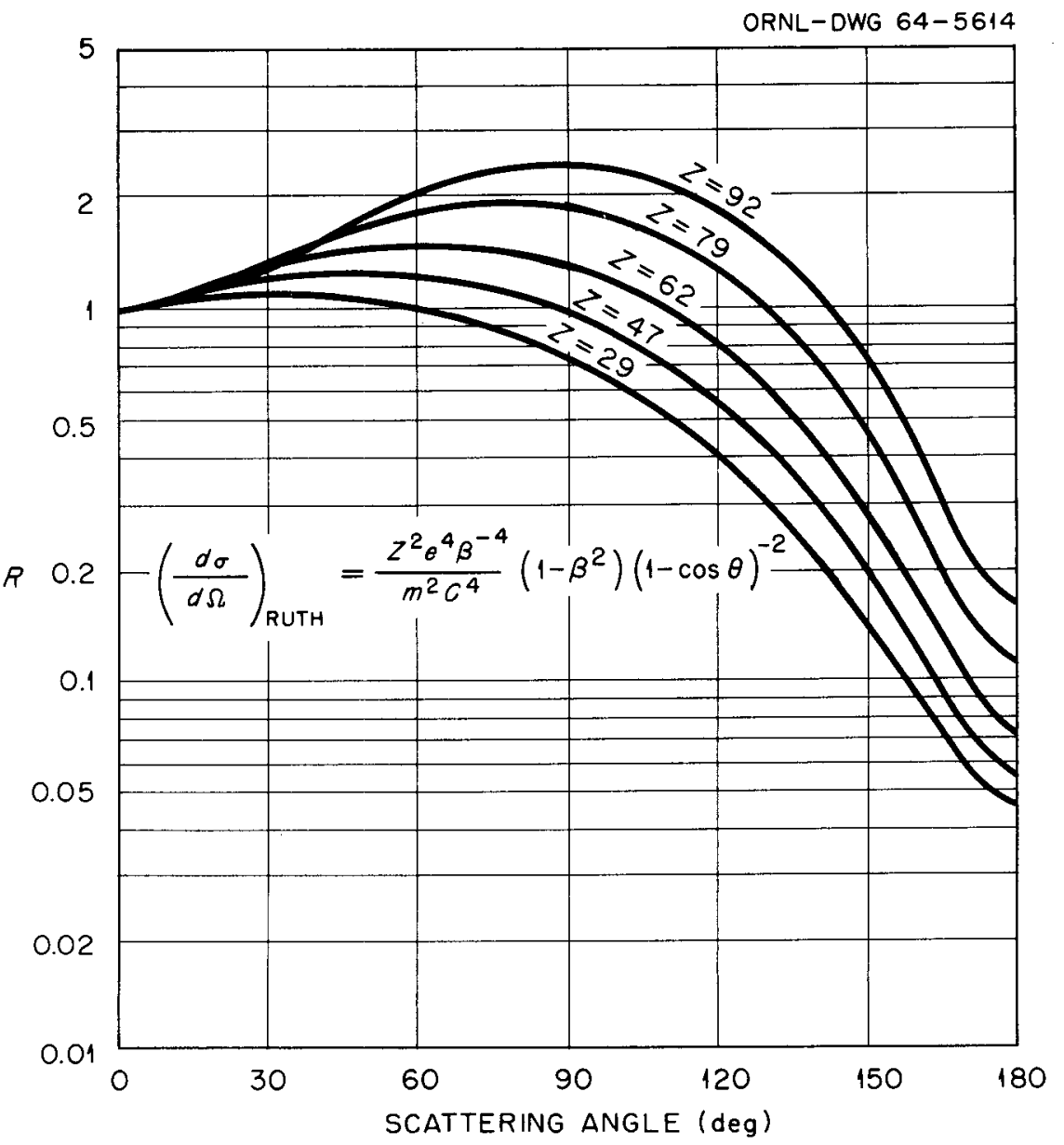

Fig. 4. The Ratio of Mott to Rutherford Scattering vs Electron Scattering Angle for $Z=92,79,62,47$, and 29 for $2 \mathrm{MeV}$ Electrons. 


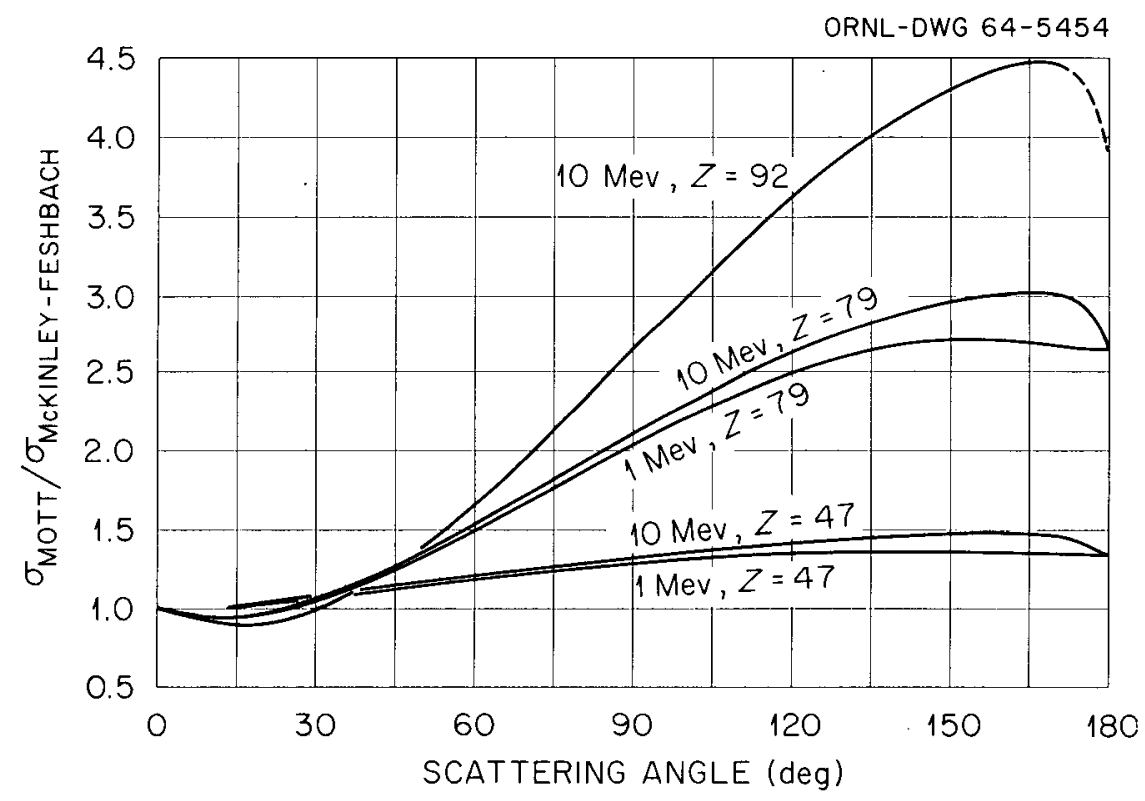

Fig. 5. The Ratio of Mott to McKinley-Feshbach Scattering vs Electron Scattering Angle for Uranium, Gold, and Silver. 
.

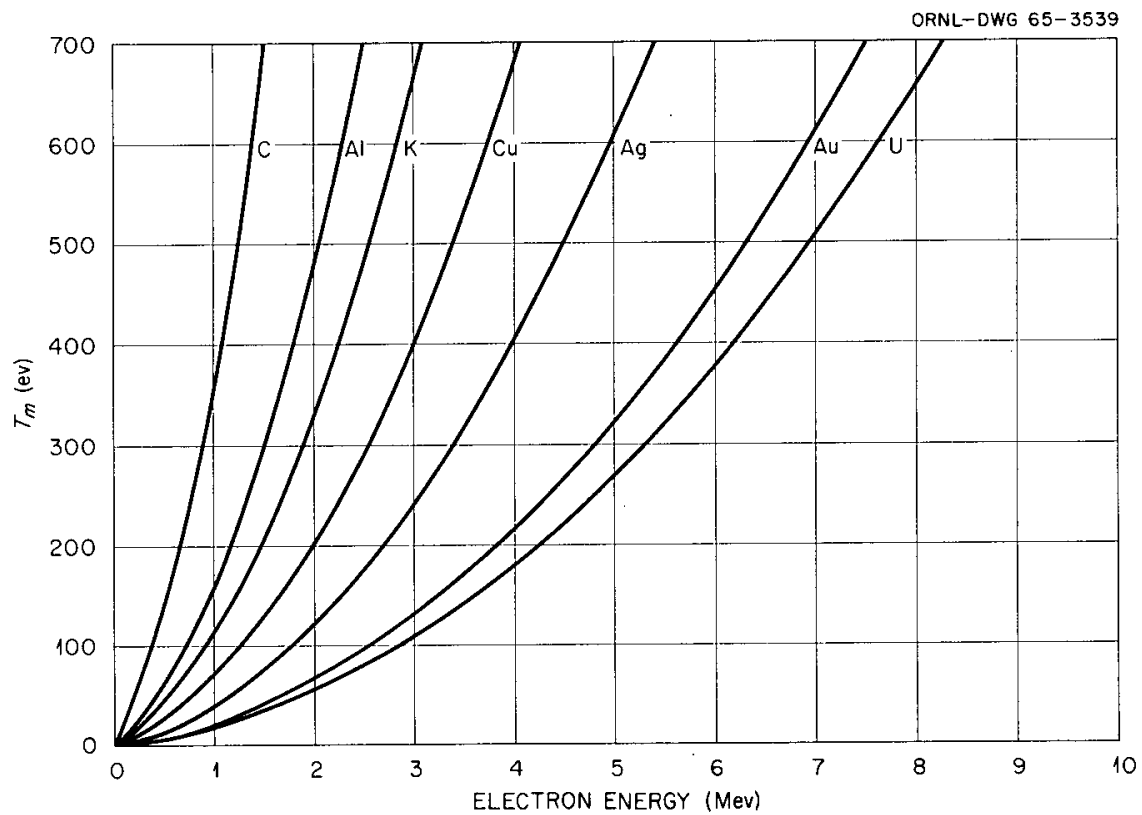

Fig. 6. The Maximum Energy Transferred to an Atomic Nucleus by an Electron in a Head-on Elastic Collision vs Electron Energy for Several Elements. 
ATOMIC DISPLAEEnENT CROSS-SECTIONS (BARNS) BY FAST ELECTRONS IN H

\begin{tabular}{rrr} 
TDE 4.0 & \multicolumn{1}{c}{ EY $=0.001834$} \\
ENERGY & MEV \\
0.00185 & PRIMARY & CASCADE \\
0.00187 & 42.27 & 42.27 \\
0.00190 & 92.09 & 92.09 \\
0.00196 & 162.90 & 162.90 \\
0.00203 & 291.61 & 291.61 \\
0.00212 & 522.53 & 422.53 \\
0.00223 & 707.10 & 565.10 \\
0.00238 & 855.87 & 707.05 \\
0.00256 & 983.75 & 855.87 \\
0.00284 & 1108.00 & 1108.75 \\
0.00320 & 1185.67 & 1185.67 \\
0.00366 & 1212.54 & 1212.54 \\
0.00421 & 1193.68 & 1203.55 \\
0.00495 & 1134.07 & 1171.22 \\
0.00586 & 1047.55 & 1120.02 \\
0.00696 & 947.82 & 1055.99 \\
0.00825 & 846.89 & 985.96 \\
0.00953 & 763.63 & 924.10 \\
0.01100 & 685.12 & 862.12 \\
0.01280 & 608.07 & 797.55 \\
0.01550 & 520.34 & 718.94 \\
0.01830 & 453.04 & 654.43 \\
0.02750 & 320.35 & 514.04 \\
0.03660 & 250.92 & 431.64 \\
0.05500 & 178.28 & 336.37 \\
0.09160 & 118.96 & 249.17 \\
0.18300 & 73.90 & 175.50 \\
0.36500 & 51.73 & 138.32 \\
0.55000 & 44.71 & 128.57 \\
0.91600 & 39.58 & 125.55 \\
1.28000 & 37.65 & 127.85 \\
1.83000 & 36.37 & 133.12 \\
3.66000 & 35.22 & 149.09 \\
7.33000 & 34.86 & 169.49 \\
12.80000 & 34.76 & 187.42 \\
18.30000 & 34.74 & 199.29
\end{tabular}

\begin{tabular}{crr} 
TD $=8.0$ & EV & \multicolumn{1}{c}{ ET $=0.003661$ MEV } \\
ENERGY (IRV) & PRTMRT & CASCADE \\
0.00369 & 9.50 & 9.50 \\
0.00373 & 22.04 & 22.04 \\
0.00380 & 42.73 & 42.73 \\
0.00391 & 72.28 & 72.28 \\
0.00406 & 107.42 & 107.42 \\
0.00424 & 142.94 & 142.94 \\
0.00446 & 178.31 & 178.31 \\
0.00475 & 214.33 & 214.33 \\
0.00512 & 247.28 & 247.28 \\
0.00567 & 277.88 & 277.88 \\
0.00640 & 297.72 & 297.72 \\
0.00732 & 304.53 & 304.53 \\
0.00841 & 300.06 & 302.62 \\
0.00988 & 285.56 & 295.00 \\
0.01170 & 264.25 & 282.64 \\
0.01390 & 239.63 & 267.08 \\
0.01640 & 215.41 & 250.53 \\
0.01900 & 194.45 & 235.18 \\
0.02190 & 175.26 & 220.26 \\
0.02560 & 155.70 & 204.10 \\
0.03110 & 133.78 & 184.74 \\
0.03660 & 117.58 & 169.47 \\
0.05490 & 85.17 & 135.92 \\
0.07320 & 68.08 & 116.25 \\
0.10900 & 50.74 & 94.50 \\
0.18300 & 36.14 & 74.64 \\
0.36600 & 25.51 & 60.07 \\
0.73200 & 20.57 & 55.56 \\
1.09000 & 19.14 & 56.50 \\
1.83000 & 18.14 & 60.25 \\
2.56000 & 17.81 & 63.86 \\
3.66000 & 17.60 & 68.44 \\
7.32000 & 17.42 & 78.58 \\
14.60000 & 17.38 & 89.86 \\
25.60000 & 17.36 & 99.29 \\
36.60000 & 17.36 & 105.37
\end{tabular}

\begin{tabular}{rrr} 
TDE12.0 & \multicolumn{1}{c}{ ET $=0.005482$ MEV } \\
ENERGY (HEV) & PRIMARY & CASCADE \\
0.00553 & 4.69 & 4.69 \\
0.00559 & 10.26 & 10.26 \\
0.00570 & 19.88 & 19.88 \\
0.00586 & 32.59 & 32.59 \\
0.00608 & 47.89 & 47.89 \\
0.00635 & 63.73 & 63.73 \\
0.00668 & 79.51 & 79.51 \\
0.00712 & 95.73 & 95.73 \\
0.00767 & 110.28 & 110.28 \\
0.00849 & 123.89 & 123.89 \\
0.00959 & 132.85 & 132.85 \\
0.01090 & 135.95 & 135.95 \\
0.01260 & 134.09 & 135.27 \\
0.01480 & 127.79 & 132.07 \\
0.01750 & 118.57 & 126.83 \\
0.02080 & 107.76 & 120.12 \\
0.02460 & 97.00 & 112.87 \\
0.02850 & 87.82 & 106.25 \\
0.03280 & 79.51 & 99.88 \\
0.03930 & 71.00 & 92.97 \\
0.04650 & 61.44 & 84.67 \\
0.05480 & 54.30 & 78.07 \\
0.08220 & 40.18 & 63.81 \\
0.10900 & 32.88 & 55.73 \\
0.16400 & 25.20 & 46.62 \\
0.27400 & 19.05 & 39.09 \\
0.54800 & 14.64 & 34.60 \\
1.09000 & 12.71 & 35.05 \\
1.64000 & 12.17 & 37.01 \\
2.74000 & 11.82 & 40.72 \\
3.83000 & 11.71 & 43.66 \\
5.48000 & 11.64 & 47.13 \\
10.90000 & 11.59 & 54.35 \\
21.90000 & 11.58 & 62.08 \\
38.30000 & 11.57 & 68.42 \\
54.80000 & 11.57 & 72.51 \\
& &
\end{tabular}

ATOMIC DISPLAzEnENT CROSS-SECTIONS (BARNS) BY PAST ELECTRONS IN H

\begin{tabular}{rrr} 
TD=16.0 & EV & \multicolumn{1}{c}{ ET=0.007296 MEV } \\
ENERGY(KEV) & PRIHARY & CASCADE \\
0.00736 & 2.63 & 2.63 \\
0.00744 & 5.77 & 5.77 \\
0.00758 & 10.97 & 10.97 \\
0.00780 & 18.38 & 18.38 \\
0.00809 & 26.92 & 26.92 \\
0.00846 & 36.11 & 36.11 \\
0.00890 & 44.98 & 44.98 \\
0.00948 & 54.03 & 54.03 \\
0.01020 & 62.12 & 62.12 \\
0.01130 & 69.91 & 69.91 \\
0.01270 & 74.87 & 74.87 \\
0.01450 & 76.81 & 76.81 \\
0.01670 & 75.91 & 76.56 \\
0.01950 & 72.53 & 74.91 \\
0.02330 & 67.28 & 72.00 \\
0.02770 & 61.29 & 68.35 \\
0.03280 & 55.28 & 64.37 \\
0.03790 & 50.29 & 60.82 \\
0.04370 & 45.62 & 57.30 \\
0.05100 & 40.93 & 53.55 \\
0.06200 & 35.61 & 49.02 \\
0.07290 & 31.73 & 45.51 \\
0.10900 & 24.00 & 37.94 \\
0.14500 & 19.95 & 33.64 \\
0.21800 & 15.77 & 29.02 \\
0.36400 & 12.46 & 25.52 \\
0.72900 & 10.15 & 24.21 \\
1.45000 & 9.20 & 25.90 \\
2.18000 & 8.94 & 27.90 \\
3.64000 & 8.79 & 31.13 \\
5.10000 & 8.74 & 33.55 \\
7.29000 & 8.71 & 36.29 \\
14.50000 & 8.69 & 41.86 \\
29.10000 & 8.68 & 47.72 \\
51.00000 & 8.68 & 52.52 \\
72.90000 & 8.68 & 55.59 \\
& &
\end{tabular}

\begin{tabular}{crr} 
TD 20.0 & EV & \multicolumn{2}{c}{ ET=0.009104 HEV } \\
ENERGY(HEV) & PRIMART & CASCADE \\
0.00919 & 1.81 & 1.81 \\
0.00928 & 3.63 & 3.63 \\
0.00946 & 7.06 & 7.06 \\
0.00974 & 11.90 & 11.90 \\
0.01010 & 17.34 & 17.34 \\
0.01050 & 22.50 & 22.50 \\
0.01110 & 28.80 & 28.80 \\
0.01180 & 34.46 & 34.46 \\
0.01270 & 39.73 & 39.73 \\
0.01410 & 44.88 & 44.88 \\
0.01590 & 48.15 & 48.15 \\
0.01820 & 49.39 & 49.40 \\
0.02090 & 48.83 & 49.27 \\
0.02450 & 46.71 & 48.28 \\
0.02910 & 43.43 & 46.50 \\
0.03450 & 39.72 & 44.28 \\
0.04090 & 35.90 & 41.80 \\
0.04730 & 32.74 & 39.59 \\
0.05460 & 29.78 & 37.39 \\
0.06370 & 26.83 & 35.09 \\
0.07730 & 23.52 & 32.32 \\
0.09100 & 21.06 & 30.16 \\
0.13600 & 16.23 & 25.58 \\
0.18200 & 13.67 & 23.00 \\
0.27300 & 11.12 & 20.41 \\
0.45500 & 9.12 & 18.70 \\
0.91000 & 7.76 & 18.70 \\
1.82000 & 7.21 & 20.74 \\
2.73000 & 7.08 & 22.59 \\
4.55000 & 7.00 & 25.39 \\
6.37000 & 6.97 & 27.41 \\
9.10000 & 6.96 & 29.66 \\
18.20000 & 6.95 & 34.23 \\
36.40000 & 6.94 & 38.93 \\
63.70000 & 6.94 & 42.77 \\
91.00000 & 6.94 & 45.23 \\
& &
\end{tabular}

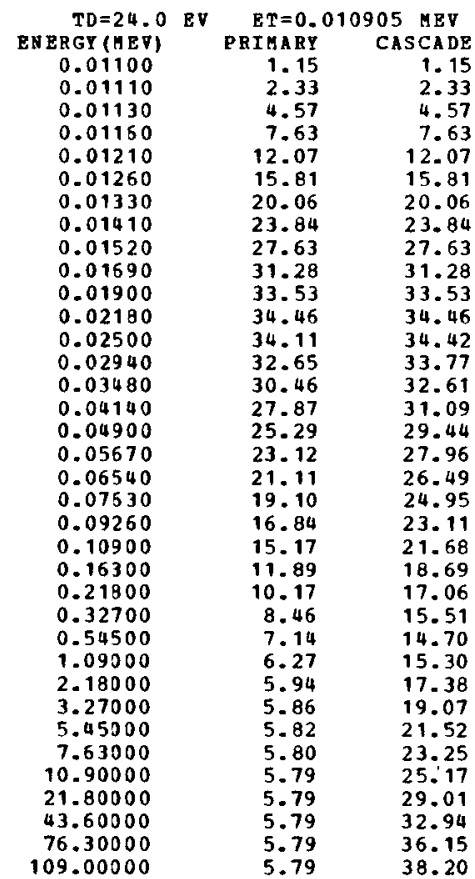


ATOHIC DISPLACEHENT CROSS-SECTIONS (BARN) BY PAST ELECTRONS IN H $=1 \mathrm{~A}=1.008$

\begin{tabular}{ccc} 
TD $=28.0$ & EV & \multicolumn{1}{c}{ ET $=0.012701$ MEV } \\
ENERGY(MEV) & PRIMAR & CASCADE \\
0.01280 & 0.77 & 0.77 \\
0.01290 & 1.52 & 1.52 \\
0.01320 & 3.63 & 3.63 \\
0.01350 & 5.56 & 5.56 \\
0.01400 & 8.41 & 8.41 \\
0.01470 & 11.75 & 11.75 \\
0.01540 & 14.46 & 14.46 \\
0.01650 & 17.76 & 17.76 \\
0.01770 & 20.35 & 20.35 \\
0.01960 & 22.97 & 22.97 \\
0.02220 & 24.76 & 24.76 \\
0.02540 & 25.43 & 25.43 \\
0.02920 & 25.18 & 25.43 \\
0.03420 & 24.17 & 25.00 \\
0.04060 & 22.56 & 24.17 \\
0.04820 & 20.71 & 23.11 \\
0.05710 & 18.84 & 21.94 \\
0.06500 & 17.28 & 20.89 \\
0.07620 & 15.82 & 19.85 \\
0.08890 & 14.37 & 18.77 \\
0.10700 & 12.81 & 17.53 \\
0.12700 & 11.55 & 16.48 \\
0.19000 & 9.20 & 14.43 \\
0.25400 & 7.97 & 13.36 \\
0.38100 & 6.77 & 12.42 \\
0.63500 & 5.86 & 12.10 \\
1.27000 & 5.27 & 13.00 \\
2.54000 & 5.06 & 15.03 \\
3.81000 & 5.01 & 16.56 \\
6.35000 & 4.98 & 18.73 \\
8.89000 & 4.97 & 20.24 \\
12.70000 & 4.96 & 21.90 \\
25.40000 & 4.96 & 25.22 \\
50.80000 & 4.96 & 28.60 \\
88.90000 & 4.96 & 31.36 \\
127.00000 & 4.96 & 33.12
\end{tabular}

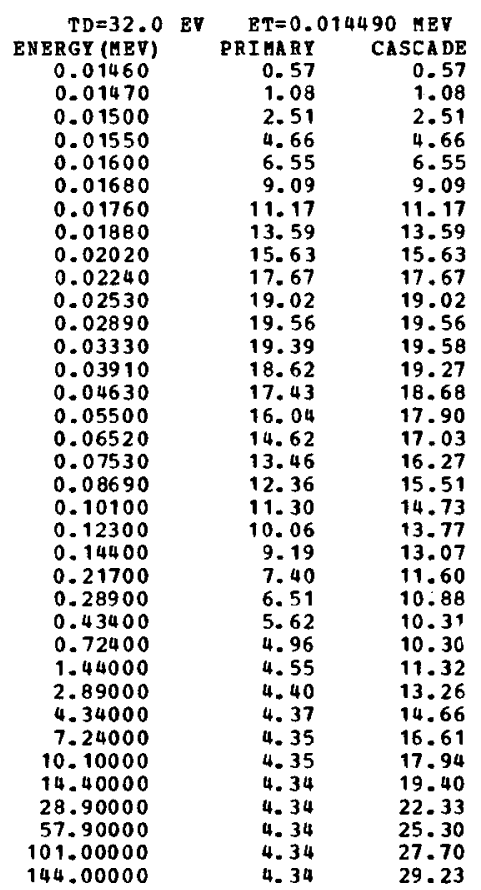

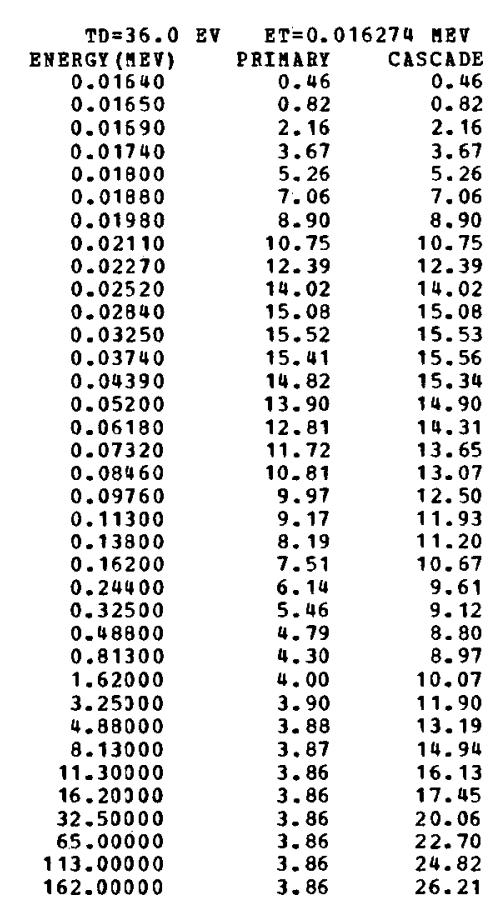

ATOMIC DISPLAZEMENT CROSS-SECTIONS (BARNS) BY FAST ELECTRONS IN H

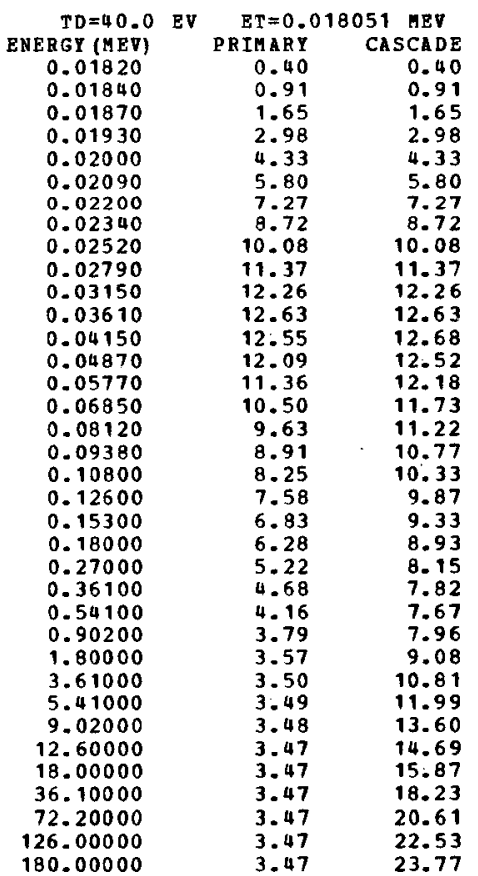

\begin{tabular}{crr} 
TD=44.0 EV & \multicolumn{2}{c}{ ET $=0.019822$ MEV } \\
ENERT (MEV) & PRIMARY & CASCADE \\
0.02000 & 0.36 & 0.36 \\
0.02020 & 0.75 & 0.75 \\
0.02060 & 1.48 & 1.49 \\
0.02120 & 2.47 & 2.47 \\
0.02200 & 3.64 & 3.64 \\
0.02290 & 4.75 & 4.75 \\
0.02410 & 5.97 & 5.97 \\
0.02570 & 7.23 & 7.23 \\
0.02770 & 8.36 & 8.36 \\
0.03070 & 9.45 & 9.45 \\
0.03460 & 10.17 & 10.17 \\
0.03960 & 10.49 & 10.49 \\
0.04550 & 10.43 & 10.54 \\
0.05350 & 10.06 & 10.42 \\
0.06340 & 9.47 & 10.16 \\
0.07530 & 8.77 & 9.80 \\
0.08920 & 8.07 & 9.40 \\
0.10300 & 7.49 & 9.05 \\
0.11800 & 6.97 & 8.72 \\
0.13800 & 6.42 & 8.35 \\
0.16800 & 5.81 & 7.93 \\
0.19800 & 5.36 & 7.62 \\
0.29700 & 4.51 & 7.05 \\
0.39600 & 4.09 & 6.83 \\
0.59400 & 3.68 & 6.79 \\
0.99100 & 3.39 & 7.17 \\
1.98000 & 3.23 & 8.29 \\
3.96000 & 3.18 & 9.92 \\
5.94000 & 3.17 & 11.01 \\
9.91000 & 3.16 & 12.49 \\
13.80000 & 3.16 & 13.48 \\
19.80000 & 3.16 & 14.57 \\
39.60000 & 3.16 & 16.71 \\
79.20000 & 3.16 & 18.88 \\
138.00000 & 3.16 & 20.62 \\
198.00000 & 3.16 & 21.76
\end{tabular}

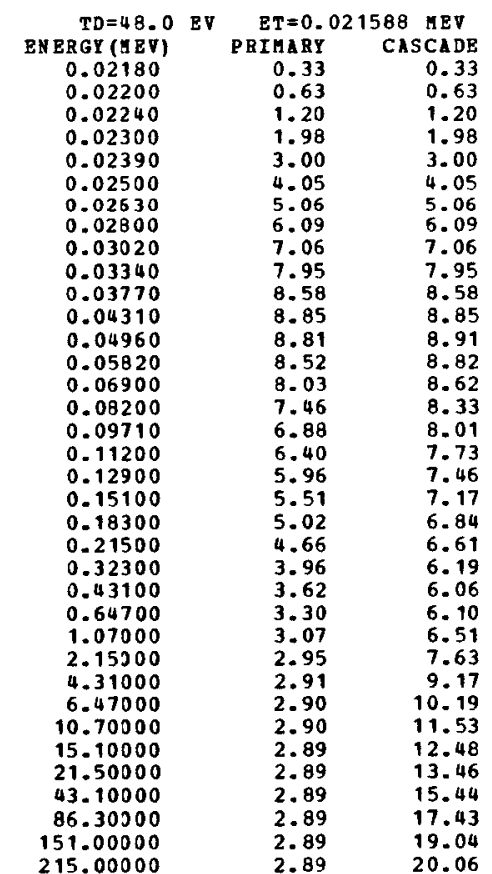


ATONIC DISPLACEHEHT CROSS-SECTIONS (BARHS) BY FAST ELECTRONS IN H

\begin{tabular}{crr} 
TD=52.0 & EV & \multicolumn{1}{c}{ ET $=0.023347$ MEV } \\
ENERGY (HEV) & PRIMARY & CASCADE \\
0.02350 & 0.19 & 0.19 \\
0.02380 & 0.55 & 0.55 \\
0.02420 & 0.99 & 0.99 \\
0.02490 & 1.71 & 1.71 \\
0.02590 & 2.60 & 2.60 \\
0.02700 & 3.43 & 3.43 \\
0.02840 & 4.30 & 4.30 \\
0.03030 & 5.21 & 5.21 \\
0.03260 & 6.01 & 6.01 \\
0.03610 & 6.79 & 5.79 \\
0.04080 & 7.34 & 7.34 \\
0.04660 & 7.58 & 7.58 \\
0.05360 & 7.55 & 7.63 \\
0.06300 & 7.31 & 7.57 \\
0.07470 & 6.99 & 7.41 \\
0.08870 & 6.43 & 7.18 \\
0.10500 & 5.94 & 6.93 \\
0.12100 & 5.55 & 6.70 \\
0.14000 & 5.17 & 6.47 \\
0.16300 & 4.81 & 6.25 \\
0.19800 & 4.39 & 5.99 \\
0.23300 & 4.10 & 5.81 \\
0.35000 & 3.52 & 5.50 \\
0.96600 & 3.24 & 5.43 \\
0.70000 & 2.98 & 5.54 \\
1.16000 & 2.81 & 5.99 \\
2.33000 & 2.71 & 7.08 \\
4.56000 & 2.68 & 8.53 \\
7.00000 & 2.68 & 9.49 \\
11.60000 & 2.67 & 10.74 \\
16.30000 & 2.67 & 11.61 \\
23.30000 & 2.67 & 12.53 \\
46.60000 & 2.67 & 14.35 \\
93.30000 & 2.67 & 16.19 \\
163.00000 & 2.67 & 17.67
\end{tabular}

\begin{tabular}{ccc} 
TD=56.0 EV & \multicolumn{2}{c}{ ET $=0.0251$ 1 MEY } \\
ENERGY (HEV) & PRIMARY & CASCADE \\
0.02530 & 0.20 & 0.20 \\
0.02560 & 0.48 & 0.48 \\
0.02610 & 0.93 & 0.93 \\
0.02680 & 1.50 & 1.50 \\
0.02780 & 2.22 & 2.22 \\
0.02910 & 3.00 & 3.00 \\
0.03060 & 3.74 & 3.74 \\
0.03260 & 4.51 & 4.51 \\
0.03510 & 5.21 & 5.21 \\
0.03890 & 5.89 & 5.89 \\
0.04390 & 6.35 & 6.35 \\
0.05020 & 6.56 & 6.57 \\
0.05770 & 6.55 & 6.62 \\
0.06770 & 6.35 & 6.58 \\
0.08030 & 6.01 & 6.45 \\
0.09530 & 5.61 & 6.27 \\
0.11200 & 5.22 & 6.07 \\
0.13000 & 4.87 & 5.87 \\
0.15000 & 4.55 & 5.69 \\
0.17500 & 4.24 & 5.51 \\
0.21300 & 3.89 & 5.30 \\
0.25100 & 3.64 & 5.16 \\
0.37600 & 3.17 & 4.95 \\
0.50200 & 2.94 & 4.92 \\
0.75300 & 2.72 & 5.08 \\
1.25000 & 2.58 & 5.55 \\
2.51000 & 2.51 & 6.61 \\
5.02000 & 2.49 & 7.99 \\
7.53000 & 2.48 & 8.88 \\
12.50000 & 2.48 & 10.05 \\
17.50000 & 2.48 & 10.85 \\
25.10000 & 2.48 & 11.72 \\
50.20000 & 2.48 & 13.41 \\
100.00000 & 2.48 & 15.11 \\
175.00000 & 2.48 & 16.49
\end{tabular}

\begin{tabular}{|c|c|c|c|}
\hline $\begin{array}{r}T D=60.0 \\
\text { ENERGY (MEV) }\end{array}$ & EV & $\begin{array}{r}\text { ET }=0.0 \\
\text { PRIABRY }\end{array}$ & $\begin{array}{l}849 \mathrm{MBV} \\
\text { CASCADE }\end{array}$ \\
\hline $0.02710^{\circ}$ & & 0.20 & 0.20 \\
\hline 0.02730 & & 0.36 & 0.36 \\
\hline 0.02790 & & 0.80 & 0.80 \\
\hline 0.02870 & & 1.33 & 1.33 \\
\hline 0.02980 & & 1.97 & 1.97 \\
\hline 0.03110 & & 2.61 & 2.61 \\
\hline 0.03270 & & 3.26 & 3.26 \\
\hline 0.03490 & & 3.95 & 3.95 \\
\hline 0.03750 & & 4.54 & 4.54 \\
\hline 0.04160 & & 5.14 & 5.14 \\
\hline 0.04690 & & 5.55 & 5.55 \\
\hline 0.05360 & & 5.74 & 5.74 \\
\hline 0.06170 & & 5.74 & 5.80 \\
\hline 0.07240 & & 5.57 & 5.77 \\
\hline 0.08590 & & 5.28 & 5.67 \\
\hline 0.10200 & & 4.94 & 5.52 \\
\hline 0.12000 & & 4.60 & 5.36 \\
\hline 0.13900 & & 4.31 & 5.20 \\
\hline 0.16100 & & 4.04 & 5.05 \\
\hline 0.18700 & & 3.78 & 4.91 \\
\hline 0.22800 & & 3.48 & 4.74 \\
\hline 0.26800 & & 3.27 & 4.64 \\
\hline 0.40200 & & 2.87 & 4.49 \\
\hline 0.53600 & & 2.68 & 4.50 \\
\hline 0.80500 & & 2.51 & 4.69 \\
\hline 1.34000 & & 2.39 & 5.17 \\
\hline 2.68000 & & 2.33 & 6.20 \\
\hline 5.36000 & & 2.32 & 7.51 \\
\hline 8.05000 & & 2.32 & 8.35 \\
\hline 13.40000 & & 2.32 & 9.46 \\
\hline 18.70000 & & 2.31 & 10.20 \\
\hline 26.80000 & & 2.31 & 11.01 \\
\hline 53.60000 & & 2.31 & 12.59 \\
\hline 107.00000 & & $\begin{array}{l}2.31 \\
2.31\end{array}$ & 14.18 \\
\hline 5000 & & & \\
\hline
\end{tabular}

ATONIC DISPLAEEHENT CROSS-SECTIONS (BARNS) BY FAST ELECTRONS IN H $z=1 \quad A=1.008$

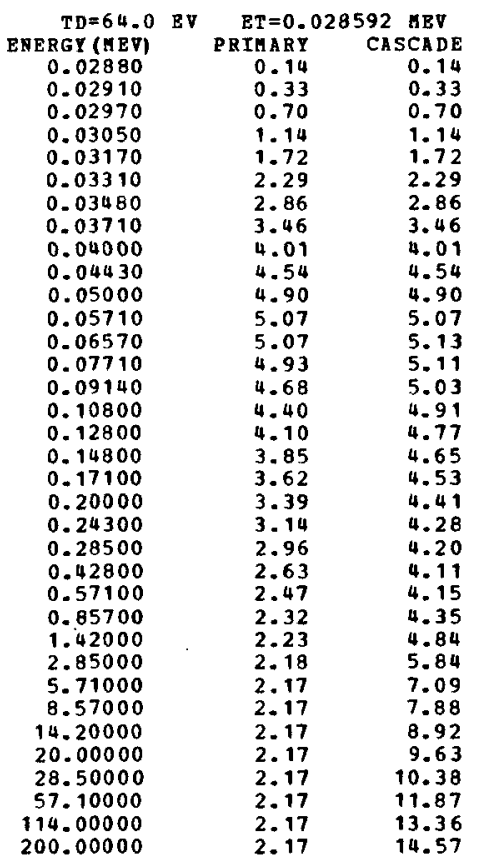

$\begin{array}{ccc}\text { TD }=68.0 & \text { BV } & \text { ET }=0.030329 \text { HEV } \\ \text { ENERGY(IIEV) } & \text { PRI IARY } & \text { CASCADR } \\ 0.03060 & 0.15 & 0.15 \\ 0.03090 & 0.31 & 0.31 \\ 0.03150 & 0.62 & 0.62 \\ 0.03240 & 1.03 & 1.03 \\ 0.03360 & 1.52 & 1.52 \\ 0.03510 & 2.03 & 2.03 \\ 0.03700 & 2.56 & 2.56 \\ 0.03940 & 3.08 & 3.08 \\ 0.04240 & 3.56 & 3.56 \\ 0.04700 & 4.03 & 4.03 \\ 0.05300 & 4.36 & 4.36 \\ 0.06060 & 4.51 & 4.51 \\ 0.06970 & 4.52 & 4.57 \\ 0.08180 & 4.40 & 4.56 \\ 0.09700 & 4.19 & 4.50 \\ 0.11500 & 3.93 & 4.40 \\ 0.13600 & 3.68 & 4.28 \\ 0.15700 & 3.47 & 4.18 \\ 0.18100 & 3.27 & 4.09 \\ 0.21200 & 3.07 & 3.99 \\ 0.25700 & 2.86 & 3.89 \\ 0.30300 & 2.70 & 3.83 \\ 0.45400 & 2.42 & 3.78 \\ 0.60600 & 2.28 & 3.84 \\ 0.90900 & 2.16 & 4.07 \\ 1.51000 & 2.09 & 4.56 \\ 3.03000 & 2.05 & 5.53 \\ 6.06000 & 2.04 & 6.71 \\ 9.09000 & 2.04 & 7.47 \\ 15.10000 & 2.04 & 8.45 \\ 21.20000 & 2.04 & 9.12 \\ 30.30000 & 2.04 & 9.83 \\ 60.60000 & 2.04 & 11.23 \\ 121.00000 & 2.04 & 12.63 \\ 212.00000 & 2.04 & 13.77 \\ & & \end{array}$

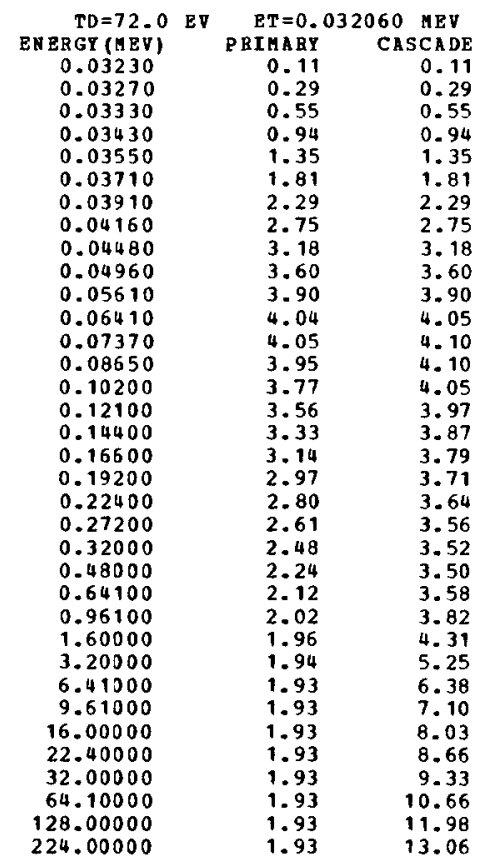


ATOMIC DISPLAEEAEN CROSS-SECTIONS (BARHS) BY FAST RLECTRONS IN H

\begin{tabular}{|c|c|c|c|}
\hline $\begin{array}{r}\text { TD }=76 . \\
\text { ENERGY (MEV) }\end{array}$ & EV & $\begin{array}{l}\text { ET }=0.0 \\
\text { PRTMARY }\end{array}$ & $\begin{array}{l}786 \text { MEV } \\
\text { CASCADE }\end{array}$ \\
\hline 0.03410 & & 0.13 & 0.13 \\
\hline 0.03440 & & 0.24 & 0.24 \\
\hline 0.03510 & & 0.50 & 0.50 \\
\hline 0.03610 & & 0.83 & 0.83 \\
\hline 0.03750 & & 1.24 & 1.24 \\
\hline 0.03910 & & 1.63 & 1.63 \\
\hline 0.04120 & & 2.06 & 2.06 \\
\hline 0.04390 & & 2.48 & 2.48 \\
\hline 0.04720 & & 2.86 & 2.86 \\
\hline 0.05230 & & 3.24 & 3.24 \\
\hline 0.05910 & & 3.51 & 3.51 \\
\hline 0.06750 & & 3.65 & 3.65 \\
\hline 0.07770 & & 3.66 & 3.70 \\
\hline 0.09120 & & 3.57 & 3.71 \\
\hline 0.10800 & & 3.41 & 3.67 \\
\hline 0.12800 & & 3.22 & 3.60 \\
\hline 0.15200 & & 3.02 & 3.52 \\
\hline 0.17500 & & 2.87 & 3.46 \\
\hline 0.20200 & & 2.72 & 3.39 \\
\hline 0.23600 & & 2.57 & 3.33 \\
\hline 0.28700 & & 2.40 & 3.27 \\
\hline 0.33700 & & 2.29 & 3.25 \\
\hline 0.50600 & & 2.08 & 3.26 \\
\hline 0.67500 & & 1.98 & 3.36 \\
\hline 1.01000 & & 1.90 & 3.60 \\
\hline 1.68000 & & 1.85 & 4.09 \\
\hline 3.37000 & & 1.83 & 5.00 \\
\hline 6.75000 & & 1.83 & 6.08 \\
\hline 10.10000 & & 1.83 & 6.76 \\
\hline 16.80000 & & 1.83 & 7.65 \\
\hline 23.50000 & & 1.83 & 8.25 \\
\hline 33.70000 & & 1.83 & 8.89 \\
\hline 67.50000 & & 1.83 & 10.14 \\
\hline 135.00000 & & 1.83 & 11.40 \\
\hline 236.00000 & & 1.83 & 12.42 \\
\hline
\end{tabular}

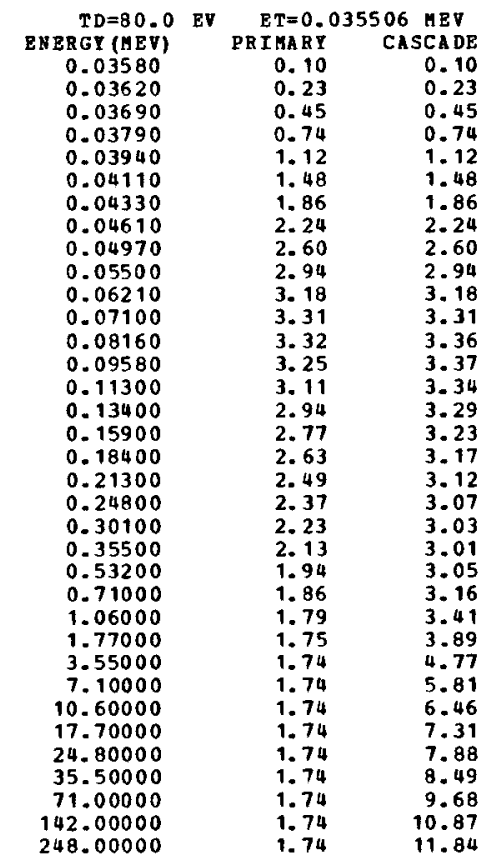

\begin{tabular}{ccr} 
TD $=34.0$ & EV & \multicolumn{1}{c}{ ET $=0.037221$} \\
PRIMARY & HEV \\
CASCADE \\
0.03750 & 0.08 & 0.08 \\
0.03790 & 0.20 & 0.20 \\
0.03870 & 0.42 & 0.42 \\
0.03980 & 0.69 & 0.69 \\
0.04130 & 1.01 & 1.01 \\
0.04310 & 1.34 & 1.34 \\
0.04540 & 1.69 & 1.69 \\
0.04830 & 2.03 & 2.03 \\
0.05210 & 2.36 & 2.36 \\
0.05760 & 2.67 & 2.67 \\
0.06510 & 2.90 & 2.90 \\
0.07440 & 3.01 & 3.01 \\
0.08560 & 3.03 & 3.07 \\
0.10000 & 2.97 & 3.08 \\
0.11900 & 2.84 & 3.06 \\
0.14100 & 2.70 & 3.02 \\
0.16700 & 2.55 & 2.97 \\
0.19300 & 2.42 & 2.92 \\
0.22300 & 2.30 & 2.88 \\
0.26000 & 2.19 & 2.84 \\
0.31600 & 2.07 & 2.82 \\
0.37200 & 1.98 & 2.81 \\
0.55800 & 1.83 & 2.87 \\
0.74400 & 1.76 & 2.98 \\
1.11000 & 1.70 & 3.24 \\
1.86000 & 1.67 & 3.72 \\
3.72000 & 1.65 & 4.57 \\
7.44000 & 1.65 & 5.57 \\
11.10000 & 1.65 & 6.18 \\
18.60000 & 1.65 & 7.00 \\
26.00000 & 1.65 & 7.54 \\
37.20000 & 1.65 & 8.12 \\
74.40000 & 1.65 & 9.25 \\
148.00000 & 1.65 & 10.38 \\
260.00000 & 1.65 & 11.31 \\
& &
\end{tabular}

A TOHIC DISPLACEHENT CROSS-SECTIONS (BARNS) BY FAST BLECTRONS IN A

$\mathrm{Z}=1 \quad \mathrm{~A}=\quad \begin{aligned} & \text { (BARNS) } \\ & 1.008\end{aligned}$

\begin{tabular}{ccr}
$\begin{array}{c}T D=88.0 \\
\text { BNERGY }\end{array}$ & \multicolumn{2}{c}{ ET $=0.038931$ MEV } \\
0.03930 & PRIMARY & CASCADE \\
0.03970 & 0.10 & 0.10 \\
0.04040 & 0.20 & 0.20 \\
0.04160 & 0.36 & 0.36 \\
0.04320 & 0.62 & 0.62 \\
0.04510 & 0.93 & 0.93 \\
0.04740 & 1.23 & 1.23 \\
0.05060 & 1.53 & 1.53 \\
0.05450 & 1.86 & 1.86 \\
0.06030 & 2.16 & 2.16 \\
0.06810 & 2.44 & 2.44 \\
0.07780 & 2.65 & 2.65 \\
0.08950 & 2.76 & 2.76 \\
0.10500 & 2.77 & 2.81 \\
0.12400 & 2.72 & 2.83 \\
0.14700 & 2.62 & 2.81 \\
0.17500 & 2.49 & 2.78 \\
0.20200 & 2.35 & 2.74 \\
0.23300 & 2.24 & 2.70 \\
0.27200 & 2.14 & 2.67 \\
0.33000 & 2.04 & 2.65 \\
0.38900 & 1.93 & 2.63 \\
0.58300 & 1.85 & 2.63 \\
0.77800 & 1.72 & 2.70 \\
1.16000 & 1.66 & 2.82 \\
1.94000 & 1.61 & 3.08 \\
3.89000 & 1.59 & 3.55 \\
7.78000 & 1.58 & 4.38 \\
11.60000 & 1.58 & 5.34 \\
19.40000 & 1.58 & 5.93 \\
27.20000 & 1.58 & 6.71 \\
38.90000 & 1.58 & 7.23 \\
77.80000 & 1.58 & 7.78 \\
155.00000 & 1.58 & 8.86 \\
& 1.58 & 9.95
\end{tabular}

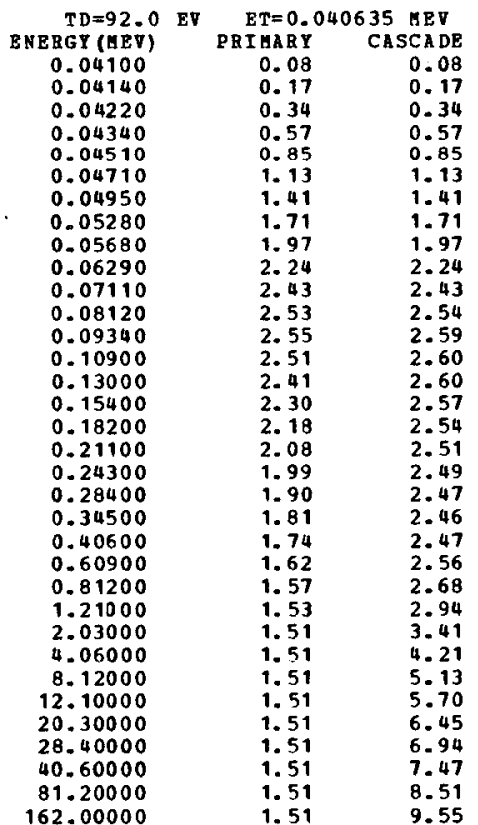

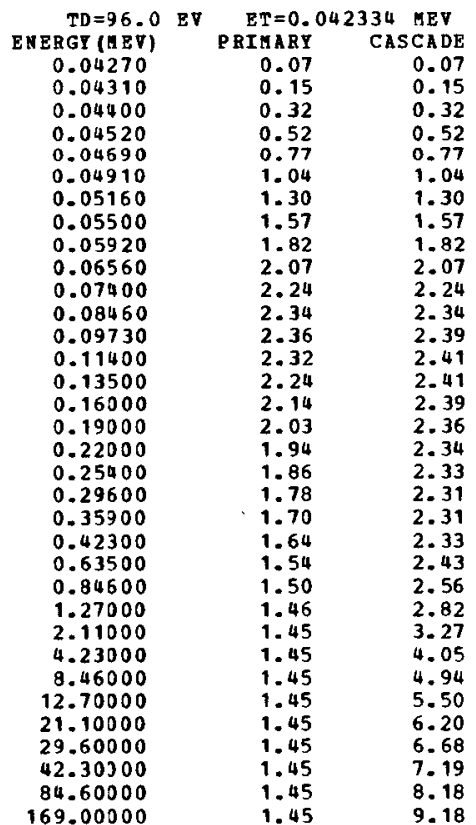


ATOHC DISPLACEHENT CROSS-SECtIONS (BARNS) BY PAST ELECTRONS IN AE

\begin{tabular}{crr}
$T D=4.0$ & EV & \multicolumn{1}{c}{ ET $=0.007244$ MEV } \\
PNBRGY $(M P V)$ & PRIMRY & CASCADE \\
0.00731 & 11.08 & 11.08 \\
0.00738 & 22.36 & 22.36 \\
0.00753 & 45.12 & 45.12 \\
0.00775 & 75.36 & 75.36 \\
0.00804 & 110.17 & 110.17 \\
0.00840 & 146.63 & 146.63 \\
0.00883 & 182.16 & 182.16 \\
0.00941 & 219.23 & 219.23 \\
0.01010 & 251.19 & 251.19 \\
0.01120 & 283.46 & 283.46 \\
0.01260 & 303.98 & 303.98 \\
0.01440 & 312.00 & 312.00 \\
0.01660 & 308.30 & 310.97 \\
0.01950 & 294.45 & 304.23 \\
0.02310 & 273.60 & 292.69 \\
0.02750 & 249.09 & 277.75 \\
0.03250 & 224.98 & 261.79 \\
0.03760 & 204.51 & 247.25 \\
0.04340 & 185.42 & 232.87 \\
0.05070 & 166.25 & 217.55 \\
0.06150 & 144.87 & 199.35 \\
0.07240 & 128.95 & 184.96 \\
0.10800 & 97.66 & 154.33 \\
0.14400 & 81.03 & 136.68 \\
0.21700 & 63.93 & 117.77 \\
0.36200 & 50.50 & 103.52 \\
0.72400 & 41.07 & 98.09 \\
1.44000 & 37.14 & 104.76 \\
2.17000 & 36.09 & 112.83 \\
3.62000 & 35.44 & 125.79 \\
5.07000 & 35.23 & 135.53 \\
7.24000 & 35.11 & 146.52 \\
14.40000 & 35.01 & 168.97 \\
28.90000 & 34.99 & 192.59 \\
50.70000 & 34.98 & 211.95 \\
72.40000 & 34.98 & 224.30
\end{tabular}

$\mathrm{Z}=2 \mathrm{~A}=4.003$

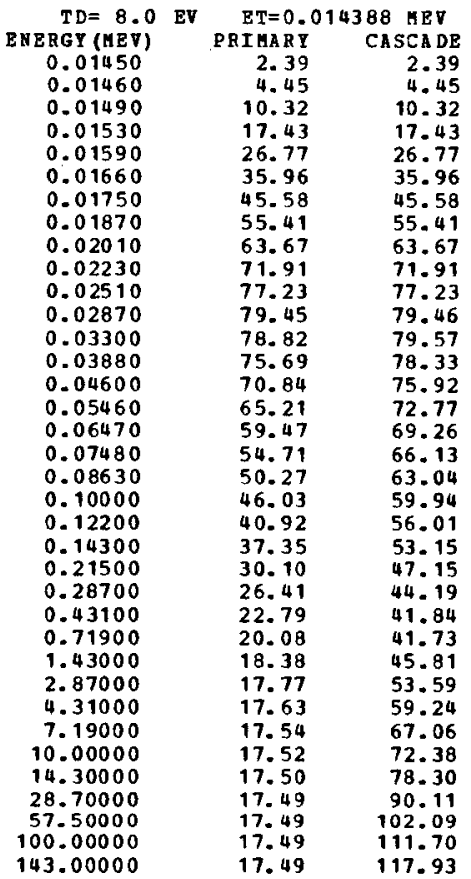

$T D=12.0 \mathrm{EV} \quad \mathrm{ET}=0.021436 \mathrm{BEV}$

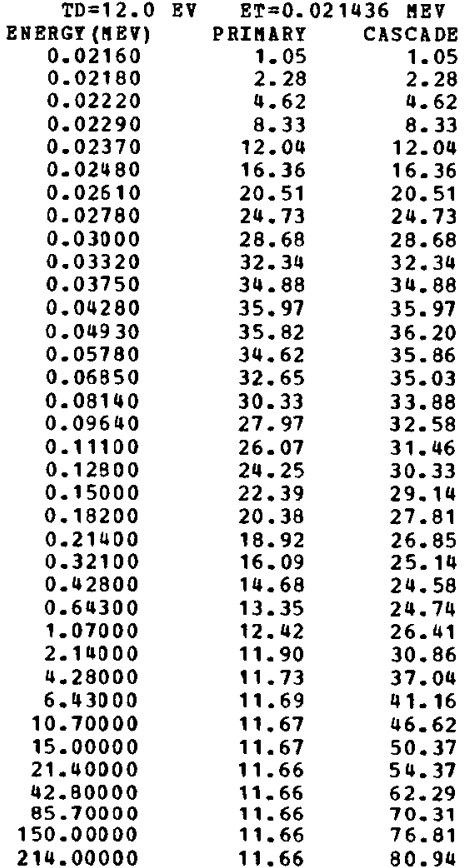

ATOMIC DISPLAERHENT CROSS-SECTIONS (BARNS) BY FAST ELECTRONS IN RE

$$
\mathrm{z}=2 \quad \mathrm{~A}=4.003
$$

\begin{tabular}{crr} 
TD=16.0 & \multicolumn{1}{c}{ ET $=0.028391$ MRV } \\
ENERGY (HEV) & PRIMARY & CASCADE \\
0.02860 & 0.57 & 0.57 \\
0.02890 & 1.36 & 1.36 \\
0.02950 & 2.85 & 2.85 \\
0.03030 & 4.65 & 4.65 \\
0.03150 & 7.02 & 7.02 \\
0.03290 & 9.35 & 9.35 \\
0.03460 & 11.67 & 11.67 \\
0.03690 & 14.11 & 14.11 \\
0.03970 & 16.28 & 16.28 \\
0.04400 & 18.43 & 18.43 \\
0.04960 & 19.90 & 19.90 \\
0.05670 & 20.61 & 20.61 \\
0.06530 & 20.61 & 20.84 \\
0.07650 & 20.03 & 20.78 \\
0.09080 & 19.04 & 20.45 \\
0.10700 & 17.91 & 19.98 \\
0.12700 & 16.68 & 19.40 \\
0.14700 & 15.66 & 18.89 \\
0.17000 & 14.71 & 18.40 \\
0.19800 & 13.80 & 17.92 \\
0.24100 & 12.77 & 17.39 \\
0.28300 & 12.05 & 17.06 \\
0.42500 & 10.66 & 16.67 \\
0.56700 & 10.00 & 16.82 \\
0.85100 & 9.40 & 17.65 \\
1.41000 & 9.02 & 19.59 \\
2.83000 & 8.82 & 23.60 \\
5.67000 & 8.77 & 28.63 \\
8.51000 & 8.76 & 31.84 \\
14.10000 & 8.75 & 36.00 \\
19.80000 & 8.75 & 38.86 \\
28.30000 & 8.75 & 41.90 \\
56.70000 & 8.74 & 47.89 \\
113.00000 & 8.74 & 53.88 \\
198.00000 & 8.74 & 58.77
\end{tabular}

\begin{tabular}{|c|c|c|c|}
\hline $\begin{array}{r}T D=20.0 \\
\text { ENERGY (MEV) }\end{array}$ & EV & $\begin{array}{r}\text { ET }=0.0 \\
\text { PRIMARY }\end{array}$ & CASCADE \\
\hline 0.03560 & & 048 & \\
\hline 0.03590 & & 0.89 & $\begin{array}{l}0.48 \\
0.89\end{array}$ \\
\hline 0.03660 & & 1.79 & 1.79 \\
\hline 0.03770 & & 3.08 & 3.08 \\
\hline 0.03910 & & 4.51 & 4.51 \\
\hline 0.04090 & & 6.06 & 6.06 \\
\hline 0.04300 & & 7.55 & 7.55 \\
\hline 0.04580 & & 9.11 & 9.11 \\
\hline 0.04930 & & 10.53 & 10.53 \\
\hline 0.05460 & & 11.93 & 11.93 \\
\hline 0.06170 & & 12.94 & 12.94 \\
\hline 0.07050 & & 13.43 & 13.44 \\
\hline 0.08100 & & 13.49 & 13.66 \\
\hline 0.09510 & & 13.20 & 13.70 \\
\hline 0.11200 & & 12.66 & 13.59 \\
\hline 0.13300 & & 11.98 & 13.38 \\
\hline 0.15800 & & 11.27 & 13. 11 \\
\hline 0.18300 & & 10.68 & 12.88 \\
\hline $\begin{array}{l}0.21100 \\
0.24600\end{array}$ & & $\begin{array}{r}10.14 \\
9.62\end{array}$ & $\begin{array}{l}12.68 \\
12.49\end{array}$ \\
\hline 0.29900 & & 9.05 & 12.31 \\
\hline 0.35200 & & 8.64 & 12.24 \\
\hline 0.52800 & & 7.89 & 12.39 \\
\hline 0.70500 & & $\begin{array}{l}7.55 \\
7.26\end{array}$ & 12.80 \\
\hline 1.76000 & & 7.09 & 15.75 \\
\hline 3.52000 & & 7.02 & 19.28 \\
\hline 7.05000 & & 7.00 & 23.48 \\
\hline 10.50000 & & 7.00 & 26.06 \\
\hline 17.60000 & & 7.00 & 29.51 \\
\hline $\begin{array}{l}24.60000 \\
35.20000\end{array}$ & & $\begin{array}{l}7.00 \\
7.00\end{array}$ & $\begin{array}{l}31.78 \\
34.24\end{array}$ \\
\hline 70.50000 & & 7.00 & 39.04 \\
\hline 141.00000 & & 7.00 & 43.87 \\
\hline & & 7.00 & .75 \\
\hline
\end{tabular}

\begin{tabular}{crr} 
TD $=24.0$ & EV & \multicolumn{1}{c}{ ET=0.042041 } \\
ENERGY (HEV) & PRIMARY & CASCADE \\
0.04240 & 0.30 & 0.30 \\
0.04280 & 0.62 & 0.62 \\
0.04370 & 1.29 & 1.29 \\
0.04490 & 2.12 & 2.12 \\
0.04660 & 3.14 & 3.14 \\
0.04870 & 4.21 & 4.21 \\
0.05120 & 5.26 & 5.26 \\
0.05460 & 6.38 & 6.38 \\
0.05880 & 7.39 & 7.39 \\
0.06510 & 8.39 & 8.39 \\
0.07350 & 9.12 & 9.12 \\
0.08400 & 9.50 & 9.51 \\
0.09660 & 9.59 & 9.71 \\
0.11300 & 9.43 & 9.80 \\
0.13400 & 9.10 & 9.79 \\
0.15900 & 8.69 & 9.71 \\
0.18900 & 8.25 & 9.61 \\
0.21800 & 7.90 & 9.52 \\
0.25200 & 7.56 & 9.45 \\
0.29400 & 7.25 & 9.41 \\
0.35700 & 6.90 & 9.40 \\
0.42000 & 6.67 & 9.46 \\
0.63000 & 6.25 & 9.85 \\
0.84000 & 6.06 & 10.37 \\
1.26000 & 5.92 & 11.43 \\
2.10000 & 5.85 & 13.26 \\
4.20000 & 5.83 & 16.38 \\
8.40000 & 5.83 & 19.97 \\
12.60000 & 5.83 & 22.19 \\
21.00000 & 5.83 & 25.06 \\
29.40000 & 5.83 & 26.97 \\
42.00000 & 5.83 & 29.02 \\
84.00000 & 5.83 & 33.02 \\
168.00000 & 5.83 & 37.04 \\
294.00000 & 5.83 & 40.30
\end{tabular}


ATONIC DISPLAZEMENT CROSS-SECTIONS (BARNS) BY FAST ELECTRONS IN HE

\begin{tabular}{|c|c|c|c|}
\hline $\begin{array}{r}T D=28.0 \\
\text { IERGY (MEV) }\end{array}$ & BV & $\begin{array}{r}E T=0.0 \\
\text { PRISARY }\end{array}$ & $\begin{array}{r}741 \mathrm{HEV} \\
\text { CASCADE }\end{array}$ \\
\hline 0.04920 & & 0.24 & 0.24 \\
\hline 0.04970 & & 0.50 & 0.50 \\
\hline 0.05060 & & 0.93 & 0.93 \\
\hline 0.05210 & & 1.58 & 1.58 \\
\hline 0.05410 & & 2.35 & 2.35 \\
\hline 0.05650 & & 3.13 & 3.13 \\
\hline 0.05940 & & 3.91 & 3.91 \\
\hline 0.06330 & & 4.73 & 4.73 \\
\hline 0.06820 & & 5.49 & 5.49 \\
\hline 0.07550 & & 6.24 & 6.24 \\
\hline $\begin{array}{l}0.08520 \\
0.09740\end{array}$ & & 7.11 & 7.12 \\
\hline 0.11200 & & 7.20 & 7.30 \\
\hline 0.13100 & & 7.13 & 7.41 \\
\hline 0.15500 & & 6.93 & 7.46 \\
\hline 0.18500 & & 6.66 & 7.46 \\
\hline 0.21900 & & 6.39 & 7.44 \\
\hline 0.25300 & & 6.16 & 7.44 \\
\hline 0.29200 & & 5.95 & 7.44 \\
\hline 0.34100 & & 5.76 & 7.47 \\
\hline 0.41400 & & 5.55 & 7.56 \\
\hline 0.48700 & & 5.40 & 7.68 \\
\hline 0.73100 & & 5.16 & 8.20 \\
\hline 0.97400 & & 5.07 & 8.75 \\
\hline 1.46000 & & 5.01 & 9.79 \\
\hline 2.43000 & & 4.99 & 11.48 \\
\hline 4.87000 & & 4.99 & 14.28 \\
\hline 9.74000 & & 5.00 & 17.42 \\
\hline 14.60000 & & 5.00 & 19.34 \\
\hline 24.30000 & & 5.00 & 21.80 \\
\hline 34.10000 & & 5.00 & 23.46 \\
\hline 48.70000 & & 5.00 & \\
\hline 194.00000 & & $\begin{array}{l}5.00 \\
5.00\end{array}$ & \\
\hline 4.00000 & & & \\
\hline
\end{tabular}

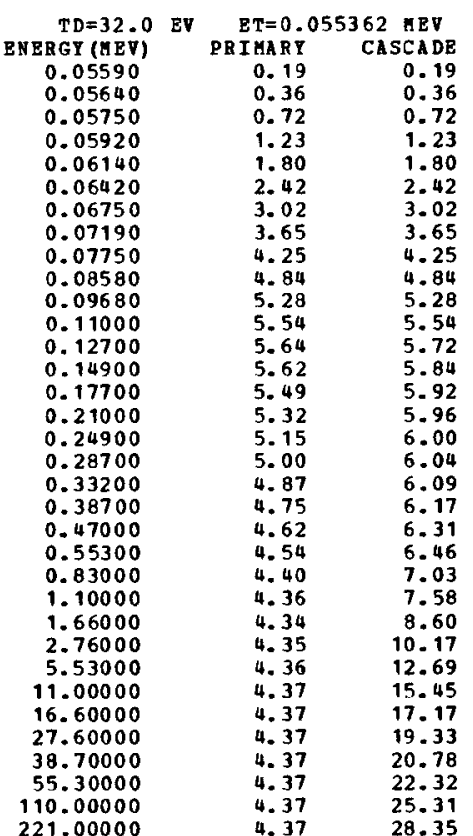

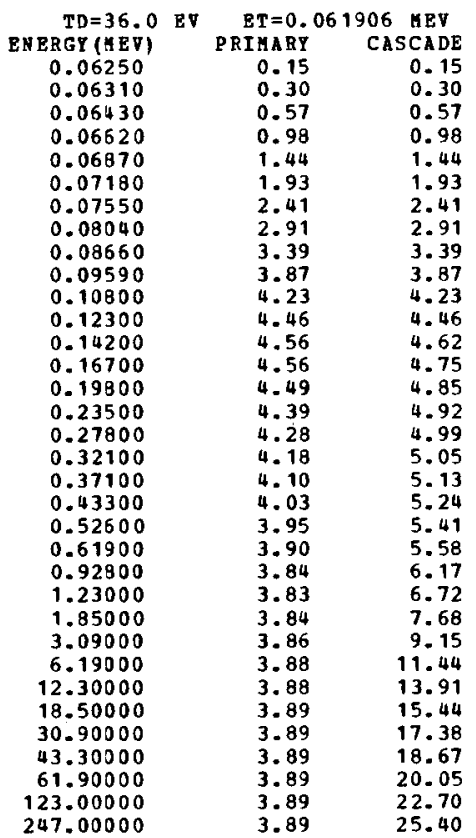

ATOMIC DISPLAZEMENT CROSS-SECTIONS (BARNS) BY PAST ELECTRONS IN HE

$=2 \quad \mathrm{~A}=4.003$

$T D=40.0 \mathrm{EV} \quad \mathrm{ET}=0.068376 \mathrm{MEV}$

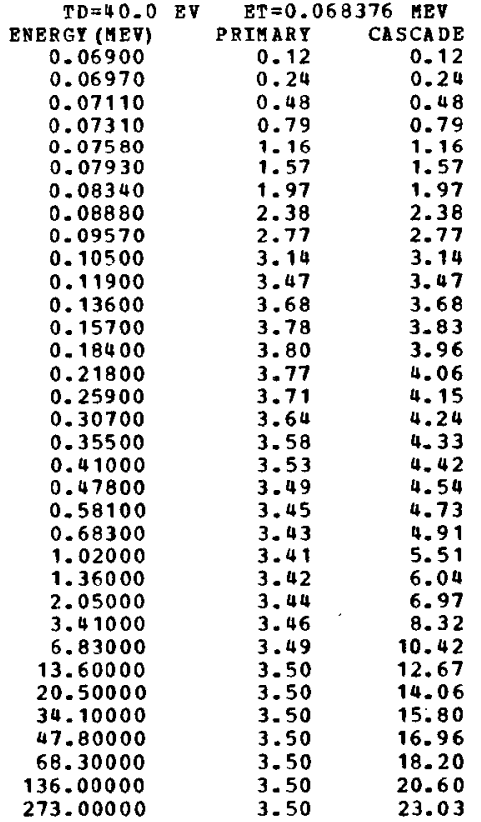

$T D=44.0 \mathrm{EV} \quad \mathrm{ET}=0.074775 \mathrm{MEV}$

\begin{tabular}{ccc} 
TD=44.0 EV & \multicolumn{1}{c}{ ET $=0.074775$ MEV } \\
ENERGY(MEV) & PRIMAY & CASCADE \\
0.07550 & 0.10 & 0.10 \\
0.07620 & 0.20 & 0.20 \\
0.07770 & 0.39 & 0.39 \\
0.08000 & 0.56 & 0.66 \\
0.08290 & 0.97 & 0.97 \\
0.08670 & 1.31 & 1.31 \\
0.09120 & 1.64 & 1.64 \\
0.09720 & 1.99 & 1.99 \\
0.10400 & 2.29 & 2.29 \\
0.11500 & 2.63 & 2.63 \\
0.13000 & 2.91 & 2.91 \\
0.14900 & 3.10 & 3.10 \\
0.17100 & 3.19 & 3.24 \\
0.20100 & 3.23 & 3.37 \\
0.23900 & 3.22 & 3.48 \\
0.28400 & 3.19 & 3.58 \\
0.33600 & 3.15 & 3.68 \\
0.38800 & 3.12 & 3.77 \\
0.44800 & 3.10 & 3.88 \\
0.52300 & 3.07 & 4.01 \\
0.63500 & 3.06 & 4.21 \\
0.74700 & 3.05 & 4.40 \\
1.12000 & 3.07 & 4.99 \\
1.49000 & 3.08 & 5.51 \\
2.24000 & 3.12 & 6.38 \\
3.73000 & 3.15 & 7.64 \\
7.47000 & 3.17 & 9.58 \\
14.90000 & 3.18 & 11.65 \\
22.40000 & 3.18 & 12.90 \\
37.30000 & 3.18 & 14.49 \\
52.30000 & 3.18 & 15.55 \\
74.70000 & 3.18 & 16.68 \\
149.00000 & 3.18 & 18.86 \\
299.00000 & 3.18 & 21.07 \\
& &
\end{tabular}

299.00000

3.18

18.86
21.07
$T D=48.0 \mathrm{EV} \quad \mathrm{ET}=0.081104 \mathrm{HEV}$

$\begin{array}{ccc}\text { TD=48.0 EV } & \begin{array}{c}\text { ET }=0.081104 \\ \text { PRTART }\end{array} \\ \text { CNERGY CASCADE } \\ 0.08190 & 0.09 & 0.09 \\ 0.08270 & 0.17 & 0.17 \\ 0.08430 & 0.33 & 0.33 \\ 0.08670 & 0.56 & 0.56 \\ 0.09000 & 0.82 & 0.82 \\ 0.09400 & 1.10 & 1.10 \\ 0.09890 & 1.38 & 1.38 \\ 0.10500 & 1.67 & 1.67 \\ 0.11300 & 1.95 & 1.95 \\ 0.12500 & 2.24 & 2.24 \\ 0.14100 & 2.48 & 2.48 \\ 0.16200 & 2.65 & 2.66 \\ 0.18600 & 2.74 & 2.79 \\ 0.21300 & 2.79 & 2.91 \\ 0.25900 & 2.80 & 3.02 \\ 0.30800 & 2.79 & 3.13 \\ 0.36400 & 2.77 & 3.24 \\ 0.42100 & 2.76 & 3.34 \\ 0.48600 & 2.75 & 3.45 \\ 0.56700 & 2.75 & 3.59 \\ 0.68900 & 2.75 & 3.79 \\ 0.81100 & 2.76 & 3.98 \\ 1.21000 & 2.79 & 4.56 \\ 1.62000 & 2.81 & 5.07 \\ 2.43000 & 2.85 & 5.89 \\ 4.05000 & 2.88 & 7.08 \\ 8.11000 & 2.91 & 8.88 \\ 16.20000 & 2.91 & 10.79 \\ 24.30000 & 2.91 & 11.93 \\ 40.50000 & 2.91 & 13.39 \\ 56.70000 & 2.91 & 14.36 \\ 81.10000 & 2.91 & 15.40 \\ 162.00000 & 2.91 & 17.41 \\ 324.00000 & 2.91 & 19.42 \\ & & \end{array}$

324.00000

2.91
2.91

19.42 
ATONIC DISPLAzEHENT CROSS-SECTIONS (BARNS) BY PAST ELECTRONS IN HE

\begin{tabular}{|c|c|c|c|}
\hline$T D=52.0$ & EV & $\begin{aligned} \mathbf{B T} & =0.08 \\
& \end{aligned}$ & $367 \mathrm{HEV}$ \\
\hline BNERGY (MEV) & & PRIMARY & CASCADE \\
\hline 0.08820 & & 0.07 & 0.07 \\
\hline 0.08910 & & 0.15 & 0.15 \\
\hline 0.09080 & & 0.29 & 0.29 \\
\hline 0.09340 & & 0.48 & 0.48 \\
\hline 0.09690 & & 0.70 & 0.70 \\
\hline 0.10100 & & 0.93 & 0.93 \\
\hline 0.10600 & & 1.17 & 1.17 \\
\hline 0.11300 & & 1.43 & 1.43 \\
\hline 0.12200 & & 1.68 & 1.68 \\
\hline 0.13500 & & 1.94 & 1.94 \\
\hline 0.15200 & & 2.15 & 2.15 \\
\hline 0.17400 & & 2.30 & 2.30 \\
\hline 0.20000 & & 2.39 & 2.43 \\
\hline 0.23500 & & 2.44 & 2.55 \\
\hline 0.27900 & & 2.47 & 2.66 \\
\hline 0.33100 & & 2.47 & 2.77 \\
\hline 0.39300 & & 2.47 & 2.89 \\
\hline $\begin{array}{l}0.45400 \\
0.52400\end{array}$ & & $\begin{array}{l}2.47 \\
2.48\end{array}$ & 2.99 \\
\hline 0.61100 & & 2.48 & 3.25 \\
\hline 0.74200 & & 2.50 & 3.45 \\
\hline 0.87300 & & 2.51 & 3.64 \\
\hline $\begin{array}{l}1.31000 \\
1.74000\end{array}$ & & $\begin{array}{l}2.56 \\
2.59\end{array}$ & $\begin{array}{l}4.21 \\
4.69\end{array}$ \\
\hline 2.62000 & & 2.63 & $\begin{array}{l}4.09 \\
5.48\end{array}$ \\
\hline 4.36000 & & 2.66 & 6.59 \\
\hline 8.73000 & & 2.68 & 8.27 \\
\hline $\begin{array}{l}17.40000 \\
26.20000\end{array}$ & & $\begin{array}{l}2.69 \\
2.69\end{array}$ & 10.03 \\
\hline 43.60000 & & $\begin{array}{l}2.09 \\
2.69\end{array}$ & 12.45 \\
\hline 61.10000 & & 2.69 & 13.35 \\
\hline $\begin{array}{r}87.30000 \\
174.00000\end{array}$ & & 2.69 & $\begin{array}{l}14.30 \\
16.15\end{array}$ \\
\hline & & & \\
\hline
\end{tabular}

\begin{tabular}{rrr} 
TD $=56.0$ & EV & \multicolumn{2}{c}{ ET $=0.093565$} & \multicolumn{1}{c}{ AEV } \\
ENERGY (EEV) & PRIMAT & CASCADE \\
0.09450 & 0.07 & 0.07 \\
0.09540 & 0.13 & 0.13 \\
0.09730 & 0.25 & 0.25 \\
0.10000 & 0.41 & 0.41 \\
0.10300 & 0.57 & 0.57 \\
0.10800 & 0.80 & 0.80 \\
0.11400 & 1.03 & 1.03 \\
0.12100 & 1.24 & 1.24 \\
0.13000 & 1.45 & 1.45 \\
0.14500 & 1.70 & 1.70 \\
0.16300 & 1.88 & 1.88 \\
0.18700 & 2.02 & 2.02 \\
0.21500 & 2.11 & 2.14 \\
0.25200 & 2.16 & 2.26 \\
0.29900 & 2.20 & 2.37 \\
0.35500 & 2.21 & 2.49 \\
0.42100 & 2.23 & 2.60 \\
0.48600 & 2.24 & 2.71 \\
0.56100 & 2.25 & 2.83 \\
0.65400 & 2.26 & 2.97 \\
0.79500 & 2.29 & 3.17 \\
0.93500 & 2.31 & 3.36 \\
1.40000 & 2.36 & 3.91 \\
1.87000 & 2.40 & 4.38 \\
2.80000 & 2.44 & 5.12 \\
4.67000 & 2.47 & 6.18 \\
9.35000 & 2.49 & 7.75 \\
18.70000 & 2.50 & 9.40 \\
28.00000 & 2.50 & 10.38 \\
46.70000 & 2.50 & 11.64 \\
65.40000 & 2.50 & 12.47 \\
93.50000 & 2.50 & 13.36 \\
187.00000 & 2.50 & 15.08 \\
& &
\end{tabular}

\begin{tabular}{crr} 
TD $=60.0$ EV & \multicolumn{2}{c}{ ET $=0.099700$ MEV } \\
ENERGY (HEV) & PRIMARY & CASCADE \\
0.10000 & 0.02 & 0.02 \\
0.10100 & 0.08 & 0.08 \\
0.10300 & 0.18 & 0.18 \\
0.10500 & 0.33 & 0.33 \\
0.11000 & 0.51 & 0.51 \\
0.11500 & 0.70 & 0.70 \\
0.12100 & 0.89 & 0.89 \\
0.12900 & 1.09 & 1.09 \\
0.13900 & 1.29 & 1.29 \\
0.15400 & 1.49 & 1.49 \\
0.17400 & 1.66 & 1.66 \\
0.19900 & 1.79 & 1.79 \\
0.22900 & 1.88 & 1.91 \\
0.26900 & 1.94 & 2.02 \\
0.31900 & 1.98 & 2.14 \\
0.37800 & 2.00 & 2.25 \\
0.44800 & 2.02 & 2.37 \\
0.51300 & 2.04 & 2.48 \\
0.59800 & 2.06 & 2.60 \\
0.69700 & 2.08 & 2.74 \\
0.94700 & 2.11 & 2.93 \\
0.99500 & 2.14 & 3.12 \\
1.49000 & 2.19 & 3.66 \\
1.99000 & 2.23 & 4.11 \\
2.99000 & 2.27 & 4.82 \\
4.98000 & 2.30 & 5.82 \\
9.96000 & 2.32 & 7.29 \\
19.90000 & 2.33 & 8.83 \\
29.90000 & 2.33 & 9.76 \\
49.80000 & 2.33 & 10.93 \\
69.70000 & 2.33 & 11.71 \\
99.60000 & 2.33 & 12.53 \\
199.00000 & 2.33 & 14.14 \\
& &
\end{tabular}

a TOMIC DISPLACEHENT CROSS-SECTIONS (BARS) BY FAST ELECTRONS IN HE

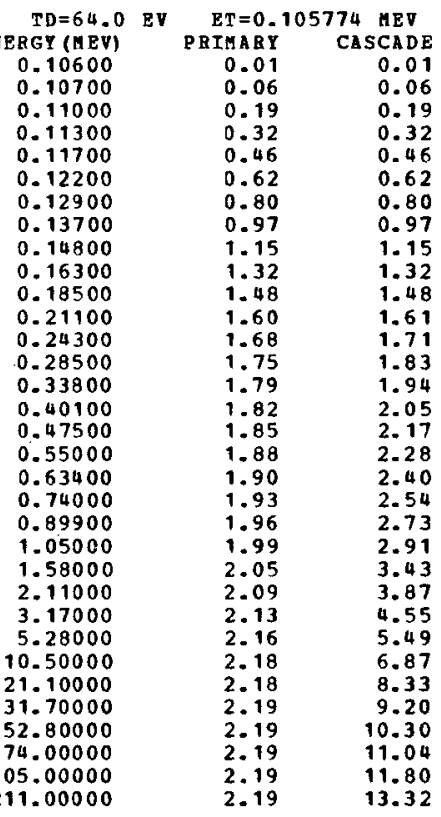

$\begin{array}{crr}\text { TD=68.0 } & \text { EV } & \begin{array}{c}\text { ET=0.111788 } \\ \text { ENERGY (HEV) } \\ \text { PRIMAR }\end{array} \\ 0.11200 & 0.01 & \text { CASCADE } \\ 0.11400 & 0.09 & 0.01 \\ 0.11600 & 0.16 & 0.16 \\ 0.11900 & 0.27 & 0.27 \\ 0.12400 & 0.42 & 0.42 \\ 0.12900 & 0.55 & 0.55 \\ 0.13600 & 0.71 & 0.71 \\ 0.14500 & 0.87 & 0.87 \\ 0.15600 & 1.02 & 1.02 \\ 0.17300 & 1.19 & 1.19 \\ 0.19500 & 1.33 & 1.33 \\ 0.22300 & 1.44 & 1.45 \\ 0.25700 & 1.52 & 1.55 \\ 0.30100 & 1.59 & 1.66 \\ 0.35700 & 1.64 & 1.77 \\ 0.42400 & 1.68 & 1.88 \\ 0.50300 & 1.71 & 2.00 \\ 0.58100 & 1.74 & 2.11 \\ 0.67000 & 1.76 & 2.23 \\ 0.78200 & 1.79 & 2.37 \\ 0.95000 & 1.83 & 2.56 \\ 1.11000 & 1.86 & 2.73 \\ 1.67000 & 1.93 & 3.24 \\ 2.23000 & 1.96 & 3.66 \\ 3.35000 & 2.00 & 4.31 \\ 5.58000 & 2.03 & 5.21 \\ 11.10000 & 2.05 & 6.51 \\ 22.30000 & 2.06 & 7.89 \\ 33.50000 & 2.06 & 8.71 \\ 55.80000 & 2.06 & 9.75 \\ 78.20000 & 2.06 & 10.44 \\ 111.00000 & 2.06 & 11.15 \\ 223.00000 & 2.06 & 12.58 \\ & & \end{array}$

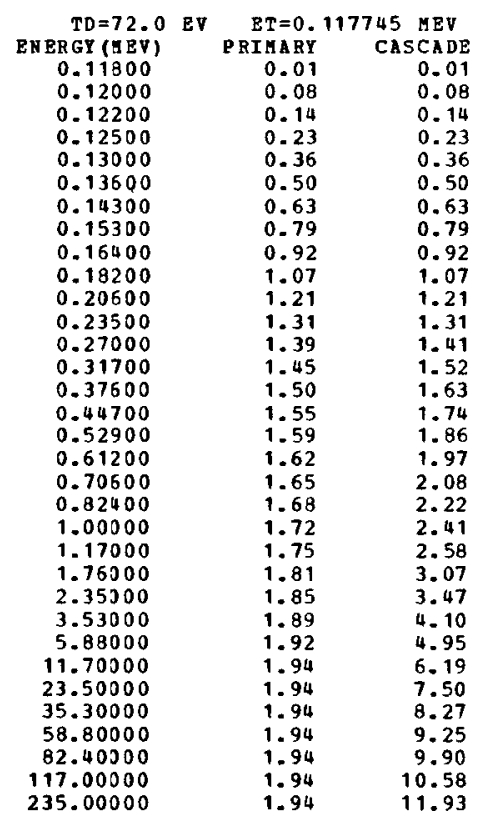


ATONIC DISPLACEHENT CROSS-SECTIONS (BABHS) BY FAST ELECTRONS IN HE

\begin{tabular}{ccr} 
TD=76.0 EV & \multicolumn{1}{c}{ ET $=0.123647$ HEV } \\
ENERGY (AEV) & PRIAARY & CASCADE \\
0.12400 & 0.01 & 0.01 \\
0.12600 & 0.07 & 0.07 \\
0.12800 & 0.12 & 0.12 \\
0.13200 & 0.23 & 0.23 \\
0.13700 & 0.34 & 0.34 \\
0.14300 & 0.45 & 0.45 \\
0.15000 & 0.57 & 0.57 \\
0.16000 & 0.70 & 0.70 \\
0.17300 & 0.84 & 0.84 \\
0.19100 & 0.97 & 0.97 \\
0.21600 & 1.10 & 1.10 \\
0.24700 & 1.20 & 1.20 \\
0.28400 & 1.27 & 1.30 \\
0.33300 & 1.34 & 1.40 \\
0.39500 & 1.39 & 1.51 \\
0.46900 & 1.44 & 1.62 \\
0.55600 & 1.48 & 1.74 \\
0.64200 & 1.51 & 1.84 \\
0.74100 & 1.54 & 1.96 \\
0.86500 & 1.58 & 2.09 \\
1.05000 & 1.62 & 2.28 \\
1.23000 & 1.65 & 2.44 \\
1.85000 & 1.72 & 2.92 \\
2.47000 & 1.75 & 3.31 \\
3.70000 & 1.79 & 3.90 \\
6.18000 & 1.82 & 4.73 \\
12.30000 & 1.84 & 5.90 \\
24.70000 & 1.84 & 7.14 \\
37.00000 & 1.84 & 7.87 \\
61.80000 & 1.84 & 8.81 \\
86.50000 & 1.84 & 9.42 \\
123.00000 & 1.84 & 10.06 \\
247.00000 & 1.84 & 11.34 \\
& &
\end{tabular}

\begin{tabular}{|c|c|c|}
\hline $\begin{array}{c}\text { TD=80.0 EV } \\
\text { ENBRY(HEV) } \\
0.13000 \\
0.13200 \\
0.13400 \\
0.13800 \\
0.14300 \\
0.15000 \\
0.15700 \\
0.16800 \\
0.18100 \\
0.20000 \\
0.22600 \\
0.25800 \\
0.29700 \\
0.34900 \\
0.41400 \\
0.49200 \\
0.58200 \\
0.67300 \\
0.77600 \\
0.90600 \\
1.10000 \\
1.29000 \\
1.94000 \\
2.58000 \\
3.88000 \\
6.47000 \\
12.90000 \\
25.80000 \\
38.80000 \\
64.70000 \\
90.60000 \\
129.00000 \\
258.00000\end{array}$ & $\begin{array}{l}\text { ET }=0.1 \\
\text { PRI IARY } \\
0.01 \\
0.06 \\
0.11 \\
0.20 \\
0.30 \\
0.42 \\
0.52 \\
0.64 \\
0.76 \\
0.89 \\
1.00 \\
1.10 \\
1.17 \\
1.24 \\
1.29 \\
1.34 \\
1.38 \\
1.42 \\
1.45 \\
1.49 \\
1.53 \\
1.56 \\
1.63 \\
1.66 \\
1.70 \\
1.73 \\
1.74 \\
1.75 \\
1.75 \\
1.75 \\
1.75 \\
1.75 \\
1.75\end{array}$ & $\begin{array}{r}493 \text { CAS } \\
\text { CADE } \\
0.01 \\
0.06 \\
0.11 \\
0.20 \\
0.30 \\
0.42 \\
0.52 \\
0.64 \\
0.76 \\
0.89 \\
1.00 \\
1.10 \\
1.20 \\
1.30 \\
1.40 \\
1.51 \\
1.63 \\
1.73 \\
1.85 \\
1.98 \\
2.16 \\
2.32 \\
2.79 \\
3.16 \\
3.73 \\
4.52 \\
5.64 \\
6.81 \\
7.52 \\
8.40 \\
8.98 \\
9.60 \\
10.81\end{array}$ \\
\hline
\end{tabular}

$T D=84.0 \quad B V$

$\begin{array}{crr}\text { TD }=84.0 & \text { EV } & \text { ET }=0.135287 \\ \text { ENERGY (MEV) } & \text { PEIAAR } & \text { CASCADE } \\ 0.13600 & 0.02 & 0.02 \\ 0.13700 & 0.04 & 0.04 \\ 0.14000 & 0.10 & 0.10 \\ 0.14400 & 0.18 & 0.18 \\ 0.15000 & 0.28 & 0.28 \\ 0.15600 & 0.37 & 0.37 \\ 0.16500 & 0.48 & 0.48 \\ 0.17500 & 0.58 & 0.58 \\ 0.18900 & 0.70 & 0.70 \\ 0.20900 & 0.81 & 0.81 \\ 0.23600 & 0.92 & 0.92 \\ 0.27000 & 1.01 & 1.02 \\ 0.31100 & 1.09 & 1.11 \\ 0.36500 & 1.15 & 1.21 \\ 0.43200 & 1.21 & 1.31 \\ 0.51400 & 1.26 & 1.42 \\ 0.60800 & 1.30 & 1.53 \\ 0.70300 & 1.34 & 1.64 \\ 0.81100 & 1.37 & 1.75 \\ 0.94700 & 1.41 & 1.88 \\ 1.14000 & 1.45 & 2.05 \\ 1.35000 & 1.48 & 2.21 \\ 2.02000 & 1.55 & 2.66 \\ 2.70000 & 1.59 & 3.02 \\ 4.05000 & 1.62 & 3.57 \\ 6.76000 & 1.65 & 4.33 \\ 13.50000 & 1.66 & 5.40 \\ 27.00000 & 1.66 & 6.52 \\ 40.50000 & 1.67 & 7.19 \\ 67.60000 & 1.67 & 8.03 \\ 94.70000 & 1.67 & 8.59 \\ 135.00000 & 1.67 & 9.18 \\ 270.00000 & 1.67 & 10.33\end{array}$

ATOHC DISPLACEHENT CROSS-SECTIONS (BARNS) BY PAST BLECTRONS IN HE

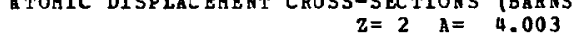

\begin{tabular}{ccc} 
TD $=88.0$ & EV & \multicolumn{1}{c}{ ET $=0.141030$ REV } \\
ENERGY (MEV) & PRIHARY & CASCADE \\
0.14200 & 0.02 & 0.02 \\
0.14300 & 0.04 & 0.04 \\
0.14600 & 0.09 & 0.09 \\
0.15000 & 0.16 & 0.16 \\
0.15600 & 0.25 & 0.25 \\
0.16300 & 0.34 & 0.34 \\
0.17200 & 0.44 & 0.44 \\
0.18300 & 0.54 & 0.54 \\
0.19700 & 0.64 & 0.64 \\
0.21800 & 0.75 & 0.75 \\
0.24600 & 0.85 & 0.85 \\
0.28200 & 0.94 & 0.95 \\
0.32400 & 1.01 & 1.03 \\
0.38000 & 1.07 & 1.13 \\
0.45100 & 1.13 & 1.23 \\
0.53500 & 1.18 & 1.33 \\
0.53400 & 1.23 & 1.45 \\
0.73300 & 1.27 & 1.55 \\
0.84600 & 1.30 & 1.66 \\
0.98700 & 1.34 & 1.79 \\
1.19000 & 1.38 & 1.95 \\
1.41000 & 1.41 & 2.12 \\
2.11000 & 1.48 & 2.55 \\
2.82000 & 1.51 & 2.90 \\
4.23000 & 1.55 & 3.43 \\
7.05000 & 1.57 & 4.15 \\
14.10000 & 1.59 & 5.19 \\
28.20000 & 1.59 & 6.26 \\
42.30000 & 1.59 & 6.89 \\
70.50000 & 1.59 & 7.70 \\
98.70000 & 1.59 & 8.23 \\
141.00000 & 1.59 & 8.79 \\
282.00000 & 1.59 & 9.89 \\
& &
\end{tabular}

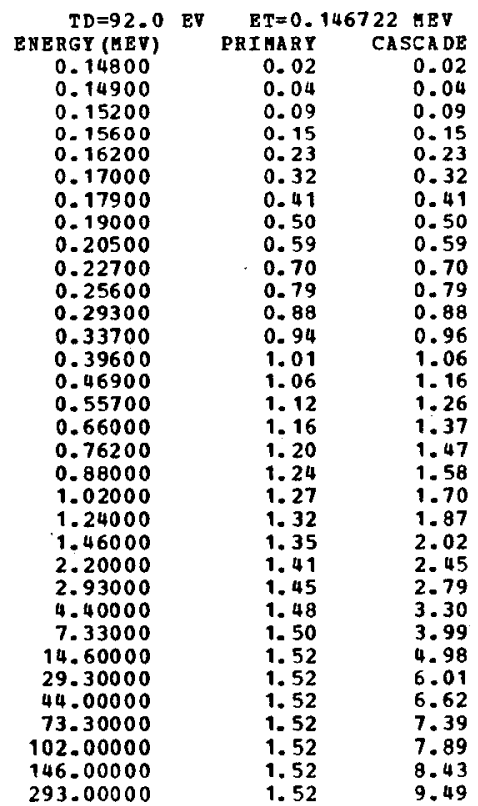

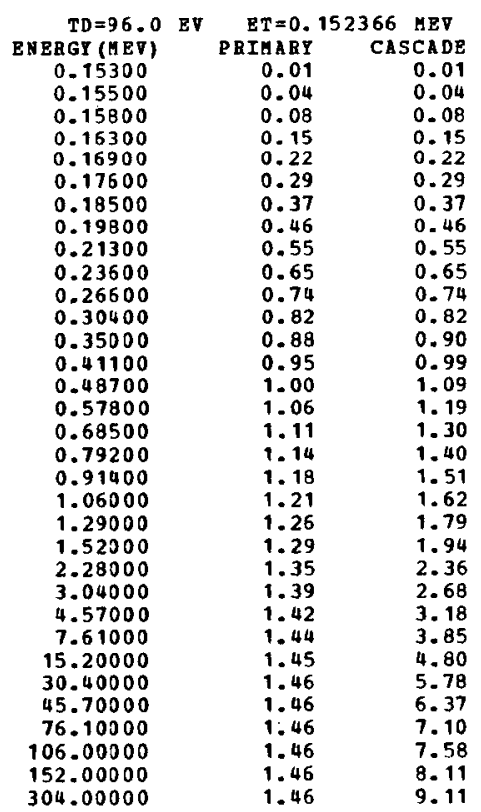


ATONIC DISPLAEELENT CROSS-SECTIONS (BARNS) BY PAST ELECTRONS IN BE

\begin{tabular}{crr} 
TD $=4.0$ & EV & \multicolumn{2}{c}{ ET $=0.016169$ HEV } \\
ENERG (MEV) & PRIMARY & CASCADE \\
0.01630 & 7.81 & 7.81 \\
0.01640 & 13.62 & 13.62 \\
0.01680 & 35.53 & 35.53 \\
0.01730 & 60.11 & 60.11 \\
0.01790 & 86.03 & 86.03 \\
0.01870 & 115.40 & 115.40 \\
0.01970 & 165.31 & 145.31 \\
0.02100 & 175.33 & 175.33 \\
0.02260 & 202.08 & 202.08 \\
0.02500 & 227.76 & 227.76 \\
0.02820 & 245.35 & 245.35 \\
0.03230 & 252.80 & 252.83 \\
0.03710 & 251.14 & 253.59 \\
0.04360 & 241.70 & 250.17 \\
0.05170 & 226.75 & 243.07 \\
0.06140 & 209.30 & 233.60 \\
0.07270 & 191.61 & 223.07 \\
0.08400 & 176.89 & 213.67 \\
0.09700 & 163.03 & 204.30 \\
0.11300 & 149.43 & 194.67 \\
0.13700 & 134.11 & 183.31 \\
0.16100 & 122.87 & 174.69 \\
0.24200 & 100.53 & 157.38 \\
0.32300 & 89.21 & 149.34 \\
0.48500 & 78.14 & 144.01 \\
0.80800 & 69.97 & 146.66 \\
1.61000 & 64.90 & 164.20 \\
3.23000 & 63.07 & 193.70 \\
4.85000 & 62.64 & 214.43 \\
8.08000 & 62.37 & 242.67 \\
11.30000 & 62.29 & 262.10 \\
16.10000 & 62.24 & 283.09 \\
32.30000 & 62.20 & 325.23 \\
64.60000 & 62.18 & 367.78 \\
113.00000 & 62.18 & 402.33 \\
161.00000 & 62.18 & 424.25
\end{tabular}

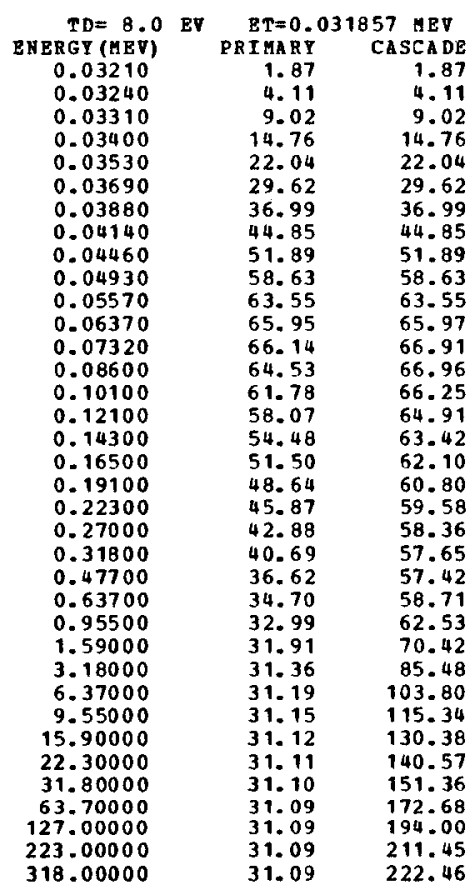

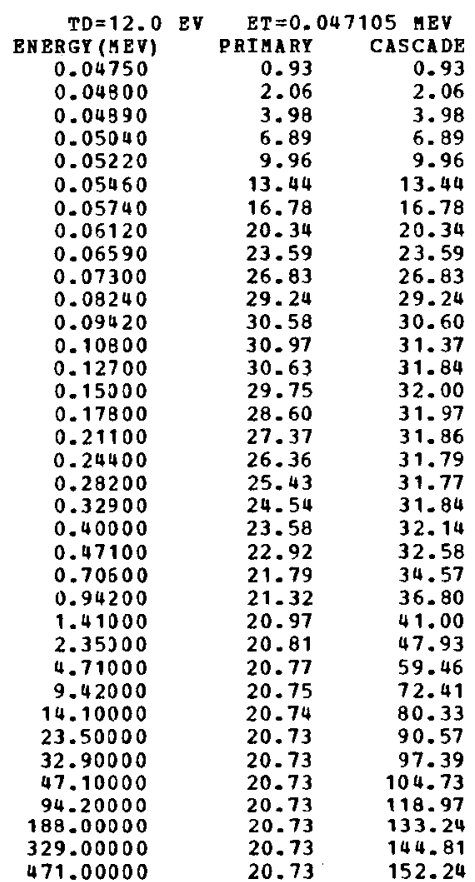

ATOHIC DISPLACEHEN ChOSS-SECTIONS (BARS) BY PAST ELECTRONS IN BE $=4 \quad \mathrm{~A}=9.013$

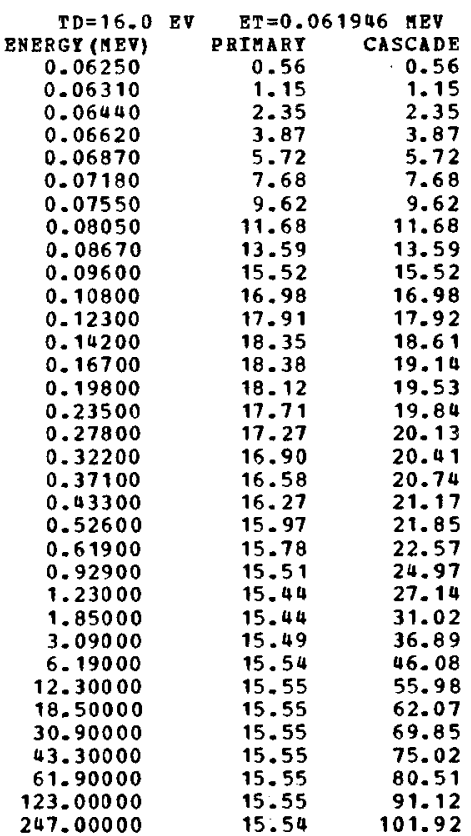

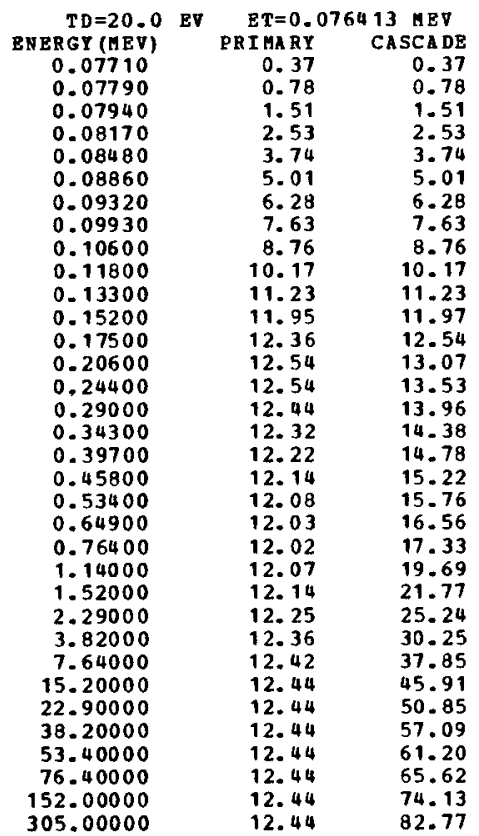

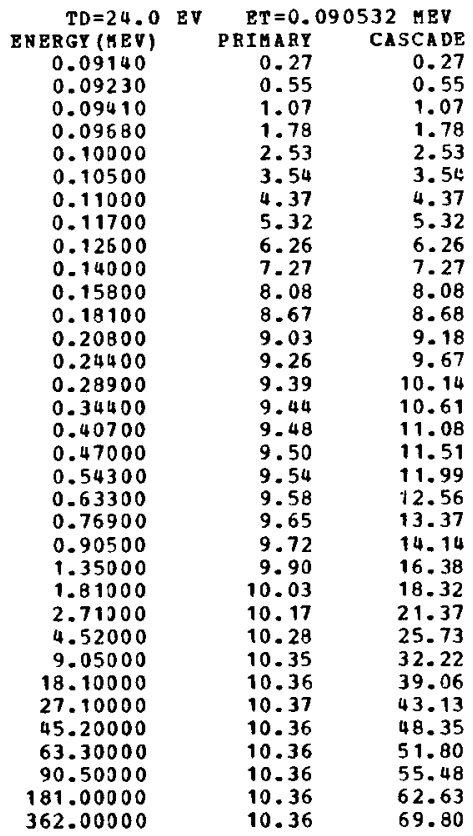


A TOMIC DISPLATEHENT CROSS-SBCTIONS (BARNS) BY FAST BLECTROHS IN BE

\begin{tabular}{|c|c|c|c|}
\hline $\mathrm{TD}=28.0$ & EV & $\begin{aligned} E T=0.1 \\
P R T M A R Y\end{aligned}$ & $\begin{array}{l}4327 \text { MEV } \\
\text { CASCADE }\end{array}$ \\
\hline 0.10500 & & 0.14 & 0.14 \\
\hline 0.10600 & & 0.34 & 0.34 \\
\hline 0.10800 & & 0.71 & 0.71 \\
\hline 0.11100 & & 1.23 & 1.23 \\
\hline 0.11500 & & 1.85 & 1.85 \\
\hline 0.12100 & & 2.64 & 2.64 \\
\hline 0.12700 & & 3.29 & 3.29 \\
\hline 0.13500 & & 4.00 & 4.00 \\
\hline 0.14600 & & 4.75 & 4.75 \\
\hline 0.16100 & & 5.47 & 5.47 \\
\hline 0.18200 & & 6.13 & 6.13 \\
\hline 0.20800 & & 6.62 & 6.64 \\
\hline $\begin{array}{l}0.23900 \\
0.28100\end{array}$ & & $\begin{array}{l}6.97 \\
7.23\end{array}$ & 7.09 \\
\hline $\begin{array}{l}0.28100 \\
0.33300\end{array}$ & & 7.41 & $\begin{array}{l}1.50 \\
8.02\end{array}$ \\
\hline $\begin{array}{l}0.33300 \\
0.39600\end{array}$ & & 7.55 & 8.49 \\
\hline 0.46900 & & 7.66 & 8.97 \\
\hline 0.54200 & & 7.76 & 9.42 \\
\hline 0.62500 & & 7.85 & 9.90 \\
\hline 0.73000 & & 7.95 & 10.47 \\
\hline 0.88600 & & 8.07 & 11.26 \\
\hline 1.04000 & & 8.17 & 11.98 \\
\hline 1.56000 & & 8.41 & 14.11 \\
\hline 2.08000 & & 8.55 & 15.86 \\
\hline 3.12000 & & 8.70 & 18.61 \\
\hline 5.21000 & & 8.81 & 22.46 \\
\hline 10.40000 & & 8.87 & 28.09 \\
\hline 20.80000 & & 8.88 & 33.99 \\
\hline .20000 & & 8.88 & 37.51 \\
\hline 00000 & & $\begin{array}{l}8.88 \\
8.88\end{array}$ & $\begin{array}{l}42.00 \\
44.97\end{array}$ \\
\hline 00000 & & 8.88 & 48.10 \\
\hline 08.00000 & & 8.88 & 54.23 \\
\hline
\end{tabular}

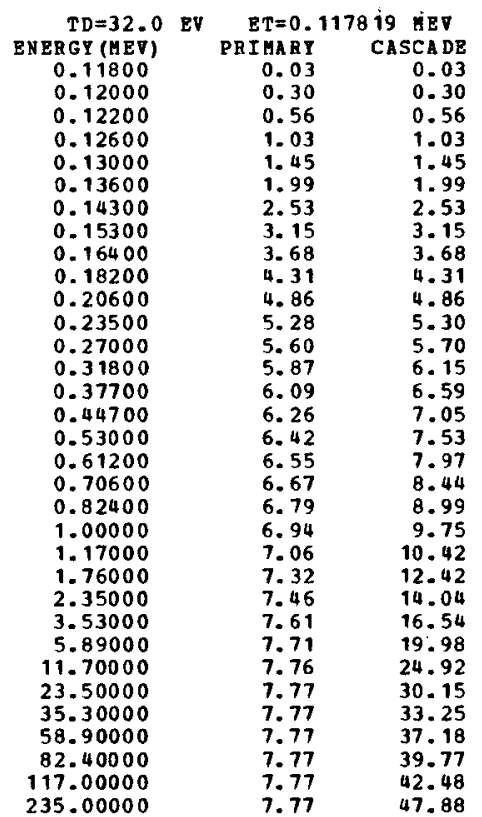

\begin{tabular}{ccc} 
TD $=36.0$ EV & \multicolumn{2}{c}{ ET $=0.131028$ MEV } \\
ENERGI (HEV) & PRISARY & CASCADE \\
0.13200 & 0.10 & 0.10 \\
0.13300 & 0.19 & 0.19 \\
0.13600 & 0.47 & 0.47 \\
0.14000 & 0.81 & 0.81 \\
0.14500 & 1.19 & 1.19 \\
0.15100 & 1.58 & 1.58 \\
0.15900 & 2.03 & 2.03 \\
0.17000 & 2.53 & 2.53 \\
0.18300 & 2.99 & 2.99 \\
0.20300 & 3.51 & 3.51 \\
0.22900 & 3.96 & 3.96 \\
0.26200 & 4.35 & 4.36 \\
0.30100 & 4.65 & 4.74 \\
0.35300 & 4.91 & 5.14 \\
0.41900 & 5.14 & 5.57 \\
0.49700 & 5.34 & 6.02 \\
0.58900 & 5.52 & 6.49 \\
0.68100 & 5.66 & 6.92 \\
0.78600 & 5.80 & 7.37 \\
0.91700 & 5.94 & 7.90 \\
1.11000 & 6.10 & 8.62 \\
1.31000 & 6.23 & 9.29 \\
1.96000 & 6.49 & 11.14 \\
2.62000 & 6.63 & 12.63 \\
3.93000 & 6.76 & 14.91 \\
6.55000 & 6.85 & 18.01 \\
13.10000 & 6.90 & 22.49 \\
26.20000 & 6.91 & 27.13 \\
39.30000 & 6.91 & 29.88 \\
65.50000 & 6.91 & 33.37 \\
91.70000 & 6.91 & 35.68 \\
131.00300 & 6.91 & 38.13 \\
262.00000 & 6.91 & 42.90 \\
& &
\end{tabular}

ATOMIC DISPLACEMENT CROSS-SECTIONS (BARNS) BY FAST ELECTRONS IN BE

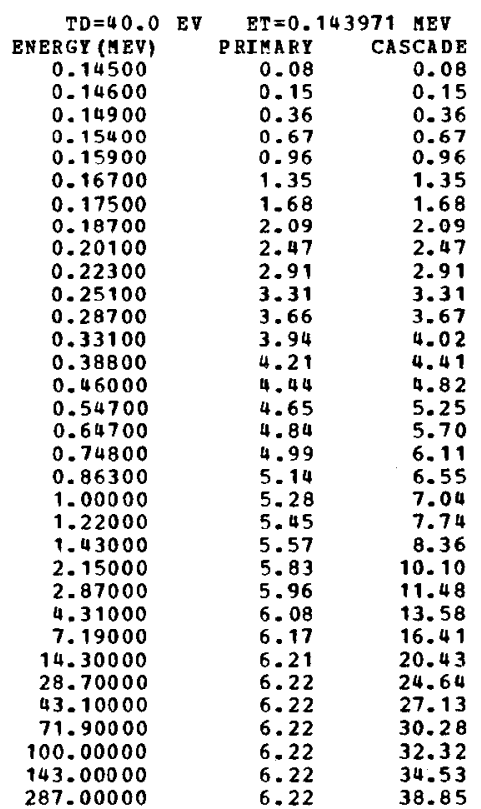

\begin{tabular}{ccc} 
TD $=44.0$ & EV & \multicolumn{1}{c}{ ET=0.156663 MEV } \\
BNERGY (HEV) & PRIMARY & CASCADE \\
0.15800 & 0.08 & 0.08 \\
0.15900 & 0.13 & 0.13 \\
0.16200 & 0.29 & 0.29 \\
0.16700 & 0.54 & 0.54 \\
0.17300 & 0.80 & 0.80 \\
0.18100 & 1.10 & 1.10 \\
0.19100 & 1.43 & 1.43 \\
0.20300 & 1.75 & 1.75 \\
0.21900 & 2.09 & 2.09 \\
0.24200 & 2.46 & 2.46 \\
0.27400 & 2.83 & 2.83 \\
0.31300 & 3.14 & 3.16 \\
0.36000 & 3.41 & 3.48 \\
0.42200 & 3.67 & 3.84 \\
0.50100 & 3.91 & 4.24 \\
0.59500 & 4.12 & 4.66 \\
0.70400 & 4.31 & 5.09 \\
0.81400 & 4.47 & 5.49 \\
0.93900 & 4.61 & 5.91 \\
1.09000 & 4.76 & 6.38 \\
1.33000 & 4.93 & 7.05 \\
1.56000 & 5.05 & 7.63 \\
2.34000 & 5.29 & 9.26 \\
3.13000 & 5.42 & 10.56 \\
4.69000 & 5.53 & 12.48 \\
7.83000 & 5.61 & 15.09 \\
15.60000 & 5.65 & 18.78 \\
31.30000 & 5.65 & 22.61 \\
46.90000 & 5.65 & 24.86 \\
78.30000 & 5.65 & 27.74 \\
109.00000 & 5.65 & 29.60 \\
156.00000 & 5.65 & 31.61 \\
313.00000 & 5.65 & 35.54 \\
& &
\end{tabular}

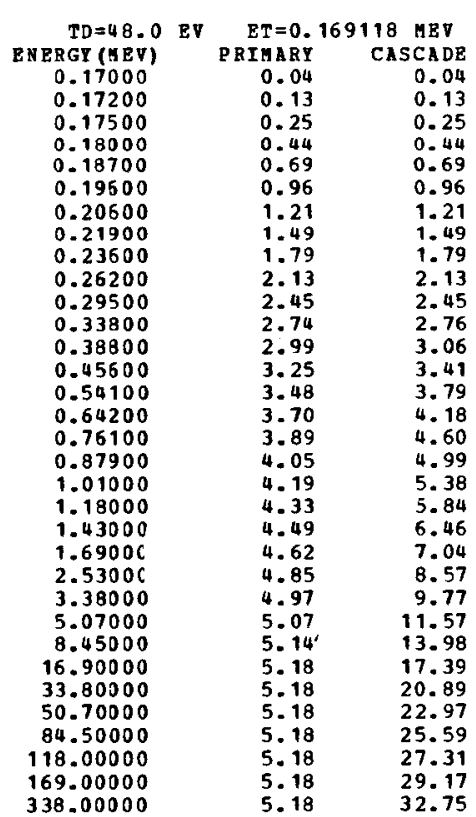


ATOHIC DISPLAZEHENT CROSS-SECTIONS (BARNS) BY PAST ELECTRONS IN BE $=4 \quad A=9.013$

$\begin{array}{rrr}\begin{array}{c}\text { TD }=52.0 \\ \text { ENERGY (HEV) }\end{array} & \begin{array}{r}\text { ET }=0.181349 \text { MEV } \\ \text { PRIAABY }\end{array} \\ 0.18300 & 0.06 & 0.06 \\ 0.18400 & 0.09 & 0.09 \\ 0.18800 & 0.23 & 0.23 \\ 0.19400 & 0.41 & 0.41 \\ 0.20100 & 0.60 & 0.60 \\ 0.21000 & 0.82 & 0.82 \\ 0.22100 & 1.05 & 1.05 \\ 0.23500 & 1.30 & 1.30 \\ 0.25300 & 1.55 & 1.55 \\ 0.28100 & 1.86 & 1.86 \\ 0.31700 & 2.15 & 2.15 \\ 0.36200 & 2.42 & 2.44 \\ 0.41700 & 2.67 & 2.73 \\ 0.48900 & 2.91 & 3.06 \\ 0.58000 & 3.14 & 3.42 \\ 0.68900 & 3.35 & 3.80 \\ 0.81600 & 3.55 & 4.21 \\ 0.94300 & 3.70 & 4.57 \\ 1.08000 & 3.84 & 4.94 \\ 1.26000 & 3.98 & 5.38 \\ 1.54000 & 4.14 & 6.00 \\ 1.81000 & 4.25 & 6.53 \\ 2.72000 & 4.48 & 7.99 \\ 3.62000 & 4.58 & 9.10 \\ 5.44000 & 4.68 & 10.79 \\ 9.05000 & 4.75 & 13.02 \\ 18.10000 & 4.78 & 16.18 \\ 36.20000 & 4.78 & 19.42 \\ 54.40000 & 4.78 & 21.34 \\ 90.60000 & 4.78 & 23.76 \\ 126.00000 & 4.78 & 25.33 \\ 181.00000 & 4.78 & 27.06\end{array}$

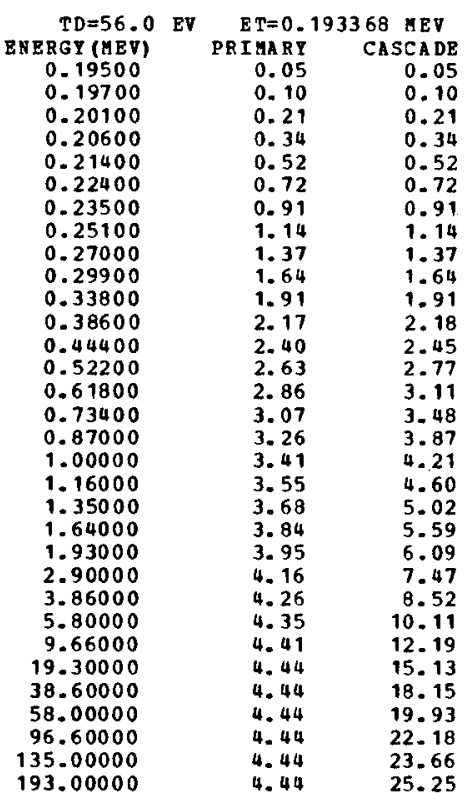

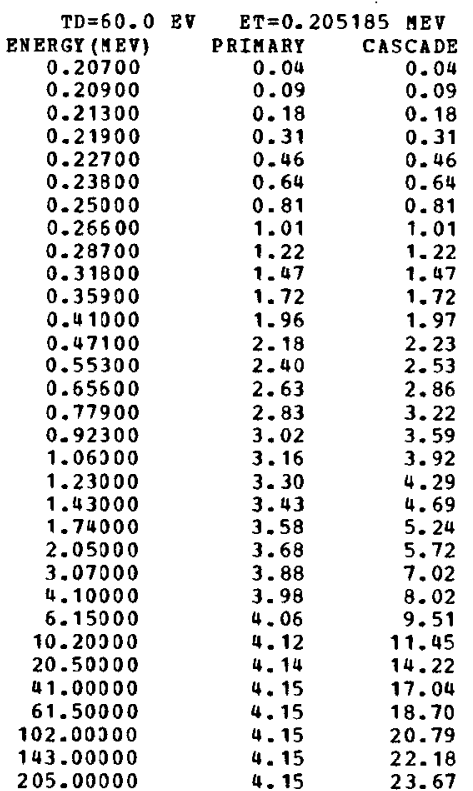

ATOAIC DISPLACEHENT CROSS-SECTIONS (BARNS) BY PAST ELECTRONS IN BE

$T D=64.0 \mathrm{EV} \quad \mathrm{BT}=0.216810 \mathrm{HEV}$

$\begin{array}{rrr}\begin{array}{r}\text { TD }=64.0 \\ \text { ENERGY }\end{array} \text { (HEV) } & \begin{array}{r}\text { ET }=0.216810 \\ \text { PRTMARY }\end{array} & \text { CASCADE } \\ 0.21800 & 0.02 & 0.02 \\ 0.22100 & 0.08 & 0.08 \\ 0.22500 & 0.16 & 0.16 \\ 0.23100 & 0.26 & 0.26 \\ 0.24000 & 0.41 & 0.41 \\ 0.25100 & 0.56 & 0.56 \\ 0.26400 & 0.72 & 0.72 \\ 0.28100 & 0.90 & 0.90 \\ 0.30300 & 1.09 & 1.09 \\ 0.33600 & 1.32 & 1.32 \\ 0.37900 & 1.55 & 1.55 \\ 0.43300 & 1.78 & 1.79 \\ 0.49800 & 1.99 & 2.04 \\ 0.58500 & 2.21 & 2.33 \\ 0.69300 & 2.43 & 2.65 \\ 0.82300 & 2.63 & 2.99 \\ 0.97500 & 2.81 & 3.35 \\ 1.12000 & 2.95 & 3.67 \\ 1.30000 & 3.09 & 4.02 \\ 1.51000 & 3.21 & 4.40 \\ 1.84000 & 3.35 & 4.93 \\ 2.16000 & 3.45 & 5.39 \\ 3.25000 & 3.64 & 6.64 \\ 4.33000 & 3.73 & 7.58 \\ 6.50000 & 3.81 & 8.98 \\ 10.80000 & 3.86 & 10.82 \\ 21.60000 & 3.88 & 13.41 \\ 43.30000 & 3.89 & 16.06 \\ 65.00000 & 3.89 & 17.62 \\ 108.00000 & 3.89 & 19.58 \\ 151.00000 & 3.89 & 20.88 \\ 216.00000 & 3.89 & 22.27 \\ & & \end{array}$

$T D=68.0 \mathrm{EV} E T=0.228252 \mathrm{MEV}$

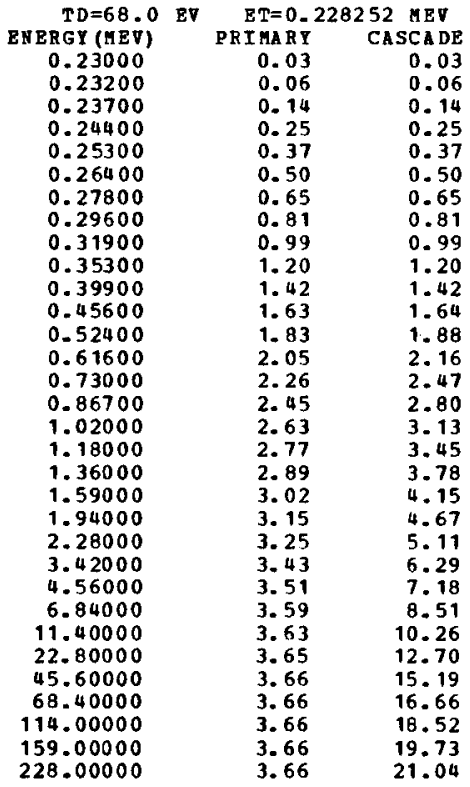

$T D=72.0 \mathrm{EV} \quad \mathrm{ET}=0.239520 \mathrm{MEV}$

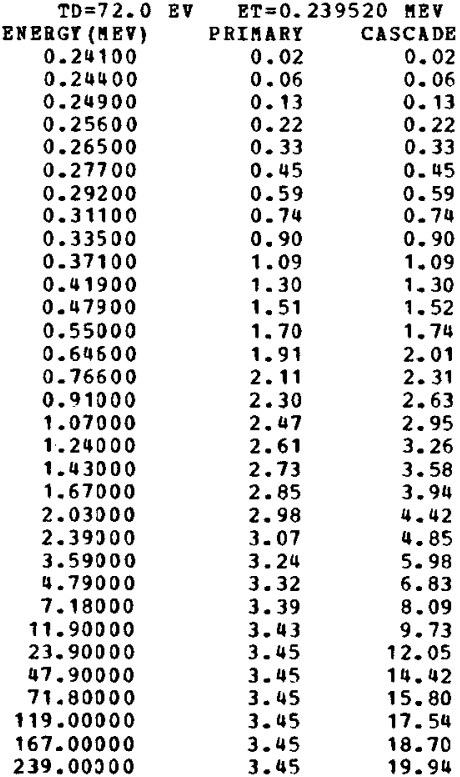


ATONIC DISPLAZEHENT CROSS-SECTIONS (BARNS) BY FAST ELECTBONS IN BE $\mathrm{z}=4 \mathrm{~A}=9.013$

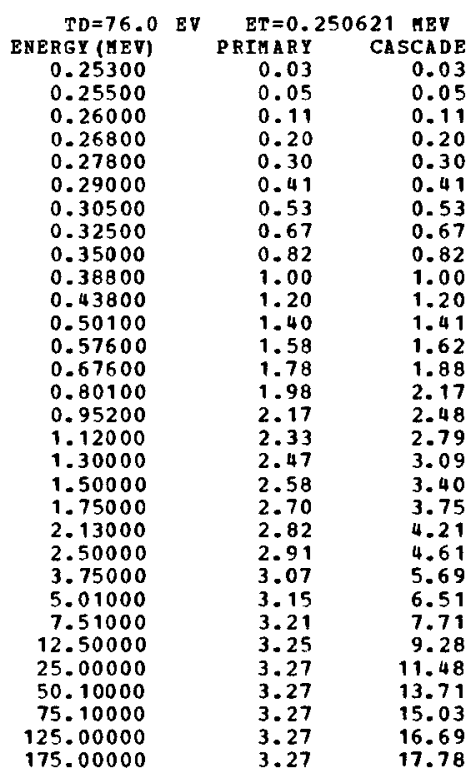

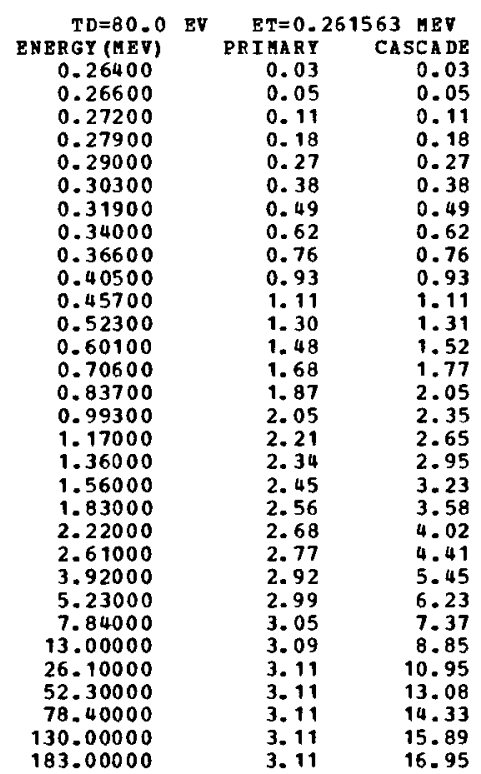

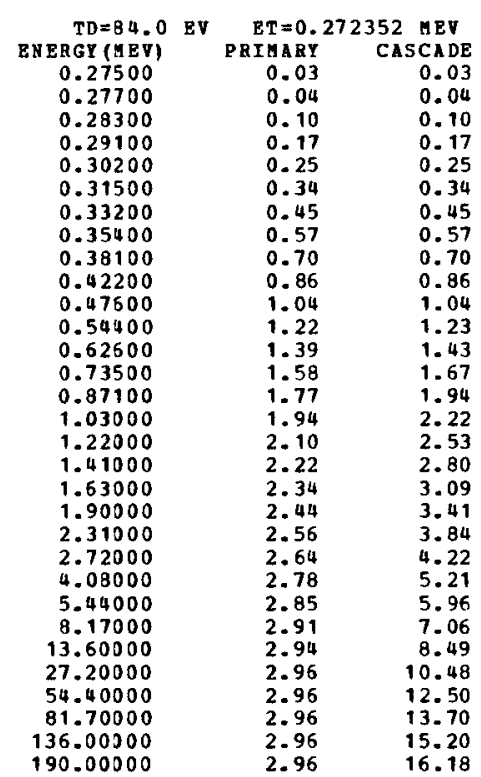

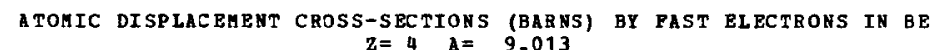

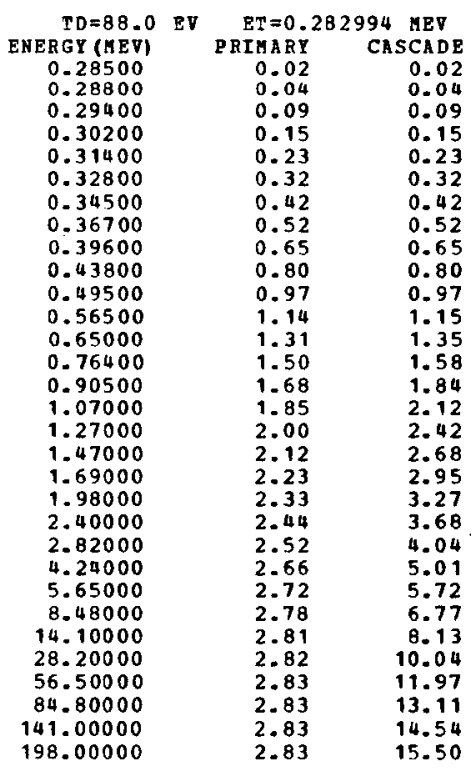

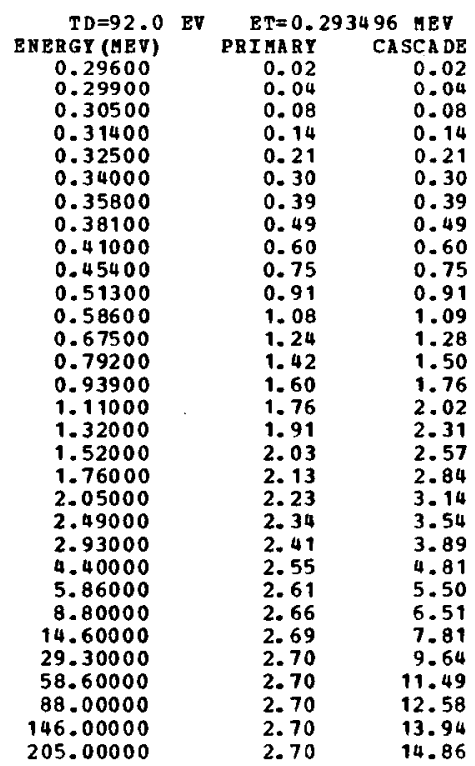

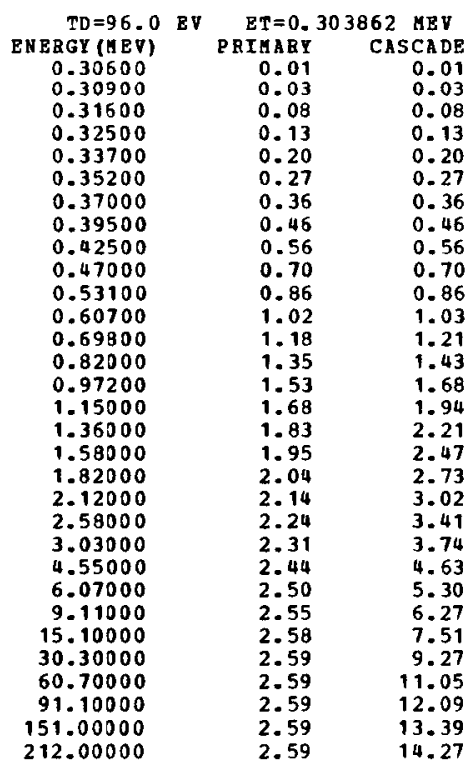


ATOMIC DISPlaCEREN CROSS-SECTIONS (BARNS) BY FAST BLECTHONS IN C

\begin{tabular}{rrr} 
TD $=4.0$ & EV & \multicolumn{1}{c}{ ET $=0.021439$ MEV } \\
PNERGY(AEV) & PRIMARY & CASCADE \\
0.02160 & 9.27 & 9.27 \\
0.02180 & 20.42 & 20.42 \\
0.02220 & 41.55 & 41.55 \\
0.02290 & 75.05 & 75.05 \\
0.02370 & 108.58 & 108.58 \\
0.02480 & 147.67 & 147.67 \\
0.02610 & 185.31 & 185.31 \\
0.02780 & 223.58 & 223.58 \\
0.03000 & 259.43 & 259.43 \\
0.03320 & 292.79 & 292.79 \\
0.03750 & 316.10 & 316.10 \\
0.04280 & 326.33 & 326.38 \\
0.04930 & 325.34 & 328.77 \\
0.05780 & 314.84 & 326.10 \\
0.06860 & 297.20 & 318.84 \\
0.08140 & 276.54 & 308.75 \\
0.09640 & 255.39 & 297.27 \\
0.11100 & 238.20 & 287.27 \\
0.12800 & 221.76 & 277.22 \\
0.15000 & 204.91 & 266.53 \\
0.18200 & 186.67 & 254.67 \\
0.21400 & 173.36 & 246.03 \\
0.32100 & 147.43 & 230.65 \\
0.42800 & 134.48 & 225.67 \\
0.64300 & 122.05 & 227.19 \\
1.07000 & 113.23 & 242.31 \\
2.14000 & 107.96 & 282.46 \\
4.28000 & 106.09 & $\mathbf{3 3 8 . 1 9}$ \\
6.43000 & 105.61 & 375.37 \\
10.70000 & 105.30 & 424.61 \\
15.00000 & 105.20 & 458.34 \\
21.40000 & 105.13 & 494.40 \\
42.80000 & 105.08 & 565.80 \\
85.70000 & 105.06 & 638.06 \\
150.00000 & 105.05 & 696.59 \\
214.00000 & 105.05 & 733.80
\end{tabular}

\begin{tabular}{rrr} 
TD= 8.0 & EV & \multicolumn{2}{c}{ ET $=0.042048$ MEV } \\
ENERGY(HEV) & PRIMARY & CASCADE \\
0.04240 & 2.63 & 2.63 \\
0.04280 & 5.52 & 5.52 \\
0.04370 & 11.64 & 11.64 \\
0.04490 & 19.07 & 19.07 \\
0.04660 & 28.34 & 28.34 \\
0.04870 & 38.05 & 38.05 \\
0.05120 & 47.56 & 47.56 \\
0.05460 & 57.71 & 57.71 \\
0.05880 & 66.93 & 66.93 \\
0.06510 & 75.05 & 76.05 \\
0.07350 & 82.79 & 82.79 \\
0.08400 & 86.43 & 86.48 \\
0.09670 & 87.35 & 88.45 \\
0.11300 & 86.12 & 89.40 \\
0.13400 & 83.23 & 89.44 \\
0.15900 & 79.55 & 88.89 \\
0.18900 & 75.63 & 88.06 \\
0.21800 & 72.48 & 87.38 \\
0.25200 & 69.49 & 86.81 \\
0.29400 & 66.61 & 86.44 \\
0.35700 & 63.48 & 86.48 \\
0.42000 & 51.29 & 87.02 \\
0.63000 & 57.33 & 90.69 \\
0.84000 & 55.58 & 95.43 \\
1.26000 & 54.12 & 105.07 \\
2.10000 & 53.27 & 121.58 \\
4.20000 & 52.85 & 149.83 \\
8.40000 & 52.68 & 182.19 \\
12.60000 & 52.62 & 202.19 \\
21.00000 & 52.57 & 228.02 \\
29.40000 & 52.55 & 245.28 \\
42.00000 & 52.54 & 263.72 \\
84.00000 & 52.53 & 299.78 \\
168.00000 & 52.53 & $\mathbf{3 3 6} .02$ \\
294.00000 & 52.52 & $\mathbf{3 6 5} .35$ \\
420.00000 & 52.52 & $\mathbf{3 8 4} .05$ \\
& &
\end{tabular}

\begin{tabular}{rrr} 
TD=12.0 & \multicolumn{1}{c}{ ET $=0.061915$ MEV } \\
ENERGY (HEV) & PRIMARY & CASCADE \\
0.06250 & 1.34 & 1.34 \\
0.06310 & 2.66 & 2.66 \\
0.06430 & 5.17 & 5.17 \\
0.06620 & 8.79 & 8.79 \\
0.06870 & 12.98 & 12.98 \\
0.07180 & 17.41 & 17.41 \\
0.07550 & 21.78 & 21.78 \\
0.08040 & 26.37 & 26.37 \\
0.08560 & 30.71 & 30.71 \\
0.09590 & 35.11 & 35.11 \\
0.10800 & 38.49 & 38.49 \\
0.12300 & 40.61 & 40.65 \\
0.14200 & 41.65 & 42.24 \\
0.16700 & 41.77 & 43.49 \\
0.19800 & 41.21 & 44.41 \\
0.23500 & 40.32 & 45.15 \\
0.27800 & 39.34 & 45.84 \\
0.32100 & 38.52 & 46.48 \\
0.37100 & 37.77 & 47.25 \\
0.43300 & 37.08 & 48.24 \\
0.52500 & 36.38 & 49.82 \\
0.61900 & 35.93 & 51.45 \\
0.92800 & 35.27 & 56.88 \\
1.23000 & 35.09 & 61.83 \\
1.85000 & 35.02 & 70.59 \\
3.09000 & 35.06 & 83.84 \\
6.19000 & 35.10 & 104.57 \\
12.30000 & 35.07 & 126.88 \\
18.50000 & 35.05 & 140.60 \\
30.90000 & 35.04 & 158.13 \\
43.30000 & 35.03 & 169.76 \\
61.90000 & 35.02 & 182.15 \\
123.00000 & 35.02 & 206.04 \\
247.00000 & 35.02 & 230.38 \\
433.00000 & 35.02 & 250.00 \\
619.00000 & 35.01 & 262.50 \\
& &
\end{tabular}

ATOMIC DISPLACEnENT CROSS-SECTIONS (BARN) BP FAST gLECTRONS IN C

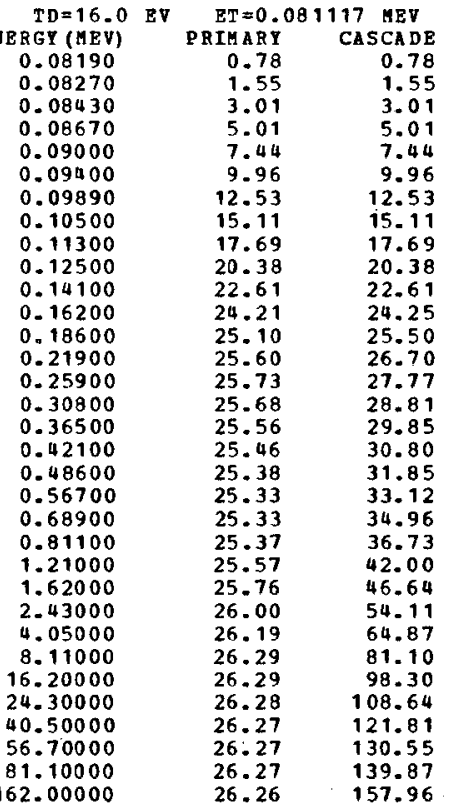

\begin{tabular}{rrr} 
TD=20.0 BV & \multicolumn{2}{c}{ ET=0.099715 HEV } \\
ENERGY(HEV) & PRIMAT & CASCADE \\
0.10000 & 0.15 & 0.15 \\
0.10100 & 0.67 & 0.67 \\
0.10300 & 1.66 & 1.66 \\
0.10600 & 3.02 & 3.02 \\
0.11000 & 4.62 & 4.62 \\
0.11500 & 6.33 & 6.33 \\
0.12100 & 8.06 & 8.06 \\
0.12900 & 9.91 & 9.91 \\
0.13900 & 11.68 & 11.68 \\
0.15400 & 13.56 & 13.56 \\
0.17400 & 15.17 & 15.17 \\
0.19900 & 16.38 & 16.41 \\
0.22900 & 17.20 & 17.50 \\
0.26900 & 17.80 & 18.59 \\
0.31900 & 18.19 & 19.67 \\
0.37800 & 18.46 & 20.73 \\
0.44800 & 18.68 & 21.85 \\
0.51800 & 18.85 & 22.87 \\
0.59800 & 19.02 & 23.98 \\
0.69800 & 19.21 & 25.28 \\
0.84700 & 19.45 & 27.10 \\
0.99700 & 19.66 & 28.80 \\
1.49000 & 20.13 & 33.69 \\
1.99000 & 20.42 & 37.79 \\
2.99000 & 20.71 & 44.26 \\
4.98000 & 20.93 & 53.27 \\
9.97000 & 21.02 & 66.57 \\
19.90000 & 21.03 & 80.48 \\
29.90000 & 21.02 & 88.83 \\
49.80000 & 21.02 & 99.39 \\
69.80000 & 21.01 & 106.42 \\
99.70000 & 21.01 & 113.87 \\
199.00000 & 21.01 & 128.33 \\
& &
\end{tabular}

\begin{tabular}{crr} 
TD=24.0 EV & \multicolumn{1}{c}{ ET $=0.117763$ MEV } \\
ENERGY (1MEV) & PRIHARY & CASCADE \\
0.11800 & 0.08 & 0.08 \\
0.12000 & 0.69 & 0.69 \\
0.12200 & 1.27 & 1.27 \\
0.12500 & 2.33 & 2.33 \\
0.13000 & 3.28 & 3.28 \\
0.13600 & 4.52 & 4.52 \\
0.14300 & 5.73 & 5.73 \\
0.15300 & 7.14 & 7.14 \\
0.16400 & 8.34 & 8.34 \\
0.18200 & 9.77 & 9.77 \\
0.20600 & 11.04 & 11.04 \\
0.23500 & 12.01 & 12.05 \\
0.27000 & 12.75 & 12.98 \\
0.31700 & 13.38 & 13.99 \\
0.37500 & 13.88 & 15.02 \\
0.44700 & 14.29 & 16.08 \\
0.52900 & 14.65 & 17.18 \\
0.61200 & 14.94 & 18.19 \\
0.70500 & 15.21 & 19.26 \\
0.82400 & 15.49 & 20.52 \\
1.00000 & 15.83 & 22.25 \\
1.17000 & 16.08 & 23.78 \\
1.76000 & 16.64 & 28.31 \\
2.35000 & 16.94 & 31.96 \\
3.53000 & 17.23 & 37.62 \\
5.88000 & 17.43 & 45.36 \\
11.70000 & 17.52 & 56.53 \\
23.50000 & 17.52 & 68.30 \\
35.30000 & 17.52 & 75.29 \\
58.80000 & 17.51 & 84.11 \\
82.40000 & 17.51 & 89.97 \\
117.00000 & 17.51 & 96.08 \\
235.00000 & 17.51 & 108.25
\end{tabular}


atonic displacement CROSS-SECTIONS (BARNS) BY PAST ELECTRONS IN C

\begin{tabular}{|c|c|c|c|}
\hline \multicolumn{4}{|l|}{$\begin{aligned} & T D=28.0 \\
&\end{aligned}$} \\
\hline $\begin{array}{c}\text { ARGY (NEV) } \\
0.13600\end{array}$ & & PHIAR & Castave \\
\hline 0.13800 & & 0.54 & 0.54 \\
\hline 0.14000 & & 0.91 & 0.91 \\
\hline 0.14400 & & 1.61 & 1.61 \\
\hline 0.15000 & & 2.54 & 2.54 \\
\hline 0.15600 & & 3.35 & 3.35 \\
\hline 0.16500 & & 4.37 & 4.37 \\
\hline 0.17500 & & 5.31 & 5.31 \\
\hline 0.18900 & & 6.34 & 6.34 \\
\hline 0.20900 & & $\begin{array}{l}7.43 \\
8.45\end{array}$ & 7.43 \\
\hline $\begin{array}{l}0.23600 \\
0.27000\end{array}$ & & $\begin{array}{l}8.45 \\
9.31\end{array}$ & 9.34 \\
\hline 0.31100 & & 10.00 & 10.19 \\
\hline 0.36500 & & 10.61 & 11.12 \\
\hline 0.43200 & & 11.15 & 12.08 \\
\hline 0.51400 & & 11.62 & 13.10 \\
\hline $\begin{array}{l}0.60800 \\
0.70300\end{array}$ & & $\begin{array}{l}12.04 \\
12.38\end{array}$ & $\begin{array}{l}14.15 \\
15.13\end{array}$ \\
\hline 0.81100 & & 12.69 & 16.15 \\
\hline 0.94700 & & 13.01 & 17.34 \\
\hline 1.15000 & & 13.37 & 18.97 \\
\hline 1.35000 & & 13.65 & 20.43 \\
\hline 2.02000 & & 14.20 & 24.52 \\
\hline 2.70000 & & 14.49 & 27.81 \\
\hline 4.05000 & & 14.76 & 32.81 \\
\hline 13.50000 & & 15.01 & $\begin{array}{l}39.61 \\
49.34\end{array}$ \\
\hline 27.00000 & & 15.02 & 59.42 \\
\hline 40.50000 & & 15.01 & 65.41 \\
\hline 67.60000 & & 15.01 & 73.01 \\
\hline 94.70000 & & 15.01 & 78.04 \\
\hline $\begin{array}{l}135.00000 \\
270.00000\end{array}$ & & $\begin{array}{l}15.01 \\
15.01\end{array}$ & $\begin{array}{l}83.34 \\
93.71\end{array}$ \\
\hline & & & \\
\hline
\end{tabular}

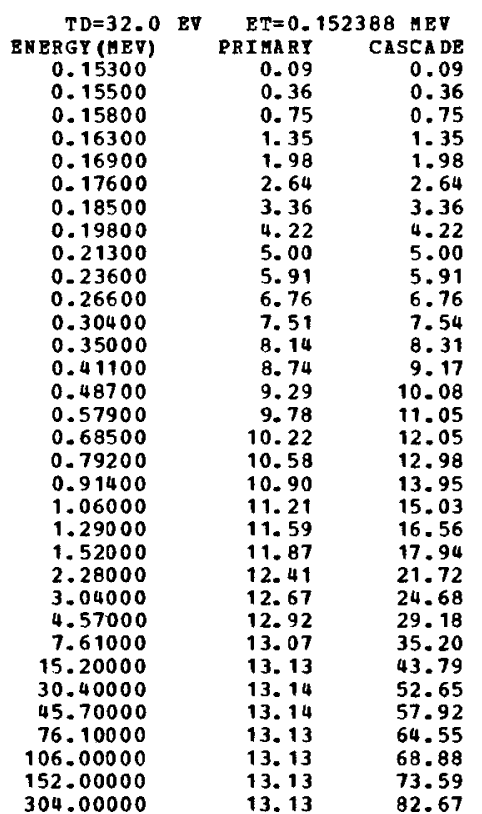

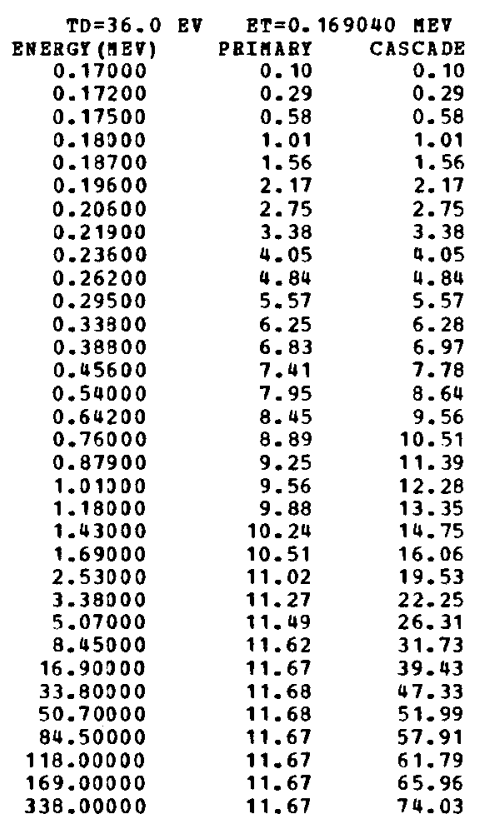

ATOHIC DISPLACEMENT CROSS-SECTIONS (BARNS) BY FAST ELECTRONS IN C $=6 \quad \mathrm{~A}=12.011$

\begin{tabular}{crr}
$\begin{array}{c}\text { TD }=40.0 \\
\text { ENERGY (MEV) }\end{array}$ & \multicolumn{2}{c}{ ET $=0.185293$ REV } \\
0.18700 & 0.13 & 0.13 \\
0.18800 & 0.20 & 0.20 \\
0.19200 & 0.48 & 0.48 \\
0.19800 & 0.87 & 0.87 \\
0.20500 & 1.28 & 1.28 \\
0.21400 & 1.75 & 1.75 \\
0.22600 & 2.28 & 2.28 \\
0.24000 & 2.80 & 2.80 \\
0.25900 & 3.38 & 3.38 \\
0.28700 & 4.05 & 4.05 \\
0.32400 & 4.71 & 4.71 \\
0.37000 & 5.31 & 5.34 \\
0.42600 & 5.87 & 6.00 \\
0.50000 & 6.42 & 6.75 \\
0.59200 & 6.94 & 7.56 \\
0.70400 & 7.43 & 8.43 \\
0.83300 & 7.87 & 9.34 \\
0.96300 & 8.22 & 10.17 \\
1.11000 & 8.53 & 11.03 \\
1.29000 & 8.84 & 12.00 \\
1.57000 & 9.19 & 13.35 \\
1.85000 & 9.44 & 14.55 \\
2.77000 & 9.91 & 17.76 \\
3.70000 & 10.14 & 20.25 \\
5.55000 & 10.34 & 23.96 \\
9.26000 & 10.46 & 28.90 \\
18.50000 & 10.51 & 35.85 \\
37.00000 & 10.51 & 42.97 \\
55.50000 & 10.51 & 47.18 \\
92.60000 & 10.51 & 52.52 \\
129.00000 & 10.51 & 55.99 \\
185.00000 & 10.51 & 59.76
\end{tabular}

\begin{tabular}{ccr} 
TD=44.0 EV & \multicolumn{2}{c}{ ET=0.201176 HEV } \\
ENERGY(MEV) & PRIMARY & CASCADE \\
0.20300 & 0.11 & 0.11 \\
0.20500 & 0.22 & 0.22 \\
0.20900 & 0.43 & 0.43 \\
0.21500 & 0.73 & 0.73 \\
0.22300 & 1.09 & 1.09 \\
0.23300 & 1.49 & 1.49 \\
0.24500 & 1.91 & 1.91 \\
0.26100 & 2.38 & 2.38 \\
0.28100 & 2.87 & 2.87 \\
0.31100 & 3.45 & 3.45 \\
0.35200 & 4.05 & 4.05 \\
0.40200 & 4.61 & 4.64 \\
0.46200 & 5.13 & 5.24 \\
0.54300 & 5.66 & 5.95 \\
0.64300 & 6.17 & 6.72 \\
0.76400 & 6.64 & 7.55 \\
0.90500 & 7.07 & 8.41 \\
1.04000 & 7.40 & 9.17 \\
1.20000 & 7.70 & 9.99 \\
1.40000 & 8.00 & 10.92 \\
1.70000 & 8.33 & 12.18 \\
2.01000 & 8.58 & 13.32 \\
3.01000 & 9.02 & 16.31 \\
4.02000 & 9.22 & 18.62 \\
6.03000 & 9.40 & 22.04 \\
10.00000 & 9.51 & 26.51 \\
20.10000 & 9.55 & 32.90 \\
40.20000 & 9.55 & 39.39 \\
60.30000 & 9.55 & 43.22 \\
100.00000 & 9.55 & 48.02 \\
140.00000 & 9.55 & 51.22 \\
201.00000 & 9.55 & 54.66
\end{tabular}

\begin{tabular}{rrr} 
TD $=48.0$ EV & \multicolumn{2}{c}{ ET=0.216712 HEV } \\
ENERGY (HEV) & PRIHARY & CASCADE \\
0.21800 & 0.06 & 0.06 \\
0.22100 & 0.19 & 0.19 \\
0.22500 & 0.36 & 0.36 \\
0.23100 & 0.60 & 0.60 \\
0.24000 & 0.92 & 0.92 \\
0.25100 & 1.27 & 1.27 \\
0.26400 & 1.64 & 1.64 \\
0.28100 & 2.04 & 2.04 \\
0.30300 & 2.48 & 2.48 \\
0.33500 & 2.99 & 2.99 \\
0.37900 & 3.54 & 3.54 \\
0.43300 & 4.06 & 4.09 \\
0.49800 & 4.55 & 4.66 \\
0.58500 & 5.06 & 5.32 \\
0.69300 & 5.55 & 6.05 \\
0.82300 & 6.01 & 6.84 \\
0.97500 & 6.42 & 7.66 \\
1.12000 & 6.74 & 8.38 \\
1.30000 & 7.04 & 9.19 \\
1.51000 & 7.32 & 10.05 \\
1.84000 & 7.64 & 11.26 \\
2.16000 & 7.86 & 12.29 \\
3.25000 & 8.27 & 15.12 \\
4.33000 & 8.46 & 17.25 \\
6.50000 & 8.62 & 20.42 \\
10.80000 & 8.72 & 24.55 \\
21.60000 & 8.76 & 30.39 \\
43.30000 & 8.76 & 36.37 \\
65.00000 & 8.76 & 39.89 \\
108.00000 & 8.76 & 44.31 \\
151.00000 & 8.76 & 47.23 \\
216.00300 & 8.75 & 50.35 \\
& &
\end{tabular}


hTOHIC DISPLAEEHENT CROSS-SECTIONS (BARNS) BY FAST ELECTRONS IN C

\begin{tabular}{crr} 
TD $=52.0$ & EV & \multicolumn{2}{c}{ ET $=0.231924$} & HEV \\
ENERGY (HEV) & PRIMARY & CASCADE \\
0.23400 & 0.08 & 0.08 \\
0.23600 & 0.15 & 0.15 \\
0.24100 & 0.32 & 0.32 \\
0.24800 & 0.54 & 0.54 \\
0.25700 & 0.80 & 0.80 \\
0.26900 & 1.11 & 1.11 \\
0.28200 & 1.41 & 1.41 \\
0.30100 & 1.78 & 1.78 \\
0.32400 & 2.17 & 2.17 \\
0.35900 & 2.64 & 2.64 \\
0.40500 & 3.13 & 3.13 \\
0.46300 & 3.62 & 3.64 \\
0.53300 & 4.08 & 4.18 \\
0.62600 & 4.57 & 4.81 \\
0.74200 & 5.05 & 5.51 \\
0.88100 & 5.49 & 6.26 \\
1.04000 & 5.88 & 7.03 \\
1.20000 & 6.19 & 7.73 \\
1.39000 & 6.48 & 8.49 \\
1.62000 & 6.75 & 9.33 \\
1.97000 & 7.05 & 10.46 \\
2.31000 & 7.25 & 11.42 \\
3.47000 & 7.64 & 14.06 \\
4.63000 & 7.81 & 16.06 \\
6.95000 & 7.96 & 19.01 \\
11.50000 & 8.05 & 22.82 \\
23.10000 & 8.09 & 28.26 \\
46.30000 & 8.08 & 33.79 \\
69.50000 & 8.08 & 37.04 \\
115.00000 & 8.08 & 41.08 \\
162.00000 & 8.08 & 43.84 \\
& &
\end{tabular}

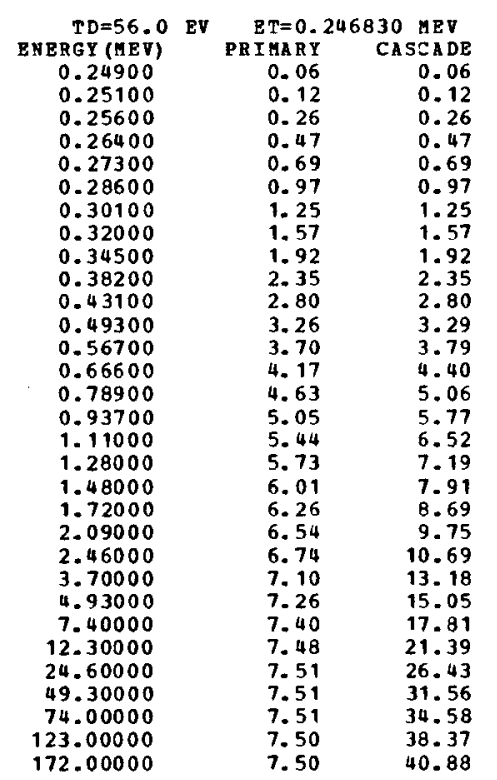

$\begin{array}{crr}\begin{array}{c}\text { TO=60.0 } \\ \text { ENERGY }\end{array} & \begin{array}{r}\text { ET }=0.261448 \text { HEV } \\ \text { PRTHARY }\end{array} & \text { CASCADE } \\ 0.26400 & 0.06 & 0.06 \\ 0.26600 & 0.11 & 0.11 \\ 0.27100 & 0.23 & 0.23 \\ 0.27900 & 0.40 & 0.40 \\ 0.29000 & 0.62 & 0.62 \\ 0.30300 & 0.86 & 0.86 \\ 0.31800 & 1.10 & 1.10 \\ 0.33900 & 1.39 & 1.39 \\ 0.36600 & 1.72 & 1.72 \\ 0.40500 & 2.11 & 2.11 \\ 0.45700 & 2.54 & 2.54 \\ 0.52200 & 2.97 & 2.99 \\ 0.60100 & 3.39 & 3.47 \\ 0.70500 & 3.83 & 4.04 \\ 0.83600 & 4.28 & 4.68 \\ 0.99300 & 4.69 & 5.37 \\ 1.17000 & 5.05 & 6.06 \\ 1.35000 & 5.33 & 6.70 \\ 1.56000 & 5.59 & 7.38 \\ 1.83000 & 5.85 & 8.17 \\ 2.22000 & 6.11 & 9.17 \\ 2.61000 & 6.29 & 10.05 \\ 3.92000 & 6.63 & 12.40 \\ 5.22000 & 6.78 & 14.16 \\ 7.84000 & 6.91 & 16.75 \\ 13.00000 & 6.98 & 20.10 \\ 26.10000 & 7.00 & 24.83 \\ 52.20000 & 7.01 & 29.61 \\ 78.40000 & 7.01 & 32.43 \\ 130.00000 & 7.00 & 35.96 \\ 183.00000 & 7.00 & 38.34 \\ & & \end{array}$

A TOMIC DISPLACENENT CROSS-SECTIONS (BARNS) BY PAST ELECTRONS IN C $z=6 \quad A=12.011$

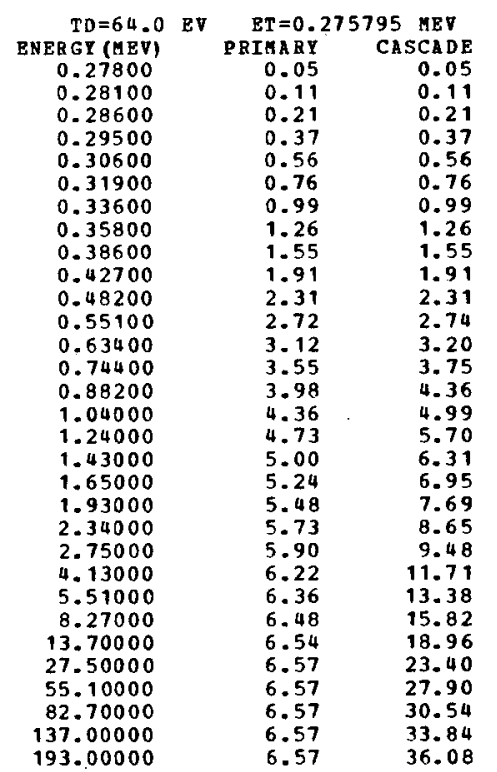

\begin{tabular}{ccc} 
TD $=68.0$ & EV & \multicolumn{2}{c}{ ET $=0.289885$ MEV } \\
ENERGY(MEV) & PRIMAR & CASCA DE \\
0.29200 & 0.04 & 0.04 \\
0.29500 & 0.09 & 0.09 \\
0.30100 & 0.19 & 0.19 \\
0.31000 & 0.33 & 0.33 \\
0.32100 & 0.49 & 0.49 \\
0.33600 & 0.69 & 0.69 \\
0.35300 & 0.89 & 0.89 \\
0.37600 & 1.13 & 1.13 \\
0.40500 & 1.40 & 1.40 \\
0.44900 & 1.75 & 1.75 \\
0.50700 & 2.12 & 2.12 \\
0.57900 & 2.51 & 2.53 \\
0.66600 & 2.89 & 2.97 \\
0.78200 & 3.31 & 3.49 \\
0.92700 & 3.72 & 4.08 \\
1.10000 & 4.10 & 4.71 \\
1.30000 & 4.43 & 5.36 \\
1.50000 & 4.70 & 5.94 \\
1.73000 & 4.93 & 6.56 \\
2.02000 & 5.16 & 7.26 \\
2.46000 & 5.40 & 8.19 \\
2.89000 & 5.56 & 8.98 \\
4.34000 & 5.86 & 11.10 \\
5.79000 & 5.99 & 12.68 \\
8.69000 & 6.10 & 14.99 \\
14.40000 & 6.16 & 17.95 \\
28.90000 & 6.18 & 22.14 \\
57.90000 & 6.18 & 26.37 \\
86.90000 & 6.18 & 28.86 \\
144.00000 & 6.18 & 31.97 \\
202.00000 & 6.18 & 34.06
\end{tabular}

\begin{tabular}{crr} 
TD=72.0 & EV & \multicolumn{1}{c}{ ET $=0.303732$ MEV } \\
ENERGY (HEV) & PRIHARY & CASCADE \\
0.30500 & 0.03 & 0.03 \\
0.30900 & 0.08 & 0.08 \\
0.31500 & 0.17 & 0.17 \\
0.32400 & 0.29 & 0.29 \\
0.33700 & 0.45 & 0.45 \\
0.35200 & 0.62 & 0.62 \\
0.37000 & 0.81 & 0.81 \\
0.39400 & 1.03 & 1.03 \\
0.42500 & 1.28 & 1.28 \\
0.47000 & 1.60 & 1.60 \\
0.53100 & 1.96 & 1.96 \\
0.60700 & 2.33 & 2.35 \\
0.69800 & 2.70 & 2.77 \\
0.82000 & 3.10 & 3.27 \\
0.97100 & 3.49 & 3.84 \\
1.15000 & 3.85 & 4.43 \\
1.36300 & 4.18 & 5.06 \\
1.57000 & 4.43 & 5.62 \\
1.82000 & 4.66 & 6.23 \\
2.12000 & 4.87 & 6.89 \\
2.58500 & 5.10 & 7.78 \\
3.03000 & 5.26 & 8.53 \\
4.55000 & 5.54 & 10.55 \\
6.07000 & 5.66 & 12.05 \\
9.11000 & 5.76 & 14.24 \\
15.10000 & 5.82 & 17.05 \\
30.30000 & 5.84 & 21.01 \\
60.70000 & 5.84 & 25.01 \\
91.10000 & 5.84 & 27.37 \\
151.00000 & 5.84 & 30.30 \\
212.00000 & 5.84 & 32.28 \\
& &
\end{tabular}


ATONIC DISPLACEHENT CROSS-SBCtIONS (BARS) BY PAST ELECTRONS IN O

\begin{tabular}{|c|c|c|}
\hline \multirow{4}{*}{$\begin{array}{r}T D=4.0 \\
\text { ENERGY (HEV) } \\
0.02860 \\
0.02890 \\
0.02950\end{array}$} & \multicolumn{2}{|c|}{$E T=0.028371 \mathrm{MEV}$} \\
\hline & $\begin{array}{r}\text { PRIHARY } \\
10.08\end{array}$ & $\begin{array}{r}\text { CASCADE } \\
10.08\end{array}$ \\
\hline & 22.81 & 22.81 \\
\hline & 46.76 & 46.76 \\
\hline 0.03030 & 75.83 & 75.83 \\
\hline 0.03140 & 111.08 & 111.08 \\
\hline 0.03290 & 151.69 & 151.69 \\
\hline 0.03460 & 189.20 & 189.20 \\
\hline 0.03680 & 227.26 & 227.26 \\
\hline 0.03970 & 263.92 & 263.92 \\
\hline 0.04390 & 298.35 & 298.35 \\
\hline 0.04960 & 323.32 & 323.32 \\
\hline 0.05670 & 335.28 & 335.38 \\
\hline 0.06520 & 335.96 & 339.71 \\
\hline 0.07660 & 327.19 & 339.30 \\
\hline 0.09070 & 311.70 & 334.60 \\
\hline 0.10700 & 293.60 & 327.39 \\
\hline 0.12700 & 273.97 & 318.50 \\
\hline 0.14700 & 257.62 & 310.53 \\
\hline 0.17000 & 242.29 & 302.80 \\
\hline 0.19800 & 227.51 & 295.29 \\
\hline $\begin{array}{l}0.24100 \\
0.28300\end{array}$ & $\begin{array}{l}210.68 \\
198.80\end{array}$ & $\begin{array}{l}287.03 \\
281.79\end{array}$ \\
\hline & $\begin{array}{l}198.80 \\
175.77\end{array}$ & $\begin{array}{r}287.19 \\
275.77\end{array}$ \\
\hline 0.56700 & 164.59 & 278.35 \\
\hline 0.85100 & 154.20 & 291.75 \\
\hline 1.41000 & 147.13 & 323.12 \\
\hline 2.83000 & 142.95 & 387.79 \\
\hline 5.67000 & 141.35 & 468.51 \\
\hline 8.51000 & 140.89 & 520.08 \\
\hline 14.10000 & 140.57 & 586.82 \\
\hline 19.80000 & 140.45 & 632.72 \\
\hline 28.30000 & 140.38 & \\
\hline 56.70000 & 140.31 & 777.67 \\
\hline 113.00000 & 140.29 & 873.74 \\
\hline 198.00000 & 140.27 & 952.14 \\
\hline
\end{tabular}

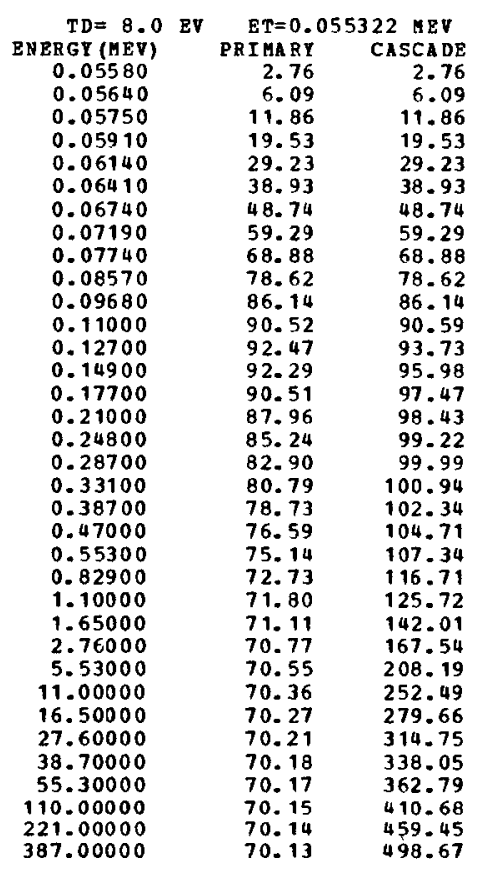

\begin{tabular}{rrr} 
TD $=12.0$ & EV & \multicolumn{2}{c}{ ET $=0.081048$ MEV } \\
ENERGY(BEV) & PRI ARY & CASCADE \\
0.08180 & 1.34 & 1.34 \\
0.08260 & 2.72 & 2.72 \\
0.08420 & 5.33 & 5.33 \\
0.08670 & 9.06 & 9.06 \\
0.08990 & 13.27 & 13.27 \\
0.09400 & 17.90 & 17.90 \\
0.09880 & 22.41 & 22.41 \\
0.10500 & 27.10 & 27.10 \\
0.11300 & 31.73 & 31.73 \\
0.12500 & 36.57 & 36.57 \\
0.14100 & 40.59 & 40.59 \\
0.16200 & 43.50 & 43.58 \\
0.18600 & 45.15 & 45.85 \\
0.21800 & 46.06 & 48.01 \\
0.25900 & 46.36 & 50.02 \\
0.30700 & 46.30 & 51.89 \\
0.36400 & 46.10 & 53.79 \\
0.42100 & 45.92 & 55.55 \\
0.48600 & 45.77 & 57.46 \\
0.56700 & 45.68 & 59.75 \\
0.68900 & 45.65 & 63.05 \\
0.81000 & 45.69 & 66.23 \\
1.21000 & 45.99 & 75.70 \\
1.62000 & 46.26 & 84.01 \\
2.43000 & 46.59 & 97.36 \\
4.05000 & 46.84 & 116.56 \\
8.10000 & 46.92 & 145.43 \\
16.20900 & 46.85 & 176.12 \\
24.30000 & 46.82 & 194.53 \\
40.50000 & 46.79 & 217.98 \\
56.70000 & 46.78 & 233.54 \\
81.00000 & 46.77 & 250.08 \\
162.00000 & 46.76 & 282.34 \\
324.00000 & 46.76 & 314.67 \\
567.00000 & 46.75 & 340.80
\end{tabular}

ATOMIC DISPLACEHENT CROSS-SBCTIONS (BARNS) BY PAST BLECTRONS IN O

$\begin{array}{rrr}\begin{array}{c}\text { TD }=16.0 \\ \text { EN }\end{array} & \begin{array}{c}\text { ET }=0.105701 \text { MEV } \\ \text { PEIMARY }\end{array} & \text { CASCADE } \\ 0.10600 & 0.24 & 0.24 \\ 0.10700 & 1.01 & 1.01 \\ 0.10900 & 2.49 & 2.49 \\ 0.11300 & 5.16 & 5.16 \\ 0.11700 & 7.51 & 7.51 \\ 0.12200 & 10.07 & 10.07 \\ 0.12800 & 12.66 & 12.66 \\ 0.13700 & 15.80 & 15.80 \\ 0.14700 & 18.49 & 18.49 \\ 0.16300 & 21.60 & 21.60 \\ 0.18400 & 24.27 & 24.27 \\ 0.21100 & 26.36 & 26.43 \\ 0.24300 & 27.83 & 28.32 \\ 0.28500 & 28.95 & 30.25 \\ 0.33800 & 29.77 & 32.19 \\ 0.40100 & 30.38 & 34.14 \\ 0.47500 & 30.89 & 36.16 \\ 0.54900 & 31.29 & 38.01 \\ 0.63400 & \mathbf{3 1 . 6 7} & 40.00 \\ 0.73900 & 32.07 & 42.30 \\ 0.89800 & 32.56 & 45.55 \\ 1.05000 & 32.94 & 48.42 \\ 1.58000 & 33.82 & 57.05 \\ 2.11000 & 34.30 & 64.10 \\ 3.17000 & 34.76 & 75.13 \\ 5.28000 & 35.06 & 90.38 \\ 10.50000 & 35.16 & 112.51 \\ 21.10000 & 35.13 & 136.02 \\ 31.70000 & 35.11 & 149.98 \\ 52.80000 & 35.09 & 167.63 \\ 73.90000 & 35.08 & 179.32 \\ 105.00000 & 35.08 & 191.56 \\ 211.00000 & 35.07 & 215.95 \\ & & \end{array}$

\begin{tabular}{|c|c|c|c|}
\hline $\begin{array}{r}T D=20.0 \\
\text { ENERGY(MEV) }\end{array}$ & EV & $\begin{aligned} & \text { ET }=0.1 \\
& \text { PRI I HARY }\end{aligned}$ & CASCADE \\
\hline & & & \\
\hline 0.13100 & & 0.66 & $\begin{array}{l}0.25 \\
0.66\end{array}$ \\
\hline 0.13400 & & 1.83 & 1.83 \\
\hline 0.13800 & & 3. 26 & 3.26 \\
\hline 0.14300 & & 4.85 & 4.85 \\
\hline 0.15000 & & 6.77 & 6.77 \\
\hline 0.15700 & & 8.40 & 8.40 \\
\hline 0.16800 & & 10.51 & 10.51 \\
\hline 0.18100 & & 12.45 & 12.45 \\
\hline 0.20000 & & 14.55 & 14.55 \\
\hline 0.22600 & & 16.53 & 16.53 \\
\hline 0.25800 & & 18.14 & 18.20 \\
\hline 0.29700 & & 19.43 & 19.80 \\
\hline 0.34900 & & 20.58 & 21.54 \\
\hline 0.41400 & & 21.55 & 23.35 \\
\hline 0.49100 & & 22.38 & 25.20 \\
\hline 0.58200 & & 23. 11 & 27.16 \\
\hline 0.67200 & & 23.69 & 28.92 \\
\hline 0.77600 & & 24.23 & 30.81 \\
\hline 0.90500 & & 24.78 & 32.99 \\
\hline 1.09000 & & 25.38 & 35.84 \\
\hline 1.29000 & & 25.87 & 38.65 \\
\hline 1.94000 & & 26.84 & 46.29 \\
\hline 2.58000 & & 27.32 & 52.28 \\
\hline 3.88000 & & 27.76 & 61.62 \\
\hline 6.47000 & & 28.03 & 74.26 \\
\hline 12.90000 & & 28.11 & 92.36 \\
\hline 25.80000 & & 28.09 & 111.19 \\
\hline 38.80000 & & & 122.43 \\
\hline 64.70000 & & 28.07 & $136-62$ \\
\hline & & & \\
\hline $\begin{array}{l}29 . \\
258 .\end{array}$ & & $\begin{array}{l}28.06 \\
28.06\end{array}$ & $\begin{array}{l}155.87 \\
175.26\end{array}$ \\
\hline 25 & & & 175.26 \\
\hline
\end{tabular}

\begin{tabular}{crr} 
TD $=24.0$ & EV & \multicolumn{1}{c}{ ET $=0.152265$ MEV } \\
ENERGY (HEV) & PEIHARY & CASCADE \\
0.15300 & 0.19 & 0.19 \\
0.15500 & 0.67 & 0.67 \\
0.15800 & 1.37 & 1.37 \\
0.16200 & 2.23 & 2.23 \\
0.16900 & 3.57 & 3.57 \\
0.17600 & 4.74 & 4.74 \\
0.18500 & 6.04 & 6.04 \\
0.19700 & 7.47 & 7.47 \\
0.21300 & 8.99 & 8.99 \\
0.23600 & 10.63 & 10.63 \\
0.26600 & 12.17 & 12.17 \\
0.30400 & 13.53 & 13.58 \\
0.35000 & 14.69 & 14.99 \\
0.41100 & 15.79 & 16.55 \\
0.48700 & 16.78 & 18.21 \\
0.57800 & 17.67 & 19.95 \\
0.68500 & 18.47 & 21.78 \\
0.79100 & 19.09 & 23.43 \\
0.91300 & 19.67 & 25.19 \\
1.06000 & 20.23 & 27.14 \\
1.29000 & 20.89 & 29.90 \\
1.52000 & 21.37 & 32.38 \\
2.28000 & 22.29 & 39.14 \\
3.04000 & 22.73 & 44.42 \\
4.56000 & 23.13 & 52.43 \\
7.61000 & 23.36 & 63.21 \\
15.20000 & 23.42 & 78.51 \\
30.40000 & 23.41 & 94.28 \\
45.60000 & 23.40 & 103.62 \\
76.10000 & 23.39 & 115.49 \\
106.00000 & 23.39 & 123.19 \\
152.00000 & 23.38 & 131.58 \\
304.00000 & 23.38 & 147.75
\end{tabular}


A TOHIC DISPLACEAET CROSS-SECTIONS (BARNS) BY FAST BLECTRONS IN O

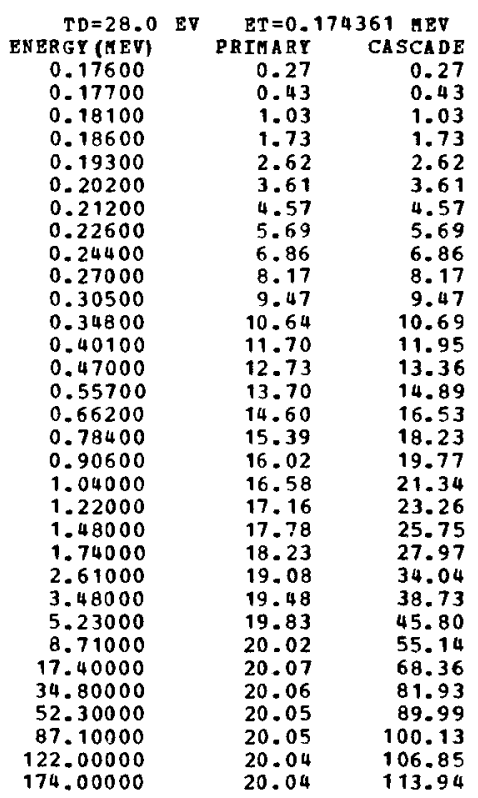

\begin{tabular}{rrr} 
TD $=32.0$ & EV & \multicolumn{1}{c}{ ET $=0.195767$ HEV } \\
ERERGY (HEV) & PRIMARY & CASCADE \\
0.19700 & 0.14 & 0.14 \\
0.19900 & 0.36 & 0.36 \\
0.20300 & 0.78 & 0.78 \\
0.20900 & 1.37 & 1.37 \\
0.21700 & 2.07 & 2.07 \\
0.22700 & 2.85 & 2.85 \\
0.23800 & 3.59 & 3.59 \\
0.25400 & 4.51 & 4.51 \\
0.27400 & 5.45 & 5.45 \\
0.30300 & 6.54 & 6.54 \\
0.34200 & 7.65 & 7.65 \\
0.39100 & 8.70 & 8.75 \\
0.45000 & 9.67 & 9.89 \\
0.52800 & 10.64 & 11.18 \\
0.62600 & 11.58 & 12.61 \\
0.74300 & 12.44 & 14.13 \\
0.88000 & 13.22 & 15.71 \\
1.01000 & 13.80 & 17.08 \\
1.17000 & 14.37 & 18.63 \\
1.37000 & 14.92 & 20.39 \\
1.66000 & 15.50 & 22.66 \\
1.95000 & 15.91 & 24.68 \\
2.93000 & 16.70 & 30.20 \\
3.91000 & 17.05 & 34.42 \\
5.87000 & 17.35 & 40.70 \\
9.78000 & 17.52 & 48.98 \\
19.50000 & 17.56 & 60.58 \\
39.10000 & 17.55 & 72.53 \\
58.70000 & 17.55 & 79.58 \\
97.80000 & 17.54 & 88.47 \\
137.00000 & 17.54 & 94.35 \\
195.00000 & 17.54 & 100.52
\end{tabular}

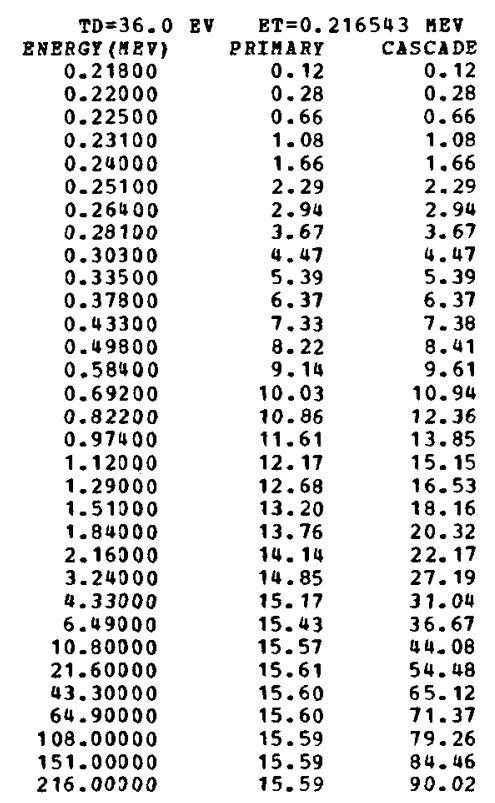
ATOHIC DISPLAzEMENT CROSS-SECTIONS (BARNS) BY PAST ELECTRONS IN 0
$\mathrm{Z}=8 \quad \mathrm{a}=16.000$

$T D=40.0 \mathrm{EV} \quad \mathrm{BT}=0.236743 \mathrm{MEV}$

\begin{tabular}{|c|c|c|}
\hline & & \\
\hline ENERGY (MEV) & PRIHARY & CASCA DE \\
\hline 0.23900 & 0.14 & 0.14 \\
\hline 0.24100 & 0.26 & 0.26 \\
\hline 0.24600 & 0.54 & 0.54 \\
\hline 0.25300 & 0.92 & 0.92 \\
\hline 0.26200 & 1.36 & 1.36 \\
\hline 0.27400 & 1.88 & 1.88 \\
\hline 0.28800 & 2.42 & 2.42 \\
\hline 0.30700 & 3.06 & 3.06 \\
\hline 0.33100 & 3.74 & 3.74 \\
\hline 0.36600 & 4.56 & 4.56 \\
\hline 0.41400 & 5.45 & 5.45 \\
\hline 0.47300 & 6.31 & 6.35 \\
\hline 0.54400 & 7.13 & 7.31 \\
\hline 0.63900 & 8.01 & 8.44 \\
\hline 0.75700 & 8.86 & 9.68 \\
\hline 0.89900 & 9.65 & 11.01 \\
\hline 1.06000 & 10.34 & 12.37 \\
\hline 1.23000 & 10.90 & 13.66 \\
\hline 1.42000 & 11.40 & \\
\hline 1.65000 & 11.86 & 16.42 \\
\hline 2.01000 & 12.37 & 18.43 \\
\hline 2.36000 & 12.73 & 20.14 \\
\hline $\begin{array}{l}3.55000 \\
4.73000\end{array}$ & $\begin{array}{l}13.38 \\
13.66\end{array}$ & $\begin{array}{r}24.79 \\
28.27\end{array}$ \\
\hline 7. 10000 & $\begin{array}{l}13.66 \\
13.89\end{array}$ & $\begin{array}{l}28.27 \\
33.42\end{array}$ \\
\hline 11.80000 & 14.02 & \\
\hline 23.60000 & 14.05 & 49.50 \\
\hline 47.30000 & 14.04 & 59.10 \\
\hline 71.00000 & 14.04 & 64.74 \\
\hline 118.00000 & 14.03 & 71.83 \\
\hline 165.00000 & 14.03 & 76.51 \\
\hline
\end{tabular}

$T D=44.0 \mathrm{EV} \quad \mathrm{ET}=0.256411 \mathrm{MEV}$

\begin{tabular}{crr}
$T D=44.0$ & \multicolumn{2}{c}{$E T=0.256411$} \\
ENERGY (HEV) & PRIMAR & CASCADE \\
0.25800 & 0.08 & 0.08 \\
0.26100 & 0.21 & 0.21 \\
0.26600 & 0.44 & 0.44 \\
0.27400 & 0.77 & 0.77 \\
0.28400 & 1.15 & 1.15 \\
0.29700 & 1.59 & 1.59 \\
0.31200 & 2.05 & 2.05 \\
0.33300 & 2.62 & 2.62 \\
0.35800 & 3.19 & 3.19 \\
0.39700 & 3.94 & 3.94 \\
0.44800 & 4.73 & 4.73 \\
0.51200 & 5.53 & 5.56 \\
0.58900 & 6.30 & 6.46 \\
0.69200 & 7.13 & 7.52 \\
0.82000 & 7.93 & 8.69 \\
0.97400 & 8.69 & 9.94 \\
1.15000 & 9.35 & 11.23 \\
1.33000 & 9.87 & 12.42 \\
1.53000 & 10.32 & 13.62 \\
1.79000 & 10.77 & 15.03 \\
2.17000 & 11.24 & 16.86 \\
2.56000 & 11.58 & 18.49 \\
3.84000 & 12.17 & 22.77 \\
5.12000 & 12.43 & 25.98 \\
7.69000 & 12.64 & 30.71 \\
12.80000 & 12.75 & 36.84 \\
25.60000 & 12.77 & 45.41 \\
51.20000 & 12.76 & 54.12 \\
76.90000 & 12.76 & 59.26 \\
128.00000 & 12.76 & 65.72 \\
179.00000 & 12.76 & 69.99
\end{tabular}

$\mathrm{TD}=48.0 \mathrm{BV}$

$\begin{array}{ccr}\text { TD=48.0 BV } & \text { ET }=0.275587 \text { MEV } \\ \text { ENERGY(AEV) } & \text { PRIARY } & \text { CASCADE } \\ 0.27800 & 0.09 & 0.09 \\ 0.28100 & 0.20 & 0.20 \\ 0.28600 & 0.37 & 0.37 \\ 0.29400 & 0.64 & 0.64 \\ 0.30500 & 0.97 & 0.97 \\ 0.31900 & 1.36 & 1.36 \\ 0.33600 & 1.78 & 1.78 \\ 0.35800 & 2.26 & 2.26 \\ 0.38500 & 2.78 & 2.78 \\ 0.42700 & 3.45 & 3.45 \\ 0.48200 & 4.18 & 4.18 \\ 0.55100 & 4.92 & 4.96 \\ 0.63300 & 5.64 & 5.78 \\ 0.74400 & 6.43 & 6.78 \\ 0.88100 & 7.19 & 7.88 \\ 1.04000 & 7.88 & 9.03 \\ 1.24000 & 8.54 & 10.31 \\ 1.43000 & 9.03 & 11.41 \\ 1.65000 & 9.46 & 12.57 \\ 1.92000 & 9.87 & 13.85 \\ 2.34000 & 10.32 & 15.60 \\ 2.75000 & 10.62 & 17.10 \\ 4.13000 & 11.17 & 21.09 \\ 5.51000 & 11.40 & 24.07 \\ 8.26000 & 11.59 & 28.41 \\ 13.70300 & 11.68 & 34.02 \\ 27.50000 & 11.71 & 41.94 \\ 55.10000 & 11.70 & 49.95 \\ 82.60000 & 11.70 & 54.64 \\ 137.00000 & 11.69 & 60.53 \\ 192.00000 & 11.69 & 64.46 \\ & & \end{array}$


ATOHIC DISPLACENENT CROSS-SECTIONS (BARS) BY PAST RLECTRONS IN O

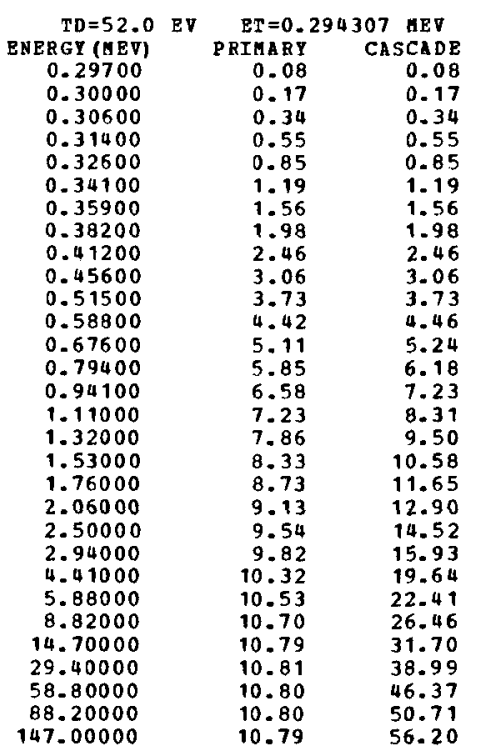

\begin{tabular}{|c|c|c|}
\hline $\begin{array}{r}T D=56.0 \\
\text { ENREY }\end{array}$ & $\begin{array}{r}\text { ET }=0 . \\
\text { PRIMART }\end{array}$ & CASCADE \\
\hline 0.31500 & 0.06 & 0.06 \\
\hline 0.31800 & 0.13 & 0.13 \\
\hline 0.32500 & 0.29 & 0.29 \\
\hline 0.33400 & 0.49 & 0.49 \\
\hline 0.34600 & 0.74 & 0.74 \\
\hline 0.36200 & 1.04 & 1.04 \\
\hline 0.38100 & 1.37 & 1.37 \\
\hline 0.40600 & 1.76 & 1.76 \\
\hline 0.43700 & 2. 18 & 2.18 \\
\hline 0.48400 & 2.74 & 2.74 \\
\hline 0.54700 & 3. 37 & 3.37 \\
\hline 0.62500 & 4.02 & 4.06 \\
\hline 0.71800 & 4.67 & 4.79 \\
\hline 0.84400 & 5.38 & 5.69 \\
\hline 1.00000 & 6.07 & 6.68 \\
\hline 1. 18000 & 6.7 & 7.71 \\
\hline 1.40000 & 7.28 & 8.83 \\
\hline 1.62000 & 7.72 & 9.84 \\
\hline 1.87000 & 8.11 & 10.88 \\
\hline 2.18000 & 8.47 & 12.03 \\
\hline 2.65000 & 8. 86 & 13.56 \\
\hline 3.12000 & 9.13 & 14.90 \\
\hline 4.68000 & 9.59 & 18.39 \\
\hline 6.25000 & 9.78 & 20.99 \\
\hline 9.37000 & 9.94 & 24.76 \\
\hline 15.60000 & 10.02 & 29.64 \\
\hline 31.20000 & 10.03 & 36.41 \\
\hline 62.50000 & 10.03 & 43.29 \\
\hline 93.70000 & 10.03 & 47.32 \\
\hline 156.00000 & 10.02 & 52.41 \\
\hline
\end{tabular}

\begin{tabular}{|c|c|c|}
\hline $\begin{array}{r}T D=60.0 \\
\text { ENERGY (MEV) }\end{array}$ & $\begin{array}{l}\text { ET }=0 \\
\text { PRTMARY }\end{array}$ & $\begin{array}{l}98 \mathrm{MEV} \\
\text { CASCADE }\end{array}$ \\
\hline $\begin{array}{c}\text { ENERGY (AEU) } \\
0.33300\end{array}$ & $\begin{array}{r}\text { PRAARY } \\
0.05\end{array}$ & $\begin{array}{r}\text { CASCADE } \\
0.05\end{array}$ \\
\hline 0.33700 & 0.13 & 0.13 \\
\hline 0.34300 & 0.25 & 0.25 \\
\hline 0.35300 & 0.43 & 0.43 \\
\hline 0.36600 & 0.66 & 0.66 \\
\hline 0.38300 & 0.93 & 0.93 \\
\hline 0.40300 & 1.22 & 1.22 \\
\hline 0.42900 & 1.57 & 1.57 \\
\hline 0.46200 & 1.96 & 1.96 \\
\hline 0.51200 & 2.48 & 2.48 \\
\hline 0.57800 & 3.07 & 3.07 \\
\hline 0.66000 & 3.68 & 3.71 \\
\hline 0.76000 & 4.30 & 4.42 \\
\hline 0.89200 & 4.97 & 5.27 \\
\hline 1.05000 & 5.61 & 6.17 \\
\hline 1.25000 & 6.24 & 7.20 \\
\hline 1.48000 & 6.79 & 8.26 \\
\hline 1.71000 & 7.20 & 9.21 \\
\hline 1.98000 & 7.58 & 10.21 \\
\hline 2.31000 & 7.92 & 11.31 \\
\hline 2.80000 & 8.28 & 12.75 \\
\hline 3.30000 & 8.53 & 14.01 \\
\hline 4.95000 & 8.96 & 17.30 \\
\hline 6.60000 & 9.14 & 19.73 \\
\hline 9.91000 & 9.28 & 23.28 \\
\hline 16.50000 & 9.35 & 27.85 \\
\hline 33.00000 & 9.36 & 34.18 \\
\hline 66.00000 & 9.36 & 40.59 \\
\hline 99.10000 & 9.36 & \\
\hline 165.00000 & 9.35 & 49.11 \\
\hline
\end{tabular}

ATOMIC DISPLACEHENT CROSS-SECTIONS (BARNS) BY PAST ELECTRONS IN O $\mathrm{Z}=\mathrm{B} \quad \mathrm{A}=16.000$

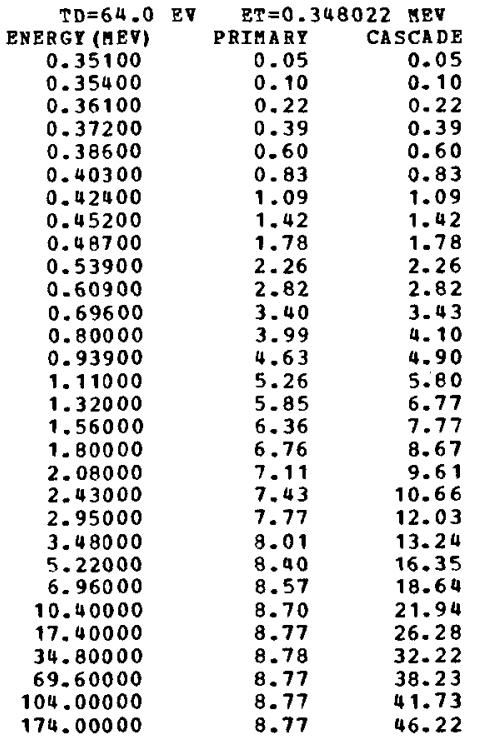

$\begin{array}{ccc}\begin{array}{c}\text { TD=68.0 } \\ \text { ENBRY }\end{array} & \begin{array}{r}\text { BT }=0.365195 \\ \text { PRIMARY }\end{array} & \text { CASCADE } \\ 0.36800 & 0.04 & 0.04 \\ 0.37200 & 0.10 & 0.10 \\ 0.37900 & 0.20 & 0.20 \\ 0.39000 & 0.34 & 0.34 \\ 0.40500 & 0.54 & 0.54 \\ 0.42300 & 0.75 & 0.75 \\ 0.44500 & 0.99 & 0.99 \\ 0.47400 & 1.29 & 1.29 \\ 0.51100 & 1.63 & 1.63 \\ 0.56600 & 2.08 & 2.08 \\ 0.63900 & 2.60 & 2.60 \\ 0.73000 & 3.15 & 3.18 \\ 0.83900 & 3.72 & 3.82 \\ 0.98600 & 4.33 & 4.60 \\ 1.16000 & 4.92 & 5.42 \\ 1.38000 & 5.49 & 6.36 \\ 1.54000 & 5.99 & 7.35 \\ 1.89000 & 6.36 & 8.19 \\ 2.19000 & 6.70 & 9.11 \\ 2.55000 & 7.01 & 10.10 \\ 3.10000 & 7.33 & 11.41 \\ 3.65000 & 7.55 & 12.54 \\ 5.47000 & 7.92 & 15.48 \\ 7.30000 & 8.07 & 17.66 \\ 10.90000 & 8.19 & 20.77 \\ 18.20000 & 8.25 & 24.84 \\ 36.50000 & 8.26 & 30.46 \\ 73.00000 & 8.26 & 36.12 \\ 109.00000 & 8.26 & 39.41 \\ 182.00000 & 8.25 & 43.63\end{array}$

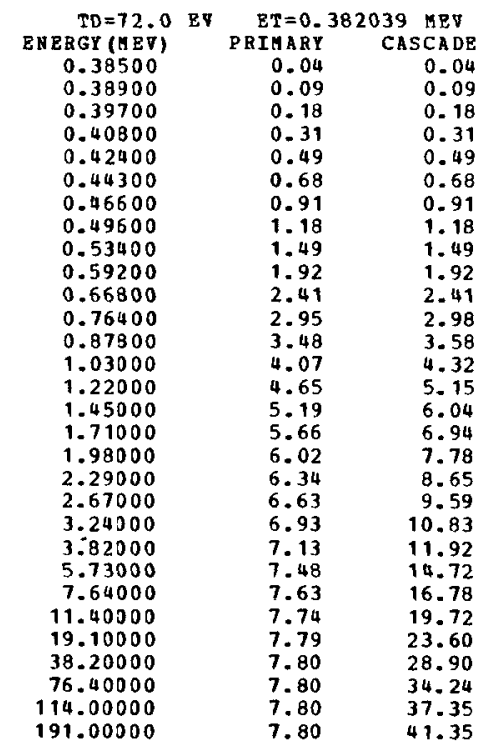


ATOHIC DISPLACEMENT CROSS-SECTIONS (BARNS) BY PAST RLECTRONS IN O

\begin{tabular}{rrr} 
TD $=76.0$ & EV & \multicolumn{2}{c}{ ET $=0.398570$ MEV } \\
ENERGY(HEV) & PRIMARY & CASCADE \\
0.40200 & 0.04 & 0.04 \\
0.40600 & 0.08 & 0.08 \\
0.41400 & 0.16 & 0.16 \\
0.42600 & 0.29 & 0.29 \\
0.44200 & 0.44 & 0.44 \\
0.46200 & 0.62 & 0.62 \\
0.48600 & 0.83 & 0.83 \\
0.51800 & 1.09 & 1.09 \\
0.55700 & 1.38 & 1.38 \\
0.61700 & 1.78 & 1.78 \\
0.69700 & 2.25 & 2.25 \\
0.79700 & 2.76 & 2.79 \\
0.91600 & 3.28 & 3.37 \\
1.07000 & 3.83 & 4.06 \\
1.27000 & 4.39 & 4.86 \\
1.51000 & 4.91 & 5.72 \\
1.79000 & 5.37 & 6.62 \\
2.07000 & 5.71 & 7.41 \\
2.39000 & 6.01 & 8.23 \\
2.78000 & 6.28 & 9.13 \\
3.38000 & 6.57 & 10.32 \\
3.98000 & 6.77 & 11.35 \\
5.97000 & 7.09 & 14.02 \\
7.97000 & 7.23 & 15.99 \\
11.90000 & 7.33 & 18.79 \\
19.90000 & 7.38 & 22.45 \\
39.80000 & 7.39 & 27.47 \\
79.70000 & 7.39 & 32.55 \\
119.00000 & 7.39 & 35.50 \\
199.00000 & 7.38 & 39.28
\end{tabular}

\begin{tabular}{rrr} 
TD $=80.0$ & EV & \multicolumn{1}{c}{ ET=0.414806 MEV } \\
ENERGY (KEV) & PRIMARY & CASCADE \\
0.41800 & 0.03 & 0.03 \\
0.42300 & 0.08 & 0.08 \\
0.43100 & 0.15 & 0.15 \\
0.44300 & 0.26 & 0.26 \\
0.46000 & 0.40 & 0.40 \\
0.48100 & 0.57 & 0.57 \\
0.50600 & 0.77 & 0.77 \\
0.53900 & 1.01 & 1.01 \\
0.58000 & 1.28 & 1.28 \\
0.64200 & 1.66 & 1.66 \\
0.72500 & 2.11 & 2.11 \\
0.82900 & 2.60 & 2.63 \\
0.95400 & 3.10 & 3.19 \\
1.11000 & 3.61 & 3.84 \\
1.32000 & 4.16 & 4.62 \\
1.57000 & 4.67 & 5.44 \\
1.86000 & 5.10 & 6.30 \\
2.15000 & 5.43 & 7.06 \\
2.48000 & 5.71 & 7.84 \\
2.90000 & 5.98 & 8.73 \\
3.52000 & 6.25 & 9.86 \\
4.14000 & 6.43 & 10.84 \\
6.22000 & 6.74 & 13.40 \\
8.29000 & 6.87 & 15.27 \\
12.40000 & 6.97 & 17.94 \\
20.70000 & 7.01 & 21.42 \\
41.40000 & 7.02 & 26.19 \\
82.90000 & 7.02 & 31.02 \\
124.00000 & 7.02 & 33.83 \\
207.00000 & 7.02 & 37.41
\end{tabular}

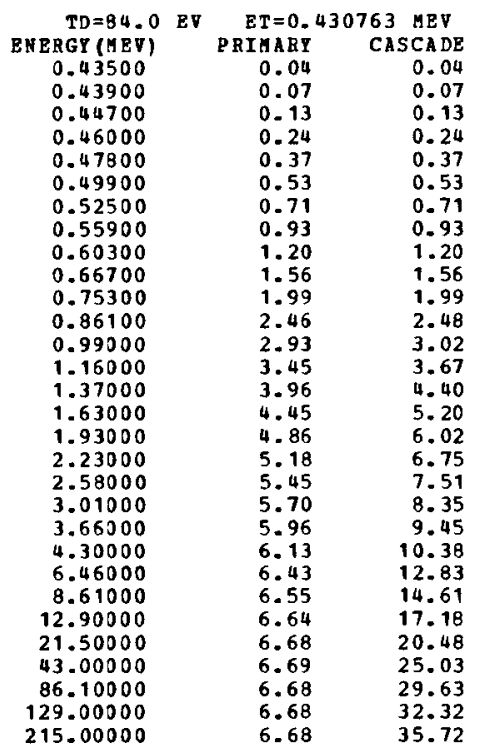

ATOHIC DISPLACEHENT CROSS-SECTIONS (BARNS) BY PAST ELECTRONS IN O $\mathrm{Z}=\mathrm{B} \quad \mathrm{A}=16.000$

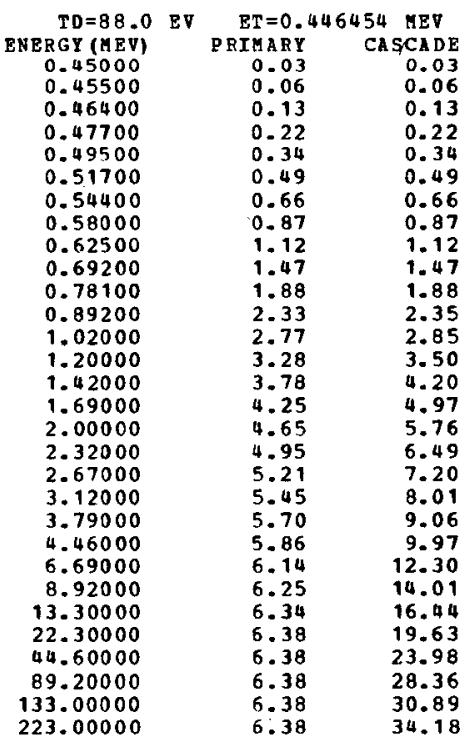

$\begin{array}{ccc}T D=92.0 & \text { EV } & \text { ET }=0.461891 \text { DEV } \\ \text { ENERGY(HEV) } & \text { PRI HARY } & \text { CASCA DE } \\ 0.46600 & 0.03 & 0.03 \\ 0.47100 & 0.06 & 0.06 \\ 0.48000 & 0.12 & 0.12 \\ 0.49400 & 0.21 & 0.21 \\ 0.51200 & 0.32 & 0.32 \\ 0.53500 & 0.45 & 0.45 \\ 0.56300 & 0.61 & 0.61 \\ 0.60000 & 0.81 & 0.81 \\ 0.64600 & 1.05 & 1.05 \\ 0.71500 & 1.38 & 1.38 \\ 0.80800 & 1.78 & 1.78 \\ 0.92300 & 2.21 & 2.24 \\ 1.06000 & 2.65 & 2.74 \\ 1.24000 & 3.13 & 3.34 \\ 1.47000 & 3.62 & 4.03 \\ 1.75000 & 4.07 & 4.77 \\ 2.07000 & 4.45 & 5.53 \\ 2.40000 & 4.74 & 6.23 \\ 2.77000 & 4.99 & 6.93 \\ 3.23000 & 5.22 & 7.70 \\ 3.92000 & 5.46 & 8.71 \\ 4.61000 & 5.61 & 9.57 \\ 6.92000 & 5.87 & 11.82 \\ 9.23000 & 5.98 & 13.46 \\ 13.80000 & 6.06 & 15.80 \\ 23.00000 & 6.10 & 18.83 \\ 46.10000 & 6.11 & 23.00 \\ 92.30000 & 6.10 & 27.20 \\ 138.00000 & 6.10 & 29.64 \\ 230.00000 & 6.10 & 32.75\end{array}$

$\begin{array}{ccc}\text { TD=96.0 BV } & \begin{array}{c}\text { ET }=0.477087 \\ \text { PRIMARY }\end{array} & \text { CASCADE } \\ \text { ENERG (HEV) } & 0.02 & 0.02 \\ 0.48100 & 0.05 \\ 0.48600 & 0.05 & 0.05 \\ 0.49600 & 0.11 & 0.11 \\ 0.51000 & 0.19 & 0.19 \\ 0.52900 & 0.30 & 0.30 \\ 0.55300 & 0.43 & 0.43 \\ 0.58200 & 0.58 & 0.58 \\ 0.62000 & 0.77 & 0.77 \\ 0.65700 & 0.99 & 0.99 \\ 0.73900 & 1.31 & 1.31 \\ 0.83400 & 1.69 & 1.69 \\ 0.95400 & 2.11 & 2.14 \\ 1.09000 & 2.52 & 2.60 \\ 1.28000 & 3.00 & 3.19 \\ 1.52000 & 3.47 & 3.87 \\ 1.81000 & 3.90 & 4.59 \\ 2.14300 & 4.27 & 5.32 \\ 2.48000 & 4.55 & 5.99 \\ 2.86000 & 4.79 & 6.67 \\ 3.33000 & 5.01 & 7.40 \\ 4.05000 & 5.23 & 8.38 \\ 4.77000 & 5.38 & 9.23 \\ 7.15000 & 5.63 & 11.38 \\ 9.54000 & 5.73 & 12.96 \\ 14.30000 & 5.81 & 15.22 \\ 23.80000 & 5.85 & 18.11 \\ 47.70000 & 5.85 & 22.11 \\ 95.40000 & 5.85 & 26.13 \\ 143.00000 & 5.85 & 28.49 \\ 238.00000 & 5.85 & 31.46 \\ & & \end{array}$


ATOMIC DISPLACEREN CROSS-SECTIONS (BARS) BY PAST ELECTRONS IN AG

\begin{tabular}{rrr} 
TD $=4.0$ & BV & \multicolumn{2}{c}{ BT=0.042549 MEV } \\
ENERGY (MEV) & PRIMARY & CASCADE \\
0.04290 & 10.24 & 10.24 \\
0.04340 & 24.29 & 24.29 \\
0.04420 & 45.51 & 45.51 \\
0.04550 & 76.96 & 76.96 \\
0.04720 & 113.08 & 113.08 \\
0.04930 & 151.07 & 151.07 \\
0.05190 & 189.79 & 189.79 \\
0.05530 & 229.55 & 229.55 \\
0.05950 & 265.93 & 265.93 \\
0.06590 & 302.81 & 302.81 \\
0.07440 & 330.28 & 330.28 \\
0.08500 & 345.58 & 345.78 \\
0.09780 & 350.10 & 354.48 \\
0.11400 & 346.27 & 359.15 \\
0.13600 & 335.26 & 360.35 \\
0.16100 & 321.49 & 359.01 \\
0.19100 & 306.54 & 356.55 \\
0.22100 & 293.99 & 354.38 \\
0.25500 & 282.41 & 352.70 \\
0.29700 & 271.14 & 351.82 \\
0.36100 & 258.55 & 352.60 \\
0.42500 & 249.64 & 355.24 \\
0.63800 & 233.21 & 370.96 \\
0.85000 & 225.63 & 390.42 \\
1.27000 & 218.86 & 429.05 \\
2.12000 & 214.19 & 495.47 \\
4.25000 & 211.07 & 608.43 \\
8.50000 & 209.36 & 736.90 \\
12.70000 & 208.77 & 815.41 \\
21.20000 & 208.33 & 918.04 \\
29.70000 & 208.16 & 986.52 \\
42.50000 & 208.05 & 1059.85 \\
85.00000 & 207.93 & 1202.59 \\
170.00000 & 207.86 & 1346.01
\end{tabular}

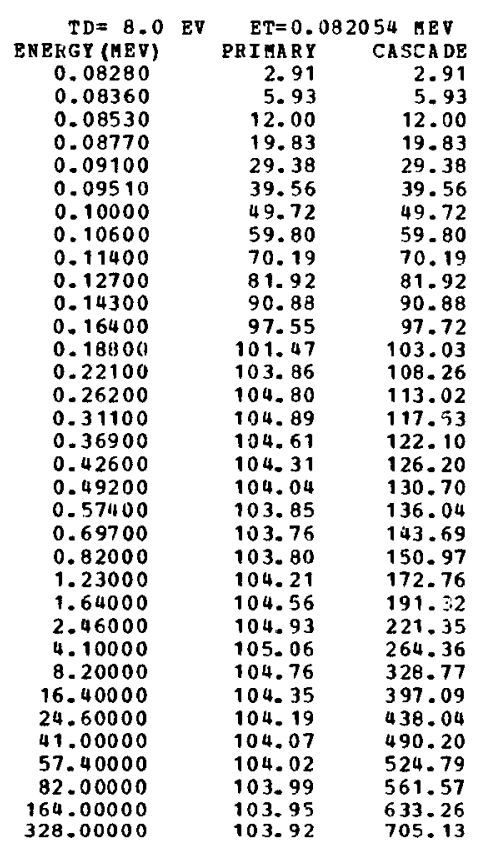

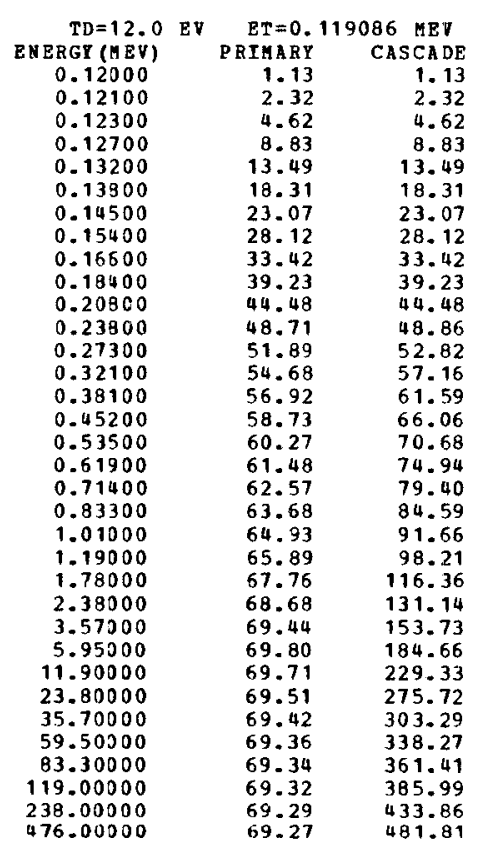

ATOMIC DISPLACEMENT CROSS-SECTIONS (BARNS) BY FAST ELECTRONS IN MS

$z=12 \quad A=24.320$

\begin{tabular}{crr} 
TD $=16.0$ & EV & \multicolumn{1}{c}{ ET $=0.154059$ HEV } \\
PNERGY (HEV) & PRIARY & CASCADE \\
0.15500 & 0.52 & 0.52 \\
0.15700 & 1.58 & 1.58 \\
0.16000 & 3.10 & 3.10 \\
0.16400 & 4.99 & 4.99 \\
0.17100 & 7.94 & 7.94 \\
0.17800 & 10.52 & 10.52 \\
0.18700 & 13.39 & 13.39 \\
0.20000 & 16.83 & 16.83 \\
0.21500 & 20.01 & 20.01 \\
0.23800 & 23.76 & 23.76 \\
0.26900 & 27.40 & 27.40 \\
0.30800 & 30.62 & 30.75 \\
0.35400 & 33.32 & 33.99 \\
0.41500 & 35.89 & 37.60 \\
0.49200 & 38.24 & 41.48 \\
0.58500 & 40.34 & 45.57 \\
0.69300 & 42.19 & 49.79 \\
0.80100 & 43.62 & 53.62 \\
0.92400 & 44.92 & 57.64 \\
1.07000 & 46.13 & 62.01 \\
1.30000 & 47.54 & 68.24 \\
1.54000 & 48.59 & 74.05 \\
2.31000 & 50.47 & 89.33 \\
3.08000 & 51.32 & 101.22 \\
4.62000 & 51.99 & 119.17 \\
7.70000 & 52.30 & 143.20 \\
15.40000 & 52.24 & 177.34 \\
30.80000 & 52.10 & 212.44 \\
46.20000 & 52.05 & 233.21 \\
77.00000 & 52.01 & 259.52 \\
107.00000 & 52.00 & 276.52 \\
154.00000 & 51.98 & 295.37
\end{tabular}

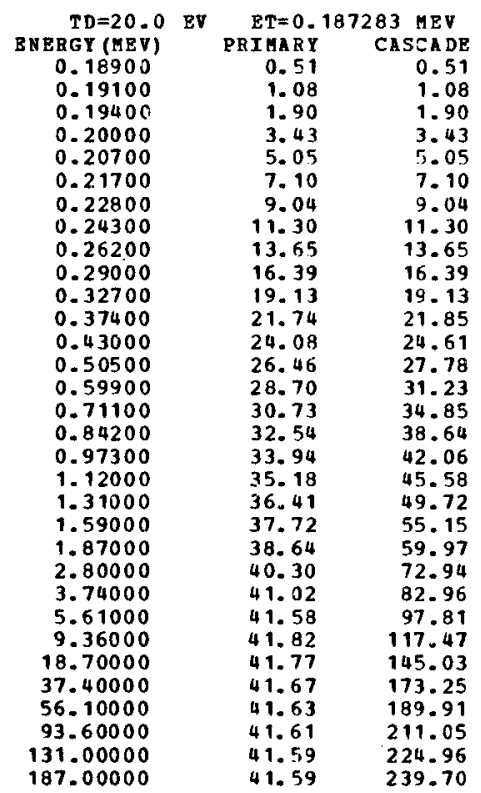

\begin{tabular}{|c|c|c|}
\hline \multirow{4}{*}{$\begin{array}{r}T D=24.0 \\
\text { ENRRGY (IEV) } \\
0.22100 \\
0.22300 \\
0.22700\end{array}$} & \multicolumn{2}{|c|}{$\mathrm{ET}=0.218997 \mathrm{MEV}$} \\
\hline & $\begin{array}{r}\text { PRIMARY } \\
0.36\end{array}$ & \\
\hline & 0,70 & 0.36 \\
\hline & 1.37 & 1.37 \\
\hline 0.23400 & 2.47 & 2.47 \\
\hline 0.24300 & 3.74 & 3.74 \\
\hline 0.25400 & 5.13 & 5.13 \\
\hline 0.26700 & 6.58 & 6.58 \\
\hline 0.28400 & 8.22 & 8.22 \\
\hline 0.30600 & 10.00 & 10.00 \\
\hline 0.33900 & 12.19 & 12.19 \\
\hline 0.38300 & 14.47 & 14.47 \\
\hline 0.43700 & 16.65 & 16.75 \\
\hline 0.50300 & 18.74 & 19.17 \\
\hline 0.59100 & 20.91 & 21.99 \\
\hline 0.70000 & 22.97 & 25.06 \\
\hline 0.83200 & 24.89 & 28.35 \\
\hline 0.98500 & 26.58 & 31.76 \\
\hline 1.13000 & 27.82 & 34.68 \\
\hline 1.31000 & 29.02 & 37.99 \\
\hline 1.53000 & 30.14 & 41.64 \\
\hline 1.86000 & 31.33 & 46.49 \\
\hline 2.18300 & 32.13 & 50.63 \\
\hline 3.28000 & 33.59 & 62.03 \\
\hline 4.37000 & 34.19 & 70.57 \\
\hline 6.56000 & 34.65 & 83.23 \\
\hline 10.90000 & 34.84 & 99.70 \\
\hline 21.80000 & 34.80 & 122.87 \\
\hline 43.70000 & 34.72 & 146.54 \\
\hline 65.60000 & 34.69 & 160.48 \\
\hline 109.00000 & 34.67 & 177.96 \\
\hline 153.00000 & 34.66 & 189.66 \\
\hline 218.00000 & 34.65 & 201.89 \\
\hline
\end{tabular}


ATONIC DISPLACEHENT CBOSS-SECTIONS (BARNS) BI PAST ELECTRONS IN HG $\mathrm{z}=12 \quad \mathrm{~A}=24.320$

\begin{tabular}{rrr}
$\begin{array}{c}\text { TD }=28.0 \\
\text { ENERGY EVEV }\end{array}$ & \multicolumn{2}{c}{ EI $=0.249389$ AET } \\
PRIARY & CASCADE \\
0.25100 & 0.19 & 0.19 \\
0.25400 & 0.53 & 0.53 \\
0.25900 & 1.08 & 1.08 \\
0.26600 & 1.81 & 1.81 \\
0.27600 & 2.76 & 2.76 \\
0.28900 & 3.88 & 3.88 \\
0.30400 & 5.02 & 5.02 \\
0.32400 & 6.36 & 6.36 \\
0.34900 & 7.79 & 7.79 \\
0.38600 & 9.56 & 9.56 \\
0.43600 & 11.50 & 11.50 \\
0.49800 & 13.43 & 13.52 \\
0.57300 & 15.30 & 15.67 \\
0.67300 & 17.28 & 18.21 \\
0.79800 & 19.20 & 21.00 \\
0.94700 & 20.96 & 23.97 \\
1.12000 & 22.51 & 27.04 \\
1.29000 & 23.68 & 29.77 \\
1.49000 & 24.75 & 32.68 \\
1.74000 & 25.76 & 35.96 \\
2.11000 & 26.81 & 40.24 \\
2.49000 & 27.55 & 44.09 \\
3.74000 & 28.81 & 54.12 \\
4.98000 & 29.33 & 61.59 \\
7.48000 & 29.71 & 72.63 \\
12.40000 & 29.86 & 86.81 \\
24.90000 & 29.82 & 106.88 \\
49.80000 & 29.76 & 127.15 \\
74.80000 & 29.73 & 139.13 \\
124.00000 & 29.72 & 154.06 \\
174.00000 & 29.71 & 164.09 \\
& &
\end{tabular}

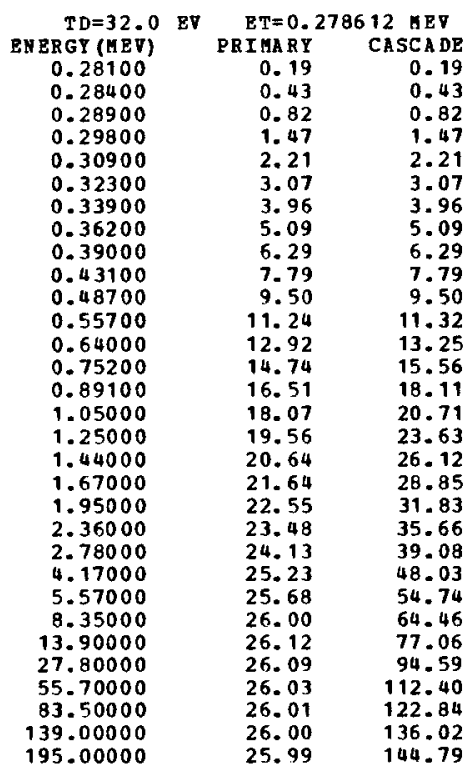

$\begin{array}{rrr}\text { ENERGY (UEV) } & \text { PBIHART } & \text { CASCADE } \\ 0.30900 & 0.13 & 0.13 \\ 0.31200 & 0.31 & 0.31 \\ 0.31900 & 0.70 & 0.70 \\ 0.32800 & 1.18 & 1.18 \\ 0.34000 & 1.78 & 1.78 \\ 0.35500 & 2.47 & 2.47 \\ 0.37400 & 3.27 & 3.27 \\ 0.39800 & 4.18 & 4.18 \\ 0.42900 & 5.22 & 5.22 \\ 0.47500 & 6.56 & 6.56 \\ 0.53600 & 8.06 & 8.06 \\ 0.61300 & 9.63 & 9.71 \\ 0.70500 & 11.19 & 11.48 \\ 0.82800 & 12.87 & 13.60 \\ 0.98100 & 14.51 & 15.95 \\ 1.16000 & 15.98 & 18.40 \\ 1.38000 & 17.35 & 21.08 \\ 1.59000 & 18.34 & 23.38 \\ 1.84000 & 19.24 & 25.85 \\ 2.14000 & 20.04 & 28.50 \\ 2.60000 & 20.90 & 32.07 \\ 3.06000 & 21.48 & 35.16 \\ 4.60000 & 22.46 & 43.30 \\ 6.13000 & 22.84 & 49.29 \\ 9.20000 & 23.12 & 58.03 \\ 15.30000 & 23.22 & 69.27 \\ 30.60000 & 23.18 & 84.89 \\ 61.30000 & 23.14 & 100.75 \\ 92.00000 & 23.12 & 110.06 \\ 153.00000 & 23.11 & 121.76 \\ 214.00000 & 23.11 & 129.49\end{array}$

ATOHIC DISPLAEEMENT CROSS-SBCTIONS (BARNS) BI FAST ELBCTRONS IN MG $=12 \quad A=24.320$

\begin{tabular}{|c|c|c|c|}
\hline $\begin{array}{r}T D=40.0 \\
\text { ENERGY (MEV) }\end{array}$ & EV & $\begin{array}{l}\mathrm{BT}=0.3 \\
\text { PRIMARY }\end{array}$ & CASCADE \\
\hline 0,33700 & & & \\
\hline 0.34000 & & 0.26 & $\begin{array}{l}0.13 \\
0.26\end{array}$ \\
\hline 0.34700 & & 0.56 & 0.56 \\
\hline 0.35700 & & 0.97 & 0.97 \\
\hline 0.37000 & & 1.47 & 1.47 \\
\hline 0.38700 & & 2.08 & 2.08 \\
\hline 0.40700 & & 2.74 & 2.74 \\
\hline 0.43400 & & 3.55 & 3.55 \\
\hline 0.46700 & & $\begin{array}{l}4.44 \\
5.63\end{array}$ & $\begin{array}{l}4.44 \\
5.63\end{array}$ \\
\hline $\begin{array}{l}0.51700 \\
0.58400\end{array}$ & & $\begin{array}{l}5.63 \\
7.00\end{array}$ & 7.00 \\
\hline 0.66800 & & 8.44 & 8.52 \\
\hline 0.76800 & & 9.88 & 10.15 \\
\hline 0.90100 & & 11.42 & 12.10 \\
\hline 1.06000 & & 12.89 & 14.18 \\
\hline 1.26000 & & 14.31 & 16.52 \\
\hline 1.50000 & & 15.58 & $\begin{array}{l}19.02 \\
21.16\end{array}$ \\
\hline $\begin{array}{l}1.73000 \\
2.00000\end{array}$ & & $\begin{array}{l}16.50 \\
17.32\end{array}$ & $\begin{array}{l}21.16 \\
23.42\end{array}$ \\
\hline 2.33000 & & 18.06 & 25.89 \\
\hline 2.83000 & & 18.84 & 29.16 \\
\hline 3.34000 & & $19 \cdot 37$ & 32.05 \\
\hline 5.01000 & & 20.23 & 39.43 \\
\hline 10.00000 & & 20.81 & 52.77 \\
\hline 16.70000 & & 20.89 & 63.01 \\
\hline 33.40000 & & 20.86 & 77.10 \\
\hline 66.80000 & & 20.82 & 91.35 \\
\hline $\begin{array}{l}100.00000 \\
167.00000\end{array}$ & & $\begin{array}{l}20.81 \\
20.80\end{array}$ & $\begin{array}{r}99.68 \\
110.30\end{array}$ \\
\hline 167.00000 & & & \\
\hline
\end{tabular}

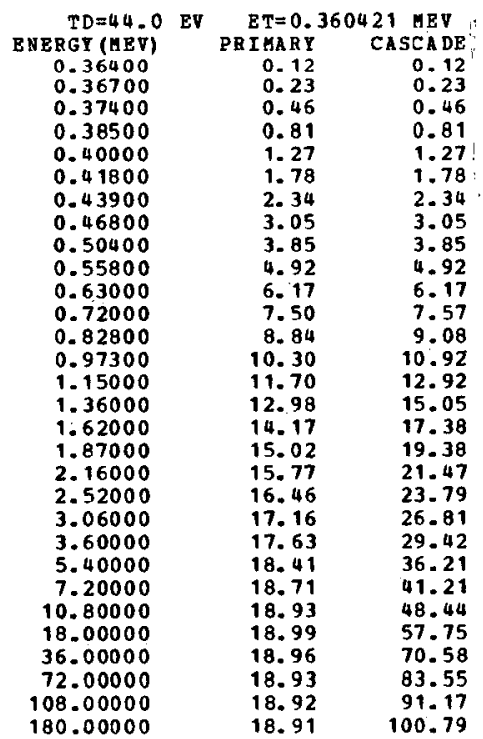

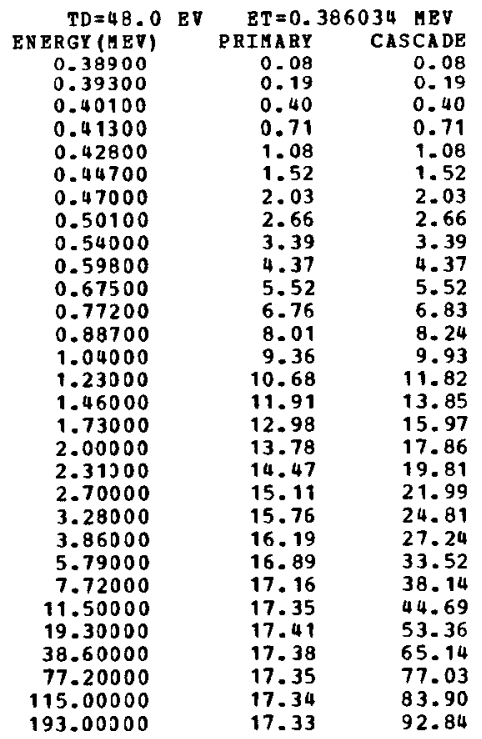


ATOHTC DISPLACEMENT CROSS-SECTIONS (BARNS) BY PAST ELECTRONS IN AG

\begin{tabular}{|c|c|c|c|}
\hline $\begin{array}{r}T D=52.0 \\
\text { BNERGY (MBV) }\end{array}$ & EV & $\begin{array}{r}\text { ET }=0.4 \\
\text { PRIMARY }\end{array}$ & $\begin{array}{l}936 \text { MEV } \\
\text { CASCADE }\end{array}$ \\
\hline 0.01500 & & $50+00$ & \\
\hline 0.41900 & & 0.09 & 0.09 \\
\hline 0.42700 & & 0.35 & 0.35 \\
\hline 0.43900 & & 0.60 & 0.60 \\
\hline 0.45600 & & 0.95 & 0.95 \\
\hline 0.47600 & & 1.33 & 1.33 \\
\hline 0.50100 & & 1.79 & 1.79 \\
\hline 0.53400 & & 2.36 & 2.36 \\
\hline 0.57500 & & 3.02 & 3.02 \\
\hline 0.63600 & & 3.92 & 3.92 \\
\hline 0.71900 & & 5.00 & 5.00 \\
\hline 0.82100 & & 6.15 & 6.21 \\
\hline 0.94500 & & 7.33 & 7.55 \\
\hline 1.10000 & & 8.55 & 9.07 \\
\hline 1.31000 & & 9.84 & 10.92 \\
\hline 1.56000 & & 11.01 & 12.87 \\
\hline 1.84000 & & 11.99 & 14.81 \\
\hline 2.13000 & & 12.74 & 16.60 \\
\hline 2.46000 & & 13.39 & 18.43 \\
\hline 2.87000 & & 13.97 & 20.44 \\
\hline 3.49000 & & 14.57 & 23.09 \\
\hline 4.10000 & & 14.96 & 25.33 \\
\hline 6.16000 & & 15.61 & 31.20 \\
\hline $\begin{array}{r}8.21000 \\
12.30000\end{array}$ & & 15.85 & 35.48 \\
\hline $\begin{array}{l}12.30000 \\
20.50000\end{array}$ & & $\begin{array}{l}16.02 \\
16.07\end{array}$ & $\begin{array}{l}41.63 \\
49.55\end{array}$ \\
\hline 41.00000 & & 16.04 & 60.44 \\
\hline 82.10000 & & 16.02 & 71.44 \\
\hline 123.00000 & & 16.01 & 77.88 \\
\hline 205.00000 & & & 86.02 \\
\hline
\end{tabular}

\begin{tabular}{rrr} 
TD=56.0 EV & \multicolumn{2}{c}{ ET=0.435183 MEV } \\
ENERGY(IIRV) & PRIMRY & CASCA DE \\
0.43900 & 0.07 & 0.07 \\
0.44300 & 0.14 & 0.14 \\
0.45200 & 0.30 & 0.30 \\
0.46500 & 0.53 & 0.53 \\
0.48300 & 0.84 & 0.84 \\
0.50400 & 1.18 & 1.18 \\
0.53000 & 1.59 & 1.59 \\
0.56500 & 2.11 & 2.11 \\
0.60900 & 2.72 & 2.72 \\
0.67400 & 3.56 & 3.56 \\
0.76100 & 4.56 & 4.56 \\
0.87000 & 5.65 & 5.71 \\
1.00000 & 6.75 & 6.96 \\
1.17000 & 7.94 & 8.45 \\
1.39000 & 9.15 & 10.17 \\
1.65000 & 10.23 & 11.98 \\
1.95000 & 11.15 & 13.83 \\
2.26000 & 11.86 & 15.53 \\
2.61000 & 12.46 & 17.25 \\
3.04000 & 13.00 & 19.13 \\
3.69000 & 13.55 & 21.59 \\
4.35000 & 13.92 & 23.73 \\
6.52000 & 14.50 & 29.19 \\
8.70000 & 14.73 & 33.20 \\
13.00000 & 14.88 & 38.90 \\
21.70000 & 14.92 & 46.28 \\
43.50000 & 14.90 & 56.44 \\
87.00000 & 14.87 & 65.65 \\
130.00000 & 14.86 & 72.58 \\
217.00000 & 14.86 & 80.17
\end{tabular}

\begin{tabular}{rrr} 
TD $=60.0$ & EV & \multicolumn{2}{c}{ ET $=0.458823$} & MEV \\
ENERGY(HEV) & PRIMARY & CASCADE \\
0.46300 & 0.06 & 0.06 \\
0.46700 & 0.12 & 0.12 \\
0.47700 & 0.28 & 0.28 \\
0.49000 & 0.47 & 0.47 \\
0.50900 & 0.74 & 0.74 \\
0.53200 & 1.06 & 1.06 \\
0.55900 & 1.43 & 1.43 \\
0.59600 & 1.91 & 1.91 \\
0.64200 & 2.47 & 2.47 \\
0.71100 & 3.26 & 3.26 \\
0.80200 & 4.19 & 4.20 \\
0.91700 & 5.22 & 5.28 \\
1.05000 & 6.24 & 6.43 \\
1.23000 & 7.37 & 7.85 \\
1.46000 & 8.51 & 9.47 \\
1.74000 & 9.55 & 11.23 \\
2.06000 & 10.43 & 13.00 \\
2.38000 & 11.09 & 14.57 \\
2.75000 & 11.65 & 16.20 \\
3.21000 & 12.16 & 18.00 \\
3.89000 & 12.66 & 20.29 \\
4.58000 & 13.00 & 22.30 \\
6.88000 & 13.55 & 27.45 \\
9.17000 & 13.75 & 31.19 \\
13.70000 & 13.89 & 36.52 \\
22.90000 & 13.93 & 43.45 \\
45.80000 & 13.90 & 52.90 \\
91.70000 & 13.88 & 62.45 \\
137.00000 & 13.87 & 67.99 \\
229.00000 & 13.86 & 75.09 \\
& &
\end{tabular}

ATOMIC DISPLACEAENT CROSS-SECTIONS (BARNS) BY PAST ELECTRONS IN HG $\mathrm{Z}=12 \quad \mathrm{~A}=24.320$

\begin{tabular}{ccr}
$\begin{array}{c}\text { TD }=64.0 \\
\text { ENERG (4EV) }\end{array}$ & \multicolumn{2}{c}{ ET $=0.481901$ AEV } \\
PRIMARY & CASCADE \\
0.48600 & 0.05 & 0.05 \\
0.49100 & 0.12 & 0.12 \\
0.50100 & 0.25 & 0.25 \\
0.51500 & 0.42 & 0.42 \\
0.53400 & 0.66 & 0.66 \\
0.55900 & 0.96 & 0.96 \\
0.58700 & 1.29 & 1.29 \\
0.62600 & 1.74 & 1.74 \\
0.67400 & 2.26 & 2.26 \\
0.74600 & 2.99 & 2.99 \\
0.84300 & 3.89 & 3.89 \\
0.96300 & 4.86 & 4.91 \\
1.10000 & 5.81 & 5.99 \\
1.30000 & 6.94 & 7.41 \\
1.54000 & 8.01 & 8.94 \\
1.83000 & 8.98 & 10.58 \\
2.16000 & 9.79 & 12.23 \\
2.50000 & 10.41 & 13.74 \\
2.89000 & 10.94 & 15.29 \\
3.37000 & 11.42 & 16.98 \\
4.09000 & 11.89 & 19.17 \\
4.81000 & 12.20 & 21.05 \\
7.22000 & 12.71 & 25.89 \\
9.63000 & 12.90 & 29.42 \\
14.40000 & 13.02 & 34.44 \\
24.00000 & 13.06 & 40.91 \\
48.10000 & 13.03 & 49.81 \\
96.30000 & 13.01 & 58.76 \\
144.00000 & 13.00 & 63.97
\end{tabular}

\begin{tabular}{rrr} 
TD $=68.0$ & EV & \multicolumn{1}{c}{ ET $=0.504455$} \\
BNRRGY $(M E V)$ & REI \\
0.50900 & 0.05 & CASCADE \\
0.51400 & 0.11 & 0.05 \\
0.52400 & 0.22 & 0.11 \\
0.53900 & 0.38 & 0.22 \\
0.55900 & 0.59 & 0.38 \\
0.58500 & 0.87 & 0.59 \\
0.61500 & 1.18 & 1.87 \\
0.65500 & 1.59 & 1.59 \\
0.70600 & 2.09 & 2.09 \\
0.78100 & 2.78 & 2.78 \\
0.88200 & 3.62 & 3.62 \\
1.00000 & 4.49 & 4.54 \\
1.16000 & 5.50 & 5.68 \\
1.36000 & 6.53 & 5.97 \\
1.61000 & 7.53 & 8.42 \\
1.91000 & 8.45 & 9.97 \\
2.27000 & 9.25 & 11.61 \\
2.62000 & 9.82 & 13.02 \\
3.02000 & 10.31 & 14.46 \\
3.53000 & 10.76 & 16.09 \\
4.28000 & 11.21 & 18.15 \\
5.04000 & 11.50 & 19.94 \\
7.56000 & 11.97 & 24.52 \\
10.00000 & 12.14 & 27.76 \\
15.10000 & 12.26 & 32.61 \\
25.20000 & 12.29 & 38.71 \\
50.40000 & 12.27 & 47.07 \\
100.00000 & 12.25 & 55.39 \\
151.00000 & 12.24 & 60.41
\end{tabular}

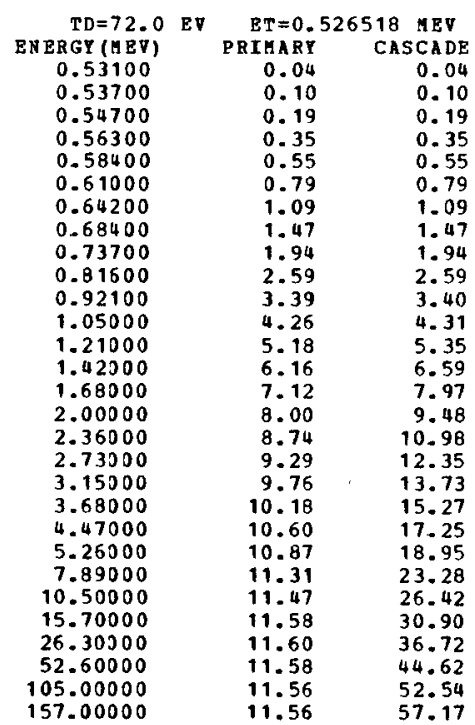


atOMIC displacenent CROSS-SECTIONS (BARS) BY FAST RLECTRONS IN AG

\begin{tabular}{rrr}
$\begin{array}{r}\text { TD }=76.0 \\
\text { ENERGY }\end{array}$ & \multicolumn{2}{c}{ ET $=0.548122$} \\
0.55300 & PRIMARY & CASCADE \\
0.55900 & 0.04 & 0.04 \\
0.55000 & 0.09 & 0.09 \\
0.578000 & 0.18 & 0.18 \\
0.60800 & 0.32 & 0.32 \\
0.63500 & 0.50 & 0.50 \\
0.66800 & 0.73 & 0.73 \\
0.71200 & 1.00 & 1.00 \\
0.76700 & 1.36 & 1.36 \\
0.84900 & 1.80 & 1.80 \\
0.95900 & 2.42 & 2.42 \\
1.09000 & 3.19 & 3.19 \\
1.26000 & 4.00 & 4.05 \\
1.47000 & 4.90 & 5.06 \\
1.75000 & 5.81 & 6.21 \\
2.08000 & 6.76 & 7.58 \\
2.46000 & 7.59 & 9.01 \\
2.85000 & 8.30 & 10.46 \\
3.28000 & 8.83 & 11.78 \\
3.83000 & 9.26 & 13.08 \\
4.65000 & 9.66 & 14.54 \\
5.48000 & 10.05 & 16.43 \\
8.22000 & 10.31 & 18.05 \\
10.90000 & 10.72 & 22.18 \\
16.40000 & 10.87 & 25.12 \\
27.40000 & 10.97 & 29.44 \\
54.80000 & 10.99 & 34.93 \\
109.00000 & 10.97 & 42.42 \\
164.00000 & 10.96 & 49.89 \\
& 10.95 & 54.34
\end{tabular}

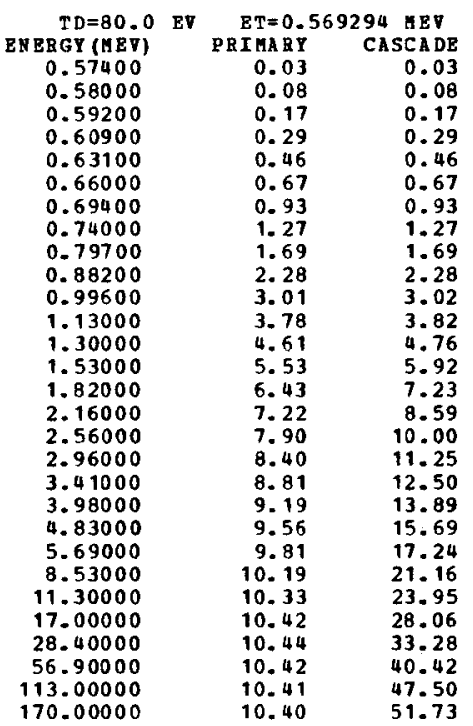

\begin{tabular}{rrr} 
TD $=84.0$ & EV & \multicolumn{2}{c}{ ET $=0.590059$ GEV } \\
ENERGI (BEV) & PRIMABY & CASCADE \\
0.59500 & 0.03 & 0.03 \\
0.60100 & 0.07 & 0.07 \\
0.61300 & 0.15 & 0.15 \\
0.63100 & 0.27 & 0.27 \\
0.65400 & 0.42 & 0.42 \\
0.68400 & 0.63 & 0.63 \\
0.71900 & 0.86 & 0.86 \\
0.76700 & 1.19 & 1.19 \\
0.82600 & 1.59 & 1.59 \\
0.91400 & 2.15 & 2.15 \\
1.03000 & 2.84 & 2.84 \\
1.18000 & 3.63 & 3.68 \\
1.35000 & 4.40 & 4.55 \\
1.59000 & 5.28 & 5.67 \\
1.88000 & 6.12 & 6.88 \\
2.24000 & 6.89 & 8.22 \\
2.65000 & 7.54 & 9.56 \\
3.06000 & 8.01 & 10.74 \\
3.54000 & 8.41 & 11.97 \\
4.13000 & 8.77 & 13.31 \\
5.01000 & 9.12 & 15.02 \\
5.90000 & 9.35 & 16.50 \\
8.85000 & 9.71 & 20.26 \\
11.80000 & 9.84 & 22.98 \\
17.70000 & 9.93 & 26.87 \\
29.50000 & 9.95 & 31.82 \\
59.00000 & 9.93 & 38.60 \\
118.00000 & 9.91 & 45.43 \\
177.00000 & 9.91 & 49.43
\end{tabular}

ATONIC DISPLACEHENT CROSS-SECTIONS (BARNS) BY PAST ELECTBONS IN HG $Z=12 \quad A=24.320$

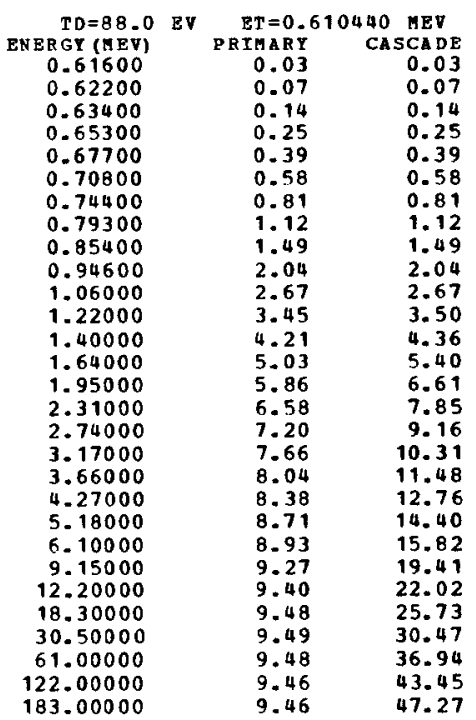

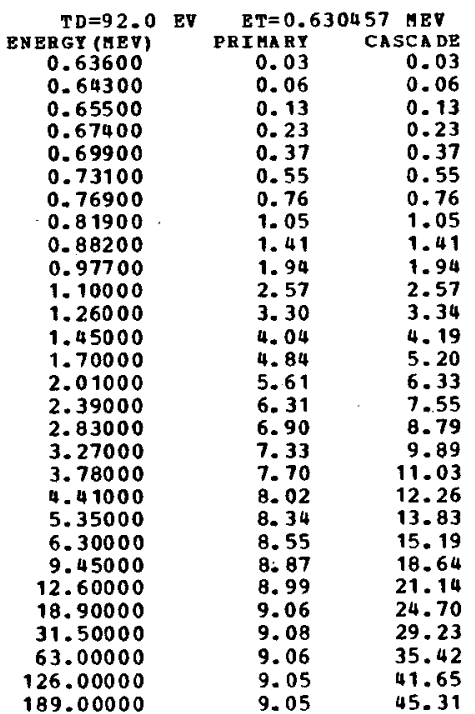

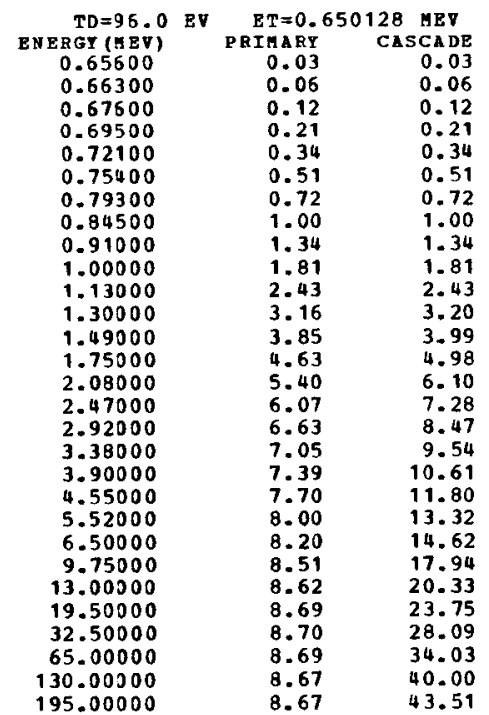


ATOHC DISPLACERENT CROSS-SECTIONS (BARNS) BI PAST ELECTRONS IN AL

\begin{tabular}{rrr} 
TD $=4.0$ & EV & \multicolumn{2}{c}{ ET $=0.047006$ MEV } \\
ENERGY(MEV) & PRIMARY & CASCADEE \\
0.04740 & 9.99 & 9.99 \\
0.04790 & 22.23 & 22.23 \\
0.04880 & 43.05 & 43.05 \\
0.05020 & 72.63 & 72.63 \\
0.05210 & 107.94 & 107.94 \\
0.05450 & 145.81 & 145.81 \\
0.05730 & 182.23 & 182.23 \\
0.06110 & 221.17 & 221.17 \\
0.06580 & 256.88 & 256.88 \\
0.07280 & 292.50 & 292.50 \\
0.08220 & 319.81 & 319.81 \\
0.09400 & 335.65 & 335.91 \\
0.10800 & 341.15 & 345.53 \\
0.12600 & 338.83 & 351.63 \\
0.15000 & 329.96 & 354.66 \\
0.17800 & 318.07 & 355.33 \\
0.21100 & 305.21 & 355.04 \\
0.24400 & 294.37 & 354.82 \\
0.28200 & 284.21 & 355.12 \\
0.32900 & 274.37 & 356.45 \\
0.39900 & 263.67 & 360.09 \\
0.47000 & 256.02 & 365.23 \\
0.70500 & 242.16 & 387.27 \\
0.94000 & 235.82 & 411.40 \\
1.41000 & 230.14 & 456.98 \\
2.35000 & 226.20 & 531.28 \\
4.70000 & 223.30 & 653.96 \\
9.40000 & 221.54 & 791.71 \\
14.10000 & 220.92 & 876.20 \\
23.50000 & 220.45 & 984.91 \\
32.90000 & 220.28 & 1057.43 \\
47.00000 & 220.16 & 1134.79 \\
94.00000 & 220.02 & 1285.97 \\
188.00000 & 219.94 & 1437.79 \\
& &
\end{tabular}

\begin{tabular}{|c|c|c|}
\hline $\begin{array}{r}\text { TDE } 8.0 \\
\text { BNERGY(1EV) } \\
0.09120 \\
0.09210 \\
0.09390 \\
0.09660 \\
0.10000 \\
0.10400 \\
0.11000 \\
0.11700 \\
0.12600 \\
0.14000 \\
0.15800 \\
0.18000 \\
0.20700 \\
0.24300 \\
0.28900 \\
0.34300 \\
0.40600 \\
0.46900 \\
0.54200 \\
0.63200 \\
0.76700 \\
0.90300 \\
1.35000 \\
1.80000 \\
2.71000 \\
4.51000 \\
9.03000 \\
18.00000 \\
27.10000 \\
45.10000 \\
63.20000 \\
90.30000 \\
180.00000 \\
361.00000\end{array}$ & $\begin{array}{c}\text { ET=0.0 } \\
\text { PRIMARY } \\
2.90 \\
5.87 \\
11.49 \\
19.21 \\
27.87 \\
36.75 \\
47.91 \\
58.32 \\
68.63 \\
79.95 \\
89.18 \\
95.89 \\
100.47 \\
103.58 \\
105.37 \\
106.29 \\
106.81 \\
107.14 \\
107.45 \\
107.81 \\
108.31 \\
108.78 \\
109.90 \\
110.55 \\
111.11 \\
111.29 \\
110.92 \\
110.45 \\
110.27 \\
110.13 \\
110.08 \\
110.04 \\
109.99 \\
109.95\end{array}$ & $\begin{array}{r}49 \text { ABV } \\
\text { CASCADE } \\
2.90 \\
5.87 \\
11.49 \\
19.21 \\
27.87 \\
36.75 \\
47.91 \\
58.32 \\
68.63 \\
79.95 \\
89.18 \\
96.07 \\
102.07 \\
108.00 \\
113.75 \\
119.23 \\
124.76 \\
129.81 \\
135.29 \\
141.68 \\
150.70 \\
159.21 \\
183.82 \\
204.51 \\
237.82 \\
284.30 \\
353.42 \\
425.78 \\
469.63 \\
524.77 \\
561.52 \\
600.48 \\
676.01 \\
752.37\end{array}$ \\
\hline
\end{tabular}

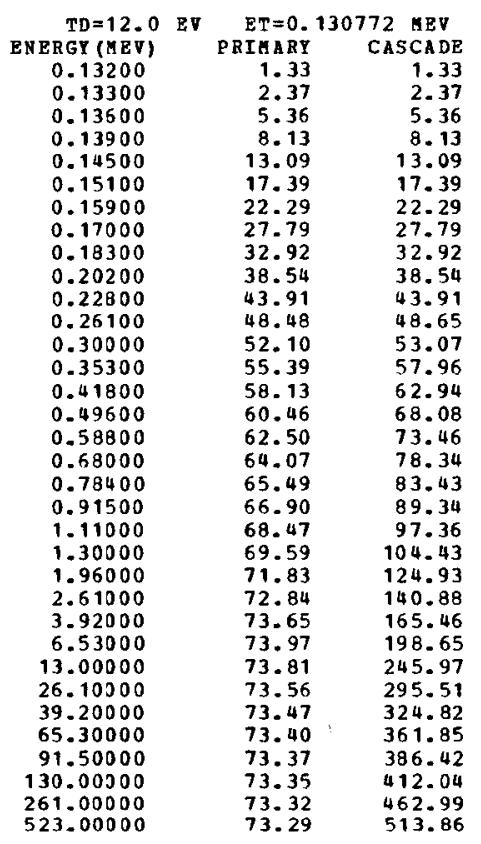

ATOMIC DISPLAEEHENT CROSS-SECTIONS (BARNS) BY FAST ELECTRONS IN AL

$T D=16.0$ EV $E T=0.168795 \mathrm{BEV}$

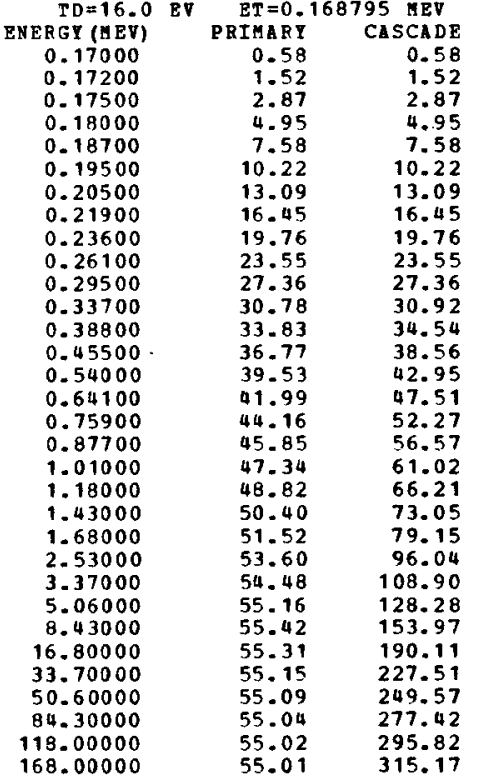

$z=13 \quad A=26.980$

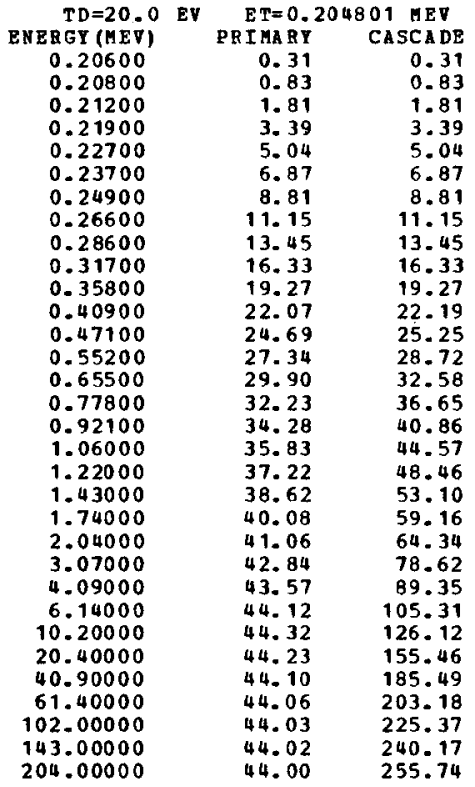

$T D=24.0 \mathrm{EV} \quad \mathrm{ET}=0.239081 \mathrm{MEV}$

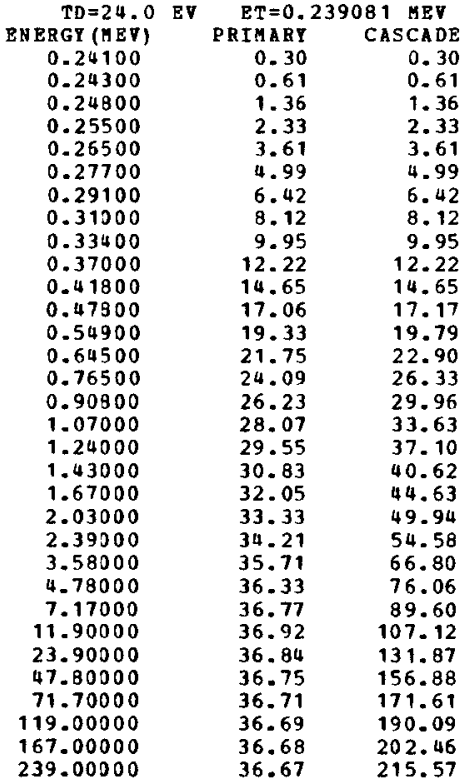


ATORIC DISPIAZENENT CROSS-SECTIONS (BARNS) BT FAST ELRCTRONS IN AL

\begin{tabular}{rrr} 
TD 28.0 & EV & \multicolumn{2}{c}{ ET $=0.271861$ MEV } \\
ENERGY (1EVV) & PRIYAR & CASCADE \\
0.27400 & 0.22 & 0.22 \\
0.27700 & 0.53 & 0.53 \\
0.28200 & 1.02 & 1.02 \\
0.29000 & 1.76 & 1.76 \\
0.30100 & 2.71 & 2.71 \\
0.31500 & 3.80 & 3.80 \\
0.33100 & 4.93 & 4.93 \\
0.35300 & 6.30 & 6.30 \\
0.38000 & 7.75 & 7.76 \\
0.42100 & 9.66 & 9.66 \\
0.47500 & 11.71 & 11.71 \\
0.54300 & 13.82 & 13.92 \\
0.62500 & 15.89 & 16.29 \\
6.73400 & 18.08 & 19.08 \\
0.86900 & 20.20 & 22.14 \\
1.03000 & 22.14 & 25.40 \\
1.22000 & 23.88 & 28.82 \\
1.41000 & 25.20 & 31.89 \\
1.63000 & 26.37 & 35.11 \\
1.90000 & 27.44 & 38.66 \\
2.31000 & 28.58 & 43.39 \\
2.71000 & 29.33 & 47.42 \\
4.07000 & 30.64 & 58.25 \\
5.43000 & 31.15 & 66.32 \\
8.15000 & 31.52 & 78.10 \\
13.50000 & 31.64 & 93.18 \\
27.10000 & 31.57 & 114.47 \\
54.30000 & 31.49 & 136.01 \\
81.50000 & 31.47 & 148.67 \\
135.00000 & 31.45 & 164.46 \\
190.00000 & 31.44 & 175.16 \\
& &
\end{tabular}

\begin{tabular}{crr} 
TD $=32.0$ & EV & \multicolumn{2}{c}{ ET $=0.303322$ MEV } \\
ENERGY (HEV) & PRTHARY & CASCADE \\
0.30600 & 0.19 & 0.19 \\
0.30900 & 0.41 & 0.41 \\
0.31500 & 0.82 & 0.82 \\
0.32400 & 1.41 & 1.41 \\
0.33600 & 2.14 & 2.14 \\
0.35100 & 2.99 & 2.99 \\
0.37000 & 3.96 & 3.96 \\
0.39400 & 5.07 & 5.07 \\
0.42400 & 6.30 & 6.30 \\
0.47000 & 7.93 & 7.93 \\
0.53000 & 9.72 & 9.72 \\
0.60600 & 11.61 & 11.70 \\
0.69700 & 13.48 & 13.83 \\
0.81800 & 15.48 & 16.36 \\
0.97000 & 17.44 & 19.18 \\
1.15000 & 19.23 & 22.15 \\
1.36000 & 20.80 & 25.24 \\
1.57000 & 21.99 & 28.02 \\
1.81000 & 23.03 & 30.88 \\
2.12000 & 24.03 & 34.19 \\
2.57000 & 25.03 & 38.39 \\
3.03000 & 25.71 & 42.12 \\
4.54000 & 26.84 & 51.73 \\
6.06000 & 27.28 & 58.91 \\
9.09000 & 27.59 & 69.30 \\
15.10000 & 27.68 & 82.65 \\
30.30000 & 27.62 & 101.34 \\
60.60000 & 27.55 & 120.16 \\
90.90000 & 27.53 & 131.24 \\
151.00000 & 27.51 & 145.13 \\
212.00000 & 27.51 & 154.43
\end{tabular}

\begin{tabular}{rrr}
$\begin{array}{r}\text { TD }=36.0 \\
\text { ENERGY(HEV) }\end{array}$ & \multicolumn{2}{c}{ ET $=0.333613$ MEV } \\
0.33600 & 0.13 & CASCADE \\
0.34000 & 0.33 & 0.13 \\
0.34600 & 0.64 & 0.64 \\
0.35500 & 1.12 & 1.12 \\
0.37000 & 1.76 & 1.76 \\
0.38600 & 2.44 & 2.44 \\
0.40700 & 3.26 & 3.26 \\
0.43300 & 4.18 & 4.18 \\
0.46700 & 5.28 & 5.28 \\
0.51700 & 6.69 & 6.69 \\
0.58300 & 8.29 & 8.30 \\
0.66700 & 10.02 & 10.10 \\
0.76700 & 11.72 & 12.04 \\
0.90000 & 13.56 & 14.36 \\
1.06000 & 15.31 & 16.85 \\
1.26000 & 16.98 & 19.62 \\
1.50000 & 18.48 & 22.57 \\
1.73000 & 19.56 & 25.10 \\
2.00000 & 20.52 & 27.77 \\
2.33000 & 21.38 & 30.69 \\
2.83000 & 22.29 & 34.55 \\
3.33000 & 22.89 & 37.90 \\
5.00000 & 23.88 & 46.62 \\
6.67000 & 24.27 & 53.06 \\
10.00000 & 24.53 & 62.36 \\
16.60000 & 24.60 & 74.26 \\
33.30000 & 24.54 & 90.91 \\
66.70000 & 24.49 & 107.70 \\
100.00000 & 24.47 & 117.54 \\
166.00000 & 24.46 & 129.88 \\
233.00000 & 24.45 & 138.14 \\
& &
\end{tabular}

ATOHIC DISPLACEMENT CROSS-SECTIONS (BARNS) BI PAST ELECTRONS IN AL

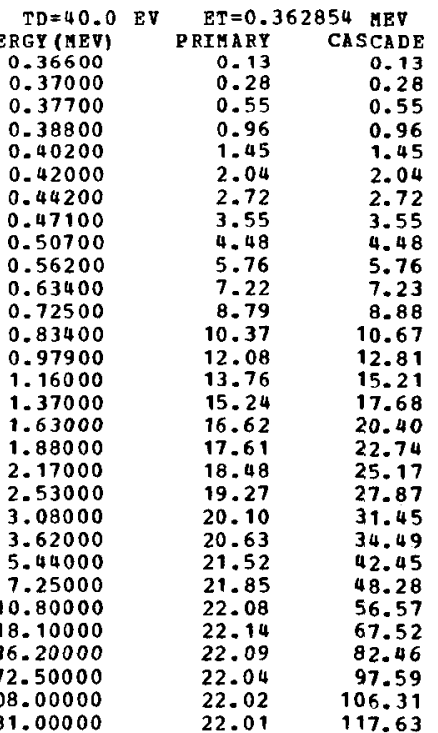

\begin{tabular}{|c|c|c|}
\hline ? & $\begin{array}{r}\text { ET }=0.3 \\
\text { PRI HARY } \\
0.12 \\
0.21 \\
0.45 \\
0.80 \\
1.25 \\
1.75 \\
2.35 \\
3.07 \\
3.91 \\
5.06 \\
6.40 \\
7.85 \\
9.31 \\
10.86 \\
12.46 \\
13.86 \\
15.13 \\
16.04 \\
16.83 \\
17.56 \\
18.30 \\
18.79 \\
19.58 \\
19.88 \\
20.07 \\
20.12 \\
20.08 \\
20.03 \\
20.02 \\
20.01\end{array}$ & $\begin{array}{r}148 \text { HEV } \\
\text { CASCA DE } \\
0.12 \\
0.21 \\
0.45 \\
0.80 \\
1.25 \\
1.75 \\
2.35 \\
3.07 \\
3.91 \\
5.06 \\
6.41 \\
7.93 \\
9.59 \\
11.52 \\
13.81 \\
16.15 \\
18.87 \\
20.84 \\
23.08 \\
25.59 \\
28.88 \\
31.71 \\
38.98 \\
44.34 \\
51.99 \\
61.88 \\
75.53 \\
89.27 \\
97.28 \\
107.47\end{array}$ \\
\hline
\end{tabular}

\begin{tabular}{rrr} 
TD $=48.0$ & EV & \multicolumn{1}{c}{ ET $=0.418581$ HEV } \\
ENERGY(HEV) & PRIHABY & CASCADE \\
0.42200 & 0.08 & 0.08 \\
0.42600 & 0.18 & 0.18 \\
0.43500 & 0.40 & 0.40 \\
0.44700 & 0.68 & 0.68 \\
0.46400 & 1.06 & 1.06 \\
0.48500 & 1.52 & 1.52 \\
0.51000 & 2.03 & 2.03 \\
0.54400 & 2.70 & 2.70 \\
0.58600 & 3.46 & 3.46 \\
0.64900 & 4.50 & 4.50 \\
0.73200 & 5.75 & 5.75 \\
0.83700 & 7.10 & 7.17 \\
0.96200 & 8.46 & 8.71 \\
1.13000 & 9.95 & 10.59 \\
1.33000 & 11.36 & 12.59 \\
1.59000 & 12.74 & 14.91 \\
1.88000 & 13.88 & 17.19 \\
2.17000 & 14.73 & 19.23 \\
2.51000 & 15.47 & 21.36 \\
2.93000 & 16.14 & 23.70 \\
3.55000 & 16.80 & 26.70 \\
4.18000 & 17.25 & 29.32 \\
6.27000 & 17.96 & 36.06 \\
8.37000 & 18.23 & 41.00 \\
12.50000 & 18.40 & 48.02 \\
20.90000 & 18.44 & 57.16 \\
41.80000 & 18.40 & 69.65 \\
83.70000 & 18.36 & 82.27 \\
125.00000 & 18.35 & 89.59 \\
209.00000 & 18.34 & 98.98
\end{tabular}


ATOMIC DISPLACEHENT CROSS-SECTIONS (BARNS) BY PAST ELECTRONS IN AL

\begin{tabular}{rrr} 
TD $=52.0$ & EV & \multicolumn{1}{c}{ ET $=0.445227$ HBV } \\
ENERGY (MEV) & PRIMARY & CASCADE \\
0.44900 & 0.08 & 0.08 \\
0.45400 & 0.17 & 0.17 \\
0.46300 & 0.35 & 0.35 \\
0.47600 & 0.60 & 0.60 \\
0.49400 & 0.94 & 0.94 \\
0.51600 & 1.33 & 1.33 \\
0.54300 & 1.80 & 1.80 \\
0.57800 & 2.39 & 2.39 \\
0.62300 & 3.09 & 3.09 \\
0.69000 & 4.06 & 4.06 \\
0.77900 & 5.22 & 5.22 \\
0.89000 & 6.47 & 6.54 \\
1.02000 & 7.73 & 7.96 \\
1.20000 & 9.15 & 9.74 \\
1.42000 & 10.50 & 11.69 \\
1.69000 & 11.77 & 13.81 \\
2.00000 & 12.83 & 15.96 \\
2.31000 & 13.62 & 17.88 \\
2.67000 & 14.31 & 19.87 \\
3.11000 & 14.92 & 22.03 \\
3.78000 & 15.54 & 24.87 \\
4.45000 & 15.95 & 27.31 \\
6.67000 & 16.59 & 33.56 \\
8.90000 & 16.84 & 38.15 \\
13.30000 & 16.99 & 44.65 \\
22.20000 & 17.02 & 53.08 \\
44.50000 & 16.98 & 64.66 \\
89.00000 & 16.95 & 76.30 \\
133.00000 & 16.94 & 83.06 \\
222.00000 & 16.93 & 91.71
\end{tabular}

$\begin{array}{rrr}\begin{array}{c}\text { TD }=56.0 \\ \text { ENERGY (MEV) }\end{array} & \begin{array}{c}\text { ET }=0.471151 \text { MEV } \\ \text { PRIMARY }\end{array} & \text { CASCADE } \\ 0.47500 & 0.06 & 0.06 \\ 0.48000 & 0.15 & 0.15 \\ 0.48900 & 0.29 & 0.29 \\ 0.50400 & 0.53 & 0.53 \\ 0.52200 & 0.81 & 0.81 \\ 0.54600 & 1.18 & 1.18 \\ 0.57400 & 1.60 & 1.60 \\ 0.61200 & 2.15 & 2.15 \\ 0.65900 & 2.79 & 2.79 \\ 0.73000 & 3.69 & 3.69 \\ 0.82400 & 4.77 & 4.78 \\ 0.94200 & 5.96 & 6.02 \\ 1.08000 & 7.14 & 7.36 \\ 1.27000 & 8.48 & 9.04 \\ 1.50000 & 9.74 & 10.86 \\ 1.79000 & 10.94 & 12.89 \\ 2.12000 & 11.95 & 14.93 \\ 2.44000 & 12.67 & 16.69 \\ 2.82000 & 13.31 & 18.56 \\ 3.29000 & 13.88 & 20.61 \\ 4.00000 & 14.45 & 23.27 \\ 4.71000 & 14.83 & 25.56 \\ 7.06000 & 15.42 & 31.40 \\ 9.42000 & 15.64 & 35.68 \\ 14.10000 & 15.78 & 41.76 \\ 23.50000 & 15.81 & 49.58 \\ 47.10000 & 15.77 & 60.34 \\ 94.20000 & 15.74 & 71.15 \\ 141.00000 & 15.73 & 77.46 \\ 235.00000 & 15.72 & 85.47\end{array}$

\begin{tabular}{rrr} 
TD $=60.0$ & EV & \multicolumn{1}{c}{ ET $=0.496408$ MEV } \\
ENERGY (HEV) & PBIHARY & CASCADE \\
0.50100 & 0.06 & 0.06 \\
0.50600 & 0.13 & 0.13 \\
0.51600 & 0.27 & 0.27 \\
0.53100 & 0.47 & 0.47 \\
0.55100 & 0.74 & 0.74 \\
0.57500 & 1.06 & 1.06 \\
0.60500 & 1.44 & 1.44 \\
0.64500 & 1.94 & 1.94 \\
0.69400 & 2.53 & 2.53 \\
0.75900 & 3.38 & 3.38 \\
0.85800 & 4.40 & 4.40 \\
0.99200 & 5.52 & 5.58 \\
1.14000 & 6.65 & 6.87 \\
1.34000 & 7.91 & 8.45 \\
1.58000 & 9.10 & 10.16 \\
1.88000 & 10.21 & 12.05 \\
2.23000 & 11.16 & 13.99 \\
2.58000 & 11.86 & 15.72 \\
2.97000 & 12.44 & 17.43 \\
3.47000 & 12.98 & 19.38 \\
4.21000 & 13.51 & 21.87 \\
4.96000 & 13.86 & 24.02 \\
7.44000 & 14.40 & 29.51 \\
9.92000 & 14.60 & 33.51 \\
14.80000 & 14.73 & 39.16 \\
24.80000 & 14.75 & 46.54 \\
49.60000 & 14.72 & 56.56 \\
99.20000 & 14.69 & 66.66 \\
148.00000 & 14.68 & 72.50 \\
248.00000 & 14.67 & 80.05
\end{tabular}

ATOMIC DISPLACENENT CROSS-SECTIONS (BARNS) BY PAST ELECTRONS IN AL $\mathrm{Z}=13 \quad \mathrm{~A}=26.980$

\begin{tabular}{rrr} 
TDE64.0 & EV & \multicolumn{1}{c}{ ET $=0.521047$ MEV } \\
ENERGY (HEV) & PRIMARY & CASCADE \\
0.52600 & 0.06 & 0.06 \\
0.53100 & 0.12 & 0.12 \\
0.54100 & 0.23 & 0.23 \\
0.55700 & 0.42 & 0.42 \\
0.57800 & 0.66 & 0.66 \\
0.60400 & 0.96 & 0.96 \\
0.63500 & 1.31 & 1.31 \\
0.67700 & 1.77 & 1.77 \\
0.72900 & 2.33 & 2.33 \\
0.80700 & 3.12 & 3.12 \\
0.91100 & 4.08 & 4.09 \\
1.04000 & 5.13 & 5.19 \\
1.19000 & 6.18 & 6.38 \\
1.40000 & 7.38 & 7.89 \\
1.66000 & 8.54 & 9.56 \\
1.97000 & 9.58 & 11.33 \\
2.34000 & 10.49 & 13.19 \\
2.70000 & 11.13 & 14.79 \\
3.12000 & 11.69 & 16.46 \\
3.64000 & 12.19 & 18.28 \\
4.42000 & 12.68 & 20.64 \\
5.21000 & 13.01 & 22.68 \\
7.81000 & 13.51 & 27.84 \\
10.40000 & 13.69 & 31.59 \\
15.60000 & 13.81 & 36.96 \\
26.00000 & 13.83 & 43.82 \\
52.10000 & 13.80 & 53.25 \\
104.00000 & 13.77 & 62.69 \\
156.00000 & 13.76 & 68.25
\end{tabular}

\begin{tabular}{rrr} 
TD=68.0 & \multicolumn{1}{c}{ EV } & \multicolumn{1}{c}{ ET=0.545111 MEV } \\
ENERGY (HEV) & PEIHARY & CASCADE \\
0.55000 & 0.05 & 0.05 \\
0.55600 & 0.11 & 0.11 \\
0.56600 & 0.21 & 0.21 \\
0.58300 & 0.38 & 0.38 \\
0.50500 & 0.60 & 0.60 \\
0.63200 & 0.87 & 0.87 \\
0.66500 & 1.20 & 1.20 \\
0.70800 & 1.63 & 1.63 \\
0.76300 & 2.16 & 2.16 \\
0.84400 & 2.89 & 2.89 \\
0.95300 & 3.81 & 3.81 \\
1.09000 & 4.82 & 4.88 \\
1.25000 & 5.83 & 6.03 \\
1.47000 & 6.97 & 7.47 \\
1.74000 & 8.06 & 9.04 \\
2.07000 & 9.06 & 10.75 \\
2.45000 & 9.89 & 12.49 \\
2.83000 & 10.51 & 14.02 \\
3.27000 & 11.03 & 15.60 \\
3.81000 & 11.49 & 17.32 \\
4.63000 & 11.96 & 19.56 \\
5.45000 & 12.26 & 21.48 \\
8.17000 & 12.72 & 26.36 \\
10.90000 & 12.89 & 29.91 \\
16.30000 & 13.00 & 34.95 \\
27.20000 & 13.02 & 41.43 \\
54.50000 & 12.98 & 50.31 \\
109.00000 & 12.96 & 59.22 \\
163.00000 & 12.95 & 64.41 \\
& &
\end{tabular}

\begin{tabular}{crr} 
TD $=72.0$ & EV & \multicolumn{2}{c}{ ET $=0.568639$ MRV } \\
ENEBGY (HEV) & PRIMARY & CASCADE \\
0.57400 & 0.05 & 0.05 \\
0.58000 & 0.10 & 0.10 \\
0.59100 & 0.19 & 0.19 \\
0.60800 & 0.34 & 0.34 \\
0.63100 & 0.55 & 0.55 \\
0.65900 & 0.80 & 0.80 \\
0.69300 & 1.10 & 1.10 \\
0.73900 & 1.51 & 1.51 \\
0.79600 & 2.00 & 2.00 \\
0.88100 & 2.71 & 2.71 \\
0.99500 & 3.58 & 3.58 \\
1.13000 & 4.49 & 4.55 \\
1.30000 & 5.48 & 5.67 \\
1.53000 & 6.57 & 7.04 \\
1.81000 & 7.61 & 8.54 \\
2.16300 & 8.57 & 10.20 \\
2.55000 & 9.35 & 11.83 \\
2.95000 & 9.94 & 13.31 \\
3.41000 & 10.43 & 14.82 \\
3.98000 & 10.87 & 16.47 \\
4.83000 & 11.31 & 18.58 \\
5.68000 & 11.59 & 20.39 \\
8.52000 & 12.02 & 25.02 \\
11.30000 & 12.18 & 28.32 \\
17.00000 & 12.28 & 33.15 \\
28.40000 & 12.29 & 39.29 \\
56.80000 & 12.26 & 47.66 \\
113.00000 & 12.24 & 56.02 \\
170.00000 & 12.23 & 60.99
\end{tabular}


ATOMIC DISPLALEMENT CROSS-SBCTIONS (BARNS) BY PAST RLBCTRONS IN AL $=13 \quad=26,980$

\begin{tabular}{rrr}
$\begin{array}{c}\text { TD }=76.0 \\
\text { ENERGY }\end{array}$ BEV & \multicolumn{1}{c}{ ET $=0.591665$} & MEV \\
PRIMARY & CASCADE \\
0.59700 & 0.04 & 0.04 \\
0.60300 & 0.08 & 0.08 \\
0.61500 & 0.18 & 0.18 \\
0.63300 & 0.32 & 0.32 \\
0.65600 & 0.50 & 0.50 \\
0.68600 & 0.74 & 0.74 \\
0.72100 & 1.02 & 1.02 \\
0.76900 & 1.40 & 1.40 \\
0.82800 & 1.87 & 1.87 \\
0.91700 & 2.54 & 2.54 \\
1.03000 & 3.33 & 3.34 \\
1.18000 & 4.27 & 4.32 \\
1.36000 & 5.22 & 5.41 \\
1.59000 & 6.22 & 6.67 \\
1.89000 & 7.24 & 8.15 \\
2.24000 & 8.12 & 9.68 \\
2.65000 & 8.89 & 11.29 \\
3.07000 & 9.43 & 12.67 \\
3.54000 & 9.90 & 14.09 \\
4.14000 & 10.32 & 15.68 \\
5.02000 & 10.72 & 17.69 \\
5.91000 & 10.99 & 19.42 \\
8.87000 & 11.40 & 23.83 \\
11.90000 & 11.54 & 26.99 \\
17.70000 & 11.63 & 31.55 \\
29.50000 & 11.64 & 37.34 \\
59.10000 & 11.62 & 45.30 \\
118.00000 & 11.59 & 53.25 \\
177.00000 & 11.59 & 57.93 \\
& &
\end{tabular}

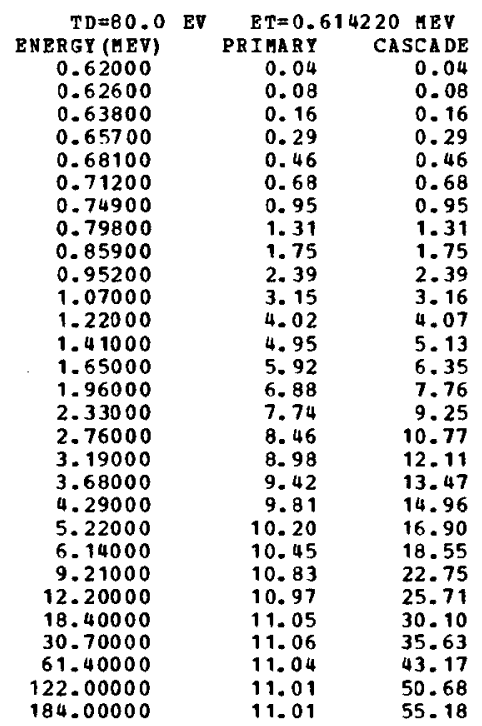

\begin{tabular}{rrr} 
TD $=34.0$ & EV & \multicolumn{2}{c}{ ET $=0.636332$} & UEV \\
ENERGY (HEV) & PRIMARY & CASCADE \\
0.64200 & 0.03 & 0.03 \\
0.64900 & 0.07 & 0.07 \\
0.66100 & 0.14 & 0.14 \\
0.68000 & 0.26 & 0.26 \\
0.70500 & 0.43 & 0.43 \\
0.73800 & 0.63 & 0.63 \\
0.77500 & 0.89 & 0.89 \\
0.82700 & 1.23 & 1.23 \\
0.89000 & 1.64 & 1.64 \\
0.98500 & 2.26 & 2.26 \\
1.11000 & 3.00 & 3.00 \\
1.27000 & 3.85 & 3.90 \\
1.46000 & 4.71 & 4.88 \\
1.71000 & 5.64 & 6.06 \\
2.03000 & 6.56 & 7.41 \\
2.41000 & 7.38 & 8.83 \\
2.86000 & 8.07 & 10.30 \\
3.30000 & 8.57 & 11.57 \\
3.81000 & 8.98 & 12.88 \\
4.45000 & 9.36 & 14.32 \\
5.40000 & 9.72 & 16.16 \\
6.36000 & 9.96 & 17.75 \\
9.54000 & 10.32 & 21.75 \\
12.70000 & 10.45 & 24.64 \\
19.00000 & 10.52 & 28.74 \\
31.80000 & 10.53 & 34.04 \\
63.60000 & 10.51 & 41.22 \\
127.00000 & 10.49 & 48.43 \\
190.00000 & 10.48 & 52.63 \\
& &
\end{tabular}

ATOMIC DISPLACEMENT CROSS-SECTIONS (BARNS) BP PAST BLECTBONS IN AL $z=13 \quad A=25.980$

\begin{tabular}{rrr} 
TD=88.0 & \multicolumn{3}{c}{ EV } & ET $=0.658025$ & MEV \\
ENERGY(HEV) & PRIMARY & CASCADE \\
0.65400 & 0.03 & 0.03 \\
0.67100 & 0.07 & 0.07 \\
0.68400 & 0.14 & 0.14 \\
0.70400 & 0.25 & 0.25 \\
0.73000 & 0.40 & 0.40 \\
0.76300 & 0.59 & 0.59 \\
0.80200 & 0.83 & 0.83 \\
0.85500 & 1.15 & 1.15 \\
0.92100 & 1.56 & 1.56 \\
1.01000 & 2.09 & 2.09 \\
1.15000 & 2.86 & 2.87 \\
1.31000 & 3.66 & 3.70 \\
1.51000 & 4.50 & 4.67 \\
1.77000 & 5.40 & 5.81 \\
2.10000 & 6.28 & 7.10 \\
2.50000 & 7.07 & 8.49 \\
2.96000 & 7.72 & 9.88 \\
3.42000 & 8.20 & 11.11 \\
3.94000 & 8.59 & 12.35 \\
4.60000 & 8.94 & 13.73 \\
5.59000 & 9.29 & 15.51 \\
6.58000 & 9.51 & 17.02 \\
9.87000 & 9.85 & 20.85 \\
13.10000 & 9.97 & 23.58 \\
19.70000 & 10.05 & 27.55 \\
32.90000 & 10.06 & 32.59 \\
65.80000 & 10.03 & 39.45 \\
131.00000 & 10.01 & 46.30 \\
197.00000 & 10.01 & 50.37
\end{tabular}

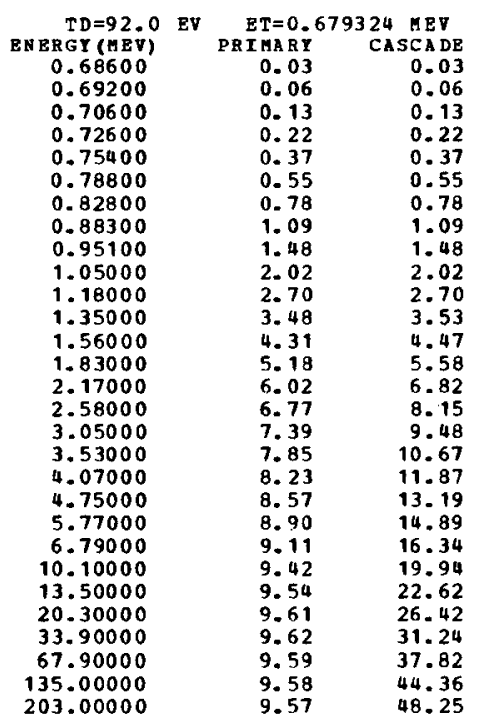

\begin{tabular}{ccc} 
TD $=96.0$ & BV & \multicolumn{2}{c}{ ET $=0.700247$} & MEV \\
ENERGY (IEV) & PRI HARY & CASCADE \\
0.70700 & 0.03 & 0.03 \\
0.71400 & 0.06 & 0.06 \\
0.72900 & 0.12 & 0.12 \\
0.74900 & 0.21 & 0.21 \\
0.77700 & 0.35 & 0.35 \\
0.81200 & 0.52 & 0.52 \\
0.85400 & 0.74 & 0.74 \\
0.91000 & 1.03 & 1.03 \\
0.98000 & 1.40 & 1.40 \\
1.08000 & 1.92 & 1.92 \\
1.22000 & 2.59 & 2.60 \\
1.40000 & 3.37 & 3.42 \\
1.61000 & 4.14 & 4.30 \\
1.89000 & 4.98 & 5.37 \\
2.24000 & 5.79 & 6.57 \\
2.66000 & 6.50 & 7.84 \\
3.15000 & 7.10 & 9.13 \\
3.64000 & 7.54 & 10.27 \\
4.20000 & 7.90 & 11.43 \\
4.90000 & 8.22 & 12.70 \\
5.95000 & 8.53 & 14.33 \\
7.00000 & 8.73 & 15.72 \\
10.50000 & 9.04 & 19.25 \\
14.00000 & 9.15 & 21.80 \\
21.00000 & 9.21 & 25.43 \\
35.00000 & 9.22 & 30.04 \\
70.00000 & 9.19 & 36.33 \\
140.00000 & 9.18 & 42.65 \\
210.00000 & 9.17 & 46.36
\end{tabular}


ATOMIC DISPLACEARNT CROSS-SBCTIONS (BARNS) BY FAST ELECTRONS IN SI $\mathrm{Z}=14 \quad \mathrm{~A}=28.090$

\begin{tabular}{|c|c|c|}
\hline $\begin{array}{r}T D=4.0 \\
\text { ENERGY }\end{array}$ & PRIMART & 856 UEV \\
\hline $\begin{array}{c}\text { ENERGY (AEV) } \\
0.04930\end{array}$ & $\begin{array}{l}\text { PRIUARY } \\
11.65\end{array}$ & CASCADE \\
\hline 0.04980 & 24.28 & 24.28 \\
\hline 0.05080 & 48.14 & 48.14 \\
\hline 0.05220 & 78.63 & 78.63 \\
\hline 0.05420 & 117.02 & 117.02 \\
\hline 0.05660 & 156.25 & 156.25 \\
\hline 0.05960 & 196.72 & 196.72 \\
\hline 0.06350 & 238.17 & 238.17 \\
\hline 0.06830 & 276.23 & 276.23 \\
\hline 0.07570 & $\begin{array}{l}315.64 \\
345.26\end{array}$ & $\begin{array}{l}315.64 \\
345.26\end{array}$ \\
\hline 0.09770 & 362.96 & 363.25 \\
\hline 0.11200 & 369.47 & 374.16 \\
\hline 0.13100 & 367.71 & 381.67 \\
\hline 0.15600 & 358.92 & 385.87 \\
\hline 0.18500 & 346.89 & 387.54 \\
\hline 0.21900 & 333.84 & 388.22 \\
\hline 0.25400 & 322.49 & 388.88 \\
\hline $\begin{array}{l}0.29300 \\
0.34100\end{array}$ & $\begin{array}{l}312.19 \\
302.22\end{array}$ & $\begin{array}{l}390.11 \\
392.51\end{array}$ \\
\hline 0.41500 & 291.01 & 397.84 \\
\hline 0.48800 & 283.24 & 404.48 \\
\hline 0.73200 & 268.99 & 431.31 \\
\hline 0.97700 & 262.45 & 459.76 \\
\hline 1.46000 & 256.58 & 511.79 \\
\hline 2.44000 & 252.33 & 596.66 \\
\hline 4.88000 & 249.03 & 734.78 \\
\hline 9.77000 & 246.95 & 889.22 \\
\hline 14.60000 & 246.22 & 982.70 \\
\hline 24.40000 & 245.67 & 1104.66 \\
\hline 34.10000 & 245.46 & 1185.10 \\
\hline 48.80000 & 245.32 & 1271.77 \\
\hline 97.70000 & 245.15 & 1440.51 \\
\hline 195.00000 & 245.04 & 1609.18 \\
\hline
\end{tabular}

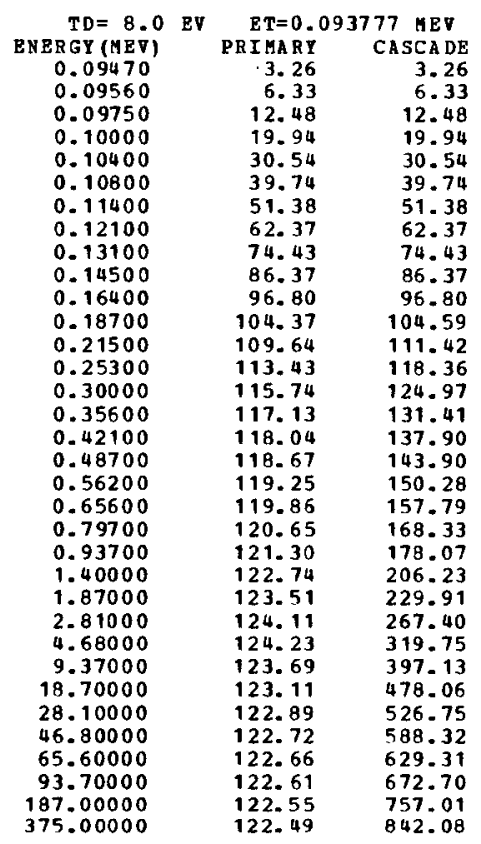

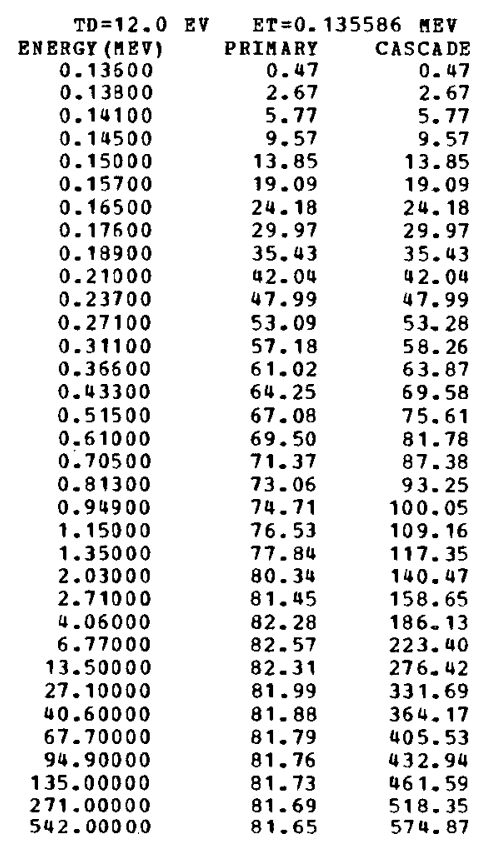

ATORIC DISPLACEMENT CROSS-SECTIONS (BARNS) BY PAST ELECTRONS IH 5 I $z=14 \quad A=28.090$

\begin{tabular}{crr} 
TD $=16.0$ & EV & \multicolumn{1}{c}{ ET $=0.174850$ MEV } \\
ENERGY (HEV) & PRIHARY & CASCADE \\
0.17600 & 0.58 & 0.58 \\
0.17800 & 1.56 & 1.56 \\
0.18100 & 2.97 & 2.97 \\
0.18700 & 5.57 & 5.57 \\
0.19400 & 8.29 & 8.29 \\
0.20200 & 11.06 & 11.06 \\
0.21300 & 14.36 & 14.36 \\
0.22700 & 17.89 & 17.89 \\
0.24400 & 21.41 & 21.41 \\
0.27100 & 25.78 & 25.78 \\
0.30500 & 29.90 & 29.90 \\
0.34900 & 33.82 & 33.98 \\
0.40200 & 37.31 & 38.11 \\
0.47200 & 40.74 & 42.74 \\
0.55900 & 43.90 & 47.71 \\
0.66400 & 45.77 & 52.97 \\
0.78600 & 49.29 & 58.40 \\
0.90900 & 51.24 & 63.36 \\
1.04000 & 52.89 & 68.20 \\
1.22000 & 54.62 & 74.26 \\
1.48000 & 56.43 & 82.08 \\
1.74000 & 57.69 & 89.03 \\
2.62000 & 59.98 & 108.12 \\
3.49000 & 60.93 & 122.59 \\
5.24000 & 61.63 & 144.35 \\
8.74000 & 61.86 & 173.17 \\
17.40000 & 61.67 & 213.46 \\
34.90000 & 61.46 & 255.17 \\
52.40000 & 61.39 & 279.76 \\
87.40000 & 61.33 & 310.87 \\
122.00000 & 61.31 & 331.21 \\
174.00000 & 61.29 & 352.88
\end{tabular}

\begin{tabular}{ccr} 
TD $=20.0$ & EV & \multicolumn{1}{c}{ ET $=0.211985$ HEV } \\
ENERGY (HEV) & PRIMARY & CASCADE \\
0.21400 & 0.55 & 0.55 \\
0.21600 & 1.07 & 1.07 \\
0.22000 & 2.09 & 2.09 \\
0.22600 & 3.51 & 3.51 \\
0.23500 & 5.46 & 5.46 \\
0.24500 & 7.39 & 7.39 \\
0.25800 & 9.60 & 9.60 \\
0.27500 & 12.08 & 12.08 \\
0.29600 & 14.66 & 14.66 \\
0.32800 & 17.85 & 17.85 \\
0.37000 & 21.13 & 21.13 \\
0.42300 & 24.32 & 24.46 \\
0.48700 & 27.32 & 27.94 \\
0.57200 & 30.39 & 31.96 \\
0.67800 & 33.32 & 36.33 \\
0.80500 & 35.99 & 40.97 \\
0.95300 & 38.35 & 45.78 \\
1.10000 & 40.15 & 50.09 \\
1.27000 & 41.76 & 54.63 \\
1.48000 & 43.27 & 59.71 \\
1.80000 & 44.91 & 66.56 \\
2.11000 & 46.00 & 72.41 \\
3.17000 & 47.94 & 88.44 \\
4.23000 & 48.73 & 100.56 \\
6.35000 & 49.29 & 118.45 \\
10.50000 & 49.45 & 141.50 \\
21.10000 & 49.31 & 174.47 \\
42.30000 & 49.15 & 207.96 \\
63.50000 & 49.10 & 227.67 \\
105.00000 & 49.06 & 252.17 \\
148.00000 & 49.04 & 268.93 \\
211.00000 & 49.03 & 286.26
\end{tabular}

\begin{tabular}{crr}
$\begin{array}{c}\text { TD }=24.0 \\
\text { ENERGY (HEV) }\end{array}$ & \multicolumn{2}{c}{ ET $=0.247304$ MBV } \\
PEIMARY & CASCADE \\
0.24900 & 0.28 & 0.28 \\
0.25200 & 0.76 & 0.76 \\
0.25700 & 1.54 & 1.54 \\
0.26400 & 2.56 & 2.56 \\
0.27400 & 3.90 & 3.90 \\
0.28600 & 5.35 & 5.35 \\
0.30100 & 6.98 & 6.98 \\
0.32100 & 8.87 & 8.87 \\
0.34600 & 10.91 & 10.91 \\
0.38300 & 13.42 & 13.42 \\
0.43200 & 16.12 & 16.12 \\
0.49400 & 18.86 & 18.98 \\
0.56800 & 21.47 & 21.98 \\
0.66700 & 24.23 & 25.52 \\
0.79100 & 26.91 & 29.43 \\
0.93900 & 29.35 & 33.57 \\
1.11000 & 31.48 & 37.83 \\
1.28000 & 33.11 & 41.64 \\
1.48000 & 34.58 & 45.71 \\
1.73000 & 35.95 & 50.28 \\
2.10000 & 37.37 & 56.24 \\
2.47000 & 38.33 & 61.45 \\
3.70000 & 39.97 & 75.21 \\
4.94000 & 40.62 & 85.60 \\
7.41000 & 41.07 & 100.76 \\
12.30500 & 41.20 & 120.34 \\
24.70000 & 41.07 & 147.95 \\
49.40000 & 40.95 & 175.84 \\
74.10000 & 40.91 & 192.26 \\
123.00000 & 40.88 & 212.85 \\
173.00000 & 40.87 & 226.74 \\
247.00000 & 40.86 & 241.24 \\
& &
\end{tabular}


ATOHIC DISPLACERENT CROSS-SECTIONS (BARNS) BY FAST ELBCTRONS IH SI

\begin{tabular}{rrr} 
TD $=28.0$ & EV & \multicolumn{1}{c}{ ET $=0.281049$ MEV } \\
ENERGY (MEV) & PRIMARY & CASCADE \\
0.28300 & 0.21 & 0.21 \\
0.28600 & 0.53 & 0.53 \\
0.29200 & 1.15 & 1.15 \\
0.30000 & 1.92 & 1.92 \\
0.31100 & 2.92 & 2.92 \\
0.32600 & 4.16 & 4.16 \\
0.34200 & 5.35 & 5.35 \\
0.36500 & 6.88 & 6.88 \\
0.39300 & 8.51 & 8.51 \\
0.43500 & 10.61 & 10.61 \\
0.49100 & 12.94 & 12.94 \\
0.56200 & 15.35 & 15.47 \\
0.64600 & 17.68 & 18.13 \\
0.75800 & 20.17 & 21.29 \\
0.89900 & 22.62 & 24.82 \\
1.06000 & 24.76 & 28.41 \\
1.26000 & 26.78 & 32.37 \\
1.46000 & 28.29 & 35.92 \\
1.68000 & 29.56 & 39.45 \\
1.96000 & 30.78 & 43.49 \\
2.38000 & 32.03 & 48.79 \\
2.81000 & 32.89 & 53.52 \\
4.21000 & 34.29 & 65.62 \\
5.62000 & 34.84 & 74.69 \\
8.43000 & 35.21 & 87.85 \\
14.00000 & 35.30 & 104.78 \\
28.10000 & 35.19 & 128.54 \\
56.20000 & 35.10 & 152.49 \\
84.30000 & 35.06 & 166.58 \\
140.00000 & 35.04 & 184.26 \\
196.00000 & 35.03 & 196.01
\end{tabular}

\begin{tabular}{rrr} 
TD $=32.0$ & EV & \multicolumn{1}{c}{ ET $=0.313415$} \\
BNERGY $(M E V)$ & PRIMARY & CASCADE \\
0.31600 & 0.20 & 0.20 \\
0.31900 & 0.42 & 0.42 \\
0.32500 & 0.85 & 0.85 \\
0.33500 & 1.54 & 1.54 \\
0.34700 & 2.31 & 2.31 \\
0.36300 & 3.27 & 3.27 \\
0.38200 & 4.30 & 4.30 \\
0.40700 & 5.54 & 5.54 \\
0.43300 & 6.91 & 6.91 \\
0.48500 & 8.72 & 8.72 \\
0.54800 & 10.78 & 10.78 \\
0.62600 & 12.91 & 13.01 \\
0.72000 & 15.03 & 15.43 \\
0.84600 & 17.32 & 18.32 \\
1.00000 & 19.52 & 21.47 \\
1.19000 & 21.59 & 24.92 \\
1.41000 & 23.38 & 28.47 \\
1.62000 & 24.68 & 31.51 \\
1.88000 & 25.89 & 34.91 \\
2.19000 & 26.96 & 38.52 \\
2.66000 & 28.08 & 43.30 \\
3.13000 & 28.82 & 47.45 \\
4.70000 & 30.04 & 58.31 \\
6.26000 & 30.50 & 66.29 \\
9.40000 & 30.81 & 77.95 \\
15.60000 & 30.88 & 92.83 \\
31.30000 & 30.79 & 113.67 \\
62.60000 & 30.71 & 134.66 \\
94.00000 & 30.68 & 147.03 \\
156.00000 & 30.66 & 162.49 \\
219.00000 & 30.65 & 172.85
\end{tabular}

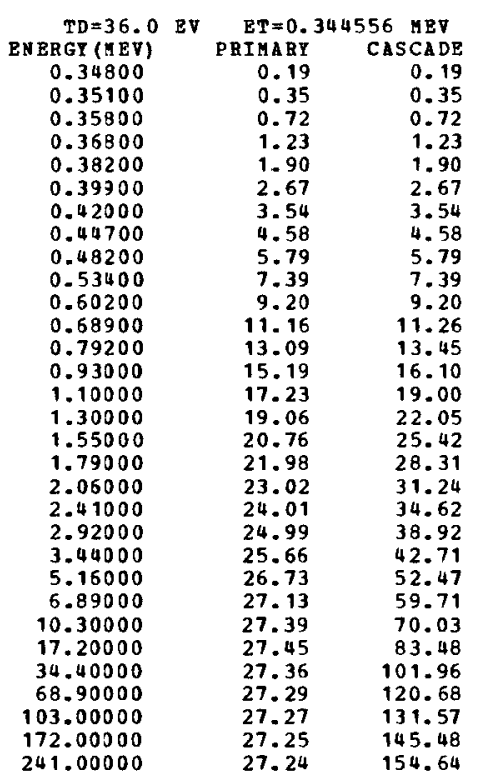

ATOMIC DISPLACEHBNT CROSS-SECTIONS (BARNS) BY FAST ELBCTRONS IN SI $\mathrm{z}=14 \quad \mathrm{~A}=28.090$

\begin{tabular}{rrr} 
TD $=40.0$ & BV & \multicolumn{1}{c}{ ET $=0.374604$ HEV } \\
ENERGY (MEV) & PRIHARY & CASCADE \\
0.37800 & 0.14 & 0.14 \\
0.38200 & 0.31 & 0.31 \\
0.38900 & 0.59 & 0.59 \\
0.40000 & 1.02 & 1.02 \\
0.41500 & 1.57 & 1.57 \\
0.43400 & 2.24 & 2.24 \\
0.45700 & 3.00 & 3.00 \\
0.48600 & 3.88 & 3.88 \\
0.52400 & 4.95 & 4.95 \\
0.58000 & 6.37 & 6.37 \\
0.65500 & 8.04 & 8.04 \\
0.74900 & 9.82 & 9.91 \\
0.86100 & 11.60 & 11.94 \\
1.01000 & 13.54 & 14.36 \\
1.19000 & 15.38 & 16.99 \\
1.42000 & 17.17 & 19.97 \\
1.68000 & 18.66 & 22.95 \\
1.94000 & 19.78 & 25.61 \\
2.24000 & 20.75 & 28.37 \\
2.62000 & 21.65 & 31.47 \\
3.18000 & 22.54 & 35.45 \\
3.74000 & 23.13 & 38.87 \\
5.61000 & 24.08 & 47.77 \\
7.49000 & 24.43 & 54.33 \\
11.20000 & 24.66 & 63.69 \\
18.70000 & 24.70 & 75.83 \\
37.40000 & 24.62 & 92.50 \\
74.90000 & 24.56 & 109.35 \\
112.00000 & 24.54 & 119.16 \\
187.00000 & 24.52 & 131.68 \\
& &
\end{tabular}

\begin{tabular}{|c|c|c|}
\hline $\begin{array}{r}T D=44.0 \\
\text { BNERGY (ABV) }\end{array}$ & $\begin{array}{l}\text { ET }=0.4 \\
\text { PRIFARY }\end{array}$ & CASCA DE \\
\hline 0,40700 & 0.11 & \\
\hline 0.41100 & & $\begin{array}{l}0.11 \\
0.24\end{array}$ \\
\hline 0.41900 & 0.49 & 0.49 \\
\hline 0.43100 & 0.86 & 0.86 \\
\hline 0.44800 & 1. 36 & 1.36 \\
\hline 0.46800 & 1.92 & 1.92 \\
\hline 0.49200 & 2.56 & 2.56 \\
\hline 0.52400 & 3.37 & 3.37 \\
\hline 0.56500 & 4.32 & 4.32 \\
\hline 0.62500 & 5.60 & 5.60 \\
\hline 0.70600 & 7.13 & 7.13 \\
\hline 0.80700 & 8.77 & \\
\hline 0.92800 & 10.44 & 10.74 \\
\hline 1.08000 & 12.15 & .89 \\
\hline 1.29000 & 14.00 & 15.53 \\
\hline 1.53000 & 15.60 & .21 \\
\hline 1.81000 & 16.98 & \\
\hline 2.09000 & 18.01 & 23.45 \\
\hline 2.42000 & 18.92 & \\
\hline 2.82000 & 19.71 & .86 \\
\hline 3.43000 & 20.53 & \\
\hline 4.03000 & 21.06 & 35.70 \\
\hline 6.05000 & 21.91 & \\
\hline 8.07000 & & \\
\hline 12.10000 & 22.42 & \\
\hline 20.10000 & $\begin{array}{l}22.43 \\
22.38\end{array}$ & \\
\hline 80,70000 & 22.33 & .00 \\
\hline 121.00000 & 22.31 & \\
\hline 201.00000 & 22.29 & 120 \\
\hline
\end{tabular}

$\begin{array}{rrr}\begin{array}{c}\text { TD }=48.0 \\ \text { ENERG (MBV) }\end{array} & \begin{array}{r}\text { ET }=0.431830 \\ \text { PRIMARY }\end{array} & \text { CASCADE } \\ 0.43600 & 0.11 & 0.11 \\ 0.44000 & 0.21 & 0.21 \\ 0.44900 & 0.44 & 0.44 \\ 0.46200 & 0.76 & 0.76 \\ 0.47900 & 1.16 & 1.16 \\ 0.50000 & 1.65 & 1.65 \\ 0.52500 & 2.23 & 2.23 \\ 0.56100 & 2.96 & 2.96 \\ 0.60400 & 3.82 & 3.82 \\ 0.66900 & 5.00 & 5.00 \\ 0.75500 & 6.40 & 6.41 \\ 0.86300 & 7.93 & 8.01 \\ 0.99300 & 9.49 & 9.78 \\ 1.16000 & 11.13 & 11.83 \\ 1.38000 & 12.82 & 14.26 \\ 1.64000 & 14.32 & 16.79 \\ 1.94000 & 15.60 & 19.38 \\ 2.24000 & 16.55 & 21.68 \\ 2.59000 & 17.38 & 24.09 \\ 3.02000 & 18.11 & 26.70 \\ 3.67000 & 18.86 & 30.13 \\ 4.31000 & 19.33 & 33.03 \\ 6.47000 & 20.10 & 40.59 \\ 8.63000 & 20.38 & 46.11 \\ 12.90000 & 20.55 & 53.96 \\ 21.50000 & 20.58 & 64.10 \\ 43.10000 & 20.51 & 78.08 \\ 86.30000 & 20.46 & 92.14 \\ 129.00000 & 20.45 & 100.32 \\ 215.00000 & 20.44 & 110.72 \\ & & \end{array}$


ATOMIC DISPLACENENT CROSS-SECTIONS (BARNS) BY FAST ELECTRONS IN SI

\begin{tabular}{|c|c|c|}
\hline $\begin{array}{r}T D=52.0 \\
\text { ENERGY }\end{array}$ & $\begin{aligned} \text { ET }=0 \\
\text { PRIMART }\end{aligned}$ & $\begin{array}{l}178 \text { MEV } \\
\text { CASCADE }\end{array}$ \\
\hline 0.46300 & $\begin{array}{r}0.08 \\
0.08\end{array}$ & 0.08 \\
\hline 0.46800 & 0.18 & 0.18 \\
\hline 0.47700 & 0.37 & 0.37 \\
\hline 0.49100 & 0.65 & 0.65 \\
\hline 0.50900 & 1.01 & 1.01 \\
\hline 0.53200 & 1.45 & 1.45 \\
\hline 0.56000 & 1.98 & 1.98 \\
\hline 0.59600 & 2.63 & 2.63 \\
\hline 0.64200 & 3.41 & 3.41 \\
\hline 0.71100 & 4.50 & 4.50 \\
\hline 0.80300 & 5.82 & 5.82 \\
\hline 0.91800 & 7.25 & 7.33 \\
\hline 1.05000 & 8.65 & 8.91 \\
\hline 1.23000 & 10.22 & 10.87 \\
\hline 1.46000 & 11.79 & 13.12 \\
\hline 1.74000 & 13,22 & 15.54 \\
\hline 2.06000 & 14.42 & 17.98 \\
\hline 2.38000 & 15.31 & 20.14 \\
\hline 2.75000 & 16.07 & 22.38 \\
\hline 3.21000 & 16.75 & 24.84 \\
\hline 3.90000 & 17.43 & 28.03 \\
\hline 4.59000 & 17.87 & 30.77 \\
\hline 6.88000 & 18.57 & 37.77 \\
\hline 9.18000 & 18.82 & 42.90 \\
\hline 13.70000 & 18.97 & 50.15 \\
\hline 22.90000 & 18.99 & 59.58 \\
\hline 45.90000 & 18.93 & 72.49 \\
\hline 91.80000 & 18.89 & 85.46 \\
\hline 137.00000 & 18.87 & 92.97 \\
\hline .00000 & 18.86 & 102.63 \\
\hline
\end{tabular}

\begin{tabular}{ccc} 
TD $=56.0$ & EV & \multicolumn{1}{c}{ ET=0.485777 MEV } \\
ENERGY (MEV) & PRIMARY & CASCADE \\
0.49000 & 0.07 & 0.07 \\
0.49500 & 0.16 & 0.16 \\
0.50500 & 0.33 & 0.33 \\
0.51900 & 0.57 & 0.57 \\
0.53900 & 0.90 & 0.90 \\
0.56300 & 1.29 & 1.29 \\
0.59200 & 1.76 & 1.76 \\
0.63100 & 2.37 & 2.37 \\
0.68000 & 3.09 & 3.09 \\
0.75200 & 4.10 & 4.10 \\
0.85000 & 5.33 & 5.34 \\
0.97100 & 6.67 & 6.75 \\
1.11000 & 7.99 & 8.24 \\
1.31000 & 9.53 & 10.18 \\
1.55000 & 10.98 & 12.26 \\
1.84000 & 12.29 & 14.49 \\
2.18000 & 13.42 & 16.80 \\
2.52000 & 14.25 & 18.84 \\
2.91000 & 14.95 & 20.94 \\
3.40000 & 15.59 & 23.26 \\
4.12000 & 16.21 & 26.21 \\
4.85000 & 16.62 & 28.77 \\
7.28000 & 17.25 & 35.34 \\
9.71000 & 17.48 & 40.11 \\
14.50000 & 17.62 & 46.87 \\
24.20000 & 17.63 & 55.62 \\
48.50000 & 17.58 & 67.62 \\
97.10000 & 17.54 & 79.68 \\
145.00000 & 17.53 & 86.67 \\
242.00000 & 17.52 & 95.62
\end{tabular}

\begin{tabular}{rrr} 
TD $=50.0$ & EV & \multicolumn{2}{c}{ ET $=0.511683$ MEV } \\
ENERGY (HEV) & PRIMARY & CASCADE \\
0.51500 & 0.06 & 0.06 \\
0.52100 & 0.13 & 0.13 \\
0.53200 & 0.29 & 0.29 \\
0.54700 & 0.51 & 0.51 \\
0.56700 & 0.79 & 0.79 \\
0.59300 & 1.16 & 1.16 \\
0.62400 & 1.59 & 1.59 \\
0.66500 & 2.15 & 2.15 \\
0.71600 & 2.82 & 2.82 \\
0.79300 & 3.77 & 3.77 \\
0.89500 & 4.92 & 4.93 \\
1.02000 & 6.16 & 6.23 \\
1.17000 & 7.44 & 7.67 \\
1.38000 & 8.89 & 9.51 \\
1.63000 & 10.24 & 11.45 \\
1.94000 & 11.50 & 13.60 \\
2.30000 & 12.56 & 15.79 \\
2.66000 & 13.34 & 17.73 \\
3.07000 & 13.99 & 19.70 \\
3.58000 & 14.57 & 21.86 \\
4.34000 & 15.15 & 24.64 \\
5.11000 & 15.53 & 27.05 \\
7.67000 & 16.11 & 33.21 \\
10.20000 & 16.32 & 37.64 \\
15.30000 & 16.44 & 44.03 \\
25.50000 & 16.46 & 52.18 \\
51.10000 & 16.41 & 63.39 \\
102.00000 & 16.37 & 74.61 \\
153.00000 & 16.36 & 81.21 \\
255.00000 & 16.35 & 89.53
\end{tabular}

ATOMIC DISPLACENENT CROSS-SECTIONS (BARNS) BY PAST BLECTRONS IN ST

\begin{tabular}{|c|c|c|c|}
\hline \multicolumn{4}{|r|}{$950 \mathrm{MEV}$} \\
\hline 0.54200 & & $\begin{array}{r}2 \pi 11 \mathrm{Ant} \\
0.06\end{array}$ & 0.06 \\
\hline 0.54700 & & 0.12 & 0.12 \\
\hline 0.55800 & & 0.26 & 0.26 \\
\hline 0.57400 & & 0.45 & 0.45 \\
\hline 0.59600 & & 0.72 & 0.72 \\
\hline 0.62200 & & 1.05 & 1.05 \\
\hline 0.65500 & & 1.45 & 1.45 \\
\hline 0.69800 & & 1.97 & 1.97 \\
\hline 0.75100 & & 2.58 & 2.58 \\
\hline 0.83200 & & 3.48 & 3.48 \\
\hline 0.93900 & & 4.57 & 4.57 \\
\hline 1.07000 & & 5.74 & 5.81 \\
\hline 1.23000 & & 6.97 & 7.20 \\
\hline 1.44000 & & 8.29 & 8.86 \\
\hline 1.71000 & & 9.61 & 10.77 \\
\hline 2.04000 & & 10.81 & 12.83 \\
\hline 2.41000 & & 11.79 & \\
\hline 2.79000 & & 12.53 & 16.71 \\
\hline 3.22000 & & 13.14 & 18.58 \\
\hline 3.75000 & & 13.68 & 20.60 \\
\hline 4.56000 & & 14.23 & 23.27 \\
\hline 5.36000 & & 14.57 & 25.52 \\
\hline 8.05000 & & 15.11 & 31.33 \\
\hline 10.70000 & & 15.30 & 35.48 \\
\hline $16=10000$ & & 15.42 & 41. \\
\hline $26^{\prime} .80000$ & & 15.43 & 49.17 \\
\hline 53.60000 & & 15.38 & \\
\hline 107.00000 & & 15.35 & \\
\hline 161.00000 & & 15.33 & \\
\hline
\end{tabular}

\begin{tabular}{ccc} 
TD $=68.0$ & EV & \multicolumn{1}{c}{ ET=0.561621 MEV } \\
ENERGY (HEV) & PRIMAY & CASCADE \\
0.56700 & 0.06 & 0.06 \\
0.57200 & 0.11 & 0.11 \\
0.58400 & 0.23 & 0.23 \\
0.60000 & 0.41 & 0.41 \\
0.62300 & 0.65 & 0.65 \\
0.65100 & 0.96 & 0.96 \\
0.68500 & 1.32 & 1.32 \\
0.73000 & 1.81 & 1.81 \\
0.78600 & 2.39 & 2.39 \\
0.87000 & 3.23 & 3.23 \\
0.98200 & 4.26 & 4.27 \\
1.12000 & 5.39 & 5.46 \\
1.29000 & 6.57 & 5.80 \\
1.51000 & 7.82 & 8.38 \\
1.79000 & 9.05 & 10.18 \\
2.13000 & 10.18 & 12.11 \\
2.52000 & 11.12 & 14.06 \\
2.92000 & 11.82 & 15.83 \\
3.36000 & 12.38 & 17.56 \\
3.93000 & 12.90 & 19.53 \\
4.77000 & 13.41 & 22.04 \\
5.61000 & 13.73 & 24.17 \\
8.42000 & 14.23 & 29.65 \\
11.20000 & 14.41 & 33.59 \\
16.80000 & 14.51 & 39.25 \\
28.00000 & 14.52 & 46.46 \\
56.10000 & 14.47 & 56.36 \\
112.00000 & 14.44 & 66.27 \\
168.00000 & 14.43 & 72.10 \\
& &
\end{tabular}

\begin{tabular}{rrr} 
TD=72.0 EV & \multicolumn{2}{c}{ ET $=0.585737$ MEV } \\
ENERGY (HEV) & PRIMARY & CASCADE \\
0.59100 & 0.05 & 0.05 \\
0.59700 & 0.10 & 0.10 \\
0.50900 & 0.21 & 0.21 \\
0.62600 & 0.37 & 0.37 \\
0.65000 & 0.60 & 0.60 \\
0.67900 & 0.88 & 0.88 \\
0.71400 & 1.21 & 1.21 \\
0.76100 & 1.67 & 1.67 \\
0.82000 & 2.23 & 2.23 \\
0.90700 & 3.02 & 3.02 \\
1.02000 & 3.97 & 3.97 \\
1.17000 & 5.09 & 5.15 \\
1.34000 & 6.17 & 6.38 \\
1.58000 & 7.42 & 7.96 \\
1.87000 & 8.58 & 9.66 \\
2.22000 & 9.63 & 11.48 \\
2.63000 & 10.53 & 13.36 \\
3.04000 & 11.17 & 15.01 \\
3.51000 & 11.72 & 16.70 \\
4.10000 & 12.21 & 18.56 \\
4.97000 & 12.68 & 20.92 \\
5.85000 & 12.98 & 22.96 \\
8.78000 & 13.45 & 28.15 \\
11.70000 & 13.61 & 31.90 \\
17.50000 & 13.71 & 37.22 \\
29.20000 & 13.71 & 44.05 \\
58.50000 & 13.67 & 53.41 \\
117.00000 & 13.64 & 62.79 \\
175.00000 & 13.63 & 68.26
\end{tabular}


A TOHIC DTSPLAZBHENT CROSS-SECTIONS (BARNS) BY FAST BLECTBOHS IN SI $=14 \quad A=28.090$

$\begin{array}{ccc}\text { TD=76.0 } & \text { BV } & \text { ET }=0.609335 \text { MEV } \\ \text { ENERGY (HEV) } & \text { PRIMARY } & \text { CASCADE } \\ 0.61500 & 0.04 & 0.04 \\ 0.62100 & 0.09 & 0.09 \\ 0.63300 & 0.19 & 0.19 \\ 0.65100 & 0.34 & 0.34 \\ 0.67600 & 0.55 & 0.55 \\ 0.70600 & 0.80 & 0.80 \\ 0.74300 & 1.13 & 1.13 \\ 0.79200 & 1.55 & 1.55 \\ 0.85300 & 2.08 & 2.08 \\ 0.94400 & 2.84 & 2.84 \\ 1.06000 & 3.73 & 3.74 \\ 1.21000 & 4.76 & 4.82 \\ 1.40000 & 5.88 & 6.09 \\ 1.64000 & 7.02 & 7.54 \\ 1.94000 & 8.13 & 9.16 \\ 2.31000 & 9.15 & 10.93 \\ 2.74000 & 10.00 & 12.73 \\ 3.16000 & 10.60 & 14.29 \\ 3.65000 & 11.12 & 15.90 \\ 4.26000 & 11.58 & 17.66 \\ 5.17000 & 12.02 & 19.92 \\ 6.09000 & 12.31 & 21.87 \\ 9.14000 & 12.75 & 26.81 \\ 12.10000 & 12.90 & 30.29 \\ 18.20000 & 12.99 & 35.40 \\ 30.40000 & 12.99 & 41.90 \\ 60.90000 & 12.95 & 50.76 \\ 121.00000 & 12.92 & 59.57 \\ 182.00000 & 12.91 & 64.82\end{array}$

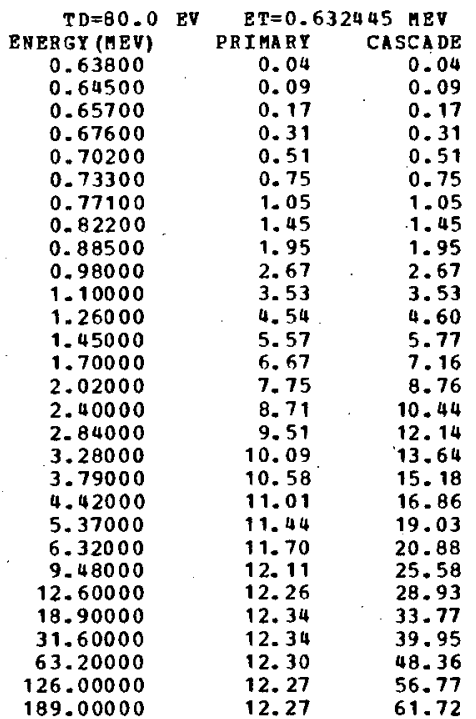

\begin{tabular}{rrr}
$\begin{array}{r}\text { TD=84.0 } \\
\text { EN ERGY (HEV) }\end{array}$ & \multicolumn{2}{c}{ ET $=0.655098$ HEV } \\
PRIMARY & CASCADE \\
0.56100 & 0.04 & 0.04 \\
0.66800 & 0.08 & 0.08 \\
0.68100 & 0.16 & 0.16 \\
0.70000 & 0.28 & 0.28 \\
0.72700 & 0.47 & 0.47 \\
0.75900 & 0.69 & 0.69 \\
0.79900 & 0.98 & 0.98 \\
0.85100 & 1.36 & 1.36 \\
0.91700 & 1.84 & 1.84 \\
1.01000 & 2.49 & 2.49 \\
1.14000 & 3.35 & 3.35 \\
1.31000 & 4.34 & 4.40 \\
1.50000 & 5.29 & 5.49 \\
1.76000 & 6.35 & 6.83 \\
2.09000 & 7.39 & 8.36 \\
2.48000 & 8.30 & 9.96 \\
2.94000 & 9.07 & 11.61 \\
3.40000 & 9.63 & 13.06 \\
3.93000 & 10.10 & 14.54 \\
4.58000 & 10.50 & 16.14 \\
5.56000 & 10.90 & 18.21 \\
6.55000 & 11.16 & 19.98 \\
9.82000 & 11.54 & 24.46 \\
13.10000 & 11.68 & 27.71 \\
19.60000 & 11.75 & 32.29 \\
32.70000 & 11.75 & 38.16 \\
65.50000 & 11.71 & 46.19 \\
131.00000 & 11.69 & 54.23 \\
196.00000 & 11.68 & 58.92
\end{tabular}

ATONIC DISPLAZEMENT CROSS-SECTIONS (BARNS) BY FAST BLECTRONS IN ST $Z=14 \quad A=28.090$

\begin{tabular}{|c|c|c|c|c|}
\hline $\begin{array}{r}T D=88.0 \\
\text { ENERG (MEV) }\end{array}$ & EV & $\begin{array}{r}\text { ET }=0 . \\
\text { PRIMARY }\end{array}$ & $\begin{array}{r}77319 \\
\text { CA }\end{array}$ & $\begin{array}{c}\mathrm{MEV} \\
\mathrm{SCADE}\end{array}$ \\
\hline 0.68400 & & 0.04 & $\therefore \therefore$ & 0.04 \\
\hline 0.69000 & & 0.07 & $\therefore$ & 0.07 \\
\hline 0.70400 & & 0.15 & & 0.15 \\
\hline 0.72400 & & 0.26 & & 0.26 \\
\hline 0.75100 & & 0.43 & & 0.43 \\
\hline 0.78500 & & 0.65 & & 0.65 \\
\hline 0.82600 & & 0.92 & $\therefore$ & 0.92 \\
\hline 0.88000 & & 1.28 & & 1.28 \\
\hline 0.94800 & & 1.74 & & 1.74 \\
\hline 1.04000 & & 2.34 & & 2.34 \\
\hline 1.18000 & & 3.19 & & 3.20 \\
\hline 1.35000 & & 4.12 & & 4.18 \\
\hline 1.55000 & & 5.05 & & 5.24 \\
\hline 1.82000 & & 6.07 & & 6.54 \\
\hline 2.16000 & & 7.07 & & 8.01 \\
\hline 2.57000 & & 7.95 & & 9.56 \\
\hline 3.04000 & & 8.68 & & 11.13 \\
\hline 3.52000 & & 9.21 & & 12.53 \\
\hline 4.06000 & & 9.65 & $\therefore$ & 13.93 \\
\hline $\begin{array}{l}4.74000 \\
5.75000\end{array}$ & & $\begin{array}{l}10.04 \\
10.42\end{array}$ & $\therefore$ & $\begin{array}{l}15.48 \\
17.46\end{array}$ \\
\hline 6.77000 & & 10.66 & & 19.15 \\
\hline 10.10000 & & 11.02 & & 23.39 \\
\hline 13.50000 & & 11.15 & “. & 26.51 \\
\hline $\begin{array}{l}20.30000 \\
33.80000\end{array}$ & & 11.22 & 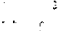 & $\begin{array}{l}30.94 \\
36.53\end{array}$ \\
\hline 67.70000 & & 11.18 & & 44.19 \\
\hline 135.00000 & & 11.16 & & 51.84 \\
\hline 203.00000 & & 11.15 & & 56.38 \\
\hline
\end{tabular}

\begin{tabular}{|c|c|c|}
\hline $\begin{array}{c}\text { TD=92.0 } \\
\text { OEGY (MEV) } \\
0.70600 \\
0.71300 \\
0.72700 \\
0.74800 \\
0.77600 \\
0.81000 \\
0.85200 \\
0.90800 \\
0.97800 \\
1.08000 \\
1.22000 \\
1.39000 \\
1.60000 \\
1.88000 \\
2.23000 \\
2.65000 \\
3.14000 \\
3.63000 \\
4.19000 \\
4.89000 \\
5.94000 \\
6.99000 \\
10.40000 \\
13.90000 \\
20.90000 \\
34.90000 \\
69.90000 \\
139.00000 \\
209.00000\end{array}$ & $\begin{array}{r}\text { ET }=0.6 \\
\text { PRI RARY } \\
0.03 \\
0.07 \\
0.14 \\
0.25 \\
0.41 \\
0.60 \\
0.86 \\
1.21 \\
1.64 \\
2.26 \\
3.06 \\
3.92 \\
4.83 \\
5.82 \\
6.77 \\
7.61 \\
8.32 \\
8.82 \\
9.24 \\
9.61 \\
9.98 \\
10.20 \\
10.54 \\
10.66 \\
10.73 \\
10.73 \\
10.69 \\
10.67 \\
10.67\end{array}$ & $\begin{array}{r}\text { CASCA DE } \\
0.03 \\
0.07 \\
0.14 \\
0.25 \\
0.41 \\
0.60 \\
0.86 \\
1.21 \\
1.64 \\
2.26 \\
3.06 \\
3.98 \\
5.02 \\
.6 .28 \\
7.68 \\
9.17 \\
10.69 \\
12.03 \\
13.38 \\
14.87 \\
16.78 \\
18.40 \\
22.43 \\
.25 .42 \\
29.66 \\
35.04 \\
42.37 \\
49.66 \\
54.00\end{array}$ \\
\hline
\end{tabular}

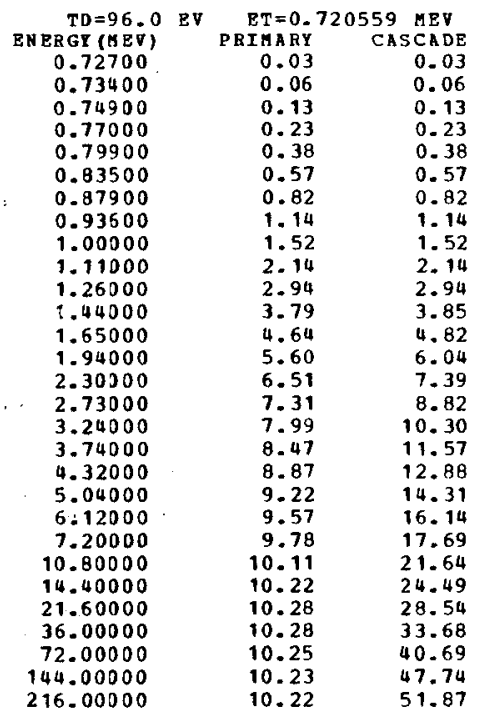




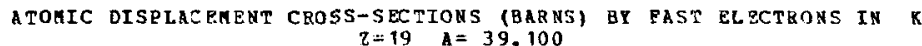

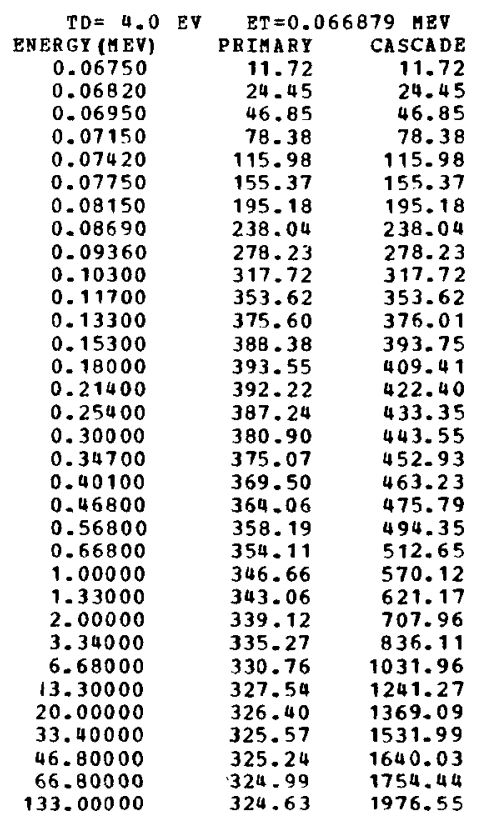

\begin{tabular}{rrr} 
TD=12.0 & \multicolumn{1}{c}{ ET $=0.181525$ MEY } \\
ENERGY (HEY) & PRIMARY & CASCADE \\
0.18300 & 1.24 & 1.24 \\
0.13500 & 2.87 & 2.87 \\
0.18800 & 5.21 & 5.21 \\
0.19400 & 9.58 & 9.58 \\
0.20100 & 14.19 & 14.19 \\
0.21000 & 19.47 & 19.47 \\
0.22100 & 25.09 & 25.09 \\
0.23500 & 31.19 & 31.19 \\
0.25400 & 38.04 & 38.04 \\
0.28100 & 45.73 & 45.73 \\
0.31700 & 53.53 & 53.53 \\
0.36300 & 60.96 & 61.27 \\
0.41700 & 67.50 & 68.93 \\
0.49000 & 74.11 & 77.76 \\
0.58000 & 80.15 & 87.13 \\
0.68900 & 95.58 & 97.04 \\
0.91500 & 90.28 & 107.25 \\
0.94300 & 93.85 & 116.45 \\
1.08000 & 96.82 & 125.49 \\
1.27000 & 99.92 & 136.86 \\
1.54000 & 102.97 & 151.20 \\
1.81000 & 105.04 & 163.88 \\
2.72000 & 108.51 & 198.31 \\
3.63000 & 109.75 & 224.53 \\
5.44000 & 110.41 & 263.30 \\
9.07000 & 110.21 & 314.56 \\
18.10000 & 109.36 & 386.25 \\
36.30000 & 108.73 & 460.02 \\
54.40000 & 108.53 & 503.30 \\
90.70000 & 108.38 & 558.23 \\
127.00000 & 108.31 & 594.48 \\
181.00000 & 108.25 & 632.66 \\
363.00000 & 108.14 & 707.74 \\
& & \\
\hline & &
\end{tabular}

ATOAIC DISPLACEMENT CROSS-SECTIONS (BARS: BY PAST ELECTRONS IN $x$ $Z=19 \quad A=39.100$

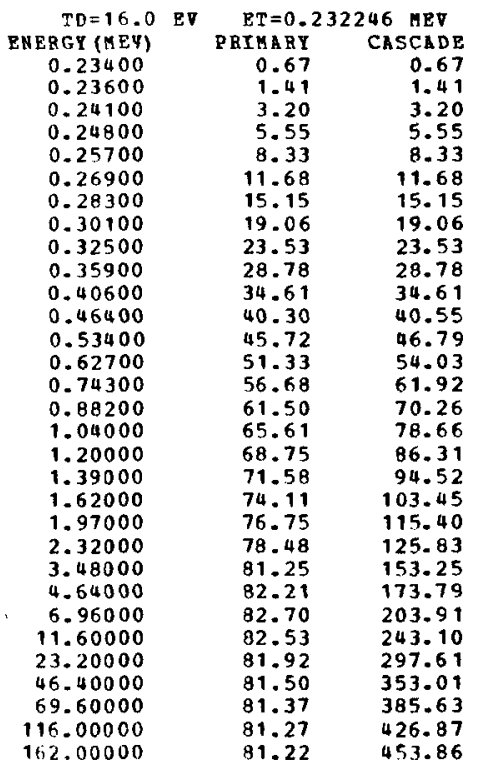

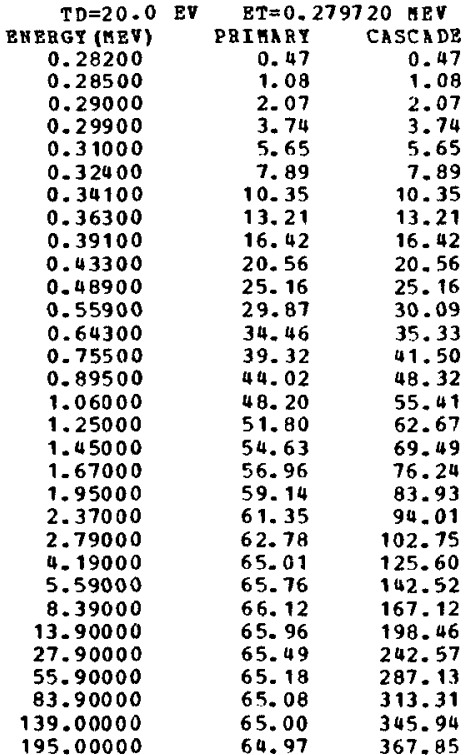

\begin{tabular}{rrr} 
TD=24.0 & EV & \multicolumn{2}{c}{ ET $=0.324500$ HEV } \\
BNERGY (1YEV) & PATARY & CASCADE \\
0.32700 & 0.32 & 0.32 \\
0.33000 & 0.69 & 0.69 \\
0.33700 & 1.54 & 1.54 \\
0.34700 & 2.71 & 2.71 \\
0.36000 & 4.13 & 4.13 \\
0.37500 & 5.77 & 5.77 \\
0.39500 & 7.58 & 7.58 \\
0.42100 & 9.85 & 9.85 \\
0.45400 & 12.44 & 12.44 \\
0.50200 & 15.76 & 15.76 \\
0.56700 & 19.62 & 19.63 \\
0.64900 & 23.70 & 23.90 \\
0.74600 & 27.69 & 28.43 \\
0.87600 & 31.98 & 33.84 \\
1.03000 & 35.94 & 39.53 \\
1.23000 & 39.82 & 46.08 \\
1.46000 & 43.09 & 52.71 \\
1.68000 & 45.42 & 58.36 \\
1.94000 & 47.47 & 54.37 \\
2.27000 & 49.36 & 71.15 \\
2.75000 & 51.19 & 79.72 \\
3.24000 & 52.39 & 87.29 \\
4.85000 & 54.21 & 106.75 \\
6.49000 & 54.81 & 121.16 \\
9.73000 & 55.08 & 141.86 \\
16.20000 & 54.93 & 168.44 \\
32.40000 & 54.54 & 205.16 \\
64.90000 & 54.30 & 242.35 \\
97.30000 & 54.22 & 264.13 \\
162.00000 & 54.16 & 291.61 \\
227.00000 & 54.13 & 309.80 \\
& &
\end{tabular}


ATOMIC DISPLACEMENT CROSS-SECTIONS (BARNS) BY PAST BLECTRONS IN K

\begin{tabular}{|c|c|c|c|}
\hline \multicolumn{4}{|l|}{$\begin{array}{r}T D=28,0 \\
E R G Y(H E V)\end{array}$} \\
\hline 0.37000 & & 0.25 & 0.25 \\
\hline 0.37400 & & 0.58 & 0.58 \\
\hline 0.38100 & & 1.15 & 1.15 \\
\hline 0.39200 & & 2.02 & 2.02 \\
\hline 0.40700 & & 3.15 & 3.15 \\
\hline 0.42500 & & 4.43 & 4.43 \\
\hline 0.44700 & & 5.91 & 5.91 \\
\hline 0.47700 & & 7.79 & 7.79 \\
\hline 0.51300 & & 9.87 & 9.87 \\
\hline 0.56800 & & 12.73 & 12.73 \\
\hline 0.64200 & & 16.09 & 16.10 \\
\hline 0.73400 & & 19.65 & 19.83 \\
\hline 0.84400 & & 23.20 & 23.85 \\
\hline 0.99000 & & 27.00 & 28.64 \\
\hline 1.17000 & & 30.65 & 33.89 \\
\hline 1.39000 & & 34.00 & 39.56 \\
\hline 1.65000 & & 36.90 & 45.47 \\
\hline 1.90000 & & 38.96 & 50.53 \\
\hline 2.20000 & & 40.78 & 55.95 \\
\hline 2.56000 & & 42.36 & 61.74 \\
\hline 3.11000 & & 43.96 & 69.39 \\
\hline 3.67000 & & 44.99 & 76.10 \\
\hline 5.50000 & & 46.50 & 93.05 \\
\hline 7.34000 & & 46.99 & 105.53 \\
\hline 11.00000 & & 47.20 & 123.41 \\
\hline 18.30000 & & 47.05 & 146.28 \\
\hline 36.70000 & & 46.73 & 177.97 \\
\hline 73.40000 & & 46.53 & 209.82 \\
\hline 110.00000 & & 46.47 & 228.49 \\
\hline 183.00000 & & 46.42 & 252.01 \\
\hline
\end{tabular}

\begin{tabular}{rrr}
$\begin{array}{r}\text { TD } D=32.0 \\
\text { ENERGY (MEV) }\end{array}$ & \multicolumn{2}{c}{ ET $=0.407536$ MEV } \\
PRIMARY & CASCADE \\
0.41100 & 0.20 & 0.20 \\
0.41500 & 0.44 & 0.44 \\
0.42300 & 0.90 & 0.90 \\
0.43500 & 1.63 & 1.63 \\
0.45200 & 2.51 & 2.51 \\
0.47200 & 3.56 & 3.56 \\
0.49700 & 4.81 & 4.81 \\
0.52900 & 6.34 & 6.34 \\
0.57000 & 8.16 & 8.16 \\
0.63100 & 10.65 & 10.65 \\
0.71300 & 13.62 & 13.63 \\
0.81500 & 16.80 & 16.96 \\
0.93700 & 19.99 & 20.58 \\
1.10000 & 23.44 & 24.93 \\
1.30000 & 26.73 & 29.67 \\
1.54000 & 29.69 & 34.71 \\
1.83000 & 32.31 & 40.07 \\
2.11000 & 34.15 & 44.66 \\
2.44000 & 35.75 & 49.49 \\
2.85000 & 37.17 & 54.80 \\
3.46000 & 38.55 & 61.62 \\
4.07000 & 39.42 & 67.48 \\
6.11000 & 40.72 & 82.57 \\
8.15000 & 41.13 & 93.58 \\
12.20000 & 41.29 & 109.28 \\
20.30000 & 41.15 & 129.38 \\
40.70000 & 40.88 & 157.15 \\
81.50000 & 40.71 & 185.10 \\
122.00000 & 40.66 & 201.40 \\
203.00000 & 40.62 & 222.00
\end{tabular}

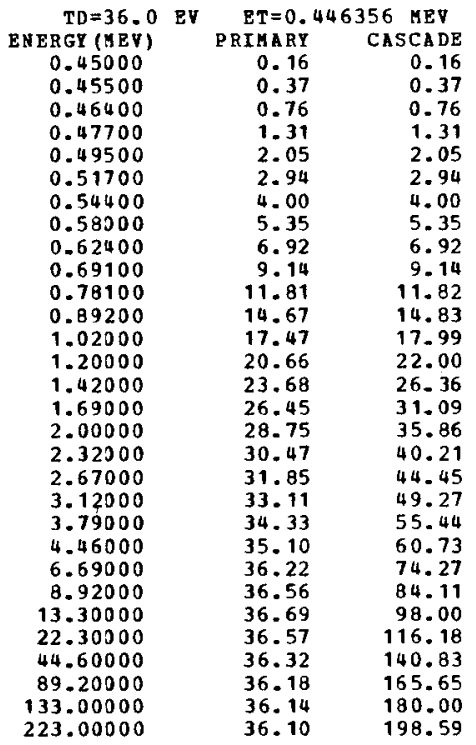

ATOHIC DISPLACEMENT CROSS-SECTIONS (BARHS) BY FAST ELECTRONS IN K $z=19 \quad=39.100$

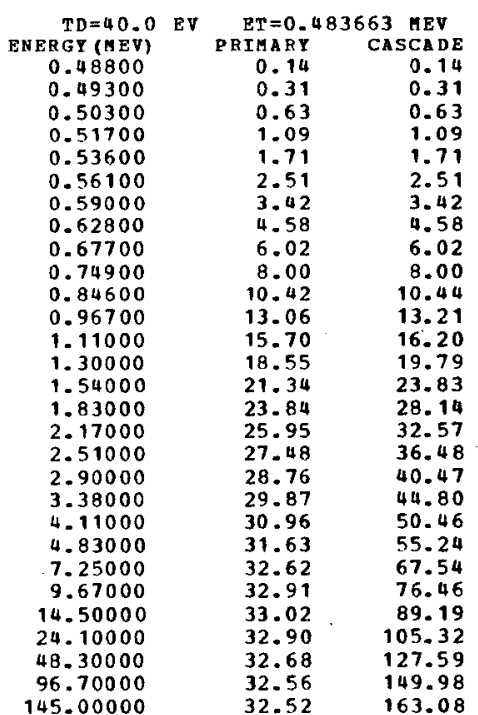

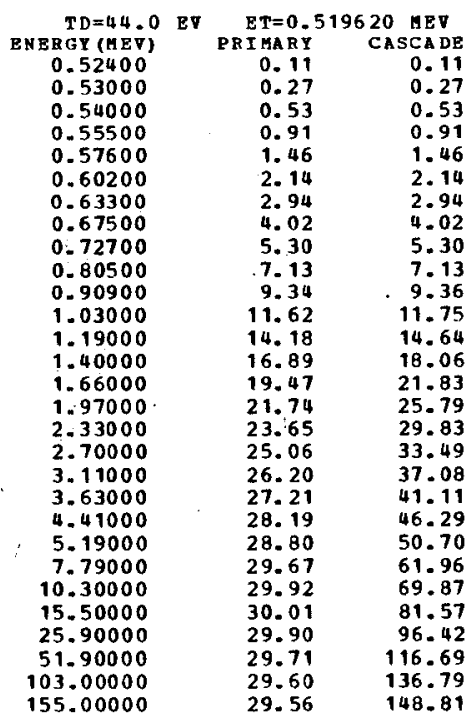

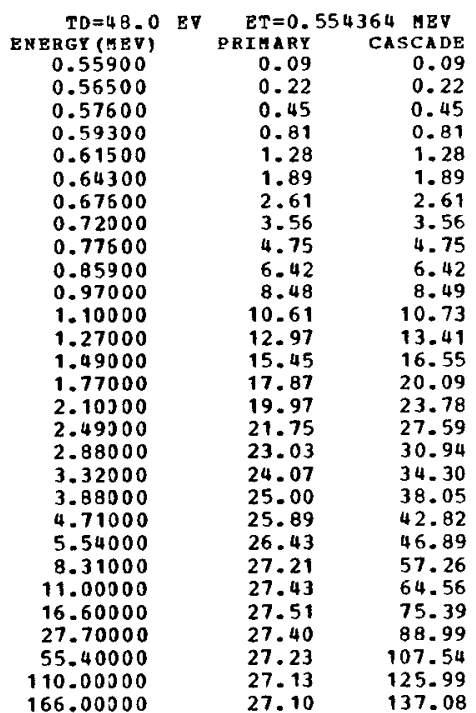


A TONIC DISPLACEAENT CROSS-SECTIONS (BARNS) BY PAST ELECTRONS IN $K$

\begin{tabular}{rrr} 
TD $=52.0$ & EV & \multicolumn{2}{c}{ ET $=0.588011$} & HEV \\
ENERGY(MEV) & PRIMARY & CASCADE \\
0.59300 & 0.08 & 0.08 \\
0.59900 & 0.18 & 0.18 \\
0.61100 & 0.39 & 0.39 \\
0.62900 & 0.71 & 0.71 \\
0.65200 & 1.12 & 1.12 \\
0.68200 & 1.67 & 1.67 \\
0.71700 & 2.32 & 2.32 \\
0.76400 & 3.20 & 3.20 \\
0.82300 & 4.29 & 4.29 \\
0.91100 & 5.84 & 5.84 \\
1.02000 & 7.62 & 7.63 \\
1.17000 & 9.79 & 9.91 \\
1.35000 & 11.99 & 12.41 \\
1.58000 & 14.27 & 15.31 \\
1.88000 & 16.55 & 18.66 \\
2.23000 & 18.50 & 22.11 \\
2.64000 & 20.13 & 25.63 \\
3.05000 & 21.30 & 28.74 \\
3.52000 & 22.27 & 31.89 \\
4.11000 & 23.12 & 35.37 \\
4.99000 & 23.93 & 39.81 \\
5.88000 & 24.43 & 43.63 \\
8.82000 & 25.13 & 53.25 \\
11.70000 & 25.33 & 60.07 \\
17.60000 & 25.39 & 70.01 \\
29.40000 & 25.29 & 82.61 \\
58.80000 & 25.13 & 99.74 \\
117.00000 & 25.04 & 116.83 \\
176.00000 & 25.01 & 127.00 \\
& &
\end{tabular}

\begin{tabular}{crr} 
TD $=56.0$ & EV & \multicolumn{2}{c}{ ET $=0.620657$ MEV } \\
ERERGY (MEV) & PRIMARY & CASCADE \\
0.62600 & 0.07 & 0.07 \\
0.63300 & 0.17 & 0.17 \\
0.64500 & 0.34 & 0.34 \\
0.66400 & 0.62 & 0.62 \\
0.68800 & 0.99 & 0.99 \\
0.71900 & 1.48 & 1.48 \\
0.75700 & 2.10 & 2.10 \\
0.80600 & 2.89 & 2.89 \\
0.86800 & 3.90 & 3.90 \\
0.96200 & 5.37 & 5.37 \\
1.08000 & 7.07 & 7.08 \\
1.24000 & 9.13 & 9.25 \\
1.42000 & 11.09 & 11.48 \\
1.67000 & 13.29 & 14.28 \\
1.98000 & 15.38 & 17.37 \\
2.35000 & 17.20 & 20.62 \\
2.79000 & 18.75 & 23.99 \\
3.22000 & 19.83 & 26.88 \\
3.72000 & 20.73 & 29.85 \\
4.34000 & 21.51 & 33.09 \\
5.27000 & 22.25 & 37.25 \\
6.20000 & 22.70 & 40.78 \\
9.30000 & 23.34 & 49.75 \\
12.40000 & 23.52 & 56.21 \\
18.60000 & 23.57 & 65.40 \\
31.00000 & 23.48 & 77.05 \\
62.00000 & 23.33 & 92.97 \\
124.00000 & 23.25 & 108.97 \\
186.00000 & 23.23 & 118.34 \\
& &
\end{tabular}

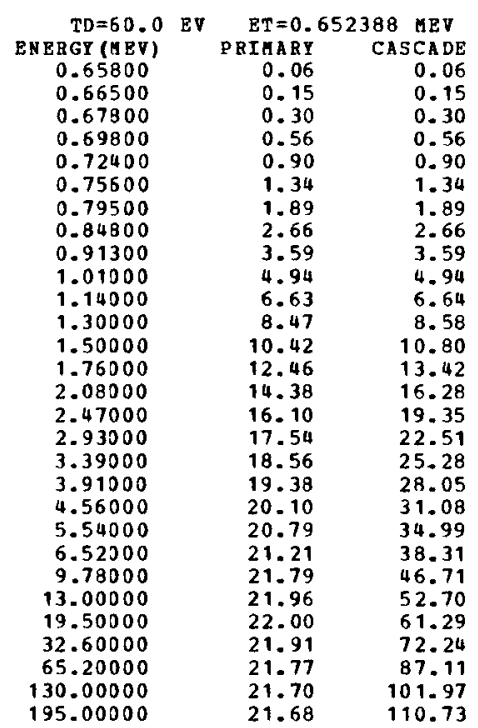
ATOHC DISPLACENENT CROSS-SBCTIONS (BARNS) BY PAST ELBCTRONS IN K
$z=19$

\begin{tabular}{rrr} 
TD $=64.0$ & EV & \multicolumn{1}{c}{ ET $=0.683276$} \\
ENERGY & MEV \\
0.59000 & PRIMARY & CASCADE \\
0.69600 & 0.07 & 0.07 \\
0.71000 & 0.13 & 0.13 \\
0.73100 & 0.27 & 0.27 \\
0.75800 & 0.50 & 0.50 \\
0.79200 & 0.81 & 0.81 \\
0.83300 & 1.22 & 1.22 \\
0.88800 & 1.74 & 1.74 \\
0.95600 & 2.44 & 2.44 \\
1.05000 & 3.32 & 3.32 \\
1.19000 & 4.50 & 4.50 \\
1.36000 & 6.14 & 6.16 \\
1.57000 & 7.91 & 8.02 \\
1.84000 & 9.76 & 10.14 \\
2.18000 & 11.68 & 12.59 \\
2.59000 & 13.52 & 15.35 \\
3.07000 & 15.14 & 18.26 \\
3.55000 & 16.48 & 21.23 \\
4.09000 & 17.43 & 23.84 \\
4.78000 & 18.20 & 26.43 \\
5.80000 & 18.88 & 29.34 \\
6.83000 & 19.51 & 33.00 \\
10.20000 & 19.90 & 36.13 \\
13.60000 & 20.44 & 43.95 \\
20.40000 & 20.59 & 49.63 \\
34.10000 & 20.62 & 57.69 \\
68.30000 & 20.54 & 67.97 \\
136.00000 & 20.41 & 81.95 \\
& 20.34 & 95.86
\end{tabular}

$\begin{array}{crr}\text { TD }=68.0 & \text { EV } & \text { ET }=0.713385 \text { MEV } \\ \text { BNERGY (MEV) } & \text { PRIMAT } & \text { CASCADE } \\ 0.72000 & 0.06 & 0.06 \\ 0.72700 & 0.12 & 0.12 \\ 0.74100 & 0.24 & 0.24 \\ 0.76300 & 0.45 & 0.45 \\ 0.79100 & 0.73 & 0.73 \\ 0.82700 & 1.12 & 1.12 \\ 0.87000 & 1.60 & 1.60 \\ 0.92700 & 2.26 & 2.26 \\ 0.99800 & 3.09 & 3.09 \\ 1.10000 & 4.25 & 4.25 \\ 1.24000 & 5.74 & 5.75 \\ 1.42000 & 7.44 & 7.55 \\ 1.64000 & 9.21 & 9.57 \\ 1.92000 & 11.01 & 11.89 \\ 2.28000 & 12.77 & 14.54 \\ 2.71000 & 14.30 & 17.31 \\ 3.21000 & 15.56 & 20.12 \\ 3.70000 & 16.43 & 22.54 \\ 4.28000 & 17.17 & 25.06 \\ 4.99000 & 17.79 & 27.76 \\ 6.06000 & 18.39 & 31.24 \\ 7.13000 & 18.74 & 34.19 \\ 10.70000 & 19.24 & 41.65 \\ 14.20000 & 19.38 & 46.93 \\ 21.40000 & 19.41 & 54.61 \\ 35.60000 & 19.33 & 64.20 \\ 71.30000 & 19.21 & 77.36 \\ 142.00000 & 19.15 & 90.46\end{array}$

\begin{tabular}{rrr} 
TD $=72.0$ & EV & \multicolumn{1}{c}{ ET $=0.742771$ HEV } \\
ENERGY (BEV) & PRIMARY & CASCADE \\
0.75000 & 0.05 & 0.05 \\
0.75700 & 0.10 & 0.10 \\
0.77200 & 0.22 & 0.22 \\
0.79400 & 0.41 & 0.41 \\
0.82400 & 0.68 & 0.68 \\
0.86100 & 1.03 & 1.03 \\
0.90600 & 1.49 & 1.49 \\
0.96500 & 2.10 & 2.10 \\
1.03000 & 2.79 & 2.79 \\
1.15000 & 4.04 & 4.04 \\
1.29000 & 5.40 & 5.41 \\
1.48000 & 7.04 & 7.14 \\
1.70000 & 8.66 & 8.99 \\
2.00000 & 10.42 & 11.27 \\
2.37000 & 12.08 & 13.76 \\
2.82000 & 13.54 & 16.42 \\
3.34000 & 14.72 & 19.09 \\
3.86000 & 15.56 & 21.43 \\
4.45000 & 16.24 & 23.77 \\
5.19000 & 16.82 & 26.34 \\
6.31000 & 17.38 & 29.66 \\
7.42000 & 17.72 & 32.44 \\
11.10000 & 18.18 & 39.45 \\
14.80000 & 18.30 & 44.52 \\
22.20000 & 18.33 & 51.71 \\
37.10000 & 18.25 & 60.86 \\
74.20000 & 18.14 & 73.26 \\
148.00000 & 18.08 & 85.67
\end{tabular}


A TONIC DISPLACBHENT CROSS-SBCTIONS (BARNS) BY FAST ELECTHONS IN $R$

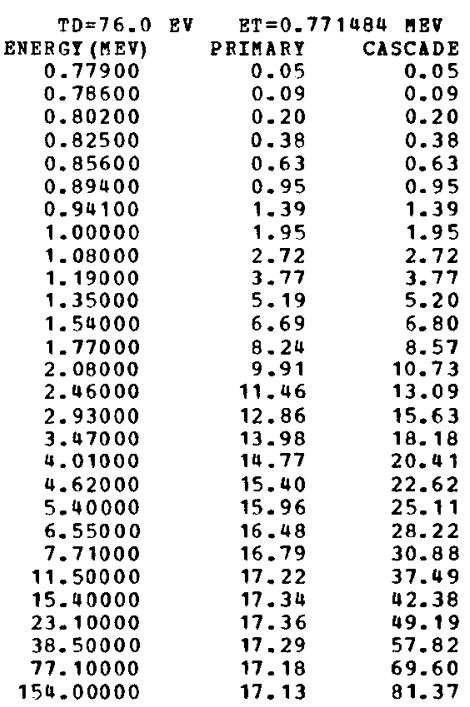

\begin{tabular}{ccc}
$T D=80.0$ & EV & \multicolumn{2}{c}{ ET $=0.799568$ IIEV } \\
ENERGY (MEV) & PRIMAR & CASCADE \\
0.80700 & 0.04 & 0.04 \\
0.81500 & 0.09 & 0.09 \\
0.83100 & 0.19 & 0.19 \\
0.85500 & 0.35 & 0.35 \\
0.88700 & 0.58 & 0.58 \\
0.92700 & 0.89 & 0.89 \\
0.97500 & 1.29 & 1.29 \\
1.03000 & 1.77 & 1.77 \\
1.11000 & 2.48 & 2.48 \\
1.23000 & 3.54 & 3.54 \\
1.39000 & 4.86 & 4.87 \\
1.59000 & 6.32 & 6.42 \\
1.83000 & 7.81 & 8.13 \\
2.15000 & 9.41 & 10.20 \\
2.55000 & 10.92 & 12.48 \\
3.03000 & 12.23 & 14.89 \\
3.59000 & 13.29 & 17.32 \\
4.15000 & 14.05 & 19.45 \\
4.79000 & 14.66 & 21.60 \\
5.59000 & 15.18 & 23.94 \\
6.79000 & 15.67 & 26.93 \\
7.99000 & 15.96 & 29.46 \\
11.90000 & 16.36 & 35.73 \\
15.90000 & 16.47 & 40.34 \\
23.90000 & 16.49 & 46.86 \\
39.90000 & 16.42 & 55.08 \\
79.90000 & 16.32 & 66.28 \\
159.00000 & 16.27 & 77.40 \\
& &
\end{tabular}

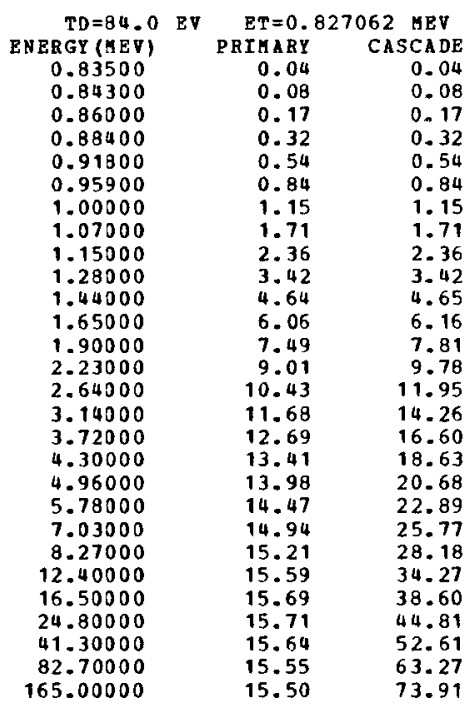

ATOMIC DISPLAZEMENT CROSS-SECTIONS (BARHS) BT FAST ELECTRONS IN K $z=19 \quad A=39.100$

\begin{tabular}{rrr} 
TD $=88.0$ & EV & \multicolumn{1}{c}{ ET $=0.854003$} \\
ENERGY (HEV) & PRIMARY & CASCADE \\
0.86200 & 0.03 & 0.03 \\
0.87100 & 0.08 & 0.08 \\
0.88800 & 0.16 & 0.16 \\
0.91300 & 0.30 & 0.30 \\
0.94700 & 0.50 & 0.50 \\
0.99000 & 0.78 & 0.78 \\
1.04000 & 1.13 & 1.13 \\
1.11000 & 1.65 & 1.65 \\
1.19000 & 2.26 & 2.26 \\
1.32000 & 3.24 & 3.24 \\
1.49000 & 4.44 & 4.46 \\
1.70000 & 5.77 & 5.86 \\
1.96000 & 7.16 & 7.46 \\
2.30000 & 8.61 & 9.35 \\
2.73000 & 9.99 & 11.47 \\
3.24000 & 11.17 & 13.66 \\
3.84000 & 12.14 & 15.90 \\
4.44000 & 12.82 & 17.86 \\
5.12000 & 13.36 & 19.81 \\
5.97000 & 13.83 & 21.95 \\
7.25000 & 14.27 & 24.68 \\
8.54000 & 14.53 & 27.00 \\
12.80000 & 14.89 & 32.82 \\
17.00000 & 14.98 & 36.93 \\
25.60000 & 14.99 & 42.89 \\
42.70000 & 14.93 & 50.36 \\
85.40000 & 14.84 & 60.52 \\
170.00000 & 14.79 & 70.65
\end{tabular}

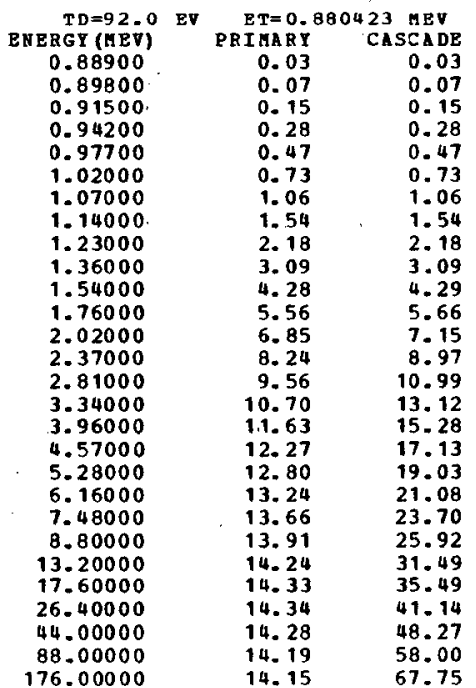

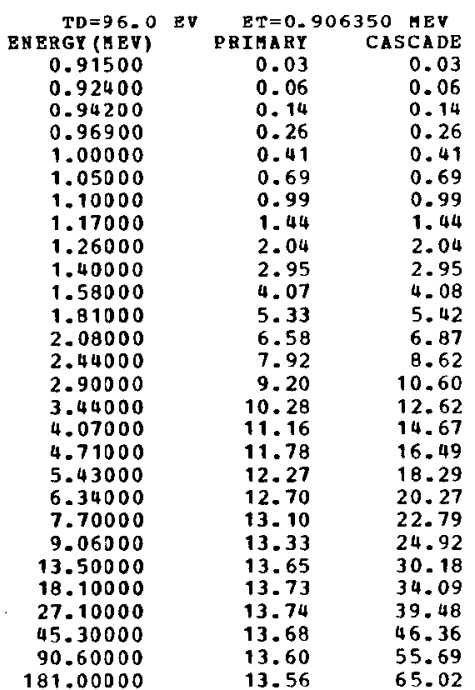


ATOHIC DISPLACRAERT CROSS-SECTIONS (BARNS) BY FAST ELECTRONS IN TI

\begin{tabular}{rrr} 
TD= 4.0 EV & \multicolumn{2}{c}{ RT $=0.080890$ MEV } \\
ENERGY (MEV) & PRIMAR & CASCADE \\
0.08160 & 10.18 & 10.18 \\
0.08250 & 22.63 & 22.63 \\
0.08410 & 43.57 & 43.57 \\
0.08650 & 72.38 & 72.38 \\
0.08970 & 106.49 & 106.49 \\
0.09380 & 144.10 & 144.10 \\
0.09860 & 180.97 & 180.97 \\
0.10500 & 220.67 & 220.67 \\
0.11300 & 258.84 & 258.84 \\
0.12500 & 299.48 & 299.48 \\
0.14100 & 334.03 & 334.03 \\
0.16100 & 359.14 & 359.69 \\
0.18600 & 375.73 & 381.54 \\
0.21800 & 385.42 & 401.48 \\
0.25800 & 389.65 & 419.77 \\
0.30700 & 390.32 & 437.24 \\
0.36400 & 389.15 & 454.31 \\
0.42000 & 387.55 & 469.37 \\
0.48500 & 385.79 & 485.68 \\
0.56600 & 383.96 & 504.91 \\
0.68700 & 381.90 & 532.06 \\
0.80800 & 380.43 & 557.67 \\
1.21000 & 377.37 & 633.50 \\
1.61000 & 375.37 & 697.43 \\
2.42000 & 372.28 & 801.71 \\
4.04000 & 368.22 & 950.37 \\
8.08000 & 362.72 & 1171.39 \\
16.10000 & 358.85 & 1404.05 \\
24.20000 & 357.54 & 1544.89 \\
40.40000 & 356.56 & 1723.93 \\
56.60000 & 356.16 & 1842.50 \\
80.80000 & 355.83 & 1968.00 \\
161.00000 & 355.31 & 2211.58
\end{tabular}

\begin{tabular}{rrr} 
TD $=8.0$ & BV & \multicolumn{2}{c}{ ET=0.151984 MEV } \\
ENERGY(MEV) & PRIMARY & \multicolumn{1}{c}{ CASCADE } \\
0.15300 & 2.04 & 2.04 \\
0.15500 & 5.94 & 5.94 \\
0.15800 & 11.50 & 11.50 \\
0.16200 & 18.40 & 18.40 \\
0.16800 & 27.79 & 27.79 \\
0.17600 & 38.78 & 38.78 \\
0.18500 & 49.42 & 49.42 \\
0.19700 & 61.35 & 61.35 \\
0.21200 & 73.49 & 73.49 \\
0.23500 & 87.88 & 87.88 \\
0.26500 & 101.59 & 101.59 \\
0.30300 & 113.98 & 114.42 \\
0.34900 & 124.65 & 127.09 \\
0.41000 & 134.69 & 141.04 \\
0.48600 & 143.54 & 155.66 \\
0.57700 & 151.13 & 170.73 \\
0.68300 & 157.57 & 186.18 \\
0.79000 & 162.42 & 200.20 \\
0.91100 & 166.59 & 214.65 \\
1.06000 & 170.43 & 230.86 \\
1.29000 & 174.56 & 253.25 \\
1.51000 & 177.19 & 272.32 \\
2.27000 & 181.59 & $\mathbf{3 2 6 . 1 5}$ \\
3.03000 & 182.96 & $\mathbf{3 6 7 . 6 8}$ \\
4.55000 & 183.32 & 430.00 \\
7.59000 & 182.33 & 512.94 \\
15.10000 & 180.29 & 629.14 \\
30.30000 & 178.89 & 749.90 \\
45.50000 & 178.44 & 821.17 \\
75.90000 & 178.10 & 911.36 \\
106.00000 & 177.94 & 970.37 \\
151.00000 & 177.80 & 1032.94 \\
303.00000 & 177.55 & 1156.22 \\
& &
\end{tabular}

\begin{tabular}{rrr} 
TD $=12.0$ & EV & \multicolumn{2}{c}{ ET $=0.216159$ MEV } \\
ENERGY (HEV) & PRIMARY & CASCADE \\
0.21800 & 1.20 & 1.20 \\
0.22000 & 2.47 & 2.47 \\
0.22400 & 4.93 & 4.93 \\
0.23100 & 8.95 & 8.95 \\
0.23900 & 13.17 & 13.17 \\
0.25000 & 18.41 & 18.41 \\
0.26300 & 23.89 & 23.89 \\
0.28100 & 30.47 & 30.47 \\
0.30200 & 37.00 & 37.00 \\
0.33500 & 45.43 & 45.43 \\
0.37800 & 54.13 & 54.13 \\
0.43200 & 62.65 & 63.02 \\
0.49700 & 70.64 & 72.24 \\
0.58300 & 78.78 & 82.81 \\
0.69100 & 86.48 & 94.34 \\
0.82100 & 93.38 & 106.49 \\
0.97200 & 99.30 & 118.95 \\
1.12000 & 103.64 & 129.90 \\
1.29300 & 107.41 & 141.28 \\
1.51300 & 110.99 & 154.52 \\
1.83000 & 114.51 & 171.47 \\
2.16000 & 116.86 & 186.75 \\
3.24000 & 120.40 & 226.44 \\
4.32000 & 121.46 & 256.24 \\
6.48000 & 121.79 & $\mathbf{3 0 0 . 0 1}$ \\
10.80000 & 121.16 & $\mathbf{3 5 7} .06$ \\
21.60000 & 119.91 & 436.51 \\
43.20000 & 119.13 & 517.32 \\
64.80000 & 118.88 & 564.92 \\
108.00000 & 118.68 & 625.10 \\
151.00000 & 118.59 & 664.63 \\
216.00300 & 118.50 & 706.88 \\
432.00300 & 118.33 & 788.72 \\
& &
\end{tabular}

ATOMIC DISPLACEHENT CROSS-SECTIONS (BARNS) BY PAST ELECTRONS IN TI $z=22 \quad A=47.900$

\begin{tabular}{rrr}
$\begin{array}{c}\text { TD }=16.0 \\
\text { ENERGY EVEV }\end{array}$ & \multicolumn{2}{c}{ ET $=0.275113$ HEV } \\
PRIMARY & CASCADE \\
0.27700 & 0.56 & 0.56 \\
0.28000 & 1.44 & 1.44 \\
0.28600 & 3.14 & 3.14 \\
0.29400 & 5.28 & 5.28 \\
0.30500 & 8.03 & 8.03 \\
0.31900 & 11.25 & 11.25 \\
0.33500 & 14.61 & 14.61 \\
0.35700 & 18.75 & 18.75 \\
0.38500 & 23.39 & 23.39 \\
0.42600 & 29.23 & 29.23 \\
0.48100 & 35.75 & 35.75 \\
0.55000 & 42.43 & 42.74 \\
0.63200 & 48.86 & 50.07 \\
0.74200 & 55.68 & 58.74 \\
0.88000 & 62.24 & 68.30 \\
1.04000 & 67.94 & 78.06 \\
1.23000 & 72.96 & 88.31 \\
1.43000 & 76.84 & 97.92 \\
1.65000 & 80.01 & 107.39 \\
1.92000 & 82.82 & 117.79 \\
2.33000 & 85.67 & 131.58 \\
2.75000 & 87.53 & 143.81 \\
4.12000 & 90.21 & 175.05 \\
5.50000 & 91.00 & 198.35 \\
8.25000 & 91.20 & $\mathbf{2 3 2 . 0 4}$ \\
13.70000 & 90.71 & 275.19 \\
27.50000 & 89.82 & $\mathbf{3 3 5 . 5 9}$ \\
55.00000 & 89.29 & $\mathbf{3 9 6 . 4 3}$ \\
82.50000 & 89.12 & $\mathbf{4 3 2 . 2 2}$ \\
137.00000 & 88.99 & 477.09 \\
192.00000 & 88.92 & 505.96
\end{tabular}

\begin{tabular}{rrr}
$T D=20.0$ & EV & \multicolumn{2}{c}{ ET $=0.329944$ HEV } \\
ENERGY (HEV) & PRI KARY & CASCADE \\
0.33300 & 0.50 & 0.50 \\
0.33600 & 0.98 & 0.98 \\
0.34300 & 2.08 & 2.08 \\
0.35300 & 3.59 & 3.59 \\
0.36600 & 5.45 & 5.45 \\
0.38200 & 7.60 & 7.60 \\
0.40200 & 10.10 & 10.10 \\
0.42800 & 13.10 & 13.10 \\
0.46100 & 16.56 & 16.56 \\
0.51100 & 21.19 & 21.19 \\
0.57700 & 26.45 & 26.46 \\
0.65900 & 31.95 & 32.21 \\
0.75800 & 37.42 & 38.42 \\
0.89000 & 43.25 & 45.78 \\
1.05000 & 48.72 & 53.68 \\
1.25000 & 53.83 & 62.38 \\
1.48000 & 58.11 & 71.19 \\
1.71000 & 61.26 & 79.01 \\
1.97000 & 63.88 & 86.93 \\
2.30000 & 66.27 & 95.88 \\
2.80000 & 68.62 & 107.61 \\
3.29000 & 70.07 & 117.52 \\
4.94000 & 72.18 & 143.41 \\
6.59000 & 72.77 & 162.42 \\
9.89000 & 72.90 & 189.81 \\
16.40000 & 72.48 & 224.55 \\
32.90000 & 71.80 & 273.06 \\
65.90000 & 71.40 & 321.96 \\
98.90000 & 71.28 & 350.66 \\
164.00000 & 71.18 & 386.48 \\
230.00000 & 71.13 & 410.44 \\
& &
\end{tabular}

\begin{tabular}{rrr} 
TD $=24.0$ & EV & \multicolumn{2}{c}{ ET $=0.381413$ MEV } \\
ENERGY (MBV) & PRIMARY & CASCADE \\
0.38500 & 0.36 & 0.36 \\
0.38900 & 0.76 & 0.76 \\
0.39600 & 1.44 & 1.44 \\
0.40800 & 2.59 & 2.59 \\
0.42300 & 3.96 & 3.96 \\
0.44200 & 5.63 & 5.63 \\
0.46500 & 7.55 & 7.55 \\
0.49500 & 9.91 & 9.91 \\
0.53300 & 12.68 & 12.68 \\
0.59100 & 16.52 & 16.52 \\
0.66700 & 20.94 & 20.95 \\
0.76200 & 25.67 & 25.91 \\
0.87700 & 30.44 & 31.31 \\
1.02000 & 35.24 & 37.34 \\
1.22000 & 40.41 & 44.80 \\
1.44000 & 44.63 & 52.03 \\
1.71000 & 48.40 & 59.83 \\
1.98000 & 51.13 & 66.74 \\
2.28000 & 53.34 & 73.58 \\
2.56000 & 55.34 & 81.26 \\
3.24000 & 57.30 & 91.37 \\
3.81000 & 58.49 & 99.89 \\
5.72000 & 60.19 & 121.91 \\
7.62000 & 60.64 & 137.89 \\
11.40000 & 60.71 & 160.77 \\
19.00000 & 60.34 & 190.18 \\
38.10000 & 59.79 & 230.72 \\
76.20000 & 59.49 & 271.44 \\
114.00000 & 59.39 & 295.20 \\
190.00000 & 59.31 & 325.36 \\
266.00000 & 59.27 & 345.23
\end{tabular}


ATOHIC DISPLACEMENT CROSS-SECTIONS (BARNS) BY PAST ELECTRONS IN II

\begin{tabular}{crr}
$\begin{array}{c}\text { TD=28.0 } \\
\text { ENER }\end{array}$ & \multicolumn{1}{c}{ ET $=0.430070$ MEV } \\
0.43400 & PRIMARY & CASCADE \\
0.43800 & 0.26 & 0.26 \\
0.44700 & 0.53 & 0.53 \\
0.46000 & 1.12 & 1.12 \\
0.47700 & 1.97 & 1.97 \\
0.49800 & 3.05 & 3.05 \\
0.52400 & 4.35 & 4.35 \\
0.55900 & 5.91 & 5.91 \\
0.60200 & 7.93 & 7.93 \\
0.65600 & 10.26 & 10.26 \\
0.75200 & 13.48 & 13.48 \\
0.86000 & 17.33 & 17.35 \\
0.98900 & 21.52 & 21.74 \\
1.16000 & 35.71 & 26.49 \\
1.37000 & 34.19 & 32.15 \\
1.63000 & 38.34 & 38.32 \\
1.93000 & 41.57 & 45.04 \\
2.23000 & 43.91 & 51.84 \\
2.58000 & 45.89 & 57.85 \\
3.01000 & 47.59 & 64.09 \\
3.65000 & 49.21 & 79.84 \\
4.30000 & 50.22 & 87.50 \\
6.45000 & 51.61 & 106.14 \\
8.50000 & 51.97 & 120.02 \\
12.90000 & 52.01 & 139.90 \\
21.50000 & 51.68 & 165.21 \\
43.00000 & 51.23 & 199.91 \\
86.00000 & 50.98 & 234.85 \\
129.00000 & 50.90 & 255.36 \\
215.00000 & 50.83 & 281.22 \\
& & \\
& &
\end{tabular}

\begin{tabular}{|c|c|c|c|}
\hline \multirow{2}{*}{\multicolumn{2}{|c|}{$\begin{array}{r}T D=32.0 \quad \mathrm{EV} \\
\text { ENERGY (MEV) }\end{array}$}} & \multicolumn{2}{|c|}{$\mathrm{ET}=0.476333 \mathrm{AEV}$} \\
\hline & & PRI I A RY & CASCADE \\
\hline 0.48100 & & 0.22 & 0.22 \\
\hline 0.48500 & & 0.41 & 0.41 \\
\hline 0.49500 & & 0.88 & 0.88 \\
\hline 0.50900 & & 1.54 & 1.54 \\
\hline 0.52800 & & 2.43 & 2.43 \\
\hline 0.55200 & & 3. 54 & 3.54 \\
\hline 0.58100 & & 4.85 & 4.85 \\
\hline 0.61900 & & 6.53 & 6.53 \\
\hline 0.66600 & & 8.53 & 8.53 \\
\hline 0.73800 & & 11. 39 & 11.39 \\
\hline 0.83300 & & 14.81 & 14.82 \\
\hline 0.95200 & & 18.53 & 18.73 \\
\hline 1.09000 & & 22.18 & 22.87 \\
\hline 1.28000 & & 26.24 & 27.99 \\
\hline 1.52000 & & 30.17 & 33.72 \\
\hline 1.81000 & & 33.65 & 39.80 \\
\hline 2.14000 & & 36.49 & 45.86 \\
\hline 2.47000 & & 38.53 & 51.21 \\
\hline 2.85000 & & 40.24 & 56.68 \\
\hline 3.33000 & & 41.73 & 62.76 \\
\hline 4.04000 & & 43.14 & 70.48 \\
\hline 4.76000 & & 44.01 & 77.17 \\
\hline 7.14000 & & 45.18 & 94.07 \\
\hline 9.52000 & & 45.48 & 106.31 \\
\hline 14.20000 & & 45.49 & 123.54 \\
\hline 23.80000 & & 45.20 & 146.01 \\
\hline 47.60000 & & 44.81 & 176.42 \\
\hline 95.20000 & & 44.60 & 207.02 \\
\hline 142.00000 & & 44.53 & 224.73 \\
\hline 238.00000 & & 44.47 & 247.61 \\
\hline
\end{tabular}

\begin{tabular}{rrr} 
TD $=36.0$ & EV & \multicolumn{2}{c}{ ET $=0.520523$ HEV } \\
ENERGY (HEV) & PRIMARY & CASCADE \\
0.52500 & 0.16 & 0.16 \\
0.53000 & 0.33 & 0.33 \\
0.54100 & 0.72 & 0.72 \\
0.55600 & 1.25 & 1.25 \\
0.57700 & 2.00 & 2.00 \\
0.60300 & 2.93 & 2.93 \\
0.63500 & 4.08 & 4.08 \\
0.67600 & 5.53 & 5.53 \\
0.72800 & 7.31 & 7.31 \\
0.80600 & 9.85 & 9.85 \\
0.91000 & 12.93 & 12.94 \\
1.04000 & 16.30 & 16.49 \\
1.19000 & 19.59 & 20.23 \\
1.40000 & 23.32 & 24.94 \\
1.66000 & 26.84 & 30.11 \\
1.97000 & 29.93 & 35.53 \\
2.34300 & 32.54 & 41.18 \\
2.70000 & 34.36 & 46.01 \\
3.12000 & 35.88 & 51.01 \\
3.64000 & 37.19 & 56.45 \\
4.42000 & 38.43 & 63.44 \\
5.20000 & 39.17 & 69.39 \\
7.80000 & 40.18 & 84.54 \\
10.40000 & 40.42 & 95.48 \\
15.60000 & 40.42 & 111.08 \\
26.00000 & 40.16 & 130.88 \\
52.00000 & 39.82 & 157.95 \\
104.00000 & 39.64 & 185.17 \\
156.00000 & 39.58 & 201.13 \\
260.00000 & 39.53 & 221.25 \\
& &
\end{tabular}

ATOMIC DISPLACRMENT CROSS-SBCTIONS (BARNS) BY PAST ELECTRONS IN TI $z=22 \quad A=47.900$

\begin{tabular}{|c|c|c|c|}
\hline$T D=40.0$ & EV & $\mathrm{ET}=0$. & $2896 \mathrm{HEV}$ \\
\hline ENERGY (MEV) & & PRIMARY & CASCADE \\
\hline 0.56800 & & 0.13 & 0.13 \\
\hline & & 0.30 & 0.30 \\
\hline & & 1.06 & 1.06 \\
\hline 0.62400 & & 1.69 & 1.69 \\
\hline 0.65200 & & 2.49 & 2.49 \\
\hline 0.68600 & & 3.48 & 3.48 \\
\hline $\begin{array}{l}0.73100 \\
0.78800\end{array}$ & & $\begin{array}{l}4.79 \\
6.41\end{array}$ & $\begin{array}{l}4.79 \\
6.41\end{array}$ \\
\hline 0.87200 & & 8.69 & 8.69 \\
\hline 0.98500 & & 11.51 & 11.53 \\
\hline 1.12000 & & 14.48 & 14.65 \\
\hline 1.29000 & & 17.64 & 18.24 \\
\hline 1.51000 & & 20.95 & 22.44 \\
\hline 1.80000 & & 24.26 & 27.33 \\
\hline 2.13000 & & 27.01 & 32.22 \\
\hline 2.53000 & & 29.38 & 37.39 \\
\hline 2.92000 & & 31.02 & 41.81 \\
\hline 3.37000 & & 32.37 & 46.33 \\
\hline 3.94000 & & 33.55 & 51.36 \\
\hline 4.78000 & & 34.64 & 57.69 \\
\hline 5.62000 & & 35.30 & 63.07 \\
\hline 8.44000 & & 36.17 & 76.84 \\
\hline 11.20000 & & 36.38 & 86.57 \\
\hline 16.80000 & & 36.37 & 100.65 \\
\hline 28.10000 & & $36 \cdot 13$ & 118.63 \\
\hline 2000 & & & \\
\hline 112.00000 & & 35.67 & 167.40 \\
\hline 168.00000 & & 35.62 & 181.77 \\
\hline
\end{tabular}

\begin{tabular}{crr} 
TD=44.0 EV & \multicolumn{2}{c}{ ET=0.6036 $=0$ MEV } \\
ENERGY(HEV) & PRIMAY & CASCA DE \\
0.60900 & 0.11 & 0.11 \\
0.61500 & 0.24 & 0.24 \\
0.62700 & 0.49 & 0.49 \\
0.64500 & 0.89 & 0.89 \\
0.67000 & 1.47 & 1.47 \\
0.70000 & 2.18 & 2.18 \\
0.73600 & 3.05 & 3.05 \\
0.78400 & 4.22 & 4.22 \\
0.84500 & 5.69 & 5.69 \\
0.93500 & 7.77 & 7.77 \\
1.05000 & 10.24 & 10.26 \\
1.20000 & 13.09 & 13.25 \\
1.38000 & 15.98 & 16.53 \\
1.62000 & 19.08 & 20.48 \\
1.93000 & 22.11 & 24.99 \\
2.29000 & 24.67 & 29.57 \\
2.71000 & 26.78 & 34.24 \\
3.13000 & 28.27 & 38.34 \\
3.62000 & 29.51 & 42.56 \\
4.22000 & 30.55 & 47.08 \\
5.13000 & 31.54 & 52.95 \\
6.03000 & 32.12 & 57.87 \\
9.05000 & 32.90 & 70.44 \\
12.00000 & 33.07 & 79.29 \\
18.10000 & 33.05 & 92.30 \\
30.10000 & 32.83 & 108.49 \\
60.30000 & 32.56 & 130.73 \\
120.00000 & 32.43 & 152.86 \\
181.00000 & 32.38 & 166.11
\end{tabular}

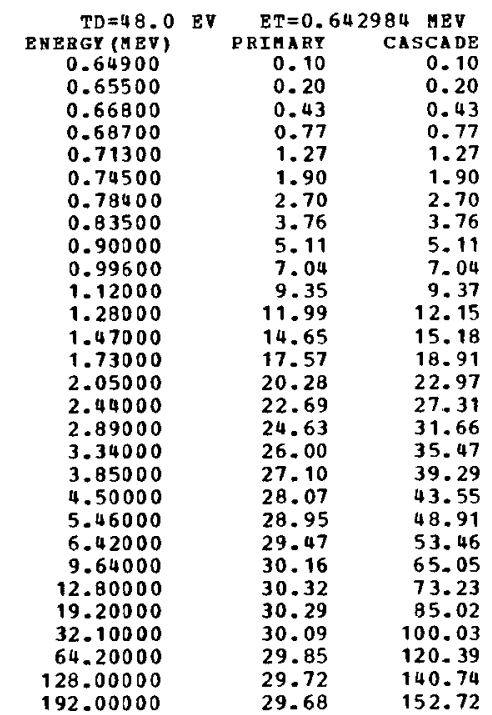


ATOMIC DISPLACEMENT CROSS-SECTIONS (BARNS) BY FAST ELECTRONS IN TI

\begin{tabular}{rrr} 
TD $=52.0$ & EV & \multicolumn{2}{c}{ ET $=0.681012$ HEV } \\
ENERGY (HEV) & PRIHARY & CASCADE \\
0.68700 & 0.08 & 0.08 \\
0.69400 & 0.18 & 0.18 \\
0.70800 & 0.38 & 0.38 \\
0.72800 & 0.68 & 0.68 \\
0.75500 & 1.12 & 1.12 \\
0.78900 & 1.69 & 1.69 \\
0.83000 & 2.41 & 2.41 \\
0.88500 & 3.41 & 3.41 \\
0.95300 & 4.64 & 4.64 \\
1.05000 & 6.35 & 6.35 \\
1.19000 & 8.65 & 8.67 \\
1.36000 & 11.11 & 11.27 \\
1.56000 & 13.57 & 14.08 \\
1.83000 & 16.23 & 17.50 \\
2.17000 & 18.77 & 21.31 \\
2.58000 & 20.98 & 25.34 \\
3.06000 & 22.80 & 29.43 \\
3.54000 & 24.07 & 33.01 \\
4.08000 & 25.08 & 36.56 \\
4.76000 & 25.95 & 40.47 \\
5.78000 & 26.76 & 45.47 \\
6.81000 & 27.24 & 49.74 \\
10.20000 & 27.85 & 60.42 \\
13.60000 & 27.99 & 68.10 \\
20.40000 & 27.95 & 79.00 \\
34.00000 & 27.77 & 92.79 \\
68.10000 & 27.55 & 111.64 \\
136.00000 & 27.43 & 130.47 \\
204.00000 & 27.40 & 141.53 \\
& &
\end{tabular}

\begin{tabular}{rrr} 
TD $=56.0$ & EV & \multicolumn{2}{c}{ ET=0.717864 MEV } \\
ENERGY (HEV) & PRI HARY & CASCA DE \\
0.72500 & 0.08 & 0.08 \\
0.73200 & 0.16 & 0.16 \\
0.74600 & 0.33 & 0.33 \\
0.76800 & 0.61 & 0.61 \\
0.79600 & 1.00 & 1.00 \\
0.83200 & 1.53 & 1.53 \\
0.87500 & 2.19 & 2.19 \\
0.93300 & 3.11 & 3.11 \\
1.00000 & 4.18 & 4.18 \\
1.11000 & 5.90 & 5.90 \\
1.25000 & 7.94 & 7.96 \\
1.43000 & 10.27 & 10.42 \\
1.65000 & 12.67 & 13.17 \\
1.93000 & 15.12 & 16.33 \\
2.29000 & 17.50 & 19.92 \\
2.72000 & 19.54 & 23.68 \\
3.23000 & 21.24 & 27.54 \\
3.73000 & 22.40 & 30.85 \\
4.30000 & 23.33 & 34.17 \\
5.02000 & 24.13 & 37.85 \\
6.10000 & 24.88 & 42.53 \\
7.17000 & 25.31 & 46.46 \\
10.70000 & 25.86 & 56.31 \\
14.30000 & 25.99 & 63.53 \\
21.50000 & 25.95 & 73.72 \\
35.80000 & 25.78 & 86.51 \\
71.70000 & 25.57 & 104.02 \\
143.00000 & 25.47 & 121.48 \\
215.00000 & 25.44 & 131.81
\end{tabular}

\begin{tabular}{rrr} 
TD=50.0 EV & \multicolumn{1}{c}{ ET $=0.753642$ MEV } \\
ENERGY (HEV) & PRIMARY & CASCADE \\
0.76100 & 0.07 & 0.07 \\
0.76300 & 0.14 & 0.14 \\
0.78300 & 0.29 & 0.29 \\
0.80600 & 0.54 & 0.54 \\
0.83500 & 0.90 & 0.90 \\
0.87400 & 1.39 & 1.39 \\
0.91900 & 2.00 & 2.00 \\
0.97900 & 2.85 & 2.85 \\
1.05000 & 3.86 & 3.86 \\
1.16000 & 5.40 & 5.40 \\
1.31000 & 7.37 & 7.38 \\
1.50000 & 9.58 & 9.72 \\
1.73000 & 11.83 & 12.30 \\
2.03000 & 14.17 & 15.34 \\
2.41000 & 16.41 & 18.74 \\
2.86000 & 18.31 & 22.26 \\
3.39000 & 19.87 & 25.86 \\
3.91000 & 20.94 & 28.94 \\
4.52000 & 21.82 & 32.13 \\
5.27000 & 22.56 & 35.54 \\
6.40000 & 23.24 & 39.93 \\
7.53000 & 23.64 & 43.63 \\
11.30000 & 24.15 & 52.98 \\
15.00000 & 24.25 & 59.57 \\
22.60000 & 24.22 & 69.14 \\
37.60000 & 24.05 & 81.08 \\
75.30000 & 23.87 & 97.42 \\
150.00000 & 23.77 & 113.69 \\
226.00000 & 23.74 & 123.39
\end{tabular}

ATONIC DISPLACEMENT CROSS-SECTIONS (BARNS) BY FAST RLECTRONS IN TI $z=22 \quad A=47.900$

\begin{tabular}{rrr} 
TD $=64.0$ & EV & \multicolumn{2}{c}{ ET $=0.788436$} & MEV \\
ENERGY (HEV) & PRIMARY & CASCADE \\
0.79600 & 0.06 & 0.06 \\
0.80400 & 0.13 & 0.13 \\
0.81900 & 0.26 & 0.26 \\
0.84300 & 0.49 & 0.49 \\
0.87500 & 0.82 & 0.82 \\
0.91400 & 1.27 & 1.27 \\
0.96100 & 1.83 & 1.83 \\
1.02000 & 2.58 & 2.58 \\
1.10000 & 3.60 & 3.60 \\
1.22000 & 5.12 & 5.12 \\
1.37000 & 6.89 & 6.91 \\
1.57000 & 8.99 & 9.13 \\
1.81000 & 11.11 & 11.57 \\
2.12000 & 13.30 & 14.41 \\
2.52000 & 15.42 & 17.65 \\
2.99000 & 17.20 & 20.97 \\
3.54000 & 18.66 & 24.34 \\
4.09000 & 19.67 & 27.29 \\
4.73000 & 20.49 & 30.30 \\
5.51000 & 21.17 & 33.50 \\
6.70000 & 21.81 & 37.65 \\
7.88000 & 22.18 & 41.13 \\
11.80000 & 22.64 & 49.87 \\
15.70000 & 22.74 & 56.11 \\
23.60000 & 22.70 & 65.05 \\
39.40000 & 22.55 & 76.32 \\
78.80000 & 22.37 & 91.62 \\
157.00000 & 22.28 & 106.88
\end{tabular}

\begin{tabular}{rrr} 
TD $=68.0$ & EV & \multicolumn{2}{c}{ ET=0.822321 MEV } \\
BNERGY (MEV) & PRIMARY & CASCADE \\
0.83000 & 0.05 & 0.05 \\
0.83800 & 0.11 & 0.11 \\
0.85500 & 0.24 & 0.24 \\
0.87900 & 0.44 & 0.44 \\
0.91200 & 0.75 & 0.75 \\
0.95300 & 1.16 & 1.16 \\
1.00000 & 1.67 & 1.67 \\
1.06000 & 2.35 & 2.35 \\
1.15000 & 3.40 & 3.40 \\
1.27000 & 4.77 & 4.77 \\
1.43000 & 6.49 & 6.51 \\
1.64000 & 8.49 & 8.63 \\
1.89000 & 10.50 & 10.94 \\
2.22000 & 12.60 & 13.69 \\
2.63000 & 14.56 & 16.70 \\
3.12000 & 16.24 & 19.85 \\
3.70000 & 17.61 & 23.08 \\
4.27000 & 18.55 & 25.84 \\
4.93000 & 19.31 & 28.66 \\
5.75000 & 19.95 & $\mathbf{3 1 . 7 1}$ \\
6.98000 & 20.54 & 35.60 \\
8.22000 & 20.88 & 38.90 \\
12.30000 & 21.31 & 47.14 \\
16.40000 & 21.40 & 53.06 \\
24.60000 & 21.36 & 61.44 \\
41.10000 & 21.22 & 72.07 \\
82.20000 & 21.05 & 86.47 \\
164.00000 & 20.97 & 100.87
\end{tabular}

\begin{tabular}{rrr} 
TD=72.0 & EV & \multicolumn{2}{c}{ ET=0.855367 MEV } \\
ENERGY(HEV) & PRIMARY & CASCADE \\
0.86300 & 0.04 & 0.04 \\
0.87200 & 0.10 & 0.10 \\
0.88900 & 0.22 & 0.22 \\
0.91500 & 0.41 & 0.41 \\
0.94900 & 0.69 & 0.69 \\
0.99200 & 1.08 & 1.08 \\
1.04000 & 1.55 & 1.55 \\
1.11000 & 2.27 & 2.27 \\
1.19000 & 3.12 & 3.12 \\
1.32000 & 4.48 & 4.48 \\
1.49000 & 6.15 & 6.17 \\
1.71000 & 8.07 & 8.20 \\
1.96000 & 9.90 & 10.32 \\
2.30000 & 11.88 & 12.91 \\
2.73000 & 13.76 & 15.81 \\
3.25000 & 15.38 & 18.87 \\
3.84000 & 16.65 & 21.86 \\
4.44000 & 17.55 & 24.52 \\
5.13000 & 18.27 & 27.21 \\
5.98000 & 18.87 & 30.09 \\
7.27000 & 19.42 & 33.80 \\
8.55000 & 19.74 & 36.91 \\
12.80000 & 20.13 & 44.71 \\
17.10000 & 20.21 & 50.35 \\
25.60000 & 20.17 & 58.23 \\
42.70000 & 20.04 & 68.24 \\
85.50000 & 19.88 & 81.88 \\
171.00000 & 19.81 & 95.52
\end{tabular}


ATOHIC DISPLACEHENT CROSS-SECTIONS (BARNS) BY PAST ELECThONS IN TI

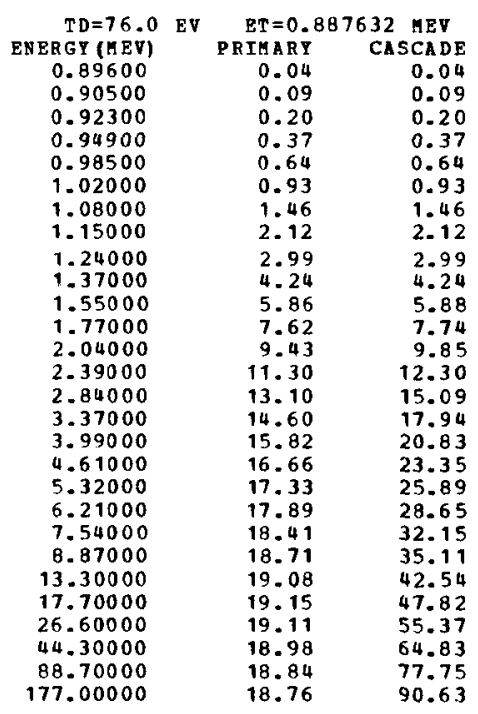

\begin{tabular}{rrr} 
TD $=80.0$ & EV & \multicolumn{2}{c}{ ET $=0.919170$ MEV } \\
ENERGY (HEV) & PRTARY & CASCADE \\
0.92800 & 0.04 & 0.04 \\
0.93700 & 0.08 & 0.08 \\
0.95500 & 0.18 & 0.18 \\
0.98300 & 0.35 & 0.35 \\
1.02000 & 0.60 & 0.60 \\
1.06000 & 0.89 & 0.89 \\
1.12000 & 1.38 & 1.38 \\
1.19000 & 1.99 & 1.99 \\
1.28000 & 2.79 & 2.79 \\
1.42000 & 4.03 & 4.03 \\
1.60000 & 5.53 & 5.54 \\
1.83000 & 7.23 & 7.35 \\
2.11000 & 8.97 & 9.37 \\
2.48000 & 10.79 & 11.76 \\
2.94000 & 12.47 & 14.39 \\
3.49000 & 13.90 & 17.12 \\
4.13000 & 15.05 & 19.87 \\
4.77000 & 15.84 & 22.27 \\
5.51000 & 16.48 & 24.70 \\
6.43000 & 17.02 & 27.33 \\
7.81000 & 17.50 & 30.68 \\
9.19000 & 17.78 & 33.49 \\
13.70000 & 18.12 & 40.47 \\
18.30000 & 18.19 & 45.55 \\
27.50000 & 18.15 & 52.72 \\
45.90000 & 18.03 & 61.75 \\
91.90000 & 17.89 & 74.03 \\
183.00000 & 17.83 & 86.24
\end{tabular}

$T D=84.0 \mathrm{BV}$

$\mathrm{BT}=0.950027 \mathrm{HEV}$

$$
\text { BNERGY }
$$

ERGY (MEV

0.95900
0.95900

0.96900

0.98800

1.01000

1.05000

1.10000

1.15000

1300

1.33000
1.47000

1.66000

1.90000

2.18000

2.56000

3.04000

3.61000

4.27000

4.94000

5.70000
6.65000

6.65000

8.07000

9.50000

14.20000

19.00000

28.50000
47.50000

47.50000
95.00000

190.00000

$\begin{array}{rr}\text { PRIMARY } & \text { CASCADE } \\ 0.04 & 0.04 \\ 0.08 & 0.08 \\ 0.17 & 0.17 \\ 0.29 & 0.29 \\ 0.53 & 0.53 \\ 0.87 & 0.87 \\ 1.24 & 1.24 \\ 1.88 & 1.88 \\ 2.70 & 2.70 \\ 3.85 & 3.85 \\ 5.31 & 5.33 \\ 6.95 & 7.07 \\ 8.56 & 8.94 \\ 10.28 & 11.22 \\ 11.91 & 13.76 \\ 13.27 & 16.39 \\ 14.36 & 19.01 \\ 15.12 & 21.33 \\ 15.72 & 23.64 \\ 16.22 & 26.14 \\ 16.68 & 29.32 \\ 16.94 & 32.02 \\ 17.26 & 38.71 \\ 17.32 & 43.59 \\ 17.28 & 50.39 \\ 17.17 & 58.97 \\ 17.04 & 70.65 \\ 16.98 & 82.35\end{array}$

A TOHIC DISPLACEHENT CROSS-SECTIONS (BARNS) BY PAST BLECTRONS IN T $\mathrm{Z}=22 \quad \mathrm{~A}=47.900$

\begin{tabular}{rrr} 
TD=88.0 & \multicolumn{2}{c}{ ET $=0.980245$} \\
ENERGY MEV \\
0.99000 & PRIMARY & CASCADE \\
0.99900 & 0.03 & 0.03 \\
1.01000 & 0.07 & 0.07 \\
1.04000 & 0.12 & 0.12 \\
1.08000 & 0.26 & 0.26 \\
1.13000 & 0.47 & 0.47 \\
1.19000 & 0.79 & 0.79 \\
1.27000 & 1.20 & 1.20 \\
1.37000 & 1.79 & 1.79 \\
1.51000 & 2.55 & 2.55 \\
1.71000 & 3.62 & 3.62 \\
1.96000 & 5.06 & 5.07 \\
2.25000 & 6.64 & 6.76 \\
2.64000 & 8.19 & 8.56 \\
3.13000 & 9.83 & 10.74 \\
3.72000 & 11.37 & 13.16 \\
4.41000 & 12.68 & 15.68 \\
5.09000 & 13.73 & 18.23 \\
5.88000 & 14.45 & 20.42 \\
6.86000 & 15.02 & 22.64 \\
8.33000 & 15.50 & 25.04 \\
9.80000 & 15.93 & 28.11 \\
14.70000 & 16.18 & 30.66 \\
19.60000 & 16.48 & 37.12 \\
29.40000 & 16.54 & 41.72 \\
49.00000 & 16.50 & 48.22 \\
98.00000 & 16.38 & 56.42 \\
196.00000 & 16.26 & 67.56 \\
& 16.20 & 78.73
\end{tabular}

\begin{tabular}{rrr} 
TD $=92.0$ & EV & \multicolumn{2}{c}{ ET=1.009863 MEV } \\
ENERGY (HEV) & PRIMAY & CASCA DE \\
1.01000 & 0.00 & 0.00 \\
1.03000 & 0.07 & 0.07 \\
1.05000 & 0.15 & 0.15 \\
1.08000 & 0.28 & 0.28 \\
1.12000 & 0.49 & 0.49 \\
1.17000 & 0.78 & 0.78 \\
1.23000 & 1.16 & 1.16 \\
1.31000 & 1.71 & 1.71 \\
1.41000 & 2.43 & 2.43 \\
1.56000 & 3.49 & 3.49 \\
1.76000 & 4.83 & 4.85 \\
2.01000 & 6.32 & 6.43 \\
2.32000 & 7.86 & 8.23 \\
2.72000 & 9.43 & 10.31 \\
3.23000 & 10.91 & 12.65 \\
3.83000 & 12.15 & 15.05 \\
4.54000 & 13.15 & 17.50 \\
5.25000 & 13.84 & 19.62 \\
6.05000 & 14.38 & 21.72 \\
7.06000 & 14.83 & 24.02 \\
8.58000 & 15.25 & 26.96 \\
10.00000 & 15.47 & 29.28 \\
15.10000 & 15.77 & 35.56 \\
20.10000 & 15.82 & 39.95 \\
30.20000 & 15.78 & 46.19 \\
50.40000 & 15.67 & 54.05 \\
100.00000 & 15.56 & 64.59 \\
201.00000 & 15.50 & 75.36
\end{tabular}

$\begin{array}{rrr}\begin{array}{c}\text { TD=96.0 } \\ \text { ENERGY (KEV) }\end{array} & \begin{array}{c}\text { ET }=1.038916 \text { MEV } \\ \text { PRIMARY }\end{array} & \text { CASCADE } \\ 1.04000 & 0.00 & 0.00 \\ 1.05000 & 0.03 & 0.03 \\ 1.08000 & 0.13 & 0.13 \\ 1.11000 & 0.26 & 0.26 \\ 1.15000 & 0.45 & 0.45 \\ 1.20300 & 0.72 & 0.72 \\ 1.26000 & 1.07 & 1.07 \\ 1.35000 & 1.65 & 1.65 \\ 1.45000 & 2.32 & 2.32 \\ 1.61000 & 3.38 & 3.38 \\ 1.81000 & 4.63 & 4.65 \\ 2.07000 & 6.08 & 6.19 \\ 2.38000 & 7.52 & 7.87 \\ 2.80000 & 9.06 & 9.92 \\ 3.32000 & 10.47 & 12.16 \\ 3.94000 & 11.67 & 14.47 \\ 4.67000 & 12.62 & 16.82 \\ 5.40000 & 13.28 & 18.87 \\ 6.23000 & 13.80 & 20.90 \\ 7.27000 & 14.23 & 23.11 \\ 8.83000 & 14.62 & 25.92 \\ 10.30000 & 14.83 & 28.16 \\ 15.50000 & 15.11 & 34.14 \\ 20.70000 & 15.16 & 38.39 \\ 31.10000 & 15.12 & 44.38 \\ 51.90000 & 15.02 & 51.91 \\ 103.00000 & 14.91 & 62.02 \\ 207.00000 & 14.85 & 72.34\end{array}$


ATOHIC DISPLACENENT CROSS-SECTIONS (BARNS) BY PAST ELECTRONS IN v

\begin{tabular}{crr} 
TD $=4.0$ & EV & \multicolumn{2}{c}{ ET $=0.085670$ MEV } \\
ENERGY (HEV) & PRIMARY & CASCADE \\
0.08650 & 10.95 & 10.95 \\
0.08730 & 21.14 & 21.14 \\
0.08900 & 41.72 & 41.72 \\
0.09160 & 70.56 & 70.56 \\
0.09500 & 104.04 & 104.04 \\
0.09930 & 140.56 & 140.56 \\
0.10400 & 174.31 & 174.31 \\
0.11100 & 215.16 & 215.16 \\
0.11900 & 251.34 & 251.34 \\
0.13200 & 293.49 & 293.49 \\
0.14900 & 328.86 & 328.86 \\
0.17100 & 355.78 & 356.42 \\
0.19700 & 373.15 & 379.04 \\
0.23100 & 384.42 & 400.62 \\
0.27400 & 390.44 & 421.03 \\
0.32500 & 392.83 & 440.22 \\
0.38500 & 393.30 & 459.35 \\
0.44500 & 392.94 & 476.50 \\
0.51400 & 392.31 & 494.83 \\
0.59900 & 391.54 & 516.03 \\
0.72800 & 390.57 & 546.14 \\
0.85600 & 389.81 & 574.06 \\
1.28000 & 387.87 & 655.43 \\
1.71000 & 386.18 & 724.38 \\
2.57000 & 383.15 & 834.27 \\
4.28000 & 378.88 & 988.70 \\
8.56000 & 372.96 & 1217.63 \\
17.10000 & 368.85 & 1458.53 \\
25.70000 & 367.48 & 1603.51 \\
42.80000 & 366.46 & 1786.86 \\
59.90000 & 366.03 & 1908.41 \\
85.60000 & 365.67 & 2037.80 \\
171.00000 & 365.08 & 2289.06
\end{tabular}

\begin{tabular}{rrr}
$T D=8.0$ & \multicolumn{1}{c}{ EV } & \multicolumn{2}{c}{ ET= 0.160497 MEV } \\
ENERGY(HEV) & PRIMAR & CASCADE \\
0.16200 & 2.79 & 2.79 \\
0.16300 & 4.60 & 4.60 \\
0.16600 & 9.82 & 9.82 \\
0.17100 & 17.90 & 17.90 \\
0.17800 & 28.06 & 28.06 \\
0.18600 & 38.26 & 38.26 \\
0.19500 & 48.26 & 48.26 \\
0.20800 & 60.49 & 60.49 \\
0.22400 & 72.79 & 72.79 \\
0.24800 & 87.20 & 87.20 \\
0.28000 & 101.45 & 101.45 \\
0.32000 & 114.34 & 114.81 \\
0.36900 & 125.77 & 128.30 \\
0.43300 & 136.52 & 143.02 \\
0.51300 & 146.16 & 158.60 \\
0.60900 & 154.50 & 174.75 \\
0.72200 & 161.65 & 191.45 \\
0.83400 & 166.92 & 206.31 \\
0.96200 & 171.48 & 221.75 \\
1.12000 & 175.66 & 239.08 \\
1.36000 & 180.03 & 262.55 \\
1.60000 & 182.89 & 283.35 \\
2.40000 & 187.36 & 339.63 \\
3.20000 & 188.66 & 382.85 \\
4.81000 & 188.85 & 447.80 \\
8.02000 & 187.64 & 533.56 \\
16.00000 & 185.37 & 653.91 \\
32.00000 & 183.89 & 777.65 \\
48.10000 & 183.41 & 851.13 \\
80.20000 & 183.04 & 943.78 \\
112.00000 & 182.87 & 1004.44 \\
160.00000 & 182.71 & 1069.25 \\
320.00000 & 182.42 & 1195.32
\end{tabular}

\begin{tabular}{|c|c|c|}
\hline $\begin{array}{r}T D=12.0 \\
\text { EN ER GY (HEV) }\end{array}$ & $\begin{aligned} & E T=0 . \\
& \text { PRTM }\end{aligned}$ & CASCAD \\
\hline $\begin{array}{c}\text { ENER GI (HEV) } \\
0.23000\end{array}$ & $\begin{array}{l}\text { PRIMARY } \\
1.34\end{array}$ & CASCA DE \\
\hline $\begin{array}{l}0.23000 \\
0.23200\end{array}$ & $\begin{array}{l}1.34 \\
2.52\end{array}$ & 1.34 \\
\hline 0.23600 & $\begin{array}{l}2.32 \\
4.80\end{array}$ & $\begin{array}{l}2.32 \\
4.80\end{array}$ \\
\hline 0.24300 & 8.57 & 8.57 \\
\hline 0.25200 & 13.02 & 13.02 \\
\hline 0.26400 & 18.38 & 18.38 \\
\hline 0.27700 & 23.57 & 23.57 \\
\hline 0.29600 & 30.20 & 30.20 \\
\hline 0.31300 & 36.79 & 36.79 \\
\hline 0.35300 & 45.50 & 45.50 \\
\hline 0.39300 & 54.47 & 54.47 \\
\hline 0.45500 & 63.44 & 63.83 \\
\hline 0.52300 & 71.86 & 73.50 \\
\hline 0.61500 & 80.66 & 84.86 \\
\hline 0.72300 & 88.82 & 97.00 \\
\hline 0.86500 & 96.19 & 109.90 \\
\hline 1.02000 & 102.34 & 122.78 \\
\hline 1.18000 & 107.07 & 134.68 \\
\hline 1.36300 & 111.05 & 146.76 \\
\hline 1.59000 & 114.75 & 160.60 \\
\hline 1.93000 & 118.41 & 178.56 \\
\hline 2.27000 & 120.76 & 194.22 \\
\hline 3.41200 & 124.29 & 235.76 \\
\hline 4.55000 & 125.27 & 266.80 \\
\hline 6.83000 & 125.47 & 312.21 \\
\hline 11.30000 & 124.69 & 370.26 \\
\hline 22.70000 & 123.29 & 452.61 \\
\hline 45.50000 & 122.45 & 536.00 \\
\hline 68.30000 & 122.19 & 585.04 \\
\hline 113.00000 & 121.98 & 646.02 \\
\hline 159.00000 & 121.87 & 687.42 \\
\hline 227.00000 & 121.77 & 730.59 \\
\hline 455.00000 & 121.57 & 814.95 \\
\hline
\end{tabular}

ATOHIC DISPLACENENT CROSS-SECTIONS (BARNS) BY FAST ELECTRONS IN $\checkmark$ $z=23 \quad A=50.950$

\begin{tabular}{rrr}
$T D=16.0$ & $E V$ & \multicolumn{2}{c}{ ET $=0.289435$ MEV } \\
ENERGY (HEV & PRIMARY & CASCADE \\
0.29200 & 0.71 & 0.71 \\
0.29500 & 1.53 & 1.53 \\
0.30100 & $\mathbf{3 . 1 1}$ & 3.11 \\
0.30900 & 5.12 & 5.12 \\
0.32100 & 7.95 & 7.95 \\
0.33500 & 11.01 & 11.01 \\
0.35300 & 14.61 & 14.61 \\
0.37600 & 18.77 & 18.77 \\
0.40500 & 23.44 & 23.44 \\
0.44800 & 29.44 & 29.44 \\
0.50600 & 36.26 & 36.26 \\
0.57800 & 43.23 & 43.55 \\
0.66500 & 50.08 & 51.35 \\
0.78100 & 57.32 & 60.53 \\
0.92600 & 64.25 & 70.61 \\
1.09000 & 70.13 & 80.67 \\
1.30000 & 75.66 & 92.03 \\
1.50000 & 79.51 & 101.64 \\
1.73000 & 82.79 & 111.55 \\
2.02000 & 85.76 & 122.70 \\
2.46000 & 88.70 & 137.39 \\
2.89000 & 90.51 & 149.80 \\
4.34000 & 93.15 & 182.47 \\
5.78000 & 93.85 & 206.41 \\
8.68000 & 93.94 & 241.33 \\
14.40000 & 93.33 & 285.75 \\
28.90000 & 92.35 & 347.91 \\
57.80000 & 91.78 & 410.50 \\
86.80000 & 91.60 & 447.39 \\
144.00000 & 91.46 & 493.43 \\
& &
\end{tabular}

\begin{tabular}{|c|c|c|c|}
\hline $\begin{aligned} T D & =20.0\end{aligned}$ & EV & $\begin{aligned} E T=0.3 \\
\text { PRTARY }\end{aligned}$ & $\begin{array}{l}66 \text { MEV } \\
\text { CASCADE }\end{array}$ \\
\hline NERGY (AEV) & & & CASCADE \\
\hline 0.35000 & & 0.51 & 0.51 \\
\hline 0.35300 & & 0.96 & 0.96 \\
\hline 0.36000 & & 1.99 & 1.99 \\
\hline 0.37000 & & 3. 41 & 3.41 \\
\hline 0.38400 & & 5. 31 & 5.31 \\
\hline 0.40200 & & 7.62 & 7.62 \\
\hline 0.42200 & & 10.03 & 10.03 \\
\hline 0.45000 & & 13. 16 & 13.16 \\
\hline 0.48500 & & 16.74 & 16.74 \\
\hline 0.53700 & & 21.49 & 21.49 \\
\hline 0.60600 & & 26.96 & 26.97 \\
\hline 0.69300 & & 32.79 & 33.08 \\
\hline 0.79700 & & 38.55 & 39.61 \\
\hline 0.93500 & & 44.67 & 47. \\
\hline 1.10000 & & 50.33 & 55.51 \\
\hline 1.31000 & & 55.72 & \\
\hline 1.55000 & & 60.18 & 73.90 \\
\hline 1.80000 & & 63.55 & $\begin{array}{l}82.40 \\
90.60\end{array}$ \\
\hline $\begin{array}{l}2.07000 \\
2.42000\end{array}$ & & $\begin{array}{l}00.22 \\
68.67\end{array}$ & $\begin{array}{r}90.60 \\
100.03\end{array}$ \\
\hline 2.94000 & & 71.03 & 112.14 \\
\hline 3.46000 & & 72.48 & 122.55 \\
\hline 5.19000 & & 74.52 & 149.36 \\
\hline 6.93000 & & 75.04 & 169.09 \\
\hline 10.30000 & & 75.08 & 196.70 \\
\hline 17.30000 & & 74.56 & 233.40 \\
\hline 34.60000 & & 73.81 & 283.12 \\
\hline 69.30000 & & 73. 39 & 333.40 \\
\hline 103.00000 & & 73.27 & 362.20 \\
\hline 173.00000 & & 73.15 & 399.95 \\
\hline
\end{tabular}

\begin{tabular}{rrr} 
TD $=24.0$ & EV & \multicolumn{1}{c}{ ET $=0.400309$ MEV } \\
ENERGY (1IEV) & PRIMARY & CASCADE \\
0.40400 & 0.35 & 0.35 \\
0.40800 & 0.72 & 0.72 \\
0.41600 & 1.45 & 1.45 \\
0.42800 & 2.53 & 2.53 \\
0.44400 & 3.93 & 3.93 \\
0.46400 & 5.62 & 5.62 \\
0.48800 & 7.55 & 7.55 \\
0.52000 & 10.00 & 10.00 \\
0.56000 & 12.87 & 12.87 \\
0.62000 & 16.81 & 16.81 \\
0.70000 & 21.45 & 21.46 \\
0.80000 & 26.43 & 26.68 \\
0.92000 & 31.42 & 32.34 \\
1.08000 & 36.77 & 39.08 \\
1.28000 & 41.90 & 46.54 \\
1.52000 & 46.45 & 54.42 \\
1.80000 & 50.29 & 62.48 \\
2.08000 & 53.06 & 69.61 \\
2.40000 & 55.36 & 76.87 \\
2.80000 & 57.38 & 84.89 \\
3.40000 & 59.32 & 95.25 \\
4.00000 & 60.50 & 104.13 \\
6.00000 & 62.13 & 126.90 \\
8.00000 & 62.52 & 143.48 \\
12.00000 & 62.52 & 167.23 \\
20.00000 & 62.08 & 197.53 \\
40.00000 & 61.47 & 239.09 \\
80.00000 & 51.15 & 280.96 \\
120.00000 & 61.04 & 305.54 \\
200.00000 & 60.95 & $\mathbf{3 3 6 . 5 4}$ \\
& &
\end{tabular}


ATOMIC DISPLAZEHENT CROSS-SECTIONS (BARS) BY PAST ELECTRONS IN $V$ $=23 \quad A=50.950$

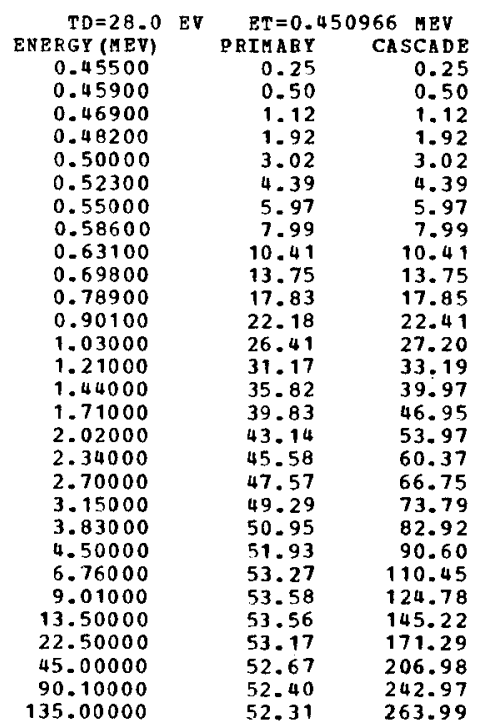

\begin{tabular}{crr} 
TD=32.0 & \multicolumn{2}{c}{ ET $=0.499085$ MEV } \\
ENERGY(AEV) & PRIARY & CASCADE \\
0.50400 & 0.22 & 0.22 \\
0.50900 & 0.44 & 0.44 \\
0.51900 & 0.88 & 0.88 \\
0.53400 & 1.55 & 1.55 \\
0.55300 & 2.40 & 2.40 \\
0.57800 & 3.52 & 3.52 \\
0.60800 & 4.85 & 4.85 \\
0.64800 & 6.59 & 6.59 \\
0.69800 & 8.69 & 8.69 \\
0.77300 & 11.67 & 11.67 \\
0.87300 & 15.26 & 15.28 \\
0.99800 & 19.18 & 19.40 \\
1.14000 & 22.94 & 23.67 \\
1.34000 & 27.23 & 29.08 \\
1.59000 & 31.32 & 35.07 \\
1.89000 & 34.91 & 41.37 \\
2.24000 & 37.89 & 47.79 \\
2.59000 & 40.01 & 53.45 \\
2.99000 & 41.75 & 59.17 \\
3.49000 & 43.25 & 65.46 \\
4.24000 & 44.67 & 73.54 \\
4.99000 & 45.51 & 80.42 \\
7.48000 & 46.63 & 97.88 \\
9.98000 & 46.88 & 110.55 \\
14.90000 & 46.84 & 128.36 \\
24.90000 & 46.50 & 151.36 \\
49.90000 & 46.07 & 182.73 \\
99.80000 & 45.84 & 214.20 \\
149.00000 & 45.77 & 232.44 \\
& &
\end{tabular}

\begin{tabular}{rrr} 
TD=36.0 RV & \multicolumn{2}{c}{ ET $=0.545014$ MEV } \\
ENERGY (HEV) & PRTHAY & CASCADE \\
0.55000 & 0.16 & 0.16 \\
0.55500 & 0.33 & 0.33 \\
0.56500 & 0.69 & 0.69 \\
0.58300 & 1.27 & 1.27 \\
0.60400 & 1.99 & 1.99 \\
0.63200 & 2.96 & 2.96 \\
0.66400 & 4.08 & 4.08 \\
0.70800 & 5.62 & 5.62 \\
0.76300 & 7.49 & 7.49 \\
0.84400 & 10.12 & 10.12 \\
0.95300 & 13.35 & 13.37 \\
1.09000 & 16.91 & 17.12 \\
1.25000 & 20.42 & 21.11 \\
1.47000 & 24.31 & 26.05 \\
1.74000 & 27.94 & 31.42 \\
2.07000 & 31.17 & 37.18 \\
2.45000 & 33.81 & 42.96 \\
2.83000 & 35.68 & 48.04 \\
3.27000 & 37.23 & 53.24 \\
3.81000 & 38.53 & 58.85 \\
4.63000 & 39.77 & 66.13 \\
5.45000 & 40.51 & 72.32 \\
8.17000 & 41.46 & 87.96 \\
10.90000 & 41.67 & 99.28 \\
16.30000 & 41.62 & 115.23 \\
27.20000 & 41.31 & 135.67 \\
54.50000 & 40.94 & 163.58 \\
109.00000 & 40.74 & 191.57 \\
163.00000 & 40.68 & 207.85
\end{tabular}

ATOHIC DISPLACERENT CROSS-SECTIONS (BARNS) BY FAST ELECTRONS IN V $z=23 \quad A=50.950$

\begin{tabular}{rrr} 
TD $=40.0$ & RV & \multicolumn{2}{c}{ RT $=0.589027$ MEV } \\
ENERGY(AEV) & PRIMARY & CASCADE \\
0.59400 & 0.12 & 0.12 \\
0.60000 & 0.27 & 0.27 \\
0.61200 & 0.58 & 0.58 \\
0.63000 & 1.06 & 1.06 \\
0.65300 & 1.68 & 1.68 \\
0.68300 & 2.53 & 2.53 \\
0.71800 & 3.52 & 3.52 \\
0.76500 & 4.87 & 4.87 \\
0.82400 & 6.54 & 6.54 \\
0.91200 & 8.93 & 8.93 \\
1.03000 & 11.89 & 11.90 \\
1.17000 & 14.98 & 15.16 \\
1.35000 & 18.34 & 18.98 \\
1.59000 & 21.92 & 23.55 \\
1.88000 & 25.20 & 28.45 \\
2.23000 & 28.10 & 33.63 \\
2.65000 & 30.54 & 39.04 \\
3.06000 & 32.22 & 43.67 \\
3.53000 & 33.58 & 48.36 \\
4.12000 & 34.76 & 53.52 \\
5.00000 & 35.85 & 60.10 \\
5.89000 & 36.50 & 65.74 \\
8.83000 & 37.33 & 79.91 \\
11.70000 & 37.50 & 89.90 \\
17.60000 & 37.44 & 104.51 \\
29.40000 & 37.16 & 122.97 \\
58.90000 & 36.83 & 148.11 \\
117.00000 & 36.66 & 173.06 \\
176.00000 & 36.61 & 187.94
\end{tabular}

$\begin{array}{crr}\begin{array}{c}\text { TD=44.0 } \\ \text { ENERGY (UEV) }\end{array} & \begin{array}{c}\text { ET=0.631345 MEV } \\ \text { PRIMARY }\end{array} & \text { CASCA DE } \\ 0.63700 & 0.11 & 0.11 \\ 0.64300 & 0.23 & 0.23 \\ 0.65600 & 0.49 & 0.49 \\ 0.67500 & 0.90 & 0.90 \\ 0.70000 & 1.45 & 1.45 \\ 0.73200 & 2.19 & 2.19 \\ 0.77000 & 3.09 & 3.09 \\ 0.82000 & 4.30 & 4.30 \\ 0.88300 & 5.81 & 5.81 \\ 0.97800 & 8.02 & 8.02 \\ 1.10000 & 10.64 & 10.66 \\ 1.26000 & 13.68 & 13.86 \\ 1.45000 & 16.71 & 17.33 \\ 1.70000 & 19.92 & 21.44 \\ 2.02000 & 23.02 & 26.09 \\ 2.39000 & 25.62 & 30.80 \\ 2.84000 & 27.85 & 35.79 \\ 3.28000 & 29.37 & 40.05 \\ 3.78000 & 30.60 & 44.33 \\ 4.41000 & 31.65 & 49.06 \\ 5.36000 & 32.64 & 55.13 \\ 6.31000 & 33.21 & 60.28 \\ 9.47000 & 33.94 & 73.26 \\ 12.60000 & 34.09 & 82.51 \\ 18.90000 & 34.03 & 95.73 \\ 31.50000 & 33.77 & 112.46 \\ 63.10000 & 33.48 & 135.34 \\ 126.00000 & 33.33 & 158.20 \\ 189.00000 & 33.28 & 171.63 \\ & & \end{array}$

\begin{tabular}{|c|c|c|}
\hline \multirow{2}{*}{$T D=4 B .0 \mathrm{EV}$} & $\mathrm{ET}=0.6$ & \\
\hline & PRIVARY & CASCADE \\
\hline 0.67800 & 0.09 & 0.09 \\
\hline 0.68500 & 0.20 & 0.20 \\
\hline 0.69900 & 0.43 & 0.43 \\
\hline 0.71900 & 0.78 & 0.78 \\
\hline 0.74600 & 1.28 & 1.28 \\
\hline 0.77900 & 1.92 & 1.92 \\
\hline 0.82000 & 2.75 & 2.75 \\
\hline 0.87300 & 3.85 & 3.85 \\
\hline 0.94100 & 5.26 & 5.26 \\
\hline 1.04000 & 7.25 & 7.25 \\
\hline 1.17000 & 9.68 & 9.70 \\
\hline 1.34000 & 12.50 & 12.67 \\
\hline 1.54000 & 15.29 & 15.86 \\
\hline 1.81000 & 18.30 & 19.74 \\
\hline 2.15300 & 21.17 & 24.06 \\
\hline 2.55000 & 23.59 & 28.49 \\
\hline 3.02000 & 25.59 & 33.03 \\
\hline 3.49000 & 26.99 & 37.00 \\
\hline 4.03000 & 28.12 & 41.01 \\
\hline 4.70000 & 29.07 & 45.37 \\
\hline 5.71000 & 29.96 & 50.96 \\
\hline 6.72000 & 30.47 & 55.70 \\
\hline 10.00000 & 31.12 & 67.40 \\
\hline 13.40000 & 31.25 & 76.11 \\
\hline 20.10000 & 31.18 & 88.25 \\
\hline 33.60000 & 30.95 & 103.70 \\
\hline 67.20000 & 30.68 & 124.63 \\
\hline 134.00000 & 30.55 & 145.56 \\
\hline 201.00000 & 30.50 & 157.87 \\
\hline
\end{tabular}


ATOMIC DISPLAEBARN CROSS-SECTIONS (BARNS) BP FAST ELECTRONS IN V

\begin{tabular}{rrr}
$\begin{array}{c}\text { TD=52.0 } \\
\text { ENERG }\end{array}$ & \multicolumn{1}{c}{ ET $=0.711596$ IEV } \\
0.71800 & PRIMART & CASCADE \\
0.72500 & 0.08 & 0.08 \\
0.74000 & 0.17 & 0.17 \\
0.75100 & 0.38 & 0.38 \\
0.78900 & 0.68 & 0.68 \\
0.82500 & 1.12 & 1.12 \\
0.86800 & 1.72 & 1.72 \\
0.92500 & 2.47 & 2.47 \\
0.99600 & 3.49 & 3.49 \\
1.10000 & 4.78 & 4.78 \\
1.24000 & 6.62 & 6.62 \\
1.42000 & 8.92 & 8.94 \\
1.63000 & 11.55 & 11.71 \\
1.92000 & 14.13 & 14.67 \\
2.27000 & 15.97 & 18.35 \\
2.70000 & 19.56 & 22.27 \\
3.20000 & 21.85 & 26.48 \\
3.70000 & 23.70 & 30.73 \\
4.26000 & 24.99 & 34.44 \\
4.98000 & 26.00 & 38.10 \\
6.04000 & 26.89 & 42.21 \\
7.11000 & 27.69 & 47.36 \\
10.60000 & 28.15 & 51.76 \\
14.20000 & 28.73 & 62.65 \\
21.30000 & 28.84 & 70.70 \\
35.50000 & 28.78 & 81.93 \\
71.10000 & 28.56 & 96.12 \\
142.00000 & 28.32 & 115.50 \\
& 28.20 & 134.86
\end{tabular}

\begin{tabular}{crr} 
TD $=56.0$ & EV & \multicolumn{2}{c}{ ET $=0.749807$ HEV } \\
ENERGY (MEV) & PRIMRY & CASCADE \\
0.75700 & 0.08 & 0.08 \\
0.76400 & 0.15 & 0.15 \\
0.77900 & 0.32 & 0.32 \\
0.80200 & 0.61 & 0.61 \\
0.83200 & 1.01 & 1.01 \\
0.86900 & 1.54 & 1.54 \\
0.91400 & 2.23 & 2.23 \\
0.97400 & 3.18 & 3.18 \\
1.04000 & 4.23 & 4.23 \\
1.16000 & 6.12 & 6.12 \\
1.31000 & 8.31 & 8.33 \\
1.49000 & 10.65 & 10.80 \\
1.72000 & 13.17 & 13.69 \\
2.02000 & 15.78 & 17.08 \\
2.39000 & 18.21 & 20.78 \\
2.84000 & 20.33 & 24.71 \\
3.37000 & 22.06 & 28.71 \\
3.89000 & 23.24 & 32.14 \\
4.49000 & 24.19 & 35.62 \\
5.24000 & 25.00 & 39.42 \\
6.37000 & 25.74 & 44.28 \\
7.49000 & 26.16 & 48.36 \\
11.20000 & 26.68 & 58.58 \\
14.90000 & 26.78 & 65.90 \\
22.40000 & 26.72 & 76.39 \\
37.40000 & 26.52 & 89.63 \\
74.90000 & 26.29 & 107.63 \\
149.00000 & 26.18 & 125.51
\end{tabular}

\begin{tabular}{rrr} 
TD $=60.0$ & EV & \multicolumn{2}{c}{ ET $=0.786894$} \\
ENERGY MEV \\
0.79400 & PRIMARY & CASCADE \\
0.80200 & 0.06 & 0.06 \\
0.81300 & 0.14 & 0.14 \\
0.84100 & 0.29 & 0.29 \\
0.87300 & 0.54 & 0.54 \\
0.91200 & 0.91 & 0.91 \\
0.96900 & 1.40 & 1.40 \\
1.02000 & 2.05 & 2.05 \\
1.10000 & 2.89 & 2.89 \\
1.21000 & 4.03 & 4.03 \\
1.37000 & 5.58 & 5.58 \\
1.57000 & 7.69 & 7.70 \\
1.80000 & 10.01 & 10.17 \\
2.12000 & 12.27 & 12.76 \\
2.51000 & 14.77 & 16.02 \\
2.99000 & 17.05 & 19.51 \\
3.54000 & 19.06 & 23.26 \\
4.09000 & 20.65 & 26.98 \\
4.72000 & 21.75 & 30.23 \\
5.50000 & 22.63 & 33.49 \\
6.68000 & 23.36 & 37.02 \\
7.86500 & 24.04 & 41.56 \\
11.80000 & 24.43 & 45.39 \\
15.70000 & 24.91 & 55.05 \\
23.60000 & 25.00 & 61.90 \\
39.30000 & 24.93 & 71.71 \\
78.60000 & 24.74 & 84.00 \\
157.00000 & 24.54 & 100.78 \\
& 24.43 & 117.57
\end{tabular}

ATOHIC DISPLAZEMENT CROSS-SECTIONS (BARNS) BY PAST ELECTRONS IN $2=23 \quad A=50.950$
$2=50 N S$ (BARNS)

\begin{tabular}{rrr} 
TD $=64.0$ & BV & \multicolumn{2}{c}{ ET $=0.822950$ MEV } \\
ENERGY (HEV) & PRIMARY & CASCADE \\
0.83100 & 0.06 & 0.06 \\
0.83900 & 0.12 & 0.12 \\
0.85500 & 0.26 & 0.26 \\
0.88000 & 0.49 & 0.49 \\
0.91300 & 0.83 & 0.83 \\
0.95400 & 1.29 & 1.29 \\
1.00000 & 1.84 & 1.84 \\
1.06000 & 2.59 & 2.59 \\
1.15000 & 3.74 & 3.74 \\
1.27000 & 5.26 & 5.26 \\
1.44000 & 7.28 & 7.30 \\
1.64000 & 9.38 & 9.53 \\
1.89000 & 11.59 & 12.08 \\
2.22000 & 13.90 & 15.11 \\
2.63000 & 16.06 & 18.43 \\
3.12000 & 17.89 & 21.89 \\
3.70000 & 19.39 & 25.43 \\
4.27000 & 20.42 & 28.46 \\
4.93000 & 21.24 & 31.55 \\
5.76000 & 21.94 & 34.93 \\
6.99000 & 22.56 & 39.19 \\
8.22000 & 22.92 & 42.78 \\
12.30000 & 23.36 & 51.78 \\
16.40000 & 23.43 & 58.26 \\
24.60000 & 23.37 & 67.42 \\
41.10000 & 23.19 & 79.03 \\
82.20000 & 23.00 & 94.76 \\
164.00000 & 22.91 & 110.48
\end{tabular}

\begin{tabular}{rrr} 
TD $=68.0$ & EV & \multicolumn{1}{c}{ ET $=0.858057$} \\
ENERGY (HEV) & PRIMARY & CASCADE \\
0.86600 & 0.05 & 0.05 \\
0.87500 & 0.11 & 0.11 \\
0.89200 & 0.24 & 0.24 \\
0.91800 & 0.45 & 0.45 \\
0.95200 & 0.76 & 0.76 \\
0.99500 & 1.19 & 1.19 \\
1.04000 & 1.67 & 1.67 \\
1.11000 & 2.46 & 2.46 \\
1.20000 & 3.51 & 3.51 \\
1.32000 & 4.89 & 4.89 \\
1.50000 & 6.84 & 6.85 \\
1.71000 & 8.84 & 8.98 \\
1.97000 & 10.93 & 11.40 \\
2.31000 & 13.10 & 14.24 \\
2.74000 & 15.14 & 17.41 \\
3.26000 & 16.90 & 20.75 \\
3.86000 & 18.30 & 24.07 \\
4.46000 & 19.26 & 26.97 \\
5.14000 & 20.02 & 29.85 \\
6.00000 & 20.67 & 33.03 \\
7.29000 & 21.25 & 37.07 \\
8.58000 & 21.58 & 40.48 \\
12.80000 & 21.98 & 48.91 \\
17.10000 & 22.05 & 55.06 \\
25.70000 & 21.99 & 63.72 \\
42.90000 & 21.82 & 74.64 \\
85.80000 & 21.65 & 89.45 \\
171.00000 & 21.55 & 104.23
\end{tabular}

\begin{tabular}{rrr} 
TD $=72.0$ & EV & \multicolumn{1}{c}{ ET $=0.892285$ MEV } \\
ENERGY (HEV) & PRIMARY & CASCADE \\
0.90100 & 0.05 & 0.05 \\
0.91000 & 0.10 & 0.10 \\
0.92700 & 0.21 & 0.21 \\
0.95400 & 0.41 & 0.41 \\
0.99000 & 0.70 & 0.70 \\
1.03000 & 1.06 & 1.06 \\
1.08000 & 1.54 & 1.54 \\
1.15000 & 2.26 & 2.26 \\
1.24000 & 3.21 & 3.21 \\
1.38000 & 4.68 & 4.68 \\
1.56000 & 6.46 & 6.48 \\
1.78000 & 8.38 & 8.52 \\
2.05000 & 10.36 & 10.81 \\
2.40000 & 12.39 & 13.49 \\
2.85000 & 14.34 & 16.52 \\
3.39000 & 16.00 & 19.69 \\
4.01000 & 17.31 & 22.83 \\
4.63000 & 18.21 & 25.56 \\
5.35000 & 18.94 & 28.35 \\
6.24000 & 19.54 & 31.35 \\
7.58000 & 20.09 & 35.17 \\
8.92000 & 20.40 & $\mathbf{3 8 . 3 9}$ \\
13.30000 & 20.77 & 46.36 \\
17.80000 & 20.83 & 52.21 \\
26.70000 & 20.77 & 60.36 \\
44.60000 & 20.61 & 70.69 \\
89.20000 & 20.44 & 84.69 \\
178.00000 & 20.36 & 98.67
\end{tabular}


ATOMIC DISPLAEEHENT CROSS-SECTIONS (BARNS) BI PAST ELECTRONS IN V

\begin{tabular}{rrr} 
TD $=76.0$ & EV & \multicolumn{1}{c}{ ET $=0.925699$ MEV } \\
ENERGY (HEV) & PRIMART & CASCADE \\
0.93400 & 0.04 & 0.04 \\
0.94400 & 0.09 & 0.09 \\
0.96200 & 0.20 & 0.20 \\
0.99000 & 0.38 & 0.38 \\
1.02000 & 0.59 & 0.59 \\
1.07000 & 1.00 & 1.00 \\
1.12000 & 1.44 & 1.44 \\
1.20000 & 2.19 & 2.19 \\
1.29000 & 3.06 & 3.06 \\
1.43000 & 4.41 & 4.41 \\
1.61000 & 6.05 & 6.06 \\
1.85000 & 7.98 & 8.11 \\
2.12000 & 9.79 & 10.23 \\
2.49000 & 11.77 & 12.83 \\
2.96000 & 13.63 & 15.74 \\
3.51000 & 15.18 & 18.70 \\
4.16000 & 16.43 & 21.73 \\
4.81000 & 17.29 & 24.36 \\
5.55000 & 17.97 & 26.98 \\
6.47000 & 18.53 & 29.82 \\
7.86000 & 19.04 & 33.45 \\
9.25000 & 19.33 & 36.51 \\
13.80000 & 19.67 & 44.09 \\
18.50000 & 19.73 & 49.67 \\
27.70000 & 19.67 & 57.36 \\
46.20000 & 19.52 & 67.12 \\
92.50000 & 19.36 & 80.40 \\
185.00000 & 19.29 & 93.70
\end{tabular}

\begin{tabular}{rrr} 
TD $=80.0$ & EV & \multicolumn{1}{c}{ ET $=0.958353$ MEV } \\
ENERGY (HEV) & PRIMARY & CASCADE \\
0.96700 & 0.04 & 0.04 \\
0.97700 & 0.08 & 0.08 \\
0.99600 & 0.18 & 0.18 \\
1.02000 & 0.32 & 0.32 \\
1.06000 & 0.58 & 0.58 \\
1.11000 & 0.95 & 0.95 \\
1.16000 & 1.35 & 1.35 \\
1.24000 & 2.04 & 2.04 \\
1.34000 & 2.94 & 2.94 \\
1.48000 & 4.18 & 4.18 \\
1.67000 & 5.78 & 5.79 \\
1.91000 & 7.55 & 7.68 \\
2.20000 & 9.35 & 9.78 \\
2.58000 & 11.22 & 12.25 \\
3.06000 & 12.97 & 14.99 \\
3.64000 & 14.46 & 17.87 \\
4.31000 & 15.64 & 20.74 \\
4.98000 & 16.45 & 23.24 \\
5.75000 & 17.09 & 25.75 \\
6.70000 & 17.62 & 28.45 \\
8.14000 & 18.10 & 31.91 \\
9.58000 & 18.37 & 34.83 \\
14.30000 & 18.69 & 42.04 \\
19.10000 & 18.74 & 47.28 \\
28.70000 & 18.68 & 54.66 \\
47.90000 & 18.54 & 63.95 \\
95.80000 & 18.39 & 76.55 \\
191.00000 & 18.32 & 89.12 \\
& &
\end{tabular}

TD=84.0 E
ENERGY(BEV)
1.00000
1.01000
1.02000
1.05000
1.09000
1.14000
1.20000
1.28000
1.38000
1.53000
1.73000
1.98000
2.27000
2.67000
3.16000
3.76000
4.45000
5.14000
5.94000
6.93000
8.41000
9.90000
14.80000
19.80000
29.70000
49.50000
99.00000
198.00000

ET $=0.990297$ MEV

PRIMARY CASCADE

$0.04 \quad 0.04$

$0.08 \quad 0.08$

$\begin{array}{ll}0.12 & 0.12 \\ 0.27 & 0.27\end{array}$

$\begin{array}{ll}0.27 & 0.27 \\ 0.51 & 0.51\end{array}$

$0.84 \quad 0.84$

$\begin{array}{ll}1.29 & 1.29 \\ 1.92 & 1.92\end{array}$

$2.75 \quad 2.75$

$\begin{array}{ll}3.99 & 3.99\end{array}$

$7.25 \quad 7.38$

$8.91 \quad 9.32$

$10.73 \quad 11.73$

$12.37 \quad 14.33$

17.09

$14.92 \quad 19.82$

$\begin{array}{ll}15.68 & 22.20 \\ 16.29 & 24.62\end{array}$

$\begin{array}{ll}16.29 & 24.62 \\ 16.80 & 27.22\end{array}$

$\begin{array}{ll}16.80 & 27.22 \\ 17.25 & 30.50\end{array}$

$17.50 \quad 33.29$

$17.81 \quad 40.20$

$17.85 \quad 45.22$

$17.79 \quad 52.22$

$17.66 \quad 61.05$

$17.52 \quad 73.05$

ATOMIC DISPLACEMENT CROSS-SECTIONS (BARNS) BY PAST ELECTRONS IN V $z=23 \quad A=50.950$

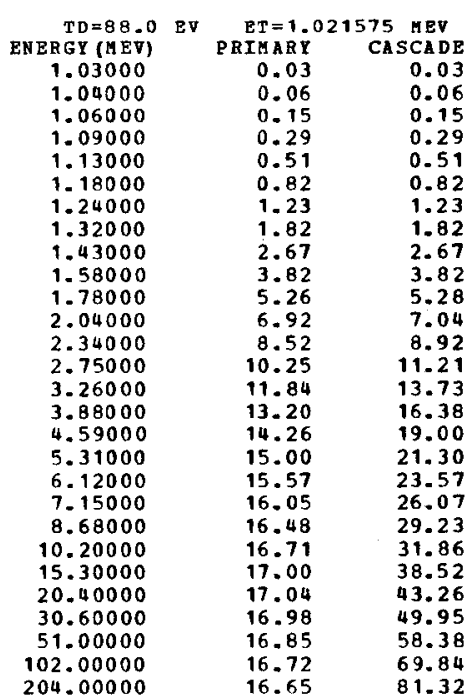

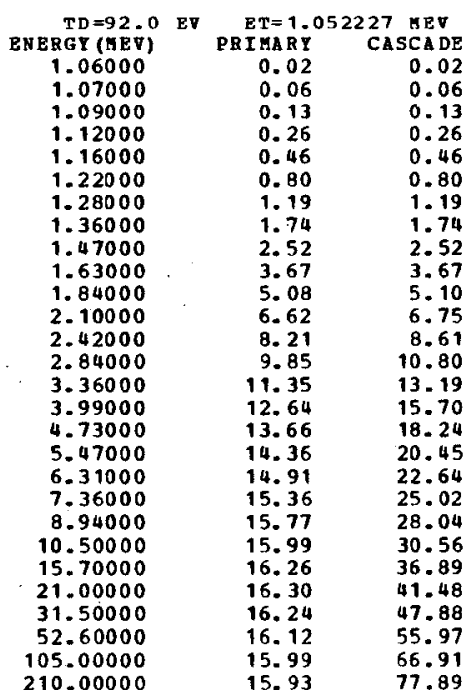

\begin{tabular}{|c|c|c|}
\hline $\begin{array}{r}T D=96.0 \\
\text { ENERGY (HEV) }\end{array}$ & $\begin{array}{r}\text { ET }=1 . \\
\text { PRIAARY }\end{array}$ & $\begin{array}{l}290 \mathrm{MEV} \\
\text { CASCADE }\end{array}$ \\
\hline 1.09000 & 0.02 & 0.02 \\
\hline 1.10000 & 0.05 & 0.05 \\
\hline 1.12000 & 0.12 & 0.12 \\
\hline 1.15000 & 0.23 & 0.23 \\
\hline 1.20000 & 0.46 & 0.46 \\
\hline 1.25000 & 0.73 & 0.73 \\
\hline 1.32000 & $\begin{array}{l}1.15 \\
1.66\end{array}$ & $\begin{array}{l}1.15 \\
1.66\end{array}$ \\
\hline $\begin{array}{l}1.40000 \\
1.51000\end{array}$ & $\begin{array}{l}1.66 \\
2.40\end{array}$ & 2.40 \\
\hline 1.67000 & 3.47 & 3.47 \\
\hline 1.89000 & 4.86 & 4.87 \\
\hline 2.16300 & 6.36 & 6.48 \\
\hline 2.48000 & 7.85 & 8.22 \\
\hline $\begin{array}{l}2.92000 \\
3.46000\end{array}$ & $\begin{array}{r}9.46 \\
10.91\end{array}$ & $\begin{array}{l}10.38 \\
12.70\end{array}$ \\
\hline 4.11000 & 12.14 & 15.12 \\
\hline 4.87000 & 13. 12 & 17.56 \\
\hline 5.62000 & 13.78 & 19.65 \\
\hline 6.49000 & 14.30 & 21.77 \\
\hline 7.57000 & 14.73 & 24.05 \\
\hline 9.19000 & 15.12 & 26.95 \\
\hline 10.80000 & 15.33 & 29.37 \\
\hline 16.20000 & 15.58 & 35.49 \\
\hline 21.60000 & 15.62 & 39.84 \\
\hline $\begin{array}{l}32.40000 \\
54.10000\end{array}$ & $\begin{array}{l}15.56 \\
15.44\end{array}$ & $\begin{array}{l}43.98 \\
53.74\end{array}$ \\
\hline 108.00000 & 15.32 & 64.22 \\
\hline .00000 & 15.27 & \\
\hline
\end{tabular}


ATOMIC DISPLAZEMENT CROSS-SECTIONS (BARNS) BY FAST ELECTRONS IN CR

\begin{tabular}{ccc} 
TD $=4.0$ & EV & \multicolumn{1}{c}{ ET $=0.087322$ HEV } \\
ENERGY(HEY) & PRIHARY & CASCADE \\
0.08810 & 10.62 & 10.62 \\
0.08900 & 22.48 & 22.48 \\
0.09080 & 44.95 & 44.95 \\
0.09340 & 74.70 & 74.70 \\
0.09690 & 110.29 & 110.29 \\
0.10100 & 146.42 & 146.42 \\
0.10600 & 183.78 & 183.78 \\
0.11300 & 226.25 & 226.25 \\
0.12200 & 268.21 & 268.21 \\
0.13500 & 311.39 & 311.39 \\
0.15200 & 348.14 & 348.14 \\
0.17400 & 376.64 & 377.31 \\
0.20000 & 395.50 & 401.57 \\
0.23500 & 408.43 & 425.53 \\
0.27900 & 415.61 & 448.11 \\
0.33100 & 418.81 & 469.32 \\
0.39200 & 419.87 & 490.42 \\
0.45400 & 419.90 & 509.62 \\
0.52300 & 419.57 & 529.41 \\
0.51100 & 419.01 & 553.05 \\
0.74200 & 418.22 & 585.87 \\
0.87300 & 417.53 & 616.43 \\
1.30000 & 415.51 & 703.74 \\
1.74000 & 413.54 & 778.61 \\
2.61000 & 410.01 & 896.21 \\
4.36000 & 405.02 & 1062.80 \\
8.73000 & 398.25 & 1308.11 \\
17.40000 & 393.66 & 1564.58 \\
26.10000 & 392.14 & 1718.61 \\
43.60000 & 391.00 & 1915.46 \\
61.10000 & 390.51 & 2045.66 \\
87.30000 & 390.09 & 2183.63 \\
174.00000 & 389.39 & 2450.75 \\
& &
\end{tabular}

$\begin{array}{rrr}\text { TD= } 8.0 & \text { EV } & \text { ET=0.163431 HEV } \\ \text { ENERGY (NEV) } & \text { PEIMARY } & \text { CASCADE } \\ 0.16500 & 3.01 & 3.01 \\ 0.16600 & 4.88 & 4.88 \\ 0.16900 & 10.29 & 10.29 \\ 0.17400 & 18.68 & 18.68 \\ 0.18100 & 29.25 & 29.25 \\ 0.18900 & 39.90 & 39.90 \\ 0.19900 & 51.47 & 51.47 \\ 0.21200 & 64.17 & 64.17 \\ 0.22800 & 77.04 & 77.04 \\ 0.25300 & 92.80 & 92.80 \\ 0.28600 & 108.22 & 108.22 \\ 0.32600 & 121.87 & 122.38 \\ 0.37500 & 134.10 & 136.78 \\ 0.44100 & 146.02 & 153.00 \\ 0.52200 & 156.51 & 169.86 \\ 0.62100 & 165.76 & 187.64 \\ 0.73500 & 173.47 & 205.62 \\ 0.84900 & 179.20 & 221.75 \\ 0.98000 & 184.15 & 238.58 \\ 1.14000 & 188.61 & 257.23 \\ 1.38000 & 193.20 & 282.18 \\ 1.63000 & 196.29 & 305.17 \\ 2.45000 & 200.88 & 366.08 \\ 3.26000 & 202.07 & 412.17 \\ 4.90000 & 202.05 & 481.77 \\ 8.17000 & 200.51 & 573.53 \\ 16.30000 & 197.90 & 702.10 \\ 32.60000 & 196.23 & 834.20 \\ 49.00000 & 195.70 & 912.61 \\ 81.70000 & 195.29 & 1011.47 \\ 114.00000 & 195.09 & 1076.01 \\ 163.00000 & 194.90 & 1145.32 \\ 326.00000 & 194.55 & 1279.78 \\ & & \\ & & \end{array}$

$\begin{array}{crr}\text { TD=12.0 EV } & \text { ET }=0.231782 \text { HEV } \\ \text { ENERGY (IIEV) } & \text { PRIMARY } & \text { CASCADE } \\ 0.23400 & 1.39 & 1.39 \\ 0.23600 & 2.61 & 2.61 \\ 0.24100 & 5.56 & 5.56 \\ 0.24800 & 9.44 & 9.44 \\ 0.25700 & 14.04 & 14.04 \\ 0.26300 & 19.16 & 19.16 \\ 0.28200 & 25.01 & 25.01 \\ 0.30100 & 31.97 & 31.97 \\ 0.32400 & 39.21 & 39.21 \\ 0.35900 & 48.41 & 48.41 \\ 0.40500 & 58.14 & 58.14 \\ 0.46300 & 67.86 & 68.28 \\ 0.53300 & 77.10 & 78.89 \\ 0.62500 & 86.49 & 91.01 \\ 0.74100 & 95.45 & 104.30 \\ 0.88000 & 103.41 & 118.24 \\ 1.04000 & 110.13 & 132.38 \\ 1.20000 & 115.12 & 145.03 \\ 1.39000 & 119.52 & 158.54 \\ 1.62000 & 123.36 & 173.19 \\ 1.97000 & 127.25 & 192.73 \\ 2.31000 & 129.64 & 209.27 \\ 3.47000 & 133.23 & 253.88 \\ 4.63000 & 134.15 & 287.15 \\ 6.95000 & 134.20 & 335.75 \\ 11.50000 & 133.22 & 397.81 \\ 23.10000 & 131.61 & 485.74 \\ 46.30000 & 130.67 & 574.75 \\ 69.50000 & 130.37 & 627.07 \\ 115.00000 & 130.13 & 692.15 \\ 162.00000 & 130.01 & 736.46 \\ 231.00000 & 129.89 & 782.35 \\ 463.00000 & 129.65 & 872.30 \\ & & \\ & & \end{array}$

ATOMIC DISPLAERENT CROSS-SECTIONS (BARNS) BY FAST ELECTROHS IN CR $z=24 \quad A=52.010$

\begin{tabular}{rrr} 
TD $=16.0$ & EV & \multicolumn{1}{c}{ ET $=\mathbf{0 . 2 9 4 3 5 3}$ MEV } \\
ENERGY (MEV) & PRIMARY & CASCADE \\
0.29700 & 0.76 & 0.76 \\
0.30000 & 1.61 & 1.61 \\
0.30600 & 3.25 & $\mathbf{3 . 2 5}$ \\
0.31400 & 5.34 & 5.34 \\
0.32600 & 8.30 & 8.30 \\
0.34100 & 11.73 & 11.73 \\
0.35900 & 15.50 & 15.50 \\
0.38200 & 19.88 & 19.88 \\
0.41200 & 24.98 & 24.98 \\
0.45600 & 31.49 & 31.49 \\
0.51500 & 38.85 & 38.86 \\
0.58800 & 46.38 & 46.74 \\
0.67700 & 53.86 & 55.24 \\
0.79400 & 61.64 & 65.10 \\
0.94100 & 69.11 & 76.00 \\
1.11000 & 75.55 & 87.03 \\
1.32000 & 81.39 & 99.10 \\
1.53000 & 85.63 & 109.80 \\
1.76000 & 89.05 & 120.30 \\
2.06000 & 92.24 & 132.50 \\
2.50000 & 95.28 & 148.02 \\
2.94000 & 97.16 & 161.44 \\
4.41000 & 99.82 & 196.38 \\
5.88000 & 100.48 & 222.13 \\
8.83000 & 100.46 & 259.49 \\
14.70000 & 99.70 & 307.27 \\
29.40000 & 98.57 & 373.31 \\
58.80000 & 97.94 & 440.10 \\
88.30000 & 97.74 & 479.47 \\
147.00000 & 97.57 & 528.91
\end{tabular}

$\begin{array}{crr}\text { TD }=20.0 & \text { EV } & \text { ET }=0.352401 \text { HEV } \\ \text { ENBRGY (MEV) } & \text { PRIMARY } & \text { CASCADE } \\ 0.35500 & 0.41 & 0.41 \\ 0.35900 & 1.04 & 1.04 \\ 0.36600 & 2.11 & 2.11 \\ 0.37700 & 3.73 & 3.73 \\ 0.39100 & 5.71 & 5.71 \\ 0.40800 & 8.00 & 9.00 \\ 0.42900 & 10.66 & 10.66 \\ 0.45800 & 14.08 & 14.08 \\ 0.49300 & 17.86 & 17.86 \\ 0.54600 & 23.01 & 23.01 \\ 0.61600 & 28.92 & 28.93 \\ 0.70400 & 35.21 & 35.51 \\ 0.81000 & 41.47 & 42.62 \\ 0.95100 & 48.13 & 51.03 \\ 1.12000 & 54.28 & 59.94 \\ 1.33000 & 59.98 & 69.70 \\ 1.58000 & 64.88 & 79.89 \\ 1.83000 & 68.41 & 88.89 \\ 2.11000 & 71.29 & 97.89 \\ 2.46000 & 73.83 & 107.86 \\ 2.99000 & 76.30 & 120.91 \\ 3.52000 & 77.81 & 132.11 \\ 5.28000 & 79.86 & 160.85 \\ 7.04000 & 80.33 & 181.86 \\ 10.50000 & 80.28 & 211.64 \\ 17.60000 & 79.65 & 250.69 \\ 35.20000 & 78.79 & 303.76 \\ 70.40000 & 78.32 & 357.31 \\ 105.00000 & 78.17 & 388.31 \\ 176.00000 & 78.04 & 428.42\end{array}$

\begin{tabular}{rrr} 
TD $=24.0$ & EV & \multicolumn{2}{c}{ ET $=0.406785$} & MEV \\
ENERGY (HEV) & PRI ARY & CASCADE \\
0.41000 & 0.31 & 0.31 \\
0.41400 & 0.70 & 0.70 \\
0.42300 & 1.56 & 1.56 \\
0.43500 & 2.69 & 2.69 \\
0.45100 & 4.15 & 4.15 \\
0.47100 & 5.92 & 5.92 \\
0.49600 & 8.05 & 8.05 \\
0.52800 & 10.64 & 10.64 \\
0.56900 & 13.76 & 13.76 \\
0.63000 & 18.01 & 18.01 \\
0.71100 & 23.02 & 23.04 \\
0.81300 & 28.44 & 28.72 \\
0.93500 & 33.85 & 34.85 \\
1.09000 & 39.39 & 41.83 \\
1.30000 & 45.15 & 50.19 \\
1.54000 & 49.99 & 58.57 \\
1.83000 & 54.18 & 67.45 \\
2.11000 & 57.09 & 75.01 \\
2.44000 & 59.56 & 82.94 \\
2.84000 & 61.66 & 91.43 \\
3.45000 & 63.70 & 102.58 \\
4.06000 & 64.93 & 112.12 \\
6.10000 & 66.56 & 136.61 \\
8.13000 & 66.92 & 154.33 \\
12.20000 & 66.84 & 179.75 \\
20.30000 & 66.31 & 212.00 \\
40.60000 & 65.61 & 256.36 \\
81.30000 & 65.24 & 301.12 \\
122.00000 & 65.13 & 327.37 \\
203.00000 & 65.02 & 360.34
\end{tabular}


ATORIC DISPLACEMENT CROSS-SECTIONS (BARN) BY FAST ELECTRONS IN CR

\begin{tabular}{crr}
$\begin{array}{c}\text { TD }=28.0 \\
\text { ENERG (MEV) }\end{array}$ & \multicolumn{2}{c}{ BT $=0.458122$ MEV } \\
PRIBAR & CASCADE \\
0.46200 & 0.25 & 0.25 \\
0.45700 & 0.58 & 0.58 \\
0.47600 & 1.16 & 1.16 \\
0.49000 & 2.06 & 2.06 \\
0.50800 & 3.21 & 3.21 \\
0.53100 & 4.65 & 4.65 \\
0.55800 & 6.31 & 6.31 \\
0.59500 & 8.52 & 8.52 \\
0.64100 & 11.14 & 11.14 \\
0.71000 & 14.81 & 14.81 \\
0.80100 & 19.16 & 19.17 \\
0.91600 & 23.92 & 24.18 \\
1.05000 & 28.59 & 29.46 \\
1.23000 & 33.64 & 35.84 \\
1.46000 & 38.58 & 43.06 \\
1.74000 & 42.97 & 50.75 \\
2.06000 & 46.54 & 58.43 \\
2.38000 & 49.09 & 65.19 \\
2.74000 & 51.16 & 71.95 \\
3.20000 & 52.99 & 79.56 \\
3.89000 & 54.71 & 89.35 \\
4.58000 & 55.74 & 97.70 \\
6.87000 & 57.07 & 118.89 \\
9.16000 & 57.35 & 134.23 \\
13.70000 & 57.26 & 155.97 \\
22.90000 & 56.79 & 183.98 \\
45.80000 & 56.21 & 222.08 \\
91.60000 & 55.91 & 260.42 \\
137.00000 & 55.82 & 282.75
\end{tabular}

\begin{tabular}{crr} 
TD=32.0 & EV & \multicolumn{2}{c}{ ET=0.506873 MEV } \\
ENERGY (HEV) & PRIARY & CASCADE \\
0.51100 & 0.19 & 0.19 \\
0.51700 & 0.47 & 0.47 \\
0.52700 & 0.93 & 0.93 \\
0.54200 & 1.63 & 1.63 \\
0.56200 & 2.57 & 2.57 \\
0.58700 & 3.75 & 3.75 \\
0.61800 & 5.20 & 5.20 \\
0.65800 & 7.05 & 7.05 \\
0.70900 & 9.33 & 9.33 \\
0.78500 & 12.53 & 12.53 \\
0.88700 & 16.44 & 16.46 \\
1.01000 & 20.57 & 20.79 \\
1.16000 & 24.81 & 25.60 \\
1.36000 & 29.36 & 31.37 \\
1.62000 & 33.86 & 37.99 \\
1.92000 & 37.64 & 44.67 \\
2.28000 & 40.86 & 51.68 \\
2.63000 & 43.07 & 57.66 \\
3.04000 & 44.92 & 63.87 \\
3.54000 & 46.48 & 70.52 \\
4.30000 & 47.96 & 79.18 \\
5.06000 & 48.83 & 86.56 \\
7.60000 & 49.95 & 105.34 \\
10.10000 & 50.17 & 118.70 \\
15.20000 & 50.07 & 138.11 \\
25.30000 & 49.66 & 162.48 \\
50.60000 & 49.17 & 195.87 \\
101.00000 & 48.91 & 229.35 \\
152.00000 & 48.83 & 249.19
\end{tabular}

\begin{tabular}{rrr}
$\begin{array}{r}\text { TD }=36.0 \\
\text { ENERGY (HEV) }\end{array}$ & \multicolumn{1}{c}{ ET $=0.553394$ MEV } \\
PRIMARY & CASCADE \\
0.55800 & 0.16 & 0.16 \\
0.56400 & 0.36 & 0.36 \\
0.57500 & 0.74 & 0.74 \\
0.59200 & 1.34 & 1.34 \\
0.61400 & 2.14 & 2.14 \\
0.64100 & 3.13 & 3.13 \\
0.67500 & 4.39 & 4.39 \\
0.71900 & 6.02 & 6.02 \\
0.77400 & 8.01 & 8.01 \\
0.85700 & 10.88 & 10.88 \\
0.96800 & 14.40 & 14.42 \\
1.10000 & 18.07 & 18.28 \\
1.27000 & 22.06 & 22.81 \\
1.49000 & 26.19 & 28.08 \\
1.77000 & 30.18 & 34.00 \\
2.10300 & 33.59 & 40.11 \\
2.49000 & 36.44 & 46.40 \\
2.87000 & 38.40 & 51.79 \\
3.32000 & 40.04 & 57.42 \\
3.87000 & 41.42 & 63.46 \\
4.70000 & 42.71 & 71.25 \\
5.53000 & 43.46 & 77.86 \\
8.30000 & 44.41 & 94.66 \\
11.00000 & 44.59 & 106.48 \\
16.60000 & 44.49 & 123.91 \\
27.60000 & 44.12 & 145.57 \\
55.30000 & 43.69 & 175.37 \\
110.00000 & 43.47 & 204.99 \\
166.00000 & 43.40 & 222.76 \\
& &
\end{tabular}

ATOMIC DISPLACEMENT CROSS-SECTIONS (BARAS) BT PAST BLECTRONS IN CR $z=24 \quad A=52.010$

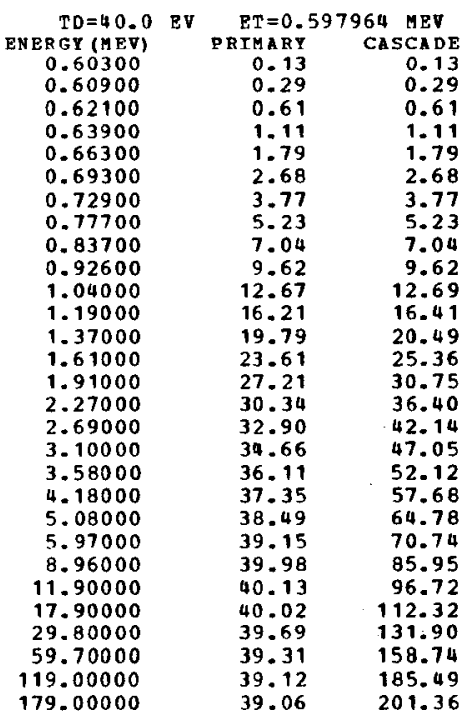

\begin{tabular}{rrr} 
TD $=44.0$ & EV & \multicolumn{2}{c}{ ET=0.640812 ABV } \\
ENERGY (HEV) & PRIMRY & CASCADE \\
0.64700 & 0.12 & 0.12 \\
0.65300 & 0.25 & 0.25 \\
0.66600 & 0.53 & 0.53 \\
0.68500 & 0.95 & 0.95 \\
0.71100 & 1.56 & 1.56 \\
0.74300 & 2.34 & 2.34 \\
0.78100 & 3.30 & 3.30 \\
0.83300 & 4.63 & 4.63 \\
0.89700 & 6.27 & 6.27 \\
0.99300 & 8.65 & 8.65 \\
1.12000 & 11.56 & 11.58 \\
1.28000 & 14.79 & 14.99 \\
1.47000 & 18.02 & 18.69 \\
1.73000 & 21.56 & 23.23 \\
2.05000 & 24.84 & 28.18 \\
2.43000 & 27.66 & 33.31 \\
2.88000 & 29.99 & 38.60 \\
3.33000 & 31.62 & 43.22 \\
3.84000 & 32.92 & 47.84 \\
4.48000 & 34.02 & 52.91 \\
5.44000 & 35.04 & 59.39 \\
6.40000 & 35.63 & 64.88 \\
9.61000 & 36.35 & 78.79 \\
12.80000 & 36.48 & 88.72 \\
19.20000 & 36.37 & 102.84 \\
32.00000 & 36.07 & 120.70 \\
64.00000 & 35.73 & 145.06 \\
128.00000 & 35.56 & 169.51 \\
192.00000 & 35.51 & 183.84 \\
& &
\end{tabular}

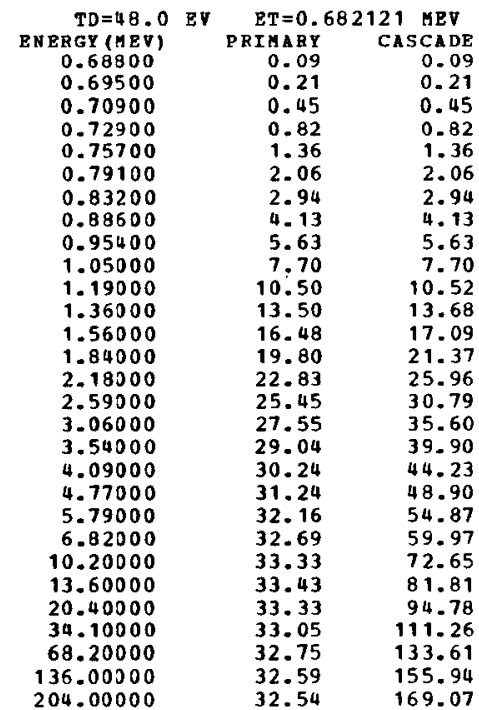


ATOMIC DISPLACEAENT CROSS-SECTIONS (BARNS) BY PAST ELECTRONS IN CR

\begin{tabular}{rrr} 
TD $=52.0$ & EV & \multicolumn{2}{c}{ ET $=0.722047$ MEV } \\
ENERGY (HEV & PRIMARY & CASCADE \\
0.72900 & 0.09 & 0.09 \\
0.73600 & 0.19 & 0.19 \\
0.75000 & 0.39 & 0.39 \\
0.77200 & 0.72 & 0.72 \\
0.80100 & 1.20 & 1.20 \\
0.83700 & 1.84 & 1.84 \\
0.88000 & 2.63 & 2.63 \\
0.93800 & 3.74 & 3.74 \\
1.01000 & 5.13 & 5.13 \\
1.11000 & 7.02 & 7.02 \\
1.26000 & 9.67 & 9.69 \\
1.44000 & 12.46 & 12.64 \\
1.66000 & 15.34 & 15.94 \\
1.94000 & 18.26 & 19.72 \\
2.31000 & 21.15 & 24.14 \\
2.74000 & 23.56 & 28.60 \\
3.24000 & 25.50 & 33.11 \\
3.75000 & 25.88 & 37.12 \\
4.33000 & 27.97 & 41.13 \\
5.05000 & 28.89 & 45.48 \\
6.13000 & 29.72 & 51.02 \\
7.22000 & 30.20 & 55.74 \\
10.80000 & 30.77 & 67.50 \\
14.40000 & 30.86 & 75.97 \\
21.60000 & 30.76 & 87.96 \\
36.10000 & 30.50 & 103.19 \\
72.20000 & 30.22 & 123.83 \\
144.00000 & 30.09 & 144.45 \\
& &
\end{tabular}

\begin{tabular}{rrr} 
TD $=56.0$ & EV & \multicolumn{2}{c}{ ET $=0.760721$ MEV } \\
ENERGY (MEV) & PRIMARY & CASCADE \\
0.76800 & 0.08 & 0.08 \\
0.77500 & 0.16 & 0.16 \\
0.79100 & 0.35 & 0.35 \\
0.81300 & 0.64 & 0.64 \\
0.84400 & 1.08 & 1.08 \\
0.88200 & 1.66 & 1.66 \\
0.92800 & 2.40 & 2.40 \\
0.98800 & 3.41 & 3.41 \\
1.06000 & 4.64 & 4.64 \\
1.17000 & 6.49 & 6.49 \\
1.33000 & 8.99 & 9.01 \\
1.52000 & 11.61 & 11.79 \\
1.74000 & 14.17 & 14.74 \\
2.05000 & 17.05 & 18.47 \\
2.43000 & 19.68 & 22.50 \\
2.89000 & 21.95 & 26.75 \\
3.42000 & 23.76 & 30.99 \\
3.95000 & 25.02 & $\mathbf{3 4} .70$ \\
4.56000 & 26.02 & $\mathbf{3 8} .44$ \\
5.32000 & 26.86 & 42.50 \\
6.46000 & 27.62 & 47.69 \\
7.60000 & 28.06 & 52.06 \\
11.40000 & 28.58 & 63.08 \\
15.20000 & 28.65 & 70.97 \\
22.80000 & 28.55 & 82.12 \\
38.00000 & 28.31 & 96.20 \\
76.00000 & 28.06 & 115.37 \\
152.00000 & 27.93 & 134.60
\end{tabular}

\begin{tabular}{rrr} 
TD $=60.0$ & EV & \multicolumn{2}{c}{ ET $=0.798252$} \\
ENERGY (HEV) & PRIBARY & CASCADE \\
0.80500 & 0.07 & 0.07 \\
0.81400 & 0.15 & 0.15 \\
0.83000 & 0.31 & 0.31 \\
0.85400 & 0.58 & 0.58 \\
0.88600 & 0.98 & 0.98 \\
0.92500 & 1.50 & 1.50 \\
0.97300 & 2.19 & 2.19 \\
1.03000 & 3.04 & 3.04 \\
1.11000 & 4.26 & 4.26 \\
1.23000 & 6.06 & 6.06 \\
1.39000 & 8.31 & 8.33 \\
1.59000 & 10.79 & 10.96 \\
1.83000 & 13.30 & 13.84 \\
2.15300 & 15.95 & 17.30 \\
2.55000 & 18.42 & 21.12 \\
3.03000 & 20.54 & 25.10 \\
3.59000 & 22.23 & 29.11 \\
4.15000 & 23.40 & 32.61 \\
4.78000 & 24.32 & 36.07 \\
5.58000 & 25.10 & 39.90 \\
6.78000 & 25.81 & 44.77 \\
7.98000 & 26.20 & 48.88 \\
11.90000 & 26.67 & 59.05 \\
15.90000 & 26.74 & 66.48 \\
23.90000 & 26.64 & 76.95 \\
39.90000 & 26.42 & 90.14 \\
79.80000 & 26.19 & 108.04 \\
159.00000 & 26.07 & 125.90
\end{tabular}

ATOAIC DISPLACEMENT CROSS-SECTIONS (BARNS) BY PAST ELECTRONS IN CR $z=24 \quad A=52.010$

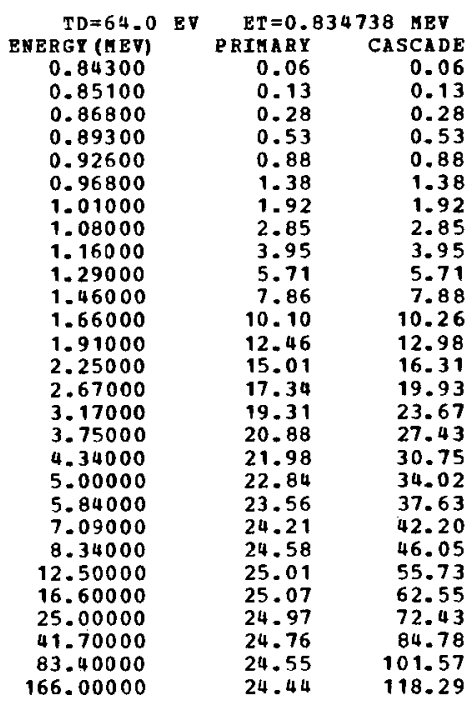

\begin{tabular}{rrr}
$T D=68.0$ & EV & \multicolumn{2}{c}{ ET $=0.870260$ HEV } \\
ENERGY (MEV) & PRIHA & CASCADE \\
0.87800 & 0.05 & 0.05 \\
0.88700 & 0.12 & 0.12 \\
0.90500 & 0.25 & 0.25 \\
0.93100 & 0.48 & 0.48 \\
0.96500 & 0.80 & 0.80 \\
1.00000 & 1.17 & 1.17 \\
1.06000 & 1.86 & 1.86 \\
1.13000 & 2.70 & 2.70 \\
1.21000 & 3.70 & 3.70 \\
1.34000 & 5.30 & 5.30 \\
1.52000 & 7.37 & 7.39 \\
1.74000 & 9.61 & 9.77 \\
2.00000 & 11.83 & 12.34 \\
2.34000 & 14.13 & 15.37 \\
2.78000 & 16.35 & 18.82 \\
3.30000 & 18.20 & 22.36 \\
3.91000 & 19.70 & 25.95 \\
4.52000 & 20.72 & 29.07 \\
5.22000 & 21.53 & 32.21 \\
6.09000 & 22.20 & 35.61 \\
7.39000 & 22.81 & 39.91 \\
8.70000 & 23.15 & 43.56 \\
13.00000 & 23.54 & 52.62 \\
17.40000 & 23.59 & 59.23 \\
26.10000 & 23.50 & 68.44 \\
43.50000 & 23.30 & 80.06 \\
87.00000 & 23.10 & 95.87 \\
174.00000 & 23.00 & 111.71
\end{tabular}

$\begin{array}{rrr}\begin{array}{r}\text { TD=72.0 EV } \\ \text { ENERGY (MEV) }\end{array} & \begin{array}{r}\text { ET }=0.904891 \text { MEV } \\ \text { PRIART }\end{array} & \text { CASCADE } \\ 0.91300 & 0.05 & 0.05 \\ 0.92200 & 0.10 & 0.10 \\ 0.94100 & 0.23 & 0.23 \\ 0.96800 & 0.44 & 0.44 \\ 1.00000 & 0.71 & 0.71 \\ 1.04000 & 1.09 & 1.09 \\ 1.10000 & 1.71 & 1.71 \\ 1.17000 & 2.48 & 2.48 \\ 1.25000 & 3.49 & 3.49 \\ 1.40000 & 5.07 & 5.07 \\ 1.58000 & 6.96 & 6.98 \\ 1.80000 & 9.01 & 9.16 \\ 2.08000 & 11.20 & 11.70 \\ 2.44000 & 13.42 & 14.63 \\ 2.89000 & 15.48 & 17.85 \\ 3.43000 & 17.23 & 21.21 \\ 4.07000 & 18.65 & 24.65 \\ 4.70000 & 19.60 & 27.59 \\ 5.42000 & 20.36 & 30.54 \\ 6.33000 & 20.99 & 33.78 \\ 7.69000 & 21.56 & 37.88 \\ 9.04000 & 21.87 & 41.31 \\ 13.50000 & 22.23 & 49.86 \\ 18.00000 & 22.28 & 56.03 \\ 27.10000 & 22.19 & 64.82 \\ 45.20000 & 22.00 & 75.81 \\ 90.40000 & 21.82 & 90.75 \\ 180.00000 & 21.72 & 105.62\end{array}$


ATOMIC DISPLACEMENT CROSS-SECTIONS (BARNS) BY PAST ELECTBONS IN CR

$T D=76.0 \mathrm{EV} \quad \mathrm{ET}=0.938695 \mathrm{MEV}$

$\begin{array}{rrr}\text { TD=76.0 EV } & \text { ET }=0.938695 \text { MEV } \\ \text { ENERGY (HEV) } & \text { PRTARY } & \text { CASCADE } \\ 0.94800 & 0.05 & 0.05 \\ 0.95700 & 0.10 & 0.10 \\ 0.97600 & 0.21 & 0.21 \\ 1.00000 & 0.37 & 0.37 \\ 1.04000 & 0.68 & 0.68 \\ 1.08000 & 1.02 & 1.02 \\ 1.14000 & 1.59 & 1.59 \\ 1.22000 & 2.39 & 2.39 \\ 1.31000 & 3.33 & 3.33 \\ 1.45000 & 4.77 & 4.77 \\ 1.54000 & 6.61 & 6.63 \\ 1.87000 & 8.58 & 8.72 \\ 2.15000 & 10.59 & 11.06 \\ 2.53000 & 12.74 & 13.90 \\ 3.00000 & 14.71 & 17.00 \\ 3.56000 & 16.36 & 20.20 \\ 4.22000 & 17.70 & 23.45 \\ 4.88000 & 18.61 & 26.28 \\ 5.63000 & 19.32 & 29.09 \\ 6.57000 & 19.91 & 32.15 \\ 7.97000 & 20.43 & 36.02 \\ 9.38000 & 20.73 & 39.30 \\ 14.00000 & 21.06 & 47.41 \\ 18.70000 & 21.11 & 53.29 \\ 28.10000 & 21.02 & 61.58 \\ 46.90000 & 20.84 & 72.02 \\ 93.80000 & 20.67 & 86.17 \\ 187.00000 & 20.58 & 100.29 \\ & & \end{array}$

$z=24 \quad A=52.010$

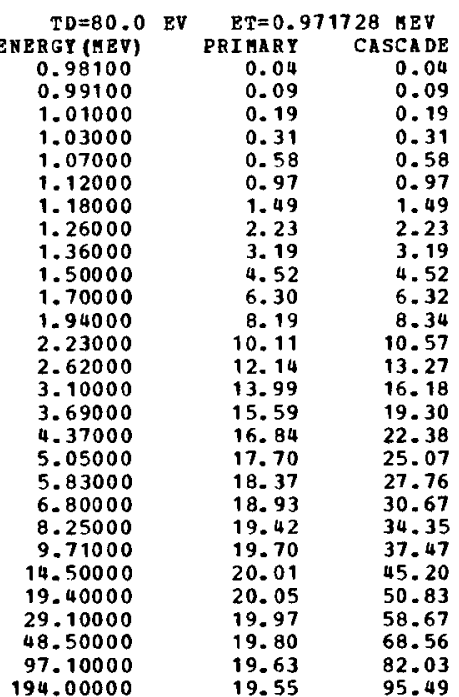

$T D=84.0 \mathrm{EV} \quad \mathrm{ET}=1.004041 \mathrm{MEV}$ ENERGT (MEV) PRIMARY CASCADE $\begin{array}{rrr}1.01000 & 0.02 & 0.02\end{array}$ $1.02000 \quad 0.06 \quad 0.06$ $\begin{array}{lll}1.04000 & 0.16 & 0.16\end{array}$ $\begin{array}{lll}1.07000 & 0.32 & 0.32 \\ 1.11300 & 0.57 & 0.57\end{array}$ $\begin{array}{lll}1.11300 & 0.57 & 0.57 \\ 1.16000 & 0.93 & 0.93\end{array}$ $\begin{array}{lll}1.22000 & 1.41 & 1.41\end{array}$ $\begin{array}{lll}1.30000 & 2.09 & 2.09\end{array}$ $\begin{array}{lll}1.40000 & 2.98 & 2.98\end{array}$ $\begin{array}{lll}1.55000 & 4.30 & 4.30 \\ 1.75000 & 5.96 & 5.98\end{array}$ $\begin{array}{lll}1.75000 & 5.96 & 5.98 \\ 2.00000 & 7.79 & 7.93\end{array}$ $\begin{array}{lll}2.00000 & 9.63 & 10.07\end{array}$ $\begin{array}{rrr}2.30500 & 9.63 & 10.07 \\ 2.71000 & 11.60 & 12.70\end{array}$ $\begin{array}{lll}2.71000 & 11.60 & 12.70 \\ 3.21000 & 13.37 & 15.51\end{array}$ $\begin{array}{lll}3.81000 & 14.87 & 18.44\end{array}$ $\begin{array}{lll}4.51000 & 16.06 & 21.38\end{array}$ $\begin{array}{lll}5.22000 & 16.88 & 23.98 \\ 6.02000 & 17.52 & 26.53\end{array}$ $\begin{array}{lll}6.02000 & 17.52 & 26.53 \\ 7.02000 & 18.04 & 29.30\end{array}$ 18.51
18.76 $\begin{array}{lll}10.00000 & 18.76 & 35.75\end{array}$ $\begin{array}{lll}15.00000 & 19.06 & 43.20\end{array}$ $\begin{array}{lll}20.00000 & 19.09 & 48.51\end{array}$ $\begin{array}{lll}30.10000 & 19.01 & 56.04\end{array}$ $\begin{array}{lll}50.20000 & 18.85 & 65.48\end{array}$ $100.00000 \quad 18.69$ $\begin{array}{lll}100.00000 & 18.69 & 78.22 \\ 200.00000 & 18.62 & 91.05\end{array}$

ATONIC DISPLACEAENT CROSS-SECTIONS (BARNS) BY FAST ELECTRONS IN CR $z=24 \quad A=52.010$

\begin{tabular}{rrr} 
TO=88.0 EV & \multicolumn{1}{c}{ ET=1.035680 MEV } \\
ENERGY(HEV) & PRIHARY & CASCADE \\
1.04000 & 0.01 & 0.01 \\
1.05000 & 0.05 & 0.05 \\
1.07000 & 0.13 & 0.13 \\
1.10000 & 0.28 & 0.28 \\
1.14000 & 0.51 & 0.51 \\
1.20000 & 0.90 & 0.90 \\
1.25000 & 1.35 & 1.35 \\
1.34000 & 1.98 & 1.98 \\
1.44000 & 2.80 & 2.80 \\
1.60000 & 4.11 & 4.11 \\
1.81000 & 5.73 & 5.75 \\
2.07000 & 7.49 & 7.63 \\
2.38000 & 9.25 & 9.69 \\
2.79000 & 11.08 & 12.13 \\
3.31000 & 12.79 & 14.86 \\
3.93000 & 14.22 & 17.67 \\
4.66000 & 15.36 & 20.52 \\
5.38000 & 16.13 & 22.96 \\
6.21000 & 16.74 & 25.42 \\
7.24000 & 17.23 & 28.07 \\
8.80000 & 17.68 & 31.46 \\
10.30000 & 17.92 & 34.21 \\
15.50000 & 18.20 & 41.39 \\
20.70000 & 18.22 & 46.49 \\
31.00000 & 18.15 & 53.60 \\
51.70000 & 17.99 & 62.61 \\
103.00000 & 17.84 & 74.77 \\
207.00000 & 17.77 & 87.11
\end{tabular}

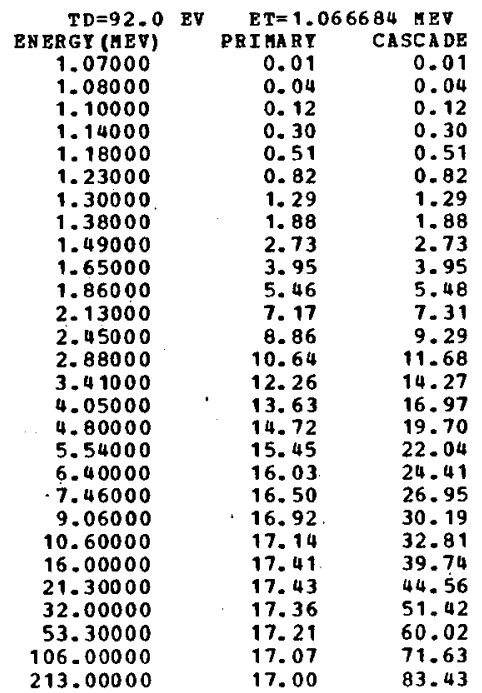

\begin{tabular}{rrr} 
TD $=96.0$ & BV & \multicolumn{1}{c}{ ET=1.097090 MEV } \\
ENERGY (HEV) & PRIMARY & CASCADE \\
1.10000 & 0.01 & 0.01 \\
1.11000 & 0.04 & 0.04 \\
1.14000 & 0.14 & 0.14 \\
1.17000 & 0.27 & 0.27 \\
1.21000 & 0.46 & 0.46 \\
1.27000 & 0.80 & 0.80 \\
1.33000 & 1.18 & 1.18 \\
1.42000 & 1.80 & 1.80 \\
1.53000 & 2.59 & 2.59 \\
1.70000 & 3.80 & 3.80 \\
1.91000 & 5.22 & 5.24 \\
2.19000 & 6.88 & 7.01 \\
2.52000 & 8.51 & 8.93 \\
2.96000 & 10.22 & 11.22 \\
3.51000 & 11.78 & 13.73 \\
4.16000 & 13.08 & 16.30 \\
4.93000 & 14.12 & 18.92 \\
5.70000 & 14.82 & 21.19 \\
6.58000 & 15.37 & 23.46 \\
7.67000 & 15.82 & 25.90 \\
9.32000 & 16.22 & 29.02 \\
10.90000 & 16.43 & 31.53 \\
16.40000 & 16.68 & 38.12 \\
21.90000 & 16.70 & 42.80 \\
32.90000 & 16.63 & 49.38 \\
54.80000 & 16.49 & 57.62 \\
109.00000 & 16.36 & 68.75 \\
219.00000 & 16.29 & 80.06
\end{tabular}


ATOMIC DISPLAZEMENT CROSS-SECTIONS (BARNS) BY FAST BLECTRONS IN PE

\begin{tabular}{crr}
$T D=4 . D$ & EV & \multicolumn{2}{c}{ EI $=0.093269$ MEV } \\
ENERGY(MEV) & PRIMARY & CASCADE \\
0.09420 & 12.31 & 12.31 \\
0.09510 & 23.80 & 23.80 \\
0.09590 & 45.65 & 45.65 \\
0.09970 & 76.90 & 76.90 \\
0.10300 & 109.91 & 109.91 \\
0.10800 & 153.24 & 153.24 \\
0.11300 & 189.88 & 189.88 \\
0.12100 & 237.58 & 237.58 \\
0.13000 & 279.08 & 279.08 \\
0.14400 & 325.88 & 325.88 \\
0.16300 & 367.42 & 367.42 \\
0.18500 & 398.19 & 398.98 \\
0.21400 & 420.12 & 426.82 \\
0.25100 & 435.96 & 454.39 \\
0.29300 & 446.08 & 481.18 \\
0.35400 & 451.85 & 506.87 \\
0.41900 & 455.05 & 532.26 \\
0.48400 & 456.64 & 554.97 \\
0.55900 & 457.62 & 579.12 \\
0.65200 & 458.22 & 606.95 \\
0.79200 & 458.51 & 645.64 \\
0.93200 & 458.43 & 681.32 \\
1.39000 & 456.97 & 782.17 \\
1.86000 & 454.78 & 867.09 \\
2.79000 & 450.48 & 999.17 \\
4.66000 & 444.26 & 1184.31 \\
9.32000 & 436.02 & 1454.33 \\
18.60000 & 430.61 & 1736.35 \\
27.90000 & 428.85 & 1905.08 \\
46.60000 & 427.51 & 2120.49 \\
65.20000 & 426.92 & 2262.23 \\
93.20000 & 426.40 & 2413.27 \\
185.00000 & 425.48 & 2705.73 \\
& &
\end{tabular}

\begin{tabular}{|c|c|c|}
\hline$T D=8.0$ & $\mathrm{ET}=0$ & $953 \mathrm{MEV}$ \\
\hline ENERGY (MEV) & PRIMARY & CASCADE \\
\hline 0.17500 & 1.97 & 1.97 \\
\hline 0.17700 & 5.64 & 5.64 \\
\hline 0.18000 & 10.91 & 10.91 \\
\hline 0.18600 & 20.70 & 20.70 \\
\hline 0.19300 & 31.01 & 31.01 \\
\hline 0.20100 & 41.53 & 41.53 \\
\hline 0.21200 & 54.19 & 54.19 \\
\hline 0.22600 & 67.85 & 67.85 \\
\hline 0.24300 & 81.64 & 81.64 \\
\hline 0.26900 & 98.43 & 98.43 \\
\hline 0.30400 & 115.53 & 115.53 \\
\hline 0.34700 & 131.12 & 131.71 \\
\hline 0.40000 & 145.35 & 149.36 \\
\hline 0.46900 & 158.94 & 166.62 \\
\hline 0.55600 & 171.36 & 186.21 \\
\hline 0.66100 & 182.17 & 206.61 \\
\hline 0.78200 & 191.17 & 227.25 \\
\hline 0.90400 & 197.85 & 245.85 \\
\hline 1.04000 & 203.41 & 264.65 \\
\hline 1.21000 & 208.48 & 285.91 \\
\hline 1.47000 & 213.67 & 374.74 \\
\hline 1.73000 & 216.94 & 340.13 \\
\hline 2.60000 & 221.50 & 408.38 \\
\hline 3.47000 & 222.56 & 460.23 \\
\hline 5.21000 & 222.05 & 537.14 \\
\hline 8.69000 & 219.87 & 638.30 \\
\hline 17.30000 & 216.62 & 778.98 \\
\hline 34.70000 & 214.63 & 924.26 \\
\hline 52.10000 & 214.00 & 1009.85 \\
\hline 86.90000 & 213.50 & 1118.05 \\
\hline 121.00000 & 213.26 & 1188.16 \\
\hline 173.00000 & 213.02 & 1263.89 \\
\hline 347.00000 & 212,55 & 1411.40 \\
\hline
\end{tabular}

\begin{tabular}{|c|c|c|}
\hline$T D=12.0$ & EV $\quad E T=0$. & $087 \mathrm{MEV}$ \\
\hline BNERGY (YEV) & PRIMARY & CASCADE \\
\hline 0.24300 & 1.18 & 1.18 \\
\hline 0.25100 & 2.98 & 2.98 \\
\hline 0.25500 & 5.30 & 5.30 \\
\hline 0.26300 & 9.70 & 9.70 \\
\hline 0.27300 & 14.78 & 14.78 \\
\hline 0.28500 & 20.35 & 20.35 \\
\hline 0.30300 & 26.64 & 26.64 \\
\hline 0.31700 & 33.71 & 33.71 \\
\hline 0.34400 & 41.82 & 41.82 \\
\hline 0.38100 & 51.95 & 51.95 \\
\hline 0.43300 & 62.89 & 62.89 \\
\hline 0.49200 & 73.98 & 74.46 \\
\hline 0.56500 & 84.47 & 86.48 \\
\hline $0.66+00$ & 95.28 & 100.35 \\
\hline 0.78700 & 105.51 & 115.50 \\
\hline 0.93500 & 194.61 & 131.43 \\
\hline 1.10300 & 122.02 & 147.09 \\
\hline 1.27000 & 127.65 & 161.48 \\
\hline 1.47000 & 132.52 & 176.67 \\
\hline 1.72000 & 136.84 & 193.61 \\
\hline 2.09300 & 140.99 & 215.46 \\
\hline 2.46000 & 143.54 & 234.42 \\
\hline 3.69200 & 147.02 & 233.93 \\
\hline 4.92300 & 147.73 & 320.69 \\
\hline 7.38000 & 147.43 & 374.21 \\
\hline 12.30300 & 146.01 & 443.44 \\
\hline 24.60000 & 144.02 & 539.21 \\
\hline 49.20300 & 142.91 & 636.34 \\
\hline 73.80000 & 142.56 & 693.48 \\
\hline 123.00300 & 142.26 & 765.66 \\
\hline 172.00000 & 142.10 & 813.05 \\
\hline 246.00500 & 141.94 & 863.62 \\
\hline 492.00300 & 141.63 & 961.58 \\
\hline
\end{tabular}

ATOMIC DISPLAZEMENT CROSS-SECTIONS (BARNS) BY FAST ELECTRONS IN PE $Z=26 \quad A=55.850$

$\begin{array}{crr}\text { TD }=16.0 & \text { EV } & \text { ET }=0.311922 \text { MEV } \\ \text { ENERGY (MEV) } & \text { PRIMARY } & \text { CASCADE } \\ 0.31500 & 0.87 & 0.87 \\ 0.31900 & 1.70 & 1.70 \\ 0.32400 & 3.32 & 3.32 \\ 0.33300 & 5.66 & 5.66 \\ 0.34600 & 9.86 & 8.86 \\ 0.35100 & 12.31 & 12.31 \\ 0.38000 & 16.38 & 16.38 \\ 0.40500 & 21.27 & 21.27 \\ 0.43600 & 25.76 & 26.76 \\ 0.48300 & 34.08 & 34.08 \\ 0.54500 & 42.31 & 42.31 \\ 0.62300 & 50.93 & 51.33 \\ 0.71700 & 59.41 & 60.97 \\ 0.84200 & 68.34 & 72.29 \\ 0.99800 & 76.83 & 84.68 \\ 1.18000 & 84.17 & 97.38 \\ 1.40000 & 90.60 & 110.86 \\ 1.52000 & 95.23 & 122.79 \\ 1.87000 & 99.06 & 134.90 \\ 2.18000 & 102.40 & 148.23 \\ 2.65000 & 105.63 & 165.73 \\ 3.11000 & 107.55 & 180.49 \\ 4.67000 & 110.13 & 219.34 \\ 6.23000 & 110.61 & 247.83 \\ 9.35000 & 110.34 & 288.90 \\ 15.50000 & 109.27 & 340.91 \\ 31.10000 & 107.87 & 413.59 \\ 62.30000 & 107.11 & 486.84 \\ 93.50000 & 106.87 & 529.84 \\ 155.00000 & 106.66 & 583.45\end{array}$

\begin{tabular}{crr} 
TD $=20.0$ & EV & \multicolumn{2}{c}{ ET=0.372867 MEV } \\
ENERGY (HEV) & PRIMARY & CASCADE \\
0.37600 & 0.49 & 0.49 \\
0.38000 & 1.10 & 1.10 \\
0.38700 & 2.16 & 2.16 \\
0.39800 & 3.79 & 3.79 \\
0.41300 & 5.93 & 5.93 \\
0.43200 & 8.53 & 8.53 \\
0.45400 & 11.39 & 11.39 \\
0.48400 & 15.06 & 15.06 \\
0.52200 & 19.37 & 19.37 \\
0.57700 & 25.02 & 25.02 \\
0.65200 & 31.78 & 31.79 \\
0.74500 & 38.90 & 39.25 \\
0.85700 & 46.01 & 47.31 \\
1.00000 & 53.28 & 56.48 \\
1.19000 & 60.66 & 67.22 \\
1.41000 & 66.94 & 78.12 \\
1.67000 & 72.27 & 89.41 \\
1.93000 & 76.09 & 99.37 \\
2.23000 & 79.27 & 109.60 \\
2.61000 & 82.05 & 121.03 \\
3.16000 & 84.58 & 135.28 \\
3.72000 & 86.12 & 147.73 \\
5.59000 & 88.08 & 179.67 \\
7.45000 & 88.41 & 202.81 \\
11.10000 & 88.15 & 235.47 \\
18.60000 & 87.27 & 278.28 \\
37.20000 & 86.21 & 336.40 \\
74.50000 & 85.65 & 395.09 \\
111.00000 & 85.47 & 428.91 \\
186.00000 & 85.31 & 472.74 \\
& &
\end{tabular}

\begin{tabular}{|c|c|c|}
\hline$T D=24.0$ & $E T=0.4$ & 373 MEV \\
\hline ENERGY (MEV) & PRIMARY & CASCADE \\
\hline 0.43400 & 0.40 & 0.40 \\
\hline 0.43300 & 0.78 & 0.78 \\
\hline 0.44700 & 1.63 & 1.63 \\
\hline 0.45700 & 2.75 & 2.76 \\
\hline 0.47700 & 4.43 & 4.43 \\
\hline 0.49300 & 6.33 & 6.33 \\
\hline 0.52700 & 8.62 & 8.62 \\
\hline 0.55300 & 11.49 & 11.49 \\
\hline 0.60100 & 14.94 & 14.94 \\
\hline 0.66500 & 19.76 & 19.76 \\
\hline 0.75200 & 25.45 & 25.47 \\
\hline 0.85900 & 31.55 & 31.86 \\
\hline 0.98300 & 37.68 & 38.82 \\
\hline 1.16300 & 44.22 & 47.11 \\
\hline 1.37000 & 50.32 & 56.04 \\
\hline 1.63500 & 55.85 & 65.74 \\
\hline 1.93300 & 60.38 & 75.51 \\
\hline 2.23300 & 63.60 & 84.11 \\
\hline 2.57000 & 66.21 & 92.77 \\
\hline 3.00300 & 68.49 & 102.41 \\
\hline 3.65000 & 70.62 & 114.91 \\
\hline 4.29000 & 71.86 & 125.41 \\
\hline 6.44300 & 73.39 & 152.42 \\
\hline 8.59000 & 73.63 & 171.99 \\
\hline 12.80300 & 73.38 & 199.43 \\
\hline 21.40000 & 72.65 & 235.13 \\
\hline 42.90000 & 71.79 & 283.85 \\
\hline 85.90000 & 71.35 & 332.82 \\
\hline 128.00000 & 71.21 & 361.03 \\
\hline 214.00000 & 71.08 & 397.40 \\
\hline
\end{tabular}


ATONIC DISPLAZBHENT CROSS-SECTIONS (BARNS) BY FAST ELBCTRONS IN PE $Z=26 \quad A=55.850$

\begin{tabular}{ccr} 
TD $=28.0$ & EV & \multicolumn{2}{c}{ ET $=0.483616$ MEV } \\
ENERGY(MEV & PRIMARY & CASCADE \\
0.48800 & 0.28 & 0.28 \\
0.49300 & 0.60 & 0.60 \\
0.50200 & 1.18 & 1.18 \\
0.51700 & 2.15 & 2.15 \\
0.53600 & 3.39 & 3.39 \\
0.56000 & 4.94 & 4.94 \\
0.59000 & 6.86 & 6.86 \\
0.52800 & 9.24 & 9.24 \\
0.67700 & 12.19 & 12.19 \\
0.74900 & 16.27 & 16.27 \\
0.84600 & 21.24 & 21.26 \\
0.96700 & 26.62 & 26.92 \\
1.11000 & 31.95 & 32.97 \\
1.30000 & 37.64 & 40.18 \\
1.54000 & 43.11 & 48.23 \\
1.83000 & 47.91 & 56.74 \\
2.17000 & 51.89 & 65.44 \\
2.51000 & 54.68 & 73.07 \\
2.90000 & 56.95 & 80.81 \\
3.38000 & 58.86 & 89.17 \\
4.11000 & 60.65 & 100.06 \\
4.83000 & 61.57 & 109.20 \\
7.25000 & 62.91 & 132.63 \\
9.67000 & 63.09 & 149.54 \\
14.50000 & 62.84 & 173.57 \\
24.10000 & 62.22 & 203.92 \\
48.30000 & 61.50 & 245.75 \\
96.70000 & 61.14 & 287.75 \\
145.00000 & 61.03 & 312.33 \\
& &
\end{tabular}

\begin{tabular}{|c|c|c|}
\hline \multirow{2}{*}{$\begin{array}{r}T D=32.0 \\
\text { ENERGY }(M E V)\end{array}$} & \multicolumn{2}{|c|}{$\mathrm{ET}=0.534601 \mathrm{MEV}$} \\
\hline & PR I MARY & CASCADE \\
\hline 0.53900 & 0.20 & 0.20 \\
\hline 0.54500 & 0.47 & 0.47 \\
\hline 0.55500 & 0.94 & 0.94 \\
\hline 0.57200 & 1.74 & 1.74 \\
\hline 0.59300 & 2.75 & 2.75 \\
\hline 0.62000 & 4.06 & 4.06 \\
\hline 0.65200 & 5.63 & 5.63 \\
\hline 0.69400 & 7.67 & 7.67 \\
\hline 0.74800 & 10.24 & 10.24 \\
\hline 0.82800 & 13.85 & 13.85 \\
\hline 0.93500 & 18.25 & 18.27 \\
\hline 1.06000 & 22.77 & 23.01 \\
\hline 1.22000 & 27.64 & 28.54 \\
\hline 1.44000 & 32.98 & 35.33 \\
\hline 1.71000 & 37.91 & 42.66 \\
\hline 2.03000 & 42.13 & 50.26 \\
\hline 2.40000 & 45.56 & 57.90 \\
\hline 2.77000 & 47.97 & 64.62 \\
\hline 3.20000 & 49.95 & 71.51 \\
\hline 3.74000 & 51.63 & 79.08 \\
\hline 4.54000 & 53.15 & 88.66 \\
\hline 5.34000 & 54.02 & 96.80 \\
\hline 8. 01000 & 55.05 & 117.45 \\
\hline 10.60000 & 55.18 & 131.93 \\
\hline 16.00000 & 54.95 & 153.39 \\
\hline 26.70000 & 54.40 & 180.22 \\
\hline 53.40000 & 53.79 & 216.77 \\
\hline 106.00000 & 53.49 & 253.09 \\
\hline 160.00000 & 53.39 & 274.95 \\
\hline
\end{tabular}

\begin{tabular}{|c|c|c|}
\hline$T D=36.0$ & $\mathrm{ET}=0$ & 213 MEV \\
\hline ENERGY (MEV) & PRIMARY & CASCADE \\
\hline 0.58900 & 0.19 & 0.19 \\
\hline 0.59400 & 0.36 & 0.36 \\
\hline 0.60500 & 0.78 & 0.78 \\
\hline 0.62400 & 1.43 & 1.43 \\
\hline 0.64700 & 2.28 & 2.28 \\
\hline 0.67500 & 3.39 & 3.39 \\
\hline 0.71100 & 4.75 & 4.75 \\
\hline 0.75300 & 6.59 & 6.59 \\
\hline 0.31500 & 8.83 & 8.83 \\
\hline 0.90300 & 12.05 & 12.05 \\
\hline 1.02300 & 15.03 & 16.05 \\
\hline 1.16000 & 20.21 & 20.45 \\
\hline 1.34300 & 24.73 & 25.60 \\
\hline 1.57300 & 29.33 & 31.50 \\
\hline 1.86000 & 33.72 & 38.06 \\
\hline 2.21300 & 37.53 & 44.98 \\
\hline 2.52000 & 40.64 & 52.02 \\
\hline 3.03000 & 42.81 & 58.17 \\
\hline 3.49300 & 44.52 & 64.26 \\
\hline 4.08300 & 45.99 & 71.09 \\
\hline 4.95000 & 47.31 & 79.67 \\
\hline 5.83000 & 48.07 & 87.03 \\
\hline 8.74000 & 48.94 & 105.49 \\
\hline 11.60000 & 49.04 & 118.57 \\
\hline 17.40000 & 48.82 & 137.41 \\
\hline 29.10000 & 48.33 & 161.41 \\
\hline 58.30000 & 47.80 & 194.02 \\
\hline 115.00000 & 47.54 & 226.43 \\
\hline 174.00000 & 47.46 & 245.58 \\
\hline
\end{tabular}

ATOMIC DISPLAZEMENT CROSS-SECTIONS (BARNS) BY FAST ELECTRONS IN ?E $\mathrm{z}=26 \quad \mathrm{~A}=55.850$

\begin{tabular}{|c|c|c|c|}
\hline $\begin{array}{r}T D=40.0 \\
\text { ENERGY (4EV) }\end{array}$ & EV & $\begin{aligned} & \text { ET }=0.6 \\
& \text { PRIMARY }\end{aligned}$ & $\begin{array}{l}55 \text { MBV } \\
\text { CASCADE }\end{array}$ \\
\hline 0.63600 & & 0.16 & 0.16 \\
\hline 0.64200 & & 0.32 & 0.32 \\
\hline 0.65400 & & 0.64 & 0.64 \\
\hline 0.67300 & & 1.17 & 1.17 \\
\hline 0.69900 & & 1.94 & 1.94 \\
\hline 0.73000 & & 2.90 & 2.90 \\
\hline 0.76800 & & 4.11 & 4.11 \\
\hline 0.81800 & & 5.73 & 5.73 \\
\hline 0.88100 & & 7.75 & 7.75 \\
\hline 0.97600 & & 10.71 & 10.71 \\
\hline 1.10000 & & 14.27 & 14.29 \\
\hline 1.25000 & & 18.07 & 18.29 \\
\hline 1.44000 & & 22.12 & 22.92 \\
\hline 1.70000 & & 26.53 & 28.58 \\
\hline 2.01000 & & 30.46 & 34.52 \\
\hline 2.39000 & & 33.92 & 40.88 \\
\hline 2.83000 & & 36.70 & 47.26 \\
\hline 3.27000 & & 38.63 & 52.83 \\
\hline 3.77000 & & 40.17 & 58.42 \\
\hline 4.40000 & & 41.46 & 64.56 \\
\hline 5.35000 & & 42.64 & 72.45 \\
\hline 6.29000 & & 43.30 & 79.05 \\
\hline 9.44000 & & 44.05 & 95.81 \\
\hline 12.50000 & & 44.13 & 107.52 \\
\hline 18.80000 & & 43.92 & 124.63 \\
\hline 31.40000 & & 43.47 & 146.21 \\
\hline 62.90000 & & 43.01 & 175.58 \\
\hline 125.00000 & & 42.78 & 204.72 \\
\hline 188.00000 & & 42.71 & 222.06 \\
\hline
\end{tabular}

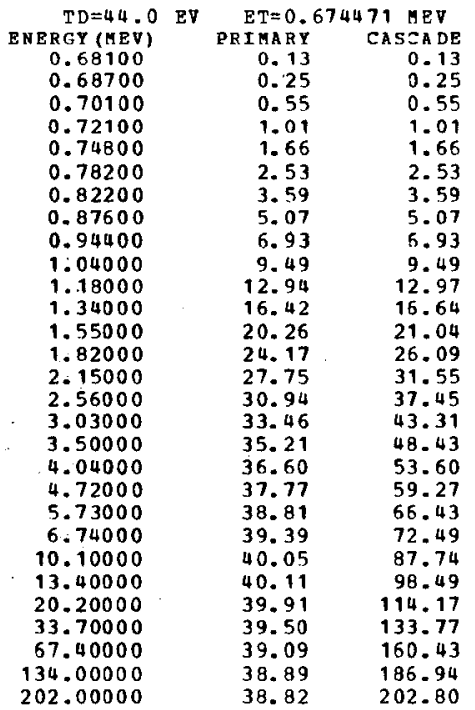

\begin{tabular}{|c|c|c|}
\hline$T D=48.0$ & $B T=0$. & $561 \mathrm{MBV}$ \\
\hline $\begin{array}{r}\text { BNERGY (GEV) } \\
0.724000\end{array}$ & $\begin{array}{r}\text { PRIMA RY } \\
0,10\end{array}$ & CASCADE \\
\hline 0.73100 & 0.22 & 0.22 \\
\hline 0.74500 & 0.48 & 0.48 \\
\hline 0.76700 & 0.87 & 0.87 \\
\hline 0.79500 & 1.46 & 1.46 \\
\hline 0.83200 & 2.23 & 2.23 \\
\hline 0.87500 & 3.21 & 3.21 \\
\hline 0.93200 & 4.55 & 4.55 \\
\hline 1.00000 & 6.16 & 6.16 \\
\hline 1.11300 & 8.70 & 8.70 \\
\hline 1.25000 & 11.71 & 11.74 \\
\hline 1.43000 & 15.13 & 15.34 \\
\hline 1.65000 & 18.62 & 19.36 \\
\hline 1.93000 & 22.15 & 23.94 \\
\hline 2.29000 & 25.55 & 29.14 \\
\hline 2.72000 & 28.44 & 34.54 \\
\hline 3.22000 & 30.75 & 39.97 \\
\hline 3.73000 & 32.37 & 44.80 \\
\hline 4.30000 & 33.63 & 49.54 \\
\hline 5.02000 & 34.68 & 54.75 \\
\hline 6.09300 & 35.61 & 51.33 \\
\hline 7.17000 & 36.14 & 66.94 \\
\hline 10.70000 & 36.71 & 80.83 \\
\hline 14.30000 & 36.76 & 90.97 \\
\hline 21.50300 & 36.57 & 105.27 \\
\hline 35.80000 & 36.20 & 123.20 \\
\hline 71.70000 & 35.83 & 147.70 \\
\hline 143.00300 & 35.64 & 172.12 \\
\hline 215.00000 & 35.58 & 186.57 \\
\hline
\end{tabular}


ATOMIC DISPLAZENENT CROSS-SECTIONS (BARN5) BY FAST ELBCTRONS IN FE

\begin{tabular}{rrr} 
TD $=52.0$ & EV & \multicolumn{2}{c}{ ER=0.759.189 HEV } \\
BNERGY (MEV) & PRIHARY & CASCADE \\
0.76600 & 0.09 & 0.09 \\
0.77400 & 0.20 & 0.20 \\
0.78900 & 0.42 & 0.42 \\
0.81200 & 0.78 & 0.78 \\
0.84200 & 1.29 & 1.29 \\
0.88000 & 1.99 & 1.99 \\
0.92600 & 2.90 & 2.90 \\
0.98600 & 4.12 & 4.12 \\
1.06000 & 5.66 & 5.66 \\
1.17000 & 7.89 & 7.89 \\
1.32000 & 10.74 & 10.76 \\
1.51000 & 13.92 & 14.13 \\
1.74900 & 17.15 & 17.84 \\
2.04000 & 20.49 & 22.18 \\
2.42000 & 23.64 & 27.03 \\
2.88000 & 26.34 & 32.12 \\
3.41000 & 28.47 & 37.19 \\
3.94000 & 29.94 & 41.60 \\
4.55000 & 31.10 & 46.06 \\
5.31000 & 32.06 & 50.89 \\
6.45000 & 32.91 & 57.04 \\
7.59000 & 33.38 & 62.23 \\
11.30000 & 33.89 & 75.01 \\
15.10000 & 33.92 & 84.39 \\
22.70000 & 33.74 & 97.61 \\
37.90000 & 33.40 & 114.25 \\
75.90000 & 33.06 & 136.88 \\
151.00000 & 32.90 & 159.35 \\
& &
\end{tabular}

$\begin{array}{crr}\text { TD }=56.0 & \text { EV } & \text { ET=0.799497 MEV } \\ \text { ENERGY(MEV) } & \text { PRIMARY } & \text { CASTADE } \\ 0.80700 & 0.08 & 0.08 \\ 0.81500 & 0.17 & 0.17 \\ 0.83100 & 0.37 & 0.37 \\ 0.85500 & 0.69 & 0.69 \\ 0.88700 & 1.16 & 1.16 \\ 0.92700 & 1.80 & 1.80 \\ 0.97500 & 2.63 & 2.63 \\ 1.03000 & 3.62 & 3.62 \\ 1.11000 & 5.09 & 5.09 \\ 1.23000 & 7.26 & 7.26 \\ 1.39000 & 9.96 & 9.98 \\ 1.59000 & 12.94 & 13.14 \\ 1.83000 & 15.94 & 16.59 \\ 2.15000 & 19.10 & 20.72 \\ 2.55000 & 22.03 & 25.26 \\ 3.03000 & 24.52 & 29.99 \\ 3.59000 & 26.50 & 34.75 \\ 4.15000 & 27.86 & 38.89 \\ 4.79000 & 28.92 & 43.04 \\ 5.59000 & 29.80 & 47.55 \\ 6.79000 & 30.58 & 53.29 \\ 7.99000 & 31.01 & 58.12 \\ 11.90000 & 31.47 & 70.04 \\ 15.90000 & 31.50 & 78.76 \\ 23.90000 & 31.33 & 91.04 \\ 39.90000 & 31.01 & 106.51 \\ 79.90000 & 30.70 & 127.53 \\ 159.00000 & 30.55 & 148.40\end{array}$

\begin{tabular}{|c|c|c|}
\hline$T D=50.0$ & $\mathbf{E T}=0.8$ & 600 MEV \\
\hline ENERGY (MEV) & PRIMABY & CASCADE \\
\hline 0.84500 & 0.07 & 0.07 \\
\hline 0.85500 & 0.15 & 0.15 \\
\hline 0.87200 & 0.33 & 0.33 \\
\hline 0.89700 & 0.62 & 0.62 \\
\hline 0.93000 & 1.04 & 1.04 \\
\hline 0.97200 & 1.64 & 1.64 \\
\hline 1.02300 & 2.37 & 2.37 \\
\hline 1.09000 & 3.49 & 3.49 \\
\hline 1.17000 & 4.80 & 4.80 \\
\hline 1.29000 & 6.74 & 6.74 \\
\hline 1.46000 & 9.32 & 9.34 \\
\hline 1.67000 & 12.12 & 12.31 \\
\hline 1.92300 & 14.92 & 15.55 \\
\hline 2.26000 & 17.92 & 19.48 \\
\hline 2.68000 & 20.65 & 23.75 \\
\hline 3.18000 & 22.95 & 28.17 \\
\hline 3.77000 & 24.80 & 32.66 \\
\hline 4.36000 & 26.06 & 36.56 \\
\hline 5.03300 & 27.05 & 40.44 \\
\hline 5.87000 & 27.85 & 44.67 \\
\hline 7.12000 & 28.57 & 50.01 \\
\hline 8.38300 & 28.96 & 54.54 \\
\hline 12.50300 & 29.37 & 65.73 \\
\hline 16.70000 & 29.39 & 73.88 \\
\hline 25.10000 & 29.23 & 85.36 \\
\hline 41.90300 & 28.93 & 99.80 \\
\hline 83.80300 & 28.65 & 119.39 \\
\hline 167.00000 & 28.51 & 138.92 \\
\hline
\end{tabular}

ATOMIC DISPLAZEMENT CROSS-SECTIONS (BARNS) BY FAST ELECTRONS IN FE $\mathrm{Z}=26 \quad \mathrm{~A}=55.850$

\begin{tabular}{rrr} 
TD $=64.0$ & EV & \multicolumn{2}{c}{ ET $=0.875603$ MEV } \\
ENERG (MEV) & PRIMARY & CASCADE \\
0.88500 & 0.06 & 0.06 \\
0.89400 & 0.14 & 0.14 \\
0.91100 & 0.29 & 0.29 \\
0.93700 & 0.55 & 0.55 \\
0.97300 & 0.96 & 0.96 \\
1.01000 & 1.42 & 1.42 \\
1.06000 & 2.10 & 2.10 \\
1.13000 & 3.10 & 3.10 \\
1.22000 & 4.43 & 4.43 \\
1.35000 & 6.33 & 6.33 \\
1.53000 & 8.79 & 8.81 \\
1.75000 & 11.43 & 11.62 \\
2.01000 & 14.05 & 14.66 \\
2.36000 & 16.83 & 18.33 \\
2.80000 & 19.41 & 22.37 \\
3.33000 & 21.59 & 26.60 \\
3.94000 & 23.30 & 30.78 \\
4.55000 & 24.47 & 34.42 \\
5.25000 & 25.38 & $\mathbf{3 8 . 0 8}$ \\
5.13000 & 26.14 & 42.08 \\
7.45000 & 26.80 & 47.15 \\
8.76000 & 27.16 & 51.39 \\
13.10000 & 27.54 & 61.97 \\
17.50000 & 27.55 & 69.62 \\
26.20000 & 27.40 & 80.29 \\
43.80000 & 27.12 & 93.87 \\
87.60000 & 26.85 & 112.24 \\
175.00000 & 26.73 & 130.62
\end{tabular}

\begin{tabular}{ccc} 
TD=68.0 & EV & \multicolumn{2}{c}{ ET $=0.913592$ HEV } \\
ENERGY (MEV) & PRIMARY & CASCADE \\
0.92200 & 0.06 & 0.06 \\
0.93100 & 0.12 & 0.12 \\
0.95000 & 0.27 & 0.27 \\
0.97700 & 0.51 & 0.51 \\
1.01000 & 0.84 & 0.84 \\
1.05000 & 1.28 & 1.28 \\
1.11000 & 2.01 & 2.01 \\
1.18000 & 2.92 & 2.92 \\
1.27000 & 4.12 & 4.12 \\
1.41000 & 5.98 & 5.98 \\
1.59000 & 8.22 & 8.24 \\
1.82000 & 10.74 & 10.92 \\
2.10000 & 13.30 & 13.90 \\
2.46000 & 15.89 & 17.33 \\
2.92000 & 18.33 & 21.17 \\
3.47000 & 20.37 & 25.16 \\
4.11000 & 21.98 & 29.14 \\
4.75000 & 23.08 & 32.61 \\
5.48000 & 23.93 & 36.06 \\
6.39000 & 24.63 & 39.81 \\
7.76000 & 25.24 & 44.58 \\
9.13000 & 25.57 & 48.59 \\
13.70000 & 25.92 & 58.65 \\
18.20000 & 25.93 & 65.72 \\
27.40000 & 25.78 & 75.91 \\
45.60000 & 25.52 & 88.59 \\
91.30000 & 25.27 & 105.91 \\
182.00000 & 25.15 & 123.16
\end{tabular}

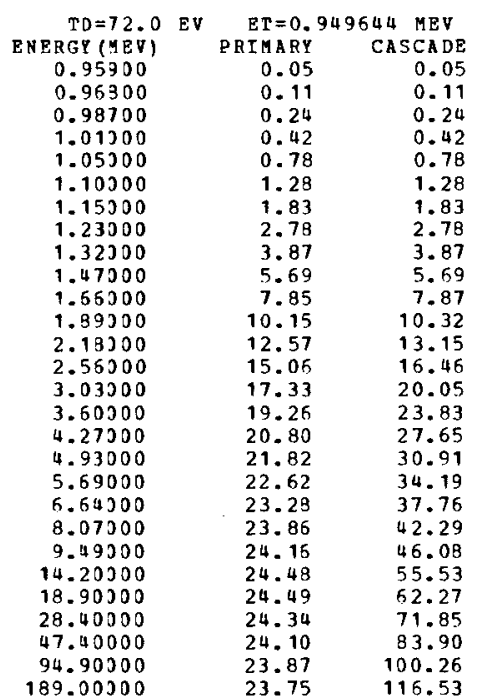


ATOMIC DISPLAZBMENT CROSS-SECTIONS (BARNS) BY FAST ELECTRONS TN FE

\begin{tabular}{rrr} 
TD $=76.0$ & EV & \multicolumn{2}{c}{ ET $=0.984828$} & MEV \\
ENERGY(HEV) & PRIMARY & CASCADE \\
0.99400 & 0.05 & 0.05 \\
1.00000 & 0.08 & 0.08 \\
1.02000 & 0.20 & 0.20 \\
1.05000 & 0.41 & 0.41 \\
1.09000 & 0.73 & 0.73 \\
1.14000 & 1.19 & 1.19 \\
1.20000 & 1.79 & 1.79 \\
1.28000 & 2.65 & 2.66 \\
1.37000 & 3.67 & 3.67 \\
1.52000 & 5.34 & 5.34 \\
1.72000 & 7.43 & 7.45 \\
1.96000 & 9.63 & 9.80 \\
2.26000 & 11.94 & 12.49 \\
2.65000 & 14.28 & 15.52 \\
3.15000 & 15.49 & 19.13 \\
3.74000 & 18.31 & 22.71 \\
4.43000 & 19.74 & 26.32 \\
5.12000 & 20.71 & 29.44 \\
5.90000 & 21.46 & 32.53 \\
6.89000 & 22.08 & 35.94 \\
8.37000 & 22.61 & 40.23 \\
9.84000 & 22.90 & 43.82 \\
14.70000 & 23.19 & 52.76 \\
19.60000 & 23.19 & 59.18 \\
29.50000 & 23.06 & 68.30 \\
49.20000 & 22.83 & 79.70 \\
98.40000 & 22.61 & 95.19 \\
196.00000 & 22.50 & 110.60
\end{tabular}

$\begin{array}{crr}\text { TD=80.0 } & \text { EV } & \text { ET }=1.019203 \text { MEV } \\ \text { ENERGY (MEV) } & \text { PRIMARY } & \text { CASEADE } \\ 1.02000 & 0.00 & 0.00 \\ 1.03000 & 0.05 & 0.05 \\ 1.05000 & 0.15 & 0.15 \\ 1.09000 & 0.40 & 0.40 \\ 1.13000 & 0.69 & 0.69 \\ 1.18000 & 1.11 & 1.11 \\ 1.24000 & 1.67 & 1.67 \\ 1.32000 & 2.47 & 2.47 \\ 1.42000 & 3.50 & 3.50 \\ 1.57000 & 5.04 & 5.04 \\ 1.78000 & 7.06 & 7.09 \\ 2.03000 & 9.18 & 9.35 \\ 2.34000 & 11.38 & 11.91 \\ 2.75000 & 13.64 & 14.94 \\ 3.26000 & 15.70 & 18.25 \\ 3.87000 & 17.43 & 21.57 \\ 4.58000 & 18.78 & 25.09 \\ 5.29000 & 19.69 & 28.05 \\ 6.11000 & 20.41 & 31.05 \\ 7.13000 & 20.99 & 34.27 \\ 8.66000 & 21.49 & 38.36 \\ 10.10000 & 21.75 & 41.61 \\ 15.20000 & 22.03 & 50.26 \\ 20.30000 & 22.03 & 56.40 \\ 30.50000 & 21.90 & 65.04 \\ 50.90000 & 21.68 & 75.89 \\ 101.00000 & 21.48 & 90.44 \\ 203.00000 & 21.38 & 105.27\end{array}$

\begin{tabular}{|c|c|c|}
\hline$T D=34.0$ & $\mathrm{ET}=1 . \mathrm{C}$ & $M E V$ \\
\hline ENERGY (YEV) & PRIMARY & CASCADE \\
\hline 1.06000 & 0.03 & 0.03 \\
\hline 1.07000 & 0.07 & 0.07 \\
\hline 1.09300 & 0.17 & 0.17 \\
\hline 1.12300 & 0.33 & 0.33 \\
\hline 1.16300 & 0.60 & 0.60 \\
\hline 1.22300 & 1.06 & 1.05 \\
\hline 1.23300 & 1.57 & 1.57 \\
\hline 1.36000 & 2.30 & 2.30 \\
\hline 1.47000 & 3.35 & 3.35 \\
\hline 1.63000 & 4.87 & 4.87 \\
\hline 1.84300 & 6.75 & 6.77 \\
\hline 2.10300 & 8.79 & 8.95 \\
\hline 2.42300 & 10.88 & 11.40 \\
\hline 2.84000 & 13.02 & 14.28 \\
\hline 3.36300 & 14.96 & 17.41 \\
\hline 4.00000 & 16.63 & 20.73 \\
\hline 4.73000 & 17.91 & 23.98 \\
\hline 5.47000 & 18.79 & 26.84 \\
\hline 6.31300 & 19.46 & 29.68 \\
\hline 7.36000 & 20.00 & 32.75 \\
\hline 8.94200 & 20.48 & 36.65 \\
\hline 10.50000 & 20.73 & 39.89 \\
\hline 15.70300 & 20.98 & 48.01 \\
\hline 21.00000 & 20.98 & 53.90 \\
\hline 31.50000 & 20.85 & 62.09 \\
\hline 52.60000 & 20.65 & 72.44 \\
\hline 105.00000 & 20.45 & 86.42 \\
\hline 210.00000 & 20.36 & 100.45 \\
\hline
\end{tabular}

A TOMIC DISPLAZEMENT CROSS-SECTIONS (BARNS) BY FAST ELECTRONS IN FE $\mathrm{Z}=26 \quad \mathrm{~A}=55.850$

$\begin{array}{ccc}T D=38.0 & \text { EV } & \begin{array}{c}\text { EY }=1.085735 \\ \text { PEI }\end{array} \\ \text { ENERGY(MEV) } & 0.01 & \text { CASCADE } \\ 1.09000 & 0.01 \\ 1.10000 & 0.05 & 0.05 \\ 1.12000 & 0.13 & 0.13 \\ 1.16000 & 0.34 & 0.34 \\ 1.20000 & 0.59 & 0.59 \\ 1.25000 & 0.94 & 0.94 \\ 1.32000 & 1.49 & 1.49 \\ 1.41000 & 2.25 & 2.25 \\ 1.52000 & 3.23 & 3.23 \\ 1.58000 & 4.65 & 4.65 \\ 1.90000 & 6.47 & 6.49 \\ 2.17000 & 8.44 & 8.60 \\ 2.49000 & 10.38 & 10.88 \\ 2.93000 & 12.46 & 13.69 \\ 3.47000 & 14.33 & 16.70 \\ 4.12000 & 15.90 & 19.84 \\ 4.88000 & 17.13 & 22.99 \\ 5.54000 & 17.95 & 25.71 \\ 6.51000 & 18.59 & 28.44 \\ 7.50000 & 19.11 & 31.39 \\ 9.22000 & 19.56 & 35.10 \\ 10.80000 & 19.79 & 38.14 \\ 16.20000 & 20.03 & 45.97 \\ 21.70000 & 20.03 & 51.62 \\ 32.50000 & 19.90 & 59.41 \\ 54.20000 & 19.70 & 69.27 \\ 108.00000 & 19.52 & 82.58 \\ 217.00000 & 19.43 & 96.07\end{array}$

\begin{tabular}{ccc}
$T D=92.0$ & EV & \multicolumn{1}{c}{ ET $=1.117982$ MEV } \\
ENERGY (1IEV) & PRIARY & CASCADE \\
1.12000 & 0.01 & 0.01 \\
1.14000 & 0.07 & 0.07 \\
1.16000 & 0.15 & 0.15 \\
1.19000 & 0.30 & 0.30 \\
1.24000 & 0.58 & 0.58 \\
1.29000 & 0.91 & 0.91 \\
1.36000 & 1.42 & 1.42 \\
1.45000 & 2.13 & 2.13 \\
1.56000 & 3.04 & 3.04 \\
1.73000 & 4.45 & 4.45 \\
1.95000 & 6.15 & 6.17 \\
2.23000 & 8.06 & 8.21 \\
2.57000 & 9.98 & 10.48 \\
3.01000 & 11.92 & 13.10 \\
3.57000 & 13.73 & 16.02 \\
4.24000 & 15.23 & 19.03 \\
5.03000 & 16.41 & 22.08 \\
5.81000 & 17.19 & 24.69 \\
6.70000 & 17.80 & 27.28 \\
7.82000 & 18.29 & 30.11 \\
9.50000 & 18.71 & 33.69 \\
11.10000 & 18.93 & 36.56 \\
16.70000 & 19.16 & 44.11 \\
22.30000 & 19.15 & 49.46 \\
33.50000 & 19.03 & 56.97 \\
55.80000 & 18.85 & 66.38 \\
111.00000 & 18.67 & 79.09 \\
223.00000 & 18.59 & 91.98
\end{tabular}

\begin{tabular}{|c|c|c|}
\hline $\mathrm{TD}=36,0$ & $E T=1$ & \\
\hline ENERGY (YEV) & EV $\begin{array}{l}\text { ET }=1 \% \\
\text { PRIMARY }\end{array}$ & $\begin{array}{l}9603 \text { MEV } \\
\text { CASCADE }\end{array}$ \\
\hline 1.16500 & 0.03 & 0.03 \\
\hline 1.17000 & 0.06 & 0.06 \\
\hline 1.19300 & 0.13 & 0.13 \\
\hline 1.23000 & 0.31 & 0.31 \\
\hline 1.27300 & 0.52 & 0.52 \\
\hline 1.33300 & 0.88 & 0.88 \\
\hline 1.40000 & 1.36 & 1.36 \\
\hline 1.49000 & 2.03 & 2.03 \\
\hline 1.60300 & 2.88 & 2.88 \\
\hline 1.78000 & 4.27 & 4.27 \\
\hline 2.01300 & 5.94 & 5.96 \\
\hline 2.29000 & 7.72 & 7.87 \\
\hline 2.64300 & 9.57 & 10.05 \\
\hline 3.10300 & 11.47 & 12.62 \\
\hline 3.67000 & 13.18 & 15.40 \\
\hline 4.36000 & 14.62 & 18.30 \\
\hline 5.17000 & 15.74 & 21.23 \\
\hline 5.97000 & 16.49 & 23.72 \\
\hline 6.89300 & 17.07 & 26.23 \\
\hline 8.04200 & 17.54 & 28.94 \\
\hline 9.77000 & 17.94 & 32.38 \\
\hline 11.40000 & 18.15 & 35.11 \\
\hline 17.20000 & 18.36 & 42.40 \\
\hline 22.90000 & 18.35 & 47.48 \\
\hline 34.40300 & 18.24 & 54.68 \\
\hline 57.40000 & 18.06 & 63.74 \\
\hline 114.00000 & 17.89 & 75.88 \\
\hline & 17.81 & \\
\hline
\end{tabular}


ATOMIC DISPLAZEMENT CROSS-SECTIONS (BARNS) BY FAST ELECTRONS IN NI $\mathrm{Z}=28 \quad \mathrm{~A}=58.710$

\begin{tabular}{crr} 
TD $=4.0$ & EV & \multicolumn{2}{c}{ BT=0.097661 MEV } \\
ENERGY (MEV) & PRIMARY & CASCADE \\
0.09850 & 12.69 & 12.69 \\
0.09960 & 25.74 & 25.74 \\
0.10100 & 43.25 & 43.25 \\
0.10400 & 78.01 & 78.01 \\
0.10800 & 119.17 & 119.17 \\
0.11300 & 153.57 & 163.57 \\
0.11900 & 209.40 & 208.40 \\
0.12600 & 251.44 & 251.44 \\
0.13600 & 299.82 & 299.82 \\
0.15100 & 352.16 & 352.16 \\
0.17000 & 396.28 & 396.28 \\
0.19500 & 432.51 & 433.46 \\
0.22400 & 457.60 & 464.99 \\
0.25300 & 476.82 & 497.16 \\
0.31200 & 489.71 & 528.43 \\
0.37100 & 497.83 & 558.93 \\
0.43900 & 502.82 & 588.90 \\
0.50700 & 505.54 & 615.66 \\
0.58500 & 507.58 & 643.84 \\
0.68300 & 508.97 & 676.57 \\
0.83000 & 509.89 & 721.62 \\
0.97600 & 510.08 & 762.60 \\
1.46000 & 508.40 & 878.81 \\
1.95000 & 505.54 & 974.39 \\
2.92000 & 499.96 & 1122.23 \\
4.88000 & 492.05 & 1329.11 \\
9.76000 & 481.94 & 1628.81 \\
19.50000 & 475.51 & 1941.39 \\
29.20000 & 473.45 & 2127.07 \\
48.80000 & 471.85 & 2365.23 \\
68.30000 & 471.13 & 2521.85 \\
97.60000 & 470.46 & 2688.35 \\
195.00000 & 469.24 & 3011.41
\end{tabular}

\begin{tabular}{rrr} 
TD $=8.0$ & \multicolumn{2}{c}{ ET $=0.181686$ MEV } \\
ENERGY (MEV) & PRIMARY & CASZADE \\
0.18300 & 2.53 & 2.53 \\
0.18500 & 6.28 & 6.28 \\
0.18800 & 11.58 & 11.68 \\
0.19400 & 21.78 & 21.78 \\
0.20100 & 32.49 & 32.49 \\
0.21000 & 44.80 & 44.80 \\
0.22100 & 58.01 & 58.01 \\
0.23600 & 73.39 & 73.39 \\
0.25400 & 88.79 & 88.79 \\
0.28100 & 107.34 & 107.34 \\
0.31700 & 126.32 & 126.32 \\
0.36300 & 144.51 & 145.20 \\
0.41700 & 160.47 & 163.79 \\
0.49000 & 176.43 & 185.06 \\
0.58100 & 190.86 & 207.60 \\
0.69000 & 203.31 & 230.92 \\
0.81700 & 213.72 & 254.71 \\
0.94400 & 221.32 & 275.94 \\
1.09000 & 227.74 & 297.96 \\
1.27000 & 233.40 & 322.41 \\
1.54000 & 238.96 & 354.83 \\
1.81000 & 242.38 & 383.30 \\
2.72000 & 246.93 & 460.05 \\
3.63000 & 247.51 & 518.05 \\
5.45000 & 246.35 & 603.74 \\
9.08000 & 243.38 & 715.77 \\
18.10000 & 239.34 & 871.74 \\
36.30000 & 236.97 & 1032.26 \\
54.50000 & 236.23 & 1126.76 \\
90.80000 & 235.62 & 1245.93 \\
127.00000 & 235.30 & 1324.34 \\
181.00000 & 234.99 & 1407.13 \\
363.00000 & 234.37 & 1569.73 \\
& &
\end{tabular}

\begin{tabular}{|c|c|c|}
\hline \multirow{2}{*}{$\begin{array}{r}T D=12.0 \\
\text { ENERGY (YRV) }\end{array}$} & $\mathrm{ET}=0$. & \\
\hline & PRIMARY & CASCADE \\
\hline 0.25900 & 1.53 & 1.53 \\
\hline 0.26100 & 2.77 & 2.77 \\
\hline 0.26500 & 5.77 & 5.77 \\
\hline 0.27400 & 10.32 & 10.32 \\
\hline 0.28400 & 15.62 & 15.62 \\
\hline 0.29700 & 21.94 & 21.94 \\
\hline 0.31300 & 28.99 & 28.99 \\
\hline 0.33300 & 36.86 & 36.86 \\
\hline 0.35900 & 45.85 & 45.85 \\
\hline 0.39700 & 57.04 & 57.04 \\
\hline 0.44300 & 69.44 & 69.44 \\
\hline 0.51300 & 82.17 & 82.72 \\
\hline 0.59000 & 94.17 & 96.44 \\
\hline 0.59200 & 106.55 & 112.29 \\
\hline 0.82100 & 118.33 & 129.71 \\
\hline $0.97 \$ 00$ & 128.58 & 147.75 \\
\hline 1.15300 & 137.12 & 165.98 \\
\hline 1.33500 & 143.45 & 182.53 \\
\hline 1.53300 & 148.59 & 199.03 \\
\hline 1.79000 & 153.31 & 218.14 \\
\hline 2.18500 & 157.78 & 243.04 \\
\hline 2.56000 & 160.39 & 264.03 \\
\hline 3.84300 & 163.77 & 319.40 \\
\hline 5.13000 & 164.22 & 360.63 \\
\hline 7.69000 & 163.50 & 420.00 \\
\hline 12.80300 & 161.57 & 496.45 \\
\hline 25.60300 & 159.11 & 602.37 \\
\hline 51.30000 & 157.78 & 709.95 \\
\hline 76.90300 & 157.35 & 772.94 \\
\hline 128.00300 & 156.99 & 852.39 \\
\hline 179.00300 & 156.78 & 904.67 \\
\hline 256.00300 & 156.58 & 960.44 \\
\hline 513.00000 & 156.16 & 1068.74 \\
\hline
\end{tabular}
ATONIC DISPLAZEMENT CROSS-SRCTIONS (BARNS) BY FAST RLECTRONS IN $N$ I
$z=28 \quad A=58.710$

\begin{tabular}{|c|c|c|}
\hline \multirow{2}{*}{$\begin{array}{r}T D=16.0 \\
\text { ENERGY (MEV) }\end{array}$} & \multicolumn{2}{|c|}{$\mathrm{ET}=0.324768 \mathrm{MEV}$} \\
\hline & PRIMARY & CASCADE \\
\hline 0.32800 & 0.94 & 0.94 \\
\hline 0.33100 & 1.80 & 1.80 \\
\hline 0.33700 & 3.47 & 3.47 \\
\hline 0.34700 & 6.17 & 6.17 \\
\hline 0.36000 & 9.52 & 9.52 \\
\hline 0.37600 & 13.39 & 13.39 \\
\hline 0.39600 & 17.92 & 17.92 \\
\hline 0.42200 & 23.35 & 23.35 \\
\hline 0.45400 & 29.44 & 29.44 \\
\hline 0.50300 & 37.72 & 37.72 \\
\hline 0.56800 & 47.13 & 47.14 \\
\hline 0.64900 & 56.94 & 57.41 \\
\hline 0.74600 & 66.56 & 68.32 \\
\hline 0.87600 & 76.75 & 81.23 \\
\hline 1.03300 & 85.96 & 94.68 \\
\hline 1.23000 & 94.77 & 109.97 \\
\hline 1.46000 & 101.95 & 125.30 \\
\hline 1.68000 & 106.89 & 138.28 \\
\hline 1.94000 & 111.11 & 151.97 \\
\hline 2.27000 & 114.81 & 167.33 \\
\hline 2.76000 & 118.22 & 187.00 \\
\hline 3.24000 & 120.19 & 20.3 .56 \\
\hline 4.87000 & 122.64 & 247.03 \\
\hline 6.49000 & 122.91 & 278.60 \\
\hline 9.74000 & 122.32 & 324.16 \\
\hline 16.20000 & 120.87 & 382.10 \\
\hline 32.40000 & 119.15 & 462.06 \\
\hline 64.90000 & 118.25 & 542.95 \\
\hline 97.40000 & 117.96 & 590.41 \\
\hline 162.00000 & 117.70 & 649.96 \\
\hline
\end{tabular}

\begin{tabular}{rrr} 
TD $=20.0$ & EV & \multicolumn{2}{c}{ ET $=0.387807$ IEV } \\
ENERGY (MEV) & PRIMA & CASEADE \\
0.39100 & 0.51 & 0.51 \\
0.39500 & 1.15 & 1.15 \\
0.40300 & 2.41 & 2.41 \\
0.41400 & 4.10 & 4.10 \\
0.43000 & 6.49 & 6.49 \\
0.44900 & 9.24 & 9.24 \\
0.47300 & 12.57 & 12.57 \\
0.50400 & 16.63 & 16.63 \\
0.54200 & 21.30 & 21.30 \\
0.60100 & 27.91 & 27.91 \\
0.67800 & 35.50 & 35.52 \\
0.77500 & 43.66 & 44.06 \\
0.89100 & 51.73 & 53.22 \\
1.04000 & 60.02 & 63.70 \\
1.24000 & 68.46 & 76.05 \\
1.47000 & 75.52 & 88.46 \\
1.74000 & 81.41 & 101.19 \\
2.01000 & 85.60 & 112.41 \\
2.32000 & 89.02 & 123.84 \\
2.71000 & 91.96 & 136.52 \\
3.29000 & 94.66 & 152.71 \\
3.87000 & 96.22 & 166.55 \\
5.81000 & 98.04 & 202.06 \\
7.75000 & 98.21 & 227.84 \\
11.50000 & 97.70 & 264.46 \\
19.30000 & 96.54 & 311.17 \\
38.70000 & 95.22 & 375.63 \\
77.50000 & 94.55 & 440.44 \\
116.00000 & 94.34 & 478.19 \\
193.00000 & 94.14 & 525.88 \\
& &
\end{tabular}

\begin{tabular}{|c|c|c|}
\hline$T D=24.0$ & $E T=0.4$ & 706 MEV \\
\hline EN ERGY (IEV) & PRIMARY & CASCADE \\
\hline 0.45100 & 0.43 & 0.43 \\
\hline 0.45500 & 0.82 & 0.82 \\
\hline $0.46+00$ & 1.71 & 1.71 \\
\hline 0.47700 & 2.99 & 2.99 \\
\hline 0.49500 & 4.75 & 4.75 \\
\hline 0.51800 & 6.96 & 6.96 \\
\hline 0.54200 & 9.41 & 9.41 \\
\hline 0.58300 & 12.70 & 12.70 \\
\hline 0.62500 & 16.62 & 16.62 \\
\hline 0.69200 & 22.05 & 22.05 \\
\hline 0.79100 & 28.51 & 28.53 \\
\hline 0.89300 & 35.52 & 35.89 \\
\hline 1.02300 & 42.17 & 43.43 \\
\hline 1.20000 & 49.71 & 52.96 \\
\hline 1.42300 & 56.69 & 63.22 \\
\hline 1.59300 & 62.90 & 74.22 \\
\hline 2.01300 & 68.05 & 85.54 \\
\hline 2.32300 & 71.55 & 95.17 \\
\hline 2.68000 & 74.41 & 105.06 \\
\hline 3.12000 & 76.79 & 115.69 \\
\hline 3.79000 & 79.00 & 129.56 \\
\hline 4.46000 & 80.26 & 141.37 \\
\hline 6.70500 & 81.68 & 171.49 \\
\hline 8.93300 & 81.78 & 193.15 \\
\hline 13.40000 & 81.31 & 224.09 \\
\hline 22.30000 & 80.34 & 263.21 \\
\hline 44.50300 & 79.30 & 316.87 \\
\hline 89.30300 & 78.77 & 370.93 \\
\hline 134.00000 & 78.59 & 402.61 \\
\hline 223.00200 & 78.43 & 442.38 \\
\hline
\end{tabular}


ATOMIC DISPLAZBHENT CROSS-SECTIONS (BARNS) BY FAST ELECTRONS IN NI $z=28 \quad A=58.710$

\begin{tabular}{rrr}
$\begin{array}{c}\text { TD } 28.0 \\
\text { ENER EY (HEV) }\end{array}$ & \multicolumn{2}{c}{ ET=0.502187 MEV } \\
PRIMARY & CASCADE \\
0.50700 & 0.32 & 0.32 \\
0.51200 & 0.65 & 0.65 \\
0.52200 & 1.32 & 1.32 \\
0.53700 & 2.34 & 2.34 \\
0.55700 & 3.72 & 3.72 \\
0.58200 & 5.44 & 5.44 \\
0.61200 & 7.51 & 7.51 \\
0.65200 & 10.22 & 10.22 \\
0.70300 & 13.58 & 13.58 \\
0.77800 & 18.24 & 18.24 \\
0.87800 & 23.86 & 23.89 \\
1.00000 & 29.84 & 30.16 \\
1.15000 & 35.99 & 37.15 \\
1.35000 & 42.53 & 45.47 \\
1.60000 & 48.70 & 54.63 \\
1.90000 & 54.05 & 64.22 \\
2.25000 & 58.40 & 73.94 \\
2.61000 & 61.52 & 82.70 \\
3.01000 & 63.94 & 91.27 \\
3.51000 & 65.98 & 100.67 \\
4.26000 & 67.82 & 112.72 \\
5.02000 & 68.87 & 123.08 \\
7.53000 & 70.00 & 149.10 \\
10.00000 & 70.05 & 167.58 \\
15.00000 & 69.63 & 194.21 \\
25.10000 & 68.80 & 228.21 \\
50.20000 & 67.93 & 274.29 \\
100.00000 & 67.50 & 320.32 \\
150.00000 & 67.36 & 347.47 \\
& &
\end{tabular}

\begin{tabular}{rrr} 
TD=32.0 EV & \multicolumn{2}{c}{ ET $=0.554784$ MEV } \\
ENERGY (MEV) & PRIMARY & CASCADE \\
0.56000 & 0.24 & 0.24 \\
0.56500 & 0.48 & 0.48 \\
0.57500 & 1.01 & 1.01 \\
0.59300 & 1.86 & 1.86 \\
0.61500 & 2.98 & 2.98 \\
0.64300 & 4.44 & 4.44 \\
0.67500 & 6.19 & 5.19 \\
0.72100 & 8.57 & 8.57 \\
0.77600 & 11.42 & 11.42 \\
0.85900 & 15.53 & 15.53 \\
0.97000 & 20.56 & 20.58 \\
1.10000 & 25.71 & 25.00 \\
1.27000 & 31.38 & 32.43 \\
1.49000 & 37.20 & 39.87 \\
1.77000 & 42.75 & 48.19 \\
2.10000 & 47.45 & 56.74 \\
2.49000 & 51.31 & 65.50 \\
2.88000 & 53.98 & 73.16 \\
3.32000 & 56.08 & 80.77 \\
3.88000 & 57.86 & 89.25 \\
4.71000 & 59.43 & 99.94 \\
5.54000 & 60.31 & 109.01 \\
8.32000 & 61.24 & 132.03 \\
11.00000 & 61.27 & 148.04 \\
16.60000 & 60.88 & 171.78 \\
27.70000 & 60.15 & 201.45 \\
55.40000 & 59.41 & 241.82 \\
110.00000 & 59.05 & 281.93 \\
166.00000 & 58.93 & 306.05
\end{tabular}

\begin{tabular}{|c|c|c|}
\hline $\mathrm{TD}=36.0$ & $\mathrm{ET}=0$. & $904 \mathrm{MEV}$ \\
\hline ENERGY (YEV) & PRIMARY & CASCADE \\
\hline 0.61300 & 0.18 & 0.18 \\
\hline 0.61700 & 0.42 & 0.42 \\
\hline 0.62300 & 0.86 & 0.86 \\
\hline 0.64700 & 1.54 & 1.54 \\
\hline 0.67100 & 2.49 & 2.49 \\
\hline 0.70100 & 3.72 & 3.72 \\
\hline 0.73700 & 5.24 & 5.24 \\
\hline 0.78500 & 7.33 & 7.33 \\
\hline 0.84500 & 9.87 & 9.87 \\
\hline 0.93700 & 13.57 & 13.57 \\
\hline 1.05000 & 17.81 & 17.83 \\
\hline 1.20300 & 22.76 & 23.04 \\
\hline 1.39000 & 28.00 & 29.01 \\
\hline 1.63000 & 33.22 & 35.74 \\
\hline 1.93000 & 38.12 & 43.15 \\
\hline 2.29300 & 42.33 & 50.89 \\
\hline 2.72000 & 45.79 & 58.90 \\
\hline 3.14300 & 48.12 & 65.72 \\
\hline 3.62300 & 49.97 & 72.59 \\
\hline 4.23000 & 51.53 & 80.20 \\
\hline 5.14000 & 52.91 & 89.86 \\
\hline 6.04300 & 53.65 & 97.94 \\
\hline 9.07000 & 54.43 & 118.54 \\
\hline 12.00000 & 54.44 & 132.87 \\
\hline 18.10300 & 54.08 & 154.01 \\
\hline 30.20000 & 53.44 & 180.43 \\
\hline 60.40200 & 52.79 & 216.35 \\
\hline 120.00300 & 52.48 & 252.05 \\
\hline 181.00000 & 52.37 & 273.47 \\
\hline
\end{tabular}

ATOMIC DISPLAZEHENT CROSS-SECTIONS (BARNS) BY PAST ELECTRONS IN NI $\mathrm{Z}=28 \quad \mathrm{~A}=58.710$

\begin{tabular}{|c|c|c|c|}
\hline $\begin{array}{r}T D=40.0 \\
E R G Y(M E V)\end{array}$ & $\mathrm{EV}$ & $\begin{aligned} & E T=0.6 \\
& \text { PRIMARY }\end{aligned}$ & $\begin{array}{l}368 \text { MEV } \\
\text { CASCADE }\end{array}$ \\
\hline 0.65900 & & $\begin{array}{r}1+16 \\
0.16\end{array}$ & $\begin{array}{r}0.16 \\
0.1\end{array}$ \\
\hline 0.66500 & & 0.32 & 0.32 \\
\hline 0.67800 & & 0.69 & 0.69 \\
\hline 0.69800 & & 1.29 & 1.29 \\
\hline 0.72400 & & 2.11 & 2.11 \\
\hline 0.75700 & & 3.20 & 3.20 \\
\hline 0.79500 & & 4.55 & 4.55 \\
\hline 0.84800 & & 6.39 & 6.39 \\
\hline 0.91400 & & 8.72 & 8.72 \\
\hline 1.01000 & & 12.01 & 12.01 \\
\hline 1.14000 & & 16.12 & 16.15 \\
\hline 1.30000 & & 20.56 & 20.83 \\
\hline 1.50000 & & 25.21 & 26.16 \\
\hline 1.76000 & & 29.99 & 32.34 \\
\hline 2.08000 & & 34.39 & 39.05 \\
\hline 2.48000 & - & 38.30 & 46.33 \\
\hline 2.93000 & & 41.31 & 53.41 \\
\hline 3.39000 & & 43.43 & 59.73 \\
\hline 3.91000 & & 45.09 & 66.00 \\
\hline 4.57000 & & 46.47 & 72.93 \\
\hline 5.54000 & & 47.66 & 81.59 \\
\hline 6.52000 & & 48.32 & 88.99 \\
\hline 9.79000 & & 48.99 & 107.62 \\
\hline 13.00000 & & 48.98 & 120.73 \\
\hline 19.50000 & & 48.64 & 139.55 \\
\hline 32.60000 & & 48.07 & 163.45 \\
\hline 65.20000 & & 47.50 & 195.81 \\
\hline 130.00000 & & 47.23 & 228.12 \\
\hline & & 47.13 & 247.14 \\
\hline
\end{tabular}

\begin{tabular}{|c|c|c|c|}
\hline $\begin{array}{r}T D=44 . \\
\text { TERGY (MEV) }\end{array}$ & 8 & עי & \\
\hline $\begin{array}{l}\text { JRGY (MEV) } \\
0.70500\end{array}$ & & $\begin{array}{r}\text { PRI IAR R } \\
0.13\end{array}$ & $\begin{array}{r}\text { CASCADE } \\
0.13\end{array}$ \\
\hline 0.71200 & & 0.28 & 0.28 \\
\hline 0.72600 & & 0.59 & \\
\hline 0.74700 & & 1.10 & 1 \\
\hline $\begin{array}{l}0.77500 \\
0.81000\end{array}$ & . & $\begin{array}{l}1.82 \\
2.78\end{array}$ & $\begin{array}{l}1.82 \\
2.78\end{array}$ \\
\hline 0.85200 & & 4.00 & 4.00 \\
\hline 0.90800 & & $\begin{array}{r}5.68 \\
7.78\end{array}$ & $\begin{array}{l}5.68 \\
7.78\end{array}$ \\
\hline $\begin{array}{l}0.97800 \\
1.08000\end{array}$ & & 10.77 & 10.77 \\
\hline & & 14.58 & \\
\hline 1.39000 & & 18.64 & 18. \\
\hline $1: 6$ & & 22. & \\
\hline 1.88 & & 88 & \\
\hline 2.23000 & & 31.40 & $\begin{array}{l}35.8 \\
42.3\end{array}$ \\
\hline $\begin{array}{l}2.65000 \\
3.14000\end{array}$ & & $\begin{array}{l}34.90 \\
37.69\end{array}$ & 49.0 \\
\hline 3.63000 & 1 & 39. & 54.7 \\
\hline 4.19000 & & 41.08 & 60. \\
\hline 4.89000 & & 42. 31 & \\
\hline 5.94000 & & 43.38 & 74.8 \\
\hline 6.98000 & & 43.96 & $\begin{array}{l}81.5 \\
98.2\end{array}$ \\
\hline 10.40000 & & 44.53 & \\
\hline 13.90000 & & $\begin{array}{l}44.51 \\
44.20\end{array}$ & $\begin{array}{l}110.4 \\
127.7\end{array}$ \\
\hline 34.90000 & & 43.68 & 14 \\
\hline & & 43.17 & \\
\hline 139.00000 & & 42.93 & 208.2 \\
\hline & & & \\
\hline
\end{tabular}

\begin{tabular}{|c|c|c|}
\hline$T D=+8.0$ & $\mathrm{ET}=0$ & 305 \\
\hline ENERGY (YEV) & PRIMARY & CASCADE \\
\hline $0.75000^{\circ}$ & 0.11 & 0.11 \\
\hline 0.75800 & 0.25 & 0.25 \\
\hline 0.77300 & 0.53 & 0.53 \\
\hline 0.79500 & 0.96 & 0.96 \\
\hline 0.82500 & 1.61 & 1.61 \\
\hline 0.86200 & 2.48 & 2.48 \\
\hline 0.90500 & 3.57 & 3.57 \\
\hline 0.96500 & 5.11 & 5.11 \\
\hline 1.04300 & 7.04 & 7.04 \\
\hline 1.15300 & 9.84 & 9.84 \\
\hline 1.30000 & 13.38 & 13.41 \\
\hline 1.48000 & 17.12 & 17.37 \\
\hline 1.70000 & 20.96 & 21.79 \\
\hline 2.00000 & 25.09 & 27.17 \\
\hline 2.37300 & 28.87 & 33.00 \\
\hline 2.82300 & 32.10 & 39.15 \\
\hline 3.34300 & 34.64 & 45.27 \\
\hline 3.86300 & 36.38 & 50.60 \\
\hline 4.45000 & 37.72 & 55.89 \\
\hline 5.20000 & 38.84 & 61.75 \\
\hline 6.31300 & 39.80 & 69.10 \\
\hline 7.43000 & 40.32 & 75.35 \\
\hline 11.10000 & 40.82 & 90.80 \\
\hline 14.80300 & 40.79 & 101.95 \\
\hline 22.20300 & 40.50 & 117.68 \\
\hline 37.10000 & 40.02 & 137.63 \\
\hline 74.30000 & 39.57 & 164.69 \\
\hline 148.00300 & 39.35 & 191.60 \\
\hline 222.00300 & 39.27 & 207.45 \\
\hline
\end{tabular}


A TOMIC DISPLAZRMENT CROSS-SECTIONS (BARNS) BY FAST ELECTRONS IN N

\begin{tabular}{rrr} 
TD $=52.0$ & EV & \multicolumn{1}{c}{ ET $=0.786162$ MEV } \\
ENERGY(MEV & PRIMARY & CASCADE \\
0.79400 & 0.11 & 0.11 \\
0.80100 & 0.21 & 0.21 \\
0.81700 & 0.45 & 0.45 \\
0.84100 & 0.85 & 0.85 \\
0.87200 & 1.42 & 1.42 \\
0.91100 & 2.21 & 2.21 \\
0.95900 & 3.23 & 3.23 \\
1.02000 & 4.60 & 4.60 \\
1.10000 & 6.43 & 6.43 \\
1.21000 & 8.89 & 8.89 \\
1.37000 & 12.24 & 12.26 \\
1.57000 & 15.90 & 16.15 \\
1.80000 & 19.42 & 20.22 \\
2.12000 & 23.29 & 25.28 \\
2.51000 & 26.76 & 30.69 \\
2.98000 & 29.71 & 36.35 \\
3.53000 & 32.05 & 42.06 \\
4.08000 & 33.64 & 47.01 \\
4.71000 & 34.89 & 51.98 \\
5.50000 & 35.90 & 57.41 \\
5.68000 & 36.77 & 64.25 \\
7.86000 & 37.23 & 70.01 \\
11.70000 & 37.67 & 84.19 \\
15.70000 & 37.64 & 94.74 \\
23.50000 & 37.37 & 109.20 \\
39.30000 & 36.93 & 127.65 \\
78.50000 & 36.52 & 152.59 \\
157.00000 & 36.32 & 177.54 \\
& &
\end{tabular}

\begin{tabular}{|c|c|c|}
\hline$T D=56.0$ & $\mathrm{ET}=0.8$ & MEV \\
\hline IERGY (MEV) & PRIMARY & CAS $\approx A D E$ \\
\hline 0.83500 & 0.08 & 0.08 \\
\hline 0.84400 & 0.19 & 0.19 \\
\hline 0.86000 & 0.40 & 0.40 \\
\hline 0.88500 & 0.75 & 0.75 \\
\hline 0.91800 & 1. 28 & 1.28 \\
\hline 0.96000 & 2.01 & 2.01 \\
\hline 1.00000 & 2.76 & 2.76 \\
\hline 1.07000 & 4.14 & 4.14 \\
\hline 1.15000 & 5.75 & 5.75 \\
\hline 1.28000 & 8.34 & 8.34 \\
\hline 1.44000 & 11.31 & 11.34 \\
\hline 1.65000 & 14.75 & 14.98 \\
\hline 1.90000 & 18.15 & 18.92 \\
\hline 2.23000 & 21.67 & 23.57 \\
\hline 2.64000 & 24.91 & 28.64 \\
\hline 3.14000 & 27.68 & 33.99 \\
\hline 3.72000 & 29.85 & 39.34 \\
\hline 4.30000 & 31.31 & 43.97 \\
\hline 4.96000 & 32.45 & 48.59 \\
\hline 5.79000 & 33.37 & 53.63 \\
\hline 7.03000 & 34.17 & 60.01 \\
\hline 8.27000 & 34.59 & 65.37 \\
\hline 12.40000 & 34.98 & 78.82 \\
\hline 16.50000 & 34.95 & 88.34 \\
\hline 24.80000 & 34.69 & 101.93 \\
\hline 41.30000 & 34.28 & 118.94 \\
\hline 82.70000 & 33.90 & 142.15 \\
\hline 165.00000 & 33.72 & 165.28 \\
\hline
\end{tabular}

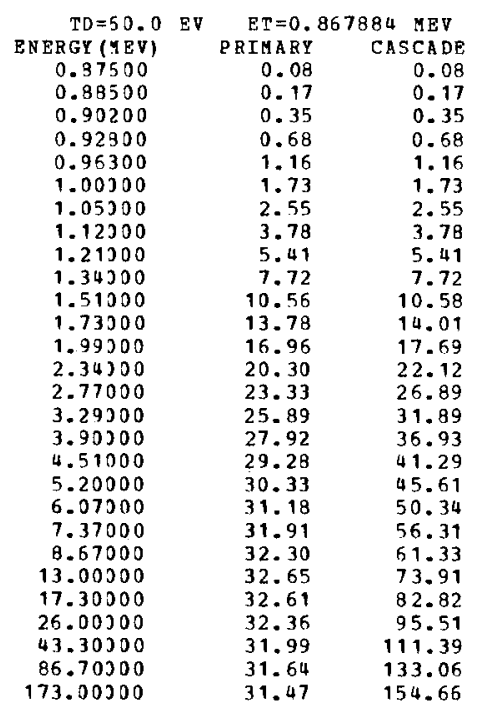

ATOMIC DISPLAZEMENT CROSS-SECTIONS (BARNS) BY FAST ELECTRONS IN NI $\mathrm{Z}=28 \quad \mathrm{~A}=58.710$

\begin{tabular}{rrr} 
TD $=64.0$ & EV & \multicolumn{1}{c}{ ET $=0.906981$} \\
ENERGY MEV \\
0.91600 & PRIMARY & CASCADE \\
0.92500 & 0.07 & 0.07 \\
0.94300 & 0.15 & 0.15 \\
0.97000 & 0.32 & 0.32 \\
1.00000 & 0.61 & 0.61 \\
1.05000 & 0.98 & 0.98 \\
1.10000 & 1.66 & 1.66 \\
1.17000 & 2.40 & 2.40 \\
1.25000 & 3.50 & 3.50 \\
1.40000 & 4.96 & 4.96 \\
1.58000 & 7.22 & 7.22 \\
1.91000 & 9.93 & 9.96 \\
2.08000 & 12.97 & 13.19 \\
2.44000 & 15.94 & 16.65 \\
2.90000 & 19.05 & 20.78 \\
3.44000 & 21.96 & 25.39 \\
4.08000 & 24.34 & 30.07 \\
4.71000 & 26.24 & 34.84 \\
5.44000 & 27.49 & 38.90 \\
6.34000 & 28.48 & 43.02 \\
7.70000 & 29.26 & 47.42 \\
9.06000 & 29.94 & 53.05 \\
13.60000 & 30.29 & 57.77 \\
18.10000 & 30.61 & 69.63 \\
27.20000 & 30.57 & 77.99 \\
45.30000 & 30.33 & 89.89 \\
90.60000 & 29.98 & 104.79 \\
181.00000 & 29.66 & 125.08 \\
& 29.50 & 145.37
\end{tabular}

$\begin{array}{crr}\text { TD }=68.0 & \text { EV } & \text { ET }=0.945027 \text { IEV } \\ \text { ENERGY (MEV) } & \text { PRIMARY } & \text { CASEADE } \\ 0.95400 & 0.06 & 0.06 \\ 0.96300 & 0.13 & 0.13 \\ 0.98200 & 0.29 & 0.29 \\ 1.01000 & 0.55 & 0.55 \\ 1.04000 & 0.87 & 0.87 \\ 1.09000 & 1.48 & 1.48 \\ 1.15000 & 2.28 & 2.28 \\ 1.22000 & 3.28 & 3.28 \\ 1.32000 & 4.76 & 4.76 \\ 1.46000 & 6.80 & 6.80 \\ 1.65000 & 9.40 & 9.43 \\ 1.89000 & 12.28 & 12.50 \\ 2.17000 & 15.07 & 15.76 \\ 2.55000 & 18.04 & 19.72 \\ 3.02000 & 20.72 & 24.00 \\ 3.59000 & 22.98 & 28.49 \\ 4.25000 & 24.74 & 32.95 \\ 4.91000 & 25.92 & 36.81 \\ 5.67000 & 26.84 & 40.70 \\ 5.61000 & 27.56 & 44.86 \\ 8.03000 & 28.19 & 50.18 \\ 9.45000 & 28.52 & 54.65 \\ 14.10000 & 28.81 & 65.65 \\ 18.90000 & 28.76 & 73.73 \\ 28.30000 & 28.54 & 84.85 \\ 47.20000 & 28.21 & 98.92 \\ 94.50000 & 27.91 & 118.05 \\ 189.00000 & 27.76 & 137.17\end{array}$

\begin{tabular}{|c|c|c|}
\hline $\mathrm{TD}=72.0$ & \multicolumn{2}{|c|}{$\mathrm{ET}=0.982105 \mathrm{MEV}$} \\
\hline ENERGY (YEV) & PRIMARY & CASCADE \\
\hline 0.99100 & 0.05 & 0.05 \\
\hline 1.00300 & 0.11 & 0.11 \\
\hline 1.02300 & 0.26 & 0.26 \\
\hline 1.05300 & 0.51 & 0.51 \\
\hline 1.09300 & 0.90 & 0.90 \\
\hline 1.13000 & 1.34 & 1.34 \\
\hline 1.19300 & 2.07 & 2.07 \\
\hline 1.27000 & 3.10 & 3.10 \\
\hline 1.37000 & 4.45 & 4.45 \\
\hline 1.52000 & 6.46 & 6.46 \\
\hline 1.71000 & 8.83 & 8.86 \\
\hline 1.96000 & 11.58 & 11.79 \\
\hline 2.25300 & 14.23 & 14.89 \\
\hline 2.65000 & 17.08 & 18.70 \\
\hline 3.14000 & 19.62 & 22.78 \\
\hline 3.73000 & 21.75 & 27.03 \\
\hline 4.41000 & 23.40 & 31.23 \\
\hline 5.10000 & 24.51 & 34.92 \\
\hline 5.39000 & 25.37 & 38.60 \\
\hline 6.87000 & 26.06 & 42.56 \\
\hline 8.34000 & 26.64 & 47.58 \\
\hline 9.82300 & 26.94 & 51.82 \\
\hline 14.70300 & 27.20 & 62.32 \\
\hline 19.60000 & 27.16 & 59.82 \\
\hline 29.40000 & 26.95 & 80.37 \\
\hline 49.10300 & 26.64 & 93.70 \\
\hline 98.20300 & 26.36 & 111.74 \\
\hline 196.00300 & 26.22 & 129.75 \\
\hline
\end{tabular}


ATOAIC DISPLACEMENT CROSS-SECTIONS (BARNS) BY FAST ELECTRONS IN NI $=28 \quad A=58.710$

\begin{tabular}{rrr} 
TDET6.0 & EV & \multicolumn{1}{c}{ ET $=1.018284$} \\
ENERGY $(Y E V)$ & PRIMARY & CASCADE \\
1.02000 & 0.01 & 0.01 \\
1.03000 & 0.06 & 0.06 \\
1.05000 & 0.18 & 0.18 \\
1.08000 & 0.40 & 0.40 \\
1.13000 & 0.84 & 0.84 \\
1.18000 & 1.34 & 1.34 \\
1.24000 & 2.01 & 2.01 \\
1.32000 & 2.96 & 2.96 \\
1.42000 & 4.20 & 4.20 \\
1.57000 & 6.04 & 6.04 \\
1.78000 & 8.46 & 8.48 \\
2.03000 & 10.98 & 11.18 \\
2.34000 & 13.57 & 14.22 \\
2.74000 & 16.18 & 17.73 \\
3.25000 & 18.51 & 21.64 \\
3.86000 & 20.63 & 25.67 \\
4.58000 & 22.21 & 29.75 \\
5.29000 & 23.26 & 33.23 \\
6.10000 & 24.06 & 36.70 \\
7.12000 & 24.70 & 40.48 \\
8.65000 & 25.25 & 45.26 \\
10.10000 & 25.52 & 49.08 \\
15.20000 & 25.77 & 59.17 \\
20.30000 & 25.73 & 66.32 \\
30.50000 & 25.53 & 76.36 \\
50.90000 & 25.23 & 88.98 \\
101.00000 & 24.97 & 105.88 \\
203.00000 & 24.84 & 123.12
\end{tabular}

\begin{tabular}{rrr} 
TD $=80.0$ & EV & \multicolumn{2}{c}{$E T=1.053626$} & MEV \\
ENERGY (HEV) & PRIMARY & CASCADE \\
1.06000 & 0.03 & 0.03 \\
1.07000 & 0.08 & 0.08 \\
1.09000 & 0.19 & 0.19 \\
1.12000 & 0.39 & 0.39 \\
1.15000 & 0.70 & 0.70 \\
1.22000 & 1.25 & 1.25 \\
1.28000 & 1.86 & 1.86 \\
1.36000 & 2.73 & 2.73 \\
1.47000 & 3.99 & 3.99 \\
1.63000 & 5.80 & 5.80 \\
1.84000 & 8.03 & 8.06 \\
2.10000 & 10.45 & 10.64 \\
2.42000 & 12.92 & 13.54 \\
2.84000 & 15.44 & 16.94 \\
3.37000 & 17.75 & 20.69 \\
4.00000 & 19.65 & 24.52 \\
4.74000 & 21.14 & 28.39 \\
5.47000 & 22.12 & 31.68 \\
6.32000 & 22.88 & 35.03 \\
7.37000 & 23.49 & 38.61 \\
8.95000 & 24.00 & 43.16 \\
10.50000 & 24.26 & 46.90 \\
15.80000 & 24.48 & 56.50 \\
21.00000 & 24.44 & 63.18 \\
31.60000 & 24.24 & 72.76 \\
52.60000 & 23.97 & 84.69 \\
105.00000 & 23.72 & 100.89 \\
210.00000 & 23.60 & 117.16
\end{tabular}

\begin{tabular}{|c|c|c|c|}
\hline \multirow{2}{*}{$\begin{array}{r}T D=34.0 \\
\text { ENERGY (MEV) }\end{array}$} & \multirow[t]{2}{*}{$\mathbf{E V}$} & \multicolumn{2}{|c|}{$E T=1.088188$ MEV } \\
\hline & & PRIMARY & CASCADE \\
\hline 1.09300 & & 0.01 & 0.01 \\
\hline 1.10000 & & 0.05 & 0.05 \\
\hline 1.13000 & & 0.20 & 0.20 \\
\hline 1.16000 & & 0.38 & 0.38 \\
\hline 1.20000 & & 0.67 & 0.67 \\
\hline 1.26000 & & 1.17 & 1.17 \\
\hline 1.32000 & & 1.74 & 1.74 \\
\hline 1.41000 & & 2.65 & 2.65 \\
\hline 1.52300 & & 3.81 & 3.81 \\
\hline 1.68000 & & 5.49 & 5.49 \\
\hline 1.90000 & & 7.65 & 7.58 \\
\hline 2.17000 & & 9.98 & 10.17 \\
\hline 2.50000 & & 12.34 & 12.95 \\
\hline 2.93000 & & 14.72 & 16.17 \\
\hline 3.48000 & & 16.94 & 19.77 \\
\hline 4.13000 & & 18.75 & 23.44 \\
\hline 4.89000 & & 20.15 & 27.12 \\
\hline 5.65000 & & 21.09 & 30.29 \\
\hline 6.52500 & & 21.81 & 33.46 \\
\hline 7.61000 & & 22.38 & 36.90 \\
\hline 9.24300 & & 22.85 & 41.23 \\
\hline 10.80300 & & 23.10 & 44.72 \\
\hline 16.30000 & & 23.32 & 53.94 \\
\hline 21.70000 & & 23.27 & 60.35 \\
\hline 32.60000 & & 23.09 & 69.44 \\
\hline 54.40000 & & 22.82 & 80.86 \\
\hline 108.00000 & & 22.59 & 96.17 \\
\hline 217.00000 & & 22.47 & 111.76 \\
\hline
\end{tabular}

ATOMIC DISPLAZEMBNT CROSS-SECTIONS (BARNS) BX FAST ELECTRONS IN N $\mathrm{z}=28 \quad \mathrm{~A}=58.710$

\begin{tabular}{|c|c|c|}
\hline \multirow{2}{*}{$\begin{array}{r}\mathrm{TD}=38.0 \\
\text { ENERGY (MEV) }\end{array}$} & \multicolumn{2}{|c|}{$\mathrm{BI}=1.122019 \mathrm{MEV}$} \\
\hline & PRIMARY & CASCADE \\
\hline 1.13000 & 0.03 & 0.03 \\
\hline 1.14000 & 0.07 & 0.07 \\
\hline 1.16000 & 0.16 & 0.16 \\
\hline 1.20000 & 0.38 & 0.38 \\
\hline 1.24000 & 0.65 & 0.65 \\
\hline 1.30000 & 1.12 & 1.12 \\
\hline 1.36000 & 1.64 & 1.64 \\
\hline 1.45000 & 2.48 & 2.48 \\
\hline 1.57000 & 3.66 & 3.66 \\
\hline 1.73000 & 5.22 & 5.22 \\
\hline 1.96000 & 7.33 & 7.35 \\
\hline 2.24000 & 9.57 & 9.76 \\
\hline 2.58000 & 11.82 & 12.41 \\
\hline 3.02000 & 14.08 & 15.48 \\
\hline 3.59000 & 16.21 & 18.95 \\
\hline 4.26000 & 17.93 & 22.47 \\
\hline 5.04000 & 19.26 & 25.97 \\
\hline 5.83000 & 20.16 & 29.03 \\
\hline 6.73000 & 20.84 & 32.07 \\
\hline 7.85000 & 21.38 & 35.35 \\
\hline 9.53000 & 21.83 & 39.49 \\
\hline 11.20000 & 22.06 & 42.94 \\
\hline 16.80000 & 22.26 & 51.62 \\
\hline 22.40000 & 22.21 & 57.77 \\
\hline 33.60000 & 22.03 & 66.42 \\
\hline 56.10000 & 21.78 & 77.34 \\
\hline 112.00000 & 21.56 & 92.08 \\
\hline 224.00000 & 21.45 & 106.86 \\
\hline
\end{tabular}

\begin{tabular}{rrr} 
TD=92.0 & EV & \multicolumn{1}{c}{ ET=1.155162 AEV } \\
ENERGY (BEV) & PRIMARY & CASCADE \\
1.16000 & 0.02 & 0.02 \\
1.17000 & 0.05 & 0.05 \\
1.20000 & 0.17 & 0.17 \\
1.23000 & 0.33 & 0.33 \\
1.26000 & 0.64 & 0.64 \\
1.33000 & 0.99 & 0.99 \\
1.40000 & 1.55 & 1.55 \\
1.50000 & 2.43 & 2.43 \\
1.61000 & 3.44 & 3.44 \\
1.79000 & 5.08 & 5.08 \\
2.02000 & 7.04 & 7.06 \\
2.31000 & 9.20 & 9.39 \\
2.65000 & 11.30 & 11.87 \\
3.11000 & 13.50 & 14.86 \\
3.69000 & 15.52 & 18.16 \\
4.38000 & 17.17 & 21.54 \\
5.19000 & 18.45 & 24.93 \\
6.00000 & 19.30 & 27.86 \\
6.93000 & 19.95 & 30.78 \\
8.08000 & 20.46 & 33.91 \\
9.81000 & 20.89 & 37.88 \\
11.50000 & 21.10 & 41.14 \\
17.30000 & 21.29 & 49.50 \\
23.10000 & 21.24 & 55.42 \\
34.60000 & 21.07 & 63.67 \\
57.70000 & 20.83 & 74.09 \\
115.00000 & 20.62 & 86.16 \\
231.00000 & 20.52 & 102.39 \\
& &
\end{tabular}

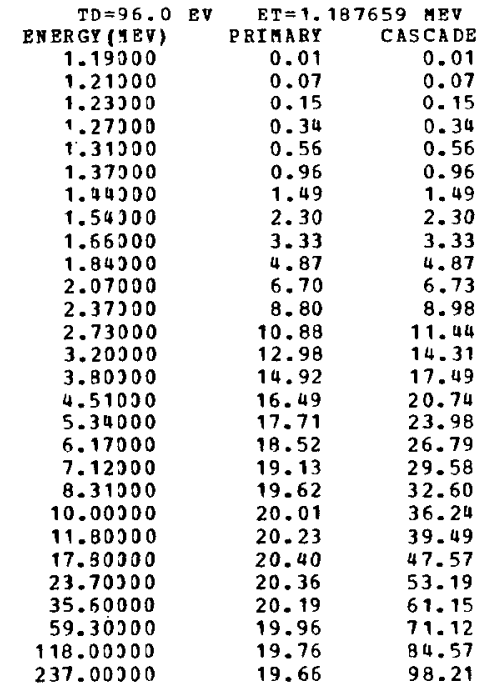


ATOMIC DISPLATEMENT CROSS-SECTIONS (BARNS) BY PAST ELECTRONS IN CU $=29 \quad A=63.540$

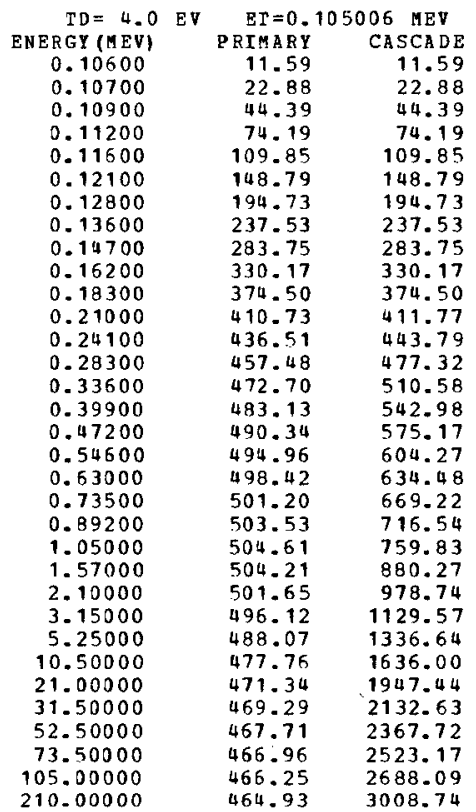

\begin{tabular}{rrr} 
TD= 8.0 & \multicolumn{2}{c}{ ET $=0.194554$ MEV } \\
ENERGY (MEV) & PRIMARY & CASCADE \\
0.19500 & 2.42 & 2.42 \\
0.19800 & 5.68 & 5.68 \\
0.20200 & 11.94 & 11.94 \\
0.20800 & 20.70 & 20.70 \\
0.21500 & 30.09 & 30.09 \\
0.22500 & 42.17 & 42.17 \\
0.23700 & 54.92 & 54.92 \\
0.25200 & 68.72 & 68.72 \\
0.27200 & 84.24 & 84.24 \\
0.30100 & 102.48 & 102.48 \\
0.34000 & 121.58 & 121.58 \\
0.38900 & 139.86 & 140.59 \\
0.44700 & 156.29 & 159.64 \\
0.52500 & 172.83 & 181.44 \\
0.62200 & 187.88 & 204.63 \\
0.73900 & 201.00 & 228.81 \\
0.87500 & 211.92 & 253.42 \\
1.01000 & 219.80 & 275.17 \\
1.16000 & 226.24 & 296.99 \\
1.36000 & 232.33 & 323.11 \\
1.65000 & 237.98 & 356.36 \\
1.94000 & 241.39 & 385.45 \\
2.91000 & 245.70 & 462.87 \\
3.89000 & 246.08 & 521.62 \\
5.83000 & 244.57 & 607.13 \\
9.72000 & 241.46 & 719.08 \\
19.40000 & 237.28 & 874.51 \\
38.90000 & 234.91 & 1033.86 \\
58.30000 & 234.17 & 1127.20 \\
97.20000 & 233.54 & 1245.54 \\
136.00000 & 233.21 & 1323.35 \\
194.00000 & 232.87 & 1405.60 \\
389.00000 & 232.20 & 1565.67 \\
& &
\end{tabular}

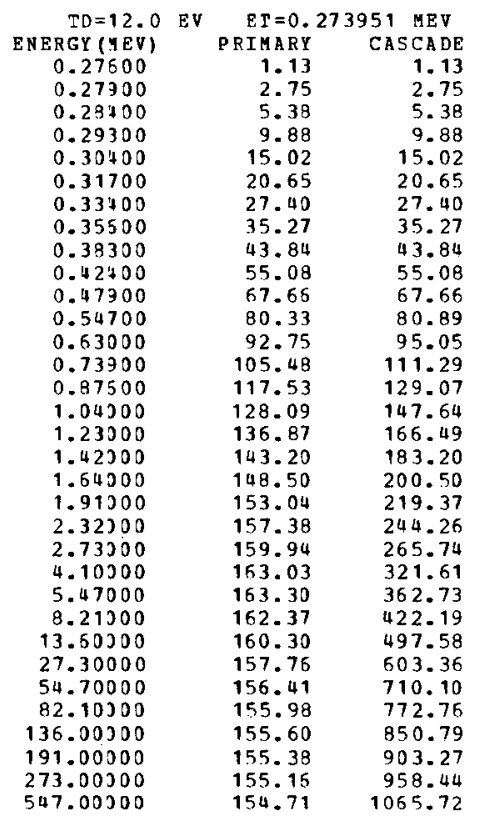

ATONIC DISPLAZEMENT CROSS-SECTIONS (BARNS) BY PAST ELECTRONS IN CU

\begin{tabular}{rrr} 
TD=16.0 & \multicolumn{2}{c}{ ET $=0.346025$ MEV } \\
ENERGY (HEV) & PRIMARY & CASCADE \\
0.34900 & 0.76 & 0.76 \\
0.35200 & 1.51 & 1.51 \\
0.35900 & 3.24 & 3.24 \\
0.37000 & 5.87 & 5.87 \\
0.38400 & 9.08 & 9.08 \\
0.40100 & 12.79 & 12.79 \\
0.42200 & 17.11 & 17.11 \\
0.44900 & 22.30 & 22.30 \\
0.48400 & 28.49 & 28.49 \\
0.53600 & 36.73 & 36.73 \\
0.60500 & 46.21 & 46.22 \\
0.69200 & 56.26 & 56.75 \\
0.79500 & 66.04 & 67.84 \\
0.93400 & 76.48 & 81.07 \\
1.10000 & 85.95 & 94.95 \\
1.31000 & 94.74 & 110.31 \\
1.55000 & 101.86 & 125.63 \\
1.79000 & 106.93 & 139.16 \\
2.07000 & 111.15 & 153.17 \\
2.42000 & 114.75 & 168.63 \\
2.94000 & 118.03 & 188.39 \\
3.45000 & 119.92 & 205.32 \\
5.19000 & 122.08 & 248.69 \\
5.92000 & 122.21 & 280.30 \\
10.30000 & 121.48 & 324.78 \\
17.30000 & 119.90 & 383.51 \\
34.60000 & 118.13 & 462.92 \\
59.20000 & 117.22 & 542.99 \\
103.00000 & 116.93 & 589.09 \\
173.00000 & 116.66 & 649.26
\end{tabular}
$\mathrm{Z}=29 \quad \mathrm{~A}=63.540$

\begin{tabular}{crr} 
TD $=20.0$ & EV & \multicolumn{1}{c}{ ET $=0.412490$ MEV } \\
ENERGY(MEV) & PRIMARY & CASEADE \\
0.41600 & 0.49 & 0.49 \\
0.42000 & 1.05 & 1.05 \\
0.42800 & 2.17 & 2.17 \\
0.44100 & 3.95 & 3.95 \\
0.45700 & 6.10 & 6.10 \\
0.47800 & 8.86 & 8.86 \\
0.50300 & 12.04 & 12.04 \\
0.53600 & 16.06 & 16.06 \\
0.57700 & 20.77 & 20.77 \\
0.63900 & 27.33 & 27.33 \\
0.72100 & 35.02 & 35.05 \\
0.82400 & 43.31 & 43.73 \\
0.94800 & 51.57 & 53.11 \\
1.11000 & 60.17 & 64.01 \\
1.31000 & 68.24 & 75.86 \\
1.56000 & 75.57 & 88.80 \\
1.85000 & 81.54 & 101.86 \\
2.14000 & 85.73 & 113.33 \\
2.47000 & 89.09 & 124.89 \\
2.88000 & 91.93 & 137.52 \\
3.50000 & 94.53 & 153.89 \\
4.12000 & 96.00 & 167.85 \\
6.18000 & 97.59 & 203.32 \\
8.24000 & 97.65 & 229.00 \\
12.30000 & 97.01 & 265.22 \\
20.60000 & 95.75 & 312.24 \\
41.20000 & 94.41 & 375.99 \\
82.40000 & 93.73 & 440.16 \\
123.00000 & 93.51 & 477.34 \\
206.00000 & 93.30 & 525.22
\end{tabular}

\begin{tabular}{|c|c|c|}
\hline$T D=24.0$ & $\mathrm{ET}=0$. & $483 \mathrm{MEV}$ \\
\hline ENBRGY (YEV) & PRIMARY & CASCADE \\
\hline 0.47900 & 0.39 & 0.39 \\
\hline 0.48300 & 0.74 & 0.74 \\
\hline 0.49350 & 1.62 & 1.62 \\
\hline 0.50700 & 2.85 & 2.85 \\
\hline 0.52500 & 4.53 & 4.53 \\
\hline 0.55300 & 6.64 & 6.64 \\
\hline 0.57300 & 9.08 & 9.08 \\
\hline 0.61500 & 12.32 & 12.32 \\
\hline $0.66+00$ & 16.26 & 16.26 \\
\hline 0.73500 & 21.72 & 21.72 \\
\hline 0.83300 & 28.30 & 28.32 \\
\hline 0.94300 & 35.36 & 35.74 \\
\hline 1.09300 & 42.45 & 43.80 \\
\hline 1.23000 & 50.00 & 53.41 \\
\hline 1.51300 & 56.90 & 63.65 \\
\hline 1.80300 & 63.18 & 74.92 \\
\hline 2.13300 & 68.16 & 86.06 \\
\hline 2.46000 & 71.63 & 95.82 \\
\hline 2.84300 & 74.44 & 105.75 \\
\hline 3.32000 & 76.81 & 116.74 \\
\hline 4.03000 & 78.90 & 130.61 \\
\hline 4.74300 & 80.07 & 142.42 \\
\hline 7.11000 & 81.29 & 172.41 \\
\hline 9.48000 & 81.30 & 194.03 \\
\hline 14.20000 & 80.73 & 224.68 \\
\hline 23.70000 & 79.70 & 263.77 \\
\hline 47.40000 & 78.62 & 317.02 \\
\hline 94.80300 & 78.09 & 370.55 \\
\hline 142.00000 & 77.91 & 401.82 \\
\hline .00300 & 77.73 & 441. \\
\hline
\end{tabular}


ATOAIC DISPLAZEHENT CROSS-SECTIONS (BARNS) BY PAST ELECTRONS IN CU

\begin{tabular}{rrr} 
TD $=28.0$ & EV & \multicolumn{2}{c}{ ET $=0.532800$ MEV } \\
ENERGY (MEV) & RRIMARY & CASCADE \\
0.53800 & 0.30 & 0.30 \\
0.54300 & 0.60 & 0.60 \\
0.55400 & 1.26 & 1.26 \\
0.57000 & 2.24 & 2.24 \\
0.59100 & 3.55 & 3.55 \\
0.61800 & 5.26 & 5.26 \\
0.65000 & 7.31 & 7.31 \\
0.69200 & 9.98 & 9.98 \\
0.74500 & 13.28 & 13.28 \\
0.82500 & 18.01 & 18.01 \\
0.93200 & 23.76 & 23.79 \\
1.05000 & 29.77 & 30.10 \\
1.22000 & 36.06 & 37.25 \\
1.43000 & 42.62 & 45.63 \\
1.70000 & 48.94 & 55.09 \\
2.02000 & 54.30 & 64.84 \\
2.39000 & 58.60 & 74.63 \\
2.77000 & 69.65 & 83.41 \\
3.19000 & 63.99 & 91.95 \\
3.72000 & 65.96 & 101.40 \\
4.52000 & 67.72 & 113.55 \\
5.32000 & 68.69 & 123.85 \\
7.99000 & 69.66 & 149.90 \\
10.60000 & 69.64 & 168.25 \\
15.90000 & 69.13 & 194.73 \\
26.60000 & 68.24 & 228.49 \\
53.20000 & 67.35 & 274.21 \\
106.00000 & 66.91 & 319.88 \\
159.00000 & 66.77 & 346.79 \\
& &
\end{tabular}

\begin{tabular}{rrr} 
TD 32.0 & \multicolumn{3}{c}{ EV } & \multicolumn{2}{c}{ ET=0.588028 MEV } \\
ENERGY (MEV) & PRIMARY & CASEADE \\
0.59300 & 0.21 & 0.21 \\
0.59900 & 0.46 & 0.46 \\
0.61100 & 0.98 & 0.98 \\
0.62900 & 1.78 & 1.78 \\
0.65200 & 2.86 & 2.86 \\
0.68200 & 4.30 & 4.30 \\
0.71700 & 6.03 & 6.03 \\
0.76400 & 8.37 & 8.37 \\
0.82300 & 11.28 & 11.28 \\
0.91100 & 15.43 & 15.43 \\
1.02000 & 20.17 & 20.20 \\
1.17000 & 25.89 & 26.20 \\
1.35000 & 31.60 & 32.72 \\
1.58000 & 37.38 & 40.15 \\
1.88000 & 43.02 & 48.68 \\
2.23000 & 47.69 & 57.31 \\
2.64000 & 51.47 & 68.08 \\
3.05000 & 54.08 & 73.73 \\
3.52000 & 56.14 & 81.45 \\
4.11000 & 57.84 & 89.90 \\
4.99000 & 59.34 & 100.62 \\
5.88000 & 60.15 & 109.79 \\
8.82000 & 60.94 & 132.70 \\
11.70000 & 60.90 & 148.83 \\
17.60000 & 60.44 & 172.25 \\
29.40000 & 59.67 & 201.77 \\
58.80000 & 58.91 & 241.82 \\
117.00000 & 58.54 & 281.73 \\
176.00000 & 58.41 & 305.45
\end{tabular}

\begin{tabular}{|c|c|c|c|}
\hline$T D=36.0$ & EV & $\mathrm{ET}=0.6$ & $\mathrm{MEV}$ \\
\hline ENERGY (IEV) & & PRIMARY & CASCADE \\
\hline 0.64700 & & 0.20 & 0.20 \\
\hline 0.65300 & & 0.38 & 0.38 \\
\hline 0.56500 & & 0.81 & 0.81 \\
\hline 0.68500 & & 1.46 & 1.46 \\
\hline 0.71100 & & 2.40 & 2.40 \\
\hline 0.74300 & & 3.62 & 3.62 \\
\hline 0.78100 & & 5.12 & 5.12 \\
\hline 0.83200 & 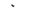 & 7.18 & 7.18 \\
\hline 0.89500 & & 9.75 & 9.75 \\
\hline 0.99200 & & 13.49 & 13.49 \\
\hline 1.12000 & & 18.07 & 18.11 \\
\hline 1.28000 & & 23.09 & 23.40 \\
\hline 1.47000 & & 28.08 & 29.12 \\
\hline 1.72300 & & 33.29 & 35.85 \\
\hline 2.04300 & & 38.26 & 43.42 \\
\hline 2.43000 & & 42.56 & 51.43 \\
\hline 2.88000 & & 45.93 & 59.40 \\
\hline 3.33000 & & 48.24 & 66.34 \\
\hline 3.84300 & & 50.04 & 73.25 \\
\hline 4.48000 & & 51.52 & 80.81 \\
\hline 5.44000 & & 52.81 & 90.44 \\
\hline 6.40000 & & 53.51 & 98.58 \\
\hline 9.60300 & & 54.16 & 119.06 \\
\hline 12.80000 & & 54.11 & 133.72 \\
\hline 19.20000 & & 53.68 & 154.45 \\
\hline 32.00000 & & 53.00 & 180.62 \\
\hline 64.00000 & & 52.34 & 216.26 \\
\hline 128.00300 & & 52.02 & 252.01 \\
\hline 192.00000 & & 51.91 & \\
\hline
\end{tabular}

A TOMIC DISPLAZEMENT CROSS-SECTIONS (BARNS) BY PAST ELECTRONS IN CU $z=29 \quad A=63.540$

$\begin{array}{rrr}\begin{array}{r}T D=40.0 \\ \text { EV }\end{array} & \begin{array}{r}\text { EY }=0.690893 \text { MEV } \\ \text { PRIMARY }\end{array} \\ \text { CASCADE } \\ 0.69700 & 0.14 & 0.14 \\ 0.70400 & 0.31 & 0.31 \\ 0.71800 & 0.67 & 0.67 \\ 0.73900 & 1.24 & 1.24 \\ 0.76600 & 2.02 & 2.02 \\ 0.80100 & 3.10 & 3.10 \\ 0.84200 & 4.44 & 4.44 \\ 0.89800 & 6.31 & 6.31 \\ 0.95700 & 8.64 & 8.64 \\ 1.07000 & 12.01 & 12.01 \\ 1.20000 & 15.96 & 15.98 \\ 1.38000 & 20.75 & 21.04 \\ 1.58000 & 25.20 & 26.15 \\ 1.86000 & 30.13 & 32.55 \\ 2.21000 & 34.67 & 39.55 \\ 2.62000 & 38.43 & 46.67 \\ 3.10000 & 41.44 & 53.87 \\ 3.59000 & 43.53 & 60.26 \\ 4.14000 & 45.13 & 66.54 \\ 4.83000 & 46.44 & 73.41 \\ 5.87000 & 47.59 & 82.18 \\ 6.90000 & 48.18 & 89.51 \\ 10.30000 & 48.74 & 107.82 \\ 13.80000 & 48.68 & 121.28 \\ 20.70000 & 48.29 & 139.97 \\ 34.50000 & 47.68 & 163.55 \\ 69.00000 & 47.10 & 195.66 \\ 138.00000 & 45.82 & 227.85 \\ 207.00000 & 46.72 & 246.70 \\ & & \end{array}$

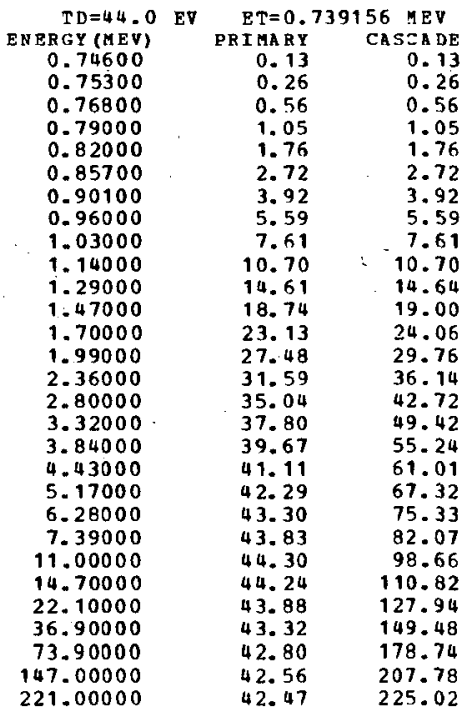

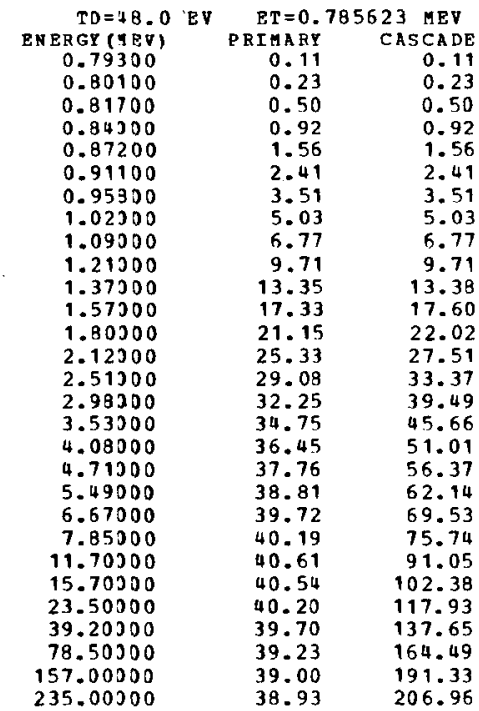


A TOMIC DISPLAZEMENT CROSS-SECTIONS (BARNS) BY FAST ELECTRONS IN CJ

\begin{tabular}{rrr}
$\begin{array}{r}\text { TD=52.0 } \\
\text { ENERGY (MEV) }\end{array}$ & \multicolumn{2}{c}{ ET=0.830482 MEV } \\
PRIMARY & CASCADE \\
0.83800 & 0.09 & 0.09 \\
0.84700 & 0.20 & 0.20 \\
0.86300 & 0.43 & 0.43 \\
0.88800 & 0.81 & 0.81 \\
0.92100 & 1.37 & 1.37 \\
0.96300 & 2.17 & 2.17 \\
1.01000 & 3.12 & 3.12 \\
1.07000 & 4.40 & 4.40 \\
1.16000 & 6.36 & 6.36 \\
1.28000 & 8.94 & 8.94 \\
1.45000 & 12.35 & 12.38 \\
1.66000 & 16.03 & 16.29 \\
1.91000 & 19.68 & 20.53 \\
2.24000 & 23.45 & 25.52 \\
2.65000 & 26.91 & 30.96 \\
3.15000 & 29.86 & 36.70 \\
3.73000 & 32.16 & 42.42 \\
4.31000 & 33.71 & 47.39 \\
4.98000 & 34.91 & 52.40 \\
5.81000 & 35.87 & 57.78 \\
7.05000 & 36.69 & 64.59 \\
8.30000 & 37.12 & 70.36 \\
12.40000 & 37.48 & 84.62 \\
16.500 .00 & 37.41 & 95.01 \\
24.90000 & 37.09 & 109.46 \\
41.50000 & 36.63 & 127.65 \\
83.00000 & 36.21 & 152.39 \\
166.00000 & 36.00 & 177.17
\end{tabular}

\begin{tabular}{rrr} 
TD $=56.0$ & EV & \multicolumn{2}{c}{ ET=0.873889 MEV } \\
ENERGY(MEV) & PRIMARY & CASEADE \\
0.88200 & 0.08 & 0.08 \\
0.89100 & 0.18 & 0.18 \\
0.90800 & 0.37 & 0.37 \\
0.93500 & 0.73 & 0.73 \\
0.97000 & 1.25 & 1.25 \\
1.01000 & 1.90 & 1.90 \\
1.06000 & 2.79 & 2.79 \\
1.13000 & 4.11 & 4.11 \\
1.22000 & 5.85 & 5.85 \\
1.35000 & 8.33 & 8.33 \\
1.52000 & 11.37 & 11.40 \\
1.74000 & 14.82 & 15.06 \\
2.00000 & 18.21 & 19.00 \\
2.35000 & 21.79 & 23.73 \\
2.79000 & 25.07 & 28.93 \\
3.32000 & 27.82 & 34.34 \\
3.93000 & 29.95 & 39.68 \\
4.54000 & 31.37 & 44.31 \\
5.24000 & 32.47 & 48.96 \\
6.11000 & $\mathbf{3 3 . 3 5}$ & 53.96 \\
7.42000 & 34.10 & 60.34 \\
8.73000 & 34.48 & 65.69 \\
13.10000 & 34.80 & 79.11 \\
17.40000 & 34.73 & 88.52 \\
26.20000 & 34.43 & 102.07 \\
43.60000 & 34.00 & 118.92 \\
87.30000 & 33.61 & 141.94 \\
174.00000 & 33.43 & 164.84
\end{tabular}

\begin{tabular}{|c|c|c|}
\hline$T D=50.0$ & $E \mathrm{~T}=0$ & HO MEV \\
\hline NERGY (MEV) & QRIMARY & CASCADE \\
\hline 0.92500 & 0.08 & 0.08 \\
\hline 0.93400 & 0.16 & 0.16 \\
\hline 0.95200 & 0.34 & 0.34 \\
\hline 0.93330 & 0.66 & 0.56 \\
\hline 1.01300 & 1.04 & 1.04 \\
\hline 1.06000 & 1.75 & 1.76 \\
\hline 1.11000 & 2.55 & 2.55 \\
\hline 1.19300 & 3.89 & 3.89 \\
\hline 1.28300 & 5.45 & 5.45 \\
\hline $1 .+1000$ & 7.63 & 7.68 \\
\hline 1.60000 & 10.72 & 10.75 \\
\hline 1.33200 & 13.94 & 14.18 \\
\hline 2.10300 & 17.09 & 17.85 \\
\hline 2.47000 & 20.45 & 22.34 \\
\hline 2.93300 & 23.51 & 27.21 \\
\hline 3.48300 & 26.04 & 32.24 \\
\hline 4.12300 & 28.01 & 37.26 \\
\hline 4.76000 & 29.33 & 41.61 \\
\hline 5.49000 & 30.34 & 45.94 \\
\hline 6.71300 & 31.16 & 50.67 \\
\hline 7.78300 & 31.84 & 56.63 \\
\hline 9.15000 & 32.19 & 61.62 \\
\hline 13.70300 & 32.48 & 74.10 \\
\hline 18.30000 & 32.40 & 83.07 \\
\hline 27.40000 & 32.13 & 95.56 \\
\hline 45.70000 & 31.73 & 111.37 \\
\hline 91.50300 & 31.37 & 132.86 \\
\hline 183.00300 & 31.20 & 154.34 \\
\hline
\end{tabular}

ATOMIC DISPLAZEMENT CROSS-SECTIONS (BARNS) BY FAST BLECTRONS IN CO $\mathrm{z}=29 \quad \mathrm{~A}=63.540$

\begin{tabular}{crr} 
TD $=64.0$ & EV & \multicolumn{2}{c}{ ET $=0.956856$ MEV } \\
ENERGY (MEV) & PRIMARY & CASCADE \\
0.96600 & 0.07 & 0.07 \\
0.97500 & 0.14 & 0.14 \\
0.99500 & 0.31 & 0.31 \\
1.02000 & 0.56 & 0.56 \\
1.06000 & 1.01 & 1.01 \\
1.10000 & 1.52 & 1.52 \\
1.16000 & 2.36 & 2.36 \\
1.24000 & 3.57 & 3.57 \\
1.33000 & 4.98 & 4.98 \\
1.48000 & 7.30 & 7.30 \\
1.57000 & 10.04 & 10.07 \\
1.91000 & 13.08 & 13.31 \\
2.20000 & 16.12 & 16.87 \\
2.58000 & 19.23 & 21.04 \\
3.06000 & 22.10 & 25.63 \\
3.63000 & 24.45 & 30.34 \\
4.30000 & 26.31 & 35.09 \\
4.97000 & 27.54 & 39.20 \\
5.74000 & 28.49 & 43.32 \\
6.69000 & 29.23 & 47.72 \\
8.13000 & 29.87 & 53.35 \\
9.56000 & 30.19 & 58.05 \\
14.30000 & 30.45 & 69.74 \\
19.10000 & 30.37 & 78.16 \\
28.70000 & 30.11 & 89.98 \\
47.80000 & 29.74 & 104.76 \\
95.60000 & 29.40 & 124.89 \\
191.00000 & 29.24 & 145.00
\end{tabular}

\begin{tabular}{rrr} 
TD=68.0 EV & \multicolumn{2}{c}{ ET $=0.996529$ MEV } \\
PNERGY (MEV) & PRIMAT & CASEADE \\
1.00000 & 0.02 & 0.02 \\
1.01000 & 0.08 & 0.08 \\
1.03000 & 0.23 & 0.23 \\
1.06000 & 0.48 & 0.48 \\
1.10000 & 0.88 & 0.88 \\
1.15000 & 1.45 & 1.46 \\
1.21000 & 2.22 & 2.22 \\
1.29000 & 3.31 & 3.31 \\
1.39000 & 4.73 & 4.73 \\
1.54000 & 6.84 & 6.84 \\
1.74000 & 9.47 & 9.50 \\
1.99000 & 12.35 & 12.57 \\
2.29000 & 15.21 & 15.93 \\
2.69000 & 18.17 & 19.91 \\
3.18000 & 20.82 & 24.18 \\
3.78000 & 23.07 & 28.69 \\
4.48000 & 24.81 & 33.20 \\
5.18000 & 25.96 & 37.09 \\
5.97000 & 26.84 & 40.93 \\
6.97000 & 27.54 & 45.13 \\
8.47000 & 28.13 & 50.45 \\
9.96000 & 28.42 & 54.87 \\
14.90000 & 28.65 & 65.90 \\
19.90000 & 28.58 & 73.83 \\
29.80000 & 28.33 & 84.87 \\
49.80000 & 27.98 & 98.88 \\
99.60000 & 27.67 & 117.83 \\
199.00000 & 27.52 & 136.76
\end{tabular}

\begin{tabular}{|c|c|c|}
\hline $\mathrm{TD}=72.0$ & EV $\quad E T=1.0$ & 379 MEV \\
\hline ENERGY ( $M E V)$ & PRIMARY & CASCADE \\
\hline 1.04000 & 0.02 & 0.02 \\
\hline 1.05000 & 0.08 & 0.08 \\
\hline 1.07300 & 0.21 & 0.21 \\
\hline 1.10000 & 0.43 & 0.43 \\
\hline 1.14000 & 0.79 & 0.79 \\
\hline 1.20000 & 1.41 & 1.41 \\
\hline 1.26300 & 2.11 & 2.11 \\
\hline 1.34300 & 3.11 & 3.11 \\
\hline 1.44300 & 4.40 & 4.40 \\
\hline 1.60030 & 6.46 & 6.46 \\
\hline 1.81300 & 8.98 & 9.02 \\
\hline 2.07000 & 11.72 & 11.94 \\
\hline 2.38000 & 14.41 & 15.11 \\
\hline 2.79000 & 17.18 & 18.85 \\
\hline 3.31300 & 19.74 & 22.99 \\
\hline 3.93000 & 21.84 & 27.24 \\
\hline 4.65000 & 23.46 & 31.48 \\
\hline 5.38000 & 24.55 & 35.19 \\
\hline 6.21300 & $25.3 B$ & 38.86 \\
\hline 7.24000 & 26.03 & 42.80 \\
\hline 8.80000 & 26.58 & 47.84 \\
\hline 10.30300 & 26.84 & 51.91 \\
\hline 15.50000 & 27.06 & 62.49 \\
\hline 20.70000 & 26.99 & 69.99 \\
\hline 31.00000 & 26.75 & 80.42 \\
\hline 51.70300 & 26.42 & 93.61 \\
\hline 103.00300 & 26.13 & 111.41 \\
\hline 207.00000 & 25.99 & 129.44 \\
\hline
\end{tabular}


ATOMIC DISPLAZEMENT CRJSS-SECTIONS (BARNS) BY PAST ELECTRONS IN CU

$\begin{array}{rrr}\text { TD=76.0 } & \text { EV } & \text { ET=1.073181 MEV } \\ \text { ENERGY(KEV) } & \text { PRIMARY } & \text { CASCADE } \\ 1.08000 & 0.03 & 0.03 \\ 1.09000 & 0.08 & 0.08 \\ 1.11000 & 0.20 & 0.20 \\ 1.14000 & 0.40 & 0.40 \\ 1.19000 & 0.81 & 0.81 \\ 1.24000 & 1.28 & 1.28 \\ 1.30000 & 1.92 & 1.92 \\ 1.39000 & 2.94 & 2.94 \\ 1.50000 & 4.25 & 4.25 \\ 1.56000 & 6.14 & 6.14 \\ 1.87000 & 8.46 & 8.49 \\ 2.14000 & 11.08 & 11.29 \\ 2.46000 & 13.64 & 14.30 \\ 2.89000 & 16.31 & 17.91 \\ 3.43000 & 18.74 & 21.87 \\ 4.07000 & 20.72 & 25.90 \\ 4.82000 & 22.26 & 29.95 \\ 5.58000 & 23.30 & 33.49 \\ 6.43000 & 24.06 & 36.94 \\ 7.51000 & 24.68 & 40.73 \\ 9.12000 & 25.19 & 45.49 \\ 10.70000 & 25.44 & 49.41 \\ 16.00000 & 25.63 & 59.30 \\ 21.40000 & 25.56 & 66.44 \\ 32.10000 & 25.34 & 76.37 \\ 53.50000 & 25.03 & 88.90 \\ 107.00000 & 24.75 & 105.81 \\ 214.00000 & 24.62 & 122.78 \\ & & \end{array}$

\begin{tabular}{rrr}
$T D=80.0$ & EV & \multicolumn{2}{c}{$E T=1.110102$} & $M E V$ \\
ENERGY (MEV) & PRIMARY & CASCADE \\
1.12000 & 0.04 & 0.04 \\
1.13000 & 0.09 & 0.09 \\
1.15000 & 0.19 & 0.19 \\
1.18000 & 0.38 & 0.38 \\
1.23000 & 0.75 & 0.75 \\
1.28000 & 1.18 & 1.18 \\
1.35000 & 1.86 & 1.86 \\
1.44000 & 2.81 & 2.81 \\
1.55000 & 4.01 & 4.01 \\
1.72000 & 5.87 & 5.87 \\
1.94000 & 8.11 & 8.14 \\
2.22000 & 10.60 & 10.81 \\
2.55000 & 13.03 & 13.68 \\
2.99000 & 15.54 & 17.09 \\
3.55000 & 17.85 & 20.87 \\
4.21000 & 19.73 & 24.71 \\
4.99000 & 21.19 & 28.59 \\
5.77000 & 22.16 & 31.93 \\
6.66000 & 22.89 & 35.26 \\
7.77000 & 23.46 & 38.84 \\
9.43000 & 23.94 & 43.36 \\
11.10000 & 24.18 & 47.17 \\
16.60000 & 24.35 & 56.57 \\
22.20000 & 24.28 & 63.36 \\
33.30000 & 24.06 & $\mathbf{7 2 . 8 0}$ \\
55.50000 & 23.77 & 84.66 \\
111.00000 & 23.51 & 100.77 \\
222.00000 & 23.39 & 116.90
\end{tabular}

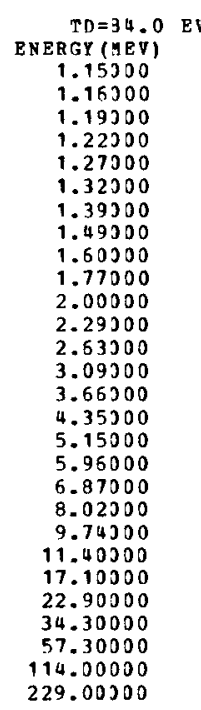

$\mathrm{ET}=1.146201 \mathrm{MEV}$ CASCADE $0.01 \quad 0.01$ $\begin{array}{ll}0.05 & 0.05 \\ 0.19 & 0.19\end{array}$ $\begin{array}{ll}0.19 & 0.19 \\ 0.36 & 0.36\end{array}$ $0.70 \quad 0.70$ $1.10 \quad 1.10$ $1.72 \quad 1.72$ $2.70 \quad 2.70$ $\begin{array}{ll}3.81 & 3.81 \\ 5.53 & 5.53\end{array}$ $\begin{array}{ll}5.53 & 5.53 \\ 7.71 & 7.74\end{array}$ $10.11 \quad 10.31$ $12.42 \quad 13.05$ $\begin{array}{ll}12.42 & 13.05 \\ 14.85 & 16.35\end{array}$ $\begin{array}{ll}14.85 & 16.35 \\ 17.02 & 19.92\end{array}$ $\begin{array}{ll}17.02 & 19.92 \\ 18.83 & 23.64\end{array}$ $\begin{array}{ll}18.83 & 23.64 \\ 20.21 & 27.32\end{array}$ $21.13 \quad 30.53$ $21.81 \quad 33.68$ $22.36 \quad 37.11$ $22.81 \quad 41.44$ $23.02 \quad 44.94$ $23.19 \quad 53.98$ $23.12 \quad 60.48$ $22.92 \quad 69.44$ $22.92 \quad 69.44$ $22.64 \quad 80.79$ $\begin{array}{rr}22.28 & 111.48\end{array}$

ATOMIC DISPIAZBMENT CROSS-SEETIONS (BARNS) BY FAST ELECTRONS IN CU $z=29 \quad=63.540$

$\begin{array}{rrr}\text { TD=38.0 } & \text { EV } & \text { ET=1.181530 MEV } \\ \text { ENERGY (MEV) } & \text { PRIMARY } & \text { CASCADE } \\ 1.19000 & 0.03 & 0.03 \\ 1.20000 & 0.06 & 0.06 \\ 1.22000 & 0.15 & 0.15 \\ 1.26000 & 0.35 & 0.35 \\ 1.31000 & 0.67 & 0.67 \\ 1.37000 & 1.11 & 1.11 \\ 1.44000 & 1.70 & 1.70 \\ 1.53000 & 2.51 & 2.51 \\ 1.65000 & 3.64 & 3.64 \\ 1.83000 & 5.34 & 5.34 \\ 2.06000 & 7.36 & 7.39 \\ 2.36000 & 9.66 & 9.86 \\ 2.71000 & 11.88 & 12.49 \\ 3.19000 & 14.23 & 15.69 \\ 3.78000 & 16.30 & 19.12 \\ 4.48000 & 17.99 & 22.63 \\ 5.31000 & 19.32 & 26.18 \\ 5.14000 & 20.19 & 29.23 \\ 7.08000 & 20.84 & 32.25 \\ 8.27000 & 21.35 & 35.55 \\ 10.00000 & 21.77 & 39.59 \\ 11.80000 & 21.98 & 43.11 \\ 17.70000 & 22.13 & 51.74 \\ 23.60000 & 22.07 & 57.86 \\ 35.40000 & 21.87 & 66.45 \\ 59.00000 & 21.60 & 77.24 \\ 118.00000 & 21.37 & 91.89 \\ 236.00000 & 21.26 & 106.55\end{array}$

$\begin{array}{crr}\text { TD }=92.0 & \text { EV } & \text { ET=1.216136 KEV } \\ \text { ENERGY (HEV) } & \text { PRIMARY } & \text { CASEADE } \\ 1.22000 & 0.01 & 0.01 \\ 1.24000 & 0.07 & 0.07 \\ 1.26000 & 0.15 & 0.15 \\ 1.30000 & 0.34 & 0.34 \\ 1.34000 & 0.58 & 0.58 \\ 1.41000 & 1.05 & 1.05 \\ 1.48000 & 1.60 & 1.60 \\ 1.58000 & 2.44 & 2.44 \\ 1.70000 & 3.50 & 3.50 \\ 1.88000 & 5.08 & 5.08 \\ 2.12000 & 7.05 & 7.08 \\ 2.43000 & 9.27 & 9.47 \\ 2.79000 & 11.40 & 11.99 \\ 3.28000 & 13.63 & 15.04 \\ 3.89000 & 15.62 & 18.35 \\ 4.62000 & 17.25 & 21.75 \\ 5.47000 & 18.50 & 25.14 \\ 6.32000 & 19.33 & 28.05 \\ 7.29000 & 19.95 & 30.95 \\ 8.51000 & 20.44 & 34.10 \\ 10.30000 & 20.83 & 37.99 \\ 12.10000 & 21.03 & 41.28 \\ 18.20000 & 21.17 & 49.59 \\ 24.30000 & 21.11 & 55.48 \\ 36.40000 & 20.92 & 63.66 \\ 60.80000 & 20.66 & 74.04 \\ 121.00000 & 20.44 & 87.95 \\ 243.00000 & 20.34 & 102.06\end{array}$

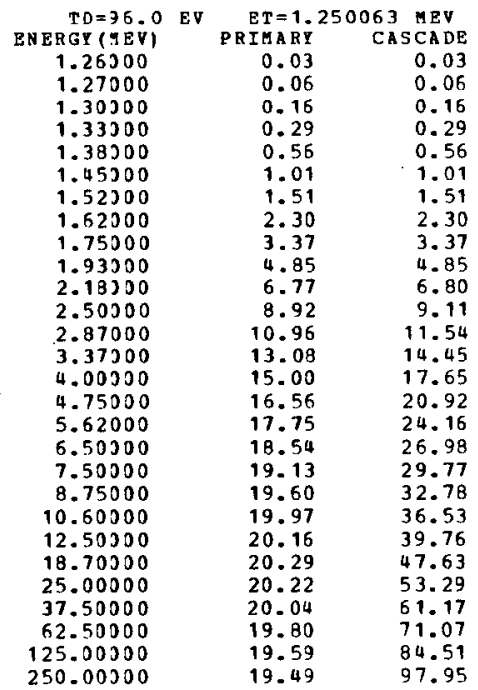


ATOMIC DISPLAZEMENY CROSS-SECTIONS (BARNS) BY FAST ELECTRONS IN $Z N$

\begin{tabular}{rrr}
$T D=4.0$ & EV & \multicolumn{2}{c}{ ET $=0.107782$ MEV } \\
ENERGY (MEV) & PRIMARY & CASCADE \\
0.10800 & 2.57 & 2.57 \\
0.10900 & 14.10 & 14.10 \\
0.11200 & 46.60 & 46.60 \\
0.11500 & 76.21 & 76.21 \\
0.11900 & 111.77 & 111.77 \\
0.12500 & 157.96 & 157.96 \\
0.13100 & 197.07 & 197.07 \\
0.14000 & 245.32 & 245.32 \\
0.15000 & 287.74 & 287.74 \\
0.16700 & 341.00 & 341.00 \\
0.18800 & 385.63 & 385.63 \\
0.21500 & 422.89 & 423.92 \\
0.24700 & 450.71 & 458.21 \\
0.29100 & 473.90 & 494.71 \\
0.34400 & 490.30 & 529.45 \\
0.40900 & 502.21 & 564.51 \\
0.48500 & 510.62 & 599.60 \\
0.56000 & 515.92 & 530.46 \\
0.64600 & 519.97 & 662.77 \\
0.75400 & 523.23 & 700.01 \\
0.91600 & 525.94 & 750.74 \\
1.07000 & 527.12 & 794.52 \\
1.51000 & 526.63 & 923.75 \\
2.15000 & 523.70 & 1026.82 \\
3.23000 & 517.45 & 1185.66 \\
5.38000 & 508.53 & 1401.95 \\
10.70000 & 497.38 & 1711.58 \\
21.50000 & 490.41 & 2038.06 \\
32.30000 & 488.21 & 2231.55 \\
53.80000 & 486.50 & 2475.86 \\
75.40000 & 485.68 & 2638.07 \\
107.00000 & 484.91 & 2806.39 \\
215.00000 & 483.41 & 3142.01
\end{tabular}

\begin{tabular}{rrr} 
TD $=8.0$ & EV & \multicolumn{1}{c}{ ET $=0.199394$ MEV } \\
ENERGI(MEV) & PRIMARY & CASCADE \\
0.20100 & 2.67 & 2.67 \\
0.20300 & 5.93 & 5.93 \\
0.20700 & 12.17 & 12.17 \\
0.21300 & 20.95 & 20.95 \\
0.22100 & 31.67 & 31.67 \\
0.23100 & 43.72 & 43.72 \\
0.24300 & 56.53 & 56.53 \\
0.25900 & 71.35 & 71.35 \\
0.27900 & 87.03 & 87.03 \\
0.30900 & 106.23 & 105.23 \\
0.34800 & 125.82 & 125.82 \\
0.39800 & 145.13 & 145.88 \\
0.45800 & 162.78 & 165.29 \\
0.53800 & 180.42 & 189.47 \\
0.63800 & 196.54 & 214.23 \\
0.75700 & 210.39 & 239.71 \\
0.89700 & 222.01 & 265.93 \\
1.03000 & 230.04 & 288.12 \\
1.19000 & 237.10 & 312.18 \\
1.39000 & 243.29 & 339.11 \\
1.69000 & 249.19 & 374.55 \\
1.99000 & 252.66 & 405.45 \\
2.99000 & 256.79 & 487.07 \\
3.98000 & 256.91 & 547.69 \\
5.98000 & 255.13 & 637.61 \\
9.96000 & 251.50 & 754.12 \\
19.90000 & 246.93 & 916.27 \\
39.80000 & 244.40 & 1081.51 \\
59.80000 & 243.59 & 1179.24 \\
99.60000 & 242.91 & 1302.09 \\
139.00000 & 242.54 & 1382.39 \\
199.00000 & 242.16 & 1468.79 \\
398.00000 & 241.41 & 1635.62
\end{tabular}

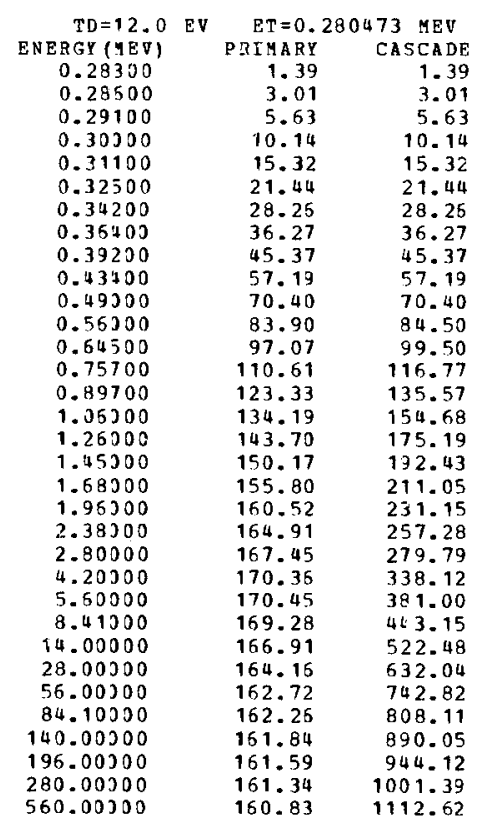

ATOMIC DISPLAEEMENT CROSS-SECTIONS (BARNS) BY FAST ELECTRONS IN ZN $Z=30 \quad A=65.380$

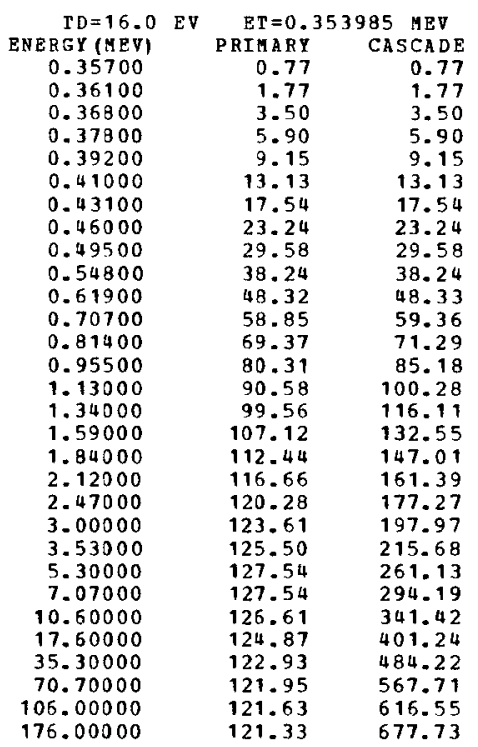

\begin{tabular}{crr}
$T D=20.0$ & EV & \multicolumn{1}{c}{ ET=0.421729 MEV } \\
ENERGY(MEV) & PRTMARY & CASZADE \\
0.42500 & 0.46 & 0.46 \\
0.43000 & 1.16 & 1.16 \\
0.43800 & 2.28 & 2.28 \\
0.45100 & 4.08 & 4.08 \\
0.46800 & 6.39 & 6.39 \\
0.48900 & 9.19 & 9.19 \\
0.51400 & 12.44 & 12.44 \\
0.54800 & 16.68 & 15.68 \\
0.59000 & 21.65 & 21.65 \\
0.65300 & 28.53 & 28.53 \\
0.73800 & 36.78 & 36.81 \\
0.84300 & 45.52 & 45.97 \\
0.96900 & 54.20 & 55.83 \\
1.13000 & 63.06 & 67.06 \\
1.34000 & 71.81 & 79.94 \\
1.60000 & 79.60 & 93.81 \\
1.89000 & 85.67 & 107.25 \\
2.19000 & 90.06 & 119.45 \\
2.53000 & 93.54 & 131.67 \\
2.95000 & 96.42 & 144.93 \\
3.58000 & 99.02 & 161.97 \\
4.21000 & 100.46 & 176.49 \\
6.32000 & 101.94 & 213.63 \\
8.43000 & 101.89 & 240.46 \\
12.60000 & 101.11 & 278.34 \\
21.00000 & 99.71 & 32.6 .85 \\
42.10000 & 98.24 & 393.42 \\
84.30000 & 97.51 & 460.30 \\
126.00000 & 97.27 & 499.10 \\
210.00000 & 97.04 & 548.43
\end{tabular}

\begin{tabular}{|c|c|c|}
\hline$T D=24.0$ & $\mathrm{ET}=0.4$ & $861 \mathrm{MBV}$ \\
\hline ENERGY (YEV) & PRI ARY & CASCADE \\
\hline 0.43900 & 0.36 & 0.36 \\
\hline 0.49400 & 0.80 & 0.80 \\
\hline $0.50 \div 00$ & 1.68 & 1.68 \\
\hline 0.51900 & 2.93 & 2.93 \\
\hline 0.53300 & 4.72 & 4.72 \\
\hline 0.55200 & 6.87 & 6.87 \\
\hline 0.59100 & 9.46 & 9.46 \\
\hline 0.63000 & 12.88 & 12.88 \\
\hline 0.57300 & 16.94 & 16.94 \\
\hline 0.75100 & 22.74 & 22.74 \\
\hline 0.34300 & 29.70 & 29.73 \\
\hline 0.96900 & 37.20 & 37.61 \\
\hline 1.11300 & 44.50 & 45.91 \\
\hline 1.30300 & 52.35 & 55.88 \\
\hline 1.55000 & 60.07 & 67.39 \\
\hline 1.84300 & 66.47 & 78.99 \\
\hline 2.18$) 00$ & 71.68 & 90.79 \\
\hline 2.52000 & 75.29 & 101.12 \\
\hline 2.90000 & 78.10 & 111.31 \\
\hline 3.39000 & 80.52 & 122.83 \\
\hline 4.12000 & 82.64 & 137.45 \\
\hline 4.84300 & 83.78 & 149.70 \\
\hline 7.27000 & 84.90 & 181.13 \\
\hline 9.69300 & 84.82 & 203.65 \\
\hline 14.50000 & 84.14 & 235.52 \\
\hline 24.20000 & 82.98 & 276.22 \\
\hline 48.40000 & 81.81 & 331.63 \\
\hline 96.90300 & 81.23 & 337.41 \\
\hline 145.00300 & 81.04 & 419.86 \\
\hline 242.00300 & 80.85 & 461.08 \\
\hline
\end{tabular}


ATOMIC DISPLAEEHENT CRJSS-SECTIONS (BARNS) BY FAST ELECTRONS IN $2 N$

\begin{tabular}{crr} 
TD $=28.0$ & EV & \multicolumn{1}{c}{ ET $=0.544229$ MPV } \\
ENERGY (MEV) & PRIMARY & CASCADE \\
0.54900 & 0.28 & 0.28 \\
0.55500 & 0.63 & 0.63 \\
0.56500 & 1.24 & 1.24 \\
0.58200 & 2.29 & 2.29 \\
0.50400 & 3.69 & 3.69 \\
0.63100 & 5.44 & 5.44 \\
0.66300 & 7.54 & 7.54 \\
0.70700 & 10.43 & 10.43 \\
0.76100 & 13.90 & 13.90 \\
0.84300 & 18.91 & 18.91 \\
0.95200 & 24.98 & 25.02 \\
1.08000 & 31.21 & 31.56 \\
1.25000 & 38.12 & 39.42 \\
1.46000 & 44.88 & 48.08 \\
1.74000 & 51.61 & 58.20 \\
2.06000 & 57.08 & 68.26 \\
2.44000 & 61.59 & 78.61 \\
2.82000 & 64.68 & 87.65 \\
3.26000 & 67.15 & 96.86 \\
3.80000 & 69.15 & 106.73 \\
4.62000 & 70.92 & 119.49 \\
5.44000 & 71.87 & 130.28 \\
8.16000 & 72.75 & 157.40 \\
10.80000 & 72.65 & 176.37 \\
15.30000 & 72.04 & 204.39 \\
27.20000 & 71.05 & 239.36 \\
54.40000 & 70.08 & 286.94 \\
108.00000 & 69.61 & 334.20 \\
163.00000 & 69.45 & 362.62
\end{tabular}

\begin{tabular}{crr} 
TD $=32.0$ & \multicolumn{3}{c}{ EV } & \multicolumn{2}{c}{ ET $=0.600430$ MEV } \\
ENERGY (KEV) & PRIMARY & CASEADE \\
0.60600 & 0.23 & 0.23 \\
0.61200 & 0.48 & 0.48 \\
0.62400 & 1.01 & 1.01 \\
0.64200 & 1.83 & 1.83 \\
0.66600 & 2.97 & 2.97 \\
0.69600 & 4.45 & 4.45 \\
0.73200 & 6.28 & 6.28 \\
0.78000 & 8.74 & 8.74 \\
0.84000 & 11.80 & 11.80 \\
0.93000 & 16.20 & 15.20 \\
1.05000 & 21.59 & 21.62 \\
1.20000 & 27.45 & 27.82 \\
1.38000 & 33.34 & 34.55 \\
1.62000 & 39.54 & 42.56 \\
1.92000 & 45.31 & 51.34 \\
2.28000 & 50.22 & 60.50 \\
2.70000 & 54.14 & 69.73 \\
3.12000 & 56.82 & 77.77 \\
3.60000 & 58.92 & 85.85 \\
4.20000 & 60.64 & 94.66 \\
5.10000 & 62.14 & 105.89 \\
6.00000 & 62.93 & 115.38 \\
9.00000 & 63.64 & 139.29 \\
12.00000 & 63.52 & 156.41 \\
18.00000 & 62.98 & 180.64 \\
30.00000 & 62.12 & 211.23 \\
60.00000 & 61.30 & 252.91 \\
120.00000 & 60.90 & 294.74 \\
180.00000 & 60.76 & 319.24
\end{tabular}

\begin{tabular}{|c|c|c|}
\hline $\mathrm{TD}=36.0$ & $\mathrm{ET}=0$ & 924 MEV \\
\hline ENERGY (YEV) & PRIMARY & CASCADE \\
\hline 0.56300 & 0.19 & 0.19 \\
\hline 0.66700 & 0.41 & 0.41 \\
\hline 0.58300 & 0.84 & 0.84 \\
\hline 0.69900 & 1.50 & 1.50 \\
\hline 0.72500 & 2.46 & 2.46 \\
\hline 0.75900 & 3.75 & 3.75 \\
\hline 0.79700 & 5.33 & 5.33 \\
\hline 0.85000 & 7.54 & 7.54 \\
\hline 0.91500 & 10.25 & 10.25 \\
\hline 1.01500 & 14.08 & 14.08 \\
\hline 1.14000 & 18.92 & 18.95 \\
\hline 1.30000 & 24.15 & 24.46 \\
\hline 1.50300 & 29.59 & 30.70 \\
\hline 1.76300 & 35.16 & 37.93 \\
\hline 2.09300 & 40.40 & 45.97 \\
\hline 2.48300 & 44.77 & 54.21 \\
\hline 2.94300 & 48.28 & 62.60 \\
\hline 3.40000 & 50.66 & 69.89 \\
\hline 3.92300 & 52.50 & 77.12 \\
\hline 4.57000 & 53.99 & 85.00 \\
\hline 5.55000 & 55.29 & 95.09 \\
\hline 6.53300 & 55.97 & 103.59 \\
\hline 9.80000 & 56.55 & 124.99 \\
\hline 13.00300 & 56.44 & 140.00 \\
\hline 19.60000 & 55.94 & 161.86 \\
\hline 32.60300 & 55.18 & 188.99 \\
\hline 65.30000 & 54.47 & 226.17 \\
\hline 130.00000 & 54.12 & 263.11 \\
\hline 196.00300 & 54.00 & 285.17 \\
\hline
\end{tabular}

ATOMIC DISPLAZEMENT CROSS-SECTIONS (BARNS) BY PAST ELECTRONS IN ZN $z=30 \quad A=65.380$

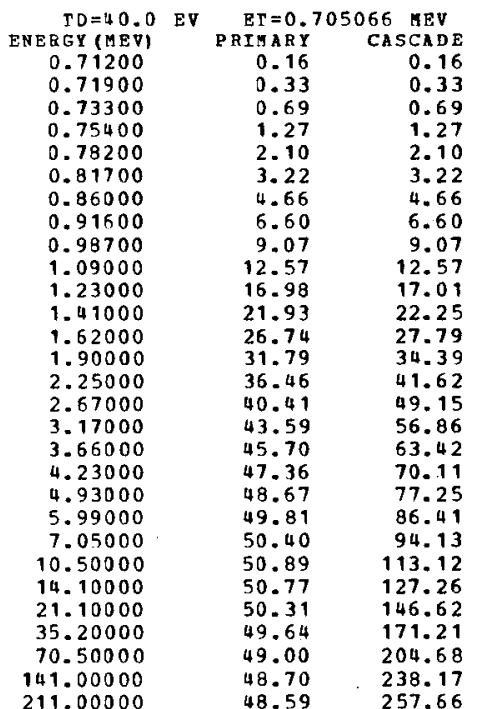

\begin{tabular}{|c|c|c|c|}
\hline & & & \\
\hline$T D=44.0$ & & $\mathrm{ET}=0.75$ & $143 \mathrm{MEV}$ \\
\hline ENERGY (MEV) & & PR I MARY Y & CASEADE \\
\hline 0.76100 & & 0.13 & 0.13 \\
\hline 0.76900 & & 0.28 & 0.28 \\
\hline 0.78400 & & 0.59 & 0.59 \\
\hline 0.80600 & & 1.08 & 1.08 \\
\hline 0.83700 & & 1.84 & 1.84 \\
\hline 0.87400 & & 2.82 & 2.82 \\
\hline 0.92000 & & 4. 12 & 4.12 \\
\hline 0.98000 & & 5.88 & 5.88 \\
\hline 1.05000 & & 7.97 & 7.97 \\
\hline 1.16000 & & 11.18 & 11.18 \\
\hline 1.31000 & & 15.25 & 15.28 \\
\hline 1.50000 & & 19.77 & 20.06 \\
\hline 1.73000 & & 24.32 & 25.30 \\
\hline 2.03000 & & 28.97 & \\
\hline 2.41000 & & 33.30 & 38.16 \\
\hline 2.86000 & & 36.89 & 45.09 \\
\hline 3.39000 & & 39.74 & 52.11 \\
\hline $\begin{array}{l}3.92000 \\
4.52000\end{array}$ & & $\begin{array}{l}41.66 \\
43.13\end{array}$ & $\begin{array}{l}38.20 \\
64.23\end{array}$ \\
\hline 5.27000 & & 44.31 & 70.78 \\
\hline 6.41000 & & 45.33 & 79.21 \\
\hline 7.54000 & & 45.84 & 86.24 \\
\hline 11.30000 & & 46.25 & 103.84 \\
\hline 15.00000 & & 46.14 & 116.23 \\
\hline 22.60000 & & 45.71 & 134.15 \\
\hline 37.70000 & & 45.10 & 156.53 \\
\hline 75.40000 & & 44.54 & 186.92 \\
\hline 150.00000 & & 44.27 & 217.14 \\
\hline 226.00000 & & 44.17 & \\
\hline
\end{tabular}

\begin{tabular}{|c|c|c|}
\hline$y=48$ & $P \mathrm{R}=0$ & \\
\hline ERGY (IEV) & PRIMARY & CASCADE \\
\hline 0.80900 & 0.11 & 0.11 \\
\hline 0.81700 & 0.24 & 0.24 \\
\hline 0.83300 & 0.51 & 0.51 \\
\hline 0.85700 & 0.95 & 0.95 \\
\hline 0.88900 & 1.61 & 1.61 \\
\hline 0.92900 & 2.51 & 2.51 \\
\hline 0.97700 & 3.67 & 3.67 \\
\hline 1.04300 & 5.26 & 5.26 \\
\hline 1.12300 & 7.33 & 7.33 \\
\hline 1.24000 & 10.37 & 10.37 \\
\hline 1.40300 & 14.14 & 14.17 \\
\hline 1.50000 & 18.26 & 18.55 \\
\hline 1.84000 & 22.38 & 23.32 \\
\hline 2.16000 & 26.68 & 29.00 \\
\hline 2.56300 & 30.63 & 35.20 \\
\hline 3.04300 & 33.93 & 41.64 \\
\hline 3.60300 & 36.52 & 48.10 \\
\hline 4.16000 & 38.26 & 53.70 \\
\hline 4.80000 & 39.60 & 59.29 \\
\hline 5.60000 & 40.67 & 65.37 \\
\hline 6.81000 & 41.58 & 73.13 \\
\hline 8.01000 & 42.04 & 79.59 \\
\hline 12.00300 & 42.39 & 95.77 \\
\hline 16.00300 & 42.28 & 107.32 \\
\hline 24.00000 & 41.89 & 123.59 \\
\hline 40.00300 & 41.33 & 144.09 \\
\hline 80.10300 & 40.82 & 172.01 \\
\hline 160.00300 & 40.58 & 199.88 \\
\hline 240.00300 & 40.49 & 216.23 \\
\hline
\end{tabular}


ATOMIC DISPLA EEMENT CROSS-SECTIONS (BARNS) BY FAST ELECTRONS IN $Z$ N $z=30 \quad A=65.380$

\begin{tabular}{rrr} 
TD $=52.0$ & EV & \multicolumn{1}{c}{ EI $=0.846986$ MEV } \\
ENERGY (HEV) & PRIMARY & CASCADE \\
0.85500 & 0.10 & 0.10 \\
0.86300 & 0.20 & 0.20 \\
0.88000 & 0.44 & 0.44 \\
0.90600 & 0.84 & 0.84 \\
0.94000 & 1.44 & 1.44 \\
0.98200 & 2.26 & 2.26 \\
1.03000 & 3.26 & 3.26 \\
1.10000 & 4.81 & 4.81 \\
1.18000 & 6.62 & 6.62 \\
1.31000 & 9.52 & 9.52 \\
1.48000 & 13.05 & 13.09 \\
1.69000 & 15.87 & 17.14 \\
1.94000 & 20.65 & 21.54 \\
2.28000 & 24.68 & 26.87 \\
2.71000 & 28.40 & 32.75 \\
3.21000 & 31.40 & 38.66 \\
3.81000 & 33.81 & 44.75 \\
4.40000 & 35.40 & 49.94 \\
5.08000 & 36.62 & 55.15 \\
5.92000 & 37.59 & 60.75 \\
7.19000 & 38.41 & 67.90 \\
8.46000 & 38.81 & 73.90 \\
12.70000 & 39.13 & 88.95 \\
16.90000 & 39.09 & 99.56 \\
25.40000 & 38.65 & 114.67 \\
42.30000 & 38.13 & 133.57 \\
84.60000 & 37.67 & 159.32 \\
169.00000 & 37.45 & 185.05
\end{tabular}

\begin{tabular}{rrr} 
TD $=56.0$ & EV & \multicolumn{2}{c}{ ET $=0.891104$ MEV } \\
ENERGY (MEV) & PRIMARY & CASEADE \\
0.90000 & 0.09 & 0.09 \\
0.90800 & 0.18 & 0.18 \\
0.92600 & 0.39 & 0.39 \\
0.95300 & 0.75 & 0.75 \\
0.98900 & 1.30 & 1.30 \\
1.03000 & 1.99 & 1.99 \\
1.08000 & 2.91 & 2.91 \\
1.15000 & 4.27 & 4.27 \\
1.24000 & 6.08 & 6.08 \\
1.38000 & 8.86 & 8.86 \\
1.55000 & 12.00 & 12.03 \\
1.78000 & 15.73 & 16.00 \\
2.04000 & 19.22 & 20.07 \\
2.40000 & 23.01 & 25.10 \\
2.85000 & 26.44 & 30.58 \\
3.38000 & 29.25 & 36.15 \\
4.00000 & 31.45 & 41.75 \\
4.63000 & 32.93 & 46.67 \\
5.34000 & 34.05 & 51.50 \\
6.23000 & 34.94 & 56.76 \\
7.57000 & 35.69 & 63.44 \\
8.91000 & 36.06 & 69.04 \\
13.30000 & 36.33 & 82.85 \\
17.80000 & 36.22 & 92.92 \\
26.70000 & 35.87 & 105.89 \\
44.50000 & 35.40 & 124.49 \\
89.10000 & 34.97 & 148.44 \\
178.00000 & 34.77 & 172.34
\end{tabular}

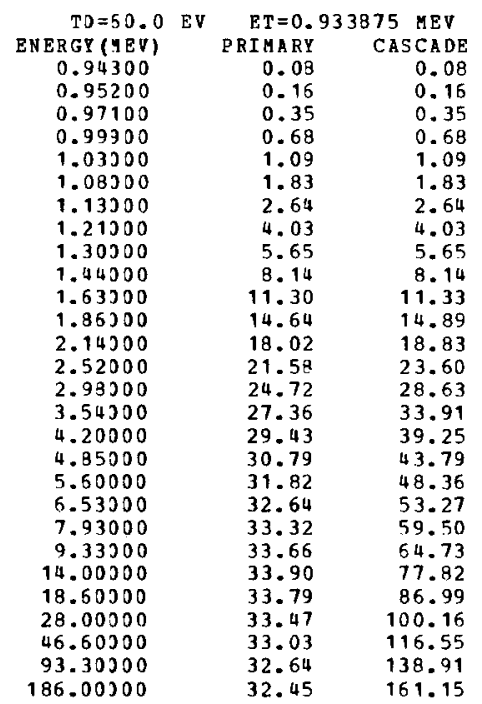

ATOMIC DISPLAEEMENT CROSS-SEETIONS (BARNS) BY FAST ELECTRONS IN ZN $Z=30 \quad A=65.380$

\begin{tabular}{rrr} 
TD=64.0 EV & \multicolumn{2}{c}{ ET $=0.975416$ MEV } \\
ENERGY (MEV) & PRIMARY & CASCADE \\
0.98500 & 0.07 & 0.07 \\
0.99400 & 0.14 & 0.14 \\
1.01000 & 0.28 & 0.28 \\
1.04000 & 0.58 & 0.58 \\
1.03000 & 1.04 & 1.04 \\
1.13000 & 1.71 & 1.71 \\
1.19000 & 2.59 & 2.59 \\
1.26000 & 3.69 & 3.69 \\
1.36000 & 5.31 & 5.31 \\
1.51000 & 7.72 & 7.72 \\
1.70000 & 10.57 & 10.60 \\
1.95000 & 13.85 & 14.10 \\
2.24000 & 15.99 & 17.79 \\
2.63000 & 20.28 & 22.20 \\
3.12000 & 23.28 & 27.05 \\
3.70000 & 25.72 & 31.98 \\
4.38000 & 27.63 & 36.94 \\
5.07000 & 28.91 & 41.29 \\
5.85000 & 29.87 & 45.57 \\
6.82000 & 30.63 & 50.18 \\
8.29000 & 31.26 & 56.08 \\
9.75000 & $\mathbf{3 1 . 5 7}$ & 60.98 \\
14.50000 & $\mathbf{3 1 . 7 8}$ & 73.21 \\
19.50000 & $\mathbf{3 1 . 6 7}$ & $\mathbf{8 1 . 9 8}$ \\
29.20000 & 31.37 & 94.18 \\
48.70000 & 30.96 & 109.61 \\
97.50000 & 30.59 & 130.58 \\
195.00000 & 30.42 & 151.53
\end{tabular}

\begin{tabular}{rrr} 
TD $=68.0$ & EV & \multicolumn{2}{c}{ ET $=1.015828$ MEV } \\
ENERGY (HEV) & PRIMARY & CASEADE \\
1.02000 & 0.03 & 0.03 \\
1.03000 & 0.09 & 0.09 \\
1.05000 & 0.24 & 0.24 \\
1.08000 & 0.50 & 0.50 \\
1.12000 & 0.91 & 0.91 \\
1.17000 & 1.50 & 1.50 \\
1.23000 & 2.29 & 2.29 \\
1.32000 & 3.56 & 3.56 \\
1.42000 & 5.04 & 5.04 \\
1.57000 & 7.23 & 7.23 \\
1.77000 & 9.96 & 9.99 \\
2.03000 & 13.06 & 13.30 \\
2.33000 & 16.01 & 16.78 \\
2.74000 & 19.15 & 21.00 \\
3.25000 & 21.97 & 25.58 \\
3.86000 & 24.29 & 30.30 \\
4.57000 & 26.07 & 34.99 \\
5.28000 & 27.25 & 39.05 \\
5.09000 & 28.14 & 43.08 \\
7.11000 & 28.85 & 47.48 \\
8.63000 & 29.43 & 53.00 \\
10.10000 & 29.71 & 57.48 \\
15.20000 & 29.91 & 69.15 \\
20.30000 & 29.80 & 77.41 \\
30.40000 & 29.52 & 88.90 \\
50.70000 & 29.13 & 103.43 \\
101.00000 & 28.79 & 123.03 \\
203.00000 & 28.63 & 142.90
\end{tabular}

\begin{tabular}{|c|c|c|}
\hline$T D=72.0$ & $\mathrm{ET}=1$. & $197 \mathrm{MEV}$ \\
\hline NERGY (IEV) & PRIMARY & CASCADE \\
\hline 1.06300 & 0.02 & 0.02 \\
\hline 1.07300 & 0.08 & 0.08 \\
\hline 1.09300 & 0.21 & 0.21 \\
\hline 1.12300 & 0.44 & 0.44 \\
\hline 1.17000 & 0.91 & 0.91 \\
\hline 1.22000 & 1.45 & 1.45 \\
\hline 1.28000 & 2.17 & 2.17 \\
\hline 1.37000 & 3.33 & 3.33 \\
\hline 1.47000 & 4.68 & 4.68 \\
\hline 1.53000 & 6.82 & 6.82 \\
\hline 1.84300 & 9.44 & 9.47 \\
\hline 2.11000 & 12.38 & 12.62 \\
\hline 2.42500 & 15.17 & 15.90 \\
\hline 2.84300 & 18.10 & 19.87 \\
\hline 3.37000 & 20.78 & 24.23 \\
\hline 4.00000 & 22.95 & 28.68 \\
\hline 4.74000 & 24.65 & 33.16 \\
\hline 5.48000 & 25.77 & 37.02 \\
\hline 6.33300 & 26.61 & 40.89 \\
\hline 7.38000 & 27.27 & 45.01 \\
\hline 8.96300 & 27.81 & 50.24 \\
\hline 10.50000 & 28.07 & 54.52 \\
\hline 15.80000 & 28.24 & 65.56 \\
\hline 21.10000 & 28.14 & 73.36 \\
\hline 31.50000 & 27.87 & 84.22 \\
\hline 52.70300 & 27.50 & 97.95 \\
\hline 105.00000 & 27.19 & 116.47 \\
\hline 211.00000 & 27.04 & 135.23 \\
\hline
\end{tabular}


ATOMIC DISPLAZEMENT CROSS-SECTIONS (BARNS) BY PAST BLECTRONS IN ZN

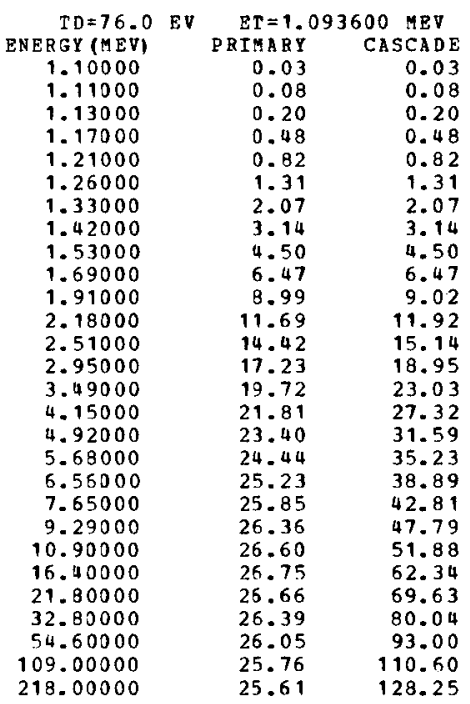

\begin{tabular}{|c|c|c|}
\hline$T D=80.0$ & $\mathrm{E} T=1$ & 06 AEV \\
\hline NERGY (MEV) & PRIMARY & CAS $=A D E$ \\
\hline 1.14000 & 0.04 & 0.04 \\
\hline 1.15000 & 0.08 & 0.08 \\
\hline 1.17000 & 0.19 & 0.19 \\
\hline 1.21000 & 0.44 & 0.44 \\
\hline 1.25000 & 0.75 & 0.75 \\
\hline 1.31000 & 1.29 & 1.29 \\
\hline 1.37000 & 1.90 & 1.90 \\
\hline 1.47000 & 2.99 & 2.99 \\
\hline 1.58000 & 4.24 & 4.24 \\
\hline 1.75000 & 6.17 & 6.17 \\
\hline 1.97000 & 8.50 & 8.53 \\
\hline 2.26000 & 11.18 & 11.41 \\
\hline 2.60000 & 13.76 & 14.46 \\
\hline 3.05000 & 16.40 & 18.06 \\
\hline 3.61000 & 18.78 & 21.97 \\
\hline 4.29000 & 20.75 & 26.04 \\
\hline 5.08000 & 22.25 & 30.09 \\
\hline 5.88000 & 23.25 & 33.62 \\
\hline 6.78000 & 23.99 & 37.07 \\
\hline 7.91000 & 24.57 & 40.81 \\
\hline 9.61000 & 25.05 & 45.56 \\
\hline 11.30000 & 25.27 & 49.50 \\
\hline 16.90000 & 25.41 & 59.31 \\
\hline 22.60000 & 25. 32 & 66.38 \\
\hline 33.90000 & 25.07 & 76.21 \\
\hline 56.50000 & 24.75 & 88.55 \\
\hline 113.00000 & 24.47 & 105.32 \\
\hline 226.00000 & 24.33 & 122.09 \\
\hline
\end{tabular}

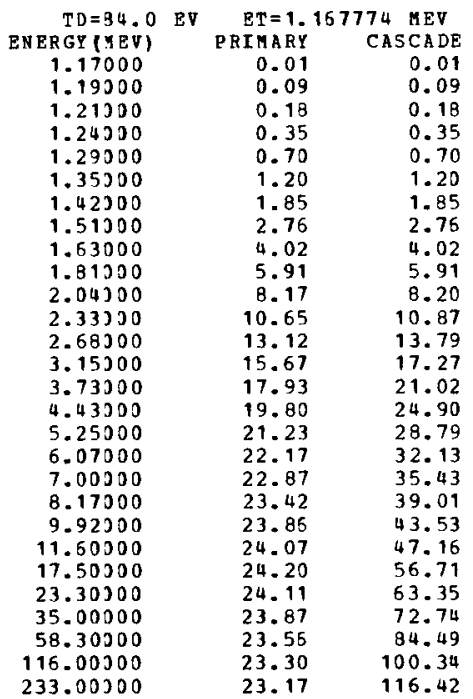

ATOMIC DISPLAZEMENT CROSS-SEZTIONS (BARNS) BY PAST ELECTRONS IN $Z$ N $\mathrm{z}=30 \quad \mathrm{~A}=65.380$

\begin{tabular}{|c|c|c|c|}
\hline $\begin{array}{r}T O=88.0 \\
\text { ENERGY (MEV) }\end{array}$ & EV & $\begin{aligned} E r & =1,2\end{aligned}$ & CASCADE \\
\hline D & & 2 & CASCADE \\
\hline 1.21000 & & 0.02 & 0.02 \\
\hline 1.22000 & & 0.06 & 0.06 \\
\hline 1. 25000 & & 0.18 & 0.18 \\
\hline 1.28000 & & 0.34 & 0.34 \\
\hline 1.33000 & & 0.66 & 0.66 \\
\hline 1.39000 & & 1.12 & 1.12 \\
\hline 1.46000 & & 1.72 & 1.72 \\
\hline 1.56000 & & 2.66 & 2.66 \\
\hline 1.58000 & & 3.84 & 3.84 \\
\hline 1.86000 & & 5.60 & 5.60 \\
\hline 2.10000 & & 7.79 & $\begin{array}{r}7.82 \\
10.79\end{array}$ \\
\hline 2.40000 & & 10.18 & 10.39 \\
\hline 2.76000 & & 12.54 & 13.19 \\
\hline 3.24000 & & 14.96 & 16.50 \\
\hline 3.85300 & & 17.17 & 20.17 \\
\hline 4.57000 & & 18.94 & 23.87 \\
\hline 5.41000 & & 20.29 & 27.57 \\
\hline 6.25000 & & 21.18 & $\begin{array}{l}30.75 \\
33.94\end{array}$ \\
\hline 8.42000 & & 22.36 & 37.36 \\
\hline 10.20000 & & 22.78 & 41.62 \\
\hline 12.00000 & & 22.98 & 45.23 \\
\hline 18.00000 & & 23.10 & 54.22 \\
\hline 24.00000 & & 23.01 & 60.60 \\
\hline 36.10000 & & 22.78 & 69.60 \\
\hline 60.10000 & & 22.49 & 80.80 \\
\hline 120.00000 & & 22.24 & .01 \\
\hline 240.00000 & & 22.12 & 26 \\
\hline
\end{tabular}

$\begin{array}{ccc}\text { TD=92.0 } & \text { EV } & \text { ET=1.238806 REV } \\ \text { ENERGY (MEV) } & \text { PRIMAY } & \text { CASCA DE } \\ 1.25000 & 0.03 & 0.03 \\ 1.26000 & 0.07 & 0.07 \\ 1.28000 & 0.14 & 0.14 \\ 1.32000 & 0.34 & 0.34 \\ 1.37000 & 0.63 & 0.63 \\ 1.43000 & 1.06 & 1.06 \\ 1.51000 & 1.70 & 1.70 \\ 1.61000 & 2.58 & 2.58 \\ 1.73000 & 3.68 & 3.68 \\ 1.92000 & 5.41 & 5.41 \\ 2.16000 & 7.45 & 7.48 \\ 2.47000 & 9.76 & 9.96 \\ 2.84000 & 12.02 & 12.65 \\ 3.34000 & 14.36 & 15.87 \\ 3.96000 & 16.45 & 19.34 \\ 4.70000 & 18.14 & 22.99 \\ 5.57000 & 19.43 & 26.46 \\ 6.44000 & 20.28 & 29.53 \\ 7.43000 & 20.91 & 32.57 \\ 8.67000 & 21.40 & 35.85 \\ 10.50000 & 21.80 & 39.93 \\ 12.30000 & 21.98 & 43.29 \\ 18.50000 & 22.09 & 51.96 \\ 24.70000 & 22.01 & 58.09 \\ 37.10000 & 21.79 & 66.67 \\ 61.90000 & 21.51 & 77.44 \\ 123.00000 & 21.27 & 91.89 \\ 247.00000 & 21.16 & 106.56\end{array}$

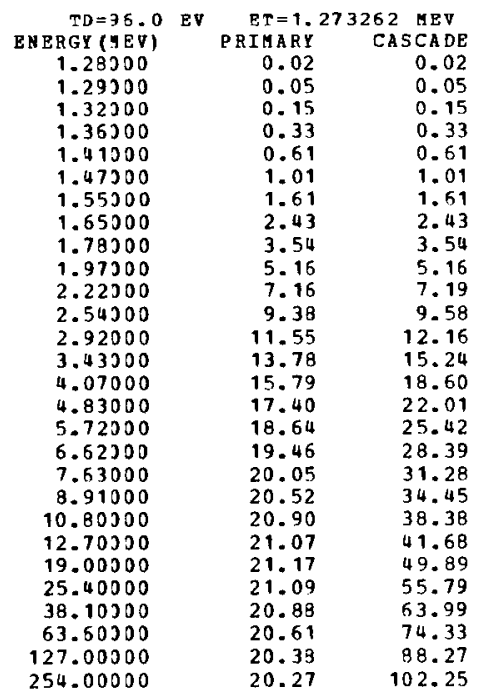


ATOMIC DISPLAZEMENT CROSS-SECTIONS (BARNS) BY PAST ELECTRONS IN GE

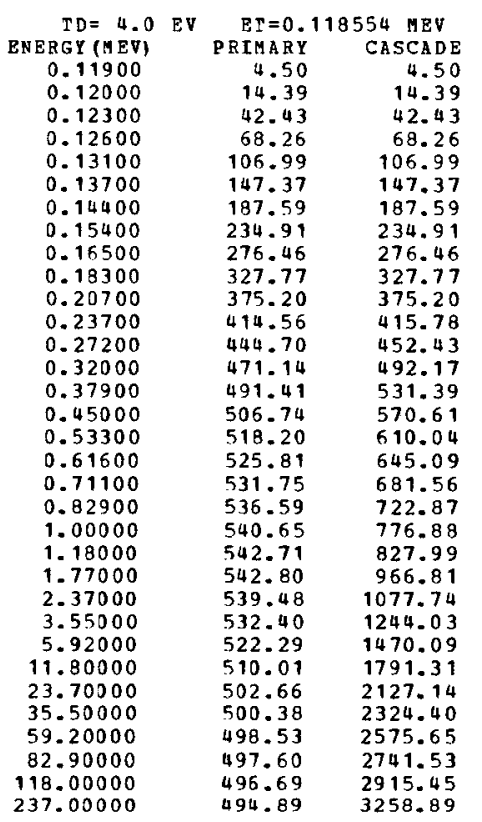

\begin{tabular}{rrr} 
TD $=8.0$ & EV & \multicolumn{2}{c}{ ET=0.218078 MEV } \\
ENERGY (MEV) & PRIMARY & CASEADE \\
0.22000 & 2.78 & 2.78 \\
0.22200 & 5.60 & 5.60 \\
0.22600 & 11.05 & 11.06 \\
0.23300 & 20.05 & 20.05 \\
0.24200 & 30.68 & 30.68 \\
0.25200 & 41.42 & 41.42 \\
0.26600 & 54.88 & 54.88 \\
0.28300 & 69.19 & 69.19 \\
0.30500 & 85.13 & 85.13 \\
0.33800 & 104.96 & 104.96 \\
0.38100 & 125.61 & 125.61 \\
0.43600 & 146.26 & 147.11 \\
0.50100 & 165.16 & 168.85 \\
0.58800 & 184.35 & 193.81 \\
0.69700 & 202.02 & 220.64 \\
0.82800 & 217.32 & 248.51 \\
0.98100 & 229.96 & 276.92 \\
1.13000 & 238.77 & 301.46 \\
1.30000 & 246.04 & 326.62 \\
1.52000 & 252.55 & 355.68 \\
1.85000 & 258.55 & 393.69 \\
2.18000 & 261.94 & 426.64 \\
3.27000 & 265.44 & 512.26 \\
4.36000 & 265.02 & 575.99 \\
6.54000 & 262.56 & 669.04 \\
10.90000 & 258.25 & 789.57 \\
21.80000 & 253.20 & 956.68 \\
43.60000 & 250.51 & 1126.36 \\
65.40000 & 249.65 & 1226.20 \\
109.00000 & 248.89 & 1352.31 \\
152.00000 & 248.46 & 1434.37 \\
218.00000 & 248.00 & 1523.28 \\
436.00000 & 247.10 & 1694.05
\end{tabular}

\begin{tabular}{rrr} 
TD=12.0 EV & \multicolumn{1}{c}{ ET $=0.305561$ MEV } \\
ENERGY(YEV) & PRIMARY & CASCADE \\
0.30300 & 1.17 & 1.17 \\
0.31100 & 2.59 & 2.59 \\
0.31700 & 5.37 & 5.37 \\
0.32500 & 9.37 & 9.37 \\
0.33900 & 14.85 & 14.85 \\
0.35100 & 20.79 & 20.79 \\
0.37200 & 27.42 & 27.42 \\
0.39700 & 35.88 & 35.88 \\
0.42700 & 45.06 & 45.05 \\
0.47300 & 57.45 & 57.45 \\
0.53100 & 71.42 & 71.42 \\
0.61100 & 85.98 & 86.65 \\
0.70200 & 99.91 & 102.49 \\
0.82500 & 114.63 & 121.24 \\
0.97700 & 128.25 & 141.40 \\
1.16300 & 140.13 & 162.52 \\
1.37000 & 149.77 & 183.68 \\
1.58300 & 156.61 & 202.36 \\
1.93300 & 162.36 & 222.13 \\
2.13300 & 167.04 & 243.10 \\
2.59000 & 171.36 & 270.85 \\
3.05300 & 173.75 & 294.64 \\
4.58000 & 176.10 & 355.74 \\
6.11300 & 175.80 & 400.37 \\
9.15000 & 174.16 & 464.37 \\
15.20000 & 171.38 & 545.59 \\
30.50300 & 168.33 & 658.71 \\
61.10300 & 166.79 & 772.66 \\
91.50000 & 166.29 & 839.32 \\
152.00300 & 165.81 & 922.77 \\
213.00300 & 165.53 & 978.30 \\
305.00300 & 165.22 & 1037.33 \\
611.00300 & 164.61 & 1151.43 \\
& &
\end{tabular}

ATONIC DISPlazenENT CRJSS-SECTIONS (BARN) BY FAST ELECTRONS IN GE $\mathrm{Z}=32 \quad \mathrm{~A}=72.600$

\begin{tabular}{rrr} 
TD $=16.0$ & \multicolumn{1}{c}{ EV } & \multicolumn{1}{c}{ EI $=0.384538$ MEV } \\
ENERGY (MEV) & PRIMARY & CASCADE \\
0.38800 & 0.77 & 0.77 \\
0.39200 & 1.56 & 1.66 \\
0.39900 & 3.19 & 3.19 \\
0.41100 & 5.77 & 5.77 \\
0.42600 & 8.92 & 8.92 \\
0.44600 & 12.97 & 12.97 \\
0.46900 & 17.46 & 17.46 \\
0.49900 & 23.02 & 23.02 \\
0.53800 & 29.78 & 29.78 \\
0.59600 & 38.95 & 38.95 \\
0.67200 & 49.50 & 49.53 \\
0.76900 & 60.94 & 61.50 \\
0.88400 & 72.08 & 74.16 \\
1.03000 & 83.31 & 88.40 \\
1.23000 & 94.84 & 105.45 \\
1.46000 & 104.33 & 122.47 \\
1.73000 & 112.10 & 139.83 \\
1.99000 & 117.34 & 154.52 \\
2.30000 & 121.71 & 170.05 \\
2.69000 & 125.36 & 187.19 \\
3.26000 & 128.51 & 208.67 \\
3.84000 & 130.24 & 227.31 \\
5.76000 & 131.80 & 274.49 \\
7.69000 & 131.51 & $\mathbf{3 0 8 . 8 2}$ \\
11.50000 & 130.24 & $\mathbf{3 5 7 . 2 6}$ \\
19.20000 & 128.17 & 419.48 \\
38.40000 & 126.05 & 504.36 \\
76.90000 & 125.00 & 589.99 \\
115.00000 & 124.65 & 639.74 \\
192.00000 & 124.30 & 703.11
\end{tabular}

\begin{tabular}{crr} 
TD $=20.0$ & EV & \multicolumn{2}{c}{ ET $=0.457093$ MEV } \\
ENERGY(MEV) & PRIMARY & CASCADE \\
0.46100 & 0.48 & 0.48 \\
0.46600 & 1.10 & 1.10 \\
0.47500 & 2.22 & 2.22 \\
0.48900 & 3.97 & 3.97 \\
0.50700 & 6.21 & 6.21 \\
0.53000 & 9.06 & 9.06 \\
0.55700 & 12.36 & 12.36 \\
0.59400 & 16.77 & 16.77 \\
0.63900 & 21.91 & 21.91 \\
0.70800 & 29.26 & 29.26 \\
0.79900 & 37.95 & 37.98 \\
0.91400 & 47.39 & 47.89 \\
1.05000 & 56.64 & 58.41 \\
1.23000 & 66.33 & 70.78 \\
1.46000 & 75.59 & 84.62 \\
1.73000 & 83.37 & 98.73 \\
2.05000 & 89.75 & 113.25 \\
2.37000 & 94.14 & 125.94 \\
2.74000 & 97.63 & 138.86 \\
3.19000 & 100.43 & 152.64 \\
3.88000 & 102.93 & 170.66 \\
4.57000 & 104.24 & 185.94 \\
6.85000 & 105.32 & 224.34 \\
9.14000 & 105.03 & 252.12 \\
13.70000 & 103.97 & 291.43 \\
22.80000 & 102.34 & 341.17 \\
45.70000 & 100.73 & 409.50 \\
91.40000 & 99.95 & 477.97 \\
137.00000 & 99.68 & 518.03 \\
228.00000 & 99.41 & 568.42
\end{tabular}

\begin{tabular}{|c|c|c|}
\hline$T D=24.0$ & $\mathrm{ET}=0.5$ & $M E V$ \\
\hline ENERGQ (MEV) & PRIMARY & CASCADE \\
\hline 0.52900 & 0.34 & 0.34 \\
\hline 0.53500 & 0.81 & 0.81 \\
\hline 0.54500 & 1.60 & 1.60 \\
\hline 0.56100 & 2.89 & 2.89 \\
\hline 0.58200 & 4.63 & 4.63 \\
\hline 0,60300 & 6.81 & 6.81 \\
\hline 0.63900 & 9.44 & 9.44 \\
\hline 0.68100 & 12.98 & 12.98 \\
\hline $0.73+00$ & 17.34 & 17.34 \\
\hline 0.81300 & 23.50 & 23.50 \\
\hline 0.91300 & 30.92 & 30.95 \\
\hline 1.04300 & 38.43 & 38.84 \\
\hline 1.20000 & 46.64 & 48.17 \\
\hline 1.41200 & 55.13 & 59.04 \\
\hline 1.67000 & 62.95 & 70.83 \\
\hline 1.99300 & 69.76 & 83.40 \\
\hline 2.36300 & 75.13 & 95.94 \\
\hline 2.72000 & 78.70 & 106.59 \\
\hline 3.14000 & 81.56 & 117.52 \\
\hline 3.57000 & 83.90 & 129.57 \\
\hline 4.45300 & 85.87 & 144.64 \\
\hline 5.24000 & 86.90 & 157.57 \\
\hline 7.86000 & 87.70 & 190.03 \\
\hline 10.40000 & 87.43 & 212.70 \\
\hline 15.70000 & 86.52 & 246.22 \\
\hline 26.20300 & 85.17 & 288.04 \\
\hline 52.40000 & 83.88 & 344.92 \\
\hline 104.00000 & 83.27 & 401.40 \\
\hline 157.00300 & 83.05 & 435.39 \\
\hline 262.00000 & 82.82 & 477.60 \\
\hline
\end{tabular}


ATOMIC DISPLAZEHENT CROSS-SECTIONS (BARNS) BY PAST BLECTRONS IN GE

\begin{tabular}{|c|c|c|c|}
\hline $\begin{array}{r}\Gamma D=28.0 \\
\text { NERGY (MEV) }\end{array}$ & EV & $\begin{aligned} & \text { ET }=0.5 \\
& \text { PRIMARY }\end{aligned}$ & $\begin{array}{l}926 \text { MEV } \\
\text { CASCADE }\end{array}$ \\
\hline 0.59300 & & 0.26 & 0.26 \\
\hline 0.59900 & & 0.58 & 0.58 \\
\hline 0.61100 & & 1.23 & 1.23 \\
\hline 0.62900 & & 2.25 & 2.25 \\
\hline 0.65200 & & 3.61 & 3.61 \\
\hline 0.58100 & & 5.38 & 5.38 \\
\hline 0.71700 & & 7.64 & 7.64 \\
\hline 0.76400 & & 10.63 & 10.63 \\
\hline 0.82300 & & 14.33 & 14.33 \\
\hline 0.91100 & & 19.63 & 19.63 \\
\hline 1.02000 & & 25.65 & 25.68 \\
\hline 1.17000 & & 32.89 & 33.29 \\
\hline 1.35000 & & 40.08 & 41.51 \\
\hline 1.58000 & & 47.31 & 50.85 \\
\hline 1.88000 & & 54.29 & 61.51 \\
\hline 2.23000 & & 60.02 & 72.27 \\
\hline 2.64000 & & 64.59 & 83.16 \\
\hline 3.05000 & & 67.69 & 92.64 \\
\hline 3.52000 & & 70.10 & 102.17 \\
\hline 4.11000 & & 72.05 & 112.59 \\
\hline 4.99000 & & 73.69 & 125.79 \\
\hline 5.87000 & & 74.52 & 136.93 \\
\hline 8.81000 & & 75.13 & 165.04 \\
\hline 11.70000 & & 74.87 & 184.85 \\
\hline 17.60000 & & 74.07 & 213.45 \\
\hline 29.30000 & & 72.93 & 249.22 \\
\hline 58.70000 & & 71.86 & 298.17 \\
\hline 117.00000 & & 71.35 & 346.91 \\
\hline & & & \\
\hline
\end{tabular}

\begin{tabular}{ccc}
$T D=32.0$ & EV & \multicolumn{2}{c}{ ET=0.647816 HEV } \\
ENERGY (MEV) & PRIMARY & CASTADE \\
0.65400 & 0.23 & 0.23 \\
0.66000 & 0.45 & 0.45 \\
0.67300 & 0.97 & 0.97 \\
0.69300 & 1.80 & 1.80 \\
0.71900 & 2.96 & 2.96 \\
0.75100 & 4.46 & 4.46 \\
0.79000 & 6.37 & 5.37 \\
0.84200 & 8.97 & 8.97 \\
0.90600 & 12.17 & 12.17 \\
1.00000 & 16.71 & 16.71 \\
1.13000 & 22.50 & 22.54 \\
1.29000 & 28.72 & 29.09 \\
1.48000 & 34.87 & 36.14 \\
1.74000 & 41.49 & 44.74 \\
2.07000 & 47.66 & 54.25 \\
2.46000 & 52.76 & 63.96 \\
2.91000 & 56.73 & 73.61 \\
3.36000 & 59.40 & 82.01 \\
3.88000 & 61.49 & 90.50 \\
4.53000 & 63.16 & 99.73 \\
5.50000 & 64.55 & 111.41 \\
6.47000 & 65.24 & 121.24 \\
9.71000 & 65.70 & 146.00 \\
12.90000 & 65.46 & 163.44 \\
19.40000 & 64.75 & 188.52 \\
32.30000 & 63.76 & 219.88 \\
64.70000 & 62.85 & 262.75 \\
129.00000 & 62.42 & 305.44 \\
194.00000 & 62.26 & 330.71
\end{tabular}

\begin{tabular}{|c|c|c|c|}
\hline $\mathrm{TD}=36.0$ & EV & $E T=0$. & 760 MEV \\
\hline ENERGY (MEV) & & PRIMARY & CASCADE \\
\hline 0.71100 & & 0.17 & 0.17 \\
\hline 0.71900 & & 0.37 & 0.37 \\
\hline 0.73200 & & 0.78 & 0.78 \\
\hline 0.75400 & & 1.49 & 1.49 \\
\hline 0.78200 & & 2.47 & 2.47 \\
\hline 0.81700 & & 3.77 & 3.77 \\
\hline 0.85900 & & 5.43 & 5.43 \\
\hline 0.91500 & & 7.74 & 7.74 \\
\hline 0.98500 & & 10.62 & 10.62 \\
\hline 1.09000 & & 14.76 & 14.76 \\
\hline 1.23000 & & 19.92 & 19.96 \\
\hline 1.40300 & & 25.41 & 25.75 \\
\hline 1.62300 & & 31.29 & 32.54 \\
\hline 1.90000 & & 37.14 & 40.20 \\
\hline 2.25000 & & 42.51 & 48.58 \\
\hline 2.67300 & & 47.02 & 57.28 \\
\hline 3.17300 & & 50.62 & 66.17 \\
\hline 3.66000 & & 52.97 & 73.72 \\
\hline 4.22000 & & 54.78 & 81.28 \\
\hline 4.93000 & & 56.23 & 89.60 \\
\hline 5.99000 & & 57.43 & 100.10 \\
\hline 7.04000 & & 58.01 & 108.86 \\
\hline 10.50000 & & 58.38 & 130.64 \\
\hline 14.00000 & & 58.15 & 146.39 \\
\hline 21.10300 & & 57.51 & 168.85 \\
\hline 35.20000 & & 56.63 & 196.88 \\
\hline 70.40300 & & 55.85 & 234.93 \\
\hline 140.00300 & & 55.47 & 272.75 \\
\hline 211.00100 & & 55.34 & 295.34 \\
\hline
\end{tabular}

ATOMIC DISPLAEBMENT CROSS-SECTIONS (BARNS) BY PAST ELECTRONS IN GE $=32 \quad A=72.600$

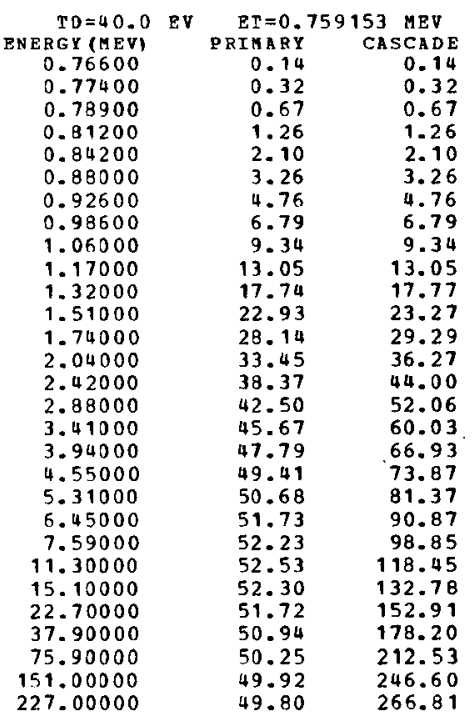

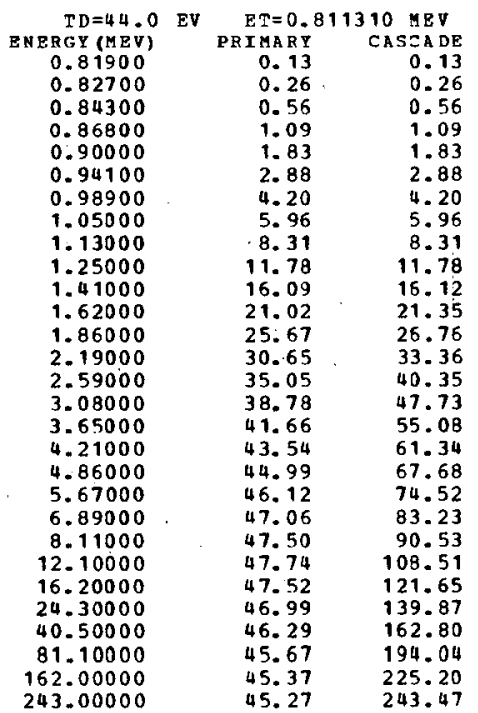

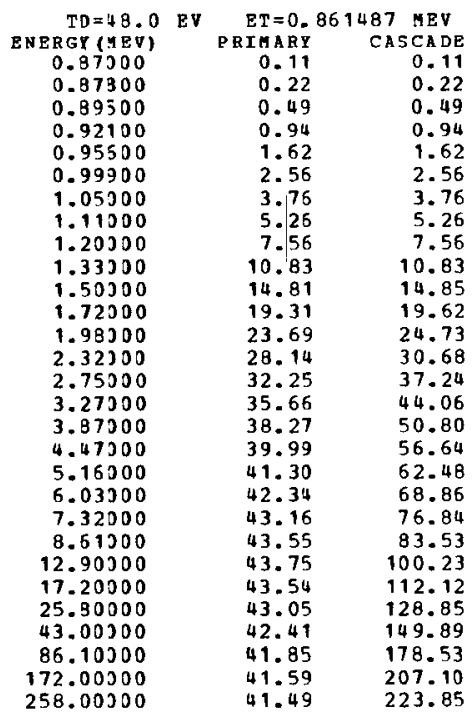


ATOMIC DISPLAzEAENT EROSS-SECTIONS (BARNS) BY FAST ELECTRONS IN GE $z=32 \quad A=72.600$

\begin{tabular}{rrr} 
PD $=52.0$ EV & \multicolumn{2}{c}{ ET $=0.909894$ MEV } \\
ENERGY (HEV) & PRIMARY & CASCADE \\
0.91800 & 0.09 & 0.09 \\
0.92300 & 0.20 & 0.20 \\
0.94600 & 0.44 & 0.44 \\
0.97300 & 0.83 & 0.83 \\
1.00000 & 1.28 & 1.28 \\
1.05000 & 2.20 & 2.20 \\
1.11000 & 3.44 & 3.44 \\
1.19000 & 4.97 & 4.97 \\
1.27000 & 6.99 & 6.99 \\
1.41000 & 10.09 & 10.09 \\
1.59000 & 13.79 & 13.83 \\
1.81000 & 17.76 & 18.05 \\
2.09000 & 21.92 & 22.92 \\
2.45000 & 26.07 & 28.47 \\
2.91000 & 29.91 & 34.65 \\
3.45000 & 33.00 & 40.90 \\
4.09000 & 35.42 & 47.23 \\
4.73000 & 37.01 & 52.70 \\
5.45000 & 38.18 & 58.05 \\
6.36000 & 39.11 & 63.92 \\
7.73000 & 39.86 & 71.35 \\
9.09000 & 40.21 & 77.54 \\
13.50000 & 40.38 & 92.94 \\
18.10000 & 40.18 & 103.87 \\
27.20000 & 39.72 & 119.39 \\
45.40000 & 39.14 & 138.88 \\
90.90000 & 38.63 & 165.33 \\
181.00000 & 38.39 & 191.59 \\
& &
\end{tabular}

\begin{tabular}{|c|c|c|}
\hline$T D=56.0$ & $\mathrm{ET}=0.9$ & 04 HEV \\
\hline ENERGY (NEV) & PR I MA R Y & CASこADE \\
\hline 0.96600 & 0.08 & 0.08 \\
\hline 0.97500 & 0.17 & 0.17 \\
\hline 0.99400 & 0.38 & 0.38 \\
\hline 1.02000 & 0.70 & 0.70 \\
\hline 1.06000 & 1.28 & 1.28 \\
\hline 1.10000 & 1.93 & 1.93 \\
\hline 1.16000 & 3.01 & 3.01 \\
\hline 1.24000 & 4.55 & 4.55 \\
\hline 1.33000 & 6.35 & 6.35 \\
\hline 1.48000 & 9.31 & 9.31 \\
\hline 1.67000 & 12.79 & 12.83 \\
\hline 1.91000 & 16.63 & 16.93 \\
\hline 2.20000 & 20.45 & 21.41 \\
\hline 2.58000 & 24.33 & 26.63 \\
\hline 3.06000 & 27.86 & 32.37 \\
\hline 3.63000 & 30.73 & 38.23 \\
\hline 4.30000 & 32.96 & 44.12 \\
\hline 4.97000 & 34.42 & 49.20 \\
\hline 5.74000 & 35.51 & 54.28 \\
\hline 6.69000 & 36.35 & 59.71 \\
\hline 8.13000 & 37.04 & 66.63 \\
\hline 9.56000 & 37.34 & \\
\hline 14.30000 & 37.48 & 86.71 \\
\hline 19.10000 & 37.30 & 97.00 \\
\hline 28.70000 & 36.87 & 111.43 \\
\hline 47.80000 & 36.33 & 129.46 \\
\hline 95.60000 & 35.86 & 153.98 \\
\hline 191.00000 & 35.64 & 178.49 \\
\hline
\end{tabular}

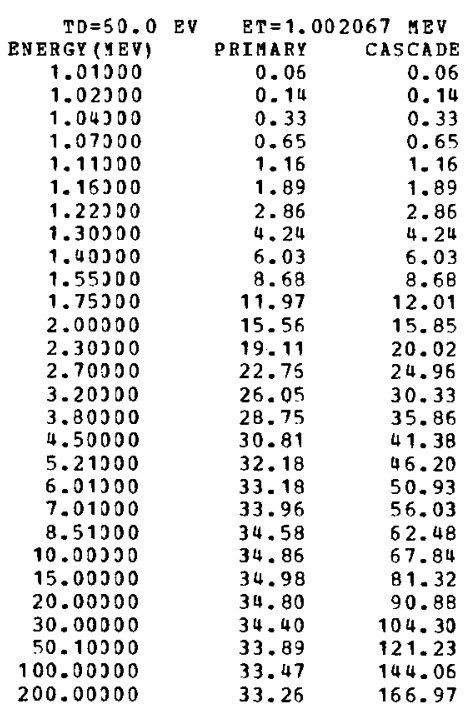

A TOMIC DISPLAZEMENT CRJSS-SEETIONS (BARNS) BY PAST ELECTRONS IN GE $z=32 \quad A=72.600$

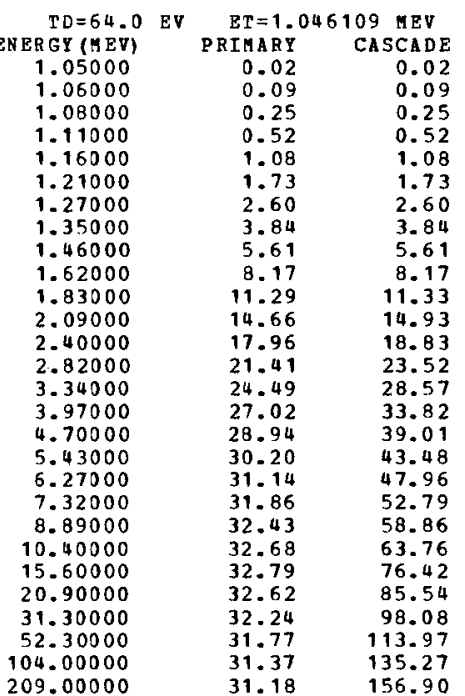

\begin{tabular}{rrr} 
TD $=68.0$ & EV & \multicolumn{2}{c}{ ET $=1.088939$ MEV } \\
ENERGY (HEV) & PRIMARY & CASCADE \\
1.09000 & 0.01 & 0.01 \\
1.11000 & 0.12 & 0.12 \\
1.13000 & 0.27 & 0.27 \\
1.16000 & 0.52 & 0.52 \\
1.20000 & 0.92 & 0.92 \\
1.26000 & 1.61 & 1.61 \\
1.32000 & 2.39 & 2.39 \\
1.41000 & 3.65 & 3.66 \\
1.52000 & 5.27 & 5.27 \\
1.68000 & 7.60 & 7.60 \\
1.90000 & 10.58 & 10.62 \\
2.17000 & 13.77 & 14.04 \\
2.50000 & 16.97 & 17.82 \\
2.94000 & 20.24 & 22.28 \\
3.48000 & 23.12 & 27.04 \\
4.13000 & 25.48 & 31.96 \\
4.90000 & 27.30 & 36.93 \\
5.66000 & 28.47 & 41.15 \\
6.53000 & 29.34 & 45.35 \\
7.62000 & 30.01 & 49.90 \\
9.25000 & 30.53 & 55.61 \\
10.80000 & 30.76 & 60.18 \\
16.30000 & 30.85 & 72.29 \\
21.70000 & 30.69 & 80.70 \\
32.60000 & 30.33 & 92.60 \\
54.40000 & 29.89 & 107.52 \\
108.00000 & 29.52 & 127.52 \\
217.00000 & 29.35 & 147.88
\end{tabular}

\begin{tabular}{|c|c|c|}
\hline $\begin{array}{r}T D=72.0 \\
\text { ENERGY }\end{array}$ & $\begin{aligned} E T & =1,1 \\
P R T A R Y & \end{aligned}$ & $\begin{array}{l}552 \mathrm{MBV} \\
\text { CASCADE }\end{array}$ \\
\hline ENERGY (IEV) & $\begin{array}{r}\text { PRIMARY } \\
0.04\end{array}$ & $\begin{array}{r}\text { CASCADE } \\
0.04\end{array}$ \\
\hline 1.14300 & 0.04 & 0.04 \\
\hline $\begin{array}{l}1.15300 \\
1.17000\end{array}$ & $\begin{array}{l}0.10 \\
0.22\end{array}$ & $\begin{array}{l}0.10 \\
0.22\end{array}$ \\
\hline 1.20000 & 0.44 & $\begin{array}{l}0.22 \\
0.44\end{array}$ \\
\hline 1.25000 & 0.89 & 0.89 \\
\hline 1.31000 & 1.52 & 1.52 \\
\hline 1.37500 & 2.23 & 2.23 \\
\hline 1.46300 & 3.39 & 3.39 \\
\hline 1.58300 & 4.99 & 4.99 \\
\hline 1.75000 & 7.26 & 7.26 \\
\hline 1.97000 & 9.98 & 10.02 \\
\hline 2.26300 & 13.11 & 13.38 \\
\hline 2.50300 & 16.11 & 16.93 \\
\hline 3.05000 & 19.16 & 21.11 \\
\hline 3.61300 & 21.83 & 25.63 \\
\hline 4.29000 & 24.12 & 30.34 \\
\hline 5.08000 & 25.81 & 34.99 \\
\hline 5.87000 & 26.91 & 39.00 \\
\hline 6.78300 & 27.73 & 43.01 \\
\hline 7.91300 & 28.36 & 47.31 \\
\hline 9.61300 & 28.85 & 52.74 \\
\hline 11.30300 & 29.06 & 57.26 \\
\hline 16.90300 & 29.14 & 68.46 \\
\hline 22.60300 & 28.98 & 76.53 \\
\hline 33.90300 & 28.64 & 87.73 \\
\hline 56.50000 & 28.22 & 101.80 \\
\hline 113.00300 & 27.88 & 120.89 \\
\hline 226.00300 & 27.71 & 139.99 \\
\hline
\end{tabular}


ATONIC DISPLAZEMENT CROSS-SECTIONS (BARNS) BY FAST ELECTRONS IN GE

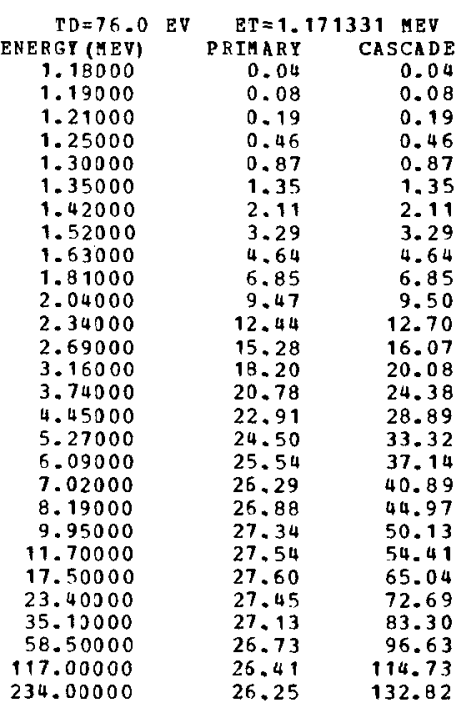

\begin{tabular}{ccr} 
TD $=80.0$ & EV & \multicolumn{2}{c}{ ET=1.211050 MEV } \\
ENERGY (MEV) & PRIMARY & CASEADE \\
1.22000 & 0.03 & 0.03 \\
1.23000 & 0.07 & 0.07 \\
1.25000 & 0.17 & 0.17 \\
1.29000 & 0.41 & 0.41 \\
1.34000 & 0.78 & 0.78 \\
1.40000 & 1.31 & 1.31 \\
1.47000 & 2.01 & 2.01 \\
1.57000 & 3.10 & 3.10 \\
1.69000 & 4.46 & 4.46 \\
1.87000 & 6.50 & 6.50 \\
2.11000 & 9.02 & 9.06 \\
2.42000 & 11.85 & 12.11 \\
2.78000 & 14.55 & 15.31 \\
3.26000 & 17.30 & 19.10 \\
3.87000 & 19.79 & 23.28 \\
4.60000 & 21.80 & 27.55 \\
5.44000 & 23.30 & 31.74 \\
6.29000 & 24.28 & 35.38 \\
7.26000 & 25.00 & 38.99 \\
8.47000 & 25.55 & 42.88 \\
10.20000 & 25.96 & 47.56 \\
12.10000 & 26.16 & 51.86 \\
18.10000 & 26.22 & 61.97 \\
24.20000 & 26.07 & 59.24 \\
36.30000 & 25.76 & 79.32 \\
60.50000 & 25.39 & 91.99 \\
121.00000 & 25.09 & 109.19 \\
242.00000 & 24.94 & 126.38
\end{tabular}

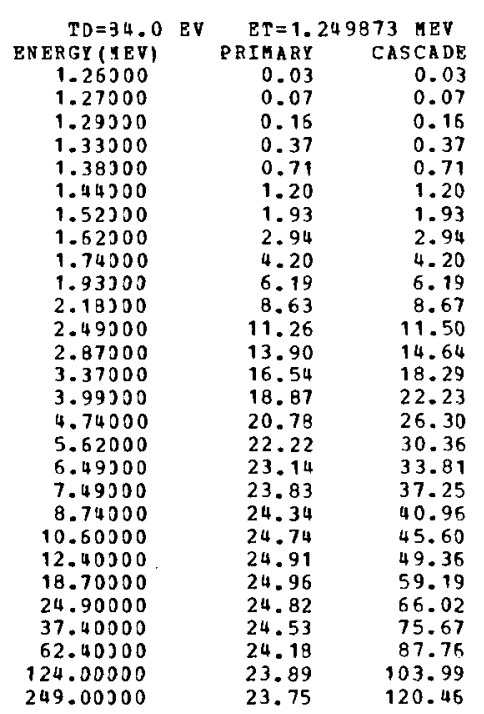

ATOMIC DISPLA

\begin{tabular}{rrr} 
TD=88.0 & EV & \multicolumn{1}{c}{ ER=1.28785B } \\
ENERG \\
1.30000 & PRIARY & CASCADE \\
1.31000 & 0.04 & 0.04 \\
1.33000 & 0.07 & 0.07 \\
1.37000 & 0.15 & 0.15 \\
1.42000 & 0.35 & 0.35 \\
1.49000 & 0.66 & 0.66 \\
1.57000 & 1.18 & 1.18 \\
1.67000 & 1.87 & 1.87 \\
1.80000 & 2.81 & 2.81 \\
1.99000 & 4.08 & 4.08 \\
2.25000 & 5.93 & 5.93 \\
2.57000 & 8.29 & 8.33 \\
2.96000 & 10.81 & 11.04 \\
3.47000 & 13.31 & 14.04 \\
4.12000 & 15.81 & 17.51 \\
4.89000 & 18.08 & 21.34 \\
5.79000 & 19.88 & 25.22 \\
6.69000 & 21.24 & 29.07 \\
7.72000 & 22.11 & 32.39 \\
9.01000 & 22.76 & 35.68 \\
10.90000 & 23.25 & 39.23 \\
12.80000 & 23.62 & 43.61 \\
19.30000 & 23.79 & 47.29 \\
25.70000 & 23.83 & 56.68 \\
38.60000 & 23.69 & 63.20 \\
64.30000 & 23.41 & 72.41 \\
128.00000 & 23.08 & 83.92 \\
257.00000 & 22.80 & 99.45 \\
& 22.67 & 115.17
\end{tabular}

\begin{tabular}{|c|c|c|c|}
\hline \multirow{2}{*}{\multicolumn{2}{|c|}{$\begin{aligned} & T D=92.0 \quad \mathrm{EV} \\
& \text { ENERGY (HEV) }\end{aligned}$}} & \multicolumn{2}{|c|}{$\mathrm{ET}=1.325057 \mathrm{MEV}$} \\
\hline & & PRI HARY & CASCA DE \\
\hline 1.33000 & & 0.01 & 0.01 \\
\hline 1.35000 & & 0.07 & 0.07 \\
\hline 1.37000 & & 0.15 & 0.15 \\
\hline 1.41000 & & 0.33 & 0.33 \\
\hline 1.47000 & & 0.68 & 0.68 \\
\hline 1.53000 & & 1.10 & 1.10 \\
\hline 1.61000 & & 1.74 & 1.74 \\
\hline 1.72000 & & 2.70 & 2.70 \\
\hline $\begin{array}{l}1.85000 \\
2.05000\end{array}$ & & $\begin{array}{l}3.89 \\
5.71\end{array}$ & $\begin{array}{l}3.89 \\
5.71\end{array}$ \\
\hline 2.31000 & & 7.91 & 7.94 \\
\hline 2.65000 & & 10.40 & 10.64 \\
\hline 3.04000 & & 12.74 & 13.43 \\
\hline 3.57000 & & 15.16 & 16.80 \\
\hline 4.24000 & & 17.33 & 20.48 \\
\hline 5.03000 & & 19.04. & 24.19 \\
\hline 5.96000 & & 20.34 & 27.91 \\
\hline 6.89000 & & 21.18 & 31.10 \\
\hline 7.95000 & & 21.79 & 34.25 \\
\hline 9.27000 & & 22.25 & 37.63 \\
\hline 11.20000 & & 22.60 & 41.79 \\
\hline 13.20000 & & 22.75 & 45.40 \\
\hline 19.80000 & & 22.79 & 54.27 \\
\hline 26.50000 & & 22.56 & 60.63 \\
\hline 39.70000 & & 22.39 & 69.38 \\
\hline 66.20000 & & 22.07 . & 80.42 \\
\hline 132.00000 & & 21.81 & 95.31 \\
\hline 265.00000 & & 21.68 & 110.34 \\
\hline
\end{tabular}

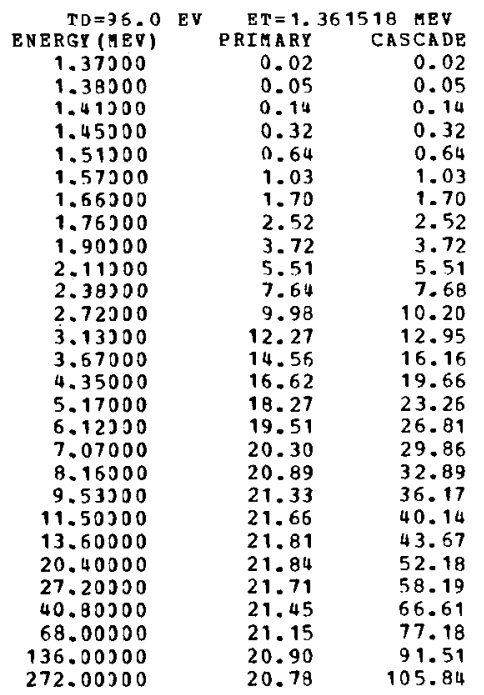


ATOMIC DISPLAZEMENT CROSS-SECTIONS (BARNS) BY FAST ELECTRONS IN RB $Z=37 \quad A=85.480$

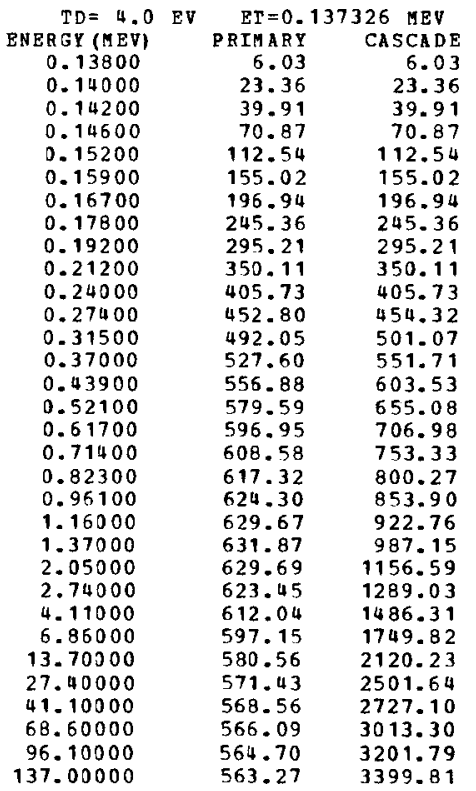

\begin{tabular}{crr} 
TD $=8.0$ & EV & \multicolumn{2}{c}{ ET $=0.250270$ MEV } \\
ENERGY(MEV) & PRIMARY & CASEADE \\
0.25200 & 2.25 & 2.25 \\
0.25500 & 6.09 & 6.09 \\
0.26000 & 12.26 & 12.26 \\
0.26700 & 20.50 & 20.50 \\
0.27700 & 31.52 & 31.52 \\
0.29000 & 44.69 & 44.69 \\
0.30500 & 58.52 & 58.52 \\
0.32500 & 75.05 & 75.05 \\
0.35000 & 93.24 & 93.24 \\
0.38700 & 116.23 & 116.23 \\
0.43700 & 141.75 & 141.75 \\
0.50000 & 167.45 & 168.52 \\
0.57500 & 191.46 & 195.99 \\
0.67500 & 215.86 & 227.50 \\
0.80000 & 238.17 & 261.29 \\
0.95100 & 257.26 & 296.44 \\
1.12000 & 272.10 & 330.62 \\
1.30000 & 283.05 & 362.67 \\
1.50000 & 291.40 & 394.29 \\
1.75000 & 298.27 & 429.29 \\
2.12000 & 304.06 & 474.17 \\
2.50000 & 307.00 & 513.85 \\
3.75000 & 308.36 & 615.21 \\
5.00000 & 306.18 & 689.75 \\
7.50000 & 301.48 & 797.61 \\
12.50000 & 294.92 & 936.09 \\
25.00000 & 288.13 & 1127.03 \\
50.00000 & 284.73 & 1320.31 \\
75.00000 & 283.61 & 1433.84 \\
125.00000 & 282.52 & 1577.02 \\
175.00000 & 281.84 & 1671.15 \\
250.00000 & 281.12 & 1770.76
\end{tabular}

\begin{tabular}{|c|c|c|}
\hline$T D=12.0$ & $\mathrm{ET}=0.3$ & MEV \\
\hline ENERGY (YEV) & PRIMARY & CASCADE \\
\hline 0.35100 & 1.09 & 1.09 \\
\hline 0.35500 & 2.82 & 2.82 \\
\hline 0.36200 & 5.80 & 5.80 \\
\hline 0.37200 & 9.95 & 9.95 \\
\hline 0.38500 & 15.58 & 15.58 \\
\hline 0.40700 & 22.52 & 22.52 \\
\hline 0.42500 & 30.20 & 30.20 \\
\hline 0.45300 & 39.83 & 39.83 \\
\hline 0.48700 & 50.65 & 50.65 \\
\hline 0.54300 & 65.82 & 65.82 \\
\hline 0.50300 & 82.91 & 82.93 \\
\hline 0.69500 & 100.94 & 101.79 \\
\hline 0.80100 & 118.58 & 121.85 \\
\hline 0.94300 & 136.72 & 145.06 \\
\hline 1.91000 & 153.17 & 169.71 \\
\hline 1.32300 & 167.59 & 196.04 \\
\hline 1.56300 & 178.88 & 222.08 \\
\hline 1.81300 & 186.88 & 245.79 \\
\hline 2.09000 & 192.93 & 269.19 \\
\hline 2.43000 & 197.68 & 294.19 \\
\hline 2.96300 & 201.76 & 327.53 \\
\hline 3.48300 & 203.65 & 355.39 \\
\hline 5.22000 & 204.36 & 426.71 \\
\hline 6.96300 & 202.87 & 478.37 \\
\hline 10.40300 & 199.80 & 551.48 \\
\hline 17.40000 & 195.57 & 645.97 \\
\hline 34.80000 & 191.52 & 774.39 \\
\hline 69.60000 & 189.56 & 903.75 \\
\hline 104.00000 & 188.89 & 978.88 \\
\hline 174.00300 & 188.17 & 1075.11 \\
\hline 243.00300 & 187.72 & 1137.39 \\
\hline 348.00000 & 187.23 & 1204.26 \\
\hline
\end{tabular}

ATOMIC DISPLAZEMENT CROSS-SECTIONS (BARNS) BY FAST ELECTRONS IN RB

\begin{tabular}{rrr} 
TD $=16.0$ & EV & \multicolumn{1}{c}{ ET $=0.436599$ MEV } \\
PNERG (MEV) & PRTMAR & CASCADE \\
0.44000 & 0.69 & 0.69 \\
0.44500 & 1.71 & 1.71 \\
0.45400 & 3.55 & 3.55 \\
0.46700 & 5.21 & 6.21 \\
0.48400 & 9.67 & 9.67 \\
0.50600 & 14.12 & 14.12 \\
0.53200 & 19.29 & 19.29 \\
0.56700 & 26.06 & 26.06 \\
0.51100 & 34.17 & 34.17 \\
0.57600 & 45.28 & 45.28 \\
0.76400 & 58.65 & 58.70 \\
0.87300 & 72.75 & 73.50 \\
1.00000 & 85.26 & 88.87 \\
1.17000 & 100.46 & 107.03 \\
1.39000 & 114.00 & 127.46 \\
1.65000 & 125.22 & 148.28 \\
1.96000 & 134.24 & 169.67 \\
2.27000 & 140.26 & 188.25 \\
2.61000 & 144.69 & 206.18 \\
3.05000 & 148.32 & 226.47 \\
3.71000 & 151.23 & 252.32 \\
4.36000 & 152.53 & 273.90 \\
6.54000 & 152.80 & 328.80 \\
8.73000 & 151.62 & 368.44 \\
13.00000 & 149.32 & 423.47 \\
21.80000 & 146.21 & 495.18 \\
43.60000 & 143.40 & 591.91 \\
87.30000 & 142.05 & 689.28 \\
130.00000 & 141.58 & 745.20 \\
218.00000 & 141.05 & 817.70
\end{tabular}

\begin{tabular}{rrr}
$T D=20.0$ & EV & \multicolumn{1}{c}{ ET $=0.517177$ MEV } \\
ENERGY(MEV) & PRIMAY & CASCADE \\
0.52200 & 0.55 & 0.55 \\
0.52700 & 1.12 & 1.12 \\
0.53700 & 2.30 & 2.30 \\
0.55300 & 4.22 & 4.22 \\
0.57400 & 6.81 & 6.81 \\
0.59900 & 9.95 & 9.95 \\
0.53000 & 13.89 & 13.89 \\
0.57200 & 19.20 & 19.20 \\
0.72400 & 25.60 & 25.60 \\
0.80100 & 34.57 & 34.57 \\
0.90500 & 45.49 & 45.55 \\
1.03000 & 56.81 & 57.44 \\
1.18000 & 68.04 & 70.24 \\
1.39000 & 80.31 & 86.06 \\
1.65000 & 91.38 & 103.04 \\
1.96000 & 100.55 & 120.47 \\
2.32000 & 107.68 & 137.88 \\
2.68000 & 112.41 & 153.00 \\
3.10000 & 116.06 & 168.45 \\
3.62000 & 118.84 & 185.06 \\
4.39000 & 121.00 & 205.93 \\
5.17000 & 121.96 & 223.78 \\
7.75000 & 122.00 & 268.32 \\
10.30000 & 121.03 & 299.88 \\
15.50000 & 119.12 & 345.38 \\
25.80000 & 115.73 & 402.18 \\
51.70000 & 114.59 & 479.98 \\
103.00000 & 113.58 & 557.40 \\
155.00000 & 113.21 & 503.34 \\
258.00000 & 112.79 & 660.50
\end{tabular}

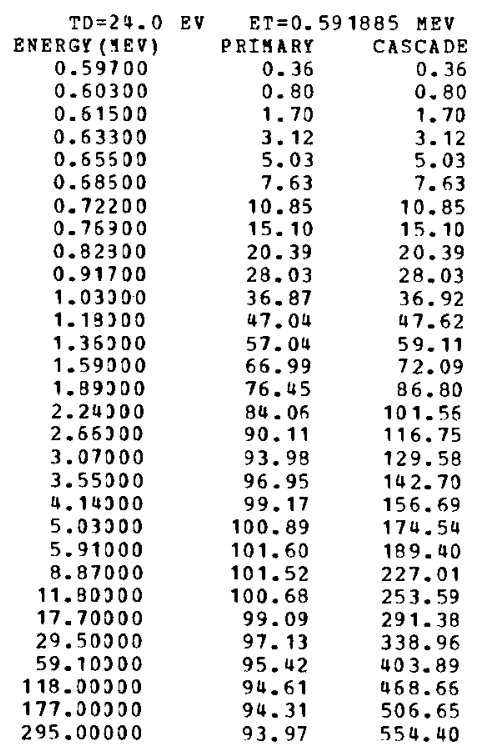


ATONIC DISPLAZEMENT CROSS-SECTIONS (BARNS) BY FAST ELECTRONS IN RB

$Z=37 \quad A=85.480$

\begin{tabular}{|c|c|c|}
\hline \multirow{2}{*}{$\begin{array}{r}T D=28.0 \\
\text { ENERGY (M EV) }\end{array}$} & \multicolumn{2}{|c|}{$\mathrm{ET}=0.661843 \mathrm{MEV}$} \\
\hline & PRIMARY & CASCADE \\
\hline 0.66800 & 0.29 & 0.29 \\
\hline 0.67500 & 0.64 & 0.64 \\
\hline 0.68800 & 1.32 & 1.32 \\
\hline 0.70800 & 2.44 & 2.44 \\
\hline 0.73400 & 4.00 & 4.00 \\
\hline 0.76700 & 6.11 & 6.11 \\
\hline 0.80700 & 8.79 & 8.79 \\
\hline 0.86000 & 12.44 & 12.44 \\
\hline 0.92600 & 16.99 & 16.99 \\
\hline 1.02000 & 23.26 & 23.26 \\
\hline 1.15000 & 31.22 & 31.27 \\
\hline 1.32000 & 40.24 & 40.78 \\
\hline 1.52000 & 48.92 & 50.81 \\
\hline 1.78000 & 57.67 & 62.33 \\
\hline 2.11000 & 65.74 & 75.03 \\
\hline 2.51000 & 72.43 & 88.24 \\
\hline 2.97000 & 77.48 & 101.24 \\
\hline 3.44000 & 80.83 & 112.71 \\
\hline 3.97000 & 83.27 & 123.98 \\
\hline 4.63000 & 85.11 & 136.14 \\
\hline 5.62000 & 86.51 & 151.54 \\
\hline 6.61000 & 87.07 & 164.48 \\
\hline 9.92000 & 86.92 & 196.95 \\
\hline 13.20000 & 86.18 & 219.87 \\
\hline 19.80000 & 84.81 & 252.36 \\
\hline 33.00000 & 83.15 & 293.23 \\
\hline 66.10000 & 81.74 & 348.94 \\
\hline 132.00000 & 81.07 & 404.49 \\
\hline 198.00000 & 80.82 & 437.07 \\
\hline
\end{tabular}

\begin{tabular}{|c|c|c|}
\hline$T D=32.0 \quad E V$ & $\mathrm{ET}=0.7$ & $57 \mathrm{AEV}$ \\
\hline ENERGY (MEV) & PRI IAA RY & CASこADE \\
\hline 0.73500 & 0.24 & 0.24 \\
\hline 0.74200 & 0.49 & 0.49 \\
\hline 0.75600 & 1.03 & 1.03 \\
\hline 0.77800 & 1.94 & 1.94 \\
\hline 0.80700 & 3. 26 & 3.26 \\
\hline 0.84400 & 5.10 & 5.10 \\
\hline 0.88700 & 7.36 & 7.36 \\
\hline 0.94500 & 10.58 & 10.58 \\
\hline 1.01000 & 14.11 & 14.11 \\
\hline 1.12000 & 20.03 & 20.03 \\
\hline 1.27000 & 27.45 & 27.50 \\
\hline 1.45000 & 35.15 & 35.65 \\
\hline 1.67000 & 42.87 & 44.61 \\
\hline 1.96000 & 50.73 & 55.03 \\
\hline 2.32300 & 57.77 & 66.26 \\
\hline 2.76000 & 63.63 & 78.02 \\
\hline 3.27000 & 68.04 & 89.66 \\
\hline 3.78000 & 70.90 & 99.69 \\
\hline 4.36000 & 72.99 & 109.63 \\
\hline 5.09000 & 74.56 & 120.45 \\
\hline 6.18000 & 75.73 & 134.07 \\
\hline 7.27000 & 76.18 & 145.49 \\
\hline 10.90000 & 75.99 & 173.99 \\
\hline 14.50000 & 75.33 & 194.09 \\
\hline 21.80000 & 74.12 & 222.75 \\
\hline 36.30000 & 72.69 & 258.50 \\
\hline 72.70000 & 71.49 & 307.28 \\
\hline 145.00000 & 70.92 & 355.82 \\
\hline 218.00000 & 70.70 & 384.49 \\
\hline
\end{tabular}

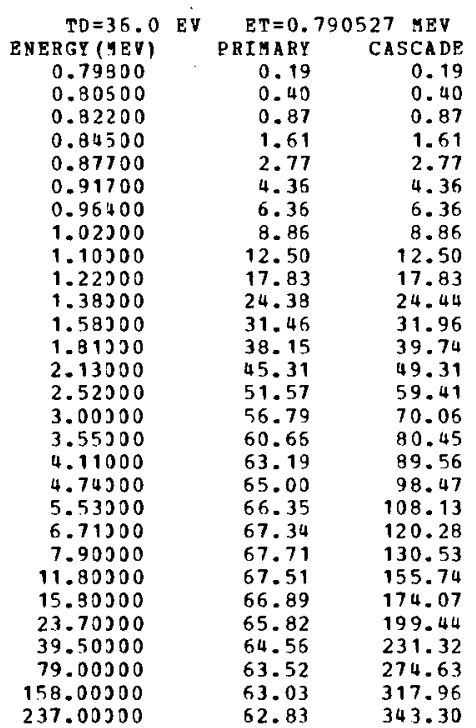

ATOMIC DISPLAZEMENT CROSS-SEETIONS (BARNS) BY PAST RLECTRONS IN RB $\mathrm{Z}=37 \quad \mathrm{~A}=85.480$

\begin{tabular}{crr} 
TD $=40.0$ & EV & \multicolumn{2}{c}{ EI $=0.850314$} & MEV \\
ENERGY (GEV) & PRIMARY & CASCADE \\
0.85800 & 0.15 & 0.15 \\
0.86700 & 0.34 & 0.34 \\
0.88400 & 0.73 & 0.73 \\
0.90900 & 1.37 & 1.37 \\
0.94300 & 2.37 & 2.37 \\
0.98600 & 3.78 & 3.78 \\
1.03000 & 5.33 & 5.33 \\
1.10000 & 7.95 & 7.95 \\
1.19000 & 11.39 & 11.39 \\
1.31000 & 15.90 & 15.90 \\
1.48000 & 21.80 & 21.86 \\
1.70000 & 28.41 & 28.88 \\
1.95000 & 34.53 & 36.05 \\
2.29000 & 40.94 & 44.67 \\
2.72000 & 46.71 & 54.09 \\
3.23000 & 51.31 & 63.64 \\
3.82000 & 54.75 & 73.06 \\
4.42000 & 56.98 & 81.29 \\
5.10000 & 58.59 & 89.40 \\
5.95000 & 59.77 & 98.15 \\
7.22000 & 60.63 & 109.15 \\
8.50000 & 60.94 & 118.42 \\
12.70000 & 60.72 & 141.20 \\
17.00000 & 60.15 & 157.72 \\
25.50000 & 59.18 & 180.58 \\
42.50000 & 58.07 & 209.30 \\
85.00000 & 57.15 & 248.30 \\
170.00000 & 56.72 & 287.31 \\
& &
\end{tabular}

\begin{tabular}{|c|c|c|c|}
\hline $\begin{array}{r}T D=44.0 \\
\text { ENERGY (HEV) }\end{array}$ & EV & $\begin{array}{r}\text { ET=0.9 } \\
\text { PRIMARY }\end{array}$ & $\begin{array}{l}585 \text {. V } \\
\text { CASZADE }\end{array}$ \\
\hline 0.91600 & & 0.13 & 0.13 \\
\hline 0.92500 & & 0.28 & 0.28 \\
\hline 0.94300 & & 0.61 & 0.61 \\
\hline 0.97100 & & 1.21 & 1.21 \\
\hline 1.00000 & & 1.91 & 1.91 \\
\hline 1.05000 & & 3. 27 & 3.27 \\
\hline 1.10000 & & 4.77 & 4.77 \\
\hline 1.17000 & & 7.00 & 7.00 \\
\hline 1.27000 & & 10.28 & 10.28 \\
\hline 1.40000 & & 14.47 & 14.47 \\
\hline 1.58000 & & 19.85 & 19.90 \\
\hline 1.81000 & & 25.80 & 25.24 \\
\hline 2.08000 & & 31.49 & 32.92 \\
\hline 2.45000 & & 37.45 & 41.00 \\
\hline 2.90000 & & 42.59 & 49.48 \\
\hline 3.44000 & & 46.75 & 58.18 \\
\hline 4.08000 & & 49.90 & 66.96 \\
\hline 4.71000 & & 51.88 & 74.39 \\
\hline 5.44000 & & 53.33 & 81.86 \\
\hline 6.35000 & & 54.38. & 89.88 \\
\hline 7.71000 & & 55.13 & 99.95 \\
\hline 9.07000 & & 55.39 & 108.37 \\
\hline 13.60000 & & 55.16 & 129.32 \\
\hline 18.10000 & & 54.65 & 144.07 \\
\hline 27.20000 & & 53.77 & 164.98 \\
\hline 45.30000 & & 52.76 & 191.07 \\
\hline 181.00000 & & $\begin{array}{l}51.94 \\
51.55\end{array}$ & $\begin{array}{l}220.59 \\
261.95\end{array}$ \\
\hline & & & \\
\hline
\end{tabular}

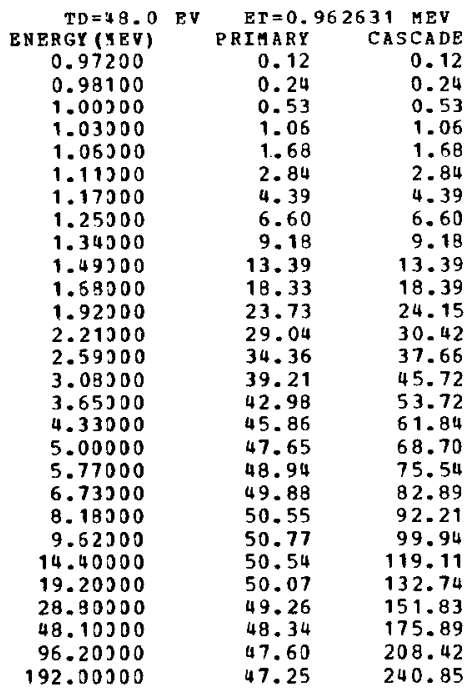


ATOMIC DISPLAEEMENT CROSS-SECTIONS (BARNS) BY FAST ELECTRONS IN RB

\begin{tabular}{rrr} 
TD $=52.0$ & EV & \multicolumn{1}{c}{ EI $=1.015694$ MEV } \\
ENERGY (MEV) & PRIMARY & CASCADE \\
1.02000 & 0.04 & 0.04 \\
1.03000 & 0.15 & 0.15 \\
1.05000 & 0.39 & 0.39 \\
1.08000 & 0.83 & 0.83 \\
1.12000 & 1.53 & 1.53 \\
1.17000 & 2.54 & 2.54 \\
1.23000 & 3.89 & 3.89 \\
1.32000 & 6.08 & 6.08 \\
1.42000 & 8.59 & 8.59 \\
1.57000 & 12.31 & 12.31 \\
1.77000 & 16.90 & 16.95 \\
2.03000 & 22.05 & 22.47 \\
2.33000 & 26.88 & 28.19 \\
2.74000 & 31.91 & 35.08 \\
3.25000 & 36.31 & 42.47 \\
3.85000 & 39.77 & 49.89 \\
4.57000 & 42.42 & 57.45 \\
5.28000 & 44.06 & 63.84 \\
6.09000 & 45.23 & 70.16 \\
7.10000 & 46.07 & 76.95 \\
8.63000 & 46.67 & 85.59 \\
10.10000 & 46.86 & 92.52 \\
15.20000 & 46.64 & 110.49 \\
20.30000 & 45.19 & 123.16 \\
30.40000 & 45.44 & 140.73 \\
50.70000 & 44.61 & 162.89 \\
101.00000 & 43.94 & 192.76 \\
203.00000 & 43.61 & 222.99 \\
& &
\end{tabular}

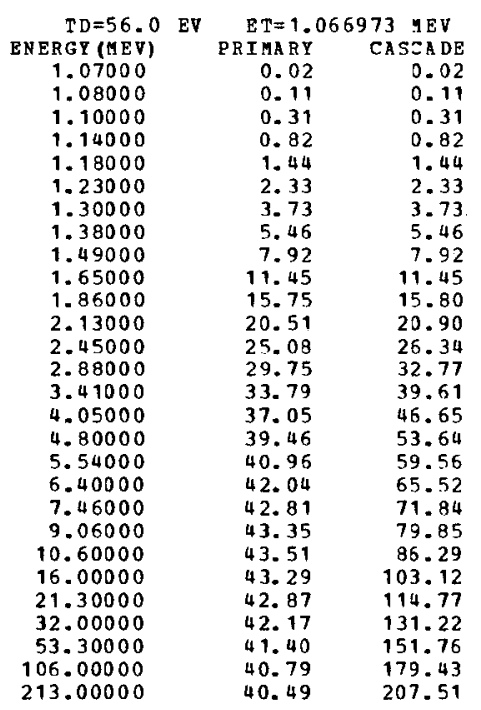

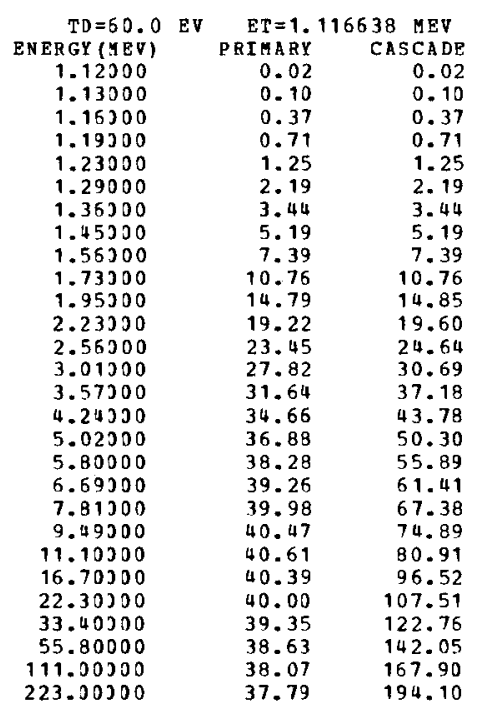

ATOMIC DISPLAEEMENT CROSS-SEZTIONS (BARNS) BY PAST BLECTRONS IN RB $Z=37 \quad A=85.480$

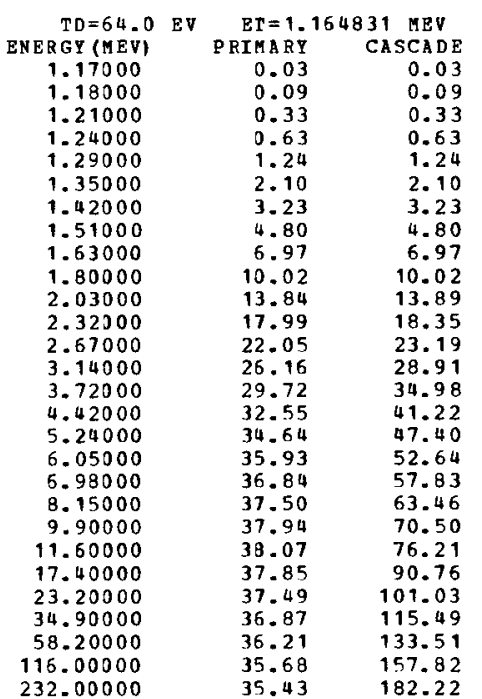

$\begin{array}{rrr}\text { TD }=68.0 & \text { EV } & \text { ET=1.211677 MEV } \\ \text { ENERGY (HEV) } & \text { PRIMARY } & \text { CASZADE } \\ 1.22300 & 0.04 & 0.04 \\ 1.23000 & 0.10 & 0.10 \\ 1.26000 & 0.31 & 0.31 \\ 1.29000 & 0.58 & 0.58 \\ 1.34000 & 1.12 & 1.12 \\ 1.40000 & 1.89 & 1.89 \\ 1.47000 & 2.91 & 2.91 \\ 1.57000 & 4.49 & 4.49 \\ 1.69000 & 6.47 & 5.47 \\ 1.87000 & 9.41 & 9.41 \\ 2.12000 & 13.18 & 13.23 \\ 2.42000 & 17.07 & 17.43 \\ 2.78000 & 20.84 & 21.95 \\ 3.27000 & 24.72 & 27.37 \\ 3.87000 & 28.04 & 33.08 \\ 4.60000 & 30.70 & 38.99 \\ 5.45000 & 32.65 & 44.81 \\ 6.30000 & 33.86 & 49.79 \\ 7.27000 & 34.70 & 54.70 \\ 8.48000 & 35.31 & 59.97 \\ 10.20000 & 35.70 & 66.28 \\ 12.10000 & 35.83 & 72.08 \\ 18.10000 & 35.52 & 85.70 \\ 24.20000 & 35.27 & 95.46 \\ 36.30000 & 34.69 & 108.98 \\ 60.50000 & 34.07 & 125.93 \\ 121.00000 & 33.58 & 149.92 \\ 242.00000 & 33.34 & 171.89\end{array}$

\begin{tabular}{|c|c|c|}
\hline $\mathrm{TD}=72.0$ & $\mathrm{ET}=1.2$ & MEV \\
\hline ENERGY (MEV) & PRIMARY & CASCADE \\
\hline 1.25300 & 0.01 & 0.01 \\
\hline 1.28300 & 0.11 & 0.11 \\
\hline 1.30500 & 0.24 & 0.24 \\
\hline 1.34300 & 0.55 & 0.55 \\
\hline 1.39300 & 1.04 & 1.04 \\
\hline 1.45000 & 1.74 & 1.74 \\
\hline 1.53000 & 2.80 & 2.80 \\
\hline 1.63000 & 4.24 & 4.24 \\
\hline 1.76000 & 6.21 & 6.21 \\
\hline 1.94300 & 8.89 & 8.89 \\
\hline 2.20000 & 12.48 & 12.54 \\
\hline 2.51000 & 16.15 & 16.50 \\
\hline 2.39300 & 19.79 & 20.87 \\
\hline 3.39000 & 23.39 & 25.93 \\
\hline 4.02000 & 26.56 & 31.41 \\
\hline 4.77000 & 29.04 & 36.95 \\
\hline 5.65000 & 30.86 & $\begin{array}{l}42.46 \\
47.17\end{array}$ \\
\hline 6.53300 & $\begin{array}{l}32.00 \\
32.80\end{array}$ & $\begin{array}{l}47.17 \\
51.84\end{array}$ \\
\hline $\begin{array}{l}7.54000 \\
8.80000\end{array}$ & $\begin{array}{l}32.80 \\
33.36\end{array}$ & 56.85 \\
\hline 10.50300 & 33.73 & 62.86 \\
\hline 12.50000 & 33.84 & 68.16 \\
\hline 18.80000 & 33.63 & 81.21 \\
\hline 25.10000 & 33.30 & 90.38 \\
\hline 37.70000 & 32.75 & 103.20 \\
\hline 62.90300 & 32,17 & 119.20 \\
\hline 125.00000 & 31.71 & 140.77 \\
\hline 251.00300 & 31.48 & 162.59 \\
\hline
\end{tabular}


ATOMIC DISPLAZENENT CROSS-SECTIONS (BARNS) BY FAST ELECTRONS IN RB

\begin{tabular}{rrr} 
TD=76.0 EV & \multicolumn{2}{c}{ ET=1.301740 MEV } \\
ENERGY (MEV & PRIMARY & CASCADE \\
1.31000 & 0.03 & 0.03 \\
1.32000 & 0.08 & 0.08 \\
1.35000 & 0.24 & 0.24 \\
1.39000 & 0.53 & 0.53 \\
1.44000 & 0.98 & 0.98 \\
1.51000 & 1.73 & 1.73 \\
1.58000 & 2.58 & 2.58 \\
1.69000 & 4.05 & 4.05 \\
1.92000 & 5.85 & 5.85 \\
2.01000 & 8.46 & 8.46 \\
2.27000 & 11.76 & 11.81 \\
2.60000 & 15.36 & 15.70 \\
2.99000 & 18.78 & 19.83 \\
3.51000 & 22.22 & 24.66 \\
4.16000 & 25.20 & 29.86 \\
4.94000 & 27.56 & 35.15 \\
5.85000 & 29.28 & 40.38 \\
6.76000 & 30.34 & 44.85 \\
7.81000 & $\mathbf{3 1 . 0 9}$ & 49.30 \\
9.11000 & $\mathbf{3 1 . 6 1}$ & 54.04 \\
11.00000 & $\mathbf{3 1 . 9 6}$ & 59.82 \\
13.00000 & $\mathbf{3 2 . 0 5}$ & 64.91 \\
19.50000 & $\mathbf{3 1 . 8 5}$ & $\mathbf{7 7 . 1 9}$ \\
26.00000 & 31.53 & 85.85 \\
39.00000 & $\mathbf{3 1 . 0 2}$ & $\mathbf{9 7 . 9 6}$ \\
65.00000 & 30.47 & $\mathbf{1 1 3 . 1 4}$ \\
130.00000 & 30.04 & 133.72 \\
260.00000 & 29.83 & 154.27 \\
& &
\end{tabular}

\begin{tabular}{crr} 
TD=80.0 EV & \multicolumn{2}{c}{ ET=1.345134 1 MV } \\
ENERGY (HEV) & PRIMAR & CASIADE \\
1.35000 & 0.02 & 0.02 \\
1.37000 & 0.10 & 0.10 \\
1.39000 & 0.20 & 0.20 \\
1.43000 & 0.45 & 0.45 \\
1.49000 & 0.94 & 0.94 \\
1.56000 & 1.63 & 1.63 \\
1.64000 & 2.53 & 2.53 \\
1.74000 & 3.76 & 3.76 \\
1.88000 & 5.55 & 5.55 \\
2.08000 & 8.09 & 8.09 \\
2.35000 & 11.25 & 11.30 \\
2.69000 & 14.66 & 15.00 \\
3.09000 & 17.89 & 18.91 \\
3.63000 & 21.17 & 23.54 \\
4.30000 & 24.00 & 28.48 \\
5.11000 & 26.23 & 33.55 \\
6.05000 & 27.85 & 38.52 \\
6.99000 & 28.85 & 42.77 \\
8.07000 & 29.55 & 46.99 \\
9.41000 & 30.04 & 51.49 \\
11.40000 & 30.37 & 57.08 \\
13.40000 & 30.45 & 61.76 \\
20.10000 & 30.25 & 73.45 \\
26.90000 & 29.95 & 81.79 \\
40.30000 & 29.46 & 93.25 \\
67.20000 & 28.94 & 107.69 \\
134.00000 & 28.53 & 127.16 \\
269.00000 & 28.33 & 146.79
\end{tabular}

\begin{tabular}{|c|c|c|c|}
\hline$T D=34.0$ & Ev & $\mathrm{ET}=1.3$ & $536 \mathrm{MEV}$ \\
\hline EN ERGY (YEV) & & PRIMARY & CASCADE \\
\hline 1.40300 & & 0.04 & 0.04 \\
\hline 1.41000 & & 0.08 & 0.08 \\
\hline 1.44300 & & 0.21 & 0.21 \\
\hline 1.43000 & & 0.45 & 0.45 \\
\hline 1.54000 & & 0.91 & 0.91 \\
\hline 1.60000 & & 1.45 & 1.45 \\
\hline 1.69000 & & 2.38 & 2.38 \\
\hline 1.80300 & & 3.64 & 3.64 \\
\hline 1.94000 & & 5.30 & 5.30 \\
\hline 2.15300 & & 7.77 & 7.77 \\
\hline 2.42300 & & 10.70 & 10.75 \\
\hline 2.77000 & & 13.96 & 14.29 \\
\hline 3.19000 & & 17.10 & 18.09 \\
\hline 3.74000 & & 20.19 & 22.45 \\
\hline 4.44300 & & 22.91 & 27.24 \\
\hline 5.27000 & & 25.02 & 32.05 \\
\hline 6.24300 & & 26.55 & 36.80 \\
\hline 7.21000 & & 27.50 & 40.86 \\
\hline 8.32000 & & 28.16 & 44.87 \\
\hline 9.71000 & & 28.62 & 49.19 \\
\hline 11.70000 & & 28.92 & 54.37 \\
\hline 13.80000 & & 29.00 & 58.93 \\
\hline 20.90300 & & 28.80 & 70.20 \\
\hline 27.70000 & & 28.52 & 78.01 \\
\hline 41.50200 & & 28.05 & 89.00 \\
\hline $69.30 J 00$ & & 27.55 & 102.73 \\
\hline 138.00300 & & 27.17 & 121.24 \\
\hline 277.00000 & & 26.98 & 139.93 \\
\hline
\end{tabular}

ATOMIC DISPLACEMENT CROSS-SECTIONS (BARN) BY FAST ELECTRONS IN RB $z=37 \quad A=85.480$

\begin{tabular}{ccc} 
TD=88.0 EV & \multicolumn{1}{c}{ ET=1.429011 MEV } \\
ENERGY(HEV) & PRIMARY & CASCADE \\
1.44000 & 0.03 & 0.03 \\
1.45000 & 0.06 & 0.06 \\
1.48000 & 0.18 & 0.18 \\
1.52000 & 0.40 & 0.40 \\
1.58000 & 0.81 & 0.81 \\
1.65000 & 1.40 & 1.40 \\
1.74000 & 2.26 & 2.26 \\
1.85000 & 3.43 & 3.43 \\
2.00000 & 5.09 & 5.09 \\
2.21000 & 7.39 & 7.39 \\
2.50000 & 10.31 & 10.36 \\
2.85000 & 13.34 & 13.65 \\
3.29000 & 16.33 & 17.28 \\
3.85000 & 19.30 & 21.50 \\
4.57000 & 21.90 & 26.07 \\
5.43000 & 23.92 & 30.71 \\
6.43000 & 25.37 & 35.25 \\
7.43000 & 26.27 & 39.14 \\
8.57000 & 26.89 & 42.96 \\
10.00000 & 27.33 & 47.08 \\
12.10000 & 27.61 & 52.14 \\
14.20000 & 27.68 & 56.37 \\
21.40000 & 27.49 & 67.13 \\
28.50000 & 27.21 & 74.59 \\
42.80000 & 26.77 & 85.08 \\
71.40000 & 26.30 & 98.22 \\
142.00000 & 25.93 & 115.86
\end{tabular}

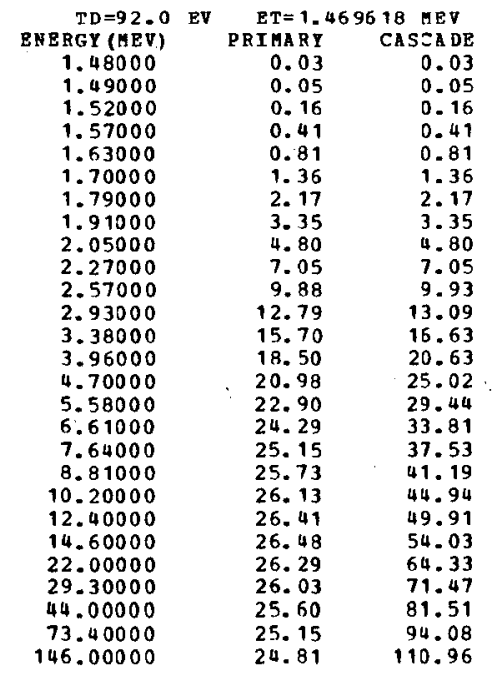

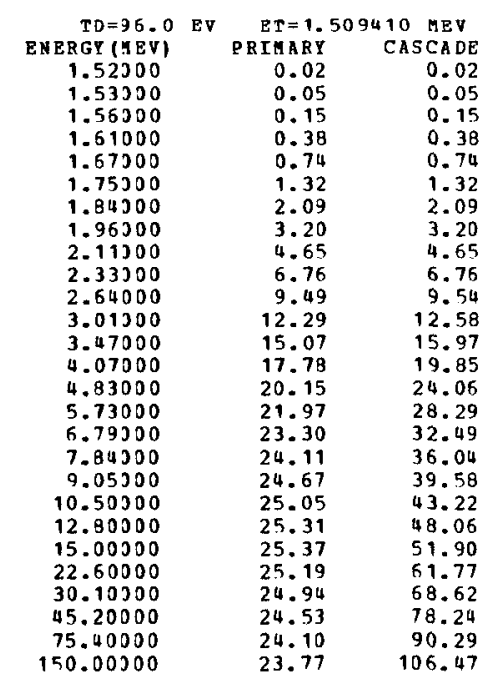


A TOMIC DISPLAZENENT CROSS-SECTIONS (BARNS) BY FAST ELECTRONS IN ZR SECTIONS (BARNS)
$=40 \quad A=91.220$

\begin{tabular}{|c|c|c|c|}
\hline$T D=4.0$ & EV & $\mathrm{ET}=0.1$ & $519 \mathrm{MEV}$ \\
\hline ENERGY (MEV) & & PRIMARY & CASCADE \\
\hline 0.14600 & & 4.35 & 4.35 \\
\hline 0.14800 & & 21.96 & 21.96 \\
\hline 0.15100 & & 47.01 & 47.01 \\
\hline 0.15500 & & 78.09 & 78.09 \\
\hline 0.16100 & & 120.30 & 120.30 \\
\hline 0.16800 & & 163.82 & 163.82 \\
\hline 0.17700 & & 212.30 & 212.30 \\
\hline 0.18900 & & 266.53 & 266.53 \\
\hline 0.20300 & & 318.31 & 318.31 \\
\hline 0.22500 & & 381.75 & 361.75 \\
\hline 0.25400 & & 443.02 & 443.02 \\
\hline 0.29100 & & 498.34 & 500.21 \\
\hline 0.33400 & & 543.36 & 553.60 \\
\hline 0.39200 & & 585.03 & 612.17 \\
\hline 0.46500 & & 619.81 & 672.52 \\
\hline 0.55200 & & 646.89 & 732.74 \\
\hline 0.65400 & & 667.41 & 793.30 \\
\hline 0.75600 & & 680.79 & 846.66 \\
\hline 0.87300 & & 690.78 & 901.58 \\
\hline 1.01000 & & 697.92 & 959.50 \\
\hline 1.23000 & & 703.48 & 1041.85 \\
\hline 1.45000 & & 704.96 & 1114.25 \\
\hline 2.18000 & & 699.55 & 1307.67 \\
\hline 2.91000 & & 690,48 & 1455.24 \\
\hline 4.36000 & & 675.38 & 1674.00 \\
\hline 7.27000 & & 656.69 & 1964.63 \\
\hline 14.50000 & & 636.80 & 2371.36 \\
\hline 29.10000 & & 626.19 & 2792.11 \\
\hline 43.60000 & & 622.85 & 3038.57 \\
\hline 72.70000 & & 619.86 & 3351.44 \\
\hline 101.00000 & & 618.12 & 3552.60 \\
\hline 145.00000 & & 616.21 & 3773.40 \\
\hline
\end{tabular}

\begin{tabular}{rrr} 
TD $=8.0$ & EV & \multicolumn{2}{c}{ ET $=0.264186$ MEV } \\
ENERGY (MEV) & PRIMARY & CASCADE \\
0.26600 & 2.40 & 2.40 \\
0.26900 & 6.29 & 6.29 \\
0.27400 & 12.60 & 12.60 \\
0.28200 & 22.25 & 22.25 \\
0.29300 & 34.70 & 34.70 \\
0.30600 & 48.36 & 48.36 \\
0.32200 & 63.80 & 63.80 \\
0.34300 & 82.14 & 82.14 \\
0.36900 & 102.33 & 102.33 \\
0.40900 & 129.08 & 129.08 \\
0.46200 & 158.41 & 158.41 \\
0.52800 & 187.79 & 189.06 \\
0.60700 & 215.48 & 220.71 \\
0.71300 & 243.71 & 257.21 \\
0.84500 & 269.22 & 296.11 \\
1.00000 & 290.28 & 335.38 \\
1.18000 & 307.02 & 374.88 \\
1.37000 & 318.97 & 411.40 \\
1.58000 & 327.81 & 447.10 \\
1.84000 & 334.78 & 486.15 \\
2.24000 & 340.45 & 537.88 \\
2.64000 & 342.85 & 582.16 \\
3.96000 & 342.35 & 595.05 \\
5.28000 & 338.75 & 777.55 \\
7.92000 & 332.31 & 896.72 \\
13.20000 & 324.04 & 1049.06 \\
26.40000 & 315.93 & 1258.67 \\
52.80000 & 311.98 & 1470.59 \\
79.20000 & 310.65 & 1594.95 \\
132.00000 & 309.27 & 1751.67 \\
184.00000 & 308.39 & 1853.27 \\
264.00000 & 307.43 & 1963.47
\end{tabular}

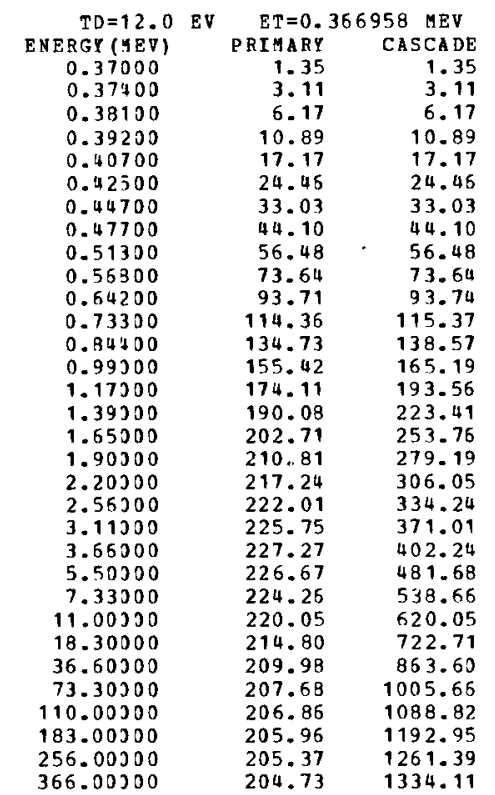

ATOMIC DISPLAZEMENT CROSS-SECTIONS (BARNS) BY PAST ELECTRONS IN ZR $z=40 \quad A=91.220$

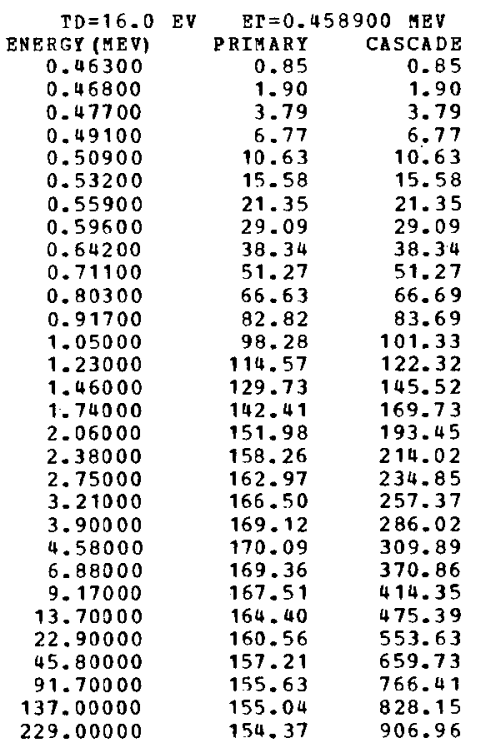

\begin{tabular}{rrr} 
TD=20.0 & EV & \multicolumn{1}{c}{ ET=0.542851 MEV } \\
ENERGY (HEV) & PRIMARY & CASZADE \\
0.54800 & 0.60 & 0.60 \\
0.55300 & 1.19 & 1.19 \\
0.56400 & 2.53 & 2.53 \\
0.58000 & 4.54 & 4.54 \\
0.50200 & 7.42 & 7.42 \\
0.52900 & 11.06 & 11.06 \\
0.66200 & 15.59 & 15.59 \\
0.70500 & 21.51 & 21.51 \\
0.75900 & 28.82 & 28.82 \\
0.84100 & 39.33 & 39.33 \\
0.94900 & 51.82 & 51.89 \\
1.08000 & 64.85 & 65.59 \\
1.24000 & 77.92 & 80.55 \\
1.46000 & 91.79 & 98.60 \\
1.73000 & 104.06 & 117.74 \\
2.06000 & 114.30 & 137.77 \\
2.44000 & 121.99 & 157.49 \\
2.82000 & 126.95 & 174.53 \\
3.25000 & 130.58 & 191.39 \\
3.79000 & 133.28 & 209.76 \\
4.61000 & 135.24 & 233.34 \\
5.42000 & 135.92 & 252.93 \\
8.14000 & 135.15 & 302.39 \\
10.80000 & 133.67 & 335.96 \\
16.20000 & 131.15 & 386.59 \\
27.10000 & 128.16 & 449.53 \\
54.20000 & 125.63 & 534.62 \\
108.00000 & 124.44 & 619.46 \\
162.00000 & 123.97 & 669.36 \\
271.00000 & 123.43 & 732.50
\end{tabular}

\begin{tabular}{|c|c|c|}
\hline$T D=24.0$ & $\mathrm{ET}=0$. & $591 \mathrm{MEV}$ \\
\hline ENERGY ( IEV) & PRIMARY & CASCADE \\
\hline 0.62600 & 0.39 & 0.39 \\
\hline 0.63300 & 0.92 & 0.92 \\
\hline 0.64500 & 1.86 & 1.86 \\
\hline 0.56400 & 3.44 & 3.44 \\
\hline 0.68900 & 5.57 & 5.57 \\
\hline 0.71900 & 8.46 & 8.46 \\
\hline 0.75700 & 12.16 & 12.16 \\
\hline 0.80500 & 17.02 & 17.02 \\
\hline 0.86300 & 23.14 & 23.14 \\
\hline 0.96100 & 31.94 & 31.94 \\
\hline 1.08300 & 42.22 & 42.28 \\
\hline 1.24000 & 54.10 & 54.81 \\
\hline 1.42300 & 65.02 & 67.40 \\
\hline 1.57300 & 76.71 & 82.80 \\
\hline 1.99300 & 87.11 & 99.27 \\
\hline 2.35300 & 95.53 & 116.11 \\
\hline 2.79000 & 102.01 & 133.18 \\
\hline 3.22300 & 106.05 & 147.56 \\
\hline 3.72300 & 109.04 & 162.14 \\
\hline 4.34000 & 111.19 & 177.76 \\
\hline 5.27000 & 112.70 & 197.54 \\
\hline 6.20300 & 113.18 & 214.13 \\
\hline 9.30300 & 112.43 & 255.61 \\
\hline 12.40300 & 111.15 & 285.12 \\
\hline 18.60000 & 109.04 & 326.64 \\
\hline 31.00000 & 106.62 & 378.85 \\
\hline 62.00000 & 104.60 & 449.87 \\
\hline 124.00000 & 103.65 & 520.99 \\
\hline 186.00300 & 103.27 & 562.59 \\
\hline 310.00000 & 102.82 & 614.81 \\
\hline
\end{tabular}


ATOKIC DISPLAZEMENT CROSS-SECTIONS (BARNS) BY PAST ELECTRONS IN Z

\begin{tabular}{rrr} 
TD=28.0 EV & \multicolumn{2}{c}{ ER=0.693323 MEV } \\
ENERGY (MEV) & PRIMARY & CASCADE \\
0.70000 & 0.33 & 0.33 \\
0.70700 & 0.69 & 0.69 \\
0.72100 & 1.45 & 1.45 \\
0.74100 & 2.63 & 2.63 \\
0.76900 & 4.43 & 4.43 \\
0.80400 & 6.86 & 6.86 \\
0.84500 & 9.87 & 9.87 \\
0.90100 & 14.11 & 14.11 \\
0.97000 & 19.36 & 19.36 \\
1.07000 & 26.73 & 26.73 \\
1.21000 & 36.16 & 36.23 \\
1.38000 & 46.04 & 46.68 \\
1.59000 & 56.00 & 58.22 \\
1.87000 & 66.14 & 71.72 \\
2.21000 & 74.95 & 85.89 \\
2.63000 & 92.28 & 100.85 \\
3.11000 & 87.66 & 115.41 \\
3.60000 & 91.15 & 128.19 \\
4.15000 & 93.60 & 140.68 \\
4.85000 & 95.39 & 154.39 \\
5.89000 & 96.61 & 171.53 \\
5.93000 & 96.96 & 185.89 \\
10.30000 & 96.26 & 220.87 \\
13.80000 & 95.13 & 246.71 \\
20.70000 & 93.33 & 782.39 \\
34.60000 & 91.28 & 327.49 \\
69.30000 & 89.61 & 388.56 \\
138.00000 & 88.82 & 449.17 \\
207.00000 & 88.50 & 484.83 \\
& &
\end{tabular}

$\begin{array}{crr}\text { TD=32.0 } & \text { EV } & \text { ET=0.761907 MEY } \\ \text { ENERGY (MEV) } & \text { PRIMARY } & \text { CASEADE } \\ 0.76900 & 0.25 & 0.25 \\ 0.77700 & 0.54 & 0.54 \\ 0.79200 & 1.14 & 1.14 \\ 0.81500 & 2.16 & 2.16 \\ 0.84500 & 3.64 & 3.64 \\ 0.88300 & 5.69 & 5.69 \\ 0.92900 & 8.34 & 8.34 \\ 0.99000 & 12.02 & 12.02 \\ 1.06000 & 16.29 & 16.29 \\ 1.18000 & 23.42 & 23.42 \\ 1.33000 & 31.57 & 31.64 \\ 1.52000 & 40.48 & 41.10 \\ 1.75000 & 49.26 & 51.34 \\ 2.05000 & 58.01 & 63.08 \\ 2.43000 & 65.89 & 75.91 \\ 2.89000 & 72.27 & 89.16 \\ 3.42000 & 76.96 & 102.14 \\ 3.96000 & 79.96 & 113.49 \\ 4.57000 & 82.05 & 124.62 \\ 5.33000 & 83.54 & 136.58 \\ 6.47000 & 84.54 & 151.67 \\ 7.51000 & 84.80 & 164.29 \\ 11.40000 & 84.11 & 195.66 \\ 15.20000 & 83.13 & 217.96 \\ 22.80000 & 81.55 & 249.25 \\ 38.00000 & 79.79 & 288.54 \\ 76.10000 & 78.37 & 342.02 \\ 152.00000 & 77.69 & 395.30 \\ 228.00000 & 77.41 & 426.50 \\ & & \end{array}$

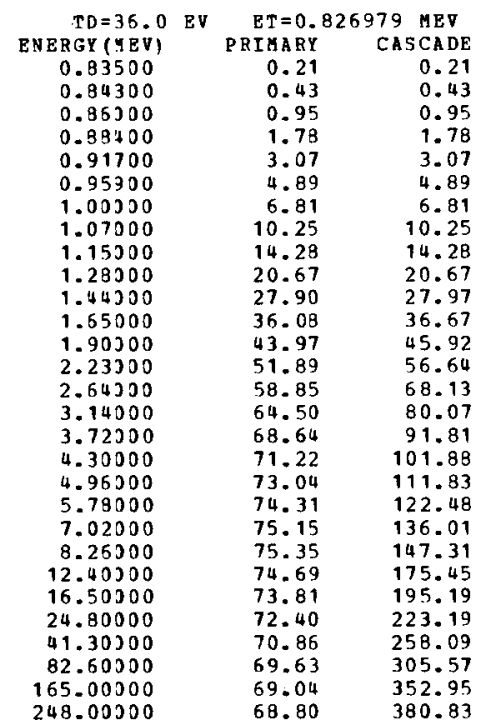

ATOMIC DISPLAFENENT CROSS-SECTIONS (BARNS) BY PAST ELECTRONS IN ZR

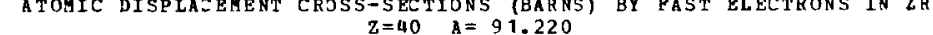

\begin{tabular}{crr} 
TD=40.0 & EV & \multicolumn{2}{c}{ ET $=0.889030$ MEV } \\
ENERGY(MEV) & PRIMARY & CASCADE \\
0.89700 & 0.16 & 0.16 \\
0.90600 & 0.35 & 0.35 \\
0.92400 & 0.79 & 0.79 \\
0.95100 & 1.54 & 1.54 \\
0.98600 & 2.65 & 2.65 \\
1.03000 & 4.23 & 4.23 \\
1.08000 & 6.18 & 6.18 \\
1.15000 & 9.08 & 9.08 \\
1.24000 & 12.90 & 12.90 \\
1.37000 & 18.31 & 18.31 \\
1.55000 & 25.21 & 25.28 \\
1.77000 & 32.46 & 33.01 \\
2.04000 & 39.68 & 41.49 \\
2.40000 & 46.95 & 51.41 \\
2.84000 & 53.20 & 61.85 \\
3.37000 & 58.20 & 72.56 \\
4.00000 & 61.93 & 83.34 \\
4.62000 & 64.21 & 92.43 \\
5.33000 & 65.81 & 101.45 \\
6.22000 & 66.92 & 111.18 \\
7.55000 & 67.64 & 123.39 \\
8.89000 & 67.79 & 133.64 \\
13.30000 & 67.17 & 158.84 \\
17.70000 & 66.38 & 176.65 \\
26.50000 & 65.11 & 201.87 \\
44.40000 & 63.73 & 233.45 \\
88.90000 & 62.65 & 276.27 \\
177.00000 & 62.13 & 318.73
\end{tabular}

\begin{tabular}{|c|c|c|c|}
\hline$n=44$ & $8 \nabla$ & & \\
\hline NERGY (NEV) & & PRIMARY & CAS $=A D E$ \\
\hline 0.95700 & & 0.13 & 0.13 \\
\hline 0.96700 & & 0.31 & 0.31 \\
\hline 0.98600 & & 0.68 & 0.68 \\
\hline 1.01000 & & 1.22 & 1.22 \\
\hline 1.05000 & & 2.27 & 2.27 \\
\hline 1.10000 & & 3.79 & 3.79 \\
\hline 1.15000 & & 5.44 & 5.44 \\
\hline 1.23000 & & 8. 28 & B. 28 \\
\hline 1.32000 & & 11.56 & 11.56 \\
\hline 1.47000 & & 16.92 & 16.92 \\
\hline 1.65000 & & 22.85 & 22.91 \\
\hline 1.89000 & & 29.66 & 30.18 \\
\hline 2.18000 & ' & 36.29 & 38.03 \\
\hline 2.56000 & & 42.85 & 47.04 \\
\hline 3.03000 & & $\begin{array}{l}48.54 \\
53.09\end{array}$ & $\begin{array}{l}56.63 \\
66.53\end{array}$ \\
\hline $\begin{array}{l}3.60000 \\
4.26000\end{array}$ & & $\begin{array}{l}53.09 \\
56.40\end{array}$ & $\begin{array}{l}66.53 \\
76.23\end{array}$ \\
\hline 4.93000 & & 58.47 & 84.66 \\
\hline 5.69000 & & 59.89 & 92.94 \\
\hline 6.63000 & & 60.87 & 101.74 \\
\hline 8.06000 & & 61.49. & 112.97 \\
\hline 9.48000 & & 61.61 & $122 \cdot 26$ \\
\hline 14.20000 & & 61.02. & 145.29 \\
\hline 18.90000 & & 60.29 & 161.52 \\
\hline $\begin{array}{l}28.40000 \\
47.40000\end{array}$ & & $\begin{array}{l}59.14 \\
57.91\end{array}$ & $\begin{array}{r}184.46 \\
213.19\end{array}$ \\
\hline 94.80000 & & .94 & 252.07 \\
\hline 189.00000 & & 56.47 & 290.75 \\
\hline
\end{tabular}

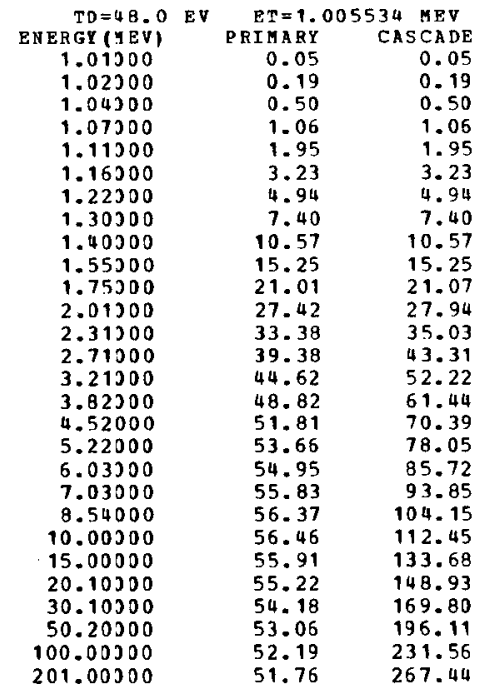


ATOMIC DISPLA $=40^{T}=91.220$

\begin{tabular}{ccr} 
TD $=52.0$ & EV & \multicolumn{1}{c}{ ET $=1.060551$ MEV } \\
ENERGY (HEVI & PRIMARY & CASCADE \\
1.07000 & 0.10 & 0.10 \\
1.08000 & 0.21 & 0.21 \\
1.10000 & 0.48 & 0.48 \\
1.13000 & 0.97 & 0.97 \\
1.17000 & 1.73 & 1.73 \\
1.23000 & 3.08 & 3.08 \\
1.29000 & 4.59 & 4.59 \\
1.37000 & 6.75 & 6.75 \\
1.48000 & 9.82 & 9.82 \\
1.64000 & 14.23 & 14.23 \\
1.85000 & 19.55 & 19.61 \\
2.12000 & 25.41 & 25.91 \\
2.43000 & 30.83 & 32.37 \\
2.86000 & 36.50 & 40.24 \\
3.39000 & 41.35 & 48.56 \\
4.03000 & 45.18 & 57.07 \\
4.77000 & 47.92 & 65.40 \\
5.51000 & 49.61 & 72.52 \\
6.36000 & 50.77 & 79.58 \\
7.42000 & 51.56 & 87.15 \\
9.01000 & 52.04 & 96.66 \\
10.60000 & 52.11 & 104.57 \\
15.90000 & 51.57 & 124.21 \\
21.20000 & 50.94 & 138.06 \\
31.80000 & 49.98 & 157.41 \\
53.00000 & 48.95 & 181.68 \\
106.00000 & 48.16 & 214.61 \\
212.00000 & 47.77 & 247.49
\end{tabular}

\begin{tabular}{|c|c|c|}
\hline$T D=56.0$ & $\mathrm{ET}=1$ & $13706 \mathrm{MEV}$ \\
\hline ENERGY (MEV) & PR I MARY & CAS $\approx A D E$ \\
\hline 1.12000 & 0.05 & 0.05 \\
\hline 1.13000 & 0.15 & 0.15 \\
\hline 1.15000 & 0.37 & 0.37 \\
\hline 1.19000 & 0.92 & 0.92 \\
\hline 1.23000 & 1.60 & 1.60 \\
\hline 1.29000 & 2. 78 & 2.78 \\
\hline 1.35000 & 4. 11 & 4.11 \\
\hline 1.44000 & 6.27 & 5.27 \\
\hline 1.55000 & 9.00 & 9.00 \\
\hline 1.72000 & 13.17 & 13.17 \\
\hline 1.94000 & 18.14 & 18.21 \\
\hline 2.22000 & 23.57 & 24.03 \\
\hline 2.56000 & 28.85 & 30.35 \\
\hline 3.00000 & 33.98 & 37.52 \\
\hline 3.56000 & 38.51 & 45.34 \\
\hline 4.23000 & 42.04 & 53.27 \\
\hline 5.01000 & 44.57 & 61.07 \\
\hline 5.79000 & 46.12 & 67.73 \\
\hline 6.68000 & 47.18 & 74.29 \\
\hline 7.79000 & 47.89 & 81.32 \\
\hline 9.46000 & 48.32 & 90.18 \\
\hline 11.10000 & 48.38 & 97.42 \\
\hline 16.70000 & 47.87 & 115.82 \\
\hline 22.20000 & 47.29 & 123.55 \\
\hline 33.40000 & 46.38 & 146.68 \\
\hline 55.60000 & 45.44 & 169.17 \\
\hline 111.00000 & 44.71 & 199.67 \\
\hline 222.00000 & 44.35 & 230.21 \\
\hline
\end{tabular}

\begin{tabular}{|c|c|c|}
\hline $\mathrm{TD}=50.0$ & $\mathrm{ET}=1$ & $176 \mathrm{MEV}$ \\
\hline ENERGY (YEV) & PRIMARY & CASCADE \\
\hline 1.17000 & 0.03 & 0.03 \\
\hline 1.19300 & 0.11 & 0.11 \\
\hline 1.21000 & 0.40 & 0.40 \\
\hline 1.24000 & 0.77 & 0.77 \\
\hline 1.29300 & 1.51 & 1.51 \\
\hline 1.35000 & 2.57 & 2.57 \\
\hline 1.42000 & 3.97 & 3.97 \\
\hline 1.51300 & 5.90 & 5.90 \\
\hline 1.53000 & 8.58 & 8.58 \\
\hline 1.80000 & 12.32 & 12.32 \\
\hline 2.03300 & 16.99 & 17.05 \\
\hline 2.33000 & 22.19 & 22.65 \\
\hline 2.57000 & 26.92 & 28.33 \\
\hline 3.14000 & 31.82 & 35.21 \\
\hline 3.72500 & 36.01 & 42.48 \\
\hline 4.42300 & 39.29 & 49.92 \\
\hline 5.24000 & 41.66 & 57.27 \\
\hline 6.05300 & 43.08 & 63.47 \\
\hline 6.99300 & 44.07 & 59.67 \\
\hline 8.15000 & 44.72 & 76.25 \\
\hline 9.90300 & 45.10 & 84.54 \\
\hline 11.60300 & 45.14 & 91.25 \\
\hline 17.40000 & 44.66 & 108.32 \\
\hline 23.30000 & 44.10 & 120.52 \\
\hline 34.90300 & 43.27 & 137.26 \\
\hline 58.20000 & 42.39 & 158.34 \\
\hline 116.00300 & 41.72 & 186.75 \\
\hline 233.00000 & 41.39 & 215.43 \\
\hline
\end{tabular}

ATOMIC DISPLAZEMENT CROSS-SECTIONS (BARNS) BY FAST RLECTRONS IN $Z$ R

\begin{tabular}{rrr} 
TD=64.0 & EV & \multicolumn{1}{c}{ ET=1.215112 MEV } \\
PERGY (HEV) & PRIARY & CASCADE \\
1.22000 & 0.03 & 0.03 \\
1.23000 & 0.10 & 0.10 \\
1.26000 & 0.35 & 0.35 \\
1.30000 & 0.78 & 0.78 \\
1.34000 & 1.32 & 1.32 \\
1.40000 & 2.25 & 2.25 \\
1.48000 & 3.68 & 3.68 \\
1.57000 & 5.43 & 5.43 \\
1.70000 & 8.05 & 8.05 \\
1.88000 & 11.63 & 11.63 \\
2.12000 & 16.02 & 16.09 \\
2.43000 & 20.87 & 21.32 \\
2.79000 & 25.38 & 26.76 \\
3.28000 & 29.97 & 33.23 \\
3.88000 & 33.84 & 40.02 \\
4.61000 & 36.91 & 47.02 \\
5.46000 & 39.10 & 53.90 \\
6.31000 & 40.43 & 59.77 \\
7.29000 & 41.34 & 65.60 \\
8.50000 & 41.94 & 71.78 \\
10.30000 & 42.28 & 79.47 \\
12.10000 & 42.31 & 85.88 \\
18.20000 & 41.85 & 102.01 \\
24.30000 & 41.33 & 113.34 \\
36.40000 & 40.55 & 129.04 \\
60.70000 & 39.73 & 148.81 \\
121.00000 & 39.11 & 175.45 \\
243.00000 & 38.80 & 202.34
\end{tabular}

$\begin{array}{rrr}\text { TD=68.0 } & \text { EV } & \text { ET=1.263643 MEV } \\ \text { ENERGY (MEV) } & \text { PRIMAY } & \text { CASEADE } \\ 1.27000 & 0.03 & 0.03 \\ 1.28000 & 0.09 & 0.09 \\ 1.31000 & 0.31 & 0.31 \\ 1.35000 & 0.70 & 0.70 \\ 1.40000 & 1.30 & 1.30 \\ 1.46000 & 2.16 & 2.16 \\ 1.54000 & 3.46 & 3.46 \\ 1.64000 & 5.23 & 5.23 \\ 1.76000 & 7.43 & 7.43 \\ 1.95000 & 10.88 & 10.88 \\ 2.21000 & 15.21 & 15.27 \\ 2.52000 & 19.61 & 20.04 \\ 2.90000 & 23.95 & 25.27 \\ 3.41000 & 28.27 & 31.40 \\ 4.04000 & 31.95 & 37.88 \\ 4.80000 & 34.82 & 44.50 \\ 5.68000 & 36.85 & 50.96 \\ 6.57000 & 38.10 & 56.53 \\ 7.58000 & 38.93 & 61.98 \\ 8.84000 & 39.48 & 67.82 \\ 10.70000 & 39.79 & 75.03 \\ 12.60000 & 39.82 & 81.16 \\ 18.90000 & 39.38 & 96.25 \\ 25.20000 & 38.89 & 106.87 \\ 37.90000 & 38.15 & 121.80 \\ 63.10000 & 37.38 & 140.35 \\ 126.00000 & 36.80 & 165.50 \\ 252.00000 & 36.52 & 190.65\end{array}$

\begin{tabular}{|c|c|c|}
\hline$T D=72.0$ & $\mathrm{ET}=1.3$ & 382 MEV \\
\hline NERGY (YEV) & PRIMARY & CASCADE \\
\hline 1.32000 & 0.04 & 0.04 \\
\hline 1.33000 & 0.09 & 0.09 \\
\hline 1.36300 & 0.29 & 0.29 \\
\hline 1.40300 & 0.64 & 0.64 \\
\hline 1.45000 & 1.19 & 1.19 \\
\hline 1.52300 & 2.10 & 2.10 \\
\hline 1.59300 & 3.13 & 3.13 \\
\hline 1.70300 & 4.99 & 4.91 \\
\hline 1.83000 & 7.09 & 7.09 \\
\hline 2.03000 & 10.41 & 10.41 \\
\hline 2.29300 & 14.37 & 14.43 \\
\hline 2.62300 & 18.65 & 19.07 \\
\hline 3.01300 & 22.70 & 23.98 \\
\hline 3.53000 & 26.72 & 29.70 \\
\hline 4.19000 & 30.24 & 35.91 \\
\hline 4.98300 & 32.94 & 42.20 \\
\hline 5.89000 & 34.84 & 48.30 \\
\hline 6.81300 & 36.00 & 53.56 \\
\hline 7.85300 & 36.78 & 58.73 \\
\hline 9.17500 & 37.30 & 64.27 \\
\hline 11.10300 & 37.58 & 71.09 \\
\hline 13.10300 & 37.60 & 76.97 \\
\hline 19.60000 & 37.18 & 91.14 \\
\hline 26.20000 & 36.71 & 101.27 \\
\hline 39.30300 & 36.01 & 115.29 \\
\hline 65.50000 & 35.30 & 132.85 \\
\hline 131.00300 & 34.75 & 156.65 \\
\hline 262.00300 & 34.49 & 180.41 \\
\hline
\end{tabular}


ATOMIC DISPLAZEMENT CROSS-SEZTIONS (BARNS) BY PAST ELECTBONS IN ZR

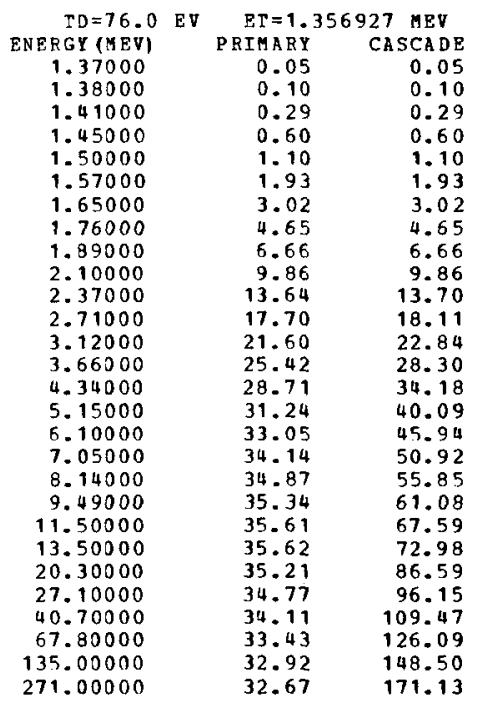

\begin{tabular}{rrr} 
TD=80.0 & EV & \multicolumn{2}{c}{ ET $=1.401863$ MEV } \\
ENERGY (HEV) & PRIMARY & CASZADE \\
1.41000 & 0.03 & 0.03 \\
1.42000 & 0.07 & 0.07 \\
1.45000 & 0.22 & 0.22 \\
1.49000 & 0.50 & 0.50 \\
1.55000 & 1.04 & 1.04 \\
1.62000 & 1.80 & 1.80 \\
1.71000 & 2.93 & 2.93 \\
1.82000 & 4.44 & 4.44 \\
1.96000 & 6.44 & 6.44 \\
2.17000 & 9.39 & 9.39 \\
2.45000 & 13.01 & 13.08 \\
2.80000 & 16.86 & 17.26 \\
3.22000 & 20.55 & 21.75 \\
3.78000 & 24.19 & 26.97 \\
4.48000 & 27.31 & 32.56 \\
5.32000 & 29.72 & 38.22 \\
6.30000 & 31.43 & 43.78 \\
7.28000 & 32.45 & 48.52 \\
8.41000 & 33.14 & 53.22 \\
9.81000 & 33.58 & 58.22 \\
11.90000 & 33.83 & 64.44 \\
14.00000 & 33.83 & 69.64 \\
21.00000 & 33.44 & 82.50 \\
28.00000 & 33.02 & 91.54 \\
42.00000 & 32.39 & 104.16 \\
70.00000 & 31.75 & 119.98 \\
140.00000 & 31.27 & 141.41 \\
280.00000 & 31.03 & 162.79
\end{tabular}

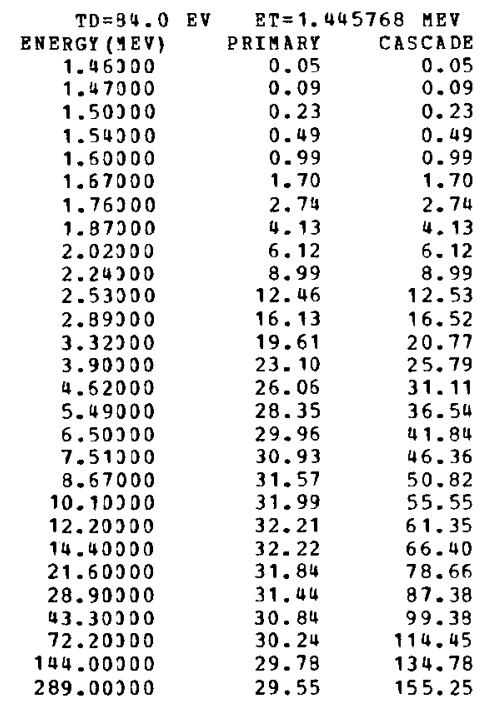

ATOMIC DISPLAZEMENT CROSS-SECTIONS (BARNS) BY FAST ELECTRONS IN ZR $\mathrm{Z}=40 \quad \mathrm{~A}=91.220$

\begin{tabular}{ccr} 
TD=88.0 EV & \multicolumn{1}{c}{ ER=1.488710 MEV } \\
ENERGY (MEV) & PRIMARY & CASCADE \\
1.50000 & 0.03 & 0.03 \\
1.51000 & 0.07 & 0.07 \\
1.54000 & 0.20 & 0.20 \\
1.59000 & 0.49 & 0.49 \\
1.65000 & 0.96 & 0.96 \\
1.72000 & 1.61 & 1.61 \\
1.81000 & 2.58 & 2.58 \\
1.93000 & 4.00 & 4.00 \\
2.08000 & 5.85 & 5.85 \\
2.30000 & 8.52 & 8.52 \\
2.60000 & 11.87 & 11.93 \\
2.97000 & 15.38 & 15.75 \\
3.42000 & 18.78 & 19.90 \\
4.01000 & 22.07 & 24.65 \\
4.76000 & 24.92 & 29.81 \\
5.65000 & 27.09 & 34.97 \\
6.59000 & 28.62 & 40.04 \\
7.74000 & 29.54 & 44.40 \\
8.93000 & 30.15 & 48.65 \\
10.40000 & 30.54 & 53.16 \\
12.60000 & 30.75 & 58.79 \\
14.80000 & 30.75 & 63.48 \\
22.30000 & 30.38 & 75.32 \\
29.70000 & 30.00 & 83.51 \\
44.60000 & 29.43 & 95.03 \\
74.40000 & 28.86 & 109.43 \\
148.00000 & 28.43 & 128.77
\end{tabular}

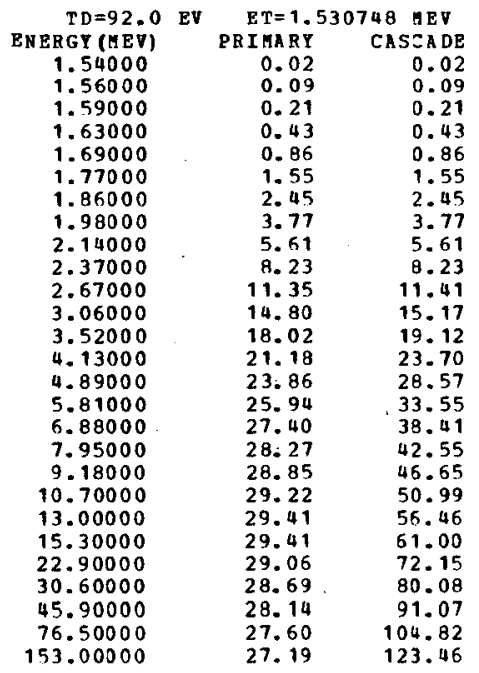

\begin{tabular}{|c|c|c|}
\hline$T D=96.0$ & $\mathrm{~g} \mathrm{~T}=1$ & HEV \\
\hline ENERGI (YEV) & PRIHARY & CASCADE \\
\hline 1.58000 & 0.02 & 0.02 \\
\hline 1.60000 & 0.07 & 0.07 \\
\hline 1.63000 & 0.19 & 0.19 \\
\hline 1.68300 & 0.45 & 0.45 \\
\hline 1.74000 & 0.85 & 0.85 \\
\hline 1.82300 & 1.50 & 1.50 \\
\hline 1.91300 & 2.35 & 2.35 \\
\hline 2.04300 & 3.69 & 3.69 \\
\hline 2.20300 & 5,41 & 5.41 \\
\hline 2.43500 & 7.86 & 7.86 \\
\hline 2.75000 & 10.98 & 11.04 \\
\hline 3.14300 & 14.20 & 14.56 \\
\hline 3.61200 & 17.28 & 18.34 \\
\hline 4.24300 & 20.33 & 22.77 \\
\hline 5.03000 & 22.93 & 27.50 \\
\hline 5.97000 & 24.89 & 32.25 \\
\hline 7.07000 & 26.28 & 36.93 \\
\hline 8.17000 & 27.11 & 40.90 \\
\hline 9.43300 & 27.66 & 44.82 \\
\hline 11.00000 & 28.01 & 49.01 \\
\hline 13.30000 & 28.19 & 54.12 \\
\hline 15.70000 & 28.18 & 58.56 \\
\hline 23.50300 & 27.84 & 69.25 \\
\hline 31.40300 & 27.49 & 76.85 \\
\hline 47.10000 & 26.97 & 87.38 \\
\hline 78.50300 & 26.44 & 100.57 \\
\hline 157.00300 & 26.05 & 118.43 \\
\hline
\end{tabular}


ATOMIC DISPLAzEMENT CROSS-SECTIONS (BARNS) BY FAST ELEZTRONS IN NB

\begin{tabular}{|c|c|c|c|c|c|c|c|c|}
\hline$T D=4.0$ & $E \Gamma=0.1$ & $912 \mathrm{MEV}$ & $T D=8.0$ & $\mathrm{ET}=0.2$ & 236 HEV & $\mathrm{TO}=12.0$ & $\mathrm{ET}=0.3$ & MEV \\
\hline ENERGY (MEV) & PRIMARY & CASCADE & ENERGY (MEV) & PRIMARY & CASEADE & ENERGY (MEV) & PRIMARY & CASCA DE \\
\hline 0.14900 & 9.88 & 9.88 & 0.27000 & 2.36 & 2.36 & 0.37500 & 1.65 & 1.65 \\
\hline 0.15000 & 18.76 & 18.76 & 0.27300 & 6.31 & 6.31 & 0.37300 & 3.00 & 3.00 \\
\hline 0.15300 & 44.34 & 44.34 & 0.27800 & 12.71 & 12.71 & 0.38700 & 6.54 & 6.54 \\
\hline 0.15800 & 83.66 & 83.66 & 0.28700 & 23.71 & 23.71 & 0.39300 & 11.34 & 11.34 \\
\hline 0.16400 & 126.06 & 126.06 & 0.29700 & 35.22 & 35.22 & 0.41300 & 17.75 & 17.75 \\
\hline 0.17100 & 169.94 & 169.94 & 0.31100 & 50.22 & 50.22 & 0.43100 & 25.21 & 25.21 \\
\hline 0.18000 & 219.01 & 219.01 & 0.32700 & 65.98 & 65.98 & $0 .+5+30$ & 34.40 & 34.40 \\
\hline 0.19200 & 274.16 & 274.16 & 0.34800 & 84.77 & 84.77 & 0.48400 & 45.78 & 45.78 \\
\hline 0.20700 & 330.48 & 330.48 & 0.37500 & 106.30 & 105.30 & 0.52100 & 58.91 & 58.91 \\
\hline 0.22900 & 394.92 & 394.92 & 0.41500 & 133.84 & 133.84 & 0.57700 & 76.93 & 76.93 \\
\hline 0.25800 & 457.73 & 457.73 & 0.46900 & 164.71 & 164.71 & 0.65100 & 97.68 & 97.72 \\
\hline 0.29500 & 514.97 & 516.84 & 0.53600 & 195.54 & 195.87 & $0.74 \$ 00$ & 119.51 & 120.58 \\
\hline 0.34000 & 563.77 & 574.59 & 0.61600 & 224.54 & 230.01 & 0.85500 & 140.75 & 144.79 \\
\hline 0.39900 & 607.57 & 636.06 & 0.72400 & 254.23 & 268.43 & 1.00300 & 161.86 & 171.95 \\
\hline 0.47300 & 644.07 & 699.26 & 0.85800 & 280.88 & 309.16 & 1.19300 & 182.15 & 202.84 \\
\hline 0.56200 & 672.69 & 762.88 & 1.01000 & 302.15 & 348.94 & 1.41300 & 198.41 & 233.55 \\
\hline 0.56500 & 694.01 & 825.99 & 1.20000 & 320.21 & 391.91 & 1.67000 & 211.27 & 264.77 \\
\hline 0.76900 & 707.96 & 882.09 & 1.39000 & 332.26 & 429.42 & 1.93300 & 219.75 & 291.93 \\
\hline 0.88700 & 718.16 & 939.16 & 1.60000 & 341.15 & 466.11 & 2.23300 & 226.15 & 319.46 \\
\hline 1.03000 & 725.53 & 1001.30 & 1.87000 & 348.30 & 507.70 & 2.60000 & 230.95 & 349.13 \\
\hline 1.25000 & 730.77 & 1085.81 & 2.28000 & 353.84 & 561.90 & 3.16300 & 234.56 & 387.38 \\
\hline 1.47000 & 731.89 & 1160.14 & 2.68000 & 355.96 & 607.13 & 3.72500 & 235.90 & 419.82 \\
\hline 2.21000 & 725.20 & 1361.16 & 4.02000 & 354.73 & 724.13 & 5.58000 & 234.78 & 501.69 \\
\hline 2.95000 & 715.04 & 1514.18 & 5.36000 & 350.59 & 809.61 & 7.44000 & 232.02 & 560.71 \\
\hline 4.43000 & 698.43 & 1742.00 & 8.04000 & 343.50 & 932.71 & 11.10300 & 227.47 & 643.59 \\
\hline 7.39000 & 678.29 & 2042.77 & 13.40000 & 334.59 & 1090.04 & 18.60300 & 221.75 & 751.07 \\
\hline 14.70000 & 657.23 & 2461.17 & 26.80000 & 326.01 & 1306.40 & 37.20000 & 216.66 & 896.47 \\
\hline 29.50000 & 646.07 & 2895.39 & 53.60000 & 321.86 & 1525.06 & 74.40000 & 214.25 & 1042.74 \\
\hline 44.30000 & 642.54 & 3151.11 & 80.40000 & 320.44 & 1653.34 & 111.00300 & 213.38 & 1127.28 \\
\hline 73.90000 & 639.33 & 3474.10 & 134.00000 & 318.95 & 1814.95 & 186.00300 & 212.39 & 1236.17 \\
\hline 103.00000 & 637.42 & 3683.55 & 187.00000 & 317.99 & 1920.05 & 260.00300 & 211.75 & 1306.56 \\
\hline 147.00000 & 635.37 & 3907.45 & 268.00000 & 315.94 & 2033.28 & 372.00000 & 211.05 & 1381.68 \\
\hline
\end{tabular}

ATOMIC DISPLAZEMENT CROSS-SECTIONS (BARNS) BY FAST ELECTRONS IN NB

\begin{tabular}{rrr} 
TD $=16.0$ & EV & \multicolumn{2}{c}{ ET $=0.465369$ MEV } \\
RRGY (MEV) & PRIMARY & CASCADE \\
0.47000 & 0.98 & 0.98 \\
0.47400 & 1.82 & 1.82 \\
0.48300 & 3.75 & 3.75 \\
0.49700 & 6.79 & 6.79 \\
0.51600 & 10.96 & 10.96 \\
0.53900 & 16.04 & 16.04 \\
0.56700 & 22.19 & 22.19 \\
0.50400 & 30.18 & 30.18 \\
0.65100 & 39.96 & 39.96 \\
0.72100 & 53.54 & 53.54 \\
0.81400 & 69.61 & 69.67 \\
0.93000 & 86.66 & 87.58 \\
1.07000 & 103.40 & 106.73 \\
1.25000 & 120.06 & 128.33 \\
1.48000 & 135.57 & 152.22 \\
1.76000 & 148.54 & 177.16 \\
2.09000 & 158.55 & 202.30 \\
2.41000 & 164.86 & 223.42 \\
2.79000 & 169.68 & 245.35 \\
3.25000 & 173.13 & 268.42 \\
3.95000 & 175.65 & 298.17 \\
4.55000 & 175.50 & 323.27 \\
6.98000 & 175.38 & 386.24 \\
9.30000 & 173.28 & 431.13 \\
13.90000 & 169.87 & 494.24 \\
23.20000 & 165.76 & 574.77 \\
46.50000 & 162.21 & 584.59 \\
93.00000 & 160.55 & 794.48 \\
39.00000 & 159.92 & 858.22 \\
32.00000 & 159.18 & 939.25 \\
& &
\end{tabular}

$$
\mathrm{Z}=41 \quad \mathrm{~A}=92.910
$$

\begin{tabular}{rrr} 
TD $=20.0$ & EV & \multicolumn{2}{c}{ ET $=0.550292$ MEV } \\
ENERGY (MEV) & PRIMARY & CASEADE \\
0.55500 & 0.55 & 0.55 \\
0.56100 & 1.28 & 1.28 \\
0.57200 & 2.64 & 2.64 \\
0.58800 & 4.70 & 4.70 \\
0.61000 & 7.65 & 7.65 \\
0.53800 & 11.53 & 11.53 \\
0.67100 & 16.21 & 15.21 \\
0.71500 & 22.48 & 22.48 \\
0.77000 & 30.17 & 30.17 \\
0.85200 & 41.06 & 41.06 \\
0.96300 & 54.36 & 54.43 \\
1.10000 & 68.40 & 69.23 \\
1.26000 & 81.82 & 84.64 \\
1.48000 & 96.05 & 103.25 \\
1.76000 & 109.04 & 123.66 \\
2.09000 & 119.40 & 144.23 \\
2.47000 & 127.18 & 164.48 \\
2.86000 & 132.30 & 182.43 \\
3.30000 & 135.95 & 200.07 \\
3.85000 & 138.60 & 219.19 \\
4.67000 & 140.44 & 243.28 \\
5.50000 & 141.01 & 263.78 \\
8.25000 & 139.94 & 314.78 \\
11.00000 & 138.23 & 351.13 \\
16.50000 & 135.47 & 402.37 \\
27.50000 & 132.29 & 466.87 \\
55.00000 & 129.62 & 554.66 \\
110.00000 & 128.36 & 642.66 \\
165.00000 & 127.87 & 594.12 \\
275.00000 & 127.28 & 758.75
\end{tabular}

$T D=24.0 \mathrm{QV} \quad \mathrm{PT}=0.628905 \mathrm{MEV}$

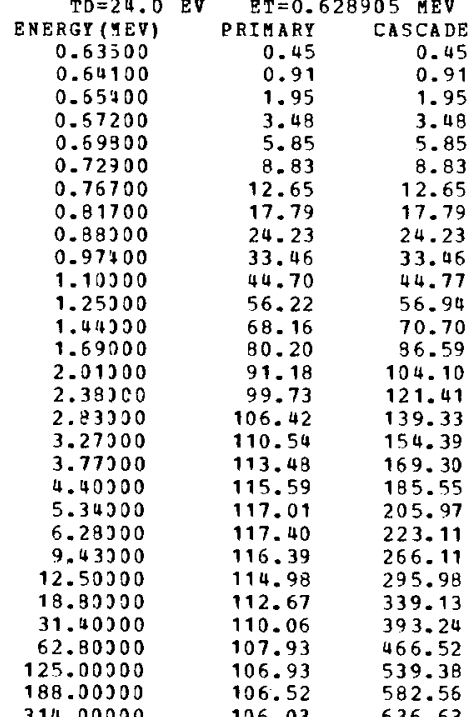


ATOMIC DISPLA ZEMEUT CROSS-SECTIONS (BARNS) BY FAST ELECTRONS IN AB

\begin{tabular}{crr} 
TO=28.J & EV & \multicolumn{1}{c}{ ET $=0.702437$ MEV } \\
ENERGY (MEV & PRIMARY & CASCADE \\
0.70900 & 0.33 & 0.33 \\
0.71600 & 0.69 & 0.69 \\
0.73000 & 1.47 & 1.47 \\
0.75100 & 2.74 & 2.74 \\
0.77900 & 4.60 & 4.60 \\
0.81400 & 7.10 & 7.10 \\
0.85600 & 10.28 & 10.28 \\
0.91300 & 14.76 & 14.76 \\
0.98300 & 20.29 & 20.29 \\
1.08000 & 27.70 & 27.70 \\
1.22000 & 37.53 & 37.59 \\
1.40000 & 48.37 & 49.05 \\
1.61000 & 58.64 & 60.98 \\
1.39000 & 69.09 & 74.93 \\
2.24000 & 78.39 & 89.96 \\
2.56000 & 85.85 & 105.35 \\
3.16000 & 91.50 & 120.90 \\
3.55000 & 94.96 & 133.99 \\
4.21000 & 97.42 & 146.99 \\
4.91000 & 99.15 & 161.02 \\
5.97000 & 100.29 & 178.88 \\
7.02000 & 100.55 & 193.69 \\
10.50000 & 99.62 & 230.43 \\
14.00000 & 98.38 & 256.68 \\
21.00000 & 96.41 & 293.52 \\
35.10000 & 94.22 & 340.05 \\
70.20000 & 92.45 & 402.93 \\
140.00000 & 91.62 & 465.59 \\
210.00000 & 91.27 & 502.36
\end{tabular}

\begin{tabular}{ccr} 
TD 32.0 & EV & \multicolumn{2}{c}{ ET $=0.771760$ MEV } \\
ENERGY (MEV) & PRIMARY & CASEADE \\
0.77900 & 0.26 & 0.26 \\
0.78700 & 0.56 & 0.56 \\
0.80200 & 1.17 & 1.17 \\
0.82500 & 2.22 & 2.22 \\
0.85600 & 3.79 & 3.79 \\
0.89500 & 5.96 & 5.96 \\
0.94100 & 8.71 & 8.71 \\
1.00000 & 12.40 & 12.40 \\
1.08000 & 17.46 & 17.46 \\
1.19000 & 24.24 & 24.24 \\
1.35000 & 33.27 & 33.34 \\
1.54000 & 42.47 & 43.12 \\
1.77000 & 51.53 & 53.71 \\
2.08000 & 60.81 & 66.22 \\
2.46000 & 68.87 & 79.43 \\
2.93000 & 75.49 & 93.35 \\
3.47000 & 80.28 & 106.90 \\
4.01000 & 83.27 & 119.53 \\
4.63000 & 85.37 & 130.11 \\
5.40000 & 86.82 & 142.50 \\
6.55000 & 87.75 & 158.06 \\
7.71000 & 87.94 & 171.18 \\
11.50000 & 87.08 & 203.26 \\
15.40000 & 85.96 & 226.65 \\
23.10000 & 84.24 & 258.96 \\
$\mathbf{3 8 . 5 0 0 0 0}$ & 82.36 & 299.50 \\
77.10000 & 80.86 & 354.67 \\
154.00000 & 80.14 & 409.64 \\
231.00000 & 79.84 & 441.82
\end{tabular}

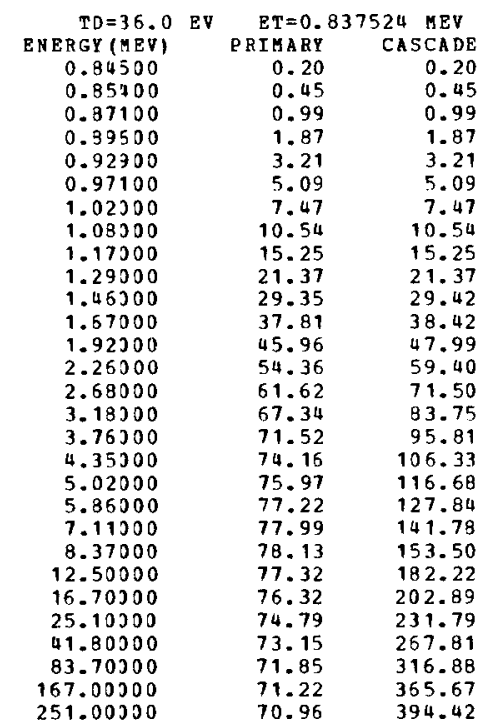

ATOHIC DISPLAZEMENT CROSS-SECTIONS (BARNS) BY FAST ELECTRONS IN NB SECTIONS (BARN)

\begin{tabular}{rrr} 
TD=40.J & EV & \multicolumn{1}{c}{ EI $=0.900226$ MEV } \\
ENERG (HEV) & PRIMARY & CASCADE \\
0.90900 & 0.18 & 0.18 \\
0.91800 & 0.38 & 0.38 \\
0.93600 & 0.82 & 0.82 \\
0.96300 & 1.50 & 1.60 \\
0.99900 & 2.78 & 2.78 \\
1.04000 & 4.30 & 4.30 \\
1.09000 & 6.32 & 6.32 \\
1.17000 & 9.75 & 9.75 \\
1.26000 & 13.72 & 13.72 \\
1.39000 & 19.33 & 19.33 \\
1.57000 & 26.47 & 26.55 \\
1.80000 & 34.29 & 34.90 \\
2.07000 & 41.59 & 43.64 \\
2.43000 & 49.15 & 53.86 \\
2.88000 & 55.57 & 64.84 \\
3.42500 & 60.81 & 76.04 \\
4.05000 & 64.56 & 87.09 \\
4.68000 & 66.87 & 96.56 \\
5.40000 & 68.46 & 105.92 \\
6.30000 & 69.54 & 115.99 \\
7.65000 & 70.20 & 128.64 \\
9.00000 & 70.29 & 139.20 \\
13.50000 & 69.51 & 165.40 \\
18.00000 & 68.62 & 183.92 \\
27.00000 & 67.25 & 209.83 \\
45.00000 & 65.78 & 242.33 \\
90.00000 & 64.64 & 286.44 \\
180.00000 & 64.09 & 330.51
\end{tabular}

\begin{tabular}{crr} 
TD=44.0 & EV & \multicolumn{1}{c}{ ET=0.960259 AEV } \\
ENERG (MEV) & PRIMARY & CASCADE \\
0.96900 & 0.14 & 0.14 \\
0.97900 & 0.32 & 0.32 \\
0.99800 & 0.70 & 0.70 \\
1.02000 & 1.21 & 1.21 \\
1.06000 & 2.28 & 2.28 \\
1.11000 & 3.83 & 3.83 \\
1.17000 & 5.90 & 5.90 \\
1.24000 & 8.48 & 8.48 \\
1.34000 & 12.26 & 12.26 \\
1.48000 & 17.46 & 17.46 \\
1.68000 & 24.29 & 24.36 \\
1.92000 & 31.29 & 31.86 \\
2.20000 & 37.89 & 39.69 \\
2.59000 & 44.83 & 49.25 \\
3.07000 & 50.77 & 59.33 \\
3.64000 & 55.39 & 69.50 \\
4.32000 & 58.82 & 79.76 \\
4.99000 & 60.88 & 88.40 \\
5.76000 & 62.29 & 96.98 \\
6.72000 & 63.25 & 106.18 \\
8.16000 & 63.81 & 117.73 \\
9.60000 & 63.87 & 127.35 \\
14.40000 & 63.15 & 151.24 \\
19.20000 & 62.33 & 168.10 \\
28.80000 & 61.09 & 191.68 \\
48.00000 & 59.77 & 221.24 \\
96.00000 & 58.75 & 261.36 \\
192.00000 & 58.25 & 301.43
\end{tabular}

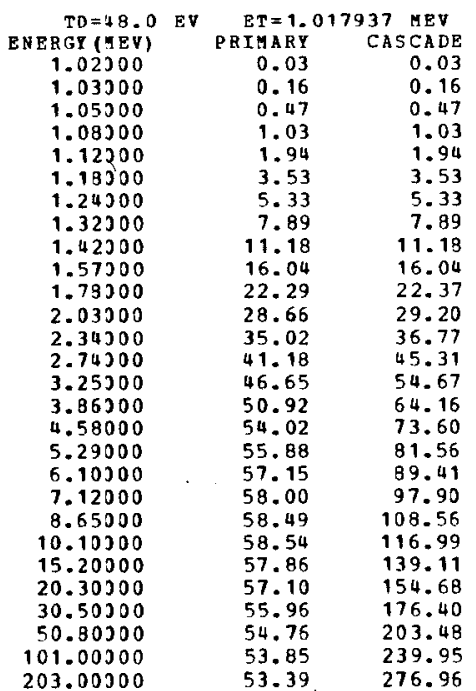


ATOMIC DISPLAZEMENT CROSS-SECTIONS (BARNS) BY RAST ELEETRONS IN NB $=41 \quad A=92.910$

$\begin{array}{rrr}\text { TD }=52.3 & \text { EV } & \text { ET=1.073516 MEV } \\ \text { ENERGY(MEV } & \text { PRIMARY } & \text { CASCADE } \\ 1.09000 & 0.07 & 0.07 \\ 1.09000 & 0.18 & 0.18 \\ 1.11000 & 0.45 & 0.45 \\ 1.14000 & 0.94 & 0.94 \\ 1.19000 & 1.93 & 1.93 \\ 1.24000 & 3.10 & 3.10 \\ 1.30000 & 4.65 & 4.65 \\ 1.39000 & 7.18 & 7.18 \\ 1.50000 & 10.37 & 10.37 \\ 1.66000 & 14.94 & 14.94 \\ 1.87000 & 20.46 & 20.53 \\ 2.14000 & 26.54 & 27.05 \\ 2.46000 & 32.32 & 33.96 \\ 2.89000 & 38.15 & 42.08 \\ 3.43000 & 43.21 & 50.81 \\ 4.07000 & 47.11 & 59.57 \\ 4.83000 & 49.95 & 68.35 \\ 5.58000 & 51.65 & 75.74 \\ 6.44000 & 52.80 & 83.06 \\ 7.51000 & 53.56 & 90.88 \\ 9.12000 & 53.99 & 100.72 \\ 10.70000 & 54.02 & 108.76 \\ 16.10000 & 53.37 & 129.21 \\ 21.40000 & 52.68 & 143.35 \\ 32.20000 & 51.62 & 163.49 \\ 53.60000 & 50.53 & 188.47 \\ 107.00000 & 49.69 & 222.35 \\ 214.00000 & 49.28 & 256.27\end{array}$

$\begin{array}{rrr}\text { TD=56.0 EV } & \text { ET=1.127211 MEV } \\ \text { ENERGY (MEV) } & \text { PRIMARY } & \text { CASCADE } \\ 1.13000 & 0.02 & 0.02 \\ 1.14000 & 0.11 & 0.11 \\ 1.17000 & 0.46 & 0.46 \\ 1.20000 & 0.89 & 0.89 \\ 1.25000 & 1.76 & 1.76 \\ 1.30000 & 2.79 & 2.79 \\ 1.37000 & 4.40 & 4.40 \\ 1.46000 & 6.64 & 6.64 \\ 1.57000 & 9.49 & 9.49 \\ 1.74000 & 13.82 & 13.82 \\ 1.97000 & 19.19 & 19.27 \\ 2.25000 & 24.78 & 25.29 \\ 2.59000 & 30.22 & 31.81 \\ 3.04000 & 35.60 & 39.36 \\ 3.60000 & 40.23 & 47.42 \\ 4.28000 & 43.87 & 55.69 \\ 5.07000 & 46.45 & 63.80 \\ 5.86000 & 48.01 & 70.71 \\ 6.76000 & 49.06 & 77.51 \\ 7.89000 & 49.75 & 94.83 \\ 9.58000 & 50.13 & 93.98 \\ 11.20000 & 50.15 & 101.30 \\ 15.90000 & 49.53 & 120.45 \\ 22.50000 & 48.88 & 133.67 \\ \mathbf{3 3 . 8 0 0 0 0} & 47.91 & 152.31 \\ 56.30000 & 46.90 & 175.54 \\ 112.00000 & 46.13 & 205.85 \\ 225.00000 & 45.75 & 238.55\end{array}$

\begin{tabular}{|c|c|c|}
\hline $\mathrm{TD}=50.0$ & EV $\quad E T=1$. & $204 \mathrm{HEV}$ \\
\hline ENERGY (YEV) & PRIMABY & CASCADE \\
\hline 1.19000 & 0.08 & 0.08 \\
\hline 1.20000 & 0.17 & 0.17 \\
\hline 1.22000 & 0.37 & 0.37 \\
\hline 1.25000 & 0.87 & 0.87 \\
\hline 1.30300 & 1.49 & 1.49 \\
\hline 1.36300 & 2.57 & 2.57 \\
\hline 1.43500 & 4.01 & 4.01 \\
\hline 1.53300 & 6.24 & 6.24 \\
\hline 1.55000 & 9.03 & 9.03 \\
\hline 1.82300 & 12.91 & 12.91 \\
\hline 2.06000 & 17.95 & 18.02 \\
\hline 2.35300 & 23.15 & 23.63 \\
\hline 2.71300 & 28.31 & 29.84 \\
\hline 3.18000 & 33.33 & 36.92 \\
\hline 3.77300 & 37.67 & 44.53 \\
\hline 4.48000 & 41.03 & 52.27 \\
\hline 5.30000 & 43.41 & 59.80 \\
\hline 6.13000 & 44.86 & 56.31 \\
\hline 7.07000 & 45.82 & 72.66 \\
\hline 8.25000 & 46.45 & 79.51 \\
\hline 10.00300 & 46.79 & 87.99 \\
\hline 11.70000 & 46.80 & 94.87 \\
\hline 17.60000 & 46.22 & 112.63 \\
\hline 23.50000 & 45.60 & 125.11 \\
\hline 35.30000 & 44.69 & 142.51 \\
\hline 58.90000 & 43.76 & 164.28 \\
\hline 117.00300 & 43.05 & 193.44 \\
\hline 235.00300 & 42.70 & 223.03 \\
\hline
\end{tabular}

ATOMIC DISPLAZEMENT CROSS-SECTIONS (BARNS) BY FAST BLECTRONS IN NB $\mathrm{Z}=41 \quad \mathrm{~A}=92.910$

\begin{tabular}{rrr} 
TO=64.0 & EV & \multicolumn{2}{c}{ ET=1.229639 MEV } \\
ENERGY (MEV) & PRIMARY & CASCADE \\
1.24000 & 0.07 & 0.07 \\
1.25000 & 0.14 & 0.14 \\
1.27000 & 0.31 & 0.31 \\
1.31000 & 0.75 & 0.75 \\
1.35000 & 1.43 & 1.43 \\
1.42000 & 2.42 & 2.42 \\
1.50000 & 3.90 & 3.90 \\
1.59000 & 5.72 & 5.72 \\
1.72000 & 8.45 & 8.45 \\
1.90000 & 12.17 & 12.17 \\
2.15000 & 16.91 & 16.98 \\
2.45000 & 21.77 & 22.23 \\
2.82000 & 26.56 & 28.01 \\
3.32000 & 31.37 & 34.82 \\
3.93000 & 35.39 & 41.93 \\
4.57000 & 38.53 & 49.21 \\
5.53000 & 40.76 & 56.35 \\
6.39000 & 42.09 & 62.42 \\
7.37000 & 42.98 & 68.40 \\
8.50000 & 43.56 & 74.83 \\
10.40000 & 43.86 & 82.70 \\
12.20000 & 43.86 & 89.27 \\
18.40000 & 43.30 & 106.04 \\
24.50000 & 42.73 & 117.64 \\
36.80000 & 41.88 & 133.95 \\
61.40000 & 41.01 & 154.37 \\
122.00000 & 40.36 & 181.73 \\
245.00000 & 40.03 & 209.46
\end{tabular}

\begin{tabular}{rrr} 
TD $=68.0$ & EV & \multicolumn{2}{c}{ ET=1.278656 MEV } \\
ENERGY (HEV) & PRIMAY & CASEADE \\
1.29000 & 0.06 & 0.06 \\
1.30000 & 0.13 & 0.13 \\
1.32000 & 0.28 & 0.28 \\
1.36000 & 0.66 & 0.66 \\
1.41000 & 1.27 & 1.27 \\
1.48000 & 2.31 & 2.31 \\
1.55000 & 3.48 & 3.48 \\
1.66000 & 5.50 & 5.50 \\
1.79000 & 7.98 & 7.98 \\
1.98000 & 11.55 & 11.55 \\
2.23000 & 15.87 & 15.94 \\
2.55000 & 20.58 & 21.04 \\
2.94000 & 25.16 & 26.57 \\
3.45000 & 29.59 & 32.89 \\
4.09000 & 33.40 & 39.66 \\
4.85000 & 36.31 & 46.47 \\
5.75000 & 38.41 & 53.25 \\
6.64000 & 39.64 & 58.96 \\
7.67000 & 40.47 & 64.66 \\
8.95000 & 41.01 & 70.72 \\
10.80000 & 41.28 & 78.06 \\
12.70000 & 41.27 & 84.34 \\
19.10000 & 40.74 & 100.04 \\
25.50000 & 40.20 & 111.06 \\
38.30000 & 39.40 & 126.42 \\
63.90000 & 38.59 & 145.64 \\
127.00000 & 37.98 & 171.40 \\
255.00000 & 37.67 & 197.50
\end{tabular}

\begin{tabular}{|c|c|c|}
\hline$T D=72.0$ & $E T=1.3$ & $365 \mathrm{MEV}$ \\
\hline ENERGY (JEV) & PRIMARY & CASCADE \\
\hline 1.33300 & 0.02 & 0.02 \\
\hline 1.35300 & 0.12 & 0.12 \\
\hline 1.37300 & 0.25 & 0.26 \\
\hline 1.41300 & 0.60 & 0.60 \\
\hline 1.47000 & 1.28 & 1.28 \\
\hline 1.53000 & 2.08 & 2.08 \\
\hline 1.61000 & 3.31 & 3.31 \\
\hline 1.72 .500 & 5.15 & 5.15 \\
\hline 1.85300 & 7.43 & 7.43 \\
\hline 2.05300 & 10.87 & 10.87 \\
\hline 2.32300 & 15.13 & 15.20 \\
\hline 2.65000 & 19.56 & 20.01 \\
\hline 3.05300 & 23.83 & 25.20 \\
\hline 3.58300 & 28.03 & 31.20 \\
\hline 4.24000 & 31.60 & 37.59 \\
\hline 5.04000 & 34.37 & 44.13 \\
\hline 5.96000 & 36.31 & 50.45 \\
\hline 6.89330 & 37.47 & 55.91 \\
\hline 7.95000 & 38.24 & 61.26 \\
\hline 9.28300 & 38.74 & 67.01 \\
\hline 11.20000 & 38.99 & 73.96 \\
\hline 13.20300 & 38.97 & 79.98 \\
\hline 19.80300 & 38.47 & 94.72 \\
\hline 26.50300 & 37.95 & 105.22 \\
\hline 39.70000 & 37.20 & 119.64 \\
\hline 66.30300 & 36.43 & 137.83 \\
\hline 132.00500 & 35.86 & 162.23 \\
\hline 265.00300 & 35.57 & 186.87 \\
\hline
\end{tabular}


ATOMIC DISPLAZEMENT CROSS-SECTIONS (BARNS) BY PAST ELECTRONS IN NB

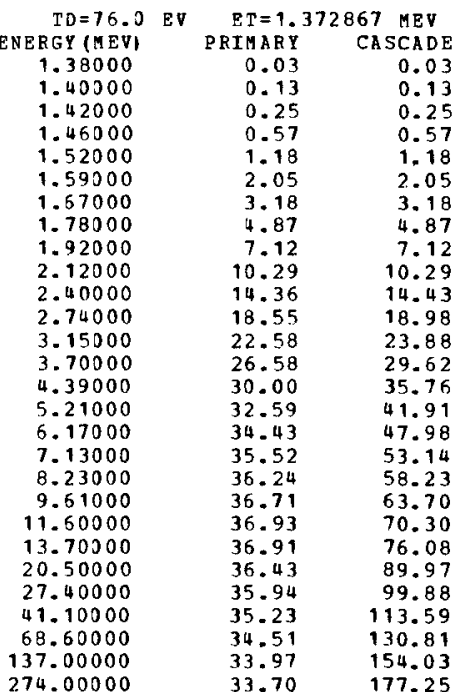

\begin{tabular}{ccr} 
TD=80.0 EV & \multicolumn{2}{c}{ ET=1.418248 MEV } \\
ENERGY (MEV) & PRIMARY & CASEADE \\
1.43000 & 0.04 & 0.04 \\
1.44000 & 0.09 & 0.09 \\
1.47000 & 0.25 & 0.25 \\
1.51000 & 0.54 & 0.54 \\
1.57000 & 1.10 & 1.10 \\
1.64000 & 1.90 & 1.90 \\
1.73000 & 3.07 & 3.07 \\
1.84000 & 4.64 & 4.64 \\
1.98000 & 6.72 & 6.72 \\
2.19000 & 9.80 & 9.80 \\
2.48000 & 13.59 & 13.75 \\
2.83000 & 17.67 & 18.09 \\
3.26000 & 21.55 & 22.83 \\
3.82000 & 25.30 & 28.22 \\
4.53000 & 28.53 & 34.05 \\
5.38000 & 31.00 & 39.94 \\
6.38000 & 32.75 & 45.76 \\
7.37000 & 33.77 & 50.66 \\
8.50000 & 34.45 & 55.48 \\
9.92000 & 34.88 & 60.67 \\
12.00000 & 35.09 & 67.01 \\
14.10000 & 35.06 & 72.34 \\
21.20000 & 34.50 & 85.71 \\
28.30000 & 34.13 & 95.09 \\
42.50000 & 33.45 & 108.15 \\
70.90000 & 32.77 & 124.50 \\
141.00000 & 32.27 & 146.43 \\
283.00000 & 32.01 & 168.60
\end{tabular}

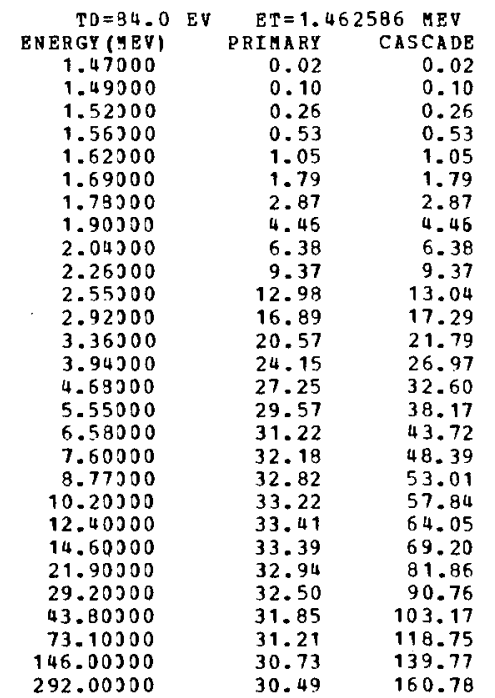

ATOMIC DISPLAZBMENT CROSS-SECTIONS (BARNS) BY FAST ELECTRONS IN NB $\mathrm{Z}=41 \quad \mathrm{~A}=92.910$

\begin{tabular}{rrr} 
TD=88.0 EV & \multicolumn{2}{c}{ ET=1.505950 NEV } \\
ENERGY (HEV) & PRIMARY & CASCADE \\
1.52000 & 0.04 & 0.04 \\
1.53000 & 0.08 & 0.08 \\
1.56000 & 0.21 & 0.21 \\
1.51000 & 0.53 & 0.53 \\
1.57000 & 1.01 & 1.01 \\
1.74000 & 1.70 & 1.70 \\
1.83000 & 2.70 & 2.70 \\
1.95000 & 4.17 & 4.17 \\
2.10000 & 6.10 & 6.10 \\
2.33000 & 9.00 & 9.00 \\
2.63000 & 12.47 & 12.53 \\
3.01000 & 16.19 & 16.59 \\
3.46000 & 19.68 & 20.87 \\
4.06000 & 23.11 & 25.85 \\
4.81000 & 26.03 & 31.16 \\
5.72000 & 28.27 & 36.58 \\
6.77000 & 29.82 & 41.83 \\
7.83000 & 30.74 & 46.34 \\
9.03000 & 31.34 & 50.73 \\
10.50000 & 31.71 & 55.35 \\
12.80000 & 31.90 & 51.36 \\
15.00000 & 31.87 & 56.14 \\
22.50000 & 31.44 & 18.23 \\
30.10000 & 31.01 & 86.83 \\
45.10000 & 30.40 & 98.64 \\
75.20000 & 29.78 & 113.49 \\
150.00000 & 29.33 & 133.52
\end{tabular}

\begin{tabular}{|c|c|c|c|}
\hline $\begin{array}{r}T D=92.0 \\
\text { ENERGY (MEV) }\end{array}$ & EV & $\begin{array}{r}\text { ET }=1.5 \\
\text { PRIMARY }\end{array}$ & $\begin{array}{l}+00 \text { UEV } \\
\text { CASEADE }\end{array}$ \\
\hline 1.56000 & & 0.03 & 0.03 \\
\hline 1.57000 & & 0.06 & 0.06 \\
\hline 1.61000 & & 0.23 & 0.23 \\
\hline 1.65000 & & 0.46 & 0.46 \\
\hline 1.71000 & & 0.90 & 0.90 \\
\hline 1.79000 & & 1.62 & 1.62 \\
\hline 1.88000 & & 2.56 & 2.56 \\
\hline 2.01000 & & 4.05 & 4.05 \\
\hline 2.16000 & & 5.85 . & 5.85 \\
\hline 2.40000 & & 8.68 & 8.68 \\
\hline 2.70000 & & 11.92 & 11.98 \\
\hline 3.09000 & & 15.48 & 15.87 \\
\hline 3.56000 & & 18.88 & 20.05 \\
\hline 4.18000 & & 22.17 & 24.84 \\
\hline 4.95000 & & 24.94 & 29.92 \\
\hline 5.88000 & & 27.07 & 35.08 \\
\hline 6.96000 & & 28.55 & 40.12 \\
\hline 8.05000 & & 29.42 & 44.44 \\
\hline 9.29000 & & 29.99 & 48.67 \\
\hline 10.80000 & & 30.34 & 53.08 \\
\hline 13.10000 & & 30.51 & 58.69 \\
\hline 15.40000 & & 30.48 & 63.35 \\
\hline 23.20000 & & 30.06 & 75.05 \\
\hline 30.90000 & & 29.65 & 83.15 \\
\hline 46.40000 & & 29.07 & 94.52 \\
\hline 77.40000 & & 28.48 & 108.74 \\
\hline 154.00000 & & 28.05 & 127.83 \\
\hline
\end{tabular}

\begin{tabular}{|c|c|c|}
\hline$T D=96.0$ & $\mathrm{ET}=1$ & $94 \mathrm{MEV}$ \\
\hline ENERGY (IEV) & PRIMARY & CASCADE \\
\hline 1.60000 & 0.02 & 0.02 \\
\hline 1.52000 & 0.08 & 0.08 \\
\hline 1.65000 & 0.20 & 0.20 \\
\hline 1.70300 & 0.47 & 0.47 \\
\hline 1.76000 & 0.89 & 0.89 \\
\hline 1.84000 & 1.57 & 1.57 \\
\hline 1.93000 & 2.45 & 2.45 \\
\hline 2.06000 & 3.84 & 3.84 \\
\hline 2.22000 & 5.63 & 5.63 \\
\hline 2.46000 & 8.29 & 8.29 \\
\hline 2.78000 & 11.52 & 11.58 \\
\hline 3.17000 & 14.85 & 15.23 \\
\hline 3.65000 & 18.10 & 19.22 \\
\hline 4.29300 & 21.28 & 23.86 \\
\hline 5.08000 & 23.93 & 28.73 \\
\hline 6.04000 & 25.97 & 33.72 \\
\hline 7.15000 & 27.38 & 38.56 \\
\hline 8.26300 & 28.21 & 42.67 \\
\hline 9.53000 & 28.74 & 46.72 \\
\hline 11.10500 & 29.08 & 51.01 \\
\hline 13.50000 & 29.24 & 56.47 \\
\hline 15.80000 & 29.20 & 60.81 \\
\hline 23.80300 & 28.80 & 72.03 \\
\hline 31.70300 & 28.41 & 79.79 \\
\hline 47.60000 & 27.85 & 90.69 \\
\hline 79.40000 & 27.29 & 104.32 \\
\hline 158.00000 & 26.88 & 122.61 \\
\hline
\end{tabular}


ATOMIC DISPLAZEHENT CROSS-SECTIONS (BARNS) BY PAST ELEZTRONS IN hO $=42 \quad A=95.950$

$\begin{array}{crr}T D=U .3 & \text { EV } & \text { ET }=0.152194 \text { MEV } \\ \text { ENERGY MEV } & \text { PRIMARY } & \text { CASCADE } \\ 0.15300 & 7.13 & 7.13 \\ 0.15500 & 24.33 & 24.33 \\ 0.15800 & 48.89 & 48.89 \\ 0.16200 & 79.50 & 79.50 \\ 0.16800 & 121.33 & 121.33 \\ 0.17600 & 170.60 & 170.60 \\ 0.18500 & 218.68 & 218.68 \\ 0.19700 & 273.13 & 273.13 \\ 0.21300 & 332.57 & 332.57 \\ 0.23500 & 396.62 & 396.62 \\ 0.26600 & 463.61 & 463.61 \\ 0.30400 & 522.37 & 524.38 \\ 0.35000 & 572.66 & 583.81 \\ 0.41000 & 618.00 & 647.04 \\ 0.48700 & 656.81 & 713.65 \\ 0.57800 & 686.73 & 779.55 \\ 0.68400 & 709.15 & 845.33 \\ 0.79100 & 723.74 & 903.72 \\ 0.91300 & 734.35 & 963.29 \\ 1.06000 & 741.86 & 1027.66 \\ 1.29000 & 747.04 & 1116.40 \\ 1.52000 & 747.82 & 1194.14 \\ 2.28000 & 739.92 & 1399.98 \\ 3.04000 & 728.83 & 1556.30 \\ 4.56000 & 717.11 & 1788.65 \\ 7.50000 & 689.90 & 2094.85 \\ 15.20000 & 667.88 & 2524.27 \\ 30.40000 & 656.52 & 2963.73 \\ 45.50000 & 652.90 & 3222.94 \\ 76.00000 & 649.52 & 3550.55 \\ 105.00000 & 647.48 & \mathbf{3 7 6 3 . 7 4} \\ 152.00000 & 645.25 & 3994.12 \\ & & \end{array}$

\begin{tabular}{|c|c|c|}
\hline$T D=8.0$ & $\mathrm{ET}=0.2$ & $68 \mathrm{MEV}$ \\
\hline VERGY (MEV) & PRIMA RY & CASZADE \\
\hline 0.27800 & 3.29 & 3.29 \\
\hline 0.28000 & 5.85 & 5.85 \\
\hline 0.28600 & 13.35 & 13.35 \\
\hline 0.29400 & 22.93 & 22.93 \\
\hline 0.30500 & 35.38 & 35.38 \\
\hline 0.31900 & 50.17 & 50.17 \\
\hline 0.33600 & 66.74 & 66.74 \\
\hline 0.35800 & 86.26 & 86.26 \\
\hline 0.38500 & 107.70 & 107.70 \\
\hline 0.42500 & 136.01 & 136.01 \\
\hline 0.48200 & 168.19 & 168.20 \\
\hline 0.55000 & 199.76 & 201.14 \\
\hline 0.63300 & 230.14 & 235.35 \\
\hline 0.74300 & 260.66 & 275.35 \\
\hline 0.88100 & 288.29 & 317.68 \\
\hline 1.04000 & 310.51 & 359.56 \\
\hline 1.23000 & 328.47 & 402.77 \\
\hline 1.43000 & 340.98 & 442.44 \\
\hline 1.65000 & 349.98 & 480.89 \\
\hline 1.92000 & 356.81 & 522.48 \\
\hline 2.34000 & 362.09 & 578.01 \\
\hline 2.75000 & 363.95 & 624.32 \\
\hline 4.13000 & 361.94 & 744.35 \\
\hline 5.50000 & 357.32 & 831.24 \\
\hline 8.26000 & 349.65 & 957.09 \\
\hline 13.70000 & 340.32 & 1115.70 \\
\hline 27.50000 & 331.34 & 1336.90 \\
\hline 55.00000 & 327.07 & 1559.18 \\
\hline 82.60000 & 325.58 & 1689.93 \\
\hline 137.00000 & 324.02 & 1852.53 \\
\hline 192.00000 & 322.98 & 1960.62 \\
\hline 275.00000 & 321.86 & 2075.39 \\
\hline
\end{tabular}

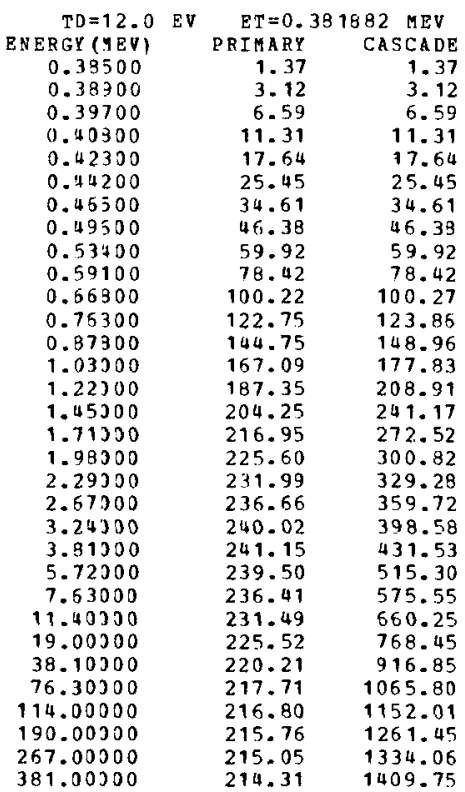
A TOMIC DISPLAzEHENT CROSS-SECTIONS (BARNS) BY FAST ELECTRONS IN HO

\begin{tabular}{|c|c|c|}
\hline$\Gamma D=16.0$ & $\mathrm{ET}=0$ & $899 \mathrm{MEV}$ \\
\hline ENERGT (MEV) & PRIMARY & CASCADE \\
\hline 0.48100 & 0.84 & 0.84 \\
\hline 0.48600 & 9.88 & 1.88 \\
\hline 0.49500 & 3.77 & 3.77 \\
\hline 0.51000 & 6.99 & 6.99 \\
\hline 0.52900 & 11.12 & 11.12 \\
\hline 0.55300 & 16.40 & 16.40 \\
\hline 0.58100 & 22.56 & 22.56 \\
\hline 0.61900 & 30.80 & 30.80 \\
\hline 0.66700 & 40.86 & 40.86 \\
\hline 0.73900 & 54.97 & 54.97 \\
\hline 0.83400 & 71.57 & 71.63 \\
\hline 0.95300 & 89.24 & 90.20 \\
\hline 1.09000 & 105.84 & 109.19 \\
\hline 1.28000 & 123.63 & 132.25 \\
\hline 1.52000 & 139.80 & 157.34 \\
\hline 1.81000 & 153.07 & 183.24 \\
\hline 2.14000 & 162.90 & 208.44 \\
\hline 2.47000 & 169.26 & 230.26 \\
\hline 2.86000 & 174.02 & 252.78 \\
\hline 3.33000 & 177.35 & 276.33 \\
\hline 4.05000 & 179.70 & 306.87 \\
\hline 4.76000 & 180.39 & 332.25 \\
\hline 7.15000 & 178.86 & 396.60 \\
\hline 9.53000 & 176.53 & 442.39 \\
\hline 14.30000 & 172.82 & 507.25 \\
\hline 23.80000 & 168.53 & 588.70 \\
\hline 47.60000 & 164.87 & 700.01 \\
\hline 95.30000 & 163.14 & 811.85 \\
\hline 143.00300 & 162.47 & 877.24 \\
\hline 238.00000 & 161.69 & 959.08 \\
\hline
\end{tabular}

\begin{tabular}{|c|c|c|}
\hline$T D=20.0$ & $\mathrm{ET}=0.5$ & $547 \mathrm{MEV}$ \\
\hline ENERGY (MEV) & PR I MA RY & CASEADE \\
\hline 0.56900 & 0.63 & 0.63 \\
\hline 0.57400 & 1. 22 & 1.22 \\
\hline 0.58600 & 2.68 & 2.68 \\
\hline 0.60200 & 4.72 & 4.72 \\
\hline 0.62500 & 7.79 & 7.79 \\
\hline 0.65300 & 11.67 & 11.67 \\
\hline 0.58700 & 16.51 & 16.51 \\
\hline 0.73200 & 22.97 & 22.97 \\
\hline 0.78800 & 30.88 & 30.88 \\
\hline 0.87300 & 42.30 & 42.30 \\
\hline 0.98600 & 56.00 & 56.07 \\
\hline 1.12000 & 69.94 & 70.75 \\
\hline 1.29000 & 84.38 & 87.33 \\
\hline 1.52000 & 99.29 & 105.93 \\
\hline 1.80000 & 112.24 & 127.46 \\
\hline 2.14000 & 122.86 & 148.77 \\
\hline 2. 53000 & 130.72 & 169.62 \\
\hline 2.93000 & 135.82 & 188.04 \\
\hline 3.38000 & 139.41 & 206.09 \\
\hline 3.94000 & 141.96 & 225.55 \\
\hline 4.79000 & 143.66 & 250.45 \\
\hline 5.63000 & 144.09 & 271.12 \\
\hline 8.45000 & 142.69 & 323.19 \\
\hline 11.20000 & 140.84 & 359.45 \\
\hline 16.90000 & 137.85 & 412.35 \\
\hline 28.10000 & 134.52 & 477.63 \\
\hline 56.30000 & 131.74 & 567.11 \\
\hline 112.00000 & 130.44 & 655.84 \\
\hline 169.00000 & 129.91 & 708.89 \\
\hline 281.00000 & 129.29 & 774.22 \\
\hline
\end{tabular}

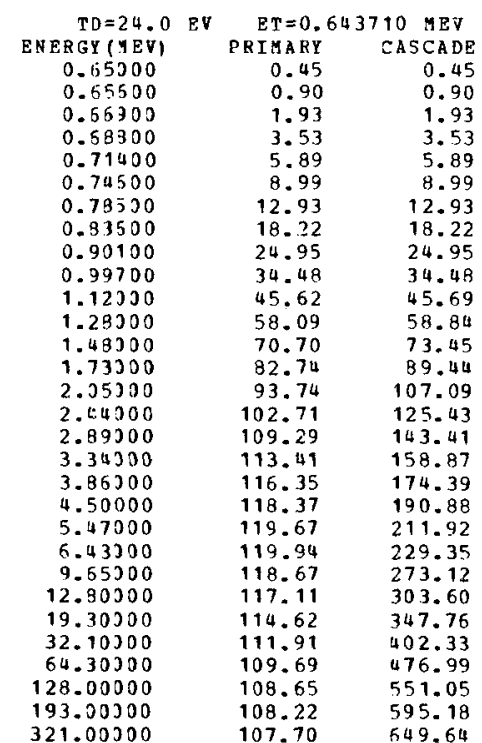


ATONIC DISPLAZEMENT CROSS-SECTIONS (BARNS) BY PAST ELEZTRONS IN MO

\begin{tabular}{crr} 
TD=28.0 & \multicolumn{2}{c}{ ER $=0.718659$ MEV } \\
ENERGY(MEV) & PRIMARY & CASCADE \\
0.72500 & 0.31 & 0.31 \\
0.73300 & 0.72 & 0.72 \\
0.74700 & 1.49 & 1.49 \\
0.76800 & 2.75 & 2.75 \\
0.79700 & 4.67 & 4.67 \\
0.83300 & 7.26 & 7.26 \\
0.87600 & 10.55 & 10.55 \\
0.93400 & 15.16 & 15.16 \\
1.00000 & 20.44 & 20.44 \\
1.11000 & 28.96 & 28.96 \\
1.25000 & 38.88 & 38.95 \\
1.43000 & 49.84 & 50.55 \\
1.55000 & 60.68 & 63.16 \\
1.94000 & 71.49 & 77.70 \\
2.29000 & 80.74 & 92.81 \\
2.73000 & 88.47 & 108.98 \\
3.23000 & 94.00 & 124.56 \\
3.73000 & 97.44 & 137.95 \\
4.31000 & 99.87 & 151.42 \\
5.03000 & 101.53 & 165.82 \\
6.10000 & 102.55 & 183.81 \\
7.18000 & 102.72 & 199.00 \\
10.70000 & 101.58 & 236.10 \\
14.30000 & 100.20 & 263.03 \\
21.50000 & 98.09 & 300.72 \\
35.90000 & 95.79 & 347.94 \\
71.80000 & 93.97 & 411.86 \\
143.00000 & 93.10 & 475.41 \\
215.00000 & 92.73 & 512.98
\end{tabular}

\begin{tabular}{ccr} 
TD=32.0 & EV & \multicolumn{1}{c}{ ET=0.789295 HEV } \\
ENERGY(MEV) & PRIMARY & CASEADE \\
0.79700 & 0.27 & 0.27 \\
0.80500 & 0.57 & 0.57 \\
0.82000 & 1.17 & 1.17 \\
0.84400 & 2.26 & 2.26 \\
0.87600 & 3.89 & 3.89 \\
0.91500 & 6.08 & 6.08 \\
0.96200 & 8.91 & 8.91 \\
1.02000 & 12.58 & 12.58 \\
1.10000 & 17.72 & 17.72 \\
1.22000 & 25.21 & 25.21 \\
1.38000 & 34.35 & 34.43 \\
1.57000 & 43.67 & 44.34 \\
1.81000 & 53.22 & 55.50 \\
2.13000 & 62.82 & 68.52 \\
2.52000 & 71.04 & 82.14 \\
2.99000 & 77.61 & 96.12 \\
3.55000 & 82.50 & 110.23 \\
4.10000 & 85.45 & 122.09 \\
4.73000 & 87.49 & 133.86 \\
5.52000 & 88.89 & 146.57 \\
6.70000 & 89.72 & 162.51 \\
7.89000 & 89.82 & 175.92 \\
11.80000 & 88.76 & 208.82 \\
15.70000 & 87.56 & 232.10 \\
23.60000 & 85.71 & 265.13 \\
39.40000 & 83.74 & 306.48 \\
78.90000 & 82.18 & 362.56 \\
157.00000 & 81.44 & 418.11 \\
236.00000 & 81.12 & 450.97
\end{tabular}

\begin{tabular}{|c|c|c|c|}
\hline$T D=36$. & EV & $\mathrm{ET}=0.8$ & $287 \mathrm{MEV}$ \\
\hline ENERGY (YEV) & & PRIMARY & CASCADE \\
\hline 0.86400 & & 0.20 & 0.20 \\
\hline 0.87300 & & 0.45 & 0.45 \\
\hline 0.39300 & & 0.98 & 0.98 \\
\hline 0.91500 & & 1.90 & 1.90 \\
\hline 0.95000 & & 3.28 & 3.28 \\
\hline 0.99300 & & 5.22 & 5.22 \\
\hline 1.04300 & & 7.53 & 7.53 \\
\hline 1.11000 & & 11.16 & 11.16 \\
\hline 1.19300 & & 15.41 & 15.41 \\
\hline 1.32300 & & 22.14 & 22.14 \\
\hline 1.49000 & & 30.23 & 30.30 \\
\hline 1.71300 & & 39.18 & 39.84 \\
\hline 1.96300 & & 47.40 & 49.51 \\
\hline 2.31300 & & 56.08 & 61.35 \\
\hline 2.74300 & & 63.50 & 73.82 \\
\hline 3.25300 & & 69.28 & 86.37 \\
\hline 3.85300 & & 73.51 & 98.87 \\
\hline 4.45000 & & 76.10 & 109.57 \\
\hline 5.13000 & & 77.86 & 120.07 \\
\hline 5.99000 & & 79.05 & 131.49 \\
\hline 7.27000 & & 79.74 & 145.73 \\
\hline 8.56000 & & 79.79 & 157.70 \\
\hline 12.80000 & & 78.81 & 187.04 \\
\hline 17.10000 & & 77.72 & 208.08 \\
\hline 25.60000 & & 76.10 & 237.18 \\
\hline 42.30300 & & 74.37 & 274.09 \\
\hline 85.60000 & & 73.02 & 323.88 \\
\hline 171.00000 & & 72.37 & 373.55 \\
\hline 256.00300 & & 72.09 & 402.46 \\
\hline
\end{tabular}

ATOMIC DISPLAZEHENT CROSS-SECTIONS (BARNS) BY FAST BLEZTRONS IN MO

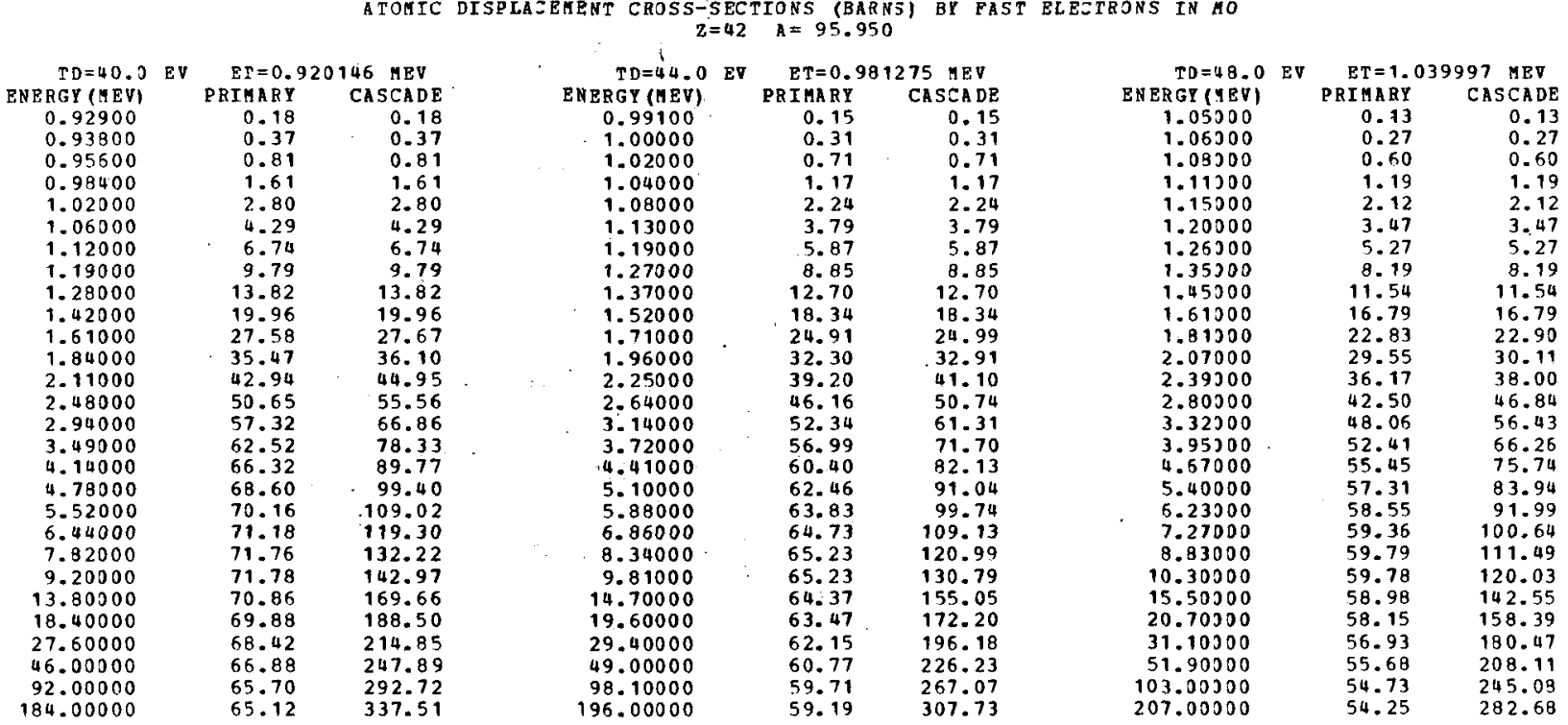


ATOMIC DISPLAZEMENT CROSS-SEZTIONS (BARNS) BY PAST BLETTRONS IN MO

\begin{tabular}{rrr} 
PD=52.0 EV & \multicolumn{1}{c}{ EI $=1.096575$ MEV } \\
ENERGY (MEV) & PRIMARY & CASCADE \\
1.10000 & 0.03 & 0.03 \\
1.11000 & 0.14 & 0.14 \\
1.14000 & 0.55 & 0.55 \\
1.17000 & 1.05 & 1.05 \\
1.21000 & 1.86 & 1.86 \\
1.27000 & 3.27 & 3.27 \\
1.33000 & 4.85 & 4.85 \\
1.42000 & 7.42 & 7.42 \\
1.53000 & 10.66 & 10.66 \\
1.69000 & 15.31 & 15.31 \\
1.91000 & 21.17 & 21.25 \\
2.19000 & 27.53 & 28.09 \\
2.52000 & 33.52 & 35.26 \\
2.96000 & 39.46 & 43.62 \\
3.50000 & 44.49 & 52.39 \\
4.16000 & 48.47 & 61.47 \\
4.93000 & 51.29 & 70.38 \\
5.70000 & 52.98 & 77.98 \\
6.57000 & 54.09 & 85.39 \\
7.57000 & 54.81 & 93.43 \\
9.32000 & 55.18 & 103.50 \\
10.90000 & 55.16 & 111.53 \\
16.40000 & 54.40 & 132.35 \\
21.90000 & 53.63 & 146.97 \\
32.80000 & 52.52 & 167.22 \\
54.80000 & 51.37 & 192.79 \\
109.00000 & 50.50 & 227.05 \\
219.00000 & 50.07 & 261.74 \\
& &
\end{tabular}

\begin{tabular}{crr} 
TD $=56.0$ & EV & \multicolumn{1}{c}{ ET $=1.151228$ MEV } \\
ENERGY(MEV) & PRIMARY & CASEADE \\
1.16000 & 0.07 & 0.07 \\
1.17000 & 0.17 & 0.17 \\
1.19000 & 0.40 & 0.40 \\
1.23000 & 0.97 & 0.97 \\
1.27000 & 1.68 & 1.68 \\
1.33000 & 2.92 & 2.92 \\
1.40000 & 4.56 & 4.56 \\
1.49000 & 6.84 & 5.84 \\
1.51000 & 9.99 & 9.99 \\
1.78000 & 14.38 & 14.38 \\
2.01000 & 19.83 & 19.90 \\
2.30000 & 25.69 & 25.21 \\
2.64000 & 31.16 & 32.80 \\
3.10000 & 36.69 & 40.60 \\
3.68000 & 41.47 & 48.99 \\
4.37000 & 45.11 & 57.42 \\
5.18000 & 47.71 & 65.75 \\
5.98000 & 49.24 & 72.76 \\
6.90000 & 50.26 & 79.71 \\
8.05000 & 50.91 & 87.16 \\
9.78000 & 51.24 & 96.53 \\
11.50000 & 51.20 & 104.26 \\
17.20000 & 50.49 & 123.33 \\
23.00000 & 49.77 & 135.99 \\
34.50000 & 48.74 & 155.87 \\
57.50000 & 47.68 & 179.52 \\
115.00000 & 46.88 & 211.58 \\
230.00000 & 46.49 & 243.59
\end{tabular}

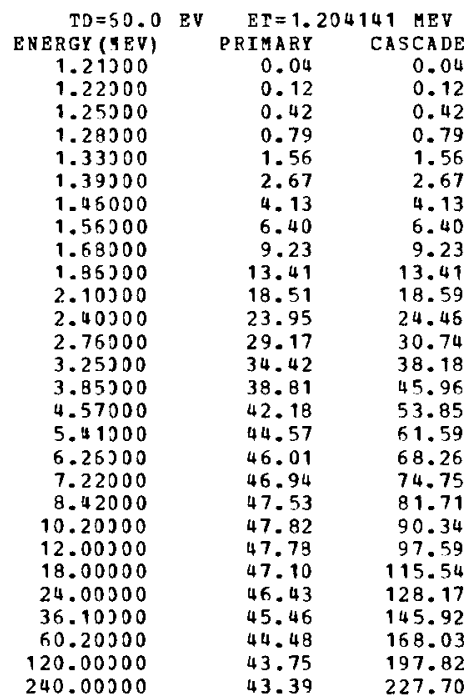

BTOMIC DISPLAZEHENT CROSS-SECTIONS (BARNS) BY FAST ELEETRONS IN MO $z=42 \quad A=95.950$

\begin{tabular}{rrr} 
TD $=64.0$ & EV & \multicolumn{1}{c}{$E T=1.255470$ MEV } \\
ENERGY (HEV) & PRIMARY & CASCADE \\
1.26000 & 0.03 & 0.03 \\
1.28000 & 0.17 & 0.17 \\
1.30000 & 0.35 & 0.35 \\
1.34000 & 0.79 & 0.79 \\
1.39000 & 1.49 & 1.49 \\
1.45000 & 2.49 & 2.49 \\
1.53000 & 4.00 & 4.00 \\
1.63000 & 6.05 & 6.06 \\
1.75000 & 8.62 & 8.62 \\
1.94000 & 12.61 & 12.61 \\
2.19000 & 17.42 & 17.49 \\
2.51000 & 22.65 & 23.15 \\
2.88000 & 27.46 & 28.98 \\
3.38000 & 32.29 & 35.85 \\
4.01000 & 36.44 & 43.25 \\
4.77000 & 39.63 & 50.76 \\
5.64000 & 41.84 & 58.00 \\
6.52000 & 43.16 & 64.23 \\
7.53000 & 44.03 & 70.39 \\
8.78000 & 44.57 & 75.92 \\
10.60000 & 44.82 & 84.87 \\
12.50000 & 44.78 & 91.78 \\
18.80000 & 44.13 & 108.74 \\
25.10000 & 43.50 & 120.65 \\
37.60000 & 42.60 & 137.13 \\
62.70000 & 41.59 & 157.85 \\
125.00000 & 41.01 & 185.80 \\
251.00000 & 40.67 & 213.97
\end{tabular}

\begin{tabular}{rrr} 
TD $=68.0$ & EV & \multicolumn{2}{c}{ ET=1.305348 MEV } \\
ENERGY (MEV) & PRIMARY & CASEADE \\
1.31000 & 0.02 & 0.02 \\
1.33000 & 0.15 & 0.15 \\
1.35000 & 0.30 & 0.30 \\
1.39000 & 0.69 & 0.69 \\
1.44000 & 1.31 & 1.31 \\
1.51000 & 2.36 & 2.36 \\
1.59000 & 3.74 & 3.74 \\
1.69000 & 5.60 & 5.60 \\
1.82000 & 8.12 & 8.12 \\
2.02000 & 11.95 & 11.95 \\
2.28000 & 16.49 & 16.57 \\
2.61000 & 21.39 & 21.88 \\
3.00000 & 25.98 & 27.46 \\
3.52000 & 30.51 & 33.96 \\
4.17000 & 34.38 & 40.88 \\
4.96000 & 37.37 & 47.98 \\
5.87000 & 39.43 & 54.85 \\
6.78000 & 40.65 & 60.70 \\
7.83000 & 41.46 & 66.50 \\
9.13000 & 41.95 & 72.66 \\
11.00000 & 42.18 & 80.08 \\
13.00000 & 42.14 & 86.68 \\
19.50000 & 41.52 & 102.55 \\
26.10000 & 40.93 & 113.86 \\
39.10000 & 40.08 & 129.38 \\
65.20000 & 39.23 & 148.89 \\
130.00000 & 38.59 & 175.20 \\
261.00000 & 38.27 & 201.71
\end{tabular}

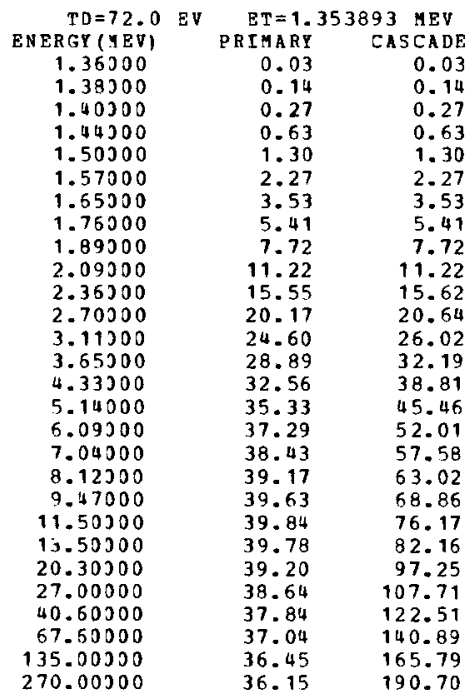


ATONIC DISPLAZEMENT CROSS-SEETIONS (BARNS) BY FAST BLECTrONS IN MO

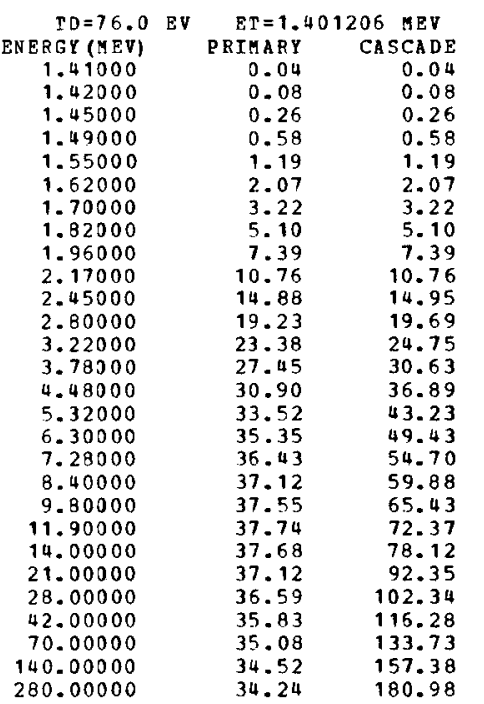

\begin{tabular}{ccc} 
TD $=80.0$ & EV & \multicolumn{1}{c}{ ET=1.447376 MEV } \\
ENERGY (MEV) & PRIMARY & CASEADE \\
1.46000 & 0.05 & 0.05 \\
1.47000 & 0.09 & 0.09 \\
1.50000 & 0.25 & 0.25 \\
1.54000 & 0.55 & 0.55 \\
1.60000 & 1.11 & 1.11 \\
1.67000 & 1.92 & 1.92 \\
1.76000 & 3.10 & 3.10 \\
1.88000 & 4.84 & 4.84 \\
2.02000 & 6.96 & 6.96 \\
2.24000 & 10.23 & 10.23 \\
2.53000 & 14.16 & 14.23 \\
2.89000 & 18.30 & 18.74 \\
3.32000 & 22.22 & 23.53 \\
3.90000 & 26.11 & 29.16 \\
4.63000 & 29.41 & 35.19 \\
5.50000 & 31.90 & 41.23 \\
6.51000 & 33.62 & 47.12 \\
7.52000 & 34.63 & 52.13 \\
8.68000 & 35.28 & 57.08 \\
10.10000 & 35.67 & 62.26 \\
12.30000 & 35.85 & 68.96 \\
14.40000 & 35.79 & 74.27 \\
21.70000 & 35.25 & 87.95 \\
28.90000 & 34.75 & 97.41 \\
43.40000 & 34.03 & 110.69 \\
72.30000 & 33.32 & 127.26 \\
144.00000 & 32.79 & 149.59 \\
289.00000 & 32.53 & 172.12
\end{tabular}

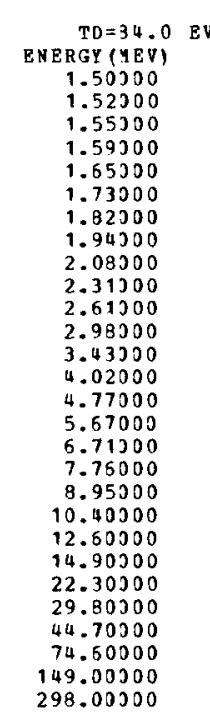

$\begin{array}{rr}\text { ET }=1.492483 \text { HEV } \\ \text { RIMARY } & \text { CASCADE } \\ 0.02 & 0.02 \\ 0.10 & 0.10 \\ 0.26 & 0.25 \\ 0.53 & 0.53 \\ 1.05 & 1.05 \\ 1.91 & 1.91 \\ 3.02 & 3.02 \\ 4.63 & 4.63 \\ 6.59 & 6.59 \\ 9.76 & 9.76 \\ 13.54 & 13.61 \\ 17.47 & 17.90 \\ 21.26 & 22.55 \\ 24.91 & 27.85 \\ 28.04 & 33.59 \\ 30.41 & 39.39 \\ 32.04 & 45.00 \\ 33.00 & 49.82 \\ 33.61 & 54.51 \\ 33.98 & 59.41 \\ 34.14 & 65.63 \\ 34.08 & 71.01 \\ 33.57 & 83.83 \\ 33.09 & 92.95 \\ 32.40 & 105.57 \\ 31.73 & 121.40 \\ 31.23 & 142.77 \\ 30.98 & 164.11\end{array}$

ATOMIC DISPLAzEMENT CROSS-SEETIONS (BARNS) BY PAST ELECTRONS IN MO $\mathrm{Z}=42 \quad \mathrm{~A}=95.950$

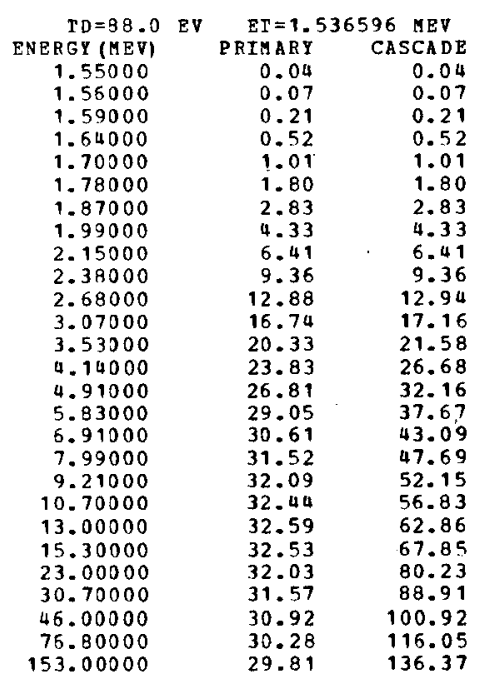

\begin{tabular}{|c|c|c|c|}
\hline$T D=92.0$ & EV & $\begin{array}{l}\text { ET=1. } \\
\text { PRIMARY }\end{array}$ & $\begin{array}{l}779 \mathrm{MEV} \\
\text { CAS }=\mathrm{ADE}\end{array}$ \\
\hline $1.59000^{\circ}$ & & 0.03 & 0.03 \\
\hline 1.61000 & & 0.09 & 0.09 \\
\hline 1.64000 & & 0.22 & 0.22 \\
\hline 1.69000 & & 0.52 & 0.52 \\
\hline 1.75000 & & 0.97 & 0.97 \\
\hline 1.83000 & & 1.71 & 1.71 \\
\hline 1.92000 & & 2.67 & 2.67 \\
\hline 2.05000 & & 4.19 & 4.19 \\
\hline 2.21000 & & 6.14 & 5.14 \\
\hline 2.44000 & & 8.89 & 8.89 \\
\hline 2.76000 & & 12.40 & 12.46 \\
\hline 3.15000 & & 16.00 & 15.40 \\
\hline 3.63000 & & 19.50 & 20.71 \\
\hline 4.26000 & & 22. 85 & 25.62 \\
\hline 5.05000 & & 25.69 & 30.86 \\
\hline 6.00000 & & 27.83 & 36.16 \\
\hline 7. 10000 & & 29.30 & 41.31 \\
\hline 8.21000 & & 30.16 & 45.71 \\
\hline $\begin{array}{r}9.47000 \\
11.00000\end{array}$ & & $\begin{array}{l}30.71 \\
31.03\end{array}$ & $\begin{array}{l}50.01 \\
54.49\end{array}$ \\
\hline 13.40000 & & 31.17 & 60.34 \\
\hline 15.70000 & & 31.11 & 64.98 \\
\hline 23.60000 & & $\begin{array}{l}30.63 \\
30.19\end{array}$ & $\begin{array}{l}76.83 \\
85.13\end{array}$ \\
\hline 47.30000 & & 29.57 & 96.68 \\
\hline 78.90000 & & 28.96 & 111.14 \\
\hline 157.00000 & & 28.51 & 130.54 \\
\hline
\end{tabular}

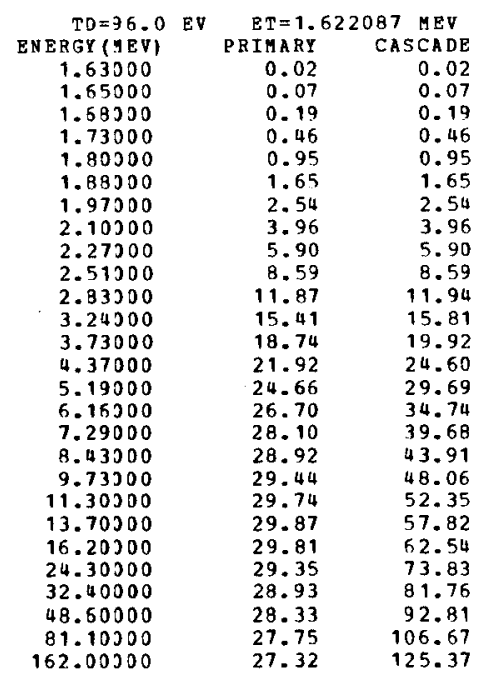


ATOMIC DISPLAZEMENT CROSS-SEzTIONS (BARNS) BY PAST ELEETRONS IN AG

$$
\mathrm{Z}=47 \quad \mathrm{~A}=107.880
$$

\begin{tabular}{|c|c|c|}
\hline$T D=4.0$ & $\mathrm{Er}=0.1$ & MEV \\
\hline ENERGY (MEV) & PRIMARY & CASCADE \\
\hline 0.17000 & .10 .77 & 10.77 \\
\hline 0.17200 & 27.38 & 27.38 \\
\hline 0.17500 & 51.27 & 51.27 \\
\hline 0.18000 & 88.55 & 88.55 \\
\hline 0.18700 & 136.03 & 136.03 \\
\hline 0.19500 & 184.52 & 184.52 \\
\hline 0.20500 & 237.93 & 237.93 \\
\hline 0.21900 & 301.87 & 301.87 \\
\hline 0.23600 & 366.38 & 366.38 \\
\hline 0.26100 & 442.19 & 442.19 \\
\hline 0.29500 & 520.32 & 520.32 \\
\hline 0.33700 & 591.09 & 593.64 \\
\hline 0.38800 & 653.04 & 566.32 \\
\hline 0.45500 & 709.90 & 744.59 \\
\hline 0.53900 & 757.69 & 825.40 \\
\hline 0.64100 & 795.20 & 907.42 \\
\hline 0.75900 & 822.29 & 988.25 \\
\hline 0.87700 & 839.03 & 1058.89 \\
\hline 1.01000 & 850.33 & 1129.71 \\
\hline 1.18000 & 857.75 & 1210.18 \\
\hline 1.43000 & 860.79 & 1313.45 \\
\hline 1.68000 & 858.98 & 1403.33 \\
\hline 2.53000 & 842.54 & 1645.07 \\
\hline 3.37000 & 825.26 & 1824.00 \\
\hline 5.06000 & 800.12 & 2089.11 \\
\hline 8.43000 & 772.04 & 2434.12 \\
\hline 16.80000 & 744.86 & 2912.84 \\
\hline 33.70000 & 731.45 & 3405.97 \\
\hline 50.60000 & 727.03 & 3695.60 \\
\hline 84.30000 & 722.53 & 4059.86 \\
\hline 118.00000 & 719.61 & 4299.16 \\
\hline 168.00000 & 716.48 & 4549.60 \\
\hline
\end{tabular}

\begin{tabular}{|c|c|c|}
\hline \multirow{2}{*}{$\begin{array}{r}T D=8.0 \\
\text { ENERGY (MEV) }\end{array}$} & \multicolumn{2}{|c|}{$\mathrm{ET}=0.303231 \mathrm{MEV}$} \\
\hline & PRIMARY & CAS $\approx A D E$ \\
\hline 0.30500 & 3.53 & 3.53 \\
\hline 0.30900 & 7.30 & 7.30 \\
\hline $0.3150 \mathrm{C}$ & 14.69 & 14.69 \\
\hline 0.32400 & 25.43 & 25.43 \\
\hline 0.33600 & 39.14 & 39.14 \\
\hline 0.35100 & 55.38 & 55.38 \\
\hline 0.36900 & 73.67 & 73.67 \\
\hline 0.39400 & 97.16 & 97.16 \\
\hline 0.42400 & 122.73 & 122.73 \\
\hline 0.47000 & 157.23 & 157.23 \\
\hline 0.53000 & 195.20 & 195.22 \\
\hline 0.60600 & 234.29 & 236.07 \\
\hline 0.69700 & 271.11 & 278.21 \\
\hline 0.81800 & 307.90 & 326.17 \\
\hline 0.97000 & 340.65 & 377.34 \\
\hline 1.15000 & 366.85 & 428.90 \\
\hline 1.36000 & 386.63 & 480.36 \\
\hline 1.57000 & 399.21 & 525.03 \\
\hline 1.81000 & 408.13 & 569.90 \\
\hline 2.12000 & 414.49 & 620.49 \\
\hline 2.57000 & 417.99 & 683.06 \\
\hline 3.03000 & 418.16 & 737.42 \\
\hline 4.54000 & 411.45 & 873.56 \\
\hline 6.06000 & 403.77 & 972.74 \\
\hline 9.09000 & 392.75 & 1114.02 \\
\hline 15.10000 & 380.41 & 1292.26 \\
\hline 30.30000 & 369.41 & 1539.29 \\
\hline 60.60000 & 364.33 & 1787.16 \\
\hline 90.90000 & 362.43 & 1932.29 \\
\hline 151.00000 & 360.24 & 2113.54 \\
\hline 212.00000 & 358.75 & 2234.09 \\
\hline 303.00000 & 357.17 & 2360.56 \\
\hline
\end{tabular}

\begin{tabular}{|c|c|c|}
\hline$T D=12.0$ & $\mathrm{ET}=0$. & MEV \\
\hline ENERGY (YEV) & PRIMARY & CASCADE \\
\hline 0.42200 & 1.53 & 1.53 \\
\hline 0.42500 & 3.26 & 3.26 \\
\hline 0.43500 & 7.17 & 7.17 \\
\hline 0.44700 & 12.41 & 12.41 \\
\hline 0.46400 & 19.81 & 19.81 \\
\hline 0.48500 & 28.90 & 28.90 \\
\hline 0.51300 & 39.52 & 39.52 \\
\hline 0.54300 & 53.12 & 53.12 \\
\hline 0.58500 & 59.57 & 69.57 \\
\hline 0.64300 & 92.24 & 92.24 \\
\hline 0.73200 & 118.74 & 118.82 \\
\hline 0.83500 & 146.17 & 147.61 \\
\hline 0.96200 & 172.77 & 178.11 \\
\hline 1.12300 & 198.21 & 211.30 \\
\hline 1.33000 & 222.31 & 249.08 \\
\hline 1.59300 & 242.07 & 288.68 \\
\hline 1.88300 & 255.96 & 326.19 \\
\hline 2.17000 & 264.60 & 358.57 \\
\hline 2.51000 & 270.71 & 391.67 \\
\hline 2.92300 & 274.63 & 426.30 \\
\hline 3.55000 & 276.75 & 471.33 \\
\hline 4.18000 & 276.65 & 509.25 \\
\hline 6.27000 & 271.80 & 604.00 \\
\hline 8.36000 & 266.81 & 671.81 \\
\hline 12.50300 & 259.78 & 767.03 \\
\hline 20.90300 & 251.94 & 888.77 \\
\hline 49.80000 & 245.49 & 1053.81 \\
\hline 83.60000 & 242.49 & 1219.49 \\
\hline 125.00000 & 241.29 & 1315.59 \\
\hline 209.00300 & 239.81 & 1437.90 \\
\hline 292.00000 & 238.82 & 1517.04 \\
\hline 418.00000 & 237.77 & 1601.69 \\
\hline
\end{tabular}

ATOMIC DISPLA:EMENT CROSS-SEETIONS (BARNS) BY FAST ELECTRONS IN AG

$\begin{array}{crr}\text { TD }=16.0 & \text { EV } & \text { ET }=0.520902 \text { MEV } \\ \text { ENERGY (MEV) } & \text { PRIMARY } & \text { CASCADE } \\ 0.52600 & 1.04 & 1.04 \\ 0.53100 & 2.08 & 2.08 \\ 0.54100 & 4.21 & 4.21 \\ 0.55700 & 7.74 & 7.74 \\ 0.57800 & 12.55 & 12.55 \\ 0.60400 & 18.67 & 18.67 \\ 0.63500 & 26.10 & 26.10 \\ 0.67700 & 35.14 & 36.14 \\ 0.72900 & 48.27 & 48.27 \\ 0.80700 & 65.38 & 65.38 \\ 0.91100 & 85.74 & 85.83 \\ 1.04000 & 107.08 & 108.32 \\ 1.19000 & 127.12 & 131.39 \\ 1.40000 & 148.37 & 159.44 \\ 1.56000 & 165.81 & 189.07 \\ 1.97000 & 181.33 & 219.05 \\ 2.34300 & 192.12 & 249.36 \\ 2.70000 & 198.48 & 274.67 \\ 3.12000 & 202.87 & 300.32 \\ 3.64000 & 205.63 & 327.73 \\ 4.42000 & 205.94 & 362.34 \\ 5.20000 & 206.67 & 391.37 \\ 7.81000 & 202.77 & 464.05 \\ 10.40000 & 199.07 & 515.39 \\ 15.60000 & 193.85 & 587.97 \\ 26.00000 & 188.26 & 679.22 \\ 52.00000 & 183.79 & 803.42 \\ 104.00000 & 181.69 & 927.83 \\ 156.00000 & 180.80 & 1000.50 \\ & & \end{array}$

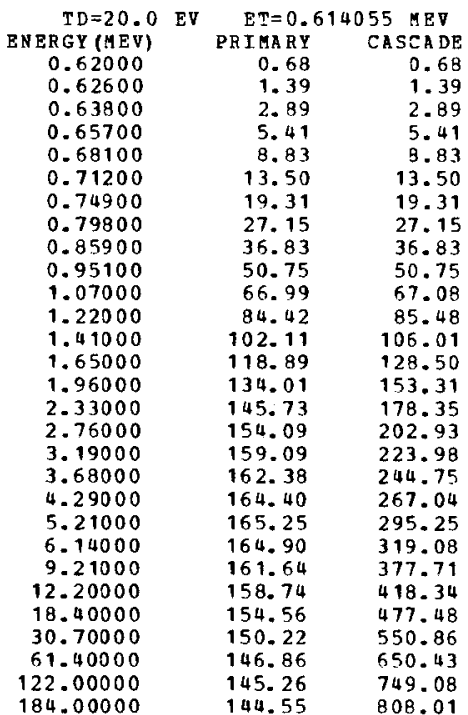

\begin{tabular}{|c|c|c|}
\hline$T D=24.0$ & $E T=0.7$ & MEV \\
\hline ENERGY (YEV) & PRIAARY & CASCADE \\
\hline 0.70700 & 0.50 & $0.50^{\circ}$ \\
\hline 0.71400 & 1.03 & 1.03 \\
\hline 0.72300 & 2.17 & 2.17 \\
\hline 0.74900 & 4.04 & 4.04 \\
\hline 0.77700 & 6.79 & 6.79 \\
\hline 0.81200 & 10.51 & 10.51 \\
\hline 0.35400 & 15.24 & 15.24 \\
\hline 0.91300 & 21.76 & 21.75 \\
\hline 0.98200 & 29.94 & 29.94 \\
\hline 1.08300 & 41.18 & 49.18 \\
\hline 1.22300 & 55.46 & 55.56 \\
\hline 1.40300 & 71.00 & 72.05 \\
\hline 1.61000 & 85.46 & 89.01 \\
\hline 1.89300 & 99.87 & 108.64 \\
\hline 2.24300 & 112.32 & 129.55 \\
\hline 2.65000 & 121.96 & 150.77 \\
\hline 3.15300 & 128.79 & 171.65 \\
\hline 3.64000 & 132.82 & 189.49 \\
\hline 4.20300 & 135.43 & 207.11 \\
\hline 4.90000 & 136.98 & 226.03 \\
\hline 5.95000 & 137.54 & 249.82 \\
\hline 7.00000 & 137.15 & 269.65 \\
\hline 10.50000 & 134.35 & 318.88 \\
\hline 14.03000 & 131.88 & 353.71 \\
\hline 21.00000 & 128.47 & 402.48 \\
\hline 35.00000 & 124.96 & 463.64 \\
\hline 70.00300 & 122.28 & 546.72 \\
\hline 140.00300 & 120.98 & 629.75 \\
\hline
\end{tabular}


ATOMIC DISPLAzEMENT CROSS-SECTIONS (BARNS) BY FAST ELECTRONS IN AG $z=47 \quad A=107.880$

\begin{tabular}{rrr} 
TD=28.0 & \multicolumn{2}{c}{ ET $=0.780355$ MEV } \\
ENERGY (MEV) & PRIMARY & CASCADE \\
0.78800 & 0.37 & 0.37 \\
0.79500 & 0.74 & 0.74 \\
0.81100 & 1.65 & 1.65 \\
0.33400 & 3.12 & 3.12 \\
0.86600 & 5.43 & 5.43 \\
0.90500 & 8.54 & 8.54 \\
0.95200 & 12.57 & 12.57 \\
1.01000 & 17.76 & 17.76 \\
1.09000 & 25.02 & 25.02 \\
1.20000 & 34.68 & 34.68 \\
1.36000 & 47.46 & 47.56 \\
1.55000 & 60.95 & 61.93 \\
1.79000 & 73.33 & 76.52 \\
2.10000 & 85.78 & 93.52 \\
2.49000 & 96.55 & 111.93 \\
2.95000 & 104.84 & 130.55 \\
3.51000 & 110.66 & 148.86 \\
4.05000 & 113.99 & 164.19 \\
4.68000 & 116.16 & 179.63 \\
5.45000 & 117.39 & 196.02 \\
6.63000 & 117.78 & 216.58 \\
7.90000 & 117.39 & 233.70 \\
11.70000 & 114.92 & 276.13 \\
15.60000 & 112.81 & 306.09 \\
23.40000 & 109.91 & 347.98 \\
39.00000 & 105.97 & 400.48 \\
78.00000 & 104.75 & 471.76 \\
156.00000 & 103.66 & 542.95
\end{tabular}

\begin{tabular}{rrr} 
TD=32.0 EV & \multicolumn{1}{c}{ ET=0.855940 REV } \\
ENERGY (IEE) & PRIMARY & CASCADE \\
0.86400 & 0.28 & 0.28 \\
0.87300 & 0.62 & 0.62 \\
0.89000 & 1.34 & 1.34 \\
0.91500 & 2.56 & 2.56 \\
0.95000 & 4.51 & 4.51 \\
0.99200 & 7.13 & 7.13 \\
1.04000 & 10.39 & 10.39 \\
1.11000 & 15.41 & 15.41 \\
1.19000 & 21.25 & 21.25 \\
1.32000 & 30.47 & 30.47 \\
1.49000 & 41.45 & 41.55 \\
1.71000 & 53.46 & 54.38 \\
1.96000 & 64.33 & 67.26 \\
2.31000 & 75.59 & 82.89 \\
2.73000 & 84.81 & 98.84 \\
3.25000 & 92.07 & 115.48 \\
3.85000 & 97.05 & 131.59 \\
4.45000 & 99.91 & 145.31 \\
5.13000 & 101.70 & 158.71 \\
5.99000 & 102.71 & 173.24 \\
7.27000 & 102.98 & 191.31 \\
8.55000 & 102.59 & 206.33 \\
12.80000 & 100.41 & 243.42 \\
17.10000 & 98.55 & 269.88 \\
25.60000 & 96.04 & 306.42 \\
42.70000 & 93.50 & 352.48 \\
85.50000 & 91.61 & 415.01 \\
171.00000 & 90.68 & 477.31
\end{tabular}

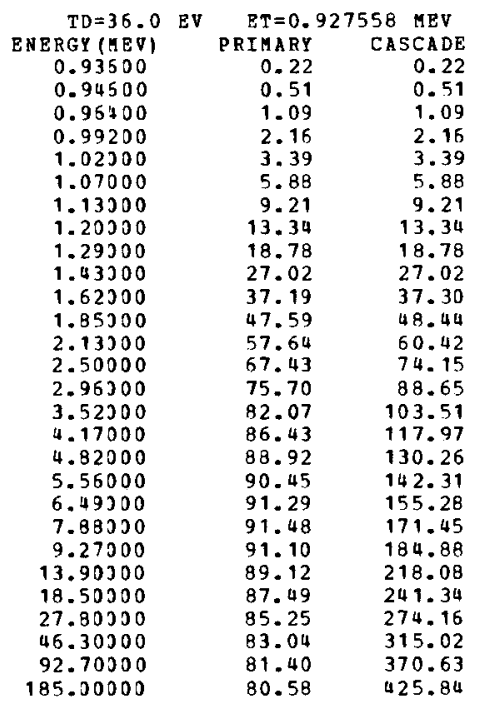

A TOMIC DISPLAZEMENT CROSS-SECTIONS (BARNS) BY FAST ELEETRONS IN AG $Z=47 \quad A=107.880$

\begin{tabular}{rrr} 
TD $=40.0$ & EV & \multicolumn{1}{c}{$E T=0.995776$ MEV } \\
BNERGY (MEV) & PRIMARY & CASCADE \\
1.00000 & 0.08 & 0.08 \\
1.01000 & 0.29 & 0.29 \\
1.03000 & 0.79 & 0.79 \\
1.05000 & 1.69 & 1.59 \\
1.10000 & 3.13 & 3.13 \\
1.15000 & 5.22 & 5.22 \\
1.21000 & 8.00 & 8.00 \\
1.29000 & 11.98 & 11.98 \\
1.39000 & 17.09 & 17.09 \\
1.54000 & 24.56 & 24.56 \\
1.74000 & 33.50 & 33.71 \\
1.99000 & 43.14 & 43.97 \\
2.29000 & 52.20 & 54.84 \\
2.59000 & 60.87 & 67.10 \\
3.18000 & 68.40 & 80.45 \\
3.78000 & 74.07 & 93.91 \\
4.48000 & 77.94 & 107.05 \\
5.17000 & 80.10 & 118.07 \\
5.97000 & 81.45 & 129.05 \\
6.97000 & 82.16 & 140.79 \\
8.46000 & 82.29 & 155.38 \\
9.95000 & 81.92 & 167.48 \\
14.90000 & 80.12 & 197.35 \\
19.90000 & 79.64 & 218.57 \\
29.80000 & 76.65 & 247.88 \\
49.70000 & 74.68 & 284.79 \\
99.50000 & 73.23 & 334.85 \\
199.00000 & 72.51 & 384.71
\end{tabular}

\begin{tabular}{rrr} 
TD $=44.0$ & EV & \multicolumn{1}{c}{ ET=1.061037 AEV } \\
ENERGY (MEV) & PRIMARY & CASEADE \\
1.07000 & 0.14 & 0.14 \\
1.08000 & 0.32 & 0.32 \\
1.10000 & 0.73 & 0.73 \\
1.13000 & 1.49 & 1.49 \\
1.17000 & 2.69 & 2.69 \\
1.23000 & 4.82 & 4.82 \\
1.29000 & 7.21 & 7.21 \\
1.37000 & 10.52 & 10.62 \\
1.48000 & 15.45 & 15.45 \\
1.64000 & 22.31 & 22.31 \\
1.85000 & 30.50 & 30.60 \\
2.12000 & 39.37 & 40.16 \\
2.44000 & 47.65 & 50.16 \\
2.86000 & 55.62 & 61.51 \\
3.39000 & 62.40 & 73.68 \\
4.03000 & 67.51 & 86.00 \\
4.77000 & 70.95 & 97.93 \\
5.51000 & 72.89 & 108.07 \\
6.36000 & 74.07 & 118.07 \\
7.42000 & 74.69 & 128.74 \\
9.01000 & 74.77 & 142.07 \\
10.60000 & 74.42 & 153.13 \\
15.90000 & 72.76 & 180.44 \\
21.20000 & 71.42 & 199.65 \\
31.80000 & 69.61 & 226.43 \\
53.00000 & 67.85 & 259.95 \\
106.00000 & 66.56 & 305.41 \\
212.00000 & 65.90 & 350.75
\end{tabular}

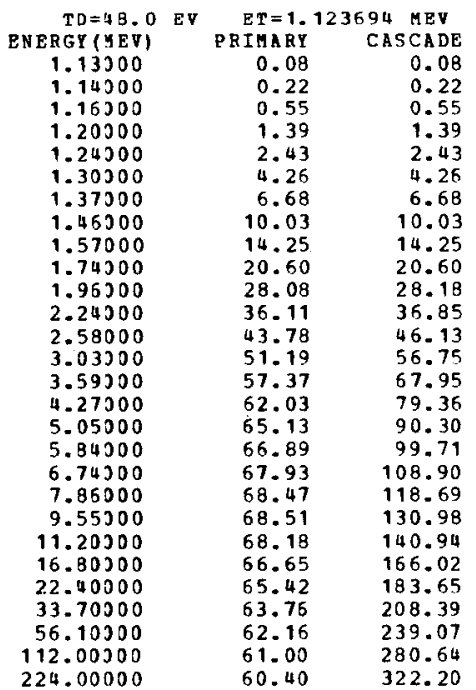


ATOMIC DISPLAZEMENT CROSS-SEZTIONS (BARNS) BY FAST ELECTRONS IN A3 $=47 \quad A=107.880$

\begin{tabular}{rrr} 
TD $=52.0$ & EV & \multicolumn{2}{c}{ ET $=1.184037$ MEV } \\
ENERGY(MEV) & PRIMARY & CASCADE \\
1.19000 & 0.06 & 0.06 \\
1.20000 & 0.17 & 0.17 \\
1.23000 & 0.61 & 0.61 \\
1.26000 & 1.15 & 1.15 \\
1.31000 & 2.28 & 2.28 \\
1.37000 & 3.89 & 3.89 \\
1.44000 & 6.01 & 6.01 \\
1.53000 & 8.95 & 8.95 \\
1.65000 & 13.00 & 13.00 \\
1.83000 & 18.95 & 18.95 \\
2.07000 & 26.14 & 26.25 \\
2.36000 & 33.45 & 34.16 \\
2.72000 & 40.59 & 42.84 \\
3.19000 & 47.38 & 52.63 \\
3.78000 & 53.08 & 63.04 \\
4.49000 & 57.33 & 73.54 \\
5.32000 & 60.19 & 83.80 \\
5.15000 & 61.78 & 92.49 \\
7.10000 & 62.73 & 101.03 \\
8.28000 & 63.20 & 110.09 \\
10.00000 & 63.23 & 121.11 \\
11.80000 & 62.90 & 130.68 \\
17.70000 & 61.47 & 153.87 \\
23.60000 & 60.34 & 170.15 \\
35.50000 & 58.81 & 192.99 \\
59.20000 & 57.35 & 221.42 \\
118.00000 & 56.29 & 259.72 \\
236.00000 & 55.75 & 298.08
\end{tabular}

\begin{tabular}{rrr} 
TD $=56.0$ & EV & \multicolumn{1}{c}{ ET=1.242304 MEV } \\
ENERGY (MEV) & PRTMAR & CASCADE \\
1.25000 & 0.07 & 0.07 \\
1.26000 & 0.16 & 0.16 \\
1.29000 & 0.53 & 0.53 \\
1.32000 & 1.01 & 1.01 \\
1.37000 & 1.98 & 1.98 \\
1.44000 & 3.64 & 3.64 \\
1.51000 & 5.52 & 5.52 \\
1.61000 & 8.43 & 8.43 \\
1.73000 & 12.04 & 12.04 \\
1.92000 & 17.64 & 17.64 \\
2.17000 & 24.31 & 24.41 \\
2.48000 & 31.26 & 31.95 \\
2.85000 & 37.77 & 39.90 \\
3.35000 & 44.15 & 49.17 \\
3.97000 & 49.43 & 58.90 \\
4.72000 & 53.36 & 68.74 \\
5.59000 & 55.98 & 78.28 \\
5.45000 & 57.41 & 86.28 \\
7.45000 & 58.26 & 94.25 \\
8.69000 & 58.68 & 102.70 \\
10.50000 & 58.69 & 112.98 \\
12.40000 & 58.37 & 121.92 \\
18.60000 & 57.04 & 143.48 \\
24.80000 & 55.99 & 158.61 \\
37.20000 & 54.58 & 179.68 \\
62.10000 & 53.23 & 206.14 \\
124.00000 & 52.26 & 241.81 \\
248.00000 & 51.75 & 277.43
\end{tabular}

\begin{tabular}{|c|c|c|}
\hline$T D=50.0$ & $\begin{array}{r}\mathrm{ET}=1 . \\
\text {. }\end{array}$ & 596 MEV \\
\hline 1.31300 & $\begin{array}{r}210.08 \\
0.08\end{array}$ & 0.08 \\
\hline 1.32500 & 0.17 & 0.17 \\
\hline 1.35300 & 0.50 & 0.50 \\
\hline 1.38000 & 0.92 & 0.92 \\
\hline 1.44300 & 1.97 & 1.97 \\
\hline 1.50000 & 3.24 & 3.24 \\
\hline 1.58000 & 5.16 & 5.15 \\
\hline 1.68000 & 7.77 & 7.77 \\
\hline 1.81300 & 11.29 & 11.29 \\
\hline 2.01000 & 16.57 & 16.57 \\
\hline 2.27300 & 22.80 & 22.90 \\
\hline 2.59300 & 29.23 & 29.90 \\
\hline 2.98300 & 35.38 & 37.42 \\
\hline 3.50300 & 41.32 & 46.08 \\
\hline 4.15200 & 46.24 & 55.23 \\
\hline 4.93000 & 49.87 & 64.41 \\
\hline 5.84000 & 52.29 & 73.36 \\
\hline 5.75000 & 53.62 & 80.93 \\
\hline 7.79000 & 54.40 & 88.35 \\
\hline 9.09000 & 54.77 & 96.27 \\
\hline 11.00300 & 54.76 & 105.96 \\
\hline 12.90000 & 54.46 & 113.96 \\
\hline 19.40500 & 53.21 & 134.23 \\
\hline 25.90000 & 52.22 & 148.43 \\
\hline 38.90500 & 50.91 & 168.17 \\
\hline 64.90300 & 49.66 & 192.85 \\
\hline 129.00300 & 48.77 & 225.92 \\
\hline 259.00300 & 48.30 & 259.36 \\
\hline
\end{tabular}

ATOHIC DISPLAZEMENT CROSS-SECTIONS (BARNS) BY PAST ELEZTRONS IN a $z=47 \quad A=107.880$

\begin{tabular}{crr} 
TD $=64.0$ & EV & \multicolumn{2}{c}{ ET $=1.353384$ MEV } \\
ENERGY(MEV) & PRIMARY & CASCADE \\
1.36000 & 0.04 & 0.04 \\
1.38000 & 0.19 & 0.19 \\
1.40000 & 0.38 & 0.38 \\
1.44000 & 0.87 & 0.87 \\
1.50000 & 1.81 & 1.81 \\
1.56000 & 2.95 & 2.95 \\
1.65000 & 4.90 & 4.90 \\
1.75000 & 7.26 & 7.26 \\
1.89000 & 10.69 & 10.69 \\
2.09000 & 15.47 & 15.47 \\
2.36000 & 21.33 & 21.43 \\
2.70000 & 27.51 & 28.16 \\
3.11000 & 33.33 & 35.31 \\
3.65000 & 38.86 & 43.43 \\
4.39000 & 43.47 & 52.06 \\
5.14000 & 46.83 & 60.57 \\
6.09000 & 49.08 & 69.09 \\
7.03000 & 50.30 & 76.15 \\
8.12000 & 51.01 & 83.16 \\
9.47000 & 51.35 & 90.57 \\
11.50000 & 51.32 & 99.83 \\
13.50000 & 51.03 & 107.39 \\
20.30000 & 49.84 & 126.41 \\
27.00000 & 48.93 & 139.55 \\
40.60000 & 47.70 & 158.12 \\
67.60000 & 46.54 & 181.16 \\
135.00000 & 45.71 & 212.39 \\
270.00000 & 45.28 & 243.57 \\
& &
\end{tabular}

$\begin{array}{ccc}\text { TD=68.0 } & \text { EV } & \text { ET=1.406512 MEV } \\ \text { ENERGY(MEV) } & \text { PRTMAY } & \text { CASEADE } \\ 1.42000 & 0.07 & 0.07 \\ 1.43000 & 0.14 & 0.14 \\ 1.46000 & 0.39 & 0.39 \\ 1.50000 & 0.84 & 0.84 \\ 1.56000 & 1.70 & 1.70 \\ 1.63000 & 2.92 & 2.92 \\ 1.71000 & 4.50 & 4.50 \\ 1.82000 & 6.86 & 5.86 \\ 1.96000 & 9.98 & 9.98 \\ 2.18000 & 14.77 & 14.77 \\ 2.46000 & 20.28 & 20.38 \\ 2.81000 & 26.04 & 26.67 \\ 3.23000 & 31.44 & 33.33 \\ 3.79000 & 36.64 & 41.00 \\ 4.50000 & 40.99 & 49.20 \\ 5.34000 & 44.13 & 57.31 \\ 6.32000 & 46.22 & 65.21 \\ 7.31000 & 47.37 & 71.95 \\ 8.43000 & 48.02 & 78.49 \\ 9.84000 & 48.32 & 85.52 \\ 11.90000 & 48.29 & 94.06 \\ 14.00000 & 48.01 & 101.28 \\ 21.00000 & 46.90 & 119.09 \\ 28.10000 & 46.03 & 131.73 \\ 42.10000 & 44.88 & 149.05 \\ 70.30000 & 43.79 & 170.87 \\ 140.00000 & 43.02 & 200.15 \\ 281.00000 & 42.61 & 229.64\end{array}$

\begin{tabular}{|c|c|c|}
\hline $\mathrm{TD}=72.0$ & $E T=1$ & $07 \mathrm{MEY}$ \\
\hline ENERGY (YEV) & PRIMARY & CASCADE \\
\hline 1.47000 & 0.05 & 0.06 \\
\hline 1.49300 & 0.11 & 0.11 \\
\hline 1.51300 & 0.33 & 0.33 \\
\hline 1.56300 & 0.84 & 0.84 \\
\hline 1.61000 & 1.49 & 1.49 \\
\hline 1.69000 & 2.74 & 2.74 \\
\hline 1.77300 & 4.19 & 4.19 \\
\hline 1.89300 & 6.54 & 6.54 \\
\hline 2.04000 & 9.60 & 9.60 \\
\hline 2.26000 & 13.98 & 13.98 \\
\hline 2.55300 & 19.20 & 19.30 \\
\hline 2.91000 & 24.62 & 25.23 \\
\hline 3.35000 & 29.79 & 31.62 \\
\hline 3.93000 & 34.69 & 38.88 \\
\hline 4.56000 & 38.76 & 46.59 \\
\hline 5.54000 & 41.74 & 54.35 \\
\hline 6.56000 & 43.70 & 61.85 \\
\hline 7.58300 & 44.76 & 58.19 \\
\hline 8.74000 & 45.36 & 74.37 \\
\hline 10.20000 & 45.64 & 81.01 \\
\hline 12.30300 & 45.60 & 38.97 \\
\hline 14.50000 & 45.33 & 95.88 \\
\hline 21.80300 & 44.27 & 112.81 \\
\hline 29.10000 & 43.45 & 124.65 \\
\hline 43.70000 & 42.37 & 141.11 \\
\hline 72.90300 & 41.35 & 161.68 \\
\hline 145.00000 & 40.63 & 189.28 \\
\hline 291.00500 & 40.24 & 217.14 \\
\hline
\end{tabular}


ATOMIC DISPLAZEMENT CROSS-SECTIONS (BARNS) BY FAST ELECTRONS IN AG

\begin{tabular}{|c|c|c|}
\hline$r D=76.0$ & $\mathrm{BT}=1$. & $580 \mathrm{MEV}$ \\
\hline ENERGY (MEV) & PRIMARY & CASCADE \\
\hline 1.52000 & 0.05 & $\begin{array}{l}0.05 \\
0.05\end{array}$ \\
\hline 1.53000 & 0.10 & 0.10 \\
\hline 1.56000 & 0.29 & 0.29 \\
\hline 1.51000 & 0.74 & 0.74 \\
\hline 1.67000 & 1.45 & 1.45 \\
\hline 1.74000 & 2.46 & 2.46 \\
\hline 1.84000 & 4.11 & 4.11 \\
\hline 1.96000 & 6.29 & 6.29 \\
\hline 2.11000 & 9.10 & 9.10 \\
\hline 2.33100 & 13.14 & 13.14 \\
\hline 2.64000 & 18.28 & 18.37 \\
\hline 3.01000 & 23.39 & 23.98 \\
\hline 3.46000 & 28.24 & 29.99 \\
\hline 4.07000 & 32.97 & 37.01 \\
\hline 4.82000 & 36.78 & 44.28 \\
\hline 5.73000 & 39.59 & 51.66 \\
\hline 6.78000 & 41.42 & 58.75 \\
\hline 7.84000 & 42.42 & 64.79 \\
\hline 9.05000 & 42.98 & 70.70 \\
\hline 10.50000 & 43.23 & 76.76 \\
\hline 12.80000 & 43.18 & 84.75 \\
\hline 15.00000 & 42.93 & 91.06 \\
\hline 22.60000 & 41.92 & 107.20 \\
\hline 30.10000 & 41.15 & 118.33 \\
\hline 45.20000 & 40.12 & 133.93 \\
\hline 75.40000 & 39.16 & 153.42 \\
\hline 150.00000 & 38.48 & 179.58 \\
\hline
\end{tabular}

\begin{tabular}{ccr} 
TD=80.0 EV & \multicolumn{2}{c}{ ET=1.557726 MEV } \\
ENERGY (MEV) & PRIMARY & CASEADE \\
1.57000 & 0.05 & 0.05 \\
1.58000 & 0.09 & 0.09 \\
1.62000 & 0.33 & 0.33 \\
1.66000 & 0.67 & 0.67 \\
1.72000 & 1.32 & 1.32 \\
1.80000 & 2.37 & 2.37 \\
1.90000 & 3.90 & 3.90 \\
2.02000 & 5.91 & 5.91 \\
2.18000 & 8.68 & 8.68 \\
2.41000 & 12.58 & 12.58 \\
2.72000 & 17.33 & 17.42 \\
3.11000 & 22.30 & 22.88 \\
3.58000 & 26.96 & 28.67 \\
4.20000 & 31.37 & 35.25 \\
4.98000 & 35.00 & 42.23 \\
5.91000 & 37.54 & 49.18 \\
7.00000 & 39.38 & 55.97 \\
8.10000 & 40.32 & 61.76 \\
9.34000 & 40.84 & 67.34 \\
10.90000 & 41.07 & 73.33 \\
13.20000 & 41.02 & 80.66 \\
15.50000 & 40.77 & 86.74 \\
23.30000 & 39.81 & 101.99 \\
31.10000 & 39.07 & 112.65 \\
45.70000 & 38.10 & 127.47 \\
77.80000 & 37.20 & 145.95 \\
155.00000 & 36.55 & 170.85
\end{tabular}

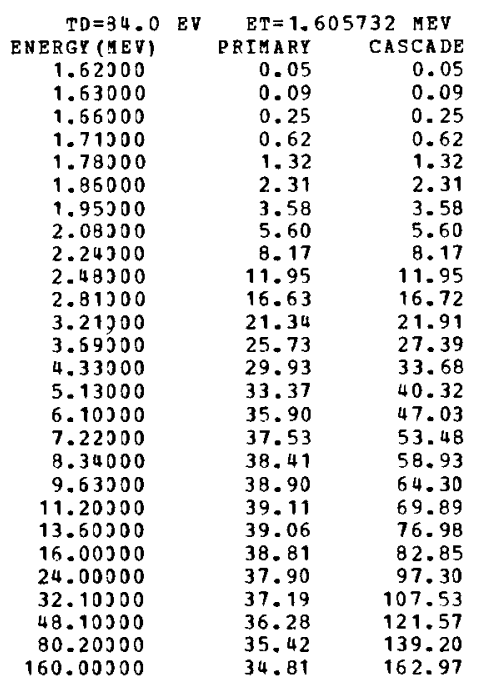

ATOMIC DISPLAZEMENT CROSS-SECTIONS (BARNS) BY FAST BLECTRONS IN AS

\begin{tabular}{rrr} 
TD $=38.0$ & EV & \multicolumn{2}{c}{ ET $=1.652672$ MEV } \\
PRIHARY & CASCADE \\
1.66000 & 0.02 & 0.02 \\
1.63000 & 0.09 & 0.09 \\
1.71000 & 0.24 & 0.24 \\
1.76000 & 0.59 & 0.59 \\
1.83000 & 1.23 & 1.23 \\
1.91000 & 2.14 & 2.14 \\
2.01000 & 3.46 & 3.46 \\
2.14000 & 5.33 & 5.33 \\
2.31000 & 7.88 & 7.88 \\
2.56000 & 11.54 & 11.54 \\
2.89000 & 15.89 & 15.98 \\
3.30000 & 20.38 & 20.93 \\
3.80000 & 24.63 & 26.24 \\
4.46000 & 28.64 & 32.27 \\
5.28000 & 31.90 & 38.60 \\
6.28000 & 34.30 & 45.02 \\
7.43000 & 35.85 & 51.18 \\
8.59000 & 36.68 & 56.42 \\
9.91000 & 37.14 & 61.52 \\
11.50000 & 37.33 & 66.78 \\
14.00000 & 37.27 & 73.65 \\
16.50000 & 37.04 & 79.31 \\
24.70000 & 36.17 & 93.04 \\
33.00000 & 35.49 & 102.77 \\
49.50000 & 34.62 & 116.22 \\
82.60000 & 33.80 & 133.07 \\
165.00000 & 33.22 & 155.80 \\
& &
\end{tabular}

\begin{tabular}{rrr} 
TD $=92.0$ & EV & \multicolumn{2}{c}{ ET=1.698615 YEV } \\
BNERGY (HEV) & PRIHAY & CASEADE \\
1.71000 & 0.03 & 0.03 \\
1.73000 & 0.10 & 0.10 \\
1.76000 & 0.24 & 0.24 \\
1.81000 & 0.56 & 0.56 \\
1.88000 & 1.16 & 1.16 \\
1.97000 & 2.13 & 2.13 \\
2.07000 & 3.36 & 3.36 \\
2.20000 & 5.11 & 5.11 \\
2.37000 & 7.49 & 7.49 \\
2.63000 & 11.04 & 11.05 \\
2.97000 & 15.23 & 15.32 \\
3.39000 & 19.53 & 20.06 \\
3.90000 & 23.57 & 25.12 \\
4.58000 & 27.43 & 30.93 \\
5.43000 & 30.56 & 37.04 \\
6.45000 & 32.83 & 43.15 \\
7.64000 & 34.31 & 49.09 \\
8.83000 & 35.09 & 54.10 \\
10.10000 & 35.51 & 58.69 \\
11.80000 & 35.71 & 63.96 \\
14.40000 & 35.55 & 70.61 \\
16.90000 & 35.42 & 75.89 \\
25.40000 & 34.58 & 89.17 \\
33.90000 & 33.94 & 98.44 \\
50.90000 & 33.10 & 111.34 \\
84.90000 & 32.32 & 127.45 \\
169.00000 & 31.78 & 149.08
\end{tabular}

\begin{tabular}{|c|c|c|}
\hline$T D=96.0$ & $\mathrm{ET}=1$ & $524 \mathrm{MEV}$ \\
\hline ENERGY (MEV) & PRIMARY & CASCADE \\
\hline 1.76000 & 0.04 & 0.04 \\
\hline 1.77000 & 0.07 & 0.07 \\
\hline 1.81300 & 0.24 & 0.24 \\
\hline 1.86300 & 0.55 & 0.55 \\
\hline 1.93000 & 1.11 & 1.11 \\
\hline 2.02000 & 2.01 & 2.01 \\
\hline 2.12300 & 3.16 & 3.16 \\
\hline 2.26000 & 4.93 & 4.93 \\
\hline 2.44000 & 7.29 & 7.29 \\
\hline 2.70000 & 10.61 & 10.61 \\
\hline 3.05000 & 14.64 & 14.74 \\
\hline 3.48000 & 18.76 & 19.28 \\
\hline 4.01000 & 22.68 & 24.20 \\
\hline 4.70000 & 26.32 & 29.71 \\
\hline 5.57000 & 29.32 & 35.56 \\
\hline 6.62300 & 31.49 & 41.45 \\
\hline 7.84000 & 32.90 & 47.14 \\
\hline 9.06300 & 33.64 & 51.95 \\
\hline 10.40300 & 34.04 & 56.47 \\
\hline 12.20300 & 34.22 & 61.65 \\
\hline 14.80000 & 34.15 & 67.84 \\
\hline 17.40000 & 33.93 & 72.96 \\
\hline 26.10300 & 33.13 & 85.62 \\
\hline 34.80000 & 32.52 & 94.48 \\
\hline 52.30300 & 31.71 & 106.87 \\
\hline 87.10000 & 30.97 & 122.25 \\
\hline 174.00000 & 30.45 & 143.11 \\
\hline
\end{tabular}


ATOMIC DISPLAZEHENT CROSS-SECTIONS (BARNS) BI FAST ELEZTRONS IN SN

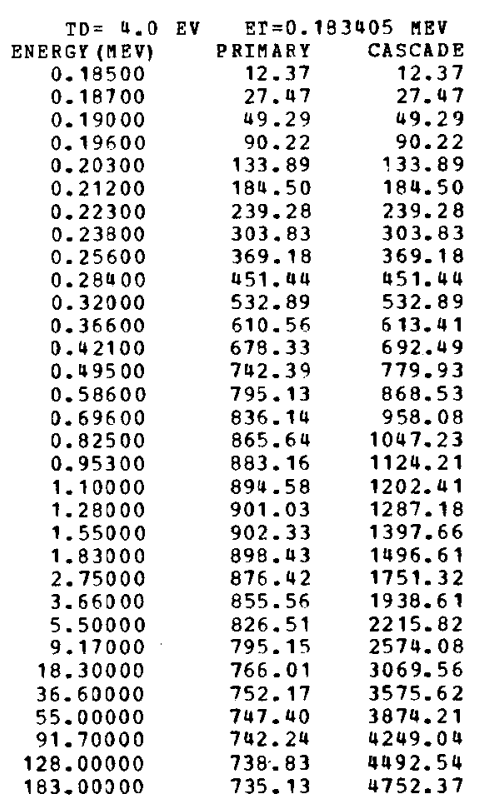

\begin{tabular}{rrr}
$T D=8.0$ & EV & \multicolumn{1}{c}{ ET $=0.327616$} \\
PNERGY $(4 E V)$ & PRIMA & CASEADE \\
0.33000 & 2.79 & 2.79 \\
0.33400 & 7.42 & 7.42 \\
0.34000 & 14.27 & 14.27 \\
0.35000 & 25.41 & 25.41 \\
0.36300 & 39.43 & 39.43 \\
0.38000 & 56.97 & 56.97 \\
0.39900 & 75.59 & 75.59 \\
0.42500 & 99.46 & 99.46 \\
0.45800 & 127.30 & 127.30 \\
0.50700 & 164.05 & 164.05 \\
0.57300 & 206.12 & 206.16 \\
0.65500 & 248.68 & 250.71 \\
0.75300 & 288.64 & 296.55 \\
0.88400 & 328.51 & 348.88 \\
1.04000 & 362.00 & 401.81 \\
1.24000 & 390.52 & 459.31 \\
1.47000 & 410.95 & 515.38 \\
1.70000 & 423.37 & 563.72 \\
1.96000 & 431.59 & 611.49 \\
2.29000 & 436.80 & 664.26 \\
2.78000 & 438.77 & 730.73 \\
3.27000 & 437.57 & 787.02 \\
4.91000 & 427.52 & 929.92 \\
6.55000 & 418.19 & 1032.78 \\
9.82000 & 405.42 & 1178.90 \\
16.30000 & 391.71 & 1362.59 \\
32.70000 & 379.99 & 1617.12 \\
55.50000 & 374.63 & 1872.79 \\
98.20000 & 372.52 & 2021.78 \\
163.00000 & 369.96 & 2207.56 \\
229.00000 & 368.20 & 2331.43 \\
327.00000 & 366.38 & 2460.76
\end{tabular}

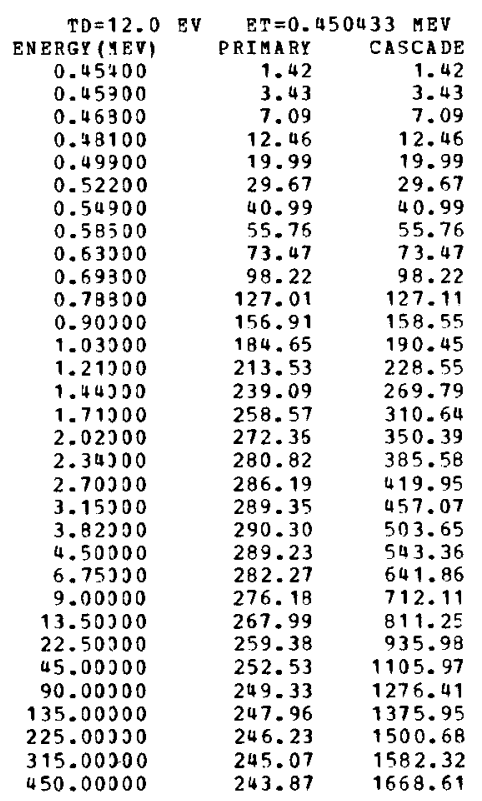

ATOMIC DISPLAZEHENT CROSS-SECTIONS (BARNS) BY FAST ELEZTRONS IN SN

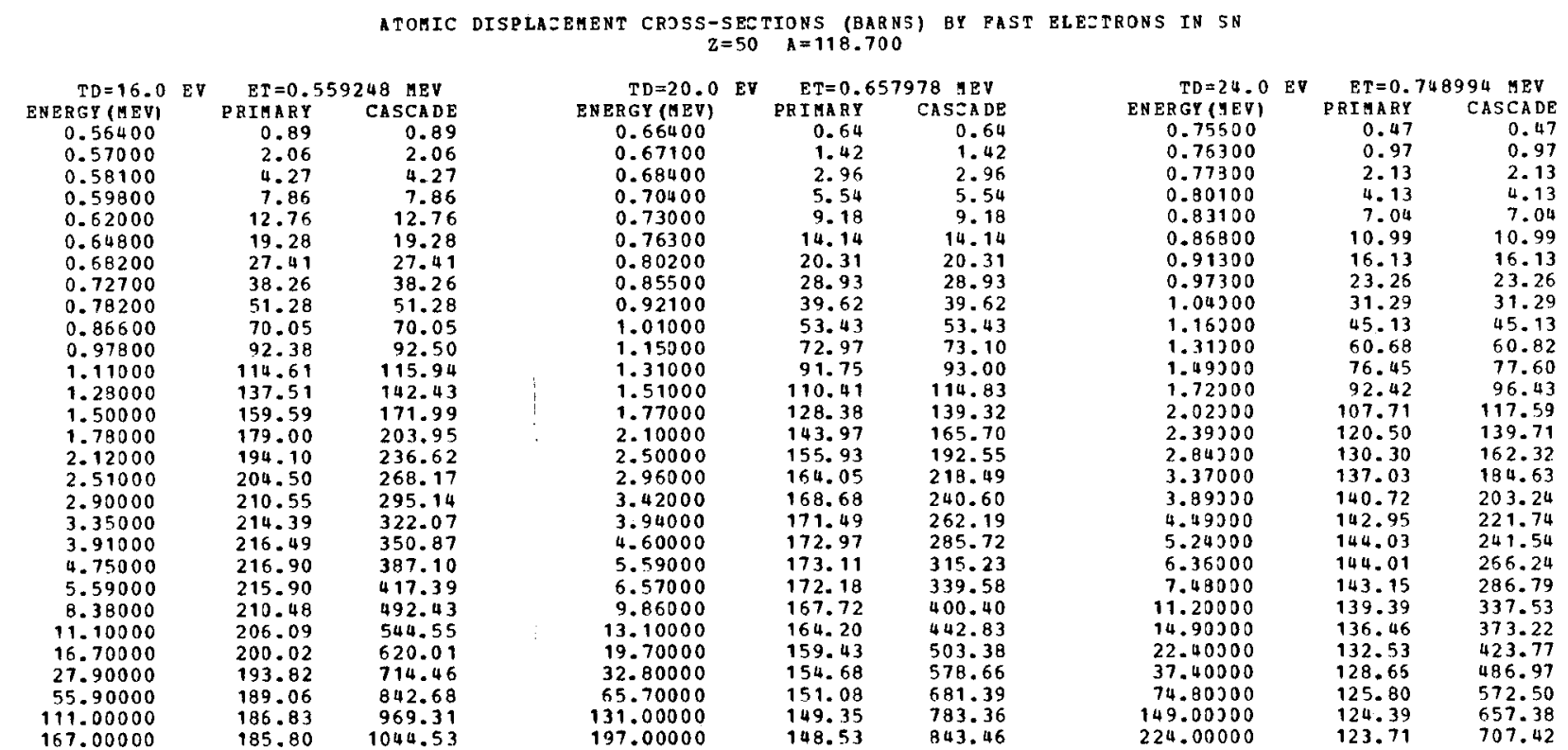


ATOMIC DISPLAzEMENT CROSS-SEETIONS (BARNS) BY PAST ELECTRONS IN SN

\begin{tabular}{rrr} 
TD=28.0 & \multicolumn{2}{c}{ EV $=0.833866$ REV } \\
PNERGY (MEV) & PRIMARY & CASCADE \\
0.84200 & 0.37 & 0.37 \\
0.85000 & 0.77 & 0.77 \\
0.86700 & 1.70 & 1.70 \\
0.89200 & 3.28 & 3.28 \\
0.92500 & 5.65 & 5.65 \\
0.96700 & 9.04 & 9.04 \\
1.01000 & 12.78 & 12.78 \\
1.08000 & 19.20 & 19.20 \\
1.16000 & 26.65 & 26.55 \\
1.29000 & 38.36 & 38.36 \\
1.45000 & 51.39 & 51.51 \\
1.56000 & 65.82 & 66.90 \\
1.91000 & 79.37 & 82.98 \\
2.25090 & 92.82 & 101.80 \\
2.66000 & 103.72 & 121.00 \\
3.15000 & 112.03 & 140.65 \\
3.75000 & 117.69 & 160.04 \\
4.33000 & 120.75 & 176.22 \\
5.00000 & 122.56 & 192.29 \\
5.83000 & 123.39 & 209.31 \\
7.08000 & 123.27 & 230.71 \\
8.33000 & 122.47 & 248.47 \\
12.50000 & 119.18 & 292.40 \\
16.60000 & 115.70 & 322.90 \\
25.00000 & 113.36 & 366.50 \\
41.60000 & 110.13 & 420.39 \\
83.30000 & 107.76 & 493.89 \\
166.00000 & 106.58 & 566.70
\end{tabular}

\begin{tabular}{crr}
$T D=32.0$ & EV & \multicolumn{2}{c}{ ET=0.913690 MEV } \\
ENERGY (MEV) & PRIMARY & CASCADE \\
0.92200 & 0.27 & 0.27 \\
0.93100 & 0.59 & 0.59 \\
0.95000 & 1.37 & 1.37 \\
0.97700 & 2.57 & 2.67 \\
1.01000 & 4.50 & 4.50 \\
1.05000 & 7.00 & 7.00 \\
1.11000 & 11.14 & 11.14 \\
1.18000 & 16.29 & 16.29 \\
1.27000 & 23.05 & 23.05 \\
1.41000 & 33.26 & 33.26 \\
1.59000 & 45.13 & 45.26 \\
1.82000 & 57.89 & 58.92 \\
2.10000 & 70.06 & 73.49 \\
2.45000 & 81.47 & 89.63 \\
2.92000 & 91.22 & 107.06 \\
3.47000 & 98.40 & 124.49 \\
4.11000 & 103.18 & 141.45 \\
4.75000 & 105.78 & 155.82 \\
5.48000 & 107.26 & 169.90 \\
6.39000 & 107.92 & 184.90 \\
7.76000 & 107.74 & 203.73 \\
9.13000 & 107.00 & 219.34 \\
13.70000 & 104.10 & 257.92 \\
18.20000 & 101.93 & 284.70 \\
27.40000 & 99.03 & 322.86 \\
45.50000 & 96.26 & 370.08 \\
91.30000 & 94.24 & 434.42 \\
182.00000 & 93.23 & 498.18
\end{tabular}

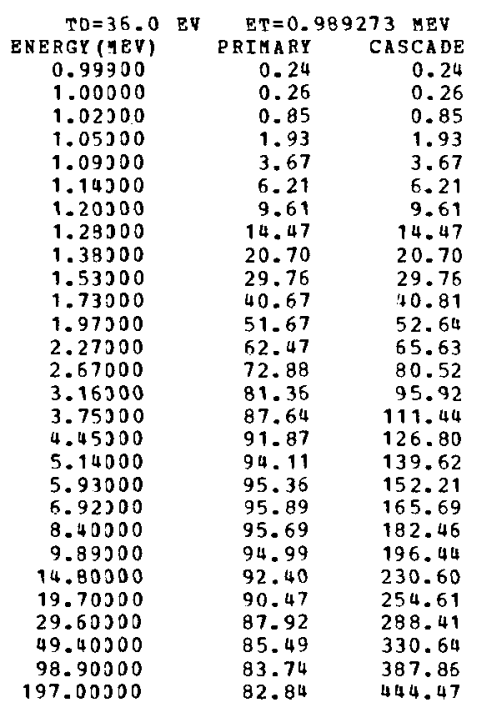

ATOMIC DISPLAZEMENT CROSS-SECTIONS (BARNS) BY PAST ELECTRONS IN SN

\begin{tabular}{|c|c|c|}
\hline$T D=40.0$ & $E T=1$ & 226 MEV \\
\hline ENERGY (MEV) & PRIMARY & CASCADE \\
\hline $1.07000^{\circ}$ & 0.16 & 0.16 \\
\hline 1.08000 & 0.37 & 0.37 \\
\hline 1.10000 & 0.87 & 0.87 \\
\hline 1.13000 & 1.77 & 1.77 \\
\hline 1.17000 & 3.22 & 3.22 \\
\hline 1.23000 & 5.79 & 5.79 \\
\hline 1.29000 & 8.67 & 8.67 \\
\hline 1.37000 & 12.77 & 12.77 \\
\hline 1.48000 & 18.56 & 18.56 \\
\hline 1.64000 & 26.76 & 26.76 \\
\hline 1.85000 & 36.49 & 36.61 \\
\hline 2.12000 & 46.95 & 47.90 \\
\hline 2.44000 & 56.61 & 59.62 \\
\hline 2.86000 & 65.79 & 72.87 \\
\hline 3.39000 & 73.47 & 86.97 \\
\hline 4.03000 & 79.11 & 101.19 \\
\hline 4.77000 & 82.79 & 114.90 \\
\hline 5.51000 & 84.76 & 126.51 \\
\hline 6.36000 & 85.84 & 137.94 \\
\hline 7.42000 & 86.28 & 150.10 \\
\hline 9.02000 & 86.05 & 165.36 \\
\hline 10.60000 & 85.40 & 177.84 \\
\hline 15.90000 & 83.05 & 208.83 \\
\hline 21.20300 & 81.30 & 230.59 \\
\hline 31.80000 & 79.03 & 260.91 \\
\hline 53.00000 & 76.88 & 298.85 \\
\hline 106.00000 & 75.34 & 350.29 \\
\hline 212.00000 & 74.54 & 401.54 \\
\hline
\end{tabular}

\begin{tabular}{crr} 
TD $=44.0$ & \multicolumn{2}{c}{ ET $=1.13002 .8$ MEV } \\
ENERGY (HEV) & PRIMARY & CASTADE \\
1.14000 & 0.15 & 0.15 \\
1.15000 & 0.32 & 0.32 \\
1.17000 & 0.72 & 0.72 \\
1.20300 & 1.46 & 1.46 \\
1.25000 & 2.99 & 2.99 \\
1.31000 & 5.19 & 5.19 \\
1.37000 & 7.64 & 7.64 \\
1.46000 & 11.60 & 11.60 \\
1.58000 & 17.04 & 17.04 \\
1.75000 & 24.54 & 24.54 \\
1.97000 & 33.30 & 33.42 \\
2.26000 & 42.95 & 43.87 \\
2.59000 & 51.50 & 54.29 \\
3.05000 & 60.11 & 66.79 \\
3.61000 & 66.99 & 79.60 \\
4.29000 & 72.06 & 92.59 \\
5.08000 & 75.36 & 105.15 \\
5.87000 & 77.11 & 115.78 \\
6.78000 & 78.06 & 126.26 \\
7.91000 & 78.41 & 137.35 \\
9.60000 & 78.17 & 151.15 \\
11.30000 & 77.56 & 162.63 \\
16.90000 & 75.43 & 190.65 \\
22.60000 & 73.83 & 210.66 \\
33.90000 & 71.78 & 238.24 \\
56.50000 & 69.84 & 272.75 \\
113.00000 & 68.47 & 319.53 \\
226.00000 & 67.75 & 365.13
\end{tabular}

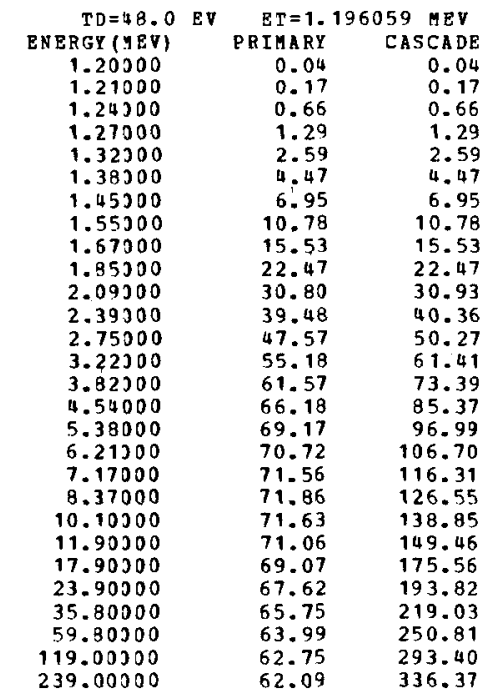


ATOMIC DISPLAZEMENT CROSS-SETTIONS (BARNS) BY PAST ELECTRONS IN SN

$z=50 \quad A=118.700$

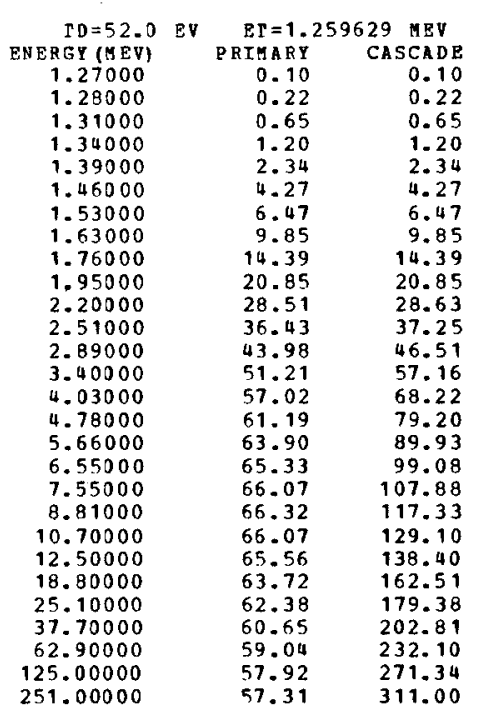

$\begin{array}{ccc}\text { TD }=56.0 & \text { EV } & \text { ET }=1.320995 \text { MEV } \\ \text { ENERGY (MEV) } & \text { PRIMARY } & \text { CASEADE } \\ 1.33000 & 0.07 & 0.07 \\ 1.34000 & 0.17 & 0.17 \\ 1.37000 & 0.53 & 0.53 \\ 1.47000 & 1.17 & 1.17 \\ 1.46000 & 2.19 & 2.19 \\ 1.53000 & 3.90 & 3.90 \\ 1.61000 & 6.14 & 6.14 \\ 1.71000 & 9.15 & 9.15 \\ 1.84000 & 13.20 & 13.20 \\ 2.04000 & 19.27 & 19.27 \\ 2.31000 & 26.64 & 2.6 .77 \\ 2.54000 & 34.11 & 34.92 \\ 3.03000 & 40.97 & 43.38 \\ 3.56000 & 47.63 & 53.25 \\ 4.22000 & 53.03 & 63.58 \\ 5.01000 & 56.90 & 73.87 \\ 5.94000 & 59.40 & 83.94 \\ 6.86000 & 60.69 & 92.34 \\ 7.92000 & 61.35 & 100.62 \\ 9.24000 & 61.57 & 109.41 \\ 11.20000 & 61.32 & 120.25 \\ 13.20000 & 60.81 & 129.38 \\ 19.80000 & 59.10 & 151.65 \\ 26.40000 & 57.86 & 167.24 \\ 39.60000 & 56.27 & 188.94 \\ 66.00000 & 54.80 & 216.09 \\ 132.00000 & 53.76 & 252.87 \\ 264.00000 & 53.21 & 289.49\end{array}$

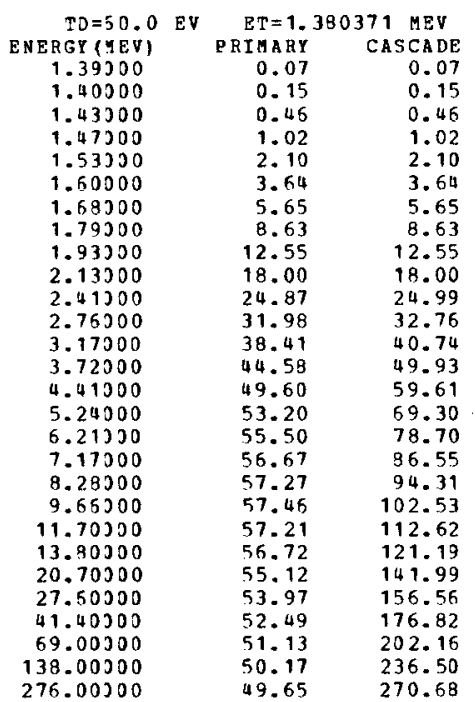

ATOMIC DISPLAZEMENT CROSS-SEETIONS (BARNS) BY PAST ELECTRONS IN SN $Z=50 \quad A=118.700$

\begin{tabular}{rrr} 
TD=64.0 EV & \multicolumn{2}{c}{ ET=1.437938 MEV } \\
ENERGY(MEV) & PRIMARY & CASCADE \\
1.45000 & 0.07 & 0.07 \\
1.46000 & 0.14 & 0.14 \\
1.49000 & 0.42 & 0.42 \\
1.53000 & 0.92 & 0.92 \\
1.59000 & 1.88 & 1.88 \\
1.66000 & 3.25 & 3.25 \\
1.75000 & 5.28 & 5.28 \\
1.86000 & 7.97 & 7.97 \\
2.01000 & 11.77 & 11.77 \\
2.22000 & 16.95 & 16.95 \\
2.51000 & 23.38 & 23.50 \\
2.87000 & 30.00 & 30.74 \\
3.30000 & 36.10 & $\mathbf{3 8 . 3 2}$ \\
3.88000 & 41.94 & 47.07 \\
4.60000 & 46.62 & 56.19 \\
5.46000 & 49.94 & 65.25 \\
6.47000 & 52.07 & 74.08 \\
7.47000 & 53.15 & 81.45 \\
8.62000 & 53.70 & 88.71 \\
10.00000 & 53.86 & 96.14 \\
12.20000 & 53.61 & 105.97 \\
14.30000 & 53.17 & 113.71 \\
21.50000 & 51.66 & 133.35 \\
28.70000 & 50.57 & 147.06 \\
43.10000 & 49.19 & 165.12 \\
71.80000 & 47.92 & 189.87 \\
143.00300 & 47.03 & 221.87 \\
287.00000 & 46.55 & 254.07
\end{tabular}

\begin{tabular}{rrr} 
TD $=68.0$ & EV & \multicolumn{2}{c}{ ET=1.493854 MEV } \\
ENERGY (HEV) & PRIMAR & CASEADE \\
1.50000 & 0.03 & 0.03 \\
1.52000 & 0.15 & 0.15 \\
1.55000 & 0.41 & 0.41 \\
1.59000 & 0.85 & 0.85 \\
1.65000 & 1.72 & 1.72 \\
1.73000 & 3.15 & 3.15 \\
1.82000 & 5.00 & 5.00 \\
1.94000 & 7.68 & 7.68 \\
2.09000 & 11.14 & 11.14 \\
2.31000 & 16.08 & 16.08 \\
2.61000 & 22.13 & 22.24 \\
2.98000 & 28.30 & 29.02 \\
3.43000 & 34.09 & 36.23 \\
4.03000 & 39.56 & 44.47 \\
4.78000 & 43.96 & 53.10 \\
5.67000 & 47.05 & 61.62 \\
6.72000 & 49.04 & 69.96 \\
7.76000 & 50.04 & 76.92 \\
8.96000 & 50.54 & 83.79 \\
10.40000 & 50.68 & 90.82 \\
12.60000 & 50.45 & 99.76 \\
14.90000 & 50.01 & 107.46 \\
22.40000 & 48.58 & 125.95 \\
29.80000 & 47.57 & 138.71 \\
44.80000 & 46.27 & 156.70 \\
74.50000 & 45.08 & 179.03 \\
149.00000 & 44.26 & 209.28 \\
298.00000 & 43.80 & 239.44
\end{tabular}

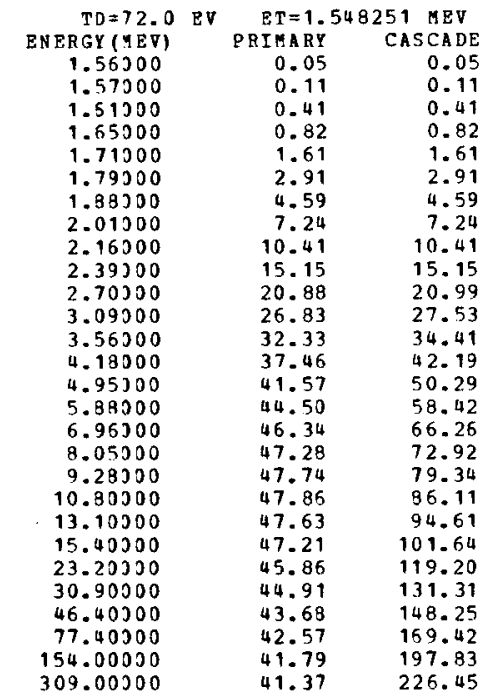


ATOMIC DISPLAZEHENT CROSS-SBCTIONS (BARNS) BY FAST ELECTRONS IN SN $z=50 \quad A=118.700$

\begin{tabular}{rrr} 
TD=76.0 EV & \multicolumn{2}{c}{ ET=1.601248 MEV } \\
ENERG (MEV) & PRIMARY & CASCADE \\
1.51000 & 0.03 & 0.03 \\
1.63000 & 0.13 & 0.13 \\
1.56000 & 0.33 & 0.33 \\
1.71000 & 0.80 & 0.80 \\
1.77000 & 1.53 & 1.53 \\
1.85000 & 2.72 & 2.72 \\
1.95000 & 4.45 & 4.45 \\
2.08000 & 6.89 & 6.89 \\
2.24000 & 10.00 & 10.00 \\
2.48000 & 14.54 & 14.54 \\
2.80000 & 19.95 & 20.07 \\
3.20000 & 25.54 & 26.23 \\
3.68000 & 30.69 & 32.69 \\
4.32000 & 35.54 & 40.07 \\
5.12000 & 39.44 & 47.81 \\
5.08000 & 42.20 & 55.52 \\
7.20000 & 43.93 & 62.98 \\
8.32000 & 44.80 & 69.26 \\
9.60000 & 45.23 & 75.39 \\
11.20000 & 45.33 & 81.91 \\
13.60000 & 45.10 & 90.02 \\
16.00000 & 44.70 & 96.71 \\
24.00000 & 43.43 & 113.17 \\
32.00000 & 42.52 & 124.69 \\
48.00000 & 41.36 & 140.71 \\
80.00000 & 40.32 & 160.73 \\
160.00000 & 39.59 & 187.86
\end{tabular}

\begin{tabular}{rrr} 
TD=80.0 & EV & \multicolumn{2}{c}{ ET $=1.652948$ IEV } \\
ENERGY (HEV) & PRIMARY & CASCADE \\
1.66000 & 0.02 & 0.02 \\
1.68000 & 0.11 & 0.11 \\
1.71000 & 0.29 & 0.29 \\
1.76000 & 0.70 & 0.70 \\
1.83000 & 1.48 & 1.48 \\
1.91000 & 2.58 & 2.58 \\
2.01000 & 4.17 & 4.17 \\
2.14000 & 6.43 & 6.43 \\
2.31000 & 9.48 & 9.48 \\
2.56000 & 13.85 & 13.85 \\
2.89000 & 19.01 & 19.12 \\
3.30000 & 24.29 & 24.95 \\
3.80000 & 29.24 & 31.17 \\
4.46000 & 33.83 & 33.20 \\
5.28000 & 37.50 & 45.52 \\
6.28000 & 40.13 & 52.92 \\
7.43000 & 41.76 & 59.98 \\
8.59000 & 42.57 & 65.98 \\
9.91000 & 42.97 & 71.80 \\
11.50000 & 43.05 & 77.80 \\
14.00000 & 42.84 & 85.60 \\
16.50000 & 42.45 & 92.04 \\
24.70000 & 41.25 & 107.61 \\
33.00000 & 40.38 & 118.63 \\
49.50000 & 39.28 & 133.85 \\
82.60000 & 38.29 & 152.93 \\
165.00000 & 37.60 & 178.65
\end{tabular}

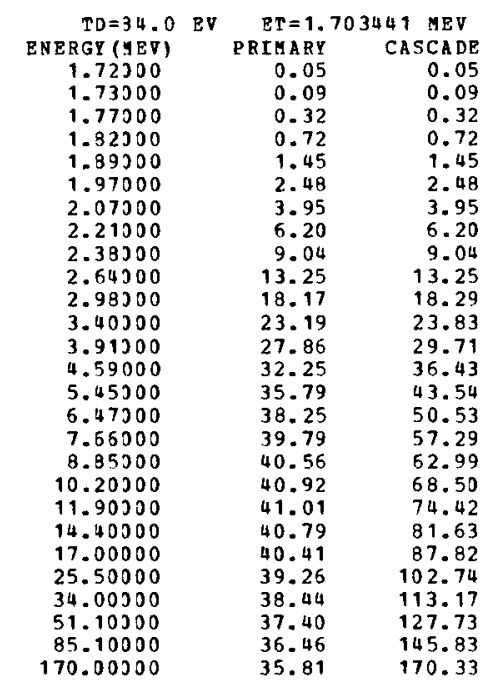

ATOMIC DISPLAZEMENT CROSS-SECTIONS (BARNS) BY PAST BLECTBONS IN $5 N$ $=50 \quad A=118.700$

\begin{tabular}{|c|c|c|c|}
\hline$T D=88.0$ & EV & $\mathrm{Er}=1.7$ & AEV \\
\hline RG I (MEV) & & PRIMARY & CASCADE \\
\hline 1.77000 & & 0.05 & 0.05 \\
\hline 1.78000 & & 0.09 & 0.09 \\
\hline 1.82000 & & 0.29 & 0.29 \\
\hline 1.87000 & & 0.65 & 0.65 \\
\hline 1.94000 & & 1.33 & 1.33 \\
\hline 2.03000 & & 2.40 & 2.40 \\
\hline 2.13000 & & 3.77 & 3.77 \\
\hline 2.27000 & & 5.87 & 5.87 \\
\hline 2.45000 & & 8.66 & 8.66 \\
\hline 2.71000 & & 12.58 & 12.59 \\
\hline 3.06000 & & 17.31 & 17.42 \\
\hline 3.50000 & & 22.20 & 22.83 \\
\hline 4.03000 & & 26.70 & 28.51 \\
\hline 4.73000 & & 30.87 & 34.93 \\
\hline 5.60000 & & 34.18 & 41.62 \\
\hline 6.66000 & & 36.55 & 48.38 \\
\hline .88000 & & 38.00 & 54.82 \\
\hline 9.11000 & & 38.72 & 60.28 \\
\hline 10.50000 & & 39.07 & 65.55 \\
\hline 12.20000 & & 39.14 & 71.05 \\
\hline 14.80000 & & 38.93 & 78.04 \\
\hline 17.50000 & & 38.56 & 84.01 \\
\hline 26.20000 & & 37.47 & $\begin{array}{r}98.19 \\
\end{array}$ \\
\hline 35.00000 & & 36.68 & 108.21 \\
\hline 52.50000 & & 35.69 & 122.05 \\
\hline 0 & & & \\
\hline
\end{tabular}

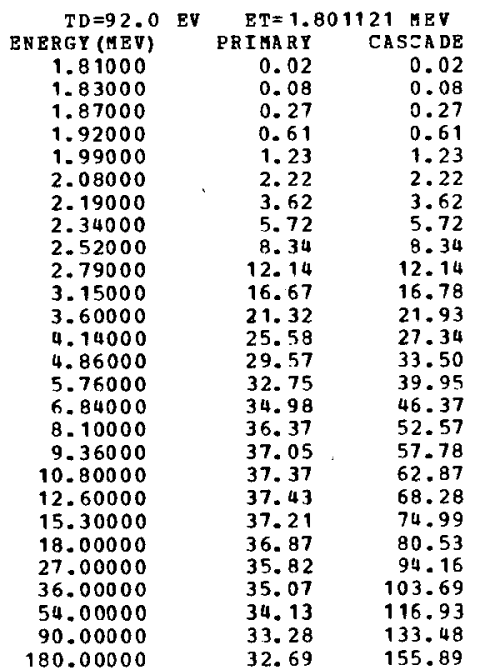

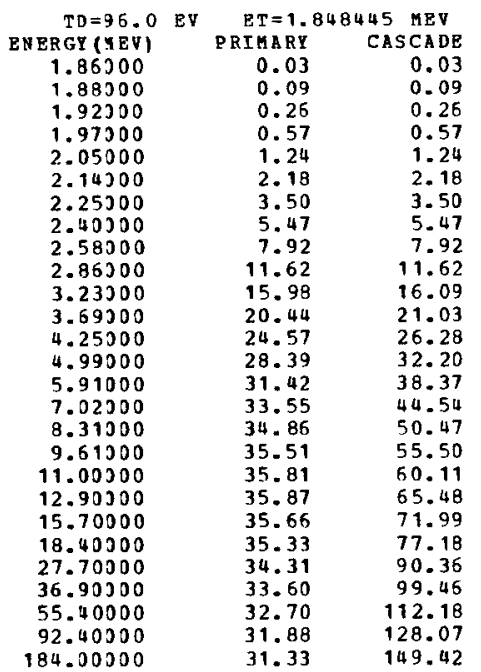


ATOHIC DISPLAZEMENT CROSS-SECTIONS (BARNS) BY PAST ELECTRONS IN CS

\begin{tabular}{rrr}
$T D=4.0$ & EV & \multicolumn{1}{c}{ ET $=0.202207$ MEV } \\
PNERGY (MEV) & PRIMARY & CASCADE \\
0.20400 & 13.41 & 13.41 \\
0.20600 & 28.00 & 28.00 \\
0.21000 & 56.14 & 56.14 \\
0.21600 & 95.88 & 95.88 \\
0.22400 & 144.78 & 144.78 \\
0.23400 & 200.24 & 200.24 \\
0.24600 & 259.79 & 259.79 \\
0.26200 & 329.43 & 329.43 \\
0.28300 & 407.41 & 407.41 \\
0.31300 & 498.83 & 498.83 \\
0.35300 & 594.43 & 594.43 \\
0.40400 & 686.39 & 590.02 \\
0.46500 & 767.05 & 784.14 \\
0.54500 & 841.44 & 885.74 \\
0.64700 & 904.14 & 992.20 \\
0.76800 & 950.50 & 1097.13 \\
0.90900 & 982.11 & 1200.34 \\
1.05000 & 999.61 & 1289.58 \\
1.29000 & 1009.36 & 1378.60 \\
1.41000 & 1012.92 & 1476.43 \\
1.71000 & 1009.06 & 1602.77 \\
2.02000 & 999.99 & 1714.60 \\
3.03000 & 965.28 & 1997.24 \\
4.04000 & 936.39 & 2205.31 \\
6.06000 & 899.29 & 2508.00 \\
10.10000 & 861.13 & 2897.90 \\
20.20000 & 827.61 & 3437.25 \\
40.40000 & 812.37 & 3985.08 \\
60.60000 & 806.73 & 4306.07 \\
101.00000 & 800.06 & 4709.69 \\
141.00000 & $\mathbf{7 9 5 . 5 3}$ & 4971.62 \\
202.00000 & 790.70 & 5252.39 \\
& &
\end{tabular}

$Z=55 \quad A=132.910$

\begin{tabular}{rrr} 
TD $=8.0$ & EV & \multicolumn{2}{c}{ ET $=0.358602$ MEV } \\
ENRRGY (MEV) & PRIMAYY & CASEADE \\
0.36200 & 3.87 & 3.87 \\
0.36500 & 7.27 & 7.27 \\
0.37200 & 15.15 & 15.16 \\
0.38300 & 27.41 & 27.41 \\
0.39800 & 43.78 & 43.78 \\
0.41500 & 61.82 & 61.82 \\
0.43700 & 84.32 & 84.32 \\
0.46600 & 112.44 & 112.44 \\
0.50200 & 144.86 & 144.86 \\
0.55500 & 187.74 & 187.74 \\
0.62700 & 237.53 & 237.61 \\
0.71700 & 288.11 & 290.66 \\
0.82400 & 334.92 & 344.67 \\
0.96800 & 381.12 & 406.29 \\
1.14000 & 418.93 & 468.23 \\
1.36000 & 449.73 & 534.70 \\
1.61000 & 470.30 & 598.22 \\
1.86000 & 481.82 & 652.61 \\
2.15000 & 488.62 & 707.38 \\
2.51000 & 491.52 & 766.13 \\
3.04000 & 490.27 & 839.15 \\
3.58000 & 486.24 & 901.86 \\
5.37000 & 469.81 & 1058.40 \\
7.17000 & 456.80 & 1171.12 \\
10.70000 & 440.64 & 1328.26 \\
17.90000 & 423.83 & 1530.50 \\
35.80000 & 410.61 & 1804.90 \\
71.70000 & 404.55 & 2081.16 \\
107.00000 & 401.94 & 2240.02 \\
179.00000 & 398.51 & 2443.04 \\
251.00000 & 396.21 & 2575.39 \\
358.00000 & 393.93 & 2713.87
\end{tabular}

\begin{tabular}{|c|c|c|}
\hline$T D=12.0$ & $\mathrm{ET}=0$ & \\
\hline NERGY (YEV) & PRIMARY & CASCADE \\
\hline 0.49500 & 1.61 & 1.61 \\
\hline 0.50000 & 3.60 & 3.60 \\
\hline 51300 & 7.67 & 7.67 \\
\hline 0.52500 & 13.98 & 13.98 \\
\hline 0.54400 & 22.23 & \\
\hline 0.56900 & 33.32 & \\
\hline 0.59300 & 46.32 & 46.32 \\
\hline 0.63300 & 64.08 & .08 \\
\hline 0.68700 & 85.10 & 85.10 \\
\hline 0.76300 & 114.21 & 114.21 \\
\hline 0.85300 & 148.85 & 149.00 \\
\hline 0.99100 & 184.16 & 186.26 \\
\hline 1.12300 & 215.94 & 223.11 \\
\hline 1.32300 & 249.57 & \\
\hline 1.57300 & 277.67 & 315.84 \\
\hline 1.85000 & 297.99 & 361.85 \\
\hline 2.20300 & 311.80 & 407.19 \\
\hline 2.55000 & 319.46 & 446.83 \\
\hline 2.94300 & 323.57 & 485.04 \\
\hline 3.43000 & 325.08 & 526.24 \\
\hline 4.17000 & 323.75 & 578.34 \\
\hline 4.90000 & 320.81 & 621.32 \\
\hline 7.36300 & 309.61 & 729.35 \\
\hline 9.81000 & 301.36 & 805.75 \\
\hline 14.70000 & 290.99 & 913.05 \\
\hline 24.50000 & 280.66 & 1048.12 \\
\hline 49.00300 & 272.90 & 1232.08 \\
\hline 98.10500 & 269.21 & 1416.43 \\
\hline 147.00000 & 267.46 & 1523.46 \\
\hline 245.00300 & 265.17 & 1657.63 \\
\hline 343.00300 & 263.66 & 1745.35 \\
\hline & .18 & \\
\hline
\end{tabular}

ATOMIC DISPLAZEMENT CROSS-SECTIONS (BARNS) BY PAST ELECTRONS IN CS $\mathrm{Z}=55 \quad \mathrm{~A}=132.910$

\begin{tabular}{rrr} 
TD $=16.0$ & EV & \multicolumn{2}{c}{ ER=0.607613 MEV } \\
ENERGY(HEV) & PRIMARY & CASCADE \\
0.61300 & 1.00 & 1.00 \\
0.61900 & 2.16 & 2.16 \\
0.63100 & 4.60 & 4.60 \\
0.65000 & 8.75 & 8.75 \\
0.67400 & 14.40 & 14.40 \\
0.70400 & 21.88 & 21.88 \\
0.74100 & 31.50 & 31.50 \\
0.78900 & 44.19 & 44.19 \\
0.85000 & 60.11 & 60.11 \\
0.94100 & 82.54 & 82.54 \\
1.06000 & 108.61 & 108.76 \\
1.21000 & 136.00 & 137.77 \\
1.39000 & 161.79 & 167.99 \\
1.54000 & 187.76 & 203.77 \\
1.94000 & 208.44 & 239.77 \\
2.30000 & 223.74 & 275.92 \\
2.73000 & 234.03 & 311.98 \\
3.15000 & 239.28 & 341.85 \\
3.64000 & 242.09 & 371.84 \\
4.25000 & 242.87 & 403.75 \\
5.16000 & 241.56 & 443.49 \\
6.07000 & 239.14 & 476.59 \\
9.11000 & 230.65 & 558.66 \\
12.10000 & 224.68 & 615.87 \\
18.20000 & 217.04 & 697.60 \\
30.30000 & 209.70 & 799.13 \\
60.70000 & 204.32 & 937.81 \\
121.00000 & 201.69 & 1075.30 \\
182.00000 & 200.37 & 1156.30
\end{tabular}

\begin{tabular}{rrr} 
TD $=20.0$ & RV & \multicolumn{2}{c}{ ET=0.713271 MEV } \\
ENERGY(MEV) & PRIMARY & CASCADE \\
0.72000 & 0.71 & 0.71 \\
0.72700 & 1.49 & 1.49 \\
0.74100 & 3.19 & 3.19 \\
0.76300 & 5.15 & 5.15 \\
0.79100 & 10.33 & 10.33 \\
0.82700 & 16.21 & 16.21 \\
0.87000 & 23.69 & 23.69 \\
0.92700 & 33.94 & 33.94 \\
0.99800 & 46.69 & 46.69 \\
1.10000 & 64.19 & 64.19 \\
1.24000 & 85.69 & 85.84 \\
1.42000 & 108.69 & 110.30 \\
1.64000 & 130.51 & 136.18 \\
1.92000 & 150.53 & 164.28 \\
2.28000 & 167.50 & 194.61 \\
2.71000 & 179.71 & 224.73 \\
3.20000 & 187.41 & 253.38 \\
3.70000 & 191.44 & 278.14 \\
4.27000 & 193.42 & 302.36 \\
4.99000 & 193.83 & 328.46 \\
6.06000 & 192.57 & 360.74 \\
7.13000 & 190.50 & $\mathbf{3 8 7 . 5 4}$ \\
10.60000 & 183.83 & 452.24 \\
14.20000 & 178.99 & 499.67 \\
21.30000 & 173.03 & 564.85 \\
35.60000 & 167.33 & 646.91 \\
71.30000 & 163.27 & 758.00 \\
142.00000 & 161.23 & 867.88 \\
213.00000 & 160.17 & 932.22 \\
& &
\end{tabular}

\begin{tabular}{|c|c|c|}
\hline$T D=24.0$ & $E T=0$. & $507 \mathrm{MEV}$ \\
\hline ENERGY (YEV) & PRIMARY & CASCADE \\
\hline 0.31300 & 0.50 & 0.50 \\
\hline 0.82500 & 1.07 & 1.07 \\
\hline 0.84200 & 2.36 & 2.36 \\
\hline 0.86700 & 4.65 & 4.65 \\
\hline 0.89700 & 8.01 & 8.01 \\
\hline 0.94000 & 12.80 & 12.80 \\
\hline 0.98900 & 18.87 & 18.87 \\
\hline 1.05$) 00$ & 27.07 & 27.07 \\
\hline 1.13300 & 37.75 & 37.75 \\
\hline 1.25300 & 53.11 & 53.11 \\
\hline 1.41300 & 71.46 & 71.62 \\
\hline 1.62300 & 91.41 & 92.95 \\
\hline 1.86000 & 109.05 & 114.11 \\
\hline 2.18300 & 125.99 & 138.21 \\
\hline 2.59000 & 140.15 & 164.07 \\
\hline 3.07000 & 150.05 & 189.24 \\
\hline 3.64300 & 156.44 & 214.09 \\
\hline 4.21000 & 159.60 & 235.06 \\
\hline 4.86000 & 161.07 & 255.53 \\
\hline 5.67000 & 161.25 & 277.28 \\
\hline 6.88300 & 160.07 & 304.32 \\
\hline 8.10300 & 158.26 & 326.90 \\
\hline 12.10300 & 152.61 & 381.79 \\
\hline 16.20300 & 148.64 & 421.39 \\
\hline 24.30500 & 143.76 & 475.81 \\
\hline 40.50300 & 139.18 & 543.94 \\
\hline 81.00300 & 135.95 & 636.43 \\
\hline 162.00300 & 134.27 & 728.57 \\
\hline 243.00100 & 133.39 & \\
\hline
\end{tabular}


ATOMIC DISPLAZEMENT CROSS-SẼTIONS (BARNS) BY PAST ELEZTRONS IN CS

\begin{tabular}{|c|c|c|}
\hline \multirow{2}{*}{$\begin{array}{r}T D=28.0 \\
\text { ENERGY (HEV) }\end{array}$} & \multicolumn{2}{|c|}{$\mathrm{ET}=0.901064 \mathrm{MEV}$} \\
\hline & PRIMARY & CASCADE \\
\hline 0.91000 & 0.41 & 0.41 \\
\hline 0.91900 & 0.86 & 0.86 \\
\hline 0.93700 & 1.89 & 1.89 \\
\hline 0.96400 & 3.71 & 3.71 \\
\hline 1.00000 & 6.53 & 6.53 \\
\hline 1.04000 & 10.07 & 10.07 \\
\hline 1.09000 & 14.89 & 14.89 \\
\hline 1.17000 & 23.08 & 23.08 \\
\hline 1.26000 & 32.44 & 32.44 \\
\hline 1.39000 & 45.45 & 45.45 \\
\hline 1.57000 & 61.59 & 61.76 \\
\hline 1.80000 & 78.64 & 80.09 \\
\hline 2.07000 & 94.07 & 98.74 \\
\hline 2.43000 & 108.74 & 119.95 \\
\hline 2.88000 & 120.61 & 142.13 \\
\hline 3.42000 & 129.04 & 164.23 \\
\hline 4.05000 & 134.29 & 185.62 \\
\hline 4.68000 & 136.85 & 203.67 \\
\hline 5.40000 & 137.98 & 221.32 \\
\hline 6.30000 & 138.03 & 240.10 \\
\hline 7.65000 & 136.92 & 263.51 \\
\hline 9.01000 & 135.32 & 283.02 \\
\hline 13.50000 & 130.43 & 330.61 \\
\hline 18.00000 & 127.10 & 364.16 \\
\hline 27.00000 & 122.97 & 410.88 \\
\hline 45.00000 & 119.13 & 469.35 \\
\hline 90.10000 & 116.45 & 548.82 \\
\hline 180.00000 & 115.04 & 627.68 \\
\hline
\end{tabular}

\begin{tabular}{rrr}
$T D=32.0$ & EV & \multicolumn{2}{c}{$E T=0.986154$} & IRV \\
ENERGY (MEV) & PRIMARY & CASEADE \\
0.99600 & 0.32 & 0.32 \\
1.00000 & 0.46 & 0.46 \\
1.02000 & 1.28 & 1.28 \\
1.05000 & 2.78 & 2.78 \\
1.09000 & 5.19 & 5.19 \\
1.14000 & 8.68 & 8.68 \\
1.20300 & 13.33 & 13.33 \\
1.28000 & 19.94 & 19.94 \\
1.38000 & 28.34 & 28.34 \\
1.52000 & 39.69 & 39.69 \\
1.72000 & 54.20 & 54.37 \\
1.97000 & 69.15 & 70.52 \\
2.25000 & 82.52 & 85.78 \\
2.66000 & 95.60 & 105.88 \\
3.15000 & 105.88 & 125.43 \\
3.74000 & 113.15 & 144.94 \\
4.43000 & 117.64 & 163.86 \\
5.12000 & 119.78 & 179.80 \\
5.91000 & 120.68 & 195.38 \\
6.90000 & 120.65 & 212.00 \\
8.38000 & 119.61 & 232.59 \\
9.86000 & 118.17 & 249.62 \\
14.70000 & 113.94 & 290.88 \\
19.70000 & 110.99 & 320.82 \\
29.50000 & 107.43 & 361.58 \\
49.30000 & 104.12 & 413.06 \\
98.60000 & 101.84 & 482.52 \\
197.00000 & 100.62 & 551.54
\end{tabular}

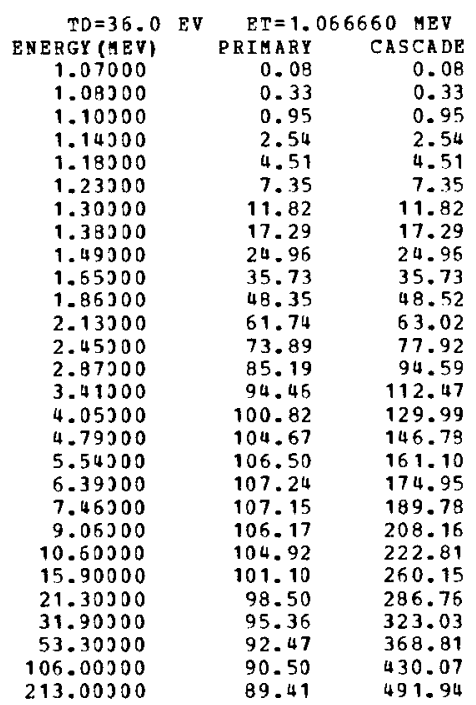

ATOMIC DISPlazeMENT CROSS-SECTIONS (BARNS) BY PAST ELECTRONS IN CS $z=55 \quad A=132.910$

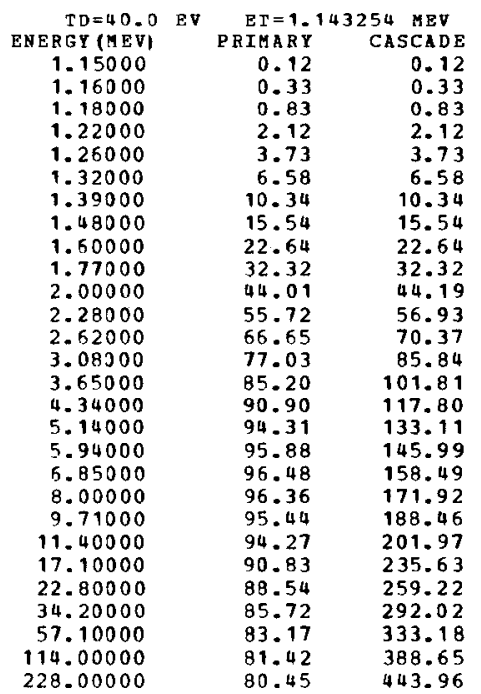

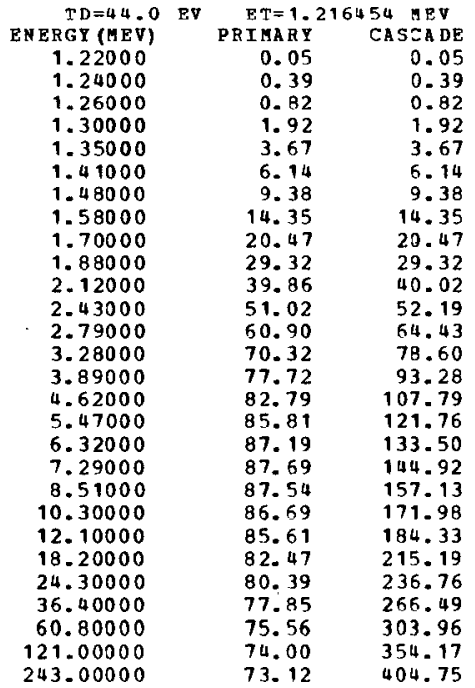

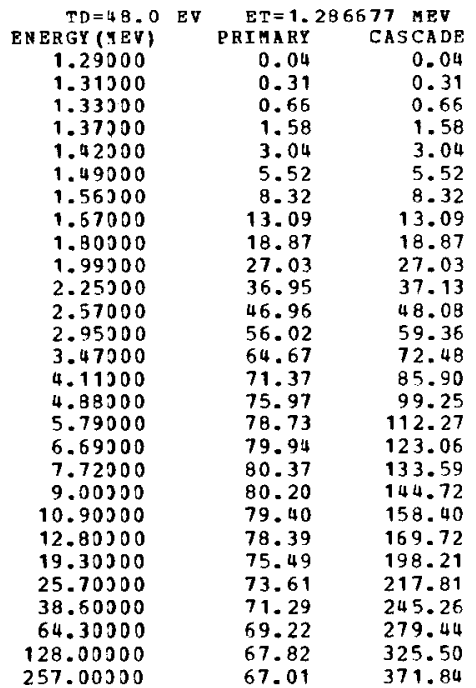


ATOHIC DISPLAEEMENT CROSS-SECTIONS (BARNS) BY PAST BLEETRONS IN CS

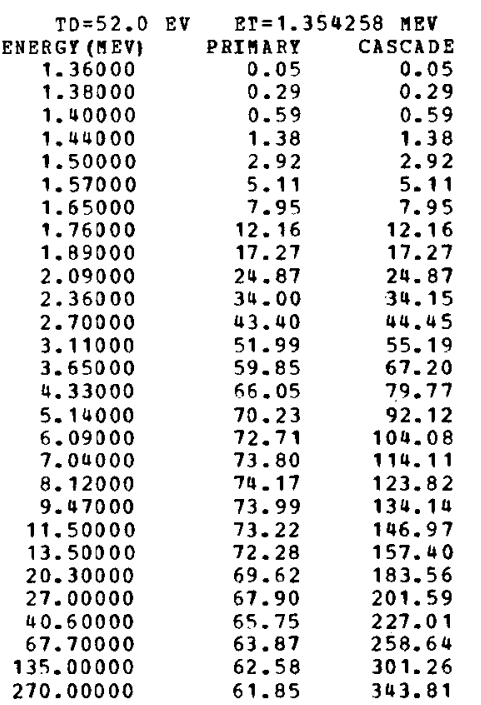

\begin{tabular}{rrr} 
TD $=56.0$ & EV & \multicolumn{2}{c}{ ET $=1.419474$ MEV } \\
ENERGY (MEV) & PRIMARY & CASTADE \\
1.43000 & 0.09 & 0.09 \\
1.44000 & 0.18 & 0.18 \\
1.47000 & 0.57 & 0.57 \\
1.51000 & 1.26 & 1.26 \\
1.57000 & 2.62 & 2.62 \\
1.64000 & 4.54 & 4.54 \\
1.73000 & 7.38 & 7.38 \\
1.84000 & 11.13 & 11.13 \\
1.98000 & 16.04 & 16.04 \\
2.20000 & 23.47 & 23.47 \\
2.48000 & 31.87 & 32.03 \\
2.83000 & 40.45 & 41.46 \\
3.26000 & 48.44 & 51.50 \\
3.83000 & 55.76 & 62.77 \\
4.54000 & 61.46 & 74.44 \\
5.39000 & 65.30 & 85.96 \\
6.38000 & 67.56 & 97.03 \\
7.38000 & 68.54 & 106.41 \\
8.51000 & 68.86 & 115.43 \\
9.93000 & 68.68 & 125.06 \\
12.00000 & 67.96 & 136.69 \\
14.90000 & 67.08 & 146.45 \\
21.20000 & 64.61 & 170.76 \\
28.30000 & 63.00 & 187.73 \\
42.50000 & 61.02 & 211.27 \\
70.90000 & 59.28 & 240.67 \\
141.00000 & 58.11 & 280.10 \\
283.00000 & 57.42 & 319.81
\end{tabular}

\begin{tabular}{|c|c|c|}
\hline$T D=50.0$ & $\mathrm{ET}=1$ & $558 \mathrm{MEV}$ \\
\hline ENERGY (MEV) & PRIMARY & CASCADE \\
\hline 1.49000 & 0.05 & 0.05 \\
\hline 1.51300 & 0.22 & 0.22 \\
\hline 1.54300 & 0.53 & 0.58 \\
\hline 1.58200 & 1.21 & 1.21 \\
\hline 1.64300 & 2.42 & 2.42 \\
\hline 1.71000 & 4.14 & 4.14 \\
\hline 1.30300 & 6.67 & 6.67 \\
\hline 1.92000 & 10.34 & 10.34 \\
\hline 2.07000 & 15.06 & 15.06 \\
\hline 2.29000 & 21.75 & 21.75 \\
\hline 2.59000 & 29.85 & 30.00 \\
\hline 2.96300 & 37.97 & 38.96 \\
\hline 3.40300 & 45.23 & 48.19 \\
\hline 4.00300 & 52.17 & 58.84 \\
\hline 4.74000 & 57.45 & 69.75 \\
\hline 5.53000 & 61.02 & 80.56 \\
\hline 6.57300 & 63.09 & 90.98 \\
\hline 7.70300 & 63.98 & 99.63 \\
\hline 8.89000 & 64.25 & 108.13 \\
\hline 10.30300 & 64.09 & 116.73 \\
\hline 12.60000 & 63.36 & 128.30 \\
\hline 14.80000 & 62.54 & 137.39 \\
\hline 22.20000 & 60.25 & 159.97 \\
\hline 29.50000 & 58.75 & 175.74 \\
\hline 44.40000 & 56.92 & 197.66 \\
\hline 74.10000 & 55.31 & 225.13 \\
\hline 148.00000 & 54.22 & 262.17 \\
\hline 296.00300 & 53.58 & 299.04 \\
\hline
\end{tabular}

ATOMIC DISPLAEEHENT CROSS-SECTIONS (BARNS) BY PAST RLECTRONS IN CS $\mathrm{Z}=55 \quad \mathrm{~A}=132.910$

\begin{tabular}{crr} 
TD $=64.0$ & EV & \multicolumn{1}{c}{ ET $=1.543706$ MEV } \\
ENERGY (AEV) & PRIMARY & CASCADE \\
1.55000 & 0.04 & 0.04 \\
1.57000 & 0.18 & 0.18 \\
1.50000 & 0.48 & 0.48 \\
1.65000 & 1.19 & 1.19 \\
1.71000 & 2.29 & 2.29 \\
1.79000 & 4.09 & 4.09 \\
1.88000 & 6.40 & 6.40 \\
2.00000 & 9.73 & 9.73 \\
2.16000 & 14.29 & 14.29 \\
2.39000 & 20.60 & 20.61 \\
2.70000 & 28.15 & 28.31 \\
3.08000 & 35.68 & 36.62 \\
3.55000 & 42.70 & 45.52 \\
4.15000 & 48.98 & 55.31 \\
4.93000 & 53.92 & 65.58 \\
5.86000 & 57.26 & 75.79 \\
6.94000 & 59.17 & 85.55 \\
8.02000 & 59.99 & 93.74 \\
9.26000 & 50.23 & 101.73 \\
10.80000 & 60.04 & 110.16 \\
13.10000 & 59.37 & 120.56 \\
15.40000 & 58.59 & 129.13 \\
23.10000 & 56.44 & 150.32 \\
30.80000 & 55.04 & 165.11 \\
46.30000 & 53.33 & 185.77 \\
$\mathbf{7 7 . 1 0 0 0 0}$ & 51.83 & 211.42 \\
154.00000 & 50.82 & 246.15 \\
308.00000 & 50.23 & 280.72
\end{tabular}

\begin{tabular}{rrr} 
TD $=68.0$ & EV & \multicolumn{2}{c}{ ET $=1.603085$ MEV } \\
ENERGY (MEV) & PRIMARY & CASEADE \\
1.61000 & 0.03 & 0.03 \\
1.63000 & 0.16 & 0.16 \\
1.66000 & 0.43 & 0.43 \\
1.71000 & 1.05 & 1.05 \\
1.77000 & 2.04 & 2.04 \\
1.85000 & 3.64 & 3.64 \\
1.95000 & 5.96 & 5.96 \\
2.08000 & 9.24 & 9.24 \\
2.24000 & 13.40 & 13.40 \\
2.48000 & 19.41 & 19.41 \\
2.80000 & 26.50 & 26.66 \\
3.20000 & 33.71 & 34.63 \\
3.68000 & 40.22 & 42.90 \\
4.32000 & 46.20 & 52.25 \\
5.12000 & 50.83 & 61.95 \\
6.09000 & 53.95 & 71.62 \\
7.21000 & 55.72 & 80.81 \\
8.33000 & 56.47 & 88.51 \\
9.61000 & 56.68 & 96.00 \\
11.20000 & 56.49 & 103.90 \\
13.60000 & 55.85 & 113.76 \\
16.00000 & 55.11 & 121.88 \\
24.00000 & 53.09 & 141.82 \\
32.00000 & 51.77 & 155.75 \\
48.00000 & 50.17 & 175.10 \\
80.10000 & 48.77 & 199.34 \\
160.00000 & 47.82 & 232.04 \\
320.00000 & 47.27 & 264.57
\end{tabular}

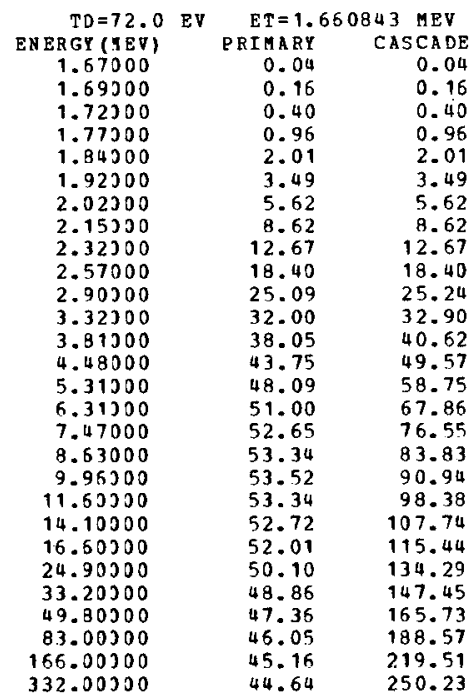


ATOMIC DISPLAZEMENT CROSS-SECTIONS (BARNS) BY PAST ELECTRONS IN CS $Z=55 \quad A=132.910$

\begin{tabular}{rrr} 
TD $=76.0$ & \multicolumn{1}{c}{ EV } & \multicolumn{1}{c}{ ET 1.717104} \\
ENERGY MEV \\
1.73000 & PRIMARY & CASCADE \\
1.75000 & 0.05 & 0.05 \\
1.78000 & 0.16 & 0.16 \\
1.83000 & 0.38 & 0.38 \\
1.90000 & 0.90 & 0.90 \\
1.99000 & 1.86 & 1.86 \\
2.09000 & 3.39 & 3.39 \\
2.23000 & 5.35 & 5.35 \\
2.40000 & 8.34 & 8.34 \\
2.65000 & 12.06 & 12.06 \\
3.00000 & 17.54 & 17.54 \\
3.43000 & 23.87 & 24.02 \\
3.94000 & 30.36 & 31.23 \\
4.63000 & 36.14 & 38.62 \\
5.49000 & 41.51 & 47.09 \\
6.52000 & 45.62 & 55.84 \\
7.72000 & 48.34 & 64.45 \\
8.92000 & 49.89 & 72.71 \\
10.30000 & 50.53 & 79.62 \\
12.00000 & 50.70 & 86.38 \\
14.50000 & 50.51 & 93.45 \\
17.10000 & 49.94 & 102.06 \\
25.70000 & 49.26 & 109.44 \\
34.30000 & 47.45 & 127.40 \\
51.50000 & 46.27 & 139.91 \\
85.80000 & 44.84 & 157.27 \\
171.00000 & 43.61 & 178.90 \\
& 42.78 & 208.07
\end{tabular}

\begin{tabular}{ccr} 
TD=80.0 EV & \multicolumn{1}{c}{ ET=1.771978 MEV } \\
ENERGY (MEV) & PRIMARY & CASZADE \\
1.78000 & 0.03 & 0.03 \\
1.80000 & 0.12 & 0.12 \\
1.84000 & 0.38 & 0.38 \\
1.89000 & 0.86 & 0.86 \\
1.96000 & 1.75 & 1.75 \\
2.05000 & 3.16 & 3.16 \\
2.16000 & 5.15 & 5.15 \\
2.30000 & 7.91 & 7.91 \\
2.48000 & 11.55 & 11.55 \\
2.74000 & 16.62 & 16.62 \\
3.10000 & 22.81 & 22.96 \\
3.54000 & 28.92 & 29.77 \\
4.07000 & 34.45 & 36.86 \\
4.78000 & 39.51 & 44.89 \\
5.67000 & 43.40 & 53.24 \\
6.73000 & 45.96 & 61.40 \\
7.97000 & 47.42 & 69.27 \\
9.21000 & 48.01 & 75.85 \\
10.60000 & 48.16 & 82.14 \\
12.40000 & 47.97 & 89.04 \\
15.00000 & 47.42 & 97.27 \\
17.70000 & 46.76 & 104.31 \\
26.50000 & 45.06 & 121.21 \\
35.40000 & 43.94 & 133.14 \\
53.10000 & 42.59 & 149.59 \\
88.50000 & 41.42 & 170.16 \\
177.00000 & 40.64 & 198.01
\end{tabular}

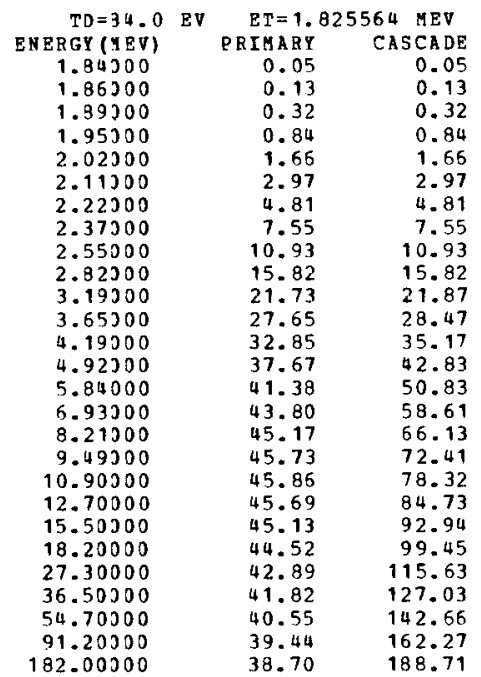

ATOMIC DISPLAZEMENT CROSS-SECTIONS (BARNS) BY FAST ELECTRONS IN CS

\begin{tabular}{rrr} 
TD $=38.0$ & EV & \multicolumn{1}{c}{ ET=1.877949 MEV } \\
PERGY (MEV) & PRIARY & CASCADE \\
1.89000 & 0.03 & 0.03 \\
1.91000 & 0.11 & 0.11 \\
1.95000 & 0.34 & 0.34 \\
2.00000 & 0.74 & 0.74 \\
2.08000 & 1.61 & 1.61 \\
2.17000 & 2.82 & 2.82 \\
2.29000 & 4.70 & 4.70 \\
2.44000 & 7.25 & 7.25 \\
2.62000 & 10.41 & 10.41 \\
2.91000 & 15.29 & 15.29 \\
3.28000 & 20.77 & 20.91 \\
3.75000 & 26.40 & 27.19 \\
4.31000 & 31.42 & 33.66 \\
5.07000 & 36.05 & 41.07 \\
6.00000 & 39.52 & 48.58 \\
7.13000 & 41.84 & 56.08 \\
8.45000 & 43.14 & 63.29 \\
9.76000 & 43.65 & 69.26 \\
11.20000 & 43.77 & 74.87 \\
13.10000 & 43.60 & 81.15 \\
15.90000 & 43.07 & 88.77 \\
18.70000 & 42.49 & 95.05 \\
28.10000 & 40.92 & 110.57 \\
37.50000 & 39.91 & 121.38 \\
56.30000 & 38.69 & 136.38 \\
93.80000 & 37.64 & 155.07 \\
187.00000 & 36.94 & 180.27
\end{tabular}

\begin{tabular}{crr} 
TD $=92.0$ & EV & \multicolumn{1}{c}{ ET=1.929209 MEV } \\
ENERGY (NEV) & PRTMAY & CASCADE \\
1.94000 & 0.03 & 0.03 \\
1.96000 & 0.09 & 0.09 \\
2.00000 & 0.30 & 0.30 \\
2.06000 & 0.75 & 0.75 \\
2.14000 & 1.57 & 1.57 \\
2.23000 & 2.71 & 2.71 \\
2.35000 & 4.46 & 4.46 \\
2.50000 & 6.84 & 6.84 \\
2.70000 & 10.11 & 10.11 \\
2.99000 & 14.67 & 14.67 \\
3.37000 & 19.92 & 20.06 \\
3.85000 & 25.29 & 26.05 \\
4.43000 & 30.12 & 32.30 \\
5.20000 & 34.49 & 39.31 \\
6.17000 & 37.85 & 46.62 \\
7.33000 & 40.05 & 53.80 \\
8.68000 & 41.27 & 60.65 \\
10.00000 & 41.75 & 65.28 \\
11.50000 & 41.86 & 71.73 \\
13.50000 & 41.68 & 77.83 \\
16.30000 & 41.19 & 84.98 \\
19.20000 & 40.62 & 91.05 \\
28.90000 & 39.12 & 105.96 \\
38.50000 & 38.15 & 116.24 \\
57.80000 & 37.00 & 130.58 \\
96.40000 & 36.00 & 148.50 \\
192.00000 & 35.33 & 172.58
\end{tabular}

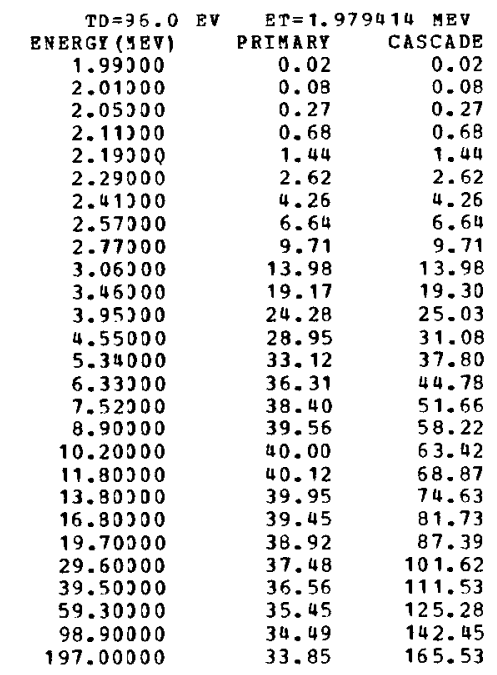


ATOMIC DISPLAZEMENT CROSS-SECTIONS (BARNS) BY FAST ELECTRONS IN ND

\begin{tabular}{rrr} 
TD $=4.0$ & \multicolumn{1}{c}{ ET $=0.216889$ MEV } \\
ENERGY(MEV & PRIMARY & CASCADE \\
0.21900 & 16.47 & 16.47 \\
0.22100 & 31.72 & 31.72 \\
0.22500 & 61.25 & 61.25 \\
0.23200 & 110.04 & 110.04 \\
0.24000 & 161.76 & 161.76 \\
0.25100 & 226.80 & 226.80 \\
0.26400 & 295.95 & 295.95 \\
0.28100 & 375.93 & 375.93 \\
0.30300 & 465.26 & 465.26 \\
0.33600 & 576.13 & 576.13 \\
0.37900 & 689.97 & 689.97 \\
0.43300 & 798.34 & 802.86 \\
0.49800 & 893.98 & 914.61 \\
0.58500 & 982.76 & 1037.00 \\
0.69400 & 1054.31 & 1162.08 \\
0.82400 & 1105.26 & 1285.10 \\
0.97500 & 1137.53 & 1405.50 \\
1.12000 & 1152.48 & 1503.71 \\
1.30000 & 1158.99 & 1610.88 \\
1.51000 & 1157.25 & 1720.01 \\
1.84000 & 1145.08 & .1866 .52 \\
2.16000 & 1129.27 & 1987.67 \\
3.25000 & 1077.17 & 2305.79 \\
4.33000 & 1038.49 & 2536.13 \\
6.50000 & 991.44 & 2871.51 \\
10.80000 & 945.59 & $\mathbf{3 2 9 8 . 1 8}$ \\
21.60000 & 907.03 & $\mathbf{3 8 9 0 . 8 3}$ \\
43.30000 & 889.94 & 4493.83 \\
65.00000 & 883.05 & 4845.79 \\
108.00000 & 874.41 & 5283.91 \\
151.00000 & 868.45 & 5570.69 \\
216.00000 & 862.39 & 5875.30
\end{tabular}

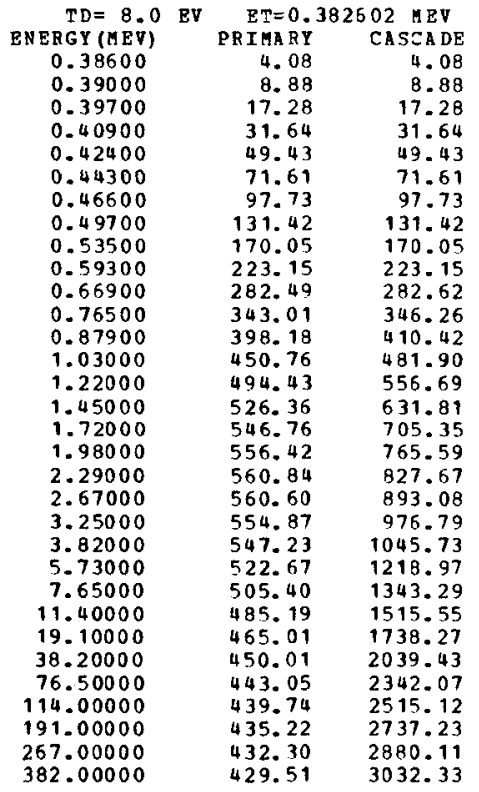

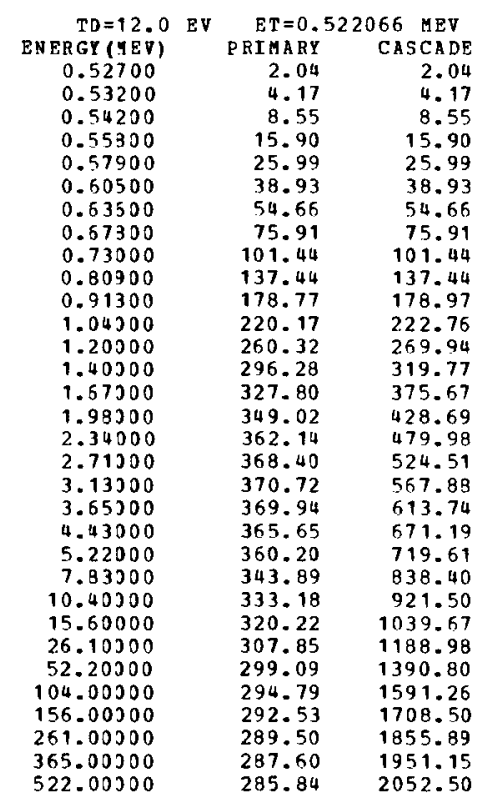

ATOMIC DISPLAZEMENT CROSS-SECTIONS (BARNS) BT PAST ELEZTRONS IN ND $\mathrm{Z}=60 \quad \mathrm{~A}=144.270$

\begin{tabular}{rrr}
$\begin{array}{r}\text { TD }=16.0 \\
\text { ENERGY }\end{array}$ & \multicolumn{1}{c}{ ET $=0.644823$ MEV } \\
0.65100 & PRTMART & CASCADE \\
0.65700 & 1.22 & 1.22 \\
0.67000 & 2.47 & 2.47 \\
0.58900 & 5.35 & 5.35 \\
0.71500 & 9.93 & 9.93 \\
0.74700 & 16.79 & 16.79 \\
0.78500 & 25.85 & 25.85 \\
0.83800 & 37.46 & 37.46 \\
0.90200 & 53.28 & 53.28 \\
0.99900 & 72.51 & 72.51 \\
1.12000 & 99.95 & 99.95 \\
1.28000 & 130.21 & 130.40 \\
1.48000 & 163.20 & 165.41 \\
1.74000 & 194.72 & 202.82 \\
2.06000 & 223.49 & 243.71 \\
2.45000 & 246.13 & 285.49 \\
2.90000 & 262.08 & 327.55 \\
3.35000 & 271.47 & 367.72 \\
3.86300 & 275.61 & 401.57 \\
4.51000 & 276.91 & 434.45 \\
5.48000 & 275.93 & 470.15 \\
6.44000 & 272.35 & 514.41 \\
9.67000 & 268.16 & 550.76 \\
12.80000 & 255.96 & 641.30 \\
19.30000 & 248.27 & 703.46 \\
32.20000 & 238.73 & 793.72 \\
64.40000 & 230.01 & 905.54 \\
128.00000 & 223.94 & 1057.30 \\
193.00000 & 220.83 & 1207.17 \\
& 219.10 & 1296.18
\end{tabular}

\begin{tabular}{rrr} 
TD $=20.0$ & EV & \multicolumn{1}{c}{ ET $=0.755739$} \\
ENERGY (IEV) & PRIMAY & CASEA DE \\
0.76300 & 0.82 & 0.82 \\
0.77000 & 1.66 & 1.66 \\
0.78500 & 3.65 & 3.65 \\
0.80800 & 7.10 & 7.10 \\
0.83800 & 12.18 & 12.18 \\
0.87500 & 19.29 & 19.29 \\
0.92200 & 28.52 & 28.52 \\
0.98200 & 41.00 & 41.00 \\
1.05000 & 55.15 & 55.15 \\
1.17000 & 78.86 & 78.86 \\
1.32000 & 104.95 & 105.19 \\
1.51000 & 131.98 & 134.15 \\
1.73000 & 155.94 & 162.97 \\
2.04000 & 179.60 & 197.22 \\
2.41000 & 197.42 & 231.09 \\
2.87000 & 210.03 & 265.80 \\
3.40000 & 217.22 & 298.78 \\
3.92000 & 220.19 & 326.01 \\
4.53000 & 220.97 & 353.30 \\
5.29000 & 219.93 & 382.17 \\
6.42000 & 216.89 & 417.80 \\
7.55000 & 213.42 & 447.30 \\
11.30000 & 203.77 & 519.74 \\
15.10000 & 197.56 & 571.42 \\
22.60000 & 190.25 & 642.53 \\
37.70000 & 183.54 & 732.18 \\
75.50000 & 178.95 & 854.00 \\
151.00000 & 176.49 & 975.00 \\
226.00000 & 175.12 & 1044.88
\end{tabular}

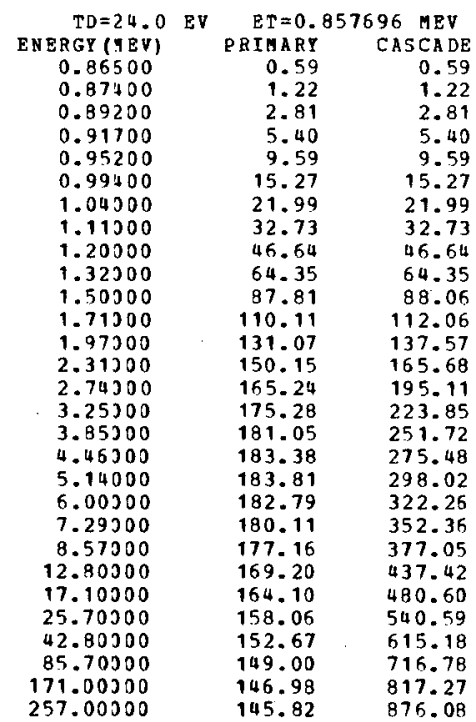


ATOMIC DISPLAZEMENT CROSS-SECTIONS (BARNS) BY PAST ELECTRONS IN ND

\begin{tabular}{rrr} 
TD $=28.0$ & EV & \multicolumn{2}{c}{ ET $=0.952568$ MEV } \\
PNERGY (MEV) & PRIMARY & CASCADE \\
0.96200 & 0.46 & 0.46 \\
0.97100 & 0.95 & 0.95 \\
0.99000 & 2.16 & 2.16 \\
1.01000 & 3.64 & 3.64 \\
1.05000 & 7.12 & 7.12 \\
1.10000 & 12.20 & 12.20 \\
1.16000 & 18.97 & 18.97 \\
1.23000 & 27.33 & 27.33 \\
1.33000 & 39.42 & 39.42 \\
1.47000 & 55.58 & 55.58 \\
1.66000 & 74.98 & 75.21 \\
1.90000 & 94.92 & 96.76 \\
2.19000 & 113.06 & 119.04 \\
2.57000 & 129.45 & 143.61 \\
3.04000 & 142.04 & 168.77 \\
3.61000 & 150.53 & 193.87 \\
4.28000 & 155.29 & 218.12 \\
4.95000 & 157.10 & 238.43 \\
5.71000 & 157.33 & 258.04 \\
6.66000 & 156.36 & 278.84 \\
8.09000 & 153.98 & 304.77 \\
9.52000 & 151.39 & 326.15 \\
14.20000 & 144.62 & 377.93 \\
19.00000 & 140.28 & 415.22 \\
28.50000 & 135.20 & 466.47 \\
47.60000 & 130.67 & 530.84 \\
95.20000 & 127.64 & 617.84 \\
190.00000 & 125.92 & 704.00
\end{tabular}

\begin{tabular}{rrr} 
TD=32.0 & \multicolumn{2}{c}{ EV $=1.041653$ MEV } \\
ENERGY (HEV) & PRIMARY & CASEADE \\
1.05000 & 0.29 & 0.29 \\
1.06000 & 0.68 & 0.68 \\
1.08000 & 1.62 & 1.62 \\
1.11000 & 3.34 & 3.34 \\
1.15000 & 6.12 & 5.12 \\
1.20000 & 10.15 & 10.15 \\
1.27000 & 16.48 & 15.48 \\
1.35000 & 24.20 & 24.20 \\
1.45000 & 33.98 & 33.98 \\
1.61000 & 48.95 & 48.95 \\
1.82000 & 66.26 & 65.49 \\
2.08000 & 83.63 & 85.38 \\
2.39000 & 99.22 & 104.69 \\
2.81000 & 113.74 & 125.70 \\
3.33000 & 124.75 & 149.18 \\
3.95000 & 131.96 & 171.19 \\
4.68000 & 135.94 & 192.50 \\
5.41000 & 137.40 & 210.34 \\
6.24000 & 137.51 & 227.57 \\
7.29000 & 136.57 & 246.67 \\
8.85000 & 134.43 & 268.77 \\
10.40000 & 132.15 & 287.39 \\
15.60000 & 126.17 & 333.46 \\
20.80000 & 122.47 & 365.75 \\
31.20000 & 118.08 & 410.64 \\
52.00000 & 114.21 & 466.78 \\
104.00000 & 111.63 & 542.94 \\
208.00000 & 110.13 & 618.56
\end{tabular}

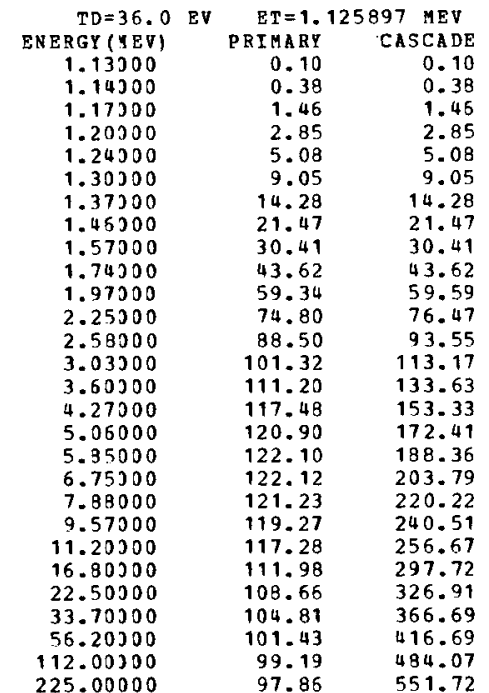

ATOMIC DISPLAzEMENT CROSS-SECTIONS (BARNS) BY FAST BLECTRONS IN N $z=60 \quad A=144.270$

\begin{tabular}{rrr} 
TD $=40.0$ & EV & \multicolumn{1}{c}{ ET $=1.206013$ ARV } \\
ENERGY (MEV) & PRIMARY & CASCADE \\
1.21000 & 0.08 & 0.08 \\
1.23300 & 0.54 & 0.54 \\
1.25000 & 1.14 & 1.14 \\
1.29000 & 2.66 & 2.66 \\
1.33000 & 4.55 & 4.55 \\
1.39000 & 7.88 & 7.88 \\
1.47000 & 12.92 & 12.92 \\
1.56000 & 19.01 & 19.01 \\
1.68000 & 27.28 & 27.28 \\
1.86000 & 39.16 & 39.16 \\
2.11000 & 53.65 & 53.89 \\
2.41000 & 67.64 & 69.23 \\
2.77000 & 80.19 & 85.00 \\
3.25000 & 91.59 & 102.68 \\
3.85000 & 100.25 & 120.90 \\
4.58000 & 105.90 & 139.05 \\
5.42000 & 108.85 & 156.16 \\
6.27000 & 109.86 & 170.64 \\
7.23000 & 109.82 & 184.51 \\
8.44000 & 108.97 & 199.34 \\
10.20000 & 107.22 & 217.19 \\
12.00000 & 105.36 & 232.26 \\
18.00000 & 100.61 & 269.27 \\
24.10000 & 97.65 & 295.53 \\
36.10000 & 94.21 & 331.37 \\
60.30000 & 91.21 & 376.54 \\
120.00000 & 89.24 & 437.07 \\
241.00000 & 88.05 & 497.93
\end{tabular}

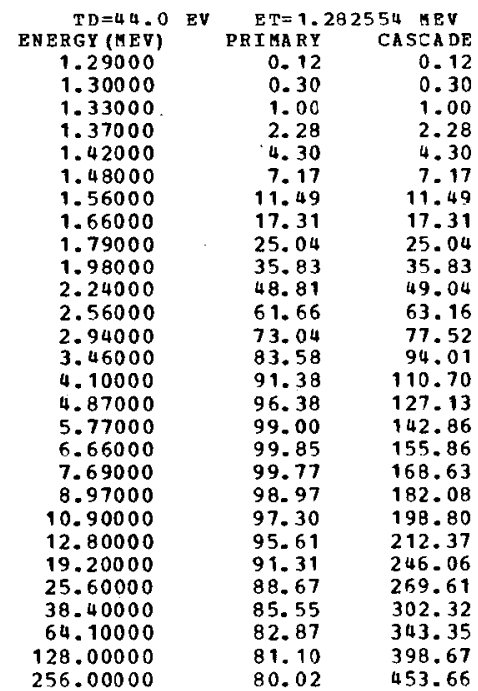

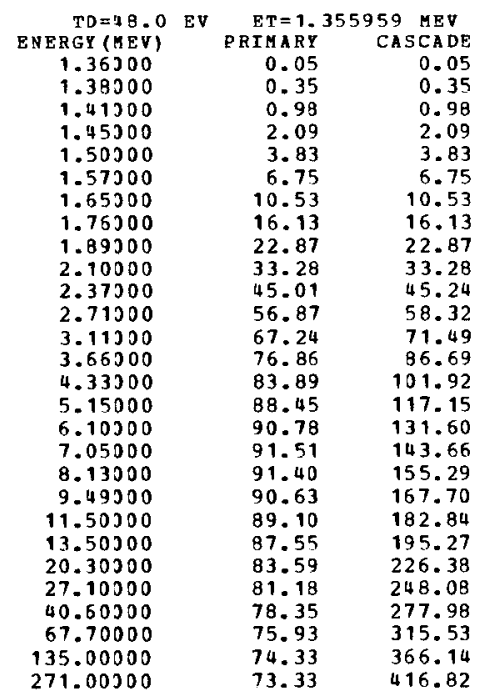


ATOMIC DESPLAzEHENT CROSS-SECTIONS (BARNS) BY PAST ELBCTRONS TN AD

\begin{tabular}{rrr}
$\begin{array}{c}\text { TD }=52.0 \\
\text { ENERGY (MEV) }\end{array}$ & \multicolumn{2}{c}{ ET $=1.426586$ MEV } \\
PRIMARY & CASCADE \\
1.44000 & 0.15 & 0.15 \\
1.45000 & 0.28 & 0.28 \\
1.48000 & 0.80 & 0.80 \\
1.52000 & 1.73 & 1.73 \\
1.58000 & 3.55 & 3.55 \\
1.65000 & 6.12 & 6.12 \\
1.74000 & 9.87 & 9.87 \\
1.85000 & 14.82 & 14.82 \\
1.99000 & 21.23 & 21.23 \\
2.21000 & 30.86 & 30.86 \\
2.49000 & 41.60 & 41.81 \\
2.85000 & 52.67 & 54.05 \\
3.28000 & 62.43 & 66.53 \\
3.85000 & 71.13 & 80.40 \\
4.56000 & 77.60 & 94.61 \\
5.42000 & 81.73 & 108.67 \\
6.41000 & 83.81 & 121.92 \\
7.41000 & 84.46 & 133.10 \\
8.55000 & 84.32 & 143.91 \\
9.98000 & 83.59 & 155.38 \\
12.10000 & 82.16 & 169.41 \\
14.20000 & 80.72 & 180.88 \\
21.30000 & 77.10 & 209.45 \\
28.50000 & 74.86 & 229.65 \\
42.70000 & 72.27 & 257.27 \\
71.30000 & 70.05 & 292.03 \\
142.00000 & 68.59 & 338.68 \\
285.00000 & 67.68 & 385.44
\end{tabular}

\begin{tabular}{rrr} 
TD=56.0 EV & \multicolumn{2}{c}{ ET=1.494727 MEV } \\
ENERGY (MEV) & PRIMARY & CASTADE \\
1.50000 & 0.04 & 0.04 \\
1.52000 & 0.26 & 0.26 \\
1.55000 & 0.71 & 0.71 \\
1.59000 & 1.52 & 1.52 \\
1.65000 & 3.10 & 3.10 \\
1.73000 & 5.69 & 5.69 \\
1.82000 & 9.03 & 9.03 \\
1.94000 & 13.84 & 13.84 \\
2.09000 & 19.97 & 19.97 \\
2.31000 & 28.56 & 28.56 \\
2.61000 & 38.81 & 39.02 \\
2.98000 & 48.94 & 50.24 \\
3.43000 & 58.04 & 61.90 \\
4.03000 & 66.15 & 74.91 \\
4.78000 & 72.19 & 88.27 \\
5.57000 & 75.93 & 101.21 \\
6.72000 & 77.85 & 113.71 \\
7.77000 & 78.41 & 124.12 \\
8.96000 & 78.26 & 134.13 \\
10.40000 & 77.60 & 144.42 \\
12.70000 & 76.20 & 157.96 \\
14.90000 & 74.87 & 168.59 \\
22.40000 & 71.50 & 195.29 \\
29.80000 & 69.47 & 213.68 \\
44.80000 & 67.05 & 239.55 \\
74.70000 & 65.02 & 271.76 \\
149.00000 & 63.68 & 315.17 \\
298.00000 & 62.84 & 358.38
\end{tabular}

\begin{tabular}{|c|c|c|}
\hline$T D=50.0$ & $\mathrm{ET}=1$. & MEV \\
\hline ENERGY (MEV) & PRIMARY & CASCADE \\
\hline 1.57000 & 0.07 & 0.07 \\
\hline 1.59300 & 0.26 & 0.26 \\
\hline 1.62000 & 0.67 & 0.67 \\
\hline 1.66000 & 1.39 & 1.39 \\
\hline 1.73000 & 3.05 & 3.05 \\
\hline 1.31000 & 5.40 & 5.40 \\
\hline 1.90000 & 8.41 & 8.41 \\
\hline 2.02000 & 12.73 & 12.73 \\
\hline 2.18300 & 18.61 & 18.61 \\
\hline 2.41300 & 26.63 & 26.69 \\
\hline 2.73000 & 36.49 & 36.71 \\
\hline 3.12300 & 46.01 & 47.30 \\
\hline 3.58300 & 54.30 & 57.98 \\
\hline 4.21300 & 61.91 & 70.25 \\
\hline 4.99300 & 67.47 & 82.70 \\
\hline 5.93000 & 70.95 & 94.94 \\
\hline 7.02500 & 72.68 & 106.55 \\
\hline 8.11000 & 73.17 & 116.23 \\
\hline 9.36300 & 73.01 & 125.64 \\
\hline 10.90000 & 72.35 & 135.47 \\
\hline 13.20300 & 71.10 & 147.59 \\
\hline 15.60000 & 69.79 & 157.97 \\
\hline 23.40300 & 66.67 & 182.78 \\
\hline 31.20000 & 64.77 & 200.08 \\
\hline 46.80300 & 62.54 & 224.10 \\
\hline 78.00000 & 60.66 & 254.14 \\
\hline 156.00300 & 59.42 & 294.82 \\
\hline & & \\
\hline
\end{tabular}

ATOHIC DISPLAZEHENT CROSS-SECTIONS (BARNS) BY FAST ELECTRONS IN ND $z=60 \quad A=144.270$

$\begin{array}{rrr}\text { TD=54.0 } & \text { EV } & \text { EI }=1.624496 \\ \text { PRIMARY } & \text { CASCADE } \\ \text { ENERGY (MEV) } & 0.10 & 0.10 \\ 1.64000 & 0.19 & 0.19 \\ 1.65000 & 0.53 & 0.53 \\ 1.68000 & 1.32 & 1.32 \\ 1.73000 & 2.82 & 2.82 \\ 1.80000 & 4.93 & 4.93 \\ 1.88000 & 7.95 & 7.95 \\ 1.98000 & 12.20 & 12.20 \\ 2.11000 & 17.52 & 17.52 \\ 2.27000 & 25.14 & 25.14 \\ 2.51000 & 34.29 & 34.50 \\ 2.84000 & 43.13 & 44.34 \\ 3.24000 & 51.09 & 54.64 \\ 3.73000 & 58.13 & 66.07 \\ 4.38000 & 63.31 & 77.76 \\ 5.19000 & 66.55 & 89.29 \\ 6.17000 & 68.15 & 100.24 \\ 7.31000 & 68.59 & 109.30 \\ 8.44000 & 68.42 & 118.13 \\ 9.74000 & 67.81 & 127.13 \\ 11.30000 & 65.57 & 139.00 \\ 13.80000 & 65.39 & 148.35 \\ 16.20000 & 65.39 \\ 24.30000 & 62.47 & 171.62 \\ 32.40000 & 60.69 & 187.85 \\ 48.70000 & 58.60 & 210.49 \\ 81.20000 & 56.85 & 238.68 \\ 162.00000 & 55.70 & 276.69 \\ 324.00000 & 54.96 & 314.49\end{array}$

\begin{tabular}{rrr} 
TD $=68.0$ & EV & \multicolumn{2}{c}{ ET $=1.686509$ REV } \\
ENERGY (HEV) & PRIMAY & CASCADE \\
1.70000 & 0.08 & 0.08 \\
1.72000 & 0.23 & 0.23 \\
1.75000 & 0.55 & 0.55 \\
1.80000 & 1.29 & 1.29 \\
1.87000 & 2.67 & 2.67 \\
1.95000 & 4.59 & 4.59 \\
2.05000 & 7.32 & 7.32 \\
2.19000 & 11.48 & 11.48 \\
2.36000 & 16.63 & 16.63 \\
2.61000 & 23.86 & 23.86 \\
2.95000 & 32.41 & 32.62 \\
3.37000 & 40.82 & 42.01 \\
3.87000 & 48.18 & 51.57 \\
4.55000 & 54.84 & 62.44 \\
5.39000 & 59.67 & 73.45 \\
5.40000 & 62.67 & 84.25 \\
7.58000 & 64.15 & 94.56 \\
8.76000 & 54.55 & 103.15 \\
10.10000 & 64.37 & 111.42 \\
11.80000 & 63.76 & 120.29 \\
14.30000 & 62.62 & 131.04 \\
16.80000 & 61.50 & 139.90 \\
25.20000 & 58.76 & 161.81 \\
33.70000 & 57.07 & 177.25 \\
50.50000 & 55.13 & 198.40 \\
84.30000 & 53.49 & 225.00 \\
168.00000 & 52.42 & 260.72 \\
337.00000 & 51.72 & 296.45
\end{tabular}

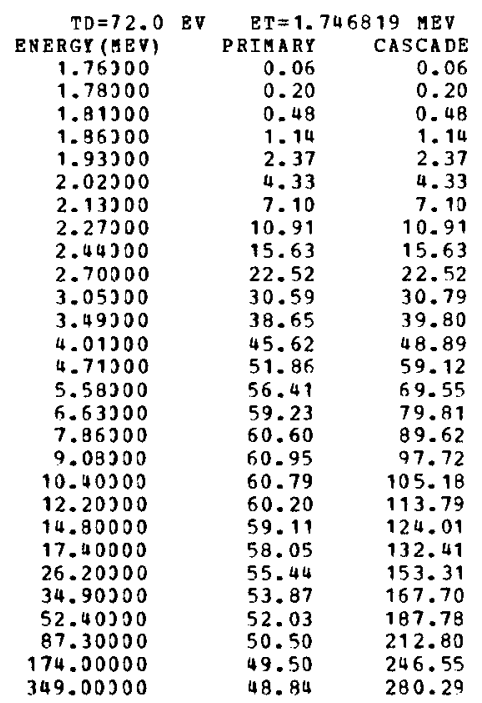


ATOMIC DISPLAZBMENT CROSS-SECTIONS (BARNS) BY FAST ELEZTRONS IN ND

\begin{tabular}{rrr} 
TD=76.0 & \multicolumn{1}{c}{ ET $=1.305559$ MEV } \\
ENERGY(MEV) & PRIMARY & CASCADE \\
1.82000 & 0.06 & 0.06 \\
1.84000 & 0.19 & 0.19 \\
1.87000 & 0.44 & 0.44 \\
1.93000 & 1.17 & 1.17 \\
2.00000 & 2.32 & 2.32 \\
2.09000 & 4.14 & 4.14 \\
2.20000 & 6.69 & 6.69 \\
2.34000 & 10.19 & 10.19 \\
2.52000 & 14.80 & 14.80 \\
2.79000 & 21.38 & 21.38 \\
3.15000 & 29.02 & 29.21 \\
3.61000 & 36.75 & 37.87 \\
4.15000 & 43.37 & 46.54 \\
4.87000 & 49.22 & 56.20 \\
5.77000 & 53.50 & 66.11 \\
6.85000 & 56.15 & 75.88 \\
8.12000 & 57.42 & 85.10 \\
9.38000 & 57.74 & 92.78 \\
10.80000 & 57.56 & 100.12 \\
12.60000 & 57.00 & 108.00 \\
15.30000 & 55.96 & 117.74 \\
18.00000 & 54.95 & 125.73 \\
27.00000 & 52.50 & 145.36 \\
36.10000 & 51.00 & 159.17 \\
54.10000 & 49.28 & 178.11 \\
90.20000 & 47.83 & 201.86 \\
180.00000 & 46.89 & 233.89
\end{tabular}

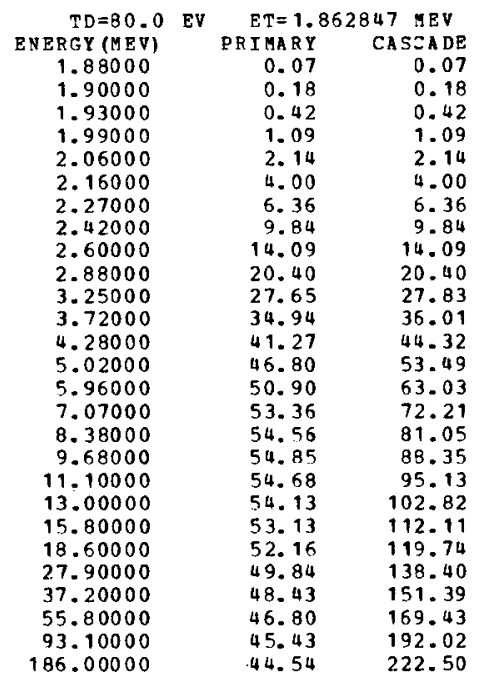

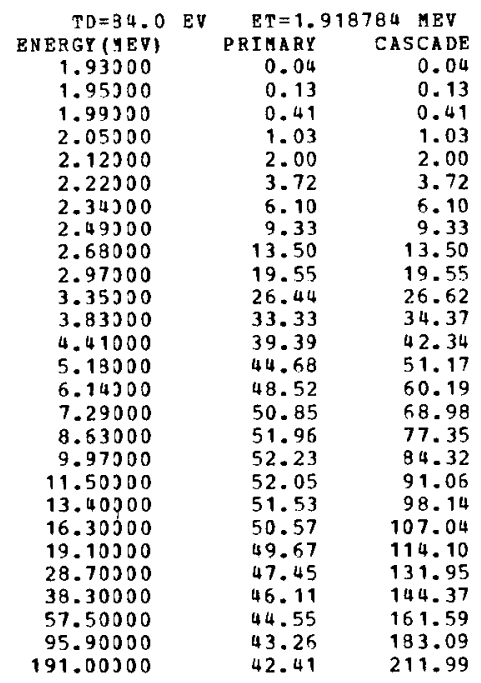

ATOMIC DISPLAZEHENT CROSS-SEETIONS (BARNS) BY FAST ELEETRONS IN ND

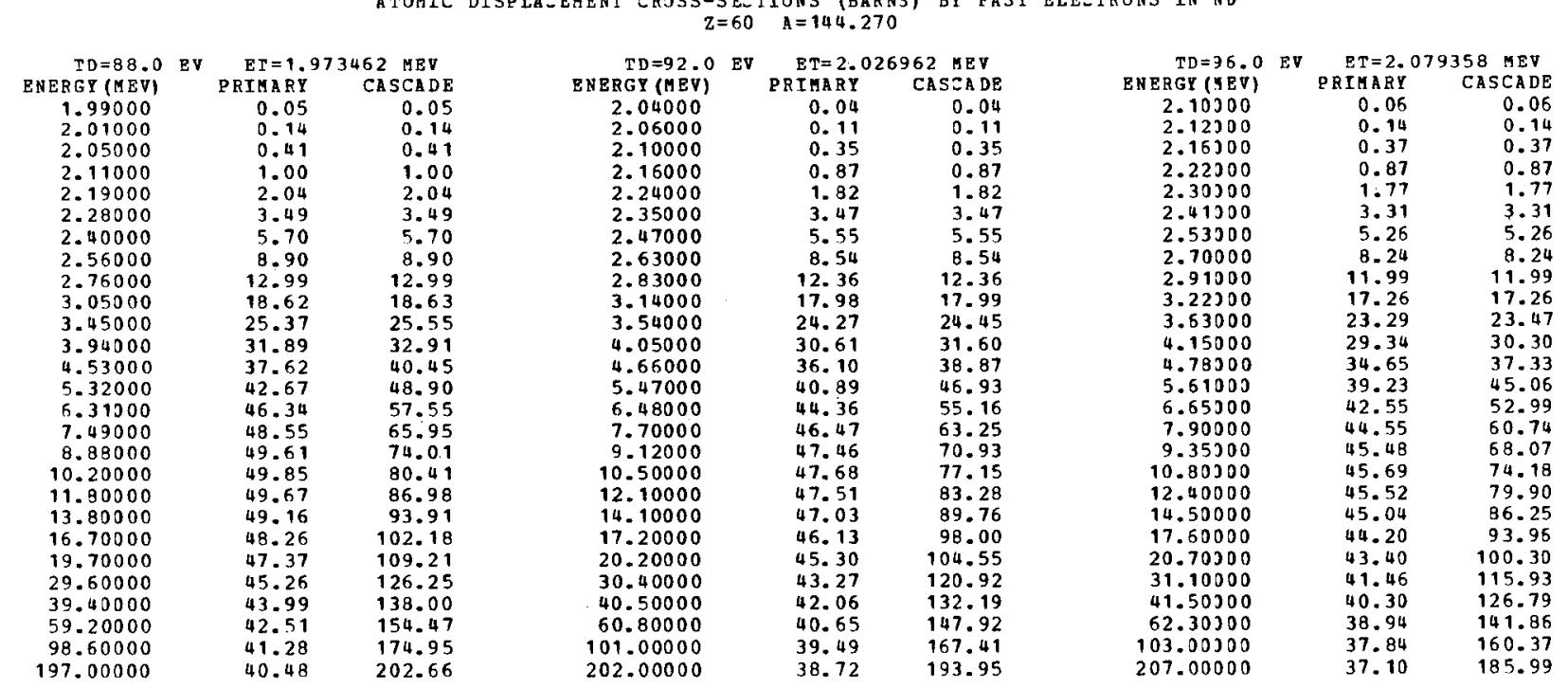


ATOMIC DISPLAZEMENT CROSS-SECTIONS (BARNS) BY PAST EC.BCTRONS IN TB $=65 \quad A=158.930$

\begin{tabular}{rrr}
$T D=4.0$ & \multicolumn{1}{c}{ ET $=0.235409$ MEV } \\
ENERGY(MEV) & PRIMARY & CASCADE \\
0.23700 & 12.45 & 12.45 \\
0.24000 & 35.41 & 35.41 \\
0.24400 & 65.07 & 65.07 \\
0.25100 & 114.53 & 114.53 \\
0.26100 & 180.33 & 180.33 \\
0.27300 & 252.70 & 252.70 \\
0.28700 & 329.38 & 329.38 \\
0.30600 & 422.20 & 422.20 \\
0.32900 & 520.24 & 520.24 \\
0.36400 & 645.53 & 645.53 \\
0.41100 & 779.21 & 779.21 \\
0.47000 & 906.31 & 911.95 \\
0.54100 & 1017.54 & 1042.57 \\
0.63500 & 1117.99 & 1183.20 \\
0.75300 & 1196.62 & 1326.18 \\
0.89400 & 1249.59 & 1465.89 \\
1.05000 & 1278.61 & 1594.75 \\
1.22000 & 1290.55 & 1714.49 \\
1.41000 & 1290.65 & 1830.09 \\
1.64000 & 1281.37 & 1951.69 \\
2.00000 & 1258.46 & 2113.01 \\
2.35000 & 1233.93 & 2246.03 \\
3.53000 & 1163.10 & 2589.34 \\
4.70000 & 1114.80 & 2836.95 \\
7.06000 & 1058.87 & 3197.68 \\
11.70000 & 1006.86 & 3651.63 \\
23.50000 & 964.91 & 4288.38 \\
47.00000 & 946.77 & 4928.82 \\
70.60000 & 938.54 & 5303.37 \\
117.00000 & 927.72 & 5765.39 \\
164.00000 & 920.33 & 6071.16 \\
235.00000 & 913.24 & 6395.11
\end{tabular}

\begin{tabular}{rrr} 
TD $=8.0$ & EV & \multicolumn{1}{c}{ ET $=0.412551$ MEV } \\
BNERGY(MEV) & PRIMARY & CASEADE \\
0.41600 & 4.06 & 4.06 \\
0.42000 & 8.93 & 8.93 \\
0.42900 & 19.99 & 19.99 \\
0.44100 & 34.86 & 34.86 \\
0.45800 & 56.04 & 56.04 \\
0.47800 & 80.86 & 80.86 \\
0.50300 & 111.37 & 117.37 \\
0.53600 & 150.27 & 150.27 \\
0.57700 & 195.79 & 195.79 \\
0.63900 & 257.96 & 257.96 \\
0.72200 & 328.68 & 328.87 \\
0.82500 & 398.75 & 402.84 \\
0.94900 & 462.33 & 477.65 \\
1.11000 & 520.18 & 558.48 \\
1.32000 & 567.76 & 645.05 \\
1.56000 & 598.55 & 726.32 \\
1.85000 & 616.75 & 807.70 \\
2.14000 & 623.58 & 876.20 \\
2.47000 & 624.10 & 943.10 \\
2.88000 & 619.46 & 1014.20 \\
3.50000 & 608.23 & 1104.04 \\
4.12000 & 596.20 & 1179.01 \\
6.18000 & 562.94 & 1364.91 \\
8.25000 & 541.61 & 1497.98 \\
12.30000 & 517.78 & 1682.18 \\
20.60000 & 495.01 & 1919.54 \\
41.20000 & 478.94 & 2240.71 \\
82.50000 & 471.21 & 2562.67 \\
123.00000 & 467.11 & 2746.54 \\
206.00000 & 461.45 & 2981.55 \\
288.00000 & 457.96 & 3132.79 \\
412.00000 & 454.88 & 3293.99
\end{tabular}

\begin{tabular}{|c|c|c|}
\hline$T D=12.0$ & $\mathrm{ET}=0$. & 0978 \\
\hline ENERGY (YEV) & PRIMARY & CASCADE \\
\hline 0.56500 & 2.11 & 2.11 \\
\hline 0.57200 & 4.73 & 4.73 \\
\hline 0.58300 & 9.75 & 9.75 \\
\hline 0.50000 & 18.00 & 18.00 \\
\hline 0.62200 & 29.34 & 29.34 \\
\hline 0.55000 & 44.49 & 44.49 \\
\hline $0.58+00$ & $\begin{array}{l}63.42 \\
88.58\end{array}$ & 63.42 \\
\hline 0.72300 & 88.58 & 88. 58 \\
\hline 0.78500 & 119.01 & 119.01 \\
\hline 0.86900 & 161.31 & 161.31 \\
\hline 0.98100 & 210.12 & 210.40 \\
\hline 1.12300 & 258.95 & 262.34 \\
\hline 1.29300 & 303.89 & 315.99 \\
\hline 1.51300 & 344.17 & 373.87 \\
\hline 1.79000 & 376.03 & 434.45 \\
\hline 2.13000 & 397.17 & 494.60 \\
\hline 2.52300 & 408.32 & 551.37 \\
\hline 2.91000 & 412.05 & 599.04 \\
\hline 3.36200 & 411.65 & 646.04 \\
\hline 3.92300 & 407.85 & 695.77 \\
\hline 4.76300 & 399.87 & 757.78 \\
\hline 5.60000 & 391.69 & 809.24 \\
\hline 8.41000 & 369.93 & 936.72 \\
\hline 11.20300 & 356.71 & 1026.35 \\
\hline 16.80300 & 341.54 & 1152.36 \\
\hline 28.00000 & 327.77 & 1310.30 \\
\hline 56.00000 & 318.36 & 1525.43 \\
\hline 112.00500 & 313.45 & 1739.78 \\
\hline 168.00000 & 310.63 & 1864.12 \\
\hline 280.00300 & 306.88 & 2018.98 \\
\hline 392.00000 & 304.64 & 2120.13 \\
\hline 560.00300 & 302.78 & 2227.21 \\
\hline
\end{tabular}

ATOAIC DISPLAZERENT CROSS-SECTIONS (BARNS) BY PAST ELECTRONS IN TB $z=65 \quad A=158.930$

\begin{tabular}{rrr} 
TD $=16.0$ & EV & \multicolumn{1}{c}{ EI $=0.691141$ MEV } \\
ENERGY (MEV) & PRIMRY & CASCADE \\
0.69800 & 1.39 & 1.39 \\
0.70400 & 2.68 & 2.68 \\
0.71800 & 5.93 & 5.93 \\
0.73900 & 11.36 & 11.36 \\
0.75700 & 19.41 & 19.41 \\
0.80100 & 30.04 & 30.04 \\
0.84300 & 43.93 & 43.93 \\
0.89800 & 62.60 & 62.60 \\
0.96700 & 85.73 & 85.73 \\
1.07000 & 118.09 & 118.09 \\
1.20000 & 153.81 & 154.07 \\
1.38000 & 193.68 & 196.70 \\
1.58000 & 226.83 & 236.74 \\
1.86000 & 258.65 & 283.48 \\
2.21000 & 282.68 & 331.21 \\
2.62000 & 297.68 & 376.87 \\
3.11000 & 305.55 & 421.62 \\
3.59000 & 307.76 & 458.28 \\
4.14000 & 306.97 & 494.06 \\
4.83000 & 303.71 & 532.17 \\
5.87000 & 297.41 & 579.76 \\
6.91000 & 291.13 & 619.10 \\
10.30000 & 275.35 & 714.11 \\
13.80000 & 265.59 & 783.33 \\
20.70000 & 254.63 & 878.29 \\
34.50000 & 244.88 & 997.20 \\
69.10000 & 238.37 & 1159.26 \\
138.00000 & 234.76 & 1319.68 \\
207.00000 & 232.62 & 1412.85 \\
& &
\end{tabular}

\begin{tabular}{rrr} 
TD=20.0 EV & \multicolumn{2}{c}{ ET=0.808526 MEV } \\
ENERG (AEV) & PRIMARY & CASEADE \\
0.81600 & 0.86 & 0.86 \\
0.82400 & 1.87 & 1.87 \\
0.84000 & 4.12 & 4.12 \\
0.86500 & 8.18 & 8.18 \\
0.89700 & 14.16 & 14.16 \\
0.93700 & 22.49 & 22.49 \\
0.98600 & 33.50 & 33.50 \\
1.05000 & 48.44 & 48.44 \\
1.13000 & 67.07 & 67.07 \\
1.25000 & 93.41 & 93.41 \\
1.41000 & 124.05 & 124.35 \\
1.61000 & 154.86 & 157.53 \\
1.85000 & 182.45 & 191.27 \\
2.18000 & 208.05 & 229.81 \\
2.58000 & 226.59 & 267.99 \\
3.07000 & 238.44 & 306.10 \\
3.63000 & 244.13 & 341.73 \\
4.20000 & 245.58 & 372.03 \\
4.85000 & 244.62 & 401.33 \\
5.65000 & 241.79 & 431.92 \\
6.87000 & 236.56 & 470.50 \\
8.08000 & 231.50 & 502.08 \\
12.10000 & 218.91 & 579.53 \\
16.10000 & 211.49 & 633.85 \\
24.20000 & 202.90 & 710.42 \\
40.40000 & 195.40 & 806.08 \\
80.80000 & 190.49 & 935.63 \\
161.00000 & 187.64 & 1063.53 \\
242.00000 & 185.90 & 1138.39
\end{tabular}

\begin{tabular}{|c|c|c|}
\hline$T O=24.0$ & $\mathrm{ET}=0$. & 289 MEV \\
\hline ENERGY (YEV) & PRIMARY & CASCADE \\
\hline 0.92500 & 0.64 & 0.64 \\
\hline $0.9340 n$ & 1.38 & 1.38 \\
\hline 0.95200 & 3.08 & 3.08 \\
\hline 0.99000 & 6.24 & 6.24 \\
\hline 1.01000 & 10.17 & 10.17 \\
\hline 1.06300 & 17.65 & 17.65 \\
\hline 1.11300 & 25.86 & 25.86 \\
\hline 1.19300 & 39.69 & 39.69 \\
\hline 1.28500 & 55.33 & 55.33 \\
\hline 1.42300 & 78.28 & 78.23 \\
\hline 1.60000 & 103.97 & 104.29 \\
\hline 1.83000 & 130.25 & 132.80 \\
\hline 2.10300 & 153.10 & 169.14 \\
\hline 2.47000 & 174.12 & 193.45 \\
\hline 2.93300 & 189.51 & 226.21 \\
\hline 3.43300 & 198.92 & 258.08 \\
\hline 4.12000 & 203.35 & 288.36 \\
\hline 4.75000 & 204.28 & 313.63 \\
\hline 5.49000 & 203.29 & 338.07 \\
\hline 6.41300 & 200.75 & 364.16 \\
\hline 7.78000 & 195.32 & 396.23 \\
\hline 9.16000 & 192.04 & 422.87 \\
\hline 13.70000 & 181.69 & 487.53 \\
\hline 18.30000 & 175.55 & 533.54 \\
\hline 27.40300 & 168.60 & 596.85 \\
\hline 45.30000 & 162.54 & 676.88 \\
\hline 91.50300 & 158.61 & 784.93 \\
\hline 183.00000 & 156.24 & 891.90 \\
\hline 274.00000 & 154.79 & 953.66 \\
\hline
\end{tabular}


ATOMIC DISPlazenENT CROSS-SECTIONS (BARNS) BY PAST ELECTRONS IN TB $Z=65 \quad A=158.930$

\begin{tabular}{rrr} 
TD $=28.0$ & EV & \multicolumn{2}{c}{ ET $=1.016468$ MEV } \\
ENERGY MEVI & PRIMARY & CASCADE \\
1.02000 & 0.17 & 0.17 \\
1.03000 & 0.70 & 0.70 \\
1.05000 & 1.98 & 1.98 \\
1.08000 & 4.37 & 4.37 \\
1.12000 & 8.27 & 8.27 \\
1.17000 & 13.96 & 13.96 \\
1.24000 & 22.88 & 22.88 \\
1.32000 & 33.69 & 33.69 \\
1.42000 & 47.28 & 47.28 \\
1.57000 & 66.58 & 66.58 \\
1.77000 & 89.03 & 89.32 \\
2.03000 & 112.25 & 114.63 \\
2.33000 & 131.94 & 139.31 \\
2.74000 & 149.86 & 167.33 \\
3.25000 & 162.86 & 195.72 \\
3.86000 & 170.68 & 223.32 \\
4.57000 & 174.24 & 249.51 \\
5.28000 & 174.86 & 271.34 \\
6.09000 & 173.87 & 292.44 \\
7.11000 & 171.59 & 314.92 \\
8.63000 & 167.72 & 342.56 \\
10.10000 & 164.16 & 364.64 \\
15.20000 & 155.23 & 421.12 \\
20.30000 & 150.05 & 460.62 \\
30.40000 & 144.18 & 514.97 \\
50.80000 & 139.13 & 583.61 \\
101.00000 & 135.89 & 675.49 \\
203.00000 & 133.84 & 767.97
\end{tabular}

\begin{tabular}{rrr} 
TD=32.0 & \multicolumn{1}{c}{ EV } & ET=1.110470 IEY \\
ENERGY (NEV) & PRIMARY & CASCADE \\
1.12000 & 0.34 & 0.34 \\
1.13000 & 0.76 & 0.76 \\
1.15000 & 1.76 & 1.75 \\
1.18000 & 3.63 & 3.63 \\
1.23000 & 7.52 & 7.52 \\
1.28000 & 12.12 & 12.12 \\
1.35000 & 19.28 & 19.28 \\
1.44000 & 29.10 & 29.10 \\
1.55000 & 41.21 & 41.21 \\
1.72000 & 58.92 & 58.92 \\
1.94000 & 78.81 & 79.12 \\
2.22000 & 98.89 & 101.15 \\
2.55000 & 116.21 & 123.10 \\
2.99000 & 131.53 & 147.42 \\
3.55000 & 142.83 & 172.64 \\
4.21000 & 149.44 & 196.76 \\
4.99000 & 152.42 & 219.99 \\
5.77000 & 152.83 & 239.32 \\
6.66000 & 151.85 & 257.96 \\
7.77000 & 149.79 & 277.63 \\
9.43000 & 146.35 & 301.89 \\
11.10000 & 143.11 & $\mathbf{3 2 1 . 9 7}$ \\
16.60000 & 135.48 & $\mathbf{3 7 0 . 7 5}$ \\
22.20000 & 130.98 & $\mathbf{4 0 5 . 5 2}$ \\
33.30000 & 125.90 & 453.32 \\
55.50000 & 121.61 & 513.12 \\
111.00000 & 118.83 & 594.24 \\
222.00000 & 117.05 & 674.57
\end{tabular}

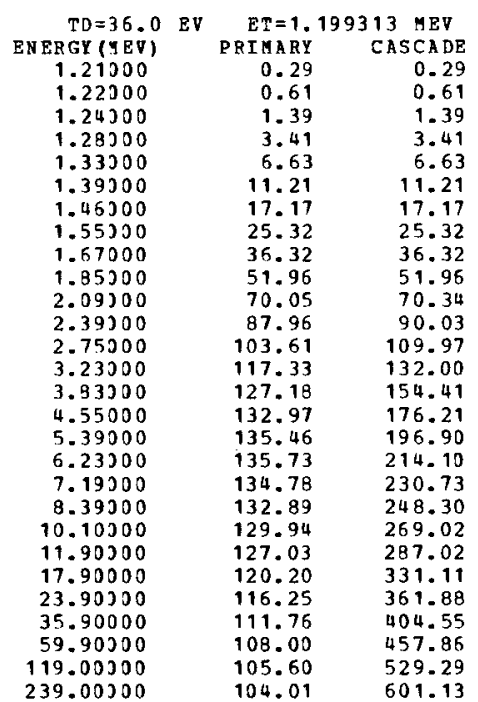

ATOMIC DISPLACEMENT CROSS-SECTIONS (BARNS) BY FAST ELECTRONS IN TB $=65 \quad A=158.930$

\begin{tabular}{rrr} 
TD=40.0 & EV & \multicolumn{2}{c}{ ET $=1.283763$ MEV } \\
PRIMARY & CASCADE \\
1.29000 & 0.12 & 0.12 \\
1.30000 & 0.36 & 0.36 \\
1.33000 & 1.28 & 1.28 \\
1.37000 & 2.96 & 2.96 \\
1.42000 & 5.63 & 5.63 \\
1.48000 & 9.43 & 9.43 \\
1.56000 & 15.16 & 15.16 \\
1.66000 & 22.83 & 22.83 \\
1.79000 & 32.94 & 32.94 \\
1.98000 & 46.93 & 46.93 \\
2.24000 & 63.52 & 63.81 \\
2.56000 & 79.61 & 81.59 \\
2.95000 & 93.82 & 99.85 \\
3.46000 & 105.96 & 119.64 \\
4.10000 & 114.68 & 139.84 \\
4.87000 & 119.76 & 159.54 \\
5.77000 & 121.90 & 178.26 \\
6.67000 & 122.06 & 193.81 \\
7.70000 & 121.14 & 208.85 \\
8.98000 & 119.41 & 224.65 \\
10.90000 & 116.59 & 244.16 \\
12.80000 & 114.02 & 260.06 \\
19.20000 & 107.95 & 299.52 \\
25.60000 & 104.45 & 327.10 \\
38.50000 & 100.44 & 365.64 \\
64.10000 & 97.13 & 413.44 \\
128.00000 & 94.99 & 178.23 \\
256.00000 & 93.58 & 542.49
\end{tabular}

\begin{tabular}{rrr} 
TD=44.0 & EV & \multicolumn{1}{c}{ ET=1.3644 15 MEV } \\
ENERGY (MEV) & PRIMAY & CASZADE \\
1.37000 & 0.09 & 0.09 \\
1.39000 & 0.49 & 0.49 \\
1.41000 & 1.01 & 1.01 \\
1.45000 & 2.38 & 2.38 \\
1.51000 & 5.07 & 5.07 \\
1.58000 & 8.91 & 8.91 \\
1.66000 & 13.86 & 13.86 \\
1.77000 & 21.14 & 21.14 \\
1.91000 & 30.52 & 30.52 \\
2.11000 & 43.18 & 43.18 \\
2.38000 & 57.98 & 58.26 \\
2.72000 & 72.66 & 74.54 \\
3.13000 & 85.47 & 91.09 \\
3.68000 & 96.63 & 109.45 \\
4.36000 & 104.44 & 127.89 \\
5.18000 & 108.96 & 145.90 \\
6.13000 & 110.80 & 162.85 \\
7.09000 & 110.88 & 177.08 \\
8.18000 & 110.01 & 190.73 \\
9.55000 & 108.39 & 205.22 \\
11.50000 & 105.92 & 222.27 \\
13.60000 & 103.48 & 237.38 \\
20.40000 & 97.99 & 273.31 \\
27.20000 & 94.83 & 298.40 \\
40.90000 & 91.22 & 333.44 \\
68.20000 & 88.24 & 377.05 \\
136.00000 & 86.33 & 435.84 \\
272.00000 & 85.05 & 494.25
\end{tabular}

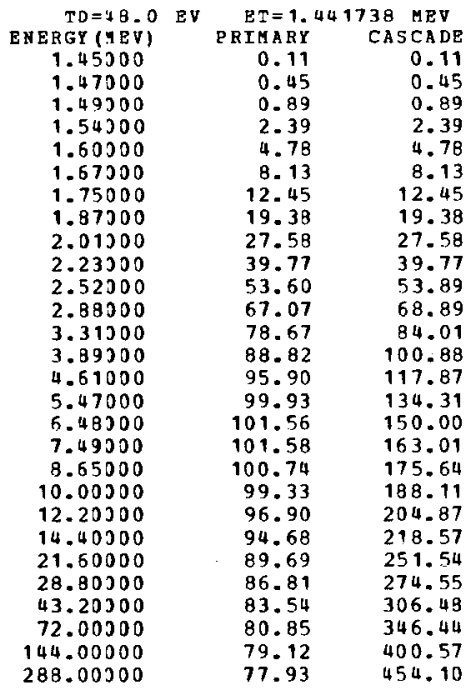


ATOMIC DISPLAZEMENT CROSS-SECTIONS (BARNS) BY FAST ELECTRONS IN TB

\begin{tabular}{rrr}
$\begin{array}{c}\text { TD }=52.0 \\
\text { ENERGY EVEV }\end{array}$ & \multicolumn{2}{c}{ ET $=1.516114$ MEV } \\
PRTMART & CASCADE \\
1.53000 & 0.16 & 0.16 \\
1.54000 & 0.30 & 0.30 \\
1.57000 & 0.86 & 0.86 \\
1.62000 & 2.18 & 2.18 \\
1.68000 & 4.27 & 4.27 \\
1.75000 & 7.20 & 7.20 \\
1.84000 & 11.46 & 11.46 \\
1.97000 & 18.10 & 18.10 \\
2.12000 & 25.86 & 25.86 \\
2.34000 & 36.62 & 36.62 \\
2.65000 & 49.68 & 49.97 \\
3.03000 & 62.18 & 63.94 \\
3.48000 & 72.83 & 77.89 \\
4.09000 & 82.16 & 93.53 \\
4.85000 & 88.66 & 109.33 \\
5.75000 & 92.31 & 124.62 \\
6.82000 & 93.74 & 139.09 \\
7.88000 & 93.72 & 151.10 \\
9.09000 & 92.92 & 162.68 \\
10.60000 & 91.51 & 174.90 \\
12.80000 & 89.36 & 189.58 \\
15.10000 & 87.32 & 202.20 \\
22.70000 & 82.70 & 232.83 \\
30.30000 & 80.05 & 254.16 \\
45.40000 & 77.06 & 283.57 \\
75.80000 & 74.59 & 320.59 \\
151.00000 & 73.02 & 370.28 \\
303.00000 & 71.92 & 419.93 \\
& &
\end{tabular}

\begin{tabular}{crr} 
TD=56.0 EV & \multicolumn{1}{c}{ ET=1.587856 YEV } \\
ENERGY (HEV) & PRIMARY & CASCADE \\
1.50000 & 0.11 & 0.11 \\
1.61000 & 0.23 & 0.23 \\
1.65000 & 0.89 & 0.89 \\
1.69000 & 1.82 & 1.82 \\
1.76000 & 3.95 & 3.95 \\
1.84000 & 6.94 & 6.94 \\
1.93000 & 10.76 & 10.76 \\
2.06000 & 16.68 & 16.68 \\
2.22000 & 24.07 & 24.07 \\
2.46000 & 34.51 & 34.52 \\
2.77000 & 46.13 & 46.40 \\
3.17000 & 57.85 & 59.52 \\
3.65000 & 67.91 & 72.77 \\
4.28000 & 76.41 & 87.13 \\
5.08000 & 82.43 & 101.94 \\
6.03000 & 85.76 & 116.13 \\
7.14000 & 87.03 & 129.50 \\
8.25000 & 86.98 & 140.77 \\
9.52000 & 86.21 & 151.57 \\
11.10000 & 84.89 & 162.92 \\
13.40000 & 82.89 & 176.54 \\
15.80000 & 80.99 & 188.24 \\
23.80000 & 76.70 & 216.85 \\
31.70000 & 74.27 & 236.51 \\
47.60000 & 71.49 & 263.98 \\
79.30000 & 69.24 & 298.21 \\
158.00000 & 67.79 & 344.37 \\
317.00000 & 66.77 & 390.46
\end{tabular}

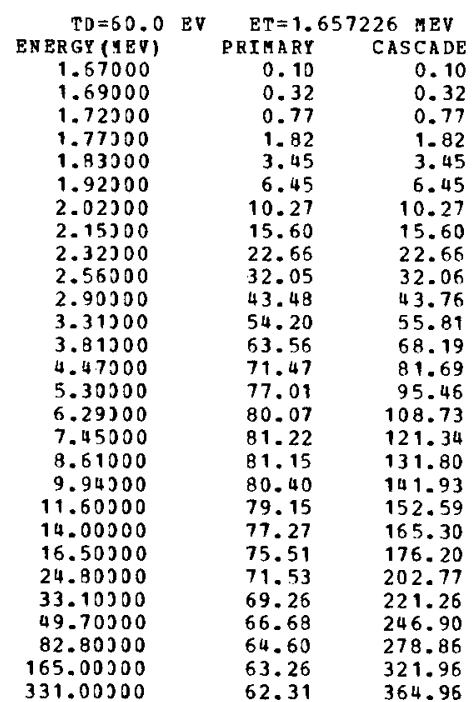

ATOMIC DISPLAZEMENT CROSS-SECTIONS (BARNS) BY FAST ELECTRONS IN TB $\mathrm{Z}=65 \quad \mathrm{~A}=158.930$

\begin{tabular}{rrr} 
TD=64.0 & EV & \multicolumn{1}{c}{ ET $=1.724444$ MEV } \\
ENERGY(HEV) & PRIMARY & CASCADE \\
1.74000 & 0.11 & 0.11 \\
1.75000 & 0.20 & 0.20 \\
1.79000 & 0.71 & 0.71 \\
1.84000 & 1.64 & 1.64 \\
1.91000 & 3.37 & 3.37 \\
2.00000 & 6.10 & 6.10 \\
2.10000 & 9.56 & 9.56 \\
2.24000 & 14.76 & 14.76 \\
2.41000 & 21.16 & 21.16 \\
2.67000 & 30.36 & 30.36 \\
3.01000 & 40.69 & 40.96 \\
3.44000 & 50.88 & 52.41 \\
3.96000 & 59.66 & 64.07 \\
4.65000 & 67.11 & 76.83 \\
5.51000 & 72.25 & 89.72 \\
6.55000 & 75.10 & 102.31 \\
7.75000 & 76.14 & 114.08 \\
8.96000 & 76.04 & 123.92 \\
10.30000 & 75.36 & 133.15 \\
12.00000 & 74.19 & 143.05 \\
14.60000 & 72.35 & 155.49 \\
17.20000 & 70.70 & 165.69 \\
25.80000 & 67.01 & 190.49 \\
34.40000 & 64.89 & 207.77 \\
51.70000 & 62.48 & 231.87 \\
86.20000 & 60.54 & 261.89 \\
172.00000 & 59.29 & 302.37 \\
344.00000 & 58.41 & 342.51
\end{tabular}

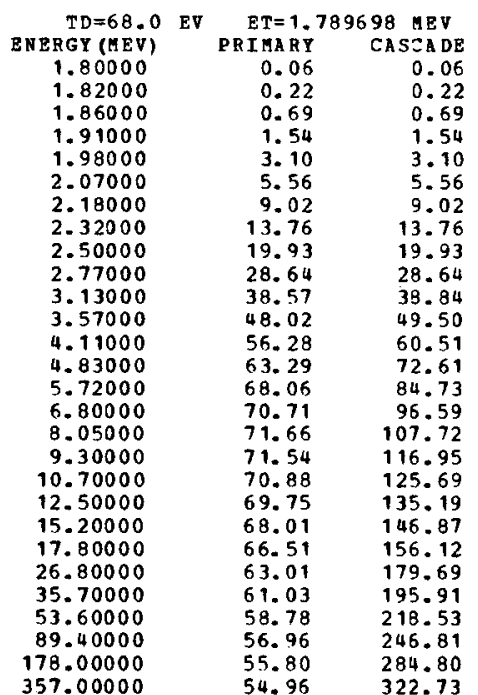

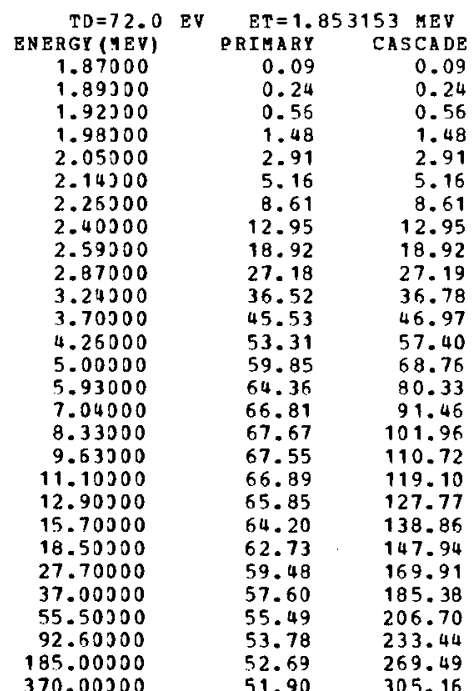


ATOMIC DISPLA ERENT CROSS-SECTIONS (BARNS) BY PAST ELECTRONS IN TB

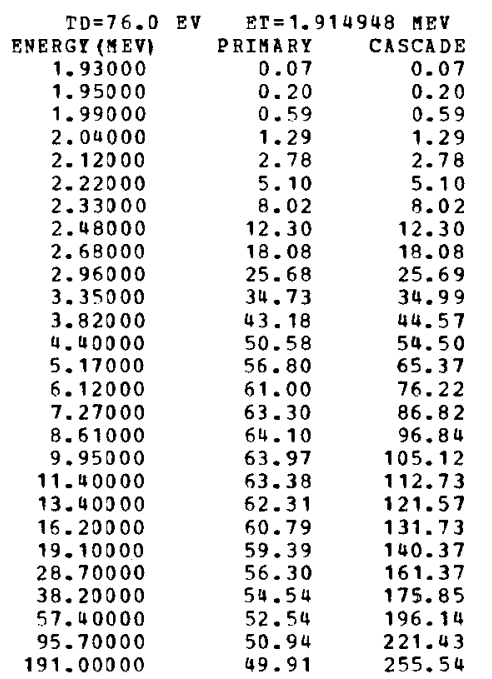

\begin{tabular}{rrr} 
TD $=80.0$ & EV & \multicolumn{2}{c}{ ET=1.975208 IEV } \\
ENERGY (MEV) & PRIMAY & CASZADE \\
1.99000 & 0.06 & 0.06 \\
2.01000 & 0.18 & 0.18 \\
2.05000 & 0.53 & 0.53 \\
2.11000 & 1.30 & 1.30 \\
2.19000 & 2.69 & 2.69 \\
2.29000 & 4.84 & 4.84 \\
2.40000 & 7.54 & 7.54 \\
2.56000 & 11.76 & 11.76 \\
2.76000 & 17.10 & 17.10 \\
3.06000 & 24.63 & 24.63 \\
3.45000 & 32.98 & 33.23 \\
3.95000 & 41.26 & 42.63 \\
4.54000 & 48.16 & 51.94 \\
5.33000 & 54.01 & 62.23 \\
6.32000 & 58.01 & 72.66 \\
7.50000 & 60.16 & 82.68 \\
8.88000 & 60.89 & 92.19 \\
10.20000 & 60.77 & 99.74 \\
11.80000 & 60.16 & 107.49 \\
13.80000 & 59.17 & 115.63 \\
16.70000 & 57.71 & 125.33 \\
19.70000 & 56.38 & 133.57 \\
29.50000 & 53.45 & 153.54 \\
39.50000 & 51.77 & 167.41 \\
59.20000 & 49.89 & 186.57 \\
98.70000 & 48.38 & 210.60 \\
197.00000 & 47.41 & 243.01
\end{tabular}

\begin{tabular}{|c|c|c|c|}
\hline \multirow{2}{*}{$\begin{array}{r}T D=34.0 \\
\text { EN ERGY (9EV) }\end{array}$} & \multirow[t]{2}{*}{ EV } & \multicolumn{2}{|c|}{$\mathrm{ET}=2.034041 \mathrm{MEV}$} \\
\hline & & PRIMARY & CASCADE \\
\hline 2.05000 & & 0.05 & 0.06 \\
\hline 2.07000 & & 0.17 & 0.17 \\
\hline 2.11$) 00$ & & 0.49 & 0.49 \\
\hline 2.17300 & & 1.19 & 1.19 \\
\hline 2.25300 & & 2.46 & 2.46 \\
\hline 2.35300 & & 4.44 & 4.44 \\
\hline 2.49000 & & 7.39 & 7.39 \\
\hline 2.64300 & & 11.32 & 11.32 \\
\hline 2.84300 & & 16.23 & 16.28 \\
\hline 3.15300 & & 23.50 & 23.50 \\
\hline 3.55000 & & 31.44 & 31.68 \\
\hline 4.05000 & & 39.28 & 40.58 \\
\hline 4.67000 & & 45.89 & 49.52 \\
\hline 5.49000 & & 51.51 & 59.42 \\
\hline 6.50300 & & 55.27 & 69.28 \\
\hline 7.72000 & & 57.30 & 78.89 \\
\hline 9.15000 & & 57.99 & 88.01 \\
\hline 10.50000 & & 57.86 & 95.16 \\
\hline 12.20000 & & 57.25 & 102.76 \\
\hline 14.20000 & & 56.33 & $110.2 B$ \\
\hline 17.20000 & & 54.93 & 119.57 \\
\hline 20.30000 & & 53.66 & 127.44 \\
\hline 30.50000 & & 50.87 & 146.46 \\
\hline 40.60000 & & 49.29 & 159.56 \\
\hline 61.00000 & & 47.50 & 177.92 \\
\hline 101.00300 & & 46.08 & 200.51 \\
\hline 203.00000 & & 45.15 & 231.68 \\
\hline
\end{tabular}

ATOMIC DISPLAZEHENT CROSS-SECTIONS (BARNS) BY FAST ELECTRONS IN TB $Z=65 \quad A=158.930$

\begin{tabular}{rrr} 
TD=38.0 EV & \multicolumn{2}{c}{ ET $=2.091545$ MEV } \\
ENERGY (MEV) & PRIMARY & CASCADE \\
2.11000 & 0.05 & 0.06 \\
2.13000 & 0.16 & 0.16 \\
2.17000 & 0.46 & 0.46 \\
2.23000 & 1.11 & 1.11 \\
2.32000 & 2.45 & 2.45 \\
2.42000 & 4.31 & 4.31 \\
2.55000 & 7.06 & 7.06 \\
2.71000 & 10.72 & 10.72 \\
2.92000 & 15.57 & 15.57 \\
3.24000 & 22.51 & 22.51 \\
3.66000 & 30.24 & 30.49 \\
4.18000 & 37.63 & 38.91 \\
4.81000 & 43.94 & 47.49 \\
5.54000 & 49.20 & 56.80 \\
6.69000 & 52.80 & 66.33 \\
7.94000 & 54.71 & 75.47 \\
9.41000 & 55.35 & 84.18 \\
10.80000 & 55.22 & 91.01 \\
12.50000 & 54.64 & 98.09 \\
14.60000 & 53.74 & 105.44 \\
17.70000 & 52.40 & 114.35 \\
20.90000 & 51.18 & 121.89 \\
31.30000 & 48.55 & 139.90 \\
41.80000 & 47.03 & 152.55 \\
62.70000 & 45.32 & 170.01 \\
104.00000 & 43.98 & 191.54 \\
209.00000 & 43.09 & 221.40 \\
& &
\end{tabular}

\begin{tabular}{rrr} 
TD $=92.0$ & EV & \multicolumn{1}{c}{ ET=2.147805 MEV } \\
ENERGY (MEV) & PRIMAY & CASEADE \\
2.16000 & 0.03 & 0.03 \\
2.19000 & 0.17 & 0.17 \\
2.23000 & 0.45 & 0.45 \\
2.29000 & 1.06 & 1.06 \\
2.38000 & 2.30 & 2.30 \\
2.49000 & 4.21 & 4.21 \\
2.62000 & 6.79 & 6.79 \\
2.79000 & 10.43 & 10.43 \\
3.00000 & 14.96 & 14.96 \\
3.32000 & 21.44 & 21.45 \\
3.75000 & 28.86 & 29.09 \\
4.29000 & 36.04 & 37.28 \\
4.93000 & 42.03 & 45.42 \\
5.79000 & 47.10 & 54.43 \\
6.87000 & 50.54 & 63.57 \\
8.16000 & 52.35 & 72.36 \\
9.66000 & 52.94 & 80.64 \\
11.10000 & 52.80 & 87.24 \\
12.80000 & 52.26 & 93.85 \\
15.00000 & 51.38 & 101.03 \\
18.20000 & 50.08 & 109.59 \\
21.40000 & 48.95 & 116.52 \\
32.20000 & 46.40 & 134.06 \\
42.90000 & 44.96 & 146.06 \\
64.40000 & 43.34 & 162.80 \\
107.00000 & 42.05 & 183.56 \\
214.00000 & 41.21 & 211.83
\end{tabular}

\begin{tabular}{|c|c|c|}
\hline \multirow{2}{*}{$\begin{array}{r}T D=36.0 \\
\text { ENERGY (3EV) }\end{array}$} & \multicolumn{2}{|c|}{$\mathrm{ET}=2.202900$} \\
\hline & PRIMARY & CASCADE \\
\hline 2.22000 & 0.05 & 0.05 \\
\hline 2.24200 & 0.13 & 0.13 \\
\hline 2.29000 & 0.45 & 0.45 \\
\hline 2.35000 & 1.02 & 1.02 \\
\hline 2.44000 & 2,19 & 2.19 \\
\hline 2.55000 & 3.97 & 3.97 \\
\hline 2.68000 & 6.38 & 6.38 \\
\hline 2.85000 & 9.98 & 9.98 \\
\hline 3.08000 & 14.43 & 14.43 \\
\hline 3.41300 & 20.69 & 20.69 \\
\hline 3.85000 & 27.78 & 28.01 \\
\hline 4.40000 & 34.60 & 35.80 \\
\hline 5.06000 & 40.36 & 43.66 \\
\hline 5.94000 & 45.19 & 52.28 \\
\hline 7.04000 & 48.45 & 69.00 \\
\hline 8.37000 & 50.18 & 69.48 \\
\hline 9.91300 & 50.73 & 77.42 \\
\hline 11.40500 & 50.59 & 33.80 \\
\hline 13.20300 & 50.04 & 90.31 \\
\hline 15.40000 & 49.21 & 97.00 \\
\hline 18.70000 & 47.96 & 105.24 \\
\hline 22.00300 & 46.87 & 112.00 \\
\hline 33.00300 & 44.45 & 128.59 \\
\hline 44.00000 & 43.07 & 140.13 \\
\hline 66.00300 & 41.52 & 156.13 \\
\hline 110.00300 & 40.29 & 176.16 \\
\hline 220.00500 & 39.49 & 203.24 \\
\hline
\end{tabular}


ATONIC DISPLACBHENT CROSS-SECTIONS (BARNS) BY PAST ELECTRONS IN YB $\mathrm{Z}=70 \quad \mathrm{~A}=173.040$

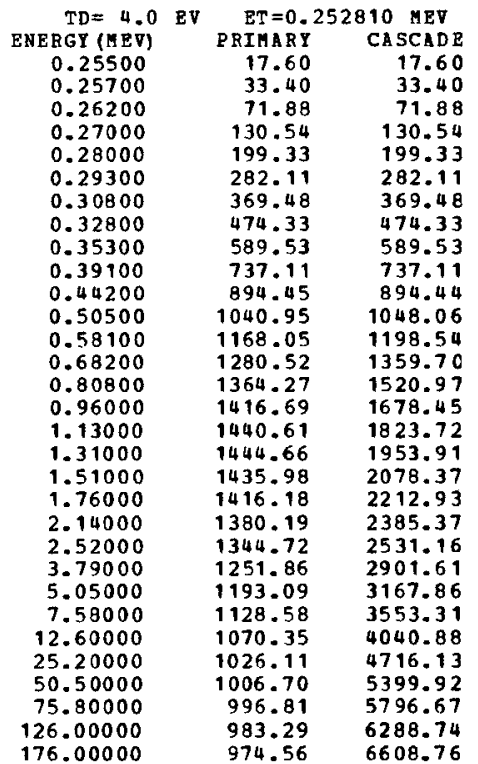

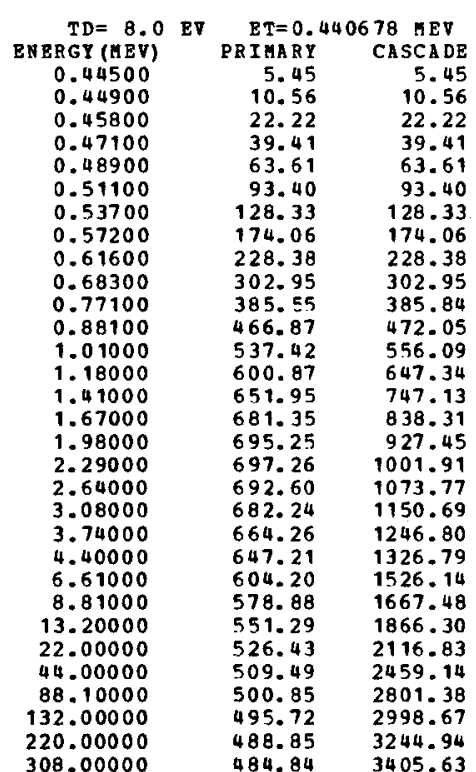

$T D=12.0 \mathrm{BV}$

\begin{tabular}{rrr} 
TD=12.0 & \multicolumn{1}{c}{ ET $=0.597141$ MEV } \\
ENERGY (HEV) & PRIMARY & CASCADE \\
0.60300 & 2.59 & 2.59 \\
0.60900 & 5.36 & 5.36 \\
0.62100 & 11.22 & 11.22 \\
0.63800 & 20.17 & 20.17 \\
0.66200 & 33.77 & 33.77 \\
0.69200 & 51.83 & 51.83 \\
0.72800 & 74.29 & 74.29 \\
0.77600 & 104.48 & 104.48 \\
0.83500 & 140.54 & 140.54 \\
0.92500 & 191.26 & 191.26 \\
1.04000 & 246.88 & 247.22 \\
1.19000 & 304.44 & 308.66 \\
1.37000 & 355.01 & 369.97 \\
1.61000 & 399.77 & 437.01 \\
1.91000 & 432.27 & 504.79 \\
2.26000 & 451.09 & 568.87 \\
2.68000 & 459.30 & 631.65 \\
3.10000 & 459.70 & 683.88 \\
3.58000 & 455.68 & 734.57 \\
4.17000 & 448.15 & 787.39 \\
5.07000 & 435.69 & 854.20 \\
5.97000 & 424.23 & 909.44 \\
8.95000 & 396.71 & 1044.78 \\
11.90000 & 381.13 & 1139.83 \\
17.90000 & 363.73 & 1274.97 \\
29.80000 & 348.63 & 1442.82 \\
59.70000 & 338.70 & 1672.57 \\
119.00000 & 333.10 & 1899.20 \\
179.00000 & 329.57 & 2031.83 \\
298.00000 & 325.05 & 2195.29 \\
417.00000 & 322.56 & 2302.20
\end{tabular}

ATONIC DISPLACEMENT CROSS-SECTIONS (BARNS) BY FAST RLECTRONS IN YB $=70 \quad A=173.040$

\begin{tabular}{rrr} 
TD $=16.0$ & EV & \multicolumn{1}{c}{ ET $=0.734095$ MEV } \\
ENERGY (MEV) & PRIMARY & CASCADE \\
0.74100 & 1.47 & 1.47 \\
0.74800 & 3.07 & 3.07 \\
0.76300 & 6.84 & 6.84 \\
0.78500 & 13.10 & 13.10 \\
0.81400 & 22.39 & 22.39 \\
0.85100 & 35.41 & 35.41 \\
0.89500 & 51.89 & 51.89 \\
0.95400 & 74.59 & 74.59 \\
1.02000 & 99.66 & 99.66 \\
1.13000 & 138.70 & 138.70 \\
1.28000 & 184.38 & 184.78 \\
1.46000 & 227.64 & 231.32 \\
1.68000 & 266.21 & 278.78 \\
1.98000 & 300.56 & 331.75 \\
2.34000 & 324.04 & 383.09 \\
2.78000 & 337.80 & 433.83 \\
3.30000 & 343.10 & 482.46 \\
3.81000 & 342.72 & 522.10 \\
4.40000 & 339.20 & 560.93 \\
5.13000 & 333.13 & 601.59 \\
6.23000 & 323.63 & 652.23 \\
7.34000 & 314.97 & 694.39 \\
11.00000 & 294.88 & 796.98 \\
14.60000 & 283.77 & 868.37 \\
22.00000 & 271.16 & 970.61 \\
36.70000 & 260.47 & 1097.45 \\
73.40000 & 253.61 & 1269.71 \\
146.00000 & 249.45 & 1439.10 \\
& &
\end{tabular}

\begin{tabular}{rrr} 
TD $=20.0$ & EV & \multicolumn{2}{c}{ ET=0.8574 10 MEV } \\
ENERGY (IIEV) & PRIARY & CASCADE \\
0.86500 & 0.92 & 0.92 \\
0.87400 & 2.13 & 2.13 \\
0.89100 & 4.74 & 4.74 \\
0.91700 & 9.44 & 9.44 \\
0.95100 & 16.58 & 16.58 \\
0.99400 & 26.75 & 26.75 \\
1.04000 & 38.49 & 38.49 \\
1.11000 & 57.08 & 57.08 \\
1.20000 & 80.85 & 80.85 \\
1.32000 & 110.52 & 110.52 \\
1.50000 & 148.70 & 149.14 \\
1.71000 & 183.59 & 187.05 \\
1.97000 & 214.76 & 226.18 \\
2.31000 & 241.20 & 268.13 \\
2.74000 & 259.93 & 310.95 \\
3.25000 & 270.19 & 351.89 \\
3.85000 & 273.81 & 390.95 \\
4.45000 & 273.10 & 423.38 \\
5.14000 & 269.96 & 454.87 \\
6.00000 & 264.84 & 488.03 \\
7.28000 & 257.14 & 528.74 \\
8.57000 & 250.25 & 562.58 \\
12.80000 & 234.60 & 644.52 \\
17.10000 & 225.78 & 703.19 \\
25.70000 & 216.04 & 784.66 \\
42.80000 & 207.88 & 886.03 \\
85.70000 & 202.67 & 1024.22 \\
171.00000 & 199.33 & 1160.30
\end{tabular}

\begin{tabular}{rrr}
$T D=24.0$ & \multicolumn{2}{c}{ ET $=0.970497 M E V$} \\
ENERGY (MEV) & PRIMARY & CASCADE \\
0.98000 & 0.74 & 0.74 \\
0.98900 & 1.53 & 1.53 \\
1.00000 & 2.63 & 2.63 \\
1.03000 & 6.20 & 6.20 \\
1.07000 & 12.04 & 12.04 \\
1.12000 & 20.55 & 20.55 \\
1.18000 & 31.83 & 31.83 \\
1.26000 & 47.66 & 47.66 \\
1.35000 & 65.44 & 65.44 \\
1.50000 & 93.21 & 93.21 \\
1.69000 & 123.35 & 123.75 \\
1.94000 & 154.30 & 157.59 \\
2.23000 & 179.91 & 190.20 \\
2.62000 & 202.00 & 226.12 \\
3.10000 & 217.01 & 261.77 \\
3.68000 & 225.18 & 296.48 \\
4.36000 & 227.80 & 329.40 \\
5.04000 & 226.92 & 356.70 \\
5.82000 & 224.09 & 383.17 \\
6.79000 & 219.71 & 410.86 \\
8.24000 & 213.22 & 445.03 \\
9.70000 & 207.47 & 473.37 \\
14.50000 & 194.61 & 542.12 \\
19.40000 & 187.39 & 591.38 \\
29.10000 & 179.50 & 659.05 \\
48.50000 & 172.92 & 743.79 \\
97.00000 & 168.76 & 858.84 \\
194.00000 & 165.96 & 972.58
\end{tabular}


ATOHIC DISPLACEMENT CROSS-SECTIONS (BARNS) BY FAST ELECTRONS IN YB $=70 \quad A=173.040$

\begin{tabular}{rrr} 
TD $=28.0$ & EV & \multicolumn{2}{c}{ ET $=1.075542$ MEV } \\
ENERGT (HEV) & PRIMARY & CASCADE \\
1.08000 & 0.23 & 0.23 \\
1.09000 & 0.80 & 0.80 \\
1.11000 & 2.19 & 2.19 \\
1.15000 & 5.85 & 5.85 \\
1.19000 & 10.40 & 10.40 \\
1.24000 & 16.99 & 16.99 \\
1.31000 & 27.22 & 27.22 \\
1.39000 & 39.59 & 39.59 \\
1.50000 & 56.62 & 56.62 \\
1.66000 & 79.83 & 79.83 \\
1.88000 & 107.07 & 107.50 \\
2.15000 & 133.00 & 136.07 \\
2.47000 & 154.89 & 164.26 \\
2.90000 & 173.54 & 195.28 \\
3.44000 & 186.42 & 226.61 \\
4.08000 & 193.05 & 256.46 \\
4.83000 & 195.01 & 284.77 \\
5.59000 & 194.05 & 308.52 \\
6.45000 & 191.50 & 331.16 \\
7.52000 & 187.67 & 354.97 \\
9.14000 & 182.01 & 384.62 \\
10.70000 & 177.25 & 408.17 \\
16.10000 & 166.20 & 468.26 \\
21.50000 & 160.16 & 510.29 \\
32.20000 & 153.53 & 568.14 \\
53.70000 & 148.03 & 640.94 \\
107.00000 & 144.58 & 739.08 \\
215.00000 & 142.16 & 837.20
\end{tabular}

\begin{tabular}{rrr}
$T D=32.0$ & EV & \multicolumn{2}{c}{ ET $=1.174053$ MEV } \\
ENERGY (MEV) & PRIMARY & CASCADE \\
1.18000 & 0.22 & 0.22 \\
1.19000 & 0.64 & 0.64 \\
1.22000 & 2.30 & 2.30 \\
1.25000 & 4.46 & 4.46 \\
1.30000 & 8.93 & 8.93 \\
1.36000 & 15.31 & 15.31 \\
1.43000 & 23.60 & 23.60 \\
1.52000 & 34.86 & 34.86 \\
1.64000 & 49.92 & 49.92 \\
1.81000 & 69.91 & 69.91 \\
2.05000 & 94.00 & 94.41 \\
2.34000 & 116.54 & 119.33 \\
2.70000 & 136.31 & 145.03 \\
3.16000 & 152.27 & 171.97 \\
3.75000 & 163.32 & 199.58 \\
4.46000 & 168.98 & 226.31 \\
5.28000 & 170.46 & 251.22 \\
6.10000 & 169.49 & 271.84 \\
7.04000 & 167.15 & 291.77 \\
8.21000 & 163.73 & 312.72 \\
9.97000 & 158.77 & 338.64 \\
11.70000 & 154.55 & 359.62 \\
17.60000 & 145.00 & 412.29 \\
23.40000 & 139.86 & 448.55 \\
35.20000 & 134.07 & 499.77 \\
58.70000 & 129.39 & 563.51 \\
117.00000 & 126.44 & 649.45 \\
234.00000 & 124.33 & 734.72
\end{tabular}

\begin{tabular}{rrr} 
TD $=36.0$ & EV & \multicolumn{2}{c}{ ET $=1.267113$ MEV } \\
ENERGY (MEV) & PRIMARY & CASCADE \\
1.27000 & 0.08 & 0.08 \\
1.29000 & 0.73 & 0.73 \\
1.31000 & 1.60 & 1.60 \\
1.35000 & 3.88 & 3.88 \\
1.40000 & 7.52 & 7.52 \\
1.46000 & 12.71 & 12.71 \\
1.54000 & 20.51 & 20.51 \\
1.64000 & 30.91 & 30.91 \\
1.77000 & 44.48 & 44.48 \\
1.96000 & 63.02 & 63.02 \\
2.21000 & 83.81 & 84.20 \\
2.53000 & 104.35 & 107.04 \\
2.91000 & 121.51 & 129.55 \\
3.42000 & 135.94 & 154.21 \\
4.05000 & 145.41 & 178.67 \\
4.81000 & 150.22 & 202.36 \\
5.70000 & 151.39 & 224.73 \\
6.58000 & 150.43 & 243.03 \\
7.60000 & 148.27 & 260.91 \\
8.86000 & 145.19 & 279.55 \\
10.70000 & 140.89 & 301.99 \\
12.60000 & 137.05 & 321.08 \\
19.00000 & 128.59 & 368.28 \\
25.30000 & 124.06 & 400.71 \\
38.00000 & 118.99 & 446.10 \\
63.30000 & 114.92 & 502.68 \\
126.00000 & 112.35 & 578.94 \\
253.00000 & 110.46 & 655.15
\end{tabular}

ATOMIC DISPLACEHENT CROSS-SECTIONS (BARNS) BY FAST ELBCTRONS IN YB $z=70 \quad A=173.040$

\begin{tabular}{rrr} 
TD $=40.0$ & EV & \multicolumn{2}{c}{ ET $=1.355540$ MEV } \\
PRIMARY & CASCADE \\
1.36000 & 0.09 & 0.09 \\
1.38000 & 0.62 & 0.62 \\
1.40000 & 1.32 & 1.32 \\
1.45000 & 3.70 & 3.70 \\
1.50000 & 6.80 & 6.80 \\
1.57000 & 11.97 & 11.97 \\
1.65000 & 18.61 & 18.61 \\
1.76000 & 28.32 & 28.32 \\
1.89000 & 39.85 & 39.85 \\
2.10000 & 57.23 & 57.23 \\
2.37000 & 76.17 & 76.57 \\
2.71000 & 94.50 & 97.08 \\
3.11000 & 109.67 & 117.14 \\
3.65000 & 122.50 & 139.28 \\
4.33000 & 131.02 & 161.65 \\
5.15000 & 135.24 & 183.24 \\
6.09000 & 136.16 & 203.20 \\
7.04000 & 135.21 & 219.88 \\
8.13000 & 133.21 & 236.00 \\
9.48000 & 130.40 & 252.84 \\
11.50000 & 126.42 & 273.55 \\
13.50000 & 123.03 & 290.43 \\
20.30000 & 115.53 & 332.68 \\
27.10000 & 111.45 & 362.15 \\
40.60000 & 106.97 & 402.76 \\
67.70000 & 103.35 & 453.81 \\
135.00000 & 101.08 & 522.64 \\
271.00000 & 99.37 & 591.19
\end{tabular}

\begin{tabular}{rrr} 
TD $=44.0$ & EV & \multicolumn{2}{c}{ ET=1.439963 HEV } \\
ENERGT(MEV) & PRTHAY & CASCADE \\
1.45000 & 0.17 & 0.17 \\
1.46000 & 0.39 & 0.39 \\
1.49000 & 1.24 & 1.24 \\
1.54000 & 3.27 & 3.27 \\
1.59000 & 5.89 & 5.89 \\
1.67000 & 10.96 & 10.96 \\
1.75000 & 16.68 & 16.68 \\
1.87000 & 25.81 & 25.81 \\
2.01000 & 36.52 & 36.52 \\
2.23000 & 52.21 & 52.21 \\
2.51000 & 69.15 & 69.53 \\
2.87000 & 85.92 & 88.30 \\
3.31000 & 100.20 & 107.30 \\
3.88000 & 111.65 & 127.37 \\
4.60000 & 119.25 & 147.71 \\
5.47000 & 122.96 & 167.38 \\
6.47000 & 123.70 & 185.61 \\
7.48000 & 122.78 & 200.82 \\
8.63000 & 120.93 & 215.41 \\
10.00000 & 118.46 & 230.13 \\
12.20000 & 114.72 & 249.56 \\
14.30000 & 111.68 & 264.78 \\
21.50000 & 104.90 & 303.23 \\
28.70000 & 101.22 & 330.03 \\
43.10000 & 97.15 & 367.19 \\
71.90000 & 93.90 & 413.66 \\
143.00000 & 91.87 & 476.01 \\
287.00000 & 90.32 & 538.30
\end{tabular}

\begin{tabular}{rrr} 
TD=48.0 EV & \multicolumn{2}{c}{ ET $=1.520882$ MEV } \\
ENERGY(MEV) & PRIAAT & CASCADE \\
1.53000 & 0.13 & 0.13 \\
1.55000 & 0.50 & 0.50 \\
1.58000 & 1.28 & 1.28 \\
1.62000 & 2.68 & 2.68 \\
1.68000 & 5.37 & 5.37 \\
1.76000 & 9.77 & 9.77 \\
1.85000 & 15.38 & 15.38 \\
1.97000 & 23.36 & 23.36 \\
2.12000 & 33.39 & 33.39 \\
2.35000 & 47.75 & 47.75 \\
2.66000 & 64.09 & 64.48 \\
3.04000 & 79.36 & 81.69 \\
3.49000 & 91.99 & 98.63 \\
4.10000 & 102.58 & 117.36 \\
4.86000 & 109.43 & 136.05 \\
5.77000 & 112.72 & 153.97 \\
6.84000 & 113.33 & 170.94 \\
7.90000 & 112.43 & 184.82 \\
9.12000 & 110.69 & 198.28 \\
10.60000 & 108.36 & 212.06 \\
12.90000 & 104.97 & 229.67 \\
15.20000 & 102.09 & 244.09 \\
22.80000 & 95.97 & 279.16 \\
30.40000 & 92.64 & 303.64 \\
45.60000 & 88.96 & 337.62 \\
76.00000 & 86.03 & 380.16 \\
152.00000 & 84.18 & 437.78 \\
304.00000 & 82.76 & 494.58
\end{tabular}


ATOMIC DISPLACEHENT CROSS-SECTIONS (BARNS) BT FAST ELECTRONS IN YB

\begin{tabular}{rrr} 
TD=52.0 EV & \multicolumn{2}{c}{ ET $=1.598699$ MEV } \\
ENERGY (HEV) & PRIMARY & CASCADE \\
1.61000 & 0.13 & 0.13 \\
1.63000 & 0.45 & 0.45 \\
1.66000 & 1.13 & 1.13 \\
1.71000 & 2.67 & 2.67 \\
1.77000 & 5.09 & 5.09 \\
1.85000 & 8.98 & 8.98 \\
1.95000 & 14.50 & 14.50 \\
2.07000 & 21.56 & 21.56 \\
2.23000 & 31.02 & 31.02 \\
2.47000 & 44.25 & 44.25 \\
2.79000 & 59.14 & 59.51 \\
3.19000 & 73.35 & 75.54 \\
3.67000 & 85.18 & 91.48 \\
4.31000 & 94.87 & 108.80 \\
5.11000 & 101.11 & 126.13 \\
6.07000 & 104.07 & 142.75 \\
7.19000 & 104.56 & 158.38 \\
8.31000 & 103.68 & 171.27 \\
9.59000 & 102.05 & 183.68 \\
11.10000 & 99.95 & 196.08 \\
13.50000 & 96.82 & 212.30 \\
15.90000 & 94.17 & 225.60 \\
23.90000 & 88.51 & 258.18 \\
31.90000 & 85.44 & 280.86 \\
47.90000 & 82.05 & 312.32 \\
79.90000 & 79.38 & 351.66 \\
159.00000 & 77.69 & 404.47
\end{tabular}

$\begin{array}{rrr}\begin{array}{c}\text { TD }=56.0 \\ \text { BNERGY EV }\end{array} & \begin{array}{c}\text { ET }=1.673746 \text { MEV } \\ 1.69000\end{array} & \begin{array}{r}\text { PRIMARY } \\ \text { CASCADE }\end{array} \\ 1.70000 & 0.17 & 0.17 \\ 1.74000 & 0.30 & 0.30 \\ 1.79000 & 1.07 & 1.07 \\ 1.85000 & 2.44 & 2.44 \\ 1.94000 & 4.57 & 4.57 \\ 2.04000 & 8.47 & 8.47 \\ 2.17000 & 13.39 & 13.39 \\ 2.34000 & 20.22 & 20.22 \\ 2.59000 & 29.18 & 29.18 \\ 2.92000 & 41.43 & 41.43 \\ 3.34000 & 55.09 & 55.45 \\ 3.84000 & 68.34 & 70.44 \\ 4.51000 & 79.25 & 85.23 \\ 5.35000 & 88.22 & 101.37 \\ 6.36000 & 93.97 & 117.55 \\ 7.53000 & 96.55 & 133.09 \\ 8.70000 & 97.05 & 147.59 \\ 10.00000 & 96.20 & 159.55 \\ 11.70000 & 94.71 & 170.77 \\ 14.20000 & 92.60 & 183.14 \\ 16.70000 & 89.71 & 198.04 \\ 25.10000 & 87.28 & 210.28 \\ 33.40000 & 82.07 & 240.55 \\ 50.20000 & 79.26 & 261.40 \\ 83.60000 & 76.13 & 290.69 \\ 167.00000 & 73.68 & 327.11 \\ & 72.12 & 376.43\end{array}$

\begin{tabular}{ccr} 
TD $=60.0$ & EV & \multicolumn{2}{c}{ ET $=1.746299$ MEV } \\
ENERGY (MEV) & PRIMARY & CASCADE \\
1.76000 & 0.12 & 0.12 \\
1.78000 & 0.36 & 0.36 \\
1.81000 & 0.86 & 0.86 \\
1.86000 & 2.04 & 2.04 \\
1.93000 & 4.23 & 4.23 \\
2.02000 & 7.72 & 7.72 \\
2.13000 & 12.58 & 12.58 \\
2.27000 & 19.20 & 19.20 \\
2.44000 & 27.26 & 27.26 \\
2.70000 & 38.72 & 38.72 \\
3.05000 & 51.71 & 52.07 \\
3.49000 & 64.10 & 66.14 \\
4.01000 & 74.20 & 79.92 \\
4.71000 & 82.50 & 95.03 \\
5.58000 & 87.77 & 110.05 \\
6.63000 & 90.21 & 124.54 \\
7.85000 & 90.54 & 138.10 \\
9.08000 & 89.72 & 149.36 \\
10.40000 & 88.35 & 159.59 \\
12.20000 & 86.33 & 171.34 \\
14.80000 & 83.64 & 185.23 \\
17.40000 & 81.38 & 196.64 \\
26.10000 & 76.55 & 224.77 \\
34.90000 & 73.90 & 244.57 \\
52.30000 & 71.02 & 271.71 \\
87.30000 & 68.74 & 305.88 \\
174.00000 & 67.30 & 351.76
\end{tabular}

ATOMIC DISPLACEMENT CROSS-SECTIONS (BARNS) BY PAST RLECTRONS IN YB $Z=70 \quad A=173.040$

\begin{tabular}{rrr}
$\begin{array}{r}\text { TD }=64.0 \\
\text { ENERG (MEV) }\end{array}$ & \multicolumn{2}{c}{ ET $=1.816592$ HEV } \\
PRIMARY & CASCADE \\
1.83000 & 0.10 & 0.10 \\
1.85000 & 0.30 & 0.30 \\
1.88000 & 0.74 & 0.74 \\
1.94000 & 2.02 & 2.02 \\
2.01000 & 4.02 & 4.02 \\
2.10000 & 7.18 & 7.18 \\
2.21000 & 11.57 & 11.57 \\
2.36000 & 17.99 & 17.99 \\
2.54000 & 25.71 & 25.71 \\
2.81000 & 36.48 & 36.48 \\
3.17000 & 48.56 & 48.90 \\
3.63000 & 60.25 & 62.21 \\
4.17000 & 69.69 & 75.15 \\
4.90000 & 77.46 & 89.39 \\
5.81000 & 82.36 & 103.57 \\
6.90000 & 84.58 & 117.13 \\
8.17000 & 84.85 & 129.86 \\
9.44000 & 84.06 & 140.36 \\
10.80000 & 82.77 & 149.87 \\
12.70000 & 80.85 & 161.07 \\
15.40000 & 78.33 & 174.07 \\
18.10000 & 76.21 & 184.75 \\
27.20000 & 71.69 & 211.26 \\
36.30000 & 69.23 & 229.70 \\
54.40000 & 66.54 & 255.16 \\
90.80000 & 64.42 & 287.19 \\
181.00000 & 63.09 & 330.21 \\
& &
\end{tabular}

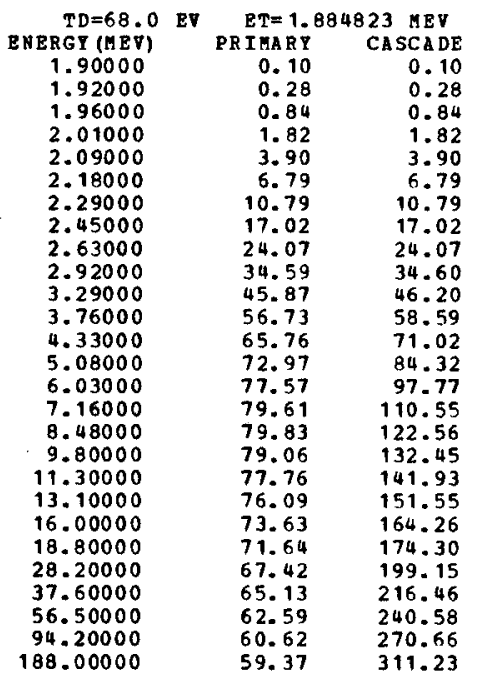

\begin{tabular}{crr} 
TD 272.0 & EV & \multicolumn{2}{c}{ ET=1.951164 HEV } \\
ENERGY (MEV) & PRIMARY & CASCADE \\
1.97000 & 0.11 & 0.11 \\
1.99000 & 0.29 & 0.29 \\
2.02000 & 0.65 & 0.65 \\
2.08000 & 1.69 & 1.69 \\
2.16000 & 3.58 & 3.58 \\
2.26000 & 6.51 & 6.51 \\
2.38000 & 10.53 & 10.53 \\
2.53000 & 15.89 & 15.89 \\
2.73000 & 23.06 & 23.06 \\
3.02000 & 32.69 & 32.69 \\
3.41000 & 43.55 & 43.88 \\
3.90000 & 53.84 & 55.67 \\
4.48000 & 62.20 & 67.23 \\
5.26000 & 69.00 & 79.87 \\
6.24000 & 73.29 & 92.56 \\
7.41000 & 75.20 & 104.64 \\
8.78000 & 75.37 & 116.03 \\
10.10000 & 74.66 & 125.09 \\
11.70000 & 73.38 & 134.34 \\
13.50000 & 71.77 & 143.59 \\
16.50000 & 69.52 & 155.20 \\
19.50000 & 67.58 & 165.03 \\
29.20000 & 63.63 & 188.41 \\
39.00000 & 61.45 & 204.86 \\
58.50000 & 59.08 & 227.54 \\
97.50000 & 57.24 & 255.94 \\
195.00000 & 56.06 & 294.37
\end{tabular}


ATOHIC DISPLACEMENT CROSS-SECTIONS (BARNS) BY FAST BLECTRONS IN YB

\begin{tabular}{|c|c|c|c|c|c|c|c|c|c|c|}
\hline \multirow{2}{*}{$\begin{array}{r}T D=76-0 \\
\text { ENERGY (MEV) }\end{array}$} & \multirow[t]{2}{*}{ EV } & \multicolumn{2}{|c|}{$\mathrm{ET}=2.015764 \mathrm{MBV}$} & $T D=80.0$ & EV & \multicolumn{2}{|c|}{$\mathrm{ET}=2.078754 \mathrm{MEV}$} & \multirow{2}{*}{$\begin{array}{r}T D=84.0 \\
\text { ENERGY (HEV) }\end{array}$} & \multicolumn{2}{|c|}{$\mathrm{ET}=2.140247 \mathrm{HEV}$} \\
\hline & & PRIMARY & CASCADE & ENERGY (MEV) & & PRIMARY & CASCADE & & PEIMARY & CASCADE \\
\hline 2.03000 & & 0.07 & 0.07 & 2.09000 & & 0.05 & 0.05 & 2.16000 & 0.08 & 0.08 \\
\hline 2.05000 & & 0.21 & 0.21 & 2.12000 & & 0.24 & 0.24 & 2.18000 & 0.21 & 0.21 \\
\hline 2.09000 & & 0.64 & 0.64 & 2.16000 & & 0.66 & 0.66 & 2.22000 & 0.58 & 0.58 \\
\hline 2.15000 & & 1.61 & 1.61 & 2.22000 & & 1.56 & 1.56 & 2.29000 & 1.55 & 1.55 \\
\hline 2.23000 & & 3.34 & 3.34 & 2.30000 & & 3.17 & 3.17 & 2.37000 & 3.06 & 3.06 \\
\hline 2.33000 & & 6.03 & 6.03 & 2.41000 & & 5.92 & 5.92 & 2.48000 & 5.61 & 5.61 \\
\hline 2.45000 & & 9.71 & 9.71 & 2.53000 & & 9.34 & 9.34 & 2.61000 & 9.05 & 9.05 \\
\hline 2.62000 & & 15.30 & 15.30 & 2.70000 & & 14.51 & 14.51 & 2.78000 & 13.86 & 13.86 \\
\hline 2.82000 & & 21.89 & 21.89 & 2.91000 & & 20.91 & 20.91 & 2.99000 & 19.80 & 19.80 \\
\hline 3.12000 & & 31.06 & 31.06 & 3.22000 & & 29.66 & 29.66 & 3.31000 & 28.19 & 28.20 \\
\hline 3.52000 & & 41.30 & 41.62 & 3.63000 & & 39.34 & 39.65 & 3.74000 & 37.61 & 37.92 \\
\hline 4.03000 & & 51.14 & 52.91 & 4.15000 & & 48.59 & 50.29 & 4.28000 & 46.47 & 48.14 \\
\hline 4.63000 & & 59.04 & 63.89 & 4.78000 & & 56.23 & 60.93 & 4.92000 & 53.62 & 58.15 \\
\hline 5.44000 & & 65.48 & 75.94 & 5.61000 & & 62.27 & 72.31 & 5.77000 & 59.33 & 68.96 \\
\hline 6.45000 & & 69.48 & 87.93 & 6.65000 & & 66.04 & 83.71 & 6.84000 & 62.91 & 79.83 \\
\hline 7.65000 & & 71.24 & 99.31 & 7.89000 & & 67.68 & 94.55 & 8.13000 & 64.47 & 90.28 \\
\hline 9.07000 & & 71.38 & 110.15 & 9.35000 & & 67.80 & 104.83 & 9.63000 & 64.55 & 100.05 \\
\hline 10.40000 & & 70.72 & 118.55 & 10.80000 & & 67.10 & 113.24 & 11.10000 & 63.89 & 107.94 \\
\hline 12.00000 & & 69.54 & 127.10 & 12.40000 & & 66.00 & 121.07 & 12.80000 & 62.80 & 115.64 \\
\hline 14.10000 & & 67.90 & 136.49 & 14.50000 & & 64.49 & 129.74 & 14.90000 & 61.40 & 123.66 \\
\hline 17.10000 & & 65.77 & 147.47 & 17.60000 & & 62.45 & 140.22 & 18.10000 & 59.45 & 133.69 \\
\hline 20.10000 & & 63.99 & 156.49 & 20.70000 & & 60.76 & 148.82 & 21.40000 & 57.79 & 142.15 \\
\hline 30.20000 & & 60.23 & 178.83 & 31.10000 & & 57.19 & 170.06 & 32.10000 & 54.42 & 162.29 \\
\hline 40.30000 & & 58.19 & 194.36 & 41.50000 & & 55.25 & 184.81 & 42.80000 & 52.59 & 176.31 \\
\hline 60.40000 & & 55.95 & 215.81 & 62.30000 & & 53.13 & 205.28 & 64.20000 & 50.58 & 195.76 \\
\hline 100.00000 & & 54.23 & 242.38 & 103.00000 & & 51.51 & 230.45 & 107.00000 & 49.03 & 220.12 \\
\hline 201.00000 & & 53.10 & 279.04 & 207.00000 & & 50.44 & 265.28 & 214.00000 & 48.03 & 253.06 \\
\hline
\end{tabular}

ATOMIC DISPLACEMENT CROSS-SECTIONS (BARNS) BY FAST ELECTRONS IN YB $Z=70 \quad A=173.040$

$\begin{array}{rrr}\text { TD }=88.0 & \text { EV } & \text { ET }=2.200345 \text { MEV } \\ \text { PRIMARY } & \text { CASCADE } \\ 2.22000 & 0.07 & 0.07 \\ 2.24000 & 0.19 & 0.19 \\ 2.28000 & 0.52 & 0.52 \\ 2.35000 & 1.41 & 1.41 \\ 2.44000 & 2.98 & 2.98 \\ 2.55000 & 5.36 & 5.36 \\ 2.68000 & 8.57 & 8.57 \\ 2.86000 & 13.31 & 13.31 \\ 3.08000 & 19.11 & 19.11 \\ 3.41000 & 27.14 & 27.14 \\ 3.85000 & 36.07 & 36.38 \\ 4.40000 & 44.44 & 46.06 \\ 5.06000 & 51.27 & 55.65 \\ 5.94000 & 56.72 & 56.05 \\ 7.04000 & 60.09 & 76.42 \\ 8.36000 & 61.54 & 86.34 \\ 9.90000 & 61.60 & 95.66 \\ 11.40000 & 60.97 & 103.15 \\ 13.20000 & 59.89 & 110.72 \\ 15.40000 & 58.52 & 118.48 \\ 18.70000 & 56.66 & 128.03 \\ 22.00000 & 55.12 & 135.87 \\ 33.00000 & 51.92 & 155.10 \\ 44.00000 & 50.17 & 158.49 \\ 66.00000 & 48.26 & 187.06 \\ 110.00000 & 46.79 & 210.31 \\ 220.00000 & 45.84 & 241.75 \\ & & \end{array}$

$\begin{array}{crr}\text { TD }=92.0 & \text { EV } & \text { ET }=2.259141 \text { MEV } \\ \text { ENERGY (MEV) } & \text { PRIBAY } & \text { CASCADE } \\ 2.28000 & 0.07 & 0.07 \\ 2.30000 & 0.18 & 0.18 \\ 2.34000 & 0.49 & 0.49 \\ 2.41000 & 1.30 & 1.30 \\ 2.50000 & 2.75 & 2.75 \\ 2.62000 & 5.17 & 5.17 \\ 2.75000 & 8.17 & 8.17 \\ 2.93000 & 12.50 & 12.60 \\ 3.16000 & 18.26 & 18.26 \\ 3.50000 & 26.00 & 26.00 \\ 3.95000 & 34.53 & 34.83 \\ 4.51000 & 42.49 & 44.04 \\ 5.19000 & 49.07 & 53.28 \\ 6.09000 & 54.27 & 63.21 \\ 7.22000 & 57.49 & 73.17 \\ 8.58000 & 58.87 & 82.71 \\ 10.10000 & 58.93 & 91.32 \\ 11.70000 & 58.29 & 98.79 \\ 13.50000 & 57.29 & 105.87 \\ 15.80000 & 55.95 & 113.45 \\ 19.20000 & 54.16 & 122.63 \\ 22.50000 & 52.73 & 129.95 \\ 33.80000 & 49.65 & 148.42 \\ 45.10000 & 47.98 & 161.26 \\ 67.70000 & 46.15 & 179.05 \\ 112.00000 & 44.76 & 200.98 \\ 225.00000 & 43.85 & 231.25\end{array}$

\begin{tabular}{rrr} 
TD=96.0 EV & \multicolumn{2}{c}{ ET $=2.316714$ HEV } \\
ENERGY (MEV) & PRIMARY & CASCADE \\
2.33000 & 0.04 & 0.04 \\
2.36000 & 0.17 & 0.17 \\
2.40000 & 0.46 & 0.46 \\
2.47000 & 1.23 & 1.23 \\
2.57000 & 2.74 & 2.74 \\
2.68000 & 4.82 & 4.82 \\
2.82000 & 7.84 & 7.84 \\
3.01000 & 12.22 & 12.22 \\
3.24000 & 17.53 & 17.53 \\
3.59000 & 24.99 & 24.99 \\
4.05000 & 33.14 & 33.44 \\
4.63000 & 40.85 & 42.37 \\
5.32000 & 47.06 & 51.14 \\
6.25000 & 52.06 & 60.74 \\
7.41000 & 55.12 & 70.28 \\
8.80000 & 56.42 & 79.40 \\
10.40000 & 56.45 & 87.85 \\
12.00000 & 55.84 & 94.82 \\
13.90000 & 54.84 & 101.79 \\
16.20000 & 53.59 & 108.86 \\
19.60000 & 51.91 & 117.46 \\
23.10000 & 50.49 & 124.73 \\
34.70000 & 47.56 & 142.44 \\
46.30000 & 45.96 & 154.74 \\
69.50000 & 44.21 & 171.79 \\
115.00000 & 42.89 & 192.81 \\
231.00000 & 42.02 & 221.82
\end{tabular}


ATOHIC DISPLACEHENT CROSS-SECTIONS (BARNS) BY FAST REBCTRONS IN HF $=72 A=178.500$

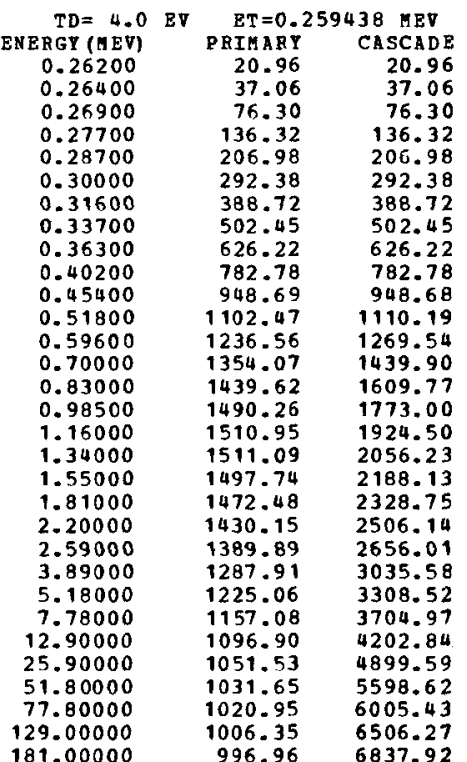

\begin{tabular}{rrr} 
TD $=8.0$ & EV & \multicolumn{2}{c}{ ET=0.451304 MEV } \\
BNERGY (HEV) & PRIARY & CASCADE \\
0.45500 & 4.77 & 4.77 \\
0.46000 & 11.31 & 11.31 \\
0.46900 & 23.32 & 23.32 \\
0.48200 & 41.08 & 41.08 \\
0.50000 & 66.19 & 66.19 \\
0.52300 & 98.64 & 98.64 \\
0.55000 & 136.56 & 136.56 \\
0.58600 & 185.81 & 185.81 \\
0.63100 & 244.00 & 244.00 \\
0.69900 & 323.22 & 323.22 \\
0.78900 & 411.37 & 411.69 \\
0.90200 & 497.93 & 503.61 \\
1.03000 & 570.06 & 589.88 \\
1.21000 & 638.13 & 689.14 \\
1.44000 & 688.49 & 790.98 \\
1.71000 & 717.25 & 887.21 \\
2.03000 & 728.96 & 980.12 \\
2.34000 & 728.63 & 1055.20 \\
2.70000 & 721.52 & 1129.67 \\
3.15000 & 708.56 & 1208.75 \\
3.83000 & 687.39 & 1308.14 \\
4.51000 & 667.99 & 1390.69 \\
6.76000 & 621.18 & 1594.04 \\
9.02000 & 594.10 & 1739.63 \\
13.50000 & 565.24 & 1943.00 \\
22.50000 & 539.49 & 2199.93 \\
45.10000 & 522.20 & 2552.19 \\
90.20000 & 513.16 & 2902.26 \\
135.00000 & 507.60 & 3103.62 \\
225.00000 & 500.21 & 3355.47 \\
315.00000 & 496.03 & 3519.86
\end{tabular}

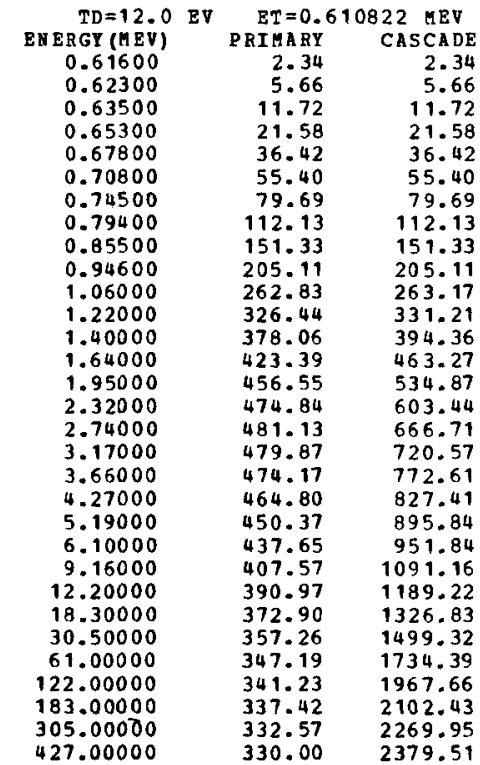

ATOMIC DISPLACEHENT CHOSS-SECTIONS (BARNS) BY PAST ELECTRONS IN HP $\mathrm{Z}=72 \quad \mathrm{~A}=178.500$

\begin{tabular}{|c|c|c|c|}
\hline \multirow{2}{*}{$\begin{array}{r}\text { TD }=16 . \\
\text { ENERGY (MBV) }\end{array}$} & EV & $\begin{aligned} & E T=0.7 \\
&\end{aligned}$ & $324 \mathrm{NBV}$ \\
\hline & & PRIHARY & CASCADE \\
\hline 0.75700 & & 1.45 & 1.45 \\
\hline 0.76500 & & 3.34 & 3.34 \\
\hline 0.78000 & & 7.27 & 7.27 \\
\hline 0.80200 & & 13.82 & 13.82 \\
\hline 0.83200 & & 23.90 & 23.90 \\
\hline 0.87000 & & $\begin{array}{l}38.00 \\
55.80\end{array}$ & $\begin{array}{l}38.00 \\
55.80\end{array}$ \\
\hline $\begin{array}{l}0.91500 \\
0.97500\end{array}$ & & $\begin{array}{l}55.80 \\
80.17\end{array}$ & $\begin{array}{l}55.80 \\
80.17\end{array}$ \\
\hline 1.05000 & & 110.14 & 110.14 \\
\hline 1.16000 & & 150.90 & 150.90 \\
\hline 1.31000 & & 198.35 & 198.81 \\
\hline $\begin{array}{l}1.50000 \\
1.72000\end{array}$ & & $\begin{array}{l}245.23 \\
284.26\end{array}$ & $\begin{array}{l}249.53 \\
298.21\end{array}$ \\
\hline 2.02000 & & 318.70 & 352.44 \\
\hline 2.40000 & & $\begin{array}{l}342.70 \\
355.32\end{array}$ & $\begin{array}{l}407.54 \\
459.88\end{array}$ \\
\hline 3.37000 & & 359.13 & 508.90 \\
\hline 3.90000 & & 357.44 & 550.35 \\
\hline 4.50000 & & 352.66 & 589.98 \\
\hline 5.25000 & & 345.25 & 631.88 \\
\hline 6.37000 & & 334.36 & 683.55 \\
\hline 7.50000 & & 324.73 & 726.59 \\
\hline 11.20000 & & 303.09 & 830.82 \\
\hline 15.00000 & & 290.96 & 906.34 \\
\hline 22.50000 & & 277.96 & 1009.99 \\
\hline 37.50000 & & 266.95 & 1139.82 \\
\hline 75.00000 & & 259.97 & 1316.47 \\
\hline 150.00000 & & 255.50 & 1491.32 \\
\hline
\end{tabular}

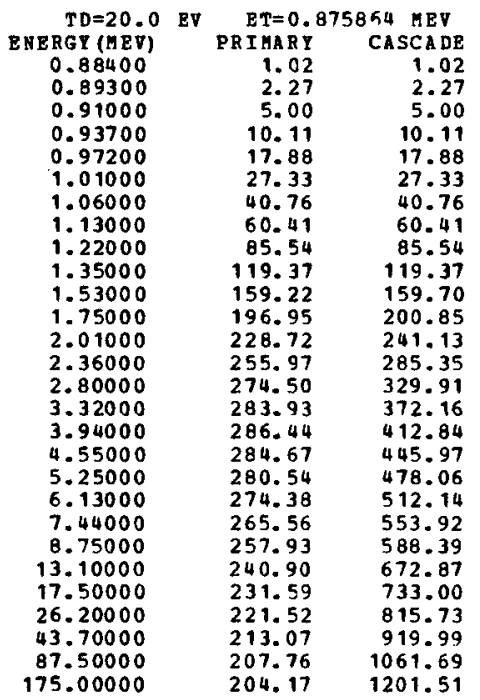

\begin{tabular}{rrr} 
TD=24.0 EV & \multicolumn{1}{c}{ ET $=0.990948$ MEV } \\
ENERGP(MEV) & PRTHARY & CASCADE \\
1.00000 & 0.72 & 0.72 \\
1.01000 & 1.64 & 1.64 \\
1.03000 & 3.81 & 3.81 \\
1.06000 & 7.80 & 7.80 \\
1.09000 & 12.49 & 12.49 \\
1.14000 & 21.43 & 21.43 \\
1.20000 & 33.33 & 33.33 \\
1.28000 & 50.05 & 50.05 \\
1.38000 & 70.92 & 70.92 \\
1.53000 & 100.11 & 100.11 \\
1.73000 & 133.17 & 133.64 \\
1.98000 & 165.05 & 168.66 \\
2.27000 & 191.26 & 202.31 \\
2.67000 & 214.07 & 240.13 \\
3.17000 & 229.15 & 277.90 \\
3.76000 & 236.54 & 313.59 \\
4.45000 & 238.20 & 347.29 \\
5.15000 & 236.43 & 375.57 \\
5.94000 & 232.79 & 402.44 \\
6.93000 & 227.56 & 430.90 \\
9.42000 & 220.11 & 466.13 \\
9.90000 & 213.78 & 494.95 \\
14.80000 & 199.89 & 565.41 \\
19.80000 & 192.26 & 615.90 \\
29.70000 & 184.02 & 685.28 \\
49.50000 & 177.24 & 772.16 \\
99.00000 & 172.99 & 890.13 \\
198.00000 & 159.99 & 1006.60 \\
& &
\end{tabular}


ATOMIC DISPLACBAENT CROSS-SECTIONS (BARNS) BY FAST RLECTRONS IN HF $=72 \quad A=178.500$

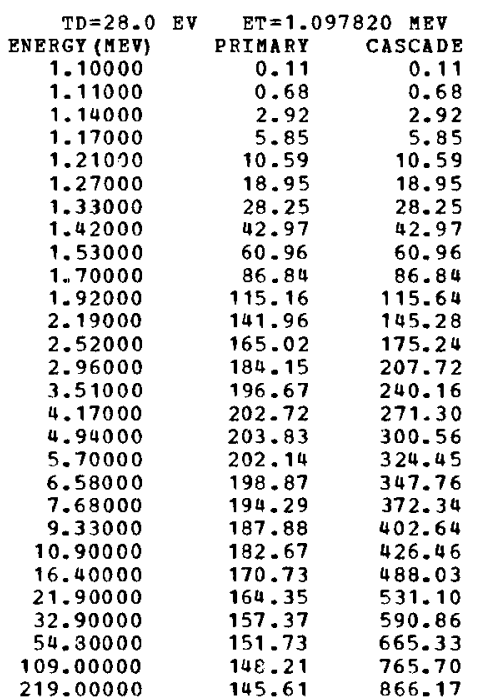

\begin{tabular}{rrr} 
TD $=32.0$ & EV & \multicolumn{1}{c}{ ET $=1.198022$ HEV } \\
ENERGY (HET) & PRIMARY & CASCADE \\
1.21000 & 0.48 & 0.48 \\
1.22000 & 0.96 & 0.96 \\
1.24000 & 2.13 & 2.13 \\
1.28000 & 5.16 & 5.16 \\
1.32000 & 8.94 & 8.94 \\
1.38000 & 15.60 & 15.60 \\
1.46000 & 25.62 & 25.62 \\
1.55000 & 37.56 & 37.56 \\
1.67000 & 53.48 & 53.48 \\
1.85000 & 75.72 & 75.72 \\
2.09000 & 100.83 & 101.28 \\
2.39000 & 124.88 & 127.99 \\
2.75000 & 145.02 & 154.45 \\
3.23000 & 161.60 & 183.09 \\
3.83000 & 172.31 & 211.68 \\
4.55000 & 177.36 & 239.10 \\
5.39000 & 178.12 & 264.83 \\
6.22000 & 176.50 & 285.85 \\
7.18000 & 173.53 & 306.33 \\
8.38000 & 169.48 & 327.91 \\
10.10000 & 164.08 & 353.44 \\
11.90000 & 159.28 & 375.47 \\
17.90000 & 148.97 & 429.45 \\
23.90000 & 143.47 & 467.17 \\
35.90000 & 137.45 & 519.48 \\
59.90000 & 132.62 & 584.92 \\
119.00000 & 129.62 & 672.62 \\
239.00000 & 127.34 & 760.45
\end{tabular}

TD=36.0
ENERGY (HEV)
1.30000
1.31000
1.34000
1.38000
1.43000
1.49000
1.57000
1.68000
1.80000
2.00000
2.26000
2.58000
2.97000
3.49000
4.13000
4.91000
5.81000
6.72000
7.75000
9.04000
10.90000
12.90000
19.30000
25.80000
38.70000
64.60000
129.00000
258.00000

$E T=1.292666$ MEV $\begin{array}{rr}0.21 & \text { CASCADE } \\ 0.25 & 0.21\end{array}$ 0.55 $1.89 \quad 1.89$

$\begin{array}{ll}4.35 & 4.35 \\ 8.25 & 8.25\end{array}$ $\begin{array}{rr}8.25 & 8.25 \\ 13.79 & 13.79\end{array}$ $13.79 \quad 13.79$ $22.08 \quad 22.08$ $\begin{array}{ll}34.21 & 34.21 \\ 47.45 & 47.45\end{array}$ $\begin{array}{ll}47.45 & 47.45 \\ 68.00 & 68.00\end{array}$ $90.48 \quad 90.94$ $111.61 \quad 114.56$ $129.51 \quad 138.32$ $144.11 \quad 164.01$ $153.33 \quad 189.27$ $157.64 \quad 213.87$ $158.16 \quad 236.69$ 153.89273 .88 $150.89 \quad 273.88$ $145.43 \quad 39.05$ $141.07 \quad 315.85$ $141.07 \quad 336.00$ $132.13 \quad 383.44$ $122.01 \quad 463.51$ $122.01 \quad 463.51$ $117.79 \quad 521.76$ $\begin{array}{ll}115.16 & 600.31 \\ 113.14 & 677.90\end{array}$

ATOHIC DISPLACEMENT CROSS-SECTIONS (BARNS) BY FAST ELECTRONS IN HF $=72 \quad A=178.500$

\begin{tabular}{crr} 
TD $=40.0$ & EV & \multicolumn{2}{c}{ ET $=1.382585$} & MEV \\
PRIMARY & CASCADE \\
1.39000 & 0.16 & 0.16 \\
1.41000 & 0.74 & 0.74 \\
1.43000 & 1.49 & 1.49 \\
1.47000 & 3.46 & 3.46 \\
1.53000 & 7.34 & 7.34 \\
1.60000 & 12.83 & 12.83 \\
1.68000 & 19.88 & 19.88 \\
1.79000 & 30.17 & 30.17 \\
1.93000 & 43.29 & 43.29 \\
2.14000 & 61.56 & 61.55 \\
2.41000 & 81.36 & 81.79 \\
2.76000 & 100.92 & 103.72 \\
3.17000 & 116.76 & 124.90 \\
3.73000 & 129.95 & 148.35 \\
4.42000 & 138.16 & 171.38 \\
5.25000 & 141.86 & 193.48 \\
6.22000 & 142.20 & 214.24 \\
7.18000 & 140.73 & 231.20 \\
8.29000 & 138.23 & 247.71 \\
9.67000 & 134.93 & 265.00 \\
11.70000 & 130.49 & 285.93 \\
13.80000 & 126.65 & 303.73 \\
20.70000 & 118.64 & 346.76 \\
27.60000 & 114.36 & 376.84 \\
41.40000 & 109.67 & 418.59 \\
69.10000 & 105.93 & 471.04 \\
138.00000 & 103.60 & 541.76 \\
276.00000 & 101.78 & 611.57
\end{tabular}

\begin{tabular}{crr} 
TD=44.0 & \multicolumn{1}{c}{ ET $=1.468424$ MEV } \\
ENERGY (MEV) & PRIMARY & CASCADE \\
1.48000 & 0.21 & 0.21 \\
1.49000 & 0.44 & 0.44 \\
1.52000 & 1.34 & 1.34 \\
1.57000 & 3.50 & 3.50 \\
1.62000 & 6.28 & 6.28 \\
1.70000 & 11.64 & 11.64 \\
1.79000 & 18.50 & 18.50 \\
1.90000 & 27.38 & 27.38 \\
2.05000 & 39.50 & 39.50 \\
2.27000 & 56.01 & 56.01 \\
2.56000 & 74.33 & 74.75 \\
2.93000 & 92.07 & 94.71 \\
3.37000 & 106.57 & 114.26 \\
3.96000 & 118.36 & 135.50 \\
4.69000 & 125.69 & 156.45 \\
5.58000 & 128.97 & 176.81 \\
6.60000 & 129.17 & 195.55 \\
7.63000 & 127.76 & 211.16 \\
8.81000 & 125.44 & 226.20 \\
10.20000 & 122.57 & 241.19 \\
12.40000 & 118.43 & 260.74 \\
14.60000 & 114.98 & 276.77 \\
22.00000 & 107.67 & 316.38 \\
29.30000 & 103.82 & 343.63 \\
44.00000 & 99.58 & 381.72 \\
73.40000 & 96.25 & 429.37 \\
146.00000 & 94.17 & 493.30 \\
293.00000 & 92.50 & 557.07
\end{tabular}

\begin{tabular}{rrr} 
TD=48.0 & \multicolumn{2}{c}{ EV $=1.550692$ MEV } \\
ENBRGY(MEV) & PRIMABY & CASCADE \\
1.56000 & 0.13 & 0.13 \\
1.58000 & 0.52 & 0.52 \\
1.61000 & 1.34 & 1.34 \\
1.65000 & 2.81 & 2.81 \\
1.72000 & 6.19 & 6.19 \\
1.79000 & 10.30 & 10.30 \\
1.89000 & 16.93 & 16.93 \\
2.01000 & 25.40 & 25.40 \\
2.17000 & 36.70 & 36.70 \\
2.40000 & 51.74 & 51.74 \\
2.71000 & 68.75 & 69.18 \\
3.10000 & 84.91 & 87.48 \\
3.56000 & 98.00 & 105.27 \\
4.18000 & 108.68 & 124.72 \\
4.96000 & 115.35 & 144.20 \\
5.89000 & 118.21 & 162.71 \\
6.97000 & 118.32 & 179.98 \\
8.06000 & 116.97 & 194.34 \\
9.30000 & 114.83 & 208.09 \\
10.80000 & 112.12 & 222.13 \\
13.10000 & 108.36 & 239.86 \\
15.50000 & 105.11 & 255.01 \\
23.20000 & 98.57 & 290.77 \\
31.00000 & 95.03 & 316.04 \\
46.50000 & 91.19 & 350.87 \\
77.50000 & 88.18 & 394.49 \\
155.00000 & 86.28 & 453.56 \\
310.00000 & 84.76 & 511.71
\end{tabular}


ATOAIC DISPLACEMENT CROSS-SECTIONS (BARNS) BY PAST BLECTHONS IN HF

\begin{tabular}{rrr} 
TD $=52.0$ & EV & \multicolumn{2}{c}{ ET $=1.629801$ HEV } \\
ENERGY(HEV) & PRIMARY & CASCADE \\
1.64000 & 0.12 & 0.12 \\
1.66000 & 0.45 & 0.45 \\
1.69000 & 1.14 & 1.14 \\
1.74000 & 2.76 & 2.76 \\
1.80000 & 5.30 & 5.30 \\
1.89000 & 9.96 & 9.96 \\
1.98000 & 15.24 & 15.24 \\
2.11000 & 23.34 & 23.34 \\
2.28000 & 33.95 & 33.95 \\
2.52000 & 47.83 & 47.83 \\
2.85000 & 63.79 & 64.21 \\
3.25000 & 78.40 & 80.80 \\
3.74000 & 90.67 & 97.54 \\
4.40000 & 100.56 & 115.77 \\
5.21000 & 106.55 & 133.58 \\
6.19000 & 109.11 & 150.74 \\
7.33000 & 109.14 & 166.79 \\
8.47000 & 107.87 & 179.99 \\
9.77000 & 105.86 & 192.68 \\
11.40000 & 103.26 & 206.07 \\
13.80000 & 99.82 & 222.28 \\
16.20000 & 96.97 & 235.62 \\
24.40000 & 90.87 & 269.17 \\
32.50000 & 87.65 & 292.25 \\
48.80000 & 84.12 & 324.49 \\
81.40000 & 81.37 & 364.84 \\
162.00000 & 79.64 & 418.98
\end{tabular}

\begin{tabular}{rrr} 
TD=56.0 EV & \multicolumn{2}{c}{ ET $=1.706089$ MEV } \\
ENERGY(MEV) & PRIMARY & CASCEDE \\
1.72000 & 0.14 & 0.14 \\
1.74000 & 0.44 & 0.44 \\
1.77000 & 1.06 & 1.06 \\
1.82000 & 2.48 & 2.48 \\
1.89000 & 5.13 & 5.13 \\
1.97000 & 8.81 & 8.81 \\
2.08000 & 14.56 & 14.56 \\
2.21000 & 21.80 & 21.80 \\
2.39000 & 31.27 & 31.27 \\
2.64000 & 44.68 & 44.68 \\
2.98000 & 59.33 & 59.73 \\
3.41000 & 73.24 & 75.59 \\
3.92000 & 84.48 & 91.06 \\
4.60000 & 93.46 & 107.77 \\
5.45000 & 98.99 & 124.41 \\
6.48000 & 101.32 & 140.45 \\
7.67000 & 101.29 & 155.34 \\
8.87000 & 100.06 & 167.69 \\
10.20000 & 98.22 & 179.23 \\
11.90000 & 95.81 & 191.67 \\
14.50000 & 92.50 & 207.25 \\
17.00000 & 89.88 & 219.54 \\
25.50000 & 84.30 & 250.41 \\
34.10000 & 81.29 & 272.15 \\
51.10000 & 78.06 & 301.95 \\
85.30000 & 75.52 & 339.47 \\
170.00000 & 73.93 & 389.86
\end{tabular}

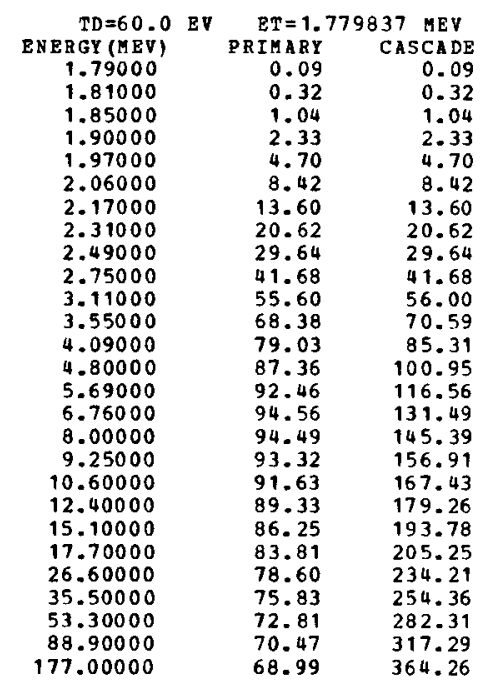

ATOMIC DISPLACEHENT CBOSS-SECTIONS (BARNS) BY PAST BLECTRONS IN HP $z=72 \quad A=178.500$

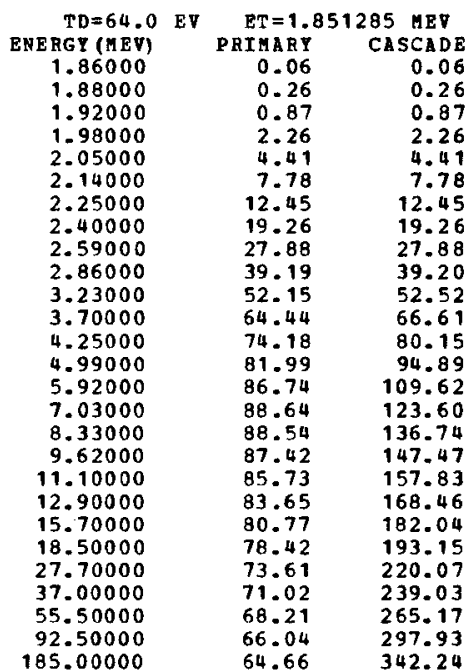

$\begin{array}{crr}\text { TD }=68.0 & \text { EV } & \text { ET=1.920634 MEV } \\ \text { ENBRGY (MEV) } & \text { PRIARY } & \text { CASCADB } \\ 1.93000 & 0.06 & 0.06 \\ 1.95000 & 0.23 & 0.23 \\ 1.99000 & 0.79 & 0.78 \\ 2.05000 & 2.01 & 2.01 \\ 2.13000 & 4.24 & 4.24 \\ 2.22000 & 7.31 & 7.31 \\ 2.34000 & 11.97 & 11.97 \\ 2.49000 & 18.18 & 18.18 \\ 2.68000 & 26.04 & 26.04 \\ 2.97000 & 37.11 & 37.12 \\ 3.36000 & 49.49 & 49.87 \\ 3.84000 & 60.83 & 62.92 \\ 4.41000 & 69.96 & 75.68 \\ 5.18000 & 77.28 & 89.62 \\ 6.14000 & 81.67 & 103.43 \\ 7.29000 & 83.43 & 116.59 \\ 8.64000 & 83.29 & 128.99 \\ 9.98000 & 82.22 & 139.11 \\ 11.50000 & 80.64 & 148.79 \\ 13.40000 & 78.64 & 158.97 \\ 16.30000 & 75.93 & 171.72 \\ 19.20000 & 73.72 & 182.16 \\ 28.80000 & 69.21 & 207.62 \\ 38.40000 & 66.79 & 225.36 \\ 57.60000 & 64.16 & 249.97 \\ 96.00000 & 62.14 & 280.80 \\ 192.00000 & 60.85 & 322.51\end{array}$

\begin{tabular}{crr} 
TD=72.0 EV & \multicolumn{2}{c}{ ET $=1.988060$ MEV } \\
ENERGY (HEV) & PRIHARY & CASCADE \\
2.00000 & 0.07 & 0.07 \\
2.02000 & 0.23 & 0.23 \\
2.06000 & 0.72 & 0.72 \\
2.12000 & 1.84 & 1.84 \\
2.20000 & 3.86 & 3.86 \\
2.30000 & 6.97 & 6.97 \\
2.42000 & 11.24 & 11.24 \\
2.58000 & 17.31 & 17.31 \\
2.78000 & 24.88 & 24.88 \\
3.08000 & 35.35 & 35.35 \\
3.47000 & 46.66 & 47.02 \\
3.97000 & 57.50 & 59.49 \\
4.57000 & 66.25 & 71.77 \\
5.36000 & 73.05 & 84.84 \\
6.36000 & 77.18 & 97.98 \\
7.55000 & 78.79 & 110.42 \\
8.94000 & 78.63 & 122.07 \\
10.30000 & 77.63 & 131.47 \\
11.90000 & 76.10 & 140.79 \\
13.90000 & 74.17 & 150.57 \\
16.80000 & 71.70 & 162.22 \\
19.80000 & 69.61 & 172.12 \\
29.80000 & 65.32 & 196.38 \\
39.70000 & 63.05 & 213.09 \\
59.60000 & 60.57 & 236.39 \\
99.40000 & 58.67 & 265.55 \\
198.00000 & 57.46 & 304.71
\end{tabular}


ATOMIC DISPLACEMENT CROSS-SECTIONS (BARSS) BY FAST ELECTRONS IN HF $z=72 \quad A=178.500$

\begin{tabular}{rrr} 
TD $=76.0$ & EV & \multicolumn{1}{c}{ ET $=2.053713$ MEV } \\
ENERGY (MEV) & PRIMARY & CASCADE \\
2.07000 & 0.08 & 0.08 \\
2.09000 & 0.24 & 0.24 \\
2.13000 & 0.70 & 0.70 \\
2.19000 & 1.73 & 1.73 \\
2.27000 & 3.57 & 3.57 \\
2.38000 & 6.73 & 6.73 \\
2.50000 & 10.67 & 10.67 \\
2.66000 & 15.26 & 16.26 \\
2.87000 & 23.58 & 23.58 \\
3.18000 & 33.53 & 33.54 \\
3.59000 & 44.45 & 44.81 \\
4.10000 & 54.57 & 56.50 \\
4.72000 & 62.85 & 68.16 \\
5.54000 & 69.29 & 80.61 \\
6.57000 & 73.15 & 93.04 \\
7.80000 & 74.64 & 104.84 \\
9.24000 & 74.46 & 115.92 \\
10.60000 & 73.53 & 124.56 \\
12.30000 & 72.03 & 133.66 \\
14.30000 & 70.26 & 142.66 \\
17.40000 & 67.83 & 154.10 \\
20.50000 & 65.86 & 163.46 \\
30.80000 & 61.83 & 186.35 \\
41.00000 & 59.70 & 202.14 \\
61.60000 & 57.35 & 224.25 \\
102.00000 & 55.58 & 251.50 \\
205.00000 & 54.43 & 289.08
\end{tabular}

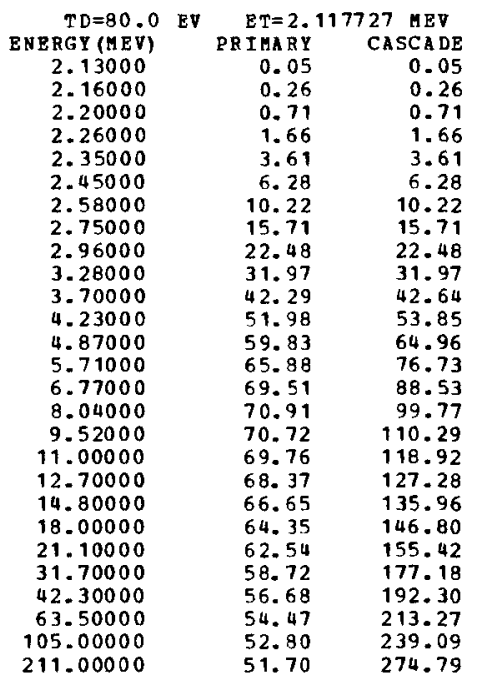

\begin{tabular}{rrr} 
TD $=84.0$ & EV & \multicolumn{1}{c}{ ET $=2.180219$ MEV } \\
ENERGY (MEV) & PRIMARY & CASCADE \\
2.20000 & 0.08 & 0.08 \\
2.22000 & 0.22 & 0.22 \\
2.26000 & 0.61 & 0.61 \\
2.33000 & 1.63 & 1.63 \\
2.42000 & 3.45 & 3.45 \\
2.52000 & 5.92 & 5.92 \\
2.65000 & 9.57 & 9.57 \\
2.83000 & 14.96 & 14.96 \\
3.05000 & 21.54 & 21.54 \\
3.37000 & 30.35 & 30.36 \\
3.81000 & 40.39 & 40.73 \\
4.36000 & 49.67 & 51.51 \\
5.01000 & 57.03 & 61.95 \\
5.88000 & 52.80 & 73.25 \\
6.97000 & 66.23 & 84.49 \\
8.28000 & 67.53 & 95.22 \\
9.81000 & 67.33 & 105.27 \\
11.30000 & 66.42 & 113.33 \\
13.00000 & 65.13 & 121.10 \\
15.20000 & 63.46 & 129.55 \\
18.50000 & 61.26 & 139.92 \\
21.80000 & 59.48 & 148.41 \\
32.70000 & 55.87 & 169.05 \\
43.60000 & 53.95 & 183.42 \\
65.40000 & 51.85 & 203.36 \\
109.00000 & 50.26 & 228.33 \\
218.00000 & 49.23 & 262.10 \\
& &
\end{tabular}
ATOMIC DISPLACENENT CROSS-SECTIONS (BARNS) BY FAST ELECTRONS IN HF
$\mathrm{Z}=72 \quad \mathrm{~A}=178.500$

\begin{tabular}{rrr} 
TD $=88.0$ & EV & \multicolumn{1}{c}{ ET $=2.241293$ MEV } \\
ENERGY (HEV) & PRIMARY & CASCADE \\
2.26000 & 0.07 & 0.07 \\
2.28000 & 0.19 & 0.19 \\
2.33000 & 0.65 & 0.65 \\
2.39000 & 1.47 & 1.47 \\
2.48000 & 3.13 & 3.13 \\
2.59000 & 5.64 & 5.64 \\
2.73000 & 9.31 & 9.31 \\
2.91000 & 14.34 & 14.34 \\
3.13000 & 20.47 & 20.47 \\
3.47000 & 29.18 & 29.19 \\
3.92000 & 38.70 & 39.04 \\
4.48000 & 47.47 & 49.24 \\
5.15000 & 54.51 & 59.26 \\
6.05000 & 60.02 & 70.12 \\
7.17000 & 63.25 & 80.84 \\
8.51000 & 64.46 & 91.04 \\
10.00000 & 64.28 & 100.19 \\
11.60000 & 63.38 & 108.28 \\
13.40000 & 62.11 & 115.91 \\
15.60000 & 60.55 & 123.76 \\
19.00000 & 58.44 & 133.70 \\
22.40000 & 56.74 & 141.83 \\
33.50000 & 53.31 & 161.54 \\
44.80000 & 51.47 & 175.25 \\
67.20000 & 49.48 & 194.29 \\
112.00000 & 47.96 & 218.13 \\
224.00000 & 46.99 & 250.36 \\
& &
\end{tabular}

\begin{tabular}{rrr}
$\begin{array}{r}\text { TD }=92.0 \\
\text { ENERGY }\end{array}$ & \multicolumn{2}{c}{ ETEV $=2.301040$ MEV } \\
PRIMARY & CASCA DE \\
2.32000 & 0.06 & 0.06 \\
2.34000 & 0.17 & 0.17 \\
2.39000 & 0.59 & 0.59 \\
2.46000 & 1.49 & 1.49 \\
2.55000 & 3.06 & 3.06 \\
2.66000 & 5.42 & 5.42 \\
2.80000 & 8.85 & 8.85 \\
2.99000 & 13.81 & 13.81 \\
3.22000 & 19.79 & 19.79 \\
3.56000 & 27.92 & 27.93 \\
4.02000 & 37.02 & 37.35 \\
4.60000 & 45.49 & 47.21 \\
5.29000 & 52.22 & 56.83 \\
6.21000 & 57.45 & 67.18 \\
7.36000 & 60.52 & 77.46 \\
8.74000 & 61.65 & 87.25 \\
10.30000 & 61.46 & 96.16 \\
11.90000 & 60.61 & 103.68 \\
13.80000 & 59.34 & 111.18 \\
16.10000 & 57.83 & 118.79 \\
19.50000 & 55.87 & 128.03 \\
23.00000 & 54.24 & 135.83 \\
34.50000 & 50.96 & 154.69 \\
46.00000 & 49.21 & 167.81 \\
69.00000 & 47.31 & 186.02 \\
115.00000 & 45.87 & 208.83 \\
230.00000 & 44.94 & 239.66 \\
& &
\end{tabular}

\begin{tabular}{rrr} 
TD=96.0 EV & \multicolumn{2}{c}{ ETI 2.359544 MEV } \\
ENERGY (HEV) & PRIMARY & CASCADE \\
2.38000 & 0.06 & 0.06 \\
2.40000 & 0.16 & 0.16 \\
2.45000 & 0.55 & 0.55 \\
2.52000 & 1.39 & 1.39 \\
2.61000 & 2.84 & 2.84 \\
2.73000 & 5.25 & 5.25 \\
2.87000 & 8.46 & 8.46 \\
3.06000 & 13.11 & 13.11 \\
3.30000 & 18.97 & 18.97 \\
3.65000 & 26.81 & 26.82 \\
4.12000 & 35.51 & 35.83 \\
4.71000 & 43.59 & 45.24 \\
5.42000 & 50.07 & 54.52 \\
6.37000 & 55.10 & 64.52 \\
7.55000 & 58.02 & 74.38 \\
8.96000 & 59.08 & 83.73 \\
10.60000 & 58.86 & 92.47 \\
12.20000 & 58.06 & 99.49 \\
14.10000 & 56.87 & 106.52 \\
16.50000 & 55.39 & 113.96 \\
20.00000 & 53.50 & 122.86 \\
23.50000 & 51.98 & 130.16 \\
35.30000 & 48.83 & 148.30 \\
47.10000 & 47.15 & 160.91 \\
70.70000 & 45.33 & 178.39 \\
117.00000 & 43.97 & 199.95 \\
235.00000 & 43.07 & 229.68
\end{tabular}


ATOHIC DISPLACEMENT CROSS-SECTIONS (BARNS) BY FAST ELECTRONS IN TA

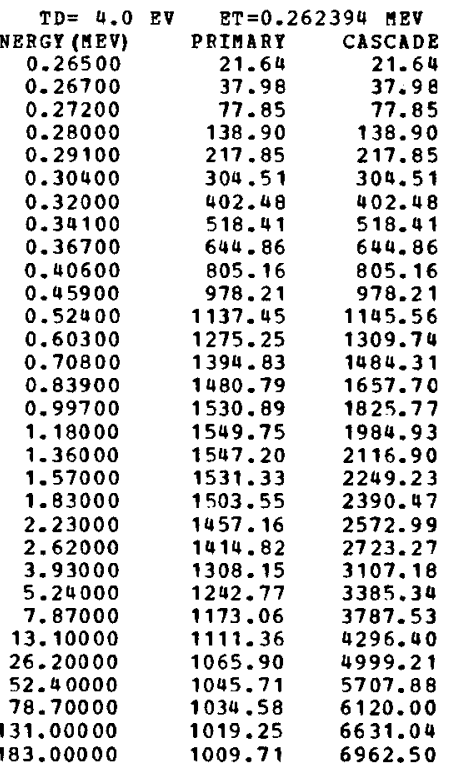

$z=73 \quad A=180.950$

\begin{tabular}{rrr} 
TDE 8.0 & EV & \multicolumn{2}{c}{ ET $=0.456035$ MEV } \\
BNERGY(NEV) & PRTMRY & CASCADE \\
0.46000 & 5.20 & 5.20 \\
0.46500 & 11.87 & 11.87 \\
0.47400 & 24.11 & 24.11 \\
0.48700 & 42.25 & 42.25 \\
0.50600 & 69.38 & 69.38 \\
0.52900 & 102.65 & 102.65 \\
0.55600 & 141.55 & 141.55 \\
0.59200 & 192.12 & 192.12 \\
0.63800 & 253.19 & 253.19 \\
0.70600 & 334.45 & 334.45 \\
0.79800 & 426.55 & 427.01 \\
0.91200 & 515.56 & 521.57 \\
1.04000 & 588.74 & 609.39 \\
1.23000 & 660.46 & 715.33 \\
1.45000 & 708.03 & 813.74 \\
1.73000 & 736.99 & 914.58 \\
2.05000 & 747.29 & 1008.13 \\
2.37000 & 745.62 & 1086.00 \\
2.73000 & 737.20 & 1160.72 \\
3.19000 & 722.62 & 1241.76 \\
3.87000 & 700.04 & 1341.42 \\
4.56000 & 679.37 & 1425.44 \\
6.84000 & 630.37 & 1631.98 \\
9.12000 & 602.52 & 1779.26 \\
13.60000 & 573.23 & 1983.61 \\
22.80000 & 546.72 & 2247.13 \\
45.60000 & 529.37 & 2603.22 \\
91.20000 & 520.10 & 2958.02 \\
136.00000 & 514.35 & 3160.13 \\
228.00000 & 506.59 & 3418.08 \\
319.00000 & 502.35 & 3584.26 \\
& &
\end{tabular}

\begin{tabular}{rrr} 
TD $=12.0$ & \multicolumn{2}{c}{ EV $=0.616907$ MEV } \\
ENERGY (KEV) & PRIMARY & CASCADE \\
0.62300 & 2.81 & 2.81 \\
0.62900 & 5.72 & 5.72 \\
0.64100 & 11.91 & 11.91 \\
0.66000 & 22.57 & 22.57 \\
0.68400 & 37.20 & 37.20 \\
0.71500 & 57.34 & 57.34 \\
0.75200 & 82.34 & 82.34 \\
0.80100 & 115.73 & 115.73 \\
0.86300 & 156.74 & 156.74 \\
0.95600 & 213.18 & 213.18 \\
1.07000 & 272.29 & 272.65 \\
1.23000 & 337.30 & 342.22 \\
1.41000 & 389.92 & 406.66 \\
1.66000 & 437.37 & 479.51 \\
1.97000 & 469.99 & 551.79 \\
2.34000 & 487.54 & 621.01 \\
2.77000 & 493.01 & 686.23 \\
3.20000 & 490.86 & 740.38 \\
3.70000 & 484.17 & 793.71 \\
4.31000 & 473.96 & 848.72 \\
5.24000 & 458.46 & 918.19 \\
6.16000 & 444.99 & 975.02 \\
9.25000 & 413.63 & 1116.23 \\
12.30000 & 396.61 & 1215.11 \\
18.50000 & 378.00 & 1355.56 \\
30.80000 & 362.14 & 1530.09 \\
61.60000 & 351.97 & 1768.45 \\
123.00000 & 345.85 & 2004.29 \\
185.00000 & 341.84 & 2141.70 \\
308.00000 & 336.83 & 2310.96 \\
431.00000 & 334.24 & 2421.79
\end{tabular}

ATOMIC DISPLACBHENT CROSS-SECTIONS (BARNS) BY FAST ELECTRONS IN TA $\mathrm{Z}=73 \quad \mathrm{~A}=180.950$

\begin{tabular}{|c|c|c|c|}
\hline \multirow{2}{*}{\multicolumn{2}{|c|}{$\begin{array}{r}T D=16.0 \mathrm{EV} \\
\text { ENERGY (MEV) }\end{array}$}} & \multicolumn{2}{|c|}{$\mathrm{BT}=0.757539 \mathrm{kEV}$} \\
\hline & & PRIUART & CASCADE \\
\hline 0.76500 & & 1.66 & 1.66 \\
\hline 0.77200 & & 3.35 & 3.35 \\
\hline 0.78700 & & 7.36 & 7.36 \\
\hline 0.81000 & & 14.38 & 14.38 \\
\hline 0.84000 & & 24.76 & 24.76 \\
\hline 0.87800 & & 39.29 & 39.29 \\
\hline 0.92400 & & 58.04 & 58.04 \\
\hline 0.98400 & & 83.15 & 83.15 \\
\hline 1.06000 & & 114.41 & 114.41 \\
\hline 1.17000 & & 156.32 & 156.32 \\
\hline 1.32000 & & 205.05 & 205.51 \\
\hline 1.51000 & & 253.07 & 257.46 \\
\hline 1.74000 & & 294.43 & 309.25 \\
\hline 2.04000 & & 328.82 & 364.16 \\
\hline 2.42000 & & 352.53 & 419.89 \\
\hline 2.87000 & & 364.65 & 472.80 \\
\hline 3.40000 & & 367.85 & 523.18 \\
\hline 3.93000 & & 365.52 & 564.92 \\
\hline 4.54000 & & 360.03 & 605.46 \\
\hline 5.30000 & & 351.90 & 648.11 \\
\hline 6.43000 & & 340.25 & 700.46 \\
\hline 7.57000 & & 330.10 & 744.06 \\
\hline 11.30000 & & 307.59 & 849.59 \\
\hline 15.10000 & & 295.19 & 925.58 \\
\hline 22.70000 & & 261.81 & 1031.23 \\
\hline 37.80000 & & 270.62 & 1162.65 \\
\hline $\begin{array}{r}75.70000 \\
151.00000\end{array}$ & & 263.55 & 1342.11 \\
\hline 151.00000 & & 258.96 & 1518.62 \\
\hline
\end{tabular}

\begin{tabular}{rrr} 
TD $=20.0$ & EV & \multicolumn{2}{c}{ ET=0.884066 MEV } \\
ERERGY (HEV) & PRIHARY & \multicolumn{1}{c}{ CASCADE } \\
0.89200 & 1.01 & 1.01 \\
0.90100 & 2.29 & 2.29 \\
0.91900 & 5.25 & 5.25 \\
0.94500 & 10.31 & 10.31 \\
0.98100 & 18.52 & 18.52 \\
1.02000 & 28.53 & 28.53 \\
1.07000 & 42.40 & 42.40 \\
1.14000 & 62.66 & 62.66 \\
1.23000 & 88.56 & 88.56 \\
1.37000 & 125.89 & 125.89 \\
1.54000 & 164.37 & 164.84 \\
1.76000 & 203.08 & 207.03 \\
2.03000 & 236.60 & 249.66 \\
2.38000 & 263.90 & 294.49 \\
2.82000 & 282.24 & 339.61 \\
3.35000 & 291.39 & 383.10 \\
3.97000 & 293.32 & 424.09 \\
4.59000 & 290.99 & 458.00 \\
5.30000 & 286.29 & 490.70 \\
6.18000 & 279.65 & 524.95 \\
7.51000 & 270.17 & 567.56 \\
8.84000 & 262.09 & 602.68 \\
13.20000 & 244.50 & 687.74 \\
17.60000 & 234.97 & 748.30 \\
26.50000 & 224.53 & 833.36 \\
44.20000 & 215.96 & 939.07 \\
88.40000 & 210.61 & 1082.51 \\
176.00000 & 205.93 & 1223.26
\end{tabular}

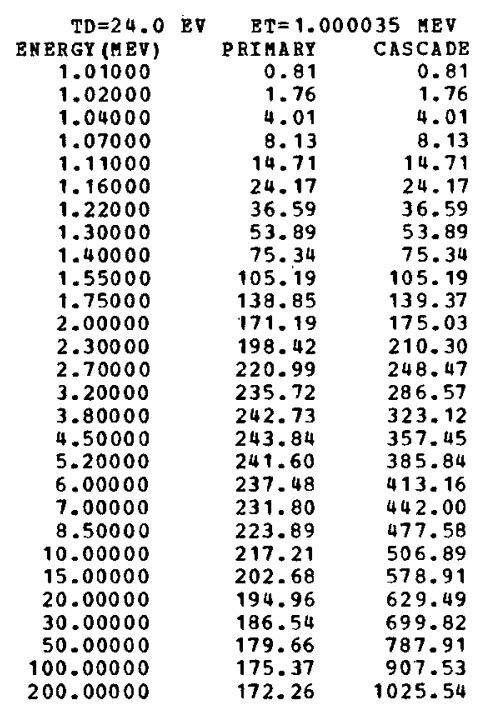


ATOMIC DISPLACEMENT CROSS-SECTIONS (EARNS) BY PAST ELECTRONS IN TA $Z=73 \quad A=180.950$

\begin{tabular}{rrr} 
TD $=28.0$ & \multicolumn{1}{c}{ ET $=1.107716$} \\
ENERGY (MEV) & PRIMARY & CASCADE \\
1.11000 & 0.12 & 0.12 \\
1.12000 & 0.70 & 0.70 \\
1.15000 & 3.00 & 3.00 \\
1.18000 & 6.01 & 6.01 \\
1.22000 & 10.88 & 10.88 \\
1.28000 & 19.50 & 19.50 \\
1.35000 & 30.74 & 30.74 \\
1.44000 & 45.97 & 45.97 \\
1.55000 & 64.47 & 64.47 \\
1.71000 & 89.50 & 89.50 \\
1.93000 & 118.68 & 119.15 \\
2.21000 & 147.10 & 150.58 \\
2.54000 & 170.43 & 181.07 \\
2.99000 & 189.99 & 214.72 \\
3.54000 & 202.24 & 247.49 \\
4.20000 & 207.93 & 278.92 \\
4.98000 & 208.63 & 308.80 \\
5.76000 & 206.51 & 333.44 \\
6.64000 & 202.87 & 356.83 \\
7.75000 & 197.91 & 381.75 \\
9.41000 & 191.11 & 412.37 \\
11.00000 & 185.62 & 436.59 \\
16.60000 & 173.15 & 499.45 \\
22.10000 & 166.66 & 542.65 \\
33.20000 & 159.54 & 603.23 \\
55.30000 & 153.81 & 678.74 \\
110.00000 & 150.25 & 780.51 \\
221.00000 & 147.56 & 882.30
\end{tabular}

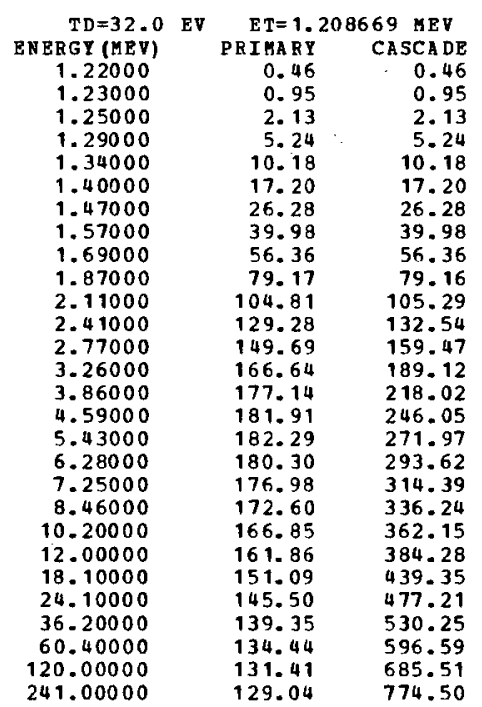

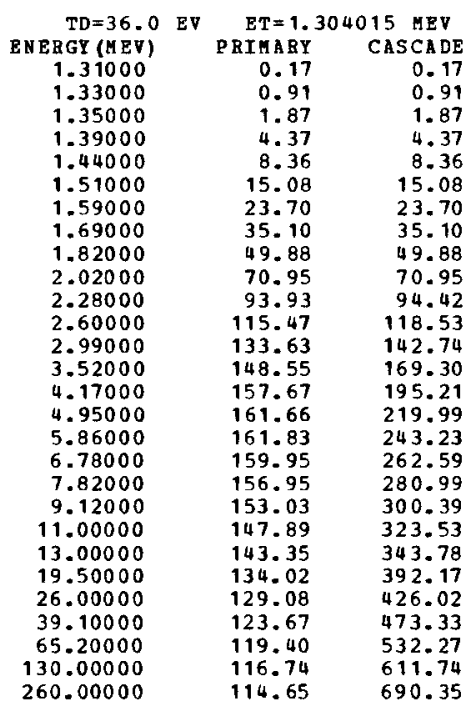

ATOHIC DISPLACEMENT CROSS-SECTIONS (BARNS) BY PAST ELECTRONS IN TA $z=73 \quad A=180.950$

\begin{tabular}{rrr} 
TD $=40.0$ & EV & \multicolumn{1}{c}{ ET $=1.394596$ MEV } \\
ENERGY(MEV) & PRIMARY & CASCADE \\
1.40000 & 0.12 & 0.12 \\
1.42000 & 0.69 & 0.69 \\
1.45000 & 1.88 & 1.88 \\
1.49000 & 4.03 & 4.03 \\
1.54000 & 7.40 & 7.40 \\
1.61000 & 13.04 & 13.04 \\
1.70000 & 21.24 & 21.24 \\
1.81000 & 31.88 & 31.88 \\
1.95000 & 45.38 & 45.38 \\
2.16000 & 64.13 & 64.13 \\
2.44000 & 85.07 & 85.55 \\
2.78000 & 104.35 & 107.24 \\
3.20000 & 120.75 & 129.31 \\
3.76000 & 133.91 & 153.06 \\
4.46000 & 142.04 & 176.66 \\
5.29000 & 145.46 & 198.95 \\
6.27000 & 145.49 & 220.08 \\
7.25000 & 143.72 & 237.48 \\
8.36000 & 140.97 & 254.06 \\
9.76000 & 137.39 & 271.68 \\
11.80000 & 132.70 & 292.80 \\
13.90000 & 128.71 & 310.69 \\
20.90000 & 120.35 & 354.57 \\
27.80000 & 115.99 & 384.80 \\
41.80000 & 111.17 & 427.38 \\
69.70000 & 107.39 & 480.46 \\
139.00000 & 105.03 & 552.01 \\
278.00000 & 103.14 & 622.74 \\
& &
\end{tabular}

\begin{tabular}{rrr} 
TD $=44.0$ & EV & \multicolumn{1}{c}{ RT $=1.481062$ MEV } \\
ENERY (HEV) & PRIMARY & CASCADE \\
1.49000 & 0.16 & 0.16 \\
1.51000 & 0.64 & 0.64 \\
1.54000 & 1.66 & 1.66 \\
1.58000 & 3.46 & 3.46 \\
1.64000 & 6.93 & 6.93 \\
1.71000 & 11.79 & 11.79 \\
1.80000 & 18.84 & 18.84 \\
1.92000 & 28.84 & 28.84 \\
2.07000 & 41.33 & 41.33 \\
2.29000 & 58.29 & 58.29 \\
2.59000 & 77.62 & 78.08 \\
2.96000 & 95.56 & 98.38 \\
3.40000 & 110.16 & 118.22 \\
3.99000 & 121.93 & 139.74 \\
4.73000 & 129.20 & 161.21 \\
5.62000 & 132.22 & 181.74 \\
6.66000 & 132.14 & 200.99 \\
7.70000 & 130.47 & 216.82 \\
8.88000 & 127.93 & 231.94 \\
10.30000 & 124.79 & 247.32 \\
12.50000 & 120.44 & 266.95 \\
14.80000 & 116.71 & 283.74 \\
22.20000 & 109.22 & 323.44 \\
29.60000 & 105.26 & 351.17 \\
44.40000 & 100.95 & 389.67 \\
74.00000 & 97.57 & 437.90 \\
148.00000 & 95.45 & 503.23 \\
296.00000 & 93.73 & 567.51
\end{tabular}

\begin{tabular}{rrr} 
TD=48.0 & \multicolumn{2}{c}{ ET $=1.563929$ MEV } \\
ENERGY (HEV) & PBI ARY & CASCADE \\
1.57000 & 0.09 & 0.09 \\
1.59000 & 0.46 & 0.46 \\
1.62000 & 1.27 & 1.27 \\
1.67000 & 3.18 & 3.18 \\
1.73000 & 6.19 & 6.19 \\
1.81000 & 11.05 & 11.05 \\
1.90000 & 17.21 & 17.21 \\
2.03000 & 26.69 & 26.69 \\
2.18000 & 37.61 & 37.61 \\
2.42000 & 53.78 & 53.78 \\
2.73000 & 71.21 & 71.65 \\
3.12000 & 87.72 & 90.36 \\
3.59000 & 101.26 & 108.86 \\
4.22000 & 112.05 & 128.86 \\
5.00000 & 118.55 & 148.53 \\
5.94000 & 121.19 & 167.38 \\
7.03000 & 121.03 & 184.93 \\
8.13000 & 119.45 & 199.55 \\
9.38000 & 117.08 & 213.42 \\
10.90000 & 114.15 & 227.72 \\
13.20000 & 110.20 & 245.53 \\
15.60000 & 106.82 & 260.77 \\
23.40000 & 100.00 & 297.21 \\
31.20000 & 96.39 & 322.64 \\
46.90000 & 92.45 & 358.13 \\
78.10000 & 89.40 & 402.28 \\
156.00000 & 87.48 & 462.06 \\
312.00000 & 85.89 & 520.98
\end{tabular}


ATOHIC DISPLACEMENT CROSS-SECTIONS (BARNS) BY FAST ELECTRONS IN TA

\begin{tabular}{rrr} 
TD $=52.0$ & EV & \multicolumn{2}{c}{ ET=1.643611 MEV } \\
PNERGY (MEV) & PRIMRY & CASCADE \\
1.66000 & 0.21 & 0.21 \\
1.67000 & 0.39 & 0.39 \\
1.70000 & 1.07 & 1.07 \\
1.75000 & 2.69 & 2.69 \\
1.82000 & 5.76 & 5.76 \\
1.90000 & 10.04 & 10.04 \\
2.00000 & 16.10 & 16.10 \\
2.13000 & 24.47 & 24.47 \\
2.30000 & 35.41 & 35.41 \\
2.54000 & 49.67 & 49.67 \\
2.87000 & 66.03 & 66.46 \\
3.28000 & 81.27 & 83.81 \\
3.78000 & 93.85 & 101.13 \\
4.43000 & 103.54 & 119.31 \\
5.25000 & 109.48 & 137.54 \\
6.24000 & 111.85 & 155.04 \\
7.39000 & 111.64 & 171.33 \\
8.54000 & 110.14 & 184.73 \\
9.86000 & 107.92 & 197.67 \\
11.50000 & 105.14 & 211.19 \\
13.90000 & 101.52 & 227.49 \\
16.40000 & 98.44 & 241.43 \\
24.60000 & 92.19 & 275.09 \\
32.80000 & 88.88 & 298.57 \\
49.30000 & 85.27 & 331.33 \\
82.10000 & 82.49 & 372.11 \\
164.00000 & 80.73 & 427.30
\end{tabular}

$\begin{array}{rrr}\begin{array}{c}\text { TD }=56.0 \\ \text { ENERGY (MBV) }\end{array} & \begin{array}{c}\text { ET }=1.720449 \text { MEV } \\ \text { PRIMARY }\end{array} & \text { CASCADE } \\ 1.73000 & 0.10 & 0.10 \\ 1.75000 & 0.38 & 0.38 \\ 1.78000 & 0.98 & 0.98 \\ 1.84000 & 2.74 & 2.74 \\ 1.90000 & 5.09 & 5.09 \\ 1.99000 & 9.36 & 9.36 \\ 2.09000 & 14.76 & 14.76 \\ 2.23000 & 22.81 & 22.81 \\ 2.40000 & 32.57 & 32.57 \\ 2.66000 & 46.36 & 46.36 \\ 3.01000 & 61.77 & 62.21 \\ 3.44000 & 75.89 & 78.35 \\ 3.95000 & 87.23 & 94.08 \\ 4.64000 & 96.31 & 111.26 \\ 5.50000 & 101.74 & 128.25 \\ 6.53000 & 103.85 & 144.42 \\ 7.74000 & 103.59 & 159.54 \\ 8.94000 & 102.17 & 172.06 \\ 10.30000 & 100.12 & 183.91 \\ 12.00000 & 97.55 & 196.40 \\ 14.60000 & 94.07 & 212.07 \\ 17.20000 & 91.25 & 224.91 \\ 25.80000 & 85.48 & 256.18 \\ 34.40000 & 82.43 & 277.99 \\ 51.60000 & 79.12 & 308.27 \\ 86.00000 & 76.56 & 346.21 \\ 172.00000 & 74.94 & 397.55\end{array}$

\begin{tabular}{rrr} 
TD=60.0 EV & \multicolumn{1}{c}{ ET $=1.794728$ MEV } \\
BNERGY (IIEV) & PRIMARY & CASCADE \\
1.81000 & 0.14 & 0.14 \\
1.83000 & 0.40 & 0.40 \\
1.86000 & 0.96 & 0.96 \\
1.92000 & 2.55 & 2.55 \\
1.99000 & 5.03 & 5.03 \\
2.08000 & 8.92 & 8.92 \\
2.18000 & 13.78 & 13.78 \\
2.33000 & 21.54 & 21.54 \\
2.51000 & 30.83 & 30.83 \\
2.78000 & 43.66 & 43.66 \\
3.14000 & 57.85 & 58.28 \\
3.58000 & 70.82 & 73.14 \\
4.12000 & 81.58 & 88.11 \\
4.84000 & 90.01 & 104.18 \\
5.74000 & 95.02 & 120.12 \\
6.81000 & 96.92 & 135.17 \\
8.07000 & 96.63 & 149.38 \\
9.33000 & 95.27 & 161.06 \\
10.70000 & 93.40 & 171.78 \\
12.50000 & 90.95 & 183.66 \\
15.20000 & 87.72 & 198.26 \\
17.90000 & 85.09 & 210.22 \\
26.90000 & 79.71 & 239.57 \\
35.80000 & 76.89 & 259.80 \\
53.80000 & 73.80 & 288.20 \\
89.70000 & 71.43 & 323.64 \\
179.00000 & 69.94 & 371.41 \\
& &
\end{tabular}
ATONIC DISPLACERENT CROSS-SECTIONS (BARNS) BY PAST ELECTRONS IN TA

\begin{tabular}{crr} 
TD $=64.0$ & EV & \multicolumn{2}{c}{ ET=1.866688 IIEV } \\
ENERGY (HEV) & PRIMARY & CASCADE \\
1.88000 & 0.10 & 0.10 \\
1.90000 & 0.32 & 0.32 \\
1.94000 & 0.99 & 0.99 \\
1.99000 & 2.18 & 2.18 \\
2.07000 & 4.71 & 4.71 \\
2.16000 & 8.21 & 8.21 \\
2.27000 & 13.05 & 13.05 \\
2.42000 & 20.08 & 20.08 \\
2.61000 & 28.97 & 28.97 \\
2.89000 & 41.01 & 41.02 \\
3.26000 & 54.22 & 54.63 \\
3.73000 & 66.71 & 68.98 \\
4.29000 & 76.70 & 82.98 \\
5.04000 & 84.53 & 98.08 \\
5.97000 & 89.12 & 112.94 \\
7.09000 & 90.85 & 127.15 \\
8.40000 & 90.54 & 140.47 \\
9.70000 & 89.25 & 151.34 \\
11.20000 & 87.39 & 161.89 \\
13.00000 & 85.17 & 172.57 \\
15.80000 & 82.15 & 186.23 \\
18.60000 & 79.69 & 197.43 \\
28.00000 & 74.65 & 225.07 \\
37.30000 & 72.02 & 244.11 \\
56.00000 & 69.15 & 270.67 \\
93.30000 & 66.95 & 303.86 \\
186.00000 & 65.56 & 348.58
\end{tabular}

\begin{tabular}{rrr} 
TD $=68.0$ & EV & \multicolumn{1}{c}{ ET=1.936533 MEV } \\
ENERGY (HEV) & PRIMAY & CASCADE \\
1.95000 & 0.09 & 0.09 \\
1.97000 & 0.28 & 0.28 \\
2.01000 & 0.87 & 0.87 \\
2.07000 & 2.17 & 2.17 \\
2.14000 & 4.19 & 4.18 \\
2.24000 & 7.69 & 7.69 \\
2.36000 & 12.51 & 12.51 \\
2.51000 & 18.93 & 18.93 \\
2.71000 & 27.45 & 27.45 \\
3.00000 & 38.79 & 38.80 \\
3.38000 & 51.13 & 51.52 \\
3.87000 & 62.95 & 65.13 \\
4.45000 & 72.31 & 78.32 \\
5.22000 & 79.59 & 92.43 \\
6.19000 & 83.91 & 105.53 \\
7.35000 & 85.50 & 119.91 \\
8.71000 & 85.17 & 132.49 \\
10.00000 & 84.00 & 142.32 \\
11.60000 & 82.19 & 152.59 \\
13.50000 & 80.07 & 162.83 \\
16.40000 & 77.22 & 175.66 \\
19.30000 & 74.92 & 186.18 \\
29.00000 & 70.22 & 212.10 \\
38.70000 & 67.73 & 230.13 \\
58.00000 & 65.05 & 255.03 \\
96.80000 & 62.99 & 286.37 \\
193.00000 & 61.69 & 328.47
\end{tabular}

\begin{tabular}{rrr} 
TD $=72.0$ & EV & \multicolumn{2}{c}{ ET $=2.004439$ MEV } \\
ENERGY (MEV) & PRIAAR & CASCADE \\
2.02000 & 0.09 & 0.09 \\
2.04000 & 0.27 & 0.27 \\
2.08000 & 0.80 & 0.80 \\
2.14000 & 1.97 & 1.97 \\
2.22000 & 4.08 & 4.08 \\
2.32000 & 7.32 & 7.32 \\
2.44000 & 11.73 & 11.73 \\
2.60000 & 18.00 & 18.00 \\
2.80000 & 25.81 & 25.81 \\
3.10000 & 36.58 & 36.58 \\
3.50000 & 48.46 & 48.85 \\
4.00000 & 59.48 & 61.56 \\
4.61000 & 68.46 & 74.25 \\
5.41000 & 75.29 & 87.63 \\
6.41000 & 79.29 & 100.90 \\
7.61000 & 80.75 & 113.54 \\
9.01000 & 80.41 & 125.36 \\
10.40000 & 79.23 & 135.01 \\
12.00000 & 77.57 & 144.37 \\
14.00000 & 75.52 & 154.20 \\
17.00000 & 72.84 & 166.29 \\
20.00000 & 70.68 & 176.21 \\
30.00000 & 66.27 & 200.60 \\
40.00000 & 63.94 & 217.58 \\
60.10000 & 61.40 & 241.25 \\
100.00000 & 59.48 & 270.68 \\
200.00000 & 58.25 & 310.62
\end{tabular}


ATOHIC DISPLACEHENT CROSS-SECTIONS (BARNS) BY FAST RLECTRONS IN TA

\begin{tabular}{rrr}
$\begin{array}{r}\text { TD }=76.0 \\
\text { ENERGY }\end{array}$ & \multicolumn{2}{c}{ ET $=2.070560$ MEV } \\
2.09000 & PRIMARY & CASCADE \\
2.11000 & 0.11 & 0.11 \\
2.15000 & 0.27 & 0.27 \\
2.21000 & 0.77 & 0.77 \\
2.29000 & 1.84 & 1.84 \\
2.40000 & 3.77 & 3.77 \\
2.52000 & 7.04 & 7.04 \\
2.69000 & 11.12 & 11.12 \\
2.89000 & 17.25 & 17.25 \\
3.20000 & 24.44 & 24.44 \\
3.62000 & 34.69 & 34.69 \\
4.14000 & 46.14 & 46.53 \\
4.76000 & 56.61 & 58.65 \\
5.59000 & 64.93 & 70.49 \\
6.62000 & 71.40 & 83.24 \\
7.86000 & 75.14 & 95.79 \\
9.31000 & 76.49 & 107.78 \\
10.70000 & 76.14 & 119.02 \\
12.40000 & 75.05 & 127.90 \\
14.40000 & 73.43 & 137.04 \\
17.50000 & 71.54 & 146.08 \\
20.70000 & 68.99 & 157.60 \\
31.00000 & 66.88 & 167.32 \\
41.40000 & 62.74 & 190.34 \\
62.10000 & 60.53 & 206.52 \\
103.00000 & 58.15 & 228.85 \\
207.00000 & 56.35 & 256.57 \\
& 55.18 & 294.67
\end{tabular}

\begin{tabular}{rrr} 
TD $=80.0$ & \multicolumn{1}{c}{ ET 2.135029 MEV } \\
ENERGY (HEV) & PRTMAY & CASCADE \\
2.15000 & 0.07 & 0.07 \\
2.17000 & 0.21 & 0.21 \\
2.22000 & 0.76 & 0.76 \\
2.28000 & 1.76 & 1.76 \\
2.36000 & 3.54 & 3.54 \\
2.47000 & 6.55 & 6.55 \\
2.60000 & 10.53 & 10.63 \\
2.77000 & 16.30 & 16.30 \\
2.98000 & 23.28 & 23.28 \\
3.30000 & 33.05 & 33.06 \\
3.73000 & 43.88 & 44.25 \\
4.27000 & 53.90 & 55.89 \\
4.91000 & 61.80 & 67.15 \\
5.76000 & 67.87 & 79.21 \\
6.83000 & 71.42 & 91.23 \\
8.11000 & 72.66 & 102.64 \\
9.60000 & 72.31 & 113.28 \\
11.10000 & 71.20 & 122.08 \\
12.80000 & 69.69 & 130.48 \\
14.90000 & 67.87 & 139.21 \\
18.10000 & 65.45 & 150.13 \\
21.30000 & 63.50 & 159.08 \\
32.00000 & 59.56 & 181.12 \\
42.70000 & 57.47 & 196.45 \\
64.00000 & 55.22 & 217.63 \\
106.00000 & 53.52 & 243.89 \\
213.00000 & 52.41 & 280.08
\end{tabular}

\begin{tabular}{rrr} 
TD $=84.0$ & EV & \multicolumn{2}{c}{ ET $=2.197964$ MEV } \\
ENERGY (MEV) & PRIHARY & CASCADE \\
2.21000 & 0.05 & 0.05 \\
2.24000 & 0.24 & 0.24 \\
2.28000 & 0.65 & 0.65 \\
2.35000 & 1.72 & 1.72 \\
2.43000 & 3.38 & 3.38 \\
2.54000 & 6.17 & 6.17 \\
2.68000 & 10.24 & 10.24 \\
2.85000 & 15.51 & 15.51 \\
3.07000 & 22.30 & 22.30 \\
3.40000 & 31.63 & 31.64 \\
3.84000 & 41.89 & 42.25 \\
4.39000 & 51.35 & 53.25 \\
5.05000 & 58.89 & 64.03 \\
5.93000 & 64.70 & 75.60 \\
7.03000 & 68.04 & 87.05 \\
8.35000 & 69.20 & 97.94 \\
9.89000 & 68.83 & 108.12 \\
11.40000 & 67.79 & 116.33 \\
13.10000 & 66.39 & 124.14 \\
15.30000 & 64.62 & 132.64 \\
18.60000 & 62.30 & 143.09 \\
21.90000 & 60.45 & 151.64 \\
32.90000 & 56.70 & 172.65 \\
43.90000 & 54.71 & 187.25 \\
65.90000 & 52.57 & 207.50 \\
109.00000 & 50.97 & 232.45 \\
219.00000 & 49.91 & 266.91 \\
& &
\end{tabular}

ATOMIC DISPLACEMENT CROSS-SECTIONS (BARNS) BY FAST ELECTRONS IN TA $\mathrm{Z}=73 \quad \mathrm{~A}=180.950$

\begin{tabular}{rrr} 
TD $=88.0$ & EV & \multicolumn{2}{c}{ ET $=2.259470$ MEV } \\
PRIMARY & CASCADE \\
2.28000 & 0.08 & 0.08 \\
2.30000 & 0.21 & 0.21 \\
2.34000 & 0.57 & 0.57 \\
2.41000 & 1.54 & 1.54 \\
2.50000 & 3.26 & 3.26 \\
2.62000 & 6.12 & 6.12 \\
2.75000 & 9.65 & 9.65 \\
2.93000 & 14.85 & 14.85 \\
3.16000 & 21.45 & 21.45 \\
3.50000 & 30.39 & 30.40 \\
3.95000 & 40.12 & 40.48 \\
4.51000 & 49.06 & 50.89 \\
5.19000 & 56.28 & 61.23 \\
6.10000 & 61.82 & 72.35 \\
7.23000 & 64.97 & 83.28 \\
8.58000 & 66.05 & 93.63 \\
10.10000 & 65.72 & 103.00 \\
11.70000 & 64.69 & 111.11 \\
13.50000 & 63.31 & 118.80 \\
15.80000 & 61.58 & 127.03 \\
19.20000 & 59.38 & 136.98 \\
22.50000 & 57.67 & 144.91 \\
33.80000 & 54.09 & 164.96 \\
45.10000 & 52.20 & 178.90 \\
67.70000 & 50.17 & 198.23 \\
112.00000 & 48.64 & 222.06 \\
25.00000 & 47.64 & 254.95 \\
& &
\end{tabular}

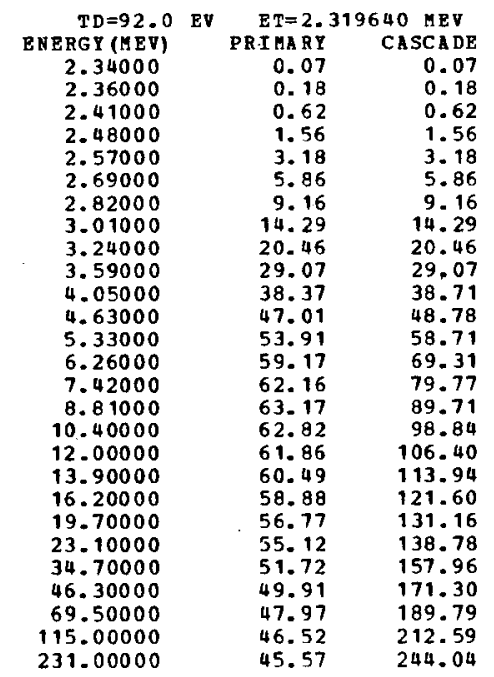

\begin{tabular}{rrr} 
TD $=96.0$ & EV & \multicolumn{2}{c}{ ET $=2.378557$ HEV } \\
ENERGY (MEV) & PRIMARY & CASCADE \\
2.40000 & 0.07 & 0.07 \\
2.42000 & 0.17 & 0.17 \\
2.47000 & 0.58 & 0.58 \\
2.54000 & 1.44 & 1.44 \\
2.64000 & 3.14 & 3.14 \\
2.75000 & 5.44 & 5.44 \\
2.90000 & 9.00 & 9.00 \\
3.09000 & 13.81 & 13.81 \\
3.32000 & 19.60 & 19.60 \\
3.68000 & 27.89 & 27.90 \\
4.16000 & 36.96 & 37.31 \\
4.75000 & 45.15 & 46.88 \\
5.47000 & 51.76 & 56.43 \\
6.42000 & 56.74 & 66.55 \\
7.61000 & 59.59 & 76.59 \\
9.03000 & 60.53 & 86.08 \\
10.70000 & 60.17 & 95.03 \\
12.30000 & 59.26 & 102.08 \\
14.20000 & 57.97 & 109.16 \\
16.60000 & 56.40 & 116.65 \\
20.20000 & 54.37 & 125.84 \\
23.70000 & 52.79 & 133.17 \\
35.60000 & 49.53 & 151.56 \\
47.50000 & 47.81 & 164.34 \\
71.30000 & 45.95 & 182.06 \\
118.00000 & 44.57 & 203.92 \\
237.00000 & 43.66 & 234.06
\end{tabular}


ATOHIC DISPLACEMENT CROSS-SECTIONS (BARNS) BY FAST ELECTRONS IN $z=74 \quad A=183.860$

\begin{tabular}{crr} 
TD $=4.0$ & \multicolumn{1}{c}{ ET $=0.265890$ MEV } \\
ENERGY (MEV) & PRIMARY & CASCADE \\
0.26800 & 17.71 & 17.71 \\
0.27100 & 42.44 & 42.44 \\
0.27600 & 82.57 & 82.57 \\
0.28400 & 144.14 & 144.14 \\
0.29500 & 223.93 & 223.93 \\
0.30800 & 311.71 & 311.71 \\
0.32400 & 411.20 & 411.20 \\
0.34500 & 529.22 & 529.22 \\
0.37200 & 662.92 & 662.92 \\
0.41200 & 829.91 & 829.91 \\
0.46500 & 1005.68 & 1005.68 \\
0.53100 & 1169.82 & 1178.31 \\
0.61100 & 1310.90 & 1346.80 \\
0.71700 & 1432.23 & 1525.05 \\
0.85000 & 1518.85 & 1702.65 \\
1.01000 & 1567.81 & 1873.91 \\
1.19000 & 1584.43 & 2031.54 \\
1.38000 & 1579.52 & 2171.45 \\
1.59000 & 1561.27 & 2303.91 \\
1.86000 & 1529.86 & 2450.48 \\
2.26000 & 1480.66 & 2632.69 \\
2.65000 & 1436.38 & 2783.01 \\
3.98000 & 1324.58 & 3172.78 \\
5.31000 & 1257.04 & 3454.90 \\
7.97000 & 1185.96 & 3861.25 \\
13.20000 & 1123.81 & 4370.92 \\
26.50000 & 1077.59 & 5085.68 \\
53.10000 & 1057.07 & 5804.13 \\
79.70000 & 1045.53 & 6219.85 \\
132.00000 & 1029.77 & 6730.90 \\
186.00000 & 1019.74 & 7074.32 \\
& & \\
18600000 & &
\end{tabular}

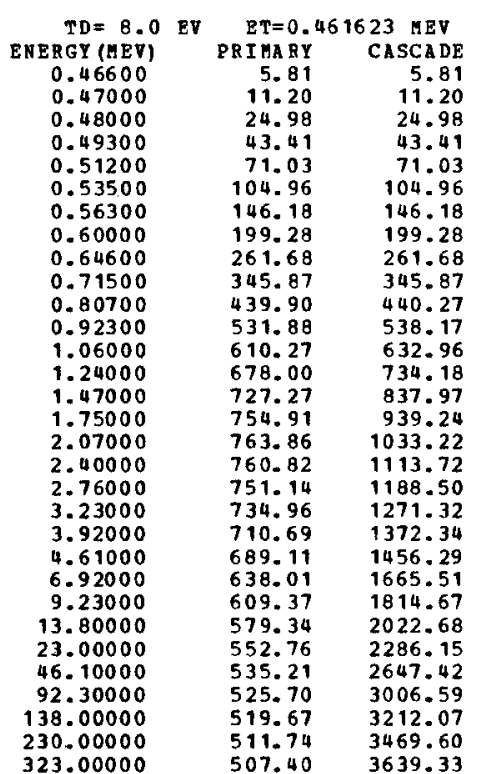

$\begin{array}{rrr}\begin{array}{r}\text { TD=12.0 EV } \\ \text { ENERGY }\end{array} \text { ETEV }=0.624092 \text { MEV } \\ 0.63000 & \text { PRIMARY } & \text { CASCADE } \\ 0.63600 & 2.75 & 2.75 \\ 0.64900 & 5.70 & 5.70 \\ 0.66700 & 12.52 & 12.52 \\ 0.69200 & 22.81 & 22.81 \\ 0.72300 & 38.36 & 38.36 \\ 0.76100 & 58.98 & 58.98 \\ 0.81100 & 85.29 & 85.29 \\ 0.87300 & 120.21 & 120.21 \\ 0.96700 & 162.22 & 162.22 \\ 1.09000 & 220.57 & 220.57 \\ 1.24000 & 285.20 & 285.68 \\ 1.43000 & 346.88 & 351.84 \\ 1.68000 & 450.97 & 420.82 \\ 1.99000 & 482.24 & 494.22 \\ 2.37000 & 499.34 & 567.04 \\ 2.80000 & 503.73 & 703.43 \\ 3.24000 & 500.59 & 759.25 \\ 3.74000 & 493.03 & 812.59 \\ 4.36000 & 481.83 & 868.52 \\ 5.30000 & 465.30 & 938.70 \\ 6.24000 & 450.98 & 996.68 \\ 9.36000 & 418.52 & 1139.05 \\ 12.40000 & 401.28 & 1237.74 \\ 18.70000 & 382.17 & 1380.69 \\ 31.20000 & 366.04 & 1557.92 \\ 62.40000 & 355.83 & 1798.98 \\ 124.00000 & 349.58 & 2035.68 \\ 187.00000 & 345.39 & 2175.37 \\ 312.00000 & 340.21 & 2347.03 \\ 436.00000 & 337.62 & 2458.54\end{array}$

ATOMIC DISPLACEMENT CROSS-SECTIONS (BARNS) BY FAST ELECTRONS IN $=74 \quad A=183.860$

\begin{tabular}{rrr}
$\begin{array}{r}\text { TD }=16.0 \\
\text { ENERGY (MEV) }\end{array}$ & \multicolumn{2}{c}{ ET $=0.766055$ MEV } \\
PRIMARY & CASCADE \\
0.77300 & 1.56 & 1.56 \\
0.78100 & 3.52 & 3.52 \\
0.79600 & 7.61 & 7.61 \\
0.81900 & 14.78 & 14.78 \\
0.85000 & 25.78 & 25.78 \\
0.88800 & 40.69 & 40.69 \\
0.93400 & 59.94 & 59.94 \\
0.99500 & 86.15 & 86.15 \\
1.07000 & 117.80 & 117.80 \\
1.18000 & 160.80 & 160.80 \\
1.34000 & 213.74 & 214.28 \\
1.53000 & 262.16 & 266.86 \\
1.76000 & 303.70 & 319.24 \\
2.06000 & 338.06 & 374.71 \\
2.45000 & 361.90 & 432.28 \\
2.91000 & 373.40 & 486.44 \\
3.44000 & 375.70 & 536.84 \\
3.98000 & 372.60 & 579.35 \\
4.59000 & 366.45 & 619.87 \\
5.36000 & 357.61 & 663.08 \\
6.51000 & 345.14 & 716.28 \\
7.66000 & 334.52 & 760.20 \\
11.40000 & 311.34 & 866.08 \\
15.30000 & 298.44 & 944.06 \\
22.90000 & 284.97 & 1049.74 \\
38.30000 & 273.54 & 1183.77 \\
76.60000 & 266.45 & 1364.90 \\
153.00000 & 261.70 & 1543.61
\end{tabular}

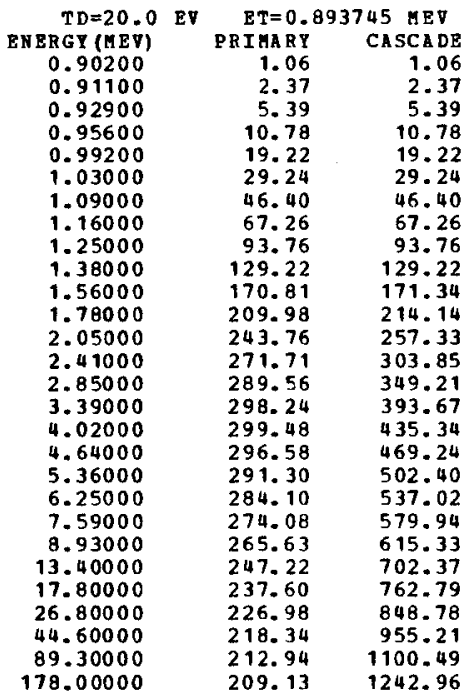

\begin{tabular}{rrr} 
TD=24.0 EV & \multicolumn{2}{c}{ ET $=1.010757$ MEV } \\
ENERGY(HEV) & PRIMABY & CASCADE \\
1.02000 & 0.76 & 0.76 \\
1.03000 & 1.71 & 1.71 \\
1.05000 & 3.99 & 3.99 \\
1.08000 & 8.17 & 8.17 \\
1.12000 & 14.89 & 14.89 \\
1.17000 & 24.57 & 24.57 \\
1.23000 & 37.30 & 37.30 \\
1.31000 & 55.06 & 55.06 \\
1.41000 & 77.12 & 77.12 \\
1.56000 & 107.81 & 107.81 \\
1.76000 & 142.41 & 142.91 \\
2.02000 & 176.74 & 180.74 \\
2.32000 & 204.25 & 216.52 \\
2.72000 & 226.92 & 255.17 \\
3.23000 & 241.72 & 294.40 \\
3.84000 & 248.35 & 331.72 \\
4.54000 & 248.93 & 366.16 \\
5.25000 & 246.20 & 395.04 \\
6.06000 & 241.62 & 422.73 \\
7.07000 & 235.50 & 451.89 \\
8.59000 & 227.09 & 487.95 \\
10.10000 & 220.11 & 517.46 \\
15.10000 & 205.18 & 589.67 \\
20.20000 & 197.17 & 641.40 \\
30.30000 & 188.60 & 712.51 \\
50.50000 & 181.63 & 801.59 \\
101.00000 & 177.31 & 922.54 \\
202.00000 & 174.10 & 1041.79
\end{tabular}


ATOMIC DISPLACEMENT CROSS-SECTIONS (BARNS) BY PAST ELECTRONS IN

\begin{tabular}{rrr} 
TD $=28.0$ & EV & \multicolumn{2}{c}{ ET $=1.119393$ HEV } \\
PRIMARY & CASCADE \\
1.13000 & 0.60 & 0.60 \\
1.14000 & 1.28 & 1.28 \\
1.16000 & 2.90 & 2.90 \\
1.19000 & 5.94 & 5.94 \\
1.24000 & 12.26 & 12.26 \\
1.29000 & 19.69 & 19.69 \\
1.36000 & 31.21 & 31.21 \\
1.45000 & 46.84 & 46.84 \\
1.56000 & 65.87 & 65.87 \\
1.73000 & 93.13 & 93.13 \\
1.95000 & 122.85 & 123.35 \\
2.23000 & 151.72 & 155.32 \\
2.57000 & 175.91 & 187.12 \\
3.02000 & 195.33 & 221.06 \\
3.58000 & 207.46 & 254.61 \\
4.25000 & 212.73 & 286.60 \\
5.03000 & 212.94 & 316.51 \\
5.82000 & 210.38 & 341.50 \\
6.71000 & 206.35 & 365.17 \\
7.83000 & 201.02 & 390.32 \\
9.51000 & 193.81 & 421.30 \\
11.10000 & 188.12 & 445.54 \\
16.70000 & 175.29 & 508.62 \\
22.30000 & 168.57 & 552.76 \\
33.50000 & 161.31 & 614.00 \\
55.90000 & 155.49 & 690.62 \\
111.00000 & 151.91 & 793.27 \\
223.00000 & 149.13 & 896.12
\end{tabular}

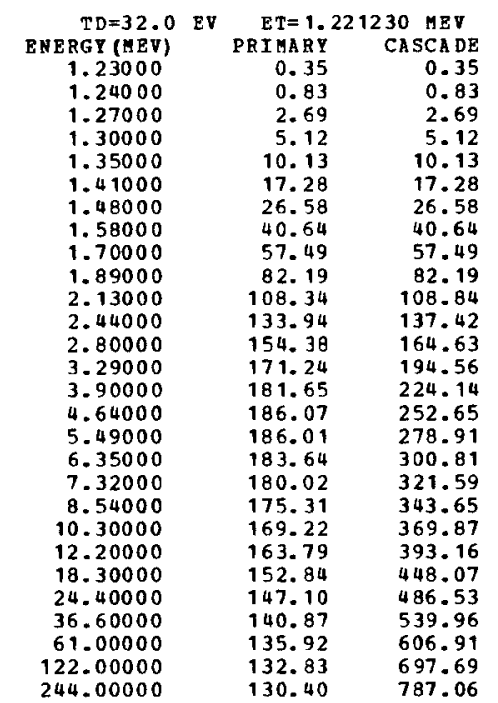

\begin{tabular}{rrr} 
TD=36.0 EV & \multicolumn{2}{c}{ ET $=1.317403$ MEV } \\
ENERGY (NEV) & PRIBARY & CASCADE \\
1.33000 & 0.39 & 0.39 \\
1.34000 & 0.78 & 0.78 \\
1.37000 & 2.27 & 2.27 \\
1.40000 & 4.22 & 4.22 \\
1.46000 & 9.15 & 9.15 \\
1.52000 & 15.08 & 15.08 \\
1.60000 & 23.91 & 23.91 \\
1.71000 & 36.79 & 36.79 \\
1.84000 & 51.96 & 51.96 \\
2.04000 & 73.53 & 73.53 \\
2.30000 & 96.99 & 97.50 \\
2.63000 & 119.50 & 122.75 \\
3.03000 & 138.10 & 147.80 \\
3.55000 & 152.59 & 174.07 \\
4.21000 & 161.64 & 200.57 \\
5.00000 & 165.32 & 225.77 \\
5.92000 & 165.12 & 249.31 \\
6.85000 & 162.90 & 268.90 \\
7.90000 & 159.60 & 287.49 \\
9.22000 & 155.38 & 307.19 \\
11.10000 & 150.00 & 330.33 \\
13.10000 & 145.29 & 350.63 \\
19.70000 & 135.59 & 399.84 \\
26.30000 & 130.51 & 434.21 \\
39.50000 & 125.03 & 481.89 \\
65.80000 & 120.72 & 541.38 \\
131.00000 & 118.04 & 621.56 \\
263.00000 & 115.86 & 701.42
\end{tabular}

ATOMIC DISPLACEMENT CROSS-SECTIONS (BARNS) BY FAST ELECTRONS IN $=74 \quad A=183.860$

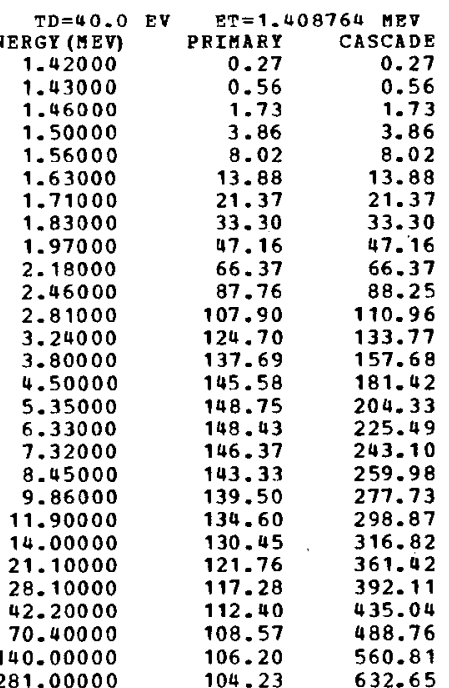

\begin{tabular}{rrr} 
TD=44.0 & \multicolumn{1}{c}{ ET $=1.495971$ MEV } \\
ENERGY(MEV) & PRIMA Y & CASCADE \\
1.51000 & 0.27 & 0.27 \\
1.52000 & 0.52 & 0.52 \\
1.55000 & 1.50 & 1.50 \\
1.60000 & 3.80 & 3.80 \\
1.66000 & 7.43 & 7.43 \\
1.73000 & 12.48 & 12.48 \\
1.82000 & 19.76 & 19.76 \\
1.94000 & 30.05 & 30.05 \\
2.09000 & 42.87 & 42.87 \\
2.31000 & 60.25 & 60.25 \\
2.61000 & 80.02 & 80.49 \\
2.99000 & 98.73 & 101.69 \\
3.44000 & 113.69 & 122.20 \\
4.03000 & 125.33 & 143.88 \\
4.78000 & 132.45 & 165.75 \\
5.68000 & 135.19 & 186.57 \\
6.73000 & 134.79 & 206.03 \\
7.77000 & 132.87 & 221.88 \\
8.97000 & 130.07 & 237.28 \\
10.40000 & 126.72 & 252.77 \\
12.70000 & 121.98 & 273.23 \\
14.90000 & 118.29 & 289.28 \\
22.40000 & 110.51 & 329.63 \\
29.90000 & 106.45 & 357.77 \\
44.80000 & 102.07 & 396.59 \\
74.70000 & 98.65 & 445.40 \\
149.00000 & 96.51 & 511.20 \\
299.00000 & 94.72 & 576.47
\end{tabular}

\begin{tabular}{rrr} 
TD $=48.0$ & EV & \multicolumn{2}{c}{ ET $=1.579543$ MEV } \\
ENERGY (HEV) & PRIMARY & CASCADE \\
1.59000 & 0.16 & 0.16 \\
1.61000 & 0.57 & 0.57 \\
1.64000 & 1.44 & 1.44 \\
1.69000 & 3.45 & 3.45 \\
1.75000 & 6.59 & 6.59 \\
1.83000 & 11.62 & 11.62 \\
1.92000 & 17.98 & 17.98 \\
2.05000 & 27.73 & 27.73 \\
2.21000 & 39.68 & 39.68 \\
2.44000 & 55.53 & 55.53 \\
2.76000 & 73.87 & 74.35 \\
3.15000 & 90.58 & 93.34 \\
3.63000 & 104.45 & 112.45 \\
4.26000 & 115.14 & 132.61 \\
5.05000 & 121.51 & 152.65 \\
6.00000 & 123.90 & 171.77 \\
7.10000 & 123.45 & 189.50 \\
8.21000 & 121.62 & 204.22 \\
9.47000 & 119.04 & 218.27 \\
11.00000 & 115.91 & 232.68 \\
13.40000 & 111.62 & 251.22 \\
15.70000 & 108.27 & 265.83 \\
23.60000 & 101.19 & 302.85 \\
31.50000 & 97.48 & 328.65 \\
47.30000 & 93.48 & 364.44 \\
78.90000 & 90.38 & 409.24 \\
157.00000 & 88.45 & 469.35 \\
315.00000 & 86.80 & 529.16
\end{tabular}


ATONIC DISPLACEHENT CROSS-SECTIONS (BARNS) BT PAST ELECTRONS IN

$z=74 \quad A=183.860$

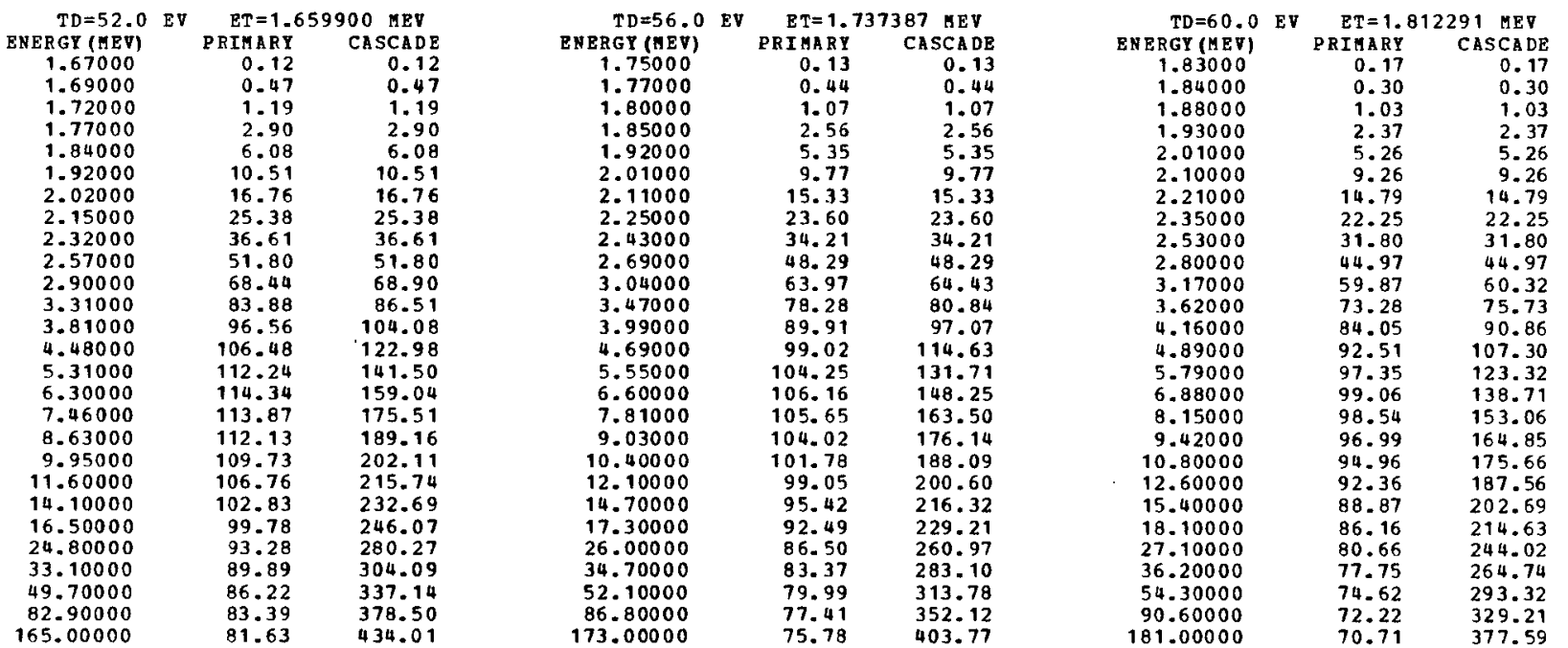

ATOHIC DISPLACEMENT CROSS-SECTIONS (BARNS) BY FAST ELECTRONS IN $=74 \quad A=183.860$

\begin{tabular}{rrr} 
TD 04.0 & EV & \multicolumn{2}{c}{ ET $=1.884854$} & MEV \\
ENERGY (HEV) & PRIMARY & CASCADE \\
1.90000 & 0.12 & 0.12 \\
1.92000 & 0.35 & 0.35 \\
1.96000 & 1.05 & 1.05 \\
2.09000 & 2.28 & 2.28 \\
2.09000 & 4.89 & 4.89 \\
2.18000 & 8.50 & 8.50 \\
2.29000 & 13.49 & 13.49 \\
2.45000 & 21.20 & 21.20 \\
2.63000 & 29.85 & 29.85 \\
2.92000 & 42.63 & 42.63 \\
3.29000 & 56.08 & 56.50 \\
3.76000 & 68.76 & 71.10 \\
4.33000 & 79.00 & 85.54 \\
5.08000 & 86.79 & 100.79 \\
6.03000 & 91.33 & 116.06 \\
7.16000 & 92.86 & 130.45 \\
8.48000 & 92.32 & 143.90 \\
9.80000 & 90.84 & 154.94 \\
11.30000 & 88.84 & 165.51 \\
13.10000 & 86.49 & 176.21 \\
16.00000 & 83.23 & 190.36 \\
18.80000 & 80.70 & 201.54 \\
28.20000 & 75.55 & 229.24 \\
37.60000 & 72.84 & 248.54 \\
56.50000 & 69.91 & 275.46 \\
94.20000 & 67.69 & 309.06 \\
188.00000 & 66.28 & 354.35
\end{tabular}

\begin{tabular}{rrr} 
TD $=68.0$ & EV & \multicolumn{1}{c}{ ET $=1.955284$} \\
ENERGY (MEV) & PRI AARY & CASCADE \\
1.97000 & 0.10 & 0.10 \\
1.99000 & 0.30 & 0.30 \\
2.03000 & 0.91 & 0.91 \\
2.09000 & 2.25 & 2.25 \\
2.17000 & 4.65 & 4.65 \\
2.26000 & 7.94 & 7.94 \\
2.38000 & 12.90 & 12.90 \\
2.54000 & 19.94 & 19.94 \\
2.73000 & 28.26 & 28.26 \\
3.03000 & 40.28 & 40.29 \\
3.42000 & 53.15 & 53.57 \\
3.91000 & 65.07 & 67.37 \\
4.49000 & 74.46 & 80.70 \\
5.27000 & 81.77 & 95.13 \\
6.25000 & 85.98 & 109.45 \\
7.43000 & 87.38 & 123.10 \\
8.79000 & 86.85 & 135.69 \\
10.10000 & 85.51 & 145.69 \\
11.70000 & 83.56 & 155.97 \\
13.60000 & 81.31 & 166.24 \\
16.60000 & 78.24 & 179.52 \\
19.50000 & 75.88 & 190.03 \\
29.30000 & 71.03 & 216.22 \\
39.10000 & 68.49 & 234.45 \\
58.60000 & 65.77 & 259.62 \\
97.70000 & 63.69 & 291.25 \\
195.00000 & 62.37 & 333.88
\end{tabular}

\begin{tabular}{ccr} 
TD $=72.0$ & EV & \multicolumn{1}{c}{ ET $=2.023757$ MEV } \\
ENERGY (MEV) & PRIARY & CASCADE \\
2.04000 & 0.10 & 0.10 \\
2.06000 & 0.28 & 0.28 \\
2.10000 & 0.83 & 0.83 \\
2.16000 & 2.04 & 2.04 \\
2.24000 & 4.20 & 4.20 \\
2.34000 & 7.53 & 7.53 \\
2.46000 & 12.07 & 12.07 \\
2.63000 & 18.93 & 18.93 \\
2.83000 & 26.94 & 26.94 \\
3.13000 & 37.95 & 37.96 \\
3.54000 & 50.34 & 50.75 \\
4.04000 & 61.46 & 63.65 \\
4.65000 & 70.47 & 76.48 \\
5.46000 & 77.34 & 90.16 \\
6.47000 & 81.23 & 103.64 \\
7.69000 & 82.52 & 116.52 \\
9.10000 & 81.98 & 128.45 \\
10.50000 & 80.65 & 138.17 \\
12.10000 & 78.86 & 147.55 \\
14.10000 & 76.69 & 157.41 \\
17.20000 & 73.81 & 169.92 \\
20.20000 & 71.58 & 179.83 \\
30.30000 & 67.04 & 204.48 \\
40.40000 & 64.66 & 221.64 \\
60.70000 & 62.08 & 245.57 \\
101.00000 & 60.14 & 275.34 \\
202.00000 & 58.89 & 315.71
\end{tabular}


ATOMIC DISPLACEMENT CROSS-SECTIONS (BARS) BY PAST ELECTRONS IN

\begin{tabular}{ccr}
$T D=76.0$ & EV & \multicolumn{2}{c}{ ET $=2.090428$ MEV } \\
ENERGY (MEV) & PRIMARY & CASCADE \\
2.11000 & 0.11 & 0.11 \\
2.13000 & 0.28 & 0.28 \\
2.17000 & 0.79 & 0.79 \\
2.23000 & 1.89 & 1.89 \\
2.32000 & 4.15 & 4.15 \\
2.42000 & 7.23 & 7.23 \\
2.55000 & 11.78 & 11.78 \\
2.71000 & 17.73 & 17.73 \\
2.92000 & 25.48 & 25.48 \\
3.24000 & 36.28 & 36.28 \\
3.65000 & 47.65 & 48.05 \\
4.18000 & 58.47 & 60.61 \\
4.80000 & 66.83 & 72.59 \\
5.64000 & 73.33 & 85.62 \\
6.68000 & 76.98 & 98.37 \\
7.94000 & 78.16 & 110.59 \\
9.40000 & 77.63 & 121.93 \\
10.80000 & 76.40 & 130.88 \\
12.50000 & 74.65 & 140.04 \\
14.60000 & 72.56 & 149.53 \\
17.70000 & 69.91 & 161.02 \\
20.90000 & 67.73 & 170.73 \\
31.30000 & 63.47 & 194.00 \\
41.80000 & 61.21 & 210.35 \\
62.70000 & 58.79 & 232.93 \\
104.00000 & 56.97 & 260.96 \\
209.00000 & 55.78 & 299.48
\end{tabular}

\begin{tabular}{crr} 
TD $=80.0$ & EV & \multicolumn{2}{c}{ ET=2.155433 REV } \\
ENERGY (NEV) & PRIMARY & CASCADE \\
2.17000 & 0.07 & 0.07 \\
2.19000 & 0.21 & 0.21 \\
2.24000 & 0.77 & 0.77 \\
2.30000 & 1.80 & 1.80 \\
2.39000 & 3.88 & 3.88 \\
2.50000 & 7.02 & 7.02 \\
2.62000 & 10.90 & 10.90 \\
2.80000 & 17.08 & 17.08 \\
3.01000 & 24.25 & 24.25 \\
3.34000 & 34.54 & 34.54 \\
3.77000 & 45.53 & 45.92 \\
4.31000 & 55.65 & 57.72 \\
4.95000 & 63.59 & 69.13 \\
5.81000 & 69.70 & 81.45 \\
6.89000 & 73.16 & 93.67 \\
8.19000 & 74.25 & 105.29 \\
9.69000 & 73.72 & 116.03 \\
11.20000 & 72.47 & 124.90 \\
12.90000 & 70.85 & 133.32 \\
15.00000 & 68.92 & 142.07 \\
18.30000 & 66.32 & 153.35 \\
21.50000 & 64.32 & 162.30 \\
32.30000 & 60.25 & 184.59 \\
43.10000 & 58.12 & 200.08 \\
64.60000 & 55.83 & 221.49 \\
107.00000 & 54.11 & 248.05 \\
215.00000 & 52.99 & 284.64 \\
& &
\end{tabular}

\begin{tabular}{crr} 
TD $=84.0$ & EV & \multicolumn{2}{c}{ ET $=2.218891$ MEV } \\
ENERGY (HEV) & PRIMARY & CASCADE \\
2.24000 & 0.10 & 0.10 \\
2.26000 & 0.24 & 0.24 \\
2.30000 & 0.65 & 0.65 \\
2.37000 & 1.75 & 1.75 \\
2.46000 & 3.68 & 3.68 \\
2.57000 & 6.59 & 6.59 \\
2.70000 & 10.49 & 10.49 \\
2.88000 & 16.23 & 16.23 \\
3.10000 & 23.20 & 23.20 \\
3.43000 & 32.75 & 32.76 \\
3.88000 & 43.44 & 43.82 \\
4.43000 & 53.00 & 54.98 \\
5.10000 & 60.68 & 66.05 \\
5.99000 & 66.47 & 77.84 \\
7.10000 & 69.71 & 89.45 \\
8.43000 & 70.70 & 100.45 \\
9.98000 & 70.18 & 110.72 \\
11.50000 & 69.01 & 119.00 \\
13.30000 & 67.41 & 127.25 \\
15.50000 & 65.54 & 135.72 \\
18.80000 & 63.14 & 146.14 \\
22.10000 & 61.23 & 154.70 \\
33.20000 & 57.36 & 175.94 \\
44.30000 & 55.33 & 190.69 \\
66.50000 & 53.15 & 211.17 \\
110.00000 & 51.53 & 236.40 \\
221.00000 & 50.46 & 271.24 \\
& &
\end{tabular}

ATOMIC DISPLACENENT CROSS-SECTIONS (BARNS) BY FAST ELECTRONS IN $\mathrm{z}=74 \quad \mathrm{~A}=183,860$

\begin{tabular}{rrr} 
TD $=88.0$ & EV & \multicolumn{2}{c}{ ET $=2.280906$ MEV } \\
PNERGY (MEV) & PRIMARY & CASCADE \\
2.30000 & 0.08 & 0.08 \\
2.32000 & 0.20 & 0.20 \\
2.37000 & 0.68 & 0.68 \\
2.44000 & 1.73 & 1.73 \\
2.53000 & 3.54 & 3.54 \\
2.64000 & 6.25 & 6.25 \\
2.78000 & 10.16 & 10.16 \\
2.96000 & 15.51 & 15.51 \\
3.19000 & 22.29 & 22.29 \\
3.53000 & 31.45 & 31.45 \\
3.99000 & 41.58 & 41.96 \\
4.56000 & 50.76 & 52.70 \\
5.24000 & 57.97 & 63.15 \\
6.15000 & 63.47 & 74.37 \\
7.29000 & 66.54 & 85.47 \\
8.66000 & 67.48 & 96.01 \\
10.20000 & 67.00 & 105.53 \\
11.80000 & 65.85 & 113.67 \\
13.60000 & 64.36 & 121.36 \\
15.90000 & 62.54 & 129.62 \\
19.30000 & 60.24 & 139.62 \\
22.80000 & 58.36 & 148.04 \\
34.20000 & 54.70 & 168.24 \\
45.60000 & 52.78 & 182.29 \\
68.40000 & 50.71 & 201.79 \\
114.00000 & 49.16 & 226.24 \\
228.00000 & 48.16 & 259.28
\end{tabular}

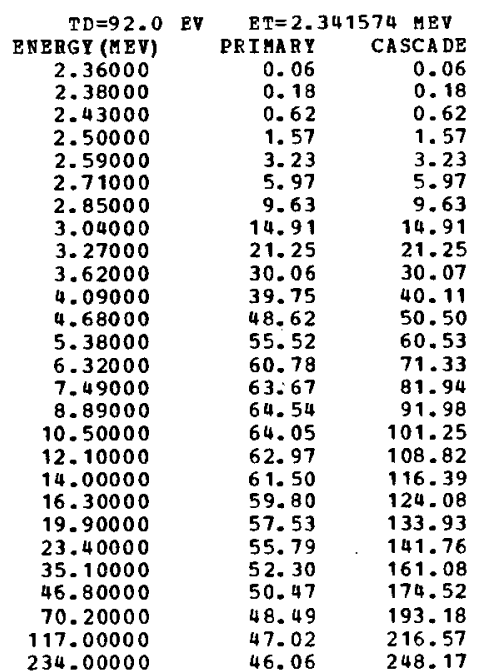

\begin{tabular}{rrr} 
TD=95.0 EV & \multicolumn{2}{c}{ ET $=2.400978$ MEV } \\
ENERGY (HEV) & PRIMARY & CASCADE \\
2.42000 & 0.06 & 0.06 \\
2.44000 & 0.16 & 0.16 \\
2.49000 & 0.57 & 0.57 \\
2.56000 & 1.44 & 1.44 \\
2.66000 & 3.17 & 3.17 \\
2.78000 & 5.76 & 5.76 \\
2.92000 & 9.19 & 9.19 \\
3.12000 & 14.39 & 14.39 \\
3.36000 & 20.59 & 20.59 \\
3.72000 & 29.05 & 29.06 \\
4.20000 & 38.27 & 38.63 \\
4.80000 & 46.68 & 48.51 \\
5.52000 & 53.29 & 58.16 \\
6.48000 & 58.28 & 68.47 \\
7.68000 & 61.04 & 78.65 \\
9.12000 & 61.85 & 88.31 \\
10.80000 & 61.34 & 97.33 \\
12.40000 & 60.32 & 104.40 \\
14.40000 & 58.87 & 111.83 \\
16.80000 & 57.21 & 119.30 \\
20.40000 & 55.10 & 128.48 \\
24.00000 & 53.43 & 136.01 \\
36.00000 & 50.10 & 154.54 \\
48.00000 & 48.34 & 167.42 \\
72.00000 & 46.46 & 185.31 \\
120.00000 & 45.05 & 207.72 \\
240.00000 & 44.14 & 238.01
\end{tabular}


ATOMIC DISPLACEMENT CROSS-SECTIONS (BARNS) BY FAST ELECTRONS IN PT

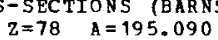

\begin{tabular}{rrr}
$T D=4.0$ & EV & \multicolumn{2}{c}{$E T=0.279237$ MEV } \\
ENERGY (MEV) & PRIMARY & CASCADE \\
0.28200 & 24.24 & 24.24 \\
0.28400 & 41.53 & 41.53 \\
0.29000 & 92.22 & 92.22 \\
0.29800 & 157.20 & 157.20 \\
0.30900 & 242.03 & 242.03 \\
0.32300 & 343.09 & 343.09 \\
0.34000 & 456.47 & 456.47 \\
0.36300 & 595.32 & 595.32 \\
0.39000 & 739.49 & 739.49 \\
0.43200 & 929.33 & 929.33 \\
0.48800 & 1129.50 & 1129.52 \\
0.55800 & 1314.80 & 1325.08 \\
0.64200 & 1469.32 & 1511.86 \\
0.75300 & 1597.76 & 1706.85 \\
0.89300 & 1684.44 & 1900.02 \\
1.06000 & 1726.46 & 2082.94 \\
1.25000 & 1732.57 & 2251.92 \\
1.45000 & 1716.01 & 2400.53 \\
1.67000 & 1686.05 & 2540.02 \\
1.95000 & 1642.13 & 2692.56 \\
2.37000 & 1577.96 & 2884.24 \\
2.79000 & 1521.70 & 3045.95 \\
4.18000 & 1390.95 & 3452.17 \\
5.58000 & 1315.39 & 3748.71 \\
8.37000 & 1238.98 & 4174.29 \\
13.90000 & 1173.26 & 4711.31 \\
27.90000 & 1126.32 & 5460.28 \\
55.80000 & 1104.68 & 6209.51 \\
83.70000 & 1091.26 & 6642.22 \\
139.00000 & 1072.94 & 7176.98
\end{tabular}

\begin{tabular}{|c|c|c|c|}
\hline $\begin{array}{r}T D=8.0 \\
\text { ENERGY (MEV) }\end{array}$ & EV & $\begin{array}{r}\text { ET }=0.5 \\
\text { PRIAARY }\end{array}$ & CASCADE \\
\hline 0.48700 & & 5.75 & 5.75 \\
\hline 0.49200 & & 12.90 & 12.90 \\
\hline 0.50200 & & 27.61 & 27.61 \\
\hline 0.51600 & & 48.98 & 48.98 \\
\hline 0.53600 & & 80.57 & 80.57 \\
\hline 0.56000 & & 119.29 & 119.29 \\
\hline 0.58900 & & 166.18 & 166.18 \\
\hline 0.62700 & & 226.25 & 226.25 \\
\hline 0.67600 & & 299.40 & 299.40 \\
\hline 0.74800 & & 395.60 & 395.60 \\
\hline 0.84500 & & 502.95 & 503.46 \\
\hline 0.96500 & & 604.29 & 611.85 \\
\hline 1.11000 & & 690.40 & 717.80 \\
\hline 1.30000 & & 761.42 & 828.91 \\
\hline 1.54000 & & 808.93 & 940.14 \\
\hline 1.83000 & & 831.48 & 1046.91 \\
\hline 2.17000 & & 833.83 & 1147.73 \\
\hline 2.51000 & & 824.44 & 1231.02 \\
\hline 2.89000 & & 808.44 & 1310.12 \\
\hline 3.38000 & & 785.84 & 1396.40 \\
\hline 4.10000 & & 754.66 & 1501.73 \\
\hline 4.82000 & & 728.29 & 1589.22 \\
\hline 7.24000 & & 668.98 & 1808.25 \\
\hline 9.65000 & & 637.52 & 1964.07 \\
\hline 14.40000 & & 605.52 & 2180.76 \\
\hline 24.10000 & & 577.42 & 2458.83 \\
\hline 48.20000 & & 559.66 & 2836.01 \\
\hline 96.50000 & & 549.14 & 3211.11 \\
\hline 144.00000 & & 542.13 & 3424.11 \\
\hline 241.00000 & & 533.09 & 3694.35 \\
\hline
\end{tabular}

\begin{tabular}{crr} 
TD $=12.0$ & \multicolumn{2}{c}{ ET $=0.651403$ MEV } \\
ENERGY (MEV) & PRIMARY & CASCADE \\
0.65700 & 2.76 & 2.76 \\
0.66400 & 6.43 & 6.43 \\
0.67700 & 13.78 & 13.78 \\
0.69700 & 26.27 & 26.27 \\
0.72300 & 44.12 & 44.12 \\
0.75500 & 67.73 & 67.73 \\
0.79400 & 97.74 & 97.74 \\
0.84600 & 138.11 & 138.11 \\
0.91100 & 186.93 & 186.93 \\
1.00000 & 248.11 & 248.11 \\
1.13000 & 323.34 & 323.84 \\
1.30000 & 397.90 & 404.26 \\
1.49000 & 455.70 & 476.90 \\
1.75000 & 504.59 & 556.55 \\
2.08000 & 535.87 & 636.22 \\
2.47000 & 549.00 & 710.73 \\
2.93000 & 548.68 & 781.23 \\
3.38000 & 541.28 & 838.16 \\
3.90000 & 529.56 & 893.78 \\
4.55000 & 514.17 & 952.47 \\
5.53000 & 493.21 & 1025.63 \\
6.51000 & 475.90 & 1086.08 \\
9.77000 & 438.61 & 1234.99 \\
13.00000 & 419.50 & 1339.86 \\
19.50000 & 399.41 & 1487.47 \\
32.50000 & 382.56 & 1672.59 \\
65.10000 & 372.12 & 1925.52 \\
130.00000 & 365.03 & 2174.34 \\
195.00000 & 360.20 & 2317.89 \\
325.00000 & 354.44 & 2496.23
\end{tabular}

ATOMIC DISPLACEHENT CROSS-SECTIONS (BARNS) BY PAST ELECTRONS IN PT $Z=78 \quad A=195.090$

\begin{tabular}{rrr} 
TD $=16.0$ & EV & \multicolumn{2}{c}{ ET $=0.798402$ MEV } \\
ENERGY (MEV) & PRIMARY & \multicolumn{1}{c}{ CASCADE } \\
0.80600 & 1.82 & 1.82 \\
0.81400 & 3.94 & 3.94 \\
0.83000 & 8.69 & 8.69 \\
0.85400 & 16.95 & 16.95 \\
0.88600 & 29.58 & 29.58 \\
0.92600 & 47.13 & 47.13 \\
0.97400 & 69.60 & 69.60 \\
1.03000 & 96.48 & 96.48 \\
1.11000 & 134.15 & 134.15 \\
1.23000 & 185.98 & 185.98 \\
1.39000 & 243.69 & 244.29 \\
1.59000 & 298.29 & 303.84 \\
1.83000 & 343.29 & 361.66 \\
2.15000 & 379.37 & 423.18 \\
2.55000 & 401.37 & 483.76 \\
3.03000 & 409.96 & 541.26 \\
3.59000 & 408.58 & 594.94 \\
4.15000 & 402.16 & 639.19 \\
4.79000 & 392.85 & 681.72 \\
5.58000 & 381.15 & 726.11 \\
6.78000 & 365.47 & 781.68 \\
7.98000 & 352.70 & 827.58 \\
11.90000 & 326.74 & 938.71 \\
15.90000 & 312.30 & 1019.03 \\
23.90000 & 297.77 & 1130.76 \\
39.90000 & 285.90 & 1270.55 \\
79.80000 & 278.65 & 1460.14 \\
159.00000 & 273.28 & 1645.97
\end{tabular}

\begin{tabular}{rrr} 
TD $=20.0$ & EV & \multicolumn{2}{c}{ ET=0.9304 37 MEV } \\
ENERGY (MEV) & PRIMARY & CASCA DE \\
0.93900 & 1.17 & 1.17 \\
0.94900 & 2.75 & 2.75 \\
0.96700 & 6.06 & 6.06 \\
0.99500 & 12.25 & 12.25 \\
1.03000 & 21.37 & 21.37 \\
1.07000 & 33.13 & 33.13 \\
1.13000 & 52.33 & 52.33 \\
1.20000 & 75.67 & 75.67 \\
1.30000 & 108.52 & 108.52 \\
1.44000 & 150.59 & 150.59 \\
1.62000 & 195.73 & 196.34 \\
1.86000 & 240.95 & 246.22 \\
2.14000 & 276.58 & 293.15 \\
2.51000 & 304.48 & 342.67 \\
2.97000 & 321.03 & 391.24 \\
3.53000 & 327.10 & 438.01 \\
4.18000 & 325.33 & 481.38 \\
4.83000 & 319.77 & 517.12 \\
5.58000 & 312.01 & 551.74 \\
6.51000 & 302.42 & 587.97 \\
7.90000 & 289.98 & 632.51 \\
9.30000 & 279.87 & 669.55 \\
13.90000 & 259.14 & 759.55 \\
18.60000 & 248.42 & 824.37 \\
27.90000 & 237.23 & 913.49 \\
46.50000 & 228.21 & 1025.21 \\
93.00000 & 222.69 & 1177.01 \\
186.00000 & 218.31 & 1326.37
\end{tabular}

$\begin{array}{rrr}\text { TD }=24.0 & \text { EY } & \text { ET }=1.051447 \text { MEV } \\ \text { ENERGY(MEV) } & \text { PRIMARY } & \text { CASCADE } \\ 1.06000 & 0.75 & 0.75 \\ 1.07000 & 1.76 & 1.76 \\ 1.09000 & 4.21 & 4.21 \\ 1.12000 & 8.79 & 8.79 \\ 1.16000 & 16.20 & 16.20 \\ 1.21000 & 26.95 & 26.95 \\ 1.28000 & 43.60 & 43.60 \\ 1.36000 & 63.54 & 53.54 \\ 1.47000 & 90.57 & 90.57 \\ 1.62000 & 124.42 & 124.42 \\ 1.84000 & 165.55 & 166.25 \\ 2.10000 & 201.70 & 206.53 \\ 2.41000 & 230.90 & 245.48 \\ 2.83000 & 254.13 & 287.52 \\ 3.36000 & 267.66 & 329.37 \\ 3.99000 & 272.09 & 368.53 \\ 4.73000 & 270.11 & 405.22 \\ 5.46000 & 265.22 & 435.02 \\ 6.30000 & 258.62 & 463.83 \\ 7.36000 & 250.49 & 494.49 \\ 8.93000 & 240.15 & 531.78 \\ 10.50000 & 231.87 & 562.57 \\ 15.70000 & 214.96 & 637.99 \\ 21.00000 & 206.26 & 692.05 \\ 31.50000 & 197.13 & 766.43 \\ 52.50000 & 189.88 & 859.67 \\ 105.00000 & 185.43 & 986.24 \\ 210.00000 & 181.75 & 1110.63\end{array}$


ATOMIC DISPLACEMENT CROSS-SECTIONS (BARNS) BY FAST ELECTRONS IN PT

\begin{tabular}{rrr} 
TD=28.0 & \multicolumn{2}{c}{ ET $=1.163692$ MEV } \\
ENERGY (MEV) & PRIMARY & CASCADE \\
1.17000 & 0.37 & 0.37 \\
1.18000 & 1.04 & 1.04 \\
1.21000 & 3.71 & 3.71 \\
1.24000 & 7.20 & 7.20 \\
1.29000 & 14.37 & 14.37 \\
1.34000 & 22.77 & 22.77 \\
1.41000 & 35.73 & 35.73 \\
1.51000 & 55.24 & 55.24 \\
1.62000 & 76.47 & 76.47 \\
1.80000 & 108.30 & 108.30 \\
2.03000 & 141.90 & 142.54 \\
2.32000 & 173.44 & 177.85 \\
2.67000 & 198.89 & 212.27 \\
3.14000 & 218.57 & 249.02 \\
3.72000 & 229.53 & 284.61 \\
4.42000 & 232.89 & 318.51 \\
5.23000 & 230.90 & 349.82 \\
6.05000 & 226.45 & 375.87 \\
6.98000 & 220.67 & 400.67 \\
8.14000 & 213.74 & 426.77 \\
9.89000 & 204.82 & 459.10 \\
11.60000 & 197.89 & 485.16 \\
17.40000 & 183.55 & 550.54 \\
23.20000 & 176.32 & 596.44 \\
34.90000 & 168.58 & 660.71 \\
58.10000 & 162.56 & 740.54 \\
116.00000 & 158.85 & 848.80 \\
232.00000 & 155.67 & 955.37
\end{tabular}

\begin{tabular}{rrr} 
TD $=32.0$ & EV & \multicolumn{2}{c}{ ET $=1.268872$ MEV } \\
ENERGY (MEV) & PRIMARY & CASCADE \\
1.28000 & 0.49 & 0.49 \\
1.29000 & 1.02 & 1.02 \\
1.31000 & 2.34 & 2.34 \\
1.35000 & 5.83 & 5.83 \\
1.40000 & 11.46 & 11.46 \\
1.47000 & 20.92 & 20.92 \\
1.54000 & 31.47 & 31.47 \\
1.64000 & 47.29 & 47.29 \\
1.77000 & 67.66 & 67.66 \\
1.96000 & 94.89 & 94.89 \\
2.22000 & 125.53 & 126.20 \\
2.53000 & 152.54 & 156.67 \\
2.91000 & 174.62 & 186.90 \\
3.42000 & 191.56 & 219.16 \\
4.06000 & 200.94 & 250.86 \\
4.82000 & 203.55 & 280.53 \\
5.70000 & 201.59 & 307.96 \\
6.59000 & 197.58 & 330.76 \\
7.61000 & 192.41 & 352.69 \\
8.88000 & 186.29 & 375.69 \\
10.70000 & 178.83 & 402.86 \\
12.60000 & 172.64 & 426.31 \\
19.00000 & 160.11 & 484.40 \\
25.30000 & 153.91 & 524.42 \\
38.00000 & 147.26 & 580.50 \\
63.40000 & 142.10 & 650.71 \\
126.00000 & 138.93 & 744.83 \\
253.00000 & 136.13 & 838.58
\end{tabular}

\begin{tabular}{rrr} 
TD $=36.0$ & EV & \multicolumn{2}{c}{ ET $=1.368175$} \\
ENERGY (MEV) & PRIMARY & CASCADE \\
1.38000 & 0.39 & 0.39 \\
1.39000 & 0.80 & 0.80 \\
1.42000 & 2.43 & 2.43 \\
1.46000 & 5.38 & 5.38 \\
1.51000 & 10.04 & 10.04 \\
1.58000 & 17.83 & 17.83 \\
1.66000 & 27.82 & 27.82 \\
1.77000 & 42.30 & 42.30 \\
1.91000 & 60.55 & 60.55 \\
2.12000 & 85.51 & 85.51 \\
2.39000 & 11.85 & 112.48 \\
2.73000 & 136.32 & 140.24 \\
3.14000 & 155.82 & 167.26 \\
3.69000 & 170.63 & 196.06 \\
4.37000 & 178.63 & 223.99 \\
5.19000 & 180.77 & 250.54 \\
6.15000 & 178.83 & 275.31 \\
7.11000 & 175.17 & 295.63 \\
8.20000 & 170.55 & 315.01 \\
9.57000 & 165.09 & 335.53 \\
11.60000 & 158.26 & 360.51 \\
13.60000 & 152.94 & 380.81 \\
20.50000 & 141.97 & 432.50 \\
27.30000 & 136.53 & 468.12 \\
41.00000 & 130.70 & 517.98 \\
68.40000 & 126.21 & 580.43 \\
136.00000 & 123.44 & 664.15 \\
273.00000 & 120.93 & 747.42
\end{tabular}

ATOMIC DISPLACEMENT CROSS-SECTIONS (BARNS) BY FAST ELECTRONS IN PT $=78 \quad \mathrm{~A}=195.090$

\begin{tabular}{crr} 
TD $=40.0$ & EV & \multicolumn{1}{c}{ ETT=1.462487 MEV } \\
ENERGY (MEV) & PRIMARY & CASCADE \\
1.47000 & 0.18 & 0.18 \\
1.49000 & 0.83 & 0.83 \\
1.52000 & 2.21 & 2.21 \\
1.56000 & 4.67 & 4.67 \\
1.62000 & 9.42 & 9.42 \\
1.59000 & 16.06 & 16.06 \\
1.78000 & 25.61 & 25.61 \\
1.90000 & 39.04 & 39.04 \\
2.04000 & 54.53 & 54.53 \\
2.26000 & 76.77 & 76.76 \\
2.55000 & 100.81 & 101.40 \\
2.92000 & 123.28 & 127.01 \\
3.36000 & 140.77 & 151.51 \\
3.94000 & 153.75 & 177.20 \\
4.67000 & 160.81 & 202.56 \\
5.55000 & 162.56 & 226.63 \\
6.58000 & 160.67 & 249.05 \\
7.50000 & 157.31 & 267.26 \\
8.77000 & 153.10 & 284.81 \\
10.20000 & 148.27 & 302.90 \\
12.40000 & 142.05 & 325.78 \\
14.60000 & 137.16 & 344.56 \\
21.90000 & 127.53 & 390.60 \\
29.20000 & 122.67 & 422.81 \\
43.80000 & 117.49 & 467.57 \\
73.10000 & 113.51 & 523.85 \\
146.00000 & 111.04 & 599.70 \\
& &
\end{tabular}

$\begin{array}{crr}\text { TD }=44.0 & \text { EV } & \text { ET=1.552493 MEV } \\ \text { ENERGY(MEV) } & \text { PRIMARY } & \text { CASCADE } \\ 1.56000 & 0.14 & 0.14 \\ 1.58000 & 0.56 & 0.66 \\ 1.61000 & 1.78 & 1.78 \\ 1.66000 & 4.40 & 4.40 \\ 1.72000 & 8.49 & 8.49 \\ 1.80000 & 15.06 & 15.06 \\ 1.89000 & 23.32 & 23.32 \\ 2.01000 & 34.91 & 34.91 \\ 2.17000 & 50.19 & 50.19 \\ 2.40000 & 70.19 & 70.19 \\ 2.71000 & 92.25 & 92.84 \\ 3.10000 & 112.50 & 116.04 \\ 3.57000 & 128.40 & 138.54 \\ 4.19000 & 110.08 & 162.09 \\ 4.96000 & 146.25 & 185.03 \\ 5.89000 & 117.68 & 206.86 \\ 6.98000 & 145.87 & 227.24 \\ 8.07000 & 112.74 & 243.94 \\ 9.31000 & 138.88 & 259.90 \\ 10.80000 & 134.56 & 276.10 \\ 13.10000 & 128.97 & 296.69 \\ 15.50000 & 124.41 & 314.31 \\ 23.20000 & 115.78 & 356.00 \\ 31.00000 & 111.36 & 385.52 \\ 46.50000 & 116.70 & 426.22 \\ 77.60000 & 113.14 & 477.40 \\ 155.00000 & 100.92 & 546.37\end{array}$

\begin{tabular}{|c|c|c|}
\hline $\mathrm{TD}=48.0$ & $E \mathrm{~T}=1$ & $M E V$ \\
\hline ENERGY (MEV) & PRIMARY & CASCADE \\
\hline 1.65000 & 0.18 & 0.18 \\
\hline 1.67000 & 0.64 & 0.64 \\
\hline 1.70000 & 1.61 & 1.61 \\
\hline 1.75000 & 3.85 & 3.85 \\
\hline 1.81000 & 7.36 & 7.36 \\
\hline 1.90000 & 13.77 & 13.77 \\
\hline 1.99000 & 20.98 & 20.98 \\
\hline 2.13000 & 32.80 & 32.80 \\
\hline 2.29000 & 46.14 & 46.14 \\
\hline 2.54000 & 65.09 & 65.09 \\
\hline 2.86000 & 84.88 & 85.45 \\
\hline 3.27000 & 103.39 & 106.74 \\
\hline 3.76000 & 117.79 & 127.23 \\
\hline 4.42000 & 128.54 & 149.11 \\
\hline 5.24000 & 134.11 & 170.38 \\
\hline 6.22000 & 135.29 & 190.38 \\
\hline 7.37000 & 133.54 & 209.08 \\
\hline 8.52000 & 130.62 & 224.40 \\
\hline 9.83000 & 127.06 & 239.07 \\
\hline 11.40000 & 123.09 & 253.90 \\
\hline 13.90000 & 117.84 & 273.31 \\
\hline 16.30000 & 113.91 & 288.61 \\
\hline 24.50000 & 105.97 & 327.27 \\
\hline 32.70000 & 101.97 & 354.24 \\
\hline 49.10000 & 97.72 & 391.66 \\
\hline 81.90000 & 94.50 & 438.55 \\
\hline 163.00000 & 92.49 & 501.45 \\
\hline
\end{tabular}


ATOMIC DISPLACEMENT CROSS-SECTIONS (BARNS) BY PAST ELECTRONS IN PT

\begin{tabular}{rrr} 
TD $=52.0$ & EV & \multicolumn{2}{c}{ BT $=1.721647$ MEV } \\
ENERGY (MEV) & PRIMARY & CASCADE \\
1.73000 & 0.11 & 0.11 \\
1.75000 & 0.47 & 0.47 \\
1.79000 & 1.58 & 1.58 \\
1.84000 & 3.57 & 3.57 \\
1.91000 & 7.23 & 7.23 \\
1.99000 & 12.27 & 12.27 \\
2.10000 & 20.07 & 20.07 \\
2.23000 & 29.78 & 29.78 \\
2.41000 & 43.05 & 43.05 \\
2.66000 & 59.80 & 59.81 \\
3.01000 & 78.90 & 79.48 \\
3.44000 & 95.88 & 99.12 \\
3.95000 & 108.97 & 117.89 \\
4.64000 & 118.76 & 138.06 \\
5.50000 & 123.81 & 157.72 \\
6.54000 & 124.82 & 176.41 \\
7.74000 & 123.13 & 193.58 \\
8.95000 & 120.40 & 207.76 \\
10.30000 & 117.15 & 221.08 \\
12.00000 & 113.37 & 235.22 \\
14.60000 & 108.59 & 252.96 \\
17.20000 & 104.87 & 267.50 \\
25.80000 & 97.65 & 303.03 \\
34.40000 & 94.01 & 327.84 \\
51.60000 & 90.13 & 362.31 \\
86.00000 & 87.20 & 405.54 \\
172.00000 & 85.35 & 464.01
\end{tabular}

\begin{tabular}{rrr} 
TD=56.0 EV & \multicolumn{2}{c}{ ET=1.801588 MEV } \\
ENERGY (NEV) & PRIMARY & CASCADE \\
1.81000 & 0.09 & 0.09 \\
1.83000 & 0.39 & 0.39 \\
1.87000 & 1.34 & 1.34 \\
1.92000 & 3.06 & 3.06 \\
1.99000 & 6.24 & 6.24 \\
2.08000 & 11.24 & 11.24 \\
2.19000 & 18.17 & 18.17 \\
2.34000 & 28.17 & 28.17 \\
2.52000 & 40.01 & 40.01 \\
2.79000 & 56.11 & 56.11 \\
3.15000 & 73.53 & 74.09 \\
3.60000 & 89.29 & 92.39 \\
4.14000 & 101.50 & 110.05 \\
4.86000 & 110.44 & 128.73 \\
5.76000 & 115.01 & 147.01 \\
6.84000 & 115.85 & 164.25 \\
8.10000 & 114.22 & 180.27 \\
9.36000 & 111.67 & 193.40 \\
10.80000 & 108.57 & 206.01 \\
12.60000 & 105.03 & 219.26 \\
15.30000 & 100.54 & 235.58 \\
18.00000 & 97.23 & 248.98 \\
27.00000 & 90.57 & 282.00 \\
36.00000 & 87.21 & 305.05 \\
54.00000 & 83.53 & 337.06 \\
90.00000 & 80.94 & 377.21 \\
180.00000 & 79.23 & 431.51
\end{tabular}

\begin{tabular}{rrr} 
TD=60.0 EV & \multicolumn{2}{c}{ ET $=1.878857 \mathrm{MEV}$} \\
ENERGY (MEV) & PRIMAY & CASCADE \\
$\mathbf{1 . 8 9 0 0 0}$ & 0.11 & 0.11 \\
1.91000 & 0.38 & 0.38 \\
1.95000 & 1.22 & 1.22 \\
2.01000 & 3.10 & 3.10 \\
2.08000 & 6.01 & 6.01 \\
2.17000 & 10.53 & 10.53 \\
2.29000 & 17.34 & 17.34 \\
2.44000 & 26.35 & 26.35 \\
2.63000 & 37.58 & 37.58 \\
2.91000 & 52.57 & 52.58 \\
3.28000 & 68.56 & 69.19 \\
3.75000 & 83.44 & 86.38 \\
4.32000 & 94.94 & 103.11 \\
5.07000 & 103.19 & 120.55 \\
6.01000 & 107.37 & 137.65 \\
$\mathbf{7 . 1 3 0 0 0}$ & 108.08 & 153.67 \\
$\mathbf{8 . 4 5 0 0 0}$ & 106.50 & 158.72 \\
9.77000 & 104.08 & 181.03 \\
11.20000 & $\mathbf{1 0 1 . 3 2}$ & 192.28 \\
13.10000 & 97.95 & 204.87 \\
$\mathbf{1 5 . 9 0 0 0 0}$ & 93.86 & 220.09 \\
$\mathbf{1 8 . 7 0 0 0 0}$ & 90.69 & 232.58 \\
29.10000 & 84.47 & 263.56 \\
$\mathbf{3 7 . 5 0 0 0 0}$ & 81.34 & 285.14 \\
$\mathbf{5 6 . 3 0 0 0 0}$ & $\mathbf{7 8 . 0 1}$ & 315.10 \\
$\mathbf{9 3 . 9 0 0 0 0}$ & $\mathbf{7 5 . 5 2}$ & 352.63 \\
$\mathbf{1 8 7 . 0 0 0 0 0}$ & $\mathbf{7 3 . 9 4}$ & 403.00
\end{tabular}

ATOMIC DISPLACEHENT CROSS-SBCTIONS (BARNS) BY FAST ELECTRONS IN PT $=78 \quad A=195.090$

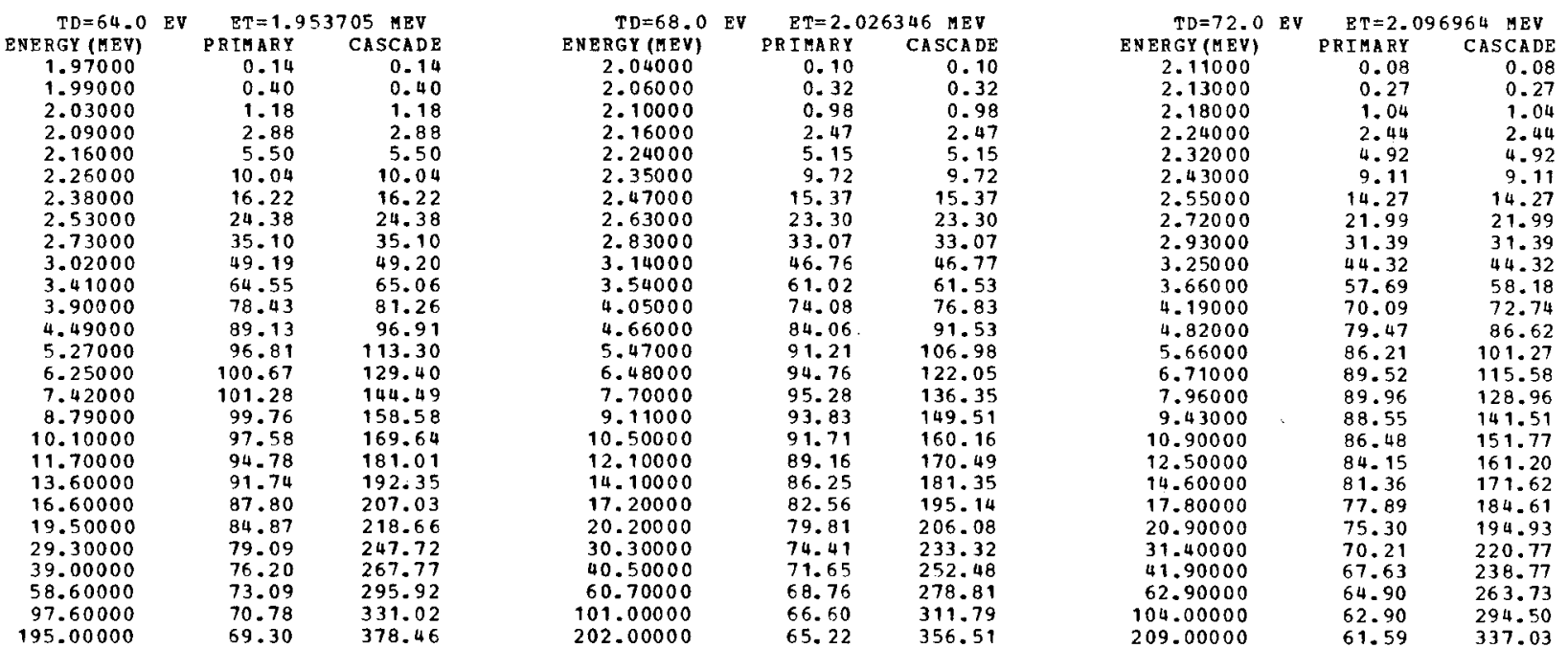


A TOMIC DISPLACEMENT CROSS-SECTIONS (BARNS) BY FAST RLECTRONS IN PT

\begin{tabular}{rrr} 
TD $=76.0$ & EV & \multicolumn{2}{c}{ ET $=2.165720$ MEV } \\
ENERGY (MEV) & PRIMARY & CASCADE \\
2.18000 & 0.08 & 0.08 \\
2.20000 & 0.25 & 0.25 \\
2.25000 & 0.95 & 0.95 \\
2.31000 & 2.21 & 2.21 \\
2.40000 & 4.78 & 4.78 \\
2.51000 & 8.65 & 8.65 \\
2.64000 & 13.81 & 13.81 \\
2.81000 & 20.93 & 20.93 \\
3.03000 & 29.99 & 29.99 \\
3.35000 & 41.88 & 41.89 \\
3.79000 & 55.05 & 55.55 \\
4.33000 & 66.58 & 69.14 \\
4.98000 & 75.41 & 82.29 \\
5.84000 & 81.70 & 96.07 \\
6.93000 & 84.82 & 109.72 \\
8.22000 & 85.20 & 122.40 \\
9.74000 & 83.84 & 134.31 \\
11.20000 & 81.94 & 143.69 \\
12.90000 & 79.66 & 152.92 \\
15.10000 & 76.98 & 162.93 \\
18.40000 & 73.70 & 175.22 \\
21.60000 & 71.26 & 184.98 \\
32.40000 & 66.47 & 209.38 \\
43.30000 & 64.03 & 226.52 \\
64.90000 & 61.46 & 250.09 \\
108.00000 & 59.57 & 279.62 \\
216.00000 & 58.34 & 319.63
\end{tabular}

\begin{tabular}{ccc} 
TD $=80.0$ & EV & \multicolumn{1}{c}{$E T=2.232753$ MEV } \\
ENERGY (MEV) & PRIMARY & CASCADE \\
2.25000 & 0.09 & 0.09 \\
2.27000 & 0.25 & 0.25 \\
2.32000 & 0.90 & 0.90 \\
2.38000 & 2.06 & 2.06 \\
2.47000 & 4.41 & 4.41 \\
2.58000 & 7.95 & 7.95 \\
2.72000 & 13.08 & 13.08 \\
2.90000 & 20.05 & 20.05 \\
3.12000 & 28.43 & 28.43 \\
3.46000 & 40.09 & 40.10 \\
3.90000 & 52.24 & 52.71 \\
4.46000 & 63.30 & 65.76 \\
5.13000 & 71.69 & 78.28 \\
6.02000 & 77.66 & 91.45 \\
7.14000 & 80.58 & 104.39 \\
8.48000 & 80.92 & 116.54 \\
10.00000 & 79.64 & 127.54 \\
11.50000 & 77.72 & 137.02 \\
13.30000 & 75.61 & 145.51 \\
15.60000 & 73.03 & 155.14 \\
18.90000 & 70.00 & 165.48 \\
22.30000 & 67.62 & 176.05 \\
33.40000 & 63.11 & 199.16 \\
44.60000 & 60.80 & 215.40 \\
66.90000 & 58.37 & 237.83 \\
111.00000 & 56.58 & 265.73 \\
223.00000 & 55.41 & 303.98 \\
& &
\end{tabular}

\begin{tabular}{rrr} 
TD=84.0 EY & \multicolumn{2}{c}{ ET=2.298188 MEV } \\
ENBRG (MEV) & PRIMARY & CASCADE \\
2.32000 & 0.11 & 0.11 \\
2.34000 & 0.27 & 0.27 \\
2.39000 & 0.88 & 0.88 \\
2.45000 & 1.96 & 1.96 \\
2.55000 & 4.40 & 4.40 \\
2.66000 & 7.72 & 7.72 \\
2.80000 & 12.49 & 12.49 \\
2.98000 & 18.96 & 18.96 \\
3.21000 & 27.10 & 27.10 \\
3.56000 & 38.24 & 38.25 \\
4.02000 & 49.99 & 50.46 \\
4.59000 & 60.37 & 62.75 \\
5.28000 & 68.35 & 74.70 \\
6.20000 & 74.03 & 87.30 \\
7.35000 & 76.75 & 99.61 \\
8.73000 & 77.04 & 111.19 \\
10.30000 & 75.80 & 121.71 \\
11.90000 & 74.01 & 130.49 \\
13.70000 & 71.94 & 138.82 \\
16.00000 & 69.54 & 147.76 \\
19.50000 & 66.58 & 158.89 \\
22.90000 & 54.37 & 167.76 \\
34.40000 & 60.06 & 189.93 \\
45.90000 & 57.88 & 205.35 \\
68.90000 & 55.57 & 226.76 \\
114.00000 & 53.88 & 253.18 \\
229.00000 & 52.77 & 289.51 \\
& &
\end{tabular}

ATOMIC DISPLACENENT CROSS-SECTIONS (BARNS) BY FAST ELECTRONS IN PT

$$
\mathrm{Z}=78 \quad A=195.090
$$

\begin{tabular}{rrr}
$T D=88.0$ & EV & \multicolumn{2}{c}{ ET $=2.362132$ MEV } \\
ENERGY (MEV) & PRIMARY & CASCADE \\
2.38000 & 0.07 & 0.07 \\
2.40000 & 0.21 & 0.21 \\
2.45000 & 0.74 & 0.74 \\
2.52000 & 1.90 & 1.90 \\
2.62000 & 4.17 & 4.17 \\
2.74000 & 7.56 & 7.56 \\
2.88000 & 12.02 & 12.02 \\
3.07000 & 18.38 & 18.38 \\
3.30000 & 25.95 & 25.95 \\
3.65000 & 36.61 & 36.62 \\
4.13000 & 47.78 & 48.24 \\
4.72000 & 57.75 & 60.07 \\
5.43000 & 65.34 & 71.49 \\
6.37000 & 70.69 & 83.46 \\
7.55000 & 73.27 & 95.20 \\
8.97000 & 73.52 & 106.28 \\
10.60000 & 72.30 & 116.42 \\
12.20000 & 70.63 & 124.59 \\
14.10000 & 68.59 & 132.77 \\
16.50000 & 66.28 & 141.41 \\
20.00000 & 63.53 & 151.74 \\
23.60000 & 61.37 & 160.46 \\
35.40000 & 57.29 & 181.55 \\
47.20000 & 55.22 & 195.24 \\
70.80000 & 53.02 & 216.64 \\
118.00000 & 51.41 & 242.23 \\
236.00000 & 50.36 & 276.78 \\
& &
\end{tabular}

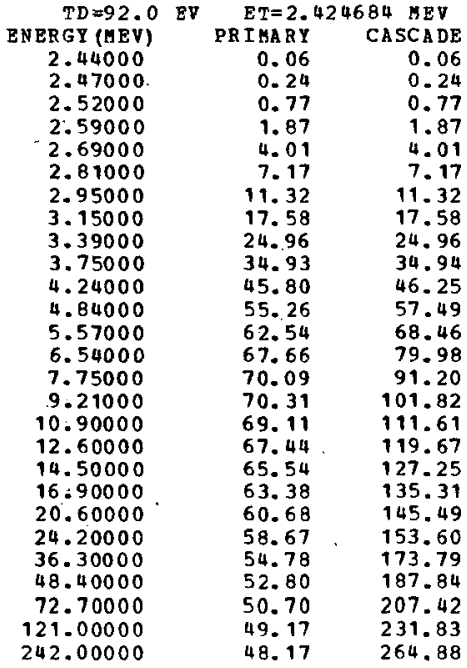

$\begin{array}{rrr}\text { TD=96.0 } & \text { EV } & \text { ET }=2.485931 \text { MEV } \\ \text { ENERGY (MEV) } & \text { PRIMARY } & \text { CASCADE } \\ 2.51000 & 0.09 & 0.09 \\ 2.53000 & 0.21 & 0.21 \\ 2.58000 & 0.69 & 0.69 \\ 2.65000 & 1.69 & 1.69 \\ 2.75000 & 3.66 & 3.66 \\ 2.88000 & 6.85 & 6.85 \\ 3.03000 & 11.03 & 11.03 \\ 3.23000 & 16.90 & 16.90 \\ 3.48000 & 24.10 & 24.10 \\ 3.85000 & 33.67 & 33.68 \\ 4.35000 & 44.02 & 44.47 \\ 4.97000 & 53.13 & 55.32 \\ 5.71000 & 59.98 & 65.71 \\ 6.71000 & 64.89 & 76.82 \\ 7.95000 & 67.18 & 87.57 \\ 9.44000 & 67.36 & 97.70 \\ 11.10000 & 66.26 & 106.73 \\ 12.90000 & 64.61 & 114.75 \\ 14.90000 & 62.74 & 122.21 \\ 17.40000 & 60.64 & 130.03 \\ 21.10000 & 58.12 & 139.53 \\ 24.80000 & 56.20 & 147.33 \\ 37.20000 & 52.48 & 166.68 \\ 49.70000 & 50.57 & 180.24 \\ 74.50000 & 48.58 & 198.92 \\ 124.00000 & 47.11 & 222.32 \\ 248.00000 & 46.16 & 253.99\end{array}$




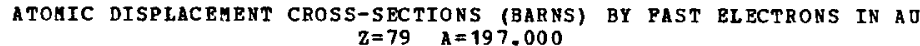

\begin{tabular}{rrr}
$T D=4.0$ & \multicolumn{1}{c}{$\begin{array}{c}\text { ET }=0.281484 \\
\text { PRV }\end{array}$} \\
ENERGY (HEV) & PRIARY & CASCADE \\
0.28400 & 22.64 & 22.64 \\
0.28700 & 49.22 & 49.22 \\
0.29200 & 92.51 & 92.51 \\
0.30100 & 167.50 & 167.50 \\
0.31200 & 254.39 & 254.39 \\
0.32600 & 358.04 & 358.04 \\
0.34300 & 474.46 & 474.46 \\
0.36500 & 611.34 & 611.34 \\
0.39400 & 770.72 & 770.72 \\
0.43600 & 965.29 & 965.29 \\
0.49200 & 1170.48 & 1170.50 \\
0.56200 & 1360.22 & 1370.87 \\
0.64700 & 1519.55 & 1563.97 \\
0.76000 & 1651.10 & 1765.79 \\
0.90000 & 1736.65 & 1961.59 \\
1.06000 & 1775.63 & 2139.85 \\
1.26000 & 1779.46 & 2320.30 \\
1.46000 & 1759.63 & 2470.24 \\
1.68000 & 1726.52 & 2611.02 \\
1.97000 & 1677.52 & 2770.11 \\
2.39000 & 1609.57 & 2962.85 \\
2.81000 & 1550.60 & 3125.71 \\
4.22000 & 1413.39 & 3540.52 \\
5.62000 & 1336.17 & 3839.22 \\
8.44000 & 1257.87 & 4272.83 \\
14.00000 & 1191.23 & 4817.12 \\
28.10000 & 1143.79 & 5578.00 \\
56.20000 & 1121.69 & 6338.82 \\
84.40000 & 1107.65 & 6779.15 \\
140.00000 & 1088.66 & 7320.35 \\
& &
\end{tabular}

\begin{tabular}{rrr} 
TD= 8.0 & \multicolumn{2}{c}{ ET=0.486468 MEV } \\
ENERGY (HEV) & PRIMARY & CASCADE \\
0.49100 & 6.52 & 6.52 \\
0.49600 & 13.89 & 13.89 \\
0.50500 & 27.51 & 27.51 \\
0.52000 & 51.09 & 51.09 \\
0.53900 & 82.04 & 82.04 \\
0.56400 & 123.70 & 123.70 \\
0.59300 & 172.18 & 172.18 \\
0.63200 & 235.90 & 235.90 \\
0.68100 & 311.44 & 311.44 \\
0.75400 & 411.97 & 411.97 \\
0.85100 & 522.30 & 522.83 \\
0.97200 & 626.90 & 634.84 \\
1.11000 & 711.07 & 738.35 \\
1.31000 & 787.15 & 857.92 \\
1.55000 & 833.99 & 970.72 \\
1.84000 & 855.23 & 1078.86 \\
2.18000 & 855.87 & 1180.89 \\
2.52000 & 844.88 & 1265.15 \\
2.91000 & 826.80 & 1347.10 \\
3.40000 & 802.52 & 1434.06 \\
4.13000 & 769.12 & 1541.68 \\
4.86000 & 741.21 & 1630.96 \\
7.29000 & 679.78 & 1852.49 \\
9.72000 & 647.44 & 2010.94 \\
14.50000 & 614.83 & 2230.84 \\
24.30000 & 586.23 & 2514.03 \\
48.60000 & 568.34 & 2897.18 \\
97.20000 & 557.51 & 3277.40 \\
145.00000 & 550.20 & 3493.31 \\
243.00000 & 540.84 & 3768.15
\end{tabular}

$\mathrm{TD}=12.0 \mathrm{EV} \quad \mathrm{ET}=0.655984 \mathrm{MEV}$

\begin{tabular}{rrr} 
TD=12.0 & \multicolumn{2}{c}{ ET $=0.655984$ MEV } \\
ENERGY (MEV) & PRIMARY & CASCADE \\
0.66200 & 3.06 & 3.06 \\
0.66900 & 6.84 & 6.84 \\
0.68200 & 14.44 & 14.44 \\
0.70100 & 26.69 & 26.69 \\
0.72800 & 45.84 & 45.84 \\
0.76000 & 70.28 & 70.28 \\
0.80000 & 102.17 & 102.17 \\
0.85200 & 143.97 & 143.97 \\
0.91800 & 195.21 & 195.21 \\
1.01000 & 260.32 & 260.32 \\
1.14000 & 337.35 & 337.91 \\
1.31000 & 413.39 & 420.13 \\
1.50000 & 472.03 & 494.23 \\
1.77000 & 522.57 & 578.06 \\
2.09000 & 552.11 & 656.21 \\
2.49000 & 564.48 & 733.50 \\
2.95000 & 562.76 & 804.59 \\
3.41000 & 553.98 & 863.18 \\
3.93000 & 541.14 & 919.12 \\
4.59000 & 524.44 & 979.06 \\
5.57000 & 502.39 & 1052.66 \\
6.55000 & 484.35 & 1113.58 \\
9.83000 & 445.66 & 1264.70 \\
13.10000 & 425.97 & 1371.77 \\
19.60000 & 405.62 & 1520.74 \\
32.70000 & 388.47 & 1709.14 \\
65.50000 & 377.91 & 1966.06 \\
131.00000 & 370.56 & 2219.18 \\
196.00000 & 365.55 & 2363.89 \\
327.00000 & 359.61 & 2545.17
\end{tabular}

ATOHIC DISPLACEAENT CROSS-SECTIONS (BARNS) BY FAST ELECTRONS IN AO

$$
\begin{aligned}
& S-S B C T I O N S \text { (BARNS } \\
& z=79 \quad A=197.000
\end{aligned}
$$

\begin{tabular}{rrr} 
TD $=16.0$ & EV & \multicolumn{2}{c}{ ET $=0.803824$ MEV } \\
ENERGY (HEV) & PRIMARY & CASCADE \\
0.81100 & 1.77 & 1.77 \\
0.81900 & 3.93 & 3.93 \\
0.83500 & 8.82 & 8.82 \\
0.86000 & 17.71 & 17.71 \\
0.89200 & 30.81 & 30.81 \\
0.93200 & 49.00 & 49.00 \\
0.98000 & 72.31 & 72.31 \\
1.04000 & 102.15 & 102.15 \\
1.12000 & 141.07 & 141.07 \\
1.24000 & 194.45 & 194.45 \\
1.40000 & 253.69 & 254.34 \\
1.60000 & 309.52 & 315.33 \\
1.84000 & 355.27 & 374.39 \\
2.17000 & 392.43 & 438.80 \\
2.57000 & 413.68 & 500.00 \\
3.05000 & 421.31 & 558.06 \\
3.61000 & 418.92 & 612.26 \\
4.17000 & 411.64 & 656.93 \\
4.82000 & 401.36 & 700.52 \\
5.62000 & 388.73 & $\mathbf{7 4 5 . 7 9}$ \\
6.83000 & $\mathbf{3 7 2 . 1 2}$ & 802.21 \\
8.03000 & $\mathbf{3 5 8 . 8 5}$ & 848.45 \\
12.00000 & $\mathbf{3 3 1 . 2 2}$ & 961.83 \\
16.00000 & $\mathbf{3 1 7 . 1 6}$ & $\mathbf{1 0 4 2 . 8 1}$ \\
24.10000 & 302.30 & 1156.85 \\
40.10000 & 290.33 & $\mathbf{1 2 9 7 . 9 1}$ \\
80.30000 & 282.99 & 1490.83 \\
160.00000 & 277.42 & 1679.45
\end{tabular}

\begin{tabular}{rrr} 
TD $=20.0$ & \multicolumn{2}{c}{ ET $=0.936644$ HEV } \\
ENERGY (HEV) & PRIARY & CASCADE \\
0.94600 & 1.33 & 1.33 \\
0.95500 & 2.80 & 2.80 \\
0.97400 & 6.42 & 6.42 \\
1.00000 & 12.36 & 12.36 \\
1.03000 & 20.34 & 20.34 \\
1.08000 & 35.51 & 35.51 \\
1.14000 & 55.50 & 55.50 \\
1.21000 & 79.71 & 79.71 \\
1.31000 & 113.69 & 113.69 \\
1.45000 & 157.07 & 157.07 \\
1.63000 & 203.48 & 204.13 \\
1.87000 & 249.78 & 255.26 \\
2.15000 & 286.05 & 303.23 \\
2.52000 & 314.18 & 353.70 \\
2.99000 & 330.72 & 404.04 \\
3.55000 & 336.05 & 451.32 \\
4.21000 & 333.41 & 495.75 \\
4.87000 & 327.03 & 532.27 \\
5.61000 & 318.72 & 566.67 \\
6.55000 & 308.42 & 603.59 \\
7.96000 & 295.17 & 649.10 \\
9.36000 & 284.68 & 686.41 \\
14.00000 & 263.21 & 777.93 \\
18.70000 & 252.32 & 843.33 \\
28.00000 & 240.95 & 933.45 \\
46.90000 & 231.73 & 1047.56 \\
93.60000 & 226.15 & 1201.74 \\
187.00000 & 221.62 & 1353.09
\end{tabular}

\begin{tabular}{rrr} 
TD=24.0 & \multicolumn{2}{c}{ ET $=1.058262$ MEV } \\
ENERGY (BEV) & PRIMARY & CASCADE \\
1.06000 & 0.15 & 0.15 \\
1.07000 & 1.08 & 1.08 \\
1.10000 & 4.80 & 4.80 \\
1.13000 & 9.64 & 9.64 \\
1.17000 & 17.43 & 17.43 \\
1.22000 & 28.65 & 28.65 \\
1.29000 & 45.99 & 45.99 \\
1.37000 & 66.67 & 66.67 \\
1.48000 & 94.65 & 94.65 \\
1.64000 & 131.75 & 131.75 \\
1.85000 & 171.92 & 172.64 \\
2.11000 & 208.97 & 213.96 \\
2.43000 & 239.47 & 254.95 \\
2.85000 & 262.54 & 297.67 \\
3.38000 & 275.64 & 339.94 \\
4.02000 & 279.47 & 380.10 \\
4.76000 & 276.77 & 417.09 \\
5.50000 & 271.20 & 447.52 \\
6.34000 & 264.07 & 476.53 \\
7.40000 & 255.44 & 507.44 \\
8.99000 & 244.46 & 545.51 \\
10.50000 & 236.15 & 575.46 \\
15.80000 & 218.35 & 653.23 \\
21.10000 & 209.50 & 707.82 \\
31.70000 & 200.17 & 783.65 \\
52.90000 & 192.80 & 878.59 \\
105.00000 & 188.34 & 1005.74 \\
211.00000 & 184.50 & 1132.86
\end{tabular}


ATONIC DISPLACEMENT CROSS-SECTIONS (BARNS) BY FAST ELECTRONS IN AU $=79 \quad A=197.000$

\begin{tabular}{rrr} 
TD $=28.0$ & EV & \multicolumn{1}{c}{ ET $=1.171110$} \\
ENERG & MEV \\
1.18000 & PRIMARY & CASCADE \\
1.19000 & 0.55 & 0.55 \\
1.21000 & 1.27 & 1.27 \\
1.25000 & 3.07 & 3.07 \\
1.29000 & 7.78 & 7.78 \\
1.35000 & 13.68 & 13.68 \\
1.42000 & 24.06 & 24.06 \\
1.52000 & 37.55 & 37.55 \\
1.63000 & 57.79 & 57.79 \\
1.81000 & 112.77 & 79.77 \\
2.04000 & 147.26 & 112.66 \\
2.34000 & 180.53 & 147.91 \\
2.65000 & 206.14 & 225.27 \\
3.16000 & 225.70 & 257.55 \\
3.74000 & 236.31 & 293.61 \\
4.45000 & 239.16 & 328.35 \\
5.26000 & 236.56 & 359.93 \\
6.08000 & 231.60 & 386.22 \\
7.02000 & 225.31 & 411.50 \\
8.19000 & 217.89 & 438.04 \\
9.95000 & 208.50 & 470.81 \\
11.70000 & 201.15 & 497.67 \\
17.50000 & 186.46 & 563.56 \\
23.40000 & 179.00 & 610.62 \\
35.10000 & 171.18 & 675.44 \\
58.50000 & 165.06 & 756.71 \\
117.00000 & 161.31 & 866.93 \\
234.00000 & 158.00 & 975.07
\end{tabular}

\begin{tabular}{rrr} 
TD $=32.0$ & EV & \multicolumn{1}{c}{ ET=1.276849 MEV } \\
BNERGY (HEV) & PRIMAR & CASCADE \\
1.28000 & 0.13 & 0.13 \\
1.30000 & 1.18 & 1.18 \\
1.32000 & 2.57 & 2.57 \\
1.36000 & 6.25 & 6.25 \\
1.41000 & 12.13 & 12.13 \\
1.48000 & 21.99 & 21.99 \\
1.55000 & 32.96 & 32.96 \\
1.65000 & 49.37 & 49.37 \\
1.78000 & 70.46 & 70.46 \\
1.97000 & 98.61 & 98.61 \\
2.23000 & 130.19 & 130.86 \\
2.55000 & 158.64 & 163.05 \\
2.93000 & 180.89 & 193.81 \\
3.44000 & 197.75 & 226.56 \\
4.08000 & 206.83 & 258.70 \\
4.85000 & 208.99 & 289.08 \\
5.74000 & 206.46 & $\mathbf{3 1 7 . 0 5}$ \\
6.63000 & 202.01 & 340.02 \\
7.66000 & 196.39 & 362.33 \\
8.93000 & 189.91 & $\mathbf{3 8 5} .51$ \\
10.80000 & 181.90 & 413.61 \\
12.70000 & 175.50 & 437.18 \\
19.10000 & 162.66 & 495.77 \\
25.50000 & 156.27 & 536.78 \\
$\mathbf{3 8 . 3 0 0 0 0}$ & 149.51 & 593.72 \\
63.80000 & 144.29 & 664.82 \\
127.00000 & 141.09 & 760.63 \\
255.00000 & 138.16 & 855.76
\end{tabular}

\begin{tabular}{rrr} 
TD $=36.0$ & EV & \multicolumn{2}{c}{ ETF1.376674 MEV } \\
ENERGY(MEV) & PRIMARY & CASCADE \\
1.39000 & 0.46 & 0.46 \\
1.40000 & 0.90 & 0.90 \\
1.43000 & 2.61 & 2.61 \\
1.47000 & 5.69 & 5.69 \\
1.52000 & 10.56 & 10.56 \\
1.59000 & 18.67 & 18.67 \\
1.67000 & 29.05 & 29.05 \\
1.78000 & 44.08 & 44.08 \\
1.92000 & 62.98 & 62.98 \\
2.13000 & 88.79 & 88.78 \\
2.40000 & 115.95 & 116.58 \\
2.75000 & 141.67 & 145.84 \\
3.16000 & 161.34 & 173.35 \\
3.71000 & 176.10 & 202.60 \\
4.40000 & 183.90 & 231.29 \\
5.23000 & 185.57 & 258.39 \\
6.19000 & 183.14 & 283.34 \\
7.15000 & 179.08 & 303.82 \\
8.26000 & 174.03 & 323.70 \\
9.63000 & 168.25 & 344.37 \\
11.70000 & 160.99 & 370.00 \\
13.70000 & 155.49 & 390.43 \\
20.60000 & 144.23 & 442.59 \\
27.50000 & 138.63 & 479.06 \\
41.30000 & 132.70 & 529.69 \\
68.80000 & 128.16 & 592.94 \\
137.00000 & 125.36 & 678.16 \\
275.00000 & 122.74 & 762.65
\end{tabular}

ATOMIC DISPLACEHEN CROSS-SECTIONS (BARNS) BY FAST ELECTRONS IN AD

\begin{tabular}{crr} 
TD=40.0 & \multicolumn{1}{c}{ ET $=1.471479$ MEV } \\
ENERGY (HEV) & PRIMARY & CASCADE \\
1.48000 & 0.22 & 0.22 \\
1.50000 & 0.90 & 0.90 \\
1.53000 & 2.34 & 2.34 \\
1.57000 & 4.91 & 4.91 \\
1.63000 & 9.85 & 9.85 \\
1.70000 & 16.76 & 16.76 \\
1.79000 & 26.58 & 26.68 \\
1.91000 & 40.62 & 40.62 \\
2.06000 & 57.78 & 57.78 \\
2.28000 & 80.62 & 80.62 \\
2.57000 & 105.21 & 105.85 \\
2.94000 & 128.05 & 131.99 \\
3.38000 & 145.70 & 156.95 \\
3.97000 & 158.80 & 183.45 \\
4.70000 & 165.53 & 209.09 \\
5.59000 & 166.86 & 233.65 \\
6.62000 & 164.52 & 256.24 \\
7.65000 & 160.78 & 274.77 \\
8.82000 & 156.25 & 292.46 \\
10.30000 & 150.98 & 311.29 \\
12.50000 & 144.50 & 334.28 \\
14.70000 & 139.45 & 353.19 \\
22.00000 & 129.57 & 399.66 \\
29.40000 & 124.56 & 432.63 \\
44.10000 & 119.30 & 478.09 \\
73.50000 & 115.28 & 535.10 \\
147.00000 & 112.77 & 612.28
\end{tabular}

\begin{tabular}{crr} 
TD $=44.0$ & EV & \multicolumn{2}{c}{ ET $=1.561953$ MEV } \\
ENERGY (MBV) & PRIMARY & CASCADE \\
1.57000 & 0.16 & 0.16 \\
1.59000 & 0.70 & 0.70 \\
1.62000 & 1.87 & 1.87 \\
1.67000 & 4.59 & 4.59 \\
1.73000 & 8.85 & 8.85 \\
1.81000 & 15.67 & 15.67 \\
1.90000 & 24.25 & 24.25 \\
2.03000 & 37.28 & 37.28 \\
2.18000 & 52.10 & 52.10 \\
2.42000 & 73.62 & 73.62 \\
2.73000 & 96.19 & 96.83 \\
3.12000 & 116.80 & 120.53 \\
3.59000 & 132.85 & 143.45 \\
4.21000 & 144.51 & 167.39 \\
4.99000 & 150.51 & 190.93 \\
5.93000 & 151.57 & 213.21 \\
7.02000 & 149.36 & 233.76 \\
8.12000 & 145.88 & 250.75 \\
9.37000 & 141.71 & 266.96 \\
10.90000 & 137.03 & 283.68 \\
13.20000 & 131.20 & 304.39 \\
15.60000 & 126.49 & $\mathbf{3 2 2 . 1 3}$ \\
$\mathbf{2 3 . 4 0 0 0 0}$ & 117.55 & $\mathbf{3 6 4 . 6 7}$ \\
31.20000 & 113.09 & $\mathbf{3 9 4 . 4 2}$ \\
$\mathbf{4 6 . 8 0 0 0 0}$ & 108.34 & 435.76 \\
78.00000 & 104.74 & 487.61 \\
156.00000 & 102.48 & 557.79
\end{tabular}

\begin{tabular}{rrr} 
TD=48.0 BV & \multicolumn{2}{c}{ ET $=1.648640$ MEV } \\
ENERGY (MEV) & PRIMARY & CASCADE \\
1.66000 & 0.19 & 0.19 \\
1.68000 & 0.66 & 0.66 \\
1.71000 & 1.67 & 1.67 \\
1.76000 & 4.00 & 4.00 \\
1.82000 & 7.64 & 7.64 \\
1.91000 & 14.29 & 14.29 \\
2.01000 & 22.64 & 22.64 \\
2.14000 & 34.04 & 34.04 \\
2.30000 & 47.87 & 47.87 \\
2.55000 & 67.47 & 67.47 \\
2.88000 & 88.45 & 89.07 \\
3.29000 & 107.30 & 110.82 \\
3.79000 & 122.08 & 132.08 \\
4.45000 & 132.70 & 154.25 \\
5.27000 & 138.00 & 175.77 \\
6.26000 & 138.84 & 196.17 \\
7.41000 & 136.72 & 215.04 \\
8.57000 & 133.49 & 230.62 \\
9.89000 & 129.64 & 245.51 \\
11.50000 & 125.36 & 260.82 \\
14.00000 & 119.89 & 280.34 \\
16.40000 & 115.82 & 295.76 \\
24.70000 & 107.60 & 335.19 \\
32.90000 & 103.55 & 362.38 \\
49.40000 & 99.22 & 400.38 \\
82.40000 & 95.96 & 448.01 \\
164.00000 & 93.93 & 511.89
\end{tabular}


ATONIC DISPLACEMENT CROSS-SECTIONS (BARNS) BY FAST ELECTRONS IN AU $Z=79 \quad A=197.000$

\begin{tabular}{rrr} 
TD $=52.0$ & EV & \multicolumn{2}{c}{ ET $=1.731979$ MEV } \\
ENERGY(HEV) & PRIMARY & CASCADE \\
1.74000 & 0.11 & 0.11 \\
1.76000 & 0.48 & 0.48 \\
1.80000 & 1.62 & 1.62 \\
1.85000 & 3.69 & 3.69 \\
1.92000 & 7.48 & 7.48 \\
2.00000 & 12.71 & 12.71 \\
2.11000 & 20.80 & 20.80 \\
2.25000 & 31.66 & 31.66 \\
2.42000 & 44.63 & 44.63 \\
2.68000 & 62.61 & 62.61 \\
3.03000 & 82.17 & 82.78 \\
3.46000 & 99.47 & 102.86 \\
3.98000 & 112.90 & 122.34 \\
4.67000 & 122.58 & 142.78 \\
5.54000 & 127.42 & 162.88 \\
6.58000 & 128.08 & 181.73 \\
7.79000 & 126.04 & 199.19 \\
9.00000 & 123.04 & 213.48 \\
10.30000 & 119.69 & 226.46 \\
12.10000 & 115.46 & 241.57 \\
14.70000 & 110.47 & 259.43 \\
17.30000 & 106.63 & 274.08 \\
25.90000 & 99.22 & 309.98 \\
34.60000 & 95.47 & 335.35 \\
51.90000 & 91.52 & 370.35 \\
86.50000 & 88.55 & 414.26 \\
173.00000 & 86.67 & 473.64
\end{tabular}

\begin{tabular}{rrr} 
TD=56.0 EV & \multicolumn{2}{c}{ ET=1.812331 HEV } \\
ENERGY (HEV) & PRIMARY & CASCA DE \\
1.83000 & 0.22 & 0.22 \\
1.84000 & 0.39 & 0.39 \\
1.88000 & 1.37 & 1.37 \\
1.93000 & 3.14 & 3.14 \\
2.01000 & 6.96 & 6.96 \\
2.10000 & 12.24 & 12.24 \\
2.21000 & 19.49 & 19.49 \\
2.35000 & 29.18 & 29.18 \\
2.53000 & 41.45 & 41.45 \\
2.80000 & 58.12 & 58.12 \\
3.17000 & 76.54 & 77.14 \\
3.62000 & 92.60 & 95.85 \\
4.16000 & 104.96 & 113.86 \\
4.89000 & 113.96 & 133.09 \\
5.79000 & 118.31 & 151.59 \\
6.88000 & 118.87 & 169.17 \\
8.15000 & 116.92 & 185.46 \\
9.42000 & 114.09 & 198.79 \\
10.80000 & 110.92 & 211.02 \\
12.60000 & 107.15 & 224.46 \\
15.40000 & 102.39 & 241.57 \\
18.10000 & 98.86 & 255.08 \\
27.10000 & 92.03 & 288.45 \\
36.20000 & 88.57 & 312.01 \\
54.30000 & 84.92 & 344.52 \\
90.60000 & 82.19 & 385.39 \\
181.00000 & 80.46 & 440.44
\end{tabular}

\begin{tabular}{rrr} 
TD $=60.0$ & EV & \multicolumn{2}{c}{ ET $=1.889995$ MEV } \\
ENERGY (HEV) & PRIMARY & CASCADE \\
1.90000 & 0.10 & 0.10 \\
1.92000 & 0.37 & 0.37 \\
1.96000 & 1.24 & 1.24 \\
2.02000 & 3.17 & 3.17 \\
2.09000 & 6.18 & 6.18 \\
2.19000 & 11.42 & 11.42 \\
2.30000 & 17.93 & 17.93 \\
2.45000 & 27.27 & 27.27 \\
2.64000 & 38.92 & 38.92 \\
2.92000 & 54.44 & 54.44 \\
3.30000 & 71.45 & 72.01 \\
3.77000 & 86.52 & 89.59 \\
4.34000 & 98.16 & 106.66 \\
5.10000 & 106.46 & 124.60 \\
6.04000 & 110.45 & 141.92 \\
7.18000 & 110.88 & 158.38 \\
8.50000 & 109.01 & 173.55 \\
9.82000 & 106.36 & 185.96 \\
11.30000 & 103.30 & 197.68 \\
13.20000 & 99.76 & 210.34 \\
15.00000 & 95.50 & 225.66 \\
18.80000 & 92.22 & 238.26 \\
28.30000 & 85.79 & 269.84 \\
37.70000 & 82.61 & 291.63 \\
56.60000 & $\mathbf{7 9 . 2 1}$ & $\mathbf{3 2 2 . 0 4}$ \\
94.40000 & $\mathbf{7 6 . 6 9}$ & $\mathbf{3 6 0 . 1 7}$ \\
188.00000 & $\mathbf{7 5 . 0 9}$ & 411.33 \\
& &
\end{tabular}

ATOHIC DISPLACEHENT CROSS-SECTIONS (BARNS) BY PAST ELECTRONS IN AO

\begin{tabular}{|c|c|c|c|}
\hline $\begin{array}{r}\text { TD }=64.0 \\
\text { ENERGY (HEV) }\end{array}$ & EV & $\begin{aligned} E T=1.5 \\
\text { RRIMARY }\end{aligned}$ & $\begin{array}{l}224 \text { KEV } \\
\text { CASCADE }\end{array}$ \\
\hline 1.98000 & & $\begin{array}{r}2.13 \\
0.13\end{array}$ & 0.13 \\
\hline 2.00000 & & 0.39 & 0.39 \\
\hline 2.04000 & & 1.19 & 1.19 \\
\hline 2.10000 & & 2.94 & 2.94 \\
\hline 2.18000 & & 6.07 & 6.07 \\
\hline 2.27000 & & 10.35 & 10.35 \\
\hline 2.39000 & & 16.76 & 16.76 \\
\hline 2.55000 & & 25.78 & 25.78 \\
\hline 2.75000 & & 36.87 & 36.87 \\
\hline 3.04000 & & 51.38 & 51.39 \\
\hline 3.43000 & & 67.13 & 67.68 \\
\hline 3.93000 & & 81.53 & 84.55 \\
\hline 4.52000 & & 92.28 & 100.46 \\
\hline 5.30000 & & 99.87 & 117.08 \\
\hline 6.28000 & & 103.55 & 133.39 \\
\hline 7.46000 & & 103.91 & 148.78 \\
\hline 8.84000 & & 102.11 & 163.09 \\
\hline 10.20000 & & 99.62 & 174.64 \\
\hline 11.70000 & & 96.81 & 185.40 \\
\hline 13.70000 & & 93.44 & 197.46 \\
\hline 16.70000 & & 89.33 & 212.25 \\
\hline 19.60000 & & 86.30 & 223.98 \\
\hline 29.40000 & & 80.36 & 253.35 \\
\hline 39.30000 & & 77.36 & 274.02 \\
\hline 58.90000 & & 74.22 & 302.42 \\
\hline 98.20000 & & 71.88 & 338.15 \\
\hline 196.00000 & & 70.38 & 386.26 \\
\hline
\end{tabular}

$\begin{array}{rrr}\begin{array}{r}\text { TD }=68.0 \\ \text { EHERGY (HEV) }\end{array} & \begin{array}{r}\text { ET }=2.038235 \\ \text { PRIMARY }\end{array} & \text { CASCADE } \\ 2.05000 & 0.09 & 0.09 \\ 2.07000 & 0.30 & 0.30 \\ 2.11000 & 0.98 & 0.98 \\ 2.18000 & 2.81 & 2.81 \\ 2.26000 & 5.66 & 5.66 \\ 2.36000 & 10.00 & 10.00 \\ 2.48000 & 15.86 & 15.86 \\ 2.64000 & 24.09 & 24.09 \\ 2.85000 & 34.71 & 34.71 \\ 3.15000 & 48.39 & 48.40 \\ 3.56000 & 63.44 & 63.98 \\ 4.07000 & 76.78 & 79.64 \\ 4.68000 & 86.88 & 94.63 \\ 5.50000 & 94.09 & 110.53 \\ 6.52000 & 97.48 & 125.93 \\ 7.74000 & 97.75 & 140.37 \\ 9.17000 & 96.02 & 153.84 \\ 10.50000 & 93.80 & 164.14 \\ 12.20000 & 90.90 & 175.22 \\ 14.20000 & 87.85 & 186.15 \\ 17.30000 & 84.00 & 200.04 \\ 20.30000 & 81.15 & 211.08 \\ 30.50000 & 75.57 & 238.85 \\ 40.70000 & 72.77 & 258.19 \\ 61.10000 & 69.81 & 285.03 \\ 101.00000 & 67.65 & 318.11 \\ 203.00000 & 66.23 & 363.84\end{array}$

\begin{tabular}{rrr} 
TD $=72.0$ & EV & \multicolumn{1}{c}{ ET $=2.109211$} \\
PNER & MEV \\
2.13000 & 0.15 & 0.15 \\
2.15000 & 0.38 & 0.38 \\
2.19000 & 1.03 & 1.03 \\
2.25000 & 2.47 & 2.47 \\
2.34000 & 5.39 & 5.39 \\
2.44000 & 9.36 & 9.36 \\
2.57000 & 15.18 & 15.18 \\
2.74000 & 23.20 & 23.20 \\
2.95000 & 32.92 & 32.92 \\
3.26000 & 45.85 & 45.86 \\
3.69000 & 60.25 & 60.79 \\
4.21000 & 72.63 & 75.38 \\
4.85000 & 82.25 & 89.74 \\
5.69000 & 88.92 & 104.61 \\
6.74000 & 92.07 & 119.11 \\
8.01000 & 92.28 & 132.85 \\
9.49000 & 90.62 & 145.58 \\
10.90000 & 88.45 & 155.53 \\
12.60000 & 85.80 & 165.65 \\
14.70000 & 82.87 & 176.13 \\
17.90000 & 79.25 & 189.23 \\
21.00000 & 76.57 & 199.64 \\
31.60000 & 71.30 & 225.98 \\
42.10000 & 68.69 & 244.15 \\
63.20000 & 65.91 & 269.51 \\
105.00000 & 63.87 & 301.06 \\
210.00000 & 62.54 & 343.95
\end{tabular}


ATOHIC DISPLACEHENT CROSS-SECTIONS (BARNS) BY FAST ELECTRONS IN AO

\begin{tabular}{rrr} 
TD $=76.0$ & EV & \multicolumn{1}{c}{ ET $=2.178316$ MEV } \\
ENERGY (MEV) & PRIMARY & CASCADE \\
2.20000 & 0.14 & 0.14 \\
2.22000 & 0.34 & 0.34 \\
2.26000 & 0.94 & 0.94 \\
2.33000 & 2.49 & 2.49 \\
2.41000 & 4.87 & 4.87 \\
2.52000 & 8.88 & 8.88 \\
2.65000 & 14.23 & 14.23 \\
2.83000 & 22.04 & 22.04 \\
3.04000 & 31.00 & 31.00 \\
3.37000 & 43.68 & 43.68 \\
3.81000 & 57.19 & 57.72 \\
4.35000 & 68.97 & 71.64 \\
5.01000 & 78.03 & 85.24 \\
5.88000 & 84.31 & 99.37 \\
6.97000 & 87.24 & 113.17 \\
8.27000 & 87.40 & 126.08 \\
9.80000 & 85.79 & 138.16 \\
11.30000 & 83.66 & 147.86 \\
13.00000 & 81.22 & 157.13 \\
15.20000 & 78.40 & 167.21 \\
18.50000 & 74.99 & 179.59 \\
21.70000 & 72.46 & 189.45 \\
32.60000 & 67.51 & 214.31 \\
43.50000 & 65.03 & 231.62 \\
65.30000 & 62.41 & 255.64 \\
108.00000 & 60.50 & 285.28 \\
217.00000 & 59.24 & 326.18
\end{tabular}

\begin{tabular}{ccr} 
TD $=80.0$ & EV & \multicolumn{2}{c}{ ET=2.245688 MEV } \\
ENERGY(MEV) & PRIMARY & CASCADE \\
2.26000 & 0.07 & 0.07 \\
2.29000 & 0.33 & 0.33 \\
2.33000 & 0.88 & 0.88 \\
2.40000 & 2.30 & 2.30 \\
2.49000 & 4.79 & 4.79 \\
2.60000 & 8.51 & 8.51 \\
2.73000 & 13.47 & 13.47 \\
2.91000 & 20.69 & 20.69 \\
3.14000 & 29.76 & 29.76 \\
3.48000 & 41.79 & 41.80 \\
3.92000 & 54.27 & 54.76 \\
4.49000 & 65.73 & 68.33 \\
5.16000 & 74.17 & 81.07 \\
6.06000 & 80.13 & 94.57 \\
7.18000 & 82.88 & 107.66 \\
8.53000 & 83.00 & 120.02 \\
10.10000 & 81.45 & 131.45 \\
11.50000 & 79.48 & 140.42 \\
13.40000 & 77.09 & 149.49 \\
15.70000 & 74.38 & 159.20 \\
19.00000 & 71.23 & 170.62 \\
22.40000 & 68.76 & 180.29 \\
33.60000 & 64.10 & 203.83 \\
44.90000 & 61.74 & 220.36 \\
67.30000 & 59.27 & 243.10 \\
112.00000 & 57.45 & 271.61 \\
224.00000 & 56.27 & 310.20 \\
& &
\end{tabular}

\begin{tabular}{rrr} 
TD=84.0 EV & \multicolumn{2}{c}{ ET=2.311453 IEV } \\
ENRRGY(MEV) & PRIMARY & CASCADE \\
2.33000 & 0.09 & 0.09 \\
2.35000 & 0.24 & 0.24 \\
2.40000 & 0.86 & 0.86 \\
2.47000 & 2.18 & 2.18 \\
2.56000 & 4.48 & 4.48 \\
2.68000 & 8.25 & 8.25 \\
2.81000 & 12.85 & 12.85 \\
3.00000 & 19.94 & 19.94 \\
3.23000 & 28.35 & 28.35 \\
3.58000 & 39.84 & 39.85 \\
4.04000 & 51.91 & 52.41 \\
4.62000 & 52.68 & 65.19 \\
5.31000 & 70.71 & 77.35 \\
6.24000 & 76.37 & 90.27 \\
7.39000 & 78.94 & 102.71 \\
8.78000 & 79.02 & 114.49 \\
10.40000 & 77.52 & 125.41 \\
12.00000 & 75.56 & 134.23 \\
13.80000 & 73.35 & 142.61 \\
16.10000 & 70.83 & 151.61 \\
19.60000 & 67.74 & 162.84 \\
23.10000 & 65.40 & 172.03 \\
34.60000 & 61.01 & 194.37 \\
46.20000 & 58.77 & 210.08 \\
69.30000 & 56.42 & 231.78 \\
115.00000 & 54.71 & 258.77 \\
231.00000 & 53.58 & 295.76
\end{tabular}

ATOMIC DISPLACBHENT CROSS-SECTIONS (BARNS) BY PAST ELECTRONS IN AO

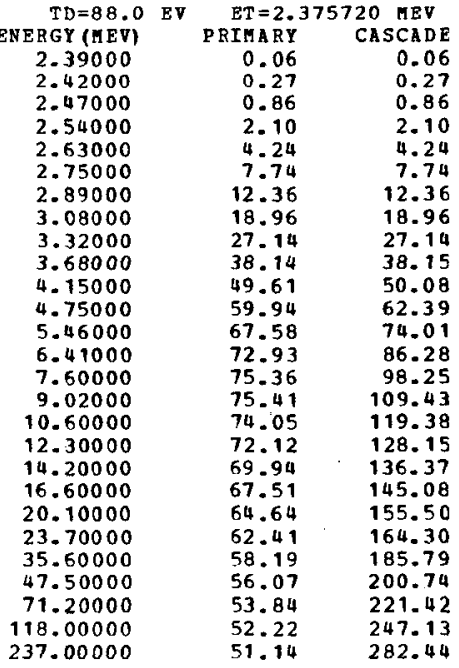

$\begin{array}{rrr}\begin{array}{c}\text { TD }=92.0 \\ \text { ENERGY (MEV) }\end{array} & \begin{array}{r}\text { ET }=2.438586 \text { MEV } \\ \text { PRIMARY }\end{array} & \begin{array}{r}\text { CASCADE } \\ 2.46000\end{array} \\ 2.48000 & 0.09 & 0.09 \\ 2.53000 & 0.22 & 0.22 \\ 2.60000 & 0.74 & 0.74 \\ 2.70000 & 1.86 & 1.86 \\ 2.82000 & 4.06 & 4.06 \\ 2.97000 & 7.33 & 7.33 \\ 3.17000 & 11.95 & 11.95 \\ 3.41000 & 18.45 & 18.45 \\ 3.77000 & 26.09 & 26.09 \\ 4.26000 & 36.37 & 36.38 \\ 4.87000 & 47.54 & 48.01 \\ 5.60000 & 57.35 & 59.69 \\ 6.58000 & 64.68 & 70.86 \\ 7.80000 & 69.79 & 82.67 \\ 9.26000 & 72.08 & 94.11 \\ 10.90000 & 72.11 & 104.83 \\ 12.60000 & 70.78 & 114.45 \\ 14.60000 & 68.96 & 122.63 \\ 17.00000 & 66.83 & 130.70 \\ 20.70000 & 64.56 & 138.81 \\ 24.30000 & 61.74 & 149.08 \\ 36.50000 & 59.66 & 157.28 \\ 48.70000 & 55.64 & 177.84 \\ 73.10000 & 53.62 & 192.14 \\ 121.00000 & 51.48 & 211.99 \\ 243.00000 & 49.94 & 236.52 \\ & 48.92 & 270.29\end{array}$

\begin{tabular}{rrr} 
TD=96.0 EV & \multicolumn{2}{c}{ ET=2.50014 1 MEV } \\
ENERGY (HEV) & PRIMARY & CASCADE \\
2.52000 & 0.07 & 0.07 \\
2.55000 & 0.26 & 0.26 \\
2.60000 & 0.78 & 0.78 \\
2.67000 & 1.86 & 1.86 \\
2.77000 & 3.94 & 3.94 \\
2.90000 & 7.27 & 7.27 \\
3.05000 & 11.63 & 11.63 \\
3.25000 & 17.72 & 17.72 \\
3.50000 & 25.17 & 25.17 \\
3.87000 & 35.05 & 35.06 \\
4.37000 & 45.69 & 46.15 \\
5.00000 & 55.12 & 57.43 \\
5.75000 & 62.10 & 68.14 \\
6.75000 & 66.93 & 79.39 \\
8.00000 & 69.09 & 90.34 \\
9.50000 & 69.08 & 100.64 \\
11.20000 & 67.77 & 109.94 \\
13.00000 & 65.96 & 118.00 \\
15.00000 & 63.97 & 125.51 \\
17.50000 & 61.77 & 133.38 \\
21.20000 & 59.14 & 142.97 \\
25.00000 & 57.10 & 151.05 \\
37.50000 & 53.28 & 170.69 \\
50.00000 & 51.36 & 184.36 \\
75.00000 & 49.32 & 203.35 \\
125.00000 & 47.84 & 227.19 \\
250.00000 & 46.87 & 259.35 \\
& &
\end{tabular}


ATOMIC DISPLACEMENT CROSS-SECTIONS (BARNS) BY PAST RLECTRONS IN PB

\begin{tabular}{rrr} 
TD $=4.0$ & EV & \multicolumn{2}{c}{ ET $=0.293393$ MEV } \\
ENERGY (HEV) & PRIMARY & CASCADE \\
0.29600 & 23.90 & 23.90 \\
0.29900 & 51.01 & 51.01 \\
0.30500 & 104.05 & 104.05 \\
0.31300 & 172.43 & 172.43 \\
0.32500 & 270.29 & 270.29 \\
0.34000 & 385.21 & 385.21 \\
0.35700 & 506.27 & 506.27 \\
0.38100 & 661.96 & 661.96 \\
0.41000 & 828.55 & 828.55 \\
0.45400 & 1042.13 & 1042.13 \\
0.51300 & 1267.30 & 1267.38 \\
0.58600 & 1471.10 & 1483.33 \\
0.67400 & 1538.28 & 1688.18 \\
0.79200 & 1773.42 & 1902.14 \\
0.93800 & 1855.69 & 2107.43 \\
1.11000 & 1886.93 & 2297.95 \\
1.32000 & 1878.13 & 2484.69 \\
1.52000 & 1848.36 & 2632.41 \\
1.76000 & 1802.68 & 2783.39 \\
2.05000 & 1744.80 & 2939.59 \\
2.49000 & 1664.46 & 3138.26 \\
2.93000 & 1597.00 & 3305.93 \\
4.40000 & 1446.42 & 3730.91 \\
5.86000 & 1364.97 & 4037.45 \\
8.80000 & 1284.57 & 4482.25 \\
14.60000 & 1216.98 & 5040.50 \\
29.30000 & 1169.95 & 5820.42 \\
58.60000 & 1146.95 & 6598.67 \\
88.00000 & 1131.34 & 7047.89 \\
146.00000 & 1110.63 & 7599.81
\end{tabular}

\begin{tabular}{rrr} 
TD $=8.0$ & EV & \multicolumn{2}{c}{ ET $=0.505353$ MEV } \\
ENERGY (HEV) & PRIMARY & CASCADE \\
0.51000 & 6.86 & 6.86 \\
0.51500 & 14.44 & 14.44 \\
0.52500 & 30.13 & 30.13 \\
0.54000 & 54.74 & 54.74 \\
0.56000 & 88.98 & 88.98 \\
0.58600 & 134.79 & 134.79 \\
0.61600 & 187.99 & 187.99 \\
0.65600 & 257.44 & 257.44 \\
0.70700 & 340.95 & 340.95 \\
0.78300 & 451.51 & 451.61 \\
0.88400 & 571.89 & 572.54 \\
1.01000 & 684.12 & 693.30 \\
1.16000 & 775.45 & 807.92 \\
1.36000 & 848.57 & 928.47 \\
1.61000 & 892.59 & 1046.26 \\
1.92000 & 908.38 & 1160.80 \\
2.27000 & 902.09 & 1264.14 \\
2.62000 & 885.34 & 1349.39 \\
3.03000 & 861.42 & 1433.93 \\
3.53000 & 832.35 & 1521.01 \\
4.29000 & 793.41 & 1631.14 \\
5.05000 & 761.90 & 1722.46 \\
7.58000 & 695.22 & 1949.32 \\
10.10000 & 661.57 & 2111.30 \\
15.10000 & 628.04 & 2337.78 \\
25.20000 & 599.33 & 2625.50 \\
50.50000 & 581.50 & 3019.15 \\
101.00000 & 569.84 & 3407.69 \\
151.00000 & 561.68 & 3629.06 \\
252.00000 & 551.79 & 3907.06
\end{tabular}

\begin{tabular}{rrr} 
TD $=12.0$ & BV & \multicolumn{2}{c}{ ET $=0.680176$ MEV } \\
ENERGY (MEV) & PRIMARY & CASCADE \\
0.68600 & 3.04 & 3.04 \\
0.69300 & 6.95 & 6.95 \\
0.70700 & 15.48 & 15.48 \\
0.72700 & 29.08 & 29.08 \\
0.75400 & 49.42 & 49.42 \\
0.78900 & 77.98 & 77.98 \\
0.82900 & 112.13 & 112.13 \\
0.88400 & 159.44 & 159.44 \\
0.95200 & 215.75 & 215.75 \\
1.05000 & 289.11 & 289.11 \\
1.19000 & 375.21 & 376.03 \\
1.36000 & 452.69 & 460.58 \\
1.56000 & 514.18 & 540.07 \\
1.83000 & 562.78 & 624.80 \\
2.17000 & 590.56 & 707.94 \\
2.58000 & 598.58 & 786.53 \\
3.06000 & 592.18 & 859.77 \\
3.53000 & 579.66 & 918.76 \\
4.08000 & 562.94 & 976.92 \\
4.76000 & 543.00 & 1037.60 \\
5.78000 & 517.52 & 1112.86 \\
6.80000 & 497.32 & 1175.19 \\
10.20000 & 455.75 & 1329.45 \\
13.60000 & 435.29 & 1439.26 \\
20.40000 & 414.38 & 1592.75 \\
34.00000 & 397.13 & 1785.33 \\
68.00000 & 386.67 & 2047.87 \\
136.00000 & 378.67 & 2306.42 \\
204.00000 & 373.10 & 2454.88 \\
340.00000 & 366.88 & 2639.42
\end{tabular}

ATOMIC DISPLACEAENT CROSS-SECTIONS (BARNS) BY FAST RLECTRONS IN PB $Z=82 \quad A=207.210$

\begin{tabular}{rrr} 
TD $=16.0$ & EV & \multicolumn{1}{c}{ ET $=0.832438$ MEV } \\
ENERGY (MEV) & PRIMARY & CASCADE \\
0.84000 & 1.92 & 1.92 \\
0.84900 & 4.48 & 4.48 \\
0.86500 & 9.65 & 9.65 \\
0.89000 & 19.10 & 19.10 \\
0.92400 & 33.99 & $\mathbf{3 3 . 9 9}$ \\
0.96500 & 54.04 & 54.04 \\
1.01000 & 77.50 & 77.50 \\
1.08000 & 114.88 & 114.88 \\
1.16000 & 156.47 & 156.47 \\
1.29000 & 217.62 & 217.62 \\
1.45000 & 279.39 & 280.16 \\
1.66000 & 339.47 & 346.31 \\
1.91000 & 386.78 & 408.95 \\
2.24000 & 422.33 & 474.03 \\
2.66000 & 441.86 & 538.41 \\
3.16000 & 446.25 & 598.40 \\
3.74000 & 440.30 & 653.77 \\
4.32000 & 430.08 & 699.32 \\
4.99000 & 417.20 & 743.49 \\
5.82000 & 402.14 & 789.71 \\
7.07000 & 383.17 & 847.06 \\
8.32000 & 368.35 & 894.51 \\
12.40000 & $\mathbf{3 3 8 . 9 0}$ & 1009.60 \\
16.60000 & 324.12 & 1093.64 \\
24.90000 & 309.07 & 1209.21 \\
41.60000 & 296.88 & 1354.84 \\
83.20000 & 289.56 & 1552.01 \\
166.00000 & 283.45 & 1745.00
\end{tabular}

\begin{tabular}{rrr}
$T D=20.0$ & EV & \multicolumn{2}{c}{ ET \pm 0.969119 MEV } \\
ENERGY(MEV) & PRIHABY & CASCADE \\
0.97800 & 1.30 & 1.30 \\
0.98800 & 3.00 & 3.00 \\
1.00000 & 5.32 & 5.32 \\
1.03000 & 12.33 & 12.33 \\
1.07000 & 23.75 & 23.75 \\
1.12000 & 40.25 & 40.25 \\
1.18000 & 61.87 & 61.87 \\
1.25000 & 87.93 & 87.93 \\
1.35000 & 124.40 & 124.40 \\
1.50000 & 173.86 & 173.86 \\
1.69000 & 225.03 & 225.83 \\
1.93000 & 272.57 & 278.72 \\
2.22000 & 310.29 & 329.62 \\
2.61000 & 338.53 & 383.34 \\
3.10000 & 353.11 & 435.64 \\
3.68000 & 355.62 & 484.06 \\
4.36000 & 350.05 & 529.15 \\
5.03000 & 341.49 & 565.68 \\
5.81000 & 330.95 & 601.37 \\
6.78000 & 318.81 & 638.85 \\
8.23000 & 303.82 & 684.93 \\
9.69000 & 292.12 & 723.29 \\
14.50000 & 269.17 & 816.92 \\
19.30000 & 258.02 & 883.04 \\
29.00000 & 246.28 & 976.10 \\
48.40000 & 237.01 & 1092.63 \\
96.90000 & 231.40 & 1250.71 \\
193.00000 & 226.45 & 1404.57 \\
& &
\end{tabular}

\begin{tabular}{rrr} 
TO=24.0 & \multicolumn{2}{c}{ ET $=1.094204$ MPV } \\
ENERGY (HEV) & PRIMARY & CASCADE \\
1.10000 & 0.52 & 0.52 \\
1.11000 & 1.57 & 1.57 \\
1.13000 & 4.14 & 4.14 \\
1.17000 & 10.92 & 10.92 \\
1.21000 & 19.36 & 19.36 \\
1.26000 & 31.50 & 31.50 \\
1.33000 & 50.20 & 50.20 \\
1.42000 & 75.26 & 75.26 \\
1.53000 & 105.22 & 105.22 \\
1.69000 & 144.71 & 144.71 \\
1.91000 & 188.95 & 189.77 \\
2.18000 & 228.49 & 234.19 \\
2.51000 & 259.86 & 277.37 \\
2.95000 & 282.71 & 322.50 \\
3.50000 & 294.06 & 366.20 \\
4.15000 & 295.52 & 406.64 \\
4.92000 & 290.42 & 444.68 \\
5.68000 & 282.98 & 475.49 \\
6.56000 & 274.06 & 505.41 \\
7.65000 & 263.96 & 536.70 \\
9.30000 & 251.46 & 575.62 \\
10.90000 & 242.10 & 606.84 \\
16.40000 & 223.19 & 686.29 \\
21.80000 & 214.21 & 741.25 \\
32.80000 & 204.62 & 819.16 \\
54.70000 & 197.20 & 916.27 \\
109.00000 & 192.69 & 1047.17 \\
218.00000 & 188.50 & 1176.06
\end{tabular}


ATONIC DISPLACEHENT CROSS-SECTIONS (BARNS) BY FAST RLECTRONS IN PB $\mathrm{z}=82 \quad \mathrm{~A}=207.210$

\begin{tabular}{rrr} 
TD $=28.0$ & EV & \multicolumn{2}{c}{ ET $=1.210222$ MEV } \\
ENERGY (MEV) & PRIMARY & CASCADE \\
1.22000 & 0.63 & 0.63 \\
1.23000 & 1.40 & 1.40 \\
1.25000 & 3.30 & 3.30 \\
1.29000 & 8.34 & 8.34 \\
1.34000 & 16.40 & 16.40 \\
1.40000 & 27.80 & 27.80 \\
1.47000 & 42.47 & 42.47 \\
1.57000 & 64.31 & 64.31 \\
1.69000 & 89.99 & 89.99 \\
1.87000 & 124.84 & 124.84 \\
2.11000 & 162.57 & 163.35 \\
2.42000 & 197.67 & 203.17 \\
2.78000 & 223.76 & 239.84 \\
3.26000 & 242.65 & 278.27 \\
3.87000 & 251.94 & 316.12 \\
4.59000 & 252.75 & 351.06 \\
5.44000 & 248.06 & 383.79 \\
6.29000 & 241.43 & 410.61 \\
7.26000 & 233.71 & 436.27 \\
8.47000 & 225.02 & 463.28 \\
10.20000 & 214.87 & 495.16 \\
12.10000 & 206.32 & 524.05 \\
18.10000 & 190.69 & 591.40 \\
24.20000 & 182.99 & 639.54 \\
36.30000 & 175.00 & 705.89 \\
60.50000 & 168.83 & 789.12 \\
121.00000 & 165.04 & 901.94 \\
242.00000 & 161.41 & 1012.33
\end{tabular}

\begin{tabular}{rrr}
$\begin{array}{r}\text { TD=32.0 } \\
\text { BNERGY }\end{array}$ & \multicolumn{1}{c}{ ET $=1.318900$ MEV } \\
1.33000 & PRIMAR & CASCADE \\
1.34000 & 0.52 & 0.52 \\
1.37000 & 1.10 & 1.10 \\
1.41000 & 3.38 & 3.38 \\
1.46000 & 7.51 & 7.51 \\
1.52000 & 14.00 & 14.00 \\
1.60000 & 23.14 & 23.14 \\
1.71000 & 36.65 & 36.65 \\
1.84000 & 56.15 & 56.15 \\
2.04000 & 78.74 & 78.74 \\
2.30000 & 110.12 & 110.12 \\
2.53000 & 173.10 & 143.87 \\
3.03000 & 196.44 & 178.23 \\
3.56000 & 212.78 & 211.23 \\
4.22000 & 220.39 & 278.44 \\
5.01000 & 220.74 & 309.29 \\
5.93000 & 216.41 & 337.83 \\
6.85000 & 210.53 & 361.22 \\
7.91000 & 203.69 & 383.84 \\
9.23000 & 196.07 & 407.58 \\
11.20000 & 186.88 & 436.69 \\
13.10000 & 180.09 & 459.94 \\
19.70000 & 166.41 & 519.80 \\
26.30000 & 159.81 & 561.75 \\
39.50000 & 152.88 & 620.03 \\
65.90000 & 147.60 & 693.07 \\
131.00000 & 144.37 & 790.94 \\
263.00000 & 141.15 & 888.04
\end{tabular}

\begin{tabular}{rrr} 
TD $=36.0$ & EV & \multicolumn{1}{c}{ ET $=1.421475$} \\
ENERGY (MEV) & PRIMART & CASCADE \\
1.43000 & 0.29 & 0.29 \\
1.44000 & 0.71 & 0.71 \\
1.47000 & 2.41 & 2.41 \\
1.52000 & 6.52 & 6.52 \\
1.57000 & 11.84 & 11.84 \\
1.64000 & 20.65 & 20.65 \\
1.73000 & 33.33 & 33.33 \\
1.84000 & 49.57 & 49.57 \\
1.99000 & 71.26 & 71.26 \\
2.20000 & 98.57 & 98.56 \\
2.48000 & 127.96 & 128.71 \\
2.84000 & 154.96 & 159.75 \\
3.26000 & 174.91 & 188.47 \\
3.83000 & 189.27 & 219.04 \\
4.54000 & 195.83 & 248.50 \\
5.40000 & 195.91 & 276.35 \\
6.39000 & 191.90 & 301.76 \\
7.39000 & 186.53 & 322.77 \\
8.52000 & 180.48 & 342.72 \\
9.95000 & 173.67 & 363.98 \\
12.00000 & 165.78 & 389.11 \\
14.20000 & 159.35 & 411.34 \\
21.30000 & 147.50 & 464.33 \\
28.40000 & 141.75 & 501.48 \\
42.60000 & 135.70 & 553.15 \\
71.00000 & 131.11 & 617.99 \\
142.00000 & 128.25 & 705.76 \\
284.00000 & 125.38 & 791.55
\end{tabular}

ATOMIC DISPLACEAENT CŔOSS-SECTIONS (BARNS) BY PAST ELECTRONS IN PB $=82 \quad A=207.210$

\begin{tabular}{rrr} 
TD $=40.0$ & EV & \multicolumn{2}{c}{ ET $=1.518873$ MEV } \\
PNERGY (NEV) & PRIMRY & CASCADE \\
1.53000 & 0.30 & 0.30 \\
1.54000 & 0.64 & 0.64 \\
1.57000 & 2.04 & 2.04 \\
1.62000 & 5.41 & 5.41 \\
1.68000 & 10.76 & 10.76 \\
1.76000 & 19.36 & 19.36 \\
1.85000 & 30.17 & 30.17 \\
1.97000 & 45.22 & 45.22 \\
2.12000 & 63.66 & 63.66 \\
2.35000 & 89.08 & 89.08 \\
2.65000 & 115.71 & 116.45 \\
3.03000 & 139.76 & 144.21 \\
3.49000 & 158.03 & 170.82 \\
4.10000 & 170.64 & 198.39 \\
4.86000 & 176.24 & 224.96 \\
5.77000 & 176.09 & 249.81 \\
6.83000 & 172.33 & 272.79 \\
7.89000 & 167.48 & 291.59 \\
9.11000 & 151.94 & 309.78 \\
10.60000 & 155.95 & 328.50 \\
12.90000 & 148.55 & 352.25 \\
15.10000 & 143.17 & 370.99 \\
22.70000 & 132.54 & 419.02 \\
30.30000 & 127.39 & 452.61 \\
45.50000 & 121.99 & 499.26 \\
75.90000 & 117.92 & 557.76 \\
151.00000 & 115.40 & 636.15
\end{tabular}

\begin{tabular}{rrr} 
TD=44.0 & \multicolumn{2}{c}{ ET $=1.611807$ MEV } \\
ENERGY (NEV) & PRIMART & CASCADE \\
1.62000 & 0.17 & 0.17 \\
1.64000 & 0.74 & 0.74 \\
1.67000 & 1.98 & 1.98 \\
1.72000 & 4.89 & 4.89 \\
1.78000 & 9.46 & 9.46 \\
1.86000 & 16.79 & 16.79 \\
1.96000 & 27.10 & 27.10 \\
2.09000 & 41.18 & 41.18 \\
2.25000 & 58.15 & 58.15 \\
2.49000 & 81.03 & 81.03 \\
2.82000 & 106.16 & 106.92 \\
3.22000 & 127.69 & 131.97 \\
3.70000 & 143.90 & 155.83 \\
4.35000 & 155.28 & 181.09 \\
5.15000 & 160.18 & 205.15 \\
6.12000 & 159.91 & 227.92 \\
7.25000 & 156.37 & 248.95 \\
8.38000 & 151.89 & 266.14 \\
9.67000 & 146.85 & 282.63 \\
11.20000 & 141.57 & 299.17 \\
13.70000 & 134.68 & 321.36 \\
16.10000 & 129.71 & 338.84 \\
24.10000 & 120.28 & 382.10 \\
32.20000 & 115.63 & 412.76 \\
48.30000 & 110.78 & 455.07 \\
80.50000 & 107.15 & 508.18 \\
161.00000 & 104.86 & 579.99
\end{tabular}

\begin{tabular}{rrr} 
TD=48.0 EV & \multicolumn{2}{c}{ ET $=1.700840$ HEV } \\
ENERGY (HEV) & PRIMARY & CASCADE \\
1.71000 & 0.16 & 0.16 \\
1.73000 & 0.63 & 0.63 \\
1.76000 & 1.67 & 1.67 \\
1.81000 & 4.12 & 4.12 \\
1.88000 & 8.73 & 8.73 \\
1.97000 & 15.99 & 15.99 \\
2.07000 & 25.04 & 25.04 \\
2.21000 & 38.30 & 38.30 \\
2.38000 & 54.03 & 54.03 \\
2.53000 & 74.79 & 74.80 \\
2.97000 & 97.37 & 98.08 \\
3.40000 & 117.49 & 121.59 \\
3.91000 & 132.31 & 143.63 \\
4.59000 & 142.47 & 166.62 \\
5.44000 & 146.82 & 188.86 \\
6.46000 & 146.44 & 209.68 \\
7.65000 & 143.11 & 228.95 \\
8.84000 & 138.97 & 244.70 \\
10.20000 & 134.33 & 259.84 \\
11.90000 & 129.26 & 275.75 \\
14.40000 & 123.31 & 295.03 \\
17.00000 & 118.64 & 311.53 \\
25.50000 & 110.05 & 351.43 \\
34.00000 & 105.86 & 379.34 \\
51.00000 & 101.46 & 418.14 \\
85.00000 & 98.18 & 466.84 \\
170.00000 & 96.09 & 532.67
\end{tabular}


ATOMIC DISPLACEMENT CROSS-SECTIONS (BARNS) BY FAST ELECTRONS IN PB $z=82 \quad A=207.210$

\begin{tabular}{rrr} 
TD $=52.0$ & EV & \multicolumn{2}{c}{ ET $=1.786426$ MEV } \\
ENERGY(HEV) & PRIMART & CASCADE \\
1.80000 & 0.20 & 0.20 \\
1.82000 & 0.63 & 0.63 \\
1.85000 & 1.55 & 1.55 \\
1.91000 & 4.21 & 4.21 \\
1.98000 & 8.36 & 8.36 \\
2.07000 & 14.81 & 14.81 \\
2.17000 & 22.80 & 22.80 \\
2.32000 & 35.37 & 35.37 \\
2.50000 & 50.10 & 50.10 \\
2.76000 & 69.17 & 69.17 \\
3.12000 & 90.24 & 90.93 \\
3.57000 & 108.72 & 112.62 \\
4.10000 & 122.23 & 132.84 \\
4.82000 & 131.61 & 154.31 \\
5.71000 & 135.50 & 174.82 \\
6.78000 & 135.06 & 194.06 \\
8.03000 & 131.93 & 211.89 \\
9.28000 & 128.07 & 226.46 \\
10.70000 & 123.80 & 240.39 \\
12.50000 & 119.08 & 255.22 \\
15.10000 & 113.65 & 272.88 \\
17.80000 & 109.40 & 287.99 \\
26.70000 & 101.50 & 324.86 \\
35.70000 & 97.62 & 350.89 \\
53.50000 & 93.59 & 386.63 \\
89.30000 & 90.59 & 431.73 \\
178.00000 & 88.68 & 492.20 \\
& &
\end{tabular}

\begin{tabular}{rrr} 
TD $=56.0$ & EV & \multicolumn{2}{c}{ ET=1.868935 HEV } \\
ENERGY (HEV) & PRIHARY & CASCADE \\
1.88000 & 0.13 & 0.13 \\
1.90000 & 0.48 & 0.48 \\
1.94000 & 1.56 & 1.56 \\
1.99000 & 3.50 & 3.50 \\
2.07000 & 7.65 & 7.65 \\
2.16000 & 13.37 & 13.37 \\
2.28000 & 21.93 & 21.93 \\
2.42000 & 32.41 & 32.41 \\
2.61000 & 46.31 & 46.31 \\
2.89000 & 64.62 & 64.63 \\
3.27000 & 84.34 & 85.03 \\
3.73000 & 101.08 & 104.75 \\
4.29000 & 113.71 & 123.78 \\
5.04000 & 122.29 & 143.67 \\
5.98000 & 125.82 & 162.92 \\
7.10000 & 125.30 & 180.80 \\
8.41000 & 122.32 & 197.38 \\
9.71000 & 118.73 & 210.83 \\
11.20000 & 114.75 & 223.81 \\
13.00000 & 110.54 & 237.03 \\
15.80000 & 105.34 & 253.98 \\
18.60000 & 101.45 & 267.90 \\
28.00000 & 94.11 & 302.47 \\
37.30000 & 90.58 & 326.34 \\
56.00000 & 86.84 & 359.68 \\
93.40000 & 84.09 & 401.52 \\
186.00000 & 82.33 & 457.59
\end{tabular}

\begin{tabular}{rrr} 
TD $=60.0$ & BV & \multicolumn{2}{c}{ ET $=1.948678$ MEV } \\
ENERGY (HEV) & PBIHARY & CASCADE \\
1.96000 & 0.12 & 0.12 \\
1.98000 & 0.41 & 0.41 \\
2.02000 & 1.35 & 1.35 \\
2.08000 & 3.44 & 3.44 \\
2.16000 & 7.20 & 7.20 \\
2.26000 & 12.96 & 12.96 \\
2.37000 & 20.03 & 20.03 \\
2.53000 & 30.80 & 30.80 \\
2.72000 & 43.29 & 43.29 \\
3.02000 & 60.89 & 60.90 \\
3.41000 & 78.98 & 79.66 \\
3.89000 & 94.58 & 98.11 \\
4.48000 & 106.40 & 116.06 \\
5.26000 & 114.24 & 134.58 \\
6.23000 & 117.42 & 152.38 \\
7.40000 & 116.87 & 169.13 \\
8.76000 & 114.06 & 184.57 \\
10.10000 & 110.72 & 197.02 \\
11.60000 & 107.11 & 208.79 \\
13.60000 & 102.92 & 221.98 \\
16.50000 & 98.13 & 237.66 \\
19.40000 & 94.54 & 250.56 \\
29.20000 & 87.75 & 282.84 \\
38.90000 & 84.47 & 305.12 \\
58.40000 & 81.00 & 336.25 \\
97.40000 & 78.46 & 375.30 \\
194.00000 & 76.83 & 427.65
\end{tabular}

ATONIC DISPLACENENT CROSS-SECTIONS (BARNS) BY FAST ELECTRONS IN PB $\mathrm{z}=82 \quad \mathrm{~A}=207.210$

\begin{tabular}{rrr}
$\begin{array}{c}\text { TD }=64.0 \\
\text { EV }\end{array}$ & \multicolumn{2}{c}{ ET $=2.025916$ MEV } \\
PRERG (MEV) & PRARY & CASCADE \\
2.04000 & 0.13 & 0.13 \\
2.06000 & 0.40 & 0.40 \\
2.10000 & 1.24 & 1.24 \\
2.16000 & 3.11 & 3.11 \\
2.24000 & 6.47 & 6.47 \\
2.35000 & 12.18 & 12.18 \\
2.47000 & 19.18 & 19.18 \\
2.63000 & 28.95 & 28.95 \\
2.83000 & 40.85 & 40.85 \\
3.14000 & 57.29 & 57.30 \\
3.54000 & 74.07 & 74.71 \\
4.05000 & 89.00 & 92.44 \\
4.65000 & 99.79 & 108.93 \\
5.46000 & 107.13 & 126.33 \\
6.48000 & 110.07 & 143.25 \\
7.69000 & 109.50 & 158.88 \\
9.11000 & 106.81 & 173.44 \\
10.50000 & 103.67 & 185.09 \\
12.10000 & 100.19 & 196.40 \\
14.10000 & 96.42 & 208.29 \\
17.20000 & 91.81 & 223.42 \\
20.20000 & 88.49 & 235.44 \\
30.30000 & 82.21 & 265.46 \\
40.50000 & 79.12 & 286.60 \\
60.70000 & 75.90 & 315.67 \\
101.00000 & 73.54 & 352.12 \\
202.00000 & 72.01 & 401.49 \\
& &
\end{tabular}

\begin{tabular}{rrr} 
TD=68.0 EV & \multicolumn{2}{c}{ ET=2.100871 TEV } \\
BNERG (MEV) & PRIMARY & CASCADE \\
2.12000 & 0.16 & 0.16 \\
2.14000 & 0.42 & 0.42 \\
2.18000 & 1.20 & 1.20 \\
2.24000 & 2.91 & 2.91 \\
2.33000 & 6.39 & 6.39 \\
2.43000 & 11.12 & 11.12 \\
2.56000 & 18.02 & 18.02 \\
2.73000 & 27.47 & 27.47 \\
2.94000 & 38.85 & 38.85 \\
3.25000 & 53.82 & 53.82 \\
3.67000 & 69.87 & 70.49 \\
4.20000 & 83.94 & 87.24 \\
4.83000 & 94.14 & 102.96 \\
5.67000 & 100.92 & 119.32 \\
6.72000 & 103.59 & 135.13 \\
7.98000 & 102.99 & 149.90 \\
9.45000 & 100.42 & 163.58 \\
10.90000 & 97.44 & 174.61 \\
12.60000 & 94.08 & 185.50 \\
14.70000 & 90.51 & 196.78 \\
17.80000 & 86.34 & 210.49 \\
21.00000 & 83.14 & 222.13 \\
31.50000 & 77.28 & 250.40 \\
42.00000 & 74.41 & 270.13 \\
63.00000 & 71.39 & 297.54 \\
105.00000 & 69.19 & 331.97 \\
210.00000 & 67.76 & 378.44 \\
& &
\end{tabular}

\begin{tabular}{rrr} 
TD=72.0 & \multicolumn{1}{c}{ ET $=2.173734$ MEV } \\
ENERGY (MEV) & PRIMARY & CASCADE \\
2.19000 & 0.12 & 0.12 \\
2.21000 & 0.33 & 0.33 \\
2.26000 & 1.22 & 1.22 \\
2.32000 & 2.80 & 2.80 \\
2.41000 & 5.99 & 5.99 \\
2.52000 & 10.77 & 10.77 \\
2.65000 & 17.11 & 17.11 \\
2.82000 & 25.78 & 25.78 \\
3.04000 & 36.71 & 36.71 \\
3.36000 & 50.87 & 50.87 \\
3.80000 & 66.23 & 66.85 \\
4.34000 & 79.32 & 82.46 \\
4.99000 & 88.94 & 97.32 \\
5.86000 & 95.34 & 112.84 \\
6.95000 & 97.82 & 127.84 \\
8.26000 & 97.21 & 141.88 \\
9.78000 & 94.75 & 154.79 \\
11.30000 & 91.88 & 165.34 \\
13.00000 & 88.82 & 175.29 \\
15.20000 & 85.39 & 186.10 \\
18.40000 & 81.47 & 199.05 \\
21.70000 & 78.46 & 210.01 \\
$\mathbf{3 2 . 6 0 0 0 0}$ & $\mathbf{7 2 . 9 3}$ & 236.83 \\
43.40000 & 70.25 & 255.37 \\
65.20000 & 67.40 & 281.36 \\
108.00000 & 65.35 & 313.49 \\
217.00000 & 63.99 & 357.67
\end{tabular}


ATOHIC DISPLACEMENT CROSS-SECTIONS (BARNS) BY PAST ELBCTBONS IN PB

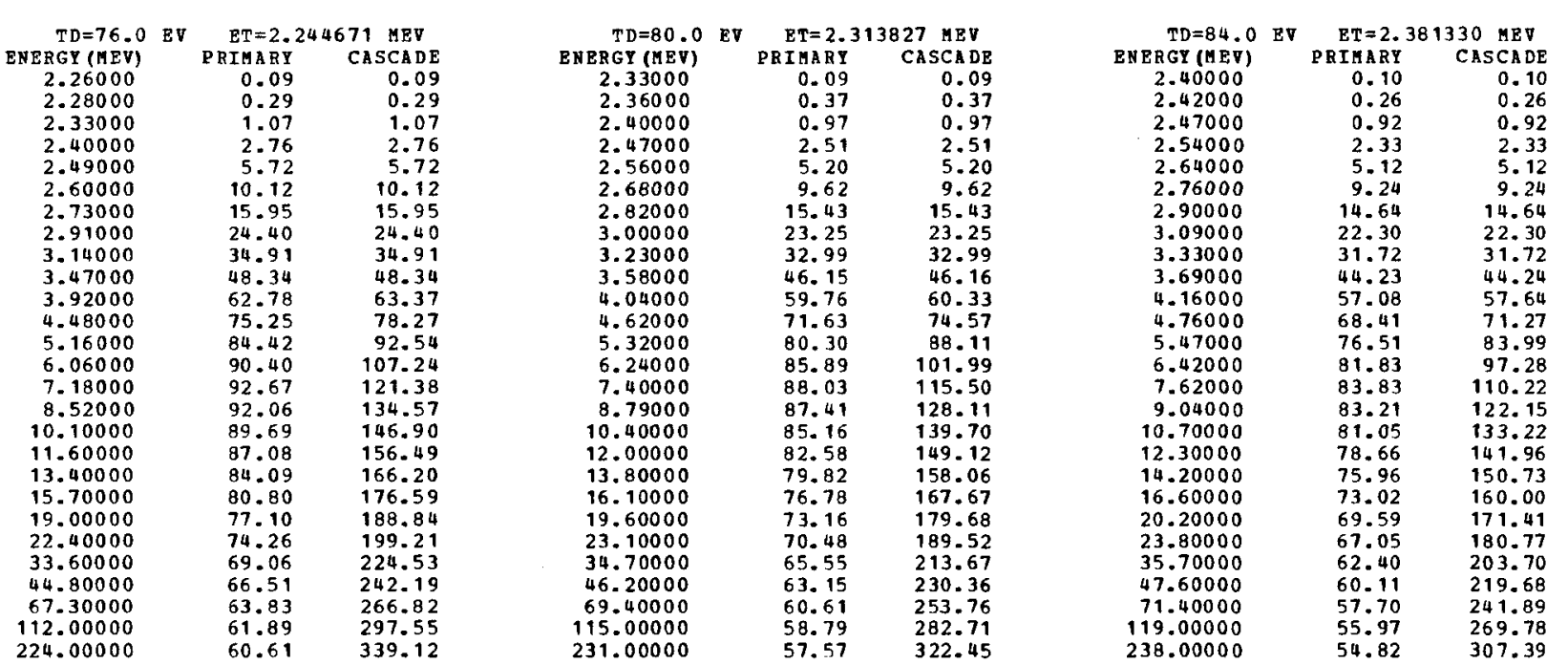

ATOMIC DISPLACEMENT CROSS-SECTIONS (BARNS) BY PAST ELECTRONS IN PB $Z=82 \quad A=207.210$

\begin{tabular}{rrr} 
TD=88.0 & EV & \multicolumn{1}{c}{ ET $=2.447294$} \\
ENERGY (MEV) & PRIMARY & CASCADE \\
2.47000 & 0.11 & 0.11 \\
2.49000 & 0.27 & 0.27 \\
2.54000 & 0.89 & 0.89 \\
2.61000 & 2.21 & 2.21 \\
2.71000 & 4.79 & 4.79 \\
2.83000 & 8.61 & 8.61 \\
2.98000 & 13.99 & 13.99 \\
3.18000 & 21.51 & 21.51 \\
3.42000 & 30.27 & 30.27 \\
3.79000 & 42.25 & 42.27 \\
4.28000 & 54.69 & 55.26 \\
4.89000 & 65.36 & 68.13 \\
5.62000 & 73.08 & 80.30 \\
6.60000 & 78.15 & 93.04 \\
7.83000 & 80.02 & 105.37 \\
9.29000 & 79.40 & 116.76 \\
11.00000 & 77.31 & 127.36 \\
12.70000 & 74.94 & 135.96 \\
14.60000 & 72.43 & 144.08 \\
17.10000 & 69.60 & 153.04 \\
20.80000 & 66.34 & 163.92 \\
24.40000 & 63.99 & 172.61 \\
36.70000 & 59.52 & 194.65 \\
48.90000 & 57.35 & 209.87 \\
73.40000 & 55.06 & 231.11 \\
122.00000 & 53.42 & 257.59 \\
244.00000 & 52.33 & 293.49 \\
& &
\end{tabular}

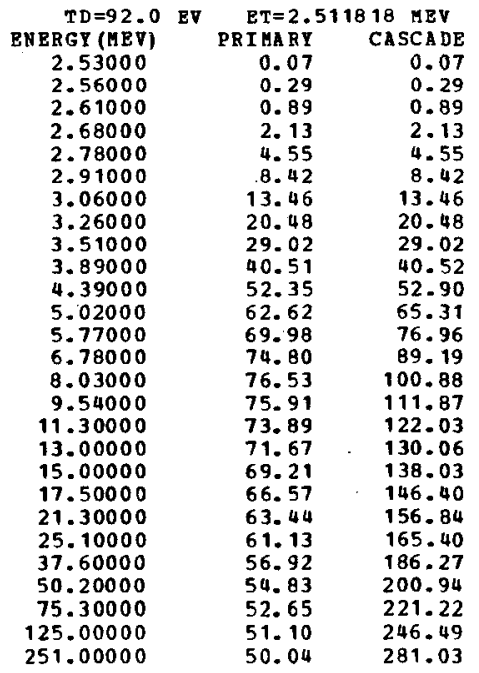

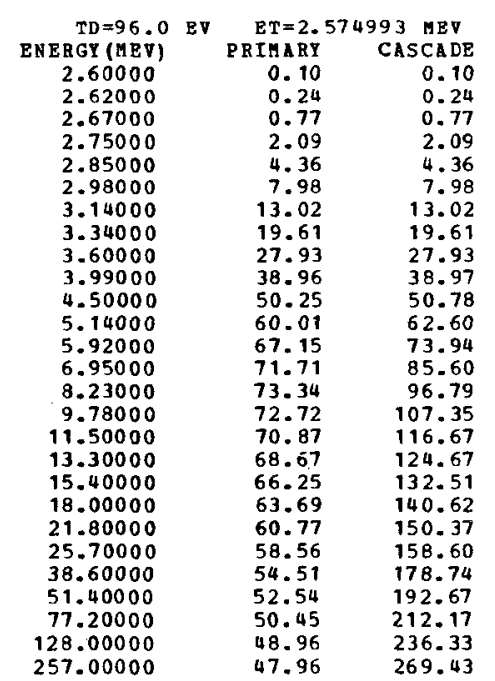


ATOMIC DISPLACEAENT CROSS-SECTIONS (BARNS) BY FAST BLECTRONS IN PA

\begin{tabular}{crr}
$T D=4.0$ & EV & \multicolumn{1}{c}{ ET $=0.311470$ MEV } \\
PNERGY (MEV) & PRIMARY & CASCADE \\
0.31400 & 24.61 & 24.61 \\
0.31700 & 53.48 & 53.48 \\
0.32300 & 110.22 & 110.22 \\
0.33300 & 201.93 & 201.93 \\
0.34500 & 307.42 & 307.42 \\
0.36100 & 440.60 & 440.60 \\
0.37900 & 580.59 & 580.59 \\
0.40400 & 758.55 & 758.55 \\
0.43600 & 960.24 & 960.24 \\
0.48200 & 1204.15 & 1204.15 \\
0.54500 & 1454.21 & 1464.42 \\
0.62200 & 1691.88 & 1707.20 \\
0.71600 & 1874.24 & 1936.07 \\
0.84000 & 2010.20 & 2166.97 \\
0.99600 & 2082.39 & 2388.37 \\
1.18000 & 2093.86 & 2590.84 \\
1.40000 & 2060.30 & 2783.52 \\
1.61000 & 2009.59 & 2935.77 \\
1.86000 & 1943.54 & 3090.11 \\
2.18000 & 1861.90 & 3258.69 \\
2.64000 & 1761.07 & 3461.85 \\
3.11000 & 1678.56 & 3637.44 \\
4.67000 & 1506.04 & 4080.77 \\
6.22000 & 1418.61 & 4402.04 \\
9.34000 & 1336.03 & 4868.18 \\
15.50000 & 1267.42 & 5453.15 \\
31.10000 & 1221.06 & 6269.69 \\
62.20000 & 1195.89 & 7081.34 \\
93.40000 & 1177.23 & 7547.72 \\
155.00000 & 1153.66 & 8120.70
\end{tabular}

\begin{tabular}{rrr} 
TD $=8.0$ & \multicolumn{3}{c}{ EV $=0.533888$ MEV } \\
ENERGY (MEV) & PRIMARY & CASCADE \\
0.53900 & 8.11 & 8.11 \\
0.54400 & 16.30 & 16.30 \\
0.55500 & 35.13 & 35.13 \\
0.57100 & 64.12 & 64.12 \\
0.59200 & 104.20 & 104.20 \\
0.61900 & 157.59 & 157.59 \\
0.65100 & 221.52 & 221.52 \\
0.69400 & 305.60 & 305.60 \\
0.74700 & 402.92 & 402.92 \\
0.82700 & 532.35 & 532.35 \\
0.93400 & 671.27 & 672.18 \\
1.06000 & 791.09 & 801.88 \\
1.22000 & 891.45 & 930.81 \\
1.44000 & 966.99 & 1066.70 \\
1.70000 & 1002.38 & 1189.53 \\
2.02000 & 1005.94 & 1307.01 \\
2.40000 & 985.72 & 1417.39 \\
2.77000 & 957.54 & 1505.45 \\
3.20000 & 923.21 & 1592.02 \\
3.73000 & 884.40 & 1682.22 \\
4.53000 & 835.93 & 1795.79 \\
5.33000 & 798.52 & 1890.18 \\
8.00000 & 723.73 & 2126.41 \\
10.60000 & 688.86 & 2292.54 \\
16.00000 & 653.66 & 2535.10 \\
26.60000 & 624.79 & 2833.81 \\
53.30000 & 607.12 & 3245.51 \\
106.00000 & 593.86 & 3646.83 \\
160.00000 & 583.98 & 3882.00 \\
266.00000 & 573.32 & 4168.60
\end{tabular}

\begin{tabular}{rrr} 
TD=12.0 EV & \multicolumn{2}{c}{ ET $=0.716651$ MEV } \\
ENERG (MEV) & PRIMARY & CASCADE \\
0.72300 & 3.59 & 3.59 \\
0.73000 & 7.85 & 7.85 \\
0.74500 & 17.96 & 17.96 \\
0.76600 & 33.98 & 33.98 \\
0.79500 & 58.75 & 58.75 \\
0.83100 & 92.24 & 92.24 \\
0.87400 & 134.15 & 134.15 \\
0.93100 & 189.97 & 189.97 \\
1.00000 & 254.67 & 254.57 \\
1.11000 & 346.45 & 346.45 \\
1.25000 & 440.34 & 441.34 \\
1.43000 & 527.38 & 537.22 \\
1.64000 & 592.53 & 624.49 \\
1.93000 & 640.56 & 717.41 \\
2.29000 & 661.94 & 805.40 \\
2.72000 & 660.93 & 886.76 \\
3.22000 & 645.25 & 961.64 \\
3.72000 & 624.99 & 1023.08 \\
4.29000 & 601.85 & 1082.05 \\
5.01000 & 575.67 & 1145.07 \\
6.09000 & 544.14 & 1223.28 \\
7.16000 & 520.52 & 1287.66 \\
10.70000 & 474.77 & 1446.79 \\
14.30000 & 453.17 & 1562.50 \\
21.40000 & 431.82 & 1722.09 \\
35.80000 & 414.18 & 1924.96 \\
71.60000 & 403.72 & 2199.42 \\
143.00000 & 394.41 & 2467.90 \\
214.00000 & 387.93 & 2621.05 \\
358.00000 & 381.39 & 2814.31
\end{tabular}

ATOMIC DISPLACEMENT CROSS-SECTIONS (BARNS) BY PAST ELECTRONS IN FR $z=87 \quad A=223.000$

\begin{tabular}{rrr} 
TD $=16.0$ & EV & \multicolumn{2}{c}{ ET $=0.875529 M \mathrm{MEV}$} \\
ENERGY (MEV) & PRIMARY & \multicolumn{1}{c}{ CASCADE } \\
0.88400 & 2.35 & 2.35 \\
0.89300 & 5.17 & 5.17 \\
0.91000 & 11.32 & 11.32 \\
0.93600 & 22.48 & 22.48 \\
0.97100 & 40.00 & 40.00 \\
1.01000 & 61.80 & 61.80 \\
1.06000 & 91.66 & 91.66 \\
1.13000 & 134.46 & 134.46 \\
1.22000 & 187.64 & 187.64 \\
1.35000 & 256.23 & 256.23 \\
1.53000 & 332.04 & 333.15 \\
1.75000 & 397.62 & 406.57 \\
2.01000 & 446.30 & 474.14 \\
2.36000 & 480.18 & 544.27 \\
2.80000 & 494.24 & 611.59 \\
3.32000 & 491.73 & 673.23 \\
3.93000 & 478.75 & 730.44 \\
4.55000 & 462.60 & 778.08 \\
5.25000 & 444.93 & 823.18 \\
6.12000 & 425.60 & 870.70 \\
7.44000 & 402.40 & 930.18 \\
8.75000 & 385.22 & 979.21 \\
13.10000 & 352.54 & 1100.44 \\
17.50000 & 337.34 & 1187.58 \\
26.20000 & 322.01 & 1307.83 \\
43.70000 & 309.71 & 1459.64 \\
87.50000 & 302.32 & 1656.03 \\
175.00000 & 295.12 & 1867.44
\end{tabular}

\begin{tabular}{|c|c|c|}
\hline $\mathrm{TD}=20.0$ & $E V$ & $M E V$ \\
\hline AERGY (MEV) & PRIMARY & CASCADE \\
\hline 1.02000 & 0.30 & 0.30 \\
\hline 9.03000 & 1.97 & 1.97 \\
\hline 1.05000 & 6.11 & 6.11 \\
\hline 1.08000 & 14.03 & 14.03 \\
\hline 1.12000 & 27.02 & 27.02 \\
\hline 1.18000 & 49.89 & 49.89 \\
\hline 1.24000 & 74.89 & 74.89 \\
\hline 1.32000 & 109.07 & 109.07 \\
\hline 1.42000 & 150.40 & 150.40 \\
\hline 1.57000 & 205.78 & 205.78 \\
\hline 1.78000 & 267.36 & 268.49 \\
\hline 2.03000 & 318.92 & 326.86 \\
\hline 2.34000 & 358.47 & 383.13 \\
\hline 2.74000 & 384.13 & 439.02 \\
\hline 3.25000 & 394.28 & 493.50 \\
\hline 3.86000 & 391.22 & 543.91 \\
\hline 4.58000 & 379.90 & 590.81 \\
\hline 5.29000 & 366.79 & 628.69 \\
\hline 6.10000 & 352.58 & 664.98 \\
\hline 7.12000 & 337.06 & 703.69 \\
\hline 8.65000 & 318.84 & 751.51 \\
\hline 10.10000 & 305.97 & 789.28 \\
\hline 15.20000 & 280.35 & 888.19 \\
\hline 20.30000 & 268.64 & 958.06 \\
\hline 30.50000 & 255.62 & 1055.31 \\
\hline 50.80000 & 247.28 & 1176.69 \\
\hline 101.00000 & 241.64 & 1340.21 \\
\hline 203.00000 & 235.74 & 1502.32 \\
\hline
\end{tabular}

\begin{tabular}{rrr} 
TD $=24.0$ & EV & \multicolumn{2}{c}{ ET $=1.148256$ MEV } \\
ENERGY (HEV) & PRI A ARY & CASCADE \\
1.15000 & 0.16 & 0.16 \\
1.17000 & 2.49 & 2.49 \\
1.19000 & 5.56 & 5.56 \\
1.22000 & 11.34 & 11.34 \\
1.27000 & 23.36 & 23.36 \\
1.33000 & 40.40 & 40.40 \\
1.40000 & 62.16 & 62.16 \\
1.49000 & 90.98 & 90.98 \\
1.60000 & 125.10 & 125.10 \\
1.77000 & 172.16 & 172.16 \\
2.00000 & 222.70 & 223.72 \\
2.29000 & 267.14 & 274.46 \\
2.64000 & 299.70 & 321.83 \\
3.10000 & 320.58 & 369.60 \\
3.67000 & 327.95 & 414.79 \\
4.36000 & 324.62 & 457.16 \\
5.16000 & 314.85 & 495.94 \\
5.97000 & 303.57 & 528.09 \\
6.88000 & 291.70 & 558.41 \\
8.03000 & 278.83 & 590.86 \\
9.76000 & 263.80 & 631.04 \\
11.40000 & 253.24 & 662.73 \\
17.20000 & 232.43 & 746.06 \\
22.90000 & 223.07 & 803.77 \\
34.40000 & 213.28 & 884.88 \\
57.40000 & 205.77 & 986.56 \\
114.00000 & 201.19 & 1122.64 \\
229.00000 & 196.22 & 1257.49
\end{tabular}


ATOMIC DISPLACEMENT CROSS-SECTIONS (BARNS) BY FAST ELECTRONS IN PR $Z=87 \quad A=223.000$

\begin{tabular}{rrr} 
TD $=28.0$ & EV & \multicolumn{2}{c}{ ET $=1.269019$} \\
ENERGY (MEV) & PRIMARY & CASCADE \\
1.28000 & 0.78 & 0.78 \\
1.29000 & 1.65 & 1.65 \\
1.31000 & 3.80 & 3.80 \\
1.35000 & 9.55 & 9.55 \\
1.40000 & 18.78 & 18.78 \\
1.47000 & 34.20 & 34.20 \\
1.54000 & 51.21 & 51.21 \\
1.64000 & 76.35 & 76.35 \\
1.77000 & 108.05 & 108.05 \\
1.96000 & 149.18 & 149.18 \\
2.22000 & 193.41 & 194.50 \\
2.53000 & 229.97 & 235.73 \\
2.91000 & 257.23 & 277.01 \\
3.42000 & 274.84 & 318.49 \\
4.06000 & 280.69 & 358.09 \\
4.82000 & 277.33 & 394.44 \\
5.71000 & 268.56 & 428.04 \\
6.59000 & 258.92 & 455.25 \\
7.61000 & 248.55 & 481.76 \\
8.88000 & 237.58 & 509.64 \\
10.70000 & 225.36 & 542.70 \\
12.60000 & 215.95 & 571.38 \\
19.00000 & 198.55 & 642.96 \\
25.30000 & 190.69 & 692.52 \\
38.00000 & 182.44 & 762.08 \\
63.40000 & 176.19 & 849.31 \\
126.00000 & 172.34 & 965.08 \\
253.00000 & 168.03 & 1081.51
\end{tabular}

\begin{tabular}{crr} 
TD=32.0 EV & \multicolumn{2}{c}{ ET=1.382093 MEV } \\
ENERGY (MEV) & PRIMARY & CASCADE \\
1.39000 & 0.39 & 0.39 \\
1.40000 & 0.99 & 0.99 \\
1.43000 & 3.45 & 3.45 \\
1.47000 & 8.03 & 8.03 \\
1.53000 & 16.98 & 16.98 \\
1.60000 & 29.49 & 29.49 \\
1.68000 & 45.27 & 45.27 \\
1.79000 & 67.73 & 67.73 \\
1.93000 & 95.41 & 95.41 \\
2.14000 & 132.13 & 132.12 \\
2.41000 & 169.23 & 170.23 \\
2.76000 & 202.42 & 208.83 \\
3.17000 & 225.69 & 243.86 \\
3.73000 & 240.73 & 280.57 \\
4.42000 & 245.31 & 314.99 \\
5.25000 & 242.01 & $3: 17.02$ \\
6.21000 & 234.19 & 376.27 \\
7.18000 & 225.56 & 400.46 \\
8.29000 & 215.46 & 423.72 \\
9.67000 & 206.92 & 448.14 \\
11.70000 & 196.11 & 477.79 \\
13.80000 & 187.92 & 503.19 \\
20.70000 & 173.24 & 565.18 \\
27.60000 & 166.45 & 608.79 \\
41.40000 & 159.37 & 669.50 \\
69.10000 & 154.04 & 745.95 \\
138.00000 & 150.69 & 848.84 \\
276.00000 & 146.90 & 949.20
\end{tabular}

\begin{tabular}{rrr} 
TD $=36.0$ & \multicolumn{1}{c}{ EV } & \multicolumn{2}{c}{ ET $=1.488784$ HEV } \\
ENERGY (MEV) & PRIMARY & CASCADE \\
1.50000 & 0.43 & 0.43 \\
1.51000 & 0.92 & 0.92 \\
1.54000 & 2.90 & 2.90 \\
1.59000 & 7.65 & 7.65 \\
1.65000 & 15.15 & 15.15 \\
1.72000 & 25.53 & 25.53 \\
1.81000 & 40.27 & 40.27 \\
1.93000 & 60.69 & 60.69 \\
2.08000 & 85.37 & 85.37 \\
2.30000 & 117.39 & 117.39 \\
2.60000 & 151.56 & 152.56 \\
2.97000 & 180.41 & 186.38 \\
3.42000 & 201.25 & 218.22 \\
4.01000 & 214.01 & 250.26 \\
4.76000 & 217.84 & 281.28 \\
5.65000 & 214.66 & 309.72 \\
6.69000 & 207.51 & 335.97 \\
7.74000 & 199.74 & 357.62 \\
8.93000 & 191.67 & 378.25 \\
10.40000 & 183.30 & 399.78 \\
12.60000 & 173.69 & 426.40 \\
14.80000 & 166.65 & 448.45 \\
22.30000 & 153.64 & 504.28 \\
29.70000 & 147.71 & 542.93 \\
44.60000 & 141.47 & 597.07 \\
74.40000 & 136.83 & 665.01 \\
148.00000 & 133.90 & 755.95 \\
297.00000 & 130.50 & 845.55
\end{tabular}

ATOMIC DISPLACEMENT CROSS-SECTIONS (BARNS) BY PAST ELECTRONS IN FR $\mathrm{z}=87 \quad \mathrm{~A}=223.000$

\begin{tabular}{rrr} 
TD & \\
ENE.0 & EV & \multicolumn{2}{c}{ ET $=1.590065$ MEV } \\
PRIMEV) & PRIMRY & CASCADE \\
1.60000 & 0.29 & 0.29 \\
1.62000 & 1.11 & 1.11 \\
1.65000 & 2.85 & 2.85 \\
1.70000 & 6.92 & 6.92 \\
1.76000 & 13.25 & 13.25 \\
1.84000 & 23.30 & 23.30 \\
1.93000 & 35.83 & 35.83 \\
2.06000 & 54.62 & 54.62 \\
2.22000 & 76.99 & 76.99 \\
2.46000 & 106.58 & 106.58 \\
2.78000 & 137.27 & 138.26 \\
3.18000 & 163.33 & 169.09 \\
3.65000 & 181.41 & 197.16 \\
4.29000 & 192.79 & 226.50 \\
5.08000 & 195.90 & 254.08 \\
6.04000 & 192.80 & 279.99 \\
7.15000 & 186.23 & 303.63 \\
8.26000 & 179.26 & 322.95 \\
9.54000 & 171.93 & 341.71 \\
11.10000 & 164.47 & 361.00 \\
13.50000 & 155.70 & 385.49 \\
15.90000 & 149.35 & 405.69 \\
23.80000 & 138.03 & 455.21 \\
31.80000 & 132.70 & 490.41 \\
47.70000 & 127.17 & 539.02 \\
79.50000 & 123.08 & 600.09 \\
159.00000 & 120.44 & 682.57
\end{tabular}

\begin{tabular}{ccc} 
TD=44.0 & \multicolumn{2}{c}{ ET $=1.686682$ MEV } \\
ENERGY (NEV) & PRIMARY & CASCADE \\
1.70000 & 0.32 & 0.32 \\
1.72000 & 1.02 & 1.02 \\
1.75000 & 2.50 & 2.50 \\
1.80000 & 5.93 & 5.93 \\
1.87000 & 12.26 & 12.26 \\
1.95000 & 20.93 & 20.93 \\
2.05000 & 32.95 & 32.95 \\
2.19000 & 50.43 & 50.43 \\
2.36000 & 70.90 & 70.90 \\
2.61000 & 97.40 & 97.40 \\
2.95000 & 125.37 & 126.34 \\
3.37000 & 148.79 & 154.19 \\
3.87000 & 165.18 & 179.88 \\
4.55000 & 175.34 & 206.67 \\
5.39000 & 177.96 & 231.86 \\
6.40000 & 175.01 & 255.27 \\
7.59000 & 168.87 & 277.04 \\
8.77000 & 162.49 & 294.66 \\
10.10000 & 155.95 & 311.40 \\
11.80000 & 149.00 & 329.45 \\
14.30000 & 141.22 & 351.34 \\
16.80000 & 135.57 & 369.44 \\
25.30000 & 125.23 & 415.20 \\
33.70000 & 120.49 & 446.87 \\
50.60000 & 115.50 & 491.19 \\
84.30000 & 111.84 & 546.70 \\
168.00000 & 109.47 & 621.29
\end{tabular}

\begin{tabular}{|c|c|c|}
\hline $\mathrm{TD}=48.0$ & $\mathrm{ET}=1$. & $228 \mathrm{MEV}$ \\
\hline ENERGY (MEY) & PRIMARY & CASCADE \\
\hline 1.79000 & 0.20 & 0.20 \\
\hline 1.81000 & 0.75 & 0.75 \\
\hline 1.85000 & 2.44 & 2.44 \\
\hline 1.90000 & 5.45 & 5.45 \\
\hline 1.97000 & 10.95 & 10.95 \\
\hline 2.06000 & 19.48 & 19.48 \\
\hline 2.17000 & 31.08 & 31.08 \\
\hline 2.31000 & 46.37 & 46.37 \\
\hline 2.49000 & 65.32 & 65.32 \\
\hline 2.75000 & 89.40 & 89.40 \\
\hline 3.11000 & 115.24 & 116.17 \\
\hline 3.55000 & 136.58 & 149.64 \\
\hline 4.09000 & 151.82 & 165.80 \\
\hline 4.80000 & 160.80 & 190.11 \\
\hline 5.69000 & 163.02 & 213.32 \\
\hline 6.76000 & 160.15 & 234.88 \\
\hline 8.00000 & 154.52 & 254.61 \\
\hline 9.25000 & 148.60 & 270.86 \\
\hline 10.60000 & 142.82 & 285.70 \\
\hline 12.40000 & 136.40 & 302.41 \\
\hline 15.10000 & 129.12 & 323.02 \\
\hline 17.70000 & 124.05 & 339.40 \\
\hline 26.60000 & 114.69 & 381.18 \\
\hline 35.50000 & 110.34 & 410.42 \\
\hline 53.30000 & 105.80 & 451.06 \\
\hline 88.90000 & 102.48 & 502.07 \\
\hline 77.00000 & 100.32 & .35 \\
\hline
\end{tabular}


ATOMIC DISPLACEMENT CROSS-SECTIONS (BARNS) BY FAST ELECTRONS IN FR

\begin{tabular}{rrr} 
TD $=52.0$ & \multicolumn{1}{c}{ ET $=1.868176$ MRV } \\
ENERGY (MEV) & PRTMARY & CASCADE \\
1.88000 & 0.19 & 0.19 \\
1.90000 & 0.65 & 0.65 \\
1.94000 & 2.09 & 2.09 \\
1.99000 & 4.68 & 4.68 \\
2.07000 & 10.18 & 10.18 \\
2.16000 & 17.71 & 17.71 \\
2.27000 & 27.94 & 27.94 \\
2.42000 & 42.43 & 42.43 \\
2.61000 & 60.15 & 60.15 \\
2.89000 & 83.04 & 83.05 \\
3.26000 & 106.45 & 107.32 \\
3.73000 & 126.47 & 131.32 \\
4.29000 & 140.27 & 153.39 \\
5.04000 & 148.49 & 176.02 \\
5.97000 & 150.40 & 197.39 \\
7.09000 & 147.67 & 217.28 \\
8.40000 & 142.37 & 235.65 \\
9.71000 & 136.90 & 250.64 \\
11.20000 & 131.30 & 265.01 \\
13.00000 & 125.69 & 279.67 \\
15.80000 & 119.05 & 298.53 \\
18.60000 & 114.27 & 314.07 \\
28.00000 & 105.70 & 352.84 \\
37.30000 & 101.75 & 379.67 \\
56.00000 & 97.58 & 417.19 \\
93.40000 & 94.56 & 464.29 \\
186.00000 & 92.58 & 527.34 \\
& &
\end{tabular}

\begin{tabular}{crr} 
TD $=56.0$ & EV & \multicolumn{1}{c}{ ET=1.953916 MEV } \\
ENERGY (UEV) & PRIMARY & CASCADE \\
1.97000 & 0.23 & 0.23 \\
1.99000 & 0.66 & 0.66 \\
2.03000 & 1.94 & 1.94 \\
2.09000 & 4.77 & 4.77 \\
2.16000 & 9.09 & 9.09 \\
2.26000 & 16.52 & 16.52 \\
2.38000 & 26.50 & 26.50 \\
2.54000 & 40.30 & 40.30 \\
2.73000 & 56.08 & 56.08 \\
3.02000 & 77.22 & 77.23 \\
3.41000 & 99.17 & 100.03 \\
3.90000 & 117.67 & 122.29 \\
4.49000 & 130.47 & 142.96 \\
5.27000 & 137.92 & 163.88 \\
6.25000 & 139.58 & 183.88 \\
7.42000 & 136.95 & 202.32 \\
8.79000 & 131.97 & 219.38 \\
10.10000 & 127.09 & 232.74 \\
11.70000 & 121.73 & 246.52 \\
13.60000 & 116.47 & 260.28 \\
16.60000 & 110.22 & 278.19 \\
19.50000 & 105.87 & 292.44 \\
29.30000 & 98.03 & 328.31 \\
39.00000 & 94.40 & 353.16 \\
58.60000 & 90.55 & 388.07 \\
97.60000 & 87.78 & 431.70 \\
195.00000 & 85.94 & 490.52
\end{tabular}

\begin{tabular}{|c|c|c|}
\hline $\mathrm{TD}=60.0$ & $\mathrm{ET}=2$ & $M E V$ \\
\hline NERGY (MEV) & PRIMARY & CASCADE \\
\hline 2.05000 & 0.15 & 0.15 \\
\hline 2.07000 & 0.50 & 0.50 \\
\hline 2.11000 & 1.58 & 1.58 \\
\hline 2.17000 & 4.00 & 4.00 \\
\hline 2.26000 & 8.98 & 8.98 \\
\hline 2.36000 & 15.73 & 15.73 \\
\hline 2.48000 & 24.72 & 24.72 \\
\hline 2.64000 & 37.15 & 37.15 \\
\hline 2.85000 & 52.84 & 52.84 \\
\hline 3.15000 & 72.44 & 72.45 \\
\hline 3.56000 & 93.06 & 93.91 \\
\hline 4.07000 & 110.17 & 114.64 \\
\hline 4.68000 & 121.92 & 133.78 \\
\hline 5.49000 & 128.75 & 153.26 \\
\hline 6.51000 & 130.22 & 171.94 \\
\hline 7.73000 & 127.69 & 189.19 \\
\hline 9.16000 & 123.00 & 205.16 \\
\hline 10.50000 & 118.51 & 217.44 \\
\hline 12.20000 & 113.41 & 230.57 \\
\hline 14.20000 & 108.47 & 243.54 \\
\hline 17.30000 & 102.71 & 260.12 \\
\hline 20.30000 & 98.70 & 273.33 \\
\hline 30.50000 & 91.43 & 306.83 \\
\hline 40.70000 & 88.02 & 330.24 \\
\hline 61.10000 & 84.46 & 362.75 \\
\hline 101.00000 & 81.93 & 402.89 \\
\hline .00000 & 80.19 & 458.26 \\
\hline
\end{tabular}

ATOMIC DISPLACEMENT CROSS-SECTIONS (BARNS) BY FAST ELECTRONS IN FR $\mathrm{Z}=87 \quad \mathrm{~A}=223.000$

\begin{tabular}{rrr} 
TD $=64.0$ & EV & \multicolumn{2}{c}{ ET $=2.117019$ MEV } \\
ENERGY (MEV) & PRIMARY & CASCADE \\
2.13000 & 0.13 & 0.13 \\
2.15000 & 0.43 & 0.43 \\
2.20000 & 1.67 & 1.67 \\
2.26000 & 3.92 & 3.92 \\
2.34000 & 7.90 & 7.90 \\
2.45000 & 14.57 & 14.57 \\
2.58000 & 23.39 & 23.39 \\
2.75000 & 35.35 & 35.35 \\
2.96000 & 49.56 & 49.56 \\
3.28000 & 68.44 & 68.46 \\
3.70000 & 87.46 & 88.29 \\
4.23000 & 103.48 & 107.76 \\
4.86000 & 114.37 & 125.63 \\
5.71000 & 120.76 & 144.09 \\
6.77000 & 122.03 & 161.60 \\
8.04000 & 119.58 & 177.79 \\
9.52000 & 115.16 & 192.71 \\
11.00000 & 110.68 & 204.88 \\
12.70000 & 106.10 & 216.68 \\
14.80000 & 101.45 & 228.94 \\
17.90000 & 96.26 & 243.92 \\
21.10000 & 92.41 & 256.67 \\
31.70000 & 85.64 & 288.09 \\
42.30000 & 82.47 & 310.04 \\
63.50000 & 79.14 & 340.53 \\
105.00000 & 76.79 & 378.18 \\
211.00000 & 75.17 & 430.08 \\
& &
\end{tabular}

\begin{tabular}{|c|c|c|}
\hline$T D=68.0$ & $E \mathrm{~T}=2.1$ & $86 \mathrm{MEV}$ \\
\hline ENERGY (NEV) & PRIMARY & CASCADE \\
\hline 2.21000 & 0.13 & 0.13 \\
\hline 2.23000 & 0.41 & 0.41 \\
\hline 2.28000 & 1.52 & 1.52 \\
\hline 2.34000 & 3.55 & 3.55 \\
\hline 2.43000 & 7.62 & 7.62 \\
\hline 2.54000 & 13.72 & 13.72 \\
\hline 2.67000 & 21.76 & 21.76 \\
\hline 2.85000 & 33.30 & 33.30 \\
\hline 3.07000 & 46.86 & 46.86 \\
\hline 3.40000 & 64.58 & 64.59 \\
\hline 3.84000 & 82.66 & 83.48 \\
\hline 4.38000 & 97.44 & 101.51 \\
\hline 5.04000 & 107.77 & 118.54 \\
\hline 5.92000 & 113.68 & 135.90 \\
\hline 7.02000 & 114.80 & 152.41 \\
\hline 8.34000 & 112.44 & 167.69 \\
\hline 9.87000 & 108.25 & 181.69 \\
\hline 11.40000 & 104.04 & 193.13 \\
\hline 13.10000 & 99.87 & 203.88 \\
\hline 15.30000 & 95.42 & 215.60 \\
\hline 18.60000 & 90.43 & 230.09 \\
\hline 21.90000 & 86.85 & 242.02 \\
\hline 32.90000 & 80.52 & 271.60 \\
\hline 43.80000 & 77.58 & 292.10 \\
\hline 65.80000 & 74.46 & 320.85 \\
\hline 109.00000 & 72.25 & 356.43 \\
\hline 219.00000 & 70.73 & 405.25 \\
\hline
\end{tabular}

\begin{tabular}{|c|c|c|}
\hline$T D=72.0$ & $\mathrm{ET}=2.2$ & $M E V$ \\
\hline NERGY (MEV) & PRIMARY & CASCADE \\
\hline 2.29000 & 0.16 & 0.16 \\
\hline 2.31000 & 0.42 & 0.42 \\
\hline 2.36000 & 1.46 & 1.46 \\
\hline 2.42000 & 3.31 & 3.31 \\
\hline 2.52000 & 7.48 & 7.48 \\
\hline 2.63000 & 13.10 & 13.10 \\
\hline 2.77000 & 21.06 & 21.06 \\
\hline 2.95000 & 31.65 & 31.65 \\
\hline 3.17000 & 44.07 & 44.07 \\
\hline 3.51000 & 60.84 & 60.86 \\
\hline 3.97000 & 78.17 & 78.96 \\
\hline 4.54000 & 92.35 & 96.34 \\
\hline 5.22000 & 101.94 & 112.33 \\
\hline 6.13000 & 107.41 & 128.70 \\
\hline 7.26000 & 108.39 & 144.18 \\
\hline 8.62000 & 106.12 & 158.57 \\
\hline 10.20000 & 102.15 & 171.80 \\
\hline 11.80000 & 98.12 & 182.73 \\
\hline 13.60000 & 94.10 & 193.11 \\
\hline 15.80000 & 90.05 & 203.80 \\
\hline 19.20000 & 85.35 & 217.47 \\
\hline 22.70000 & 81.90 & 229.02 \\
\hline 34.00000 & 76.01 & 256.77 \\
\hline 45.40000 & 73.21 & 276.34 \\
\hline 68.10000 & 70.29 & 303.39 \\
\hline 113.00000 & 68.22 & 337.11 \\
\hline 227.00000 & 66.79 & 383.21 \\
\hline
\end{tabular}


A TOMIC DISPLACEMENT CROSS-SECTIONS (BARNS) BY FAST ELECTRONS IN ER $\mathrm{Z}=87 \quad \mathrm{~A}=223.000$

\begin{tabular}{rrr} 
TD $=76.0$ & EV & \multicolumn{1}{c}{ ST $=2.344256$} \\
PNERGY (MEV) & PRIMARY & CASCADE \\
2.36000 & 0.11 & 0.11 \\
2.39000 & 0.46 & 0.46 \\
2.43000 & 1.22 & 1.22 \\
2.50000 & 3.17 & 3.17 \\
2.60000 & 7.01 & 7.01 \\
2.71000 & 12.16 & 12.16 \\
2.85000 & 19.47 & 19.47 \\
3.04000 & 29.76 & 29.76 \\
3.28000 & 42.23 & 42.23 \\
3.63000 & 58.04 & 58.06 \\
4.10000 & 74.25 & 75.02 \\
4.68000 & 87.50 & 91.28 \\
5.39000 & 96.66 & 106.64 \\
5.32000 & 101.76 & 122.02 \\
7.50000 & 102.64 & 136.87 \\
8.90000 & 100.46 & 150.47 \\
10.50000 & 96.75 & 162.79 \\
12.10000 & 93.03 & 172.89 \\
14.00000 & 89.11 & 183.00 \\
16.40000 & 85.08 & 193.69 \\
19.90000 & 80.68 & 206.55 \\
23.40000 & 77.54 & 217.14 \\
35.10000 & 71.96 & 243.54 \\
45.80000 & 69.33 & 261.99 \\
70.30000 & 66.56 & 287.71 \\
117.00000 & 64.61 & $\mathbf{3 1 9 . 8 5}$ \\
234.00000 & 63.26 & $\mathbf{3 6 3 . 2 4}$ \\
& &
\end{tabular}

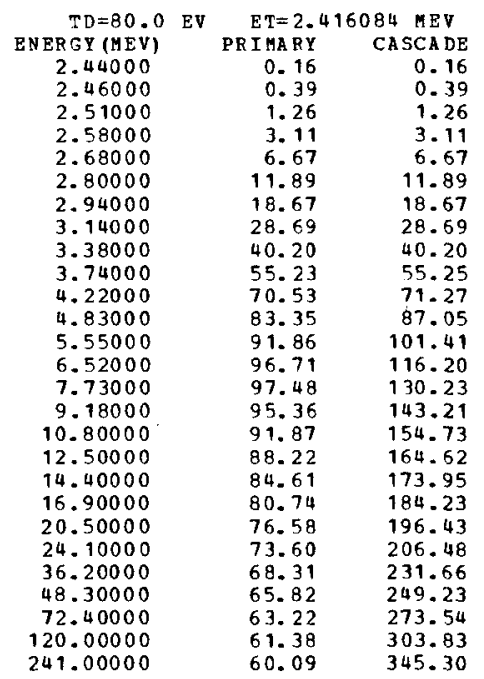

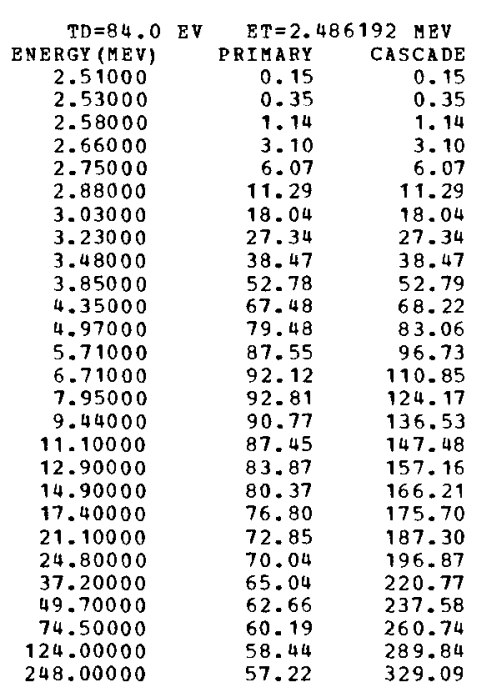

ATOMIC DISPLACEMENT CROSS-SECTIONS (BARNS) BY PAST ELECTRONS IN FR $\mathrm{Z}=87 \quad \mathrm{~A}=223.000$

\begin{tabular}{rrr} 
TD=88.0 & EV & \multicolumn{1}{c}{ ET $=2.554696$} \\
ENERGY MEV \\
2.58000 & PRIMARY & CASCADE \\
2.60000 & 0.14 & 0.14 \\
2.65000 & 0.33 & 0.33 \\
2.73000 & 1.06 & 1.06 \\
2.83000 & 2.86 & 2.86 \\
2.96000 & 5.94 & 5.94 \\
3.11000 & 10.82 & 10.82 \\
3.32000 & 17.10 & 17.10 \\
3.57000 & 26.20 & 26.20 \\
3.95000 & 36.58 & 36.58 \\
4.47000 & 50.29 & 50.31 \\
5.10000 & 64.53 & 65.26 \\
5.87000 & 75.87 & 79.29 \\
6.89000 & 83.65 & 92.52 \\
8.17000 & 87.94 & 105.91 \\
9.70000 & 88.57 & 118.70 \\
11.40000 & 86.59 & 130.49 \\
13.20000 & 83.41 & 140.91 \\
15.30000 & 80.08 & 149.94 \\
17.80000 & 76.66 & 158.79 \\
21.70000 & 73.33 & 167.64 \\
25.50000 & 59.47 & 179.03 \\
38.30000 & 56.79 & 188.15 \\
51.00000 & 52.03 & 217.05 \\
76.60000 & 59.79 & 226.91 \\
127.00000 & 57.43 & 249.13 \\
& 55.78 & 276.69
\end{tabular}

\begin{tabular}{rrr} 
TD=92.0 & EV & \multicolumn{1}{c}{ ET=2.621703 MEV } \\
ENERGY (HEV) & PRIMARY & CASCADE \\
2.64000 & 0.08 & 0.08 \\
2.67000 & 0.33 & 0.33 \\
2.72000 & 1.01 & 1.01 \\
2.80000 & 2.69 & 2.69 \\
2.91000 & 5.87 & 5.87 \\
3.04000 & 10.45 & 10.45 \\
3.19000 & 16.33 & 16.33 \\
3.40000 & 24.84 & 24.84 \\
3.67000 & 35.31 & 35.31 \\
4.06000 & 48.40 & 48.42 \\
4.58000 & 61.68 & 62.37 \\
5.24000 & 72.74 & 76.09 \\
6.02000 & 80.04 & 88.58 \\
7.07000 & 84.13 & 101.44 \\
8.38000 & 84.70 & 113.55 \\
9.96000 & 82.77 & 125.01 \\
11.70000 & 79.72 & 134.94 \\
13.60000 & 76.44 & 143.81 \\
15.70000 & 73.26 & 152.04 \\
18.30000 & 70.04 & 160.61 \\
22.20000 & 66.44 & 171.24 \\
26.20000 & 63.82 & 180.20 \\
39.30000 & 59.30 & 202.04 \\
52.40000 & 57.16 & 217.29 \\
78.60000 & 54.92 & 238.47 \\
131.00000 & 53.34 & 265.11
\end{tabular}

\begin{tabular}{rrr} 
TD $=96.0$ & EV & \multicolumn{2}{c}{ ET $=2.687306$} \\
ENERGY (MBV) & PRIMARY & CASCADE \\
2.71000 & 0.10 & 0.10 \\
2.74000 & 0.34 & 0.34 \\
2.79000 & 1.00 & 1.00 \\
2.87000 & 2.57 & 2.57 \\
2.98000 & 5.54 & 5.54 \\
3.11000 & 9.82 & 9.82 \\
3.27000 & 15.69 & 15.69 \\
3.49000 & 24.04 & 24.04 \\
3.76000 & 33.85 & 33.85 \\
4.16000 & 46.43 & 46.45 \\
4.70000 & 59.31 & 59.99 \\
5.37000 & 69.77 & 73.01 \\
5.18000 & 76.81 & 85.14 \\
7.25000 & 80.64 & 97.38 \\
8.59000 & 81.15 & 109.05 \\
10.20000 & 79.29 & 119.88 \\
12.00000 & 76.33 & 129.49 \\
13.90000 & 73.26 & 137.80 \\
16.10000 & 70.14 & 145.88 \\
18.80000 & 67.03 & 154.19 \\
22.80000 & 63.59 & 164.36 \\
26.80000 & 61.15 & 172.74 \\
40.30000 & 56.80 & 193.81 \\
53.70000 & 54.76 & 208.39 \\
80.60000 & 52.61 & 228.72 \\
134.00000 & 51.11 & 254.13
\end{tabular}


ATOMIC DISPLACBMENT CROSS-SECTIONS (BARNS) BY PAST ELECTRONS IN U
$\mathrm{Z}=92 \quad \mathrm{~A}=238.000$

\begin{tabular}{|c|c|c|c|}
\hline $\begin{array}{r}T D=4 . \\
\text { ENERGY (MEV }\end{array}$ & EV & $\begin{array}{r}\text { ET }=0 \\
\text { PRIMARY }\end{array}$ & CASCADE \\
\hline 0.33100 & & 28.67 & 28.67 \\
\hline 0.33400 & & 60.04 & 60.04 \\
\hline 0.34100 & & 132.16 & 132.16 \\
\hline 0.35100 & & 232.58 & 232.58 \\
\hline 0.36400 & & 358.46 & 358.46 \\
\hline 0.38000 & & 506.05 & 506.05 \\
\hline 0.40000 & & 679.02 & 679.02 \\
\hline 0.42600 & & 884.94 & 884.94 \\
\hline 0.45900 & & 1116.55 & 1116.55 \\
\hline 0.50800 & & 1404.15 & 1404.15 \\
\hline 0.57400 & & 1701.04 & 1701.42 \\
\hline 0.65600 & & 1957.77 & 1977.26 \\
\hline 0.75500 & & 2151.25 & 2228.20 \\
\hline 0.88600 & & 2283.00 & 2476.40 \\
\hline 1.05000 & & 2335.42 & 2708.54 \\
\hline 1.24000 & & 2318.99 & 2914.56 \\
\hline 1.47000 & & 2253.67 & 3111.89 \\
\hline 1.70000 & & 2173.31 & 3274.23 \\
\hline 1. 96000 & & 2083.05 & 3430.32 \\
\hline 2.29000 & & 1979.13 & 3600.12 \\
\hline 2.79000 & & 1851.18 & 3816.65 \\
\hline 3.28000 & & 1755.36 & 3996.44 \\
\hline 4.92000 & & $1564 \cdot 20$ & 4457.87 \\
\hline 6.56000 & & 1473.08 & $\begin{array}{l}4796.44 \\
5780.76\end{array}$ \\
\hline $\begin{array}{r}9.84000 \\
16.40000\end{array}$ & & $\begin{array}{l}1391.08 \\
1322.02\end{array}$ & $\begin{array}{l}5284.76 \\
5903.74\end{array}$ \\
\hline 32.80000 & & 1276.79 & 6756.29 \\
\hline 65.60000 & & 1248.52 & 7603.52 \\
\hline $\begin{array}{r}98.40000 \\
164.00000\end{array}$ & & $\begin{array}{l}1226.37 \\
1200.12\end{array}$ & $\begin{array}{l}8086.85 \\
8687.63\end{array}$ \\
\hline 164.00000 & & $1200 \cdot 12$ & \\
\hline
\end{tabular}

\begin{tabular}{rrr}
$\begin{array}{c}\text { TD } \\
\text { ENERGY }\end{array}$ EV & \multicolumn{2}{c}{ ET $=0.560292$ HEV } \\
0.56500 & PRIARY & CASCADE \\
0.57100 & 8.18 & 8.18 \\
0.58200 & 19.02 & 19.02 \\
0.59900 & 39.98 & 39.98 \\
0.62100 & 12.60 & 74.60 \\
0.64900 & 185.44 & 122.24 \\
0.68300 & 263.12 & 185.42 \\
0.72800 & 363.59 & 363.12 \\
0.78400 & 480.25 & 480.25 \\
0.86800 & 632.35 & 632.35 \\
0.98000 & 791.40 & 792.63 \\
1.12000 & 930.44 & 945.37 \\
1.28000 & 1029.30 & 1078.62 \\
1.51000 & 1099.99 & 1222.93 \\
1.79000 & 1123.30 & 1354.16 \\
2.12000 & 1109.96 & 1472.74 \\
2.52000 & 1071.95 & 1585.91 \\
2.91000 & 1029.94 & 1676.19 \\
3.36000 & 983.69 & 1764.42 \\
3.92000 & 934.12 & 1857.59 \\
4.76000 & 875.89 & 1974.66 \\
5.60000 & 832.91 & 2072.43 \\
8.40000 & 751.96 & 2318.44 \\
11.20000 & 715.88 & 2496.52 \\
16.80000 & 681.46 & 2747.00 \\
28.00000 & 652.40 & 3061.59 \\
56.00000 & 634.95 & 3491.69 \\
112.00000 & 619.31 & 3913.29 \\
168.00000 & 607.95 & 4153.81 \\
280.00000 & 596.81 & 4453.81
\end{tabular}

$\mathrm{TD}=12.0 \mathrm{EV} \quad \mathrm{ET}=0.750324 \mathrm{MEV}$

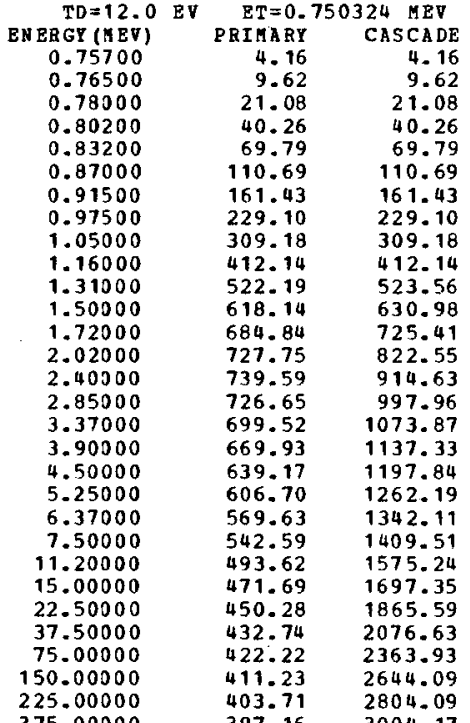

ATOAIC DISPLACEMENT CROSS-SECTIONS (BARNS) BY FAST RLECTRONS IN O

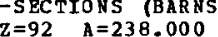

\begin{tabular}{crr} 
TD=16.0 & EV & \multicolumn{1}{c}{ ET $=0.915258$ MEV } \\
ENERGY (MEV) & PRIMARY & \multicolumn{1}{c}{ CASCADE } \\
0.92400 & 2.69 & 2.69 \\
0.93300 & 5.85 & 5.85 \\
0.95100 & 13.26 & 13.26 \\
0.97900 & 27.14 & 27.14 \\
1.01000 & 45.05 & 45.05 \\
1.06000 & 77.59 & 77.59 \\
1.11000 & 112.45 & 112.45 \\
1.18000 & 161.98 & 161.98 \\
1.28000 & 229.49 & 229.49 \\
1.41000 & 306.40 & 306.40 \\
1.60000 & 393.81 & 395.28 \\
1.83000 & 465.28 & 476.74 \\
2.10000 & 514.54 & 549.29 \\
2.47000 & 544.75 & 624.10 \\
2.92000 & 551.06 & 692.37 \\
3.47000 & 539.50 & 756.36 \\
4.11000 & 517.75 & 814.91 \\
4.75000 & 495.21 & 862.95 \\
5.49000 & 471.87 & 909.64 \\
6.40000 & 448.00 & 958.51 \\
7.77000 & 420.90 & 1019.48 \\
9.15000 & 401.41 & 1070.79 \\
13.70000 & 366.65 & 1197.35 \\
18.30000 & 351.38 & 1288.58 \\
27.40000 & 336.01 & 1414.62 \\
45.70000 & 323.66 & 1573.69 \\
91.50000 & 316.15 & 1789.64 \\
183.00000 & 307.65 & 1999.31
\end{tabular}

\begin{tabular}{rrr} 
TD $=20.0$ & EV & \multicolumn{2}{c}{ ET $=1.063002$ MEV } \\
ENERGI (HEV) & PRI AA & CASCA DE \\
1.07000 & 1.21 & 1.21 \\
1.08000 & 3.24 & 3.24 \\
1.10000 & 8.21 & 8.21 \\
1.13000 & 17.61 & 17.61 \\
1.17000 & 32.90 & 32.90 \\
1.23000 & 59.65 & 59.65 \\
1.29000 & 88.78 & 88.78 \\
1.38000 & 133.34 & 133.34 \\
1.48000 & 180.69 & 180.69 \\
1.64000 & 247.15 & 247.15 \\
1.86000 & 317.34 & 318.84 \\
2.12000 & 373.04 & 383.17 \\
2.44000 & 412.63 & 443.19 \\
2.87000 & 435.36 & 503.67 \\
3.40000 & 438.85 & 559.50 \\
4.03000 & 428.48 & 610.43 \\
4.78000 & 410.21 & 658.15 \\
5.52000 & 391.99 & 696.76 \\
6.37000 & 373.51 & 734.10 \\
7.44000 & 354.43 & 774.08 \\
9.03000 & 333.26 & 823.22 \\
10.60000 & 318.39 & 863.78 \\
15.90000 & 291.59 & 966.21 \\
21.20000 & 279.92 & 1038.96 \\
31.80000 & 267.88 & 1140.44 \\
53.10000 & 258.46 & 1268.26 \\
106.00000 & 252.65 & 1440.32 \\
212.00000 & 245.72 & 1607.83 \\
& &
\end{tabular}

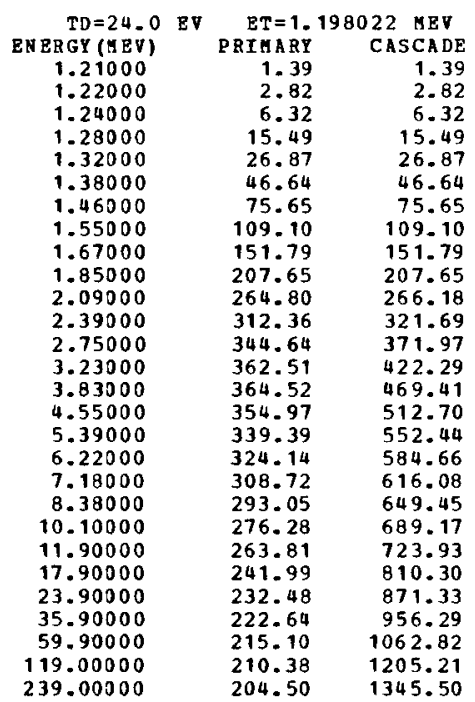


ATONIC DISPLACEHENT CROSS-SECTIONS (BARNS) BY PAST ELECTRONS IN D

\begin{tabular}{rrr} 
TD $=28.0$ & RV & \multicolumn{2}{c}{ ET $=1.323129$} \\
ENERGY (MEV) & PRTMARY & CASCADE \\
1.33000 & 0.52 & 0.52 \\
1.34000 & 1.42 & 1.42 \\
1.37000 & 5.14 & 5.14 \\
1.47000 & 12.04 & 12.04 \\
1.46000 & 22.99 & 22.99 \\
1.53000 & 41.13 & 41.13 \\
1.61000 & 63.93 & 63.93 \\
1.72000 & 96.04 & 96.04 \\
1.85000 & 132.22 & 132.22 \\
2.05000 & 180.55 & 180.54 \\
2.31000 & 228.52 & 229.89 \\
2.64000 & 268.88 & 277.52 \\
3.04000 & 296.14 & 321.06 \\
3.57000 & 310.65 & 364.32 \\
4.23000 & 311.64 & 404.70 \\
5.02000 & 303.01 & 441.76 \\
5.95000 & 289.33 & 476.12 \\
6.88000 & 276.00 & 504.24 \\
7.93000 & 262.93 & 531.04 \\
9.26000 & 249.57 & 559.86 \\
11.20000 & 235.16 & 594.71 \\
13.20000 & 224.69 & 624.65 \\
19.80000 & 206.68 & 698.45 \\
26.40000 & 198.76 & 750.58 \\
39.60000 & 190.48 & 823.21 \\
66.10000 & 184.20 & 914.70 \\
132.00000 & 180.18 & 1037.66 \\
264.00000 & 175.10 & 1157.06
\end{tabular}

\begin{tabular}{rrr} 
TD $=32.0$ & EV & \multicolumn{1}{c}{ ET $=1.440231$} \\
BNERGY & MEV \\
1.45000 & PRIMARY & CASCADE \\
1.46000 & 0.55 & 0.55 \\
1.49000 & 1.25 & 1.25 \\
1.54000 & 1.13 & 4.13 \\
1.59000 & 11.05 & 11.05 \\
1.67000 & 35.94 & 19.94 \\
1.75000 & 55.31 & 36.79 \\
1.87000 & 83.79 & 55.31 \\
2.01000 & 115.48 & 115.49 \\
2.23000 & 158.64 & 158.64 \\
2.52000 & 201.67 & 203.07 \\
2.54000 & 236.60 & 244.83 \\
3.31019 & 259.63 & 282.50 \\
3.88000 & 271.70 & 320.06 \\
4.60000 & 272.14 & 355.66 \\
5.47000 & 264.12 & 388.59 \\
6.48000 & 251.97 & 418.66 \\
7.48000 & 240.39 & 443.06 \\
8.64000 & 228.81 & 466.97 \\
10.00000 & 217.84 & 490.83 \\
12.20000 & 204.77 & 522.83 \\
14.40000 & 195.68 & 549.33 \\
21.60000 & 180.31 & 614.08 \\
28.80000 & 173.51 & 659.74 \\
43.20000 & 166.38 & 723.32 \\
72.00000 & 161.05 & 803.21 \\
144.00000 & 157.55 & 911.02 \\
288.00000 & 153.07 & 1015.41
\end{tabular}

\begin{tabular}{crr} 
TD=36.0 & \multicolumn{2}{c}{ ET $=1.550692$ MEV } \\
ENERGY (HEV) & PRIARY & CASCADE \\
1.56000 & 0.39 & 0.39 \\
1.58000 & 1.55 & 1.55 \\
1.51000 & 4.05 & 4.05 \\
1.65000 & 8.55 & 8.55 \\
1.72000 & 18.82 & 18.82 \\
1.79000 & 31.09 & 31.09 \\
1.89000 & 50.35 & 50.35 \\
2.01000 & 74.09 & 74.09 \\
2.17000 & 104.22 & 104.22 \\
2.40000 & 141.62 & 141.63 \\
2.71000 & 179.76 & 181.08 \\
3.10000 & 210.94 & 218.63 \\
3.56000 & 231.04 & 252.04 \\
4.18000 & 241.49 & 285.87 \\
4.96000 & 241.44 & 317.73 \\
5.89000 & 234.06 & 346.83 \\
6.97000 & 223.19 & 373.47 \\
8.06000 & 212.74 & 395.49 \\
9.30000 & 202.53 & 416.65 \\
10.80000 & 192.63 & 438.40 \\
13.10000 & 181.47 & 466.10 \\
15.50000 & 173.36 & 490.04 \\
23.20000 & 159.99 & 547.42 \\
31.00000 & 153.98 & 588.35 \\
46.50000 & 147.71 & 644.89 \\
77.50000 & 143.07 & 715.96 \\
155.00000 & 139.98 & 811.78 \\
310.00000 & 135.97 & 904.52
\end{tabular}

ATONIC DISPLAEEHENT CROSS-SECTIONS (BARNS) BY PAST ELECTRONS IN U CLACEEMT CROSS-SECTIONS (BRR:

\begin{tabular}{crr} 
TD=40.0 EV & \multicolumn{2}{c}{ ET=1.655528 MEV } \\
ENERG (HEV & PRIHARY & CASCADE \\
1.67000 & 0.50 & 0.50 \\
1.68000 & 0.96 & 0.96 \\
1.72000 & 3.62 & 3.62 \\
1.77000 & 8.47 & 8.47 \\
1.83000 & 15.95 & 15.95 \\
1.92000 & 29.34 & 29.34 \\
2.01000 & 44.09 & 44.09 \\
2.15000 & 67.62 & 67.62 \\
2.31000 & 93.24 & 93.24 \\
2.56000 & 127.82 & 127.82 \\
2.89000 & 162.19 & 163.44 \\
3.31000 & 190.41 & 197.66 \\
3.80000 & 208.22 & 227.73 \\
4.46000 & 217.30 & 258.17 \\
5.29000 & 216.99 & 286.84 \\
6.29000 & 210.10 & 313.28 \\
7.44000 & 200.23 & 337.25 \\
8.60000 & 190.83 & 357.06 \\
9.93000 & 181.61 & 376.25 \\
11.50000 & 172.90 & 395.50 \\
14.00000 & 162.75 & 420.97 \\
16.50000 & 155.69 & 442.05 \\
24.80000 & 143.71 & 494.31 \\
33.10000 & 138.39 & 531.02 \\
49.60000 & 132.81 & 581.80 \\
82.70000 & 128.70 & 645.85 \\
165.00000 & 125.94 & 731.79 \\
& &
\end{tabular}

\begin{tabular}{ccr} 
TD=44.0 EV & \multicolumn{2}{c}{ ET=1.755521 VEV } \\
ENERGY (AEV) & PRIARY & CASCA DE \\
1.77000 & 0.40 & 0.40 \\
1.79000 & 1.21 & 1.21 \\
1.82000 & 2.94 & 2.94 \\
1.87000 & 6.95 & 6.95 \\
1.94000 & 14.35 & 14.35 \\
2.03000 & 25.84 & 25.84 \\
2.14000 & 41.41 & 41.41 \\
2.28000 & 61.74 & 61.74 \\
2.45000 & 85.22 & 85.22 \\
2.72000 & 117.30 & 117.31 \\
3.07000 & 148.40 & 149.66 \\
3.51000 & 173.56 & 180.40 \\
4.03000 & 189.55 & 207.81 \\
4.73000 & 197.53 & 235.52 \\
5.61000 & 197.00 & 261.62 \\
6.67000 & 190.57 & 285.68 \\
7.89000 & 181.51 & 307.52 \\
9.12000 & 172.94 & 325.56 \\
10.50000 & 164.74 & 342.70 \\
12.20000 & 156.68 & 360.62 \\
14.90000 & 147.39 & 384.19 \\
17.50000 & 141.18 & 402.97 \\
26.30000 & 130.44 & 450.54 \\
35.10000 & 125.65 & 483.93 \\
52.60000 & 120.63 & 530.11 \\
87.70000 & 116.95 & 588.37 \\
175.00000 & 114.45 & 666.49 \\
& &
\end{tabular}

\begin{tabular}{rrr} 
TD=48.0 EV & \multicolumn{1}{c}{ ET=1.851285 MEV } \\
ENERGY (KEV) & PEIMARY & CASCADE \\
1.86000 & 0.18 & 0.18 \\
1.88000 & 0.78 & 0.78 \\
1.92000 & 2.67 & 2.67 \\
1.98000 & 6.95 & 6.95 \\
2.05000 & 13.52 & 13.52 \\
2.14000 & 23.60 & 23.60 \\
2.25000 & 37.20 & 37.20 \\
2.40000 & 56.26 & 56.26 \\
2.59000 & 79.19 & 79.19 \\
2.96000 & 107.20 & 107.21 \\
3.23000 & 136.03 & 137.19 \\
3.70000 & 159.41 & 165.88 \\
4.25000 & 173.96 & 191.12 \\
4.99000 & 181.06 & 216.60 \\
5.92000 & 180.36 & 240.59 \\
7.03000 & 174.37 & 262.52 \\
8.33000 & 165.91 & 282.77 \\
9.62000 & 158.09 & 299.22 \\
11.10000 & 150.48 & 315.21 \\
12.90000 & 143.12 & 331.68 \\
15.70000 & 134.82 & 352.95 \\
18.50000 & 129.07 & 370.52 \\
27.70000 & 119.44 & 413.79 \\
37.00000 & 115.07 & 444.50 \\
55.50000 & 110.49 & 486.95 \\
92.50000 & 107.17 & 540.33 \\
185.00000 & 104.87 & 612.17
\end{tabular}


ATOMIC DISPLAZEAENT CROSS-SBCTIONS (BARNS) BY FAST ELECTRONS IN

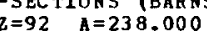

\begin{tabular}{crr} 
TD $=52.0$ & EV & \multicolumn{2}{c}{ ET=1.943315 MEV } \\
ENERGY (HEV) & PRIMARY & CASCADE \\
1.96000 & 0.32 & 0.32 \\
1.98000 & 0.90 & 0.90 \\
2.02000 & 2.65 & 2.65 \\
2.07000 & 5.75 & 5.75 \\
2.15000 & 12.27 & 12.27 \\
2.25000 & 22.18 & 22.18 \\
2.37000 & 35.34 & 35.34 \\
2.52000 & 52.21 & 52.21 \\
2.72000 & 73.53 & 73.53 \\
3.01000 & 99.98 & 99.99 \\
3.40000 & 126.49 & 127.67 \\
3.88000 & 147.33 & 153.4 .3 \\
4.46000 & 160.72 & 176.89 \\
5.24000 & 167.13 & 200.54 \\
6.21000 & 166.32 & 222.56 \\
7.38000 & 160.67 & 242.92 \\
8.74000 & 152.83 & 261.59 \\
10.10000 & 145.58 & 276.86 \\
11.60000 & 138.78 & 291.16 \\
13.60000 & 131.62 & 307.27 \\
16.50000 & 124.14 & 326.62 \\
19.40000 & 118.95 & 342.64 \\
29.10000 & 110.11 & 382.80 \\
38.80000 & 106.13 & 410.98 \\
58.20000 & 101.93 & 450.17 \\
97.10000 & 98.89 & 499.56 \\
194.00000 & 96.78 & 565.78
\end{tabular}

\begin{tabular}{crr} 
TD=56.0 EV & \multicolumn{2}{c}{ ET=2.032017 HEV } \\
ENERGY (HEV) & PRIMARY & CASCADE \\
2.05000 & 0.29 & 0.29 \\
2.07000 & 0.80 & 0.80 \\
2.11000 & 2.32 & 2.32 \\
2.17000 & 5.64 & 5.64 \\
2.25000 & 11.52 & 11.52 \\
2.35000 & 20.36 & 20.36 \\
2.47000 & 32.09 & 32.09 \\
2.64000 & 49.15 & 49.15 \\
2.84000 & 68.16 & 68.16 \\
3.14000 & 92.63 & 92.65 \\
3.55000 & 117.53 & 118.65 \\
4.06000 & 137.15 & 143.01 \\
4.67000 & 149.47 & 164.90 \\
5.48000 & 155.18 & 186.69 \\
6.50000 & 154.28 & 207.26 \\
7.72000 & 148.95 & 226.10 \\
9.14000 & 141.63 & 243.42 \\
10.50000 & 135.16 & 257.05 \\
12.10000 & 128.70 & 270.68 \\
14.20000 & 122.02 & 285.75 \\
17.20000 & 115.16 & 303.59 \\
20.30000 & 110.25 & 318.83 \\
30.40000 & 102.15 & 356.00 \\
40.50000 & 98.46 & 382.33 \\
60.90000 & 94.58 & 418.73 \\
101.00000 & 91.82 & 464.07 \\
203.00000 & 89.84 & 526.08
\end{tabular}

\begin{tabular}{crr} 
TD=60.0 & \multicolumn{2}{c}{ ET $=2.117727$ MEV } \\
ENERGY (HEV) & PEIMARY & CASCADE \\
2.13000 & 0.16 & 0.16 \\
2.16000 & 0.80 & 0.80 \\
2.20000 & 2.18 & 2.18 \\
2.26000 & 5.15 & 5.15 \\
2.35300 & 11.11 & 11.11 \\
2.45000 & 19.10 & 19.10 \\
2.58000 & 30.56 & 30.56 \\
2.75000 & 45.91 & 45.91 \\
2.96000 & 63.87 & 63.87 \\
3.28000 & 87.24 & 87.25 \\
3.70000 & 110.00 & 111.09 \\
4.23000 & 128.20 & 133.79 \\
4.87000 & 139.66 & 154.37 \\
5.71000 & 144.82 & 174.61 \\
6.77000 & 143.88 & 193.78 \\
8.04000 & 138.85 & 211.38 \\
9.52000 & 131.98 & 227.58 \\
11.00000 & 125.68 & 240.83 \\
12.70000 & 119.59 & 253.73 \\
14.80000 & 113.67 & 267.18 \\
18.00000 & 107.17 & 284.20 \\
21.10500 & 102.82 & 297.84 \\
31.70000 & 95.25 & 332.85 \\
42.30500 & 91.83 & 357.35 \\
63.50000 & 88.23 & 391.40 \\
105.00000 & 85.68 & 433.48 \\
211.00000 & 83.84 & 491.34 \\
& &
\end{tabular}

ATOHIC DISPLACEMENT CROSS-SECTIONS (BARNS) BY FAST ELECTRONS IN O $=92 \quad A=238.000$

\begin{tabular}{|c|c|c|c|}
\hline $\begin{array}{r}T D=64=0 \\
\text { (a) }\end{array}$ & EV & $\begin{array}{r}E T=2.2 \\
\text { PRTMARY }\end{array}$ & $\begin{array}{l}730 \text { MEV } \\
\text { CASCADE }\end{array}$ \\
\hline $\begin{array}{c}\text { TERGY (AEV) } \\
2-22000\end{array}$ & & $\begin{array}{r}\text { PRIAARY } \\
0.23\end{array}$ & $\begin{array}{r}\text { CASCADE } \\
0.23\end{array}$ \\
\hline 2.24000 & & 0.62 & 0.62 \\
\hline 2.28000 & & 1.79 & 1.79 \\
\hline 2.35000 & & 4.87 & 4.87 \\
\hline 2.44000 & & 10.27 & 10.27 \\
\hline 2.55000 & & 18.24 & 18.24 \\
\hline 2.68000 & & 28.63 & 28.63 \\
\hline 2.86000 & & 43.36 & 43.36 \\
\hline 3.08000 & & 60.37 & 60.37 \\
\hline 3.41000 & & 82.12 & 82.14 \\
\hline 3.85000 & & 103.57 & 104.65 \\
\hline 4.40000 & & 120.48 & 125.88 \\
\hline 5.06000 & & 131.02 & 145.02 \\
\hline 5.94000 & & 135.78 & 164.15 \\
\hline 7.04000 & & 134.77 & 182.08 \\
\hline 8.36000 & & 129.98 & 198.58 \\
\hline 9.90000 & & 123.50 & 213.78 \\
\hline 11.40000 & & 117.73 & 225.93 \\
\hline 13.20000 & & 111.92 & 238.28 \\
\hline 15.40000 & & 106.34 & 250.99 \\
\hline 18.70000 & & 100.33 & 266.83 \\
\hline 22.00000 & & 96.19 & 279.92 \\
\hline $\begin{array}{l}33.00000 \\
44.00000\end{array}$ & & $\begin{array}{l}89.20 \\
86.03\end{array}$ & $\begin{array}{l}312.63 \\
335.55\end{array}$ \\
\hline 66.00000 & & 82.67 & 367.41 \\
\hline 110.00000 & & 80.29 & 407.49 \\
\hline & & 78.57 & \\
\hline
\end{tabular}

\begin{tabular}{rrr} 
TD $=68.0$ & EV & \multicolumn{1}{c}{ ET $=2.281267$ LEV } \\
ENERGY (HEV) & PRIMAR & CASCA DE \\
2.30000 & 0.20 & 0.20 \\
2.32000 & 0.53 & 0.53 \\
2.37000 & 1.88 & 1.88 \\
2.44000 & 4.76 & 4.76 \\
2.53000 & 9.70 & 9.70 \\
2.64000 & 16.96 & 16.96 \\
2.78000 & 27.16 & 27.16 \\
2.96000 & 40.59 & 40.59 \\
3.19000 & 56.82 & 56.82 \\
3.53000 & 77.26 & 77.27 \\
3.99000 & 97.67 & 98.72 \\
4.56000 & 113.54 & 118.71 \\
5.24000 & 123.36 & 136.66 \\
6.15000 & 127.77 & 154.66 \\
7.30000 & 126.75 & 171.70 \\
8.66000 & 122.21 & 187.15 \\
10.20000 & 116.30 & 201.02 \\
11.80000 & 110.69 & 212.85 \\
13.60000 & 105.38 & 224.12 \\
15.90000 & 100.06 & 236.27 \\
19.30000 & 94.41 & 251.16 \\
22.80000 & 90.43 & 263.81 \\
34.20000 & 83.89 & 294.61 \\
45.60000 & 80.92 & 316.19 \\
68.40000 & 77.78 & 346.18 \\
114.00000 & 75.55 & 383.91 \\
228.00000 & 73.93 & 434.60
\end{tabular}

\begin{tabular}{|c|c|c|}
\hline$T D=72.0 \mathrm{EV}$ & $\mathrm{ET}=2$ & 544 MEV \\
\hline NERGY (MEV) & PRIMARY & CASCADE \\
\hline 2.38300 & 0.19 & 0.19 \\
\hline 2.40000 & 0.50 & 0.50 \\
\hline 2.45000 & 1.72 & 1.72 \\
\hline 2.52000 & 4.31 & 4.31 \\
\hline 2.61300 & 8.78 & 8.78 \\
\hline 2.73000 & 16.00 & 16.00 \\
\hline 2.87000 & 25.35 & 25.35 \\
\hline 3.06000 & 38.35 & 38.35 \\
\hline 3.30000 & 53.86 & 53.86 \\
\hline 3.65000 & 73.11 & 73.12 \\
\hline 4.12000 & 92.21 & 93.20 \\
\hline 4.71000 & 107.26 & 112.18 \\
\hline 5.42000 & 116.58 & 129.32 \\
\hline 6.37000 & 120.68 & 146.46 \\
\hline 7.55000 & 119.63 & 162.42 \\
\hline 8.96000 & 115.29 & 177.05 \\
\hline 10.60000 & 109.52 & 190.50 \\
\hline 12.20000 & 104.40 & 201.28 \\
\hline 14.10000 & 99.30 & 212.14 \\
\hline 16.50000 & 94.27 & 223.68 \\
\hline 20.00000 & 89.01 & 237.66 \\
\hline 23.50000 & 85.38 & 249.22 \\
\hline 35.30000 & 79.20 & 278.43 \\
\hline 47.10000 & 76.39 & 298.87 \\
\hline 70.70000 & 73.43 & 327.24 \\
\hline 117.00000 & 71.36 & 362.39 \\
\hline 235.00000 & 69.82 & 410.56 \\
\hline
\end{tabular}


ATOMIC DISPLAEEMENT CROSS-SECTIONS (BARNS) BT FAST ELECTRONS IN O

\begin{tabular}{rrr} 
TD $=76.0$ & EV & \multicolumn{2}{c}{ ET $=2.435744$ MEV } \\
ENERGY (HEV) & PRIMARY & CASCADE \\
2.46000 & 0.21 & 0.21 \\
2.48000 & 0.51 & 0.51 \\
2.53000 & 1.64 & 1.64 \\
2.60000 & 4.02 & 4.02 \\
2.70000 & 8.61 & 8.61 \\
2.82000 & 15.29 & 15.29 \\
2.97000 & 24.53 & 24.53 \\
3.16000 & 36.50 & 36.50 \\
3.41000 & 51.36 & 51.36 \\
3.77000 & 69.53 & 69.55 \\
4.26000 & 87.73 & 88.73 \\
4.87000 & 101.88 & 106.68 \\
5.60000 & 110.55 & 122.82 \\
6.57000 & 114.31 & 138.89 \\
7.79000 & 113.27 & 154.06 \\
9.25000 & 109.11 & 167.97 \\
10.90000 & 103.76 & 180.43 \\
12.60000 & 98.76 & 190.97 \\
14.60000 & 93.85 & 201.45 \\
17.00000 & 89.25 & 212.04 \\
20.70000 & 84.17 & 225.60 \\
24.30000 & 80.77 & 236.50 \\
36.50000 & 74.97 & 264.18 \\
48.70000 & 72.32 & 283.54 \\
73.00000 & 69.54 & 310.33 \\
121.00000 & 67.59 & 343.75 \\
243.00000 & 66.13 & 389.36 \\
& &
\end{tabular}

\begin{tabular}{rrr}
$\begin{array}{c}\text { TD }=80.0 \\
\text { ENERGY (HEV) }\end{array}$ & \multicolumn{2}{c}{ ET $=2.510021$ MEV } \\
2.53000 & 0.15 & CASCADE \\
2.56000 & 0.54 & 0.15 \\
2.61000 & 1.62 & 1.62 \\
2.68000 & 3.83 & 3.83 \\
2.78000 & 8.07 & 8.07 \\
2.91000 & 14.77 & 14.77 \\
3.06000 & 23.33 & 23.33 \\
3.26000 & 34.98 & 34.98 \\
3.51000 & 48.72 & 48.72 \\
3.89000 & 65.41 & 66.44 \\
4.39000 & 83.50 & 84.47 \\
5.02000 & 96.93 & 101.57 \\
5.77000 & 105.08 & 116.86 \\
6.77000 & 108.59 & 132.14 \\
8.03000 & 107.54 & 146.58 \\
9.53000 & 103.56 & 159.76 \\
11.20000 & 98.55 & 171.42 \\
13.00000 & 93.66 & 181.73 \\
15.00000 & 89.14 & 191.40 \\
17.50000 & 84.71 & 201.60 \\
21.30000 & 79.90 & 214.47 \\
25.10000 & 76.62 & 225.07 \\
37.60000 & 71.18 & 251.21 \\
50.20000 & 68.67 & 269.65 \\
75.30000 & 66.03 & 295.14 \\
125.00000 & 64.19 & 326.98 \\
251.00000 & 62.81 & 370.31 \\
& &
\end{tabular}

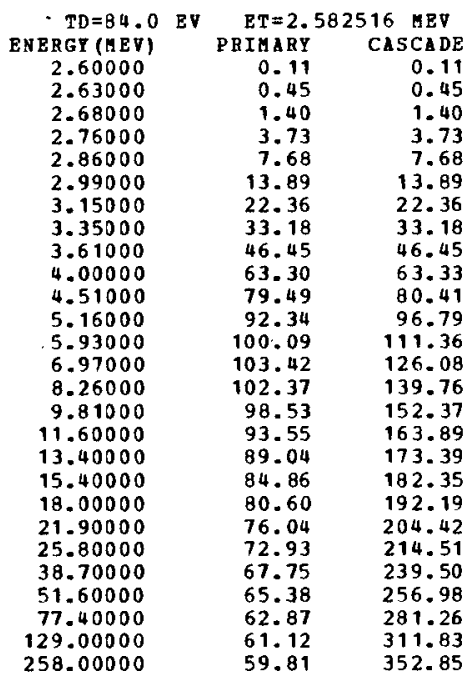
ATOMIC DISPLACEAENT CROSS-SECTIONS (BARNS) BY PAST ELRCTRONS IN 0
$\mathrm{Z}=92 \quad \mathrm{~A}=238.000$

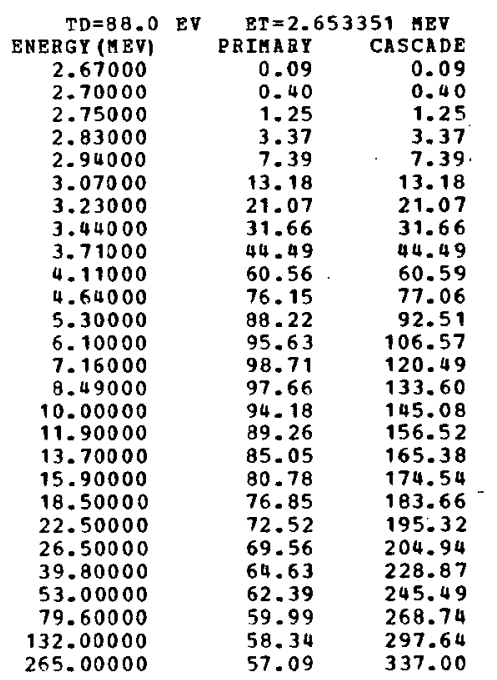

\begin{tabular}{rrr} 
TD $=92.0$ & EV & \multicolumn{1}{c}{ ET $=2.722634$ HEV } \\
PNERGY (HEV) & PRIMAY & CASCADE \\
2.74000 & 0.09 & 0.09 \\
2.77000 & 0.37 & 0.37 \\
2.83000 & 1.36 & 1.36 \\
2.91000 & 3.40 & 3.40 \\
3.02000 & 7.20 & 7.20 \\
3.15000 & 12.62 & 12.62 \\
3.32000 & 20.46 & 20.46 \\
3.53000 & 30.36 & 30.36 \\
3.81000 & 42.78 & 42.78 \\
4.22000 & 58.13 & 58.16 \\
4.76000 & 72.92 & 73.81 \\
5.44000 & 84.48 & 88.66 \\
6.26000 & 91.52 & 102.08 \\
7.35000 & 94.42 & 115.43 \\
8.71000 & 93.38 & 127.92 \\
10.30000 & 89.93 & 139.17 \\
12.20000 & 85.32 & 149.81 \\
14.10000 & 81.19 & 158.52 \\
16.30000 & 77.22 & 167.06 \\
19.00000 & 73.41 & 175.89 \\
23.10000 & 69.29 & 187.03 \\
27.20000 & 66.48 & 196.22 \\
40.80000 & 61.80 & 219.05 \\
54.40000 & 59.65 & 235.01 \\
81.60000 & 57.37 & 257.19 \\
136.00000 & 55.79 & 285.11 \\
272.00000 & 54.60 & 322.55
\end{tabular}

\begin{tabular}{rrr} 
TD=96.0 EV & \multicolumn{2}{c}{ ET=2.790463 MEV } \\
ENERGY (MEV) & PRIARY & CASCADE \\
2.81000 & 0.09 & 0.09 \\
2.84000 & 0.36 & 0.36 \\
2.90000 & 1.28 & 1.28 \\
2.98000 & 3.19 & 3.19 \\
3.09000 & 6.72 & 6.72 \\
3.23000 & 12.18 & 12.18 \\
3.40000 & 19.53 & 19.53 \\
3.62000 & 29.25 & 29.25 \\
3.90000 & 40.90 & 40.90 \\
4.32000 & 55.66 & 55.68 \\
4.88000 & 70.01 & 70.89 \\
5.58000 & 81.09 & 85.17 \\
6.41000 & 87.71 & 97.88 \\
7.53000 & 90.48 & 110.72 \\
8.92000 & 89.46 & 122.67 \\
10.60000 & 86.02 & 133.76 \\
12.50000 & 81.70 & 143.69 \\
14.50000 & 77.64 & 152.25 \\
16.70000 & 73.94 & 160.22 \\
19.50000 & 70.26 & 168.78 \\
23.70000 & 66.33 & 179.44 \\
27.90000 & 63.66 & 188.24 \\
41.80000 & 59.20 & 210.06 \\
55.80000 & 57.14 & 225.43 \\
83.70000 & 54.97 & 246.68 \\
139.00000 & 53.46 & 273.25 \\
279.00000 & 52.32 & 309.32 \\
& &
\end{tabular}


ATOHIC DISPLAzEHENT CROSS-SECTIONS (BARNS) BI PAST ELBCTRONS IN PU $z=94 \quad A=242.000$

\begin{tabular}{rrr} 
TD $=4.0$ & EV & \multicolumn{1}{c}{ ET $=0.332708$ IEV } \\
BNERGY (HEV) & PRIMARY & CASCADE \\
0.33600 & 36.72 & 36.72 \\
0.33900 & 69.89 & 69.89 \\
0.34600 & 146.26 & 146.26 \\
0.35500 & 242.21 & 242.21 \\
0.36900 & 386.38 & 386.38 \\
0.39500 & 543.32 & 543.32 \\
0.40500 & 727.47 & 727.47 \\
0.43200 & 954.82 & 954.82 \\
0.46500 & 1200.49 & 1200.49 \\
0.51500 & 1510.57 & 1510.57 \\
0.58200 & 1826.13 & 1826.60 \\
0.66500 & 2094.52 & 2116.05 \\
0.76500 & 2291.96 & 2376.18 \\
0.89800 & 2420.73 & 2632.11 \\
1.06000 & 2462.59 & 2863.62 \\
1.26000 & 2430.98 & 3080.77 \\
1.49000 & 2350.40 & 3277.26 \\
1.73000 & 2253.90 & 3445.81 \\
1.99000 & 2153.29 & 3601.26 \\
2.32000 & 2039.87 & 3771.10 \\
2.82000 & 1902.57 & 3988.87 \\
3.32000 & 1799.31 & 4173.93 \\
4.99000 & 1598.94 & 4648.79 \\
6.65000 & 1506.70 & 4995.96 \\
9.98000 & 1424.77 & 5498.37 \\
16.60000 & 1355.57 & 6131.61 \\
33.20000 & 1310.03 & 7007.10 \\
66.50000 & 1279.97 & 7877.36 \\
99.80000 & 1256.08 & 8372.47 \\
166.00000 & 1228.85 & 8985.11
\end{tabular}

\begin{tabular}{|c|c|c|c|}
\hline \multirow{2}{*}{\multicolumn{2}{|c|}{$\begin{array}{r}T D=8.0 \mathrm{EV} \\
\text { BNERGY }\end{array}$}} & \multicolumn{2}{|c|}{$\mathrm{ET}=0.567223 \mathrm{MEV}$} \\
\hline & & PRIMAR Y & CASCADE \\
\hline 0.57200 & & $\begin{array}{r}8.82 \\
20.36\end{array}$ & 8.82 \\
\hline 0.5890 .0 & & 42.72 & 42.72 \\
\hline 0.60600 & & 79.73 & 79.73 \\
\hline 0.62900 & & 133.13 & 133.13 \\
\hline 0.65700 & & 200.95 & 200.95 \\
\hline 0.692 .00 & & 286.76 & 286.76 \\
\hline 0.73700 & & $\begin{array}{l}394.33 \\
520.99\end{array}$ & 394.33 \\
\hline 0.87900 & & $\begin{array}{l}280.99 \\
684.09\end{array}$ & $\begin{array}{l}520.95 \\
684.09\end{array}$ \\
\hline 0.99200 & & 852.41 & 853.78 \\
\hline 1. 13000 & & 994.64 & 1010.50 \\
\hline 1.30000 & & 1100.24 & 1155.78 \\
\hline 1.53000 & & 1166.48 & 1301.50 \\
\hline $\begin{array}{l}1.81000 \\
2.15000\end{array}$ & & $\begin{array}{l}1183.13 \\
1161.26\end{array}$ & $\begin{array}{l}1433.48 \\
1555.65\end{array}$ \\
\hline 2.55000 & & 1115.29 & 1668.57 \\
\hline 2.94000 & & 1067.43 & 1758.93 \\
\hline 3.40000 & & 1015.09 & 1849.33 \\
\hline 4.82000 & & 897.95 & 2063.71 \\
\hline 5.67000 & & 852.59 & $2163 \cdot 52$ \\
\hline 8.50000 & & 769.15 & 2415.22 \\
\hline 11.30000 & & 733.16 & 2596.25 \\
\hline $\begin{array}{l}17.00000 \\
28.30000\end{array}$ & & $\begin{array}{l}698.45 \\
669.16\end{array}$ & 2855.41 \\
\hline 56.70000 & & 651.47 & 3620.25 \\
\hline 113.00000 & & 634.78 & 4049.94 \\
\hline 170.00000 & & 622.56 & 4297.79 \\
\hline 283.00000 & & 611.30 & 4604.50 \\
\hline
\end{tabular}

\begin{tabular}{rrr} 
TD $=12.0$ & \\
ENERGY & \multicolumn{1}{c}{ ET $=0.759153$ MEV } \\
0.76600 & PRIMARY & CASCADE \\
0.77400 & 4.55 & 4.55 \\
0.78900 & 10.39 & 10.39 \\
0.81200 & 22.66 & 22.66 \\
0.84200 & 44.25 & 44.25 \\
0.88000 & 76.12 & 76.12 \\
0.92600 & 120.21 & 120.21 \\
0.98600 & 176.04 & 176.04 \\
1.06000 & 248.69 & 248.69 \\
1.17000 & 333.28 & 333.28 \\
1.32000 & 443.08 & 443.08 \\
1.51000 & 559.58 & 560.95 \\
1.74000 & 659.90 & 673.34 \\
2.04000 & 730.36 & 774.95 \\
2.42500 & 770.69 & 873.53 \\
2.88000 & 778.09 & 966.45 \\
3.41000 & 759.45 & 1051.97 \\
3.94000 & 726.63 & 1129.33 \\
4.55000 & 658.21 & 1192.87 \\
5.31000 & 623.36 & 1254.60 \\
6.45000 & 583.53 & 1320.19 \\
7.59000 & 555.25 & 1470.73 \\
11.30000 & 505.25 & 1639.37 \\
15.10000 & 483.34 & 1763.80 \\
22.70000 & 461.70 & 1937.55 \\
37.90000 & 443.89 & 2154.96 \\
75.90000 & 433.19 & 2450.35 \\
151.00000 & 421.46 & 2735.09 \\
227.00000 & 413.44 & 2899.70 \\
379.00000 & 406.89 & 3105.55 \\
& &
\end{tabular}

ATOHC DISPLACEMENT CROSS-SECTIONS (BARNS) BY PAST ELECTRONS IN PU $\mathrm{z}=94 \quad \mathrm{~A}=242.000$

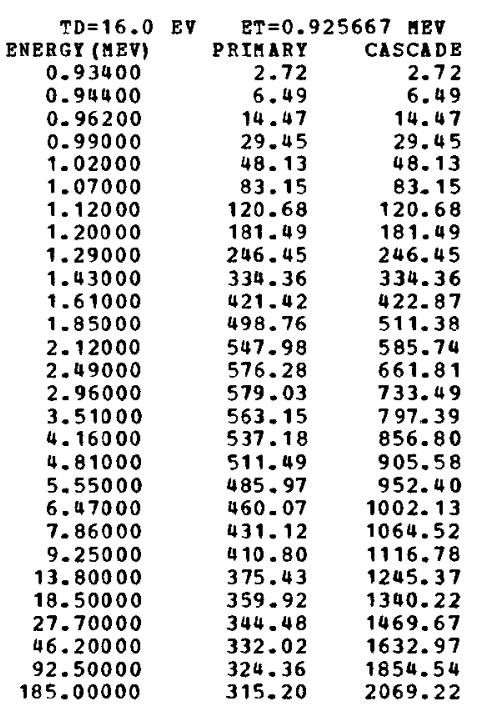

\begin{tabular}{rrr} 
TD $=20.0$ & EV & \multicolumn{2}{c}{ ET $=1.074791$ MEV } \\
ENERGY(MEV) & PRIMAY & CASCADE \\
1.08000 & 0.95 & 0.95 \\
1.09000 & 3.04 & 3.04 \\
1.11000 & 8.27 & 8.27 \\
1.15000 & 22.05 & 22.05 \\
1.19000 & 39.10 & 39.10 \\
1.24000 & 63.34 & 63.34 \\
1.31000 & 100.02 & 100.02 \\
1.39000 & 142.65 & 142.65 \\
1.50000 & 198.40 & 198.40 \\
1.66000 & 268.67 & 268.67 \\
1.88000 & 342.07 & 343.72 \\
2.14000 & 399.38 & 410.39 \\
2.47000 & 439.87 & 473.68 \\
2.90000 & 460.44 & 534.81 \\
3.43000 & 460.93 & 591.00 \\
4.08000 & 446.80 & 643.61 \\
4.83000 & 425.47 & 691.32 \\
5.58000 & 404.83 & 730.53 \\
6.44000 & 384.44 & 768.47 \\
7.52000 & 363.83 & 809.10 \\
9.13000 & 341.31 & 859.31 \\
10.70000 & 325.93 & 900.39 \\
16.10000 & 298.35 & 1006.23 \\
21.40000 & 286.80 & 1080.11 \\
32.20000 & 274.61 & 1185.12 \\
53.70000 & 265.14 & 1316.04 \\
107.00000 & 259.21 & 1492.09 \\
214.00000 & 251.75 & 1653.59
\end{tabular}

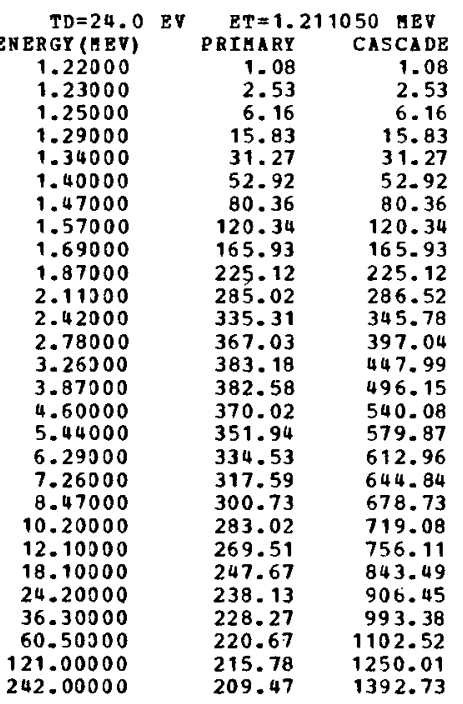


ATONIC DISPLACEMENT CROSS-SECTIONS (BARNS) BY PAST ELECTRONS IN PU

$\begin{array}{rrr}\text { TD }=28.0 & \text { EV } & \text { ET }=1.337291 \text { ABV } \\ \text { ENERGY (IIEV) } & \text { PRIMARY } & \text { CASCADE } \\ 1.35000 & 1.09 & 1.09 \\ 1.36000 & 2.18 & 2.18 \\ 1.39000 & 6.47 & 6.47 \\ 1.43000 & 14.22 & 14.22 \\ 1.48000 & 26.32 & 26.32 \\ 1.55000 & 46.12 & 46.12 \\ 1.63000 & 70.81 & 70.81 \\ 1.73000 & 102.25 & 102.25 \\ 1.87000 & 144.03 & 144.03 \\ 2.07000 & 195.30 & 195.29 \\ 2.34000 & 247.26 & 248.86 \\ 2.67000 & 288.29 & 297.90 \\ 3.07000 & 315.14 & 342.35 \\ 3.61000 & \mathbf{3 2 8 . 2 8} & \mathbf{3 8 6} .90 \\ 4.27000 & 326.96 & 427.45 \\ 5.08000 & 315.62 & 465.47 \\ 6.01000 & 299.82 & 499.84 \\ 6.95000 & 284.83 & 528.36 \\ 8.02000 & 270.38 & 555.80 \\ 9.36000 & 256.04 & 585.04 \\ 11.30000 & 240.93 & 620.27 \\ 13.30000 & 230.08 & 650.66 \\ 20.00000 & 211.59 & 726.81 \\ 26.70000 & 203.63 & 780.57 \\ 40.10000 & 195.28 & 855.36 \\ 66.80000 & 188.98 & 948.94 \\ 133.00000 & 184.85 & 1074.53 \\ 267.00000 & 179.37 & 1197.42 \\ & & \end{array}$

$\mathrm{SECTIONS}$ (BARNS)
$z=94 \quad A=242.000$

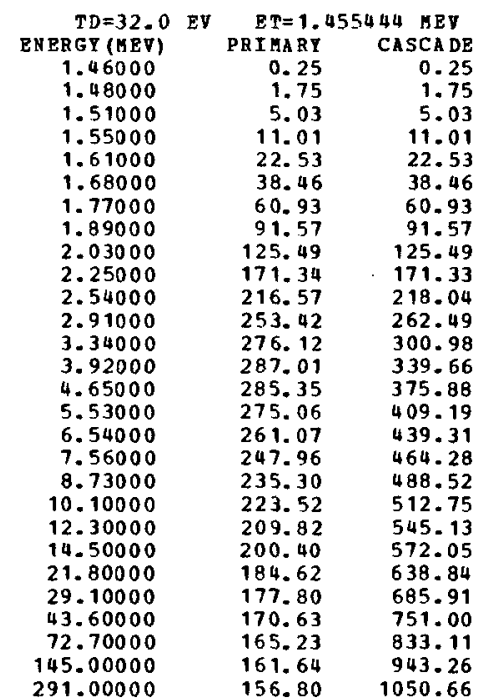

$T D=36.0 \mathrm{EV}$

\begin{tabular}{crr} 
TD $=36.0$ & EV & \multicolumn{1}{c}{ ET $=1.566889$ MEV } \\
ENERGY(HEV) & PRIMARY & CASCADE \\
1.58000 & 0.62 & 0.62 \\
1.59000 & 1.23 & 1.23 \\
1.62000 & 3.73 & 3.73 \\
1.67000 & 9.75 & 9.75 \\
1.73000 & 19.24 & 19.24 \\
1.81000 & 34.34 & 34.34 \\
1.91000 & 55.18 & 55.18 \\
2.03000 & 80.73 & 80.73 \\
2.19000 & 112.99 & 112.99 \\
2.42000 & 152.76 & 152.76 \\
2.74000 & 193.95 & 195.44 \\
3.13000 & 225.77 & 234.19 \\
3.60000 & 245.89 & 269.00 \\
4.23000 & 255.05 & 303.65 \\
5.01000 & 253.09 & 335.60 \\
5.95000 & 243.72 & 365.04 \\
7.05000 & 231.01 & 392.19 \\
8.14000 & 219.44 & 414.27 \\
9.40000 & 208.21 & 435.90 \\
10.90000 & 197.67 & 457.83 \\
13.30000 & 185.54 & 486.98 \\
15.60000 & 177.55 & 510.21 \\
23.50000 & 163.74 & 570.01 \\
31.30000 & 157.80 & 511.56 \\
47.00000 & 151.47 & 669.76 \\
78.30000 & 146.78 & 742.67 \\
156.00000 & 143.61 & 840.42 \\
313.00000 & 139.29 & 935.79
\end{tabular}

ATOHIC DISPLATEAENT CROSS-SECTIONS (BARNS) BY PAST ELECTRONS IN PU $\mathrm{Z}=94 \quad \mathrm{~A}=242.000$

\begin{tabular}{rrr}
$\begin{array}{c}\text { TD }=40.0 \\
\text { EN }\end{array}$ & \multicolumn{1}{c}{ ET $=1.672654$} & \multicolumn{1}{c}{ MEV } \\
ENERGI (HEV) & PRIMARY & CASCADE \\
1.68000 & 0.25 & 0.25 \\
1.70000 & 1.19 & 1.19 \\
1.73000 & 3.29 & 3.29 \\
1.78000 & 8.30 & 8.30 \\
1.85000 & 17.65 & 17.65 \\
1.94000 & 32.19 & 32.19 \\
2.04000 & 49.96 & 49.96 \\
2.17000 & 73.48 & 73.48 \\
2.34000 & 102.55 & 102.55 \\
2.59000 & 139.03 & 139.03 \\
2.92000 & 174.82 & 176.22 \\
3.34000 & 203.68 & 211.57 \\
3.84000 & 221.49 & 242.89 \\
4.51000 & 229.43 & 274.06 \\
5.35000 & 227.35 & 303.14 \\
6.35000 & 218.74 & 329.60 \\
7.52000 & 207.24 & 354.02 \\
8.69000 & 196.75 & 374.06 \\
10.00000 & 186.91 & 393.11 \\
11.70000 & 176.92 & 414.07 \\
14.20000 & 166.44 & 439.67 \\
16.70000 & 159.23 & 460.96 \\
25.00000 & 147.18 & 514.05 \\
33.40000 & 141.83 & 551.88 \\
50.10000 & 136.20 & 604.14 \\
83.60000 & 132.04 & 670.01 \\
167.00000 & 129.18 & 758.30
\end{tabular}

\begin{tabular}{rrr} 
TD $=44.0$ & EV & \multicolumn{2}{c}{ ET=1.773528 MEV } \\
ENERGY (IEEV) & PRIART & CASCADE \\
1.79000 & 0.50 & 0.50 \\
1.80000 & 0.92 & 0.92 \\
1.84000 & 3.31 & 3.31 \\
1.89000 & 7.70 & 7.70 \\
1.96000 & 15.76 & 15.76 \\
2.05000 & 28.23 & 28.23 \\
2.16000 & 45.05 & 45.05 \\
2.30000 & 66.94 & 66.94 \\
2.48000 & 93.51 & 93.51 \\
2.74000 & 126.27 & 126.27 \\
3.10000 & 159.81 & 161.19 \\
3.54000 & 185.55 & 192.98 \\
4.07000 & 201.54 & 221.51 \\
4.78000 & 208.50 & 249.88 \\
5.67000 & 206.38 & 276.35 \\
6.73000 & 198.39 & 300.45 \\
7.98000 & 187.77 & 322.87 \\
9.22000 & 178.25 & 341.09 \\
10.60000 & 169.39 & 358.33 \\
12.40000 & 160.36 & 377.41 \\
15.00000 & 151.05 & 400.33 \\
17.70000 & 144.41 & 420.11 \\
26.60000 & 133.53 & 468.91 \\
35.40000 & 128.79 & 502.86 \\
53.20000 & 123.69 & 550.60 \\
88.60000 & 119.99 & 610.28 \\
177.00000 & 117.39 & 690.54
\end{tabular}

$T D=48.0 \mathrm{BV}$

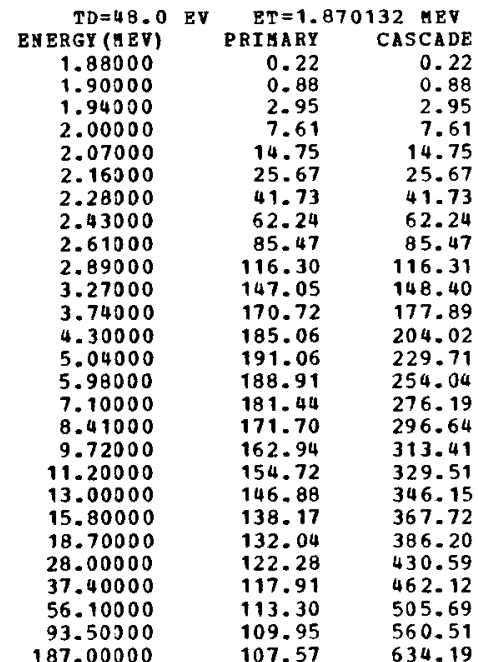


ATONIC DISPLAEEMENT CROSS-SECTIONS (BARNS) BY FAST ELECTROHS IN PO

\begin{tabular}{rrr} 
TD $=52.0$ & \multicolumn{2}{c}{ ET $=1.962967$ HEV } \\
ENERGY (MEV) & PRIMARY & CASCADE \\
1.98000 & 0.35 & 0.35 \\
2.00000 & 0.98 & 0.98 \\
2.04000 & 2.88 & 2.88 \\
2.10000 & 7.02 & 7.02 \\
2.17000 & 13.31 & 13.31 \\
2.27000 & 24.03 & 24.03 \\
2.39000 & 38.24 & 38.24 \\
2.55000 & 57.59 & 57.59 \\
2.74000 & 79.27 & 79.27 \\
3.04000 & 108.32 & 108.34 \\
3.43000 & 136.02 & 137.31 \\
3.92000 & 157.71 & 164.44 \\
4.51000 & 170.92 & 188.73 \\
5.30000 & 176.33 & 212.84 \\
6.28000 & 174.15 & 235.13 \\
7.45000 & 167.18 & 255.50 \\
8.83000 & 158.10 & 274.47 \\
10.20000 & 150.05 & 289.91 \\
11.70000 & 142.70 & 304.33 \\
13.70000 & 135.08 & 320.61 \\
16.60000 & 127.23 & 340.25 \\
19.60000 & 121.70 & 357.08 \\
29.40000 & 112.74 & 398.28 \\
39.20000 & 108.76 & 427.22 \\
58.80000 & 104.52 & 467.45 \\
98.10000 & 101.46 & 518.16 \\
196.00000 & 99.27 & 586.07
\end{tabular}

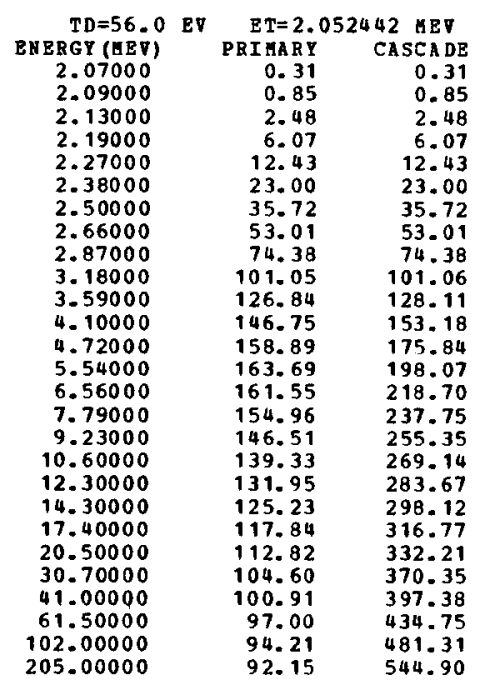

\begin{tabular}{rrr}
$\begin{array}{r}\text { TD }=60.0 \\
\text { ENE }\end{array}$ & \multicolumn{1}{c}{ ET $=2.138898$ MEV } \\
RHGY (HEV) & PRIMARY & CASCADE \\
2.16000 & 0.33 & 0.33 \\
2.18000 & 0.83 & 0.83 \\
2.22000 & 2.30 & 2.30 \\
2.28000 & 5.49 & 5.49 \\
2.37000 & 11.93 & 11.93 \\
2.48000 & 21.48 & 21.48 \\
2.60000 & 32.92 & 32.92 \\
2.78000 & 50.41 & 50.41 \\
2.99000 & 69.59 & 69.59 \\
3.31300 & 94.35 & 94.37 \\
3.74000 & 118.63 & 119.85 \\
4.27000 & 137.12 & 143.24 \\
4.91000 & 148.31 & 164.25 \\
5.77000 & 152.74 & 185.18 \\
6.84000 & 150.62 & 204.58 \\
8.12000 & 144.40 & 222.34 \\
9.62000 & 136.48 & 238.78 \\
11.10000 & 129.55 & 252.09 \\
12.80000 & 122.97 & 265.10 \\
14.90000 & 116.66 & 278.71 \\
18.10000 & 109.85 & 295.99 \\
21.30000 & 105.22 & 310.31 \\
32.00000 & 97.54 & 346.22 \\
42.70000 & 94.12 & 371.38 \\
64.10000 & 90.48 & 406.33 \\
106.00000 & 87.91 & 449.55 \\
213.00000 & 86.00 & 508.88
\end{tabular}

ATOHC DISPLACBHENT CROSS-SECTIONS (BARNS) BY PAST BLECTRONS IN PU $\mathrm{Z}=94 \quad \mathrm{~A}=242.000$

\begin{tabular}{rrr} 
TD $=64.0$ & EV & \multicolumn{2}{c}{ ET=2.222620 HEV } \\
ENERGY (MEV) & PRIMAY & CASCADE \\
2.24000 & 0.22 & 0.22 \\
2.26000 & 0.62 & 0.62 \\
2.31000 & 2.25 & 2.25 \\
2.37000 & 5.17 & 5.17 \\
2.46000 & 10.98 & 10.98 \\
2.57000 & 19.58 & 19.58 \\
2.71000 & 31.67 & 31.67 \\
2.88000 & 46.65 & 46.65 \\
3.11000 & 65.70 & 65.70 \\
3.44000 & 88.75 & 88.76 \\
3.88000 & 111.21 & 112.37 \\
4.44000 & 128.81 & 134.70 \\
5.11000 & 139.21 & 154.51 \\
5.00000 & 143.17 & 174.03 \\
7.11000 & 141.06 & 192.17 \\
8.44000 & 135.17 & 208.82 \\
10.00000 & 127.71 & 224.26 \\
11.50000 & 121.36 & 236.46 \\
13.30000 & 115.08 & 248.92 \\
15.50000 & 109.14 & 261.79 \\
18.80000 & 102.84 & 277.88 \\
22.20000 & 98.44 & 291.59 \\
33.30000 & 91.35 & 325.16 \\
44.40000 & 88.18 & 348.69 \\
66.60000 & 84.79 & 381.39 \\
111.00000 & 82.38 & 422.55 \\
222.00000 & 80.59 & 477.78
\end{tabular}

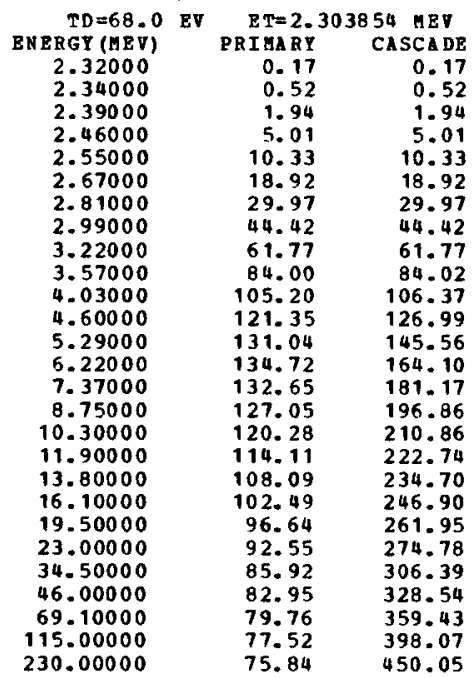

\begin{tabular}{crr} 
TD=72.0 & BV & \multicolumn{1}{c}{ ET $=2.382808$ MEV } \\
ENERGY(HEV) & PRIMARY & CASCADE \\
2.40000 & 0.17 & 0.17 \\
2.43000 & 0.68 & 0.68 \\
2.47000 & 1.75 & 1.75 \\
2.54000 & 4.52 & 4.52 \\
2.64000 & 9.91 & 9.91 \\
2.76000 & 17.79 & 17.79 \\
2.90000 & 27.91 & 27.91 \\
3.09000 & 41.90 & 41.90 \\
3.33000 & 58.49 & 58.49 \\
3.69000 & 79.42 & 79.44 \\
4.16000 & 99.28 & 100.38 \\
4.76000 & 114.80 & 120.24 \\
5.48000 & 123.89 & 137.91 \\
6.43000 & 127.21 & 155.19 \\
7.62000 & 125.19 & 171.34 \\
9.05000 & 119.84 & 186.20 \\
10.70000 & 113.26 & 199.77 \\
12.30000 & 107.63 & 210.61 \\
14.20000 & 102.12 & 221.57 \\
16.60000 & 96.76 & 233.26 \\
20.20000 & 91.11 & 247.83 \\
23.80000 & 87.30 & 259.87 \\
35.70000 & 81.09 & 289.74 \\
47.60000 & 78.29 & 310.67 \\
71.40000 & 75.30 & 339.75 \\
119.00000 & 73.20 & 376.35 \\
238.00000 & 71.61 & 425.43 \\
& &
\end{tabular}


ATOMIC DISPLAEEMENT CROSS-SECTIONS (BARSS) BY PAST BLECTRONS IN PU

$\begin{array}{rrr}\begin{array}{r}\text { TD }=76.0 \\ \text { ENERGY EV (HEV) }\end{array} & \begin{array}{r}\text { ET }=2.459654 \\ \text { PRIMARY }\end{array} & \text { CASCADE } \\ 2.48000 & 0.18 & 0.18 \\ 2.50000 & 0.48 & 0.48 \\ 2.55000 & 1.65 & 1.65 \\ 2.63000 & 4.61 & 4.61 \\ 2.73000 & 9.66 & 9.66 \\ 2.85000 & 16.95 & 16.95 \\ 3.00000 & 26.95 & 26.95 \\ 3.19000 & 39.83 & 39.83 \\ 3.44000 & 55.72 & 55.72 \\ 3.81000 & 75.47 & 75.50 \\ 4.30000 & 94.41 & 95.51 \\ 4.91000 & 108.83 & 114.04 \\ 5.65000 & 117.38 & 130.74 \\ 6.64000 & 120.50 & 147.28 \\ 7.87000 & 118.51 & 162.60 \\ 9.34000 & 113.42 & 176.62 \\ 11.00000 & 107.31 & 189.19 \\ 12.70000 & 101.81 & 199.79 \\ 14.70000 & 96.51 & 210.38 \\ 17.20000 & 91.43 & 221.52 \\ 20.90000 & 86.16 & 235.23 \\ 24.50000 & 82.68 & 246.29 \\ 36.80000 & 76.79 & 274.69 \\ 49.10000 & 74.14 & 294.57 \\ 73.70000 & 71.32 & 322.17 \\ 122.00000 & 69.35 & 356.39 \\ 245.00000 & 67.84 & 403.17 \\ & & \end{array}$

\begin{tabular}{rrr} 
TD $=80.0$ & \multicolumn{1}{c}{ EV } & \multicolumn{1}{c}{ ET=2.534582 MEV } \\
ENERGY (IEV) & PRIMAY & CASCADE \\
2.55000 & 0.11 & 0.11 \\
2.58000 & 0.51 & 0.51 \\
2.63000 & 1.62 & 1.62 \\
2.71000 & 4.37 & 4.37 \\
2.81000 & 9.02 & 9.02 \\
2.94000 & 16.32 & 16.32 \\
3.09000 & 25.58 & 25.58 \\
3.29000 & 38.11 & 38.11 \\
3.54000 & 52.81 & 52.81 \\
3.92000 & 71.60 & 71.62 \\
4.43000 & 89.82 & 90.88 \\
5.06000 & 103.51 & 108.54 \\
5.82000 & 111.55 & 124.36 \\
6.84000 & 114.46 & 140.09 \\
8.11000 & 112.51 & 154.67 \\
9.63000 & 107.62 & 168.04 \\
11.40000 & 101.61 & 180.36 \\
13.10000 & 96.56 & 190.10 \\
15.20000 & 91.45 & 200.32 \\
17.70000 & 86.79 & 210.59 \\
21.50000 & 81.80 & 223.60 \\
25.30000 & 78.44 & 234.36 \\
38.00000 & 72.89 & 261.36 \\
50.60000 & 70.40 & 280.12 \\
76.00000 & 67.72 & 306.37 \\
126.00000 & 65.87 & 338.98 \\
253.00000 & 64.43 & 383.41 \\
& &
\end{tabular}

TO= 84.0
ENERGY (11EV)
2.63000
2.65000
2.71000
2.79000
2.89000
3.02000
3.18000
3.39000
3.65000
4.04000
4.56000
5.21000
5.99000
7.04000
8.34000
9.90000
11.70000
13.50000
15.60000
18.20000
22.10000
26.00000
39.10000
52.10000
78.20000
130.00000
260.00000

\begin{tabular}{rr}
\multicolumn{2}{c}{$\begin{array}{r}\text { ET }=2.607700 \text { YEV } \\
\text { PRIMARY }\end{array}$} \\
0.16 & CASCADE \\
0.41 & 0.16 \\
1.64 & 0.41 \\
4.22 & 1.64 \\
8.54 & 4.22 \\
15.30 & 8.54 \\
24.46 & 15.30 \\
36.68 & 36.46 \\
50.83 & 50.83 \\
68.62 & 68.64 \\
85.74 & 86.79 \\
98.74 & 103.63 \\
106.30 & 118.65 \\
108.99 & 133.63 \\
107.09 & 147.45 \\
102.42 & 160.17 \\
96.75 & 171.79 \\
91.79 & 181.35 \\
87.07 & 190.83 \\
82.58 & 200.73 \\
77.85 & 213.11 \\
74.66 & 223.35 \\
69.38 & 249.15 \\
67.02 & 267.06 \\
64.48 & 292.03 \\
62.71 & 323.26 \\
61.35 & 365.32
\end{tabular}
ATOMIC DISPLACENENT CROSS-SECTIONS (BARNS) BY PAST RLECTRONS IN PU

\begin{tabular}{rrr} 
TD $=88.0$ & EV & \multicolumn{2}{c}{ ET $=2.679143$ HEV } \\
ENERGY (MEV) & PRIMARY & CASCADE \\
2.70000 & 0.13 & 0.13 \\
2.73000 & 0.49 & 0.49 \\
2.78000 & 1.45 & 1.45 \\
2.86000 & 3.80 & 3.80 \\
2.97000 & 8.20 & 8.20 \\
3.10000 & 14.49 & 14.49 \\
3.26000 & 23.02 & 23.02 \\
3.48000 & 34.95 & 34.95 \\
3.75000 & 48.63 & 48.63 \\
4.15000 & 65.61 & 65.63 \\
4.68000 & 81.85 & 82.85 \\
5.35000 & 94.31 & 99.02 \\
6.16000 & 101.54 & 113.50 \\
7.23000 & 104.02 & 127.68 \\
8.57000 & 102.16 & 140.93 \\
10.10000 & 97.89 & 152.57 \\
12.00000 & 92.31 & 164.04 \\
13.90000 & 87.45 & 173.42 \\
16.00000 & 83.07 & 182.23 \\
18.70000 & 78.74 & 191.80 \\
22.70000 & 74.25 & 203.60 \\
26.70000 & 71.22 & 213.37 \\
40.10000 & 66.21 & 237.93 \\
53.50000 & 63.95 & 255.10 \\
80.30000 & 61.53 & 278.93 \\
133.00000 & 59.86 & 308.53 \\
267.00000 & 58.56 & $\mathbf{3 4 8 . 9 0}$ \\
& &
\end{tabular}

\begin{tabular}{ccc} 
TD $=92.0$ & EV & \multicolumn{2}{c}{ ET=2.749021 MEV } \\
ENERGY (HEV) & PRIBAY & CASCADE \\
2.77000 & 0.12 & 0.12 \\
2.80000 & 0.44 & 0.44 \\
2.85000 & 1.33 & 1.33 \\
2.94000 & 3.80 & 3.80 \\
3.05000 & 7.95 & 7.95 \\
3.18000 & 13.84 & 13.84 \\
3.35000 & 22.32 & 22.32 \\
3.57000 & 33.47 & 33.47 \\
3.84000 & 46.27 & 46.27 \\
4.26000 & 62.94 & 62.97 \\
4.81000 & 78.59 & 79.59 \\
5.49000 & 90.29 & 94.86 \\
6.32000 & 97.16 & 108.70 \\
7.42000 & 99.48 & 122.29 \\
8.79000 & 97.67 & 134.92 \\
10.40000 & 93.45 & 146.33 \\
12.30000 & 88.24 & 157.00 \\
14.20000 & 83.70 & 165.77 \\
16.40000 & 79.41 & 174.40 \\
19.20000 & 75.23 & 183.66 \\
23.30000 & 70.95 & 194.94 \\
27.40000 & 68.07 & 204.27 \\
41.20000 & 63.29 & 227.84 \\
54.90000 & 61.14 & 244.20 \\
82.40000 & 58.84 & 267.00 \\
137.00000 & 57.25 & 295.53 \\
274.00000 & 56.00 & 333.93
\end{tabular}

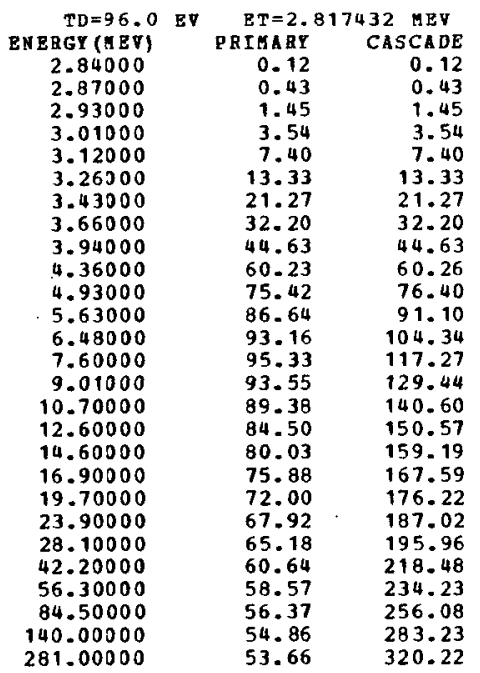


A TONIC DISPLACEHENT CROSS-SECTIONS (BARNS) BY PAST ELECTRONS IN ES

\begin{tabular}{rrr} 
TD $=4.0$ & \multicolumn{1}{c}{ EV } & EI $=0.345851$ HEV \\
ENERGY (HEV) & PRIMARY & CASCADE \\
0.34900 & 39.75 & 39.75 \\
0.35200 & 77.37 & 77.37 \\
0.35900 & 164.25 & 164.25 \\
0.37000 & 297.95 & 297.95 \\
0.38300 & 451.11 & 451.11 \\
0.40100 & 653.73 & 653.73 \\
0.42100 & 865.06 & 865.06 \\
0.44900 & 1135.62 & 1135.62 \\
0.48400 & 1432.66 & 1432.66 \\
0.53600 & 1795.36 & 1795.36 \\
0.60500 & 2153.03 & 2153.77 \\
0.69100 & 2447.96 & 2475.13 \\
0.79500 & 2650.95 & 2756.16 \\
0.93300 & 2762.48 & 3023.90 \\
1.10000 & 2759.65 & 3258.96 \\
1.31000 & 2691.62 & 3480.14 \\
1.55000 & 2564.57 & 3677.81 \\
1.79000 & 2435.31 & 3841.41 \\
2.07000 & 2299.14 & 4005.06 \\
2.42000 & 2156.06 & 4181.85 \\
2.93000 & 1997.15 & 4402.62 \\
3.45000 & 1880.12 & 4595.67 \\
5.18000 & 1666.94 & 5094.48 \\
6.91000 & 1575.16 & 5463.99 \\
10.30000 & 1497.95 & 5988.03 \\
17.20000 & 1428.19 & 6663.43 \\
34.50000 & 1382.14 & $\mathbf{7 5 9 3 . 0 4}$ \\
69.10000 & 1347.16 & $\mathbf{8 5 0 8 . 2 2}$ \\
103.00000 & 1319.39 & 9017.96 \\
172.00000 & 1290.06 & 9666.22
\end{tabular}

\begin{tabular}{rrr} 
TD $=8.0$ & EV & \multicolumn{2}{c}{ ET $=0.587756$ HEV } \\
ENERGY(MEV) & PRIARY & CASCADE \\
0.59300 & 11.09 & 11.09 \\
0.59900 & 24.39 & 24.39 \\
0.61100 & 52.72 & 52.72 \\
0.62800 & 96.02 & 96.02 \\
0.65200 & 161.49 & 161.49 \\
0.68100 & 244.24 & 244.24 \\
0.71700 & 348.06 & 348.06 \\
0.76400 & 479.51 & 479.51 \\
0.82200 & 628.88 & 628.88 \\
0.91100 & 823.35 & 823.35 \\
1.02000 & 1004.68 & 1006.10 \\
1.17000 & 1169.99 & 1190.16 \\
1.35000 & 1277.94 & 1348.96 \\
1.58000 & 1330.03 & 1495.47 \\
1.88000 & 1325.33 & 1634.34 \\
2.23000 & 1278.52 & 1756.07 \\
2.64000 & 1209.53 & 1868.25 \\
3.05000 & 1143.78 & 1960.88 \\
3.52000 & 1078.14 & 2051.59 \\
4.11000 & 1012.06 & 2149.42 \\
4.99000 & 940.01 & 2273.04 \\
5.87000 & 889.99 & 2377.40 \\
8.81000 & 803.22 & 2643.50 \\
11.70000 & 768.57 & 2834.64 \\
17.60000 & 734.99 & 3109.10 \\
29.30000 & 705.40 & 3449.77 \\
58.70000 & 687.31 & 3917.10 \\
117.00000 & 567.47 & 4368.40 \\
176.00000 & 653.42 & 4627.86 \\
293.00000 & 642.49 & 4950.90
\end{tabular}

\begin{tabular}{rrr}
$\begin{array}{r}\text { TD=12.0 } \\
\text { ENERGY(18V) }\end{array}$ & \multicolumn{2}{c}{ ET $=0.785278$ MEV } \\
0.79300 & PRIMARY & CASCADE \\
0.80000 & 5.94 & 5.94 \\
0.81500 & 21.87 & 11.87 \\
0.84000 & 53.17 & 27.17 \\
0.87100 & 93.02 & 53.84 \\
0.91000 & 146.90 & 93.02 \\
0.95800 & 216.04 & 146.90 \\
1.02000 & 304.46 & 316.04 \\
1.09000 & 397.97 & 397.46 \\
1.21000 & 535.41 & 535.41 \\
1.37000 & 671.90 & 673.88 \\
1.57000 & 780.53 & 798.78 \\
1.80000 & 846.50 & 902.87 \\
2.12000 & 877.54 & 1007.61 \\
2.51000 & 868.66 & 1100.47 \\
2.99000 & 833.03 & 1185.13 \\
3.53000 & 784.71 & 1263.15 \\
4.08000 & 740.18 & 1327.61 \\
4.71000 & 697.30 & 1390.47 \\
5.49000 & 655.44 & 1457.53 \\
6.67000 & 610.15 & 1542.75 \\
7.85000 & 579.43 & 1614.75 \\
11.70000 & 528.17 & 1793.08 \\
15.70000 & 506.93 & 1926.74 \\
23.50000 & 486.04 & 2108.72 \\
39.20000 & 468.05 & 2338.12 \\
78.50000 & 456.94 & 2649.79 \\
157.00000 & 442.84 & 2950.83 \\
235.00000 & 433.88 & 3121.44 \\
392.00000 & 427.99 & 3338.19
\end{tabular}

ATOMIC DISPLACEMENT CROSS-SECTIONS (BARNS) BY FAST RLECTRONS IN ES $z=99 \quad=254.000$

\begin{tabular}{rrr}
$\begin{array}{r}\text { TD }=16.0 \\
\text { BNERGY (NEV) }\end{array}$ & $\begin{array}{c}\text { BT }=0.956450 \text { MEV } \\
\text { PRIMARY }\end{array}$ & \multicolumn{1}{c}{ CASCADE } \\
0.96600 & 3.64 & 3.64 \\
0.97500 & 7.62 & 7.62 \\
0.99400 & 17.57 & 17.57 \\
1.02000 & 34.03 & $\mathbf{3 4 . 0 3}$ \\
1.06000 & 64.03 & 64.03 \\
1.10000 & 97.67 & 97.67 \\
1.16000 & 151.31 & 151.31 \\
1.24000 & 223.18 & 223.18 \\
1.33000 & 299.01 & 299.01 \\
1.48000 & 406.40 & 406.40 \\
1.67000 & 506.63 & 508.73 \\
1.91000 & 586.41 & 602.44 \\
2.19000 & 634.28 & 681.75 \\
2.58000 & 654.58 & 761.61 \\
3.06000 & 644.80 & 833.08 \\
3.63000 & 615.99 & 897.36 \\
4.30000 & 578.75 & 957.00 \\
4.97000 & 545.19 & 1006.32 \\
5.73000 & 513.82 & 1053.95 \\
6.69000 & 483.00 & 1105.79 \\
8.12000 & 450.59 & 1170.40 \\
9.56000 & 428.68 & 1225.35 \\
14.30000 & 392.67 & 1361.97 \\
19.10000 & 378.03 & 1461.12 \\
28.60000 & 362.81 & 1598.12 \\
47.80000 & 350.13 & 1771.45 \\
95.60000 & 342.09 & 2004.77 \\
191.00000 & 331.21 & 2229.62
\end{tabular}

\begin{tabular}{rrr} 
TD $=20.0$ & EV & \multicolumn{2}{c}{ ET=1. } & 109643 MEV \\
ENERGY (HEV) & PRIRARY & CASCA DE \\
1.12000 & 2.29 & 2.29 \\
1.13000 & 4.96 & 4.96 \\
1.15000 & 11.48 & 11.48 \\
1.18000 & 23.77 & 23.77 \\
1.23000 & 49.13 & 49.13 \\
1.28000 & 78.33 & 78.33 \\
1.35000 & 122.15 & 122.15 \\
1.44000 & 178.87 & 178.87 \\
1.55000 & 243.69 & 243.69 \\
1.71000 & 323.86 & 323.86 \\
1.94000 & 408.35 & 410.50 \\
2.21000 & 469.80 & 483.93 \\
2.55000 & 508.29 & 550.75 \\
2.99000 & 521.85 & 613.18 \\
3.55000 & 512.19 & 671.23 \\
4.21000 & 487.96 & 723.10 \\
4.99000 & 457.54 & 771.53 \\
5.77000 & 430.62 & 811.56 \\
6.65000 & 405.88 & 850.05 \\
7.76000 & 381.86 & 891.85 \\
9.43000 & 356.58 & 944.40 \\
11.00000 & 340.59 & 986.22 \\
16.60000 & 312.45 & 1098.76 \\
22.10000 & 301.32 & 1177.40 \\
33.20000 & 289.30 & 1288.06 \\
55.40000 & 279.66 & 1426.61 \\
110.00000 & 273.40 & 1611.31 \\
221.00000 & 264.49 & 1792.36
\end{tabular}

\begin{tabular}{rrr} 
TD $=24.0$ & EV & \multicolumn{2}{c}{ ET $=1.249555$ HEV } \\
ENERGY (HEV) & PRIMARY & CASCADE \\
1.26000 & 1.48 & 1.48 \\
1.27000 & 3.22 & 3.22 \\
1.29000 & 7.58 & 7.58 \\
1.33000 & 19.21 & 19.21 \\
1.38000 & 37.75 & 37.75 \\
1.44000 & 63.69 & 63.69 \\
1.52000 & 101.20 & 101.20 \\
1.62000 & 148.56 & 148.56 \\
1.74000 & 201.80 & 201.80 \\
1.93000 & 273.04 & 273.04 \\
2.18000 & 341.18 & 343.20 \\
2.49000 & 393.03 & 406.04 \\
2.87000 & 423.78 & 461.54 \\
3.37000 & 433.80 & 514.24 \\
3.99000 & 424.72 & 562.10 \\
4.74000 & 403.60 & 606.08 \\
5.62000 & 377.90 & 646.83 \\
6.49000 & 355.69 & 680.15 \\
7.49000 & 335.07 & 712.82 \\
8.74000 & 315.42 & 747.90 \\
10.60000 & 295.05 & 791.57 \\
12.40000 & 281.85 & 827.26 \\
18.70000 & 259.37 & 921.39 \\
24.90000 & 250.36 & 987.08 \\
37.40000 & 240.51 & 1079.33 \\
62.40000 & 232.78 & 1194.95 \\
124.00000 & 227.60 & 1349.01 \\
249.00000 & 220.08 & 1499.56
\end{tabular}

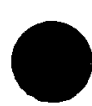


ATOMIC DISPLACENET CROSS-SECTIONS (BARAS) BY PAST ELECTRONS IN ES

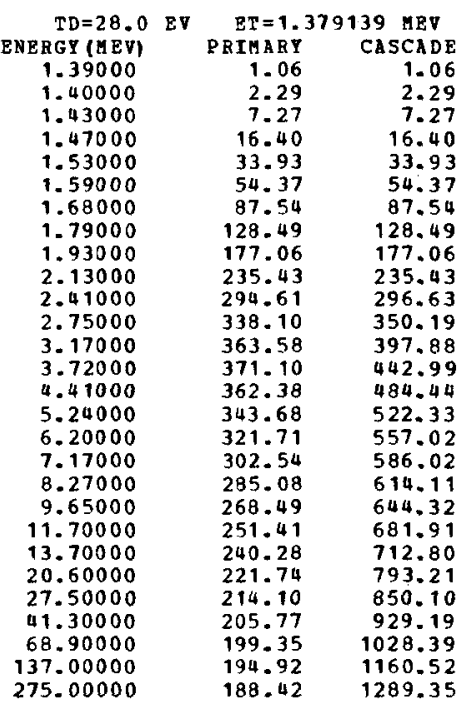

\begin{tabular}{crr}
$T D=32.0$ & \multicolumn{2}{c}{ ET $=1.500392$ KEV } \\
ENERGY (HEV) & PRIMABY & CASCADE \\
1.51000 & 0.67 & 0.67 \\
1.53000 & 2.60 & 2.60 \\
1.56000 & 6.74 & 6.74 \\
1.60000 & 14.12 & 14.12 \\
1.66000 & 28.17 & 28.17 \\
1.74000 & 50.32 & 50.32 \\
1.83000 & 77.38 & 77.38 \\
1.95000 & 113.67 & 113.67 \\
2.10000 & 155.87 & 155.87 \\
2.32000 & 207.74 & 207.74 \\
2.62000 & 258.71 & 260.65 \\
3.00000 & 297.25 & 308.79 \\
3.45000 & 318.37 & 349.80 \\
4.05000 & 324.19 & 389.40 \\
4.80000 & 315.93 & 425.70 \\
5.70000 & 299.26 & 458.85 \\
6.75000 & 279.81 & 489.49 \\
7.80000 & 263.17 & 514.83 \\
9.00000 & 247.96 & 539.60 \\
10.50000 & 233.63 & $\mathbf{5 6 6 . 1 1}$ \\
12.70000 & 219.10 & 598.72 \\
15.00000 & 209.05 & 627.31 \\
22.50000 & 193.47 & 697.48 \\
30.00000 & 186.95 & 747.09 \\
45.00000 & 179.77 & 816.11 \\
75.00000 & 174.31 & 902.83 \\
150.00000 & 170.40 & 1019.37 \\
300.00000 & 164.69 & 1131.38
\end{tabular}

\begin{tabular}{rrr} 
TD $=36.0$ & BV & \multicolumn{2}{c}{ ET $=1.614740$ HEV } \\
ENERGY (MEV) & PRIMARY & CASCADE \\
1.63000 & 0.86 & 0.86 \\
1.64000 & 1.61 & 1.61 \\
1.67000 & 4.67 & 4.67 \\
1.72000 & 11.97 & 11.97 \\
1.79000 & 25.56 & 25.56 \\
1.87000 & 44.02 & 44.02 \\
1.96000 & 66.53 & 66.53 \\
2.09000 & 99.36 & 99.36 \\
2.26000 & 139.29 & 139.29 \\
2.50000 & 186.17 & 186.18 \\
2.82000 & 230.94 & 232.82 \\
3.22000 & 264.35 & 274.88 \\
3.71000 & 283.10 & 311.96 \\
4.35000 & 287.82 & 346.99 \\
5.16000 & 280.07 & 379.51 \\
6.13000 & 264.98 & 409.14 \\
7.26000 & 247.59 & 436.48 \\
8.39000 & 232.83 & 459.11 \\
9.68000 & 219.40 & 481.20 \\
11.30000 & 206.74 & 504.92 \\
13.70000 & 193.86 & 534.38 \\
16.10000 & 185.34 & 559.08 \\
24.20000 & 171.67 & 621.91 \\
32.20000 & 165.98 & 665.73 \\
48.40000 & 159.62 & 727.42 \\
80.70000 & 154.86 & 804.62 \\
161.00000 & 151.40 & 907.82 \\
322.00000 & 146.30 & 1007.33
\end{tabular}
ATONIC DISPLACBHENT CROSS-SECTIONS (BARNS) BY PAST BLECTRONS IN ES

$\begin{array}{rrr}\text { TD }=40.0 & \text { EV } & \text { ET=1.723243 REV } \\ \text { ENERGY (MEV) } & \text { PRIMARY } & \text { CASCADE } \\ 1.74000 & 0.75 & 0.75 \\ 1.75000 & 1.36 & 1.36 \\ 1.79000 & 4.87 & 4.87 \\ 1.84000 & 11.24 & 11.24 \\ 1.91000 & 22.82 & 22.82 \\ 1.99000 & 38.44 & 38.44 \\ 2.10000 & 61.82 & 61.82 \\ 2.24000 & 91.83 & 91.83 \\ 2.41000 & 125.64 & 125.64 \\ 2.67000 & 168.64 & 168.65 \\ 3.01000 & 208.66 & 210.49 \\ 3.44000 & 238.61 & 248.60 \\ 3.96000 & 254.94 & 281.77 \\ 4.65000 & 258.72 & 313.58 \\ 5.51000 & 251.38 & 342.69 \\ 6.54000 & 237.67 & 369.26 \\ 7.75000 & 221.90 & 394.03 \\ 8.96000 & 208.61 & 414.50 \\ 10.30000 & 196.89 & 433.95 \\ 12.00000 & 185.69 & 455.08 \\ 14.60000 & 173.98 & 482.16 \\ 17.20000 & 166.30 & 504.76 \\ 25.80000 & 154.28 & 561.13 \\ 34.40000 & 149.18 & 600.88 \\ 51.60000 & 143.53 & 656.13 \\ 86.10000 & 139.31 & 725.76 \\ 172.00000 & 135.19 & 818.79\end{array}$

\begin{tabular}{rrr} 
TD=44.0 & \multicolumn{1}{c}{ ET } & \multicolumn{1}{c}{ ET=1.826716 MEV } \\
ENERGY (AEV) & PRIMAR & CASCA DE \\
1.84000 & 0.45 & 0.45 \\
1.86000 & 1.46 & 1.46 \\
1.89000 & 3.66 & 3.66 \\
1.95000 & 10.04 & 10.04 \\
2.02000 & 19.93 & 19.93 \\
2.11000 & 35.04 & 35.04 \\
2.22000 & 55.23 & 55.23 \\
2.37000 & 83.02 & 83.02 \\
2.55000 & 114.00 & 114.00 \\
2.83000 & 153.93 & 153.95 \\
3.19000 & 190.26 & 192.02 \\
3.65000 & 217.48 & 226.99 \\
4.20000 & 231.90 & 257.04 \\
4.93000 & 234.96 & 285.90 \\
5.84000 & 228.03 & 312.34 \\
6.94000 & 215.32 & 336.70 \\
8.22000 & 200.97 & 359.20 \\
9.49000 & 189.03 & 377.67 \\
10.90000 & 178.48 & 395.29 \\
12.70000 & 168.35 & 414.56 \\
15.50000 & 157.65 & 439.62 \\
18.20000 & 150.90 & 459.78 \\
27.40000 & 140.03 & 511.57 \\
36.50000 & 135.46 & 547.62 \\
54.80000 & 130.35 & 597.96 \\
91.30000 & 126.60 & 661.10 \\
182.00000 & 123.77 & 745.40
\end{tabular}

\begin{tabular}{rrr} 
TD=48.0 & \multicolumn{1}{c}{ EV } & \multicolumn{1}{c}{ ET $=1.925799$ MEV } \\
ENERGY (HEY) & PRIHARY & CASCADE \\
1.94000 & 0.40 & 0.40 \\
1.96000 & 1.25 & 1.25 \\
2.00000 & 3.85 & 3.85 \\
2.06000 & 9.58 & 9.58 \\
2.13000 & 18.28 & 18.28 \\
2.23000 & 33.01 & 33.01 \\
2.34000 & 50.71 & 50.71 \\
2.50000 & 76.65 & 76.65 \\
2.69000 & 105.20 & 105.20 \\
2.98000 & 141.28 & 141.30 \\
3.37000 & 175.41 & 177.17 \\
3.85000 & 199.77 & 208.82 \\
4.42000 & 212.57 & 235.94 \\
5.19000 & 215.22 & 262.51 \\
6.16000 & 208.59 & 287.05 \\
7.31000 & 196.91 & 309.24 \\
8.66000 & 183.70 & 329.93 \\
10.00000 & 172.76 & 346.92 \\
11.50000 & 163.05 & 363.26 \\
13.40000 & 153.84 & 380.95 \\
16.30000 & 144.27 & 403.59 \\
19.20000 & 138.02 & 422.46 \\
28.80000 & 128.27 & 469.58 \\
38.50000 & 124.05 & 503.04 \\
57.70000 & 119.42 & 548.99 \\
96.20000 & 116.01 & 606.98 \\
192.00000 & 113.41 & 684.37
\end{tabular}


ATONIC DISPLACEHENT CROSS-SFCTIONS (BARNS) BY FAST RLECTRONS IN ES $Z=99 \quad A=254.000$

$\begin{array}{rrr}\begin{array}{c}\text { TD=52.0 } \\ \text { ENERGY (MEV) }\end{array} & \begin{array}{c}\text { ET }=2.021007 \text { MEV } \\ \text { PRIHART }\end{array} & \text { CASCADE } \\ 2.04000 & 0.47 & 0.47 \\ 2.06000 & 1.25 & 1.25 \\ 2.10000 & 3.57 & 3.57 \\ 2.16000 & 8.60 & 8.60 \\ 2.24000 & 17.40 & 17.40 \\ 2.34000 & 30.48 & 30.48 \\ 2.46000 & 47.57 & 47.57 \\ 2.62000 & 70.53 & 70.53 \\ 2.82000 & 97.13 & 97.13 \\ 3.13000 & 131.14 & 131.16 \\ 3.53000 & 161.95 & 163.61 \\ 4.04000 & 184.68 & 193.27 \\ 4.64000 & 196.32 & 218.38 \\ 5.45000 & 198.50 & 242.94 \\ 6.46000 & 192.25 & 265.44 \\ 7.67000 & 181.34 & 286.01 \\ 9.09000 & 169.09 & 305.18 \\ 10.50000 & 158.99 & 320.93 \\ 12.10000 & 149.95 & 336.27 \\ 14.10000 & 141.51 & 352.63 \\ 17.10000 & 132.90 & 373.24 \\ 20.20000 & 127.10 & 390.98 \\ 30.30000 & 118.24 & 434.53 \\ 40.40000 & 114.41 & 465.15 \\ 60.60000 & 110.16 & 507.66 \\ 101.00000 & 107.05 & 561.17 \\ 202.00000 & 104.64 & 632.81\end{array}$

\begin{tabular}{rrr} 
TD $=56.0$ & EV & \multicolumn{2}{c}{ ET=2.112763 HEV } \\
BNERGY (ABV) & PRIMRY & CASCADE \\
2.13000 & 0.35 & 0.35 \\
2.15000 & 0.99 & 0.99 \\
2.19000 & 2.94 & 2.94 \\
2.26000 & 8.10 & 8.10 \\
2.34000 & 15.96 & 15.96 \\
2.45000 & 28.82 & 28.82 \\
2.57000 & 44.09 & 44.09 \\
2.74000 & 65.86 & 65.86 \\
2.95000 & 90.75 & 90.75 \\
3.27000 & 122.00 & 122.03 \\
3.69000 & 150.74 & 152.34 \\
4.22000 & 171.64 & 179.77 \\
4.85000 & 182.35 & 203.21 \\
5.70000 & 184.18 & 226.11 \\
6.76000 & 178.19 & 247.06 \\
8.02000 & 168.02 & 266.09 \\
9.50000 & 156.65 & 283.87 \\
10.90000 & 147.71 & 297.84 \\
12.60000 & 139.12 & 312.41 \\
14.70000 & 131.24 & 327.75 \\
17.90000 & 123.13 & 347.32 \\
21.10000 & 117.86 & 363.60 \\
31.60000 & 109.73 & 403.91 \\
42.20000 & 106.17 & 432.52 \\
63.30000 & 102.24 & 472.00 \\
105.00000 & 99.40 & 521.24 \\
211.00000 & 97.14 & 588.21
\end{tabular}

\begin{tabular}{rrr} 
TD $=60.0$ & BV & \multicolumn{2}{c}{ ET=2.201417 REV } \\
ENERGY(HEV) & PRIMARY & CASCA.DE \\
2.22000 & 0.33 & 0.33 \\
2.24000 & 0.89 & 0.89 \\
2.28000 & 2.61 & 2.61 \\
2.35000 & 7.15 & 7.15 \\
2.44000 & 15.06 & 15.06 \\
2.55000 & 26.63 & 26.63 \\
2.68000 & 41.53 & 41.53 \\
2.86300 & 62.25 & 62.25 \\
3.08000 & 85.62 & 85.62 \\
3.41000 & 114.43 & 114.46 \\
3.85000 & 141.26 & 142.85 \\
4.40000 & 160.47 & 168.28 \\
5.06000 & 170.30 & 190.24 \\
5.94000 & 171.79 & 211.44 \\
7.04000 & 166.09 & 230.93 \\
8.36000 & 156.49 & 248.81 \\
9.90000 & 145.88 & 265.39 \\
11.40000 & 137.33 & 278.79 \\
13.20000 & 129.29 & 292.57 \\
15.40000 & 122.01 & 306.89 \\
18.70000 & 114.64 & 324.93 \\
22.00000 & 109.83 & 339.94 \\
33.00000 & 102.31 & 377.75 \\
44.00000 & 99.03 & 404.31 \\
66.00000 & 95.37 & 441.17 \\
110.00000 & 92.73 & 487.57 \\
220.00000 & 90.63 & 549.63
\end{tabular}

ATOATC DISPLACEHENT CROSS-SECTIONS (BARNS) BY PAST ELECTRONS IN ES $z=99 \quad A=254.000$

\begin{tabular}{crr} 
TD $=64.0$ & EV & \multicolumn{1}{c}{ ET $=2.287263$} \\
ENERGY (AEV) & PRIFARY & CASCADE \\
2.31000 & 0.37 & 0.37 \\
2.33000 & 0.90 & 0.90 \\
2.37000 & 2.47 & 2.47 \\
2.44000 & 6.56 & 6.56 \\
2.53000 & 13.66 & 13.66 \\
2.65000 & 25.07 & 25.07 \\
2.79000 & 39.62 & 39.62 \\
2.97000 & 58.40 & 58.40 \\
3.20000 & 80.52 & 80.52 \\
3.54000 & 107.38 & 107.41 \\
4.00000 & 132.70 & 134.24 \\
4.57000 & 150.59 & 158.04 \\
5.26000 & 159.72 & 178.75 \\
6.17000 & 160.96 & 198.53 \\
7.31000 & 155.54 & 216.78 \\
8.69000 & 146.42 & 233.65 \\
10.20000 & 136.98 & 248.41 \\
11.80000 & 128.69 & 261.41 \\
13.70000 & 121.02 & 274.59 \\
16.00000 & 114.18 & 288.12 \\
19.40000 & 107.36 & 304.92 \\
22.80000 & 102.90 & 318.92 \\
34.30000 & 95.84 & 354.65 \\
45.70000 & 92.78 & 379.49 \\
68.60000 & 89.36 & 414.11 \\
114.00000 & 86.93 & 457.37 \\
228.00000 & 84.96 & 515.56 \\
& &
\end{tabular}

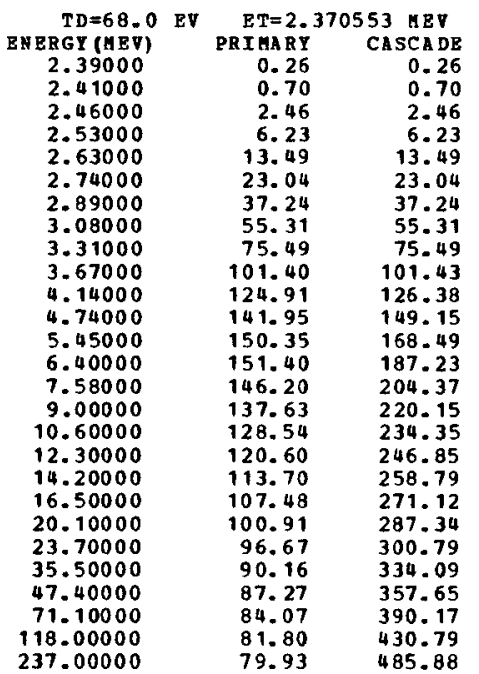

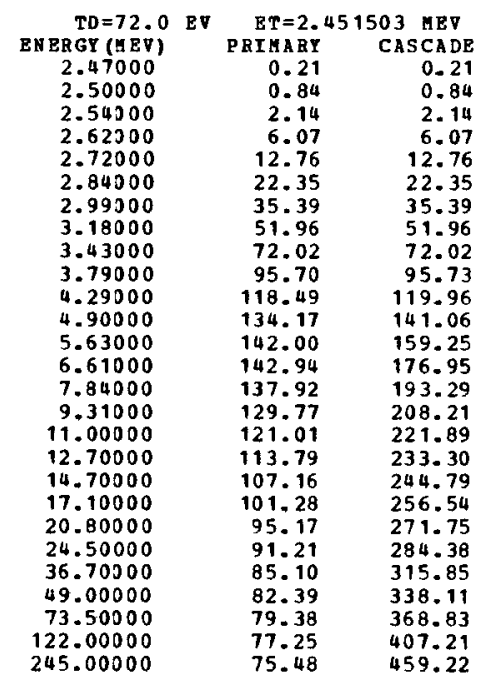


ATOMIC DISPLAZEHENT CROSS-SECTIONS (BARNS) BY FAST ELECTRONS IN ES

\begin{tabular}{|c|c|c|c|}
\hline $\begin{array}{r}T D=76.0 \\
\text { ENERGY (MEV) }\end{array}$ & EV & $\begin{array}{r}\text { ET }=2.5 \\
\text { PRIMARY }\end{array}$ & $\begin{array}{l}298 \text { ИBV } \\
\text { CASCADE }\end{array}$ \\
\hline & & & \\
\hline 2.58000 & & 0.20 & $\begin{array}{l}0.20 \\
0.77\end{array}$ \\
\hline 2.63000 & & 2.32 & 2.32 \\
\hline 2.70000 & & 5.52 & 5.52 \\
\hline 2.80000 & & 11.59 & 11.59 \\
\hline 2.93000 & & 21.11 & 21.11 \\
\hline 3.08000 & & 33.11 & 33.11 \\
\hline 3.28000 & & 49.19 & 49.19 \\
\hline 3.54000 & & 68.40 & 68.40 \\
\hline 3.92000 & & 91.30 & 91.34 \\
\hline 4.42000 & & 112.19 & 113.57 \\
\hline 5.06000 & & 127.26 & 133.93 \\
\hline 5.81000 & & 134.55 & 151.07 \\
\hline 6.83000 & & 135.34 & 167.96 \\
\hline 8.09000 & & 130.54 & 183.32 \\
\hline 9.61000 & & 122.77 & 197.49 \\
\hline 11.30000 & & 114.70 & 210.09 \\
\hline 13.10000 & & 107.66 & 221.23 \\
\hline $\begin{array}{l}15.10000 \\
17.70000\end{array}$ & & $\begin{array}{r}101.56 \\
95.72\end{array}$ & $\begin{array}{l}231.82 \\
243.52\end{array}$ \\
\hline 21.50000 & & 90.02 & 257.84 \\
\hline 25.30000 & & 86.32 & 269.74 \\
\hline 37.90000 & & 80.58 & 299.58 \\
\hline 50.60000 & & 78.01 & $\begin{array}{r}320.66 \\
349.76\end{array}$ \\
\hline 126.00000 & & 73.17 & 386.14 \\
\hline 253.00000 & & 71.49 & 435.40 \\
\hline
\end{tabular}

\begin{tabular}{crr} 
TD $=80.0$ & EV & \multicolumn{2}{c}{ ET $=2.607103$ MEV } \\
ENERGY (HEV) & PRIMARY & CASCADE \\
2.63000 & 0.22 & 0.22 \\
2.65000 & 0.55 & 0.55 \\
2.71000 & 2.20 & 2.20 \\
2.78000 & 5.14 & 5.14 \\
2.89000 & 11.33 & 11.33 \\
3.02000 & 20.17 & 20.17 \\
3.18000 & 32.03 & 32.03 \\
3.38000 & 46.89 & 46.89 \\
3.64000 & 64.68 & 64.68 \\
4.04000 & 86.97 & 87.01 \\
4.56000 & 106.92 & 108.30 \\
5.21000 & 120.95 & 127.35 \\
5.99000 & 127.87 & 143.78 \\
7.03000 & 128.53 & 159.67 \\
8.34000 & 123.87 & 174.40 \\
9.90000 & 116.49 & 187.81 \\
11.70000 & 108.58 & 200.16 \\
13.50000 & 102.12 & $\mathbf{2 1 0 . 4 0}$ \\
15.60000 & 96.25 & 220.65 \\
18.20000 & 90.89 & 231.44 \\
22.10000 & 85.48 & 245.03 \\
26.00000 & 81.98 & 256.33 \\
39.10000 & 76.50 & 284.96 \\
52.10000 & 74.09 & $\mathbf{3 0 4} .85$ \\
78.20000 & 71.39 & $\mathbf{3 3 2 . 5 5}$ \\
130.00000 & 69.50 & $\mathbf{3 6 7 . 2 1}$ \\
260.00000 & 67.91 & 413.73 \\
& &
\end{tabular}

\begin{tabular}{rrr}
$\begin{array}{r}\text { TD }=34.0 \\
\text { ENER }\end{array}$ & \multicolumn{1}{c}{ ET=2.682061 MEV } \\
PRIARY & CASCADE \\
2.70000 & 0.14 & 0.14 \\
2.73000 & 0.59 & 0.59 \\
2.78000 & 1.83 & 1.83 \\
2.86000 & 4.89 & 4.89 \\
2.97000 & 10.62 & 10.62 \\
3.11000 & 19.46 & 19.46 \\
3.27000 & 30.48 & 30.48 \\
3.48000 & 44.96 & 44.96 \\
3.75000 & 62.06 & 62.06 \\
4.15000 & 82.73 & 82.76 \\
4.69000 & 101.94 & 103.28 \\
5.36000 & 115.29 & 121.46 \\
6.16000 & 121.79 & 137.08 \\
7.24000 & 122.34 & 152.33 \\
8.58000 & 117.87 & 166.27 \\
10.10000 & 111.19 & 178.45 \\
12.00000 & 103.41 & 190.61 \\
13.90000 & 97.09 & 200.64 \\
16.00000 & 91.66 & 210.15 \\
18.70000 & 86.50 & 220.55 \\
22.70000 & 81.37 & 233.48 \\
26.80000 & 77.98 & 244.48 \\
40.20000 & 72.83 & 271.59 \\
53.60000 & 70.53 & 290.58 \\
80.40000 & 67.97 & $\mathbf{3 1 6 . 9 2}$ \\
134.00000 & 66.17 & 350.10 \\
268.00000 & 64.66 & 394.40 \\
& &
\end{tabular}

ATOMIC DISPLACEMENT CROSS-SECTIONS (BARNS) BY PAST RLECTRONS IN ES $\mathrm{Z}=99 \quad \mathrm{~A}=\mathbf{2 5 4 . 0 0 0}$

\begin{tabular}{rrr} 
TD=88.0 & \multicolumn{1}{c}{ ET $=2.755299$ MEV } \\
ENERGY (MEV) & $\begin{array}{r}\text { RRIMARY } \\
2.78000\end{array}$ & $\begin{array}{c}\text { CASCADE } \\
2.20\end{array}$ \\
2.81000 & 0.65 & 0.65 \\
2.86000 & 1.86 & 1.86 \\
2.94000 & 4.74 & 4.74 \\
3.05000 & 10.09 & 10.09 \\
3.19000 & 18.30 & 18.30 \\
3.36000 & 29.20 & 29.20 \\
3.58000 & 43.33 & 43.33 \\
3.85000 & 59.25 & 59.25 \\
4.27000 & 79.39 & 79.43 \\
4.82000 & 97.49 & 98.81 \\
5.51000 & 110.17 & 116.18 \\
6.33000 & 116.28 & 131.03 \\
7.43000 & 116.75 & 145.48 \\
8.81000 & 112.44 & 158.84 \\
10.40000 & 105.92 & 170.66 \\
12.30000 & 98.68 & 181.96 \\
14.30000 & 92.51 & 191.79 \\
16.50000 & 87.26 & 201.02 \\
19.20000 & 82.49 & 210.68 \\
23.40000 & 77.52 & 223.29 \\
27.50000 & 74.40 & 233.50 \\
41.30000 & 69.48 & 259.46 \\
55.10000 & 67.30 & 277.63 \\
82.60000 & 64.87 & 302.74 \\
137.00000 & 63.17 & 334.11 \\
275.00000 & 61.71 & 376.62
\end{tabular}

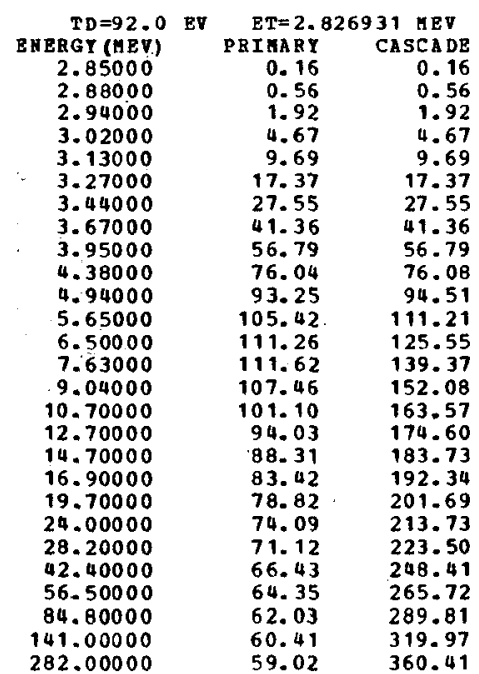

\begin{tabular}{rrr}
$\begin{array}{c}\text { TD=96.0 } \\
\text { EN }\end{array}$ & \multicolumn{2}{c}{ ET $=2.897057$ MEV } \\
PNERGY (HEV) & PRIMARY & CASCADE \\
2.92000 & 0.15 & 0.15 \\
2.95000 & 0.51 & 0.51 \\
3.01000 & 1.76 & 1.76 \\
3.09000 & 4.29 & 4.29 \\
3.21000 & 9.41 & 9.41 \\
3.36000 & 17.16 & 17.16 \\
3.53000 & 26.72 & 26.72 \\
3.76000 & 39.67 & 39.67 \\
4.05000 & 54.63 & 54.63 \\
4.49000 & 73.04 & 73.08 \\
5.06000 & 89.41 & 90.63 \\
5.79000 & 101.09 & 106.70 \\
6.66000 & 106.64 & 120.44 \\
7.82000 & 106.93 & 133.69 \\
9.27000 & 102.89 & 145.91 \\
11.00000 & 96.67 & 157.09 \\
13.00000 & 90.06 & 167.40 \\
15.00000 & 84.69 & 175.96 \\
17.30000 & 79.90 & 184.41 \\
20.20000 & 75.45 & 193.47 \\
24.60000 & 70.95 & 205.00 \\
28.90000 & 68.12 & 214.35 \\
43.40000 & 63.65 & 238.16 \\
57.900000 & 61.65 & 254.82 \\
86.90000 & 59.43 & 277.90 \\
144.00000 & 57.89 & 306.62 \\
289.00000 & 56.56 & 345.57
\end{tabular}


FAMTO OF MOTT TO ROTHERFOPD STATMERTNG TN H, $\mathrm{z}=1$

$\begin{array}{cc}\text { ENERGY (MEV) } & 100 \\ \text { ANGLE (DEG.) } & \\ 0 & 1.0000 \\ 5 & 0.9990 \\ 10 & 0.9943 \\ 15 & 0.9856 \\ 20 & 0.9732 \\ 25 & 0.9571 \\ 30 & 0.9375 \\ 35 & 0.9144 \\ 40 & 0.8882 \\ 45 & 0.8590 \\ 50 & 0.8271 \\ 55 & 0.7926 \\ 60 & 0.7558 \\ 65 & 0.7171 \\ 70 & 0.6767 \\ 75 & 0.6350 \\ 80 & 0.5922 \\ 85 & 0.5487 \\ 90 & 0.5048 \\ 95 & 0.4609 \\ 100 & 0.4174 \\ 105 & 0.3744 \\ 110 & 0.3325 \\ 115 & 0.2918 \\ 120 & 0.2527 \\ 125 & 0.2156 \\ 130 & 0.1806 \\ 135 & 0.1481 \\ 140 & 0.1183 \\ 145 & 0.0915 \\ 150 & 0.0678 \\ 155 & 0.0474 \\ 160 & 0.0305 \\ 165 & 0.0173 \\ 170 & 0.0077 \\ 175 & 0.0019 \\ 180 & 0.0000 \\ & \end{array}$

10.
1.0000
0.9990
0.9943
0.9856
0.9732
0.9572
0.9376
0.9147
0.8885
0.8594
0.8275
0.7931
0.7564
0.7178
0.6775
0.6358
0.5931
0.5497
0.5060
0.4622
0.0187
0.3759
0.3340
0.2934
0.2545
0.2174
0.1825
0.1501
0.1204
0.0936
0.0700
0.0496
0.0328
0.0196
0.0100
0.0043
0.0024

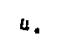

1.0000

0.9990
0.9943

0.9943

0.9858
0.9735

0.9576
0.9383

0.9156
0.8897

0.8897

0.8609
0.8293

0.7953

0.7590

0.6809

0.6397

0.5974

0.5545
0.5112

0.4679

0.4248
0.3825

0.3825

0.3410

0.2623

0.2256

0.1911
0.1590

0.1296

0.1031

0.0596

0.0596

0.0429
0.0298

0.0298

0.0204

\begin{abstract}
2.
\end{abstract}
1.0000

0.9991

0.9945
0.9862

0.9743

0.9589

0.9401
0.9181

0.8930

0.8650

0.8343

0.8013
0.7660

0.7289

0.6902

0.6502

0.6092
0.5675

0.5254

0.4833
0.4416

0.4416
0.4004

0.3602

0.3212

0.2481

0.2146

0.1548

0.1291

0.1064

0.0869

0.0869
0.070579

0.0488

0.0433
0.0414

$$
1.5
$$

1.0000

0.0991
0.9947

0.9947

0.9750

0.9600
0.9416

0.9416
0.0201

0.8955

0.8683

0.8061

0.7718

0.7355
0.6977
0.6587

0.6187

0.5780

0.5369

0.455

0.1149

0.3757

0.3010

0.2663

0.2336
0.2031

0.1753

0.1502

0.1280

0.1089

0.0931
0.0807

0.0807
0.0718

0.0664
0.0646
1.0

1.0000

0.9992

0.9950
0.9874

0.9764

0.9622

0.9245

0.9013

0.8755

0.8166

0.7841

0.7140

0.6770

0.6006

0.6006
0.5617
0.5228

0.5228
0.4842

0.4842
0.4462

0.0090
0.3729

0.3383

0.3054

0.2744

0.2192

0.1954

0.1564

0.1414

0.1414
0.1297

0.1212

0.1161
0.1144

$$
0.8
$$

1.0000

0.9992
0.9953

0.0880

0.9775

0.9639

0.9473
0.9278

0.9056

0.8808

0.8537

0.8245

0.7933

0.7262
0.75908

0.6545

0.6176

0.5804
0.5432

0.5062

0.4697

0.4341
0.3996
0.3664

0.3664

0.3349

0.3052

0.2776

0.2296

0.2095

0.1922
0.1778

0.1778
0.1666

0.1585

0.1536
0.1519
0.6

1.0000

0.9993

0.9957

0.9889

0.9792
0.9565

0.9511
0.9330

0.9124

0.8894

0.8642

0.8080

0.7775

0.7457

0.7127

0.51447

0.6191

0.5754

0.5410

0.5071
0.4740

0.4419

0.4111

0.3817

0.354

0.3285

0.2838

0.2651

0.2490

0.2357

0.2252
0.2176

0.2131
0.2116
0.1

1.0000 0.9995

0.9963

0.9905

0.9711

0.9578
0.9429

0.9242

0.9042

0.8823

$0.858^{7}$

0.8335

0.7792

0.7792

0.7506

0.6914

0.6613

0.6013

0.5718

0.5430

0.5151

0.4882

0.4627

0.4387

0.3959

0.3775

0.3612

0.3356

0.3265

0.3199

0.3160
0.3147
0.2

1.0000

0.9997

0.9976
0.9936

0.9877

0.9809

0.9707

0.9597

0.99330

0.9176

0.9009

0.8645

0.8449

0.8247

0.8040

0.7829

0.7616
0.7004

0.7192

0.5984

0.6780

0.6583

0.6213

0.6043

0.5885

0.5744

0.574

0.5610

0.5305

0.5314

0.5250

0.5203

0.5175
0.5166
0.1

1.2300 0.9909 0.9963 0 0.9823 0.9755 0.9677 0.9590 0.9309 0.9281 0.9164 0.9917 0.8789 0.8657 0.8391 0.8263 0.8130 0.3203 0.7880
0.7761 0.7649 0.7144 0.7354 0.7201 0.7130 0.7588 0.75 $0 .-001$ $0.699=$

RATTO OF MOTT TO RUTHFRFORD SCATTERING JN FE, $z=2$

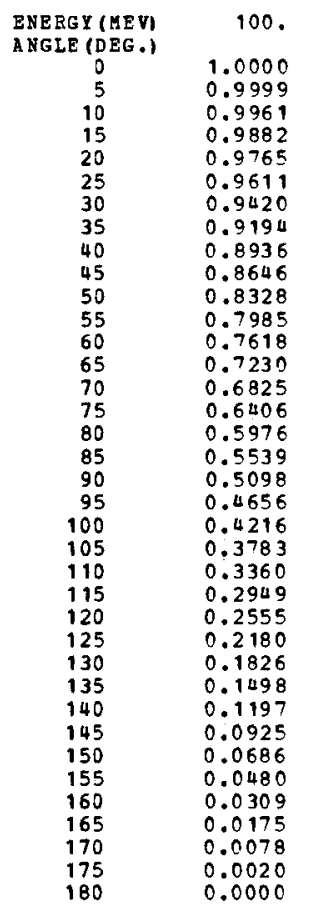

10. 
RATIO OF MOTT TO PUTHEPFOFD SCATIFRING IN BE, $z=4$

\begin{tabular}{|c|c|c|c|c|c|c|c|c|c|c|c|}
\hline $\begin{array}{l}\text { ENERGY (MEV) } \\
\text { ANGLE (DEG.) }\end{array}$ & 100. & 10. & 4. & 2. & 1.5 & 1.0 & 0.8 & 0.6 & 0.4 & 0.2 & 0.1 \\
\hline 0 & 1.0000 & 1.0000 & 1.0000 & 1.0000 & 1.0000 & 1.0000 & 1.0000 & 1.0000 & 1.0000 & 9.0000 & 1.3000 \\
\hline $\begin{array}{r}5 \\
10\end{array}$ & 1.0018 & $\begin{array}{r}1.0018 \\
0.9999\end{array}$ & $\begin{array}{l}1.0018 \\
1.0000\end{array}$ & 1.0018 & $\begin{array}{l}1.0018 \\
1.000 ?\end{array}$ & $\begin{array}{l}1.0048 \\
1.0003\end{array}$ & $\begin{array}{l}1.0018 \\
1.0005\end{array}$ & $\begin{array}{l}1.0018 \\
1.0007\end{array}$ & $\begin{array}{l}1.0078 \\
1.0010\end{array}$ & $\begin{array}{l}1.0017 \\
1.0016\end{array}$ & $\begin{array}{l}1.0015 \\
1.0019\end{array}$ \\
\hline $\begin{array}{l}10 \\
15\end{array}$ & $\begin{array}{l}0.9999 \\
0.9936\end{array}$ & $\begin{array}{l}0.9999 \\
0.9936\end{array}$ & $\begin{array}{l}1.0000 \\
0.9938\end{array}$ & $\begin{array}{l}1.0001 \\
0.9041\end{array}$ & $\begin{array}{l}1.0002 \\
0.9044\end{array}$ & $\begin{array}{l}1.0003 \\
0.9949\end{array}$ & $\begin{array}{l}1.0005 \\
0.9954\end{array}$ & $\begin{array}{l}1.0007 \\
0.9960\end{array}$ & $\begin{array}{l}1.0010 \\
0.9972\end{array}$ & $\begin{array}{l}1.0016 \\
0.9902\end{array}$ & $\begin{array}{l}1.0019 \\
1.0008\end{array}$ \\
\hline 20 & 0.9834 & 0.9835 & 0.9837 & 0.9844 & 0.9849 & 0.9861 & 0.9870 & 0.9883 & 0.9006 & 0.9950 & 0.9985 \\
\hline 25 & 0.9692 & 0.9693 & 0.9697 & 0.9708 & 0.9717 & 0.9736 & 0.9759 & 0.9774 & 0.9813 & 0.9896 & 0.9940 \\
\hline 30 & 0.9513 & 0.9514 & 0.9520 & 0.9537 & 0.9550 & 0.9579 & 0.9600 & 0.9634 & 0.9693 & 0.9905 & 0.9901 \\
\hline 35 & 0.9296 & 0.9298 & 0.9306 & 0.9329 & 0.0348 & 0.9388 & 0.9418 & 0.9466 & 0.9547 & 0.9704 & 0.9841 \\
\hline 40 & 0.9045 & 0.9048 & 0.9059 & 0.9089 & 0.9114 & 0.9167 & 0.9207 & 0.9269 & 0.9377 & 0.9586 & 0.9769 \\
\hline 45 & 0.8762 & 0.8765 & 0.8779 & 0.8818 & 0.8849 & 0.8915 & 0.8967 & 0.9047 & 0.9185 & 0.9452 & $0.968^{7}$ \\
\hline 50 & 0.8448 & 0.8452 & 0.8470 & 0.8517 & 0.8556 & 0.8630 & 0.8702 & 0.8801 & 0.8971 & 0.9302 & 0.9595 \\
\hline 55 & 0.8107 & 0.8111 & 0.8133 & 0.8190 & 0.8237 & 0.8337 & 0.8412 & 0.8532 & 0.8738 & 0.9438 & 0.9404 \\
\hline 60 & 0.7741 & 0.7746 & 0.7771 & 0.7839 & 0.7894 & 0.8013 & 0.8102 & 0.8244 & 0.8487 & 0.8962 & 0.9385 \\
\hline 65 & 0.7353 & 0.7359 & 0.7388 & 0.7467 & 0.7532 & 0.7669 & 0.7773 & 0.7938 & 0.8222 & 0.8774 & 0.9269 \\
\hline 70 & 0.6946 & 0.6954 & 0.6987 & 0.7078 & 0.7151 & 0.7309 & 0.7428 & 0.7617 & 0.7943 & 0.8577 & 0.9146 \\
\hline 75 & 0.6524 & 0.6533 & 0.6570 & 0.6673 & 0.6756 & 0.6935 & $0.70>0$ & 0.7284 & $0 .{ }^{-7} 653$ & 0.8372 & 0.9018 \\
\hline 80 & 0.6090 & 0.6100 & 0.6142 & 0.6257 & 0.6350 & 0.6551 & 0.5702 & 0.6941 & 0.7354 & 0.8161 & $0 . B B 8 F$ \\
\hline 85 & 0.5648 & 0.5659 & 0.5705 & 0.5833 & 0.5936 & 0.6158 & 0.6326 & 0.6591 & 0.7050 & 0.7945 & 0.8751 \\
\hline 90 & 0.5201 & 0.5212 & 0.5264 & 0.5104 & 0.5517 & 0.5762 & 0.5946 & 0.5238 & 0.67112 & 0.7727 & 0.8514 \\
\hline 95 & 0.4752 & 0.4765 & 0.4821 & 0.0994 & 0.5098 & 0.5364 & 0.5564 & 0.5883 & 0.6433 & 0.7507 & 0.8476 \\
\hline 100 & 0.4306 & 0.4379 & 0.4380 & 0.4545 & 0.4679 & 0.4968 & 0.5185 & 0.5530 & $0.612^{5}$ & 0.7289 & 0.8330 \\
\hline 105 & 0.3865 & 0.3880 & 0.3945 & 0.4123 & $0.426^{7}$ & 0.4577 & 0.4810 & 0.5181 & 0.5821 & 0.7073 & 0.8203 \\
\hline 110 & 0.3434 & 0.3450 & 0.3519 & $0.3^{-09}$ & 0.3863 & 0.0194 & 0.0443 & 0.4839 & 0.5524 & 0.6951 & $0.30>0$ \\
\hline 115 & 0.3016 & 0.3032 & 0.3106 & 0.3308 & 0.3471 & 0.3823 & 0.4088 & 0.4508 & 0.5235 & 0.6656 & 0.7949 \\
\hline 120 & 0.2613 & 0.2631 & 0.2709 & 0.2922 & 0.3094 & 0.3465 & 0.3745 & 0.11189 & 0.4957 & 0.6458 & 0.7815 \\
\hline 125 & 0.2230 & 0.2248 & 0.2330 & 0.2554 & 0.2735 & 0.3125 & 0.3419 & 0.3886 & 0.4692 & 0.6270 & $0.7<98$ \\
\hline 130 & 0.1869 & 0.1888 & 0.1974 & 0.2208 & 0.2307 & 0.2805 & 0.3112 & 0.3600 & 0.4443 & 0.6093 & 0.7586 \\
\hline 135 & 0.1533 & 0.1553 & 0.1642 & 0.1886 & 0.2083 & 0.2507 & 0.2826 & 0.3334 & 0.4211 & 0.5928 & 0.7481 \\
\hline 140 & 0.1225 & 0.1246 & 0.1338 & 0.1590 & 0,1794 & 0.2233 & 0.2560 & 0.3090 & 0.3998 & 0.5776 & 0.7386 \\
\hline 145 & 0.0948 & 0.0969 & 0.1064 & 0.1324 & 0.1534 & 0.1987 & 0.2328 & $0.28>0$ & 0.3806 & 0.5640 & 0.7300 \\
\hline 150 & 0.0702 & 0.0724 & 0.0822 & 0.1088 & 0.1304 & 0.1769 & 0.2119 & 0.2675 & 0.3636 & $0.5 \times 19$ & 0.7223 \\
\hline 155 & 0.0491 & 0.0514 & 0.0613 & 0.0886 & 0,1107 & 0.1581 & 0.1939 & 0.2508 & 0.3491 & 0.5115 & 0.7158 \\
\hline 160 & 0.0316 & 0.0339 & 0.0441 & 0.0798 & 0.0943 & 0.1426 & 0.1791 & 0.2369 & 0.3370 & 0.5329 & 0.7903 \\
\hline 165 & 0.0179 & 0.0202 & 0.0305 & 0.0586 & 0,0814 & 0.1304 & 0.1674 & 0.2260 & 0.3274 & 0.5261 & 0.7051 \\
\hline 170 & 0.0080 & 0.0103 & 0.0207 & 0.0491 & 0.0721 & 0.1216 & 0.1589 & 0.2182 & 0.3206 & 0.5212 & 0.7030 \\
\hline 175 & 0.0020 & 0.0042 & 0.0148 & 0.0434 & 0.0665 & 0.1163 & 0.1538 & 0.2134 & $0.316^{5}$ & 0.5183 & ก. ᄀก11 \\
\hline 180 & 0.0000 & 0.0024 & 0.0129 & 0.0415 & 0.0647 & 0.1145 & 0.1521 & 0.2119 & 0.3151 & 0.5173 & 0.7005 \\
\hline
\end{tabular}

SATTC OF MOMT TC RUTHERFADD SCATTEPING IN $C, z=6$

ENE RGY (HEV)
BNGLE (DEG )
0
5
10
15
20
25
30
35
40
45
50
55
60
65
70
75
80
85
90
95
100
105
110
115
120
125
130
135
140
145
150
155
160
165
170
175
180

$\begin{array}{cccccc}100 . & 10 . & 4 . & 2 . & 1.5 & 1.0 \\ 1.0000 & 1.0000 & 1.0000 & 1.0000 & 1.0000 & 1.0700 \\ 1.0037 & 1.0037 & 1.0037 & 1.0037 & 1.0036 & 1.0036 \\ 1.0038 & 1.0038 & 1.0038 & 1.0039 & 1.0039 & 1.0040 \\ 0.9991 & 0.9997 & 0.9992 & 0.9995 & 0.9997 & 1.0001 \\ 0.9905 & 0.9906 & 0.9908 & 0.9913 & 0.9918 & 0.9928 \\ 0.9776 & 0.9777 & 0.9781 & 0.9791 & 0.9799 & 0.9816 \\ 0.9609 & 0.9610 & 0.9616 & 0.9631 & 0.9643 & 0.9670 \\ 0.9402 & 0.9404 & 0.9412 & 0.9433 & 0.9451 & 0.9488 \\ 0.9159 & 0.9162 & 0.9172 & 0.9201 & 0.9225 & 0.9275 \\ 0.8882 & 0.8885 & 0.8899 & 0.8936 & 0.8966 & 0.9030 \\ 0.8573 & 0.8577 & 0.8594 & 0.8640 & 0.8677 & 0.8757 \\ 0.8235 & 0.8239 & 0.8260 & 0.8316 & 0.8361 & 0.8458 \\ 0.7870 & 0.7875 & 0.7900 & 0.7966 & 0.8020 & 0.8135 \\ 0.7482 & 0.7488 & 0.7516 & 0.7594 & 0.7657 & 0.7791 \\ 0.7073 & 0.7081 & 0.7113 & 0.7203 & 0.7275 & 0.7430 \\ 0.6649 & 0.6657 & 0.6694 & 0.6795 & 0.5877 & 0.7053 \\ 0.6211 & 0.6220 & 0.6261 & 0.6375 & 0.6467 & 0.6665 \\ 0.5763 & 0.5773 & 0.5820 & 0.5946 & 0.6048 & 0.6268 \\ 0.5310 & 0.5321 & 0.5372 & 0.5511 & 0.5623 & 0.5865 \\ 0.4854 & 0.4867 & 0.4922 & 0.5074 & 0.5197 & 0.5461 \\ 0.4401 & 0.4414 & 0.4474 & 0.4639 & 0.41772 & 0.5058 \\ 0.3952 & 0.3967 & 0.4031 & 0.4208 & 0.4352 & 0.4660 \\ 0.3513 & 0.3528 & 0.3597 & 0.3787 & 0.3940 & 0.1269 \\ 0.3086 & 0.3102 & 0.3176 & 0.3377 & 0.3540 & 0.3890 \\ 0.2675 & 0.2692 & 0.2770 & 0.2983 & 0.3155 & 0.3525 \\ 0.2283 & 0.2302 & 0.2383 & 0.2607 & 0.2788 & 0.3177 \\ 0.1914 & 0.1933 & 0.2019 & 0.2253 & 0.22442 & 0.2849 \\ 0.1571 & 0.1591 & 0.1680 & 0.1923 & 0.2120 & 0.2544 \\ 0.1256 & 0.1276 & 0.1368 & 0.1620 & 0.1825 & 0.2264 \\ 0.0971 & 0.0992 & 0.1088 & 0.1347 & 0.1558 & 0.2011 \\ 0.0720 & 0.0742 & 0.0839 & 0.1106 & 0.1322 & 0.1787 \\ 0.0504 & 0.0526 & 0.0626 & 0.0899 & 0.1120 & 0.1595 \\ 0.0324 & 0.0347 & 0.0449 & 0.0727 & 0.0952 & 0.1436 \\ 0.0183 & 0.0206 & 0.0310 & 0.0591 & 0.0820 & 0.1310 \\ 0.0082 & 0.0105 & 0.0209 & 0.0494 & 0.0724 & 0.1220 \\ 0.0021 & 0.0044 & 0.0149 & 0.0435 & 0.0667 & 0.1166 \\ 0.0000 & 0.0024 & 0.0129 & 0.0416 & 0.0648 & 0.1148\end{array}$

$\begin{array}{cc}0.8 & 0.6 \\ 1.0000 & 1.0000 \\ 1.0036 & 1.0035 \\ 1.0040 & 1.0041 \\ 1.0005 & 1.0010 \\ 0.9935 & 0.9946 \\ 0.9829 & 0.9849 \\ 0.9689 & 0.9720 \\ 0.9516 & 0.9560 \\ 0.9312 & 0.9372 \\ 0.9078 & 0.9155 \\ 0.8817 & 0.8913 \\ 0.8531 & 0.8647 \\ 0.8222 & 0.8360 \\ 0.7893 & 0.8054 \\ 0.7546 & 0.7732 \\ 0.7186 & 0.7396 \\ 0.6813 & 0.7050 \\ 0.6433 & 0.6695 \\ 0.6047 & 0.6336 \\ 0.5660 & 0.5976 \\ 0.5274 & 0.5616 \\ 0.4892 & 0.5261 \\ 0.4518 & 0.4912 \\ 0.4154 & 0.4573 \\ 0.3804 & 0.4247 \\ 0.3471 & 0.3936 \\ 0.3156 & 0.3643 \\ 0.2863 & 0.3370 \\ 0.2595 & 0.3120 \\ 0.2352 & 0.2894 \\ 0.2138 & 0.2694 \\ 0.1954 & 0.2523 \\ 0.1801 & 0.2380 \\ 0.1681 & 0.2258 \\ 0.1594 & 0.2188 \\ 0.1542 & 0.2139 \\ 0.1524 & 0.2123\end{array}$

0.4
1.0000
1.0033
1.0042
1.0018
0.9065
0.9883
0.9773
0.9636
0.9473
0.9286
0.9076
0.8846
0.8596
0.8331
0.8050
0.7758
0.7457
0.7148
0.5835
0.5521
0.6207
0.5897
0.5593
0.5297
0.5012
0.4741
0.4485
0.4247
0.4028
0.3831
0.3657
0.3506
0.3382
0.3280
0.3214
0.3971
0.3157

0.2

0.1

1.0030

1.0043

1.0031

1.0000

0.9946

0.9946

0.9780
0.9668

0.9668
0.9538

0.9538
0.9392

$0.923 n$
0.9055

0.8868

0.8670

0.8463

0.8249
0.8030

C. 8030
0.7808

0.7584

ก. 7361

0.7140
0.6023

0.5023

0.6712

0.6509

0.6133

0.6133
0.5052

0.5806

0.5806
0.5665

0.5540

0.5433

0.5344

0.5274

0.5224

0.5193
0.5193

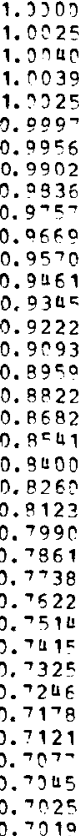


RATTO OF MOMT TO POTHESFORD SCRTTERING IN $0, \mathrm{z}=8$

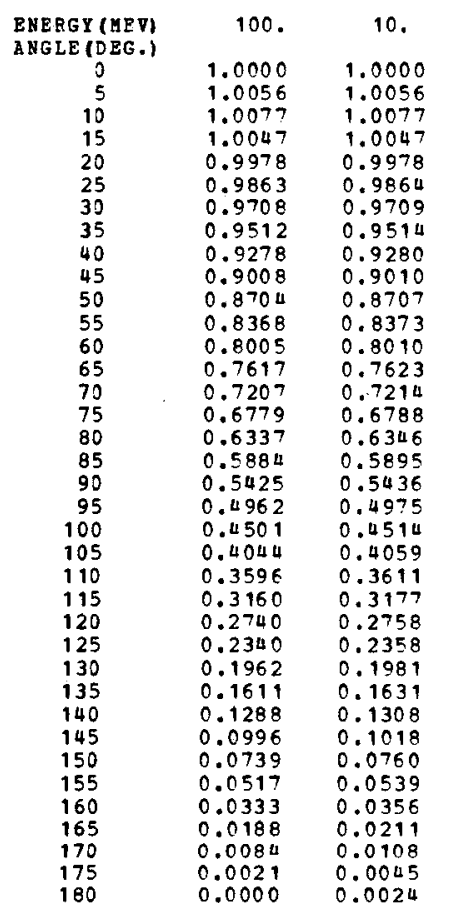

$\begin{array}{cc}4 & 2 . \\ 1.0000 & 1.0000 \\ 1.0056 & 1.0055 \\ 1.0077 & 1.0077 \\ 1.0048 & 1.0050 \\ 0.9980 & 0.9985 \\ 0.9867 & 0.9876 \\ 0.9714 & 0.9728 \\ 0.9521 & 0.9541 \\ 0.9290 & 0.9318 \\ 0.9023 & 0.9059 \\ 0.8724 & 0.8768 \\ 0.8393 & 0.8447 \\ 0.8034 & 0.8099 \\ 0.7651 & 0.7727 \\ 0.7246 & 0.7334 \\ 0.6824 & 0.6924 \\ 0.6387 & 0.6499 \\ 0.5940 & 0.6065 \\ 0.5486 & 0.5624 \\ 0.5030 & 0.5180 \\ 0.4574 & 0.4737 \\ 0.4123 & 0.4299 \\ 0.3680 & 0.3869 \\ 0.3250 & 0.3451 \\ 0.2835 & 0.3048 \\ 0.2440 & 0.2663 \\ 0.2067 & 0.2301 \\ 0.1720 & 0.1963 \\ 0.1401 & 0.1653 \\ 0.1113 & 0.1373 \\ 0.0858 & 0.1126 \\ 0.0639 & 0.0913 \\ 0.0458 & 0.0736 \\ 0.0315 & 0.0597 \\ 0.0212 & 0.0497 \\ 0.0150 & 0.0437 \\ 0.01129 & 0.0017\end{array}$

1.5
1.0000
1.0055
1.0077
1.0051
0.9988
0.9883
0.9740
0.9557
0.9340
0.9087
0.8804
0.8491
0.8151
0.7788
0.7405
0.7004
0.6590
0.6166
0.5736
0.5302
0.4870
0.4442
0.4022
0.3613
0.3219
0.2844
0.2490
0.2160
0.1857
0.1584
0.1342
0.1134
0.0962
0.0826
0.0728
0.0669
0.0650

1.0
1.0000
1.0054
1.0077
1.0054
0.9996
0.9898
0.9764
0.9592
0.9387
0.9140
0.8881
0.8585
0.8264
0.7920
0.7557
0.7178
0.6785
0.6383
0.5975
0.5564
0.5154
0.4748
0.4350
0.3962
0.3589
0.3233
0.2897
0.2584
0.2297
0.2037
0.1808
0.1611
0.1447
0.1318
0.1225
0.1169
0.11511

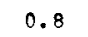

1.0000

1.0053

1.0057

1.0002

0.9909

0.9781
0.9618

0.9618
0.9422

0.9195

0.8655

0.8348

0.8019
0.7671

0.7308

0.6932

0.6547

0.5156

0.5762

0.5368
0.4979

0.14597

0.4226
0.3867

0.3526

0.3204

0.2904
0.2628

0.2379

0.2159

0.197

0.1813
0.1680

0.1680
0.1600

0.1600
0.1546

0.1546
0.1529

0.6
1.0000
1.0052
1.0076
1.0060
1.0011
0.9927
0.9809
0.9659
0.9478
0.9268
0.0030
0.8767
0.8482
0.8175
0.7853
0.7515
0.7155
0.6806
0.6442
0.6075
0.5708
0.5346
0.4990
0.4643
0.4309
0.3991
0.3691
0.3411
0.3154
0.2922
0.2716
0.2540
0.2393
0.2278
0.2195
0.2145
0.2128

0.4
1.0000
1.0049
1.0075
1.0065
1.0026
0.9056
0.9857
0.9729
0.9573
0.9392
0.9187
0.8959
0.8711
0.8446
0.8165
0.7870
0.7566
0.7253
0.6035
0.5615
0.6295
0.5979
0.5667
0.5365
0.5073
0.4795
0.4532
0.4287
0.4063
0.3860
0.3680
0.3526
0.3398
0.3297
0.3224
0.3180
0.3166

0.2

1.0000

1.0043

1.0071

1.0071
1.0052

1. 0008

0.9045

0.9859

0.9754
0.9629

0.9487

0.9328

0.9155

0.8958

$0.875 \%$

0.8561

0.8345

0.8122

0.7896

0.7658
0.7440

0.7214

0.6991

0.6775
0.6556

ก. 6366

0.6178

0.6003

0.5841

0.5696

$0.556^{7}$

0.5456

0.5364
$0.52 \circ 2$

0.5240

0.5208
0.5198
0.1

1. 290 ?

1.0262
1.0371

1.0067

1. 004?

1.09711
0.9965

0.9906

0.9932

0.9747

0.9550

0.9428

0.9305

0.9530

0.8890

0.8890

0.8613

0.8468

0.8325
0.8183

0.8046

0.7013

0.7786

0.7566
0.7554

0.7459

0.7358

0.7276

0.7205

0.7146
0.7100

0.7265

0.7046
0.730

RATTO OP MOTT TO ROTHEREOPD SCATTERTNG TN MG, $z=12$

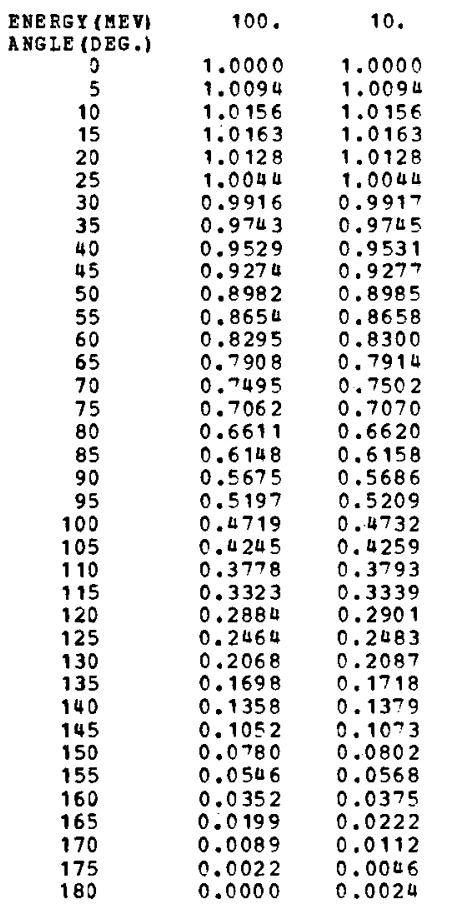

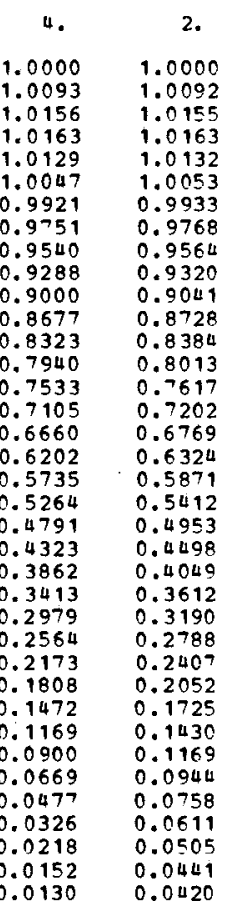

1.5
1.0000
1.0091
1.0154
1.0163
1.0134
1.0058
0.9942
0.9782
0.9584
0.9346
0.9074
0.8769
0.8433
0.8071
0.7685
0.7280
0.6858
0.6423
0.5980
0.5532
0.5084
0.4639
0.4201
0.3774
0.3362
0.2968
0.2596
0.2249
0.1930
0.1642
0.1387
0.1167
0.0985
0.0841
0.0738
0.0676
0.0655

1.0
1.0000
1.0089
1.0152
1.0163
1.0138
1.0069
0.9961
0.9811
0.9625
0.9402
0.9145
0.8856
0.8539
0.8197
0.7831
0.7447
0.7047
0.6635
0.6215
0.5791
0.5365
0.4943
0.4527
0.4127
0.3731
0.3357
0.3004
0.2674
0.2371
0.2098
0.1855
0.1647
0.1474
0.1337
0.1239
0.1180
0.1160

0.8
1.0000
1.0088
1.0150
1.0163
1.0142
1.0077
0.9975
0.9833
0.9656
0.9443
0.9198
0.8922
0.8619
0.8291
0.7941
0.7573
0.7190
0.6795
0.6392
0.5985
0.5577
0.5172
0.4773
0.4384
0.4009
0.3650
0.3311
0.2995
0.2704
0.2441
0.2209
0.2008
0.1842
0.1711
0.1617
0.1560
0.1549 0.6
1.0000 1. 0085 1.0147
1.0163 1.0163 1.0089 0.9997 0.9867 0.9704 0.9508 0.9282 0.9026 0.8745 0.8440 0.8115 0.7773 0.7416
0.7048 0.7048
0.6673 0.6673
0.6293 0.5913 0.5535 0.5163 0.4800 0.4450 0.4195 0.3798 0.3503 0.3232 0.2985 0.2769 0.2582 0.2427 0.2305 0.2217 0.2164
0.2146

$\begin{array}{cc}0.2 & 0.1 \\ 1.0000 & 1.9200 \\ 1.0069 & 1.0055 \\ 1.0126 & 1.0105 \\ 1.0153 & 1.0136 \\ 1.0159 & 1.2153 \\ 1.0139 & 1.0152 \\ 1.0095 & 1.9136 \\ 1.0027 & 1.0104 \\ 0.9937 & 1.0056 \\ 0.9825 & 0.9903 \\ 0.9692 & 0.0917 \\ 0.9540 & 0.9827 \\ 0.9371 & 0.9725 \\ 0.9186 & 0.9612 \\ 0.8997 & 0.9189 \\ 0.8777 & 0.9358 \\ 0.8556 & 0.9220 \\ 0.8328 & 0.0076 \\ 0.8094 & 0.8920 \\ 0.7857 & 0.8778 \\ 0.7619 & 0.8527 \\ 0.7382 & 0.8476 \\ 0.7148 & 0.8327 \\ 0.6920 & 0.8191 \\ 0.6699 & 0.8039 \\ 0.6488 & 0.7904 \\ 0.6288 & 0.7775 \\ 0.6101 & 0.7556 \\ 0.5930 & 0.7545 \\ 0.5774 & 0.7145 \\ 0.5637 & 0.7357 \\ 0.5519 & 0.7280 \\ 0.5420 & 0.7217 \\ 0.5343 & 0.7167 \\ 0.5287 & 0.7131 \\ 0.5253 & 0.7100 \\ 0.5542 & 0.7102\end{array}$


RATTO OF MOTM TO BUTHERFCDD SCATTFRTNG IN AL, $Z=13$

\begin{tabular}{|c|c|c|c|c|c|c|c|c|c|c|c|}
\hline $\begin{array}{l}\text { ENERGY (NEV) } \\
\text { ANGLE (DEG.) }\end{array}$ & 100. & 10. & 4. & 2. & 1.5 & 1.0 & 0.8 & 0.6 & 0.4 & 0.2 & 3.1 \\
\hline 0 & 1.0000 & 1.0000 & 1.0000 & 1.0000 & 1.0000 & 1.0000 & $1.000 n$ & 1.0000 & 1.0000 & 1.0000 & 1.0200 \\
\hline 5 & 1.0103 & 1.0103 & 1.0103 & 1.0101 & 1.0100 & 1.0098 & 1.0096 & 1.0093 & 1.0088 & 1.0075 & 1.0060 \\
\hline 10 & 1.0176 & 1.0176 & 1.0176 & 1.0174 & 1.0173 & 1.0171 & 1.0168 & 1.0165 & 1.0158 & 1.0140 & 1.3116 \\
\hline 15 & 1.0192 & 1.0192 & 1.0192 & 1.0192 & 1.0192 & 1.0191 & 1.0190 & 1.0189 & 1.0186 & 1.0973 & 1. $ก 152$ \\
\hline 20 & 1.0167 & 1.0167 & 1.0168 & 1.0170 & 1.0172 & 1.0175 & 1.0177 & 1.0181 & 1.0185 & 1.0186 & 1.0175 \\
\hline 25 & 1.0090 & 1.0091 & 1.0093 & 1.0099 & 1.0103 & 1.0113 & 1.0120 & 1.0131 & 1.0147 & 1.0172 & 1.0170 \\
\hline 30 & 0.9970 & 0.9971 & 0.9975 & 0.9986 & 0.0994 & 1.0012 & 1.0025 & 1.0045 & 1.0078 & 1.0134 & 1.0168 \\
\hline 35 & 0.9804 & 0.9805 & 0.9811 & 0.9827 & 0.0940 & 0.9868 & 0.9880 & 0.9922 & 0.9075 & 1.0071 & 1.0140 \\
\hline 40 & 0.9595 & 0.9597 & 0.9605 & 0.9629 & 0.9647 & 0.9687 & $0.971=$ & 0.9754 & 0.9842 & 0.9085 & $1.009 \mathrm{~F}$ \\
\hline 45 & 0.9344 & 0.9346 & 0.9358 & 0.9389 & 0.9414 & 0.9468 & 0.9508 & 0.9571 & 0.9578 & 0.9875 & 1.3036 \\
\hline 55 & 0.8730 & 0.8734 & 0.8752 & 0.8802 & 0.8842 & 0.8928 & 0.8903 & 0.9095 & 0.9268 & 0.9597 & $0.987 \mathrm{~L}$ \\
\hline 60 & 0.8372 & 0.8377 & 0.8399 & 0.8459 & 0.8508 & 0.8613 & 0.8691 & 0.8815 & 0.9026 & 0.9429 & 0.9773 \\
\hline 65 & 0.7985 & 0.7991 & 0.8017 & 0.8088 & 0.8146 & 0.8270 & 0.9363 & 0.8511 & $0.8^{7} 63$ & 0.9245 & 0.9561 \\
\hline 70 & 0.7572 & 0.7570 & 0.7609 & 0.7693 & 0.7760 & 0.7905 & 0.8013 & 0.8185 & 0.8480 & 0.9046 & $0.95,39$ \\
\hline 75 & 0.7137 & 0.7145 & 0.7180 & 0.7276 & 0.7353 & 0.7519 & 0.7644 & 0.7842 & 0.8181 & 0.8835 & 0.01108 \\
\hline 80 & 0.6684 & 0.6693 & 0.6733 & 0.6841 & 0.6929 & 0.7117 & $0.725^{\circ}$ & 0.7483 & 0.7869 & 0.8613 & 0.9260 \\
\hline 85 & 0.6218 & 0.6228 & 0.6272 & 0.6394 & 0.6092 & 0.6703 & 0.6862 & 0.7113 & 0.7546 & 0.8384 & 0.0125 \\
\hline 90 & 0.5742 & 0.5753 & 0.5802 & 0.5937 & 0.6045 & 0.6279 & 0.5056 & 0.6735 & 0.7216 & 0.8148 & 0.3976 \\
\hline 95 & 0.5260 & $0.52^{\ulcorner} 2$ & 0.5327 & 0.5474 & 0.5594 & 0.5851 & 0.6045 & 0.6352 & 0.5881 & 0.7909 & 0.8825 \\
\hline 100 & 0.4778 & 0.4791 & 0.4850 & 0.5011 & 0.5141 & 0.5422 & 0.5633 & 0.5968 & 0.6545 & $0.7 E 5 B$ & 0.8572 \\
\hline 105 & 0.4299 & 0.4313 & 0.4377 & 0.4551 & 0.4692 & 0.4995 & 0.5224 & 0.5586 & 0.5211 & 0.7428 & 0.8519 \\
\hline 110 & 0.3827 & 0.3842 & 0.3911 & 0.4098 & 0.4240 & 0.4575 & 0.0821 & 0.5210 & 0.5882 & 0.7192 & 0.8368 \\
\hline $\begin{array}{l}115 \\
120\end{array}$ & $\begin{array}{l}0.3367 \\
0.2922\end{array}$ & $\begin{array}{l}0.3383 \\
0.2940\end{array}$ & $\begin{array}{l}0.3456 \\
0.3017\end{array}$ & $\begin{array}{l}0.3656 \\
0.3229\end{array}$ & $\begin{array}{l}0.3818 \\
0.3401\end{array}$ & $\begin{array}{l}0.1165 \\
0.3769\end{array}$ & $\begin{array}{l}0.4427 \\
0.4047\end{array}$ & $\begin{array}{l}0.4843 \\
0.4488\end{array}$ & $\begin{array}{l}0.5560 \\
0.5250\end{array}$ & $\begin{array}{l}0.6950 \\
0.6737\end{array}$ & 0.8220 \\
\hline $\begin{array}{l}120 \\
125\end{array}$ & 0.2498 & 0.2516 & 0.2598 & 0.2821 & 0.3002 & 0.3391 & 0.3684 & 0.4149 & 0.4952 & 0.6522 & 0.7938 \\
\hline 130 & 0.2096 & 0.2115 & 0.2201 & 0.2435 & 0.2625 & 0.3033 & 0.3340 & 0.3828 & 0.4671 & 0.6319 & 0.7908 \\
\hline 135 & 0.1722 & 0.1742 & 0.1832 & 0.2076 & 0.2274 & 0.2699 & 0.3020 & 0.3529 & 0.4009 & $0.613 n$ & $0.768 F$ \\
\hline 140 & 0.1378 & 0.1398 & 0.1491 & 0.1745 & 0.1950 & 0.2392 & 0.2725 & 0.3254 & 0.4167 & 0.5056 & 0.7574 \\
\hline 145 & 0.1066 & 0.1088 & 0.1184 & 0.1446 & 0.1658 & 0.2114 & 0.2459 & 0.3005 & 0.3949 & 0.5798 & 0.7472 \\
\hline 150 & 0.0791 & 0.0813 & 0.0912 & 0.1181 & 0.1399 & 0.1869 & 0.2223 & 0.2784 & 0.3756 & 0.5658 & 0.7382 \\
\hline 155 & $0.055 \mathrm{a}$ & 0.0576 & 0.0677 & 0.0053 & 0.1177 & 0.1657 & 0.2019 & 0.2595 & 0.3589 & 0.5538 & 0.7304 \\
\hline 160 & 0.0357 & 0.0380 & 0.0483 & $0.0^{-7} 64$ & 0.0992 & 0.1481 & 0.1851 & 0.2437 & 0.3451 & 0.5438 & 0.7240 \\
\hline 165 & 0.0202 & 0.0225 & 0.0330 & 0.0615 & 0.0846 & 0.1343 & 0.1718 & 0.2313 & 0.3342 & 0.5359 & 0.7180 \\
\hline 170 & 0.0090 & 0.0114 & 0.0219 & 0.0508 & 0.0741 & 0.1243 & 0.1622 & 0.2224 & 0.3264 & 0.5302 & 0.7152 \\
\hline 175 & 0.0023 & 0.0046 & 0.0153 & 0.0443 & 0.0678 & 0.1183 & 0.1564 & 0.2170 & 0.3216 & 0.5268 & 0.7130 \\
\hline 180 & 0.0000 & 0.0024 & 0.0130 & 0.0421 & 0.0657 & 0.1163 & 0.1545 & 0.2152 & 0.3201 & 0.5257 & 0.7123 \\
\hline
\end{tabular}

RATIO OF MOTT TO RUTHERFORD SCATTERING IN SI, $z=14$

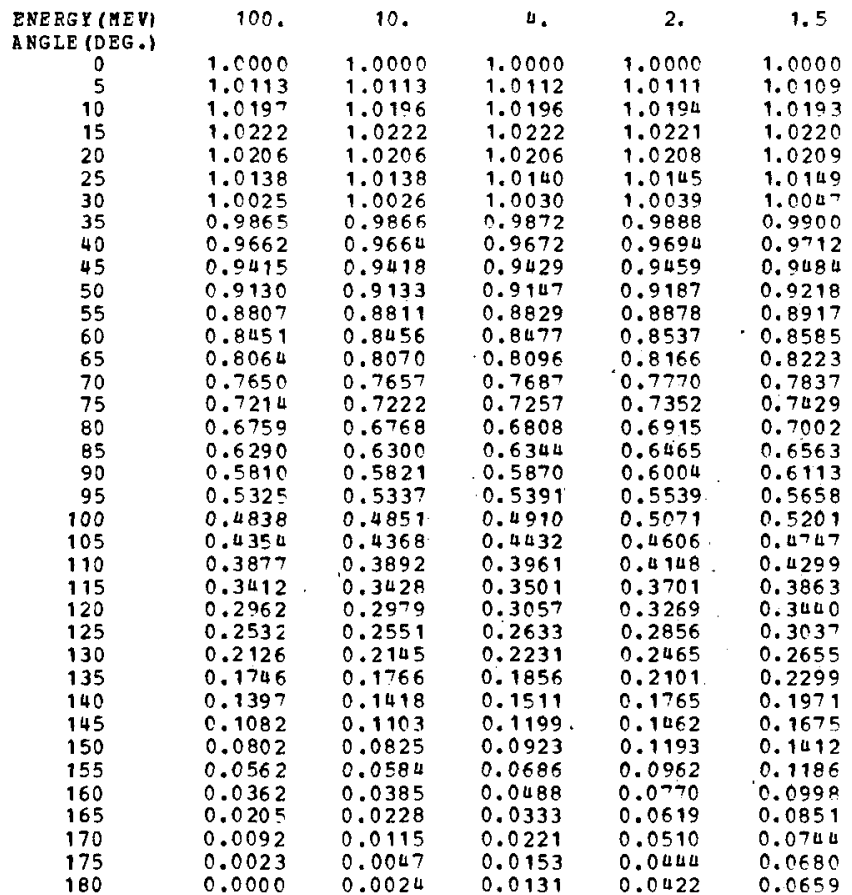

$\begin{array}{ccc}1.0 & 0.8 & 0.5 \\ 1.0000 & 1.0000 & 1.0000 \\ 1.0107 & 1.0105 & 1.0102 \\ 1.0189 & 1.0187 & 1.0183 \\ 1.0219 & 1.0218 & 1.0215 \\ 1.0212 & 1.0213 & 1.0215 \\ 1.0158 & 1.0164 & 1.0173 \\ 1.0064 & 1.0076 & 1.0095 \\ 0.9927 & 0.9946 & 0.9977 \\ 0.9751 & 0.9779 & 0.9824 \\ 0.9536 & 0.9575 & 0.9636 \\ 0.9286 & 0.9336 & 0.9416 \\ 0.9002 & 0.9065 & 0.9165 \\ 0.8688 & 0.8765 & 0.8886 \\ 0.8346 & 0.8438 & 0.8583 \\ 0.7980 & 0.8087 & 0.8257 \\ 0.7593 & 0.7717 & 0.7913 \\ 0.7189 & 0.7330 & 0.7552 \\ 0.6772 & 0.5930 & 0.7180 \\ 0.6346 & 0.6521 & 0.6799 \\ 0.5014 & 0.6107 & 0.6413 \\ 0.5480 & 0.5691 & 0.6025 \\ 0.5050 & 0.5278 & 0.5639 \\ 0.4625 & 0.4870 & 0.5259 \\ 0.4210 & 0.4472 & 0.4887 \\ 0.3809 & 0.4087 & 0.4528 \\ 0.3426 & 0.3719 & 0.4185 \\ 0.3063 & 0.3371 & 0.3859 \\ 0.2725 & 0.3046 & 0.3556 \\ 0.2413 & 0.2747 & 0.3277 \\ 0.2132 & 0.2477 & 0.3024 \\ 0.1883 & 0.2237 & 0.2800 \\ 0.1668 & 0.2031 & 0.2608 \\ 0.1490 & 0.1860 & 0.2448 \\ 0.1349 & 0.1725 & 0.2322 \\ 0.1248 & 0.1628 & 0.2231 \\ 0.1187 & 0.1569 & 0.2176 \\ 0.1167 & 0.1550 & 0.2158\end{array}$

0.4

1.0000

1.0096

1. 0974

1. 0210

1.0217
1.0187

1.0187
1.0125

1.0027

0.9890

0.9739

0.0551
0.9335

0.9094

0.8831

0.8548

0.8249

0.7935

0.751 त

0.6940

0.6900

0.6263

0.6263

0.5930

0.5604

0.5289

0.4703

0.4703
0.4437

0.4192

0.397 ?

0.3774

0.3605

0.3465

0.3354

0.3274

0.3226
0.321 ?
0.2

1.0000

1.0081

1.0194

1.0194

1.0276

1.0175

1.0035

.9929

0.9803

0.9655

0.9489
0.9305

$0.910^{7}$

0.8895

ก. $86^{73}$

0.8442

0.7953

0.7719
0.7477

0.7237

0.7003

0.6776

0.6353

0.6161

.5983

0.5823
0.5681

0.5559

0.5457

$0.53^{77}$

0.5319

0.5284
0.5273
0.1

1.3000
1.0054

1. 2127

1. 1168

1. 5197

1.0206
1.0200

1.0176

1. 1936

1. 0080

0.9922

0.0924

0. 9712

0.9591

0.9321

0.9176

0.0026

0.8873

0.8718
$0.8=64$

0.8411

0.8211

0.3115

0.7803

0.7803

0.7710

0.7501

0.7110

0.7331
0.7265

0.7213

0.7178

0.7153 
RATIO OF MOTT TO ROTHEAFORD SCPTTERING IN $K, \quad z=19$

\begin{tabular}{|c|c|c|c|c|c|c|c|c|c|c|c|}
\hline $\begin{array}{l}\text { ENERGY (MEV) } \\
\text { ANGLE (DEG.) }\end{array}$ & 100 & 10. & 4. & 2. & 1.5 & 1.0 & 0.8 & 0.6 & 0.0 & 0.2 & 0.9 \\
\hline 0 & 1.0000 & 1.0000 & 1.0000 & 1.0000 & 1.0000 & 1.0000 & 1.0000 & 1.0000 & $1.020 \mathrm{C}$ & 1.0000 & 1.9900 \\
\hline 5 & 1.0159 & 1.0158 & 1.0158 & 1.0155 & 1.0154 & 1.0150 & 1. 0147 & 1.0142 & 1.0132 & 1.0110 & 1.0086 \\
\hline 10 & 1.0297 & 1.0297 & 1.0296 & 1.0293 & 1.0200 & 1.0284 & 1.0279 & 1.0271 & 1.0256 & 1.0221 & 1.9176 \\
\hline 15 & 1.0372 & 1.0372 & 1.0371 & 1.0368 & 1.0366 & 1.0360 & 1.0356 & 1.0349 & 1.0334 & $1.029^{7}$ & 1.0246 \\
\hline 20 & 1.0405 & 1.0405 & 1.0005 & 1.0404 & 1.0403 & 1.0400 & 1.0398 & 1.0394 & 1.0384 & 1.0354 & 1.0306 \\
\hline 25 & 1.0382 & 1.0382 & 1.0383 & 1.0385 & 1.0386 & 1.0389 & 1.0390 & 1.0392 & 1.0392 & 1.0390 & 1.0340 \\
\hline 30 & 1.0311 & 1.0311 & 1.0314 & 1.0320 & 1.0324 & 1.0334 & 1.0341 & 1.0351 & 1.0366 & 1.0380 & 1.5366 \\
\hline 35 & 1.0186 & 1.0187 & 1.0191 & 1.0203 & 1.0212 & 1.0231 & 1.0245 & 1.0266 & 1.0300 & 1.0350 & 1. 2366 \\
\hline 40 & 1.0014 & 1.0015 & 1.0022 & 1.0040 & 1.0055 & 1.0085 & 1.0108 & 1.0142 & 1.0190 & 1.0294 & 1.0349 \\
\hline 45 & 0.9793 & 0.97 .95 & 0.9804 & 0.9830 & 0.9851 & 0.9895 & 0.9927 & 0.9978 & 1.0062 & 1.0210 & 1.0311 \\
\hline 50 & 0.9527 & 0.9530 & 0.9543 & 0.9577 & 0.9605 & 0.9664 & 0.9708 & 0.9777 & 0.9892 & 1.0101 & 1.2257 \\
\hline 55 & 0.9219 & 0.9222 & 0.9238 & 0.9283 & 0.9318 & 0.9394 & 0.9451 & 0.9540 & 0.9690 & 0.9956 & 1.0184 \\
\hline 60 & 0.8871 & 0.8876 & 0.8896 & 0.8951 & 0.8995 & 0.9089 & 0.9160 & 0.9271 & 0.9459 & 0.9810 & 1.0005 \\
\hline 65 & 0.8488 & 0.8494 & 0.8518 & 0.8584 & 0.8637 & 0.8751 & $0.883^{7}$ & 0.8971 & 0.9201 & 0.9633 & 0.9992 \\
\hline 70 & 0.8073 & 0.8080 & 0.8108 & 0.8187 & 0.8250 & 0.8385 & 0.8486 & $0.86 \square 6$ & 0.8919 & 0.9437 & 0.9875 \\
\hline 75 & 0.7631 & 0.7639 & 0.7672 & 0.7763 & 0.7836 & 0.7993 & 0.8111 & 0.8298 & 0.8616 & 0.9225 & $0.9>46$ \\
\hline 80 & 0.7166 & 0.7174 & 0.7212 & 0.7316 & 0.7400 & 0.7580 & 0.7716 & 0.7929 & 0.8296 & 0.8999 & $0.950^{\circ}$ \\
\hline 85 & 0.6682 & 0.6692 & 0.6735 & 0.6852 & 0.6947 & 0.7151 & 0.7304 & 0.7546 & 0.7962 & 0.8761 & 0.9159 \\
\hline 90 & 0.6184 & 0.6195 & 0.6243 & 0.6374 & 0.6480 & 0.6708 & 0.6879 & 0.7150 & 0.7616 & 0.8515 & 0.9305 \\
\hline 95 & 0.5678 & 0.5689 & 0.5743 & 0.5888 & 0.6005 & 0.6257 & $0.644=$ & 0.6747 & 0.7264 & 0.8253 & 0.9147 \\
\hline 100 & 0.5167 & 0.5180 & 0.5238 & 0.5397 & 0.5525 & 0.5802 & 0.6010 & 0.6339 & 0.6907 & 0.8007 & 0.8985 \\
\hline 105 & 0.4657 & 0.4671 & 0.4735 & 0.4907 & 0.5047 & 0.5348 & 0.5574 & 0.5933 & 0.6551 & 0.7751 & 0.8822 \\
\hline 110 & 0.4153 & 0.4168 & 0.4236 & 0.4423 & 0.4573 & 0.4898 & 0.5142 & 0.5529 & 0.6197 & 0.7497 & 0.8650 \\
\hline 115 & 0.3659 & 0.3676 & 0.3749 & 0.3948 & 0.4110 & 0.4458 & 0.11719 & 0.5135 & 0.5851 & 0.7247 & 0.8500 \\
\hline 120 & 0.3181 & 0.3198 & 0.3276 & 0.3488 & 0.3660 & 0.4030 & 0.4309 & 0.4751 & 0.5514 & 0.7000 & 0.9344 \\
\hline 125 & 0.2722 & 0.2741 & 0.2823 & 0.3048 & 0.3230 & 0.3621 & 0.3916 & 0.4384 & 0.5192 & 0.6770 & 0.8194 \\
\hline 130 & 0.2287 & 0.2306 & 0.2393 & 0.2629 & 0.2821 & 0.3232 & 0.3543 & 0.4035 & 0.4885 & $0.65+9$ & 0.9551 \\
\hline 135 & 0.1881 & 0.1901 & 0.1992 & 0.2239 & 0.2439 & 0.2869 & 0.3194 & 0.3709 & 0.4599 & 0.6349 & 0.7918 \\
\hline 140 & 0.1506 & 0.1527 & 0.1621 & 0.1878 & 0.2086 & 0.2534 & 0.2872 & 0.3408 & 0.4335 & 0.6119 & 0.7794 \\
\hline 145 & 0.1167 & 0.1188 & 0.1286 & 0.1552 & 0.1768 & 0.2231 & 0.2589 & 0.3136 & 0.4095 & 0.5976 & 0.7682 \\
\hline 150 & 0.0866 & 0.08888 & 0.0989 & 0.1263 & 0.1485 & 0.1962 & 0.2322 & 0.2894 & 0.3883 & 0.5821 & 0.7582 \\
\hline 155 & 0.0607 & 0.0630 & 0.0732 & 0.1013 & 0.1241 & 0.1730 & $0.210 n$ & 0.2686 & 0.3700 & C. 5688 & 0.9406 \\
\hline 160 & 0.0391 & 0.0494 & 0.0519 & 0.0806 & 0.1038 & 0.1538 & 0.1994 & 0.2513 & 0.3547 & $0.55^{7} 8$ & 0.7425 \\
\hline 165 & 0.0221 & 0.0245 & 0.0352 & 0.0643 & 0.0878 & 0.1386 & 0.1768 & 0.2376 & 0.3427 & 0.5490 & 0.7368 \\
\hline 170 & 0.0099 & 0.0123 & 0.0231 & 0.0525 & 0.0764 & 0.1276 & 0.1663 & 0.2278 & 0.3349 & $C .5428$ & 0.7328 \\
\hline 175 & 0.0025 & 0.0049 & 0.0157 & 0.0454 & 0.0694 & 0.1210 & 0.1600 & 0.2218 & 0.3289 & 0.5390 & 0.7303 \\
\hline 180 & 0.0000 & 0.0025 & 0.0133 & 0.0130 & 0.0671 & 0.1188 & 0.1579 & 0.2198 & 0.3271 & 0.5377 & 0.7295 \\
\hline
\end{tabular}

KATIO OF MOTT TO ROTHERFCPE SCMTMPRTNG IN TI, $\mathrm{z}=22$

\begin{tabular}{|c|c|c|c|c|c|c|c|c|c|c|c|}
\hline $\begin{array}{l}\text { ENERGY (MEV) } \\
\text { ANGLE (DEG.) }\end{array}$ & 100 & 10. & $\Delta$. & 2. & 1.5 & 1.0 & 0.8 & 0.6 & 0.4 & 0.2 & 0.1 \\
\hline $\begin{array}{l}0 \\
0\end{array}$ & 1.0000 & 1.0000 & 1.0000 & 1.0000 & 1.0000 & 1.0000 & 1.0000 & 1.0000 & 1.0000 & 1.0030 & 1.2909 \\
\hline 5 & 1.0185 & 1.0185 & 1.0184 & 1.0181 & 1.0179 & 1.0174 & 1.0170 & 1.0164 & 1.0152 & 1.0125 & 1.0093 \\
\hline 10 & 1.0357 & 1.0357 & 1.0355 & 1.0351 & 1.0348 & 1.0340 & 1.0334 & $1.032 \mathrm{u}$ & 1.0304 & 1.0259 & 1.3202 \\
\hline 15 & 1.0463 & 1.0463 & 1.0461 & $1.045^{7}$ & 1.0454 & 1. 0446 & 1.0440 & 1.0429 & 1.0408 & 1.0357 & 1.0289 \\
\hline 20 & 1.0529 & 1.0528 & 1.0527 & 1.0524 & 1.0522 & 1.0516 & 1.0511 & 1.0503 & 1.0486 & 1.0038 & 1.3369 \\
\hline 25 & 1.0534 & 1.0534 & 1.0534 & 1.0534 & 1.0534 & 1.0532 & 1.0531 & 1.0528 & 1.0519 & 1.0486 & 1.2426 \\
\hline 30 & 1.0491 & 1.0491 & 1.0492 & 1.0496 & 1.0499 & 1.0504 & 1.0508 & 1.0513 & 1.3517 & 1.0508 & $1.2 \div 66$ \\
\hline 35 & 1.0390 & 1.0391 & 1.0394 & 1.0403 & 1.0410 & 1.0425 & 1.0435 & 1.0450 & 1.0473 & 1.0498 & 1.0484 \\
\hline 40 & 1.0240 & 1.0241 & 1.0246 & 1.0262 & 1.0274 & 1.0299 & 1.0318 & 1.0346 & 1.0391 & 1.0460 & 1.5483 \\
\hline 45 & 1.0036 & 1.0038 & 1.0046 & 1.0069 & 1.0087 & 1.0126 & 1.0155 & 1.0199 & $1.027 n$ & 1.0390 & 1.0459 \\
\hline 50 & 0.9785 & 0.9788 & 0.9799 & 0.9831 & 0.9856 & 0.9910 & 0.9949 & 1.0011 & 1.0114 & 1.0294 & 1.2417 \\
\hline 55 & $0.948^{7}$ & 0.9490 & 0.9506 & 0.9547 & 0.9580 & 0.9650 & 0.9703 & 0.9785 & 0.9022 & 1.0170 & 1,0354 \\
\hline 60 & 0.9147 & 0.9151 & 0.9170 & 0.9222 & 0.9260 & 0.9353 & 0.9419 & 0.9523 & $0.9 \in 09$ & 1.0022 & $\begin{array}{l}1.9274 \\
1.9177\end{array}$ \\
\hline 65 & 0.8767 & 0.8773 & 0.8796 & 0.8859 & 0.8910 & 0.9019 & 0.9100 & 0.9228 & 0.9445 & $0.9 R 50$ & $\begin{array}{l}1.0977 \\
1.0965\end{array}$ \\
\hline $\begin{array}{l}70 \\
75\end{array}$ & 0.8353 & 0.8359 & 0.8387 & 0.8462 & 0.8523 & 0.8653 & 0.8751 & 0.8904 & 0.9166 & 0.9657 & $\begin{array}{l}1.0265 \\
0.0039\end{array}$ \\
\hline $\begin{array}{l}75 \\
80\end{array}$ & $\begin{array}{l}0.7908 \\
0.7436\end{array}$ & $\begin{array}{l}0.7915 \\
0.7444\end{array}$ & $\begin{array}{l}0.7947 \\
0.7482\end{array}$ & $\begin{array}{l}0.8035 \\
0.7583\end{array}$ & $\begin{array}{l}0.8107 \\
0.7665\end{array}$ & $\begin{array}{l}0.8259 \\
0.7849\end{array}$ & $\begin{array}{l}0.8374 \\
0.7973\end{array}$ & $\begin{array}{l}0.8554 \\
0.8182\end{array}$ & $\begin{array}{l}0.8862 \\
0.8539\end{array}$ & $\begin{array}{l}0.9446 \\
0.9210\end{array}$ & $\begin{array}{l}0.0939 \\
0.9901\end{array}$ \\
\hline 85 & 0.6944 & 0.6953 & 0.6995 & 0.7111 & 0.7204 & 0.7404 & 0.7554 & 0.7992 & 0.8199 & 0.8979 & 0.9654 \\
\hline 90 & 0.6434 & 0.6405 & 0.6492 & 0.6622 & 0.6726 & 0.6951 & 0.7120 & $0.738^{7}$ & 0.7846 & 0.8728 & 0.9198 \\
\hline 95 & 0.5914 & 0.5926 & 0.5979 & 0.6123 & 0.6239 & 0.6488 & 0.6676 & 0.6973 & 0.7484 & $0.84>0$ & $0.033^{7}$ \\
\hline 100 & 0.5388 & 0.5401 & 0.5359 & 0.5617 & 0.5745 & 0.6019 & 0.6226 & 0.6553 & 0.7117 & $0.820^{7}$ & 0.9172 \\
\hline 105 & 0.4861 & 0.4876 & 0.4939 & 0.5111 & 0.5250 & 0.5550 & 0.5776 & 0.5133 & 0.6748 & 0.7943 & 0.0005 \\
\hline 110 & 0.4339 & 0.4354 & 0.4422 & 0.4609 & 0.4760 & 0.5084 & 0.5328 & 0.5715 & 0.6382 & 0.7679 & 0.8837 \\
\hline 115 & 0.3827 & 0.3843 & 0.3916 & 0.4117 & 0.4279 & $0.462^{7}$ & 0.4889 & 0.5305 & 0.6022 & 0.7419 & 0.8672 \\
\hline 120 & 0.3329 & 0.3346 & 0.3424 & 0.3638 & 0.3810 & 0.4182 & 0.4462 & 0.4905 & 0.5672 & 0.7166 & 0.8511 \\
\hline 125 & 0.2851 & 0.2869 & 0.2952 & 0.3178 & 0.3361 & 0.3755 & 0.4052 & 0.4522 & 0.5335 & 0.6923 & 0.8355 \\
\hline 130 & 0.2397 & 0.2416 & 0.2504 & 0.2742 & 0.2935 & 0.3349 & 0.3662 & 0.1158 & 0.5015 & 0.6691 & $0.820 \mathrm{~F}$ \\
\hline 135 & 0.1972 & 0.1993 & 0.2084 & 0.2333 & 0.2535 & 0.2969 & 0.3297 & 0.3817 & $0 . b>15$ & 0.6474 & $0.806^{7}$ \\
\hline 140 & 0.1580 & 0.1601 & 0.1696 & 0.1956 & 0.2166 & 0.2619 & 0.2960 & 0.3501 & 0.4438 & 0.6273 & 0.7938 \\
\hline 145 & 0.1225 & 0.1247 & 0.1345 & 0.1614 & 0.1832 & 0.2301 & 0.2655 & 0.3216 & $0.1118^{7}$ & 0.6090 & 0.7821 \\
\hline 150 & 0.0909 & 0.0932 & 0.1033 & 0.1311 & 0.1535 & 0.2019 & 0.2383 & 0.2962 & $0.396 \mathrm{u}$ & 0.5928 & $0.771^{7}$ \\
\hline 155 & 0.0637 & 0.0660 & 0.0765 & 0.1049 & 0.1280 & 0.1775 & 0.2149 & 0.2743 & 0.3771 & $0.5^{-89}$ & $0.762^{7}$ \\
\hline 160 & 0.0411 & 0.0435 & 0.0541 & 0.0831 & 0.1067 & 0.1573 & 0.1955 & 0.2561 & 0.3611 & 0.5672 & 0.7552 \\
\hline 165 & 0.0232 & 0.0257 & 0.0365 & $0.06 E 0$ & 0.0899 & 0.1413 & 0.1801 & 0.2418 & 0.3485 & 0.5580 & 0.7493 \\
\hline 170 & 0.0104 & 0.0128 & 0.0238 & 0.0536 & 0.0778 & 0.1298 & 0.1691 & 0.2314 & $0.339 \mathrm{u}$ & 0.5510 & 0.7450 \\
\hline 175 & 0.0026 & 0.0051 & 0.0161 & 0461 & 0.0705 & 0.1229 & 0.1624 & 0.2252 & 0.3336 & $0.54>a$ & $0.7 \cup 24$ \\
\hline 180 & 0.0000 & 0.0025 & 0.0135 & 0436 & 0.0680 & 0.1205 & 0.1602 & 0.2231 & 0.3320 & 0.5461 & 0.7415 \\
\hline
\end{tabular}




ENERGY (MEV)
ANGLE (DEG.)
0
5
10
15
20
25
30
35
40
45
50
55
60
65
70
75
80
85
90
95
100
105
110
115
120
125
130
135
140
145
150
155
160
165
170
175
180

100.
1.0000
1.0193
1.0377
1.0494
1.0570
1.0586
1.0552
1.0460
1.0317
1.0120
0.9874
0.9580
0.9243
0.8865
0.8451
0.8005
0.7531
0.7036
0.6522
0.5998
0.5466
0.0934
0.4405
0.3886
0.3381
0.2897
0.2436
0.2005
0.1606
0.1245
0.0925
0.0648
0.0418
0.0236
0.0106
0.0026
0.0000

10.
1.0000
1.0193
1.0377
1.0493
1.0570
1.0586
1.0552
1.0461
1.0318
1.0122
0.9877
0.9584
0.9247
0.8870
0.8457
0.8012
0.7539
0.7045
0.6533
0.6010
0.5479
0.4948
0.4420
0.3903
0.3399
0.2915
0.2455
0.2025
0.1628
0.1267
0.0947
0.0671
0.0442
0.0261
0.0130
0.0051
0.0025
0

11
1.0000
1.0192
.0375
1.0491
1.0569
1.0586
1.0553
1.0464
1.0324
1.0130
0.9888
0.9598
0.9266
0.8893
0.8484
0.8044
0.7576
0.7087
0.6580
0.6062
0.5537
0.5011
0.4488
0.3976
0.3477
0.2998
0.2543
0.2117
0.1723
0.1366
0.1049
0.0776
0.0549
0.0369
0.0240
0.0162
0.0136

2.
1.0000
1.0189
1.0370
1.0487
1.0565
1.0585
1.0556
1.0472
1.0338
1.0152
0.9918
0.9638
0.9316
0.8955
0.8558
0.8131
0.7677
0.7202
0.6709
0.6205
0.5695
0.5183
0.4675
0.4176
0.3691
0.3225
0.2782
0.2367
0.1984
0.1636
0.1328
0.1062
0.0841
0.0666
0.0540
0.0464
0.0439

1.5
1.0000
1.0187
1.0367
1.0483
1.0562
1.0584
1.0558
1.0478
1.0349
1.0169
0.9943
0.9671
0.0357
0.9005
0.8619
0.8202
0.7758
0.7294
0.6813
0.6321
0.5822
0.5322
0.4826
0.4338
0.3864
0.3408
0.2975
0.2570
0.2195
0.1855
0.1554
0.1294
0.1077
0.0905
0.0783
0.0709
0.0584

1.0
1.0000
1.0182
1.0358
1.0474
1.0555
1.0581
1.0562
1.0491
1.0373
1.0206
0.9995
0.9739
0.9444
0.9112
0.8747
0.8353
0.7933
0.7493
0.7037
0.6570
0.5096
0.5622
0.5150
0.4687
0.4236
0.3803
0.3391
0.3005
0.2649
0.2326
0.2039
0.1792
0.1586
0.1123
0.1307
0.1235
0.1212

0.8
1.0000
1.0178
1.0352
1.0467
1.0549
1.0579
1.0565
1.0500
1.0390
1.0233
1.0033
0.9990
0.9509
0.9192
0.8843
0.8466
0.8064
0.7642
0.7205
0.6757
0.5303
0.5847
0.5394
0.4949
0.4516
0.4100
0.3704
0.3334
0.2992
0.2681
0.2406
0.2168
0.1970
0.1814
0.1701
0.1633
0.1610

0.5
1.0000
1.0171
1.0341
1.0456
1.0540
1.0574
1.0568
1.0513
1.0416
1.0275
1.0092
0.9870
0.9611
0.9318
0.8995
0.8644
0.8271
0.7878
0.7471
0.7053
0.6629
0.6204
0.5781
0.5365
0.4961
0.4572
0.11202
0.3856
0.3535
0.3245
0.2987
0.2765
0.2579
0.2433
0.2328
0.2264
0.2243

0.4
1.0000
1.0159
1.0320
1.0433
1.0520
1.0562
1.0569
1.0532
1.0457
1.0342
1.0191
1.0003
0.9782
0.9531
0.9252
0.8949
0.8624
0.8283
0.7927
0.7562
0.7191
0.6819
0.6448
0.5083
0.5728
0.5387
0.5062
0.4757
0.4475
0.4220
0.3693
0.3798
0.3635
0.3506
0.3410
0.3357
0.3339

\begin{tabular}{|c|c|}
\hline 0.2 & 3.1 \\
\hline 1.0000 & 1.0500 \\
\hline $1.013 n$ & 1.0096 \\
\hline 1.0271 & 1.3210 \\
\hline 1.0377 & 1.0303 \\
\hline 1. OEE 6 & 1.0389 \\
\hline 1.0522 & 1.0453 \\
\hline 1.0552 & 1.3500 \\
\hline 1.0548 & 1.0524 \\
\hline 1.0516 & 1.0528 \\
\hline 1.0453 & 1.0510 \\
\hline 1.0361 & 1. $90-2$ \\
\hline 1.0249 & 1.0413 \\
\hline 1.0096 & 1.0336 \\
\hline 9026 & 1.0242 \\
\hline $9-35$ & 1.5131 \\
\hline 0524 & 1.0007 \\
\hline 0.9297 & $0.9 B>0$ \\
\hline 0.9056 & 0.9723 \\
\hline O. RRDU & 0.9567 \\
\hline 0.8544 & 0.0405 \\
\hline $82^{79}$ & 0.9230 \\
\hline 8011 & $0.90>0$ \\
\hline 7944 & 0.8902 \\
\hline 7482 & $0.9^{7} 35$ \\
\hline 7225 & $0.8=72$ \\
\hline 0.6978 & 0.849 \\
\hline $0.6>13$ & 0.8264 \\
\hline 0.6522 & 0.8122 \\
\hline 0.6318 & 0.7992 \\
\hline 0.6133 & 0.7973 \\
\hline 0.5968 & 0.7767 \\
\hline 0.5826 & 0.7676 \\
\hline 0.5708 & 0.7500 \\
\hline & 0.7540 \\
\hline 0.5547 & 0.7496 \\
\hline 0.5506 & 0.7470 \\
\hline
\end{tabular}

$$
\text { RATIO OF MOTT TO ROTHERFOAD SCMTTPRTNG IN CR, } z=24
$$

$\begin{array}{cc}\text { ENERGY (MEV) } & 100 \\ \text { ANG LE (DEG.) } & \\ 0 & 1.0000 \\ 5 & 1.0202 \\ 10 & 1.0397 \\ 15 & 1.0524 \\ 20 & 1.0612 \\ 25 & 1.0638 \\ 30 & 1.0614 \\ 35 & 1.0531 \\ 40 & 1.0396 \\ 45 & 1.0206 \\ 50 & 0.9965 \\ 55 & 0.9676 \\ 60 & 0.9341 \\ 65 & 0.8965 \\ 70 & 0.8551 \\ 75 & 0.8104 \\ 80 & 0.7628 \\ 85 & 0.7130 \\ 90 & 0.6613 \\ 95 & 0.6080 \\ 100 & 0.5546 \\ 105 & 0.5008 \\ 110 & 0.0473 \\ 115 & 0.3947 \\ 120 & 0.3435 \\ 125 & 0.2944 \\ 130 & 0.2476 \\ 135 & 0.2038 \\ 140 & 0.1633 \\ 145 & 0.1267 \\ 150 & 0.0941 \\ 155 & 0.0659 \\ 160 & 0.0425 \\ 165 & 0.0241 \\ 170 & 0.0108 \\ 175 & 0.0027 \\ 180 & 0.0000 \\ & \\ & \end{array}$

10.
.0000
.0202
.0396
.0524
.0611
.0638
.0614
.0532
.0397
.0207
.9968
.9679
.9345
.8970
.8557
.8111
.7637
.7139
.6623
.6095
.05559
.5022
.4488
.3964
.3453
.2963
.2496
.2059
.1655
.1289
.0963
.0683
.0449
.0265
.0132
.0052
.0025

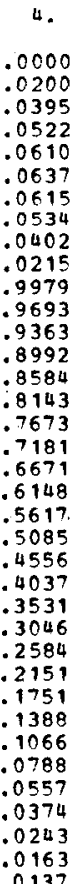

2.
1.0000
1.0197
1.0390
1.0517
1.0606
1.0636
1.05177
1.0541
1.0415
1.0236
1.0008
0.9732
0.9413
0.9053
0.8657
0.8229
0.7773
0.7295
0.6799
0.6291
0.5775
0.5257
0.0743
0.4238
0.3746
0.3273
0.2823
0.2402
0.2012
0.1660
0.1346
0.1075
0.0850
0.0672
0.0545
0.0467
0.0441

1.5
1.0000
1.0195
1.0386
1.0512
1.0602
1.0634
1.0619
1.0547
1.0426
1.0252
1.0031
0.9763
0.9453
0.9103
0.8717
0.8299
0.7854
0.7387
0.6903
0.6406
0.5902
0.5397
0.4894
0.4400
0.3919
0.3457
0.3017
0.2605
0.2224
0.1879
0.1573
0.1308
0.1088
0.0914
0.0789
0.0713
0.0688

1.0
1.0000
1.0190
1.0377
1.0503
1.0594
1.0630
1.0621
1.0558
1.0448
1.0287
1.0081
0.9830
0.9538
0.9208
0.8843
0.8448
0.8027
0.7585
0.7125
0.6654
0.6175
0.5696
0.5218
0.4749
0.4292
0.3852
0.3434
0.3043
0.2681
0.2352
0.2061
0.1809
0.1599
0.1434
0.1315
0.1243
0.1219

0.8
1.0000
1.0185
1.0370
1.0495
1.0588
1.0627
1.0622
1.0565
1.0464
1.0313
1.0118
0.9880
0.9602
0.9287
0.8938
0.8560
0.8157
0.7733
0.7293
0.6849
0.6382
0.5921
0.5462
0.5012
0.4572
0.4151
0.3749
0.3373
0.3024
0.2709
0.2429
0.2187
0.1985
0.1827
0.1712
0.1643
0.1620

0.6
1.0000
1.0178
1.0358
1.0482
1.0576
1.0620
1.0523
1.0577
1.0488
1.0352
1.0175
0.9957
0.9701
0.9410
0.9087
0.8737
0.8362
0.7968
0.7557
0.7136
0.6708
0.6278
0.5849
0.5428
0.5018
0.4524
0.4248
0.3896
0.3571
0.3276
0.3013
0.2787
0.2598
0.2450
0.2343
0.2278
0.2256

0.6

.0000

.0482

1.0576
1.0620

1.0523

1.0488

1.0352

0.9957

0.9701

0.9087

0.8737
0.8362

0.7968
0.7557

0.7557
0.7136

0.6708

0.5849

0.5428

0.4524

0.3896

0.3571
0.3276

0.3013

0.2787

0.2598

0.2343
0.2278

0.2256 1.0165
1.0336

1.0336
1.0457

1.0554

1. 0605

1.0620
1.0592

1.0592

1.0415

1.0269

1.0085
0.0958

. 961

0.9341

0.9037

0.8712
0.8369

0.8369

0.7643

0.7643
0.7268

0.6892

0.6516

0.6147
0.5787

0.5440

0.5190
0.4801

0.4515

0.4255
0.4025

0.3826

0.3660

0.3435

0.3378

0.3378
0.3358
0.2

1.0000 1.0135

1.0283

1.0396

1.0494
1.0558

1. 0595

1.0509
1.0573

1. 0516

1.0429

1.0313
1.0179

1.0004

0.9814

0.9604

0.9377

0.9135

0.8882

0.8620

0.8352

0.8082
0.7813

0.7813
0.7547

C. 7287

0.7036

0.6797
0.6573

0.6366

0.6178

0.6010

0.5866
0.5745

0.5745

0.5582

0.5540

0.5540
0.5526

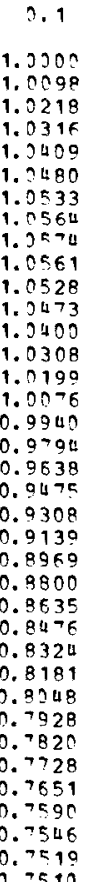

1.3900 1. 0218 1.0316
1.3409 1. 2480 1.0533
1.0564 . 3574 1.0561
1.0528 1. 3473 1.0308 1.0076 0.9940 0.9638 0.9475 0.9308 0.8969 0.9800 0.8635
0.8476 .8324 0.8181 0.3248
ก. 7928 0.7820 0.7728 0.7590
0.7546 0.7546 0.7510 
RATIO OF MOTT TO BUTHEGFOPD SCAMTRRTNG IN FE, $\mathrm{z}=26$

\begin{tabular}{|c|c|c|}
\hline $\begin{array}{l}\text { ENEFEY (HEV) } \\
\text { ANGLE (DEG.) }\end{array}$ & 100. & 10. \\
\hline & 1.0000 & 1.0000 \\
\hline 5 & 1.0218 & 1.0218 \\
\hline 10 & 1.0436 & 1.0435 \\
\hline 15 & 1.0585 & 1.0584 \\
\hline 20 & 1.0695 & 1.0695 \\
\hline 25 & 1.0743 & 1.0743 \\
\hline 30 & 1.0740 & 1.0740 \\
\hline 35 & 1.0576 & 1.0676 \\
\hline 40 & 1.0557 & 1.0558 \\
\hline 45 & 1.0381 & 1.0382 \\
\hline 50 & 1.0152 & 1.0155 \\
\hline 55 & 0.9872 & 0.9875 \\
\hline 60 & 0.9544 & 0.9548 \\
\hline 65 & 0.9171 & 0.9176 \\
\hline 70 & 0.8759 & 0.8764 \\
\hline 75 & 0.8310 & 0.8317 \\
\hline 80 & 0.7831 & 0.7839 \\
\hline 85 & 0.7327 & 0.7336 \\
\hline 90 & 0.6802 & 0.6812 \\
\hline $\begin{array}{r}95 \\
100\end{array}$ & $\begin{array}{l}0.6263 \\
0.5715\end{array}$ & $\begin{array}{l}0.6275 \\
0.5727\end{array}$ \\
\hline 105 & 0.5164 & 0.5178 \\
\hline 110 & 0.4615 & 0.4630 \\
\hline 115 & 0.4075 & 0.4092 \\
\hline 120 & 0.3549 & 0.3567 \\
\hline 125 & 0.3043 & 0.3062 \\
\hline 130 & 0.2561 & 0.2580 \\
\hline 135 & 0.2109 & 0.2130 \\
\hline 140 & 0.1691 & 0.1712 \\
\hline 145 & 0.1311 & 0.1334 \\
\hline 150 & 0.0974 & 0.0997 \\
\hline 155 & 0.0683 & 0.0707 \\
\hline 160 & 0.0441 & 0.0465 \\
\hline 165 & 0.0249 & 0.0274 \\
\hline 170 & 0.0112 & 0.0137 \\
\hline 175 & 0.0028 & 0.0053 \\
\hline 180 & 0.0000 & 0.0025 \\
\hline
\end{tabular}

\begin{tabular}{|c|c|c|c|}
\hline $\begin{array}{l}0000 \\
0217\end{array}$ & $\begin{array}{l}1.0000 \\
1.0213\end{array}$ & $\begin{array}{l}1.0000 \\
1.0210\end{array}$ & $\begin{array}{r}1.0000 \\
1.0204\end{array}$ \\
\hline .0433 & 1.0428 & 1.0423 & 1.0413 \\
\hline .0582 & 1.0576 & 1.0571 & 1.0559 \\
\hline .0693 & 1.0688 & 1.0683 & 1.0573 \\
\hline .0742 & 1.0739 & 1.0736 & 1.0729 \\
\hline .0740 & 1.0741 & 1.0741 & 1.0740 \\
\hline .0678 & 1.0683 & 1.0687 & 1.0695 \\
\hline .0562 & 1.0570 & 1.0582 & 1.0601 \\
\hline 389 & 1.0408 & 1.0423 & 1.0454 \\
\hline .0165 & 1.0192 & 1.0210 & 1.0260 \\
\hline 888 & 0.9925 & 0.9955 & 1.0017 \\
\hline 565 & 0.9613 & 0.9651 & 0.9732 \\
\hline 9198 & 0.9257 & 0.9304 & 0.9406 \\
\hline .8791 & 0.8862 & 0.8920 & 0.9043 \\
\hline 348 & 0.8433 & 0.8501 & 0.8647 \\
\hline 375 & 0.7974 & 0.8053 & 0.8223 \\
\hline 377 & 0.7490 & 0.7581 & 0.7776 \\
\hline 5859 & 0.6986 & 0.7089 & 0.7310 \\
\hline 327 & 0.6469 & 0.6584 & 0.6830 \\
\hline 5785 & 0.5942 & 0.6060 & 0.6342 \\
\hline 41 & 0.5413 & 0.5552 & 0.5851 \\
\hline 699 & 0.4885 & 0.5036 & 0.5361 \\
\hline 166 & 0.4367 & 0.4529 & 0.4879 \\
\hline .3645 & 0.3861 & 0.4035 & 0.4409 \\
\hline 3145 & 0.3374 & 0.3559 & 0.3957 \\
\hline .2669 & 0.2910 & 0.3105 & 0.3526 \\
\hline 2222 & 0.2475 & 0.2680 & 0.3121 \\
\hline 1809 & 0.2073 & $0.228^{7}$ & 0.2747 \\
\hline . 1434 & 0.1708 & 0.1930 & 0.2408 \\
\hline $\begin{array}{l}.11 \\
.08\end{array}$ & 0.1384 & $\begin{array}{r}0.1613 \\
0.1330\end{array}$ & $\begin{array}{l}0.2106 \\
0.1846\end{array}$ \\
\hline 574 & $0.08>0$ & 0.1111 & 0.1628 \\
\hline 385 & 0.0686 & 0.0931 & 0.1457 \\
\hline 248 & 0.0554 & 0.0801 & 0.1334 \\
\hline $\begin{array}{l}.0166 \\
.0138\end{array}$ & $\begin{array}{l}0.0473 \\
0.0447\end{array}$ & $\begin{array}{l}0.0723 \\
0.0697\end{array}$ & $\begin{array}{l}0.1259 \\
0.1234\end{array}$ \\
\hline & & & \\
\hline
\end{tabular}

0.8
1.0000
1.0200
1.0404
1.0550
1.0664
1.0723
1.0739
1.0700
1.0614
1.0476
1.0293
1.0064
0.9792
0.9482
0.9135
0.8757
0.8351
0.7922
0.7475
0.7016
0.6547
0.6076
0.5605
0.5143
0.4691
0.4257
0.3842
0.3454
0.3094
0.2768
0.2478
0.2228
0.2019
0.1854
0.1736
0.1664
0.1640

0.6
1.0000
1.0192
1.0391
1.0535
1.0650
1.0713
1.0735
1.0706
1.0633
1.0511
1.0345
1.0136
0.9887
0.9500
0.9279
0.8929
0.8552
0.8153
0.7737
0.7309
0.6872
0.6432
0.5993
0.5561
0.5139
0.4732
0.4345
0.3982
0.3646
0.3340
0.3069
0.2835
0.2640
0.2486
0.2375
0.2307
0.2285

0.4
1.0000
1.0177
1.0366
1.0506
1.0522
1.0692
1.0725
1.0713
1.0561
1.0565
1.0430
1.0255
1.0044
0.9800
0.9524
0.9222
0.8895
0.8549
0.8186
0.7812
0.7430
0.7045
0.6660
0.6281
0.5911
0.5554
0.5214
0.4895
0.0599
0.4331
0.4092
0.3886
0.3714
0.3578
0.3481
0.3422
0.3402

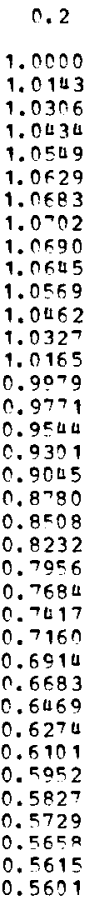

3.1

1.2200 1. 0101 1. .232 1.0341 1. 1148 1. 0532 1.0643 1. 0666 1.3641 1. 0506 1. 0506 1. 2530 1. 2349 1. 0221 0.9042 0.9986 0.0623 0.0155 0.9283 $0.011 n$ 0.8939 0.8 .71 0.8608 0.9152 0.8170 0.8946 0.8946
0.7936 0.7849 0.7762
0.7699 D. 7654 0.7627

SATIO OF MOTM TO EUTHERFCPD SCATTERING IN NT, $z=28$

$\begin{array}{ccc}\text { ENEFGY (IEV) } & 100 . & 10 . \\ \text { ANGIE (DEG.) } & & \\ 0 & 1.0000 & 1.0000 \\ 5 & 1.0234 & 1.0233 \\ 10 & 1.0474 & 1.0473 \\ 15 & 1.0645 & 1.0644 \\ 20 & 1.0779 & 1.0779 \\ 25 & 1.0850 & 1.0849 \\ 30 & 1.0868 & 1.0868 \\ 35 & 1.0823 & 1.0824 \\ 40 & 1.0723 & 1.0724 \\ 45 & 1.0562 & 1.0563 \\ 50 & 1.0347 & 1.0349 \\ 55 & 1.0076 & 1.0079 \\ 60 & 0.9755 & 0.9759 \\ 65 & 0.9387 & 0.9392 \\ 70 & 0.8976 & 0.8982 \\ 75 & 0.8527 & 0.8534 \\ 80 & 0.8045 & 0.8053 \\ 85 & 0.7535 & 0.7544 \\ 90 & 0.7002 & 0.7012 \\ 95 & 0.6453 & 0.6464 \\ 100 & 0.5893 & 0.5906 \\ 105 & 0.5329 & 0.5343 \\ 110 & 0.4766 & 0.4782 \\ 115 & 0.4212 & 0.4228 \\ 120 & 0.3670 & 0.3688 \\ 125 & 0.3148 & 0.3167 \\ 130 & 0.2651 & 0.2671 \\ 135 & 0.2184 & 0.2205 \\ 140 & 0.1752 & 0.1773 \\ 145 & 0.1359 & 0.1382 \\ 150 & 0.1010 & 0.1033 \\ 155 & 0.0708 & 0.0732 \\ 160 & 0.0457 & 0.0482 \\ 165 & 0.0259 & 0.0284 \\ 170 & 0.0196 & 0.0149 \\ 175 & 0.0029 & 0.0051 \\ 180 & 0.0000 & 0.0026\end{array}$

4.
1.0000
1.0232
1.0471
1.0642
1.0776
1.0847
1.0868
1.0825
1.0727
1.0570
1.0358
1.0092
0.9776
0.9413
0.9008
0.8564
0.8088
0.7585
0.7058
0.6516
0.5963
0.5406
0.4850
0.4302
0.3767
0.3252
0.2760
0.2299
0.1871
0.1483
0.1138
0.0840
0.0592
0.0396
0.0254
0.0169
0.0140

2.
1.0000
1.0228
1.0465
1.0634
1.0770
1.0843
1.0866
1.0828
1.0736
1.0586
1.0383
1.0126
0.9821
0.9470
0.9077
0.8647
0.8185
0.7696
0.7184
0.6657
0.6120
0.5578
0.5037
0.4504
0.3983
0.3482
0.3003
0.2554
0.2138
0.1760
0.1424
0.1134
0.0892
0.0701
0.0564
0.0480
0.0453

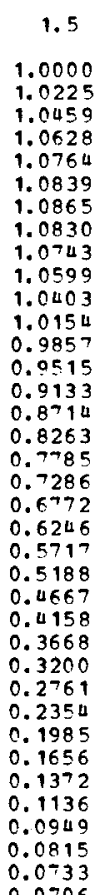

1.0
1.0000
1.0218
1.0448
1.0615
1.0751
1.0829
1.0861
1.0834
1.0758
1.0626
1.0444
1.0212
0.9934
0.9693
0.9252
0.8857
0.8430
0.7978
0.7505
0.7017
0.6518
0.6016
0.5513
0.5019
0.4535
0.4068
0.3624
0.3206
0.2819
0.2468
0.2155
0.1885
0.1660
0.1483
0.1355
0.1277
0.1251

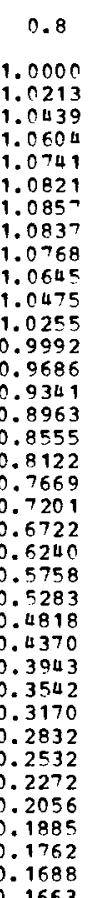

0.1663

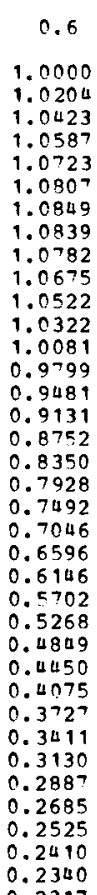

0.2317

0.4
1.0000
1.0188
1.0395
1.0553
1.0689
1.0779
1.0831
1.0837
1.0801
1.0719
1.0596
1.0432
1.0229
0.9989
0.9717
0.9416
0.9088
0.8739
0.8372
0.7991
0.7602
0.7208
0.6814
0.6425
0.6044
0.5677
0.5326
0.4996
0.4690
0.4413
0.4166
0.3952
0.3774
0.3634
0.3532
0.3471
0.3450

0.3450

\begin{tabular}{|c|c|}
\hline 0.2 & 0.1 \\
\hline 1.0000 & 1.0900 \\
\hline 1.0150 & 1. $010 \mathrm{u}$ \\
\hline $1.032^{7}$ & 1.2244 \\
\hline 1.0471 & 1. 0364 \\
\hline 1.0602 & 1. 3484 \\
\hline $1.0=00$ & 1.0582 \\
\hline 1.0771 & 1.0664 \\
\hline 1.0805 & 1.0722 \\
\hline 1.0809 & 1. 0758 \\
\hline $1.0^{-777}$ & 1.0769 \\
\hline $1.0-12$ & 1.2759 \\
\hline 1.0615 & 1.0722 \\
\hline 1.0488 & 1.9664 \\
\hline 1.0333 & 1.0585 \\
\hline 1.0151 & 1.0388 \\
\hline 0.9946 & 1.0373 \\
\hline $0.9 \div 20$ & 1. ?242 \\
\hline $0.94-7$ & 1.0098 \\
\hline 0.9219 & 0.9944 \\
\hline 0.8950 & 0.9781 \\
\hline $0.86^{73}$ & 0.9611 \\
\hline 0.8393 & 0.9438 \\
\hline 0.8111 & 0.9253 \\
\hline 0.7832 & 0.9099 \\
\hline 0.7558 & 0.3918 \\
\hline 0.7293 & 0.8752 \\
\hline 0.7000 & 0.8593 \\
\hline 0.6802 & 0.8443 \\
\hline $0.6\ulcorner 32$ & 0.9304 \\
\hline 0.6381 & $0.81^{77}$ \\
\hline 0.6202 & 0.8265 \\
\hline 0.6048 & $0.796^{7}$ \\
\hline 0.5919 & 0.7886 \\
\hline 0.5817 & 0.7821 \\
\hline 0.5704 & \\
\hline 0.5699 & 0.7749 \\
\hline & \\
\hline
\end{tabular}

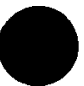


RATTO OP MOTM TO RUMHERFOPD SCATTERTMG IN CU, $Z=20$

$\begin{array}{cc}\text { ENERGY (MEVI } & 100 \\ \text { ANGLE (DEG.) } & \\ 0 & 1.0000 \\ 5 & 1.0241 \\ 10 & 1.0493 \\ 15 & 1.0675 \\ 20 & 1.0821 \\ 25 & 1.0903 \\ 30 & 1.0933 \\ 35 & 1.0899 \\ 40 & 1.0808 \\ 45 & 1.0655 \\ 50 & 1.0446 \\ 55 & 1.0181 \\ 60 & 0.9865 \\ 65 & 0.9499 \\ 70 & 0.9089 \\ 75 & 0.8640 \\ 80 & 0.8156 \\ 85 & 0.7643 \\ 90 & 0.7106 \\ 95 & 0.6552 \\ 100 & 0.5986 \\ 105 & 0.5416 \\ 110 & 0.4845 \\ 115 & 0.4283 \\ 120 & 0.3734 \\ 125 & 0.3204 \\ 130 & 0.2698 \\ 135 & 0.2224 \\ 140 & 0.1784 \\ 145 & 0.1384 \\ 150 & 0.1029 \\ 155 & 0.0722 \\ 160 & 0.0466 \\ 165 & 0.0264 \\ 170 & 0.0118 \\ 175 & 0.0029 \\ 180 & 0.0000 \\ & \\ 150 & \\ 150 & \end{array}$

10.
1.0000
1.0241
1.0492
1.0674
1.082 .1
1.0903
1.0933
1.0899
1.0808
1.0656
1.0448
1.0184
0.9868
0.9504
0.9095
0.8647
0.8163
0.7652
0.7116
0.6563
0.5999
0.5430
0.4861
0.4300
0.3751
0.3223
0.2718
0.2245
0.1806
0.1407
0.1052
0.0746
0.0490
0.0289
0.0144
0.0055
0.0026

$\begin{array}{cc}4 & 2 . \\ 1.0000 & 1.0000 \\ 1.0239 & 1.0236 \\ 1.0490 & 1.0483 \\ 1.0671 & 1.0563 \\ 1.0818 & 1.0811 \\ 1.0901 & 1.0895 \\ 1.0932 & 1.0930 \\ 1.0900 & 1.0902 \\ 1.0811 & 1.0819 \\ 1.0662 & 1.0677 \\ 1.0457 & 1.0481 \\ 1.0196 & 1.0229 \\ 0.9884 & 0.9928 \\ 0.9524 & 0.9580 \\ 0.9120 & 0.9188 \\ 0.8677 & 0.8758 \\ 0.8199 & 0.8294 \\ 0.7692 & 0.7803 \\ 0.7162 & 0.7288 \\ 0.6615 & 0.0756 \\ 0.6056 & 0.6212 \\ 0.5493 & 0.5664 \\ 0.4929 & 0.5116 \\ 0.4374 & 0.4576 \\ 0.3839 & 0.4048 \\ 0.3307 & 0.3538 \\ 0.2808 & 0.3052 \\ 0.2339 & 0.2595 \\ 0.1904 & 0.2172 \\ 0.1509 & 0.1788 \\ 0.1158 & 0.1446 \\ 0.0854 & 0.1150 \\ 0.0601 & 0.0904 \\ 0.0402 & 0.0710 \\ 0.0258 & 0.0669 \\ 0.0170 & 0.0484 \\ 0.0141 & 0.0456 \\ & \\ 0.0149 & \end{array}$

1.5
1.0000
1.0232
1.0477
1.0657
1.0804
1.0890
1.0927
1.0903
1.0826
1.0689
1.0500
1.0256
0.9964
0.9625
0.9243
0.8824
0.8372
0.7892
0.7389
0.6870
0.6339
0.5803
0.5268
0.4740
0.4223
0.3725
0.3250
0.2803
0.2389
0.2014
0.1679
0.1390
0.1149
0.0959
0.0822
0.0739
0.0711

1.0
1.0000
1.0225
1.0465
1.0643
1.0790
1.0979
1.0922
1.0906
1.0838
1.0714
1.0539
1.0312
1.0039
0.9720
0.9361
0.8965
0.8537
0.8083
0.7506
0.7114
0.6610
0.6102
0.5593
0.5092
0.0601
0.4127
0.3675
0.3251
0.2857
0.2500
0.2182
0.1907
0.1677
0.1496
0.1366
0.1287
0.1261

0.8
1.0000
1.0219
1.0455
1.0631
1.0779
1.0870
1.0917
1.0906
1.0846
1.0732
1.0568
1.0354
1.0094
0.9791
0.9448
0.9070
0.8661
0.8226
0.7770
0.7298
0.8814
0.6327
0.5838
0.5357
0.4885
0.4430
0.3996
0.3588
0.3210
0.2866
0.2561
0.2296
0.2076
0.1902
0.1777
0.1701
0.1675

0.6
1.0000
1.0210
1.0439
1.0612
1.0760
1.0854
1.0907
1.0906
1.0858
1.0758
1.0612
1.0418
1.0181
0.9902
0.9586
0.9235
0.8856
0.8452
0.8027
0.7588
0.7137
0.6682
0.6227
0.5777
0.5336
0.4091
0.4505
0.4124
0.3770
0.3449
0.3163
0.2915
0.2709
0.2547
0.2429
0.2358
0.2334

0.2

1.0000 1.0153 1.0338
1.0488 1.0488 1.0629 1.0735
1.0814 1. 0858 1.0869 1.0844 1.0786 1. 0694 1.0572 1.0240 1. 0240 1.0037 C. 9 . 12 0.9568 0.9309 0.9039 $0.8^{-60}$ 0.8477 0.8192 0.7010 c. 7633 0.7108 0.5866 0.6642 0.6438 0.6256 0.5256
0.6099 0.6099
0.5058 0.5865 0.5765
0.5790 0.5790
0.5745 0.5729

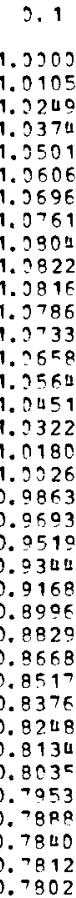

RATTO OF MOTT TO RUTHERFCRD SCATTERTNG IN $\mathrm{zN}, \mathrm{z}=30$

$\begin{array}{cc}\text { ENERGY (MEVI } & 100 \\ \text { ANGIE (DEG.) } & \\ 0 & 1.0000 \\ 5 & 1.0248 \\ 10 & 1.0511 \\ 15 & 1.0704 \\ 20 & 1.0863 \\ 25 & 1.0957 \\ 30 & 1.0999 \\ 35 & 1.0975 \\ 40 & 1.0893 \\ 45 & 1.0749 \\ 50 & 1.0548 \\ 55 & 1.0288 \\ 60 & 0.9976 \\ 65 & 0.9613 \\ 70 & 0.9205 \\ 75 & 0.8755 \\ 80 & 0.8270 \\ 85 & 0.7754 \\ 90 & 0.7213 \\ 95 & 0.6654 \\ 100 & 0.6082 \\ 105 & 0.5505 \\ 110 & 0.4927 \\ 115 & 0.4357 \\ 120 & 0.3799 \\ 125 & 0.3261 \\ 130 & 0.2747 \\ 135 & 0.2264 \\ 140 & 0.1817 \\ 145 & 0.1410 \\ 150 & 0.1048 \\ 155 & 0.0735 \\ 160 & 0.0475 \\ 165 & 0.0269 \\ 170 & 0.0120 \\ 175 & 0.0030 \\ 185 & 0.0000 \\ & \\ 130 & \end{array}$

1.0000$$
1.0248
$$

1.0511

1.0704

1.0956

1.0998

1.0975

1.089

1.0750
1.0550

1.0550
1.0291

0.9980

0.9618

0.9210
0.8762

0.8762
0.8277

0.7763

0.7223
0.6665

0.6665

0.6095

0.4942

0.4370

$0.3280^{\circ}$

0.2767

0.2286
0.1839

0.1433

0.1072

0.0760

0.0499
.0 .0294

0.0146

0.0056
0.0026

2.
1.0000
1.0243
1.0501
1.0692
1.0852
1.0948
1.0994
1.0976
1.0904
1.0770
1.0580
1.0335
1.0038
0.9692
0.9302
0.8872
0.8407
0.7913
0.7394
0.6857
0.6308
0.5754
0.5198
0.4650
0.4114
0.3597
0.3102
0.2638
0.2208
0.1816
0.1468
0.1167
0.0916
0.0718
0.0575
0.0488
0.0460

1.5

1.0000

$1.023^{\circ}$

1. 0685

1.0645

1. 0942

1.0991

1.0977

1.0909
1.0781

1.0781
1.0598

1.0598
1.0361

1.0072

0.9736
0.9356

0.8937

0.8484

0.8001

0.7495
0.6971

0.6434

0.6434
0.5893

0.5350

0.4815

0.4290

0.3784

0.3301
0.2847

0.2847

0.2426
0.2044

0.2044
0.1703

0.1703
0.1409

0.1163
0.0969

0.0969
0.0830

0.0830
0.0745
0.0717

1.0
1.0000
1.0232
1.0482
1.0670
1.0829
1.0930
1.0983
1.0977
1.0919
1.0803
1.0636
1.0415
1.0145
0.9830
0.9472
0.9076
0.8648
0.8191
0.7711
0.7210
0.6705
0.6191
0.5676
0.5167
0.4669
0.0188
0.3729
0.3297
0.2897
0.2533
0.2209
0.1929
0.1695
0.1511
0.1378
0.1297
0.1270

0.0261

0.0172
0.0142

0.0460
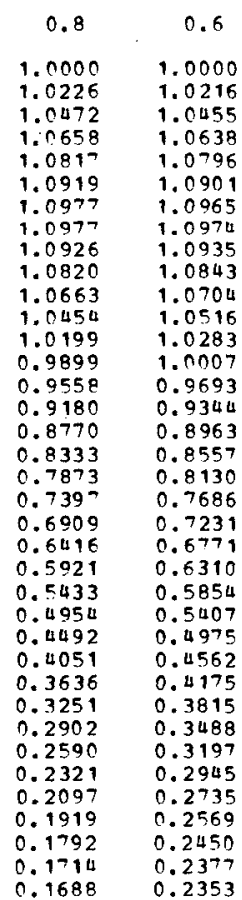

0.4
1.0000
1.0198
1.0423
1.0599
1.0756
1.0865
1.0938
1.0962
1.0944
1.0878
1.0768
1.0615
1.0420
1.0188
0.9919
0.9620
0.9291
0.8940
0.8568
0.8182
0.7785
0.7383
0.6979
0.6579
0.6187
0.5809
0.5447
0.5106
0.4790
0.4503
0.4247
0.4025
0.3841
0.3695
0.3590
0.3526
0.3505

0.2

1.0000 1.0156

1.0347

1.0555

1.0655

1.0769

1.0858
1.0010

1.002

1.0912
1.0860

1.0860
$1.09-4$

1.0656

.0508

1.0332
1.0130

1.0130
0.9905

0.9905
0.9562

0.9403

0.913

0.8850

0.8564
0.8277

0.799

0.799
0.7710

0.7438

0.717

0.5033

0.6755

0.5313

0.5313
0.5153

$0.602 \mathrm{n}$

$0.602 n$
0.5015

0.5839

0.5793
0.5777
0.1

1.3300 1. 1905

1. 3383

1. 0629

1. 0727

1. 0800

1. 2950

1. 2875
1.3875

1. 0850

1.5302
1.0732

1. OEU1

1.0531
1.2404

1. 0263

1. 0111

0.9948
0.9778

0.9504

0.9427

0.0251

0.8908

0.8746

0.8503

0.8452

0.9322

$0.820^{7}$

0.8223

0.7957

0.7910

0.7881
0.7879 
RATTO OF MOTT TO RUTHPRFOPD SCATTERING IN GE, $z=32$

\begin{tabular}{|c|c|c|c|c|c|c|c|c|c|c|c|}
\hline $\begin{array}{l}\text { BNEEGY (KEV) } \\
\text { ANGLE (DEG.) }\end{array}$ & 100 & 10. & 4. & 2. & 1.5 & 1.0 & 0.8 & 0.6 & 0.4 & 0.2 & 0.1 \\
\hline 0 & 1.0000 & 1.0000 & 1.0000 & 1.0000 & 1.0000 & 1.0000 & 1.0000 & 1.0000 & 1.0000 & 1.0000 & 1.3900 \\
\hline 5 & 1.0262 & 1.0262 & 1.0260 & 1.0256 & 1.0252 & 1.0244 & 1.0237 & 1.0227 & 1.0207 & 1.0161 & 1.2106 \\
\hline 10 & 1.0547 & 1.0547 & 1.0544 & 1.0536 & 1.0529 & 1.0515 & 1.0503 & 1.0485 & 1.0049 & 1.0365 & 1.3261 \\
\hline 15 & 1.0763 & 1.0762 & 1.0759 & 1.0749 & 1.0742 & 1.0724 & 1.0710 & 1.0687 & 1.0603 & $1.053^{7}$ & 1.0400 \\
\hline 20 & 1.0947 & 1.0946 & 1.0943 & 1.0933 & 1.0925 & 1.0907 & 1.0893 & 1.0868 & 1.0822 & $1.0>05$ & 1.0547 \\
\hline 25 & 1.1065 & 1.1065 & 1.1062 & 1.1053 & 1.1046 & $1: 1031$ & 1.1018 & 1.0996 & 1.0052 & 1. ก.838 & 1. DE74 \\
\hline 30 & 1.1139 & 1.1130 & 1.1129 & 1.1123 & 1.1118 & 1.1107 & 1.1098 & 1. 1081 & 1.1046 & 1.0045 & 1.2787 \\
\hline 35 & 1.1129 & 1.1129 & 1.1129 & 1.1128 & 1.1126 & 1. 1123 & 1.1119 & 1.1111 & 1.1090 & 1. 1015 & 1.3875 \\
\hline 40 & 1.1068 & 1.1069 & 1.1071 & 1.1075 & 1.1078 & 1. 1084 & 1.1088 & 1.1091 & 1.1090 & 1.1051 & 1.0942 \\
\hline 45 & 1.0942 & 1.0943 & 1.0947 & 1.0959 & 1.0968 & 1.0986 & 1.0999 & 1.1017 & 1.1040 & $1.1 \times 49$ & 1.0981 \\
\hline 50 & 1.0756 & 1.0758 & 1.0765 & 1.0785 & 1.0801 & 1.0833 & 1.0857 & 1.0892 & 9.0946 & 1.1012 & 1.3994 \\
\hline 55 & 1.0509 & 1.0511 & 1.0522 & 1.0552 & 1.0575 & 1.0625 & 1.0661 & 1.0716 & 1.0801 & 1.0038 & 1.9082 \\
\hline 60 & 1.0206 & 1.0209 & 1.0224 & 1.0265 & 1.0297 & 1.0365 & 1.0415 & 1.0493 & 1.0620 & 1.0831 & 1.0944 \\
\hline 65 & 0.9850 & 0.9854 & 0.9873 & 0.9925 & 0.9967 & 1.0056 & 1.0122 & 1.0224 & 1.0394 & 1.0690 & 1.0883 \\
\hline 70 & 0.9446 & 0.9450 & 0.9474 & 0.9539 & 0.9591 & 0.9702 & 0.9785 & 0.9914 & 1.0131 & $1.0=20$ & 1.3900 \\
\hline & & $\begin{array}{l}0.9001 \\
0.8514\end{array}$ & & 0.9109 & 0.9172 & & 0.9409 & 0.9567 & & $\begin{array}{r}1.0322 \\
1.0101\end{array}$ & $\begin{array}{l}1.0696 \\
1.0571\end{array}$ \\
\hline $\begin{array}{l}80 \\
85\end{array}$ & 0.7985 & $\begin{array}{l}0.8514 \\
0.7994\end{array}$ & $\begin{array}{l}0.8548 \\
0.8034\end{array}$ & 0.8142 & $\begin{array}{l}0.8716 \\
0.8229\end{array}$ & $\begin{array}{l}0.8877 \\
0.8416\end{array}$ & $\begin{array}{l}0.8997 \\
0.8556\end{array}$ & $\begin{array}{l}0.9186 \\
0.8777\end{array}$ & $\begin{array}{l}0.9506 \\
0.9152\end{array}$ & $\begin{array}{l}1.0101 \\
0.9828\end{array}$ & $\begin{array}{l}1.35711 \\
1.343^{7}\end{array}$ \\
\hline 90 & 0.7436 & 0.7446 & 0.7491 & 0.7615 & 0.7715 & 0.7929 & 0.8090 & 0.8343 & 0.8776 & 0.9597 & 1.3286 \\
\hline 95 & 0.6867 & 0.6878 & 0.6929 & 0.7069 & 0.7182 & 0.7424 & 0.7606 & 0.7893 & 0.8384 & 0.9323 & 1.0125 \\
\hline 100 & 0.6282 & 0.6295 & 0.6352 & 0.6508 & 0.6634 & 0.6900 & $0.710^{7}$ & 0.7428 & 0.7980 & 0.9039 & 0.9055 \\
\hline $\begin{array}{l}115 \\
120\end{array}$ & 0.3512 & 0.3954 & 0.4035 & 0.4254 & 0.4431 & 0.4812 & 0.5099 & 0.5555 & 0.6341 & 0.7874 & 0.9249 \\
\hline 125 & 0.3381 & 0.3400 & 0.3486 & 0.3720 & 0.3909 & 0.4316 & 0.4623 & 0.5110 & 0.5951 & 0.7595 & 0.9077 \\
\hline 130 & 0.2850 & 0.2870 & 0.2961 & 0.3209 & 0.3410 & 0.3841 & 0.4167 & 0.4684 & 0.5577 & 0.7327 & 0.3913 \\
\hline 135 & 0.2350 & 0.2372 & 0.2467 & 0.2728 & 0.2940 & 0.3395 & 0.3738 & 0.4283 & 0.5225 & 0.7075 & 0.8757 \\
\hline 140 & 0.1887 & 0.1909 & 0.2009 & 0.2282 & 0.2504 & 0.2980 & 0.3339 & 0.3910 & 0.4898 & 0.5800 & 0.8513 \\
\hline 145 & 0.1465 & 0.1488 & 0.1592 & 0.1877 & 0.2107 & 0.2603 & 0.2977 & 0.3571 & 0.4601 & 0.5626 & 0.8480 \\
\hline 150 & 0.1089 & 0.1113 & 0.1221 & 0.1515 & 0.1750 & 0.2267 & 0.2654 & 0.3269 & 0.1335 & 0.6435 & 0.8363 \\
\hline 155 & $0.076 \mathrm{a}$ & 0.0789 & 0.0900 & 0.1203 & 0.1408 & 0.1976 & 0.2374 & 0.3008 & 0.4106 & $0.62=0$ & 0.8260 \\
\hline 160 & 0.0493 & 0.0519 & 0.0632 & 0.0942 & 0.1193 & 0.1733 & 0.2141 & 0.2790 & 0.3914 & 0.6132 & 0.8175 \\
\hline 165 & 0.0279 & 0.0305 & 0.0421 & 0.0736 & 0.0991 & 0.1542 & 0.1957 & 0.2617 & 0.3763 & 0.6023 & 0.8108 \\
\hline 170 & 0.0125 & 0.0151 & 0.0268 & 0.0587 & 0.0846 & 0.1003 & 0.1824 & 0.2493 & 0.3653 & 0.5944 & 0.3950 \\
\hline 175 & 0.0031 & 0.0058 & 0.0175 & 0.0497 & 0.0758 & 0.1319 & 0.1744 & 0.2418 & 0.3587 & 0.5897 & 0.8030 \\
\hline 180 & 000 & 0.0027 & 0.0145 & 0.0467 & 0.0729 & 0.1292 & 0.1717 & 0.2393 & 0.3565 & $0.5,881$ & 0.8320 \\
\hline
\end{tabular}

RATIO OF MOTT TO RUTHERFOPD SCATTPRING IN RB, $z=3$ ?

$\begin{array}{ccc}\text { BNERGY (MEV) } & 100 & 10 \\ \text { ANG IE (DEG.) } & & \\ 0 & 1.0000 & 1.0000 \\ 5 & 1.0293 & 1.0293 \\ 10 & 1.0632 & 1.0632 \\ 15 & 1.0905 & 1.0904 \\ 20 & 1.1154 & 1.1153 \\ 25 & 1.1337 & 1.1336 \\ 30 & 1.1468 & 1.1467 \\ 35 & 1.1528 & 1.1527 \\ 40 & 1.1525 & 1.1524 \\ 45 & 1.1450 & 1.1450 \\ 50 & 1.1308 & 1.1309 \\ 55 & 1.1098 & 1.1100 \\ 60 & 1.0824 & 1.0827 \\ 65 & 1.0488 & 1.0492 \\ 70 & 1.0094 & 1.0099 \\ 75 & 0.9648 & 0.9655 \\ 80 & 0.9155 & 0.9162 \\ 85 & 0.8621 & 0.8629 \\ 90 & 0.8052 & 0.8061 \\ 95 & 0.7456 & 0.7467 \\ 100 & 0.6838 & 0.6851 \\ 105 & 0.6210 & 0.6224 \\ 110 & 0.5575 & 0.5590 \\ 115 & 0.4943 & 0.4960 \\ 120 & 0.4321 & 0.4340 \\ 125 & 0.3718 & 0.3737 \\ 130 & 0.3139 & 0.3159 \\ 135 & 0.2592 & 0.2614 \\ 140 & 0.2083 & 0.2106 \\ 145 & 0.1619 & 0.1643 \\ 150 & 0.1205 & 0.1230 \\ 155 & 0.0846 & 0.0872 \\ 160 & 0.0547 & 0.0573 \\ 165 & 0.0310 & 0.0337 \\ 170 & 0.0139 & 0.0166 \\ 175 & 0.0035 & 0.0062 \\ 180 & 0.0000 & 0.0028\end{array}$

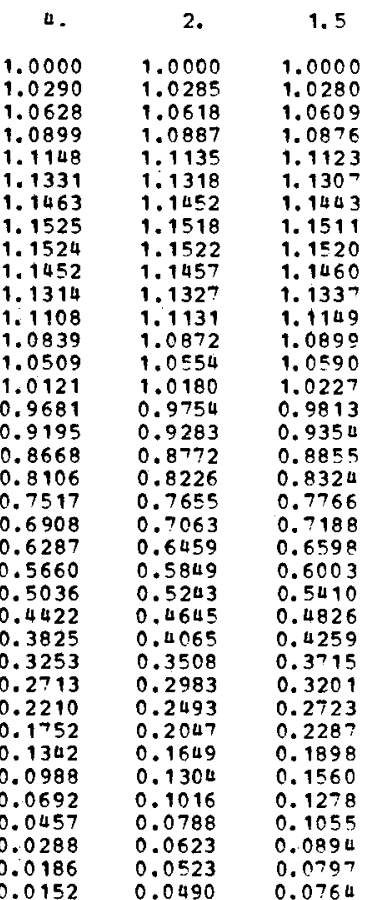

\begin{tabular}{|c|c|}
\hline 1.0 & 0.8 \\
\hline $\begin{array}{l}1.0000 \\
1.0270\end{array}$ & $\begin{array}{l}1.0000 \\
1.0262\end{array}$ \\
\hline 1.0591 & 1.0576 \\
\hline 1.0853 & 1.0834 \\
\hline 1. 1098 & 1.1078 \\
\hline 1. 1283 & 1.1263 \\
\hline 1. 1422 & 1.1405 \\
\hline 1. 1497 & 1.1484 \\
\hline 1.1514 & 1.1508 \\
\hline 1. 1466 & 1.1469 \\
\hline 1.1357 & 1.1371 \\
\hline 1.1185 & 1. 1211 \\
\hline $\begin{array}{l}1.0955 \\
1.0667\end{array}$ & $\begin{array}{l}1.0995 \\
1.0723\end{array}$ \\
\hline $\begin{array}{l}1.0667 \\
1.0326\end{array}$ & 1.0400 \\
\hline 0.9938 & 1.0030 \\
\hline 0.9505 & 0.9618 \\
\hline 0.9035 & 0.9169 \\
\hline 0.8532 & 0.8688 \\
\hline 0.8004 & 0.8182 \\
\hline 0.7456 & 0.7658 \\
\hline 0.6897 & 0.7122 \\
\hline 0.6333 & 0.6581 \\
\hline $\begin{array}{l}0.5770 \\
0.5216\end{array}$ & $\begin{array}{r}0.6041 \\
0.5500\end{array}$ \\
\hline $\begin{array}{l}0.5216 \\
0.4677\end{array}$ & 0.4992 \\
\hline 0.4160 & 0.4496 \\
\hline 0.3672 & 0.4027 \\
\hline 0.3217 & 0.3590 \\
\hline 0.2803 & 0.3192 \\
\hline 0.2433 & 0.2837 \\
\hline 0.2112 & $0.252 B$ \\
\hline 0.18 u & 0.2271 \\
\hline $\begin{array}{l}0.1632 \\
0.1479\end{array}$ & $\begin{array}{l}0.2067 \\
0.1920\end{array}$ \\
\hline 0.1386 & 0.1831 \\
\hline 0.1355 & 0.1801 \\
\hline
\end{tabular}

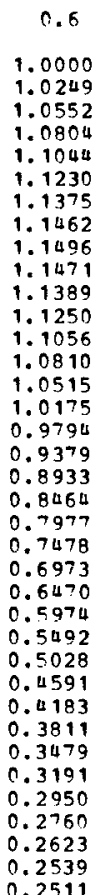

0.4
1.0000
1.0224
1.0507
1.0746
1.0979
1.1164
1.1315
1.1414
1.1466
1.1464
1.1412
1.1307
1.1153
1.0950
1.0704
1.0416
1.0092
0.9735
0.9351
0.8945
0.8522
0.8088
0.7649
0.7210
0.6776
0.6355
0.5950
0.5566
0.5210
0.0984
0.4593
0.4341
0.4130
0.3963
0.3842
0.3770
0.3745

0.2

1.0000 1.0168 1. 0401 $1.060^{7}$ 1.0819 1. 0999 1.1156 1. 1275 1.1360 1. 1402 1.1405 1.1368 1. 1291 1.1176 1.1026
1.0843 1.0843
1.0631 1.0631
1.0393 1.0133 0.9854 0.9552 0.9260 0.8953 0.8645 0.8340 0.8042 0.7755 0.7484 0.7231
0.5999 0.6999
0.6793 0.6793
0.6613 0.6463 0.6345 0.6259 0.6189 


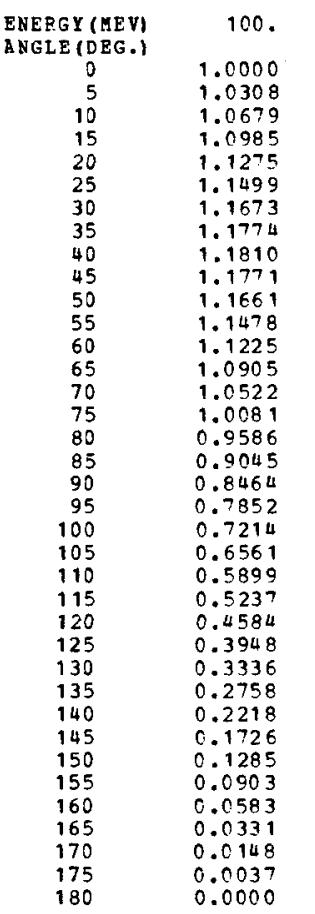

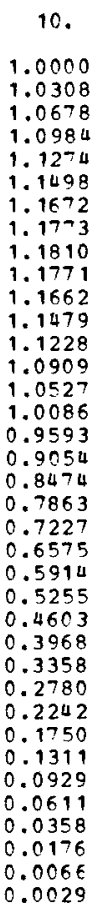

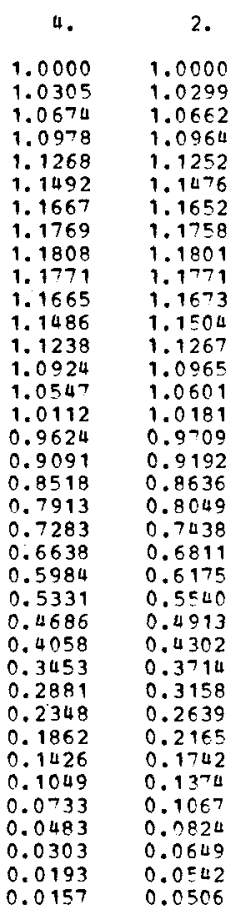

$\begin{array}{cc}1.5 & 1.0 \\ 1.0000 & 1.0000 \\ 1.0290 & 1.0282 \\ 1.0653 & 1.0631 \\ 1.0951 & 1.0924 \\ 1.1238 & 1.1208 \\ 1.1462 & 1.1432 \\ 1.1640 & 1.1612 \\ 1.1748 & 1.1726 \\ 1.1795 & 1.1782 \\ 1.1771 & 1.1768 \\ 1.1670 & 1.1690 \\ 1.1517 & 1.1545 \\ 1.1290 & 1.1336 \\ 1.0997 & 1.1066 \\ 1.0645 & 1.0736 \\ 1.0236 & 1.0354 \\ 0.9777 & 0.9922 \\ 0.9273 & 0.9447 \\ 0.8731 & 0.8936 \\ 0.8159 & 0.8394 \\ 0.7562 & 0.7830 \\ 0.6951 & 0.7250 \\ 0.6330 & 0.6662 \\ 0.5710 & 0.6073 \\ 0.5097 & 0.5492 \\ 0.4500 & 0.14925 \\ 0.3925 & 0.4380 \\ 0.3382 & 0.3863 \\ 0.2875 & 0.3382 \\ 0.2412 & 0.2942 \\ 0.1698 & 0.2549 \\ 0.1639 & 0.2207 \\ 0.1338 & 0.1922 \\ 0.1101 & 0.1596 \\ 0.0929 & 0.1533 \\ 0.0825 & 0.1430 \\ 0.0790 & 0.1401 \\ & \\ 0.090 & \end{array}$
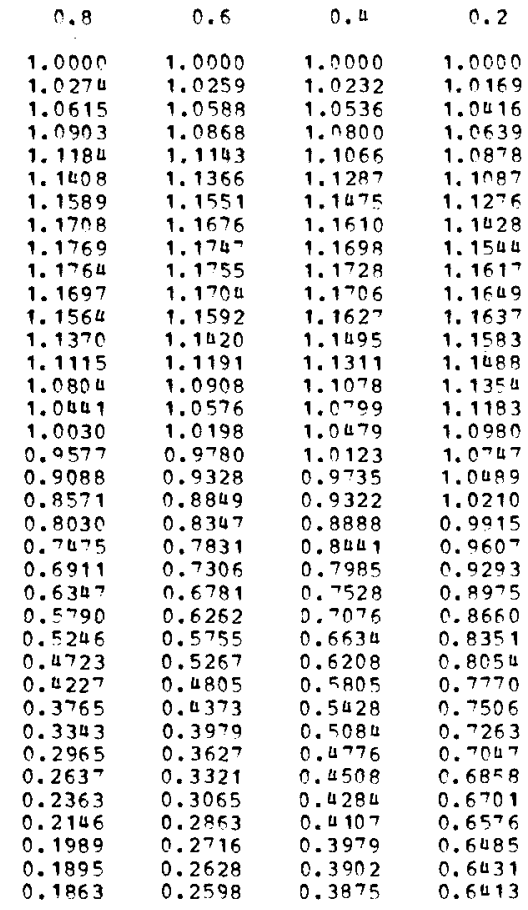

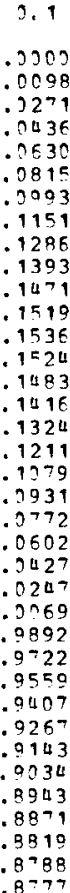

RATIO OP MOET TO RUTHEEFCPD SCATTERING IN NB, $\mathrm{z}=41$

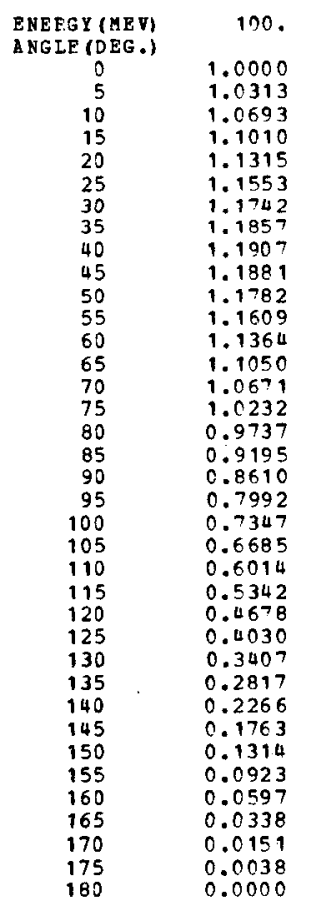

10.
1.0000
1.0312
1.0692
1.1009
1.1313
1.1552
1.1741
1.1856
1.1907
1.1880
1.1783
1.1610
1.1367
1.1054
1.0676
1.0238
0.9744
0.9203
0.8619
0.8003
0.7360
0.6699
0.6029
0.5359
0.4696
0.4050
0.3429
0.2840
0.2290
0.1789
0.1340
0.0950
0.0624
0.0366
0.0180
0.0067
0.0029

4.
1.0000
1.0310
1.0688
1.1004
1.1307
1.1545
1.1735
1.1852
1.1904
1.1880
1.1785
1.1616
1.1377
1.1068
1.0695
1.0262
0.9775
0.9240
0.8663
0.8053
0.7416
0.6763
0.6099
0.5436
0.4780
0.4140
0.3525
0.2942
0.22398
0.1901
0.1456
0.1071
0.0748
0.0493
0.0308
0.0196
0.0159

2.
1.0000
1.0303
1.0676
1.0988
1.1290
1.1528
1.1719
1.1839
1.1896
1.1879
1.1792
1.1532
1.1404
1.1107
1.0748
1.0330
0.9858
0.9340
0.8780
0.8188
0.7570
0.6936
0.6291
0.5646
0.5008
0.4386
0.3787
0.3220
0.2691
0.2208
0.1775
0.1400
0.1086
0.0937
0.0658
0.0549
0.0512

\begin{tabular}{|c|c|}
\hline 1.5 & 1.0 \\
\hline $\begin{array}{l}1.0000 \\
1.0298\end{array}$ & $\begin{array}{l}1.0000 \\
1.0286\end{array}$ \\
\hline $\begin{array}{l}1.0666 \\
1.0975\end{array}$ & $\begin{array}{l}1.0644 \\
1.0947\end{array}$ \\
\hline 1.1275 & 1.1243 \\
\hline 1. 1513 & 1.1481 \\
\hline 1.1706 & 1. 1675 \\
\hline 1.1828 & 1.1803 \\
\hline 1,1889 & 1.1872 \\
\hline 1.1877 & 1. 1871 \\
\hline 1.1796 & 1.1805 \\
\hline 1,1644 & 1. 1669 \\
\hline 1.1425 & 1.1469 \\
\hline 1.1139 & 1. 1204 \\
\hline 1.0790 & 1.0879 \\
\hline 1.0384 & 1.0409 \\
\hline 0.9925 & 1.0068 \\
\hline 0.9420 & 0.9592 \\
\hline 0.8875 & 0.9078 \\
\hline 0,8298 & 0.8532 \\
\hline 0.7695 & 0.7962 \\
\hline 0.7075 & 0.7375 \\
\hline 0.6446 & 0.6779 \\
\hline 0.5816 & 0.6181 \\
\hline 0.5193 & \\
\hline 0.4585 & 0.5014 \\
\hline 0.4000 & 0.4458 \\
\hline 0.3446 & 0.3932 \\
\hline 0.2929 & 0.3441 \\
\hline 0.2457 & 0.2992 \\
\hline $0.2034^{\circ}$ & 0.2591 \\
\hline 0.1657 & 0.2242 \\
\hline 0.1360 & 0.1950 \\
\hline 0.1117 & 0.1720 \\
\hline 0.0941 & 0.1553 \\
\hline & 0.1452 \\
\hline & \\
\hline
\end{tabular}

0.8
1.1000
1.0277
1.0627
1.0925
1.1218
1.1455
1.1651
1.1783
1.1858
1.1865
1.1809
1.1686
1.1500
1.1251
1.0945
1.0584
1.0174
0.9721
0.9229
0.8708
0.8162
0.7600
0.7029
0.16456
0.5890
0.5336
0.4804
0.4299
0.3828
0.3397
0.3011
0.2676
0.2396
0.2175
0.2015
0.1918
0.1885

0.6
1.0000
1.0262
1.0598
1.0888
1.1175
1.1411
1.1609
1.1747
1.1832
1.1852
1.1812
1.1709
1.1546
1.1323
1.1045
1.0716
1.0339
0.9921
0.9467
0.8984
0.8478
0.7956
0.7425
0.6892
0.6364
0.5849
0.5352
0.0881
0.0442
0.0040
0.3680
0.3368
0.3107
0.2900
0.2750
0.2660
0.2629

0.4
1.0000
1.0233
1.0545
1.0817
1.1094
1.1327
1.1527
1.1675
1.1775
1.1818
1.1806
1.1737
1.1613
1.1436
1.1209
1.0933
1.0615
1.0259
0.9870
0.9455
0.9018
0.8566
0.8105
0.7642
0.7183
0.5734
0.5301
0.5890
0.5507
0.5156
0.4842
0.4569
0.4340
0.4160
0.4029
0.3950
0.3923

0.2

1. 0 กก

1.0169

1.0420

1. 06109

1.111

1.1315

1. 1605

1. 1689

1. 1731

1. 1729

1. 1505

1. 1505

1. 13 ก 1

1. 1101

1.0871
1.0614

1.0614
1.0335

1. 0040

0.9730
0.9414

0.9093

0.8775

0.8462

C. 8160

0.7873

0.760

0.7359
0.7139

0.6047

0.6787

0.6660

0.6568
0.6513

0.6494
0.1

1. 390 ?

1. 0096

1.0270
1.0436

1. $) 636$

1. $282^{7}$

1.1313
1.1180

1.1180
1.1325

1. 1442

1.1529
1.1586

1. 1612

$1.160^{\circ}$

1. 1573

1. 1425

1. 1316

1. 1189
1. 1043

1. 3986

1.0718
1.5501

1. 0365

$1.218^{7}$

1.0013

0.9340

0.9525

0.938 a

0.9259

$0.915 \mathrm{C}$

0.998

0.9986

0.8934

0.8903
0.8893 
RATIO OF MOTT TO BOTHERFORT SCATTERTNG IN MO, $\mathrm{z}=42$

\begin{tabular}{|c|c|c|c|c|c|c|c|c|c|c|c|}
\hline $\begin{array}{l}\text { ENERGY (MEV) } \\
\text { ANGLE (DEG.) }\end{array}$ & 100 & 10. & 4. & 2. & 1.5 & 1.0 & 0.8 & 0.6 & 0.4 & 0.2 & 3.1 \\
\hline 0 & 1.0000 & 1.0000 & 1.0000 & 1.0000 & 1.0000 & 1.0000 & 1.0000 & 1.0000 & 1.0000 & 1.0000 & 1.0200 \\
\hline 5 & 1.0317 & 1.0316 & 1.0314 & 1.0307 & 1.0301 & 1.0289 & 1.0280 & 1.0264 & 1.0235 & 1.0168 & 1.0094 \\
\hline 10 & 1.0707 & 1.0706 & 1.0702 & 1.0689 & 1.0679 & 1.0656 & 1.0638 & 1.0609 & 1.0553 & 1.0423 & 1.9260 \\
\hline 15 & 1.1035 & 1.1034 & 1.1028 & 1.1012 & 1.0099 & 1.0969 & 1.0946 & 1.0907 & 1.0833 & 1.0657 & 1.0136 \\
\hline 20 & 1.1354 & 1.1352 & 1.1345 & 1.1327 & 1.1312 & 1.1278 & 1. 1251 & 1.1207 & 1.1121 & 1.0912 & 1.3640 \\
\hline 25 & 1.1606 & 1.1605 & 1.1598 & 1.1580 & 1. 1564 & 1.1530 & 1.1502 & 1.1455 & 1.1366 & 1.1141 & 1.0838 \\
\hline 30 & 1.1810 & 1.1809 & 1.1803 & 1.1786 & 1. 1771 & 1.1738 & 1.1712 & 1.1667 & 1.1579 & 1.1352 & 1. 1532 \\
\hline 35 & 1.1941 & 1.1940 & 1.1935 & 1.1920 & 1.1908 & 1. 1881 & 1.1858 & 1.1819 & $1.17 \mathrm{ac}$ & 1. 1527 & 1.1209 \\
\hline 40 & 1.2005 & 1.2004 & 1.2001 & 1.1991 & 1.1983 & 1. 1963 & 1.1947 & 1.1917 & 1.1853 & 1.1565 & 1.1363 \\
\hline $\begin{array}{l}45 \\
50\end{array}$ & $\begin{array}{l}1.1992 \\
1.1905\end{array}$ & $\begin{array}{l}1.1991 \\
1.1906\end{array}$ & $\begin{array}{l}1.1990 \\
1.1908\end{array}$ & & $\begin{array}{l}1.1984 \\
1.1915\end{array}$ & $\begin{array}{l}1.1975 \\
1.1920\end{array}$ & $\begin{array}{r}1.1967 \\
1.1922\end{array}$ & $\begin{array}{l}1.1950 \\
1.1921\end{array}$ & $\begin{array}{l}1.1908 \\
1.190^{\circ}\end{array}$ & $\begin{array}{l}1.1751 \\
1.1813\end{array}$ & $\begin{array}{l}1.1490 \\
1.15 R^{7}\end{array}$ \\
\hline $\begin{array}{l}50 \\
55\end{array}$ & $\begin{array}{l}1.1905 \\
1.1742\end{array}$ & $\begin{array}{l}1.1906 \\
1.1743\end{array}$ & $\begin{array}{l}1.1908 \\
1.1749\end{array}$ & $\begin{array}{l}1.1912 \\
1.1763\end{array}$ & $\begin{array}{l}1.1915 \\
1.1774\end{array}$ & $\begin{array}{l}1.7920 \\
1.1795\end{array}$ & $\begin{array}{l}1.1922 \\
1.1809\end{array}$ & 1.1821 & 1.1908 & $\begin{array}{l}1.1813 \\
1.1821\end{array}$ & $\begin{array}{l}1.15 R^{7} \\
1.1653\end{array}$ \\
\hline 60 & 1.1506 & 1.1508 & 1.1518 & 1.1543 & 1. 1562 & 1. 1603 & 1.1632 & 1.1674 & 1.1733 & 1.1784 & $1.168^{7}$ \\
\hline 65 & 1.1199 & 1.1202 & 1.1216 & 1.1253 & 1.1283 & 1. 1345 & 1.1390 & 1.1458 & 1.1563 & 1.1703 & 1. 1691 \\
\hline 70 & 1.0824 & 1.0828 & 1.0847 & 1.0898 & 1.0939 & 1. 1025 & 1.1089 & 1.1185 & 1.1341 & 1.1582 & 1.1664 \\
\hline 75 & 1.0387 & 1.0392 & 1.0417 & 1.0483 & 1.0536 & 1.0648 & 1.0731 & 1.0859 & 1.1069 & 1. 1421 & 1.1609 \\
\hline 80 & 0.0892 & 0.9899 & 0.9929 & 1.0011 & 1.0077 & 1.0218 & 1.0322 & 1.0481 & 1.0754 & 1. 1225 & 1. $1 \leq 28$ \\
\hline & & & 0.9393 & 0.9492 & 0.9571 & 0.9741 & 0.9868 & 1.0066 & 1.0399 & 1.0097 & 1. 142 \\
\hline $\begin{array}{l}90 \\
95\end{array}$ & $\begin{array}{l}0.8759 \\
0.8136\end{array}$ & $\begin{array}{l}0.8769 \\
0.8147\end{array}$ & $\begin{array}{l}0.8812 \\
0.8197\end{array}$ & $\begin{array}{l}0.8929 \\
0.8332\end{array}$ & $\begin{array}{l}0.9023 \\
0.8441\end{array}$ & $\begin{array}{l}0.9224 \\
0.8674\end{array}$ & $\begin{array}{l}0.9374 \\
0.8840\end{array}$ & 0.9610 & 1.0009 & 1.0742 & 1.1309 \\
\hline 100 & 0.7484 & 0.7497 & 0.7553 & 0.7707 & 0.7831 & 0.8098 & 0.8298 & 0.8613 & 0.9151 & $\begin{array}{l}1.0464 \\
1.0967\end{array}$ & $\begin{array}{l}1.1158 \\
1.1203\end{array}$ \\
\hline 115 & 0.5450 & 0.5467 & 0.5544 & 0.5755 & 0.5926 & 0.6293 & 0.6569 & 0.7006 & 0.7759 & 0.9214 & 1.2486 \\
\hline 120 & 0.4774 & 0.4793 & 0.4877 & 0.5107 & 0.5292 & 0.5692 & 0.5993 & 0.6470 & 0.7293 & 0.8893 & 1.2309 \\
\hline $\begin{array}{l}125 \\
130\end{array}$ & $\begin{array}{l}0.4115 \\
0.3480\end{array}$ & $\begin{array}{l}0.4135 \\
0.3502\end{array}$ & $\begin{array}{l}0.4226 \\
0.3599\end{array}$ & $\begin{array}{l}0.4474 \\
0.3863\end{array}$ & $\begin{array}{l}0.4674 \\
0.4078\end{array}$ & $\begin{array}{l}0.5105 \\
0.4540\end{array}$ & $\begin{array}{l}0.5430 \\
0.4888\end{array}$ & $\begin{array}{l}0.5946 \\
0.5441\end{array}$ & $\begin{array}{l}0.6837 \\
0.639^{7}\end{array}$ & $\begin{array}{l}0.8576 \\
0.8271\end{array}$ & $\begin{array}{l}1.0133 \\
0.9963\end{array}$ \\
\hline 135 & 0.2878 & 0.2901 & 0.3004 & 0.3285 & 0.3513 & 0.4003 & 0.4373 & 0.4961 & 0.5979 & $\begin{array}{l}0.8271 \\
0.7980\end{array}$ & $\begin{array}{l}0.9963 \\
0.9700\end{array}$ \\
\hline 140 & 0.2316 & 0.2341 & 0.2449 & 0.2745 & 0.2985 & 0.3502 & 0.3893 & 0.4513 & 0.5588 & 0.7708 & 0.9647 \\
\hline 145 & 0.1803 & 0.1828 & 0.1942 & 0.2252 & 0.2503 & 0.3044 & 0.3453 & 0.4103 & 0.5230 & 0.7458 & $0.950 \mathrm{~F}$ \\
\hline 150 & 0.1343 & 0.1370 & 0.1488 & 0.1810 & 0.2071 & 0.2634 & 0.3059 & 0.3736 & 0.4910 & 0.7234 & 0.9380 \\
\hline 155 & $0.094 \mathrm{a}$ & 0.0971 & 0.1093 & 0.1426 & 0.1696 & 0.2278 & $0.2^{717}$ & 0.3417 & 0.0632 & 0.7040 & 0.9271 \\
\hline $\begin{array}{l}160 \\
165\end{array}$ & 0.0610 & 0.0638 & 0.0763 & 0.1105 & 0.1382 & 0.1980 & 0.2431 & 0.3150 & 0.4399 & 0.6877 & 0.9179 \\
\hline $\begin{array}{l}165 \\
170\end{array}$ & $\begin{array}{l}0.0346 \\
0.0155\end{array}$ & $\begin{array}{l}0.0374 \\
0.0184\end{array}$ & $\begin{array}{l}0.0502 \\
0.0313\end{array}$ & $\begin{array}{l}0.0851 \\
0.0667\end{array}$ & $\begin{array}{l}0.1134 \\
0.0954\end{array}$ & $\begin{array}{l}0.1744 \\
0.1573\end{array}$ & $\begin{array}{l}0.2205 \\
0.2041\end{array}$ & $\begin{array}{l}0.2939 \\
0.2786\end{array}$ & $\begin{array}{l}0.4215 \\
0.4081\end{array}$ & $\begin{array}{l}0.6748 \\
0.6654\end{array}$ & $\begin{array}{l}0.9107 \\
0.9054\end{array}$ \\
\hline $\begin{array}{l}170 \\
175\end{array}$ & 0.0039 & 0.0058 & 0.0199 & 0.0556 & 0.0846 & & 0.1942 & $\begin{array}{l}0.2786 \\
0.2693\end{array}$ & $\begin{array}{l}0.4081 \\
0.4000\end{array}$ & 0.6598 & 0.9023 \\
\hline 180 & 0.0000 & 0.0030 & 0.0161 & 0.0519 & 0.0809 & 0.1435 & 0.1909 & 0.2662 & 0.3973 & 0.6579 & 0.9012 \\
\hline
\end{tabular}

RATIO OP MOTT TO RUTHERFOPD SCATTEPTNG IN $B G, z=47$

BNERGY (MEV)
ANGLE (DEG.)
5
5
10
15
20
25
30
35
40
45
50
55
60
65
70
75
80
85
90
95
100
105
110
115
120
125
130
135
140
145
150
155
160
165
170
175
180

$\begin{array}{cc}100, & 10 . \\ 1.0000 & 1.0000 \\ 1.0333 & 1.0332 \\ 1.0769 & 1.0768 \\ 1.1151 & 1.1149 \\ 1.1539 & 1.1537 \\ 1.1867 & 1.1865 \\ 1.2151 & 1.2149 \\ 1.2362 & 1.2360 \\ 1.2505 & 1.2503 \\ 1.2565 & 1.2564 \\ 1.2546 & 1.2545 \\ 1.2442 & 1.2442 \\ 1.2255 & 1.2257 \\ 1.1987 & 1.1989 \\ 1.1640 & 1.1644 \\ 1.1220 & 1.1224 \\ 1.0730 & 1.0736 \\ 1.0179 & 1.0187 \\ 0.9573 & 0.9583 \\ 0.8922 & 0.8933 \\ 0.8234 & 0.8246 \\ 0.7518 & 0.7532 \\ 0.6785 & 0.6801 \\ 0.6045 & 0.6063 \\ 0.5308 & 0.5328 \\ 0.4585 & 0.4606 \\ 0.3885 & 0.3908 \\ 0.3219 & 0.3243 \\ 0.2595 & 0.2620 \\ 0.2022 & 0.2049 \\ 0.1508 & 0.1536 \\ 0.1061 & 0.1090 \\ 0.0686 & 0.0716 \\ 0.0389 & 0.0420 \\ 0.0174 & 0.0205 \\ 0.0044 & 0.0075 \\ 0.0000 & 0.0032 \\ & \\ 0.003 & \end{array}$

4.
1.0000
1.0329
1.0762
1.1142
1.1529
1.1856
1.2141
1.2352
1.2497
1.2560
1.2544
1.2444
1.2263
1.2000
1.1659
1.1246
1.0764
1.0221
0.9624
0.8982
0.8302
0.7596
0.6873
0.6142
0.5414
0.4700
0.4009
0.3351
0.2734
0.2169
0.1661
0.1220
0.0849
0.0556
0.0344
0.0215
0.0172

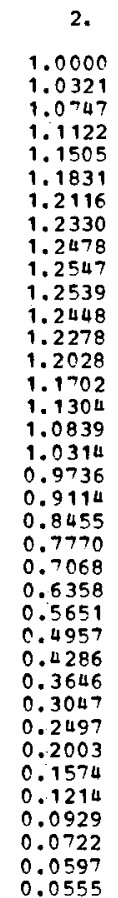

1.5
1.0000
1.0315
1.0735
1.1105
1.1486
1.1811
1.2096
1.2311
1.2463
1.2536
1.2534
1.2451
1.2289
1.2050
1.1735
1.1350
1.0899
1.0389
0.9826
0.9220
0.8578
0.7910
0.7225
0.6533
0.5843
0.5165
0.4510
0.3885
0.3300
0.2762
0.2281
0.1861
0.1509
0.1231
0.1029
0.0907
0.0866

1.0
1.0000
1.0301
1.0708
1.1069
1.1443
1.1764
1.2049
1.2269
1.2427
1.2510
1.2521
1.2454
1.2312
1.2094
1.1805
1.1447
1.1026
1.0547
1.0019
0.9448
0.8842
0.8211
0.7563
0.6908
0.6255
0.5613
0.4992
0.4399
0.3845
0.3335
0.2878
0.2480
0.2147
0.1882
0.1601
0.1575
0.1536

$\begin{array}{cc}0.8 & 0.6 \\ 1.0000 & 1.0000 \\ 1.0290 & 1.0271 \\ 1.0686 & 1.0651 \\ 1.1040 & 1.0991 \\ 1.1408 & 1.1351 \\ 1.1728 & 1.1666 \\ 1.2013 & 1.1950 \\ 1.2234 & 1.2176 \\ 1.2397 & 1.2346 \\ 1.2488 & 1.2449 \\ 1.2509 & 1.2485 \\ 1.2454 & 1.2450 \\ 1.2327 & 1.2345 \\ 1.2126 & 1.2171 \\ 1.1855 & 1.1930 \\ 1.1518 & 1.1626 \\ 1.1119 & 1.1263 \\ 1.0665 & 1.0848 \\ 1.0162 & 1.0386 \\ 0.9618 & 0.9884 \\ 0.9040 & 0.9351 \\ 0.8437 & 0.8792 \\ 0.7817 & 0.8219 \\ 0.7190 & 0.7637 \\ 0.6565 & 0.7057 \\ 0.5950 & 0.6486 \\ 0.5355 & 0.5933 \\ 0.4788 & 0.5405 \\ 0.4256 & 0.4911 \\ 0.3768 & 0.4456 \\ 0.3330 & 0.4049 \\ 0.2948 & 0.3694 \\ 0.2629 & 0.3396 \\ 0.2375 & 0.3160 \\ 0.2191 & 0.2989 \\ 0.2081 & 0.2986 \\ 0.2043 & 0.2851 \\ & \end{array}$

0.4
1.0000
1.0237
1.0584
1.0001
1.1243
1.1548
1.1930
1.2060
1.2243
1.2365
1.2427
1.2425
1.2360
1.2232
1.2044
1.1798
1.1499
1.1151
1.0761
1.0334
0.0879
0.9400
0.8907
0.8405
0.7904
0.7410
0.6932
0.6474
0.6046
0.5652
0.5298
0.4990
0.0731
0.4527
0.4378
0.4289
0.4258

0.2
1.0900
1.0162
1.0431
1.0685
1.0980
1.1257
1.1524
1.1759
1.1059
1.2116
1.2227
1.2290
1.2303
1.2267
1.2183
1.2554
1.1883
1.1673
1.1430
1.1157
1.0861
1.0545
1.0218
1.9882
0.9545
0.9210
0.8887
0.8575
0.8284
0.8115
0.7774
0.7563
0.7387
0.7247
0.7145
0.7094
0.7064
0.1

1. 3203 1. 0 1. 82 1. 0255 1. 26 प 1. 7872 1.1329 1. 1329 1.1532
1.1713 1. 1713 1. 1990 1. 2363
1. 2112 1.2126
1.2100 1. 2109
1. 2062 1. 1986 1. 188 P 1. 1756 1. 1530 1. 1316 1. 1147 1. 2077 1.05038 1. 0638
1.0476 1. 3325 1. 0185 1.0560
0.0949 0.0858 0.9784 0.9731 0.9700 
RATYO OP MOTM TC RUTHERFOFI SCRTTPRTNG IN $S N, Z=50$

\begin{tabular}{|c|c|c|c|c|c|c|c|c|c|c|c|}
\hline $\begin{array}{l}\text { ENERGY (MEV) } \\
\text { ANGLE (DEG,) }\end{array}$ & 100. & 10. & 4. & 2. & 1.5 & 1.0 & 0.8 & 0.6 & 0.4 & 0.2 & 0.1 \\
\hline $\begin{array}{l}0 \\
5\end{array}$ & $\begin{array}{r}1.0000 \\
1.0339\end{array}$ & $\begin{array}{l}1.0000 \\
1.0338\end{array}$ & & 1.0000 & 1.0000 & 1.0000 & 1.0000 & 1.0000 & 9.0000 & 1.0000 & 1.0000 \\
\hline $\begin{array}{r}5 \\
10\end{array}$ & $\begin{array}{l}1.0339 \\
1.0709\end{array}$ & $\begin{array}{l}1.0338 \\
1.0798\end{array}$ & $\begin{array}{l}1.0335 \\
1.0792\end{array}$ & $\begin{array}{l}1.0326 \\
1.0775\end{array}$ & $\begin{array}{l}1.0319 \\
1.0762\end{array}$ & $\begin{array}{l}1.0304 \\
1.0731\end{array}$ & $\begin{array}{l}1.0292 \\
1.0708\end{array}$ & $\begin{array}{l}1.0272 \\
1.0668\end{array}$ & 1.0236 & $\begin{array}{r}1.0156 \\
1.0429\end{array}$ & $\begin{array}{l}1.0075 \\
1.3244\end{array}$ \\
\hline 15 & 1.1211 & 1.1209 & 1.1201 & 1.1178 & 1.01160 & 1. 1118 & 1.1086 & $\begin{array}{l}1.0668 \\
1.1032\end{array}$ & $\begin{array}{l}1.0596 \\
1.0930\end{array}$ & $\begin{array}{l}1.0429 \\
1.0601\end{array}$ & $\begin{array}{l}1.3244 \\
1.3405\end{array}$ \\
\hline 20 & 1.1641 & 1.1639 & 1.1629 & 1.1603 & 1.1580 & 1.1531 & 1.1492 & 1.1427 & 1.1303 & 1.1005 & 1.0532 \\
\hline 25 & 1.2016 & 1.2014 & 1.2003 & 1.1974 & 1.1950 & 1. 1896 & 1.1854 & 1.1782 & 1.1546 & 1.1310 & 1.0875 \\
\hline 30 & 1.2352 & 1.2350 & 1.2339 & 1.2310 & 1.2285 & 1.2230 & 1.2186 & 1. 2112 & 1.1969 & 1.1611 & 1. 1128 \\
\hline 35 & 1.2615 & 1.2613 & 1.2603 & 1.2576 & 1. 2552 & 1.2500 & 1.2457 & 1.2386 & 1.2245 & 1.1884 & 1.1380 \\
\hline 40 & 1.2811 & 1.2809 & 1.2800 & 1.2776 & 1.2755 & 1.2708 & $1.26>0$ & 1.2605 & $1.2 u>u$ & 1.2124 & 1.1514 \\
\hline 45 & 1.2922 & 1.2920 & 1.2914 & 1.2895 & 1.2879 & 1. 2841 & 1.2810 & 1. 2755 & 1.2642 & 1.2323 & 1.1830 \\
\hline 50 & 1.2950 & 1.2949 & 1.2945 & 1.2933 & 1.2923 & 1.2898 & 1.2876 & 1.2836 & $1.2^{747}$ & 1.2074 & 1. 2313 \\
\hline 55 & 1.2888 & 1.2888 & 1. 2887 & 1.2885 & 1.2882 & $1.28>3$ & 1.2863 & 1.2842 & 1.2786 & 1.2575 & 1.2166 \\
\hline 60 & 1.2738 & 1.2739 & 1.2742 & 9.2751 & 1.2757 & $1.276^{7}$ & 1.2771 & 1.2773 & 1.2757 & 1.2623 & 1.2282 \\
\hline 65 & 1.2499 & 1.2501 & 1.2509 & 1.2530 & 1.2547 & 1.2579 & 1.2601 & 1.2630 & 1.2660 & 1.2520 & 1.2363 \\
\hline 70 & 1.2175 & 1.2178 & 1.2191 & 1.2227 & 1.2255 & 1.2313 & 1.2354 & 1.2413 & 1.2498 & 1.2564 & 1.2408 \\
\hline 75 & 1.1769 & 1.1773 & 1.1792 & 1.1844 & 1. 1886 & 1. 1972 & 1.2034 & 1.2128 & 1.2272 & 1.2459 & 1. 2418 \\
\hline 80 & 1.1286 & 1. 1292 & 1. 1317 & 1.1387 & 1.1443 & 1. 1560 & 1.1646 & 1.1777 & $1.198^{\circ}$ & 1. 2308 & 1.2395 \\
\hline 85 & 1.0734 & 1.0741 & 1.0774 & 1.0862 & 1.0933 & 1.1084 & 1.1195 & 1.1367 & 1.1648 & 1.2113 & 1. 2342 \\
\hline 90 & 1.0149 & 1.0128 & 1.0168 & 1.0277 & 1.0364 & 1.0550 & 1.0689 & 1.0904 & 1.1261 & 1. 1882 & 1.2264 \\
\hline 95 & 0.9452 & 0.9463 & 0.9510 & 0.9640 & 0.9745 & 0.9968 & $1.013^{\circ}$ & 1.0394 & 1.0831 & 1. 1615 & 1.2160 \\
\hline 100 & 0.8741 & 0.8753 & 0.8809 & 0.8961 & 0.9083 & 0.0345 & 0.9541 & 0.9848 & 1.0368 & 1. 1323 & 1.2038 \\
\hline 115 & 0.6452 & 0.6470 & 0.6550 & $0.6>70$ & 0.6947 & 0.7328 & 0.7615 & 0.8058 & 0.8245 & 1.0333 & 1. $158^{7}$ \\
\hline 120 & 0.5676 & 0.5694 & 0.5783 & 0.6025 & 0.6220 & 0.6641 & 0.6958 & 0.7059 & 0.9323 & 0.9989 & 1.1425 \\
\hline 125 & 0.4908 & 0.4929 & 0.5026 & 0.5290 & 0.5503 & 0.5962 & 0.6308 & 0.6857 & 0.7804 & 0.9644 & 1. 1258 \\
\hline 130 & 0.4164 & 0.4187 & 0.4292 & 0.4576 & 0.4807 & $0.530 u$ & 0.5678 & 0.6273 & 0.7301 & 0.0310 & $1.179^{\circ}$ \\
\hline 135 & 0.3453 & 0.3478 & 0.3590 & 0.3895 & 0.4142 & 0.4673 & 0.5075 & 0.5713 & 0.5817 & 0.8986 & 1.0939 \\
\hline 140 & 0.2787 & 0.2813 & 0.2932 & 0.3255 & 0.3517 & 0.4082 & $0.450^{\circ}$ & 0.5187 & 0.6364 & 0.8684 & 1.0701 \\
\hline 145 & 0.2173 & 0.2201 & 0.2326 & 0.2666 & 0.2943 & 0.3538 & 0.3987 & 0.4703 & 0.5045 & 0.8403 & 1.0653 \\
\hline 150 & 0.1623 & 0.1652 & 0.1782 & 0.2138 & 0.2427 & 0.3049 & 0.3519 & 0.4268 & $0.557 \mathrm{C}$ & $O . R 1=1$ & 1.3539 \\
\hline 155 & 0.1142 & 0.1172 & 0.1307 & 0.1677 & 0.1976 & 0.2622 & 0.3110 & 0.3888 & 0.5241 & 0.7030 & 1.0421 \\
\hline 160 & 0.0739 & 0.0770 & 0.0909 & 0.1290 & 0.1599 & 0.2264 & 0.2768 & 0.3570 & 0.4966 & 0.7745 & 1.0330 \\
\hline 165 & 0.0420 & $0.045^{\circ}$ & 0.0594 & 0.0983 & 0.1299 & 0.1980 & 0.2496 & 0.3317 & 0.4748 & 0.7598 & 1. .258 \\
\hline 170 & 0.0188 & $0.022 \mathrm{C}$ & 0.0365 & 0.0760 & 0.1082 & 0.1774 & 0.2298 & 0.3133 & 0.4588 & 0.7499 & 1.3205 \\
\hline 175 & 0.0048 & $0.008 C$ & 0.0227 & 0.0626 & 0.0951 & 0.1550 & 0.2179 & 0.3023 & 0.4494 & 0.7428 & 1.0174 \\
\hline 180 & 0.0000 & 0.0033 & 0.0180 & 0.0581 & 0.0906 & 0.1608 & 0.2139 & 0.2985 & 0.4461 & 0.7406 & 1.2165 \\
\hline
\end{tabular}

FMTIO OP MOTT TO RUTHERFOFD SCATMRRING TN CS, $z=55$

ENE EGY (MEV)
ANGIE $(D E G)$ )
0
5
10
15
20
25
30
35
40
45
50
55
60
65
70
75
80
85
90
95
100
105
110
115
120
125
130
135
140
145
150
155
160
165
170
175
180

$\begin{array}{cc}100 . & 10 . \\ 1.0000 & 1.0000 \\ 1.0342 & 1.0349 \\ 1.0836 & 1.0835 \\ 1.1292 & 1.1290 \\ 1.1791 & 1.1789 \\ 1.2246 & 1.2243 \\ 1.2673 & 1.2670 \\ 1.3033 & 1.3030 \\ 1.3326 & 1.3323 \\ 1.3534 & 1.3531 \\ 1.3653 & 1.3651 \\ 1.3675 & 1.3674 \\ 1.3598 & 1.3598 \\ 1.3421 & 1.3422 \\ 1.3145 & 1.3147 \\ 1.2773 & 1.2776 \\ 1.2310 & 1.2315 \\ 1.1761 & 1.1768 \\ 1.1137 & 1.1145 \\ 1.0444 & 1.0454 \\ 0.9696 & 0.9708 \\ 0.8901 & 0.8915 \\ 0.8074 & 0.8091 \\ 0.7227 & 0.7245 \\ 0.6374 & 0.6394 \\ 0.5526 & 0.5548 \\ 0.4700 & 0.4724 \\ 0.3905 & 0.3931 \\ 0.3157 & 0.3186 \\ 0.2466 & 0.2496 \\ 0.1844 & 0.1875 \\ 0.1299 & 0.1332 \\ 0.0842 & 0.0875 \\ 0.0478 & 0.0513 \\ 0.0214 & 0.0249 \\ 0.0055 & 0.0090 \\ 0.0000 & 0.0036 \\ & \\ 0.3759 & \end{array}$

$\begin{array}{cc}4 & 2 . \\ 1.0000 & 1.0000 \\ 1.0338 & 1.0328 \\ 1.0828 & 1.0808 \\ 1.1280 & 1.1254 \\ 1.1777 & 1.1744 \\ 1.2230 & 1.2194 \\ 1.2656 & 1.2618 \\ 1.3016 & 1.2978 \\ 1.3311 & 1.3275 \\ 1.3520 & 1.3489 \\ 1.3642 & 1.3617 \\ 1.3668 & 1.3652 \\ 1.3596 & 1.3591 \\ 1.3425 & 1.3433 \\ 1.3156 & 1.3179 \\ 1.2792 & 1.2832 \\ 1.2337 & 1.2396 \\ 1.1798 & 1.1877 \\ 1.1183 & 1.1284 \\ 1.0500 & 1.0625 \\ 0.9763 & 0.9912 \\ 0.8979 & 0.9153 \\ 0.8164 & 0.8364 \\ 0.7328 & 0.7553 \\ 0.6486 & 0.6737 \\ 0.5650 & 0.5926 \\ 0.4834 & 0.5135 \\ 0.4050 & 0.4374 \\ 0.3312 & 0.3658 \\ 0.2630 & 0.2995 \\ 0.2015 & 0.2399 \\ 0.1478 & 0.1877 \\ 0.1026 & 0.1438 \\ 0.0667 & 0.1090 \\ 0.0406 & 0.0836 \\ 0.0249 & 0.0684 \\ 0.0195 & 0.0632 \\ & \\ 0.1039\end{array}$

\begin{tabular}{|c|c|}
\hline 1.5 & 1.0 \\
\hline $\begin{array}{l}1.0000 \\
1.0320 \\
1.0793\end{array}$ & $\begin{array}{l}1.0000 \\
1.0303 \\
1.0757\end{array}$ \\
\hline 1.1231 & 1.1182 \\
\hline 1.1717 & 1.1656 \\
\hline $\begin{array}{l}1.2163 \\
1.2586\end{array}$ & $\begin{array}{l}1.2095 \\
1.2514\end{array}$ \\
\hline 1.2946 & 1.2875 \\
\hline 1.3245 & 1.3176 \\
\hline 1.3462 & 1,3402 \\
\hline 1.3596 & 1. 3547 \\
\hline $\begin{array}{r}1.3638 \\
1.3586\end{array}$ & $\begin{array}{l}1.3605 \\
1.3572\end{array}$ \\
\hline 1.3439 & 1.3447 \\
\hline 1.3197 & 1.3231 \\
\hline 1.2863 & 1.2928 \\
\hline 1.2443 & 1.2540 \\
\hline 1. 1941 & 1.2074 \\
\hline 1.1366 & 1.1538 \\
\hline 1.0726 & 1.0939 \\
\hline 1.0032 & 1.0289 \\
\hline 0.8526 & 0.8871 \\
\hline 0.7736 & 0.8127 \\
\hline 0.6941 & 0.7377 \\
\hline $\begin{array}{l}0.6150 \\
0.5378\end{array}$ & $\begin{array}{l}0.6630 \\
0.5902\end{array}$ \\
\hline 0.4636 & 0.5201 \\
\hline 0.3938 & 0.4541 \\
\hline 0.3291 & 0.3330 \\
\hline & 0.3380 \\
\hline 0.2201 & 0.2899 \\
\hline 0.1773 & 0.2494 \\
\hline $\begin{array}{l}0.1433 \\
0.1185\end{array}$ & 0.2172 \\
\hline $\begin{array}{l}185 \\
037\end{array}$ & $\begin{array}{l}0.1938 \\
0.1798\end{array}$ \\
\hline 0.0986 & 0.1750 \\
\hline
\end{tabular}

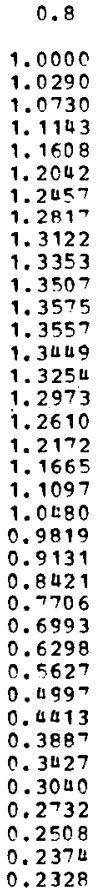

0.6
1.0000
1.0268
1.0685
1.1079
1.1529
1.1952
1.2361
1.2721
1.3029
1.3269
1.3435
1.3522
1.3526
1.3446
1.3283
1.3037
1.2716
1.2321
1.1862
1.1344
1.0779
1.0172
0.9539
0.8885
0.8225
0.7567
0.6925
0.6305
0.5722
0.5181
0.4695
0.4268
0.3910
0.3625
0.3417
0.3294
0.3251

0.0

1.0000 1.0228 1.0602 1.0059
1.1378

1.1378
1.1781

1.1781

1.2534

1.2846

1.3100

1.3288

1. 3406

1. 3449

1.3416
1.3308

1.3308
1.3126

1.3126
1.2875

1.2557

1.2557
1.2182

1. 1752

1.1280
1.0769

1.0769
1.0234

0.9678
0.9118

0.9118
0.8556

0.8008

0.7477

0.6978

0.6515

0.6098

0.5733
0.5425

0.5181

0.5003

0.5003
0.4897

0.4860
0.2

1.0000

1. 0143

1. 0415

1.0680
1.1021

1. 1368

1.1723

1. 2063

1.2373

1. $264^{7}$

1. 2873

1.3048
1.3166

1.3166
1. 3226

1. 3229

1. 3176

1. 2905

1. 2905
1. 2001

1. 2453

1.2173

1. 1860

1.1529
1.1179

1.1179
1.0825

1. 0465

1. 0115

0.9772

ก. 9451

0.9150

0.8880

0.8643

C. 8443

0.8284

0.8168

0.8100
0.8077
0.1

1. 3009 1.0064
1.2222 1. 0364 1.0589 1.3848 1.1130 1. 1425 1. 1711 1. 1986 1.2234
1.2449 1. 2628 1. $2>71$ 1.2875
1.2940 1. 2969 1. 2962 1. 2859 1. 2859 1. 2560 1. 2536 1. $239^{7}$ 1. 2100 1. 1958 1. 1811 1.1574 1. 1544 .1428
1.1325 1.1239 1.1170 1.1120 1. 1383 
RATIO OF MOTM TC RUTHERTOBD SCATMERTNG IN ND, $\mathrm{z}=60$

\begin{tabular}{|c|c|c|c|c|c|c|c|c|c|c|c|}
\hline $\begin{array}{l}\text { BNERGY (MEV) } \\
\text { ANGLE (DEG.) }\end{array}$ & 100. & 10. & 4. & 2. & 1.5 & 1.0 & 0.8 & 0.6 & 0.4 & 0.2 & $\therefore 1$ \\
\hline $\begin{array}{l}0 \\
5\end{array}$ & $\begin{array}{l}1.0000 \\
1.0338\end{array}$ & $\begin{array}{l}1.0000 \\
1.0337\end{array}$ & $\begin{array}{l}1.0000 \\
1.0333\end{array}$ & $\begin{array}{l}1.0000 \\
1.0323\end{array}$ & $\begin{array}{l}1.0000 \\
1.0314\end{array}$ & $\begin{array}{l}1.0000 \\
1.0295\end{array}$ & $\begin{array}{l}1.0000 \\
1.0281\end{array}$ & $\begin{array}{l}1.0000 \\
1.0257\end{array}$ & $\begin{array}{l}1.0000 \\
1.0215\end{array}$ & $\begin{array}{l}1.0000 \\
1.0128\end{array}$ & $\begin{array}{l}1.0200 \\
1.0054\end{array}$ \\
\hline 10 & 1.0856 & $1.085 \mathrm{U}$ & 1.0846 & 1.0824 & 1.0806 & 1.0766 & 1.0736 & 1.0685 & 1.0502 & 1.0392 & 1.2201 \\
\hline 15 & 1.1347 & 1,1344 & 1.1333 & 1.1302 & 1.1276 & 1.1218 & 1.1173 & 1.1098 & 1.0960 & 1.0647 & 1.0314 \\
\hline 20 & 1.1910 & 1.1907 & 1.1893 & 1.1853 & 1. 1820 & 1.1748 & 1.1690 & 1.1596 & $1.149^{7}$ & 1. 1no? & $1.0<21$ \\
\hline 25 & 1.2045 & 1.2442 & 1.2425 & 1.2380 & 1,2343 & 1.2259 & 1.2194 & 1.2084 & 1.9876 & 1. 1382 & 1.7784 \\
\hline 30 & 1.2968 & 1.2964 & 1.2947 & 1.2898 & 1. 2857 & 1.2766 & 1.2694 & 1.2570 & 1.2344 & 1.1786 & 1.1082 \\
\hline 35 & 1.3434 & 1.3430 & 1.3412 & 1.3362 & 1.3320 & 1. 3226 & 1. 3152 & 1.3027 & 1. 2787 & 1.2192 & $1.141 \mathrm{~F}$ \\
\hline 40 & 1.3838 & 1.3834 & 1.3816 & 1.3767 & 1. 3725 & 1.3633 & 1.3559 & 1.3434 & 1.3191 & 1.2576 & 1.1749 \\
\hline 45 & 1.4158 & 1.4154 & 1.4138 & 1.4092 & 1.4053 & 1.3966 & 1. 3896 & 1.3777 & 1. 3542 & 1.2034 & 1.2084 \\
\hline 50 & 1.4385 & 1.4382 & 1.4368 & 1.4328 & 1.4294 & 1.4216 & 1.4153 & 1.4044 & 1.3827 & 1.3240 & 1.2394 \\
\hline 55 & 1.4510 & 1.4508 & 1.4496 & 1.4464 & 1.4437 & 1.4374 & 1.4321 & 1.4229 & 1.10039 & 1.3505 & 1. 2683 \\
\hline 60 & 1.4526 & 1.4525 & 1.4517 & 1.4496 & 1.4477 & 1.4432 & 1.4394 & 1.4323 & 1.4171 & 1.3706 & $1.2 \div 35$ \\
\hline 65 & 1.4430 & 1.4429 & 1.4427 & 1.4419 & 1.7411 & 1.4389 & 1. $436^{7}$ & 1.4325 & 1.4219 & 1.3846 & 1. 3159 \\
\hline 70 & 1.4220 & 1.4221 & 1.4224 & 1.4231 & 1. 11236 & 1.4241 & 1. 4241 & 1.4231 & 1.4182 & 1.3921 & 1. 3325 \\
\hline 75 & 1.3898 & 1.3900 & 1.3910 & 1.3935 & 1.3954 & 1. 3991 & 1.4010 & 1.0042 & 1.4060 & 1.3932 & 1. 3258 \\
\hline 80 & 1.3467 & 1.3071 & 1.3488 & 1.3534 & 1.3569 & 1.3641 & 1. 3692 & 1.3763 & 1.3857 & 1.3881 & 1.3552 \\
\hline 85 & 1.2933 & 1.2939 & 1.2964 & 1.3032 & 1.3086 & 1.3197 & 1.3277 & 1.3396 & 1.3573 & 1.3768 & 1. 3604 \\
\hline 90 & 1.2306 & 1.2313 & 1.2348 & 1.2440 & 1.2513 & 1. 2667 & 1.2780 & 1.2951 & 1.3220 & $1.360 \mathrm{u}$ & 1.3624 \\
\hline 95 & 1.1592 & 1.1602 & 1.1645 & 1.1763 & 1.1858 & 1. 2057 & 1.2204 & 1.2431 & 1.2799 & 1.3386 & 1.3608 \\
\hline 100 & 1.0807 & 1.0819 & 1.0872 & 1.1018 & 1.1134 & 1. 1383 & 1.1566 & 1.1852 & 1.2325 & 1.3128 & 1.3566 \\
\hline 105 & 0.9959 & 0.9973 & 1.0037 & 1.0211 & 1.0351 & 1.0649 & 1.0871 & 1.1218 & 1.1799 & 1. 2828 & 1. 3497 \\
\hline 115 & 0.8142 & 0.8161 & 0.8246 & 0.8478 & 0.8666 & 0.9068 & 0.9369 & 0.9844 & 1.0651 & 1.2154 & 1.3308 \\
\hline 120 & 0.7203 & 0.7225 & 0.7321 & 0.7583 & 0.7794 & 0.8249 & 0.8590 & 0.9129 & 1.0052 & 1.1704 & 1.3193 \\
\hline 125 & 0.6262 & 0.6286 & 0.6392 & 0.6683 & 0.6919 & 0.7425 & 0.7805 & 0.8408 & 0.9443 & 1. 1423 & $1.30>0$ \\
\hline 130 & 0.5340 & 0.5366 & 0.5483 & 0.5802 & 0.6061 & 0.6617 & 0.7036 & 0.7701 & 0.8816 & 1.1059 & 1.2946 \\
\hline 135 & 0.4446 & 0.4475 & 0.4601 & 0.4948 & 0.5229 & 0.5833 & 0.6289 & 0.7013 & 0.8264 & 1.0609 & 1.2322 \\
\hline 140 & 0.3603 & 0.3633 & 0.3769 & 0.4149 & 0.4443 & 0.5092 & 0.5583 & 0.6363 & 0.7794 & 1.0360 & $1.2^{702}$ \\
\hline 145 & 0.2818 & 0.2851 & 0.2995 & 0.3391 & 0.3712 & 0.4403 & 0.4925 & 0.5757 & 0.7200 & 1.0040 & 1.2589 \\
\hline 150 & 0.2111 & 0.2145 & 0.2297 & 0.2714 & 0.3052 & 0.3789 & 0.4332 & 0.5210 & 0.6736 & $0.9-53$ & 9.2486 \\
\hline 155 & 0.1489 & 0.1525 & 0.1684 & 0.2119 & 0.2472 & 0.3234 & 0.3811 & 0.4729 & $0.632^{7}$ & 0.9498 & 1.2399 \\
\hline 160 & 0.0966 & 0.1002 & 0.1167 & 0.1618 & 0.1984 & 0.2774 & 0.3371 & 0.4324 & 0.5983 & 0.9284 & 1.2318 \\
\hline 165 & 0.0549 & 0.0587 & 0.0756 & 0.1220 & 0.1596 & 0.2407 & 0.3022 & 0.4002 & 0.5709 & 0.9113 & 1. 2256 \\
\hline 170 & 0.0245 & 0.0284 & 0.0456 & 0.0929 & 0.1312 & 0.2139 & 0.2766 & 0.3765 & 0.5509 & 0.8987 & 1. 2211 \\
\hline 175 & 0.0064 & 0.0103 & 0.0277 & 0.0755 & 0.1143 & 0.1980 & 0.2614 & 0.3626 & 0.5390 & 0.8914 & 1.2184 \\
\hline 180 & 0.0000 & 0.0040 & 0.0215 & 0.0695 & $0.108 \mathrm{a}$ & 0.1924 & 0.2569 & 0.3577 & 0.5340 & 0.8990 & 1.2175 \\
\hline
\end{tabular}

RATIO OF MOTT TO ROTHERFORD SCATTRRING IN TB, $\mathrm{z}=65$

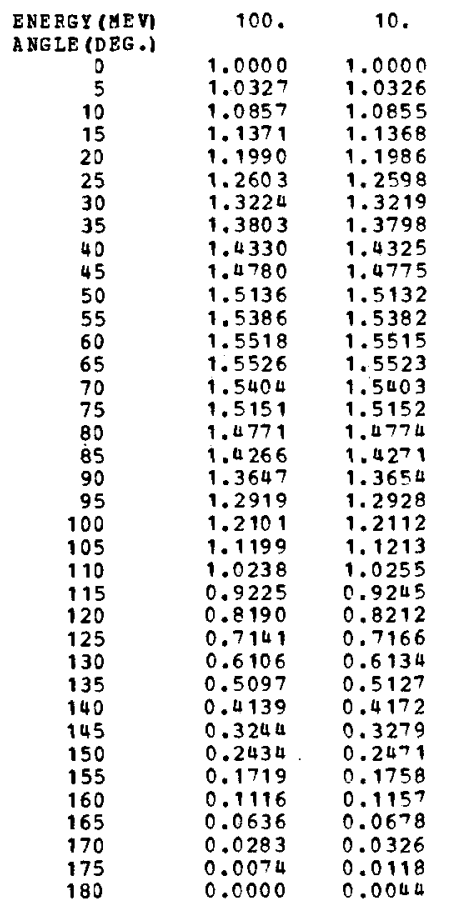

\begin{tabular}{|c|c|c|c|c|}
\hline 4. & 2. & 1.5 & 1.0 & 0.8 \\
\hline $\begin{array}{l}1.0000 \\
1.0322\end{array}$ & $\begin{array}{l}1.0000 \\
1.0311\end{array}$ & $\begin{array}{l}1.0000 \\
1.0302\end{array}$ & $\begin{array}{l}1.0000 \\
1.0282\end{array}$ & $\begin{array}{l}1.0000 \\
1.0267\end{array}$ \\
\hline 1.0846 & 1.0822 & 1.0802 & 1.0758 & 1.0724 \\
\hline 1.1355 & 1.1319 & 1.1289 & 1. 1224 & 1.1173 \\
\hline 1.1969 & 1.1923 & 1.1884 & 1. 1799 & 1.1732 \\
\hline 1.2579 & 1.2524 & 1.2479 & 1.2379 & 1.2300 \\
\hline 1.3197 & 1.3136 & 1.3086 & 1. 2974 & 1.2886 \\
\hline 1.3775 & 1.3712 & 1.3658 & 1.3540 & 1.3446 \\
\hline 1.4302 & 1.4237 & 1.4183 & 1.4062 & 1.3965 \\
\hline 1.4753 & 1.4689 & 1.4637 & 1.4518 & 1.4423 \\
\hline 1.5111 & 1.5052 & 1.5002 & 1. 4891 & 1.4801 \\
\hline 1.5364 & 1.5313 & 1.5269 & 1. 5170 & 1.5089 \\
\hline 1.5500 & 1.5459 & 1.5423 & 1.5341 & 1.5274 \\
\hline 1.5514 & 1.5485 & 1.5460 & 1.5401 & 1.5350 \\
\hline $\begin{array}{l}1.5399 \\
1.5155\end{array}$ & 9.5386 & $\begin{array}{l}1.5374 \\
1.5164\end{array}$ & 1.5343 & $\begin{array}{l}1.5313 \\
1.5161\end{array}$ \\
\hline 1.4785 & 1.8813 & 1.4834 & $\begin{array}{l}1.5160 \\
1.4873\end{array}$ & $\begin{array}{l}1.5167 \\
1.489 ?\end{array}$ \\
\hline 1.4290 & 1.4342 & 1.4383 & 1.4465 & 1.4522 \\
\hline 1.3683 & 1.3762 & 1.3825 & 1.3954 & 1.404 \\
\hline 1. 2968 & 1.3076 & 1.3162 & 1.3342 & 1.3474 \\
\hline 1.2164 & 1.2303 & 1.2494 & 1. 2649 & 1.2822 \\
\hline 1. 1276 & 1.1447 & 1. 1585 & 1.1877 & 1.2093 \\
\hline 1.0330 & 1.0535 & 1.0700 & 1. 1051 & 1.1313 \\
\hline 0.9333 & 0.9572 & $0.9^{7} 64$ & 1.0177 & 1.0485 \\
\hline 0.8313 & 0.8586 & 0.8807 & 0.9281 & 0.9636 \\
\hline 0.7279 & 0.7586 & 0.7835 & 0.8369 & 0.8770 \\
\hline 0.6259 & 0.6600 & $0.68^{77}$ & 0.7470 & 0.7917 \\
\hline 0.5264 & 0.5637 & 0.5940 & 0.6590 & 0.7080 \\
\hline 0.4320 & 0.4724 & 0.5051 & 0.5756 & 0.6287 \\
\hline 0.3437 & 0.3869 & 0.4219 & 0.4974 & 0.5543 \\
\hline 0.2638 & 0.3096 & 0.3467 & 0.4266 & 0.4871 \\
\hline 0.1934 & 0.2413 & 0.2802 & 0.3642 & 0.4276 \\
\hline 0.1339 & 0.1837 & $0.22 \div 2$ & 0.3114 & 0.3774 \\
\hline 0.0865 & 0.1378 & 0.1795 & 0.2694 & 0.3375 \\
\hline 0.0518 & 0.1042 & 0.1467 & 0.2386 & 0.3081 \\
\hline 0.0312 & 0.0842 & 0.1273 & 0.2203 & 0.2908 \\
\hline 0.0239 & $0.0>72$ & 0.1205 & 0.2139 & 0.2846 \\
\hline
\end{tabular}

0.6
1.0000
1.0242
1.0669
1.1088
1.1622
1.2169
1.2738
1.3289
1.3803
1.4263
1.4648
1.4950
1.5155
1.5258
1.5255
1.5142
1.4925
1.4501
1.4184
1.3673
1.3088
1.2429
1.1722
1.0968
1.0195
0.9404
0.8624
0.7858
0.7132
0.6450
0.5833
0.5287
0.4827
0.0460
0.4190
0.4031
0.3975

0.4
1.0000
1.0199
1.0570
1.0933
1.1416
1.1923
1.2459
1.2989
1.3491
1.3951
1.4347
1.4672
1.4910
1.5067
1.5114
1.5071
1.4934
1.4701
1.4385
1.3985
1.3518
1.2984
1.2407
1.1785
1.1146
1.0488
0.9839
0.9197
0.8592
0.8020
0.7503
0.7045
0.6659
0.6351
0.6124
0.5991
0.5944

0.2
1.0000
1.0114
1.0363
1.0593
1.0945
1.1348
1.1792
1.2260
1.2719
1.3165
1.3568
1.3928
1.4226
1.4462
1.4627
1.4721
1.4745
1.4698
1.4589
1.4415
1.4993
1.3998
1.3611
1.3268
1.2910
1.2534
1.2160
1.1787
1.9432
1.1096
1.0791
1.0520
1.0291
1.0108
0.9973
0.9904
0.9869


QATTO OF MOTT TO RUTHFRFCRD SCATTERTNG IN YB, $\mathrm{Z}=7$ ?

\begin{tabular}{|c|c|c|c|c|c|c|c|c|c|c|c|}
\hline $\begin{array}{l}\text { ENEGGY (HEV) } \\
\text { ANGLE (DEG) }\end{array}$ & 100. & 10. & 4. & 2. & 1.5 & 1.0 & 0.8 & 0.6 & 0.4 & 0.2 & 0.1 \\
\hline 0 & 1.0000 & 1.0000 & 1.0000 & 1.0000 & 1.0000 & 1.0000 & 1.0000 & 1.0000 & 1.0000 & 1.0000 & 1.5300 \\
\hline 5 & 1.0311 & 1.0310 & 1.0306 & 1.0295 & 1.0285 & 1.0265 & 1.0249 & 1.0224 & 1.0182 & 1.0102 & 1.0042 \\
\hline 10 & 1.0840 & 1.0838 & 1.0829 & 1.0803 & 1.0781 & 1.0735 & 1.0699 & 1.0641 & $1.053^{7}$ & 1.0333 & 1.2184 \\
\hline 15 & 1.1362 & 1.1358 & $1.134 \%$ & 1.1304 & 1. 1270 & 1. 1197 & 1.1181 & 1. 1048 & 1.0880 & 1.0526 & 1.0218 \\
\hline 20 & 1.2023 & 1.2019 & 1.1999 & 1.1946 & 1.1902 & 1.1804 & 1.1728 & 1.1602 & 1.1370 & 1.0857 & $9.33 u 4$ \\
\hline 25 & 1.2707 & 1.2702 & 1.2679 & 1.2614 & 1.2561 & 1.2443 & 1.2351 & 1.2198 & 1.1914 & 1.1265 & 1.0566 \\
\hline 30 & 1.3424 & 1.3418 & 1.3392 & 1.3399 & 1.3258 & 1.3123 & 1.3017 & 1.2841 & 1.2509 & 1.1734 & 1.2946 \\
\hline 35 & 1.4125 & 1.4118 & 1.4090 & 1.4010 & 1.3945 & 1.3798 & 1.3683 & 1.3490 & 1.3125 & 1. 2257 & 1. $121^{7}$ \\
\hline 40 & 1.4786 & 1.4780 & 1.4750 & 1.4667 & 1.4598 & 1.4444 & 1.4322 & 9.4118 & $1.3-29$ & 1. $2^{-86}$ & 1.1615 \\
\hline 45 & 1.5383 & 1.5377 & 1.5347 & 1.5263 & 1.5194 & 1.5038 & 1.4915 & 1.4707 & $1.430^{7}$ & 1. 3323 & 1.2058 \\
\hline 50 & 1.5890 & 1.5883 & 1.5854 & 1.5773 & 1.5705 & 1.5553 & 1.5432 & 1.5226 & 1.4828 & 1. 3826 & 1.2490 \\
\hline 55 & 1.6290 & 1.6284 & 1.6257 & 1.6182 & 1.6119 & 1.5976 & 1.5862 & 1.5667 & 1.5283 & 1.4296 & 1. 2941 \\
\hline 60 & 1.6564 & 1.6559 & 1.6536 & 1.6470 & 1.6414 & $1.628^{7}$ & 1.6183 & 1.6006 & 1.5650 & 1.4707 & 1.3359 \\
\hline 65 & 1.6704 & 1.6700 & 1.6681 & 1.6627 & 1.6581 & 1.6476 & 1.5389 & 1.6236 & 1.5024 & 1.5056 & 1. 3740 \\
\hline 70 & 1.6697 & 1.6695 & 1.6682 & 1.6604 & 1.6612 & 1.6534 & 1.6468 & 1.6349 & 1.6093 & 1.5330 & 1.4101 \\
\hline 75 & 1.6541 & 1.6539 & 1.6534 & 1.6516 & 1.6499 & 1.6456 & 1.6415 & $1.633^{7}$ & 1.6152 & 1. 5527 & 1.0411 \\
\hline 80 & 1.6235 & 1.6236 & 1.6238 & 1.6244 & 1.6246 & 1.6243 & 1.6233 & 1.6204 & 1.5104 & 1.5607 & 1.1457 \\
\hline 85 & 1.5778 & 1.5781 & 1.5793 & 1.5825 & 1.5848 & 1. 5892 & 1.5918 & 9.5945 & 1.5944 & 1.5695 & 1.4897 \\
\hline 90 & $1.518 \mathrm{a}$ & 1.5189 & 1.5212 & 1.5273 & 1.5321 & 1.5417 & 1.5482 & 1.5573 & $1.568 \mathrm{u}$ & 1.5651 & 1.5575 \\
\hline 95 & 1.4452 & 1.4460 & 1.4495 & 1.4589 & 1. 4663 & 1.4816 & 1.4925 & 1.5085 & 1.5320 & 1.5500 & 1.5211 \\
\hline 100 & 1.3607 & 1.3618 & 1.3666 & $1.3^{-95}$ & 1. 3897 & 1.4112 & 1.4269 & 1.4505 & 1.4874 & 1.5370 & 1.5308 \\
\hline 105 & 1.2652 & 1.2665 & 1.2727 & 1.2893 & 1.3026 & 1.3306 & 1.3513 & 1.3830 & 1.4342 & 1.5136 & $9.53+3$ \\
\hline 110 & 1. 1618 & 1.1634 & 1.1710 & 1.1915 & 1.2080 & 1.2429 & 1.2688 & 1.3090 & 1.3752 & 1.4859 & 1.5008 \\
\hline 115 & 1.0510 & 1.0530 & 1.0620 & 1.0865 & 1.1062 & 1.1483 & 1.1796 & 1.2285 & 1.3103 & 1.4535 & 1.5419 \\
\hline 120 & 0.9366 & 0.9389 & 0.9494 & 0.9780 & 1.0010 & 1.0503 & 1.0871 & 1.1449 & 1.2425 & 1.4188 & 1.5408 \\
\hline 125 & 0.8192 & 0.8219 & 0.8339 & 0.8665 & 0.8928 & 0.9493 & 0.9916 & 1.0583 & 1.1717 & 1.3815 & 1.5384 \\
\hline 130 & 0.7028 & 0.7058 & 0.7192 & 0.7558 & 0.7854 & 0.8490 & 0.8967 & 0.9722 & 1.1012 & $1.343 B$ & 1.5346 \\
\hline 135 & 0.5881 & 0.5914 & 0.6062 & 0.6467 & 0.6795 & 0.7408 & 0.8028 & 0.8867 & 1.0309 & 1.3057 & 1.5303 \\
\hline 140 & 0.4788 & 0.4824 & 0.4986 & 0.5427 & 0.5785 & 0.6554 & 0.7133 & 0.8053 & 0.9638 & 1.2691 & 1.5253 \\
\hline 145 & 0.3760 & 0.3799 & 0.3973 & 0.4448 & 0.4833 & 0.5663 & 0.6288 & 0.7283 & 0.9002 & 1.2340 & 1.5205 \\
\hline 150 & 0.2826 & 0.2868 & 0.3053 & 0.3559 & 0.3969 & 0.4853 & 0.5521 & 0.6584 & 0.8424 & 1.2020 & 1.5156 \\
\hline 155 & 0.2000 & 0.2043 & 0.2238 & 0.2779 & 0.3204 & 0.4136 & 0.4841 & 0.5962 & 0.7910 & $1.1 \div 34$ & 1.5113 \\
\hline 160 & 0.1300 & 0.1345 & 0.1548 & 0.2104 & 0.2555 & 0.3528 & 0.4264 & 0.5437 & 0.7474 & 1.1401 & 1.52711 \\
\hline 165 & 0.0741 & 0.0788 & 0.0998 & 0.1572 & 0.2038 & 0.3043 & 0.3804 & 0.5017 & 0.7126 & 1. 1297 & 1.5012 \\
\hline 170 & 0.0330 & 0.0378 & 0.0593 & 0.1180 & 0.1657 & 0.2686 & 0.3465 & 0.4707 & 0.6870 & 1.1153 & 1.5025 \\
\hline 175 & 0.0087 & 0.0136 & 0.0354 & 0.0949 & 0.1433 & 0.2475 & 0.3266 & 0.4525 & 0.6719 & 1.1058 & 1.5004 \\
\hline 180 & 0.0001 & 0.0049 & 0.0268 & 0.0866 & 0.1352 & 0.2001 & 0.3195 & 0.4461 & $0.666^{7}$ & 1.1041 & 1.1096 \\
\hline
\end{tabular}

EATIO OF MOTT TO PUTHERFCRD SCATTERING IN HF, $\mathrm{Z}=>2$

$\begin{array}{cc}\text { ENERGY (MEV) } & 100 \\ \text { ANGLE IDEG.) } & \\ 5 & 1.0000 \\ 5 & 1.0304 \\ 10 & 1.0829 \\ 15 & 1.1348 \\ 20 & 1.2022 \\ 25 & 1.2731 \\ 30 & 1.3486 \\ 35 & 1.4235 \\ 40 & 1.4953 \\ 45 & 1.5614 \\ 50 & 1.6187 \\ 55 & 1.5655 \\ 60 & 1.6995 \\ 65 & 1.7196 \\ 70 & 1.7245 \\ 75 & 1.7136 \\ 80 & 1.6868 \\ 85 & 1.6437 \\ 90 & 1.5858 \\ 95 & 1.5129 \\ 100 & 1.4276 \\ 105 & 1.3300 \\ 110 & 1.2237 \\ 115 & 1.1089 \\ 120 & 0.9897 \\ 125 & 0.8669 \\ 130 & 0.7447 \\ 135 & 0.6239 \\ 140 & 0.5085 \\ 145 & 0.3996 \\ 150 & 0.3007 \\ 155 & 0.2128 \\ 160 & 0.1384 \\ 165 & 0.0790 \\ 170 & 0.0352 \\ 175 & 0.0093 \\ 180 & 0.0001\end{array}$

$\begin{array}{ccc}10 . & 4 . & 2 . \\ 1.0000 & 1.0000 & 1.0000 \\ 1.0303 & 1.0299 & 1.0287 \\ 1.0827 & 1.0817 & 1.0791 \\ 1.1345 & 1.1330 & 1.1288 \\ 1.2017 & 1.1997 & 1.1941 \\ 1.2726 & 1.2701 & 1.2633 \\ 1.3479 & 1.3451 & 1.3372 \\ 1.4228 & 1.4197 & 1.4111 \\ 1.4946 & 1.4913 & 1.4823 \\ 1.5607 & 1.5574 & 1.5481 \\ 1.6179 & 1.6147 & 1.5056 \\ 1.6648 & 1.5618 & 1.5532 \\ 1.6989 & 1.6961 & 1.6884 \\ 1.7191 & 1.7168 & 1.7103 \\ 1.7242 & 1.7224 & 1.7175 \\ 1.7133 & 1.7123 & 1.7094 \\ 1.6867 & 1.6866 & 1.6860 \\ 1.6439 & 1.6448 & 1.6470 \\ 1.5863 & 1.5883 & 1.5935 \\ 1.5137 & 1.5169 & 1.5256 \\ 1.4287 & 1.4333 & 1.4456 \\ 1.3314 & 1.3374 & 1.3537 \\ 1.2253 & 1.2329 & 1.2533 \\ 1.1109 & 1.1200 & 1.1447 \\ 0.9921 & 1.0028 & 1.0319 \\ 0.8697 & 0.8819 & 0.9153 \\ 0.7478 & 0.7616 & 0.7993 \\ 0.6273 & 0.6426 & 0.6845 \\ 0.5122 & 0.5290 & 0.5748 \\ 0.4037 & 0.4218 & 0.4713 \\ 0.3050 & 0.3243 & 0.3771 \\ 0.2174 & 0.2378 & 0.2935 \\ 0.1431 & 0.1644 & 0.2227 \\ 0.0839 & 0.1059 & 0.1661 \\ 0.0402 & 0.0628 & 0.1240 \\ 0.0144 & 0.0373 & 0.0998 \\ 0.0052 & 0.0282 & 0.0910\end{array}$

1.5
1.0000
1.0278
1.0769
1.1254
1.1894
1.2576
1.3307
1.4040
1.4747
1.5404
1.5980
1.6460
1.6819
1.7047
1.7133
1.7068
1.6853
1.6485
1.5976
1.5324
1.4554
1.3667
1.2697
1.1646
1.0553
0.9423
0.8298
0.7183
0.6119
0.5114
0.4199
0.3387
0.2699
0.2150
0.1745
0.1506
0.1420

1.0
1.0000
1.0257
1.0721
1.1178
1.1792
1.2451
1.3163
1.3882
1.4579
1.5233
1.5810
1.6298
1.6671
1.6920
1.7034
1.7003
1.5830
1.6509
1.6055
1.5463
1.4759
1.3941
1.3044
1.2069
1.1053
1.0000
0.8951
0.7911
0.6917
0.5977
0.5122
0.4362
0.3718
0.3204
0.2824
0.2601
0.2521

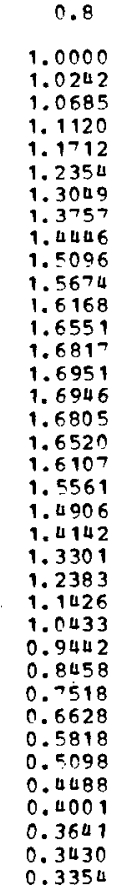

0.6
1.0000
1.0217
1.0626
1.1024
1.1581
1.2192
1.2861
1.3549
1.4224
1.4868
1.5445
1.5947
1.6347
1.6636
1.6803
1.6840
1.6749
1.6522
1.6175
1.5702
1.5128
1.4449
1.3698
1.2873
1.2011
1.1113
1.0216
0.9324
0.8470
0.7651
0.6925
0.6270
0.5715
0.5271
0.1944
0.4751
0.4683

0.4
1.0000
1.0175
1.0523
1.0853
1.1340
1.1893
1.2508
1.3157
1.3801
1.4430
1.5004
1.5517
1.5941
1.6272
1.6494
1.5602
1.6598
1.5473
1.5242
1.5898
1.5465
1.4937
1.4346
1.3687
1.2994
1.2266
1.1537
1.0808
1.0110
0.9446
0.8842
0.8302
0.7846
0.7480
0.7210
0.7051
0.6996

0.2

1.0000 1.0098 1.0323 1.0814 1. 1218 1.1218 1. 1692 1. 2233 $1.278 \mathrm{~B}$ 1.3361
1.3906 1.3906
1.4423 1. 4823 1. 48281 1.5281
1.5605 1. 5850 1.6015
1.6093 1.6093
1.6095 1. 6016 1.5872 1. .5659 1.5399 1. 5088
1. 4748 1.4380 1.4380
1.4004 1. 4004 1. 3621 1. 3252 $1.289^{7}$ 1.2572 1.2280
1.2033 1.2033
1.1833 1.1833
1.1687 1. 1600 1. 1600
1. 1571

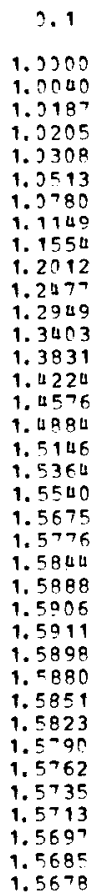


RATIO OF MOTT TO RUTHERFCRD SCATTERING IN TA, $\mathrm{z}=-3$

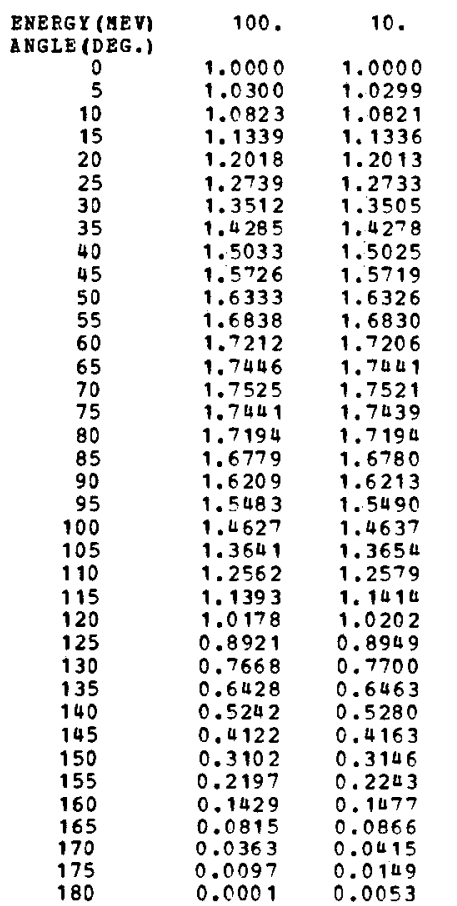

$\begin{array}{ccc}4 . & 2 . & 1.5 \\ 1.0000 & 1.0000 & 1.0000 \\ 1.0295 & 1.0283 & 1.0274 \\ 1.0811 & 1.0784 & 1.0762 \\ 1.1321 & 1.1278 & 1.1243 \\ 1.1993 & 1.1935 & 1.1888 \\ 1.2708 & 1.2638 & 1.2580 \\ 1.3476 & 1.3395 & 1.3327 \\ 1.4246 & 1.4157 & 1.4083 \\ 1.4991 & 1.4896 & 1.4817 \\ 1.5684 & 1.5587 & 1.5506 \\ 1.6291 & 1.6195 & 1.6115 \\ 1.5798 & 1.6705 & 1.6630 \\ 1.7176 & 1.7093 & 1.7023 \\ 1.7416 & 1.7344 & 1.7284 \\ 1.7502 & 1.7446 & 1.7399 \\ 1.7427 & 1.7391 & 1.7360 \\ 1.7190 & 1.7179 & 1.7167 \\ 1.6787 & 1.6803 & 1.6814 \\ 1.6232 & 1.6279 & 1.6316 \\ 1.5521 & 1.5603 & 1.5668 \\ 1.4681 & 1.4802 & 1.4898 \\ 1.3714 & 1.3875 & 1.4003 \\ 1.2654 & 1.2858 & 1.3021 \\ 1.1505 & 1.1753 & 1.1952 \\ 1.0309 & 1.0602 & 1.0838 \\ 0.9073 & 0.9411 & 0.9684 \\ 0.7840 & 0.8223 & 0.8532 \\ 0.6619 & 0.7045 & 0.7389 \\ 0.5451 & 0.5918 & 0.6296 \\ 0.4348 & 0.4853 & 0.5262 \\ 0.3344 & 0.3884 & 0.4321 \\ 0.2452 & 0.3022 & 0.3485 \\ 0.1696 & 0.2292 & 0.2776 \\ 0.1091 & 0.1708 & 0.2209 \\ 0.0646 & 0.1278 & 0.1791 \\ 0.0383 & 0.1024 & 0.1545 \\ 0.0289 & 0.0933 & 0.1456\end{array}$

$\begin{array}{cc}1.0 & 0.8 \\ 1.0000 & 1.0000 \\ 1.0254 & 1.0238 \\ 1.0714 & 1.0677 \\ 1.1166 & 1.1107 \\ 1.1783 & 1.1701 \\ 1.2451 & 1.2351 \\ 1.3178 & 1.3061 \\ 1.3918 & 1.3789 \\ 1.4642 & 1.4503 \\ 1.5326 & 1.5183 \\ 1.5935 & 1.5792 \\ 1.6457 & 1.6319 \\ 1.6864 & 1.6736 \\ 1.7145 & 1.7033 \\ 1.7288 & 1.7196 \\ 1.7284 & 1.7218 \\ 1.7133 & 1.7098 \\ 1.0828 & 1.6831 \\ 1.6386 & 1.6431 \\ 1.5799 & 1.5891 \\ 1.5096 & 1.5239 \\ 1.4273 & 1.4471 \\ 1.3367 & 1.3622 \\ 1.2376 & 1.2691 \\ 1.1342 & 1.1718 \\ 1.0268 & 1.0705 \\ 0.9195 & 0.9692 \\ 0.8129 & 0.8685 \\ 0.7109 & 0.7721 \\ 0.6143 & 0.5807 \\ 0.5264 & 0.5975 \\ 0.4482 & 0.5235 \\ 0.3819 & 0.4607 \\ 0.3288 & 0.4105 \\ 0.2897 & 0.3735 \\ 0.2667 & 0.3517 \\ 0.2585 & 0.3439 \\ & \\ 1.969 & \end{array}$

0.6
1.0000
1.0214
1.0618
1.1011
1.1567
1.2185
1.2866
1.3574
1.4272
1.4943
1.5551
1.6085
1.6517
1.6838
1.7034
1.7097
1.7028
1.6820
1.5486
1.5022
1.5452
1.4772
1.4015
1.3181
1.2306
1.1392
1.0477
0.9564
0.8691
0.7861
0.7106
0.6433
0.5853
0.5406
0.5069
0.4471
0.4801

0.4
1.0000
1.0172
1.0515
1.0837
1.1322
1.1879
1.2503
1.3168
1.3832
1.4486
1.5087
1.5630
1.6085
1.6445
1.6696
1.6831
1.6849
1.6744
1.6529
1.6197
1.5771
1.5247
1.4659
1.3992
1.3292
1.2550
1.1813
1.1071
1.0359
0.9680
0.9062
0.8510
0.8942
0.7667
0.7390
0.7227
0.7170

0.2
1.0000
1.0096
1.0318
1.0482
1.0791
1.1192
1.1667
1.2216
1.2784
1.3374
1.3940
1.4481
1.4965
1.5390
1.5741
1.5011
1.6199
1.5299
1.5321
1.5259
1.6130
1.5929
1.5678
1.5374
1.5039
1.4674
1.4299
1.3916
1.3545
1.3198
1.2861
1.2567
1.2317
1.2116
1.1968
1.1879
1.1851

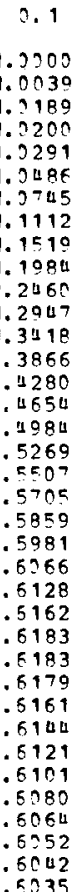

RATIC OF MOTY TO BUTHERFORD SCATTERING IN

\begin{tabular}{|c|c|}
\hline $\begin{array}{c}\text { ENE RGY (MEV) } \\
\text { ANGLE (DEG .) } \\
0 \\
5 \\
10 \\
15 \\
20 \\
25 \\
30 \\
35 \\
40 \\
45 \\
50 \\
55 \\
60 \\
65 \\
70 \\
75 \\
80 \\
85 \\
90 \\
95 \\
100 \\
105 \\
110 \\
115 \\
120 \\
125 \\
130 \\
135 \\
140 \\
145 \\
150 \\
155 \\
160 \\
165 \\
170 \\
175 \\
180\end{array}$ & $\begin{array}{l}100 . \\
1.0000 \\
1.0296 \\
1.0816 \\
1.1329 \\
1.2012 \\
1.2744 \\
1.3534 \\
1.4333 \\
1.5109 \\
1.5836 \\
1.6479 \\
1.7020 \\
1.7431 \\
1.7699 \\
1.7810 \\
1.7753 \\
1.7528 \\
1.7129 \\
1.6569 \\
1.5846 \\
1.4987 \\
1.3992 \\
1.2898 \\
1.1709 \\
1.0468 \\
0.9182 \\
0.7898 \\
0.6625 \\
0.5405 \\
0.4252 \\
0.3202 \\
0.2268 \\
0.1475 \\
0.0842 \\
0.0375 \\
0.0100 \\
0.0001\end{array}$ \\
\hline
\end{tabular}

10
1.0000
1.0295
1.0814
1.1326
1.2007
1.2738
1.3528
1.4325
1.5101
1.5828
1.6471
1.7012
1.7424
1.7693
1.7805
1.7750
1.7527
1.7130
1.6573
1.5853
1.4997
1.4005
1.2915
1.1729
1.0492
0.9211
0.7930
0.6660
0.5444
0.4294
0.3247
0.2315
0.1525
0.0894
0.0428
0.0153
0.0055

$\begin{array}{cccc}4 . & 2 & 1.5 & 1.0 \\ 1.0000 & 1.0000 & 1.0000 & 1.0000 \\ 1.0291 & 1.0280 & 1.0270 & 1.0250 \\ 1.0804 & 1.0777 & 1.0754 & 1.0706 \\ 1.1310 & 1.1267 & 1.1231 & 1.1154 \\ 1.1986 & 1.1927 & 1.1879 & 1.1771 \\ 1.2712 & 1.2640 & 1.2580 & 1.2448 \\ 1.3497 & 1.3413 & 1.3344 & 1.3189 \\ 1.4292 & 1.4199 & 1.4122 & 1.3952 \\ 1.5065 & 1.4966 & 1.4884 & 1.4701 \\ 1.5791 & 1.5690 & 1.5605 & 1.5416 \\ 1.6434 & 1.6333 & 1.6248 & 1.6059 \\ 1.6977 & 1.6880 & 1.6798 & 1.6615 \\ 1.7392 & 1.7302 & 1.7227 & 1.7056 \\ 1.7666 & 1.7588 & 1.7522 & 1.7372 \\ 1.7783 & 1.7721 & 1.7668 & 1.7545 \\ 1.7735 & 1.7693 & 1.7656 & 1.7568 \\ 1.7521 & 1.7503 & 1.7486 & 1.7441 \\ 1.7134 & 1.7145 & 1.7151 & 1.7154 \\ 1.6590 & 1.6632 & 1.6664 & 1.6724 \\ 1.5882 & 1.5961 & 1.6022 & 1.6145 \\ 1.5041 & 1.5158 & 1.5251 & 1.5443 \\ 1.4064 & 1.4223 & 1.4350 & 1.4616 \\ 1.2990 & 1.3193 & 1.3356 & 1.3699 \\ 1.1821 & 1.2069 & 1.2269 & 1.2694 \\ 1.0601 & 1.0896 & 1.1134 & 1.1641 \\ 0.9336 & 0.9678 & 0.9954 & 1.0544 \\ 0.8073 & 0.8461 & 0.8775 & 0.9447 \\ 0.6819 & 0.7252 & 0.7602 & 0.78354 \\ 0.5619 & 0.6095 & 0.6480 & 0.7308 \\ 0.4483 & 0.4099 & 0.5417 & 0.6316 \\ 0.3449 & 0.4001 & 0.4448 & 0.5411 \\ 0.2529 & 0.3113 & 0.3586 & 0.4606 \\ 0.1749 & 0.2360 & 0.2855 & 0.3923 \\ 0.1125 & 0.1757 & 0.2270 & 0.3377 \\ 0.0665 & 0.1313 & 0.1830 & 0.2974 \\ 0.0394 & 0.1051 & 0.1585 & 0.2736 \\ 0.0296 & 0.0957 & 0.1494 & 0.2651 \\ & & & \end{array}$

1.0000
1.0234
1.0669
1.1094
1.1688
1.2345
1.3069
1.3818
1.4557
1.5267
1.5908
1.6469
1.6920
1.7250
1.7444
1.7493
1.7397
1.7149
1.6762
1.6230
1.5581
1.4809
1.3953
1.3009
1.2019
1.0986
0.9951
0.8920
0.7932
0.6993
0.6139
0.5377
0.4731
0.4214
0.3832
0.3607
0.3527

0.6
1.0000
1.0210
1.0610
1.0996
1.1552
1.2175
1.2868
1.3594
1.1316
1.5016
1.5655
1.6222
1.6686
1.7039
1.7266
1.7356
1.7312
1.7123
1.6804
1.6350
1.5784
1.5104
1.4343
1.3499
1.2611
1.1680
1.0745
0.9814
0.8919
0.8069
0.7294
0.6602
0.6016
0.5546
0.5190
0.4996
0.4923

0.4
1.0000
1.0169
1.0507
1.0821
1.1303
1.1862
1.2495
1.3175
1.3859
1.4538
1.5168
1.5741
1.8227
1.6618
1.6899
1.7061
1.7104
1.7020
1.6822
1.6502
1.6085
1.5565
1.4973
1.4305
1.3599
1.2851
1.2099
1.1342
1.0616
0.9922
0.9290
0.8725
0.8245
0.7860
0.7576
0.7409
0.7351

0.2

1.0000

1.0095

1.0314

1. 0467

1.0767

1.1164

1. 1600

1.2196
1.2775

1.2775
1.3383

1. 3960

1. 4535

1.5497

1. 5874

$1.61 ? 0$

1.6384
1.6507

1.6507
1.6550

1.6550
1.6505

1.6392

1.6204
1.5963

1.5963
$? .5567$

1.5667
1.5337

1. 4976

1. 4602

1. 4220

1. 3848

1. 3160

1. 2864

1. 2612

1. 2612
1. 2409

1. 2408

1.2169
1.2140
1.3005

1.0038

1. 2192

1. 195

1.3274
1.0459

1. $)>09$

1. 1072

1.1180
1.1952

1.2439

1.2941

1. 3429

1. 3898

1. 4729

1.5381
1.5389

1.5389

1. 5650

1.5869
1.5044

1. 6186

1. 5201

1.6379
1.6422

1.6460

1. 6475

1. 6485

1. 5479

1.5461

1. 6450

1.6450
1.6436

1.6436
1.6426

1.6426
$1.6 \pm 18$

1.6118
1.6410

1.6410
1.5403 
RATIO OF MOTT TO RUTHERFORD SCATTRRING IN AT, $2=79$

\begin{tabular}{|c|c|c|c|c|c|c|c|c|c|c|c|}
\hline $\begin{array}{l}\text { ENEPGY (HEV) } \\
\text { ANGLE (DEG.) }\end{array}$ & 100 & 10. & 4. & 2. & 1.5 & 1.0 & 0.8 & 0.6 & 0.4 & 0.2 & 0.1 \\
\hline $\begin{array}{l}0 \\
5\end{array}$ & $\begin{array}{l}1.0000 \\
1.0277\end{array}$ & $\begin{array}{l}1.0000 \\
1.0275\end{array}$ & $\begin{array}{l}1.0000 \\
1.0272\end{array}$ & $\begin{array}{l}1.0000 \\
1.0260\end{array}$ & $\begin{array}{l}1.0000 \\
1.0251\end{array}$ & $\begin{array}{l}1.0000 \\
1.0231\end{array}$ & $\begin{array}{l}1.0000 \\
1.0217\end{array}$ & $\begin{array}{l}1.0000 \\
1.0194\end{array}$ & $\begin{array}{l}1.0000 \\
1.0156\end{array}$ & $\begin{array}{l}1.0000 \\
1.0089\end{array}$ & $\begin{array}{l}1.0005 \\
1.0029\end{array}$ \\
\hline 10 & 1.0774 & 1.0772 & 1.0762 & $1.0 \div 34$ & $1.0^{-12}$ & 1.0663 & 1.0627 & 1.0569 & 9.0070 & 1.0299 & 1.5212 \\
\hline 15 & 1.1258 & 1.1255 & 1.1238 & 1.1192 & 1.1155 & $1.10^{73}$ & 1.1010 & 1.0909 & 1.0732 & 1.0396 & 1.2188 \\
\hline 20 & 1.1948 & 1.1942 & 1.1919 & 1.1854 & 1.1801 & 1.1683 & 1.1593 & 1.1446 & 9.1181 & 1.0638 & 1.0202 \\
\hline 25 & 1.2723 & 1.2717 & 1. 2687 & 1.2605 & 1.2538 & 1.2389 & 1.2274 & 1.2085 & 9.1740 & 1.1001 & 1.0328 \\
\hline 30 & 1.3594 & 1.3586 & 1.3550 & 1.3452 & $1.33^{7} 2$ & 1.3193 & 1.3054 & 1.2824 & 1.2409 & 1.1462 & 1.5518 \\
\hline 35 & 1.4512 & 1.4503 & 1.4463 & 1.4353 & 1.4261 & 1.4059 & 1.3900 & 1.3638 & 1.3150 & 1.2041 & 1.0849 \\
\hline 40 & 1.5436 & 1.5426 & 1.5383 & 1.5262 & 1.5161 & 1.4939 & 1.4764 & 1.4474 & $1.3 \div 30$ & 1.2670 & 1.1248 \\
\hline 45 & 1.6340 & 1.6330 & 1.6284 & 1.6156 & 1.5050 & 1.5815 & 1.5629 & 1.5320 & $1.4>36$ & 1.3360 & $1.1-30$ \\
\hline 55 & 1.7917 & 1.7907 & 1.7861 & 1.7731 & 1. 7623 & 1.7380 & 1.7189 & 1.5866 & 1.6247 & 1.4738 & 1. 2839 \\
\hline 60 & 1.8531 & 1.8521 & 1.8476 & 1.8352 & 1.8248 & 1.8014 & 1.7828 & 1.7513 & 1.6903 & 1.5385 & 1.3415 \\
\hline 65 & 1.8995 & 1.8987 & 1.8946 & 1.8831 & 1.8735 & 1.8518 & 1.8344 & 1.8048 & 1.7466 & 1.5982 & 1. 3083 \\
\hline 70 & 1.9288 & 1.9281 & 1.9245 & 1.9146 & 1.9062 & 1.8870 & 1.8715 & 1.8448 & 1.7914 & 1.6507 & $1.453 ?$ \\
\hline 75 & 1.9392 & 1.9386 & 1.9358 & 1.9279 & 1.9211 & 1.9054 & 1. 8925 & 1.8698 & 1.9234 & 1.5949 & $1.50 \mathrm{bs}$ \\
\hline 80 & 1.9303 & 1.9298 & 1.9280 & 1.9226 & 1.9178 & 1.9066 & 1.8970 & 1.8795 & 1.8421 & 1.7303 & 1.5520 \\
\hline 85 & 1.9005 & 1.9004 & 1.8996 & 1.8973 & 1.8950 & 1.8891 & 1.8835 & 1.8725 & 1.8461 & 1.7558 & 1.5953 \\
\hline 90 & 1.8516 & 1.8518 & 1.8523 & 1.8535 & 1.8541 & 1.8544 & 1.8535 & 1.8500 & $1.836^{7}$ & 1.7721 & $1.533^{7}$ \\
\hline 95 & 1.7824 & 1.7829 & 1.7849 & 1.7901 & 1.7940 & 1.8013 & 1.8059 & 1.8110 & 1.8128 & 1.7786 & 1.6680 \\
\hline 100 & 1.6962 & 1.6970 & 1.7007 & 1.7103 & 1.7179 & 1.7331 & 1.7435 & 1.7582 & 1.7768 & 1.7765 & 1.5974 \\
\hline 105 & 1.5924 & 1.5936 & 1.5990 & 1.6135 & 1.6250 & 1.6487 & 1.6657 & 1.6909 & 1.7280 & 1.7658 & 1.7234 \\
\hline 115 & 1.3459 & 1.3479 & 1.3572 & 1.3823 & 1.4023 & 1.4448 & 1.4760 & 1.5241 & 1.6019 & 1. 7236 & 1.7638 \\
\hline 120 & 1.2086 & 1.2111 & 1.2224 & 1.2530 & 1.2776 & 1.3299 & 1.3687 & 1.4289 & 1.5281 & 1.6003 & 1.7788 \\
\hline 125 & 1.0643 & 1.0673 & $1.0 B 06$ & 1.1168 & 1.1461 & 1.2083 & 1.2548 & 1.3273 & 1.4485 & 1.5610 & 1.7925 \\
\hline 130 & 0.9188 & 0.9222 & 0.9376 & 0.9794 & 1.0132 & 1.0854 & 1.1394 & 1.2242 & 1.3672 & 1. 6251 & 1.3928 \\
\hline 135 & 0.7730 & 0.7769 & 0.7942 & 0.8416 & 0.8798 & 0.9617 & 1.0232 & 1.1200 & 1.2845 & 1.5879 & 1.8123 \\
\hline 140 & 0.6326 & 0.6369 & 0.6561 & $0.708^{7}$ & 0.7512 & 0.8424 & 0.9110 & 1.0192 & 1.2042 & 1.5506 & 1.8193 \\
\hline 145 & 0.4988 & 0.5035 & 0.5245 & 0.5820 & 0.6285 & 0.7284 & 0.8037 & 0.9228 & 1.1270 & 1. 5105 & 1.8259 \\
\hline 150 & 0.3764 & 0.3814 & 0.4041 & 0.4660 & 0.5162 & 0.6241 & 0.7054 & 0.8343 & 1.0561 & 1.4807 & 1.9306 \\
\hline 155 & 0.2670 & 0.2724 & 0.2965 & 0.3624 & 0.4158 & 0.5307 & 0.6174 & 0.7550 & 0.9925 & 1.4502 & 1.8349 \\
\hline 160 & 0.1740 & 0.1797 & 0.2050 & 0.2742 & 0.3303 & 0.4512 & 0.5425 & 0.6875 & 0.9382 & 1.4200 & 1.9380 \\
\hline 165 & 0.0994 & 0.1053 & 0.1316 & 0.2035 & 0.2618 & 0.3875 & 0.4824 & 0.6333 & 0.3945 & 1. 4028 & 1.8404 \\
\hline 170 & 0.0443 & 0.0503 & 0.0774 & 0.1512 & 0.2111 & 0.3403 & 0.4379 & 0.5931 & 0.8622 & 1.3872 & 1. 9422 \\
\hline 175 & 0.0118 & 0.0180 & 0.0454 & 0.1204 & 0.1813 & 0.3125 & 0.4117 & 0.5695 & 0.8431 & 1.3776 & 1.8429 \\
\hline 180 & 0.0001 & 0.0062 & 0.0338 & 0.1093 & 0.1706 & 0.3026 & 0.4023 & 0.5611 & 0.8365 & 1.3744 & 1.3423 \\
\hline
\end{tabular}

RATIO OF MOTT TO RUTHERFORE SCATTERING IN DB, $\mathrm{z}=82$

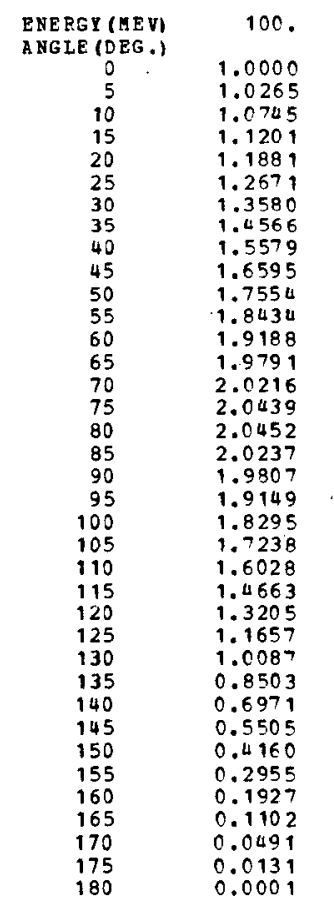

10.
1.0000
1.0265
1.0742
1.1197
1.1875
1.2664
1.3572
1.4557
1.5568
1.6583
1.7542
1.8422
1.9176
1.9780
2.0206
2.0430
2.0446
2.0233
1.9807
1.9151
1.8302
1.7249
1.6044
1.4684
1.3230
1.1688
1.0123
0.8544
0.7017
0.5556
0.4214
0.3013
0.1988
0.1166
0.0557
0.0198
0.0068

4.
1.0000
1.0260
1.0732
1.1180
1.1851
1.2633
1.3533
1.4513
1.5520
1.6531
1.7488
1.8368
1.9123
1.9730
2.0161
2.0393
2.0418
2.0217
1.9804
1.9164
1.8332
1.7299
1.6114
1.4775
1.3345
1.1826
1.0283
0.8728
0.7222
0.5781
0.4458
0.3273
0.2263
0.1451
0.0850
0.0496
0.0368

2.
1.0000
1.0249
1.0705
1.1133
1.1783
1.2545
1.3427
1.4391
1.5385
1.6387
1.7337
1.8216
1.8974
1.9590
2.0035
2.0286
2.0337
2.0168
1.9792
1.9196
1.8412
1.7432
1.6304
1.5026
1.3657
1.2201
1.0721
0.9228
0.7781
0.6396
0.5124
0.3984
0.3012
0.2231
0.1653
0.1312
0.1189

$\begin{array}{cc}1.5 & 1.0 \\ 1.0000 & 1.0000 \\ 1.0241 & 1.0222 \\ 1.0683 & 1.0635 \\ 1.1095 & 1.1012 \\ 1.1727 & 1.1605 \\ 1.2474 & 1.2315 \\ 1.3340 & 1.3147 \\ 1.4291 & 1.4069 \\ 1.5273 & 1.5026 \\ 1.6267 & 1.6001 \\ 1.7212 & 1.6934 \\ 1.8090 & 1.7808 \\ 1.8850 & 1.8572 \\ 1.0473 & 1.9209 \\ 1.9929 & 1.9688 \\ 2.0196 & 1.9990 \\ 2.0268 & 2.0106 \\ 2.0124 & 2.0018 \\ 1.9779 & 1.9738 \\ 1.9218 & 1.9253 \\ 1.8474 & 1.8593 \\ 1.7536 & 1.7750 \\ 1.5455 & 1.6768 \\ 1.5225 & 1.5645 \\ 1.3907 & 1.4437 \\ 1.2503 & 1.3144 \\ 1.1074 & 1.1828 \\ 0.9631 & 1.0494 \\ 0.8233 & 0.9201 \\ 0.6893 & 0.7961 \\ 0.5663 & 0.6821 \\ 0.4560 & 0.5798 \\ 0.3619 & 0.4925 \\ 0.2863 & 0.4223 \\ 0.2303 & 0.3704 \\ 0.1973 & 0.3398 \\ 0.1854 & 0.3288 \\ & \\ 0.8934 & \end{array}$

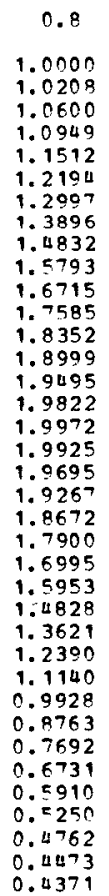

0.6
1.0000
1.0186
1.0544
1.0848
1.1361
1.1996
1.2752
1.3610
1.4511
1.5446
1.6350
1.7212
1.7981
1.8642
1.9165
1.9531
1.9734
1.9753
1.9602
1.9266
1.8774
1.8116
1.7334
1.6422
1.5432
1.4363
1.3269
1.2156
1.1073
1.0031
0.9072
0.8210
0.7474
0.5881
0.5443
0.5183
0.6092

1.4
1.0000
1.0150
1.0450
1.0674
1.1092
1.1637
1.2301
1.3082
1.3914
1.4795
1.5661
1.6502
1.7270
1.7959
1.8516
1.8948
1.9239
1.9372
1.9358
1.9182
1.8871
1.8415
1.7848
1.7168
1.6416
1.5594
1.4743
1.3873
1.3021
1.2198
1.1439
1.0754
1.0169
0.9697
0.9348
0.9140
0.9069

0.2
1.0000
1.0086
1.0297
1.0359
1.0558
1.0888
1.1326
1.1906
1.2554
1.3286
1.4035
1.4798
1.5532
1.6223
1.5847
1.7390
1.7849
1.8191
1.8441
1.8536
1.8635
1.8590
1.8463
1.8263
1.8002
1.7694
1.7352
1.6092
1.6624
1.6256
1.5026
1.5619
1.5353
1.5137
1.4998
1.4890
1.4845

3.1

1.3900 1.0922 1. 0197 1.3172 1.0257 1.01900
1.3598 1.1578 1.1568 1.2118 1.2710 1.3343
1.3971 1.3971
1.1588 1. 5179 1.5734
1.5252 1. $5>19$ 1.7120
1.7526 1.7526
1.7870 1. 3163 1.8433 1. 8866 1.9035 1. 9322 1. 1.9442 1. 9620 1. 9685 1. $9-36$ 1. 9792 1. 9790 
RATIO OF MOTM TO RUTHERFCBD SCA TTPRTNG IN FR, $Z=87$

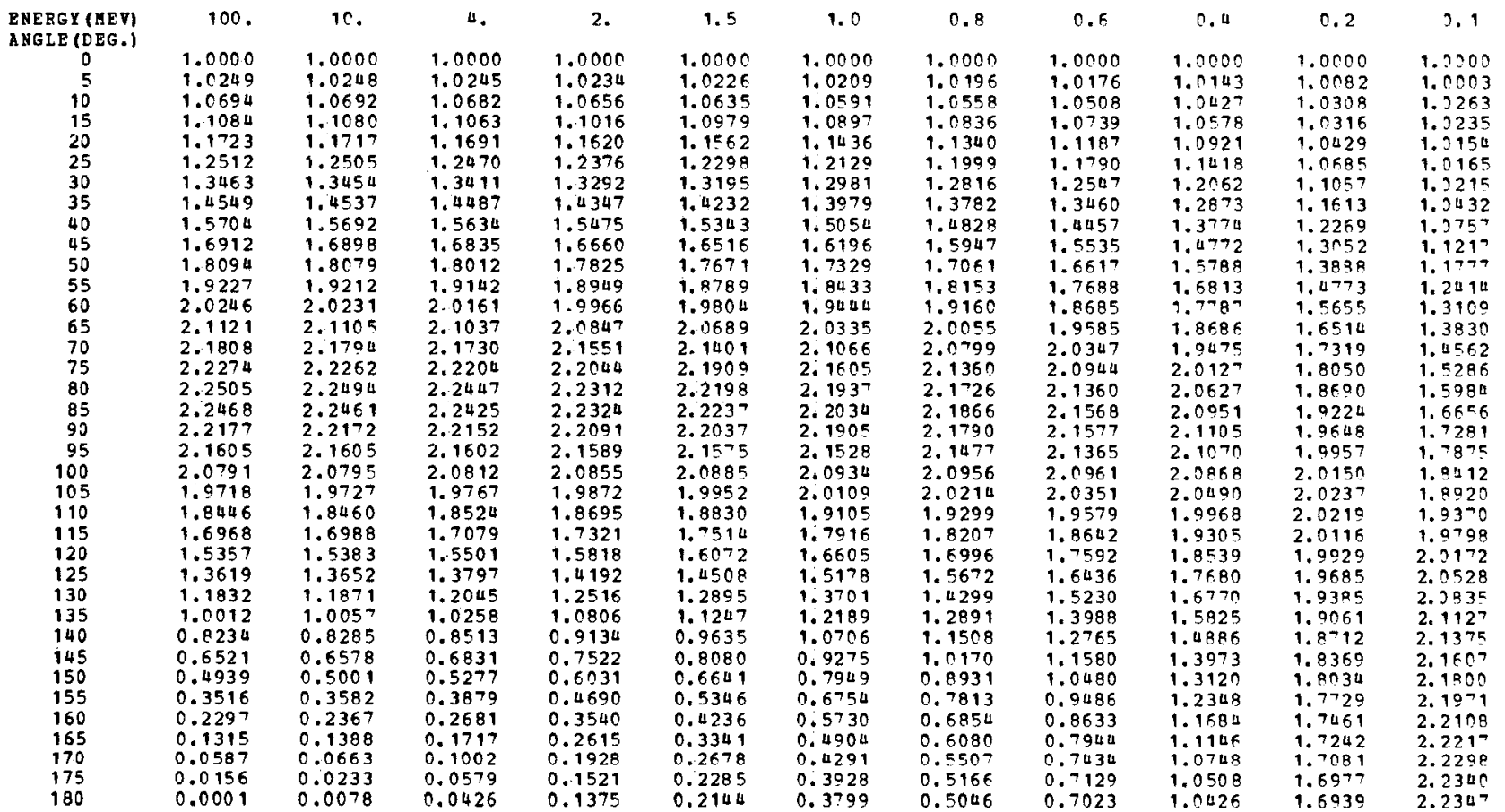

RATIO OF NOTT TO RUTHEFFORD SCATIERING IN $0, \mathrm{z}=92$

\begin{tabular}{|c|c|c|c|c|c|c|c|c|c|c|c|}
\hline $\begin{array}{l}\text { ENERGY (MEV) } \\
\text { A NGIE (DEG ) }\end{array}$ & 100 & 10. & a. & 2. & 1.5 & 1.0 & 0.8 & 0.5 & 0.4 & 0.2 & $\therefore .1$ \\
\hline 0 & 1.0000 & 1.0000 & 1.0000 & 1.0000 & 1.0000 & 1.0000 & 1.0000 & 1.0000 & 1.0000 & 1.0000 & 1.3909 \\
\hline & 1.0238 & 1.0237 & 1.0234 & 1.0224 & 1.0217 & 1.0201 & 1.0190 & 1.0171 & 1.0141 & 1.0077 & 0.9976 \\
\hline $\begin{array}{l}10 \\
15\end{array}$ & $\begin{array}{l}1.0647 \\
1.0950\end{array}$ & 1.0645 & 1.0637 & 1.0613 & 1.0595 & 1.0556 & 1.0528 & 1.0486 & 1.0421 & 1.0338 & 1.0295 \\
\hline $\begin{array}{l}15 \\
20\end{array}$ & $\begin{array}{l}1.0950 \\
1.1511\end{array}$ & 1.0946 & $\begin{array}{r}1.0930 \\
1.1480\end{array}$ & $\begin{array}{l}1.0886 \\
1.1408\end{array}$ & $\begin{array}{l}1.0850 \\
1.1351\end{array}$ & $\begin{array}{l}1.0774 \\
1.1226\end{array}$ & $\begin{array}{l}1.0718 \\
1.1133\end{array}$ & $\begin{array}{l}1.0632 \\
1.0985\end{array}$ & $\begin{array}{l}1.0404 \\
1.0737\end{array}$ & $\begin{array}{l}1.0304 \\
1.0325\end{array}$ & $\begin{array}{l}1.0300 \\
1.2182\end{array}$ \\
\hline 25 & 1.2261 & 1.2253 & 1.2217 & 1.2118 & 1.2037 & 1. 1862 & $1.1>30$ & 1.1518 & 1.1151 & 1. กบ8 82 & 1.3120 \\
\hline 30 & 1.3215 & 1.3205 & 1.3158 & 1.3029 & 1.2924 & 1.2694 & 1.2519 & 1.2235 & 1.1734 & 1.0753 & 1.0066 \\
\hline 35 & 1.4370 & 1.4358 & 1.4301 & 1. 4145 & 1.4016 & 1.3735 & 1. 3519 & 1.3167 & 1.2536 & 1. 1244 & 1.3174 \\
\hline 40 & 1.5651 & 1.5636 & 1.5570 & 1.5387 & 1. 5236 & 1.4005 & 1.4650 & 1.4232 & 1.3140 & 1.1870 & 1.0408 \\
\hline 45 & 1.7048 & 1.7039 & 1.6956 & 1.6750 & 1.6580 & 1. 6206 & 1.5916 & 1.5439 & 1.4567 & $1.26>0$ & 1.0000 \\
\hline 50 & 1.8466 & 1.8448 & 1.8367 & 1.8141 & 1.7955 & 1.7540 & 1.7224 & $1.669^{7}$ & $1.5^{7} 26$ & 1.3571 & 1.1334 \\
\hline 55 & 1.9880 & 1.9861 & 1.9775 & 1.9535 & 1.9337 & 1.8898 & 1.8555 & 1.7989 & 1.6939 & 1.4562 & 1.1977 \\
\hline 60 & 2.1207 & 2.1187 & 2. 1098 & 2.0850 & 2.0645 & 2.0189 & 1.9832 & 1.9240 & 1.8134 & 1.5590 & $1.2 \div 19$ \\
\hline 65 & 2.2405 & 2.2385 & 2.2296 & 2.2046 & 2.1840 & 2. 1380 & 2.1018 & 2.0416 & 1.9281 & 1.6623 & 1,3518 \\
\hline 70 & 2.3417 & 2.3397 & 2.3310 & 2.3068 & 2.2866 & 2.2415 & 2.2059 & 2.1464 & 2.0331 & 1.7627 & 1.4358 \\
\hline 75 & 2.4194 & 2.4176 & 2.4094 & 2.3867 & 2.3677 & 2. 3252 & 2. 2913 & 2.2344 & 2.1248 & 1.8571 & 1.5295 \\
\hline 80 & 2.4710 & 2.4694 & 2.4621 & 2.4418 & 2.4248 & 2.3863 & $2.355^{5}$ & 2.3030 & 2.2007 & 1.9431 & 1.6564 \\
\hline 85 & 2.4918 & 2.4905 & 2.4845 & 2.4676 & 2.4533 & 2.4207 & 2.3942 & 2.3486 & 2.2575 & $2.018^{7}$ & 1.6905 \\
\hline 90 & 2.4825 & 2.4816 & 2.4772 & 2.4646 & 2.4538 & 2.4287 & 2.0079 & 2.3710 & 2.2947 & 2.0823 & 1.7707 \\
\hline 95 & 2.4394 & 2.0389 & 2.4365 & 2.4293 & 2.4229 & 2.4071 & 2. 3933 & 2.3676 & 2.3104 & 2.1338 & 1.8480 \\
\hline 100 & 2.3662 & 2.3661 & 2.3660 & 2.3650 & 2.3637 & 2. 3587 & 2. 3531 & 2.3404 & 2.3056 & 2.1717 & 1.9216 \\
\hline 105 & 2.2605 & 2.2691 & 2.2636 & 2.2698 & 2.2742 & 2.2819 & 2.2858 & 2.2882 & 2.2798 & 2.1979 & 1.9919 \\
\hline 110 & 2.1287 & 2.1299 & 2.1352 & 2.11493 & 2.1601 & 2.1815 & 2.1950 & 2.2149 & 2.2355 & 2.2108 & 2.0563 \\
\hline 115 & 1.9701 & 1.9720 & 1.9805 & 2.0031 & 2.0210 & 2.0575 & 2.0833 & 2.1208 & 2.1737 & 2.2139 & $2.118 \mathrm{u}$ \\
\hline 120 & 1.7928 & 1.7955 & 1.8072 & 1.8388 & 1.8640 & 1.9163 & 1.9541 & 2.0109 & & 2.2058 & 2.1747 \\
\hline 125 & 1.5979 & 1.6013 & 1.6165 & 1.6576 & 1.6903 & 1.7593 & 1.8098 & 1.8869 & 2.0094 & 2.1900 & $2.228^{7}$ \\
\hline 130 & 1.3944 & 1.3985 & $1.417 \%$ & 1.4676 & 1.5081 & 1. 5938 & 1.6570 & 1.7544 & 1.9129 & 2. 1677 & 2.2772 \\
\hline 135 & 1.1846 & 1.1896 & 1.2116 & 1.2716 & 1.3198 & 1.4222 & 1.4982 & 1.6163 & 1.8113 & 2. 1412 & 2. 3232 \\
\hline 140 & 0.9776 & 0.9832 & 1.0087 & 1.0778 & 1.1334 & 1.2520 & 1.3403 & 1.4781 & 1.7081 & 2.1101 & 2.3630 \\
\hline 145 & 0.7767 & 0.7831 & 0.8117 & 0.8895 & 0.9522 & 1.0863 & 1.1864 & 1.3433 & $1.50>0$ & $2.0^{-90}$ & 2.4015 \\
\hline 150 & 0.5897 & 0.5968 & 0.6283 & 0.7141 & 0.7833 & 0.9315 & 1.0424 & 1.2167 & 1.5113 & 2.0471 & 2.11330 \\
\hline 155 & 0.4207 & 0.4283 & 0.4624 & 0.5554 & 0.6305 & 0.7914 & 0.9120 & 1.1019 & 1.4243 & 2.0181 & $2.462=$ \\
\hline 160 & 0.2753 & 0.2834 & 0.3198 & 0.4188 & 0.4989 & 0.6706 & 0.7995 & 1.0028 & 1.3488 & 1.9918 & 2.4859 \\
\hline 165 & 0.1578 & 0.1663 & 0.2044 & 0.3084 & 0.3925 & 0.5729 & 0.7084 & 0.9223 & 1.2873 & 1.9701 & 2.5044 \\
\hline 170 & 0.0706 & 0.0794 & 0.1189 & 0.2265 & 0.3135 & 0.5000 & 0.6409 & 0.8528 & 1.2420 & 1.9543 & 2.5181 \\
\hline 175 & 0.0186 & 0.0276 & 0.0678 & 0.1776 & 0.2663 & 0.4569 & 0.6002 & $0.826^{7}$ & 1.2141 & 9.9436 & 2.5257 \\
\hline 180 & 0.0001 & 0.0091 & 0.0497 & 0.1602 & 0.2497 & 0.4437 & 0.5861 & 0.8143 & 1.2046 & 1.9395 & 2. 5282 \\
\hline
\end{tabular}




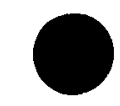

FATTO OF MOTT TO RUTPERFORD SCATTERING IN PO, $z=94$

\begin{tabular}{|c|c|c|c|c|c|c|c|c|c|c|c|}
\hline $\begin{array}{l}\text { ENERGY (GEV) } \\
\text { ANGLE (DEG.) }\end{array}$ & 100. & 10. & u. & 2. & 1.5 & 1.0 & 0.8 & 0.6 & 0.0 & 0.2 & $\therefore 1$ \\
\hline $\begin{array}{l}0 \\
5\end{array}$ & 1.0000 & 1.0000 & 1.0000 & 1.0000 & 1.0000 & 1. 0000 & 1.0000 & 1.0000 & 1.0000 & 1.0000 & 1.2505 \\
\hline $\begin{array}{r}5 \\
10\end{array}$ & $\begin{array}{l}1.0235 \\
1.0632\end{array}$ & 1.0235 & 1.0231 & 1.0222 & 1.0215 & 1.0200 & 1.0189 & 1.0171 & 1.0141 & 1.0073 & 0.0063 \\
\hline 10 & 1.0632 & 1.0630 & 1.0622 & 1.0600 & 1.0583 & 1.0547 & 1.0521 & 1.0483 & 1.0425 & 1.0355 & 1.9305 \\
\hline 15 & 1.0895 & 1.0891 & 1.0876 & 1.0833 & 1.0799 & 1.0727 & 1.0674 & 1.0593 & 1.01468 & 1.0310 & 1.0332 \\
\hline 20 & 1.1414 & 1.1409 & 1.1383 & 1.1313 & 1.1256 & 1.1134 & 1.1043 & 1.0900 & 1.0665 & 1.0294 & 1.0207 \\
\hline 25 & 1.2135 & 1.2126 & 1.2090 & 1.1991 & 1. 1910 & 1.1735 & 1. 1603 & 1. 1393 & 1.1035 & 1.0407 & 1.2125 \\
\hline 30 & 1.3076 & 1.3066 & 1.3018 & 1.2886 & 1.2779 & 1.2545 & $1.236^{7}$ & 1.2080 & 1.1580 & 1.0627 & 1.0323 \\
\hline 35 & 1.4248 & 1.4235 & 1.4476 & 1.4014 & 1.3881 & 1. 3590 & 1.3368 & 1. 3007 & 1.2365 & 1.1079 & 1.0080 \\
\hline 40 & 1.5570 & $1.555 \mathrm{~d}$ & 1.5485 & 1.5293 & 1.5135 & 1.4789 & 1.4522 & 1.4088 & 1.3306 & 1.1680 & $1.226 \mathrm{~B}$ \\
\hline 45 & 1.7038 & 1.7021 & 1.6941 & 1.6722 & 1.6542 & $1.614^{-}$ & 1.5840 & 1.5339 & $1.442^{7}$ & 1.2475 & 1.0621 \\
\hline 50 & 1.8553 & 1.8533 & 1.8446 & 1.8204 & 1.8005 & 1.7565 & 1.7224 & 1.6664 & 1.5638 & 1.3392 & 1.1132 \\
\hline 55 & 2.0086 & 2.0065 & 1.9971 & & 1.9407 & 1.9023 & 1.8650 & 1.8046 & 1.6924 & 1. 4418 & $\begin{array}{l}1.176 E \\
1.2517\end{array}$ \\
\hline 60 & $\begin{array}{l}2.1547 \\
2.2890\end{array}$ & $\begin{array}{l}2.1525 \\
2.2868\end{array}$ & $\begin{array}{l}2.1427 \\
2.2769\end{array}$ & $\begin{array}{l}2.1156 \\
2.2493\end{array}$ & $\begin{array}{l}2.0932 \\
2.2265\end{array}$ & $\begin{array}{l}2.0435 \\
2.1758\end{array}$ & $\begin{array}{l}2.0047 \\
2.1360\end{array}$ & $\begin{array}{l}1.9405 \\
2.0700\end{array}$ & & 1.5501 & $\begin{array}{l}1.2517 \\
1.3341\end{array}$ \\
\hline $\begin{array}{l}65 \\
70\end{array}$ & $\begin{array}{l}2.2890 \\
2.4050\end{array}$ & 2.4029 & $\begin{array}{l}2.2769 \\
2.3931\end{array}$ & 2.3659 & $\begin{array}{l}2.2265 \\
2.3434\end{array}$ & $\begin{array}{l}2.1758 \\
2.2932\end{array}$ & $\begin{array}{l}2.1360 \\
2.2536\end{array}$ & $\begin{array}{l}2.0700 \\
2.1876\end{array}$ & $\begin{array}{l}1.9563 \\
2.0629\end{array}$ & $\begin{array}{l}1.6605 \\
1.7692\end{array}$ & $\begin{array}{l}1.3341 \\
1.4220\end{array}$ \\
\hline 75 & 2.4973 & 2.4953 & 2.4860 & 2.4602 & 2.4387 & 2.3906 & 2.3525 & 2.2885 & 2.1665 & 1.8727 & 1.5128 \\
\hline 80 & 2.5625 & 2.5607 & 2.5523 & 2.5288 & 2.5091 & 2.4648 & 2.0295 & 2.3698 & 2.2542 & 1.9683 & 1.5030 \\
\hline 85 & 2.5955 & 2.5939 & 2.5867 & 2.5666 & 2.5497 & 2.5112 & 2.4802 & 2.4272 & 2.3225 & 2.0539 & 1.6949 \\
\hline 90 & 2.5962 & 2.5950 & 2.5895 & 2.5738 & 2. $560 \mathrm{H}$ & 2.5296 & 2.5043 & 2.4600 & 2.3701 & $2.12\urcorner 2$ & 1.7328 \\
\hline 95 & 2.5607 & 2.5599 & 2.5564 & 2.5463 & 2.5375 & 2. 5163 & 2.4983 & 2. 4655 & 2.3951 & 2.1883 & 1.8590 \\
\hline 100 & 2.4922 & 2.4920 & 2.4909 & 2.4873 & 2. 4838 & 2.4740 & 2.4645 & 2.4452 & 2.3981 & 2.2309 & 1.9502 \\
\hline 105 & 2.3885 & 2.3889 & 2.3905 & 2.3946 & 2.3972 & 2. 4007 & 2.4012 & 2.3980 & $2.3>86$ & 2. 2694 & 2. .292 \\
\hline 110 & 2.2555 & 2.2566 & 2.2613 & 2.2737 & 2.2831 & 2.3013 & 2.3129 & 2.3274 & 2.3386 & 2.2894 & 2.1324 \\
\hline 115 & 2.0930 & 2.0948 & 2.1029 & 2.1246 & 2. 1415 & 2.1758 & 2.1998 & 2.2339 & 2.2797 & 2.2992 & 2,1733 \\
\hline 120 & 1.9090 & 1.9916 & 1.9233 & 1.9546 & 1.9794 & 2.0308 & $2.067^{7}$ & 2.1225 & 2.2044 & 2.2964 & 2.2384 \\
\hline 125 & 1.7052 & 1.7086 & 1.7240 & 1.7655 & 1.7986 & 1.8680 & 1.9186 & 1.9954 & 2.1159 & 2. 2863 & 2. 3009 \\
\hline 130 & 1.4907 & 1.4950 & 1.5141 & 1.5659 & 1.6073 & 1.6948 & 1.7592 & 1.8581 & 2.0175 & 2.2667 & $2.35^{7} 8$ \\
\hline 135 & 1.2687 & 1.2738 & 1.2967 & 1.3588 & 1.4087 & 1.5145 & 1.5928 & 1.7141 & 1.9131 & 2.2434 & 2.1116 \\
\hline 140 & 1.0484 & 1.0543 & 1.0809 & 1.1530 & 1.2110 & 1.3344 & 1.4262 & 1.5690 & 1.8061 & 2.2145 & 2.4590 \\
\hline 145 & 0.8341 & 0.8408 & 0.8708 & 0.9525 & 1.0183 & 1.1587 & 1. 2633 & 1.4270 & 1.7009 & 2. 1852 & 2.5944 \\
\hline 150 & 0.6340 & 0.5414 & 0.6746 & 0.7651 & 0.8380 & 0.9939 & $1.110 \mathrm{u}$ & 1.2931 & 1.6007 & 2.1505 & 2.5432 \\
\hline 155 & 0.4527 & 0.4608 & 0.4969 & 0.5952 & 0.6746 & 0.34 a 4 & 0.9715 & 1.1714 & $1.509 \mathrm{~d}$ & 2.1264 & 2.5771 \\
\hline 160 & 0.2965 & 0.3051 & 0.3436 & 0.4487 & 0.5335 & 0.7153 & 0.8515 & 1.0660 & 1.0300 & 2.1006 & 2. 6053 \\
\hline 165 & 0.1700 & 0.1791 & 0.2196 & 0.3300 & 0.4193 & 0.6106 & 0.7541 & 0.9803 & 1. 3552 & $2.0>93$ & 2.6274 \\
\hline 170 & 0.0762 & 0.0855 & 0.1275 & 0.2420 & 0.3345 & 0.5329 & 0.6819 & 0.9169 & $1.397 \mathrm{u}$ & 2.0637 & 2. 5138 \\
\hline 175 & 0.0200 & 0.0296 & 0.0724 & 0.1892 & 0.2836 & 0.4861 & 0.6382 & 0.8783 & 1. 2877 & 2.0530 & 2.6531 \\
\hline 180 & 0.0001 & 0.0097 & 0.0529 & 0.1706 & 0.2657 & 0.4699 & 0.6231 & 0.8651 & 1.2776 & 2.0488 & 2.5566 \\
\hline
\end{tabular}

RATIO OP MOTT TO ROTHERFCPD SCATMPRING IN ES, $\mathrm{z}=99$

\begin{tabular}{|c|c|c|c|c|c|c|c|c|c|c|c|}
\hline $\begin{array}{l}\text { ENERGY (MEV) } \\
\text { A NGLE (DEG.) }\end{array}$ & 100 & 10. & 4. & 2. & 1.5 & 1.0 & 0.8 & 0.6 & 0.4 & 0.2 & 0,1 \\
\hline 0 & 1.0000 & 1.0000 & 1.0000 & 1.0000 & 1.0000 & 1.0000 & $1.000 ?$ & 1.0000 & $1.0 \cap 00$ & 1.0000 & 1.3000 \\
\hline 5 & 1.0233 & 1.0233 & 1.0230 & 1.0221 & 1.0215 & 1.0200 & 1.0189 & 1.0172 & 1.0140 & 1.0058 & 0.9927 \\
\hline $\begin{array}{l}10 \\
15\end{array}$ & $\begin{array}{l}1.0609 \\
1.0762\end{array}$ & $\begin{array}{l}1.0607 \\
1.0759\end{array}$ & $\begin{array}{l}1.0600 \\
1.0746\end{array}$ & $\begin{array}{l}1.0583 \\
1.0709\end{array}$ & $\begin{array}{l}1.0569 \\
1.0681\end{array}$ & $\begin{array}{l}1.0501 \\
1.0621\end{array}$ & $\begin{array}{l}1.0522 \\
1.0579\end{array}$ & $\begin{array}{l}1.0493 \\
1.0517\end{array}$ & $\begin{array}{l}1.0453 \\
1.0429\end{array}$ & $\begin{array}{l}1.0405 \\
1.0357\end{array}$ & $\begin{array}{l}1.3319 \\
1.0418\end{array}$ \\
\hline 20 & 1.1151 & 1.1145 & 1.1121 & 1.1057 & 1.1005 & 1.0896 & 1.0815 & 1.0693 & 1.0502 & $1.025^{7}$ & 1.2302 \\
\hline 25 & 1.1760 & 1.1752 & 1.1717 & 1.1620 & 1.1541 & $1 .+374$ & 1.1250 & 1. 1055 & 1.0738 & 1.0252 & 1.3182 \\
\hline $\begin{array}{l}25 \\
30\end{array}$ & 1.2627 & 1.2616 & 1.2567 & 1.2433 & 1.2323 & 1.2087 & 1.1910 & 1.1628 & 1.1150 & 1.0322 & 0.9075 \\
\hline 35 & 1.3799 & 1.3785 & 1. 3722 & 1.3549 & 1.3008 & 1.3102 & 1.2870 & 1. 2498 & 1.1852 & 1.0642 & 0.9992 \\
\hline 40 & 1.5192 & 1.5175 & 1.5098 & 1.4886 & $1.4-13$ & 1.4335 & 1.4048 & 1.3581 & 1.2760 & 1.1143 & 0.9941 \\
\hline 45 & 1.6819 & 1.6799 & 1.6708 & 1.6459 & 1.6255 & 1.5810 & $1.546^{7}$ & 1.4911 & 1.3019 & 1.1885 & 1.0163 \\
\hline 50 & 1.8567 & 1.8544 & 1.8441 & 1.8159 & 1.7927 & 1.7418 & 1.7025 & 1.6386 & 1.5233 & 1.2809 & 1.2582 \\
\hline 55 & 2.0405 & 2.0380 & 2.0267 & 1.9956 & 1.9700 & 1.9136 & 1.8700 & 1.7987 & 1.6689 & 1.3896 & 1.1160 \\
\hline 60 & 2.2228 & 2.2201 & 2.2081 & 2.1747 & $2.10>1$ & 2.0865 & 2.0395 & 1.9622 & 1.8206 & 1.5099 & 9.1900 \\
\hline 65 & 2.3973 & 2.3945 & 2.3819 & 2.3471 & 2. 3184 & 2. 2549 & 2.2054 & 2.1239 & $1.9-3 b$ & 1.6370 & $1.2^{-63}$ \\
\hline 70 & 2.5557 & 2.5528 & 2.5401 & 2.5048 & 2. 4756 & 2.4110 & 2.3604 & 2.2767 & 2.1211 & 1.7666 & 1.3720 \\
\hline 75 & 2.6904 & 2.6876 & 2.6751 & 2.6405 & 2.6117 & 2.5479 & 2.4978 & 2.10144 & 2.2579 & 1.8943 & 1. $11-45$ \\
\hline 80 & 2.7962 & 2.7936 & 2.7818 & 2.7490 & 2.7217 & 2.6608 & 2.6127 & 2.5321 & 2.3793 & 2.0159 & 1.5804 \\
\hline 85 & 2.8661 & 2.8637 & 2.8531 & 2.8235 & 2.7988 & 2.7433 & 2.6990 & 2.6244 & 2.4807 & 2. 1292 & 1.6891 \\
\hline 90 & 2.8984 & 2.8964 & 2.8875 & 2.8624 & 2.8413 & 2.7934 & 2.7549 & 2.6889 & 2.5593 & 2.2297 & 1.7988 \\
\hline 95 & 2.8878 & 2.8864 & 2.8797 & 2.8606 & 2.8444 & 2.8068 & 2.7760 & 2.7221 & 2.6127 & 2.3182 & 1.0047 \\
\hline 100 & 2.8365 & 2.8356 & 2.8316 & 2.8198 & 2.8094 & 2.7845 & 2.7630 & 2.7240 & 2.6395 & 2.3903 & 2.2090 \\
\hline 105 & 2.7416 & 2.7415 & 2.7 .406 & 2.7376 & 2.7343 & 2.7246 & 2.7146 & 2.6936 & 2.6402 & 2.4404 & 2.1117 \\
\hline 110 & 2.6087 & 2.6093 & 2.6121 & 2.6188 & 2.6235 & 2. 6308 & $2.633^{7}$ & 2.5331 & 2.6148 & 2.4910 & 2.2090 \\
\hline 115 & 2.4379 & 2.4394 & 2.4462 & 2.4640 & 2.4776 & $2.50 \div 2$ & 2.5217 & 2.5443 & 2.5663 & 2.5209 & 2.3053 \\
\hline 120 & 2.2373 & 2.2398 & 2.2509 & 2.2804 & 2.3034 & 2.3505 & 2.3835 & 2.4309 & 2.4957 & 2.5348 & 2.3958 \\
\hline 125 & 2.0101 & 2.0136 & 2.0292 & 2.0712 & 2. 1044 & 2,1735 & 2.2231 & 2.2971 & 2.4084 & 2.5397 & $2.482^{7}$ \\
\hline 130 & 1.7658 & 1.7704 & 1.7906 & 1.8453 & 1.8888 & 1.9801 & 2.0466 & 2.1476 & 2.3061 & 2.5320 & 2.5642 \\
\hline 135 & 1.5099 & 1.5154 & 1.5404 & 1.6080 & 1.6620 & 1.7760 & 1.8598 & 1.9884 & 2.1955 & 2.5190 & 2.6008 \\
\hline 140 & 1.2524 & 1.2590 & 1.2885 & 1.3686 & 1.4328 & 1.5689 & 1.6694 & 1.8248 & 2.0789 & 2.4977 & 2.7117 \\
\hline 145 & 1.0000 & 1.0076 & 1.0415 & 1.1337 & 1.2077 & 1. 3651 & 1.4818 & 1.5632 & 1.9631 & 2.11748 & 2.7762 \\
\hline 150 & 0.7622 & 0.7707 & 0.8086 & 0.9119 & 0.9950 & 1.1720 & 1.3036 & 1.5089 & 1.8509 & 2,4485 & 2.8340 \\
\hline 155 & 0.5457 & 0.5550 & 0.5966 & 0.7099 & 0.8012 . & 0.9959 & 1.1410 & 1.3680 & 1.7482 & 2.4238 & 2.8840 \\
\hline 160 & 0.3589 & 0.3681 & 0.4129 & 0.5347 & 0.6330 & 0.8428 & 0.9995 & 1.2450 & 1.6579 & 2.4003 & 2.0263 \\
\hline $\begin{array}{l}100 \\
165\end{array}$ & 0.2055 & 0.2161 & 0.2634 & 0.3922 & 0.4961 & 0.7181 & 0.8841 & 1.1445 & 1.5838 & 2.3805 & 2.9594 \\
\hline 170 & 0.0924 & 0.1034 & 0.1526 & 0.2865 & 0.3946 & 0.6257 & 0.7987 & 1.0703 & 1.5292 & 2.3661 & 2.9839 \\
\hline 175 & 0.0239 & 0.0351 & 0.0854 & 0.2223 & 0.3328 & 0.5692 & 0.7462 & 1.0242 & 1.4946 & 2.3554 & 2.9983 \\
\hline 180 & 0.0001 & 0.0115 & 0.0621 & 0.2002 & 0.3116 & 0.5500 & 0.7284 & 1.0088 & 1.4830 & 2.3515 & 3.2247 \\
\hline
\end{tabular}


RATIO OF MOTT TO MCKTNLET-FESHBACH SCATTERTNG IN H, $\mathrm{z}=1$

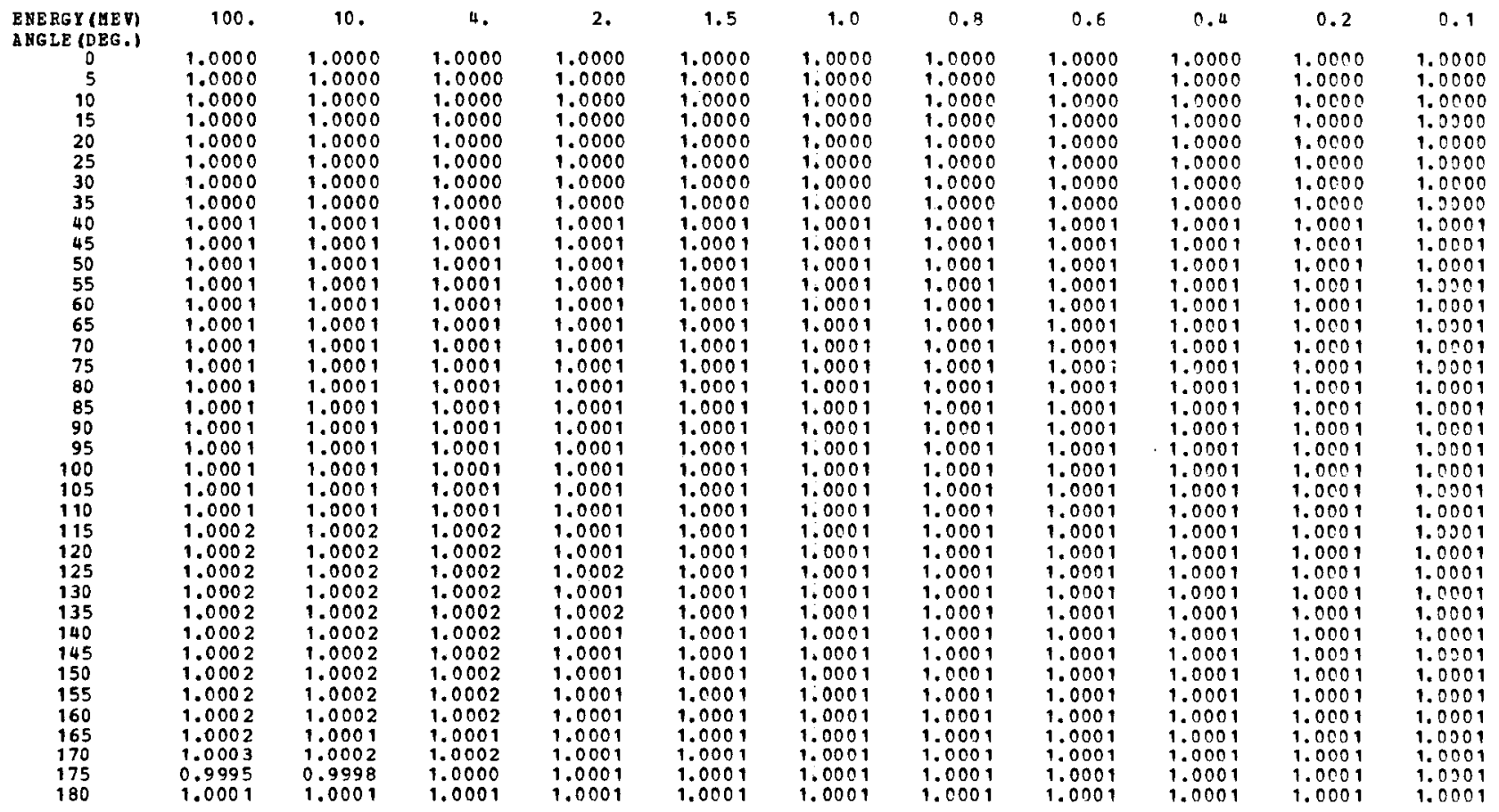

RATTO OF MOTT TO MCKINLEY-FESHBACH SCATTERTNG IN HE, $\mathrm{z}=2$

ENERG (HEV)
ANGLE (DEG.)
0
5
10
15
20
25
30
35
40
45
50
55
60
65
70
75
80
85
90
95
100
105
110
115
120
125
130
135
140
145
150
155
160
165
170
175
180

$\begin{array}{cc}100 & 10 \\ 1.0000 & 1.0000 \\ 0.9999 & 0.9999 \\ 1.0001 & 1.0001 \\ 1.0000 & 1.0000 \\ 1.0001 & 1.0001 \\ 1.0001 & 1.0001 \\ 1.0002 & 1.0002 \\ 1.0002 & 1.0002 \\ 1.0002 & 1.0002 \\ 1.0003 & 1.0003 \\ 1.0003 & 1.0003 \\ 1.0003 & 1.0003 \\ 1.0004 & 1.0004 \\ 1.0004 & 1.0004 \\ 1.0004 & 1.0004 \\ 1.0005 & 1.0004 \\ 1.0005 & 1.0005 \\ 1.0005 & 1.0005 \\ 1.0005 & 1.0005 \\ 1.0005 & 1.0005 \\ 1.0006 & 1.0006 \\ 1.0006 & 1.0006 \\ 1.0006 & 1.0006 \\ 1.0006 & 1.0006 \\ 1.0006 & 1.0006 \\ 1.0006 & 1.0006 \\ 1.0006 & 1.0006 \\ 1.0007 & 1.0007 \\ 1.0007 & 1.0007 \\ 1.0007 & 1.0007 \\ 1.0007 & 1.0007 \\ 1.0007 & 1.0007 \\ 1.0007 & 1.0007 \\ 1.0007 & 1.0006 \\ 1.0009 & 1.0008 \\ 0.9994 & 0.9999 \\ 1.0004 & 1.0004 \\ & \\ & \end{array}$

$\begin{array}{cc}4 & 2 \\ 1.0000 & 1.0000 \\ 0.9999 & 0.9999 \\ 1.0001 & 1.0001 \\ 1.0000 & 1.0000 \\ 1.0001 & 1.0001 \\ 1.0001 & 1.0001 \\ 1.0002 & 1.0002 \\ 1.0002 & 1.0002 \\ 1.0002 & 1.0002 \\ 1.0003 & 1.0003 \\ 1.0003 & 1.0003 \\ 1.0003 & 1.0003 \\ 1.0004 & 1.0004 \\ 1.0004 & 1.0004 \\ 1.0004 & 1.0004 \\ 1.0004 & 1.0004 \\ 1.0005 & 1.0005 \\ 1.0005 & 1.0005 \\ 1.0005 & 1.0005 \\ 1.0005 & 1.0005 \\ 1.0006 & 1.0005 \\ 1.0006 & 1.0006 \\ 1.0006 & 1.0006 \\ 1.0006 & 1.0006 \\ 1.0006 & 1.0006 \\ 1.0006 & 1.0006 \\ 1.0006 & 1.0006 \\ 1.0006 & 1.0006 \\ 1.0006 & 1.0006 \\ 1.0007 & 1.0006 \\ 1.0006 & 1.0006 \\ 1.0006 & 1.0005 \\ 1.0006 & 1.0005 \\ 1.0005 & 1.0004 \\ 1.0006 & 1.0004 \\ 1.0002 & 1.0003 \\ 1.0004 & 1.0004\end{array}$

1.5
1.0000
0.9999
1.0001
1.0000
1.0001
1.0001
1.0002
1.0002
1.0002
1.0003
1.0003
1.0003
1.0004
1.0004
1.0004
1.0004
1.0005
1.0005
1.0005
1.0005
1.0005
1.0005
1.0005
1.0006
1.0006
1.0006
1.0006
1.0006
1.0006
1.0005
1.0005
1.0005
1.0005
1.0004
1.0004
1.0003
1.0004

1.0
1.0000
0.9999
1.0001
1.0000
1.0001
1.0001
1.0002
1.0002
1.0002
1.0003
1.0003
1.0003
1.0004
1.0004
1.0004
1.0004
1.0004
1.0005
1.0005
1.0005
1.0005
1.0005
1.0005
1.0005
1.0005
1.0005
1.0005
1.0005
1.0005
1.0005
1.0005
1.0004
1.0004
1.0004
1.0004
1.0003
1.0004

0.8
1.0000
0.9999
1.0001
1.0000
1.0001
1.0001
1.0002
1.0002
1.0002
1.0003
1.0003
1.0003
1.0003
1.0004
1.0004
1.0004
1.0004
1.0005
1.0005
1.0005
1.0005
1.0005
1.0005
1.0005
1.0005
1.0005
1.0005
1.0005
1.0005
1.0005
1.0004
1.0004
1.0004
1.0004
1.0004
1.0003
1.0004

0.6
1.0000
0.9999
1.0001
1.0001
1.0001
1.0001
1.0002
1.0002
1.0002
1.0003
1.0003
1.0003
1.0003
1.0004
1.0004
1.0004
1.0004
1.0004
1.0004
1.0005
1.0005
1.0005
1.0005
1.0005
1.0005
1.0005
11.0005
1.0005
1.0004
1.0004
1.0004
1.0004
1.0004
1.0004
1.0004
1.0004
1.0004

0.4
1.0000
0.9999
1.0001
1.0001
1.0001
1.0001
1.0002
1.0002
1.0002
1.0002
1.0003
1.0003
1.0003
1.0003
1.0004
1.0004
1.0004
1.0004
1.0004
1.0004
1.0004
1.0004
1.0004
1.0005
1.0004
1.0004
1.0004
1.0004
1.0004
1.0004
1.0004
1.0004
1.0004
1.0004
1.0004
1.0004
1.0004

0.2
1.0000
1.0000
1.0001
1.0001
1.0001
1.0001
1.0002
1.0002
1.0002
1.0002
1.0003
1.0003
1.0003
1.0003
1.0003
1.0004
1.0004
1.0004
1.0004
1.0004
1.0004
1.0004
1.0004
1.0004
1.0004
1.0004
1.0004
1.0004
1.0004
1.0004
1.0004
1.0004
1.0004
1.0004
1.0004
1.0004
1.0004

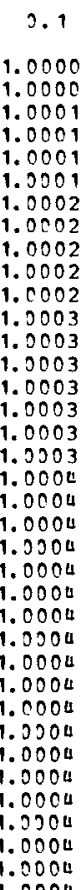

1.0000 .0000 1. 0001 1.0001 1.0002 1.0002 1.0002 1.0003 1.0003
1.0003 1.0003 1.0003 1.0503 1.0004 1.0004 1.0004 1.0008 1.0004 .0000 1.0004
1.0004

1.2000 1.0004 1.000 1. 9204 1.0004 
RETIO OF MOTT TO MCKINLEY-FESHBACH SCATTERING IN BE, $z=4$

\begin{tabular}{|c|c|c|c|c|c|c|c|c|c|c|c|}
\hline $\begin{array}{l}\text { ENERGY (MEV) } \\
\text { ANGLE (DEG.) }\end{array}$ & 100. & 10. & 4. & 2. & 1.5 & 1.0 & 0.8 & 0.6 & 0.4 & 0.2 & 0.1 \\
\hline 0 & 1.0000 & 1.0000 & 1.0000 & 1.0000 & 1.0000 & 1.0000 & 1.0000 & 1.0000 & 1.0000 & 1.0000 & 1.0000 \\
\hline 5 & 0.9999 & 0.9999 & 0.9999 & 0.9999 & 0.9999 & 0.9999 & 0.9999 & 0.9999 & 0.9999 & 0.9099 & 1.0000 \\
\hline 10 & 1.0002 & 1.0002 & 1.0002 & 1.0002 & 1.0002 & 1.0002 & 1.0002 & 1.0002 & 1.0002 & 1.0002 & 1.0002 \\
\hline 15 & 1.0002 & 1.0002 & 1.0002 & 1.0002 & 1.0002 & 1.0002 & 1.0002 & 1.0002 & 1.0002 & 1.0002 & 1.0002 \\
\hline 20 & 1.0004 & 1.0004 & 1.0004 & 1.0004 & 1.0004 & 1.0004 & 1.0004 & 1.0004 & 1.0004 & 1.0004 & 1.0004 \\
\hline 25 & 1.0005 & 1.0005 & 1.0005 & 1.0005 & 1.0005 & 1.0005 & 1.0005 & 1.0005 & 1.0005 & 1.0005 & 1.0005 \\
\hline 30 & 1.0007 & 1.0007 & 1.0007 & 1.0007 & 1.0007 & 1.0007 & $1.000^{7}$ & 1.0007 & 1.0006 & 1.0006 & 1.0006 \\
\hline 35 & 1.0008 & 1.0008 & 1.0008 & 1.0008 & 1.0008 & 1.0008 & 1.0008 & 1.0008 & 1.0008 & 1.0007 & $1.00 \mathrm{c}^{7}$ \\
\hline 40 & 1.0010 & 1.0010 & 1.0010 & 1.0010 & 1.0009 & 1.0009 & 1.0009 & 1.0009 & 1.0009 & 1.0008 & 1.0008 \\
\hline 45 & 1.0011 & 1.0011 & 1.0011 & 1.0011 & 1.0011 & 1.0010 & 1.0010 & 1.0010 & 1.0010 & 1.0009 & 1.0009 \\
\hline 50 & 1.0012 & 1.0012 & 1.0012 & 1.0012 & 1.0012 & 1.0012 & 1.0012 & 1.0011 & 1.0011 & 1.0010 & 1.0010 \\
\hline 55 & 1.0013 & 1.0013 & 1.0013 & 1.0013 & 1.0013 & 1.0013 & 1.0013 & 1.0012 & 1.0012 & 1.0011 & 1.2011 \\
\hline 60 & 1.0015 & 1.0015 & 1.0015 & 1.0014 & 1.0014 & 1.0014 & $1.001 \mathrm{u}$ & 1.0014 & 1.0013 & 1.0012 & 1.0011 \\
\hline 65 & 1.0016 & 1.0016 & 1.0016 & 1.0016 & 1.0015 & 1.0015 & 1.0015 & 1.0014 & 1.0014 & 1.0013 & 1.0012 \\
\hline 70 & 1.0017 & 1.0017 & 1.0017 & 1.0017 & 1.0016 & 1.0016 & 1.0016 & 1.0015 & 1.0015 & 1.0044 & 1.0013 \\
\hline 75 & 1.0018 & 1.0018 & 1.0018 & 1.0018 & 1.0017 & 1.0017 & 1.0017 & 1.0016 & 1.0015 & 1.0014 & 1.0013 \\
\hline 80 & 1.0019 & 1.0019 & 1.0019 & 1.0018 & 1.0018 & 1.0018 & 1.0017 & 1.0017 & 1.0016 & 1.0015 & 1.0014 \\
\hline 85 & 1.0020 & 1.0020 & 1.0020 & 1.0019 & 1.0019 & 1.0019 & 1.0018 & 1.0018 & 1.0017 & 1.0015 & 1.0014 \\
\hline 90 & 1.0021 & 1.0021 & 1.0021 & 1.0020 & 1.0020 & 1.0019 & 1.0019 & 1.0018 & 1.0017 & 1.0015 & 1.0014 \\
\hline 95 & 1.0022 & 1.0022 & 1.0021 & 1.0021 & 1.0021 & 1.0020 & 1.0019 & 1.0019 & 1.0017 & 1.0016 & 1.0015 \\
\hline 100 & 1.0022 & 1.0022 & 1.0022 & 1.0022 & 1.0021 & 1.0020 & 1.0020 & 1.0019 & 1.0018 & 1.0016 & 1.0015 \\
\hline 115 & 1.0025 & 1.0025 & 1.0024 & 1.0023 & 1.0023 & 1.0021 & 1.0021 & 1.0020 & 1.0018 & 1.0016 & 1.0015 \\
\hline 120 & 1.0025 & 1.0025 & 1.0024 & 1.0023 & 1.0023 & 1.0021 & 1.0020 & 1.0019 & 1.0018 & 1.0016 & 1.0015 \\
\hline 125 & 1.0026 & 1.0026 & 1.0025 & 1.0024 & 1.0023 & 1.0021 & 1.0021 & 1.0019 & 1.0018 & 1.0016 & 1.0015 \\
\hline 130 & 1.0026 & 1.0026 & 1.0025 & 1.0024 & 1.0023 & 1.0021 & 1.0020 & 1.0019 & 1.0018 & 1.0016 & 1.0015 \\
\hline 135 & 1.0027 & 1.0027 & 1.0026 & 1.0024 & 1.0023 & 1.0021 & 1.0020 & 1.0019 & 1.0017 & 1.0016 & 1.0015 \\
\hline 140 & 1.0027 & 1.0027 & 1.0026 & 1.0024 & 1.0022 & 1.0020 & 1.0019 & 1.0018 & 1.0017 & 1.0016 & 1.0015 \\
\hline 145 & 1.0028 & 1.0027 & 1.0026 & 1.0023 & 1.0022 & 1.0020 & 1.0019 & 1.0018 & 1.0017 & 1.0016 & 1.0015 \\
\hline 150 & 1.0027 & 1.0027 & 1.0025 & 1.0023 & 1.0021 & 1.0019 & 1.0018 & 1.0017 & 1.0016 & 1.0015 & 1.0015 \\
\hline 155 & 1.0028 & 1.0027 & 1.0025 & 1.0022 & 1.0020 & 1.0018 & 1.0017 & 1.0017 & 1.0016 & 1.0015 & 1.0015 \\
\hline 160 & 1.0028 & 1.0027 & 1.0024 & 1.0020 & 1.0019 & 1.0017 & 1.0017 & 1.0016 & 1.0015 & 1.0015 & 1.0015 \\
\hline 165 & 1.0027 & 1.0026 & 1.0022 & 1.0018 & 1.0017 & 1.0016 & 1.0016 & 1.0015 & 1.0095 & 1.0015 & 1.0015 \\
\hline 170 & 1.0032 & 1.0028 & 1.0021 & 1.0017 & 1.0016 & 1.0016 & 1.0015 & 1.0015 & 1.0015 & 1.0015 & 1.0015 \\
\hline 175 & 1.0003 & 1.0009 & 1.0013 & 1.0014 & 1.0014 & 1.0014 & 1.0014 & 1.0015 & 1.0015 & 1.0015 & 1.0015 \\
\hline 180 & 1.0015 & 1.0015 & 1.0015 & 1.0015 & 1.0015 & 1.0015 & 1.0015 & 1.0015 & 1.0015 & 1.0015 & 1.0015 \\
\hline
\end{tabular}

RATIO OF MOTT IO MCKINIEY-FESHBACR SCATTERING IN $C, z=6$

ENERGY
ANGLE

1
1
20
25
3
4
45
5
55
6
65
7
75
8
8
9
9
10
105
110
115
120
125
130
135
140
145
150
155
160
165
170
175
180

$\begin{array}{lllc}100 . & 10 . & 4 & 2 . \\ 1.0000 & 1.0000 & 1.0000 & 1.0000 \\ 0.9999 & 0.9999 & 0.9999 & 0.9999 \\ 1.0004 & 1.0004 & 1.0004 & 1.0004 \\ 1.0005 & 1.0005 & 1.0005 & 1.0005 \\ 1.0009 & 1.0009 & 1.0009 & 1.0009 \\ 1.0012 & 1.0012 & 1.0012 & 1.0012 \\ 1.0015 & 1.0015 & 1.0015 & 1.0015 \\ 1.0018 & 1.0018 & 1.0018 & 1.0018 \\ 1.0021 & 1.0021 & 1.0021 & 1.0021 \\ 1.0024 & 1.0024 & 1.0024 & 1.0024 \\ 1.0027 & 1.0027 & 1.0027 & 1.0027 \\ 1.0030 & 1.0030 & 1.0030 & 1.0030 \\ 1.0033 & 1.0033 & 1.0033 & 1.0032 \\ 1.0035 & 1.0035 & 1.0035 & 1.0035 \\ 1.0038 & 1.0038 & 1.0038 & 1.0037 \\ 1.0040 & 1.0040 & 1.0040 & 1.0040 \\ 1.0043 & 1.0043 & 1.0042 & 1.0042 \\ 1.0045 & 1.0045 & 1.0044 & 1.0044 \\ 1.0047 & 1.0047 & 1.0046 & 1.0045 \\ 1.0049 & 1.0049 & 1.0048 & 1.0047 \\ 1.0050 & 1.0050 & 1.0050 & 1.0049 \\ 1.0052 & 1.0052 & 1.0052 & 1.0050 \\ 1.0054 & 1.0053 & 1.0053 & 1.0051 \\ 1.0055 & 1.0055 & 1.0054 & 1.0052 \\ 1.0056 & 1.0056 & 1.0055 & 1.0053 \\ 1.0058 & 1.0058 & 1.0057 & 1.0054 \\ 1.0059 & 1.0058 & 1.0057 & 1.0054 \\ 1.0060 & 1.0060 & 1.0058 & 1.0054 \\ 1.0061 & 1.0060 & 1.0058 & 1.0053 \\ 1.0062 & 1.0061 & 1.0058 & 1.0053 \\ 1.0062 & 1.0061 & 1.0058 & 1.0051 \\ 1.0063 & 1.0062 & 1.0057 & 1.0049 \\ 1.0064 & 1.0061 & 1.0055 & 1.0046 \\ 1.0063 & 1.0059 & 1.0050 & 1.0042 \\ 1.0069 & 1.0061 & 1.0047 & 1.0039 \\ 1.0027 & 1.0030 & 1.0033 & 1.0033 \\ 1.0033 & 1.0033 & 1.0033 & 1.0033 \\ & & & \\ 1.0053 & \end{array}$

$\begin{array}{ccc}1.5 & 1.0 & 0.8 \\ 1.0000 & 1.0000 & 1.0000 \\ 0.9999 & 0.9999 & 0.9999 \\ 1.0004 & 1.0004 & 1.0004 \\ 1.0005 & 1.0005 & 1.0005 \\ 1.0009 & 1.0009 & 1.0009 \\ 1.0012 & 1.0011 & 1.0011 \\ 1.0015 & 1.0015 & 1.0015 \\ 1.0018 & 1.0018 & 1.0018 \\ 1.0021 & 1.0021 & 1.0021 \\ 1.0024 & 1.0024 & 1.0023 \\ 1.0027 & 1.0026 & 1.0026 \\ 1.0029 & 1.0029 & 1.0029 \\ 1.0032 & 1.0032 & 1.0031 \\ 1.0035 & 1.0034 & 1.0033 \\ 1.0037 & 1.0036 & 1.0035 \\ 1.0039 & 1.0038 & 1.0037 \\ 1.0041 & 1.0040 & 1.0039 \\ 1.0043 & 1.0042 & 1.0041 \\ 1.0045 & 1.0043 & 1.0042 \\ 1.0046 & 1.0045 & 1.0044 \\ 1.0048 & 1.0046 & 1.0045 \\ 1.0049 & 1.0047 & 1.0046 \\ 1.0050 & 1.0048 & 1.0046 \\ 1.0051 & 1.0048 & 1.0047 \\ 1.0051 & 1.0048 & 1.0047 \\ 1.0052 & 1.0049 & 1.0047 \\ 1.0052 & 1.0048 & 1.0046 \\ 1.0052 & 1.0048 & 1.0045 \\ 1.0051 & 1.0046 & 1.0044 \\ 1.0050 & 1.0045 & 1.0043 \\ 1.0048 & 1.0043 & 1.0041 \\ 1.0046 & 1.0042 & 1.0040 \\ 1.0043 & 1.0039 & 1.0038 \\ 1.0040 & 1.0037 & 1.0036 \\ 1.0037 & 1.0036 & 1.0035 \\ 1.0033 & 1.0033 & 1.0033 \\ 1.0034 & 1.0034 & 1.0034\end{array}$

0.6

1.0000 0.9999 1.0004 1.0005 1.0009 1.0011 1.0015 1.0017 1.0020 1.0023 1.0026 1.0028 1.0030 1.0033 1.0035 1.0036 1.0038 1.0940 1.0041 1.0042 1.0043 1.0044 1.0044
1.0044 1.0044
1.0044 1.0044
1.0044 1.0044
1.0043 1.0043
1.0043 1.0043 1.0042 1.0041 1.0039
1.0038 1.0038 1.0037
1.0035 1.0035 1.0035 1.0034
1.0034

0.4
1.0000
0.9999
1.0004
1.0005
1.0009
1.0011
1.0014
1.0017
1.0020
1.0022
1.0025
1.0027
1.0029
1.0031
1.0033
1.0035
1.0036
1.0038
1.0039
1.0040
1.0040
1.0041
1.0041
1.0041
1.0041
1.0041
1.0040
1.0040
1.0039
1.0038
1.0037
1.0036
1.0036
1.0035
1.0034
1.0034
1.0034

0.2

0.1

1.0000 $1.0004 \quad 1.0003$ $1.0005 \quad 1.0005$ $1.0008 \quad 1.0008$ $1.0011 \quad 1.0010$
1.0015 $1.0014 \quad 1.0013$ $1.0015 \quad 1.0015$ $1.0019 \quad 1.0018$ $1.0021 \quad 1.0020$ $\begin{array}{ll}1.0023 & 1.0022 \\ 1.0025 & 1.0022\end{array}$ $\begin{array}{ll}1.0025 & 1.002 \\ 1.0027 & 1.002\end{array}$ $1.0029 \quad 1.002$ ? $\begin{array}{ll}1.0031 & 1.0029\end{array}$ $1.0032 \quad 1.0039$ 1.0034 $1.0034 \quad 1.0032$ $1.0036 \quad 1.0033$ $\begin{array}{ll}1.0036 & 1.0033 \\ 1.0036 & 1.0034\end{array}$ $1.0037 \quad 1.0334$ $\begin{array}{ll}1.0037 & 1.0334 \\ 1.0037 & 1.0035\end{array}$ $1.0037 \quad 1.0035$ $1.0037 \quad 1.0035$ $\begin{array}{ll}1.0037 & 1.0235\end{array}$ $\begin{array}{ll}1.0037 & 1.0035 \\ 1.0037 & 1.0035\end{array}$ $\begin{array}{ll}1.0037 & 1.0035\end{array}$ $\begin{array}{ll}1.0036 & 1.0035 \\ 1.0036 & 1.0335\end{array}$ $1.0036 \quad 1.0035$ $1.0035 \quad 1.0035$ $1.0035 \quad 1.0035$ $1.0034 \quad 1.0035$ 1.0034 $1.0034 \quad 1.0035$ 
BATIO OF MOTT TO MCKINLEY-FESHBACH SCATTERTNG IN $0, z=8$

\begin{tabular}{|c|c|c|c|c|c|c|c|c|c|c|c|}
\hline $\begin{array}{l}\text { ENERGY (HEV) } \\
\text { ANGLE (DEG.) }\end{array}$ & 100 & 10. & 4. & 2. & 1.5 & 1.0 & 0.8 & 0.6 & 0.4 & 0.2 & 3.1 \\
\hline 0 & 1.0000 & 1.0000 & 1.0000 & 1.0000 & 1.0000 & 1.0000 & 1.0000 & 1.0000 & 1.0000 & 1.0000 & 1.0000 \\
\hline 5 & 0.9998 & 0.9998 & 0.9998 & 0.9999 & 0.9999 & 0.9999 & 0.9999 & 0.9999 & 0.9990 & 0.9999 & 0.9900 \\
\hline 10 & 1.0007 & 1.0007 & 1.0007 & 1.0007 & 1.0007 & 1.0007 & 1.0007 & 1.0006 & 1.0006 & 1.0006 & 1.0005 \\
\hline 15 & 1.0009 & 1.0009 & 1.0009 & 1.0009 & 1.0009 & 1.0009 & 1.0009 & 1.0009 & 1.0009 & 1.0009 & 1.0008 \\
\hline 20 & 1.0016 & 1.0016 & 1.0016 & 1.0016 & 1.0016 & 1.0016 & 1.0016 & 1.0015 & 1.0015 & 1.0014 & 1.0013 \\
\hline 25 & 1.0021 & 1.0021 & 1.0021 & 1.0021 & 1.0020 & 1.0020 & 1.0020 & 1.0020 & 1.0019 & 1.0019 & 1.0017 \\
\hline 30 & 1.0027 & 1.0027 & 1.0027 & 1.0027 & 1.0027 & 1.0026 & 1.0026 & 1.0026 & 1.0025 & 1.0024 & 1.0023 \\
\hline 35 & 1.0032 & 1.0032 & 1.0032 & 1.0032 & 1.0032 & 1.0031 & 1.0031 & 1.0031 & 1.0030 & 1.0028 & $1.032^{7}$ \\
\hline 40 & 1.0038 & 1.0038 & 1.0038 & 1.0038 & 1.0037 & 1.0037 & 1.0037 & 1.0036 & 1.0035 & 1.0033 & 1.0031 \\
\hline 45 & 1.0043 & 1.0043 & 1.0043 & 1.0043 & 1.0042 & 1.0042 & 1.0041 & 1.0041 & 1.0039 & 1.0037 & 1.0035 \\
\hline 50 & 1.0048 & 1.0048 & 1.0048 & 1.0048 & 1.0048 & 1.0047 & 1.0046 & 1.0045 & 1.0044 & 1.0061 & 1.0039 \\
\hline 55 & 1.0053 & 1.0053 & 1.0053 & 1.0053 & 1.0052 & 1.0051 & 1.0051 & 1.0050 & 1.0048 & 1.0045 & 1.0543 \\
\hline 60 & 1.0058 & 1.0058 & 1.0058 & 1.0057 & 1.0057 & 1.0056 & 1.0055 & 1.0054 & 1.0052 & 1.0049 & 1.0046 \\
\hline 65 & 1.0063 & 1.0063 & 1.0063 & 1.0062 & 1.0061 & 1.0060 & 1.0059 & 1.0058 & 1.0056 & 1.0052 & 1.0040 \\
\hline 70 & 1.0067 & 1.0067 & 1.0067 & 1.0066 & 1.0066 & 1.0064 & 1.0063 & 1.0062 & 1.0059 & 1.0055 & 1.0051 \\
\hline 75 & 1.0072 & 1.0072 & 1.0071 & 1.0070 & 1.0070 & 1.0068 & 1.0067 & 1.0065 & 1.0062 & 1.0057 & 1.0050 \\
\hline 80 & 1.0076 & 1.0076 & 1.0075 & 1.0074 & 1.0073 & 1.0071 & 1.0070 & 1.0068 & 1.0065 & 1.0059 & 1.0056 \\
\hline 85 & 1.0080 & 1.0080 & 1.0079 & 1.0078 & 1.0077 & 1.0074 & 1.0073 & 1.0071 & 1.0067 & 1.0061 & 1.0057 \\
\hline 90 & 1.0083 & 1.0083 & 1.0082 & 1.0081 & 1.0080 & 1.0077 & 1.0075 & 1.0073 & 1.0069 & 1.0063 & 1.0059 \\
\hline 95 & 1.0087 & 1.0087 & 1.0086 & 1.0084 & 1.0083 & 1.0080 & 1.0078 & 1.0075 & 1.0071 & 1.0064 & $1.006 \mathrm{C}$ \\
\hline 100 & 1.0090 & 1.0090 & 1.0089 & 1.0087 & 1.0085 & 1.0082 & 1.0080 & 1.0077 & 1.0072 & $1.0 \cap 65$ & 1.0061 \\
\hline 105 & 1.0093 & 1.0093 & 1.0092 & 1.0089 & 1.0088 & 1.0080 & 1.0081 & 1.0078 & 1.0073 & 1.0065 & 1.0062 \\
\hline 110 & 1.0096 & 1.0095 & 1.0094 & 1.0091 & 1.0089 & 1.0085 & 1.0082 & 1.0079 & 1.0074 & 1.0067 & 1.0063 \\
\hline 115 & 1.0099 & 1.0098 & 1.0097 & 1.0093 & 1.0091 & 1.0086 & 1.0083 & 1.0079 & 1.0074 & 1.0067 & 1.9563 \\
\hline 120 & 1.0101 & 1.0100 & 1.0099 & 1.0095 & 1.0092 & 1.0087 & 1.0083 & 1.0079 & 1.0074 & 1.0067 & 1.0064 \\
\hline 125 & 1.0103 & 1.0103 & 1.0101 & 1.0096 & 1.0093 & 1.0087 & 1.0084 & 1.0079 & 1.0074 & 1.0067 & $1.005 \mathrm{t}$ \\
\hline 130 & 1.0105 & 1.0104 & 1.0102 & 1.0096 & 1.0093 & 1.0086 & 1.0083 & 1.0078 & 1.0073 & 1.0067 & 1.0064 \\
\hline 135 & 1.0107 & 1.0106 & 1.0103 & 1.0097 & 1.0093 & 1.0086 & 1.0082 & 1.0077 & 1.0072 & 1.0066 & 1.0064 \\
\hline 140 & 1.0108 & 1.0107 & 1.0104 & 1.0096 & 1.0091 & 1.0084 & 1.0080 & 1.0075 & 1.0070 & 1.0066 & 1.0064 \\
\hline 145 & 1.0110 & 1.0109 & 1.0104 & 1.0095 & 1.0089 & 1.0082 & 1.0078 & 1.0074 & 1.0069 & 1.0065 & 1.0065 \\
\hline 150 & 1.0111 & 1.0109 & 1.0103 & 1.0092 & 1.0086 & 1.0078 & 1.0075 & 1.0071 & 1.0068 & 1.0065 & 1. 0060 \\
\hline 155 & 1.0112 & 1.0110 & 1.0102 & 1.0088 & $1.00 B 2$ & $1.00^{75}$ & 1.0072 & 1.0069 & 1.0066 & 1.0064 & 1.0060 \\
\hline 160 & 1.0113 & 1.0110 & 1.0098 & 1.0083 & 1.0078 & 1.0071 & 1.0069 & 1.0067 & 1.0065 & 1.0064 & $1.006 \mathrm{u}$ \\
\hline 165 & 1.0112 & 1.0106 & 1.0091 & 1.0076 & 1.0072 & $1.006^{7}$ & 1.0066 & 1.0064 & 1.0063 & 1.0063 & 1.0060 \\
\hline 170 & 1.0120 & 1.0107 & 1.0084 & 1.0070 & 1.0067 & 1.0065 & 1.0064 & 1.0063 & 1.0063 & 1.0063 & 1.0064 \\
\hline 175 & 1.0065 & 1.0063 & 1.0061 & 1.0061 & 1.0061 & 1.0061 & 1.0061 & 1.0051 & 1.0062 & 1.0063 & 1.0064 \\
\hline 180 & 1.0061 & 1.0061 & 1.0061 & 1.0061 & 1.0061 & 1.0061 & 1.0061 & 1.0061 & 1.0062 & 1.0063 & 1.0064 \\
\hline
\end{tabular}

RATTO OF MOTT TC MCKINIFY-FESHBACH SCATTERING IN MG, $z=12$

\begin{tabular}{|c|c|c|c|c|c|c|c|c|c|c|c|}
\hline $\begin{array}{l}\text { ENERGY (HEV) } \\
\text { ANGLE (DEG.I }\end{array}$ & 100. & 10. & 4. & 2. & 1.5 & 1.0 & 0.8 & 0.6 & 0.0 & 0.2 & 0.1 \\
\hline $\begin{array}{l}0 \\
5\end{array}$ & $\begin{array}{l}1.0000 \\
0.9998\end{array}$ & $\begin{array}{l}1.0000 \\
0.9998\end{array}$ & $\begin{array}{l}1.0000 \\
0.9998\end{array}$ & $\begin{array}{l}1.0000 \\
0.9998\end{array}$ & $\begin{array}{l}1.0000 \\
0.9998\end{array}$ & $\begin{array}{l}1.0000 \\
0.9998\end{array}$ & $\begin{array}{l}1.0000 \\
0.9998\end{array}$ & $\begin{array}{l}1.0000 \\
0.9998\end{array}$ & $\begin{array}{l}1.0000 \\
0.9998\end{array}$ & $\begin{array}{l}1.0000 \\
0.9998\end{array}$ & $\begin{array}{l}1.0000 \\
0.9998\end{array}$ \\
\hline 10 & 1.0013 & 1.0013 & 1.0013 & 1.0013 & 1.0013 & 1.0013 & 1.0013 & 1.0012 & 1.0012 & 1.0010 & $\begin{array}{l}0.9998 \\
1.0008\end{array}$ \\
\hline 15 & 1.0021 & 1.0021 & 1.0021 & 1.0020 & 1.0020 & 1.0020 & 1.0020 & 1.0020 & 1.0019 & 1.0018 & 1.0515 \\
\hline 20 & 1.0034 & 1.0034 & 1.0034 & 1.0034 & 1.0034 & 1.0034 & 1.0033 & 1.0033 & 1.0032 & 1.0030 & 1.0027 \\
\hline 25 & 1.0046 & 1.0046 & 1.0045 & 1.0045 & 1.0045 & 1.0044 & 1.0044 & 1.0043 & 1.0042 & $1.00<0$ & $1.003^{7}$ \\
\hline 30 & 1.0059 & 1.0059 & 1.0059 & 1.0059 & 1.0058 & 1.0058 & 1.0057 & 1.0056 & 1.0055 & 1.0052 & 1.0048 \\
\hline 35 & 1.0071 & 1.0071 & 1.0071 & 1.0071 & 1.0070 & 1.0069 & 1.0069 & 1.0068 & 1.0066 & 1.0062 & 1.0058 \\
\hline 40 & 1.0084 & 1.0084 & 1.0084 & 1.0083 & 1.0083 & 1.0082 & 1.0081 & 1.0080 & 1.0078 & 1.0073 & 1. 0068 \\
\hline 45 & 1.0096 & 1.0096 & 1.0096 & 1.0095 & 1.0095 & 1.0093 & 1.0092 & 1.0091 & 1.0088 & 1.0083 & 1.0078 \\
\hline 50 & 1.0108 & 1.0108 & 1.0108 & 1.0107 & 1.0106 & 1.0105 & 1.0104 & 1.0102 & 1.0099 & 1.0093 & 1.0087 \\
\hline 55 & 1.0120 & 1.0120 & 1.0119 & 1.0118 & 1.0117 & 1.0115 & 1.0114 & 1.0112 & 1.0108 & 1.0101 & 1.0095 \\
\hline 60 & 1.0131 & 1.0131 & 1.0130 & 1.0129 & 1.0128 & 1.0126 & 1.0124 & 1.0122 & 1.0117 & 1.0110 & 1.0103 \\
\hline 65 & 1.0142 & 1.0142 & 1.0141 & 1.0139 & 1.0138 & 1.0136 & 1.0134 & 1.0131 & 1.0126 & 1.0117 & 1.0110 \\
\hline 70 & 1.0152 & 1.0152 & 1.0151 & 1.0149 & 1.0148 & 1.0145 & 1.0142 & 1.0139 & 1.0134 & 1.0124 & 1.0116 \\
\hline 75 & 1.0162 & 1.0162 & 1.0161 & 1.0159 & 1.0157 & 1.0153 & 1.0151 & 1.0147 & 1.0141 & 1.0130 & 1.0122 \\
\hline 80 & 1.0171 & 1.0171 & 1.0170 & 1.0167 & 1.0165 & 1.0161 & 1.0158 & 1.0154 & 1.0147 & 1.0135 & 1.0127 \\
\hline 85 & 1.0180 & 1.0180 & 1.0179 & 1.0176 & 1.0173 & 1.0169 & 1.0165 & 1.0160 & 1.0153 & 1.0100 & 1.0131 \\
\hline 90 & 1.0188 & 1.0188 & 1.0186 & 1.0183 & 1.0180 & 1.0175 & 1.0171 & 1.0166 & 1.0157 & 1.0144 & 1. $013^{c}$ \\
\hline 95 & 1.0196 & 1.0196 & 1.0194 & 1.0190 & 1.0187 & 1.0181 & 1.0177 & 1.0171 & 1.0162 & 1.0148 & 1.0139 \\
\hline 100 & 1.0203 & 1.0203 & 1.0201 & 1.0196 & 1.0193 & 1.0186 & 1.0189 & 1.0175 & 1.0165 & 1.0150 & 1.0141 \\
\hline 105 & 1.0210 & 1.0210 & 1.0208 & 1.0203 & 1.0199 & 1.0191 & 1.0186 & 1.0178 & 1.0168 & 1.0153 & 1.3144 \\
\hline 110 & 1.0216 & 1.0216 & 1.0213 & 1.0207 & 1.0203 & 1.0194 & 1.0188 & 1.0180 & 1.0169 & 1.0154 & 1.0146 \\
\hline 115 & 1.0223 & 1.0222 & 1.0219 & 1.0212 & 1.0207 & 1.0197 & 1.0191 & 1.0182 & 1.0170 & 1.0155 & 1.0148 \\
\hline 120 & 1.0228 & 1.0227 & 1.0224 & 1.0215 & 1.0209 & 1.0198 & 1.0191 & 1.0182 & 1.0170 & 1.0156 & 1.0149 \\
\hline 125 & 1.0234 & 1.0233 & 1.0229 & 1.0219 & 1.0212 & 1.0199 & 1.0192 & 1.0182 & 1.0170 & 1.0156 & 1.0151 \\
\hline 130 & 1.0237 & 1.0236 & 1.0231 & 1.0219 & 1.0212 & 1.0198 & 1.0190 & 1.0180 & 1.0168 & 1.0156 & 1.0152 \\
\hline 135 & 1.0242 & 1.0241 & 1.0235 & 1.0221 & 1.0212 & 1.0197 & 1.0188 & 1.0178 & 1.0167 & 1.0156 & 1.0152 \\
\hline 140 & 1.0245 & 1.0243 & 1.0236 & 1.0219 & 1.0209 & 1.0193 & 1.0184 & 1.0175 & 1.0164 & 1.0155 & 1.0153 \\
\hline 145 & 1.0249 & 1.0247 & 1.0237 & 1.0217 & 1.0205 & 1.0188 & 1.0180 & 1.0171 & 1.0162 & 1.0154 & 1.0153 \\
\hline 150 & 1.0251 & 1.0248 & 1.0235 & 1.0211 & 1.0199 & 1.0182 & 1.0170 & 1.0166 & 1.0158 & 1.0153 & $1.015 u$ \\
\hline 155 & 1.0254 & 1.0250 & 1.0232 & 1.0204 & 1.0191 & 1.0175 & 1.0168 & 1.0161 & 1.0155 & 1.0152 & 1.0154 \\
\hline 160 & 1.0256 & 1.0249 & 1.0225 & 1.0192 & 1.0180 & 1.0166 & 1.0161 & 1.0156 & 1.0152 & 1.0151 & $1.015 \mathrm{~b}$ \\
\hline 165 & 1.0255 & 1.0243 & 1.0210 & 1.0177 & 1.0167 & 1.0157 & 1.0154 & 1.0151 & 1.0149 & 1.0150 & 1.3154 \\
\hline 170 & 1.0268 & 1.0241 & 1.0193 & 1.0163 & 1.0157 & 1.0151 & 1.0149 & 1.0148 & 1.0147 & 1.0140 & 1.0154 \\
\hline 175 & 1.0188 & 1.0164 & 1.0149 & 1.0145 & 1.0144 & 1.0144 & 1.0144 & 1.0140 & 1.0145 & 1.0149 & 1.0154 \\
\hline 180 & 1.0142 & 1.0142 & 1.0142 & 1.0143 & 1.0143 & 1.0143 & 1.0144 & 1.0144 & 1.0145 & 1.0149 & 1.0154 \\
\hline
\end{tabular}


RATIO OF MOTT TO MCKINLEY-FESHBACH SCATTERING IN AL, $z=13$

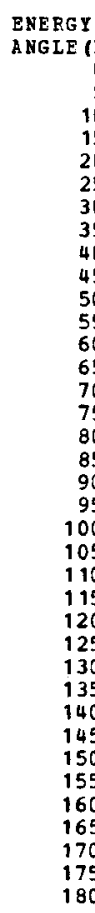

100
1.0000
0.9998
1.0015
1.0024
1.0040
1.0053
1.0069
1.0083
1.0099
1.0113
1.0127
1.0141
1.0154
1.0166
1.0178
1.0190
1.0201
1.0211
1.0221
1.0231
1.0239
1.0247
1.0254
1.0262
1.0268
1.0275
1.0279
1.0285
1.0288
1.0293
1.0296
1.0299
1.0302
1.0301
1.0314
1.0228
1.0169

$\begin{array}{cc}4 . & 2 . \\ 1.0000 & 1.0000 \\ 0.9998 & 0.9998 \\ 1.0015 & 1.0015 \\ 1.0024 & 1.0024 \\ 1.0040 & 1.0040 \\ 1.0053 & 1.0053 \\ 1.0069 & 1.0069 \\ 1.0083 & 1.0083 \\ 1.0098 & 1.0098 \\ 1.0112 & 1.0111 \\ 1.0127 & 1.0126 \\ 1.0140 & 1.0139 \\ 1.0153 & 1.0152 \\ 1.0166 & 1.0164 \\ 1.0177 & 1.0175 \\ 1.0189 & 1.0186 \\ 1.0199 & 1.0196 \\ 1.0210 & 1.0206 \\ 1.0219 & 1.0215 \\ 1.0228 & 1.0224 \\ 1.0236 & 1.0231 \\ 1.0244 & 1.0238 \\ 1.0251 & 1.0244 \\ 1.0258 & 1.0250 \\ 1.0263 & 1.0253 \\ 1.0269 & 1.0257 \\ 1.0272 & 1.0259 \\ 1.0276 & 1.0260 \\ 1.0277 & 1.0258 \\ 1.0279 & 1.0256 \\ 1.0279 & 1.0249 \\ 1.0273 & 1.0240 \\ 1.0265 & 1.0227 \\ 1.0248 & 1.0210 \\ 1.0227 & 1.0193 \\ 1.0178 & 1.0172 \\ 1.0169 & 1.0169\end{array}$

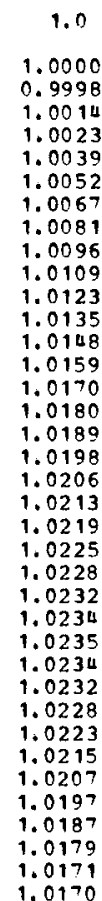

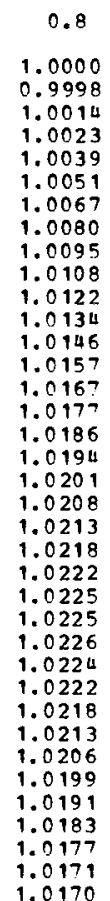

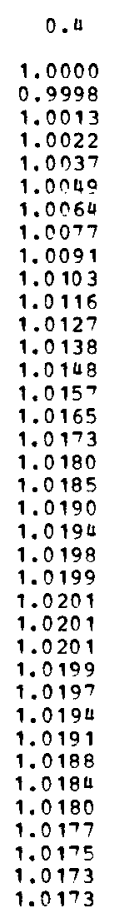

0.2

1.0000 0.9998 1.0011 1.0020 1.0034
1.0046 1.0046 1.0060 1.0072 1.0085
1.0097 1.0108 1.0108
1.0119 1.0119
1.0129 1.0129
1.0137 1.0137 1.0153 1.0159 1.0165 1.0170 1.0174 1.0179 1.0180 1.0182 1.0184
1.0184 1.0185 1.0185 1.0184 1.0183 1.0183 1.0181
1.0180 1.0179 1.0178 1.0178 1.0177 1.0177

0.1
1.0000
0.9999
1.0000
1.0017
1.0030
1.0042
1.0055
1.0069
1.0080
1.0091
1.0101
1.0111
1.0120
1.0129
1.0136
1.0143
1.0140
1.0154
1.0159
1.0163
1.0167
1.0170
1.0173
1.0175
1.0177
1.0179
1.0180
1.0181
1.0182
1.0182
1.0183
1.0183
1.0183
1.0183
1.0184
1.2183
1.0183

1.0183
RATTO OF MOTT TO MCKINLEY-FESHBACH SCATTERING IN SI, $z=14$

ENERGY (MEV)
ANG LE (DEG.)
0
5
10
15
20
25
30
35
40
45
50
55
60
65
70
75
80
85
90
95
100
105
110
115
120
125
130
135
140
145
150
155
160
165
170
175
180

\begin{tabular}{|c|c|}
\hline 4. & 2. \\
\hline $\begin{array}{l}1.0000 \\
0.9998 \\
1.0017 \\
1.0027 \\
1.0046 \\
1.0061 \\
1.0080 \\
1.0096 \\
1.0114 \\
1.0130 \\
1.0147 \\
1.0162 \\
1.0178 \\
1.0192 \\
1.0206 \\
1.0219 \\
1.0232 \\
1.0244 \\
1.0254 \\
1.0265 \\
1.0275 \\
1.0284 \\
1.0292 \\
1.0300 \\
1.0306 \\
1.0313 \\
1.0317 \\
1.0321 \\
1.0323 \\
1.0325 \\
1.0322 \\
1.0319 \\
1.0309 \\
1.0289 \\
1.0265 \\
1.0209 \\
1.0198\end{array}$ & $\begin{array}{l}1.0000 \\
0.9998 \\
1.0017 \\
1.0027 \\
1.0045 \\
1.0061 \\
1.0079 \\
1.0095 \\
1.0113 \\
1.0129 \\
1.0145 \\
1.0161 \\
1.0176 \\
1.0190 \\
1.0203 \\
1.0216 \\
1.0228 \\
1.0240 \\
1.0250 \\
1.0260 \\
1.0269 \\
1.0277 \\
1.0284 \\
1.0290 \\
1.0295 \\
1.0299 \\
1.0301 \\
1.0303 \\
1.0301 \\
1.0298 \\
1.0291 \\
1.0281 \\
1.0266 \\
1.0245 \\
1.0226 \\
1.0202 \\
1.0198\end{array}$ \\
\hline
\end{tabular}

1.5
1.0000
0.9998
1.0016
1.0027
1.0045
1.0060
1.0079
1.0095
1.0112
1.0128
1.0144
1.0160
1.0174
1.0188
1.0201
1.0210
1.0226
1.0237
1.0246
1.0256
1.0264
1.0272
1.0277
1.0283
1.0287
1.0290
1.0290
1.0291
1.0287
1.0283
1.0274
1.0263
1.0249
1.0232
1.0217
1.0201
1.0199

1.0
1.0000
0.9998
1.0016
1.0027
1.0045
1.0060
1.0078
1.0094
1.0111
1.0126
1.0142
1.0157
1.0171
1.0185
1.0197
1.0209
1.0220
1.0230
1.0239
1.0248
1.0255
1.0261
1.0266
1.0270
1.0272
1.0270
1.0272
1.0271
1.0266
1.0260
1.0251
1.0242
1.0230
1.0219
1.0210
1.0201
1.0199

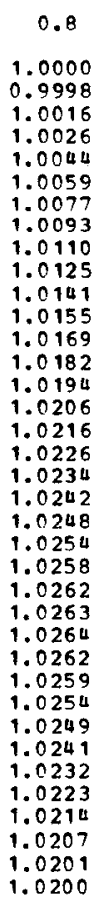

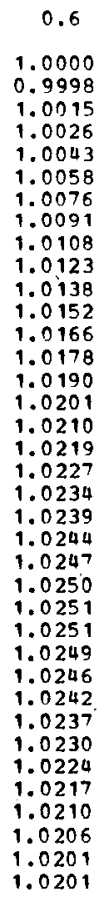

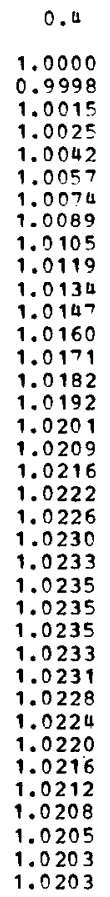

$\begin{array}{cc}0.2 & 1.1 \\ 1.0000 & 1.0000 \\ 0.9997 & 0.9997 \\ 1.0013 & 1.0009 \\ 1.0023 & 1.0019 \\ 1.0039 & 1.0034 \\ 1.0053 & 1.0048 \\ 1.0069 & 1.0063 \\ 1.0083 & 1.0077 \\ 1.0098 & 1.0091 \\ 1.0112 & 1.0104 \\ 1.0125 & 1.0117 \\ 1.0138 & 1.0128 \\ 1.0149 & 1.0139 \\ 1.0159 & 1.0149 \\ 1.0169 & 1.0158 \\ 1.0178 & 1.0166 \\ 1.0185 & 1.0173 \\ 1.0192 & 1.0180 \\ 1.0198 & 1.0185 \\ 1.0203 & 1.0191 \\ 1.0207 & 1.0195 \\ 1.0210 & 1.0199 \\ 1.0213 & 1.0202 \\ 1.0215 & 1.0205 \\ 1.0216 & 1.0207 \\ 1.0216 & 1.0209 \\ 1.0216 & 1.0211 \\ 1.0216 & 1.0212 \\ 1.0215 & 1.0213 \\ 1.0214 & 1.0214 \\ 1.0213 & 1.0215 \\ 1.0212 & 1.0215 \\ 1.0210 & 1.0216 \\ 1.0209 & 1.0216 \\ 1.0209 & 1.0216 \\ 1.0208 & 1.0216 \\ 1.0208 & 1.0216 \\ & \\ 1.029 & \end{array}$


RATIO OF MOTT TO MCKINLEY-FESHBACH SCATTERING TN $K, z=19$

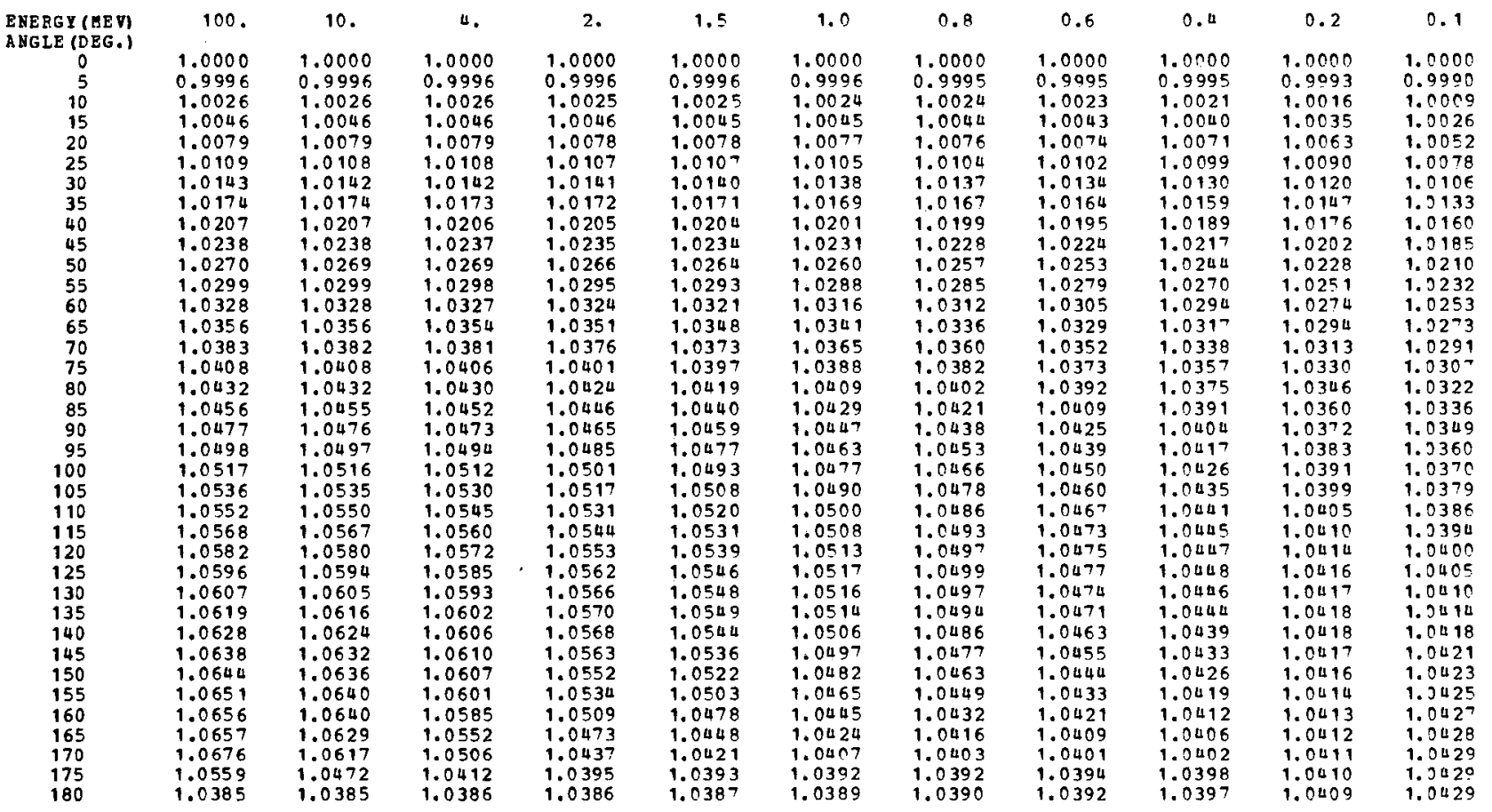

RATIO OF MOTT TO MCKINIFY-FESHBACH SCATTERING IN TI, $\mathrm{z}=22$
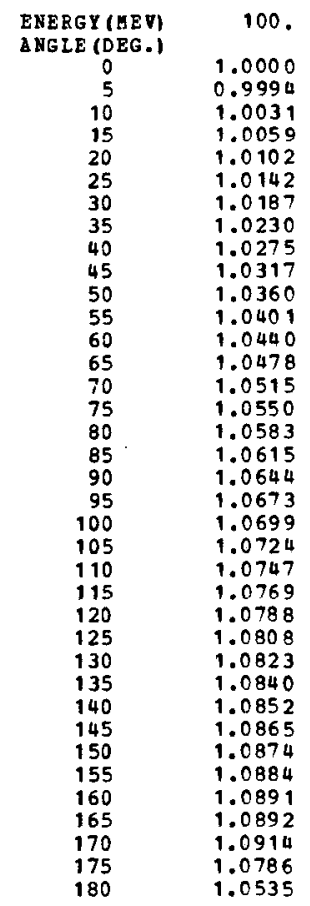

10
1.0000
0.9994
1.0031
1.0059
1.0102
1.0142
1.0187
1.0230
1.0275
1.0317
1.0360
1.0600
1.0440
1.0478
1.0514
1.0549
1.0582
1.0614
1.0643
1.0672
1.0698
1.0723
1.0745
1.0767
1.0786
1.0805
1.0820
1.0835
1.0846
1.0858
1.0864
1.0870
1.0870
1.0856
1.0839
1.0662
1.0535

$\begin{array}{cc}4 & 2 . \\ 1.0000 & 1.0000 \\ 0.9994 & 0.9993 \\ 1.0031 & 1.0030 \\ 1.0058 & 1.0058 \\ 1.0102 & 1.0101 \\ 1.0141 & 1.0140 \\ 1.0187 & 1.0185 \\ 1.0229 & 1.0227 \\ 1.0274 & 1.0272 \\ 1.0316 & 1.0314 \\ 1.0359 & 1.0356 \\ 1.0399 & 1.0395 \\ 1.0438 & 1.0434 \\ 1.0476 & 1.0471 \\ 1.0512 & 1.0506 \\ 1.0547 & 1.0540 \\ 1.0579 & 1.0571 \\ 1.0610 & 1.0601 \\ 1.0639 & 1.0629 \\ 1.0667 & 1.0655 \\ 1.0692 & 1.0678 \\ 1.0717 & 1.0701 \\ 1.0738 & 1.0719 \\ 1.0759 & 1.0737 \\ 1.0776 & 1.0751 \\ 1.0793 & 1.0763 \\ 1.0805 & 1.0770 \\ 1.0817 & 1.0776 \\ 1.0824 & 1.0774 \\ 1.0829 & 1.0769 \\ 1.0827 & 1.0754 \\ 1.0819 & 1.0732 \\ 1.0798 & 1.0699 \\ 1.0756 & 1.0652 \\ 1.0695 & 1.0604 \\ 1.0574 & 1.0550 \\ 1.0535 & 1.0536 \\ & \\ & \end{array}$

1.5
1.0000
0.9993
1.0030
1.0057
1.0100
1.0139
1.0184
1.0226
1.0270
1.0311
1.0353
1.0392
1.0431
1.0467
1.0502
1.0534
1.0565
1.0594
1.0621
1.0646
1.0668
1.0689
1.0706
1.0721
1.0733
1.0742
1.0746
1.0748
1.0743
1.0734
1.0716
1.0692
1.0659
1.0619
1.0582
1.0546
1.0537

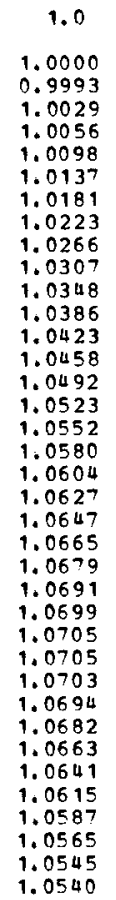

0.8
1.0000
0.9993
1.0028
1.0055
1.0097
1.0135
1.0179
1.0220
1.0263
1.0303
1.0344
1.0381
1.0418
1.0452
1.0485
1.0515
1.0543
1.0569
1.0592
1.0614
1.0632
1.0649
1.0661
1.0672
1.0678
1.0682
1.0680
1.0677
1.0668
1.0656
1.0639
1.0619
1.0598
1.0577
1.0560
1.0545
1.0542

$\begin{array}{cc}0.6 & 0.4 \\ 1.0000 & 1.0000 \\ 0.9992 & 0.9991 \\ 1.0026 & 1.0024 \\ 1.0053 & 1.0050 \\ 1.0094 & 1.0090 \\ 1.0132 & 1.0127 \\ 1.0176 & 1.0169 \\ 1.0216 & 1.0209 \\ 1.0258 & 1.0250 \\ 1.0298 & 1.0288 \\ 1.0337 & 1.0326 \\ 1.0374 & 1.0361 \\ 1.0409 & 1.0395 \\ 1.0442 & 1.0426 \\ 1.0473 & 1.0455 \\ 1.0502 & 1.0482 \\ 1.0529 & 1.0506 \\ 1.0554 & 1.0529 \\ 1.0575 & 1.0548 \\ 1.0595 & 1.0566 \\ 1.0611 & 1.0580 \\ 1.0626 & 1.0592 \\ 1.0636 & 1.0601 \\ 1.0645 & 1.0609 \\ 1.0649 & 1.0612 \\ 1.0652 & 1.0615 \\ 1.0650 & 1.0513 \\ 1.0646 & 1.0511 \\ 1.0637 & 1.0605 \\ 1.0627 & 1.0598 \\ 1.0613 & 1.0590 \\ 1.0598 & 1.0581 \\ 1.0583 & 1.0572 \\ 1.0568 & 1.0564 \\ 1.0557 & 1.0558 \\ 1.0548 & 1.0554 \\ 1.0545 & 1.0552\end{array}$

$\begin{array}{lc}0.2 & 1.1 \\ 1.0000 & 1.0000 \\ 0.9988 & 0.9984 \\ 1.0016 & 1.0005 \\ 1.0040 & 1.0026 \\ 1.0078 & 1.0061 \\ 1.0114 & 1.0095 \\ 1.0154 & 1.0133 \\ 1.0192 & 1.0160 \\ 1.0231 & 1.0206 \\ 1.0267 & 1.0241 \\ 1.0302 & 1.0275 \\ 1.0335 & 1.0306 \\ 1.0366 & 1.0336 \\ 1.0394 & 1.0364 \\ 1.0421 & 1.0389 \\ 1.0445 & 1.0413 \\ 1.0467 & 1.0434 \\ 1.0487 & 1.0454 \\ 1.0504 & 1.0472 \\ 1.0520 & 1.0489 \\ 1.0533 & 1.0504 \\ 1.0545 & 1.0517 \\ 1.0554 & 1.0520 \\ 1.0562 & 1.0540 \\ 1.0568 & 1.0550 \\ 1.0573 & 1.0559 \\ 1.0575 & 1.0567 \\ 1.0578 & 1.0574 \\ 1.0578 & 1.0580 \\ 1.0578 & 1.0585 \\ 1.0577 & 1.0590 \\ 1.0576 & 1.0594 \\ 1.0575 & 1.0597 \\ 1.0574 & 1.0599 \\ 1.0573 & 1.0501 \\ 1.0572 & 1.0602 \\ 1.0571 & 1.0602 \\ & \\ 1.057 & \end{array}$


RATIO OF MOTT TO MCKINIEY-FESHBQCH SCATTERING IN $v, z=23$

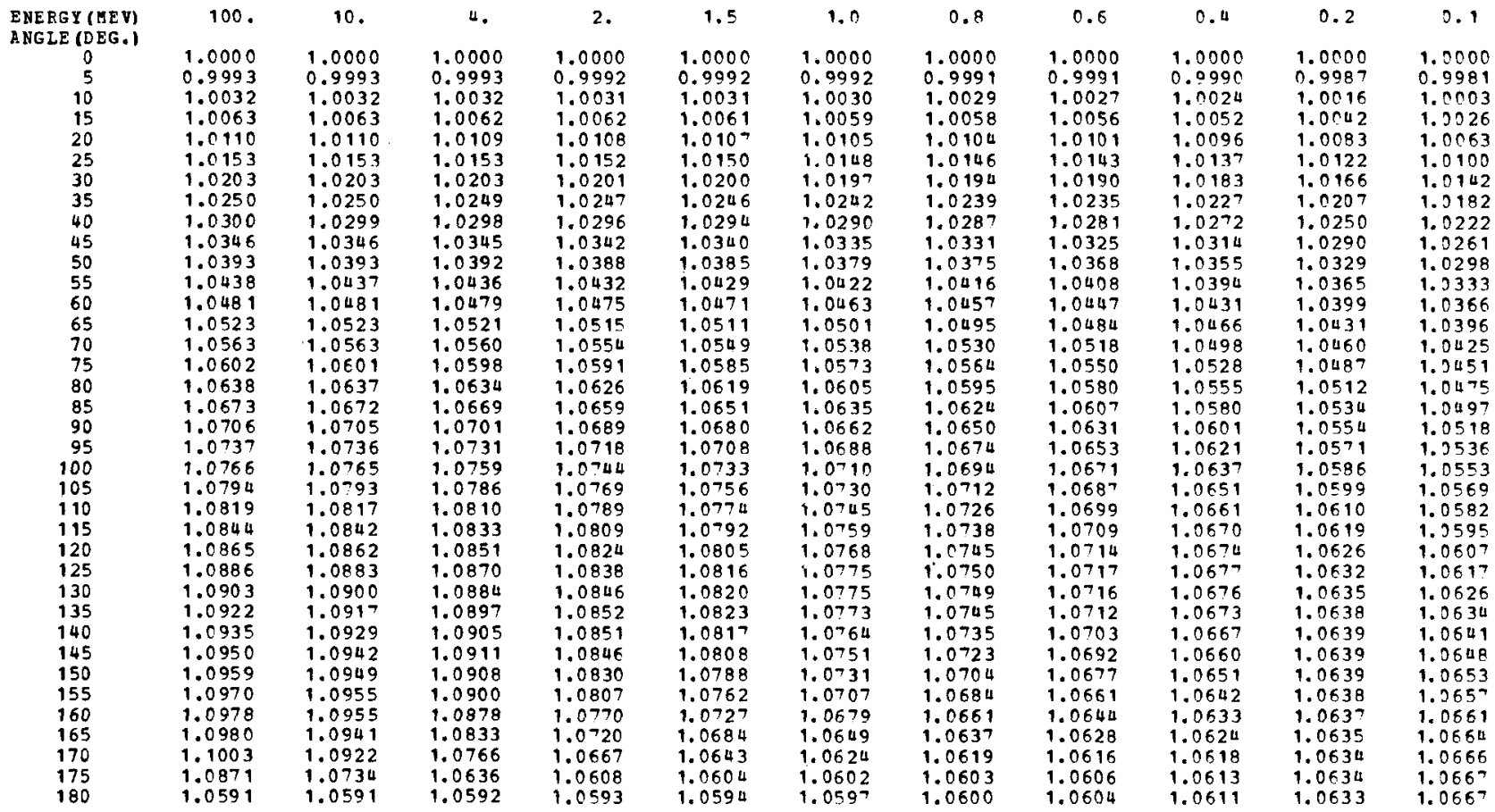

RATIO OF MOT! TO MCRINIEY-FFSHBACH SCATTERING IN CR, $Z=24$

ENERGY (HEV)
ANGLE (DEG.)
0
5
10
15
20
25
30
35
40
45
50
55
60
65
70
75
80
85
90
95
100
105
110
115
120
125
130
135
140
145
150
155
160
165
170
175
180

$\begin{array}{cccc}100 . & 10 . & 4 & 2 . \\ 1.0000 & 1.0000 & 1.0000 & 1.0000 \\ 0.9991 & 0.9991 & 0.9991 & 0.9991 \\ 1.0034 & 1.0034 & 1.0033 & 1.0033 \\ 1.0067 & 1.0067 & 1.0066 & 1.0065 \\ 1.0118 & 1.0118 & 1.0117 & 1.0116 \\ 1.0165 & 1.0165 & 1.0165 & 1.0163 \\ 1.0220 & 1.0220 & 1.0219 & 1.0217 \\ 1.0271 & 1.0271 & 1.0270 & 1.0268 \\ 1.0325 & 1.0325 & 1.0324 & 1.0321 \\ 1.0376 & 1.0376 & 1.0375 & 1.0372 \\ 1.0427 & 1.0427 & 1.0426 & 1.0422 \\ 1.0476 & 1.0476 & 1.0474 & 1.0470 \\ 1.0524 & 1.0524 & 1.0522 & 1.0517 \\ 1.0570 & 1.0569 & 1.0567 & 1.0561 \\ 1.0614 & 1.0613 & 1.0611 & 1.0604 \\ 1.0656 & 1.0656 & 1.0653 & 1.0645 \\ 1.0696 & 1.0695 & 1.0692 & 1.0683 \\ 1.0735 & 1.0734 & 1.0730 & 1.0719 \\ 1.0771 & 1.0769 & 1.0765 & 1.0753 \\ 1.0805 & 1.0804 & 1.0799 & 1.0785 \\ 1.0837 & 1.0835 & 1.0829 & 1.0813 \\ 1.0868 & 1.0866 & 1.0859 & 1.0840 \\ 1.0895 & 1.0893 & 1.0885 & 1.0863 \\ 1.0922 & 1.0920 & 1.0910 & 1.0885 \\ 1.0945 & 1.0943 & 1.0931 & 1.0902 \\ 1.0969 & 1.0966 & 1.0952 & 1.0917 \\ 1.0988 & 1.0984 & 1.0967 & 1.0926 \\ 1.0008 & 1.0903 & 1.0982 & 1.0933 \\ 1.1023 & 1.1016 & 1.0990 & 1.0932 \\ 1.1039 & 1.1030 & 1.0997 & 1.0927 \\ 1.0050 & 1.1038 & 1.0995 & 1.0910 \\ 1.1069 & 1.1045 & 1.0996 & 1.0885 \\ 1.1070 & 1.1045 & 1.0962 & 1.0846 \\ 1.1072 & 1.1031 & 1.0914 & 1.0791 \\ 1.1096 & 1.1009 & 1.0841 & 1.0734 \\ 1.0962 & 1.0810 & 1.0701 & 1.0670 \\ 1.0651 & 1.0651 & 1.0652 & 1.0654\end{array}$

$\begin{array}{ccc}1.5 & 1.0 & 0.8 \\ 1.0000 & 1.0000 & 1.0000 \\ 0.9991 & 0.9990 & 0.9990 \\ 1.0032 & 1.0031 & 1.0030 \\ 1.0065 & 1.0063 & 1.0062 \\ 1.0115 & 1.0113 & 1.0111 \\ 1.0162 & 1.0159 & 1.0157 \\ 1.0216 & 1.0212 & 1.0210 \\ 1.0266 & 1.0262 & 1.0259 \\ 1.0319 & 1.0314 & 1.0311 \\ 1.0369 & 1.0363 & 1.0359 \\ 1.0419 & 1.0412 & 1.0408 \\ 1.0466 & 1.0459 & 1.0453 \\ 1.0513 & 1.0504 & 1.0497 \\ 1.0557 & 1.0546 & 1.0539 \\ 1.0598 & 1.0587 & 1.0578 \\ 1.0638 & 1.0625 & 1.0615 \\ 1.0675 & 1.0560 & 1.0649 \\ 1.0711 & 1.0694 & 1.0681 \\ 1.0743 & 1.0724 & 1.0710 \\ 1.0774 & 1.0752 & 1.0736 \\ 1.0801 & 1.0776 & 1.0759 \\ 1.0826 & 1.0798 & 1.0779 \\ 1.0847 & 1.0816 & 1.0795 \\ 1.0866 & 1.0831 & 1.0808 \\ 1.0880 & 1.0841 & 1.0816 \\ 1.0893 & 1.0849 & 1.0822 \\ 1.0898 & 1.0850 & 1.0821 \\ 1.0901 & 1.0848 & 1.0817 \\ 1.0896 & 1.0838 & 1.0807 \\ 1.0886 & 1.0825 & 1.0794 \\ 1.0865 & 1.0803 & 1.0774 \\ 1.0839 & 1.0778 & 1.0752 \\ 1.0799 & 1.0747 & 1.0727 \\ 1.0752 & 1.0715 & 1.0702 \\ 1.0708 & 1.0687 & 1.0682 \\ 1.0666 & 1.0664 & 1.0665 \\ 1.0655 & 1.0658 & 1.0661\end{array}$

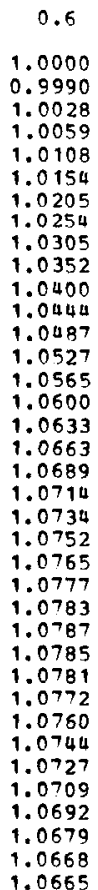

0.4
1.0000
0.9988
1.0024
1.0055
1.0102
1.0147
1.0197
1.0245
1.0294
1.0340
1.0386
1.0428
1.0469
1.0507
1.0543
1.0576
1.0606
1.0633
1.0657
1.0679
1.0697
1.0713
1.0724
1.0734
1.0739
1.0743
1.0742
1.0740
1.0734
1.0726
1.0717
1.0707
1.0697
1.0688
1.0681
1.0676
1.0674

0.2 0.1 1.0000 0.9994 1.0015
1.0043 1.0087 1.0130 1.0130 1.0223 1.0270 1.0314 1.031 1.0356 1.0396 1.0430 1.0469 1.0502
1.0531 1. 0559 1.0583 1. 0605 1.0625 1. 0642 $1.065^{7}$ 1.0669 1.0679 1.0694 1.0698 1.0702 1.0702 1.0703 1.0704 1.070 1.0702 1.0702
1.0701 1.0700 1.0699 1.0699
1.0699

1.0000 0.9978 1.0001
1.0024 1.0055 1.0065 1. 3105 1. 0150 1. 0239 1. 0322 1.0322
1.0360 1.0397 1.039 ? 1. 0462 1.0491 1. 0518 1.0543 1.0555 1.0586 1.0605 1.0623 1. 0639 1.0653 1.0566
1.0578 1.0578
1.0588 1.0588 1.0698
1.0706 1. 79 1. 3714 1.0720 1.0725 1.0730
1.2733 1.2733 1. $0^{7} 35$ 1.0737
1.0737 
RATIO OF MOTT TO MCKTNIEY-FESHBACH SCATTERTNG IN PE, $z=25$

\begin{tabular}{|c|c|c|c|c|c|c|c|c|c|c|c|}
\hline $\begin{array}{l}\text { ENERG Y (MEV) } \\
\text { ANGLE (DEG.) }\end{array}$ & 100 & 10. & 4. & 2. & 1.5 & 1.0 & 0.8 & 0.6 & 0.4 & 0.2 & 0.1 \\
\hline $\begin{array}{l}0 \\
5\end{array}$ & 1.0000 & 1.0000 & 1.0000 & 1.0000 & 1.0000 & 1.0000 & 1.0000 & 1.0000 & 1.0000 & 1.0000 & 1.0000 \\
\hline 5 & 0.9989 & 0.9989 & 0.9989 & 0.9988 & 0.9988 & 0.9987 & 0.9987 & 0.9986 & 0.9984 & 0.9979 & \\
\hline 10 & 1.0036 & 1.0036 & 1.0035 & 1.0035 & 1.0034 & 1.0032 & 1.0031 & 1.0029 & 1.0024 & 1.0013 & 0.9995 \\
\hline 15 & 1.0075 & 1.0074 & 1.0074 & 1.0073 & 1.0072 & 1.0070 & 1.0068 & 1.0065 & 1.0060 & 1.0045 & 1.3021 \\
\hline 20 & 1.0134 & 1.0134 & 1.0133 & 1.0132 & 1.0131 & 1.0128 & 1.0125 & 1.0122 & 1.0114 & 1.0096 & 1.0067 \\
\hline 25 & 1.0190 & 1.0190 & 1.0189 & 1.0188 & 1.0186 & 1.0183 & 1.0180 & 1.0176 & 1.0167 & 1.0146 & 1.0113 \\
\hline 30 & 1.0254 & 1.0254 & 1.0253 & 1.0251 & 1.0249 & 1.0245 & 1.0242 & 1.0236 & 1.0226 & 1.0202 & $1.016^{7}$ \\
\hline 35 & 1.0315 & 1.0315 & 1.0314 & 1.0311 & 1.0309 & 1.0304 & 1.0300 & 1.0294 & 1.0283 & 1.0256 & 1.5219 \\
\hline 40 & 1.0379 & 1.0378 & 1.0377 & 1.0374 & 1.0371 & 1.0366 & 1.0361 & 1.0354 & 1.0341 & 1.0319 & 1.0271 \\
\hline 45 & 1.0439 & 1.0439 & 1.0438 & 1.0434 & 1.0431 & 1.0824 & 1.0419 & 1.0411 & 1.0396 & 1.0363 & 1.0322 \\
\hline 50 & 1.0500 & 1.0500 & 1.0498 & 1.0494 & 1.0490 & 1.0483 & 1.0477 & 1.0467 & 1.0451 & 1.0414 & 1.0371 \\
\hline 55 & 1.0558 & 1.0558 & 1.0556 & 1.0551 & 1.0547 & 1.0538 & 1.0531 & 1.0520 & 1.0502 & 1.0462 & 1.3417 \\
\hline 60 & 1.0615 & 1.0615 & 1.0613 & 1.0607 & 1.0602 & 1.0591 & 1.0584 & 1.0579 & 1.0550 & 1.0508 & 1.0461 \\
\hline $\begin{array}{l}65 \\
70\end{array}$ & $\begin{array}{l}1.0670 \\
1.0722\end{array}$ & $\begin{array}{l}1.0669 \\
1.0722\end{array}$ & & 1.0660 & 1.0654 & 1.0642 & 1.0634 & 1.0620 & 1.0596 & 1.0550 & $\begin{array}{l}1.0502 \\
1.0540\end{array}$ \\
\hline $\begin{array}{l}70 \\
75\end{array}$ & $\begin{array}{l}1.0722 \\
1.0773\end{array}$ & $\begin{array}{l}1.0722 \\
1.0772\end{array}$ & $\begin{array}{l}1.0719 \\
1.0769\end{array}$ & $\begin{array}{l}1.0711 \\
1.0759\end{array}$ & $\begin{array}{l}1.0704 \\
1.0752\end{array}$ & $\begin{array}{l}1.0691 \\
1.0736\end{array}$ & $\begin{array}{l}1.0681 \\
1.0725\end{array}$ & $\begin{array}{l}1.0665 \\
1.0708\end{array}$ & $\begin{array}{l}1.0639 \\
1.0679\end{array}$ & $\begin{array}{l}1.0589 \\
1.0625\end{array}$ & $\begin{array}{l}1.0540 \\
1.2576\end{array}$ \\
\hline 80 & 1.0821 & 1.0820 & 1.0816 & 1.0805 & 1.0797 & 1.0779 & 1.0766 & 1.0747 & 1.0715 & 1.0659 & 1.0609 \\
\hline 85 & 1.0867 & 1.0866 & 1.0861 & 1.0849 & 1.0839 & 1.0819 & 1.0804 & 1.0783 & 1.0748 & 1.0689 & 1.0639 \\
\hline 90 & 1.0909 & 1.0908 & 1.0903 & 1.0889 & 1.0878 & 1.0855 & 1.0839 & 1.0815 & 1.0778 & 1.0716 & $1.066^{7}$ \\
\hline 95 & 1.0951 & 1.0950 & 1.0943 & 1.0927 & 1.0914 & 1.0889 & 1.0871 & 1.0844 & 1.0804 & 1.0740 & 1.3693 \\
\hline 100 & 1.0989 & 1.0987 & 1.0980 & 1.0961 & 1.0947 & 1.0918 & 1.0898 & 1.0869 & 1.0826 & 1.0761 & 1.0717 \\
\hline 105 & 1.1026 & 1.1024 & 1.1016 & 1.0994 & 1.0977 & 1.0945 & 1.0923 & 1.0892 & 1.0846 & 1.0780 & 1.3739 \\
\hline 110 & 1.1059 & 1.1056 & 1.1047 & 1. 1022 & 1.1003 & 1.0967 & 1.0942 & 1.0909 & 1.0861 & 1.0796 & 1.0759 \\
\hline 115 & 1.1091 & 1.1089 & 1.1077 & 1.1048 & 1.1027 & 1.0986 & 1.0959 & 1.0923 & 1.0873 & 1.0809 & 1.0778 \\
\hline 120 & 1.1119 & 1.1116 & 1.1102 & 1.1069 & 1.1044 & 1.0999 & 1.0970 & 1.0931 & 1.0881 & 1.0820 & 1.0795 \\
\hline 125 & $1.114 ?$ & 1.1143 & 1.1127 & 1.1087 & 1.1059 & 1.1008 & 1.0977 & 1.0937 & 1.0885 & 1.0829 & 1.0810 \\
\hline $\begin{array}{l}130 \\
135\end{array}$ & $\begin{array}{l}1.1170 \\
1.1193\end{array}$ & $\begin{array}{l}1.1165 \\
1.1188\end{array}$ & $\begin{array}{l}1.1146 \\
1.1164\end{array}$ & $\begin{array}{l}1.1099 \\
1.1108\end{array}$ & $\begin{array}{l}1.1067 \\
1.1071\end{array}$ & $\begin{array}{l}1.1011 \\
1.1009\end{array}$ & $\begin{array}{l}1.0978 \\
1.0974\end{array}$ & $\begin{array}{l}1.0936 \\
1.0932\end{array}$ & $\begin{array}{l}1.0886 \\
1.0884\end{array}$ & $\begin{array}{l}1.0835 \\
1.0840\end{array}$ & $\begin{array}{l}1.0824 \\
1.383^{7}\end{array}$ \\
\hline $\begin{array}{l}135 \\
140\end{array}$ & $\begin{array}{l}1.1193 \\
1.1212\end{array}$ & $\begin{array}{l}1.1188 \\
1.1204\end{array}$ & $\begin{array}{l}1.1164 \\
1.1174\end{array}$ & 1.1108 & $\begin{array}{l}1.10^{71} \\
1.1066\end{array}$ & $\begin{array}{l}1.1009 \\
1.0999\end{array}$ & $\begin{array}{l}1.0974 \\
1.0963\end{array}$ & $\begin{array}{l}1.0932 \\
1.0922\end{array}$ & 1.0878 & 1.0843 & 1.0848 \\
\hline 145 & 1.1230 & 1.1221 & 1.1183 & 1.1102 & 1.1055 & 1.0984 & 1.0948 & 1.0909 & 1.0870 & 1.0845 & 1.0858 \\
\hline 150 & 1.1244 & 1.1231 & 1.1181 & 1.1084 & 1.1032 & 1.0960 & 1.0926 & 1.0891 & 1.0860 & 1.0846 & 1.0866 \\
\hline 155 & 1.1258 & 1.1239 & 1.1171 & 1.1055 & 1.0999 & 1.0930 & 1.0901 & 1.0872 & 1.0849 & 1.0946 & $1.2 B>4$ \\
\hline $\begin{array}{l}160 \\
165\end{array}$ & $\begin{array}{r}1.1268 \\
1.1272\end{array}$ & 1. 1240 & 1. 1145 & 1.1010 & 1.0955 & 1.0895 & 1.0872 & 1.0851 & 1.0838 & 1.0845 & 1.0880 \\
\hline $\begin{array}{l}165 \\
170\end{array}$ & $\begin{array}{l}1.1272 \\
1.1297\end{array}$ & 1.1224 & 1.1089 & $\begin{array}{l}1.0947 \\
1.0879\end{array}$ & 1.0901 & 1.0858 & 1.0843 & 1.0832 & 1.0828 & 1.0844 & 1.0884 \\
\hline $\begin{array}{l}170 \\
175\end{array}$ & $\begin{array}{l}1.1297 \\
1.1159\end{array}$ & $\begin{array}{r}1.1198 \\
1.0977\end{array}$ & $\begin{array}{l}1.1004 \\
1.0844\end{array}$ & $\begin{array}{l}1.0879 \\
1.0806\end{array}$ & $\begin{array}{l}1.0850 \\
1.0801\end{array}$ & 1.0826 & 1. 0819 & 1.0815 & 1.0820 & 1.0844 & 1.0888 \\
\hline $\begin{array}{l}175 \\
180\end{array}$ & $\begin{array}{l}1.1159 \\
1.0783\end{array}$ & $\begin{array}{l}1.0977 \\
1.0783\end{array}$ & $\begin{array}{l}1.0844 \\
1.0784\end{array}$ & $\begin{array}{l}1.0806 \\
1.0786\end{array}$ & $\begin{array}{l}1.0801 \\
1.0788\end{array}$ & 1.0799 & 1.0800 & 1.0804 & 1.0814 & 1.0843 & 1.3890 \\
\hline 180 & 1.0783 & 1.0783 & 1.0784 & 1.0786 & 1.0788 & 1.0792 & 1.0795 & 1.0801 & 1.0812 & 1.0843 & 1.0890 \\
\hline
\end{tabular}

EATIO OF MOTT TO MCKINLEY-FESHBACH SCATTERING IN vT, $z=28$

BNERGY (MEV)
ANGLE (DEG.)
0
5
10
15
20
25
30
35
40
45
50
55
60
65
70
75
80
85
90
95
100
105
110
115
120
125
130
135
140
145
150
155
160
165
170
175
180

$\begin{array}{ccc}100 . & 10 & 1 \\ 1.0000 & 1.0000 & 1.0000 \\ 0.9985 & 0.9985 & 0.9985 \\ 1.0037 & 1.0037 & 1.0037 \\ 1.0082 & 1.0082 & 1.0081 \\ 1.0150 & 1.0150 & 1.0149 \\ 1.0216 & 1.0216 & 1.0215 \\ 1.0290 & 1.0290 & 1.0289 \\ 1.0361 & 1.0361 & 1.0360 \\ 1.0436 & 1.0436 & 1.0434 \\ 1.0507 & 1.0507 & 1.0505 \\ 1.0579 & 1.0578 & 1.0576 \\ 1.0647 & 1.0647 & 1.0644 \\ 1.0714 & 1.0714 & 1.0711 \\ 1.0778 & 1.0778 & 1.0775 \\ 1.0840 & 1.0840 & 1.0836 \\ 1.0900 & 1.0899 & 1.0895 \\ 1.0956 & 1.0955 & 1.0951 \\ 1.1011 & 1.1009 & 1.1004 \\ 1.1061 & 1.1060 & 1.1054 \\ 1.1110 & 1.1109 & 1.1102 \\ 1.1155 & 1.1153 & 1.1145 \\ 1.1199 & 1.1197 & 1.1187 \\ 1.1238 & 1.1236 & 1.1224 \\ 1.1276 & 1.1273 & 1.1260 \\ 1.1310 & 1.1306 & 1.1291 \\ 1.1343 & 1.1338 & 1.1320 \\ 1.1370 & 1.1365 & 1.1343 \\ 1.1398 & 1.1391 & 1.1364 \\ 1.1419 & 1.1411 & 1.1377 \\ 1.1442 & 1.1431 & 1.1388 \\ 1.1458 & 1.1444 & 1.1387 \\ 1.1474 & 1.1453 & 1.1376 \\ 1.1487 & 1.1455 & 1.1347 \\ 1.1491 & 1.1438 & 1.1284 \\ 1.1518 & 1.1406 & 1.1186 \\ 1.1378 & 1.1163 & 1.1004 \\ 1.0930 & 1.0930 & 1.0931 \\ & & \end{array}$

1.5
1.0000
0.9984
1.0035
1.0079
1.0146
1.0211
1.0284
1.0354
1.0427
1.0497
1.0567
1.0633
1.0698
1.0760
1.0819
1.0876
1.0928
1.0979
1.1025
1.1068
1.1107
1.1144
1.1174
1.1203
1.1224
1.1243
1.1253
1.1258
1.1254
1.1242
1.1216
1.1180
1.1130
1.1068
1.1008
1.0952
1.0936

1.1
1.0000
0.9984
1.0033
1.0076
1.0143
1.0207
1.0279
1.0348
1.0420
1.0489
1.0558
1.0623
1.0686
1.0746
1.0803
1.0858
1.0908
1.0956
1.0999
1.1039
1.1074
1.1107
1.1133
1.1156
1.1172
1.1185
1.1189
1.1188
1.1178
1.1161
1.1134
1.1101
1.1060
1.1018
1.0980
1.0950
1.0941

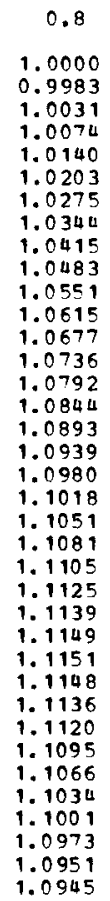

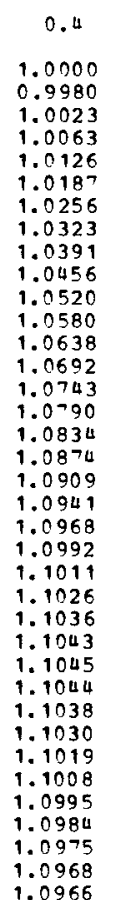

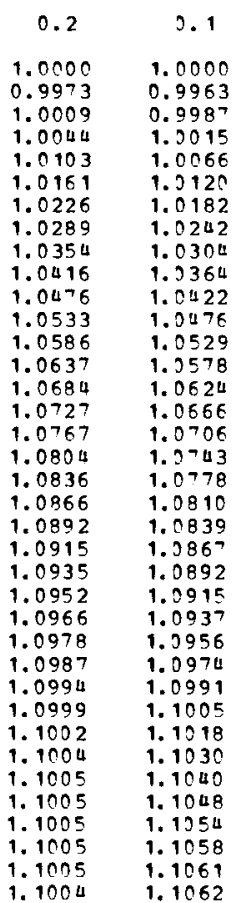

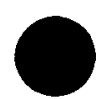

.0890 
FATIO OF MOTT TO MCKINLEX-FESHEACH SCATTERING IN CU, $\mathrm{z}=29$

\begin{tabular}{|c|c|c|c|c|c|c|c|c|c|c|c|}
\hline $\begin{array}{l}\text { ENERGY (MEV) } \\
\text { ANGIE (DEG.) }\end{array}$ & 100. & 10. & 4. & 2. & 1.5 & 1.0 & 0.8 & 0.6 & 0.4 & 0.2 & 0.1 \\
\hline 0 & 1.0000 & 1.0000 & 1.0000 & 1.0000 & 1.0000 & 1.0000 & 1.0000 & 1.0000 & 1.0000 & 1.0000 & 1.0000 \\
\hline 5 & 0.9983 & 0.9983 & 0.9983 & 0.9983 & 0.9982 & 0.9981 & 0.9981 & 0.9979 & 0.9977 & 0.9970 & 0.9959 \\
\hline 10 & 1.0038 & 1.0038 & 1.0037 & 1.0036 & 1.0035 & 1.0033 & 1.0031 & 1.0028 & 1.0022 & 1.0006 & 0.9982 \\
\hline 15 & 1.0085 & 1.0085 & 1.0085 & 1.0083 & 1.0082 & 1.0079 & 1.0077 & 1.0073 & 1.0065 & 1.0044 & 1.2011 \\
\hline 20 & 1.0158 & 1.0158 & $1.015 ?$ & 1.0155 & 1.0154 & 1.0150 & 1.0147 & 1.0142 & 1.0132 & 1.0106 & 1.0065 \\
\hline 25 & 1.0229 & 1.0229 & 1.0228 & 1.0225 & 1.0223 & 1.0219 & 1.0215 & 1.0209 & 1.0197 & 1.0168 & 1.0123 \\
\hline 30 & 1.0309 & 1.0308 & 1.0307 & 1.0304 & 1.0302 & 1.0296 & 1.0292 & 1.0285 & 1.0272 & 1.0238 & 1.0188 \\
\hline 35 & 1.0386 & 1.0385 & 1.0384 & 1.0381 & 1.0378 & 1.0371 & 1.0366 & 1.0358 & 1.0343 & 1.0306 & 1.0254 \\
\hline 40 & 1.0466 & 1.0465 & 1.0464 & 1.0460 & 1.0456 & 1.0449 & 1.0443 & 1.0434 & 1.0417 & 1.0376 & 1.0320 \\
\hline 45 & 1.0543 & 1.0542 & 1.0540 & 1.0536 & 1.0532 & 1.0523 & 1.0517 & 1.0506 & 1.0487 & 1.0443 & 1.0385 \\
\hline 50 & 1.0620 & 1.0619 & 1.0617 & 1.0612 & 1.0607 & 1.0597 & 1.0590 & 1.0577 & 1.0556 & 1.0508 & 1.0047 \\
\hline 55 & 1.0694 & 1.0693 & 1.0691 & 1.0684 & 1.0679 & 1.0668 & 1.0659 & 1.0645 & 1.0621 & 1.0569 & $1.050^{\circ}$ \\
\hline 60 & 1.0766 & 1.0766 & 1.0763 & 1.0755 & 1.0749 & 1.0736 & 1.0726 & 1.0711 & 1.0684 & $1.0 \in 28$ & 1.0564 \\
\hline 65 & 1.0836 & 1.0835 & 1.0832 & 1.0823 & 1.0816 & 1.0801 & 1.0790 & 1.0773 & 1.0743 & 1.0683 & 1.0617 \\
\hline 70 & 1.0903 & 1.0902 & 1.0898 & 1.0888 & 1.0880 & $1.0 B \in 3$ & 1.0851 & 1.0831 & 1.0798 & 1.0734 & $1.066^{7}$ \\
\hline 75 & 1.0967 & 1.0966 & 1.0962 & 1.0950 & 1.0941 & 1.0922 & 1.0908 & 1.0886 & 1.0849 & 1.0781 & $1.5-14$ \\
\hline 80 & 1.1028 & 1. 1027 & 1.1022 & 1.1009 & 1.0999 & 1.0977 & 1.0961 & 1.0936 & $1.089^{7}$ & 1.0825 & 1.0758 \\
\hline 85 & 1.1087 & 1.1086 & 1.1080 & 1.1065 & 1.1053 & 1.1028 & 1.1010 & 1.0983 & 1.0900 & 1.0865 & 1.0798 \\
\hline 90 & 1.1142 & 1.1141 & 1.1134 & 1.1117 & 1.1103 & 1. 1075 & 1.1055 & 1.1026 & 1.0979 & 1.0901 & 1.0836 \\
\hline 95 & 1.1195 & 1.1194 & 1.1186 & 1.1166 & 1,1151 & 1.1119 & $1.109^{7}$ & 1.1064 & 1.1014 & 1.0933 & 1.3871 \\
\hline 100 & 1.1244 & 1. 1242 & 1.1233 & 1.1210 & 1.1193 & 1.1158 & 1.1133 & 1. 1098 & 1. 1044 & 1.0962 & $1.090 u$ \\
\hline 115 & 1.1375 & 1.1372 & 1.1358 & 1.1323 & 1.1297 & 1.1247 & 1.1215 & 1.1170 & 1.1109 & 1.1029 & 1.0988 \\
\hline 120 & 1.1412 & 1.1408 & 1.1394 & 1.1350 & 1.1321 & 1. 1265 & 1.1230 & 1.1183 & 1.1120 & 9.1045 & 1.1013 \\
\hline 125 & 1.1447 & 1.1443 & 1.1423 & 1.1375 & 1.1341 & 1.1279 & 1.1249 & 1.1191 & 1.1128 & 1.1058 & 1.1035 \\
\hline 130 & 1.1477 & 1.1472 & 1.1448 & 1.1392 & 1.1353 & 1. 1284 & 1. 1244 & 1.1192 & 1.1131 & 1.1069 & 1.1055 \\
\hline 135 & 1.1507 & 1.1500 & 1.1471 & 1.1404 & 1.1359 & 1. 1284 & 1.1241 & 1.1189 & 1.1130 & 1.1077 & 1. 1073 \\
\hline 140 & 1.1531 & 1.1522 & 1.1486 & 1.1406 & 1. 1355 & 1.1273 & 1.1230 & 1. 1179 & 1.1125 & 1.1083 & 1.1090 \\
\hline 145 & 1.1555 & 1.1544 & 1.1497 & 1.1000 & 1.1342 & 1.1256 & 1.1212 & 1.1164 & 1.1117 & 1.1087 & 1.1105 \\
\hline 150 & 1.1573 & 1.1557 & 1.1497 & 1.1380 & 1.1316 & 1. 1228 & 1.1187 & 1.1144 & 1.1106 & 1.1090 & 1.1118 \\
\hline 155 & 1.1590 & 1.1568 & 1.1486 & 1.1345 & 1.1277 & 1.1193 & 1.1156 & 1.1121 & 1.1093 & 1.1092 & 1.1120 \\
\hline 160 & 1.1604 & 1.1570 & 1.1456 & 1.1291 & 1.1224 & 1.1150 & 1.1121 & 1.1096 & 1.1081 & 1.1092 & 1.1139 \\
\hline 165 & 1.1609 & 1.1553 & 1.1389 & 1.1215 & 1,1158 & 1.1104 & 1.1086 & 1.1073 & 1.1069 & 1.1093 & 1.1146 \\
\hline 170 & 1.1637 & 1.1518 & 1.1284 & 1.1130 & 1.1094 & 1. 1064 & 1.1057 & 1.1054 & 1.1059 & 1. 1092 & 1.1151 \\
\hline 175 & 1.1497 & 1.1264 & 1.1091 & 1.1041 & 1.1034 & 1. 1032 & 1.1034 & 1.1039 & 1.1053 & 1.1092 & 1.1154 \\
\hline 180 & 1.1010 & 1.1010 & 1.1011 & 1.1014 & 1.1017 & 1. 1022 & 1.1027 & 1. 1035 & 1.1050 & 1. 1092 & 1. 1155 \\
\hline
\end{tabular}

BATIO OF MOTT TO MCRTNLEY-FESHBACH SCATMERENG IN $Z N, Z=3 n$

ENERGY (HEV)
ANGLE (DEG.)
0
5
10
15
20
25
30
35
40
45
50
55
60
65
70
75
80
85
90
95
100
105
110
115
120
125
130
135
140
145
150
155
160
165
170
175
180

$\begin{array}{lccc}100 . & 10 . & 4 . & 2 . \\ 1.0000 & 1.0000 & 1.0000 & 1.0000 \\ 0.9981 & 0.9981 & 0.9981 & 0.9980 \\ 1.0038 & 1.0038 & 1.0037 & 1.0036 \\ 1.0089 & 1.0088 & 1.0088 & 1.0086 \\ 1.0166 & 1.0166 & 1.0165 & 1.0163 \\ 1.0242 & 1.0242 & 1.0241 & 1.0238 \\ 1.0328 & 1.0327 & 1.0326 & 1.0323 \\ 1.0410 & 1.0410 & 1.0409 & 1.0405 \\ 1.0496 & 1.0496 & 1.0494 & 1.0490 \\ 1.0579 & 1.0579 & 1.0577 & 1.0572 \\ 1.0562 & 1.0662 & 1.0650 & 1.0653 \\ 1.0742 & 1.0741 & 1.0739 & 1.0732 \\ 1.0820 & 1.0820 & 1.0817 & 1.0808 \\ 1.0895 & 1.0895 & 1.0891 & 1.0882 \\ 1.0968 & 1.0967 & 1.0953 & 1.0952 \\ 1.1037 & 1.1036 & 1.1032 & 1.1019 \\ 1.1103 & 1.1102 & 1.1097 & 1.1083 \\ 1.1167 & 1.1166 & 1.1160 & 1.1143 \\ 1.1226 & 1.1225 & 1.1218 & 1.1199 \\ 1.1284 & 1.1282 & 1.1274 & 1.1253 \\ 1.1337 & 1.1335 & 1.1325 & 1.1301 \\ 1.1388 & 1.1385 & 1.1375 & 1.1347 \\ 1.1434 & 1.1431 & 1.1419 & 1.1386 \\ 1.1479 & 1.1475 & 1.1461 & 1.1423 \\ 1.1518 & 1.1514 & 1.1497 & 1.1453 \\ 1.1557 & 1.1552 & 1.1531 & 1.1480 \\ 1.1589 & 1.1583 & 1.1558 & 1.1498 \\ 1.1622 & 1.1614 & 1.1584 & 1.1512 \\ 1.1647 & 1.1638 & 1.1600 & 1.1515 \\ 1.1673 & 1.1661 & 1.1613 & 1.1509 \\ 1.1693 & 1.1677 & 1.1613 & 1.1488 \\ 1.1712 & 1.1688 & 1.1502 & 1.1452 \\ 1.1726 & 1.1691 & 1.1569 & 1.1395 \\ 1.1733 & 1.1673 & 1.1499 & 1.1314 \\ 1.1760 & 1.1636 & 1.1387 & 1.1223 \\ 1.1621 & 1.1371 & 1.1183 & 1.1129 \\ 1.1095 & 1.1095 & 1.1096 & 1.1099\end{array}$

$\begin{array}{ccc}1.5 & 1.0 & 0.8 \\ 1.0000 & 1.0000 & 1.0000 \\ 0.9980 & 0.9979 & 0.9978 \\ 1.0035 & 1.0033 & 1.0031 \\ 1.0085 & 1.0081 & 1.0079 \\ 1.0161 & 1.0157 & 1.0154 \\ 1.0236 & 1.0231 & 1.0227 \\ 1.0320 & 1.0314 & 1.0310 \\ 1.0402 & 1.0395 & 1.0389 \\ 1.0486 & 1.0478 & 1.0472 \\ 1.0567 & 1.0558 & 1.0551 \\ 1.0549 & 1.0638 & 1.0630 \\ 1.0726 & 1.0714 & 1.0705 \\ 1.0802 & 1.0788 & 1.0777 \\ 1.0874 & 1.0858 & 1.0846 \\ 1.0943 & 1.0925 & 1.0912 \\ 1.1009 & 1.0989 & 1.0974 \\ 1.1071 & 1.1048 & 1.1031 \\ 1.1131 & 1.1100 & 1.1085 \\ 1.1185 & 1.1155 & 1.1134 \\ 1.1236 & 1.1203 & 1.1179 \\ 1.1282 & 1.1245 & 1.1218 \\ 1.1325 & 1.1283 & 1.1254 \\ 1.1362 & 1.1315 & 1.1283 \\ 1.1395 & 1.1343 & 1.1308 \\ 1.1422 & 1.1363 & 1.1325 \\ 1.1444 & 1.1378 & 1.1337 \\ 1.1457 & 1.1384 & 1.1341 \\ 1.1465 & 1.1385 & 1.1339 \\ 1.1460 & 1.1374 & 1.1327 \\ 1.1448 & 1.1356 & 1.1309 \\ 1.1420 & 1.1327 & 1.1283 \\ 1.1380 & 1.1289 & 1.1250 \\ 1.1323 & 1.1244 & 1.1214 \\ 1.1253 & 1.1195 & 1.1176 \\ 1.1184 & 1.1153 & 1.1145 \\ 1.1121 & 1.1118 & 1.1120 \\ 1.1102 & 1.1108 & 1.1113\end{array}$

0.6

1.0000 0.9977

1.0027

1.0074

1.0148

1.0220
1.0302 1.0302
1.0380 1.0462 1.0539 1.0617 1.0690 1.0761
1.0827 1.0827 1.0891
1.0950 1.1005 1. 1056 1. 1102 1. 1180 1. 1213 1. 1239 1. 1260 1. 1275 1. 1284 1.1286 1. 1284
1. 1273 1.1273
1. 1258 1. 1237 1. 1213 1. 1187 1. 1162 1. 1142 1. 1126

0.4

1.0000 0.9974 1.0021 1.0066 1.0137 1.0207
1.0287
1.0364 1.0443 1.0443 1.0593 1.0663 1.0731 1.0795 1.0855 1.0911 1.0962 1.1010 1.1090 1.1123 1.1152 1.1175 1. 1195 1.1208 1.1221
1.1221 1.1221
1.1216 .120 1.1196
1.1184 1.1184
1.1171 1.1158 1. 1148 1. 1139
0.2 1.0000 .9966 1.0003 1.0043 1. 0108 1.0174
1.0250 1.0323 1.0398 1.0470 1.0540 $1.060^{\circ}$ 1.0670 1.0730 1.0785 1.0837 1.0884 1.0928 1.0967 1.1003 1. 1035 1.1063
1.1088 1.1088
1.1109 1.1109
1.1127 1. 1127 1.1143
1.1155 1164 1.1171
1.1177 1.1177
1.1180 1. 1183 1. 1184
1. 1185 1. 1185
1. 1185 1. 1185 1. 1184
2.1
1.0000
0.9955
0.9979
1.0006
1.0064
1.0125
1.0195
1.0265
1.0336
1.0106
1.0474
1.0538
1.0600
1.0657
1.0712
1.0763
1.0811
1.0855
1.0897
1.2936
1.0972
1.1005
1.1036
1.1065
1.1092
1.1117
1.1140
1.1160
1.1179
1.1196
1.1211
1.1224
1.1234
1.1243
1.1249
1.1253
1.1253
109 
RATIO OF MOTT TO MCKTNLEY-PESHBACH SCATTERING IN GE, $z=32$

\begin{tabular}{|c|c|c|c|c|c|c|c|c|c|c|c|}
\hline $\begin{array}{l}\text { ENERGY (HEV) } \\
\text { ANGLE (DEG.) }\end{array}$ & 100 & 10. & 4. & 2. & 1.5 & 1.0 & 0.8 & 0.6 & 0.4 & 0.2 & 0.1 \\
\hline $\begin{array}{l}0 \\
5\end{array}$ & $\begin{array}{l}1.0000 \\
0.9976\end{array}$ & $\begin{array}{l}1.0000 \\
0.9976\end{array}$ & 1.0000 & 1.0000 & $\begin{array}{l}1.0000 \\
0.9975\end{array}$ & $\begin{array}{l}1.0000 \\
0.9973\end{array}$ & $\begin{array}{l}1.0000 \\
0.9972\end{array}$ & $\begin{array}{l}1.0000 \\
0.9971\end{array}$ & 1.0000 & 1.0000 & $\begin{array}{l}1.0000 \\
0.9945\end{array}$ \\
\hline 10 & 1.0038 & 1.0038 & 1.0037 & 1.0035 & 1.0034 & 1.0031 & & $\begin{array}{l}0.9971 \\
1.0025\end{array}$ & $\begin{array}{l}0.9967 \\
1.0017\end{array}$ & $\begin{array}{l}0.9958 \\
0.9096\end{array}$ & $\begin{array}{l}0.9945 \\
0.9965\end{array}$ \\
\hline 15 & 1.0094 & 1.0094 & 1.0094 & 1.0092 & 1.0090 & 1.0086 & 1.0083 & 1.0077 & $1.006^{7}$ & 1.0039 & 0.9995 \\
\hline 20 & 1.0182 & 1.0182 & 1.0181 & 1.0178 & 1.0176 & 1.0171 & 1.0167 & 1.0160 & 1.0147 & 1.0112 & 1.0058 \\
\hline 25 & 1.0268 & 1.0268 & 1.0267 & 1.0264 & 1.0261 & 1.0255 & 1.0250 & 1.0242 & $1.022^{7}$ & $1.018^{7}$ & 1.0126 \\
\hline 30 & 1.0366 & 1.0366 & 1.0364 & 1.0361 & 1.0357 & 1.0350 & 1.0345 & 1.0335 & 1.0317 & 1.0272 & 1.0205 \\
\hline 35 & 1.0461 & 1.0461 & 1.0459 & 1.0455 & 1.0451 & 1.0443 & 1.0436 & 1.0426 & 1.0406 & 1.0357 & 1.2285 \\
\hline 40 & 1.0560 & 1.0560 & 1.0558 & 1.0552 & 1.0548 & 1.0539 & 1.0531 & 1.0519 & 1.0497 & 1.0443 & 1.0368 \\
\hline 45 & 1.0655 & 1.0655 & 1.0653 & 1.0647 & 1.0642 & 1.0631 & 1.0623 & 1.0609 & 1.0584 & 1.0526 & 1.3448 \\
\hline 50 & 1.0751 & 1.0751 & 1.0748 & 1.0741 & 1.0735 & 1.0723 & 1.0713 & 1.0698 & $1.06^{70}$ & 1.0607 & 1.0526 \\
\hline 55 & 1.0843 & 1.0843 & 1.0840 & 1.0832 & 1.0825 & 1.0811 & 1.0800 & 1.0783 & 1.0752 & 1.0685 & $\begin{array}{l}1.2601 \\
1.0673\end{array}$ \\
\hline 60 & & 1.0933 & 1.0930 & 1.0920 & 1.0913 & 1.0897 & 1.0884 & 1.0865 & 1.0831 & 1.0759 & 1.0673 \\
\hline $\begin{array}{l}65 \\
70\end{array}$ & $\begin{array}{l}1.1021 \\
1.1105\end{array}$ & $\begin{array}{l}1.1020 \\
1.1104\end{array}$ & $\begin{array}{l}1.1016 \\
1.1099\end{array}$ & $\begin{array}{l}1.1005 \\
1.1087\end{array}$ & $\begin{array}{l}1.0997 \\
1.1077\end{array}$ & $\begin{array}{l}1.0978 \\
1.1056\end{array}$ & $\begin{array}{l}1.0964 \\
1.1041\end{array}$ & $\begin{array}{l}1.0943 \\
1.1016\end{array}$ & $\begin{array}{l}1.0905 \\
1.0975\end{array}$ & $\begin{array}{l}1.0828 \\
1.0803\end{array}$ & $\begin{array}{l}1.2741 \\
1.0805\end{array}$ \\
\hline 75 & 1.1185 & 1.1184 & 1.1179 & 1.1165 & 1.1154 & 1.1130 & 1.1113 & 1.1086 & 1.1001 & 1.0954 & 1.0865 \\
\hline 80 & 1. 1262 & 1.1261 & 1. 1255 & 1.1239 & 1. 1226 & 1.1190 & 1.1180 & 1.1150 & 1.1101 & 1.1010 & 1.0922 \\
\hline 85 & 1.1336 & 1.1334 & 1.1328 & 1.1309 & 1.1295 & 1.1265 & 1.1243 & 1.1210 & 1.1157 & 1. 1062 & 1.0975 \\
\hline 90 & 1.1405 & 1.1403 & 1.1396 & 1.1375 & 1. 1358 & 1. 1324 & 1.1300 & 1.1264 & 1.1207 & 1.1109 & 1.1024 \\
\hline 95 & 1.1472 & 1.1470 & 1.1461 & 1.1437 & 1.1418 & 1.1380 & 1.1353 & 1.1314 & 1.1252 & 1.1152 & 1.1071 \\
\hline 100 & 1.1534 & 1.1531 & 1.1521 & 1.1493 & 1. 1472 & 1. 1430 & 1.1400 & 1.1357 & 1.1292 & 1.1190 & 1.1114 \\
\hline 105 & 1.1593 & 1.1590 & 1.1578 & 1.1547 & 1.1523 & 1. 1475 & 1.1442 & 1.1395 & 1.1327 & 1.1224 & 1.1155 \\
\hline 110 & 1.1647 & 1.1644 & 1.1630 & 1.1593 & 1.1566 & 1.1513 & 1.1477 & 1.1427 & 1.1355 & 1.1255 & 1.1193 \\
\hline 115 & 1.1699 & 1.1695 & 1.1679 & 1.1637 & 1.1606 & 1.1546 & 1.1507 & 1.1453 & 1.1379 & 1.1281 & $1.122 \mathrm{e}$ \\
\hline 120 & 1.1745 & 1.1741 & 1.1721 & 1.1673 & 1.1637 & 1. 1571 & 1.1528 & 1.1471 & 1.1396 & 1.1304 & 1. 1262 \\
\hline 125 & 1.1790 & 1.1785 & 1.1762 & 1.1704 & 1.1664 & 1. 1590 & 1.1544 & 1.1484 & 1.1408 & 1.137 .3 & 1.1292 \\
\hline 130 & 1.1828 & 1.1822 & 1. 1794 & 1.1727 & 1.1680 & 1. 1599 & 1.1550 & 1.1488 & 1.1414 & 1. 1339 & 1.1321 \\
\hline 135 & 1.1866 & 1.1858 & 1.1824 & 1.1744 & 1.1690 & 1.1600 & 1.1549 & 1.1486 & 1.1415 & 1.1352 & 1.1347 \\
\hline 140 & 1.1896 & 1.1886 & 1.1844 & 1.1748 & 1.1687 & 1. 1590 & $1.153^{7}$ & 1.1476 & 1.1411 & 1.1362 & 1.1371 \\
\hline 145 & 1.1926 & 1.1913 & 1.1859 & 1.1743 & 1.1674 & 1. 1571 & 1.1518 & 1.1461 & 1.1404 & 1.1370 & 1. 1392 \\
\hline 150 & 1.1949 & 1.1931 & 1. 1860 & 1.1721 & 1.1645 & 1.1539 & 1.1489 & 1.1438 & 1.1392 & 1.1375 & 1.1411 \\
\hline 155 & 1.1971 & 1.1945 & 1.1849 & 1.1681 & 1.1600 & 1. 1498 & 1.1454 & 1. 1412 & 1.1379 & 1.1379 & 1.1428 \\
\hline 160 & 1.1988 & 1.1949 & 1.1814 & 1.1617 & 1. 1537 & 1. 1447 & 1.1413 & 1.1383 & 1.1365 & 1.1382 & 1.1401 \\
\hline 165 & 1.1997 & 1.1930 & 1.1736 & 1.1527 & $1.145 \mathrm{~B}$ & 1.1393 & 1.1371 & 1.1355 & 1.1351 & 1.1384 & 1.1452 \\
\hline 170 & 1.2025 & 1.1887 & 1. 1609 & 1.1424 & 1.1379 & 1.1344 & 1.1335 & 1.1332 & 1.1341 & 1.1385 & 1.1450 \\
\hline 175 & 1.1890 & 1.1601 & 1.1382 & 1.1318 & 1.1308 & 1.1305 & 1.1308 & 1.1315 & 1.1333 & 1.1385 & 1. 1465 \\
\hline 180 & 1.1277 & 1.1277 & 1.1279 & 1.1283 & 1.1286 & 1.1293 & 1.1299 & 1.1310 & 1.1330 & 1.1385 & 1. 1466 \\
\hline
\end{tabular}

RATIO OF MOTT TO MCKINIFY-FESHBACH SCATTERING IN RB, $\mathrm{Z}=37$

ENERGY (MEV)
ANGLE (DEG.)
0
5
10
15
20
25
30
35
40
45
50
55
60
65
70
75
80
85
90
95
100
105
110
115
120
125
130
135
140
145
150
155
160
165
170
175
180

\begin{tabular}{|c|c|c|c|c|c|c|c|}
\hline 100. & 10. & 4. & 2 . & 1.5 & 1.0 & 0.8 & 0.6 \\
\hline $\begin{array}{l}1.0000 \\
0.9959\end{array}$ & $\begin{array}{l}1.0000 \\
0.9959\end{array}$ & $\begin{array}{l}1.0000 \\
0.9959\end{array}$ & $\begin{array}{l}1.0000 \\
0.9958\end{array}$ & $\begin{array}{l}1.0000 \\
0.9957\end{array}$ & $\begin{array}{l}1.0000 \\
0.9955\end{array}$ & $\begin{array}{l}1.0000 \\
0.9954\end{array}$ & $\begin{array}{l}1.0000 \\
0.9951\end{array}$ \\
\hline 1.0031 & 1.0031 & 1.0030 & 1.0028 & 1.0026 & 1.0022 & 1.0018 & 1.0012 \\
\hline 1.0104 & 1.0104 & 1.0103 & 1.0099 & 1.0097 & 1.0091 & 1.0086 & 1.0078 \\
\hline 1.0218 & 1.0218 & 1.0217 & 1.0213 & 1.0209 & 1.0201 & 1.0195 & 1.0185 \\
\hline 1.0334 & 1.0334 & 1.0332 & 1.0327 & 1.0323 & 1.0314 & $1.030^{7}$ & 1.0295 \\
\hline 1.0466 & 1.0465 & 1.0463 & 1.0457 & 1.0453 & 1.0442 & 1.0434 & 1.0420 \\
\hline 1.0596 & 1.0595 & 1.0593 & 1.0586 & 1.0581 & 1.0569 & 1.0559 & 1.0543 \\
\hline 1.0731 & 1.0730 & 1.0728 & 1.0720 & 1.0714 & 1.0700 & 1.0689 & 1.0671 \\
\hline 1.0863 & 1.0862 & 1.0859 & 1.0851 & 1.0843 & 1.0828 & 1.0816 & 1.0796 \\
\hline 1.0996 & 1.0995 & 1.0991 & 1.0981 & 1.0973 & 1.0955 & 1.0942 & 1.0920 \\
\hline 1.1124 & 1.1123 & 1.1119 & 1.1108 & 1.1099 & 1. $10 ? 9$ & 1.1063 & 1.1039 \\
\hline 1.1250 & 1.1249 & 1. 1245 & 1. 1232 & 1.1221 & 1. 1199 & 1. 1182 & 1.1154 \\
\hline 1.1372 & 1.1371 & 1.1366 & 1.1351 & 1.1339 & 1.1314 & 1.1295 & 1.1264 \\
\hline 1.1490 & 1.1489 & 1.1483 & 1.1466 & 1. 1453 & 1. $1 \pm 24$ & 1.1403 & 1. 1369 \\
\hline 1.1604 & 1.1602 & 1. 1595 & 1.1576 & 1.1561 & 1. 1529 & 1.1505 & 1.1468 \\
\hline 1.1712 & 1.1711 & 1. 1703 & 1.1681 & 1. 1664 & 1.1628 & 1. 1602 & 1.1561 \\
\hline 1.1817 & 1.1815 & 1. 1806 & 1.1781 & 1. 1762 & 1.1722 & 1.1692 & 1.1647 \\
\hline 1.1915 & 1.1913 & 1.1903 & 1.1875 & 1. 1853 & 1. $18 \cap 8$ & 1.1775 & 1. $1^{727}$ \\
\hline 1.2010 & 1.2008 & 1.1996 & 1.1964 & 1.1939 & 1.1889 & 1.1853 & 1.1799 \\
\hline 1.2098 & 1.2095 & 1.2082 & 1.2045 & 1.2017 & 1.1961 & 1.1922 & 1. 1864 \\
\hline 1.2183 & 1.2179 & 1.2163 & 1.2122 & 1.2090 & 1.2027 & 1.1984 & 1. 1922 \\
\hline 1.2260 & 1.2256 & 1.2238 & 1.2191 & 1.2155 & 1.2085 & 1.2037 & 1.1970 \\
\hline 1.2335 & 1.2330 & 1.2308 & 1.2254 & 1.2213 & 1.2135 & 1.2083 & 1.2011 \\
\hline 1.2401 & 1.2396 & 1.2370 & 1.2307 & 1.2261 & 1. 2174 & 1.2118 & 1.2043 \\
\hline 1.2465 & 1.2458 & 1.2428 & 1.2354 & 1.2301 & 1.2205 & 1.2144 & 1. 2065 \\
\hline 1.2521 & 1.2512 & 1.2477 & 1.2390 & 1.2329 & 1.2223 & 1.2159 & 1. 2077 \\
\hline 1.2574 & 1.2563 & 1. 2520 & 1.2417 & 1.2347 & 1.2230 & 1.2163 & 1.2080 \\
\hline 1.2619 & 1.2606 & 1. 2551 & 1.2428 & 1.2350 & 1.2223 & 1. 2153 & 1.2073 \\
\hline 1.2661 & 1.2644 & 1.2575 & 1.2426 & 1.2337 & 1.2202 & 1.2133 & 1. 2056 \\
\hline 1.2695 & 1.2672 & 1.2581 & 1.2402 & 1. 2303 & 1.2164 & 1.2099 & 1.2031 \\
\hline 1.2726 & 1.2693 & 1.2570 & 1.2354 & 1.2247 & 1.2113 & 1.2055 & 1.1999 \\
\hline 1.2750 & 1.2701 & 1. 2528 & 1.2273 & 1.2167 & 1.2049 & 1. 2003 & 1.1964 \\
\hline 1.2765 & 1.2681 & 1.2432 & 1.2156 & 1.2065 & 1.1978 & 1.1950 & 1.1929 \\
\hline 1.2794 & 1.2620 & 1.2262 & 1.2019 & 1. 1960 & 1. 1914 & 1. 1903 & 1.1900 \\
\hline 1.2685 & 1.2287 & 1.1975 & 1.1882 & 1. 1868 & 1.1864 & 1.1868 & 1.1879 \\
\hline 1,1822 & 1.1823 & 1. 1825 & 1.1831 & 1. 1836 & 1.1847 & 1. 1856 & 1.1872 \\
\hline
\end{tabular}

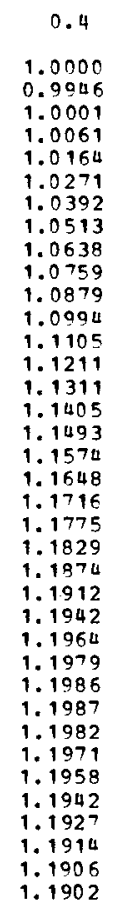

0.2
1.0000
0.9033
0.9970
1.0018
1.0110
1.0209
1.0323
1.0437
1.0555
1.0671
1.0784
1.0893
1.0998
1.1098
1.1192
1.1280
1.1352
1.1438
1.1509
1.1573
1.1633
1.1686
1.1734
1.1777
1.1815
1.1848
1.1877
1.1901
1.1921
1.1938
1.1952
1.1962
1.1970
1.1976
1.1980
1.1983
1.1982
0.1 1.7500 0.9916 $0.992^{7}$ 0.9954
1.0029 1.0115
1.0218 1.0325 1. 0437 1.0547 1.0656 1.0762
1.0861 1.0861
1.0961 1. 1054 1.1054
1.1142 1. 1225 1. 1304
1. 1379 1. 1409 1. 1517 1. 1579 1. 1696 1. 1750 1. 1800 1. 1847 1.1891 1.1932
1.1968 1.1968
1.2001 1.2001
1.2029 1. 2052 1. 2071 1.2084 1.2004
1.2096 
RATIO OF MOTT TO MCKINIEY-FESHBACH SCATTERING IN ZR, $z=40$

\begin{tabular}{|c|c|c|c|c|c|c|c|c|c|c|c|}
\hline $\begin{array}{l}\text { ENERGY (MEV) } \\
\text { ANGLE (DEG.) }\end{array}$ & 100. & 10. & u. & 2. & 1.5 & 1.0 & 0.8 & 0.6 & 0.4 & 0.2 & 0.1 \\
\hline $\begin{array}{l}0 \\
5\end{array}$ & 1.0000 & 1.0000 & & 1.0000 & 1.0000 & 1.0000 & 1.0000 & 1.0000 & 1.0000 & 1.0000 & 1.0000 \\
\hline & 0.9946 & 0.9946 & 0.9946 & 0.9945 & 0.9944 & 0.9941 & 0.9939 & 0.9936 & 0.9930 & 0.9914 & $\begin{array}{l}0.9896 \\
0.9898\end{array}$ \\
\hline $\begin{array}{l}10 \\
15\end{array}$ & $\begin{array}{l}1.0023 \\
1.0105\end{array}$ & $\begin{array}{l}1.0023 \\
1.0105\end{array}$ & $\begin{array}{l}1.0022 \\
1.0103\end{array}$ & $\begin{array}{l}1.0019 \\
1.0099\end{array}$ & $\begin{array}{l}1.0017 \\
1.0096\end{array}$ & $\begin{array}{l}1.0011 \\
1.0088\end{array}$ & $\begin{array}{l}1.0007 \\
1.0082\end{array}$ & $\begin{array}{l}1.0000 \\
1.0072\end{array}$ & $\begin{array}{l}0.9985 \\
1.0052\end{array}$ & $\begin{array}{l}0.9948 \\
0.9998\end{array}$ & $\begin{array}{l}0.9898 \\
0.9920\end{array}$ \\
\hline 20 & 1.0237 & 1.0236 & 1.0234 & 1.0229 & 1.0225 & 1.0215 & 1.0207 & 1.0194 & 1.0168 & 1.0101 & 1.0000 \\
\hline 25 & 1.0372 & 1.0371 & 1.0369 & 1.0363 & 1.0358 & 1.0347 & 1.0337 & 1.0322 & 1.0292 & 1.0214 & 1.0096 \\
\hline 30 & 1.0526 & 1.0526 & 1.0523 & 1.0516 & 1.0510 & 1.0097 & 1.0486 & 1.0468 & 1.0434 & 1.0346 & 1.0213 \\
\hline 35 & 1.0680 & 1.0679 & 1.0676 & 1.0668 & 1.0661 & 1.0646 & 1.0635 & 1.0615 & 1.0577 & 1.0481 & 1.0338 \\
\hline 40 & 1.0841 & 1.0840 & 1.0837 & 1.0827 & 1.0819 & 1.0802 & 1.0789 & 1.0767 & 1.0725 & 1.0620 & 1.0469 \\
\hline 45 & 1.0998 & 1.0997 & 1.0994 & 1.0983 & $1.097 \mathrm{t}$ & 1.0955 & 1.0940 & 1.0916 & 1.0870 & 1.0758 & 1.0601 \\
\hline 50 & 1.1157 & 1.1156 & 1.1151 & 1.1139 & 1.1129 & 1.1108 & 1. 1091 & 1.1064 & 1.1013 & 1.0894 & 1.0731 \\
\hline 55 & 1.1311 & 1.1310 & 1.1305 & 1.1291 & 1.1280 & 1.1256 & $1.123^{7}$ & 1.1207 & 1.1152 & 1. 1026 & 1.2858 \\
\hline 60 & 1.1463 & 1.1461 & 1.1456 & 1.1440 & 1. 1428 & 1.1400 & 1. 1380 & 1.1346 & $1.128^{7}$ & 1.1153 & 1.0981 \\
\hline 65 & 1.1610 & 1.1608 & 1. 1602 & 1.1584 & 1. 1570 & 1.1540 & 1.1517 & 1.1480 & 1.1415 & 1.1274 & 1.1090 \\
\hline 70 & 1.1752 & 1.1750 & 1.1743 & 1.1723 & 1.1707 & 1. 1673 & 1. 1648 & 1. 1607 & 1. 1537 & 1.1389 & 1.1213 \\
\hline 75 & 1.1890 & 1.1888 & 1.1879 & $1.185^{7}$ & 1.1839 & 1. 1801 & 1. 1772 & 1.1728 & 1.1652 & 1.1497 & 1.1321 \\
\hline 80 & 1.2021 & 1.2019 & 1.2010 & 1.1984 & 1. 1964 & 1.1921 & 1.1890 & 1. 1841 & 1.1759 & 1.1598 & 1.1424 \\
\hline 85 & 1.2148 & 1.2145 & 1.2135 & 1.2106 & 1.2083 & 1.2035 & 1.2001 & 1.1947 & 1.1859 & 1.1693 & 1.1522 \\
\hline 90 & 1.2268 & 1.2265 & 1.2253 & 1.2220 & 1.2195 & 1.2142 & 1.2103 & 1.2045 & 1.1951 & 1.1781 & 1.1615 \\
\hline 95 & 1.2383 & 1.2380 & 1.2366 & 1.2329 & 1.2300 & 1.2241 & 1.2198 & 1.2135 & 1.2035 & 1.1862 & 1.1703 \\
\hline 100 & 1.2490 & 1.2487 & 1.2471 & 1.2429 & 1.2396 & 1.2330 & 1.2284 & 1. 2216 & 1.2111 & 1.1937 & 1. 1789 \\
\hline 115 & 1.2779 & 1.2773 & 1.2748 & 1.2685 & 1. 2638 & 1.2547 & 1.2487 & 1.2403 & 1.2285 & 1.2123 & 1.2018 \\
\hline 120 & 1.2861 & 1.2854 & 1.2825 & 1.2752 & 1,2699 & 1.2598 & 1.2533 & 1.2445 & 1.2326 & 1.2174 & 1.2088 \\
\hline 125 & 1.2938 & 1.2930 & 1.2896 & 1.2811 & 1.2750 & 1.2638 & 1.2568 & 1.2476 & $1.235^{7}$ & 1.2218 & 1.2153 \\
\hline 130 & 1.3007 & 1.2998 & 1. 2957 & 1.2857 & 1.2787 & 1.2664 & 1.2589 & 1.2495 & 1.2379 & 1.2257 & 1.2216 \\
\hline 135 & 1.3072 & 1.3060 & 1.3010 & 1.2892 & 1.2812 & 1.2677 & 1.2598 & 1. 2502 & 1.2392 & 1.2291 & 1.2273 \\
\hline 140 & 1.3127 & 1.3113 & 1. 3050 & 1.2909 & 1.2819 & $1,26>2$ & 1. 2592 & 1. 2498 & 1.2397 & 1.2320 & $1.232^{7}$ \\
\hline 145 & 1.3179 & 1.3159 & 1. 3080 & 1.2910 & $1.280^{7}$ & 1. 2651 & 1.2571 & 1.2482 & 1.2395 & 1.2344 & 1.2376 \\
\hline 150 & 1.3221 & 1.3195 & 1.3092 & 1.2886 & 1.2772 & 1. 2611 & 1.2535 & 1. 2456 & 1.2387 & 1.2364 & 1.2420 \\
\hline 155 & 1.3259 & 1.3222 & 1.3082 & 1.2833 & 1.2710 & 1.2554 & 1.2486 & 1. 2422 & 1.2374 & 1.2381 & 1.2457 \\
\hline 160 & 1.3289 & 1.3232 & 1.3035 & 1.2742 & 1.2619 & 1.2481 & 1. 2428 & 1. 2383 & 1.2358 & 1.2393 & 1. 2489 \\
\hline 165 & 1.3309 & 1.3213 & 1. 2928 & 1.2609 & 1.2503 & 1.2001 & 1.2368 & 1.2345 & 1.2343 & 1.2403 & 1.2515 \\
\hline 170 & 1.3337 & 1.3140 & 1.2730 & 1.2448 & 1.2379 & 1. 2326 & 1.2313 & 1.2311 & 1.2330 & 1.2409 & 1. 2533 \\
\hline 175 & 1.3256 & 1.2783 & 1.2405 & 1.2291 & 1.2274 & 1.2270 & 1.2275 & 1.2289 & 1.2322 & 1.2414 & 1.2545 \\
\hline 180 & 1.2218 & 1.2219 & 1. 2221 & 1.2229 & 1.2235 & 1.2249 & 1.2260 & 1. 2279 & 1.2317 & 1.2414 & 1.2549 \\
\hline
\end{tabular}

RATTO OF MOTT TO MCRINLFY -FESHBACH SCATTERING IN NB, $z=41$

ENERGY (MFV)
ANGIE (DEG.)
0
5
10
15
20
25
30
35
40
45
50
55
60
65
70
75
80
85
90
95
100
105
110
115
120
125
130
135
140
145
150
155
160
165
170
175
180

$\begin{array}{cccccc}100 . & 10 . & 4 . & 2 . & 1.5 & 1.0 \\ 1.0000 & 1.0000 & 1.0000 & 1.0000 & 1.0000 & 1.0000 \\ 0.9942 & 0.9949 & 0.9941 & 0.9940 & 0.9939 & 0.9936 \\ 1.0020 & 1.0020 & 1.0018 & 1.0015 & 1.0013 & 1.0007 \\ 1.0104 & 1.0104 & 1.2103 & 1.0098 & 1.0095 & 1.0086 \\ 1.0242 & 1.0241 & 1.0239 & 1.0234 & 1.0229 & 1.0219 \\ 1.0384 & 1.0383 & 1.0381 & 1.0374 & 1.0369 & 1.0357 \\ 1.0546 & 1.0546 & 1.0543 & 1.0535 & 1.0529 & 1.0514 \\ 1.0709 & 1.0708 & 1.0705 & 1.0696 & 1.0689 & 1.0673 \\ 1.0878 & 1.0877 & 1.0874 & 1.0864 & 1.0855 & 1.0837 \\ 1.1045 & 1.1044 & 1.1040 & 1.1029 & 1.1019 & 1.0999 \\ 1.1213 & 1.1212 & 1.1207 & 1.1194 & 1.1184 & 1.1161 \\ 1.1376 & 1.1375 & 1.1370 & 1.1355 & 1.1343 & 1.1318 \\ 1.1537 & 1.1536 & 1.1530 & 1.1513 & 1.1500 & 1.1471 \\ 1.1693 & 1.1692 & 1.1685 & 1.1666 & 1.1651 & 1.1619 \\ 1.1844 & 1.1843 & 1.1835 & 1.1814 & 1.1797 & 1.1761 \\ 1.1991 & 1.1989 & 1.1980 & 1.1956 & 1.1937 & 1.1897 \\ 1.2131 & 1.2128 & 1.2119 & 1.2092 & 1.2070 & 1.2025 \\ 1.2265 & 1.2263 & 1.2252 & 1.2221 & 1.2197 & 1.2147 \\ 1.2393 & 1.2390 & 1.2378 & 1.2343 & 1.2316 & 1.2260 \\ 1.2516 & 1.2513 & 1.2498 & 1.2459 & 1.2429 & 1.2366 \\ 1.2631 & 1.2627 & 1.2610 & 1.2566 & 1.2532 & 1.2463 \\ 1.2740 & 1.2736 & 1.2717 & 1.2666 & 1.2628 & 1.2550 \\ 1.2842 & 1.2837 & 1.2815 & 1.2757 & 1.2713 & 1.2628 \\ 1.2938 & 1.2932 & 1.2906 & 1.2840 & 1.2791 & 1.2695 \\ 1.3026 & 1.3019 & 1.2989 & 1.2912 & 1.2856 & 1.2751 \\ 1.3108 & 1.3100 & 1.3064 & 1.2975 & 1.2911 & 1.2794 \\ 1.3182 & 1.3172 & 1.3129 & 1.3025 & 1.2952 & 1.2823 \\ 1.3251 & 1.3239 & 1.3186 & 1.3063 & 1.2980 & 1.2838 \\ 1.3311 & 1.3295 & 1.3230 & 1.3083 & 1.2988 & 1.2835 \\ 1.3365 & 1.3345 & 1.3263 & 1.3085 & 1.2978 & 1.2814 \\ 1.3411 & 1.3384 & 1.3276 & 1.3061 & 1.2941 & 1.2773 \\ 1.3451 & 1.3412 & 1.3266 & 1.3007 & 1.2878 & 1.2714 \\ 1.3483 & 1.3424 & 1.3219 & 1.2912 & 1.2783 & 1.2639 \\ 1.3505 & 1.3405 & 1.3108 & 1.2774 & 1.2662 & 1.2555 \\ 1.3532 & 1.3328 & 1.2900 & 1.2604 & 1.2532 & 1.2476 \\ 1.3464 & 1.2965 & 1.2562 & 1.2440 & 1.2423 & 1.2498 \\ 1.2363 & 1.2363 & 1.2366 & 1.2374 & 1.2381 & 1.2396\end{array}$

0.8
1.0000
0.9934
1.0002
1.0080
1.0210
1.0347
1.0503
1.0660
1.0823
1.0983
1.1143
1.1298
1.1449
1.1595
1.1734
1.1867
1.1992
1.2110
1.2220
1.2322
1.2414
1.2497
1.2570
1.2632
1.2682
1.2720
1.2745
1.2755
1.2750
1.2730
1.2693
1.2643
1.2583
1.2520
1.2453
1.2423
1.2408

0.6
1.0000
0.9931
0.9994
1.0069
1.0196
1.0330
1.0484
1.0638
1.0799
1.0959
1.1114
1.1266
1.1414
1.1555
1.1691
1.1820
1.1940
1.2054
1.2159
1.2255
1.2342
1.2419
1.2487
1.2544
1.2589
1.2623
1.2645
1.2655
1.2652
1.2637
1.2610
1.2576
1.2536
1.2496
1.2461
1.2438
1.2428

0.4 0.0924 0.9924 0.9979 1.0169 1.0298 1.0447 1.0598 1.0754 1.0907 1.1060 1. 1350 1.1350
1.1486 1.1616 1.1616
1.1738 1.1738 1. 1853 1.1960 1.2059 1.2149
1.2231 1.2231
1.2303 1.2303
1.2366 1.2420 1.2464 1.2498 1.2523
1.2539 1.2546 1.2545 1.2538 1.2525
1.2510 1.2510
1.2495 1.2495 1.2481
1.2473 1.2473
1.2468
0.2

0.1

1.0000 0.9908 0.9940 0.990

1.0096

1.0214

1.0352

1.0494

1.0641
1.0787

1.078

1. 0931

1. 1206

1. 1335

1. 1573

1. 1681

1.1681
1.1783

1. 1878

1. 1965

1. 2046

1.2119
$1.218^{7}$

$1.218^{7}$
$1.224^{7}$

1.2247
1.2303

1.2303
1.2351

1.2351

1.243

1. 2464

1. 2491

1.2514
1.2533

1. 2533

1.2547
1.2559

1.2559
1.2566

1.2566
1.2572

1. 2572
1. 2572
1.0000 0.9889 0.9887
0.0008 0.9988 1. 008 ? 1.0210 1.0341 1. 0478 1.0617 1.0754
1.0889 1. 1020 1. 1146 1. 1382 1. 1492 1. 1597 1. 1698 1. 1884 1. 1971 1. 2055 1. 2133 1. 2200 1. 2280 1. 2348 1. 2410 1. 2523 1. 2571 1. 2612 1. 2648 1.2648
1.2676 1.2676 1. 2696 1.2709
1.2713 
RATIO OF MOTT TO MCKINLFY-FESHBACH SCATTERING IN MO, $z=42$

\begin{tabular}{|c|c|c|c|c|c|c|c|c|c|c|c|}
\hline $\begin{array}{l}\text { ENERGY (HEV) } \\
\text { ANGLE (DEG.) }\end{array}$ & 100. & 10. & a. & 2. & 1.5 & 1.0 & 0.8 & 0.6 & 0.4 & 0.2 & 0.1 \\
\hline 0 & 1.0000 & 1.0000 & 1.0000 & 1.0000 & 1.0000 & 1.0000 & 1.0000 & 1.0000 & 1.0000 & 1.0000 & 1.0000 \\
\hline 5 & 0.9936 & 0.9936 & 0.9936 & 0.9934 & 0.9933 & 0.9930 & 0.9928 & 0.9925 & 0.9918 & 0.9901 & 0.9882 \\
\hline 10 & 1.0016 & 1.0016 & 1.0014 & 1.0011 & 1.0008 & 1.0002 & 0.9997 & 0.9989 & 0.9972 & 0.9931 & 0.9876 \\
\hline 15 & 1.0103 & 1.0103 & 1.0101 & 1.0097 & 1.0093 & 1.0084 & 1.0077 & 1.0065 & 1.0042 & 0.9981 & 0.9894 \\
\hline 20 & 1.0246 & 1.0246 & 1.0244 & 1.0238 & 1.0233 & 1.0222 & 1.0213 & 1.0198 & 1.0968 & 1.0090 & 0.9975 \\
\hline 25 & 1.0396 & 1.0395 & 1.0392 & 1.0385 & 1.0379 & 1.0366 & 1.0356 & 9.0338 & 1.0303 & 1.0213 & 1.0078 \\
\hline 30 & 1.0566 & 1.0565 & 1.0562 & 1.0554 & 1.0547 & 1.0532 & 1.0520 & 1.0499 & 1.0460 & $1.035^{\circ}$ & 1.0204 \\
\hline 35 & 1.0737 & 1.0736 & 1.0733 & 1.0724 & 1.0716 & 1.0699 & 1.0685 & 1.0662 & 1.0618 & 1.0507 & 1.0342 \\
\hline 40 & 1.0916 & 1.0915 & 1.0911 & 1.0901 & 1.0892 & 1.0872 & 1.0857 & 1.0831 & 1.0783 & 1.0662 & 1.0486 \\
\hline 45 & 1.1092 & 1.1091 & $1.108^{7}$ & 1.1075 & 1. 1065 & 1.1043 & 1.1026 & 1.0998 & 1.0945 & 1.0816 & 1.0632 \\
\hline 50 & 1. 1270 & 1. 1269 & 1.1264 & 1.1250 & 1. 1239 & 1. 1214 & 1.1195 & 1.1164 & 1. 1106 & 1.0968 & 1.0777 \\
\hline 55 & 1.1443 & 1.1442 & 1.1436 & 1.1421 & 1.1408 & 1.1381 & 1.1360 & 1.1325 & 1.1262 & 1.1117 & 1.0920 \\
\hline 60 & 1.1613 & 1.1612 & 1.1606 & 1.1588 & 1.1574 & 1. 1543 & 1.1520 & 1.1482 & 1.1414 & 1.1260 & 1.1050 \\
\hline 65 & 1.1779 & 1.1777 & 1.1770 & 1.1750 & 1.1735 & 1.1700 & 1.1674 & 1.1633 & 1.1559 & 1.1397 & 1.1193 \\
\hline 70 & 1.1939 & 1.1938 & 1.1930 & 1.1907 & 1. 1890 & 1. 1851 & 1.1823 & 1.1777 & 1.1697 & 1.1527 & 1. 1321 \\
\hline 75 & 1.2095 & 1.2093 & 1.2083 & 1.2058 & 1.2038 & 1.1096 & $1.196 \mathrm{u}$ & 1.1914 & 1.1828 & 1.1650 & 1.1040 \\
\hline 80 & 1.2244 & 1.2241 & 1.2231 & 1. 2203 & 1.2180 & 1.2133 & 1.2097 & 1.2043 & 1.1950 & 1.1766 & 1. 1562 \\
\hline 85 & 1.2387 & 1.2384 & 1.2372 & 1.2341 & 1. 2315 & 1.2262 & 1.2223 & 1.2154 & 1.2065 & 1.1875 & 1.1674 \\
\hline 90 & 1.2523 & 1.2520 & 1.2506 & 1.2470 & 1.2442 & 1.2383 & 1.2341 & 1. 2276 & 1.2170 & 1.1977 & 1.1782 \\
\hline 95 & 1.2653 & 1.2650 & 1.2635 & 1.2594 & 1.2562 & 1.2496 & 1.2449 & 1.2379 & 1.2267 & 1.2070 & 1.1884 \\
\hline 100 & 1.2776 & 1.2772 & 1.2754 & 1.2708 & 1.2672 & 1. 2599 & 1.2548 & 1.2472 & 1.2354 & 1.2158 & 1. 1983 \\
\hline 105 & 1.2892 & 1.2888 & 1.2868 & 1.2815 & 1.2774 & 1.2693 & 1.2637 & 1.2555 & 1.2432 & 1.2237 & 1.2976 \\
\hline 110 & 1.3001 & 1.2995 & 1. 2972 & 1.2912 & 1. 2866 & 1. 2776 & 1.2715 & 1.2628 & 1. 2501 & 1.2310 & 1. 2166 \\
\hline 115 & 1.3103 & 1.3097 & 1.3070 & 1.3001 & 1.2949 & 1.2849 & 1.2782 & 1.2690 & 1.2559 & 1.2376 & 1.2251 \\
\hline 120 & 1.3197 & 1.3190 & 1.3158 & 1.3078 & 1.3020 & 1. 2909 & $1.283^{7}$ & 1.2740 & $1.260^{\circ}$ & 1.2436 & 1. 2334 \\
\hline 125 & 1.3285 & 1.3276 & 1.3239 & 1.3146 & 1.3079 & 1.2956 & 1.2879 & 1.2777 & 1.2605 & 1.2489 & 1.2410 \\
\hline 130 & 1.3364 & 1.3354 & 1.3309 & 1.3200 & 1. 3124 & 1.2989 & 1.2906 & 1. 2802 & 1.2674 & 1.2537 & ท. 2485 \\
\hline 135 & 1.3437 & 1.3424 & 1. 3370 & 1.3241 & 1.3154 & 1. 3005 & 1.2919 & 1.2814 & 1.2692 & 1.2577 & 1.2553 \\
\hline 140 & 1.3501 & 1.3485 & 1.3417 & 1.3264 & 1.3165 & 1. 3004 & 1.2915 & 1. 2812 & 1.2701 & 1. 2614 & 1. 2618 \\
\hline 145 & 1.3559 & 1.3538 & 1.3452 & 1.3267 & 1.3155 & 1.2984 & 1.2895 & 1.2798 & 1.2701 & 1.2644 & 1. $26-5$ \\
\hline 150 & 1.3608 & 1.3580 & 1.3468 & 1.3244 & 1.3118 & 1. 2942 & 1.2858 & $1.2>72$ & 1.2695 & 1.2670 & $1.2 \div 29$ \\
\hline 155 & 1.3651 & 1.3611 & 1.3459 & 1.3188 & 1.3053 & 1. 2881 & $1.280^{7}$ & 1.2736 & 1.2683 & 1. 2691 & 1.2774 \\
\hline 160 & 1.3685 & 1.3624 & 1.3410 & 1.3090 & 1.2955 & 1.2803 & 1.2745 & 1.2695 & 1.2668 & 1. 2708 & 1. 2813 \\
\hline 165 & 1.3709 & 1.3605 & 1.3295 & 1.2946 & 1.2829 & 1.2716 & 1.2679 & 1. 2654 & 1.2653 & 1.2721 & 1.2843 \\
\hline 170 & 1.3736 & 1.3524 & 1.3078 & 1.2767 & 1.2692 & 1.2633 & 1.2620 & 1.2618 & 1.2639 & 1.2729 & 1. 2865 \\
\hline 175 & 1.3682 & 1.3154 & 1.2727 & 1.2597 & 1. 2578 & 1.2573 & 1.2579 & 1.2590 & 1.2632 & 1.2736 & 1.2880 \\
\hline 180 & 1.2514 & 1.2515 & 1. 2518 & 1.2526 & 1.2533 & 1.2549 & 1.2562 & 1.2584 & 1.2627 & $1.2^{7} 3^{7}$ & 1. 2885 \\
\hline
\end{tabular}

RATTO OF MOTT TO MCKINIFY-FESHBACH SCATTERING IN AG, $\mathrm{Z}=47$

\begin{tabular}{|c|c|c|c|c|c|c|c|c|c|c|c|}
\hline $\begin{array}{l}\text { ENERGY (MEV) } \\
\text { ANGLE (DEG..) }\end{array}$ & 100 & 10. & 4. & 2. & 1.5 & 1.0 & 0.8 & 0.6 & 0.4 & 0.2 & 0.1 \\
\hline 0 & 1.0000 & 1.0000 & 1.0000 & 1.0000 & 1.0000 & 1.0000 & 1.0000 & 1.0000 & 1.0000 & 1.0000 & 1.0000 \\
\hline 5 & 0.9906 & 0.9906 & 0.9905 & 0.9904 & 0.9902 & 0.9899 & 0.9896 & 0.9891 & 0.9882 & 0.9863 & 0.9845 \\
\hline 10 & 0.9989 & 0.9988 & 0.9987 & 0.9982 & 0.9978 & 0.9970 & 0.9964 & 0.9953 & 0.9931 & 0.9878 & 0.9816 \\
\hline 15 & 1.0089 & 1.0088 & 1.0086 & 1.0080 & 1.0074 & 1.0062 & 1.0053 & 1.0036 & 1.0004 & 0.9923 & 0.9815 \\
\hline 20 & 1.0262 & 1.0261 & 1.0258 & 1.0250 & 1.0243 & 1.0227 & 1.0215 & 1.0194 & 1.0153 & 1.0046 & 0.9895 \\
\hline 25 & 1.0447 & 1.0446 & 1.0443 & 1.0433 & 1.0425 & 1.0006 & 1.0392 & 1.0367 & 1.0318 & 1.0192 & 9.0010 \\
\hline 30 & 1.0661 & 1.0660 & 1.0656 & 1.0645 & 1.0635 & 1.0614 & 1.0597 & 1.0569 & 1.0513 & 1.0369 & 1.0158 \\
\hline 35 & 1.0880 & 1.0879 & 1.0874 & 1.0861 & 1.0851 & 1.0827 & 1.0808 & 1.0776 & 1.0714 & $1.055^{7}$ & 1.2326 \\
\hline 40 & 1.1110 & 1.1109 & $1 .: 103$ & 1.1089 & 1.1076 & 1.1049 & 1.1028 & 1.0993 & 1.0925 & 1.0754 & 1.0505 \\
\hline 45 & 1.1339 & 1.1337 & 1.1331 & 1.1315 & 1.1301 & 1.1272 & 1.1208 & 1.1209 & 1.1135 & 1.0953 & 1.0691 \\
\hline 50 & 1.1570 & 1. 1568 & 1.1561 & 1.1543 & 1.1528 & 1.1495 & 1. 1469 & 1,1426 & 1.1346 & 1.1151 & $1.08-8$ \\
\hline 55 & 1.1797 & 1.1796 & 1.1788 & 1.1767 & 1.1751 & 1.1714 & 1.1685 & 1.1638 & 1.1552 & 1.1346 & $1,106 \mathrm{~b}$ \\
\hline 60 & 1.2022 & 1.2020 & 1.2011 & 1.1988 & 1.1970 & 1. 1929 & 1.1897 & 1.1846 & 1.1752 & 1.1536 & 1.1247 \\
\hline 65 & 1.2241 & 1.2239 & 1.2230 & 1.2204 & 1.2183 & 1.2137 & 1.2103 & 1.2047 & 1.1946 & 1.1719 & 1.1425 \\
\hline 70 & 1.2455 & 1.2452 & 1.2442 & 1.2413 & 1.2389 & 1. 2339 & 1.2301 & 1.2240 & 1.2132 & 1. 1895 & 1.1597 \\
\hline 75 & 1.2662 & 1.2659 & 1.2647 & 1.2615 & 1.2569 & 1.2533 & 1.2491 & 1.2424 & 1.2308 & 1.2062 & 1. 1764 \\
\hline 80 & 1.2861 & 1.2858 & 1.2845 & 1.2809 & $1.2^{779}$ & 1. 2718 & 1.2672 & 1. 2599 & 1.2476 & 1.2221 & 1. 1925 \\
\hline 65 & 1.3054 & 1.3050 & 1.3035 & 1.2994 & 1.2962 & 1.2893 & 1.2843 & 1.2764 & 1.2632 & 1.2371 & 1.2079 \\
\hline 90 & 1.3237 & 1.3234 & 1.3216 & 1.3170 & 1.3134 & 1.3058 & 1.3003 & 1. 2918 & 1.2779 & 1.2513 & 1. 2229 \\
\hline 95 & 1.3413 & 1.3409 & 1.3389 & 1.3337 & 1.3297 & 1.3213 & 1.3152 & 1.3061 & 1.2994 & 1.2645 & 1.2372 \\
\hline 100 & 1.3579 & 1.3574 & 1.3552 & 1.3494 & 1.3448 & 1.3356 & 1.3290 & 1. 3192 & 1.3038 & 1.2769 & 1.2511 \\
\hline 105 & 1.3737 & 1.3731 & 1.3706 & 1.3640 & 1.3589 & 1.3486 & 1.3414 & 1.3309 & 1.3148 & 1.2883 & 1.2644 \\
\hline 110 & 1.3885 & 1.3879 & 1.3850 & 1.3774 & 1.3717 & 1.3603 & 1. 3526 & 1.3414 & 1.3248 & 1.2990 & 1. $2^{774}$ \\
\hline 115 & 1.4024 & 1.4017 & 1.3983 & 1.3897 & 1.3832 & 1.3706 & 1.3622 & 1.3504 & 1.3334 & 1.3087 & $1.289^{\circ}$ \\
\hline 120 & 1.4153 & 1.4144 & 1.4105 & 1.4006 & 1.3933 & 1.3794 & 1.3704 & 1.3579 & 1.3409 & 1.3178 & 1.3019 \\
\hline 125 & 1.4272 & 1.4262 & 1.4216 & 1.4101 & 1.4018 & 1.3865 & 1.3767 & 1.3638 & 1.3469 & 1.3258 & 1.3132 \\
\hline 130 & 1.4382 & 1.4369 & 1.4314 & $1.4180^{\circ}$ & 1.4086 & 1.3917 & 1.3814 & 1.3682 & 1.3517 & 1.3333 & 1.3243 \\
\hline 135 & 1.4481 & 1.4465 & 1.4399 & 1.4241 & 1.4133 & 1.3949 & 1.3840 & 1.3707 & 1.3551 & 1.3398 & 1.3345 \\
\hline 140 & 1.4570 & 1.4551 & 1.4468 & 1.4280 & 1.4158 & 1.3958 & 1. 3847 & 1.3717 & 1.3575 & 1.3458 & 1.3443 \\
\hline 145 & 1.4649 & 1.4623 & 1.4519 & 1.4293 & 1.4354 & 1. 3942 & 1. 3831 & 1.3708 & 1.3586 & 1.3508 & 1.3531 \\
\hline 150 & 1.4717 & 1.4683 & 1.4547 & 1.4273 & 1.4119 & 1.3900 & 1.3794 & 1.3685 & $1.358 B$ & 1.3553 & 1. 3612 \\
\hline 155 & 1.4775 & 1.4727 & 1.4543 & 1.4211 & 1.4045 & 1.3831 & 1.3737 & 1.3648 & 1.3582 & 1.3590 & 1. 3682 \\
\hline 160 & 1.4822 & 1.4749 & 1.4490 & 1.4097 & 1.3929 & 1.3739 & 1. 3666 & 1.3604 & 1.3570 & 1.3620 & 1.3742 \\
\hline 165 & 1.4859 & 1.4734 & 1.4357 & 1.3924 & 1.3777 & 1. 3635 & 1.3589 & 1.3558 & 1.3558 & 1.3644 & 1.3789 \\
\hline 170 & 1.4880 & 1.4626 & 1.4084 & 1. 3699 & 1.3605 & 1.3532 & 1. 3516 & 1.3515 & 1.3544 & 1.3660 & 1.3823 \\
\hline 175 & 1.4921 & 1.4239 & 1.3669 & 1.3494 & 1. 3468 & 1.3460 & 1. 3468 & 1.3489 & 1.3538 & $1.36>2$ & 1.3845 \\
\hline 180 & 1.3381 & 1. 3382 & 1.3386 & 1.3398 & 1.3407 & 1. 3429 & 1. 3447 & 1.3476 & 1.3533 & 1.3675 & $1.385 u$ \\
\hline
\end{tabular}


BATIO OF HOTT TO MCKINIEY-FESHBACH SCATTERTNG IN $S N, z=50$

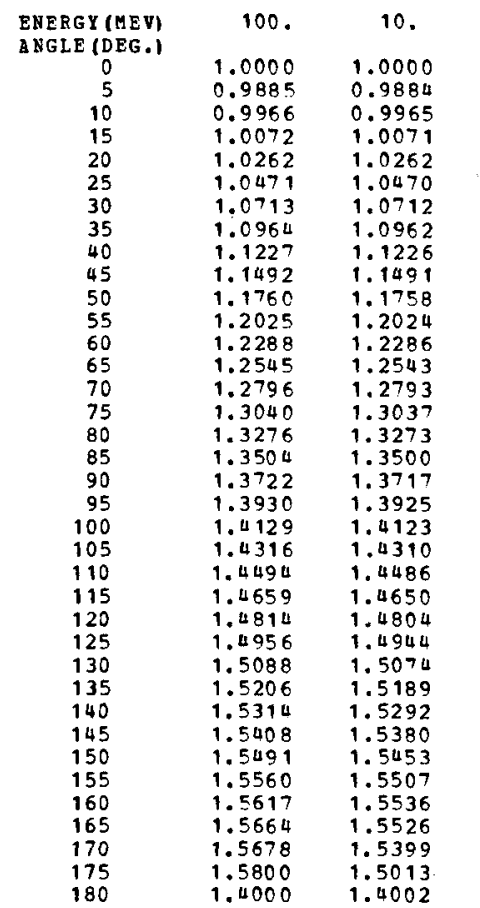

$\begin{array}{cc}4 & 2 . \\ 1.0000 & 1.0000 \\ 0.9884 & 0.9882 \\ 0.9964 & 0.9958 \\ 1.0059 & 1.0061 \\ 1.0258 & 1.0248 \\ 1.0465 & 1.0454 \\ 1.0707 & 1.0694 \\ 1.0957 & 1.0941 \\ 1.1220 & 1.1202 \\ 1.1484 & 1.1464 \\ 1.1751 & 1.1729 \\ 1.2015 & 1.1990 \\ 1.2276 & 1.2249 \\ 1.2532 & 1.2501 \\ 1.2781 & 1.2747 \\ 1.3023 & 1.2986 \\ 1.3257 & 1.3215 \\ 1.3482 & 1.3435 \\ 1.3698 & 1.3645 \\ 1.3903 & 1.3843 \\ 1.4098 & 1.4031 \\ 1.4281 & 1.4205 \\ 1.4453 & 1.4367 \\ 1.4612 & 1.4515 \\ 1.4750 & 1.4648 \\ 1.4893 & 1.4763 \\ 1.5012 & 1.4861 \\ 1.5114 & 1.4937 \\ 1.5201 & 1.4990 \\ 1.5264 & 1.5010 \\ 1.5302 & 1.4995 \\ 1.5302 & 1.4931 \\ 1.5248 & 1.4807 \\ 1.5105 & 1.4616 \\ 1.4796 & 1.4361 \\ 1.4343 & 1.4134 \\ 1.4006 & 1.4020\end{array}$

1.5
1.0000
0.9880
0.9954
1.0055
1.0240
1.0444
1.0682
1.0928
1.1188
1.1448
1.1711
1.1970
1.2226
1.2477
1.2720
1.2955
1.3181
1.3397
1.3603
1.3797
1.3979
1.4147
1.4302
1.4441
1.4565
1.4669
1.4755
1.4816
1.4852
1.4854
1.4821
1.4743
1.4617
1.4449
1.4254
1.4103
1.4031

1.0
1.0000
0.9875
0.9945
1.0040
1.0221
1.0422
1.0657
1.0900
1.1155
1.1412
1.1671
1.1927
1.2178
1.2423
1.2661
1.2890
1.3109
1.3318
1.3515
1.3700
1.3872
1.4029
1.4172
1.4298
1.4407
1.4495
1.4564
1.4607
1.4626
1.4614
1.4573
1.4501
1.4401
1.4287
1.4171
1.4093
1.4057

0.6
1.0000
0.9868
0.9924
1.0000
1.0181
1.0374
1.0692
1.0838
1.1087
1.1337
1.1589
1.1837
1.2080
1.2316
1.2544
1.2762
1.2971
1.3167
1.3352
1.3523
1.3682
1.3825
1.3954
1.4064
1.4160
1.4236
1.4295
1.4331
1.4350
1.4348
1.4329
1.4293
1.4247
1.4199
1.4152
1.4126
1.4111

0.4
1.0000
0.9858
0.9900
0.9997
1.0132
1.0316
1.0535
1.0764
1.1005
11248
1.1492
1.1733
1.1968
1.2196
1.2415
1.2625
1.2824
1.3011
1.3188
1.3350
1.3509
1.3639
1.3760
1.3867
1.3962
1.4039
1.4104
1.4151
1.2187
1.4206
1.4217
1.4216
1.4209
1.4199
1.4186
1.4182
1.4179

1.0177

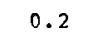

1. 0000 0.9838

0.9841

0.9878

1.0007

1.0166
1.0362

1.0362
1.0574

1.0798

1. 1027

1. 1256

1.1484
1.1705

1.1921

1. 2129

1. 2327

1.2517
1.2697

1. 2868

1.2868
1.3027

1.3027
1.3179

1.3179
1.3318

1.3318
1.3451

1.3451
1.3571

1. 3685

1. 3787

1. 3883

1. 3967

1. 4045

1. 4111

1.4221

1. 4263

1. 4295

1. 4334

1. 4334
1. 4338
0.1

1. 0000 0.9823
0.0777

0.9759

0.9834

0.9954

1.0113
1.0290

1.0490

1. 2711
1.0924

1.1140

1. 1351

1. 1763

1. 1960

1.2151
1.2335

1. 2515

1. 2688

1. 2688
1. 2857

1. 2857

1.3519
1.3179

1. 3330

1. 3480

1.3621
1.3750

1. $388^{7}$

1. 4010

1. 4121

1. 4224

1. 1313

1. 4389

1. 4493

1. 4529

RATTO OF MOTM TO MCK INLEY-FESHBACH SCATTERING IN CS, $z=55$

ENERGY (HEV)
A NGLE (DEG.)
0
5
10
15
20
25
30
35
40
45
50
55
60
65
70
75
80
85
90
95
100
105
110
115
120
125
130
135
140
145
150
155
160
165
170
175
180

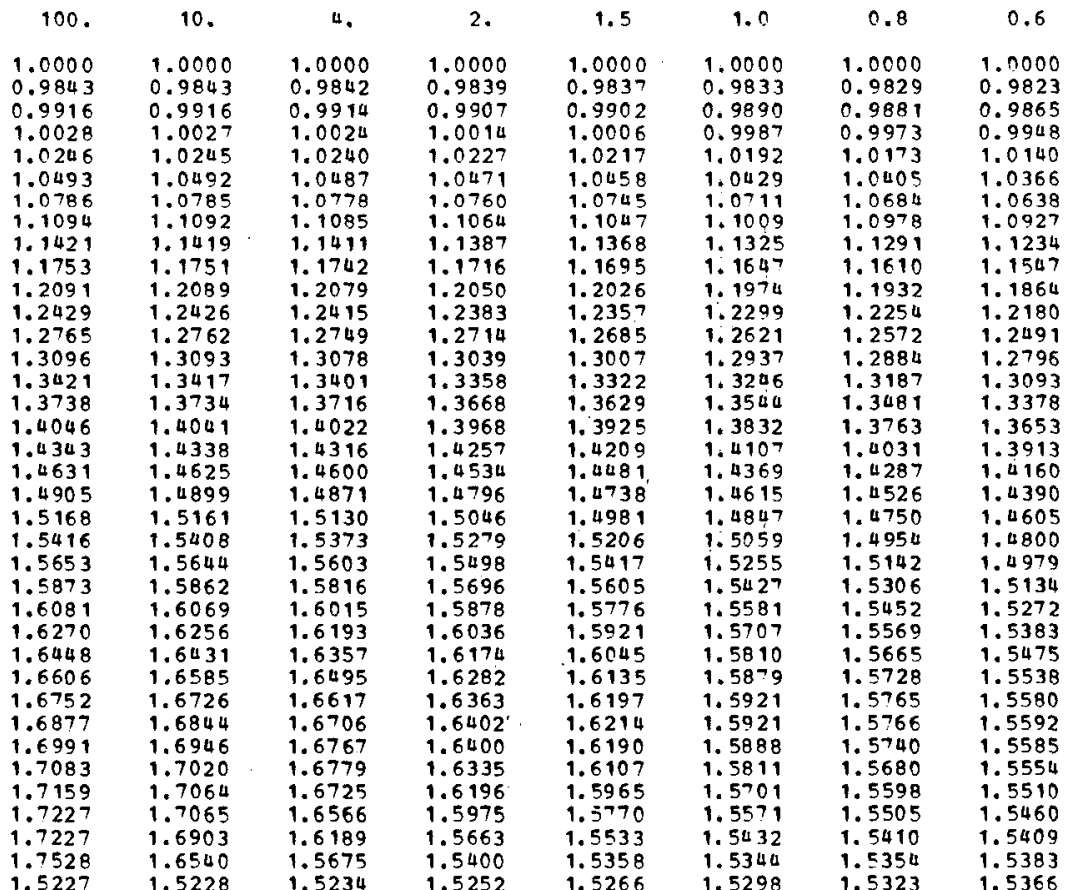

0.4
1.0000
0.9813
0.9836
0.9901
1.0077
1.0290
1.0549
1.0828
1.1124
1.1428
1.1735
1.2041
1.2342
1.2636
1.2922
1.3196
1.3460
1.3709
1.3945
1.4164
1.4370
1.4557
1.4730
1.4881
1.5018
1.5132
1.5232
1.5309
1.5372
1.5413
1.5442
1.5456
1.5460
1.5458
1.5450
1.5451
1.5447

0.2

0.1

1.0000 0.9793 0.9770

0.9918

1.0095
1.0321

1.0321
1.0575

1.0846

1.1130

1.1416
1.1704

1.1704
1.1987

1.226

1.2534

1.3045

1.3284

1. 3513

1.3936

1.3936

1,4313

1.4313

1.4645

1.4645
1.4792

1.0933

1.5057

1.5175
1.5276

1.5276
1.5370

1.5370
1.5447

1.5514

1. 5566

1.5602

1.5628
1.5637

1.0000

0.9710

0.9656

0.9833

1.0004

1.0218
1.0452

1.0452
1.0708

1. 0969

1. 1238

1. 1504

1. 1769

1.2030
1.2285

1. 2536

1. 2779

1. 3018

1.3249
1.3478

1.3478

1.3598
1.3917

1. 1917

1. 1927

1. 4336

1. 4534

1. 4729

1. $508^{7}$

$1.52 \mathrm{t}$ -

1. 5395

1. 5635

1. 5723

1. $5>88$

1. 5828 
RATIC OF MOTT TC MCKINIFY-FESHBACH SCA PTFRING IN ND, $z=60$

\begin{tabular}{|c|c|c|c|c|c|c|c|c|c|c|c|}
\hline $\begin{array}{l}\text { ENERGY (NEV) } \\
\text { ANGLE (DEG.) }\end{array}$ & 100 & 10. & 4. & 2. & 1.5 & $1 . n$ & 0.8 & 0.6 & 0.4 & 0.2 & 0.1 \\
\hline 0 & 1.0000 & 1.0000 & 1.0000 & 1.0000 & 1.0000 & 1.0000 & 1.0000 & 1.0000 & 1.0000 & 1.0000 & 1.0000 \\
\hline 5 & 0.9794 & 0.9794 & 0.9793 & 0.9790 & 0.9788 & 0.9783 & 0.9780 & 0.9774 & 0.9763 & 0.9748 & 0.9753 \\
\hline 10 & 0.9852 & 0.9851 & 0.9849 & 0.9841 & 0.9835 & 0.9821 & 0.9810 & 0.9792 & 0.9759 & 0.9690 & 0.9644 \\
\hline 15 & 0.9961 & 0.9960 & 0.9956 & 0.9944 & 0.9934 & 0.9911 & 0.9893 & 0.9863 & 0.9807 & 0.9676 & 0.9505 \\
\hline 20 & 1.0203 & 1.0202 & 1.0196 & 1.0180 & 1.0166 & 1.0135 & 1.0111 & 1.0070 & 0.9991 & 0.9799 & 0.9572 \\
\hline 25 & 1.0489 & 1.0488 & 1.0480 & 1.0460 & 1.0444 & 1.0406 & 1.0376 & 1.0326 & 1.0229 & 0.9988 & 0.9682 \\
\hline 30 & 1.0834 & 1.0833 & 1.0824 & 1.0800 & 1.0781 & $1.0^{73} 6$ & 1.0701 & 1.0642 & 1.0528 & 1.0239 & 0.9855 \\
\hline 35 & 1.1205 & 1.1203 & 1.1193 & 1.1167 & 1. 1144 & 1.1094 & 1.1055 & 1.0988 & 1.0859 & 9.0533 & 1.0090 \\
\hline 40 & 1.1603 & 1.1600 & 1.1589 & 1.1559 & 1. 1534 & 1. 1478 & 1. 1433 & 1.1359 & 1. 1215 & 1.0854 & 1.0355 \\
\hline 45 & 1.2013 & 1.2010 & 1.1998 & 1.1964 & 1.1936 & 1.1874 & 1.1825 & 1.1743 & 1.1587 & 1.1196 & 1.0654 \\
\hline 50 & 1.2432 & 1.2429 & 1.2416 & 1.2378 & 1. 2347 & 1. 2279 & 1.2225 & 1.2136 & 1.1966 & 1.1546 & 1.2965 \\
\hline 55 & 1.2855 & 1.2852 & 1.2837 & 1.2796 & 1.2762 & $1.268^{7}$ & 1.2628 & 1.2531 & 1.2348 & 1.1902 & 1.1290 \\
\hline 60 & 1.3279 & 9.3275 & 1.3258 & 1.3213 & 1.3175 & 1.3093 & 1.3029 & 1.2924 & 1.2727 & 1.2256 & 1. 1617 \\
\hline 65 & 1.3699 & 1.3695 & 1.3677 & 1.3626 & 1.3585 & 1.3495 & 1.3426 & 1.3312 & 1.3102 & $1.260^{7}$ & 1.1947 \\
\hline 70 & 1.4114 & 1.4109 & 1.4089 & 1.4034 & 1. 3988 & 1. 3890 & 1.3814 & 1.3699 & 1. $3116^{7}$ & 1.2951 & 1.2274 \\
\hline 75 & 1.4521 & 1.4516 & 1.4493 & 1.4432 & 1.4382 & 1.4274 & 1.4192 & 1.4059 & 1.3821 & 1.3285 & 1.2598 \\
\hline 80 & 1.4918 & 1.4913 & 1.4888 & 1.4820 & 1.4765 & 1.4647 & 1.4558 & 1.4415 & 1.4163 & 1.3610 & 1. 2918 \\
\hline 85 & 1.5303 & 1.5297 & 1.5269 & 1.5194 & 1.5133 & 1.5004 & 1.4908 & 1.4755 & 1.4489 & 1.3922 & 1. 3232 \\
\hline 90 & 1.5677 & 1.5670 & 1. 5639 & 1.5556 & 1.5489 & 1.5348 & 1.5244 & 1.5080 & 1.4801 & 1. 4224 & 1.3543 \\
\hline 95 & 1.6035 & 1.6027 & 1. 5992 & 1.5899 & 1.5826 & 1.5671 & 1.5559 & 1.5384 & 1.5092 & 1.4510 & 1.3847 \\
\hline 100 & 1.6380 & 1.6371 & 1.6333 & 1.6229 & 1.6148 & 1.5979 & 1. 5858 & 1. 5672 & 1.5369 & 1.4788 & 1. 4148 \\
\hline 105 & 1.6705 & 1.6696 & 1.6652 & 1.6536 & 1.6446 & 1.6261 & 1.6130 & 1. 5934 & 1.5621 & 1.5047 & 1.4443 \\
\hline 110 & 1.7018 & 1.7007 & 1.6957 & $1.582^{7}$ & 1.6727 & 1.6526 & 1.6385 & 1.6179 & 1.5858 & 1.5300 & 1. 11736 \\
\hline 115 & 1.7307 & 1.7294 & 1.7238 & 1.7091 & 1.6980 & 1.6760 & 1.6609 & 1.6392 & 1.6057 & 1.5532 & 1.5020 \\
\hline 120 & 1.7584 & 1.7569 & 1.7504 & 1.7338 & 1.7213 & 1.6973 & 1.6812 & 1.6587 & 1.6261 & 1. 5759 & 1.5303 \\
\hline 125 & 1.7834 & 1.7817 & 1.7741 & 1.7551 & 1.7411 & 1.7148 & 1.6978 & 1.6746 & 1.6425 & 1.5965 & 1.5576 \\
\hline 130 & 1.8072 & 1.8052 & 1.7963 & 1.7743 & 1.7586 & 1.7299 & 1.7120 & 1.6884 & $1.657 \mathrm{tt}$ & 1.6165 & 1.5843 \\
\hline 135 & 1.8280 & 1.8256 & 1.8149 & 1.7893 & 1.7716 & 1.7405 & 1.7219 & 1.6982 & 1.6691 & 1.6344 & 1.6097 \\
\hline 140 & 1.8478 & 1.8447 & 1.8317 & 1.8015 & 1.7814 & 1.7479 & 1. 7288 & 1.7058 & 1.6793 & 1.6515 & 1.6341 \\
\hline 145 & 1.8643 & 1.8603 & 1.8441 & 1.8080 & 1.7855 & 1.7500 & 1.7310 & 1.7090 & 1.5865 & 1.6665 & 1.5566 \\
\hline 150 & 1.8796 & 1.8744 & 1.8534 & 1.8098 & 1.7846 & 1.7480 & 1.7299 & 1.7106 & 1.6923 & 1.6804 & 1.6773 \\
\hline 155 & 1.8918 & 1.8845 & 1.8563 & 1.8036 & 1.7764 & 1.7405 & 1.7244 & 1.7087 & 1.6959 & 1.6921 & 1.6956 \\
\hline 160 & 1.9021 & 1.8910 & 1.8513 & 1.7886 & 1.7608 & 1. 7287 & 1.7160 & 1.7049 & 1.6982 & 1.7021 & 1.7113 \\
\hline 165 & 1.9117 & 1.8928 & 1.8342 & 1.7634 & 1.7386 & 1.7142 & 1.7060 & 1.7003 & 1.6994 & 1.7101 & 1.7238 \\
\hline 170 & 1.9097 & 1.8725 & 1.7888 & 1.7260 & 1.7102 & 1.6979 & 1.6951 & 1.6948 & 1.6994 & 1.7157 & 1.7330 \\
\hline 175 & 1.9638 & 1.8417 & 1.7317 & 1.6960 & 1.6904 & 1.6883 & 1.6893 & 1.6925 & 1.7003 & 1.7196 & 1.7387 \\
\hline 180 & 1.6740 & 1.6741 & 1.6749 & 1.6770 & 1.6787 & 1.6826 & 1.6856 & 1.6907 & 1.7001 & 1.7210 & 1.7409 \\
\hline
\end{tabular}

QATIO OF MOTT TO MCKINLEY-PESHBACH SCATTERING IN TB, $z=65$

\begin{tabular}{|c|c|c|c|c|c|c|c|c|c|c|c|}
\hline $\begin{array}{l}\text { ENERGY (HEV) } \\
\text { ANGLE (DEG }\end{array}$ & 100 & 10. & 4. & 2. & 1.5 & 1.0 & 0.8 & 0.6 & 0.4 & 0.2 & 0.1 \\
\hline $\begin{array}{l}0 \\
5\end{array}$ & $\begin{array}{l}1.0000 \\
0.9740\end{array}$ & $\begin{array}{l}1.0000 \\
0.9740\end{array}$ & $\begin{array}{l}1.0000 \\
0.9739\end{array}$ & $\begin{array}{l}1.0000 \\
0.9736\end{array}$ & $\begin{array}{l}1.0000 \\
0.0734\end{array}$ & $\begin{array}{l}1.0000 \\
0.9720\end{array}$ & $\begin{array}{l}1.0000 \\
0.0725\end{array}$ & $\begin{array}{l}1.0000 \\
0.9720\end{array}$ & $\begin{array}{l}1.0000 \\
0.0711\end{array}$ & 1.0000 & 1.0000 \\
\hline 10 & 0.9772 & 0.9771 & 0.9768 & $\begin{array}{l}0.976 \\
0.9760\end{array}$ & 0.9753 & 0.9737 & 0.9725 & 0.9706 & 0.9671 & 0.9606 & 0.9586 \\
\hline 15 & 0.9870 & 0.9869 & 0.9863 & 0.9849 & 0.9837 & 0.9810 & 0.9789 & 0.9754 & 0.9690 & 0.9549 & 0.9435 \\
\hline 20 & 1.0129 & 1.0127 & 1.0120 & 1.0100 & 1.0083 & 1.0045 & 1.0016 & 0.9966 & 0.9872 & 0.9651 & 0.9418 \\
\hline 25 & 1.0451 & 1.0449 & 1.0440 & 1.0415 & 1.0394 & 1.0347 & 1.0310 & 1.0248 & 1.0129 & 0.9841 & 0.9504 \\
\hline 30 & 1.0848 & 1.0846 & 1.0835 & 1.0805 & 1.0780 & 1.0725 & 1.0680 & 1.0606 & 1.0463 & 1.0110 & 0.9666 \\
\hline 35 & 1.1287 & 1.1284 & 1.1272 & 1.1237 & 1.1209 & 1.1145 & 1.1095 & 1.1010 & 1.0847 & 1.0441 & 0.9913 \\
\hline 40 & 1.1761 & 1.1758 & 1.1744 & 1.1706 & 1.1673 & 1. 1601 & 1.1545 & 1.1449 & 1.1266 & 1.0809 & 1.0201 \\
\hline 45 & 1.2259 & 1.2256 & 1.2240 & 1.2197 & 1.2161 & 1. 2082 & 1.2019 & 1.1914 & 1.1713 & 1.1214 & 1.0541 \\
\hline 50 & 1.2772 & 1.2769 & 1.2751 & 1.2703 & 1.2664 & 1. 2576 & 1.2507 & 1.2391 & 1.2172 & 1. 1631 & 1.0900 \\
\hline 55 & 1.3296 & 1.3292 & 1.3273 & 1.3220 & 1.3176 & 1. 3080 & 1.3004 & 1. 2879 & 1. 2642 & 1.2065 & 1.1285 \\
\hline 60 & 1.3823 & 1.3818 & 1.3797 & 1.3739 & 1.3691 & 1. 3585 & 1.3503 & 1.3367 & 1. 3112 & 1.2499 & 1.1679 \\
\hline 65 & 1.4351 & 1.4346 & 1.4322 & 1.4258 & 1.4206 & 1.4090 & 1.4001 & 1.3854 & 1.3581 & 1.2935 & 1.2078 \\
\hline 70 & 1.4875 & 1.4869 & 1.4843 & 1.4773 & 1.0715 & 1.4589 & 1. 4092 & 1.4333 & 1.4082 & 1.3365 & 1.2481 \\
\hline 75 & 1.5391 & 1.5385 & 1. 5356 & 1.5279 & 1.5215 & 1.5078 & 1.4972 & 1.4802 & 1.4493 & 1.3788 & 1.2883 \\
\hline 80 & 1.5899 & 1.5892 & 1.5861 & 1.5775 & 1.5705 & 1.5555 & 1.5442 & 1.5259 & 1.4932 & 1.4203 & 1. 3285 \\
\hline 85 & 1.6393 & 1.6385 & $1.635 t$ & 1.6256 & 1.6179 & 1.6016 & 1.5893 & 1.5697 & 1.5352 & 1.4603 & 1. 3682 \\
\hline 90 & 1.6876 & 1.6867 & 1.6828 & 1.6724 & 1.6640 & 1.6462 & 1.6329 & 1.6120 & 1.5758 & 1.0095 & 1.4078 \\
\hline 95 & 1.7338 & 1.7328 & 1.7285 & 1.7169 & 1.7077 & 1.6883 & 1.6740 & 1.6518 & 1.6140 & 1.5369 & 1. $4<>0$ \\
\hline 100 & 1.7787 & 1.7777 & 1.7729 & 1.7600 & 1.7499 & 1.7287 & 1.7134 & 1.6898 & 1.6506 & $1.5>34$ & 1. 11860 \\
\hline 105 & 1.8211 & 1.8199 & 1. 8145 & 1.8002 & 1.7890 & 1.7660 & 1.7495 & 1.7246 & 1.6842 & 1.6079 & 1.5245 \\
\hline 110 & 1.8621 & 1.8607 & 1.8546 & 1.8386 & 1.8263 & 1.8013 & 1.7836 & 1.7575 & 1.7162 & 1.6416 & 1. 5629 \\
\hline 115 & 1.8999 & 1.8983 & 1.8915 & 1.8735 & 1.8598 & 1.8326 & 1.8138 & 1.7865 & 1.7448 & 1.6732 & 1.6008 \\
\hline 120 & 1.9364 & 1.9346 & 1.9268 & 1.9065 & 1.8913 & 1.8616 & 1.8417 & 1.8133 & 1.7716 & 1.7040 & 1.6383 \\
\hline 125 & 1.9692 & 1.9672 & 1.9581 & 1.9350 & 1.9181 & 1.8859 & 1.8648 & 1.8357 & 1.7946 & 1.7324 & 1.6751 \\
\hline 130 & 2.0009 & 1.9984 & 1.9878 & 1.9613 & 1.9023 & 1.9073 & 1. 8852 & 1.8556 & 1.8159 & 1.7601 & 1.7910 \\
\hline 135 & 2.0283 & 2.0253 & 2.0127 & 1.9820 & 1.9607 & 1.9229 & 1.9000 & 1.8706 & 1.8333 & 1.7853 & $1.745^{7}$ \\
\hline 140 & 2.0546 & 2.0510 & 2.0356 & 1.9996 & 1.9755 & 1.9349 & 1.9115 & 1.8828 & 1.8488 & 1.8094 & $1.778^{7}$ \\
\hline 145 & 2.076 & 2.0718 & 2.0526 & 2.0099 & 1.9829 & 1.9400 & 1.9168 & 1.8899 & 1.8605 & 1.8309 & 1.8098 \\
\hline 150 & 2.0970 & 2.0909 & 2.0662 & 2.0147 & 1.9847 & 1.9404 & 1.9182 & 1.8942 & 1.8704 & 1.8509 & 1. 8382 \\
\hline 155 & 2.1131 & 2.1046 & 2.0716 & 2.0096 & 1.9770 & $1.933^{7}$ & 1.9140 & 1.8943 & 1.8773 & 1.8679 & 1.8636 \\
\hline 160 & 2.1268 & 2.1140 & 2.0678 & 1.9937 & 1.9605 & 1.9216 & 1.9059 & 1.8919 & 1.8823 & 1.8827 & 1.8852 \\
\hline 165 & 2.1399 & 2.1181 & 2.0497 & 1.9657 & 1.9357 & 1.9058 & 1.8956 & 1.8889 & 1.8857 & 1.8944 & $1.902^{\circ}$ \\
\hline 170 & 2.1357 & 2.0931 & 1.9959 & 1.9213 & 1.9022 & 1.8871 & 1.8835 & 1.8827 & 1.8871 & 1.9028 & 1.9156 \\
\hline 175 & 2.2197 & 2.0709 & 1.9332 & 1.8874 & 1.8801 & 1.8768 & 1.8777 & 1.8810 & 1.8892 & 1.9084 & 1.9234 \\
\hline 180 & 1.8600 & 1.8602 & 1.8611 & 1.8635 & 1.8655 & 1.8599 & 1.8734 & 1.8790 & 1.8893 & 1.9105 & 1.9260 \\
\hline
\end{tabular}


QTTIO OF MOTT TO MCKINLEY -FESHBACH SCATTFRING IN YB, $Z=70$

$\begin{array}{cccccc}\text { ENERGY (MEV) } & 100 . & 10 . & 4 . & 2 . & 1.5 \\ \text { ANGLE (DEG.1 } & & & & & \\ 0 & 1.0000 & 1.0000 & 1.0000 & 1.0000 & 1.0000 \\ 5 & 0.9681 & 0.9681 & 0.9680 & 0.9678 & 0.9676 \\ 10 & 0.9678 & 0.9677 & 0.9674 & 0.9655 & 0.9657 \\ 15 & 0.9751 & 0.9750 & 0.9744 & 0.9727 & 0.9714 \\ 20 & 1.0018 & 1.0016 & 1.0007 & 0.9983 & 0.9963 \\ 25 & 1.0370 & 1.0367 & 1.0356 & 1.0326 & 1.0301 \\ 30 & 1.0818 & 1.0815 & 1.0802 & 1.0765 & 1.0734 \\ 35 & 1.1326 & 1.1323 & 1.1307 & 1.1264 & 1.1229 \\ 40 & 1.1884 & 1.1880 & 1.1862 & 1.1814 & 1.1773 \\ 45 & 1.2479 & 1.2475 & 1.2455 & 1.2401 & 1.2355 \\ 50 & 1.3099 & 1.3094 & 1.3072 & 1.3011 & 1.2961 \\ 55 & 1.3739 & 1.3733 & 1.3709 & 1.3642 & 1.3587 \\ 60 & 1.4388 & 1.4382 & 1.4355 & 1.4281 & 1.4220 \\ 65 & 1.5044 & 1.5037 & 1.5008 & 1.4926 & 1.4859 \\ 70 & 1.5699 & 1.5692 & 1.5659 & 1.5570 & 1.5497 \\ 75 & 1.6349 & 1.6341 & 1.6306 & 1.6207 & 1.6127 \\ 80 & 1.6993 & 1.6984 & 1.6945 & 1.6837 & 1.6749 \\ 85 & 1.7622 & 1.7612 & 1.7568 & 1.7449 & 1.7353 \\ 90 & 1.8240 & 1.8229 & 1.8181 & 1.8050 & 1.7945 \\ 95 & 1.8834 & 1.8822 & 1.8768 & 1.8624 & 1.8508 \\ 100 & 1.9415 & 1.9402 & 1.9342 & 1.9182 & 1.9056 \\ 105 & 1.9963 & 1.9948 & 1.9882 & 1.9705 & 1.9566 \\ 110 & 2.0497 & 2.0480 & 2.0406 & 2.0209 & 2.0056 \\ 115 & 2.0990 & 2.0971 & 2.0887 & 2.0667 & 2.0499 \\ 120 & 2.1469 & 2.1447 & 2.1352 & 2.1105 & 2.0918 \\ 125 & 2.1899 & 2.1874 & 2.1764 & 2.1485 & 2.1278 \\ 130 & 2.2316 & 2.2287 & 2.2159 & 2.1840 & 2.1609 \\ 135 & 2.2676 & 2.2641 & 2.2490 & 2.2123 & 2.1866 \\ 140 & 2.3025 & 2.2982 & 2.2800 & 2.2371 & 2.2082 \\ 145 & 2.3312 & 2.3258 & 2.3033 & 2.2526 & 2.2204 \\ 150 & 2.3586 & 2.3515 & 2.3226 & 2.2618 & 2.2259 \\ 155 & 2.3799 & 2.3701 & 2.3317 & 2.2586 & 2.2198 \\ 160 & 2.3980 & 2.3833 & 2.3297 & 2.2425 & 2.2029 \\ 165 & 2.4158 & 2.3907 & 2.3112 & 2.2118 & 2.1757 \\ 170 & 2.4092 & 2.3606 & 2.2480 & 2.1597 & 2.1367 \\ 175 & 2.5281 & 2.3492 & 2.1794 & 2.1217 & 2.1122 \\ 180 & 2.0883 & 2.0885 & 2.0895 & 2.0921 & 2.0943 \\ & & & & & \end{array}$

$\begin{array}{ccc}1.0 & 0.8 & 0.6 \\ 1.0000 & 1.0000 & 1.0000 \\ 0.9672 & 0.9669 & 0.9664 \\ 0.9641 & 0.9628 & 0.9609 \\ 0.9683 & 0.9660 & 0.9621 \\ 0.9919 & 0.9884 & 0.9826 \\ 1.0244 & 1.0199 & 1.0125 \\ 1.0565 & 1.0611 & 1.0520 \\ 1.1150 & 1.1087 & 1.0982 \\ 1.1683 & 1.1612 & 1.1492 \\ 1.2255 & 1.2175 & 1.2043 \\ 1.2850 & 1.2762 & 1.2616 \\ 1.3664 & 1.3368 & 1.3208 \\ 1.4086 & 1.3981 & 1.3807 \\ 1.4712 & 1.4598 & 1.4410 \\ 1.5336 & 1.5212 & 1.5010 \\ 1.5952 & 1.5818 & 1.5600 \\ 1.6559 & 1.6414 & 1.6180 \\ 1.7146 & 1.6990 & 1.6740 \\ 1.7720 & 1.7552 & 1.7285 \\ 1.8264 & 1.8083 & 1.7800 \\ 1.8791 & 1.8597 & 1.8297 \\ 1.9278 & 1.9071 & 1.8755 \\ 1.9745 & 1.9523 & 1.9192 \\ 2.0161 & 1.9926 & 1.9581 \\ 2.0552 & 2.0303 & 1.9946 \\ 2.0882 & 2.0620 & 2.0255 \\ 2.1181 & 2.0906 & 2.0536 \\ 2.1405 & 2.1123 & 2.0755 \\ 2.1588 & 2.1300 & 2.0941 \\ 2.1685 & 2.1399 & 2.1063 \\ 2.1724 & 2.1452 & 2.1151 \\ 2.1674 & 2.1431 & 2.1183 \\ 2.1557 & 2.1362 & 2.1183 \\ 2.1391 & 2.1262 & 2.1161 \\ 2.1180 & 2.1132 & 2.1113 \\ 2.1072 & 2.1076 & 2.1105 \\ 2.0991 & 2.1027 & 2.1085\end{array}$

0.4
1.0000
0.9659
0.9575
0.9551
0.9718
0.9984
1.0346
1.0781
1.1263
1.1790
1.2338
1.2907
1.3482
1.4061
1.4636
1.5202
1.5759
1.6296
1.6819
1.7314
1.7992
1.8235
1.8661
1.9045
1.9409
1.9728
2.0025
2.0275
2.0502
2.0681
2.0838
2.0054
2.1044
2.1110
2.1145
2.1182
2.1189

\begin{tabular}{|c|c|}
\hline 0.2 & 0.1 \\
\hline 1.0000 & 1.0000 \\
\hline 0.9661 & 0.9692 \\
\hline 0.9523 & 0.9538 \\
\hline 0.9411 & 0.9334 \\
\hline 0.9477 & 0.9259 \\
\hline 0.9656 & 0.9308 \\
\hline 0.9930 & 0.9443 \\
\hline 1.0291 & 0.9588 \\
\hline 1.0703 & 0.9989 \\
\hline 1.1169 & 1.3360 \\
\hline 1. 1558 & 1.0765 \\
\hline 1.2174 & 1.1208 \\
\hline 1.26599 & 1. 1669 \\
\hline 1.3231 & 1.2946 \\
\hline 1.3763 & 1.2631 \\
\hline 1.4289 & 1.3122 \\
\hline 1.4810 & 1.3617 \\
\hline 1.5318 & 1.4112 \\
\hline 1.5818 & 1. 4608 \\
\hline 1.6300 & 1.5100 \\
\hline 1.6774 & 1.5599 \\
\hline 1.7226 & 1.5096 \\
\hline 1.7659 & 1.6591 \\
\hline 1.8089 & 1.7086 \\
\hline 1.8501 & \\
\hline 1.8887 & 1.8963 \\
\hline 1.9262 & $1.853^{7}$ \\
\hline 1.9610 & 1.9502 \\
\hline 1.9943 & 1,9444 \\
\hline 2.0245 & 1.9854 \\
\hline 2.0524 & 2.0247 \\
\hline $2.076^{7}$ & 2.0593 \\
\hline 2.0977 & 2.0887 \\
\hline 2.1145 & 2.1126 \\
\hline $2.126^{7}$ & 2.1302 \\
\hline 2.1346 & $2.940 B$ \\
\hline & \\
\hline
\end{tabular}

PATIO OF MOTT TO MCKTNLPY-FESHBACH SCATTERING IN HF, $z=72$

$\begin{array}{cc}\text { ENERGY (MEV) } & 100 \\ \text { ANGLE (DEG.) } & \\ 0 & 1.0000 \\ 5 & 0.9657 \\ 10 & 0.9637 \\ 15 & 0.9697 \\ 20 & 0.9962 \\ 25 & 1.0324 \\ 30 & 1.0791 \\ 35 & 1.1327 \\ 40 & 1.1919 \\ 45 & 1.2556 \\ 50 & 1.3222 \\ 55 & 1.3913 \\ 60 & 1.4616 \\ 65 & 1.5330 \\ 70 & 1.5045 \\ 75 & 1.6756 \\ 80 & 1.7463 \\ 85 & 1.8154 \\ 90 & 1.8836 \\ 95 & 1.9491 \\ 100 & 2.0134 \\ 105 & 2.0741 \\ 110 & 2.1334 \\ 115 & 2.1881 \\ 120 & 2.2414 \\ 125 & 2.2893 \\ 130 & 2.3358 \\ 135 & 2.3760 \\ 140 & 2.4150 \\ 145 & 2.4470 \\ 150 & 2.4776 \\ 155 & 2.5015 \\ 160 & 2.5218 \\ 165 & 2.5417 \\ 170 & 2.5343 \\ 175 & 2.6679 \\ 180 & 2.1935 \\ & \\ 185 & \\ 150 & \\ 150 & \\ 150 & \\ 150 & \end{array}$

\begin{tabular}{|c|c|}
\hline 10. & 4. \\
\hline $\begin{array}{l}1.0000 \\
0.0657\end{array}$ & $\begin{array}{l}1.0000 \\
0.9656\end{array}$ \\
\hline 0.9636 & 0.9632 \\
\hline 0.9695 & \\
\hline 0.9960 & 0.9951 \\
\hline 1.0321 & 1.0310 \\
\hline 1.0788 & 1.0773 \\
\hline 1.1323 & 1.1306 \\
\hline 1.1915 & 1.1896 \\
\hline 1.2551 & 1.2530 \\
\hline 1.3216 & 1.3192 \\
\hline 1.3907 & 1.3880 \\
\hline 1.4610 & 1.4581 \\
\hline 1.5322 & 1.5290 \\
\hline 1.6037 & 1.6001 \\
\hline 1.6748 & $\begin{array}{r}1.6708 \\
17410\end{array}$ \\
\hline 1.8143 & 1.8096 \\
\hline 1.8824 & 1.8771 \\
\hline 1.9478 & 1.9419 \\
\hline 2.0119 & 2.0054 \\
\hline 2.0725 & 2.0652 \\
\hline $\begin{array}{l}2.1 .315 \\
2.1860\end{array}$ & 2.1769 \\
\hline 2.2391 & 2.2287 \\
\hline 2.2866 & 2.2747 \\
\hline 2.3326 & 2. 3189 \\
\hline & \\
\hline $\begin{array}{l}2.4104 \\
2.4412\end{array}$ & $\begin{array}{r}2.3909 \\
2.4172\end{array}$ \\
\hline 2.4701 & 2.4393 \\
\hline 2.4911 & 2.4503 \\
\hline 2.5062 & 2.4493 \\
\hline 2.5152 & 2.4308 \\
\hline 2.4831 & 2. 3637 \\
\hline 2.4763 & 2.2925 \\
\hline 2.1937 & 2.1946 \\
\hline
\end{tabular}

2.
1.0000
0.9654
0.9623
0.9672
0.9925
1.0277
1.0733
1.1260
1.1843
1.2470
1.3126
1.3807
1.4499
1.5201
1.5903
1.6600
1.7291
1.7965
1.8627
1.9261
1.9880
2.0459
2.1020
2.1531
2.2020
2.2446
2.2845
2.3165
2.3448
2.3629
2.3743
2.3723
2.3563
2.3245
2.2692
2.2294
2.1973

1.5
1.0000
0.9652
0.9616
0.9657
0.9904
1.0250
1.0700
1.1221
1.1798
1.2421
1.3071
1.3745
1.4433
1.5127
1.5823
1.6512
1.7195
1.7860
1.8512
1.9135
1.9742
2.0308
2.0854
2.1347
2.1817
2.2222
2.2595
2.2888
2.3136
2.3283
2.3358
2.3306
2.3137
2.2857
2.2444
2.2188
2.1995

1.0
1.0000
0.9648
0.9599
0.9626
0.9857
1.0189
1.0626
1.1135
1.1700
1.2311
1.2949
1.3612
1.4285
1.4966
1.5647
1.6320
1.6986
1.7633
1.8266
1.8868
1.9452
1.9993
2.0513
2.0979
2.1418
2.1791
2.2130
2.2389
2.2603
2.2722
2.2780
2.2740
2.2627
2.2459
2.2240
2.2129
2.2043

0.8
1.0000
0.9646
0.9587
0.9602
0.9821
1.0142
1.0568
1.1067
1.1622
1.2224
1.2853
1.3506
1.4170
1.4841
1.5510
1.6173
1.6827
1.7461
1.8081
1.8669
1.9239
1.9766
2.0271
2.0722
2.1146
2.1505
2.1831
2.2081
2.2289
2.2412
2.2484
2.2476
2.2415
2.2318
2.2185
2.2130
2.2079

0.6

1.0000 0.9642 0.9568 0.9562 0.9760 1.0062 1.0470
1.0954 1.0954
1.1492 1. 2079 1.2079
1.2693 1.3331 1.3979 1.4634 1.5287
1.5933 1.5933
1.6569 1. 7185 1. 7787 1.8357 1.8909 1.9419 1.9906 2.0343 2.1104 2. 1425 2. 1678 2. 1896 2.2044
2.2153 2.2153
2.2203 2. 2203 2.2216
2.2202 2.2202 2.2159
2.2156 2.2156
2.2137

0.2 0.1

1.0000 0.9638 0.9535 0.9647 0.9913 1.0284
1.0737 1. 1244 1.1803

1. 2389

1.3000
1.3621

1.3621
1.4249

1. 4249

1. 5494

1.6104
1.6694

1.7271

1.7819
1.8350

1. 83844

1.9319

1.9750

2.0521

2.0859

2.1146
2.1408

2. 1618

2. 1803

2.1943

2.2054
2.2136

2.2136
2.2182

2.2227
2.2236
1.0000 0.9645 0.9491 0.9355 0.9402 0.9571 0.9843 1.0214 1.0641 1. 1649 1.2200 1.2200
1.2762 1.2762 1.3336
1.3912 1.4485 1.5054 1.5610
1.6160 1.6160
1.6691 1.6691
1.7214 1.7716 1.8209
1.8579 1.8679 1.9139
1.9573 1.9995 2.0389 2.0765 2. 1110 2.1427 2.1706 2.1946 2.2138 2.2369 2.2369
2.2402
1.0000 0.9680
0.9523 0.9298 $0.919^{-}$ 0.9227 0.9346 0.9888 1.0269 1.0690

1. 1155

1.1643
1.2151 1. 2151
1. 2672 1. 3201 1.3201
1.3735 1.4273 1.4813
1.5356 1. 5900 1.8447 1. 6992 1.7541
1.8083 1.8626 1.9155 1. 9675 2. 3169 2. 1071 2. 1460 2.1791 2.2061 2. 2260
2.2379 2.2379
2.2414 
RATTO OF MOTT TO MCKINLPY-FESHBACP SCPTTERING IN TA, $z=73$

\begin{tabular}{|c|c|c|c|c|c|c|c|c|c|c|c|}
\hline $\begin{array}{l}\text { ENERGY (HEV) } \\
\text { ANGLE (DEG.) }\end{array}$ & 100. & 10 & 4. & 2. & 1,5 & 1.0 & 0.8 & 0.6 & 0.4 & 0.2 & 0.1 \\
\hline $\begin{array}{l}0 \\
5\end{array}$ & 1.0000 & 1.0000 & 1.0000 & 1.0000 & 1.0000 & 1.0000 & 1.0000 & 1.0000 & 1.0000 & 1.0000 & 1.0000 \\
\hline 5 & 0.9645 & 0.9645 & 0.9644 & 0.9642 & 0.9640 & 0.9637 & $0.963 \mathrm{u}$ & 0.9631 & 0.9528 & 0.9637 & 0.9674 \\
\hline 10 & 0.9515 & 0.9615 & 0.9611 & 0.9602 & 0.9594 & 0.9578 & 0.9566 & 0.9547 & 0.9516 & 0.0476 & 0.9516 \\
\hline 15 & 0.9668 & 0.9666 & 0.9660 & 0.9642 & 0.9628 & 0.9596 & 0.9571 & 0.9531 & 0.9460 & 0.9327 & 0.9281 \\
\hline 20 & 0.9932 & 0.9930 & 0.9920 & 0.9894 & 0.9872 & 0.9824 & 0.9787 & 0.9725 & 0.9510 & 0.9363 & 0.9167 \\
\hline 25 & 1.0298 & 1.0295 & 1.0283 & 1.0249 & 1.0221 & 1.0159 & 1.0110 & 1.0028 & 0.9875 & $0.952^{7}$ & 0.9186 \\
\hline 30 & 1.0774 & 1.0771 & 1.0756 & 1.0714 & 1.0680 & 1.0603 & 1.0542 & 1.0441 & 1.0249 & 0.9797 & 0.9296 \\
\hline 35 & 1.1324 & 1.1320 & 1.1302 & 1.1254 & 1. 1214 & 1. 1124 & 1.1054 & 1.0935 & 1.0711 & 1.0171 & 0.9531 \\
\hline 40 & 1.1933 & 1.1929 & 1.1909 & 1. 1853 & $1.180^{7}$ & 1. 1705 & 1.1624 & 1.1488 & 1. 1230 & 1.0606 & 0.9834 \\
\hline 45 & 1.2591 & 1.2586 & 1.2564 & 1.2502 & 1. 2450 & 1.2335 & 1.2245 & 1.2094 & 1.1806 & 1.1107 & 1.2210 \\
\hline 50 & 1.3281 & 1.3275 & 1.3250 & 1.3181 & 1.3123 & 1.2996 & 1.2895 & 1.2728 & 1.2410 & 1.1639 & 1.06118 \\
\hline $\begin{array}{l}55 \\
60\end{array}$ & $\begin{array}{l}1.3999 \\
1.4731\end{array}$ & $\begin{array}{l}1.3992 \\
1.4724\end{array}$ & $\begin{array}{l}1.3965 \\
1.4693\end{array}$ & $\begin{array}{l}1.3888 \\
1.4608\end{array}$ & $\begin{array}{l}1.3824 \\
1.4538\end{array}$ & $\begin{array}{l}1.3683 \\
1.4384\end{array}$ & $\begin{array}{l}1.3573 \\
1.4263\end{array}$ & $\begin{array}{l}1.3390 \\
1.0063\end{array}$ & $\begin{array}{l}1.3044 \\
1.3688\end{array}$ & $\begin{array}{l}1.2208 \\
1.2790\end{array}$ & $\begin{array}{l}1.1123 \\
1.1625\end{array}$ \\
\hline $\begin{array}{l}60 \\
65\end{array}$ & $\begin{array}{l}1.4731 \\
1.5474\end{array}$ & 1.5467 & 1.5433 & 1.5339 & 1.5262 & 1.5093 & 1.4962 & $\begin{array}{l}1.0063 \\
1.4745\end{array}$ & $\begin{array}{l}1.3688 \\
1.4342\end{array}$ & $\begin{array}{l}1.2790 \\
1.3386\end{array}$ & $\begin{array}{l}1.1625 \\
1.2140\end{array}$ \\
\hline 70 & 1.6221 & 1.6213 & 1.6175 & 1.6072 & 1.5988 & 1. 5804 & 1.5661 & 1.5427 & 1.4995 & 1.3984 & 1. $268^{7}$ \\
\hline 75 & 1.6965 & 1.6956 & 1.6915 & 1.6802 & 1.6709 & 1.6508 & 1.6353 & 1.6102 & $1.56 \mathrm{ul}$ & 1.4581 & 1. 3236 \\
\hline 80 & 1.7705 & 1.7695 & 1.7649 & 1.7525 & 1.7424 & 1.7205 & 1.7038 & 1.6768 & 1.6279 & 1.5175 & 1. 3791 \\
\hline 85 & 1.8429 & 1.8418 & 1.8368 & 1.8231 & 1.8121 & 1.7983 & 1.7703 & 1.7414 & 1.6898 & 1.5757 & 1.4351 \\
\hline 90 & 1.9144 & 1.9132 & 1.9077 & 1.8927 & 1.8806 & 1.8548 & 1.8354 & 1.8045 & 1.7503 & 1.6332 & 1.4911 \\
\hline 95 & 1.9833 & 1.9819 & 1.9758 & 1.9593 & 1.9461 & 1.9180 & 1.8972 & 1.8645 & 1.8079 & 1.6890 & 1.5082 \\
\hline 100 & 2.0509 & 2.0494 & 2.0426 & 2.0244 & 2.0099 & 1.9795 & 1.9572 & 1.9226 & 1.8638 & 1.7440 & 1.6050 \\
\hline 105 & 2.1147 & 2.1130 & 2.1055 & 2.0854 & 2.0695 & 2.0365 & 2.0128 & 1.9764 & 1.9159 & 1.7968 & 1.6624 \\
\hline 110 & 2.1772 & 2.1753 & 2.1668 & 2.1445 & 2.1271 & 2.0915 & 2.0661 & 2.0279 & 1.9661 & 1.8488 & 1. 7196 \\
\hline 115 & 2.2349 & 2.2327 & 2.2232 & 2.1984 & 2.1792 & 2.1408 & 2.1139 & 2.0741 & $2.011^{7}$ & 1.8984 & 1.7773 \\
\hline 120 & 2.2911 & 2.2886 & 2.2779 & 2.2500 & 2.2289 & 2. $18>3$ & 2. 1588 & 2.1177 & 2.0552 & 1.9469 & 1. 8344 \\
\hline $\begin{array}{l}125 \\
130\end{array}$ & $\begin{array}{l}2.3416 \\
2.3907\end{array}$ & $\begin{array}{r}2.3388 \\
2.3874\end{array}$ & $\begin{array}{l}2.3265 \\
2.3731\end{array}$ & $\begin{array}{r}2.2951 \\
2.3374\end{array}$ & $\begin{array}{l}2.2718 \\
2.3114\end{array}$ & 2.2269 & & 2.1550 & $\begin{array}{l}2.0936 \\
2.1296\end{array}$ & 1.9930 & \\
\hline $\begin{array}{l}130 \\
135\end{array}$ & 2.4331 & 2.4293 & 2.4125 & 2.3715 & 2.3426 & $\begin{array}{l}2.2630 \\
2.290^{7}\end{array}$ & $\begin{array}{l}2.2317 \\
2.2585\end{array}$ & $\begin{array}{l}2.1891 \\
2.2163\end{array}$ & $\begin{array}{l}2.1296 \\
2.1603\end{array}$ & $\begin{array}{l}2.0377 \\
2.0796\end{array}$ & $\begin{array}{l}1.9473 \\
2.0023\end{array}$ \\
\hline 140 & 2.4743 & 2.4696 & 2.4494 & 2.4016 & 2.3693 & 2. 3137 & 2.2810 & 2.2398 & 2.1884 & 2.1196 & 2.0545 \\
\hline 145 & 2.5082 & 2.5022 & 2.4774 & 2.4212 & 2.3853 & 2.3270 & 2.2946 & 2.2561 & 2.2192 & 2.1563 & 2.1045 \\
\hline 150 & 2.5406 & 2.5328 & 2.5010 & 2.4338 & 2.3939 & 2.3338 & 2.3029 & 2.2683 & 2.2311 & 2.1901 & 2.1500 \\
\hline 155 & 2.5659 & 2.5551 & 2.5131 & 2.4324 & 2.3893 & 2.3304 & 2.3028 & 2.2742 & 2.2465 & 2.2199 & 2.1913 \\
\hline 160 & 2.5873 & 2.5713 & 2.5127 & 2.4166 & 2.3724 & 2.3193 & 2.2971 & 2.2762 & 2.2587 & 2.2455 & 2.2264 \\
\hline 165 & 2.6083 & 2.5811 & 2.4941 & 2.3842 & 2.3439 & 2.3025 & 2.2876 & 2.2754 & 2.2678 & 2.2661 & 2. 2550 \\
\hline 170 & 2.6006 & 2.5480 & 2.4250 & 2.3273 & 2.3015 & 2.2802 & 2.2743 & 2.2713 & 2.2731 & 2.2812 & 2.2762 \\
\hline 175 & 2.7417 & 2.5435 & 2.3524 & 2.2865 & 2.2753 & 2.2690 & 2.2689 & 2.2713 & $2.2^{7} 80$ & 2.2908 & 2.2888 \\
\hline 180 & 2.2493 & 2.2495 & 2.2505 & 2.2532 & 2.2554 & 2.2601 & $2.263^{7}$ & 2.2694 & 2.2790 & 2.2942 & 2.2924 \\
\hline
\end{tabular}

RATTO OF MOTT TO MCKINIFY-FESHBACH SCATTERTNG IN

ENERGY (HEV)
ANGIE (DEG.)
0
5
10
15
20
25
30
35
40
45
50
55
60
65
70
75
80
85
90
95
100
105
110
115
120
125
130
135
140
145
150
155
160
165
170
175
180

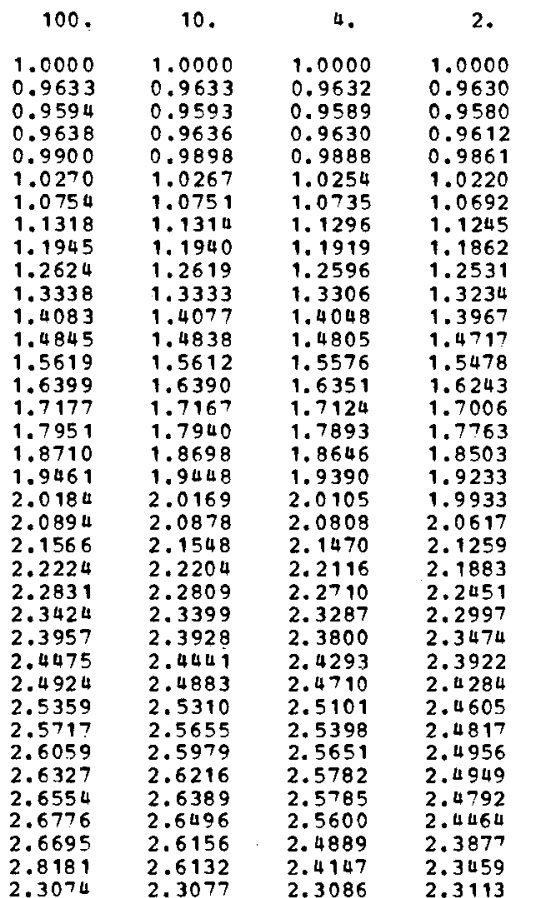

1.5
1.0000
0.9628
0.9573
0.9597
0.9839
1.0191
1.0657
1.1204
1.1814
1.2477
1.3174
1.3901
1.4643
1.5398
1.6155
1.6909
1.7657
1.8388
1.9106
1.9794
2.0466
2.1094
2.1701
2.2252
2.2777
2.3231
2.3652
2.3984
2.4270
2.4444
2.4542
2.4502
2.4334
2.4045
2.3610
2.3342
2.3135

1.0
1.0000
0.9625
0.9556
0.9565
0.9790
1.0126
1.0577
1.1111
1.1707
1.2357
1.3040
1.3754
1.4482
1.5221
1.5962
1.6698
1.7428
1.8138
1.8836
1.9501
2.0148
2.0750
2.1329
2.1850
2.2342
2.2762
2.3147
2.3443
2.3692
2.3838
2.3917
2.3890
2.3782
2.3614
2.3386
2.3273
2.3182

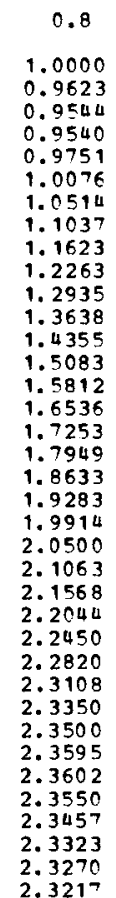

0.6
1.0000
0.9620
0.9526
0.9499
0.9688
0.9992
1.0410
1.0915
1.1482
1.2105
1.2760
1.3447
1.4146
1.4856
1.5567
1.6272
1.6969
1.7646
1.8309
1.8939
1.9551
2.0118
2.0662
2.1151
2.1613
2.2010
2.2374
2.2665
2.2919
2.3096
2.3232
2.3301
2.3329
2.3326
2.3289
2.3291
2.3272

$\begin{array}{cc}0.2 & 0.1 \\ 1.0000 & 1.0900 \\ 0.9630 & 0.9568 \\ 0.9461 & 0.9509 \\ 0.9298 & 0.9265 \\ 0.9324 & 0.9137 \\ 0.9482 & 0.9145 \\ 0.9749 & 0.9245 \\ 1.0126 & 0.9476 \\ 1.0567 & 0.9777 \\ 1.1079 & 1.9166 \\ 1.1626 & 1.0602 \\ 1.2212 & 1.1088 \\ 1.2814 & 1.1503 \\ 1.3432 & 1.2143 \\ 1.4054 & 1.2699 \\ 1.4676 & 1.3267 \\ 1.5295 & 1.3844 \\ 1.5904 & 1.4427 \\ 1.6506 & 1.5014 \\ 1.7092 & 1.5607 \\ 1.7669 & 1.6201 \\ 1.8225 & 1.6802 \\ 1.8772 & 1.7402 \\ 1.9295 & 1.9009 \\ 1.9808 & 1.8608 \\ 2.0296 & 1.9212 \\ 2.0769 & 1.9799 \\ 2.1214 & 2.2379 \\ 2.1639 & 2.0931 \\ 2.2030 & 2.1459 \\ 2.2390 & 2.1941 \\ 2.2708 & 2.2379 \\ 2.2982 & 2.2751 \\ 2.3202 & 2.3055 \\ 2.3364 & 2.3279 \\ 2.3466 & 2.3413 \\ 2.3502 & 2.3451 \\ & \\ 1.979 & \end{array}$


RATTC OF MOTT TO MCKTNLEX-FESHBACH SCATTFRING IN AD, $z=79$

\begin{tabular}{|c|c|c|c|c|c|c|c|c|c|c|c|}
\hline $\begin{array}{l}\text { ENERGY (MEV) } \\
\text { ANGLE (DEG.) }\end{array}$ & 100. & 10. & 4. & 2. & 1.5 & 1.0 & 0.8 & 0.6 & 0.4 & 0.2 & 0.1 \\
\hline 0 & 1.0000 & 1.0000 & 1.0000 & 1.0000 & 1.0000 & 1.0000 & 1.0000 & 1.0000 & 1.0000 & 1.0000 & 1.0000 \\
\hline 5 & 0.9572 & 0.9572 & 0.9571 & 0.9570 & 0.9569 & 0.9567 & 0.9567 & 0.9566 & 0.9569 & 0.9593 & 0.9636 \\
\hline 10 & 0.9480 & 0.9479 & 0.9476 & 0.9467 & 0.9460 & 0.9446 & 0.9435 & 0.9420 & 0.9398 & 0.9392 & 0.9484 \\
\hline 15 & 0.9472 & 0.9471 & 0.9464 & 0.9445 & 0.9430 & 0.9397 & 0.9371 & 0.9331 & 0.9264 & 0.9162 & 0.9108 \\
\hline 20 & 0.9715 & 0.9713 & 0.9702 & 0.9672 & 0.9647 & 0.9593 & 0.9551 & 0.9483 & 0.9361 & 0.9123 & 0.9001 \\
\hline 25 & 1.0095 & 1.0092 & 1.0077 & 1.0037 & 1.0004 & 0.9930 & 0.9873 & 0.9779 & 0.9605 & $0.923^{7}$ & 0.8947 \\
\hline 30 & 1.0616 & 1.0612 & 1.0593 & 1.0543 & 1.0501 & 1.0407 & 1.0334 & 1.0213 & 0.9986 & $0.9 u>9$ & $0.898^{7}$ \\
\hline 35 & 1.1245 & 1.1241 & 1.1219 & 1.1158 & 1.1108 & 1.0999 & 1.0910 & 1.0765 & 1.0492 & 0.9861 & 0.9181 \\
\hline 40 & 1.1960 & 1.1954 & 1.1929 & 1.1858 & 1.1800 & 1. 1671 & 1. 1569 & 1.1399 & 1.1079 & 1.0327 & 0.9465 \\
\hline 45 & 1.2751 & 1.2744 & 1.2716 & 1.2636 & 1.2570 & 1.2423 & 1.2308 & 1.2115 & 1.1752 & 1.0890 & 0.9859 \\
\hline 50 & 1.3593 & 1.3586 & 1.3553 & 1.3464 & 1.3389 & 1.3224 & 1.3095 & 1.2880 & 1.2474 & 1.1506 & 1.0324 \\
\hline 55 & 1.4485 & 1.4477 & 1.4440 & 9.4340 & 1.4258 & 1.4075 & 1.3932 & 1.3694 & 1.3246 & 1.2181 & 1.2855 \\
\hline 60 & 1.5406 & 1.5397 & 1.5357 & 1.5246 & $1.515 \mathrm{~b}$ & 1.4952 & 1.4794 & 1.4533 & 1.4044 & 1. 2885 & 1.1435 \\
\hline 65 & 1.6352 & 1.6342 & 1.6298 & 1.6176 & 1.6075 & 1.5853 & 1.5680 & 1.5395 & 1.4865 & 1.3618 & 1.2053 \\
\hline 70 & 1.7313 & 1.7302 & 1.7254 & 1.7119 & 1.7008 & 1.6765 & 1.6577 & 1.6268 & 1.5696 & 1.4366 & 1.2701 \\
\hline 75 & 1.8280 & 1.8268 & 1.8214 & 1.8065 & 1.7944 & 1.7678 & $1.747 \mathrm{a}$ & 1.7140 & 1.6528 & 1.5122 & $1.33^{7} 2$ \\
\hline 80 & 1.9249 & 1.9236 & 1.9177 & 1.9013 & 1.8880 & 1.8591 & 1.8370 & 1.8011 & 1.7359 & 1. 5882 & 1,4060 \\
\hline 85 & 2.0206 & 2.0191 & 2.0125 & 1.9946 & 1.9801 & $1.948^{7}$ & 1.9248 & 1.8863 & 1.8172 & 1.6637 & $1.4 \div 65$ \\
\hline 90 & 2.1157 & 2.1141 & 2.1069 & 2.0872 & 2.0713 & 2.0372 & 2.0115 & 1.9704 & 1.8976 & 1.7389 & 1.5479 \\
\hline 95 & 2.2079 & 2.2061 & 2.1981 & 2.1765 & 2.1599 & 2.1222 & 2.0946 & 2.0510 & 1.9748 & $1.812^{7}$ & 1.5211 \\
\hline 100 & 2.2989 & 2.2969 & 2.2881 & 2.2643 & 2.2450 & 2.2054 & 2.1759 & 2.1296 & 2.0504 & 1.8858 & 1.6946 \\
\hline 105 & 2.3853 & 2.3832 & 2.3734 & 2.3472 & 2.3265 & 2.2834 & 2.2519 & 2.2033 & 2.1216 & 1.9572 & 1.7701 \\
\hline 110 & 2.4703 & 2.4679 & 2.4570 & 2.4281 & 2.4054 & 2.3588 & 2.3253 & 2.2743 & 2.1906 & 2.0274 & 1.8450 \\
\hline 115 & 2.5499 & 2.5464 & 2.5343 & 2.5023 & 2.4775 & 2.4273 & 2.3919 & 2.3390 & 2.2543 & 2.0957 & 1.9227 \\
\hline 120 & 2.6263 & 2.6232 & 2.6095 & 2.5738 & 2.5466 & 2.4925 & 2.4551 & 2.4003 & 2.3154 & 2.1624 & 1.9991 \\
\hline 125 & 2.6959 & 2.6923 & 2.6768 & 2.6369 & 2.6070 & 2.5490 & 2.5098 & 2.4540 & 2.3705 & 2.2269 & 2.2769 \\
\hline 130 & 2.7637 & 2.7596 & 2.7416 & 2.6965 & 2.6634 & 2.6010 & 2. 5602 & 2.5037 & 2.4224 & 2.2894 & 2. 1527 \\
\hline 135 & 2.8226 & 2.8178 & 2.7969 & 2.7455 & 2.7090 & 2.6425 & 2.6006 & 2.5446 & 2.4679 & 2.3492 & 2.2284 \\
\hline 140 & 2.8797 & 2.8739 & 2.8489 & 2.7895 & 2.7488 & 2.6779 & 2.6354 & 2.5808 & 2.5098 & 2.4050 & 2.3003 \\
\hline 145 & 2.9270 & 2.9198 & 2.8994 & 2.8200 & 2.7750 & 2.7010 & 2.6590 & $2.60^{78}$ & 2.5452 & 2.4592 & 2.3690 \\
\hline 150 & 2.9721 & 2.9627 & 2.9241 & 2.8416 & 2.7918 & 2.7157 & 2.6756 & 2.6293 & 2.5765 & 2.5080 & 2. 4334 \\
\hline 155 & 3.0076 & 2.9947 & 2.9440 & 2.8455 & 2.7919 & 2.7173 & 2.6813 & 2.6426 & 2.5018 & 2.5517 & 2.4910 \\
\hline 160 & 3.0376 & 3.0186 & 2.9483 & 2.8312 & 2.7763 & 2.7087 & 2.6795 & 2.6504 & 2.6225 & 2.5893 & 2.5408 \\
\hline 165 & 3.0668 & 3.0345 & 2.9304 & 2.7960 & 2.7456 & 2.6922 & 2.6720 & 2.6538 & 2.6384 & 2.6198 & 2. 5813 \\
\hline 170 & 3.0580 & 2.9962 & 2.8490 & 2.7287 & 2.6961 & 2.6676 & 2.6587 & 2.6522 & 2.6486 & 2.6423 & 2.5112 \\
\hline 175 & 3.2423 & 3.0030 & 2.7654 & 2.6810 & 2.6659 & 2.6558 & 2.6539 & 2.6540 & 2.6565 & 2.6569 & 2.6291 \\
\hline 180 & 2.6361 & 2.6363 & 2.6372 & 2.6305 & 2.6414 & 2.6454 & 2.6483 & 2.6525 & 2.6586 & $2.660^{7}$ & 2.6339 \\
\hline
\end{tabular}

RATTO OF MOTT TO MCKTNLEY-FESHBACH SCATTERTNG IN PB, $z=82$

ENERGY
ANGLE (
1
1
2
2
3
3
4
4
5
5
6
6
7
7
8
8
9
9
10
10
11
115
120
12
130
135
140
14
15
15
16
16
17
17
18

\begin{tabular}{|c|c|c|c|}
\hline 100 & 10. & 4. & 2. \\
\hline 1.0000 & 1.0000 & 1.0000 & 1.0000 \\
\hline 0.9536 & 0.9536 & 0.9535 & 0.9535 \\
\hline 0.9409 & 0.9408 & 0.9405 & 0.9397 \\
\hline 0.9363 & 0.9361 & 0.9354 & 0.9335 \\
\hline 0.9584 & 0.9581 & 0.9570 & 9539 \\
\hline 0.9961 & 0.9958 & 0.9942 & 0.9899 \\
\hline 1.0497 & 1.0493 & 1.0473 & 1.0417 \\
\hline 1.1162 & 1.1157 & 1.1133 & 1.1066 \\
\hline 1.1928 & 1.1921 & 1.1893 & 1.1815 \\
\hline 1.2788 & 1.2781 & 1.2748 & 1.2659 \\
\hline 1.3712 & 1.3704 & 1.3667 & 1.3566 \\
\hline 1.6699 & 1.4690 & 1.4649 & 1.4536 \\
\hline 1.5727 & 1.5717 & 1.5671 & 1.5545 \\
\hline 1.6790 & 1.6779 & 1.6728 & 1.6589 \\
\hline 1.7876 & 1.7863 & 1.7807 & 1.7653 \\
\hline 1.8973 & 1.8959 & 1.8898 & 1.8728 \\
\hline 2.0080 & 2.0064 & 1.9996 & 1.9810 \\
\hline 2.1175 & 2.1159 & 2.1084 & 2.0879 \\
\hline 2.2270 & 2.2252 & 2.2169 & 2.1944 \\
\hline 2.3334 & 2.3313 & 2.3222 & 2.2975 \\
\hline 2.4388 & 2.4366 & 2.4265 & 2.3993 \\
\hline 2.5393 & 2.5367 & 2.5256 & 2.4957 \\
\hline 2.6382 & 2.6354 & 2.6230 & 2.5900 \\
\hline 2.7303 & 2.7272 & 2.7134 & 2.6770 \\
\hline 2.8205 & 2.8169 & 2.8014 & 2.7609 \\
\hline 2.9021 & 2.8981 & 2.8806 & 2.8355 \\
\hline 2.9817 & 2.9770 & 2.9569 & 2.9060 \\
\hline 3.0511 & 3.0457 & 3.0224 & 2.9646 \\
\hline 3.1183 & 3.1118 & 3.0840 & 3.0174 \\
\hline 3.1742 & 3.1662 & 3.1324 & 3.0551 \\
\hline 3.2273 & 3.2169 & 3.1743 & 3.0826 \\
\hline 3.2694 & 3.2553 & 3.1995 & 3.0903 \\
\hline 3.3050 & 3.2841 & 3.2071 & 3.0774 \\
\hline 3.3390 & 3.3038 & 3.1899 & 3.0409 \\
\hline 3.3309 & 3.2638 & 3.1022 & 2.9683 \\
\hline $\begin{array}{l}3.5335 \\
2.8674\end{array}$ & $\begin{array}{l}3.2730 \\
2.8675\end{array}$ & $\begin{array}{l}3.0106 \\
2.8683\end{array}$ & $\begin{array}{l}2.9159 \\
2.8702\end{array}$ \\
\hline
\end{tabular}

1.5
1.0000
0.9534
0.9391
0.9320
0.9513
0.9864
1.0372
1.1011
1.1750
1.2585
1.3482
1.4442
1.5441
1.6474
1.7527
1.8589
1.9657
2.0712
2.1761
2.2776
2.3776
2.4721
2.5642
2.6487
2.7300
2.8015
2.8685
2.9234
2.9716
3.0046
3.0268
3.0303
3.0160
2.9843
2.9313
2.8984
2.8717

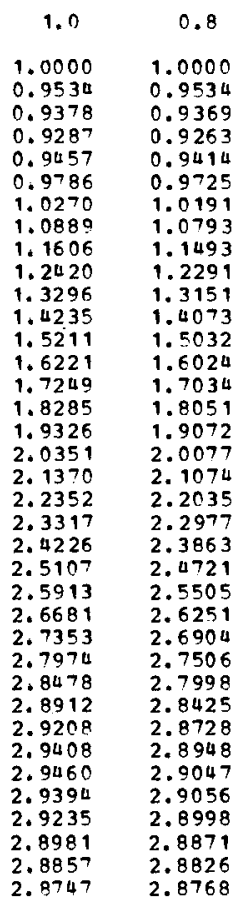

0.6

1.0000

0.9536
0.9357

0.9225

0.9344

0.9626

1.0061
1.0635

1.0635
1.1306

1.1306
1.2077

1.2909
1.3804

1.3804
1.4735

1.4735
1.5699

1.5699
1.6680

1. 7668

1.8659
1.9634

1.9634
2.0600

2.1531

2.2443

2.3302

2.4132
2.4894

2.4894
2.5518

2. 5259

2.6853

2.7352

2.7794

2.8135
2.8411

2.8594

2. 8711

2.8774
2.8778

2.87807
2.88796

2.8807
2.8796

0.4
1.0000
0.9542
0.9342
0.9163
0.9222
0.9446
0.9820
1.0339
1.0955
1.1674
1.2455
1.3300
1.4181
1.5095
1.6028
1.6967
1.7909
1.8838
1.9759
2.0649
2.1523
2.2353
2.3158
2.3908
2.4627
2.5283
2.5901
2.6451
2.6957
2.7393
2.7779
2.8097
2.8359
2.8562
2.8698
2.8796
2.8823

0.2

1.0000 0.9573 0.9358 0.9086 0.908 ? 0.9087 0.9295 0.9671 1.0143 1.0731 1. 1385 1.2111 1. 3685 1. 4515 1. 5359 1.6213 1. 7067 1. 7921 1. 8756 $1.960 \mathrm{~L}$ 2.0428 2. 1240 2.2036 2.2814 2.3572 2.3572 2. 4306 2.5015 2.5687
2.6322 2.6322
2.6904 2.6904
2.7429 2.7880 2. 8247 2.8520 2.8685 2.8738

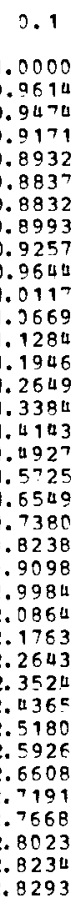


RATIO OF MOTT TO MCKINLEY-FFSHBACH SCATTERING IN FP, $z=87$

\begin{tabular}{|c|c|c|c|c|c|c|c|c|c|c|c|}
\hline $\begin{array}{l}\text { ENERGY (HEV) } \\
\text { ANGLE (DEG.) }\end{array}$ & 100 & 10. & 4. & 2. & 1.5 & 1.0 & 0.8 & 0.6 & 0.4 & 0.2 & 0.1 \\
\hline 0 & 1.0000 & 1.0000 & 1.0000 & 1.0000 & 1.0000 & 1.0000 & 1.0000 & 1.0000 & 1.0000 & 1.0000 & 1.0000 \\
\hline 5 & 0.9478 & 0.9478 & 0.9479 & 0.9479 & 0.9480 & 0.9482 & 0.9484 & 0.9489 & 0.9501 & 0.9539 & 0.9572 \\
\hline 10 & 0.9290 & 0.9289 & 0.9287 & 0.9281 & 0.9277 & 0.9269 & 0.9264 & 0.9258 & 0.9259 & 0.9315 & 0.9461 \\
\hline 15 & 0.9165 & 0.9164 & 0.9157 & 0.9140 & 0.9126 & 0.9096 & 0.9075 & 0.9043 & 0.8997 & 0.8976 & 0.9146 \\
\hline 20 & 0.9333 & 0.9330 & 0.9318 & 0.9286 & 0.9260 & 0.9203 & 0.9160 & 0.9092 & 0.8978 & 0.8805 & 0.8846 \\
\hline 25 & 0.9689 & 0.9685 & 0.9668 & 0.9621 & 0.9583 & 0.9499 & 0.9435 & 0.9334 & 0.9149 & 0.8812 & 0.8678 \\
\hline 30 & 1.0233 & 1.0228 & 1.0205 & 1.0143 & 1.0092 & 0.9979 & 0.9892 & 0.9749 & 0.9491 & 0.8962 & 0.8586 \\
\hline 35 & 1.0947 & 1.0940 & 1.0913 & 1.0836 & 1.0773 & 1.0633 & 1.0524 & 1.0345 & 1.0016 & 0.9306 & 0.8673 \\
\hline 40 & 1.1790 & 1.1783 & 1.1750 & 1.1658 & 1.1582 & 1.1414 & 1.1283 & 1.1066 & 1.0665 & $0.9>72$ & 0.8883 \\
\hline 45 & 1.2765 & 1.2757 & 1.2718 & 1.2611 & 1.2523 & 1.2328 & 1.2175 & 1.1923 & 1.1453 & 1.0384 & 0.9237 \\
\hline 50 & 1.3831 & 1.3822 & 1.3778 & 1.3655 & 1. 3554 & 1.3331 & 1.3156 & 1.2867 & 1.2329 & 1. 1092 & $0.9^{7} 0^{-}$ \\
\hline 55 & 1.4991 & 1.4980 & 1.4930 & 1.4792 & 1.4678 & 1.4426 & 1.4229 & 1.3904 & 1.3298 & 1. 1898 & 1.3279 \\
\hline 60 & 1. 6214 & 1.6201 & 1.6145 & 1.5990 & 1.5863 & 1. 5581 & 1.5361 & 1.4999 & 1.4326 & 1.2770 & $\begin{array}{l}1.0940 \\
1.1670\end{array}$ \\
\hline 65 & & 1.7481 & $\begin{array}{l}1.7418 \\
1.8732\end{array}$ & 1.7246 & & $\begin{array}{r}1.6791 \\
1\end{array}$ & & 1.6149 & 1.5408 & $\begin{array}{r}1.3701 \\
1.675\end{array}$ & $\begin{array}{l}1.1670 \\
1.2461\end{array}$ \\
\hline $\begin{array}{l}70 \\
75\end{array}$ & $\begin{array}{l}1.8817 \\
2.0166\end{array}$ & $\begin{array}{l}1.8801 \\
2.0149\end{array}$ & $\begin{array}{l}1.8732 \\
2.0072\end{array}$ & $\begin{array}{l}1.8541 \\
1.9861\end{array}$ & $\begin{array}{l}1.8384 \\
1.9687\end{array}$ & $\begin{array}{l}1.8039 \\
1.9308\end{array}$ & $\begin{array}{l}1.7772 \\
1.9016\end{array}$ & $\begin{array}{l}1.7333 \\
1.8539\end{array}$ & $\begin{array}{l}1.5526 \\
1.7665\end{array}$ & $\begin{array}{l}1.4675 \\
1.5679\end{array}$ & $\begin{array}{l}1.2461 \\
1.3302\end{array}$ \\
\hline 80 & 2.1538 & 2.1519 & 2.1434 & 2.1201 & 2.1010 & 2.0596 & 2.0277 & 1.9760 & 1.8820 & 1.6706 & 1. 1184 \\
\hline 85 & 2.2908 & $2.288^{7}$ & 2.2793 & 2.2537 & 2.2328 & 2.1876 & 2.1531 & 2.0973 & 1.9970 & 1.7745 & $1.510^{7}$ \\
\hline 90 & 2.4285 & 2.4262 & 2.4159 & 2.3877 & 2.3648 & 2. 3156 & 2.2782 & 2.2184 & 2.1118 & 1.8792 & 1.6057 \\
\hline 95 & 2.5634 & 2.5608 & 2.5494 & 2.5184 & 2.4935 & 2.4401 & 2.4000 & 2.3362 & 2.2241 & 1.9840 & 1.7048 \\
\hline $\begin{array}{l}100 \\
105\end{array}$ & 2.6976 & 2.6948 & 2. 6822 & 2.6481 & 2.6209 & 2.5631 & 2.5200 & 2.4522 & 2.3347 & 2.0883 & 1.8057 \\
\hline $\begin{array}{l}105 \\
110\end{array}$ & $\begin{array}{l}2.8264 \\
2.9535\end{array}$ & $\begin{array}{l}2.8233 \\
2.9501\end{array}$ & $\begin{array}{l}2.8094 \\
2.9346\end{array}$ & $\begin{array}{l}2.7720 \\
2.8935\end{array}$ & $\begin{array}{l}2.7423 \\
2.8611\end{array}$ & $\begin{array}{l}2.6800 \\
2.7938\end{array}$ & $\begin{array}{l}2.6344 \\
2.7449\end{array}$ & 2.5626 & 2.4410 & 2. 1923 & $\begin{array}{l}1.9109 \\
2.0170\end{array}$ \\
\hline $\begin{array}{l}110 \\
115\end{array}$ & $\begin{array}{l}2.9535 \\
3.0728\end{array}$ & $\begin{array}{l}2.9501 \\
3.0689\end{array}$ & $\begin{array}{l}2.9346 \\
3.0518\end{array}$ & $\begin{array}{l}2.8935 \\
3.0065\end{array}$ & $\begin{array}{l}2.8611 \\
2.9712\end{array}$ & $\begin{array}{l}2.7938 \\
2.8990\end{array}$ & $\begin{array}{l}2.7449 \\
2.8474\end{array}$ & $\begin{array}{l}2.6598 \\
2.7594\end{array}$ & $\begin{array}{l}2.5445 \\
2.6422\end{array}$ & $\begin{array}{l}2.2948 \\
2.3967\end{array}$ & $\begin{array}{l}2.0170 \\
2.1273\end{array}$ \\
\hline 120 & 3.1896 & 3.1852 & 3. 1661 & 3.1158 & 3.0772 & 2. 9995 & 2.9451 & 2.8643 & 2.7359 & 2.4963 & 2.2376 \\
\hline 125 & 3.2963 & 3.2914 & 3.2698 & 3.2141 & 3.1719 & 3.0890 & 3.0322 & 2.9498 & 2.8229 & 2.5948 & 2.3511 \\
\hline 130 & 3.3998 & 3.3942 & 3. 3695 & 3.3069 & 3. 2606 & 3.1718 & 3.1126 & 3.0291 & 2.9049 & 2.6899 & 2. $6 \in 28$ \\
\hline 135 & 3.4913 & 3.4848 & 3.4563 & 3.3858 & 3.3350 & 3.2408 & 3.1803 & 3.0976 & 2.9794 & 2.7831 & 2.5753 \\
\hline 140 & 3.5791 & 3.5713 & 3.5377 & 3.4567 & 3.4006 & 3.3008 & 3.2394 & 3.1584 & 3.0479 & $2,8>13$ & 2.6833 \\
\hline 145 & 3.6532 & 3.6437 & 3.6032 & 3.5098 & 3.4481 & 3.3443 & 3.2838 & 3.2075 & 3.1085 & 2.9557 & 2.7883 \\
\hline 150 & 3.7228 & 3.7105 & 3.6598 & 3.5097 & 3.4819 & 3.3755 & 3.3175 & 3.2479 & 3.1622 & 3.0330 & 2.8851 \\
\hline 155 & 3.7788 & 3.7622 & 3.6963 & 3.5659 & 3.4931 & 3. 3888 & 3.3364 & 3.2771 & 3.2077 & 3. 1034 & 2.9737 \\
\hline 160 & 3.8259 & 3.8016 & 3.7112 & 3.5567 & 3.4822 & 3.3871 & 3.3437 & 3.2973 & 3.2455 & 3.1639 & 3.0499 \\
\hline 165 & 3.8695 & 3.8289 & 3.6957 & 3.5183 & 3.4493 & 3.3730 & 3.3417 & 3.3100 & 3.2752 & 3.2135 & 3. 1123 \\
\hline 170 & 3.8658 & 3.7879 & 3.5980 & 3.4365 & 3. 3905 & $3.34>0$ & 3.3309 & 3. 3150 & 3.2960 & 3.2506 & 3.1589 \\
\hline 175 & 877 & 3.7920 & 3.4869 & 3.3737 & 3.3515 & 3.3332 & 3.3268 & 3.3201 & 3.3096 & 3.2725 & 3.1866 \\
\hline 180 & 199 & 3.3200 & 3.3202 & 3.3208 & 3.3211 & 3.3214 & 3.3211 & 3.3197 & 3.3136 & 3. 2793 & 3.1950 \\
\hline
\end{tabular}

RATIO OF MOTT TO MCKINIEY-FESHBACH SCATTERING IN $0, z=92$

\begin{tabular}{|c|c|c|c|c|c|c|c|c|c|c|c|}
\hline $\begin{array}{l}\text { ENERGY (HEV) } \\
\text { ANGLE (DEG.) }\end{array}$ & 100 & 10. & 4. & 2. & 1.5 & 1.0 & 0.8 & 0.6 & 0.4 & 0.2 & 0.1 \\
\hline $\begin{array}{l}0 \\
5\end{array}$ & $\begin{array}{l}1.0000 \\
0.9426\end{array}$ & $\begin{array}{l}1.0000 \\
0.9427\end{array}$ & $\begin{array}{l}1.0000 \\
0.9427\end{array}$ & $\begin{array}{l}1.0000 \\
0.9429\end{array}$ & $\begin{array}{l}1.0000 \\
0.9431\end{array}$ & $\begin{array}{l}1.0000 \\
0.9436\end{array}$ & $\begin{array}{l}1.0000 \\
0.9440\end{array}$ & $\begin{array}{l}9.0000 \\
0.9447\end{array}$ & $\begin{array}{l}1.0000 \\
0.9464\end{array}$ & $\begin{array}{l}1.0000 \\
0.9504\end{array}$ & $\begin{array}{l}1.0000 \\
0.9522\end{array}$ \\
\hline 10 & 0.9177 & 0.9177 & 0.9175 & 0.9173 & 0.9171 & 0.9170 & 0.9170 & 0.9174 & $\begin{array}{l}0.9464 \\
0.9192\end{array}$ & $\begin{array}{l}0.9504 \\
0.9288\end{array}$ & $\begin{array}{l}0.9522 \\
0.9447\end{array}$ \\
\hline 15 & 0.8958 & 0.8957 & 0.8951 & 0.8937 & 0.8926 & 0.8903 & 0.8887 & 0.8866 & 0.8845 & 0.8896 & 0.9146 \\
\hline 20 & 0.9046 & 0.9043 & 0.9032 & 0.9001 & 0.8976 & 0.8923 & 0.8883 & 0.8823 & 0.8730 & 0.8633 & 0.8801 \\
\hline 25 & 0.9353 & 0.9349 & 0.9331 & 0.9283 & 0.9244 & 0.9159 & 0.9095 & 0.8993 & 0.8821 & 0.8549 & 0.8565 \\
\hline 30 & 0.9879 & 0.9874 & 0.9849 & 0.9782 & $0.9^{7} 27$ & 0.9606 & 0.9514 & 0.9364 & 0.9102 & 0.8609 & $0.837 E$ \\
\hline 35 & 1.0620 & 1.0613 & 1.0582 & 1.0496 & 1.0425 & 1.0270 & 1.0150 & 0.9955 & 0.9604 & 0.8890 & $0.836^{-}$ \\
\hline 40 & 1.1527 & 1.1519 & 1.1481 & 1.1375 & 1.1288 & 1. 1097 & 1.0949 & 1.0706 & 1.0263 & 0.9321 & 0.8496 \\
\hline 45 & 1.2610 & 1.2600 & 1.2555 & 1.2430 & $1.232^{7}$ & 1.2100 & 1.1924 & 1.1634 & 1.1109 & 0.9932 & $0.8^{7} 86$ \\
\hline 50 & 1.3821 & 1.3809 & 1.3757 & 1.3611 & 1.3492 & 1.3228 & 1.3023 & 1.2685 & 1.2062 & 1.0673 & 0.9225 \\
\hline 55 & 1.5163 & 1.5150 & 1.5090 & 1.4924 & 1.4787 & 1.4486 & 1.4251 & 1.3865 & 1.3152 & 1.1544 & 0.9790 \\
\hline 60 & 1.6602 & 1.6587 & 1.6519 & 1.6331 & 1.6176 & 1.5836 & 1.5571 & 1.5136 & 1.4332 & 1.2513 & 1.0477 \\
\hline 65 & 1.8131 & 1.8114 & 1.8037 & 1.7826 & 1.7653 & 1.7271 & 1.6975 & 1.6490 & 1.5595 & 1.3569 & 1.1258 \\
\hline 70 & 1.9728 & 1.9709 & 1.9623 & 1.9388 & 1.9194 & 1.8770 & 1.8442 & 1.7905 & 1.6919 & 1.4693 & 1. 2127 \\
\hline 75 & 2.1376 & 2.1354 & 2.1259 & 2.0997 & 2.0783 & 2.0314 & 1.9952 & 1.9363 & $1.828^{7}$ & 1.5871 & 1.3970 \\
\hline 80 & 2.3067 & 2.3043 & 2.2938 & 2.2647 & 2.2410 & 2.1894 & 2.1498 & 2.0854 & $1.968^{7}$ & 1.7089 & 1.0075 \\
\hline 85 & 2.4771 & 2.4745 & 2.4628 & 2.4308 & 2.4047 & 2. 3482 & 2.3050 & 2.2353 & 2.1099 & 1.8339 & 1.5144 \\
\hline 90 & 2.6497 & 2.6468 & 2.6338 & 2.5985 & 2.5699 & 2.5081 & 2.4612 & 2.3860 & 2.2519 & 1.9606 & 1.6256 \\
\hline 95 & 2.8200 & 2.8168 & 2.8025 & 2.7637 & 2.7324 & 2.6653 & 2.6147 & 2.5342 & 2.3923 & 2.0893 & 1.7432 \\
\hline 100 & 2.9904 & 2.9868 & 2.9711 & 2.9283 & 2.8941 & 2. 8212 & 2.7667 & 2.6808 & 2.5314 & 2.2179 & 1.8640 \\
\hline 105 & 3.1552 & 3.1513 & 3.1339 & 3.0870 & 3.0497 & 2.9710 & 2.9129 & 2.8221 & 2.6667 & 2.3477 & 1.9910 \\
\hline 110 & 3.3182 & 3.3139 & 3.2946 & 3.2430 & 3.2022 & 3.1172 & 3.0552 & 2.9594 & 2.7985 & 2.4760 & 2. $120^{7}$ \\
\hline 115 & 3.4726 & 3.4677 & 3.4464 & 3.3897 & 3.3453 & 3.2542 & 3.1886 & 3.0889 & 2.9249 & 2.6051 & 2.2561 \\
\hline 120 & 3.6235 & 3.6181 & 3.5942 & 3.5315 & 3.4830 & 3. 3850 & 3. 3158 & 3.2123 & 3.0461 & 2.7314 & 2. 393 n \\
\hline 125 & 3.7629 & 3.7568 & 3.7301 & 3.6607 & 3.6080 & 3.5034 & 3. 4312 & 3.3255 & 3.1605 & 2.8578 & 2.5342 \\
\hline 130 & 3.8975 & 3.8906 & 3.8602 & 3.7825 & 3.7247 & 3.6130 & 3.5378 & 3.4305 & 3.2680 & 2.9799 & 2.6748 \\
\hline 135 & 4.0181 & 4.0100 & 3.9752 & 3.8881 & 3.8250 & 3.7068 & 3.6299 & 3.5233 & 3.3678 & 3. 1009 & 2. B166 \\
\hline 140 & 4.1326 & 4. 1231 & 4.0822 & 3.9829 & 3.9134 & 3.7884 & 3.7103 & 3.6058 & 3.4592 & 3.2956 & 2.9543 \\
\hline 145 & 4.2311 & 4.2195 & 4.1706 & 4.0567 & 3.9807 & 3.8509 & 3.7739 & 3.6748 & 3.5420 & 3.3264 & 3.0880 \\
\hline 150 & 4.3220 & 4.3074 & 4.2466 & 4.1131 & 4.0299 & 3.8970 & 3.8230 & 3.7320 & 3.6152 & 3.4279 & 3.2125 \\
\hline 155 & 4.3967 & 4.3770 & 4.2985 & 4.1412 & 4.0521 & 3.9217 & 3.8544 & 3.7758 & 3.6788 & 3.5211 & 3. 3263 \\
\hline 160 & 4.4592 & 4.4306 & 4.3236 & 4.1379 & 4.0065 & 3.9270 & 3.8706 & 3.8077 & 3.7317 & 3.5013 & 3. 4250 \\
\hline 165 & 4.5147 & 4.4673 & 4.3106 & 4.0977 & 4.0128 & 3.9159 & 3.8740 & 3.8290 & 3.7738 & 3.6673 & $3.505^{\circ}$ \\
\hline 170 & 4.5209 & 4.4297 & 4.2038 & 4.0066 & 3.9485 & 3.8904 & 3. 8668 & 3.8406 & 3.8044 & 3.7170 & 3. 5661 \\
\hline $\begin{array}{l}175 \\
180\end{array}$ & 4.7389 & 4.4095 & 4.0615 & 3.9281 & 3.9003 & $3.87 \cup 2$ & 3.8628 & 3.8482 & 3.8228 & 3.7460 & 3.6523 \\
\hline 180 & 3.8712 & 3.8710 & 3.8704 & 3.8684 & 3.8666 & 3.8620 & 3.8576 & 3.8491 & 3.8286 & 3.7548 & 3.6146 \\
\hline
\end{tabular}


RETIC OF MOTT TO MCKINIFY-FESHBACR SCATTERING IN PD, $z=94$

\begin{tabular}{|c|c|c|c|c|c|c|c|c|}
\hline \multirow{2}{*}{$\begin{array}{c}\text { ENERGY (MEV) } \\
\text { ANGLE (DEG.) } \\
0 \\
5\end{array}$} & 100 & 10. & 4. & 2. & 1.5 & 1.0 & 0.8 & 0.6 \\
\hline & $\begin{array}{l}1.0000 \\
0.9407\end{array}$ & $\begin{array}{l}1.0000 \\
0.9008\end{array}$ & $\begin{array}{l}1.0000 \\
0.9408\end{array}$ & $\begin{array}{l}1.0000 \\
0.9411\end{array}$ & $\begin{array}{l}1.0000 \\
0.9413\end{array}$ & 1.0000 & 1.0000 & 1.0000 \\
\hline $\begin{array}{r}5 \\
10\end{array}$ & $\begin{array}{l}0.9407 \\
0.9135\end{array}$ & 0.9135 & $\begin{array}{l}0.9408 \\
0.9134\end{array}$ & $\begin{array}{l}0.9471 \\
0.9133\end{array}$ & $\begin{array}{l}0.9413 \\
0.9133\end{array}$ & $\begin{array}{l}0.9419 \\
0.9134\end{array}$ & $\begin{array}{l}0.9423 \\
0.9137\end{array}$ & $\begin{array}{l}0.9432 \\
0.9145\end{array}$ \\
\hline 15 & 0.8875 & 0.8874 & 0.8869 & 0.8856 & 0.8847 & 0.8828 & 0.8815 & 0.8800 \\
\hline 20 & 0.8923 & 0.8921 & 0.8910 & 0.8880 & 0.8856 & 0.8806 & 0.8769 & 0.8714 \\
\hline 25 & 0.9202 & 0.9198 & 0.9180 & 0.9132 & 0.9093 & 0.9010 & 0.8947 & 0.8848 \\
\hline 30 & 0.9711 & 0.9706 & 0.9681 & 0.9612 & 0.9556 & 0.9434 & 0.9341 & 0.9191 \\
\hline 35 & 1.0455 & 1.0447 & 1.0415 & 1.0326 & 1.0253 & 1.0093 & 0.9971 & 0.9771 \\
\hline 40 & 1.1381 & 1.1372 & 1.1332 & 1.1222 & 1.1131 & 1.0931 & 1.0777 & 1.0525 \\
\hline 45 & 1.2503 & 1.2492 & 1.2445 & 1.2313 & 1.2204 & 1.1965 & 1.1779 & 1.1475 \\
\hline 50 & 1.3770 & 1.3758 & 1.3702 & 1.3547 & 1.3420 & 1.3140 & 1.2923 & 1. 2565 \\
\hline 55 & 1.5188 & 1.5173 & 1.5909 & 1.4931 & 1.4785 & 1.4463 & 1.4213 & 1.3801 \\
\hline 60 & 1.6718 & 1.6702 & 1. 5628 & 1.6426 & 1.6260 & 1.5894 & 1.5610 & 1.5144 \\
\hline 65 & 1.8354 & 1.8335 & 1.8253 & 1.8025 & 1.7837 & 1.7426 & 1.7107 & 1.6584 \\
\hline 70 & 2.0072 & 2.0052 & 1.9959 & 1.9703 & 1.9494 & 1.9034 & 1.8679 & 1.8098 \\
\hline 75 & 2.1853 & 2.1830 & 2.1727 & 2.1442 & 2.1209 & 2.0699 & 2.0307 & 1.9667 \\
\hline 80 & 2.3689 & 2.3663 & 2.3548 & 2.3232 & 2.2974 & 2.2411 & 2.1980 & 2.1279 \\
\hline 85 & 2.5546 & 2.5518 & 2.5390 & 2.5040 & 2.0756 & 2.4139 & 2.3667 & 2.2906 \\
\hline 90 & 2.7432 & 2.7401 & 2.7259 & 2.6873 & 2. $656 \mathrm{C}$ & 2.5884 & 2.5371 & 2.4548 \\
\hline 95 & 2.9301 & 2.9266 & 2.9110 & 2.8685 & 2.8342 & 2.7606 & 2.7052 & 2.6169 \\
\hline 100 & 3.1173 & 3.1134 & 3.0962 & 3.0494 & 3.0118 & 2.9318 & 2.8721 & 2.7776 \\
\hline 105 & 3.2991 & 3.2949 & 3. 2758 & 3.2245 & 3.1835 & 3.0971 & 3.0333 & 2.9333 \\
\hline 110 & 3.4790 & $3.4>43$ & 3.4532 & 3.3966 & 3.3519 & 3.2585 & 3.1903 & 3.0848 \\
\hline 115 & 3.6502 & 3.6449 & 3.6215 & 3.5594 & 3.5108 & 3.4106 & 3.3384 & 3.2284 \\
\hline 120 & 3.8173 & 3.8114 & 3.7853 & 3.7166 & 3.6635 & 3.5558 & 3.4795 & 3.3652 \\
\hline 125 & 3.9725 & 3.9659 & 3.9367 & 3.8608 & 3.8030 & 3.6881 & 3.6085 & 3.4916 \\
\hline 130 & 4.1219 & 4. 1144 & 4.0812 & 3.9964 & 3.9332 & 3.8104 & 3.7275 & 3.6087 \\
\hline 135 & 4.2566 & 4.2479 & 4.2100 & 4.1151 & 4.0460 & 3., 163 & 3.8314 & 3.7132 \\
\hline 140 & 4.3840 & 4.3736 & 4. 3292 & 4.2212 & 4.1454 & 4.0082 & 3. 9221 & 3.8060 \\
\hline 145 & 4.4943 & 4.4818 & 4.0289 & 4.3052 & 4.2223 & 4.0800 & 3.9049 & 3.8848 \\
\hline 150 & 4.5956 & 4.5798 & 4.5142 & 4.3697 & 4. 2790 & 4. 1334 & 4.0515 & 3.9500 \\
\hline 155 & 4.6793 & 4.6582 & 4.5738 & 4.4038 & 4.3067 & 4. 1638 & 4.0891 & 4.0009 \\
\hline 160 & 4.7494 & 4.7188 & 4.6040 & 4.4035 & 1.3040 & 4.1726 & a. 1096 & 4.0384 \\
\hline 165 & 4.8104 & 4.7599 & 4.5922 & 4.3625 & 4.2700 & $a .1630$ & a. 1159 & 0.0640 \\
\hline 170 & 4.8228 & 4.7252 & 4.4821 & 4.2678 & 4.2036 & a. 1381 & 4.1105 & 4.0789 \\
\hline 175 & 5.0301 & 4.6878 & 4. 3229 & 4.1810 & 4. 1505 & a. 1204 & 4.1064 & 4.0876 \\
\hline 180 & 4.1235 & 0.1233 & 4,1221 & 4.1187 & 4.1157 & H. 1082 & 4.1016 & 4.0892 \\
\hline
\end{tabular}

$\begin{array}{ccc}0.4 & 0.2 & 0.1 \\ 1.9000 & 1.0000 & 1.0000 \\ 0.9450 & 0.9488 & 0.9500 \\ 0.9171 & 0.9282 & 0.9439 \\ 0.8790 & 0.8874 & 0.9151 \\ 0.8633 & 0.8575 & 0.8795 \\ 0.8685 & 0.8451 & 0.8535 \\ 0.8932 & 0.8467 & 0.8307 \\ 0.9416 & 0.8714 & 0.8254 \\ 1.0070 & 0.9120 & 0.8343 \\ 1.0920 & 0.9722 & 0.8599 \\ 1.1910 & 1.0468 & 0.9016 \\ 1.3045 & 1.1358 & 0.9569 \\ 1.4286 & 1.2363 & 1.0258 \\ 1.5624 & 1.3467 & 1.1053 \\ 1.7035 & 1.4651 & 1.1948 \\ 1.8501 & 1.5901 & 1.2929 \\ 2.0010 & 1.7200 & 1.3989 \\ 2.1539 & 1.8541 & 1.5109 \\ 2.3081 & 1.9005 & 1.6280 \\ 2.4614 & 2.1297 & 1.7541 \\ 2.6134 & 2.2692 & 1.8835 \\ 2.7621 & 2.4105 & 2.9200 \\ 2.9071 & 2.5506 & 2.1599 \\ 3.0469 & 2.6921 & 2.3064 \\ 3.1809 & 2.8306 & 2.4552 \\ 3.3082 & 2.9699 & 2.6988 \\ 3.4278 & 3.1045 & 2.7626 \\ 3.5395 & 3.2384 & 2.9176 \\ 3.5418 & 3.3653 & 3.0589 \\ 3.7352 & 3.4885 & 3.2157 \\ 3.8177 & 3.6013 & 3.3530 \\ 3.9899 & 3.7053 & 3.4785 \\ 3.9502 & 3.7948 & 3.5896 \\ 3.9982 & 3.8685 & 3.5768 \\ 4.0335 & 3.9242 & 3.7437 \\ 4.0541 & 3.9565 & 3.7839 \\ 4.0607 & 3.9664 & 3.7981\end{array}$

RATIC OF MOTT TO MCKINLEY-FESHBACH SCAMTEPING IN ES, $z=99$

$\begin{array}{cc}\text { ENERGY (MEV) } & 100 \\ \text { ANGLE (DEG.) } & \\ 0 & 1.0000 \\ 5 & 0.9364 \\ 10 & 0.9044 \\ 15 & 0.8675 \\ 20 & 0.8607 \\ 25 & 0.8788 \\ 30 & 0.9227 \\ 35 & 0.9949 \\ 40 & 1.0899 \\ 45 & 1.2101 \\ 50 & 1.3500 \\ 55 & 1.5104 \\ 60 & 1.6871 \\ 65 & 1.8793 \\ 70 & 2.0842 \\ 75 & 2.2993 \\ 80 & 2.5235 \\ 85 & 2.7529 \\ 90 & 2.9876 \\ 95 & 3.2225 \\ 100 & 3.4590 \\ 105 & 3.6911 \\ 110 & 3.9211 \\ 115 & 4.1423 \\ 120 & 4.3578 \\ 125 & 4.5606 \\ 130 & 4.7547 \\ 135 & 4.9324 \\ 140 & 5.0984 \\ 145 & 5.2453 \\ 150 & 5.3778 \\ 155 & 5.4896 \\ 160 & 5.5827 \\ 165 & 5.6599 \\ 170 & 5.6945 \\ 175 & 5.8452 \\ 180 & 4.8455 \\ & \\ 115 & \\ 115 & \end{array}$

$\begin{array}{cc}10 . & 4 \\ 1.0000 & 1.0000 \\ 0.9365 & 0.9366 \\ 0.9044 & 0.9045 \\ 0.8674 & 0.8672 \\ 0.8605 & 0.8595 \\ 0.8785 & 0.8768 \\ 0.9222 & 0.9196 \\ 0.9942 & 0.9907 \\ 1.0889 & 1.0845 \\ 1.2089 & 1.2035 \\ 1.3486 & 1.3421 \\ 1.5087 & 1.5012 \\ 1.6852 & 1.6765 \\ 1.8771 & 1.8671 \\ 2.0816 & 2.0704 \\ 2.2965 & 2.2838 \\ 2.5203 & 2.5061 \\ 2.7494 & 2.7335 \\ 2.9836 & 2.9660 \\ 3.2182 & 3.1986 \\ 3.4542 & 3.4325 \\ 3.6858 . & 3.6618 \\ 3.9151 & 3.8886 \\ 4.1356 & 4.1063 \\ 4.3504 & 4.3177 \\ 4.5523 & 4.5158 \\ 4.7452 & 4.7039 \\ 4.9215 & 4.8744 \\ 5.0857 & 5.0309 \\ 5.2300 & 5.1650 \\ 5.3585 & 5.2786 \\ 5.4641 & 5.3620 \\ 5.5461 & 5.4080 \\ 5.6001 & 5.3999 \\ 5.5778 & 5.2829 \\ 5.4727 & 5.0660 \\ 4.8449 & 4.8419\end{array}$

2.
1.0000
0.9370
0.9049
0.8665
0.8571
0.8723
0.9127
0.9813
1.0724
1.1887
1.3244
1.4804
1.6525
1.8397
2.0393
2.2489
2.4671
2.6901
2.9178
3.1454
3.3737
3.5972
3.8173
4.0280
4.2312
4.4204
4.5976
4.7558
4.8966
5.0118
5.1007
5.1535
5.1629
5.1200
5.0156
4.9015
4.8334

\begin{tabular}{cc}
1.5 & 1.0 \\
1.0000 & 1.0000 \\
0.9373 & 0.9380 \\
0.9052 & 0.9062 \\
0.8661 & 0.8654 \\
0.8552 & 0.8513 \\
0.8687 & 0.8611 \\
0.9071 & 0.8950 \\
0.9736 & 0.9570 \\
1.0625 & 1.0409 \\
1.1765 & 1.1498 \\
1.3098 & 1.2778 \\
1.4633 & 1.4258 \\
1.6328 & 1.5895 \\
1.8172 & 1.7679 \\
2.0139 & 1.9583 \\
2.2204 & 2.1581 \\
2.4353 & 2.3660 \\
2.6548 & 2.5783 \\
2.8788 & 2.7945 \\
3.1025 & 3.0103 \\
3.3265 & 3.2259 \\
3.5456 & 3.4366 \\
3.7609 & 3.6427 \\
3.9666 & 3.8395 \\
4.1641 & 4.0273 \\
4.3474 & 4.2015 \\
4.5179 & 4.3619 \\
4.6690 & 4.5042 \\
4.8014 & 4.6274 \\
4.9081 & 4.7275 \\
4.9877 & 4.8029 \\
5.0326 & 4.8508 \\
5.0388 & 4.8707 \\
5.0043 & 4.8658 \\
4.9326 & 4.8433 \\
4.8631 & 4.8204 \\
4.8260 & 4.8087 \\
& \\
\hline .8969 &
\end{tabular}

0.8
1.0000
0.9386
0.9079
0.8651
0.8487
0.8556
0.8860
0.9443
1.0244
1.1292
1.2531
1.3968
1.5561
1.7299
1.9154
2.1103
2.3129
2.5199
2.7305
2.9408
3.1506
3.3557
3.5560
3.7476
3.9299
4.0996
4.2557
4.3953
4.5167
4.6178
4.6970
4.7535
4.7874
4.8019
4.8027
4.7976
4.7943

0.6
1.0000
0.9396
0.9090
0.8654
0.8450
0.8472
0.8718
0.9200
0.9976
1.0958
1.2129
1.3495
1.5015
1.6578
1.8456
2.0325
2.2269
2.4257
2.6278
2.8301
3.0314
3.2290
3.4216
3.6069
3.9832
3.9490
4.1021
4.2418
4.3654
4.4732
4.5627
4.6352
4.6895
4.7279
4.7530
4.7646
4.7684

0.4

1.0000 0.9414 0.9135 0.8408 0.8408 0.8346 0.8482 0.889 0.9503 1.0359 1.1401
1.2634 1. 4021 1.4021
1.5547 1.5547
1.7186 1.7186
1.8916 2.0719 2.2571 2.4455
2.6351 2. 6351 2.8241 3.0115 3.1945 3.3735
3.5409 3.5409 3.7103
3.8652 3.8652
4.0124 4.1469 4.2717 4.2717
4.3819 4.4799 4.5619 4.6276 4.6771 4.7041
4.7135

\begin{tabular}{|c|c|}
\hline 0.2 & 0.1 \\
\hline $\begin{array}{l}1.0000 \\
0.9445\end{array}$ & $\begin{array}{l}1.0000 \\
0.0442\end{array}$ \\
\hline $\begin{array}{l}0.9275 \\
0.8845\end{array}$ & $\begin{array}{l}0.9409 \\
0.9170\end{array}$ \\
\hline 0.8463 & 0.8809 \\
\hline $\begin{array}{l}0.8234 \\
0.8125 \\
0.8251\end{array}$ & $\begin{array}{l}0.8507 \\
0.8186 \\
0.9913\end{array}$ \\
\hline $\begin{array}{l}0.8261 \\
0.8581\end{array}$ & $\begin{array}{l}0.8213 \\
0.7985\end{array}$ \\
\hline 0.9128 & 0.8130 \\
\hline $\begin{array}{l}0.9862 \\
1.0779\end{array}$ & 0.8465 \\
\hline $\begin{array}{l}1.0779 \\
1.1854\end{array}$ & 0.9631 \\
\hline $\begin{array}{l}1.3066 \\
1.4398\end{array}$ & $\begin{array}{l}1.0439 \\
1.1381\end{array}$ \\
\hline 1.5829 & 1.2043 \\
\hline 1.7339 & 1. 3606 \\
\hline $\begin{array}{l}1.8929 \\
2.0547\end{array}$ & $\begin{array}{l}1.4875 \\
1.5225\end{array}$ \\
\hline 2.2228 & $1 .-6>0$ \\
\hline 2.3923 & 1.9194 \\
\hline 2.5662 & 2.0808 \\
\hline 2.7391 & 2.2488 \\
\hline 2.9157 & 2.4252 \\
\hline 3.0892 & 2.6071 \\
\hline 3.2652 & 2.7050 \\
\hline 3.4359 & 2.9857 \\
\hline 3.6070 & 3.1780 \\
\hline 3.7698 & 3.3679 \\
\hline 3.9287 & 3.5521 \\
\hline 4.0748 & 3.7262 \\
\hline 4.2100 & 3.9851 \\
\hline 4.3269 & \\
\hline 4.4233 & 4.1383 \\
\hline 4.4966 & 4. 2238 \\
\hline 4.5388 & 4. 2758 \\
\hline 4.5524 & 4.2958 \\
\hline
\end{tabular}


DIFFERENTIAL BUTHERPORD SCATTERING WITHOOT Z SQUARED FACTOR (BARNS PER STERADIAN)

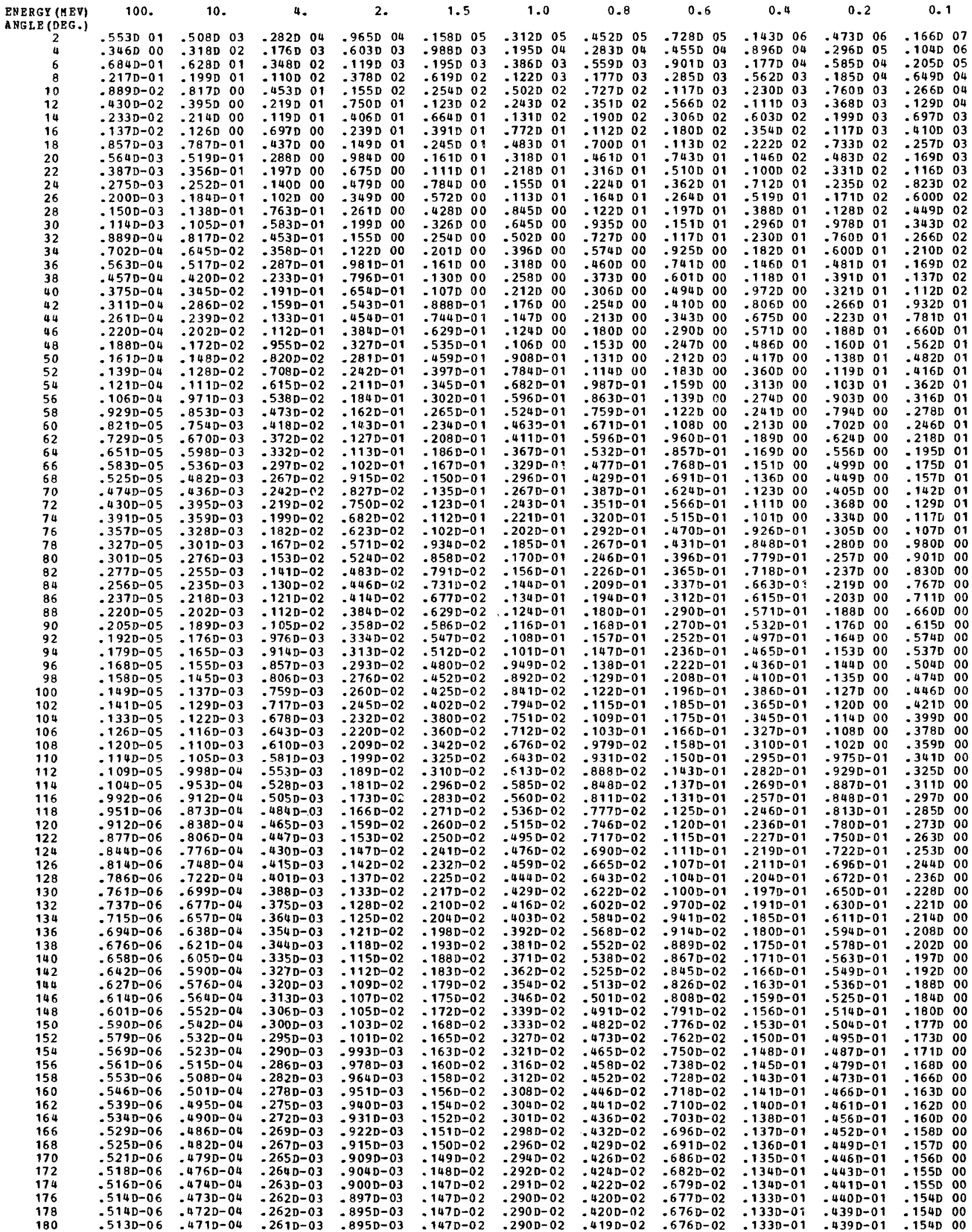




$$
\begin{gathered}
\text { ORNL }-4897 \\
\text { UC- } 34 \text { - Physics }
\end{gathered}
$$

INTERNAL DISTRIBUTION

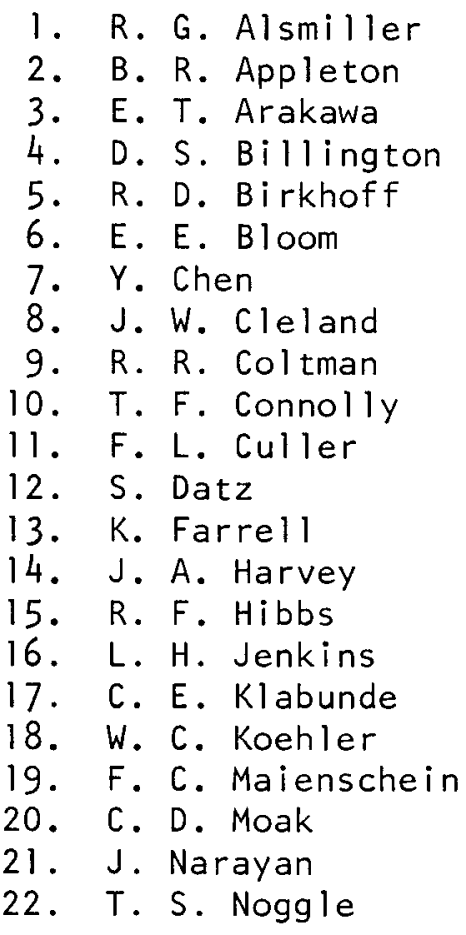

\author{
23-52. 0. S. Oen \\ 53. S. M. Ohr \\ 54. J. C. Pigg \\ 55. R. H. Ritchie \\ 56. M. T. Robinson \\ 57. 0. E. Schow \\ 58. M. J. Skinner \\ 59. E. Sonder \\ 60. J. 0. Stiegler \\ 61. J.E. Turner \\ 62. A. M. Weinberg \\ 63. F. W. Wiffen \\ 64. M. K. Wilkinson \\ 65. J. M. Williams \\ 66: R. F. Wood \\ 67. Man H. Yoo \\ 68. F. W. Young \\ 69. A. Zucker \\ 70-72. Central Research Library \\ 73. ORNL-Y-12 Technical Library, \\ Document Reference Section \\ 74-123. Laboratory Records \\ 124. Laboratory Records, ORNL, RC
}

\title{
EXTERNAL DISTRIBUTION
}

125. S. Amelinckx, Solid State Physics Department, C.E.N., Mol-Donk, Belgium

126. H. H. Andersen, Danish Atomic Energy Commission Research Establishment Risö, DK-4000, Roskilde, Denmark

127. G. W. Arnold, Sandia Corporation, Albuquerque, New Mexico 87115

128. R. W. Balluffi, Materials Science and Engineering, 228 Bard Hall, Cornell University, I thaca, New York 14850

129. P. C. Banbury, University of Reading, 'Reading, England

130. P. Baruch, Laboratorie de Physique, Ecole Normale Superieure, Paris V, France

131. R. Bauerlein, Forschungslaboratorium, Siemens-Schuckertwerke $A G$, Erlangen, W. Germany

132. J. R. Beeler, Nuclear Engineering Department, North Carolina State University, P. 0. Box 5636, State College Station, Raleigh, North Carolina 27607

133. R. Behrisch, Obteilung Oberfilachenphysik, Max-Planck-Institut für Plasmaphysik, D-8046 Garching bei München, Germany

134. T. H. Blewitt, Argonne National Laboratory, Argonne, 111 inois 60439

135. F. Brown, Solid State Sciences Branch, Chemical and Materials Division, Chalk River NUCLEAR Laboratory, Chalk River, Ontario, Canada 
136. W. L. Brown, Bell Telephone Laboratories, Murray Hill, New Jersey 07974

137. R. L. Chaplin, Department of Physics, Clemson College, Clemson, South Carolina 29631

138. W. D. Compton, Director, Scientific Research Staff, 20000 Rotunda Drive, Dearborn, Michigan 48121

139. J. W. Corbett, Physics Department, New York State University, Albany, New York 12203

140. A. Cordier, Laboratoire D'Optique Electronique Du Centre National de la Recherche Scientifique, Toulouse, France

141. A. F. J. Cox, Physics Department, The University, Hull, East Yorkshire, England

142. James H. Crawford, Jr., Chairman, Department of Physics, University of North Carolina, Chapel Hill, North Carolina 27514

143. 0. L. Curtis, Jr., Northrop-Ventura, Newbury Park, California 91320

144. D. Dautreppe, Head, Solid State Physics, Centre D'Etude Nucleaires de Grenoble, P. 0. Box 269, Grenoble, France

145. J. Diehl, Max Planck Institut für Metallforschung, Seestrasse 75, Stuttgart, Germany

146. D. G. Doran, Battelle Memorial Institute, Pacific Northwest Laboratories, P. 0. Box 999, Richland, Washington 99352

147. H. Ehrenreich, Department of Engineering and Applied Physics, Harvard University, Cambridge, Massachusetts 02138

148. B. D. Evans, Naval Research Laboratory (Code 6440), Washington, D. C. 20390

149. M. J. Fluss, Argonne National Laboratory, Argonne, 111 inois 60439

150. Konrad Gartner, Technisch-Physikalisches Institut der FriedrichSchiller-Universitat, DDR 69 Jena, Helmholtzweg 3, Germany

151. A. N. Goland, Brookhaven National Laboratory, Upton, New York 11973

152. C. Gomez, Max-Planck-Institut für Metallforschung, 7000 Stuttgart I, Seestrasse 92, Germany

153. Walter Green, Los Alamos Scientific Laboratory, P. 0. Box 1663, Los Alamos, New Mexico 97544

154. Mike Guinan, L-503, Lawrence Livermore Laboratory, Livermore, California 94550

155. R. Hanada, The Research Institute for Iron, Steel and Other Metals, Tohoku University, Sendai, Japan

156. P. L. F. Hemment, United Kingdom Atomic Energy Authority, Aldermaston, Berkshire, England

157. Peter Jung, Institut für Festkorperforschung, Kernforschungsanlage Jülich, D-517 Jülich, Postfach 365, Germany

158. Edward A. Kenik, Case-Western Reserve University, Division of Metallurgy and Material Science, University Circle, Cleveland, Ohio 44106

159. J. S. Koehler, Physics Department, University of 111 inois, Urbana, llinois 60801

160. C. Lehmann, Institut für Festkorperforschung, Kernforschungsanlage Jülich, D-517 Jülich, Postfach 365, Germany

161. G. Leibfried, Institut für Festkorperforschung, Kernforschungsanlage Jülich, D-517 Jülich, Postfach 365, Germany

162. J. Lindhard, Institute of Physics, University of Aarhus, Aarhus, Denmark 
163. J. Lomer, University of Reading, Reading, England

164. P. Lucasson, Laboratoire de Chimie Physique, Centre D'Orsay, Orsay, France

165. J. W. MacKay, Purdue University, Lafayette, Indiana 46207

166. M. J. Makin, Atomic Energy Research Establishment, Harwell, Didcot, Berks., England

167. A. Merlini, Solid State Physics Department, C.C.R.-EURATOM, Ispra (Varese), Italy

168. E. W. J. Mitchell, University of Reading, Reading, England

169. Terry E. Mitchel1, Case Western Reserve University, University Circle, Cleveland, Ohio 44106

170. V. A. Molchanov, Scientific Institute of Nuclear Physics, Moscow State University, Moscow B-234, U.S.S.R.

171. G. L. Montet, Building 200, Al57, Argonne National Laboratory, Argonne, 111 inois 60439

172. R. S. Nelson, Atomic Energy Research Establishment, Harwell, Didcot, Berks., England

173. D. I. R. Norris, Berkeley Nuclear Laboratories, Berkeley, Gloucestershire, United Kingdom

174. B. Perovic, Institute "Boris Kidrich," P. 0. Box 522, Belgrade, Yugoslavia

175. M. 0. Ruault, Service de Physique des Solides, Faculte des Science D'Orsay, 91-Orsay, France

176. W. F. Schilling, Institut für Festkörperforschung, Kernforschungsanlage Jülich, D-517 Jülich, Postfach 365, Germany

177. C. G. Shul1, Department of Physics, Massachusetts Institute of Technology, Cambridge, Massachusetts 02139

178. W. A. Sibley, Department of Physics, Oklahoma State University, Stillwater, Oklahoma 74074

179. P. Sigmund, H. C. Ørsted Institute, DK-2100 Copenhagen, Denmark

180. R. Sizmann, Sektion Physik, Universität München, 8 München 13, Amalienstrasse 54/111, Germany

181. A. Sosin, University of Utah, Salt Lake City, Utah 84102

182. J. T. Stanley, Arizona State University, Tempe, Arizona 85281

183. P. R. C. Stevens, United Kingdom Atomic Energy Authority, Aldermaston, Berkshire, England

184. M. L. Swanson, Atomic Energy of Canada Ltd., Chalk River, Ontario, Canada

185. J. A. Swartout, Union Carbide Corporation, New York, N. Y. 10017

186. D. 0. Thompson, North American Aviation Science Center, Thousand Oaks, California 91360

187. M. W. Thompson, University of Sussex, Brighton, Sussex, England

188. M. S. Wechsler, Department of Metallurgy, lowa State University, Ames, lowa 50010

189. H. F. Wenzl, Institut für Festkörperforschung, Kernforschungsanlage, D-517 Jülich, Postfach 365, Germany

190. K. B. Winterbon, Chalk River Nuclear Laboratories, Chalk River, Ontario, Canada

191. Alan Wolfenden, Physics and Engineering Laboratory, Private Bag, Lower Hutt, New Zealand 
192. H. Wollenberger, Institute für Festkörperforschung, Kernforschungsanlage Jülich, D-517 Jülich, Post Fach 365, Germany

193. P. Vajda, Laboratoire de Chimie Physique, Centre D'Orsay, Orsay, France

194. F. L. Vook, Sandia Corporation, Albuquerque, N. M. 87115

195. G. D. Watkins, General Electric Company, Research and Development Center, P. 0. Box 8, Schenectady, New York 12301

196. V. E. Yurasova, Physical Department, Moscow State University, Moscow, U.S.S.R.

197. Research and Technical Support Division, A.E.C., ORO

198. Patent Office, A.E.C., ORO

199-338. Given distribution as shown in TID-4500 under Physics Category (25 copies - NTIS) 